\title{
Investigation of Fundamental Thermal- Hydraulic Phenomena in Advanced Gas-Cooled Reactors
}

Donald M. McEligot Glenn E. McCreery Richard R. Schultz Jeongik Lee Pavel Hejzlar Peter Stahle Pradip Saha

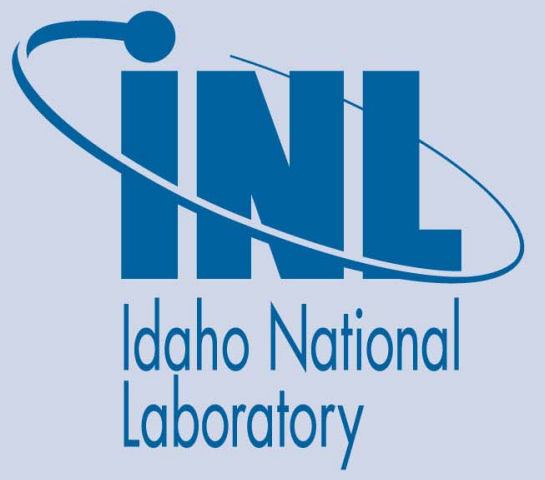

September 2006

The INL is a U.S. Department of Energy National Laboratory operated by Battelle Energy Alliance 
INL/EXT-06-11801

MIT-GFR-042

\title{
Investigation of Fundamental Thermal-Hydraulic Phenomena in Advanced Gas-Cooled Reactors
}

\author{
Donald M. McEligot ${ }^{\mathrm{a}}$ \\ Glenn E. McCreery ${ }^{a}$ \\ Richard R. Schultz \\ Jeongik Lee ${ }^{b}$ \\ Pavel Hejzlar ${ }^{b}$ \\ Peter Stahle \\ Pradip Sahab \\ ${ }^{a}$ INL
${ }^{b}$ MIT
}

September 2006

Idaho National Laboratory

Idaho Falls, Idaho 83415

Prepared for the

U.S. Department of Energy

Office of Nuclear Energy

Under DOE Idaho Operations Office

Contract DE-AC07-05ID14517 


\section{Abstract}

INL LDRD funded research was conducted at MIT in collaboration with INL personnel to experimentally characterize mixed convection heat transfer in gascooled fast reactor (GFR) core channels. The gas-cooled fast reactor (GFR) for Generation IV has generated considerable interest and is under development in the U.S., France, and Japan. One of the key candidates is a block-core configuration first proposed by MIT, has the potential to operate in Deteriorated Turbulent Heat Transfer (DTHT) regime or in the transition between the DTHT and normal forced or laminar convection regime during post-loss-of-coolant accident (LOCA) conditions. The DTHT regime is defined as a regime where the turbulent heat transfer deteriorates due to either a streamwise flow acceleration effect or a high buoyancy effect.

This is contrary to most industrial applications where operation is in a well-defined and well-known turbulent forced convection regime. As a result, important new need emerged to develop heat transfer correlations that make possible rigorous and accurate predictions of Decay Heat Removal (DHR) during post LOCA in these regimes.

Extensive literature review on these regimes was performed and a number of the available correlations was collected in: (1) forced laminar, (2) forced turbulent, (3) mixed convection laminar, (4) buoyancy driven DTHT and (5) acceleration driven DTHT regimes. Preliminary analysis on the GFR DHR system was performed and using the literature review results and GFR conditions. It confirmed that the GFR block type core has a potential to operate in the DTHT regime. Further, a newly proposed approach proved that gas, liquid and super critical fluids all behave differently in single channel under DTHT regime conditions, thus making it questionable to extrapolate liquid or supercritical fluid data to gas flow heat transfer.

Suggested upgrades for the experimental facility from the $2^{\text {nd }}$ annual report were successfully installed and the control and data reduction software for facility operation was developed and tested. An extensive development of programming and controlling techniques were introduced for the software development that allowed substantial speed up of data collection and analysis process.

Experimental data were collected for three different gases (nitrogen, helium and carbon dioxide) in various heat transfer regimes under a fully developed flow and reasonably uniform wall heat flux conditions. The following ranges of non-dimensional numbers, were covered in the experiment: (1) Reynolds number from 1,800 to 42,700 (2) Jackson' buoyancy parameter up to $10^{-5}$ (3) Acceleration parameter up to $5 \times 10^{-6}$ and (4) wall to bulk temperature ratio up to 1.88 . The data were obtained at various pressures up to $1 \mathrm{MPa}$.

Each gas unveiled different physical phenomena. All data basically covered the forced turbulent heat transfer regime, nitrogen data covered the acceleration driven DTHT and buoyancy driven DTHT, helium data covered the mixed convection laminar, acceleration 
driven DTHT and the laminar to turbulent transition regimes and carbon dioxide data covered the returbulizing buoyancy driven DTHT and non-returbulizing buoyancy induced DTHT. A newly observed phenomenon, namely "returbulization", occurs when the buoyancy driven DTHT regime recovers back to the normal forced convection heat transfer due to the strong dependency of gas properties on the temperature. The validity of the data was established using heat balance and the uncertainty analysis. Based on the experimental data, the traditional threshold for the DTHT regime was updated to account for phenomena observed in the facility and a new heat transfer regime map was proposed. Overall, substantial reduction of the heat transfer coefficient was observed in the DTHT regime, which will have significant impact on the core and DHR design of passive GFRs.

The MIT data were compared to a large number of existing correlations. None of the mixed convection laminar correlations agreed with the data. The forced turbulent and the DTHT regime, Celeta et al.'s correlation showed the best fit with the data. However, due to the larger $L / D$ ratio of MIT's facility compared to Celeta et al.'s facility and the returbuliziation due to the gas characteristics, the correlation sometimes under predicts the heat transfer coefficient. Also, since Celeta et al.'s correlation requires information about the wall temperature to evaluate the heat transfer coefficient, it is difficult to apply this correlation directly for predicting the wall temperature.

Three new sets of correlations that cover all heat transfer regimes were developed. The basic idea of the new correlation is to use the best available and most widely recommended Gnielinski correlation for forced turbulent convection and modify its empirically fitted constant (the Re- 1000 part) by an empirically fitted function that accounts for physical phenomena driving the DTHT regimes. Thus, the function is dependent on the acceleration parameter or the buoyancy parameter depending on the heat transfer regime. Type-1 correlation set is the most accurate fit suitable for scientific applications, Type- 2 simplifies the calculations by eliminating an iteration process on the wall temperature and Type- 3 is the simplest form suitable for industrial applications. The first two types show very good fit with the data, while the third type exhibits slightly less accuracy, which was traded off for simplicity. Three correlations cover most of the MIT experimental data within $\pm 20 \%$ range.

Finally, it is noted that more data on the laminar-turbulent transition are required to understand the flow phenomena more thoroughly. A number of research issues for future work are listed, based on the phenomena uncovered in this project. 


\section{Table of Contents}

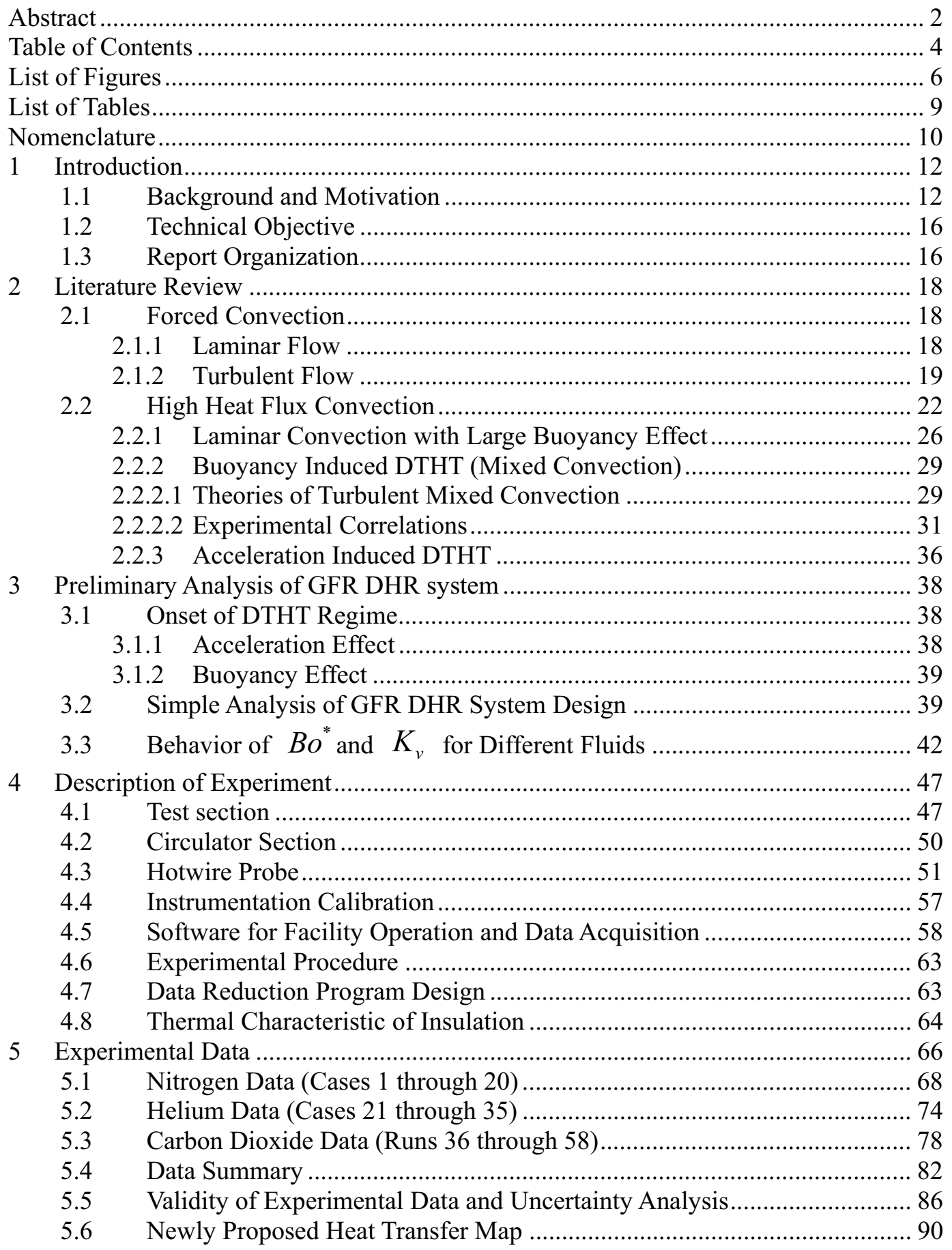




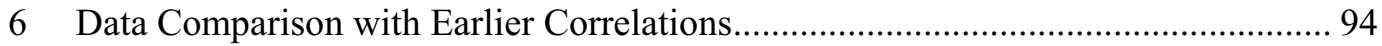

6.1 Mixed Convection Laminar Correlations Comparison ............................... 94

$6.2 \quad$ DTHT Correlations Comparison …….................................................... 98

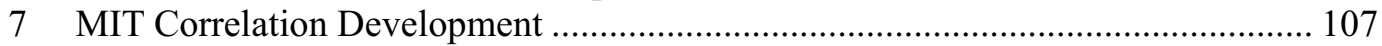

7.1 Correlation Development Criteria and Basic Ideas ................................. 107

7.2 Type-1 MIT Correlation ......................................................................... 110

7.3 Type-2 MIT Correlation ........................................................................... 113

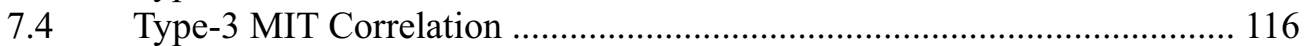

7.5 MIT Correlations Comparison.................................................................... 118

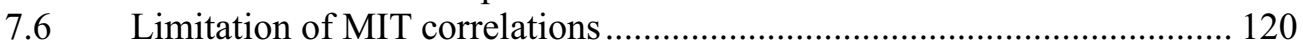

7.7 Smoothness of Developed Correlations .............................................. 122

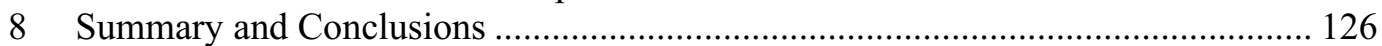

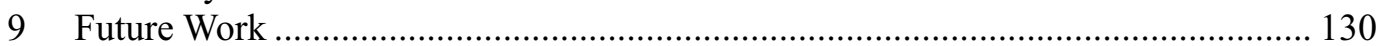

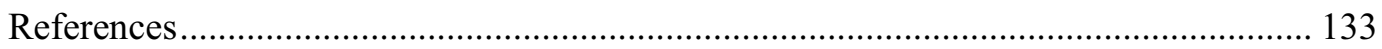

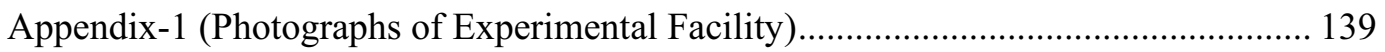

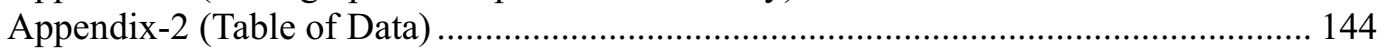




\section{List of Figures}

Figure 1-1 GFR Configuration with Block-Type Core

Figure 1-2 Convection Flow Regimes at Various Operating Pressures for both Helium and $\mathrm{CO}_{2}$ (from Williams et al. 2003) 14

Figure 2-1 Determining Truncation Term for Laminar Correlation 19

Figure 2-2 Turbulent Forced Convection Correlations Comparison 22

Figure 2-3 Comparisons between Forced Laminar Flow and Laminar Flow with High

Buoyancy Effect Velocity Profile 23

Figure 2-4 Buoyancy Effect on Turbulent Heat Transfer 24

Figure 2-5 "Laminarization" of the Turbulent Heat Transfer due to the Acceleration Effect

Figure 2-6 Acceleration Effect on the Turbulent Flow 26

Figure 2-7 Conceptual Diagrams of Two Theories on the Turbulent Mixed Convection 30

Figure 3-1 Acceleration Properties Group 40

Figure 3-2 Buoyancy Properties Group $\quad 40$

Figure 3-3 GFR DHR Operation Range 41

Figure 3-4 Nitrogen Buoyancy Properties Group 43

Figure 3-5 Nitrogen Acceleration Properties Group 43

Figure 3-6 Liquid Water Buoyancy Properties Group 44

Figure 3-7 Liquid Water Acceleration Properties Group 44

Figure 3-8 Super-Critical $\mathrm{CO}_{2}$ Buoyancy Properties Group 45

Figure 3-9 Super-Critical $\mathrm{CO}_{2}$ Acceleration Properties Group 45

Figure 4-1 Test Section Cross Section $\quad 47$

Figure 4-2 Simplified Wiring Diagram of Guard Heaters and Controllers 49

Figure 4-3 Flow Measurements and Circulator System 50

Figure 4-4 Close-up of Assembly Piece for Calibration Loop 51

Figure 4-5 Pitot Tube vs. Hotwire Signal 52

Figure 4-6 Schematic Diagram of Online Hot Wire Calibration System 54

Figure 4-7 Hotwire Calibration Results at Different Flow Rate 55

Figure 4-8 Some Problems with Hotwire Calibration Scheme 56

Figure 4-9 Digital Volt Meter Calibration Results $\quad 57$

Figure 4-10 Power Supply Current Signal Calibration Results 58

Figure 4-11 Facility Operation and Data Acquisition Software (1/3) 60

Figure 4-12 Facility Operation and Data Acquisition Software (2/3) 61

Figure 4-13 Facility Operation and Data Acquisition Software (3/3) 62

Figure 4-14 Thermal Resistance of the Insulation for Different Outer Wall Temperatures

$\begin{array}{ll}\text { Table 5-1 Operating Conditions (continued) } & 67\end{array}$

$\begin{array}{ll}\text { Table 5-1 Operating Conditions (continued) } & 68\end{array}$

Figure 5-1 Nitrogen Nusselt Number vs. Reynolds Number 69

Figure 5-2 Case 6 and 7 Wall, Bulk and Predicted Temperature Profiles 70

Figure 5-3 Case 16 and 17 Wall, Bulk and Predicted Temperature Profiles 71

Figure 5-4 Nitrogen Nusselt Number Ratio vs. Buoyancy Parameter 72

Figure 5-5 Nitrogen Nusselt Number Ratio vs. Acceleration Parameter 72 
Figure 5-6 Map of DTHT Runs: Inlet Acceleration Parameter vs. Inlet Buoyancy Parameter

Figure 5-7 Helium Nusselt Number vs. Reynolds Number. 75

Figure 5-8 Nusselt Number Ratio vs. Helium Buoyancy Parameter 76

Figure 5-9 Helium Nusselt Number Ratio vs. Acceleration Parameter 76

Figure 5-10 Helium Laminar Nusselt Number Ratio vs. Laminar Buoyancy Parameter 78

Figure 5-11 Carbon Dioxide Nusselt Number vs. Reynolds Number 79

Figure 5-12 Carbon Dioxide Nusselt Number Ratio vs. Buoyancy Parameter $\quad 79$

Figure 5-13 Carbon Dioxide Nusselt Number Ratio vs. Acceleration Parameter $\quad 80$

Figure 5-14 Temperature Profile of Re-turbulizing Flow (Run 50 and 52) 81

Figure 5-15 Temperature Profile of non Re-turbulizing Flow (Run 50 and 57) 81

Figure 5-16 Reynolds Number vs. Nusselt Number $\quad 83$

Figure 5-17 Nusselt Number Ratio vs. Buoyancy Parameter 83

Figure 5-18 Nusselt Number Ratio vs. Acceleration Parameter $\quad 84$

Figure 5-19 Location of Maximum Reduction vs. Inlet Buoyancy Parameter $\quad 85$

Figure 5-20 Inlet Acceleration Parameter vs. Inlet Buoyancy Parameter 85

Figure 5-21 Inlet Re vs. Heat Balance and maximum Reduction in Nusselt Number

Observed 87

Figure 5-22 Uncertainty of Experimental Measurement considering the properties $\begin{array}{ll}\text { uncertainty and without considering the properties uncertainty vs. Inlet Re } & 88\end{array}$

Figure 5-23 Inverse Temperature Difference of Wall and Bulk vs. Inlet Re 89

Figure 5-24 Newly Proposed Heat Transfer Map 92

Figure 5-25 Experimental data plotted on the Newly Proposed Map 93

Figure 6-1 Experimental Nu vs. Laminar Correlation Predicted Nu 95

Figure 6-2 Experimental Nu vs. Hallman Correlation Predicted $\mathrm{Nu}$

Figure 6-3 Experimental Nu vs. Worsøe-schmidt \& Leppert Correlation Predicted Nu 96

Figure 6-4 Experimental Nu vs. Worsøe-schmidt Correlation Predicted Nu 97

Figure 6-5 Experimental Nu vs. Churchill Correlation Predicted Nu 97

Figure 6-6 Experimental Nu vs. Gnielinski \& Laminar Correlation Predicted Nu 99

Figure 6-7 Experimental Nu vs. Petukhov \& Strigin Correlation Predicted Nu 99

Figure 6-8 Experimental Nu vs. Petukhov \& Polyakov Correlation Predicted Nu $\quad 100$

Figure 6-9Experimental $\mathrm{Nu}$ vs. Herbert \& Sterns Correlation Predicted Nu $\quad 100$

Figure 6-10 Experimental Nu vs. Jackson et al. Correlation Predicted Nu 101

Figure 6-11 Experimental Nu vs. Parlatan Correlation Predicted Nu 101

Figure 6-12 Experimental $\mathrm{Nu}$ vs. Vilemas et al. Correlation Predicted Nu 102

Figure 6-13 Experimental Nu vs. Celeta et al. Correlation Predicted Nu 102

Figure 6-14 Experimental Nu vs. Symolon et al. Correlation Predicted Nu 103

Figure 6-15 Experimental Nu vs. McEligot Correlation Predicted Nu 103

Figure 6-16 Experimental $\mathrm{Nu}$ vs. Perkins \& Worsøe-schmidt Correlation Predicted $\mathrm{Nu}$

Figure 6-17al Experiment Nu vs. Taylor Correlation Predicted Nu 104

$\begin{array}{ll}\text { Figure 7-1 Functional Form for the New Correlation } & 108\end{array}$

Figure 7-2 $2^{\text {nd }}$ Threshold of Re-turbulization 110

Figure 7-3 Experimental Nu vs. First Kind MIT Correlation Predicted Nu 112

Figure 7-4 Experimental Nu vs. Second Kind MIT Correlation Predicted Nu 116

Figure 7-5 Nu Ratio vs. New Non-Dimensional Number 117

Figure 7-6 Experimental Nu vs. Third Kind MIT Correlation Predicted Nu 118 
$\begin{array}{ll}\text { Figure 7-7 Nusselt Number Ratio vs. Buoyancy Parameter } & 122\end{array}$

Figure 7-8 Nusselt Number Ratio vs. Acceleration Parameter 123

Figure 7-9 MIT1 Inlet Nusselt Number vs. Reynolds Number 124

Figure 7-10 MIT2 Inlet Nusselt Number vs. Reynolds Number 125

Figure 7-11 MIT3 Inlet Nusselt Number vs. Reynolds Number 125

Figure 8-1 Inlet Experimental Conditions (Markers Depict Different Gases) 127

Figure 8-2 Inlet Experimental Conditions (Markers Depict Different Flow Regimes) 128

Figure 9-1 Governing Physical Phenomena Variation with the Test Section Diameter Size 


\section{List of Tables}

Table 1-1 Possible Flow and Heat Transfer Regimes ..................................................... 15

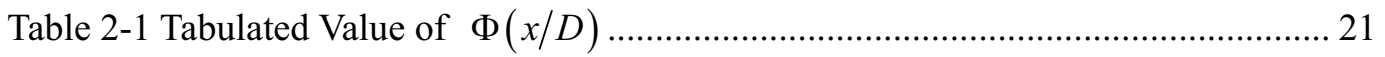

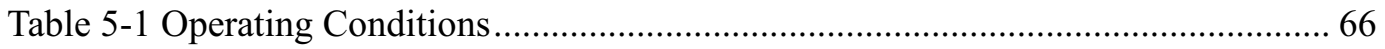

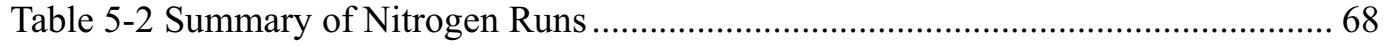

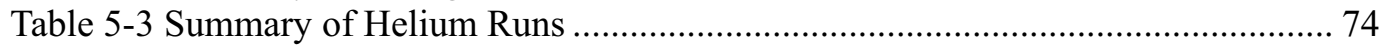

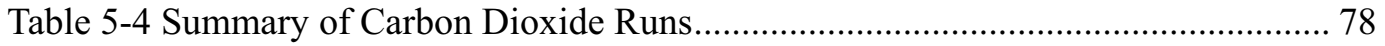

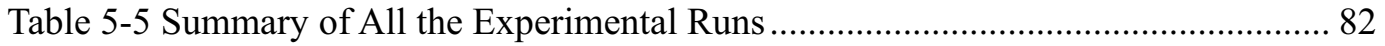

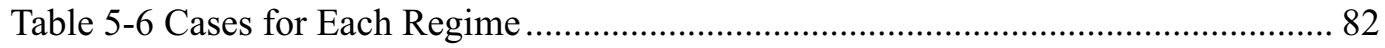

Table 5-7 Threshold Values for Each Regime.............................................................. 84

Table 5-8 Thermo-Physical Properties Uncertainty (from Lemmon et al., 2002) ............ 89

Table 6-1 Mixed Convection Laminar Correlation Fitness Indicators ................................ 97

Table 6-2 Tested Correlations with Equation Numbers .................................................. 98

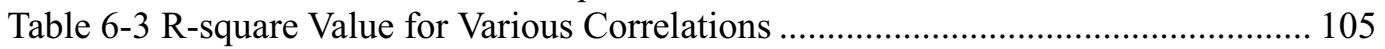

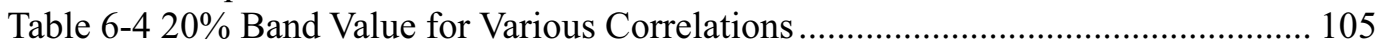

Table 7-1 Summary of MIT-Laminar Correlation Performance ..................................... 119

Table 7-2 Summary of MIT Correlations Performance (R-square) ................................ 119

Table 7-3 Summary of MIT Correlations Performance (20\% band) ............................ 120

Table 7-4 Summary of All the Experimental Runs ....................................................... 121

Table A2-1. Summary of All the Experimental Runs .................................................... 144 


\section{Nomenclature}

$c_{p}:$ specific heat at constant pressure $(\mathrm{J} / \mathrm{kg} K)$

$g$ : gravitational acceleration $\left(\mathrm{m}^{2} / \mathrm{sec}\right)$

$h$ : heat transfer coefficient $\left(\mathrm{W} / \mathrm{m}^{2} K\right)$

$k$ : thermal conductivity of gas $(\mathrm{W} / \mathrm{m} K)$

$\dot{m}$ : mass flow rate $(\mathrm{kg} / \mathrm{sec})$

$q^{\prime \prime}:$ heat flux $\left(\mathrm{W} / \mathrm{m}^{2}\right)$

$x$ : axial direction and distance $(\mathrm{m})$

$y:$ radial direction

$A:$ area $\left(\mathrm{m}^{2}\right)$

$D$ : pipe diameter $(\mathrm{m})$

$E_{v}$ : electrical signal of hotwire (V)

$G$ : mass flux $\left(\mathrm{kg} / \mathrm{m}^{2} \mathrm{sec}\right)$

$H$ : enthalpy $(\mathrm{J} / \mathrm{kg})$

$L$ : length (m)

$P$ : system pressure $(\mathrm{MPa})$

$Q$ : volumetric flow rate $\left(\mathrm{CFM}=\mathrm{ft}^{3} / \mathrm{min}=0.000471947 \mathrm{~m}^{3} / \mathrm{sec}\right)$

$T$ : temperature $(K)$

$U$ : velocity $(\mathrm{m} / \mathrm{sec})$

$\alpha:$ thermal diffusivity $=\frac{k}{\rho c_{p}}\left(\mathrm{~m}^{2} / \mathrm{sec}\right)$

$\beta$ : thermal expansion coefficient $=-\frac{1}{\rho}\left(\frac{\partial \rho}{\partial T}\right)_{P}\left(K^{-1}\right)$

$\mu$ : dynamic viscosity $(\mathrm{kg} / \mathrm{m} \mathrm{sec})$

$v:$ kinematic viscosity $\left(\mathrm{m}^{2} / \mathrm{sec}\right)$

$\rho$ : density $\left(\mathrm{kg} / \mathrm{m}^{3}\right)$

Non-Dimensional Number

$\mathrm{Bo}^{*}$ : buoyancy parameter $=\frac{\mathrm{Gr}_{\mathrm{q}}}{\operatorname{Re}^{3.425} \operatorname{Pr}^{0.8}}$

$\mathrm{Gr}_{\Delta \mathrm{T}}:$ Grashof number $=\frac{g \beta\left(T_{w}-T_{b}\right) D^{3}}{v^{2}}$ 
$\mathrm{Gr}_{\mathrm{q}}$ : Grashof number based on heat flux $=\mathrm{Gr}_{\Delta \mathrm{T}} \mathrm{Nu}=\frac{g \beta q_{w}^{\prime \prime} D^{4}}{k v^{2}}$

$\mathrm{K}_{\mathrm{v}}:$ acceleration parameter $=\frac{v}{U_{b}^{2}} \frac{d U_{b}}{d x} \approx \frac{4 q^{+}}{\mathrm{Re}}$

$\mathrm{Nu}:$ Nusselt number $=\frac{h D}{k}$

Pr: Prandtl number $=\frac{v}{\alpha}$

Ra: Rayleigh number $=\mathrm{Gr}_{\Delta \mathrm{T}} \mathrm{Pr}$

Re: Reynolds number $=\frac{U D}{v}$

$q^{+}:$nondimensional heat flux $=\frac{q_{w}^{\prime \prime}}{G H_{b}} \approx \frac{q_{w}^{\prime \prime}}{G c_{p} T_{b}} \approx \frac{\beta q_{w}^{\prime \prime}}{G c_{p}}$

\section{Subscripts}

b: bulk

in: inlet

o: reference state

th: threshold

w: wall

$F$ : forced convection

$N$ : natural convection

$\infty$ : infinite from the inlet or the wall 


\section{Introduction}

\subsection{Background and Motivation}

The Gas-Cooled Fast Reactor (GFR) is one of the leading advanced reactor designs among the Generation IV concepts, under development in the U.S., France, and Japan. It is widely recognized that one of the major design challenges of this type of reactor is to ensure sufficient decay heat removal in loss of coolant accidents, because gases have inherently mediocre heat transfer coefficients and specific heat capacities at low pressures. This shortcoming of gas coolants has been circumvented in gas-cooled thermal reactors by reliance on conduction and radiation of decay heat through the solid matrix or pebble bed core to the vessel and ultimately to the heat sink. Thus, no coolant is required to maintain the core within acceptable temperature limits. Although this approach is also possible for the GFR core, unacceptably high fuel cycle cost is unavoidable as a result of higher enrichment and core heavy metal loading [Hejzlar et al. 2002], making it extremely difficult to achieve good economy - an important Generation IV goal and the key requirement of the current electricity generation market. Therefore, fast reactors must be operated at significantly higher power densities than their thermal counterparts to offset the cost associated with higher enrichment and heavy metal requirements. Because the required power density is an order of magnitude higher than the typical 4 to $8 \mathrm{~kW} / 1$ for thermal rectors, use of the conduction and radiation heat transfer modes cannot solely provide sufficient decay heat removal rates. Thus, different approaches for dissipation of decay heat must be adopted.

Traditionally, active gas cooling loops using electrically driven blowers and heat exchangers were used in earlier gas-cooled fast reactor designs [Gratton, 1981]. However, in the last decade, an increased emphasis has been made on passive decay heat removal systems due to their simplicity and higher reliability (since they do not depend on energy sources). Also, there is a strong preference for passive systems for decay heat removal in the Generation IV program. Therefore, there is a strong incentive for GFR designs having the capability to dissipate decay heat by passive means. To ensure decay heat removal from the GFR core using natural mechanisms is, however, a very ambitious and difficult task, given the aforementioned need for high power density. The ongoing U.S.-French International-Nuclear Engineering Research Initiative (I-NERI) Program of Generation IV GFR design, which studies various options of post-LOCA passive decay heat removal schemes, identified the convection loops connecting the core and elevated heat exchangers that provide heat sinks as the most promising approach, if elevated pressure inside the guard containment can be ensured after the primary system depressurization [Garnier 2003]. Since, such a system requires very low core pressure drop, candidates for low-pressure core are block type core with circular channels (MIT and Japanese design) and plate-type core (CEA design). An example of GFR with block type core is shown in Figure 1-1. Thermal-hydraulic performance of such naturally circulating system has been evaluated at CEA and at MIT and it has been found that these loops can operate in atypical heat transfer and flow regimes, which are challenging for currently available system analysis codes because of the lack of appropriate correlations implemented in the codes. 

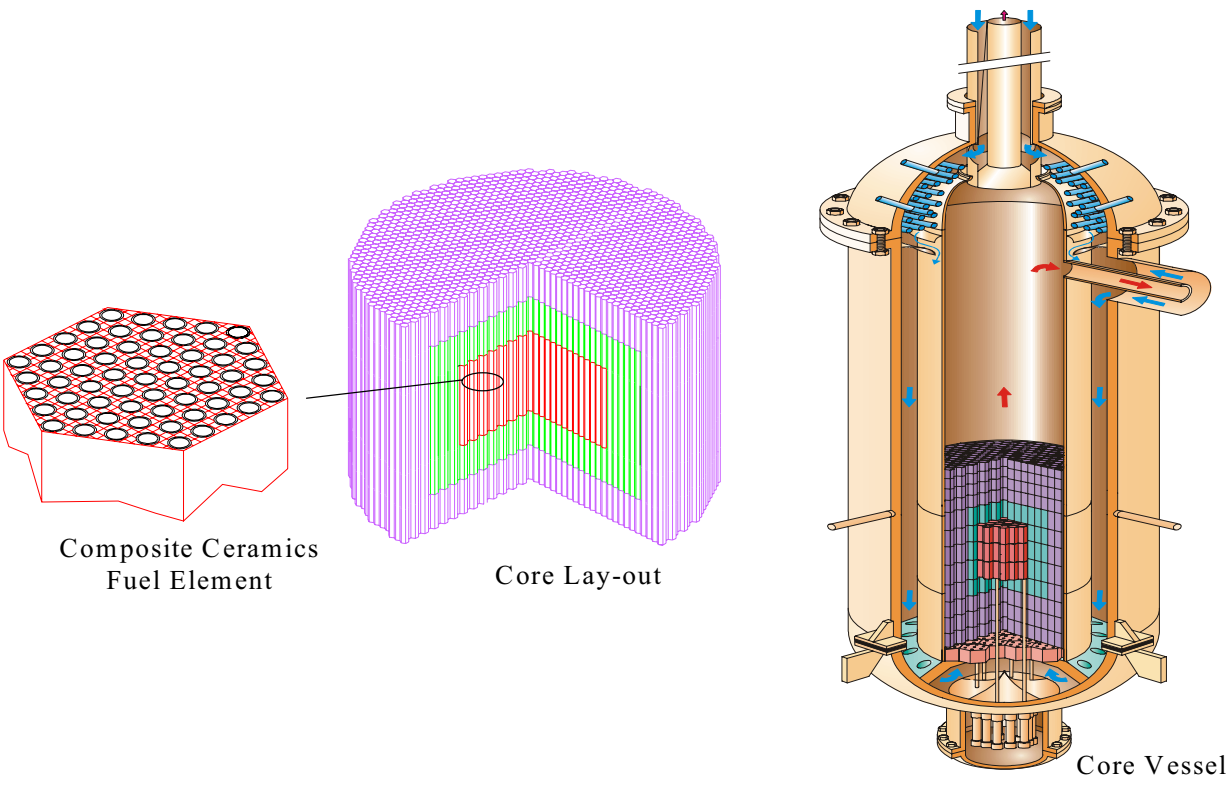

Figure 1-1 GFR Configuration with Block-Type Core

Figure 1-2 shows an example of convection flow regimes along the heater (reactor core) and cooler (heat exchanger providing ultimate heat sink) at various pressures in a simplified Reynolds-Rayleigh ${ }^{1}$ number map [Williams et al. 2003]. Figure 1-2 was generated for a typical GFR core having hexagonal blocks with circular coolant holes, shown in Figure 1-1. Both helium and $\mathrm{CO}_{2}$ coolants were studied as the means for postLOCA decay heat removal. The operating pressure of the convection loop was varied to explore how the flow regime changes with pressure. It can be observed that unlike in most industrial applications, which operate in well defined and well-known turbulent forced convection regime, the GFR loop operates in the mixed convection or in the transition between the mixed and forced or laminar convection regimes.

\footnotetext{
${ }^{1}$ Rayleigh number is the product of the Grashof number and the Prandtl number.
} 


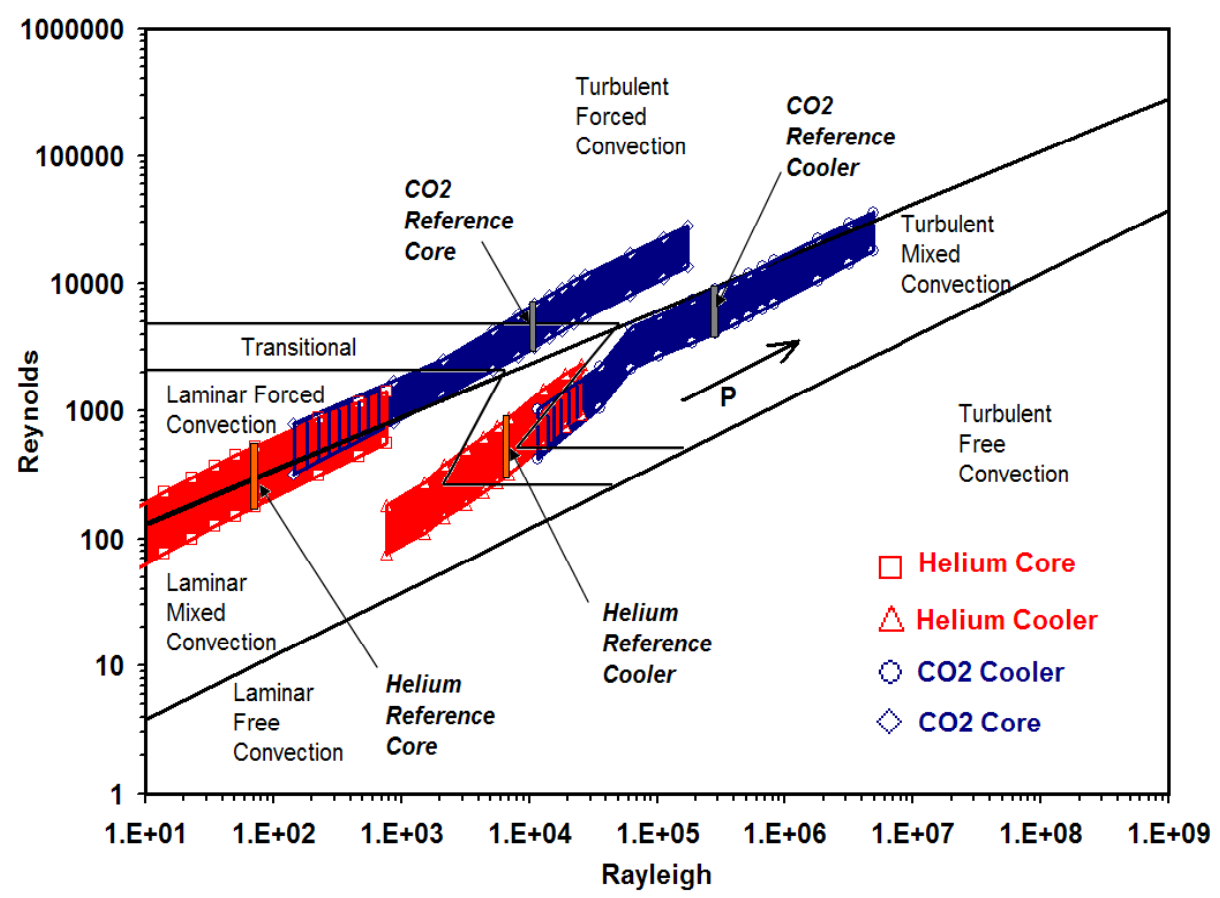

Figure 1-2 Convection Flow Regimes at Various Operating Pressures for both Helium and $\mathrm{CO}_{2}$ (from Williams et al. 2003)

To design the core and heat exchangers and to ensure that fuel and cladding limits are not exceeded during post-LOCA, firm knowledge of heat transfer coefficients and friction factors in these regions is necessary. In addition, system analysis codes need to have the capability to simulate all the regimes with reasonably low uncertainties, hence the heat transfer and friction factor correlations will have to be available in the codes and will have to be implemented in such a way that code stability is ensured when transition among individual regimes occurs. When the existing system analysis codes, such as RELAP5-3D, are applied to the analysis of the GFR convection decay heat removal loop, three needs for additional research and development become apparent:

1. Currently available system analysis codes do not cover the whole flow regime map in terms of heat transfer and friction factor correlations, because they were originally designed for active systems with forced convection cooling. For example, RELAP5-3D employs forced convection correlations in the DittusBoelter form where the user can supply heat transfer coefficients and natural convection correlation. Clearly, there is a need to cover the whole flow regime map, if the code is to be used for the analysis of the GFR passive decay heat removal system.

2. Although a large number of heat transfer correlations for one-phase flow is available in the literature [Kakac et al., 1987], a unified approach designed to cover the entire range of possible flow regimes that can be implemented into the computer code is missing. However, the boundaries between the regions cannot 
be always clearly defined, or if defined, large discontinuities in the heat transfer coefficient correlations exist. Moreover, different authors use different nondimensional numbers to define these boundaries. For example Metais and Eckert (1964) present a flow regime map in terms of a Grashof (Gr)-Prandtl number group times diameter over length ratio and Reynolds (Re) number groups while Tanaka et. al. (1987) use a Gr-Re plot. Transition criteria to define the boundaries between forced, mixed and natural convection also vary among the investigators (compare Aicher \& Martin (1997) and Burmeister (1993)). Therefore, there is an acute need to develop a robust set of heat transfer correlations that (i) covers the entire flow regime map, (ii) has clearly defined boundaries using a consistent set of dimensionless numbers for the whole map, and (iii) ensures continuous transitions of correlations on regime boundaries if the real physical phenomena occur continuously, which is a necessary condition for numerical stability of the large system codes, such as RELAP5-3D.

3. There are nine possible heat transfer and flow regimes, as indicated in Table 1-1. Heat transfer correlations are generally available for forced and natural convection regimes in both turbulent and laminar flows and more recently mixed convection regime correlations were also developed. However, there are gaps in transition regions between various flow regimes, where no heat transfer correlations are available. In some regimes, correlations have a small range of validity that does not cover the whole region and require more experimental data to extend their range or reduce large uncertainties. Therefore, there is a need to obtain more experimental data to close the gaps in heat transfer prediction capability.

Table 1-1 Possible Flow and Heat Transfer Regimes

\begin{tabular}{|c|c|c|c|}
\hline Regime & Turbulent & Transition & Laminar \\
\hline Forced & $\mathrm{x}$ & $\mathrm{x}$ & $\mathrm{x}$ \\
\hline Transition & $\mathrm{x}$ & & $\mathrm{x}$ \\
\hline Mixed & $\mathrm{x}$ & $\mathrm{x}$ & $\mathrm{x}$ \\
\hline Transition & $\mathrm{x}$ & & $\mathrm{x}$ \\
\hline Free & $\mathrm{x}$ & $\mathrm{x}$ & $\mathrm{x}$ \\
\hline
\end{tabular}

A collaborative INL/MIT project was initiated to satisfy the needs mentioned above, since it has been recognized that GFR has a potential to operate in Deteriorated Turbulent Heat Transfer (DTHT) regime, which will be explained in much detail in the following section, or in the transition between the DTHT and normal forced turbulent or laminar convection regime during post-loss-of-coolant accident (LOCA) conditions. This is to acquire experimental data (to the extent possible) to develop heat transfer correlation that would have a smooth transition at the boundaries and covers all regimes including the transition regions to the largest extent possible. This final report summarizes the results of this project. 


\subsection{Technical Objective}

To address the aforementioned needs the project has the following objectives:

1 Acquire experimental data needed to fill the gaps (to the extent possible) in heat transfer correlations and identification of the boundaries for transitions between individual flow regimes.

2 Develop heat transfer correlations for all regimes including the transition regions in such a manner that there is a smooth transition on the boundaries to the largest extent possible, so that it can be easily implemented into RELAP5-3D.

\subsection{Report Organization}

Earlier work throughout this 3-year project was documented in three reports. The first report on scaling [Cochran et al., 2004a] documented how a proposed experimental loop might encompass flow regimes as they go from laminar to turbulent flow and from free to forced convection. Based upon non-dimensional analysis, the following dimensionless parameters were chosen to characterize the flow regimes; Re for the transition between laminar and turbulent flow, and $\mathrm{Gr}_{\Delta T}, \mathrm{Ra}_{\Delta \mathrm{T}}, B o^{*}$, and $K_{v}$ for the transition between forced to free convection and laminar to turbulent flow. Using a steady state convective loop heat transfer computer code LOCA-COLA, developed at MIT, it has been shown that using a blower in the experimental loop, three coolants, helium, nitrogen, and carbon dioxide in a range of system pressures between $0.1 \mathrm{MPa}$ and $1.0 \mathrm{MPa}$ and two test section diameters, 16 and $32 \mathrm{~mm}$, the transition between free and forced convection and laminar to turbulent flow can be mapped. It was confirmed that the proposed experimental loop could encompass the GFR prototype loop conditions for these parameters.

The first annual report focused on the assessment of measurement uncertainties and identified that the heat transfer coefficients to be obtained from the proposed experimental loop can have relatively large errors, especially in low flow rate cases. The performed analyses have shown the need to reduce heat losses to environment and proposed to implement guard heaters to minimize these losses.

The second annual report [Lee et al., 2005c] provided the first description of the experimental loop as designed, and presented the preliminary test results. The first runs were performed in the forced convection regime to verify facility operation against wellestablished forced convection correlations. The results of the three runs at Reynolds numbers $6,700,8,000$ and 12,800 showed good agreement with the Gnielinski correlation, which is considered the best available heat transfer correlation in the forced convection regime and is valid for a large range of Reynolds and Prandtl numbers. The very good agreement between the experimental data with the Gnielinski correlation confirmed that the loop facility and the instrumentation performed correctly. Improvements to further reduce the uncertainty for the highest wall temperature case have been outlined and were planned to be implemented early in this fiscal year together with the installation of the 
hot-wire calibration facility for velocity profile measurements for very low flow rate.

This final report does not detail the material covered in the above mentioned progress reports. The major focus of this report will be the summary of the final experimental data that were taken in all flow regimes and the correlation development to satisfy the project objectives. The report is organized as follows: First, an expanded literature review that was performed in addition to the review presented in the earlier reports is presented in Chapter 2. The review mainly covers literature related to the gas flow in the Deteriorated Heat Transfer (DTHT) regimes and the correlations that have been developed in the past. Chapter 3 presents a preliminary analysis on GFR decay heat removal system, which is performed using a different method in Williams et al., (2003). This chapter will show more thoroughly why GFR system during accident situations can be susceptible to operate in the DTHT regime. Chapter 4 will briefly summarize the upgrades that have been made to the experimental facility and the software to resolve the problems that were brought up in the second annual report. Chapter 5 will present the experimental data that have been collected under various operating conditions and working fluids. Chapters 6 and 7 will compare the experimental data to the existing correlations in the literature and present a new correlation set developed at MIT. Summary, conclusions and suggestions for future work will be covered in Chapters 8 through 9 . 


\section{Literature Review}

Extensive literature survey was performed and documented in the $2^{\text {nd }}$ annual report [Lee et al., 2005c]. The literature review in the $2^{\text {nd }}$ annual report was rather general with respect to the heat transfer in deteriorated heat transfer regime and covered experimental data available as well as the attempts of various investigators to express their observations in correlations. In this chapter, literature review will be mainly focused on the correlations in different heat transfer regimes in an effort to select the most relevant correlations for comparison with our experimental data. Therefore, only a limited number of references will be added to the extensive review of the $2^{\text {nd }}$ annual report. This chapter is divided into two sections. The first section will address the studies of the forced convection gas flow and will be divided into two sub-sections: laminar flow and turbulent flow. The second section will be divided into three short subsections covering mixed convection laminar, buoyancy force induced DTHT and acceleration induced DTHT respectively. The literature will be presented with descending order in timeline to show the historical development of the correlations.

\subsection{Forced Convection}

\subsubsection{Laminar Flow}

For laminar forced convection, selecting the best correlation is rather simple compared to other regimes. This is because Seigel et al., (1958) solved the laminar forced convection Nusselt number analytically and Kays \& Crawford (1993) simplified the solution for the case when uniform heat flux is applied, fluid properties are regarded as a constant and thermal developing length was considered. The basic procedure for obtaining the analytical solution employed the method of separation of variables and the SturmLiouville theory to obtain an eigen-value solution. Simplified solution form is given in Equation 2-1. It should be noted that $\gamma_{m}$ and $A_{m}$ are an approximation to the true value.

$$
\begin{aligned}
& \mathrm{Nu}_{\text {Laminar }}=\left(\frac{1}{\mathrm{Nu}_{\infty}}-\frac{1}{2} \sum_{m=1}^{\infty} \frac{\exp \left(-\gamma_{m}^{2} x^{+}\right)}{A_{m} \gamma_{m}^{4}}\right)^{-1} \\
& \text { where } \mathrm{Nu}_{\infty}=4.364, \quad x^{+}=\frac{2 x / D}{\operatorname{RePr}}, \quad \gamma_{m}=4 m+\frac{4}{3}, \quad A_{m}=0.4165 \gamma_{m}{ }^{-7 / 3}
\end{aligned}
$$

However, for an actual engineering application, $m$ needs to be truncated at some point. Since the maximum Reynolds number for laminar case is 2,300 and the typical gas Prandtl number is 0.7 , setting $x / D$ equal to unity will yield $x^{+}$of $\sim 0.001$. If we set our conditions for choosing maximum $m$ such that the Nusselt number changes remain below $1 \%$ after adding one more term at $x^{+}$equal to 0.001 , it can be found that one can start to neglect the summation terms after $m$ is larger than 10 (see Figure 2-1). This 
correlation, shown as Equation 2-2, will be used as a reference laminar forced convection correlation for the data comparison purpose.

$$
\mathrm{Nu}_{\text {Laminar }}=\left(\frac{1}{\mathrm{Nu}_{\infty}}-\frac{1}{2} \sum_{m=1}^{\infty} \frac{\exp \left(-\gamma_{m}^{2} x^{+}\right)}{A_{m} \gamma_{m}^{4}}\right)^{-1} \approx\left(\frac{1}{\mathrm{Nu}_{\infty}}-\frac{1}{2} \sum_{m=1}^{10} \frac{\exp \left(-\gamma_{m}^{2} x^{+}\right)}{A_{m} \gamma_{m}^{4}}\right)^{-1}
$$

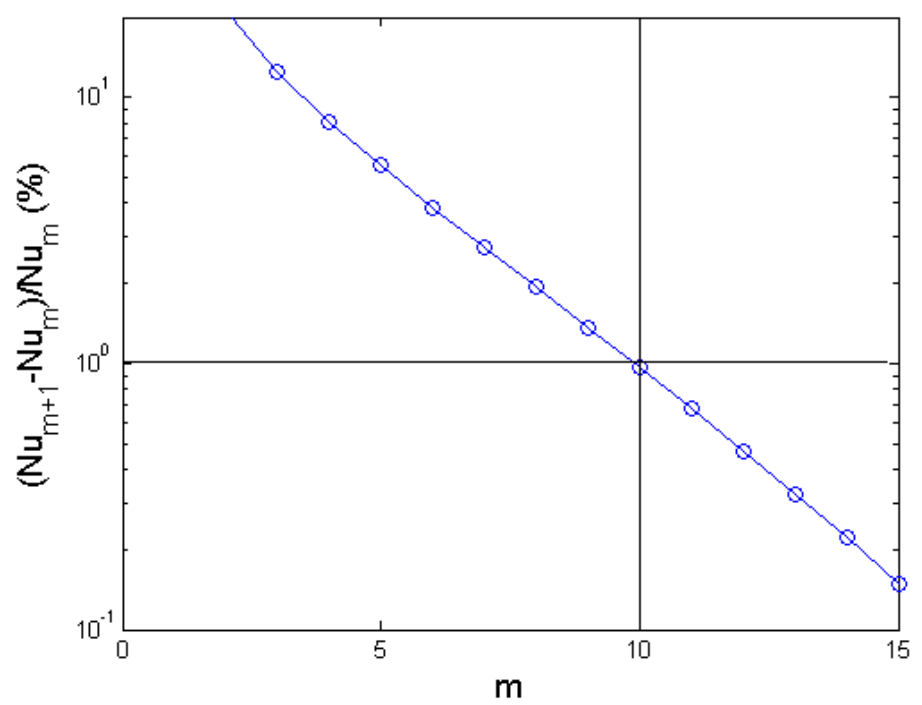

Figure 2-1 Determining Truncation Term for Laminar Correlation

\subsubsection{Turbulent Flow}

A large number of correlations for the heat transfer in forced turbulent flow exist. Since the goal of this project is to obtain and correlate data in the transition regime between forced turbulent convection and laminar flow to cover deteriorated heat transfer due to suppressed turbulence, correlations that cover this transition region are of main interest. Three correlations were identified as the most promising candidates for comparison with our data. These include McEligot et al. (1966), Petukhov et al. (1973), and Gnielinski (1976) and will be desribed next.

\section{McEligot et al. (1966) correlation}

In McEligot et al. (1966) work, the low Reynolds number gas flow experimental data were used to develop a heat transfer correlation based on the Nusselt number power law or the modified Dittus-Boelter formulation (Eq. 2-3).

$$
\mathrm{Nu}_{\text {Dittus-Boelter }}=0.021 \mathrm{Re}^{0.8} \operatorname{Pr}^{0.4}
$$

For a moderate heating case $\left(\mathrm{q}^{+} \sim 0.004\right)$, Equation 2-4 was suggested for a better fit 
with the data.

$$
\mathrm{Nu}_{\text {McEligot }}=0.021 \operatorname{Re}^{0.8} \operatorname{Pr}^{0.4}\left(\frac{T_{w}}{T_{b}}\right)^{-0.5}
$$

The correlation is valid for $x / D>30,4,000<\operatorname{Re}<15,000,0<q^{+}<0.004$. The experimental data used for developing this correlation were based on air, helium and nitrogen gas flow at inlet Reynolds number from 1,500 to 45,000 and maximum wall to bulk temperature ratio around four.

\section{Petukhov et al. (1973) correlation}

Petukhov et al. [1973] started to develop a correlation by questioning the power law form of a heat transfer correlation, which can be found in various references. In his opinion, the major problem with the power law form was that the leading constant (in case of McEligot correlation, 0.021) and in the properties variation function can have multiple combinations. He also noted that the power law formula only approximately expresses the Nusselt, Reynolds and Prandtl numbers relationship. Therefore, Petukhov et al., developed a different correlation form (Eq. 2-5).

$$
\begin{aligned}
& \mathrm{Nu}_{\text {Petukhov }}=\frac{(f / 8) \operatorname{Re} \operatorname{Pr}}{1.07+900 / \operatorname{Re}^{-[0.63 /(1+10 \operatorname{Pr})]+12.7 \sqrt{f / 8}\left(\operatorname{Pr}^{2 / 3}-1\right)}} \\
& \text { where } 10,000<\operatorname{Re}, f=\left(1.82 \log _{10} \operatorname{Re}-1.64\right)^{-2} \quad \text { (Filonenko formula) } \\
& 4,000<\operatorname{Re}<10,000, f=0.3164 / \operatorname{Re}^{0.25} \quad \text { (Blaisus Formula) }
\end{aligned}
$$

The properties variation and the thermal developing length effect for the Petukhov et al. correlation are evaluated from Equation 2-6.

$$
\begin{aligned}
\mathrm{Nu}_{\text {Petukhov } P M \text { \& TDL }}=\mathrm{Nu}_{\text {Petukhov }} & \left(\left[\frac{k_{w}}{k_{b}}\right]^{\frac{1}{3}}\left[\frac{c_{p_{w}}}{c_{p_{b}}}\right]^{\frac{1}{4}}\left[\frac{T_{w}}{T_{b}}\right]^{\left.-0.53+\Phi\left(\frac{x}{D}\right) \log \left(\frac{\mu_{w}}{\mu_{b}}\right)\right)}\right. \\
& \times\left(1+\frac{0.48(1+3600 / \operatorname{Re} \sqrt{x / D})}{(x / D)^{0.25}} \exp (-0.17 x / D)\right)
\end{aligned}
$$

where $\Phi(x / D)$ is tabulated value in Table 2-1. 
Table 2-1 Tabulated Value of $\Phi(x / D)$

\begin{tabular}{||c|c|c|c|c|c|c|c|c|c|c|c||}
\hline \hline$x / D$ & 10 & 20 & 30 & 40 & 50 & 60 & 70 & 80 & 90 & 100 & $\infty$ \\
\hline$\Phi$ & 0.11 & 0.4 & 0.38 & 0.55 & 0.73 & 0.89 & 1.02 & 1.13 & 1.21 & 1.27 & 1.50 \\
\hline
\end{tabular}

This correlation was tested against air, argon, carbon dioxide, nitrogen and ammonia. The range of validity covers the Reynolds number above 7,000, $q^{+}$below 0.007 and wall to bulk temperature ratio below 4.0 and above 0.5 .

\section{Gnielinski (1976)}

Although the Petukhov et. al. correlation showed better agreement with a wide range of experimental data than the power law correlations, its complicated form makes industrial applications difficult. Gnielinski correlation is a simplified form of the Petukhov et al. correlation with small modification to fit the experimental data better in the transition regime $(2,300<\operatorname{Re}<7,000)$. Correlation form is given in Equation 2-7.

$\mathrm{Nu}_{\text {Gnielinski }}=\frac{(f / 8)(\mathrm{Re}-1000) \operatorname{Pr}}{1+12.7 \sqrt{f / 8}\left(\operatorname{Pr}^{2 / 3}-1\right)}\left(\frac{T_{w}}{T_{b}}\right)^{-0.45}\left(1+\left(\frac{x}{D}\right)^{-\frac{2}{3}}\right)$

where $f=\left(1.82 \log _{10} \mathrm{Re}-1.64\right)^{-2}$

The correlation is valid for Reynolds number above 2,300 and below $10^{6}$, Prandtl number above 0.6 and below $10^{6}$ and the wall to bulk temperature ratio is in between 0.667 and 2.0. However, Gnielinski did not discuss the limitation of the correlation in terms of the non-dimensional heat flux $q^{+}$. The Gnielinski correlation was tested against water, oil, gasoline, kerosene, acetone, air, nitrogen and carbon dioxide. All these fluids' heat transfer coefficients were successfully predicted with this correlation. Currently, Gnielinski correlation is recommended in most heat transfer handbooks and the heat exchanger design guidelines as the best correlation for the forced turbulent heat transfer.

Even though there are many more references that discuss correlations for the turbulent forced convection heat transfer regime, only the above three correlations were selected for further comparison with MIT data. This is because these three papers summarize the forced turbulent convection correlation development history well and it is necessary to limit the number of correlation for comparison.

In summary, the turbulent forced convection heat transfer correlation was developed first based on the power law (e. g. McEligot et al.), the next step was to improve the correlation based on the theoretical development of the turbulent boundary layer theory (e. g. Petukhov et al.) and finally a simplification and an improvement on the transition regime were made to the correlation (e. g. Gnielinski). Figure 2-2 shows the comparison between these three correlations as a function of Reynolds number for fixed $\operatorname{Pr}=0.7$. It 
is noted that only constant properties and fully developed part of the correlation are used for the comparison purpose. Even though McEligot et al. and Petukhov et al. correlations do not claim to fit the experimental data around Reynolds number 2,300, it is presented in the figure only to show the difference between the fully turbulent heat transfer correlation and the correlation that claims to cover the transition regime.

From the figure, it is clearly shown that due to $(\mathrm{Re}-1000)$ part in the Gnielinski correlation, the correlation tends to bend towards the laminar Nusselt number than the other two correlations and this makes this correlation to fit the experimental data better in the transition regime and has smoother performance for the numerical analysis when the flow regime is crossing from the laminar to turbulent flow. For fully turbulent flow, the Gnielinski correlation approaches the McEligot et al. correlation value and stays with the Petukhov et al. correlation within 5\%. Since our experimental setup covers large range of Reynolds number encompassing the laminar, transitional and turbulent flows, the Gnielinski correlation is used to compare with the experimental data for the forced turbulent heat transfer cases.

One interesting fact worthy of noting is that only few experimental data were used to verify the correlations for the case of forced turbulent heat transfer near the transition region in pressurized gas systems. Therefore, the MIT data will also contribute to the technical community by extending experimental database for testing the well-known forced turbulent correlation in relatively rare operating conditions.

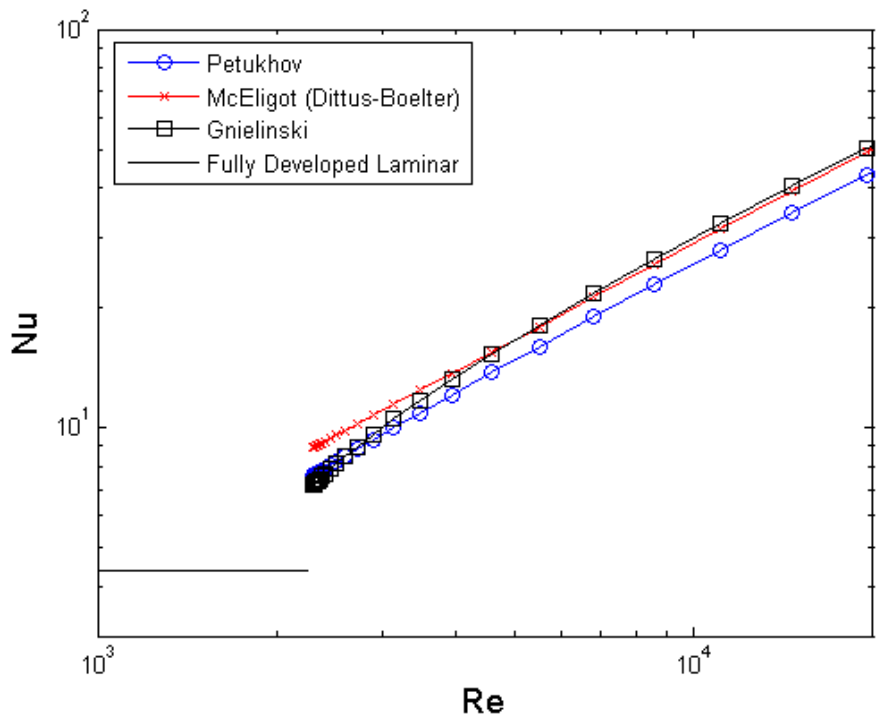

Figure 2-2 Turbulent Forced Convection Correlations Comparison

\subsection{High Heat Flux Convection}

When the heat flux is high enough to affect the flow pattern, the characteristic of the 
convective heat transfer starts to depart from the forced convection theory and correlation predictions. High heat flux usually induces density change in the fluid and its transport properties, such as viscosity and thermal conductivity, and affects a radial velocity and temperature profile within the flow. However, multiplication of the forced convection correlation by the wall to bulk temperature ratio with an appropriate power is typically sufficient to account for the variation of transport properties due to heating. In contrast, the axial change in density can cause more complicated phenomena.

The first major effect is the buoyancy effect. The buoyancy effect can have different effects on the flow with different orientation of the flow and heating. Under GFR condition, upward heated flow is the case that requires attention, thus from now on all the buoyancy effects discussed here will be assuming upward heated flow and the literature is selected based on this orientation.

The buoyancy effect can alter the heat transfer characteristics of both laminar and turbulent flow. In laminar flow, since the buoyancy force results in a steeper velocity gradient than the normal flow near the heated wall (See Figure 2-3), the amount of heat that is convected near the wall increases; therefore the laminar heat transfer is enhanced due to the buoyancy force. Typically, the situation where a strong buoyancy force is acting on the forced convection flow is called "Mixed Convection".

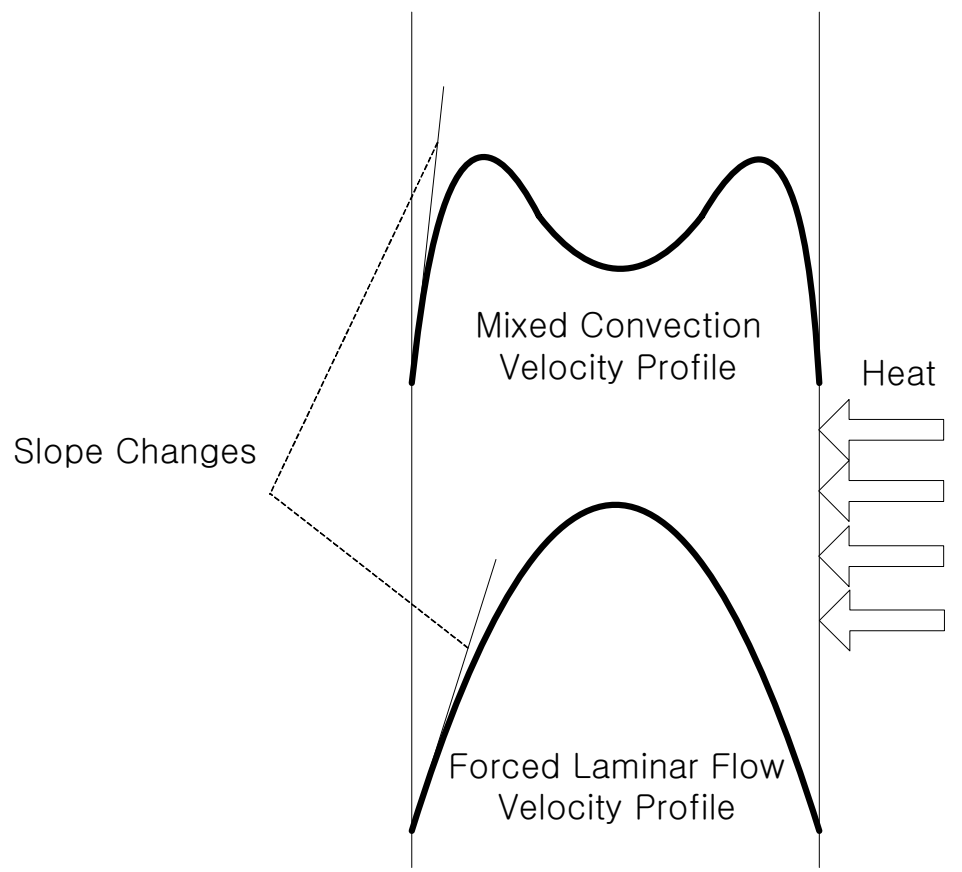

Figure 2-3 Comparisons between Forced Laminar Flow and Laminar Flow with High Buoyancy Effect Velocity Profile

For the turbulent flow, there are two theories for strong buoyancy effect on the turbulence. One was introduced by Hall \& Jackson (1969) and the other was introduced by Petukhov 
\& Polyakov (1988). These two theories will be discussed in more details in the following subsection. To summarize the phenomenon briefly, we will just say at this point that the turbulent heat transfer deteriorates for moderate buoyancy effect, recovers back to the normal turbulent heat transfer for higher buoyancy forces and finally the heat transfer is enhanced above that of the forced turbulent heat transfer for very high-buoyancy flows and asymptotically approaches the free turbulent convection heat transfer. Figure 2-4 depicts the buoyancy effect on the turbulent heat transfer. $\mathrm{X}$-axis is the buoyancy number $\left(B o^{*}\right)$, which is the measure of the buoyancy effect and Y-axis is the ratio between the measured Nusselt number and the forced turbulent flow Nusselt number. The buoyancy number will be defined more thoroughly in the later part of this report.

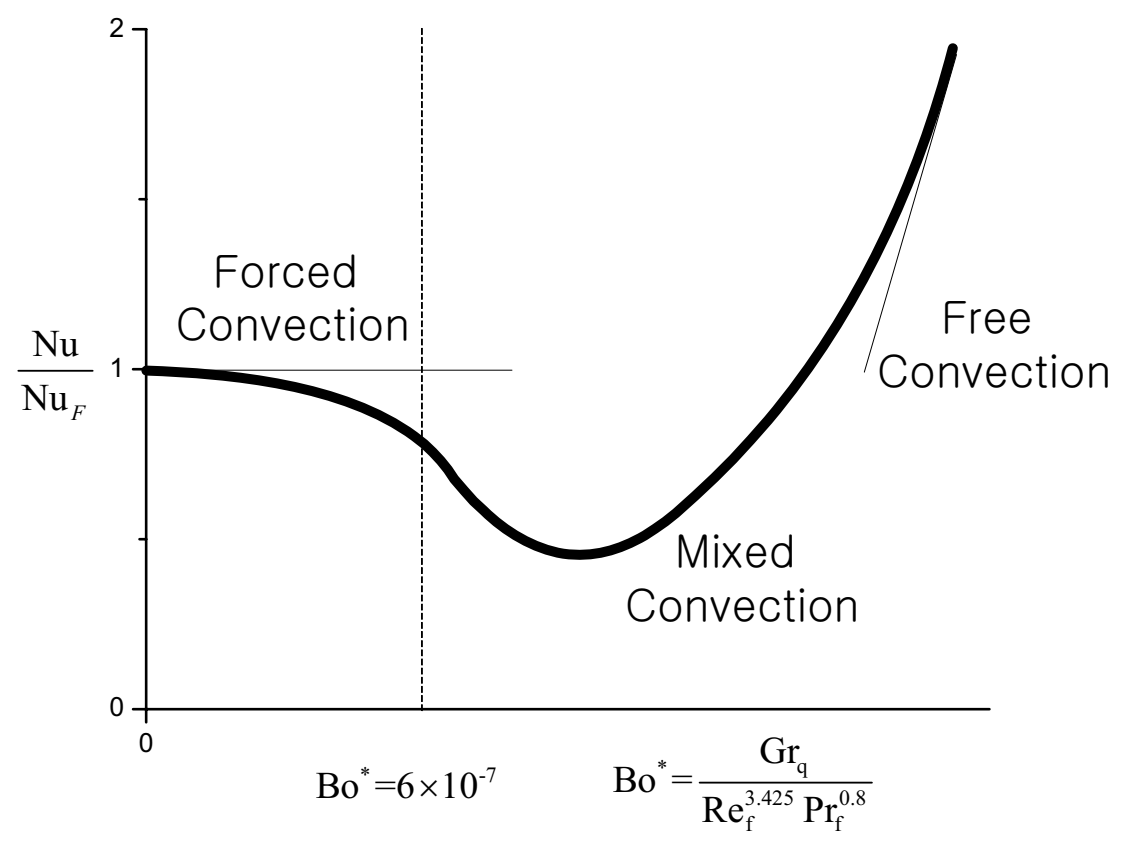

Figure 2-4 Buoyancy Effect on Turbulent Heat Transfer

One issue that has to be discussed before moving forward is the selection of appropriate non-dimensional number for describing the buoyancy force in the channel. When one attempts to non-dimensionalize the momentum equation, the temperature in the buoyancy force term, written using the Boussinesq approximation (Eq. 2-8), is the most controversial term for selecting the reference parameter. It is noted that only twodimensional steady state $\mathrm{x}$-momentum equation is presented here for simplicity.

$$
\frac{\partial u}{\partial t}+u \frac{\partial u}{\partial x}+v \frac{\partial u}{\partial y}=-\frac{1}{\rho} \frac{\partial p}{\partial x}+v\left(\frac{\partial^{2} u}{\partial x^{2}}+\frac{\partial^{2} u}{\partial y^{2}}\right)+\underset{\text { Boussinesq Approximation }}{g \beta\left(T-T_{\infty}\right)}
$$

From Petukhov et al. (1988) discussion (p. 20) the reference parameter for nondimensionalizing the temperature should be dependent on boundary conditions. Since we are limiting ourselves with the prescribed heat flux boundary condition only, the heat flux based non-dimensional group will be adopted for our study. Therefore, reference 
parameter for non-dimensionalizing the buoyancy force term will be Equation 2-9 and the resulting non-dimensionalized momentum equation is presented in Equation 2-10.

$$
\begin{aligned}
& T_{\text {ref }}=\frac{q_{w}^{\prime \prime} D}{k} \\
& \frac{\partial u^{*}}{\partial t^{*}}+u^{*} \frac{\partial u^{*}}{\partial x^{*}}+v^{*} \frac{\partial u^{*}}{\partial y^{*}}=-\frac{\partial p^{*}}{\partial x^{*}}+\frac{1}{\operatorname{Re}}\left(\frac{\partial^{2} u^{*}}{\partial x^{* 2}}+\frac{\partial^{2} u^{*}}{\partial y^{* 2}}\right)+\frac{\mathrm{Gr}_{q}}{\operatorname{Re}^{2}} T^{*} \\
& \text { where } t^{*}=\frac{t}{\left(D / U_{b}\right)}, x^{*}=\frac{x}{D}, y^{*}=\frac{y}{D}, u^{*}=\frac{u}{U_{b}}, v^{*}=\frac{v}{U_{b}}, p^{*}=\frac{p}{\rho_{o} U_{b}^{2}}, T^{*}=\frac{k\left(T-T_{o}\right)}{q_{w}^{\prime \prime} D} \\
& \operatorname{Re}=\frac{\rho_{o} U_{b} D}{\mu}, \mathrm{Gr}_{q}=\frac{g \beta q_{w}^{\prime \prime} D^{4}}{k v^{2}}
\end{aligned}
$$

The other phenomenon, which is induced by fluid density change, is the acceleration effect. As the flow temperature increases, gas density is reduced. Since mass is conserved, density increase is accompanied by velocity rise and accelerating the flow along the channel. The flow acceleration in axial direction decreases the turbulence in the flow. Therefore, the turbulent flow can become laminar flow (some time it is called "laminarization") and the heat transfer decreases drastically. Figure 2-5 shows the laminarization of the turbulent heat transport. X-axis is the acceleration number $\left(K_{v}\right)$, which is the measure of the axial acceleration of the flow and Y-axis is the ratio of the measured Nusselt number to the laminar uniform-heat-flux forced-convection Nusselt number.

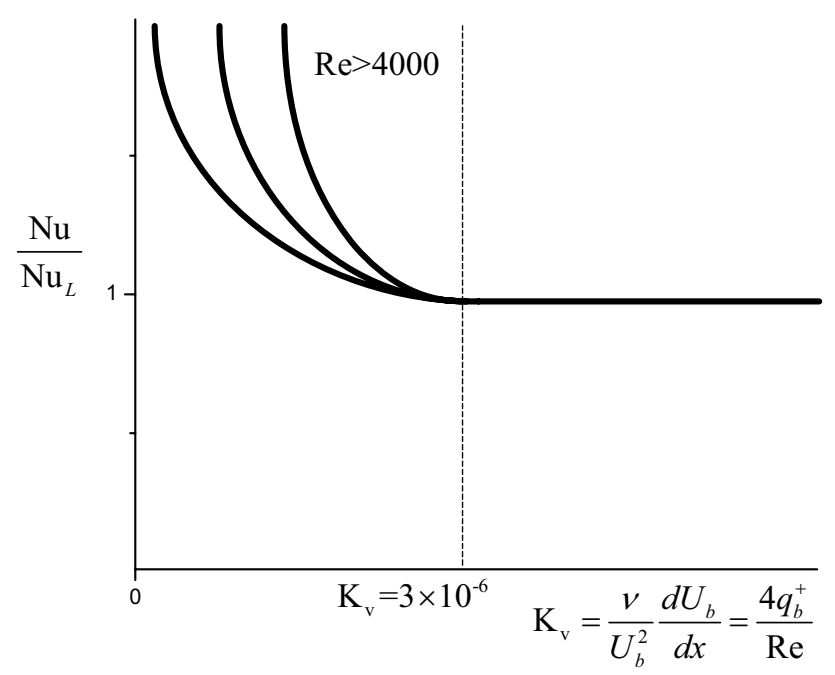

Figure 2-5 "Laminarization" of the Turbulent Heat Transfer due to the Acceleration Effect

The acceleration number was covered in detail in $2^{\text {nd }}$ annual report [Lee et al., 2005c]. The flow acceleration was well studied in the converging channels, where the mean 
velocity of the flow needs to increase due to the decrease in the flow area. The strongly heated flow, where the density decrease due to the heating is accompanied by the increase of the mean flow velocity, exhibits similar behavior as converging channels. Therefore, strongly heated flow also can have acceleration effect and the turbulent flow can be "laminarized". Figure 2-6 shows the similarity of the converging channel and the strongly heated flow.
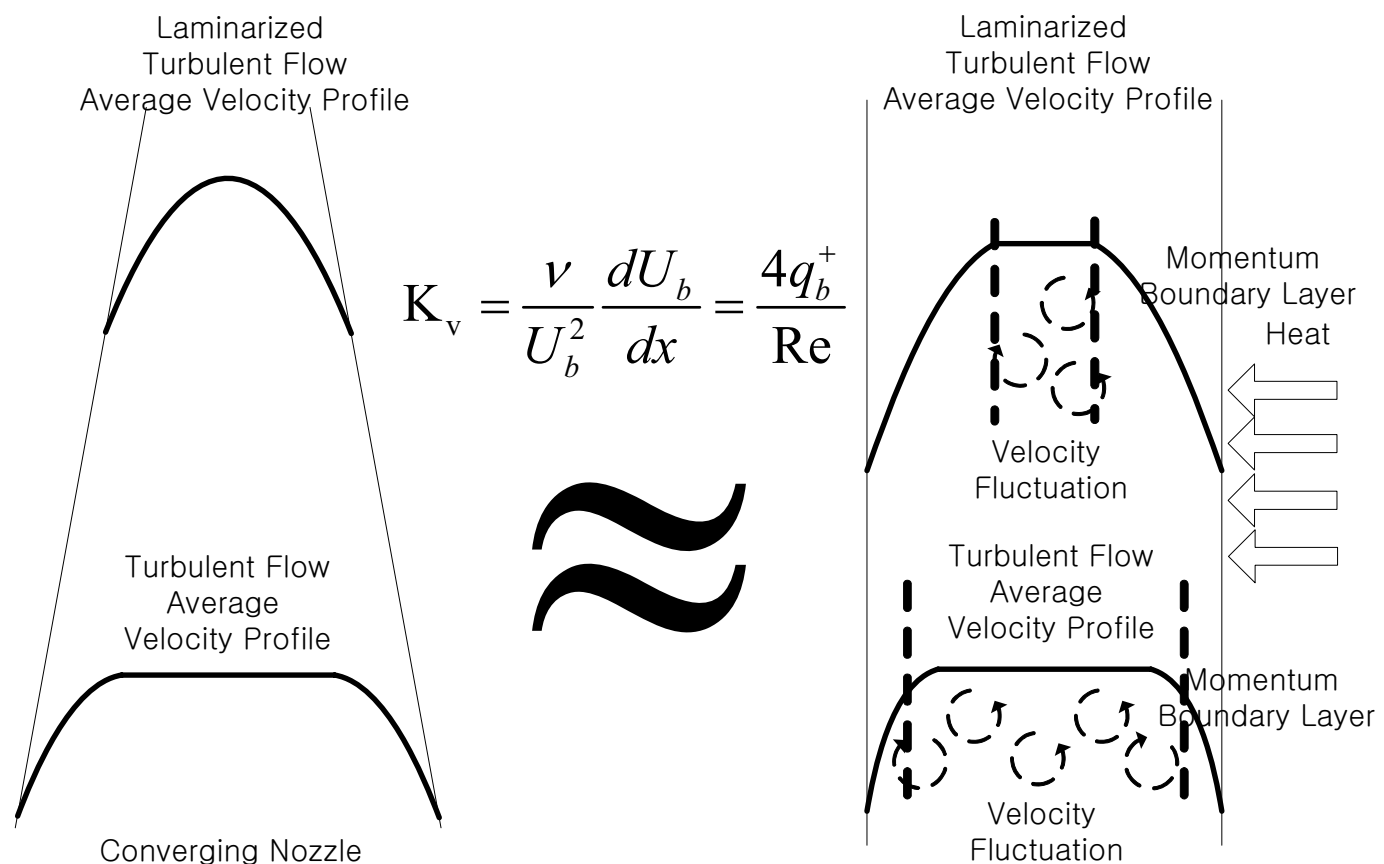

Figure 2-6 Acceleration Effect on the Turbulent Flow

The threshold values for both effects to move the forced turbulent heat transfer to the DTHT regime are indicated in Figure 2-4 and 2-5 $\left(B o_{t h}^{*} \sim 6 \times 10^{-7}\right.$ and $\left.K_{v} \sim 3 \times 10^{-6}\right)$ The threshold values are adopted from McEligot and Jackson presentation (2004), which summarizes the two different physical effects well: (1) Flow acceleration (2) Buoyancy effect. The regime, where normal turbulent convective heat transfer is hindered by either of these two phenomena to cause drastic reduction of the fluid's heat transfer capability (as shown in Figure 2-4 and 5), is called deteriorated turbulent heat transfer regime. Even though the onset of this regime due to the acceleration and buoyancy effects is well defined, the heat transfer coefficient for this regime did not reach agreement among the researchers, as it will be shown in the following sections.

\subsubsection{Laminar Convection with Large Buoyancy Effect}

\section{Hallman (1961)}


In his earlier paper [Hallman, 1955], Hallman solved the fully developed laminar velocity and temperature profile analytically when the forced and free convection are combined. Furthermore, he concluded that the governing non-dimensional number is the Rayleigh number defined with the axial temperature gradient (Eq. 2-11). If we transform the axial temperature gradient as a function of heat flux by applying heat balance, the Hallman's definition of the Rayleigh number can be represented with the combination of the following numbers (Eq. 2-11).

$$
\begin{aligned}
\mathrm{Ra}_{d T_{b} / d x} & =\frac{\rho^{2} \beta g c_{p} D^{4}}{16 \mu k} \frac{d T_{b}}{d x}=\frac{\rho^{2} \beta g c_{p} D^{4}}{16 \mu k} \frac{\pi D q_{w}^{\prime \prime}}{\rho U_{b} c_{p} \frac{\pi}{4} D^{2}} \\
& =\frac{\rho \beta q_{w}^{\prime \prime} g D^{3}}{4 \mu k U_{b}}=\frac{g \beta q_{w}^{\prime \prime} D^{4}}{k v^{2}} \frac{\rho v^{2}}{4 \mu U_{b} D}=\frac{\mathrm{Gr}_{q}}{4 \operatorname{Re}}
\end{aligned}
$$

Hallman's work in 1961 presented some experimental data taken with water and a modified heat transfer correlation based on his theoretical development in 1955 (Eq. 212). The correlation is valid between $100 \leq \mathrm{Ra}_{d T_{b} / d x}=\mathrm{Gr}_{q} / 4 \mathrm{Re} \leq 10,000$.

$$
\mathrm{Nu}_{\text {Hallman }}=1.40 \mathrm{Ra}_{d T_{b} / d x} 0.28=1.40\left(\frac{\mathrm{Gr}_{q}}{4 \mathrm{Re}}\right)^{0.28}
$$

\section{Worsøe-schmidt \& Leppert (1965)}

Worsøe-schmidt and Leppert work is based on numerical analysis. They developed an implicit finite difference scheme for solving the gas laminar flow in heated circular tube with large variations of gas properties. Based on their numerical scheme the friction factor and Nusselt number correlation were given for air as the working fluid (Eq. 2-13). It is noted that they gave the heat transfer and friction factor correlation for uniform wall heat flux and wall temperature condition, but since we are only interested in the uniform heat flux case, only the uniform heat flux correlation is given here.

When $0<q^{*}<10$

$$
\mathrm{Nu}_{\text {Worsoe-schmidt \& Leppert }}=4.36\left[1-\exp \left(-17 x^{+}\right)+a x^{+-\frac{1}{3}} \exp \left(-b x^{+-\frac{5}{4}}\right)\right]
$$

When $10<q^{*}<20$

$$
\begin{aligned}
& \mathrm{Nu}_{\text {Worsoe-schmidt \& Leppert }}=4.36\left[1-\exp \left(-17 x^{+}\right)+c x^{+-\frac{1}{3}} \exp \left(-d x^{+}\right)\right] \\
& \text {where } q^{*}=\frac{D q_{w}^{\prime \prime}}{2 k_{i n} T_{b_{i n}}}, x^{+}=\frac{2 x / D}{\operatorname{Re}_{\text {in }} \operatorname{Pr}_{\text {in }}}, a=1.53+0.11 q^{* 0.4}, b=20+5 \sqrt{q^{*}} \\
& c=1.74+0.011 q^{*}, d=10+2.7 \sqrt{q^{*}}
\end{aligned}
$$


Worsøe-schmidt later performed calculation with this finite difference scheme for helium and carbon dioxide and proposed a heat transfer correlation based on the calculation results [Worsøe-schmidt, 1966] (Eq. 2-14 \& 15).

Air and Helium

When $3<G z<1000,0<q^{*} \leq 20$

$$
\mathrm{Nu}_{\text {Worsoe-schmidt }}=4.36+\frac{0.025 q^{* \frac{1}{2}}(G z-3)(G z-20)}{G z^{3 / 2}}
$$

When $\quad G z<3$

$$
\mathrm{Nu}_{\text {Worsoe-schmidt }}=4.36
$$

Carbon Dioxide

When $10<G z<1000,0<q^{*} \leq 5$

$$
\mathrm{Nu}_{\text {Worsoe-schmidt }}=4.36+\frac{0.07 q^{* \frac{1}{2}}(G z-8)}{G z^{1 / 2}}
$$

When $G z<10$

$$
\mathrm{Nu}_{\text {Worsoe-schmidt }}=4.36
$$

where $G z=\frac{\pi D}{4 x} \operatorname{Re} \operatorname{Pr}$

\section{Churchill (1988)}

Churchill presented a correlation for laminar mixed convection by combining a free convection correlation with a forced convection correlation for uniform heat flux (Eq.2$16)$.

$$
\begin{gathered}
\mathrm{Nu}_{\text {Churchill }}^{6}=\mathrm{Nu}_{F}^{6}+\mathrm{Nu}_{N}^{6} \\
\text { where } \mathrm{Nu}_{F}=\frac{48}{11} \approx 4.364, \mathrm{Nu}_{N}=0.846\left(\mathrm{Ra}_{d T_{b} / d x}\right)^{\frac{1}{4}}=0.846\left(\frac{\mathrm{Gr}_{q}}{4 \mathrm{Re}}\right)^{\frac{1}{4}}
\end{gathered}
$$

The correlation was tested against Hallman's experimental data and some numerical analysis results.

It is rare to find in the vast amount of references different correlations from three correlations presented above. Hallman's correlation (Eq. 2-12) is based on the analytical solution and backed up with the experimental data and Worsøe-schmidt correlations (Eq. $2-14 \& 15)$ are based on the numerical results. Even for the laminar convection with a significant buoyancy effect, which is relatively simpler than the turbulent flow, the governing non-dimensional numbers are different among the various literature sources (Hallman used $\mathrm{Gr}_{q} / 4 \mathrm{Re}$ and Worsøe-schmidt used $x^{+}$and $q^{*}$ ). This means that there is no universal governing non-dimensional number that we can utilize to investigate the 
phenomenon. Thus, a different non-dimensional number can be proposed for the gas heat transfer experiments, since it is hard to find a correlation based on the gas laminar heat transfer experiment with high buoyancy effect.

\subsubsection{Buoyancy Induced DTHT (Mixed Convection)}

This section will be further divided into two subsections. The first section will describe the theoretical development on mixed convection and the second section will briefly summarize the experimental correlations that were identified for different working fluids in the open literature.

\subsubsection{Theories of Turbulent Mixed Convection}

As mentioned in earlier section, there are two thorough theoretical developments in the turbulent mixed convection theory. One is Jackson \& Hall Theory (1969) and the other is Petukhov \& Polyakov theory (1988). However, we will try to focus more on the results and conclusions from the theory rather than the mathematical detail. Figure 2-7 shows the two theories conceptually.

Jackson \& Hall first developed their theory to explain a heat transfer deterioration observed in the super-critical fluid turbulent heat transfer. Their theory is based on the fact that the turbulence is generated by the shear stress near the wall. Since the buoyancy force accelerates the flow near the wall relatively higher than the bulk flow due to higher temperature near the wall than the bulk fluid, the shear stress on the fluid element near the heated wall starts to decrease due to the velocity profile change. As a result, the flow starts to stabilize due to the reduction in the shear stress, which causes decrease in the turbulence generation near the wall and turbulent heat transport. However, after body force reaches certain point, it will start to induce larger shear stress into opposite direction, since the maximum velocity point moves to near the heated wall and steeper velocity gradient is achieved. Therefore, after certain point the buoyancy effect will start to destabilize the flow and generates turbulence and enhances the turbulent heat transfer. More details can be found in [Hall \& Jackson, 1969].

The resulting governing non-dimensional number that was developed within Jackson \& Hall theoretical framework is the buoyancy parameter $B o^{*}$ (Eq. 2-17).

$$
B o^{*}=\frac{\mathrm{Gr}_{q}}{\operatorname{Re}^{3.425} \operatorname{Pr}^{0.8}}
$$



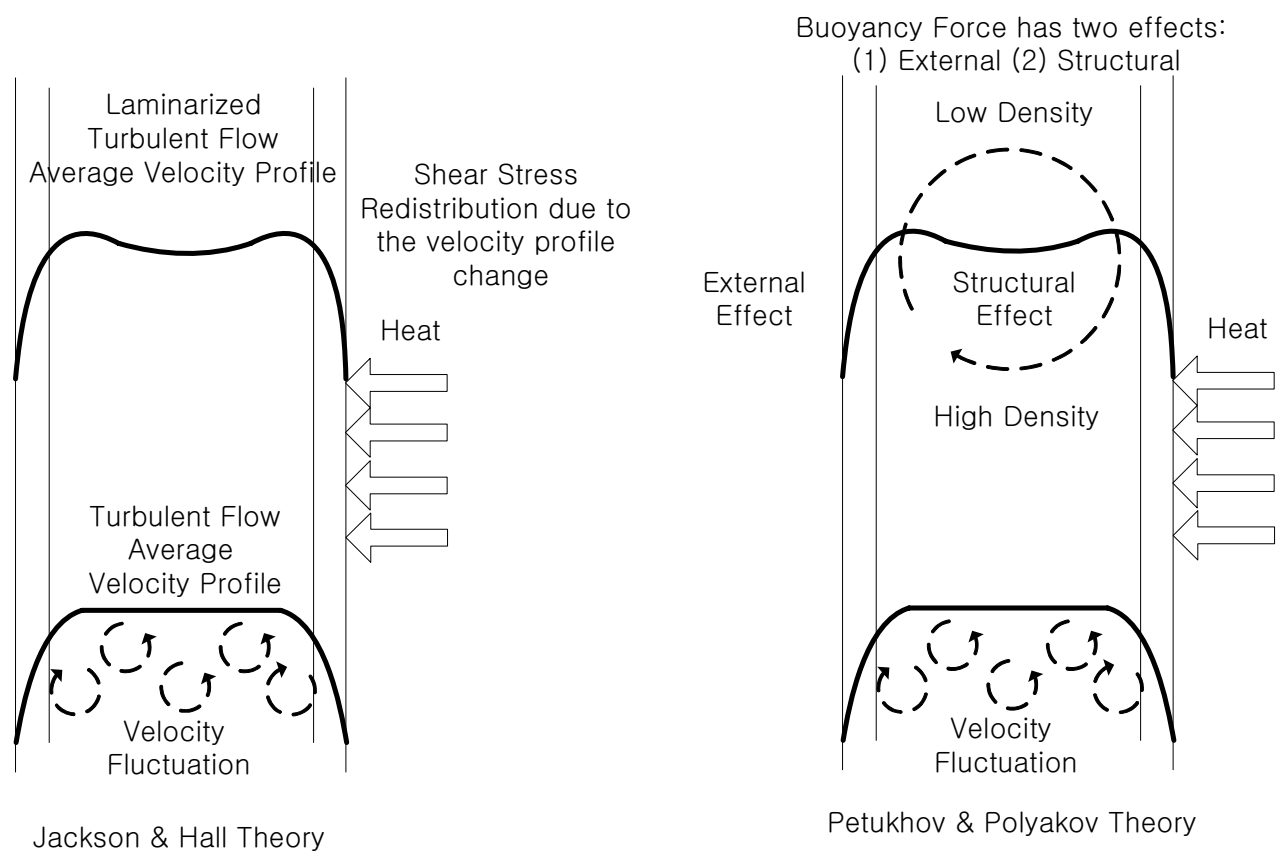

Figure 2-7 Conceptual Diagrams of Two Theories on the Turbulent Mixed Convection

On the other hand, Petukhov \& Polyakov theory adopted concepts of the turbulence interaction with the buoyancy force, which was developed in the meteorology area first [Monin \& Yaglom, 1979]. Petukhov \& Polyakov explain that the heating for upward flow can cause two effects. One is the external effect and the other is the structural effect. The external effect is defined as the mean velocity field change due to the buoyancy force and the structural effect is defined as an additional work for the turbulence to overcome the stabilized density gradient for upward heated flow. For the intermediate heating power, the structural effect is much stronger than the external effect, which leads to decrease in the turbulent intensity due to the additional energy loss of the turbulence to work against the stabilized density distribution. As heating power increases further, the external effect starts to induce steeper velocity gradient and, as a result, more turbulence is generated near the wall. The governing non-dimensional parameter developed within this framework is the buoyancy number $E$ (Eq. 2-18).

$$
E=\frac{\mathrm{Gr}_{q}}{\mathrm{Re}^{4} \operatorname{Pr}}
$$

As a summary, even though two theoretical frameworks have different explanation for the mixed convection effect on the turbulent flow, the resulting non-dimensional number (Eq. 2-17 \& 18) are not that far apart (only slight difference in the power of Reynolds and Prandtl number). Therefore, Hall \& Jackson non-dimensional numbers will be used for explaining the MIT data for buoyancy force induced DTHT cases, since more literature used to Hall \& Jackson parameter to correlate the data to develop correlations. Therefore, from here on, the buoyancy parameter will indicate Hall \& Jackson's parameter. 


\subsubsection{Experimental Correlations}

\section{Petukhov \& Strigin (1968)}

Petukhov \& Strigin developed a correlation based on the water experiment. Their selected non-dimensional number to capture the buoyancy effect was $\mathrm{Ra}_{d T_{b} / d x}$. The experimental data was taken at the Reynolds number from 300 to 30,000, the axial temperature gradient Rayleigh number from 300 to $8 \times 10^{5}$, the Prandtl number from 2 to 6 and $x / D$ below 99. Equation 2-19 gives the correlation. $\mathrm{Ra}_{d T_{b} / d x}$ is transformed in terms of the heat flux based Grashof number and Reynolds number, according to Equation 2-11. All the forced convection Nusselt numbers in this section $\left(\mathrm{Nu}_{F}\right)$ will be calculated from the Gnielinski correlation, even though the original correlations for the mixed convection are developed from different forced convection correlations. This is possible since most of the general forced convection correlations overlie with each other within few percents (e. g. Figure 2-2). However, since this correlation is developed from the forced convection correlation for constant properties without any developing length, Equation 2-20 will be used instead of Equation 2-7.

$$
\begin{aligned}
& \text { When } \mathrm{Ra}_{d T_{b} / d x} / \mathrm{Re}^{2}=\mathrm{Gr}_{q} / 4 \mathrm{Re}^{3}<10^{-4} \\
& \mathrm{Nu}_{\text {Petukhov-Strigin }}=\mathrm{Nu}_{F}\left(1+1.15 \times 10^{4} \frac{\mathrm{Gr}_{q}}{4 \mathrm{Re}^{3}}\right)^{-1} \\
& \text { When } 10^{-4}<\mathrm{Gr}_{q} / 4 \mathrm{Re}^{3}<1 \\
& \mathrm{Nu}_{\text {Petukhov-Strigin }}=\mathrm{Nu}_{F} 10\left(\frac{\mathrm{Gr}_{q}}{4 \mathrm{Re}^{3}}\right)^{\frac{1}{3}} \\
& \mathrm{Nu}_{F}=\frac{(f / 8)(\mathrm{Re}-1000) \mathrm{Pr}}{1+12.7 \sqrt{f / 8}\left(\mathrm{Pr}^{2 / 3}-1\right)} \\
& \text { where } f=\left(1.82 \log _{10} \mathrm{Re}-1.64\right)^{-2}
\end{aligned}
$$

\section{Herbert \& Sterns (1972)}

Herbert \& Sterns obtained data with water in the range of the Reynolds number from 5,800 to 65,000 , the wall to bulk temperature difference based Grashof number $\left(\mathrm{Gr}_{\Delta T}\right)$ from $1.9 \times 10^{7}$ to $2.6 \times 10^{7}$, the Prandtl number from 1.79 to 2.22 and $x / D$ below 80 . Equation 2-21 shows the correlation. 
When $\operatorname{Re} \geq \operatorname{Re}_{a c}=3000+0.00027 \mathrm{Gr}_{\Delta T} \operatorname{Pr}$

$\mathrm{Nu}_{\text {Herbert-Sterns }}=0.0225 \operatorname{Re}^{0.795} \operatorname{Pr}^{0.495-0.0225 \ln (\operatorname{Pr})}$

When $\operatorname{Re}<\operatorname{Re}_{a c}$

$\mathrm{Nu}_{\text {Herbert-Sterns }}=8.5 \times 10^{-2}\left(\mathrm{Gr}_{\Delta T} \operatorname{Pr}\right)^{1 / 3}$

\section{Petukhov \& Polyakov (1988)}

From the theoretical development, which was presented in the previous section, semiempirical correlation (Equation 2-22) was developed. The validity of the correlation is the Reynolds number above 3,000, the heat flux based Grashof number below $10^{11}$ and $x / D$ above 40 . The correlation was tested against experimental data from other literature sources. However, some questions still remain, since the experimental data chosen to validate the correlation were not tabulated in the original reference.

$$
\begin{aligned}
& \mathrm{Nu}_{\text {Petukhov-Polyakov }}=\frac{f}{8} \operatorname{Re} \operatorname{Pr}\left[\frac{1+0.83 e^{2}}{1+0.042 e^{2}\left[E^{1 / 4} \log _{10}(\mathrm{Re} / 8)\right]^{-1}}\right. \\
& \left.+12.7 \sqrt{\frac{f}{8}}\left\{\operatorname{Pr}^{2 / 3}\left(1+\frac{0.72 e^{3}(1+0.28 \sqrt{e})}{1+0.43 e^{4}}\right)-\frac{1+0.58 e^{2}}{1+0.83 e^{2}}\right\}\right]^{-1} \\
& \text { where } e=\frac{10^{3} \mathrm{Gr}_{q}}{\operatorname{Re}^{2.75} \operatorname{Pr}}, \quad f=\left[\frac{1+0.83 e^{2}}{1.82 \log _{10}(\mathrm{Re} / 8)+0.076 e^{2} E^{-0.25}}\right]^{2}
\end{aligned}
$$

\section{Jackson et al. (1989)}

A review paper on mixed convection in vertical tubes written by Jackson et al. in 1989 summarizes the large body of research work performed in the area until then. Equation 223 gives the correlation that was suggested in their review.

$$
\frac{\mathrm{Nu}_{\text {Jackson }}}{\mathrm{Nu}_{F}}=\left(1-\frac{8 \times 10^{4} B o^{*}}{\left(\mathrm{Nu}_{\text {Jackson }} / \mathrm{Nu}_{F}\right)^{2}}\right)^{0.46}
$$

The limitation of the correlation is not explicitly described in the paper. However, experimental data with water, air, mercury and super critical carbon dioxide are presented and the range of buoyancy parameter $\left(B \mathrm{O}^{*}\right)$ spans from $10^{-7}$ to $10^{-2}$. It should be noted that the correlation is discontinuous near $B o^{*} \sim 3 \times 10^{-6}$ and the correlation form is implicit. 


\section{Parlatan (1989)}

Parlatan developed a correlation (Eq. 2-24) based on his experimental data. The experiments were performed with water in the Reynolds range of 4,000 9,000 and buoyancy number of $B o^{*}<1.25 \times 10^{-5}$.

When $B o^{*}<1.875 \times 10^{-6}$

$\frac{\mathrm{Nu}_{\text {Parlatan }}}{\mathrm{Nu}_{F}}=1+6.104 \times 10^{3} B o^{*}-2.1768 \times 10^{6} B^{* 2}$

When $1.875 \times 10^{-6}<B o^{*}<1.25 \times 10^{-5}$

$\frac{\mathrm{Nu}_{\text {Parlatan }}}{\mathrm{Nu}_{F}}=1+1.768 \times 10^{4} B o^{*}-1.608 \times 10^{4} B o^{* 2}$

Vilemas et al. (1992)

Vilemas et al. performed experiment with air in mixed convection regime and developed correlation based on their data (Eq. 2-26). The experimental data range covers the inlet Reynolds number from 3,000 to 50,000 , inlet $q^{+}$from 0.00035 to 0.0024 and a new buoyancy parameter defined in Eq. (2-25) from $8 \times 10^{-6}$ to $3.37 \times 10^{-3}$ (inlet values).

$$
K=\frac{\mathrm{Gr}_{q}}{4 \mathrm{Re}^{3} \mathrm{Pr}}
$$

When $K_{i n}<K_{i n 1}$

$$
\begin{aligned}
\mathrm{Nu}_{\text {Villemas }} & =\mathrm{Nu}_{F} \\
\text { When } K_{\text {in } 1} & <K_{\text {in }}<K_{\text {in } 2} \\
\mathrm{Nu}_{\text {Villemas }} & =\frac{\mathrm{Nu}_{F}}{0.98+0.54 K_{\text {in }}^{0.25}(x / D)^{0.975}}
\end{aligned}
$$

When $K_{\text {in } 2}<K_{\text {in }}<K_{\text {in } 3}$

Not Available

When $K_{\text {in } 3}<K_{\text {in }}$

If $0<x / D<x_{\min } / D$

$$
\begin{aligned}
\mathrm{Nu}_{\text {Villemas }} & =\mathrm{Nu}_{F}\left[11.3 K_{\text {in }}^{1 / 3}+\left(5.39 \times 10^{-9}+5.12 \times 10^{-4} \ln \left(K_{\text {in }}\right)\right)\left(\frac{x}{D}-\frac{x_{\text {min }}}{D}\right)^{2}\right] \\
\text { If } x_{\text {min }} / D \leq x / D & \leq x_{\text {max }} / D \\
\mathrm{Nu}_{\text {Villemas }} & =\mathrm{Nu}_{F}\left[11.3 K_{\text {in }}^{1 / 3}+\left(3.865 \times 10^{-9}+3.672 \times 10^{-4} \ln \left(K_{\text {in }}\right)\right)\left(\frac{x}{D}-\frac{x_{\text {min }}}{D}\right)\right]
\end{aligned}
$$




$$
\begin{gathered}
\text { If } x_{\max } / D<x / D \\
\mathrm{Nu}_{\text {Villemas }}=\mathrm{Nu}_{F}\left[14.5 K_{\text {in }}^{1 / 3}-3.555 K_{\text {in }}^{0.92} q_{\text {in }}^{+0.26}\left(\frac{x}{D}-\frac{x_{\max }}{D}\right)^{0.46}\right] \\
\text { where } K_{\text {in } 1}=2-4 \times 10^{-6}, K_{\text {in } 2}=2.5 \times 10^{-5}, K_{\text {in } 3}=1.5 \times 10^{-3} q_{\text {in }}^{+0.45} \\
x_{\text {min }} / D=5.19+0.0059 / K_{\text {in }}^{0.9}, x_{\text {max }} / D=8.8+0.063 / K_{\text {in }}^{0.7}
\end{gathered}
$$

It should be noted that this correlation has a gap in the area of $K_{i n 2}<K_{i n}<K_{i n 3}$ due to the high sensitivity of the flow for a small change in buoyancy parameter. Therefore, when we evaluate the Nusselt number in this region to compare to the MIT data we will take the $K_{i n 1}<K_{i n}<K_{i n 2}$ region Nusselt number as an approximate value for $K_{\text {in } 2}<K_{\text {in }}<K_{\text {in } 3}$ region.

\section{Celeta et al. (1998) and Aicher \& Martin (1996)}

Celeta et al. correlation combined Aicher \& Martin's work (1996) with Jackson \& Hall work. When Aicher \& Martin were developing a correlation, they introduced rather a new idea to this area, which was not shown in the previous works. They correlated the upward heated flow Nusselt number with the downward heated flow Nusselt number to get a smooth functional form. This idea is later adopted by Celeta et al. who modified Aicher $\&$ Martin's correlation form to fit their data better and included $x / D$ effect, which was mentioned in Aicher \& Martin's work but not included in their correlation. Aicher \& Martin's correlation is not shown here, since Celeta et al. correlation encompasses Aicher $\&$ Martin's work as they collected larger amount of data than Aicher \& Martin originally did. After Celeta et al. obtained the functional form by modifying Aicher \& Martin's idea; they utilized the buoyancy parameter $\left(B o^{*}\right.$, developed by Jackson \& Hall) as their governing non-dimensional number. Equation 2-27 is the correlation form. It should be noted that Celeta et al. originally developed the correlation by using the Dittus-Boelter correlation with the water properties variation correction factor as a forced convection Nusselt number. Therefore, within this study, we will use the Gnielinski correlation with the gas properties variation correction factor as our forced convection Nusselt number when we evaluate the Celeta et al. Nusselt number.

$$
\begin{aligned}
& \mathrm{Nu}_{\text {Celeta }}=\sqrt{\mathrm{Nu}_{F}^{2}+\mathrm{Nu}_{N}^{2}}\left\{1-\left(0.36+0.0065 \frac{x}{D}\right) \exp \left(-0.8 \ln \frac{8 \times 10^{4} B o^{*}}{869(x / D)^{-2.16}}\right)\right\} \\
& \text { where } \mathrm{Nu}_{F}=\frac{(f / 8)(\mathrm{Re}-1000) \mathrm{Pr}}{1+12.7 \sqrt{f / 8}\left(\operatorname{Pr}^{2 / 3}-1\right)}\left(\frac{T_{w}}{T_{b}}\right)^{-0.45}, \mathrm{Nu}_{N}=\frac{0.15\left(\mathrm{Gr}_{\Delta T, w} \operatorname{Pr}_{w}\right)^{1 / 3}}{\left(1+\left(\frac{0.437}{\operatorname{Pr}_{w}}\right)^{9 / 16}\right)^{16 / 27}}
\end{aligned}
$$

The limitation of the correlation is not explicitly described in Celeta et al. work. However, 
the experimental data covers the Reynolds number from 800 to 23,000 , the buoyancy parameter $B o^{*}<0.156$ and $x / D<60$.

\section{Symolon et al. (2005)}

Symolon et al. correlation (Eq. 2-29) is also based on the water experiment. The range of the data cover the Reynolds numbers from 2,600 to 70,000, $x / D$ up to 90 and a new buoyancy number $(B o)$ (Eq. 2-28) below $10^{-3}$. Since, the correlation was developed from the Dittus-Boelter correlation without the thermal properties modification factor, Eq. $(2-20)$ will be used to evaluate the forced convection Nusselt number.

$$
\begin{gathered}
B o=\frac{\mathrm{Gr}_{\Delta T}}{\operatorname{Re}^{3} \operatorname{Pr}^{0.5}} \\
\mathrm{Nu}_{\text {Symolon }}=\mathrm{Nu}_{F}\left[\left(\frac{\left(\frac{5.9 \times 10^{-6}}{B o}\right)^{2.174}}{1+\left(\frac{5.9 \times 10^{-6}}{B o}\right)^{2.174}}\right)^{5}+\left(76.08 B o^{0.426}\right)^{5}\right]^{0.2}
\end{gathered}
$$

As a brief summary of this section, eight correlations were selected from the literature and will be tested against the MIT data to see which correlation performs the best with the gas heat transfer. Interesting fact is that except for the Vilemas et al. and Jackson et al. correlations (Villemas et al. used air and Jackson et al. showed some air data along with the supercritical fluid and water data), most of the correlations in the turbulent mixed convection regime are based on the liquid experiments. Another fact is that the buoyancy parameter developed by Jackson \& Hall is the most frequently used parameter for explaining the turbulent mixed convection phenomenon (e .g. Jackson et al., Parlatan and Celeta et al.). However, the correlation form is very different for each correlation presented in this section and most of the time even the governing non-dimensional parameter is different. This leads us to a conclusion that even though the turbulent mixed convection was studied extensively, the investigators have not reached final agreement on a correlation form or a governing non-dimensional number. This is more critical problem for the gas heat transfer cases, since turbulent heat convection experimental data for pressurized gas can be rarely found.

Literature search have shown that it is hard to find experimental heat transfer data for a pressurized gas flow for any regime, i.e. forced, mixed and to free convection. Therefore, the MIT loop data will also expand the database in the regions with lack of data and hopefully will serve to further enhance the understanding of the forced convection and mixed convection turbulent flow behavior and applicability of correlations for the pressurized gas systems. 


\subsubsection{Acceleration Induced DTHT}

Even though the "laminarization" process was known for a half century, heat transfer correlation that has explicit acceleration effect and can successfully predict the Nusselt number in this regime is rarely found. This is because most literature sources focus more on the threshold value for the acceleration driven laminarization process, rather than on the development of heat transfer correlations. Since, most of the literature for the development of the acceleration parameter threshold was covered in the $2^{\text {nd }}$ annual report [Lee et al., 2005c], this section will just add few more heat transfer correlations that have a potential to be related to the acceleration induced DTHT. This means that any heat transfer correlation that was developed with a gas heat transfer experiment, with high heat loads and relatively low Reynolds number are going to be covered in this short section as much as possible.

\section{McEligot (1963)}

The experimental data were collected in the Reynolds number from 1,500 to 200,000 and $q^{+}$up to 0.006 . For the low Reynolds number runs this is equivalent to $K_{v} \sim 10^{-6}$, which is near the threshold value for the acceleration induced DTHT. In his report, a preliminary correlation was developed for such cases (Eq. 2-30).

$\mathrm{Nu}_{\text {McEligot }_{\text {Acc }}}=0.021 \mathrm{Re}_{\text {inlet }}^{-1.2} \operatorname{Re}^{2} \operatorname{Pr}^{0.4}$

This correlation is valid for the Reynolds number between 1,500 and 8,000, $q^{+}$from 0.004 to 0.006 and $x / D$ from 15 to 49 . If we transform this validation range in terms of the acceleration effect by using the definition of acceleration parameter, the range of $K_{v}$ is from $5 \times 10^{-7}$ to $4 \times 10^{-6}$. For the Reynolds number higher than 15,000 and $q^{+}$lower than 0.004 , the Nusselt number can be successfully predicted with Eq. 2-4. The data were obtained with air, helium and nitrogen.

\section{Perkins \& Worsøe-schmidt (1965)}

The experimental data were obtained at maximum wall to bulk temperature ratio up to 7.5 and minimum exit Reynolds number 4,300 with nitrogen. This indicates that the heat load was exceptionally high, even though they didn't indicate $q^{+}$value. Thus, it is reasonable to think that their data might have entered acceleration DTHT regime. The correlation that was developed with the data is shown in Eq. 2-31. 
When $x / D<40$

$\mathrm{Nu}_{\text {Perkins-Worsoe-schmidt }}=0.024 \operatorname{Re}^{0.8} \operatorname{Pr}^{0.4}\left(\frac{T_{w}}{T_{b}}\right)^{-0.7}\left[1+\left(\frac{x}{D}\right)^{-0.7}\left(\frac{T_{w}}{T_{b}}\right)^{0.7}\right]$

When $x / D>40$

$\mathrm{Nu}_{\text {Perkins-Worsoe-schmidt }}=0.024 \operatorname{Re}^{0.8} \operatorname{Pr}^{0.4}\left(\frac{T_{w}}{T_{b}}\right)^{-0.7}$

This correlation is valid for the inlet Reynolds number from 4,300 to 359,000 and $x / D$ below 144.

\section{Taylor (1965)}

Taylor's work was also selected, since the maximum wall to bulk temperature ratio that he had achieved is around eight and the Reynolds number range was from 5,700 to 48,400 . He used pre-cooled hydrogen and helium as working fluids. The correlation developed from the experimental data is shown in Equation 2-32.

$\mathrm{Nu}_{\text {Taylor }}=0.021 \operatorname{Re}^{0.8} \operatorname{Pr}^{0.4}\left(\frac{T_{w}}{T_{b}}\right)^{-[0.29+0.0019 x / D]}$

The validity of the correlation holds for $x / D$ below 250 . 


\section{Preliminary Analysis of GFR DHR system}

In this chapter, the two effects responsible for heat transfer deterioration will be briefly discussed in addition to Chapter 2. A newly developed methodology for the preliminary analysis will be presented first. This will be followed by an analysis of GFR decay heat removal (DHR) system to evaluate which effects would play key role in the GFR DHR system performance. Lastly, some observations of the behavior of different fluids using the analysis methods developed in this chapter will be presented.

\subsection{Onset of DTHT Regime}

\subsubsection{Acceleration Effect}

The Acceleration effect is also known as a "laminarization due to the favorable pressure gradient". The decrease in turbulence transport occurs whenever a flow directional acceleration exceeds a certain value. The stream wise acceleration can be quantified by using the definition of acceleration parameter, $K_{v}=\frac{v}{U_{b}^{2}} \frac{d U_{b}}{d x}$. In a heated system, the acceleration occurs from gas expansion due to heating. This effect is different from the buoyancy effect, since the effect is not related to the gravitational force. When a flow condition satisfies the onset of laminarization due to the acceleration, the heat transfer coefficient of the fluid quickly drops to the laminar heat transfer coefficient value (see Figure 2-5), even though the Reynolds number is well above the adiabatic turbulent flow criterion. Applying energy balance and continuity equation with perfect gas and constant cross section assumptions to the original definition of $K_{v}$, an alternative definition of acceleration parameter can be obtained for the heated case in the form of nondimensional number $K_{v} \approx \frac{4 q^{+}}{\operatorname{Re}}$ [McEligot et. al., 1969].

Based on McEligot \& Jackson work (2004), we can normalize the non-dimensional number that represents acceleration effect with the onset criterion $\left(N K_{v}=K_{v} / 3 \times 10^{-6}\right)$. This is done in order to determine that whenever the normalized value exceeds one, it indicates that the heat transfer regime is changing from normal forced convection regime to the DTHT regime due to the acceleration effect (Eq. 3-1). Equation 3-1 also separates the normalized acceleration parameter into two groups. We will designate the first one as the controlled group $\left(C K_{v}\right)$, because it involves geometry, heat flux and the volumetric flow rate. The second one will be called the fluid properties group $\left(P K_{v}\right)$, because it depends on system conditions, such as system pressure and operating temperature and it is not controlled directly. Later in this chapter, analysis will be focused on the properties 
group to determine which system pressure and operating temperature will have higher impact on the heat transfer fluids entrance into the DTHT regime.

$$
N K_{v}=\frac{K_{v}}{3 \times 10^{-6}}=\frac{4 q^{+}}{3 \times 10^{-6} \operatorname{Re}}=\left(\frac{q^{\prime \prime} A P_{w}}{Q^{2}}\right)\left(\frac{\mu \beta}{3 \times 10^{-6} c_{p} \rho^{2}}\right)=C K_{v} \cdot P K_{v}
$$

\subsubsection{Buoyancy Effect}

Buoyancy effect originates from the density gradient due to heating, and reduces the turbulent heat transport when the flow direction is the same as the Buoyancy force. Hall \& Jackson (1969) explained the decrease in turbulence by the shear stress re-distribution in the flow. Contrary to Hall \& Jackson (1969), according to Petukhov \& Polyakov (1988) the phenomenon is governed by two terms that are competing in the turbulent energy equation. One is the velocity gradient and the other is the turbulence work that needs to be provided to work against stable density gradient. Two theories were explained in more detail in Chapter 2. Nevertheless, the onset of buoyancy induced DTHT criterion form is similar in both cases. Therefore, in this report we will follow McEligot and Jackson presentation (2004), which is an extended version of Hall and Jackson (1969) discussion.

Similar to the acceleration effect, the buoyancy group will be normalized with the onset criterion $\left(N B o^{*}=B o^{*} / 6 \times 10^{-7}\right)$. Similarly as for the case of acceleration effect, the buoyancy effect can be viewed as a multiplication of control group $\left(C B o^{*}\right)$ and properties group $\left(P B o^{*}\right)($ Eq. 3-2). The buoyancy properties group will be used to provide information on which system pressure and operating temperature combination will have a stronger influence on the system to operate in DTHT regime.

$$
\begin{aligned}
N B o^{*} & =\frac{B o^{*}}{6 \times 10^{-7}}=\frac{\mathrm{Gr}_{q}}{6 \times 10^{-7} \operatorname{Re}^{3.425} \operatorname{Pr}^{0.8}} \\
& =\left(\frac{q^{\prime \prime} A^{4}}{Q^{3.425} P_{w}^{0.575}}\right)\left(\frac{g \mu^{0.625} \beta}{2.704 \times 10^{-7} c_{p}^{0.8} k^{0.2} \rho^{1.425}}\right)=C B o^{*} \cdot P B o^{*}
\end{aligned}
$$

\subsection{Simple Analysis of GFR DHR System Design}

Using Equations (3-1) and (3-2), one can perform a simple parametric study without exact design parameters (e. g. geometry, heat flux, flow rate etc.) and only with the gas properties to investigate general trend of the gases in various operating conditions. Since two GFR coolants under consideration are helium and carbon dioxide, the two gases are evaluated. Figure 3-1 and Figure 3-2 show both helium and carbon dioxide acceleration properties groups and buoyancy properties groups for different pressures and temperatures, respectively. 


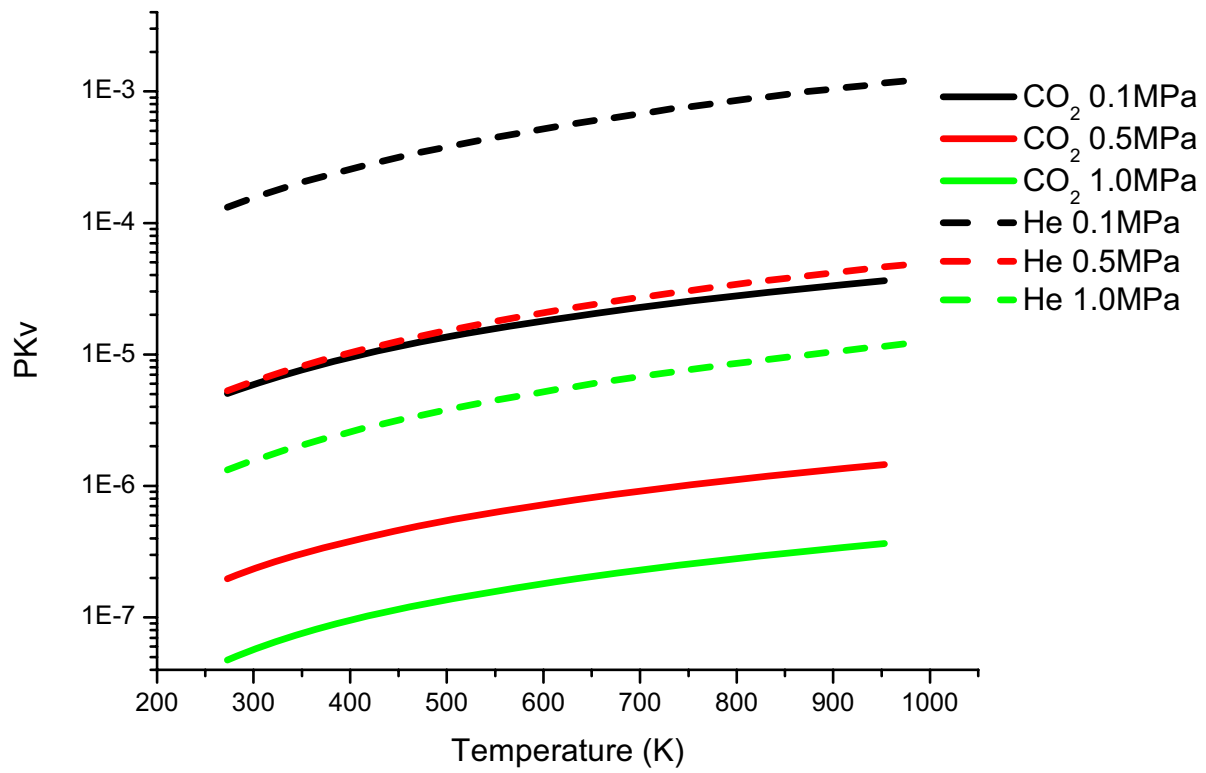

Figure 3-1 Acceleration Properties Group

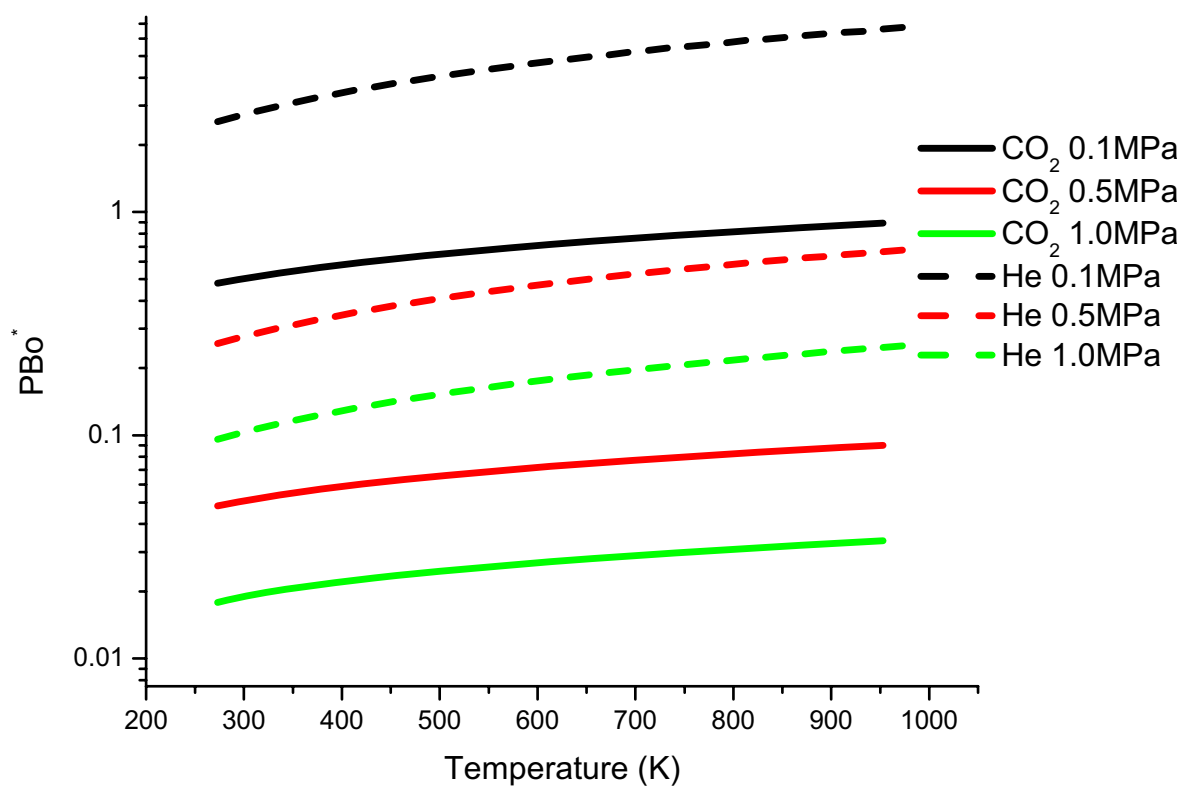

Figure 3-2 Buoyancy Properties Group

It is clear from the figures that as temperature increases and pressure decreases, helium and carbon dioxide properties groups increase. This indicates that the normal gas turbulent heat transfer has stronger tendency to fall into DTHT regime when the system depressurizes and the operating temperature increases. This situation occurs in the GFR system during the loss of coolant accident. Therefore, both acceleration and buoyancy DTHT criteria need to be checked when designing the GFR DHR system. 
The above figures also show that for the same controlled variable group, helium is more susceptible to the DTHT regime than carbon dioxide. Thus, more attention is needed when designing a helium-cooled system.

Figure 3-3 shows one of the possible operating ranges of a GFR DHR system calculated with an in-house code LOCA-COLA [Williams et al., 2003]. The details on LOCACOLA simulation and the results of GFR DHR are documented well in the scaling analysis report [Cochran et. al., 2004a].

The Reynolds number is normalized to 4,000 where fully developed adiabatic turbulent flow regime starts, and normalized DTHT parameters are $N K_{v}$ and $N B o^{*}$. Curves in Figure 3-3 show the axial variation of $N K_{v}$ and $N B o^{*}$ in a channel for given system pressure and heated diameter of the hot channel with helium and carbon dioxide as an operating fluid.

Figure 3-3 shows that there is a possibility for GFR DHR system to operate not only in the DTHT regime but also in the laminar to turbulent transition regime. The laminar to turbulent transition criteria is not fully understood even in the adiabatic flow situation and a complete set of study for heated flow is rarely found [Lee at al., 2005b].

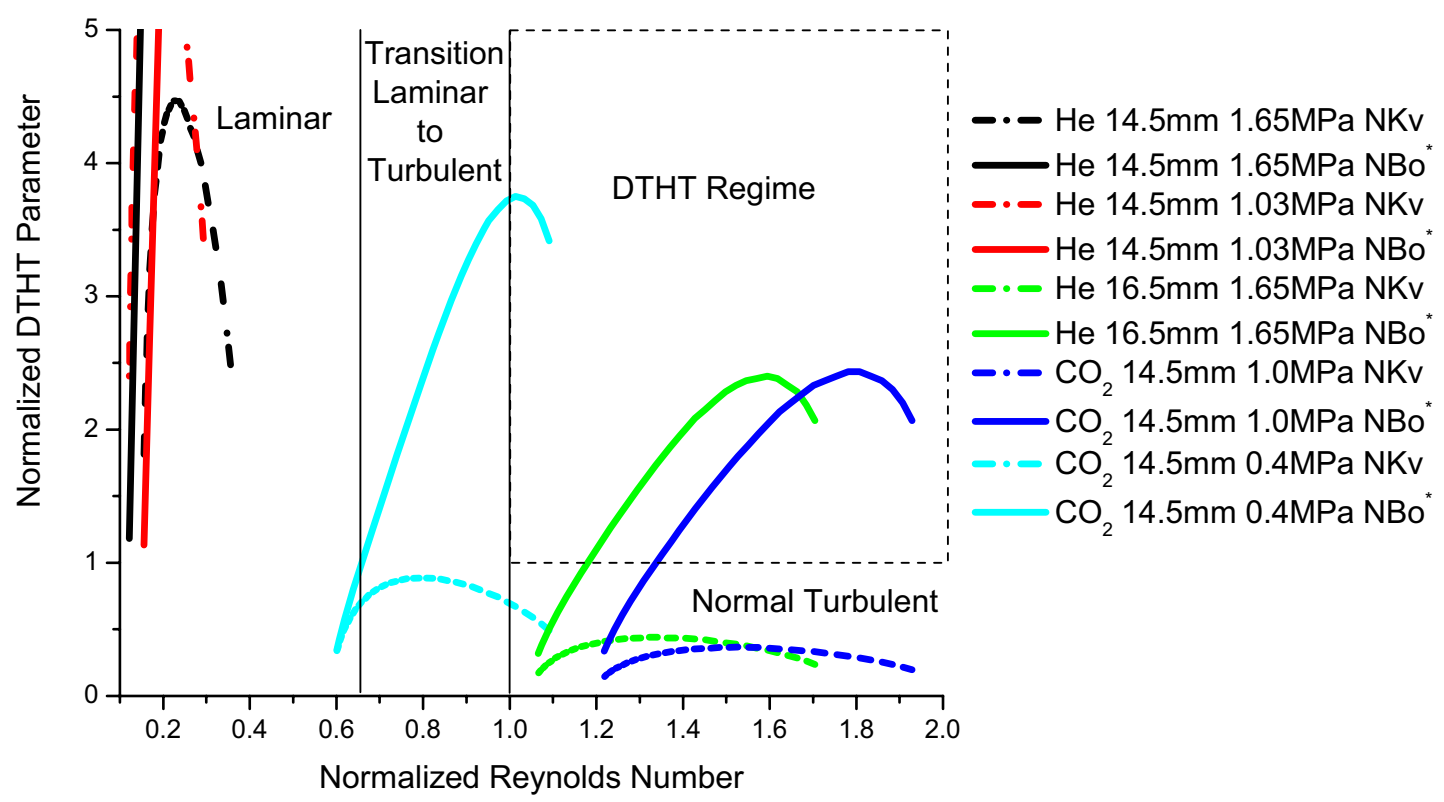

Figure 3-3 GFR DHR Operation Range

As was mentioned before, the general heat transfer coefficient and friction factor in DTHT regime and all the transitions from normal turbulent flow to the DTHT regime is not well defined yet. Therefore, experimental studies in these heat transfer regimes are necessary to develop reliable correlation for the design of GFR DHR system. 


\subsection{Behavior of $\mathrm{Bo}^{*}$ and $K_{v}$ for Different Fluids}

In this subsection, behavior of the buoyancy parameter and the acceleration parameter during the experimental runs will be discussed. Since, the MIT experimental facility is a single channel, Equation 3-1 and 3-2 needs to be rearranged to fit for the single channel analysis. This is because along the channel the mass flow rate is constant due to the mass conservation, rather than the volumetric flow rate. Equation 3-3 and 3-4 are the rearranged equations of Eqs. 3-1 and 3-2.

$$
\begin{aligned}
& N B o^{*}=\left(\frac{q^{\prime \prime} A^{4}}{\dot{m}^{3.425} P_{w}^{0.575}}\right)\left(\frac{\rho^{2} g \mu^{0.625} \beta}{2.704 \times 10^{-7} c_{p}^{0.8} k^{0.2}}\right)=C_{\dot{m}} B o^{*} \cdot P_{\dot{m}} B o^{*} \\
& N K_{v}=\left(\frac{q^{\prime \prime} A P_{w}}{\dot{m}^{2}}\right)\left(\frac{\mu \beta}{3 \times 10^{-6} c_{p}}\right)=C_{\dot{m}} K_{v} \cdot P_{\dot{m}} K_{v}
\end{aligned}
$$

Figure 3-4 and 3-5 plot the buoyancy properties group and acceleration properties group behaviors of nitrogen at three different pressures with varying temperature. Since, most of the gas thermal properties behave similarly with the temperature, nitrogen is chosen as an exemplary case.

First observation is that if we compare Figure 3-1 to 3-4 and Figure 3-2 to 3-5, the trend is reversed. This is because the density term in the properties group moved to the controlled group side (since $\dot{m}=\rho Q$ ). Since bulk temperature of the fluid will increase as it flows toward the downstream due to the heating, it can be predicted from the figures that both the buoyancy parameter and the acceleration parameter of the gas flow will decrease along the downstream. Therefore, it can be concluded that the maximum buoyancy number and acceleration number for the gas flow will be at the inlet of the channel when the control group is fixed as a constant. The control group is a constant for the MIT facility since the test section has circular tube shape with the same diameter along the channel and is operated with uniform heat flux.

Another interesting observation is that the buoyancy parameter properties group of nitrogen increases with the pressure while the acceleration parameter properties group is pressure-independent. This indicates that by increasing the operating pressure of the facility, the buoyancy effect will increase relative to the acceleration effect. 


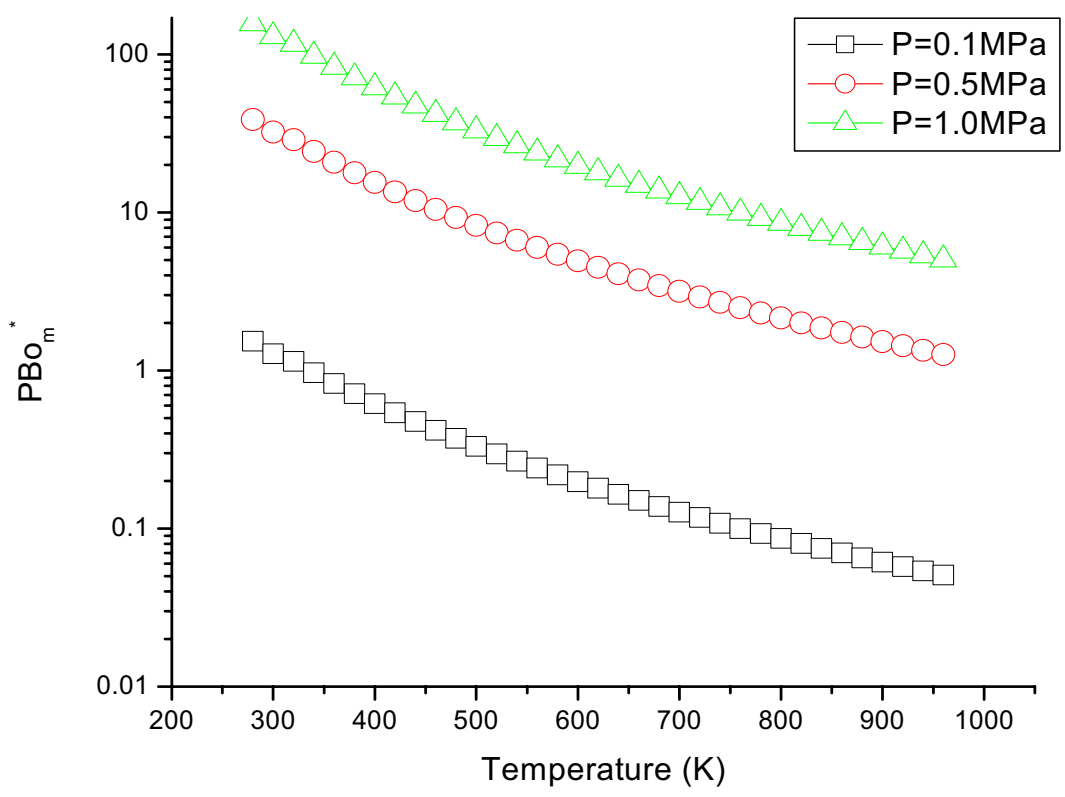

Figure 3-4 Nitrogen Buoyancy Properties Group

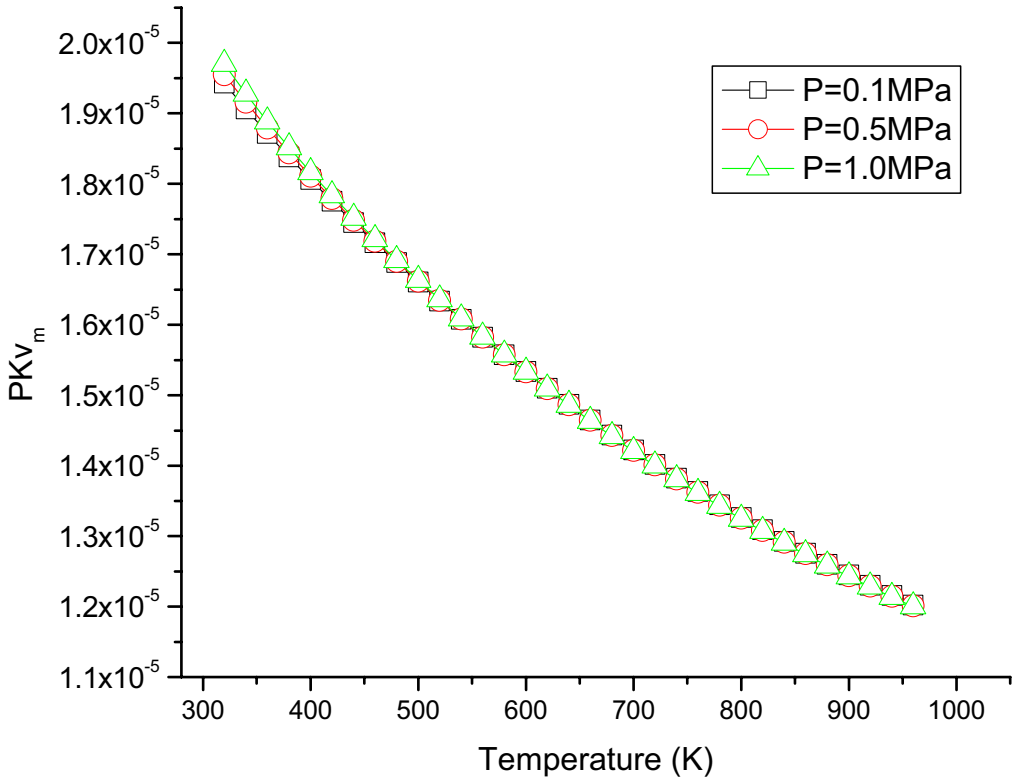

Figure 3-5 Nitrogen Acceleration Properties Group

As it was summarized in Chapter 2, various correlations were developed from the experimental data collected with working fluid of water and super critical phase fluid. Thus, it is of interest to check how these fluids behave compared to gases. Figures 3-6 to 3-9 show the properties group trends for liquid water and super critical $\mathrm{CO}_{2}$. 


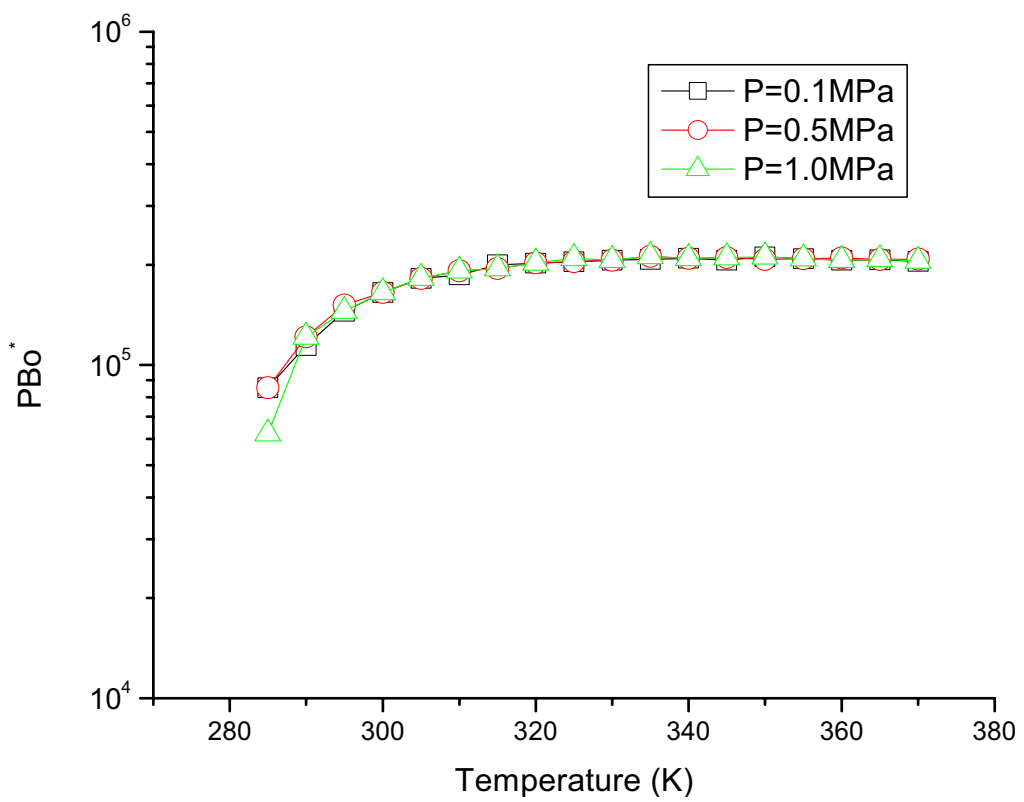

Figure 3-6 Liquid Water Buoyancy Properties Group

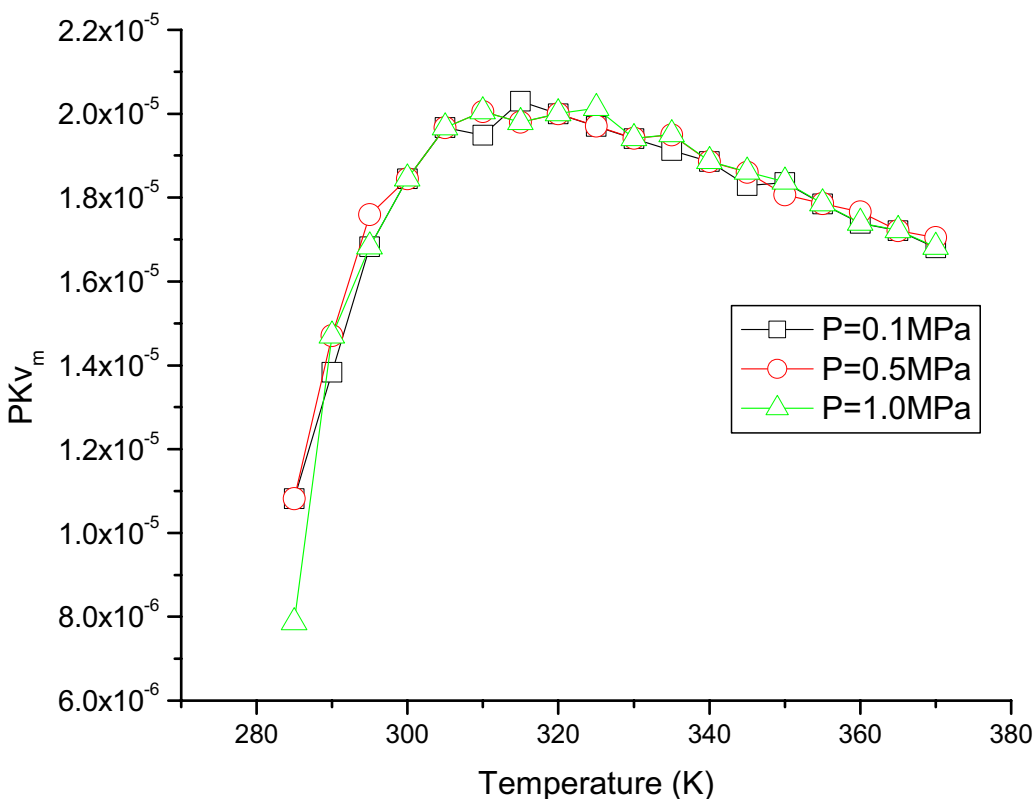

Figure 3-7 Liquid Water Acceleration Properties Group 


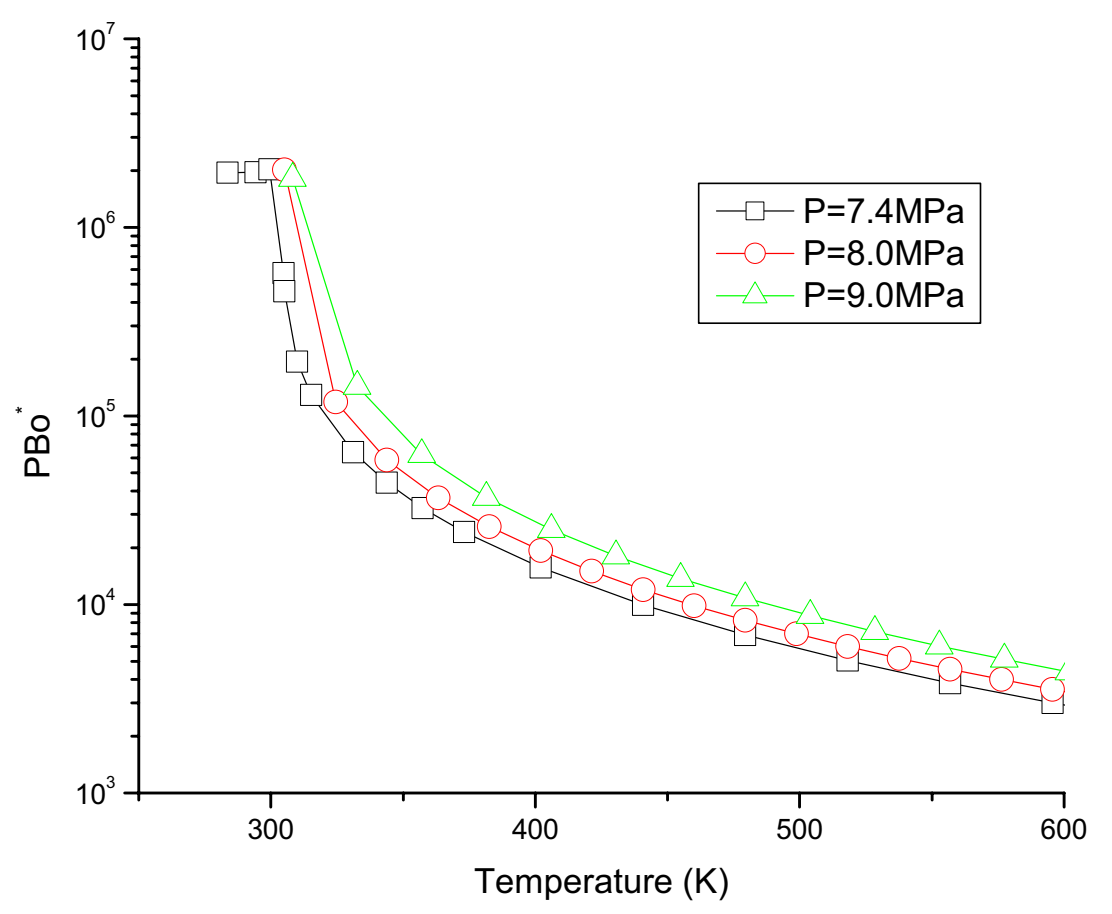

Figure 3-8 Super-Critical $\mathrm{CO}_{2}$ Buoyancy Properties Group

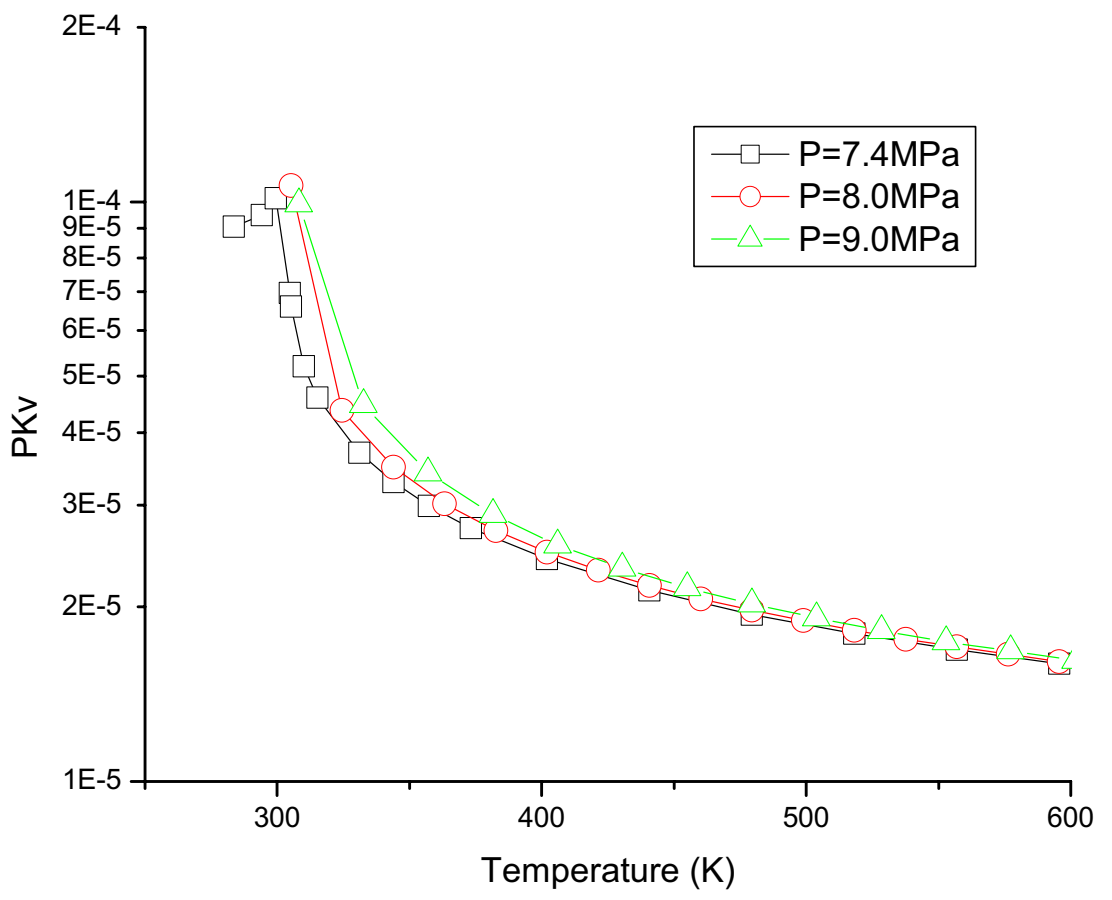

Figure 3-9 Super-Critical $\mathrm{CO}_{2}$ Acceleration Properties Group 
From the liquid water buoyancy properties group behavior (Figure 3-6), one can see that the trend is totally different from that of the gas. The liquid water buoyancy parameter will increase or stay the same downstream the channel, since the properties group increases with the fluid bulk temperature. This is because water is incompressible fluid and the thermal properties variation due to the temperature change in the low-pressure range is insignificant compared to gas. One can also conclude from the figures that the order of magnitude for the water buoyancy properties group is four times higher than that of the low-pressure gas. Therefore, it can be predicted that water experiment can reach buoyancy induced DTHT regime relatively easier than in the gas experiment if the control group is the same. In addition, the channel wise behavior due to the buoyancy force will be different also.

If we compare the acceleration number properties group between water and nitrogen (Figure 3-5 \& 3-7), their behavior is also different. The nitrogen case shows steady decrease with increasing temperature, but water shows parabolic behavior with the temperature. However, in contrast to the buoyancy parameter case, since the order of magnitude in acceleration property group is the same for both water and nitrogen cases, the integral performance of both fluids will be the same.

When we compare the super critical carbon dioxide to nitrogen, situation is different from the comparison of water to nitrogen case. The trend is the same with the temperature for both fluids but the order of magnitude change with the temperature is different. This is because of a dramatic jump in both property groups for the super critical $\mathrm{CO}_{2}$ near at the critical point (Figure 3-8 \& 3-9), where the properties undergo a steep change. Therefore, it can be predicted that the experiment using a super critical fluid will usually enter the DHTH regime, induced by either buoyancy effect or acceleration effect, where the temperature is near the critical point.

From the comparison of each fluid, the following observations can be made:

1. The channel behavior of buoyancy number will be very different between liquid, gas and super critical fluids.

2. For the gases, both maximum buoyancy number and acceleration number will occur at the inlet of the heated channel.

3. For water, the maximum buoyancy number will be at the outlet of the heated channel and the maximum acceleration number will depend on the bulk fluid temperature.

4. For the super critical carbon dioxide, both maximum buoyancy number and acceleration number will be at the point where the bulk temperature is near critical point.

5. By pressurizing the gas, buoyancy effect can be more pronounced while the acceleration effect is immune to the pressure change for the same control group. 


\section{Description of Experiment}

In the $2^{\text {nd }}$ annual report [Lee et al., 2005c], several upgrades of the experimental facility were suggested after obtaining preliminary data. This section will describe major upgrades of the facility in detail and the facility performance after their implementation.

\subsection{Test section}

It was suggested at the end of the first year of the project that to reduce an uncertainty in the heat transfer coefficient measurement, guard heaters should be installed to minimize heat loss from the test section and maintain natural circulation potential in the chimney section [Cochran et al., 2004b]. Moreover, preliminary runs at the end of second year indicated that more insulation thermocouples are needed to quantify the heat loss more accurately [Lee et al, 2005c]. Therefore, in the third fiscal year, more insulation thermocouples were installed along the test section, between the guard heaters and secondary insulation. Figure 4-1 shows the test section cross-section with the new thermocouples installed.

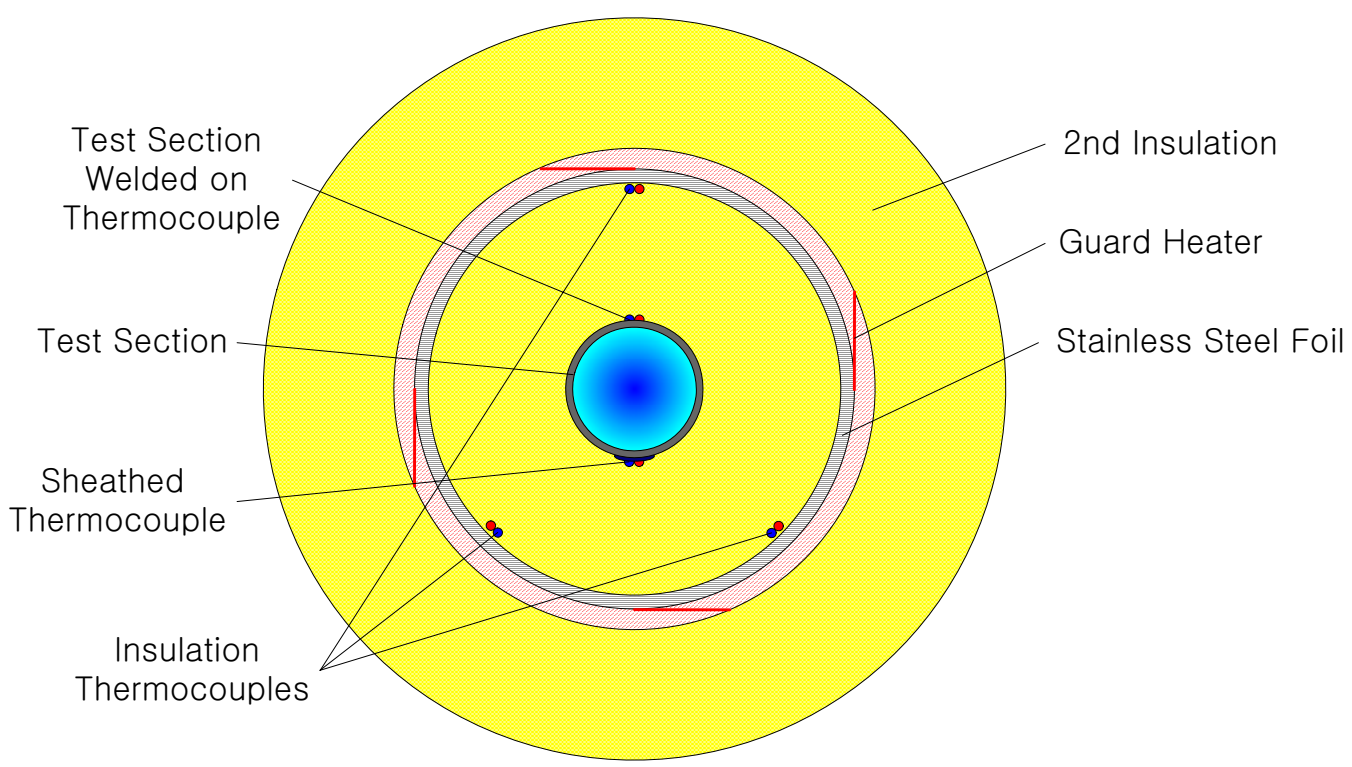

Figure 4-1 Test Section Cross Section

Three insulation thermocouples were installed azimuthally at corresponding test section axial thermocouples locations facing the wall thermocouples. Since, there were twenty test section thermocouples axially, a total of sixty insulation thermocouples were installed at the surface of the test section primary insulation. A greater number of thermocouples are required on the insulation material (glass wool) due to poor heat conduction of the insulation. As a result greater azimuthal temperature variations are possible within the 
insulation than on the stainless steel test section, with its higher heat conductivity.

In addition, two thermocouples have been attached to the power taps inside the primary insulation. These power taps are made from a solid copper block on the inlet to the test section and a stainless steel block on the test section outlet. Each tap is affixed directly to the test section resulting in a significant test section heat loss due to heat conduction into the power tap. Measuring the temperature of each power tap is important for evaluating axial heat losses more accurately.

A thin stainless steel foil, shown in Figure 4-1, is wrapped around the outer surface of the primary insulation to conduct the heat from guard heater coils more uniformly into the outer surface of primary insulation material. The foil is wrapped along the entire height of both the test section and the chimney section. This is a necessary measure to provide a uniform heating to the largest extent possible, since the insulation is a weak conductor and the guard heater is a tape heater that coils around the test section creating hot and cold spot (non-uniform heating).

The guard heater is powered by 240 VAC and controlled by an OMEGA six-zone PID temperature controller and two single zone PID temperature controllers from the same company. Test section guard heaters are divided into four zones and connected to the sixzone controller and the chimney section guard heaters are separated into two zones and each zone is connected to the single zone controller respectively. Since, the guard heaters on the test section were wrapped around more finely than the guard heaters on the chimney section ${ }^{*}$, different PID settings were necessary. Thus, multiple PID temperature controllers were used and each controller is controlled by PC. Figure 4-2 shows the simplified wiring diagram of the guard heater and its controllers. The actual wiring is more complicated since three-phase 209 VAC was used to power 240VAC guard heater and combining six solid-state relays with controllers are wired together.

\footnotetext{
${ }^{*}$ This is because the chimney section requires only minimum heat input to compensate for heat losses to maintain sufficient buoyancy for the natural circulation runs, while the heating section has axially variable temperature profile and heating losses. Thus, the heating section requires finer tuning axially.
} 


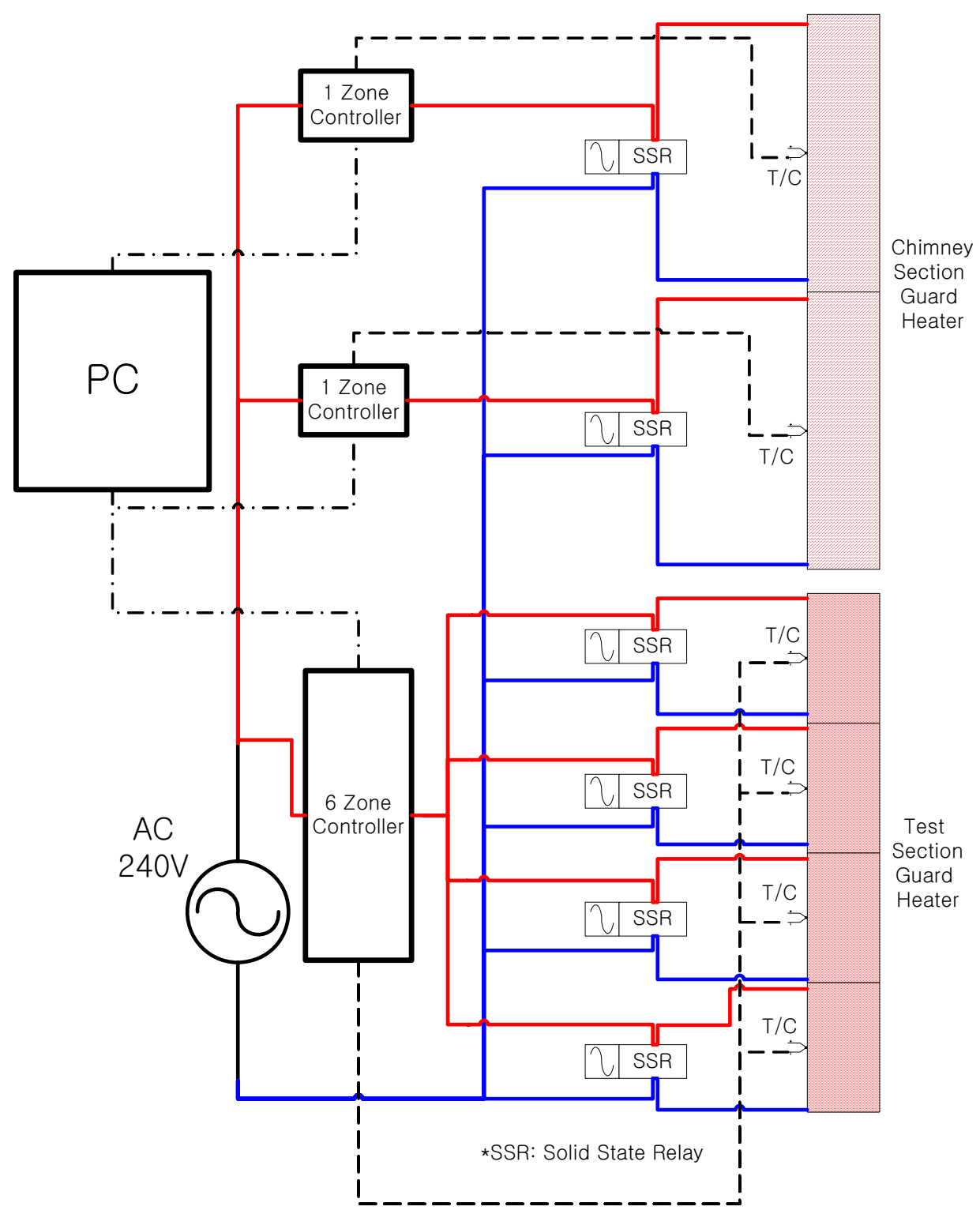

Figure 4-2 Simplified Wiring Diagram of Guard Heaters and Controllers

Finally, the secondary insulation was installed to minimize any heat loss from the guard heaters and to provide a safety measure to the operator when high temperature operation was necessary.

Photographs of the facility are provided in the Appendix-1. 


\subsection{Circulator Section}

Preliminary facility tests during the second fiscal year showed that operation at higher system pressure requires higher power motor to operate all three cylinders of the compressor. At higher pressures, gas density lowers the volumetric flow rate required to reach desired Reynolds numbers. Therefore, for the high-pressure operation only one or two compressor cylinders are required to generate the needed flow. To make possible easy and fast disconnection of the cylinders from the compressor with minimum moving parts, multiple valve systems were designed and installed to the compressor section. Figure 4-3 shows the final arrangement of the valve system used in all runs to gather data.

In addition, flow measurement instrumentation was upgraded from a single rotameter system to combined rotameter and turbine meter system. Addition of the turbine flow meter reduced the amount of signal processing necessary to produce accurate flow readings, the turbine meter being less sensitive to operating fluid, system pressure and temperature. After installation of the turbine meter the rotameter was used for approximate indication of flow when setting up the experimental conditions. In this experimental facility, two turbine meters are installed to accurately match measurement ranges to system operating conditions. One turbine meter is from OMEGA and it can measure volumetric flow rate between 1 and 10 cubic feet per minute (CFM), and the second turbine meter, manufactured by FLOWMETRICS, measures flow from 0.2 to 2 CFM. Photographs of the facility are provided in the Appendix-1.

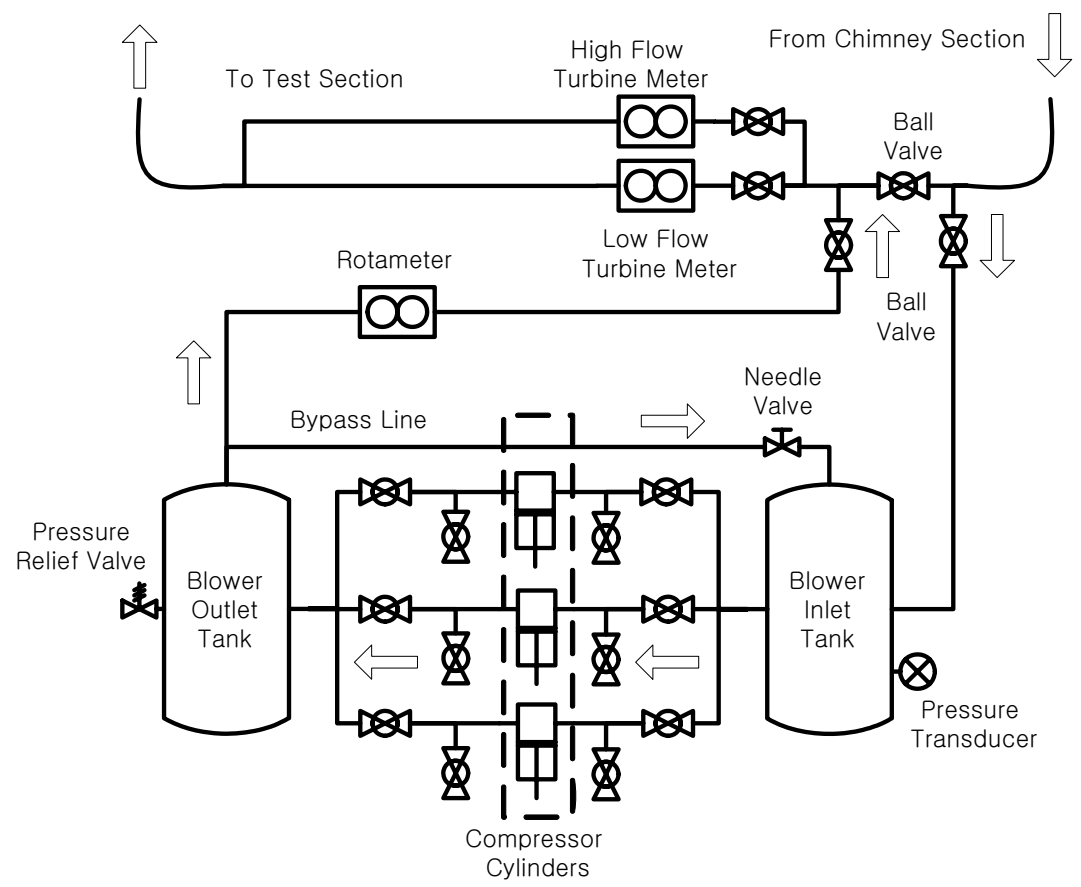

Figure 4-3 Flow Measurements and Circulator System 


\subsection{Hotwire Probe}

Very small flow rates of low density gas are very difficult to measure reliably. Therefore, we evaluated potential of using hot wire probe to measure velocity profile in the downcomer adiabatic flow region with fully developed flow and obtain flow rate by integration of the velocity profile. The major challenge of such system is how to place the wire probe in a pressurized system and how to calibrate it without need of frequent dismantling the probe when conditions in the loop change. Since all hot wire probe measurements we could find in the literature occurred in open flow systems, the concept of online calibration facility using the mini Pitot tube proposed in this project is a first such attempt to obtain velocity profile in pressurized system. Figure 4-4 shows a conceptual diagram of assembly piece that was manufactured for the hotwire online calibration system and installed in the down comer section (see Figure 4-6 for its placement in the loop).

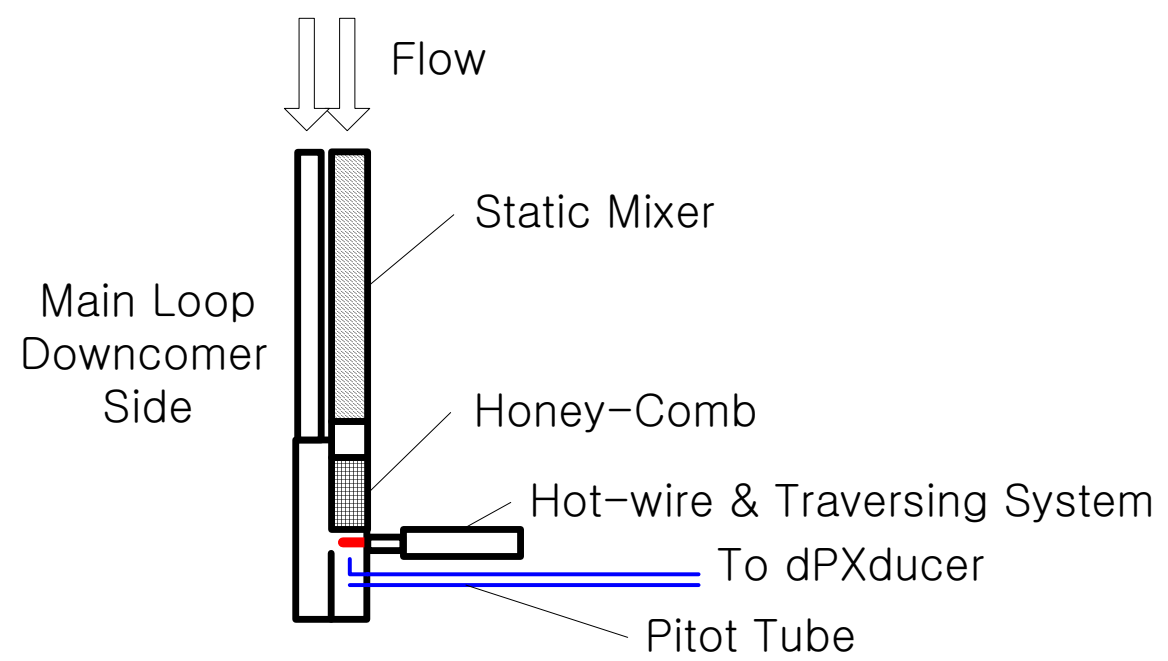

Figure 4-4 Close-up of Assembly Piece for Calibration Loop

To calibrate the hotwire, output from the hot wire needs to be correlated to the Pitot tube velocity measurement. To minimize temperature gradient and flow turbulence entering the calibration stage, a static mixer and a flow straightener are installed upstream of the hotwire and Pitot tube. Both the main loop side and the calibration loop side have 2.35 $\mathrm{cm}$ inner diameter. The hotwire and Pitot tube are separated from each other by $2 \mathrm{~cm}$. This distance was selected based on the analyses described in the $2^{\text {nd }}$ annual report [Lee et al., 2005c] that strived for the minimization of developing velocity profile effect and upstream effect using FLUENT and simple experiments.

In operation the hot wire is positioned within the calibration flow stream and after calibration completion, the probe traverses to the main loop downcomer, where measurements of the flow rate are performed. A $5 \mathrm{~mm}$ hole drilled between the calibration and main flow channel allowed the hot wire probe to extend into the main flow channel. Hole size was chosen to accommodate passage of the hotwire support, yet minimize flow 
disturbance in both the calibration section and main flow channel.

Before installation of the calibration stage into the main loop, preliminary experimental runs with air and using a small compressor outside the loop were performed. This was to check if the King's law (Eq. 4-1) can be used as an interpolation scheme between the lookup table values and if the Pitot tube can successfully measure the true velocity with a differential pressure transducer (see more details on calibration in the $1^{\text {st }}$ Annual report [Chochran et al., 2004b]). Figure 4-5 is the result.

$$
E_{V}^{2}=A+B U_{x}^{0.5}(\text { King's Law })
$$

Figure 4-5 clearly shows that the King's law is valid and even the King's law alone shows a reasonable agreement with the data since $\mathrm{R}$-square value is near unity.

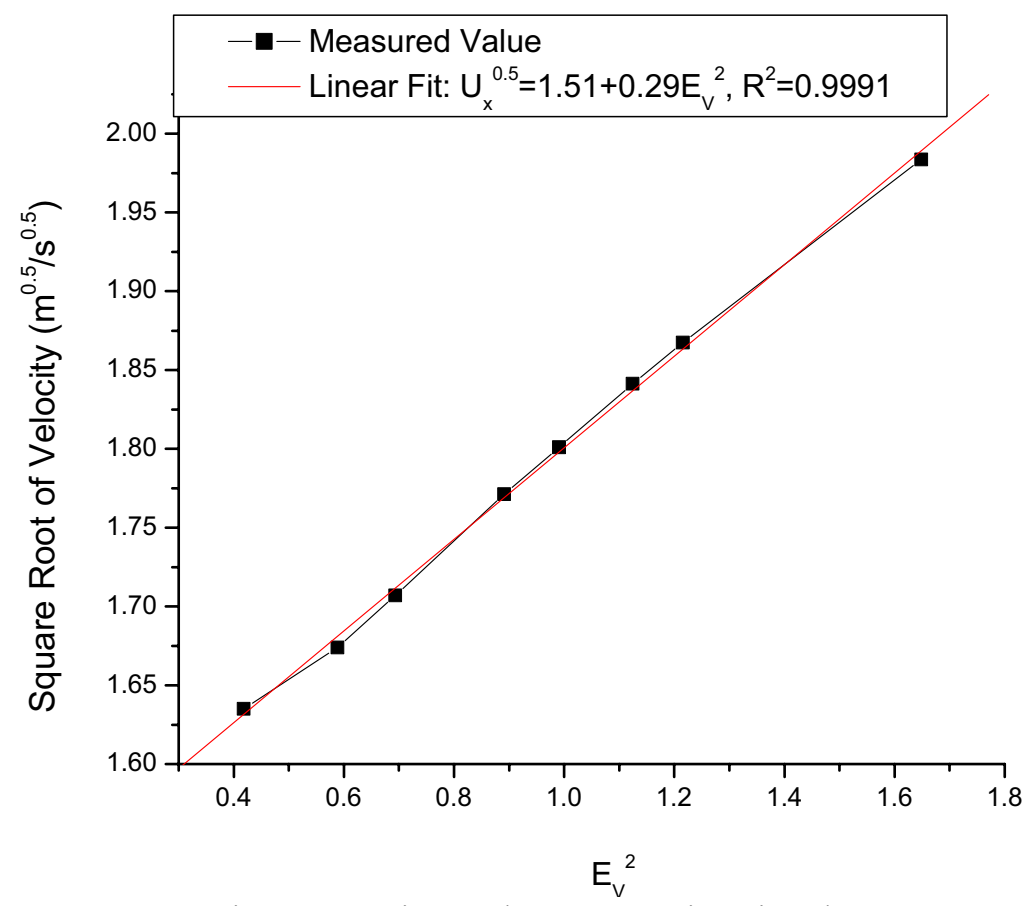

Figure 4-5 Pitot Tube vs. Hotwire Signal

Another engineering challenge we faced was how to know the exact position of the hotwire when traversing. Since the actuator is a stepper motor and can move with accuracy of 1/2400 inch per step, step count from the fully retracted position was used as information of the hotwire position. Thus, the number of steps was converted into the position inside the calibration loop and the main loop by checking the position optically before the assembly piece was integrated into the facility.

Figure 4-6 shows the online calibration loop of the hotwire measurement system after it was installed into the facility. The hotwire was calibrated by following the next procedure during the experiment: First, the system pressure is set to the experimental condition with 
the working fluid. Next, the downcomer section of the main loop side is isolated and the calibration loop side is connected to the system by utilizing the four ball valves on the loop (see Figure 4-6). After starting the circulator, the hotwire measures the electrical signal at the middle point and returns back to the fully retracted position to minimize the down stream effect for the Pitot tube measurement. This procedure is repeated for different flow rates by controlling the circulator system. During the process, average and variance of the hotwire signal is recorded to the computer with the flow temperature, since hotwire is sensitive to the temperature change.

After the calibration is finished, a lookup table is produced to correlate a hotwire signal to a true velocity value. Four valves will be operated to isolate the calibration loop side and reconnect downcomer section to the main loop.

Photographs of the facility are provided in the Appendix-1. 


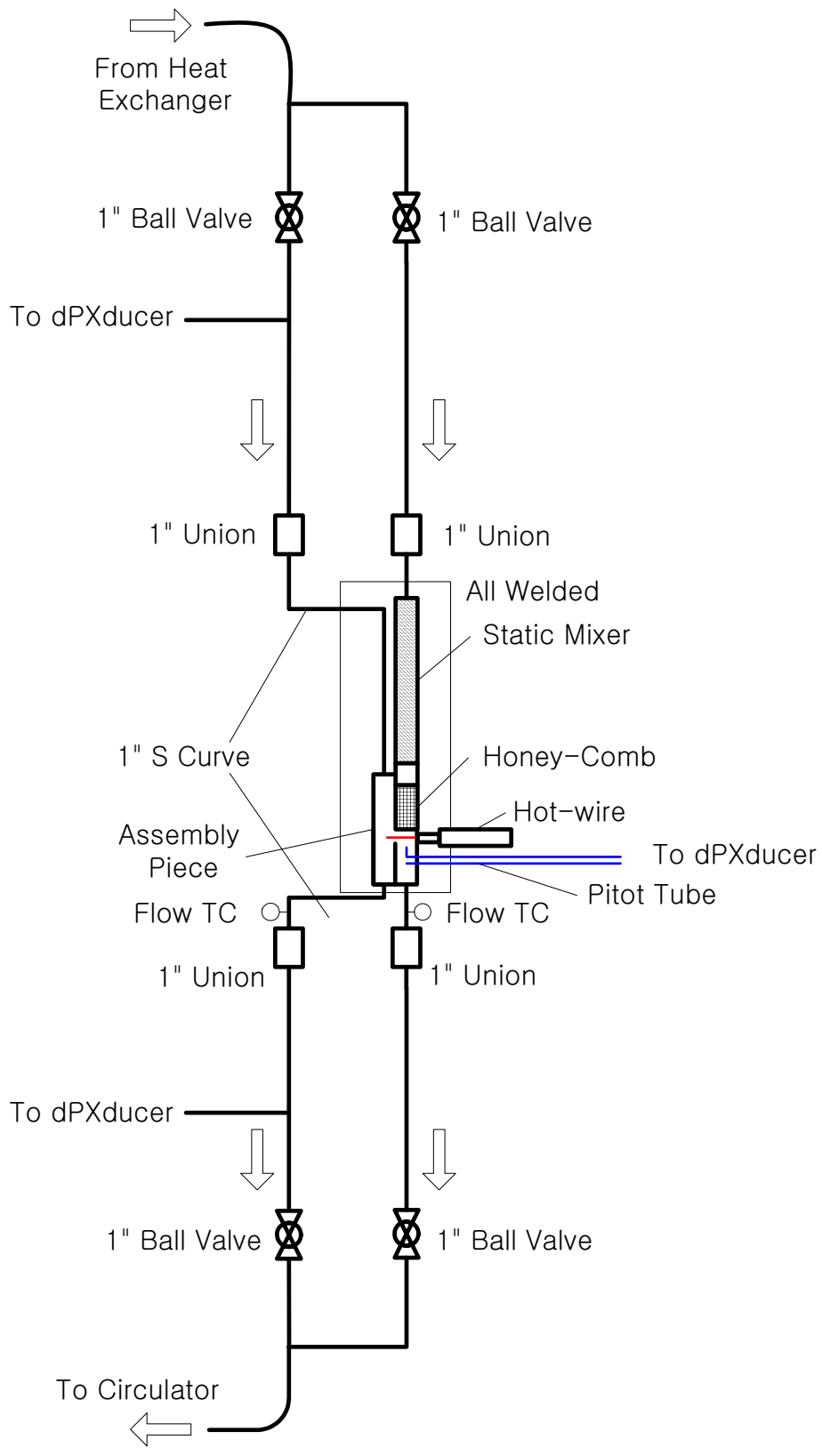

Figure 4-6 Schematic Diagram of Online Hot Wire Calibration System

A few sample runs were performed with air for different flow rates after the installation of the hotwire calibration system to check if the calibration procedure can yield acceptable result. Figure 4-7 shows the data.

Figure 4-7 data were taken by measuring the hotwire signal for every 10 steps $(1 / 240$ inch) inside the calibration loop to check if the optimization procedure used to establish the balanced distance between the mini-Pitot tube and hotwire could provide good results. 
It should be noted that the markers in the figure are only to help the understanding of the readers do not represent actual data. The data are all connected through the line.

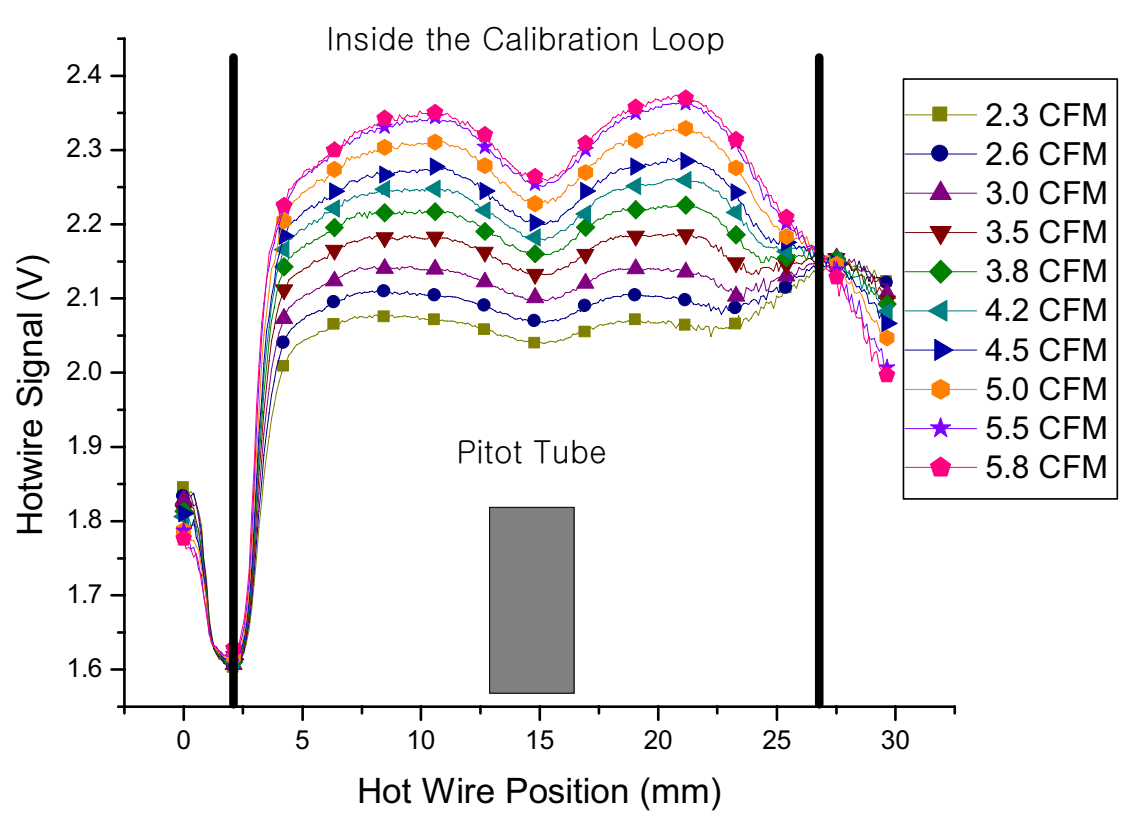

Figure 4-7 Hotwire Calibration Results at Different Flow Rate

Figure 4-7 shows that for high flow rate cases the upstream effect of the Pitot tube is significant. It is noted that the high flow rates in these cases are higher than the range of conditions used in the $2^{\text {nd }}$ annual report for optimum distance identification. These results were unexpected, since from the $2^{\text {nd }}$ annual report analysis, if the flow becomes turbulent when the flow rate is high, the upstream effect decreases compared to the laminar flow due to the turbulent mixing. However, as the flow rate decreases the upstream effect diminishes in the experiment. Therefore, from the experimental data, it can be concluded that the hotwire flow transducer system can be valuable when the flow rate is small enough.

Another drawback on this hotwire arrangement is that when operating in high flow rate, some flow penetrates sideways through the opening for hot wire stem to the calibration loop and induces asymmetric velocity profile in the main loop. Figure 4-8 shows this problem of sideway leakage. Again, same note should be made that the markers are added to aid the readability of plots, not actual data. In Figure 4-8 legend, "Main Loop Flow" depicts the case when only the main loop side is connected to the facility (and the hot wire was measuring also inside the main loop) and "Calibration Loop Flow" means that the hotwire signal was measured when only the calibration loop was connected to the loop. 


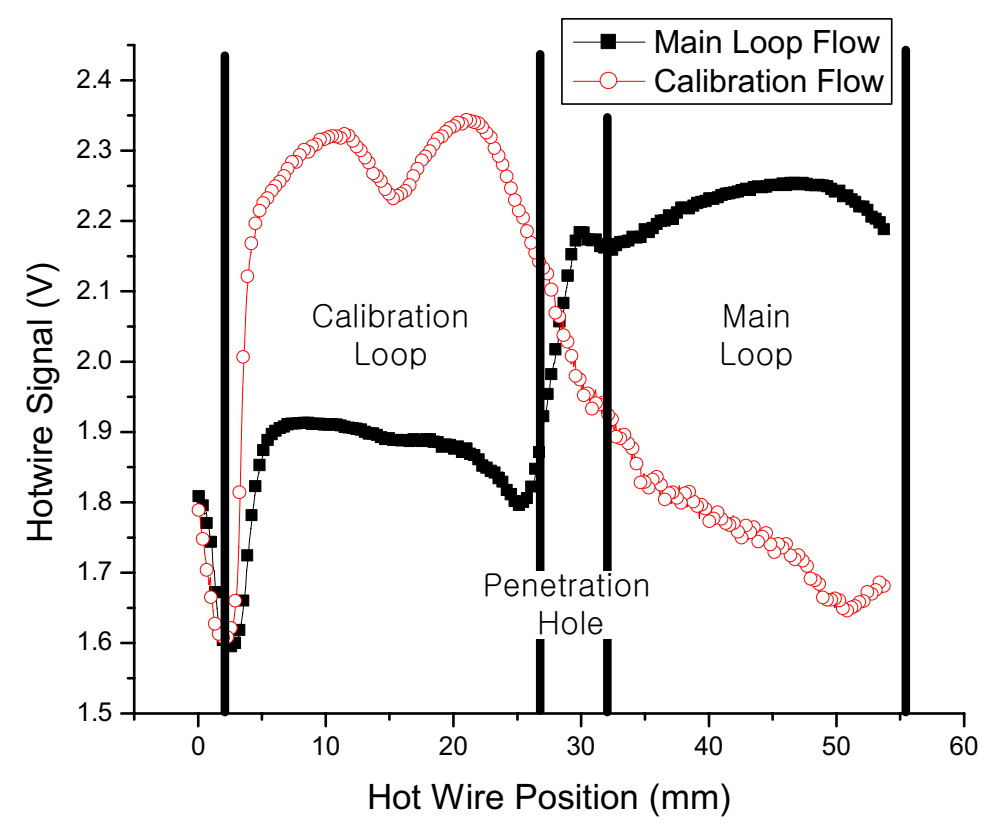

Figure 4-8 Some Problems with Hotwire Calibration Scheme

Sideway penetration of the hotwire to the main loop is inevitable if the facility is to be built for operating at elevated pressure. Even though the penetration hole was small, the skewed velocity profile can be readily observed from the figure. This can cause a significant error when the total volumetric flow rate is measured by measuring the one dimensional velocity profile, since the flow rate is calculated from the integration of the velocity profile. Two or Three dimensional asymmetric velocity distributions can't be captured by one-dimension velocity measurement. However, one can predict that the effect of asymmetric velocity profile effect will be less for the low velocity flow cases, since the skewed maximum velocity will be smaller for those cases and smaller error can be achieved due to the asymmetric velocity profile integration.

Thus, it was concluded that due to the upstream effect of the Pitot tube and inducing asymmetrical velocity profile, the hotwire system is not a reliable measurement device in a high flow rate pressurized loop operation cases. However, the hotwire system may have a potential to provide more accurate measurements for low flow rates, but it would have to undergo thorough time-consuming validation process.

However, the low flow rate turbine meter was selected as the primary flow rate measurement device due to time constraints and the low flow turbine meter from the FLOWMETRICS can cover flow rates down to 0.2 CFM. In addition, since most of the data will be taken with circulator operation, high pressure drop is not a major concern in this case. In the future, after resolving the problems discussed above, the hotwire system could be utilized to measure the flow rate in the facility reliably. 


\subsection{Instrumentation Calibration}

Since all the signals collected by the data acquisition system are voltage signals, the digital volt meter (DVM) on the data acquisition system required a calibration check to ensure accuracy of voltage readings and thus of correct conversion from temperatures, pressures and all other voltage-related signals. Figure 4-9 is the result of this calibration. Universal voltage source calibrated from the NIST was used to supply voltage to the HP3852 digital voltmeter. The digital voltmeter readings agree with the supplied voltage within $0.004 \%$ (maximum error).

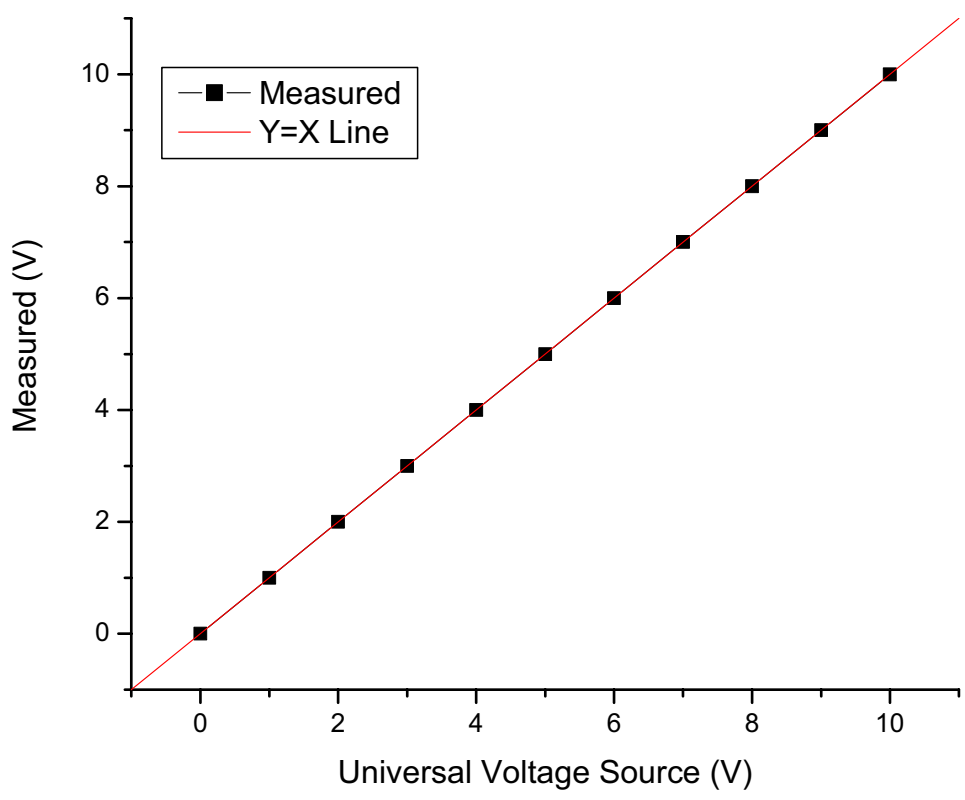

Figure 4-9 Digital Volt Meter Calibration Results

Calibration of the output current signal from the DC power supply, used for heating the test section, was checked using a 2 kiloamp calibrated shunt connected across the output terminals of the DC supply. For this calibration procedure bus bars to the loop experiment were disconnected from the DC supply. Voltage drop across the shunt was compared to the current signal from the DC supply at different current levels. Figure 4-10 displays the results of this calibration

The figure clearly shows that a current value calculated from the voltage measurement agrees well with the current value read from the voltage signal of the power supply. The maximum error between the measured value and the voltage signal readings is below $0.8 \%$.

The turbine flow meter and differential pressure transducer were calibrated by their manufacturers. For each instrument, manufacturer provided calibration table, which were used in the data reduction program using lookup table method. The interpolation between the table values was automatically done by the facility operation software, which is 
presented in the next section.

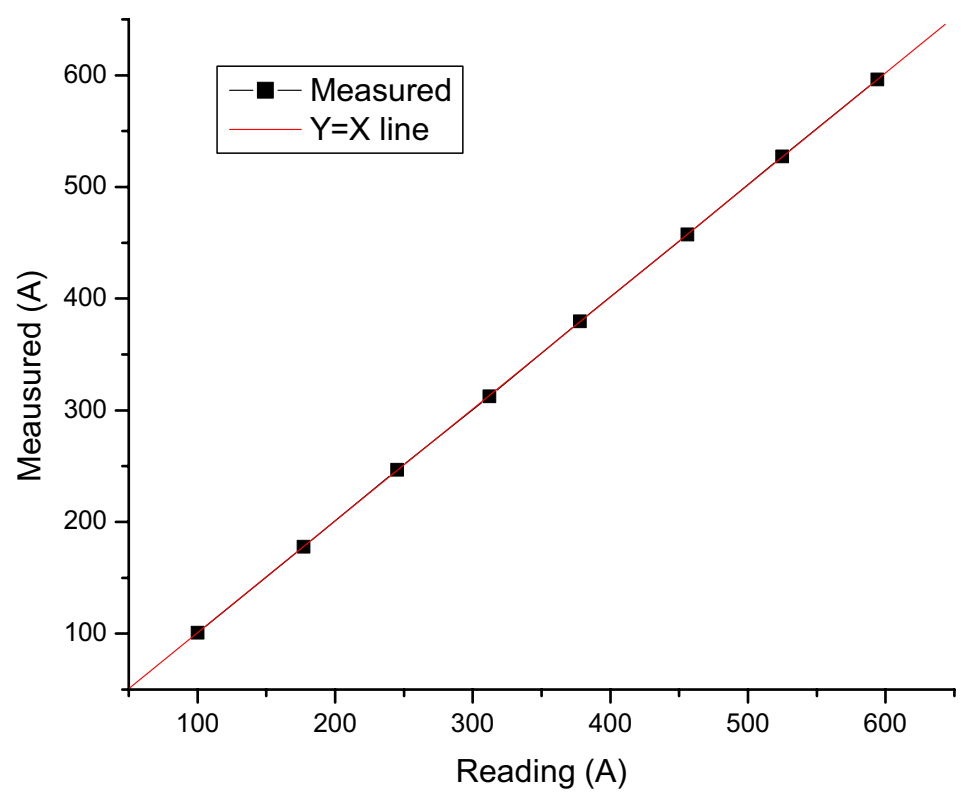

Figure 4-10 Power Supply Current Signal Calibration Results

\subsection{Software for Facility Operation and Data Acquisition}

Facility operation and data acquisition were all done using a Visual Basic program, developed by MIT for this project. The advantage of writing our own program for the facility operation and data acquisition is that it can significantly reduce the load on the computer compared to general commercial programs, such as LABVIEW. In addition, writing an in-house code gives more flexibility than just utilizing existing programs. Figures 4-11 to 13 are screen captures of the operation and data acquisition program.

The most complicated issues in designing the software are: (1) selecting measurement quantities that need to be averaged and integrated, (2) filtering the raw data to extract meaningful information, (3) displaying selection of the data representation, and (4) integrating online calibration capability. For example, system pressure is integrated over "integration time" (see Figure 4-11) and displayed in a time vs. system pressure graph. Also, since system pressure transducer needed to be calibrated for every experiment, the online calibration was also possible with this software. The other example is the temperature display. Since, spatial temperature profile is an important indicator for the heat transfer deterioration and temperature history is an important indicator for the steady state, both were displayed on the same screen. The software is also capable of monitoring the primary insulation surface temperature and setting the guard heater temperature.

As explained in the $2^{\text {nd }}$ annual report [Lee et al., 2005c], the data were taken when the power supply was off for the short time to minimize the electrical noise on the 
ungrounded T/Cs (welded T/Cs). Therefore, a steady state indicator was necessary to decide if the system reached the steady state or not and turn off the power supply to take data. This indicator is designed to compare previous time averaged temperature of the sheathed T/Cs, which can be measured at all times due to their invulnerability to the power supply electrical noise, to the current time averaged temperature. The temperature variation rate is calculated for each sheath $\mathrm{T} / \mathrm{C}$ using an averaging scheme, which reduces electrical noise induced to the sheathed $\mathrm{T} / \mathrm{C}$ 's signal. The averaging time is decided by changing the integration time. The default averaging time is 30 seconds and steady state temperature readings are taken when the maximum temperature variation ratio was below $6{ }^{\circ} \mathrm{C}$ per hour.

The differential pressure is measured by a MKS Type 120A high accuracy pressure transducer and the raw signal is filtered to eliminate pressure pulses introduced by the circulating compressor. Both raw data and filtered signal are displayed. All flow measurement data, rotameter, turbine meter and hotwire is displayed on screen in real time.

The software saves data to two files. One file contains all the data of sheathed T/Cs and flow thermocouples gathered while the power supply is on, and the other file includes all the data taken when the power supply is off.

For safety purposes, the heating DC power supply is automatically turned off when any sheathed $\mathrm{T} / \mathrm{C}$ reading is above $1050 \mathrm{~K}$. Additional safety functions include interrupting DC heating current when the data acquisition system is not operating or there is some difficulty in acquiring data.

The software provides a dedicated summary tab for display of key operating parameters such as power, heat flux, flow rate and system pressure. In addition inlet non-dimensional numbers are displayed providing easy determination of the current loop operational flow regime.

The hotwire calibration tab consists of four sections. First section is for measuring and setting the hotwire characteristic parameters such as probe resistance and the aspect ratio. The second tab is the motion tab, which provides stepper motor control. The third tab is for the calibration. In this tab the hotwire signal and velocity measured by the Pitot tube are correlated automatically and saved to a file. Fourth tab measures the flow rate with calibrated hotwire and stores the measured signal to a different file and displays the velocity profile inside the main loop. 


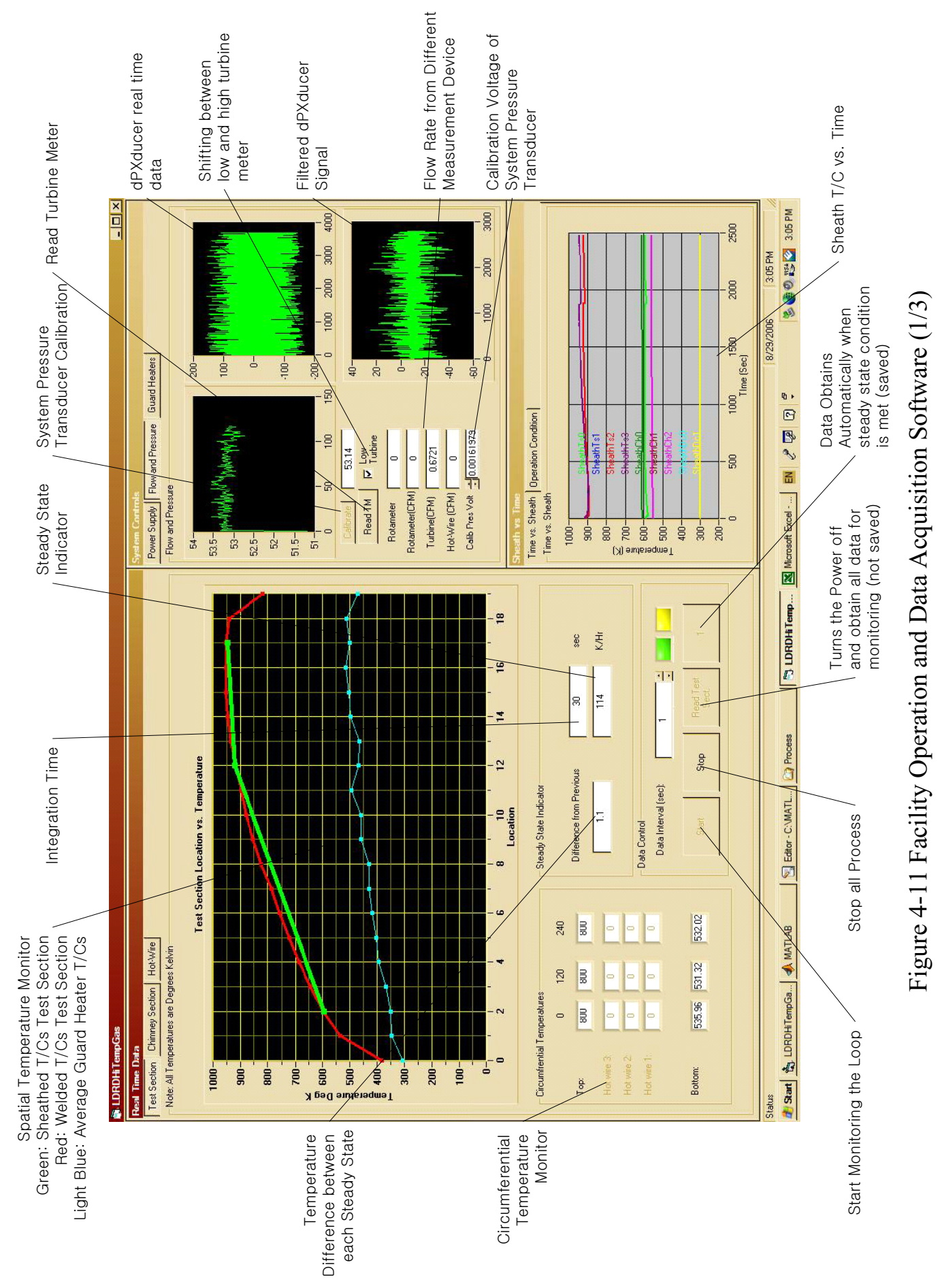

ㅇำ 


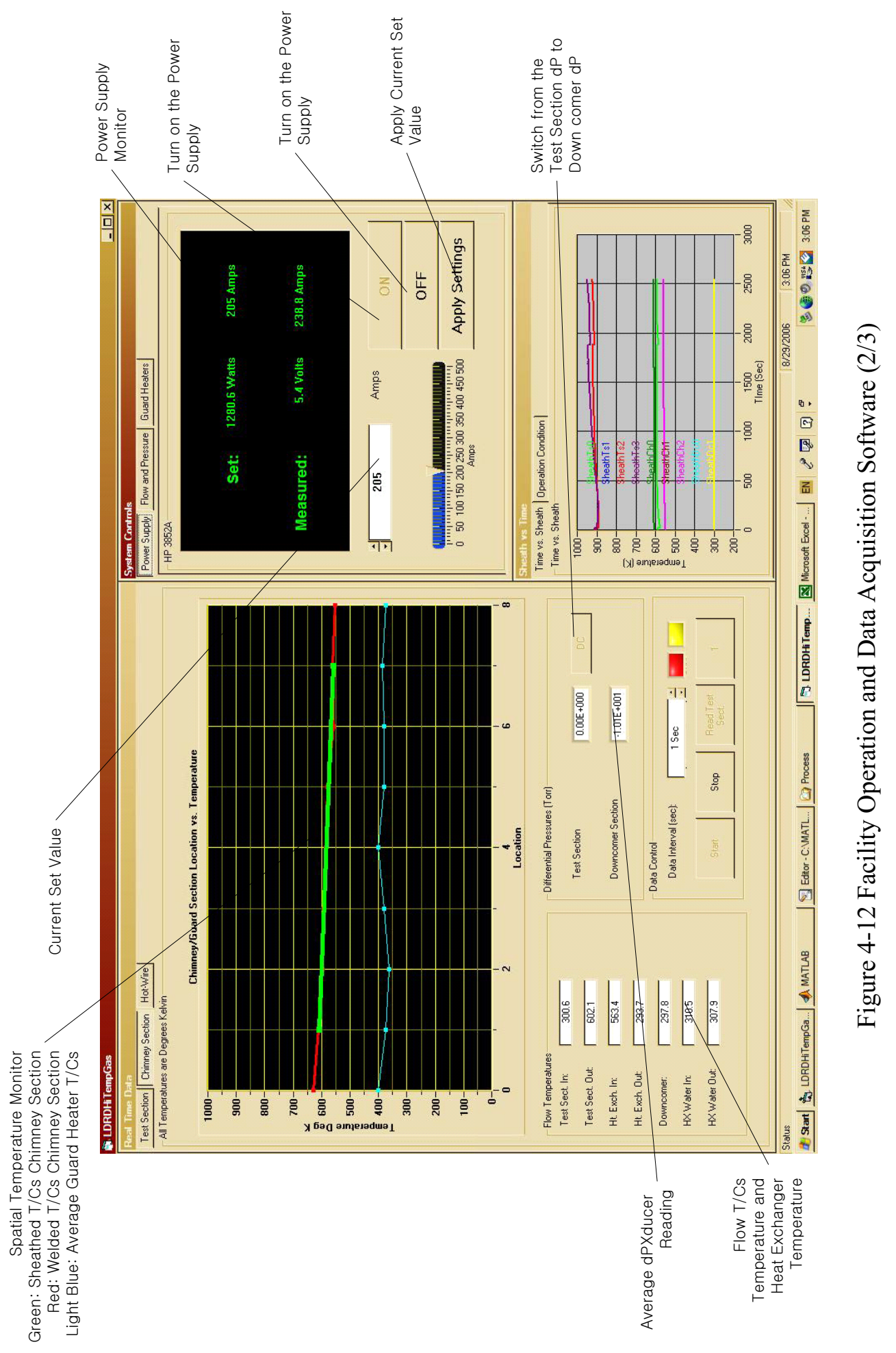

6 


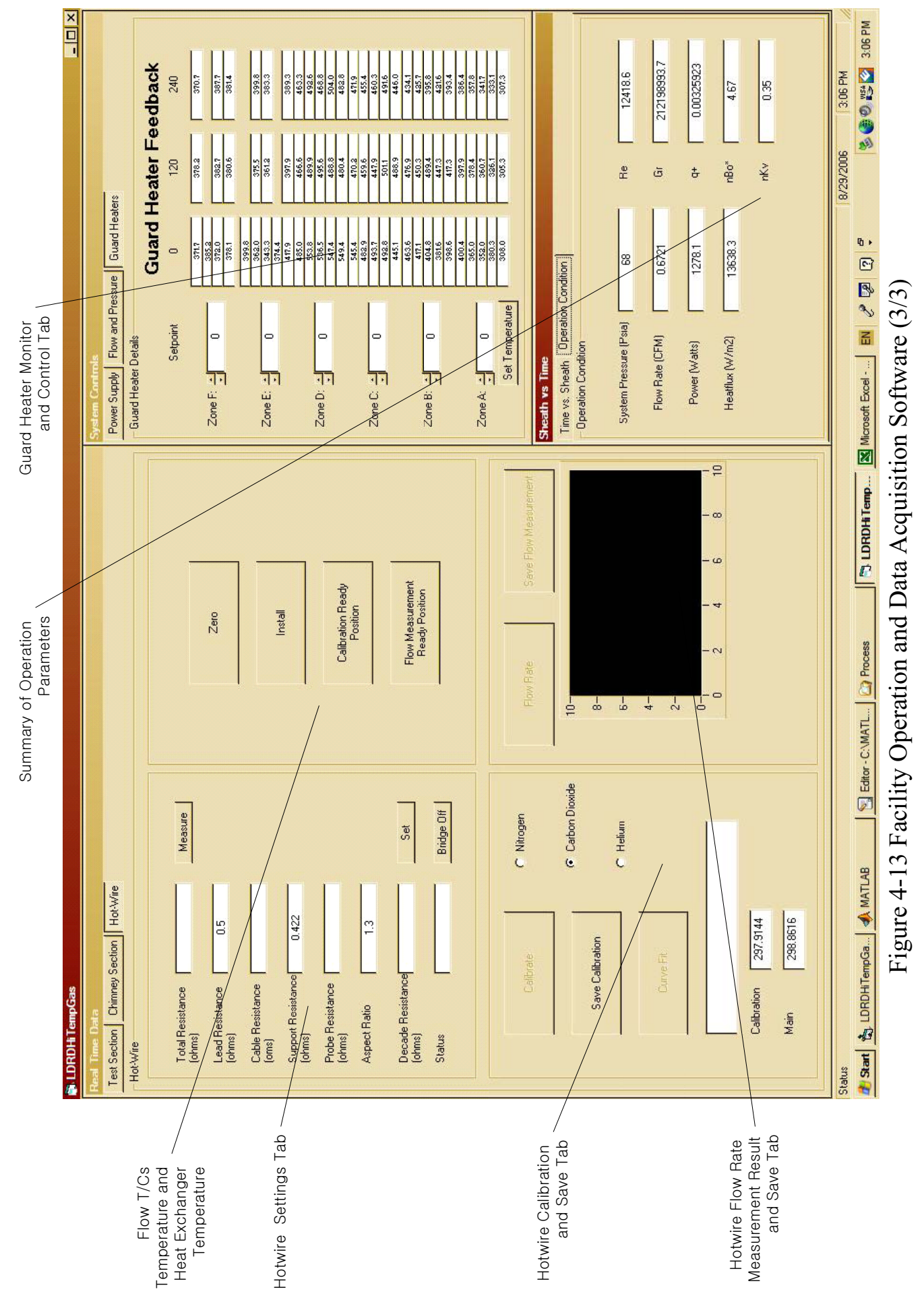

$\widetilde{ช}$ 


\subsection{Experimental Procedure}

This section provides a step-by-step description of the method for acquiring data during each experiment.

1. Five hours prior to testing turn on the differential pressure transducer for warm up period.

2. After the warm up period, connect both ports of the differential pressure transducer to each other to create zero differential pressure. Execute "auto zero" function for the differential pressure transducer. Auto zero sets the transducer output at a known zero differential pressure.

3. Start the facility operation and measurement software.

4. Calibrate the pressure transducer for zero gage pressure, by insuring that the transducer is exposed to atmospheric pressure, then measuring the transducer output for 20 seconds and averaging the readings. This value is subtracted from the pressure transducer signal during the test.

5. Isolate the compressor from the main loop. This procedure is necessary, since the compressor sealing is not designed to withstand vacuum condition.

6. Open all the valves in main loop and start the vacuuming process.

7. After vacuum state is reached, charge the loop with the gas required for the experiment: nitrogen, helium or carbon dioxide.

8. Set the system pressure with the pressure regulator on the gas bottle and monitor the system pressure with the calibrated pressure transducer.

9. Valve in the compressor and select the number of cylinders required for proper loop operation. Gas volume required to fill the compressor is made up automatically from the gas supply bottle.

10. Start the compressor and turn on the heat exchanger water supply.

11. Measure the flow rate to check if desired operating condition is achieved.

12. Set the DC current supply value and start the power supply.

13. Set the guard heater temperature value.

14. Set the steady condition and monitor the temperature history and profile to check the steady state and the operating flow regime. Also a lot of information can be obtained from the operation summary tab.

15. Obtain steady state data five times and move to next case. The data reduction program averages five data points and reduces raw data.

\subsection{Data Reduction Program Design}

There was no major upgrade for the data reduction program in terms of the reduction procedure in comparison to the $2^{\text {nd }}$ annual report. Minor upgrades in the procedure were:

1. Zoning process of the insulation for estimating a radial heat loss is eliminated. The zoning process was necessary, since number of axial insulation T/Cs (6 axial $\mathrm{T} / \mathrm{Cs})$ were smaller than the test section $\mathrm{T} / \mathrm{Cs}(20 \mathrm{~T} / \mathrm{Cs})$ last year. After the additional thermocouples were installed within the insulation adjacent to the test 
section, each axial point on the test section has three corresponding primary insulation $\mathrm{T} / \mathrm{Cs}$ at the same axial point.

2. The thermal resistance of the insulation is after the update dependent on test section outer wall temperature. Therefore, lookup table method is used to evaluate the thermal resistance of the insulation as a function of outer wall temperature.

3. Stainless steel conductivity is now linearly dependent on temperature. This affects the calculation for the inner wall temperature from the measured outer wall temperature value.

4. The flow rate does not require system pressure dependent corrections, since turbine meter measures the actual volumetric flow rate while pressure modification is needed for the rotameter readings.

5. Various non-dimensional numbers, such as buoyancy parameter, acceleration parameter, non-dimensional heat flux and so forth are calculated during the process.

6. Helium and carbon dioxide properties are added to the gas properties database.

This year major upgrades of the data reduction program focused on code modularity. These involved addition of the gas properties function, thermal resistance database of the insulation material, geometrical features and the main processor, which were all separated and can be called as an intrinsic function in MATLAB. In addition, format of output file was designed in such a manner that the reduced data can be easily used to verify various existing correlations and produce new correlations.

\subsection{Thermal Characteristic of Insulation}

Before we present the experimental data, thermal resistance of the insulation needs to be determined. Measurements of thermal resistance will be presented in this section. Thermal resistance is measured by (1) vacuuming the main loop, (2) applying heat flux to the test section, (3) measuring the temperature of the test section outlet wall temperature and the outer surface of the primary insulation, and (4) calculating the thermal resistance from the data. The measurement procedure is documented in more detail in the $2^{\text {nd }}$ annual report [Lee et al., 2005c].

It was pointed out in the $2^{\text {nd }}$ annual report that to estimate the radial heat loss more accurately, the thermal resistance of the insulation needs to be temperature dependent. Therefore, six measurements with different average test section wall temperatures were performed. The results are presented in Figure 4-14. In the figure, number indicates the axial position (e. g. ' 0 ' is the inlet of the test section and '19' is the outlet of the test section) and different markers are for different azimuthal sections (e. g. circle is for 0 degrees, cross is for 120 degrees and square is for 240 degrees T/C positions). Since three azimuthal $\mathrm{T} / \mathrm{Cs}$ are placed on twenty different axial positions, the insulation is divided into three azimuthal sections and twenty axial sections (total 60 sections).

However, one interesting observation can be made from the figure. The ' 0 ', ' 1 ', '18' and '19' axial insulations have different trend versus temperature compared to the rest of the insulation sections (' 3 ' to ' 17 '). Since, the same material is used for all the insulation, the 
discrepancy in the behavior is mainly due to the uncertainty in the axial conduction estimation rather than the difference in the insulation itself. Even by installing T/Cs on the power tap improved the accuracy of the axial heat loss estimate, the heat flux calculations at this position is not as reliable as for other positions. This can be observed by comparing ' 0 ' to ' 1 ' and ' 19 ' to ' 18 ' insulation data, since as soon as the insulation moves further from the inlet or the outlet (from ' 0 ' to ' 1 ' and from ' 19 ' to ' 18 '), where the axial heat loss is maximum due to the power tap, the behavior becomes similar to the rest of the insulation. Therefore, insulation ' 0 ' and ' 1 ' data will be substituted with ' 2 ' insulation data and '18' and '19' will be substituted with '17' data. This will provide better estimation of the heat flux and the bulk temperature than using the insulation data itself.

From the insulation ' 3 ' to ' 17 ' behavior, most of the insulations respond linearly to the test section outer wall temperature. Thus, when the test section outer wall temperature rises slightly higher than the calibration cases for the insulation, the thermal resistance is evaluated with an extrapolation based on the linear fitting of the data.

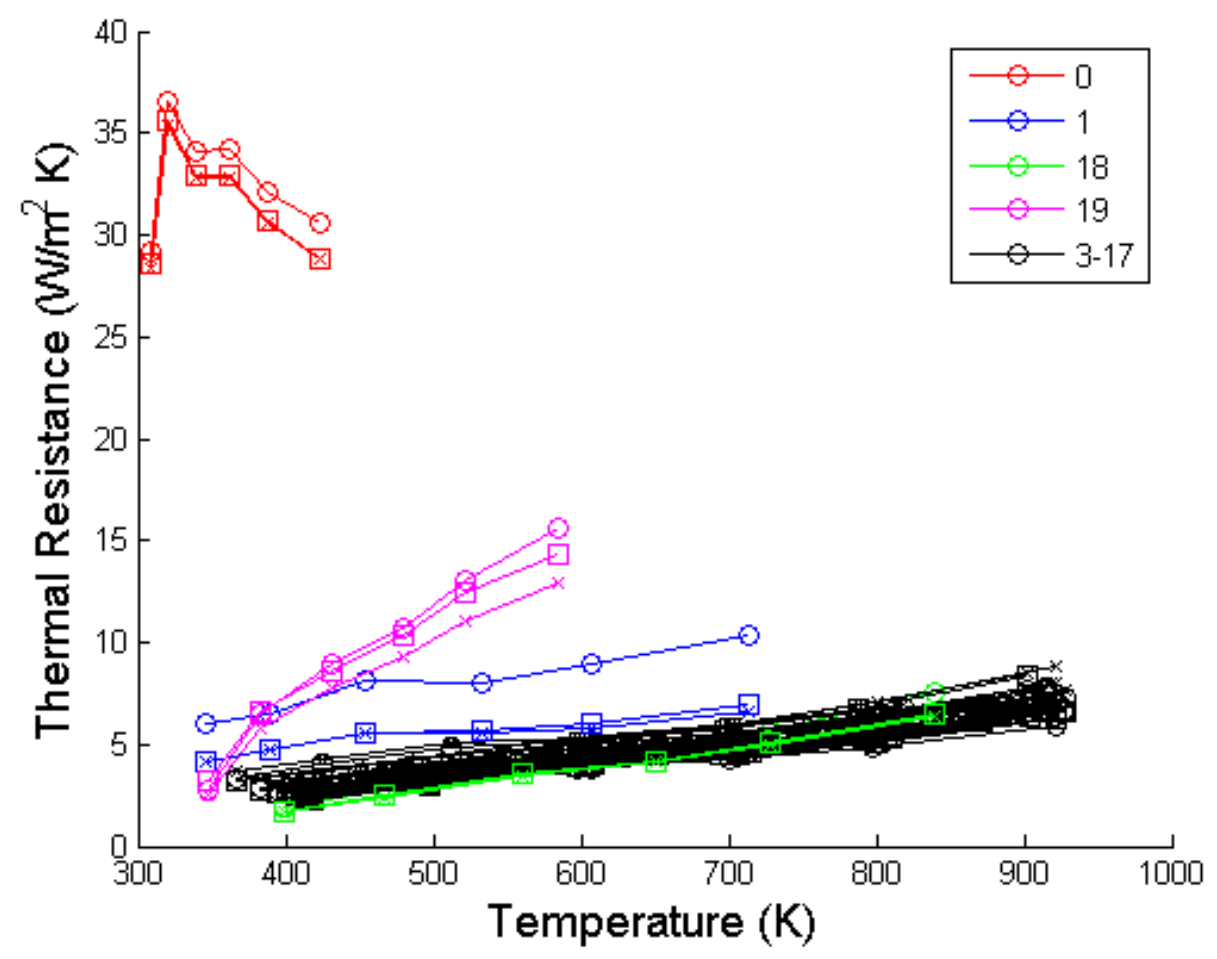

Figure 4-14 Thermal Resistance of the Insulation for Different Outer Wall Temperatures 


\section{Experimental Data}

This chapter presents experimental data obtained from the loop facility operation. The chapter will be divided into six sections. First three sections show the experimental data for each gas: nitrogen, helium and carbon dioxide, and fourth last section will summarize the entire set of experimental data. The last two chapters are to show the validation of the data and present a newly proposed heat transfer map.

The data presented in this chapter will be all given in non-dimensionalized form, as obtained through the data reduction process, except for few cases where temperature profile can provide some insight. Since, the thermal resistance of insulation locations ' 0 ', '1', '18' and '19' could not be determined with sufficient accuracy due to axial heat conduction, four data points at these channel inlet and outlet locations are all omitted from the heat transfer data presentation, even though the data of these points are used for calculating the fluid bulk temperature and the heat balance. Thus, one case provides 16 Nusselt numbers along the channel. All the data presented here was taken with $15.7 \mathrm{~mm}$ small diameter test section only.

Before describing the experimental data obtained at the lop facility, all the operating conditions are summarized in Table 5-1. Within this work, when we present the data, each regime is defined as:

1. The laminar regime and mixed convection laminar regime are when the inlet Reynolds number is smaller than 2,300 (yellow in Table 5-1).

2. The transition regime is when the inlet Reynolds number is higher than 2,300 but the outlet Reynolds number is lower than 2,300 (green in Table 5-1).

3. The turbulent regime is when both the inlet and outlet Reynolds number is above 2,300 (light blue in Table 5-1).

4. The DTHT regime is when both the inlet and outlet Reynolds number are above 2,300 and the reduction in the heat transfer coefficient is over $20 \%$ (red in Table 5-1).

It should be noted that all the gases were operated far from the critical point.

Table 5-1 Operating Conditions

\begin{tabular}{||c||c||c||c|c|c||c||c||c||c||}
\hline \hline case & Gas & P(MPa) & CFM & $q^{\prime \prime}\left(\mathrm{W} / \mathrm{m}^{2}\right)$ & Inlet Re & Inlet $q^{+}$ & Tw/Tb & $\max B o^{*}$ & $\max K_{v}$ \\
\hline \hline 1 & $\mathrm{~N} 2$ & 0.33 & 1.1 & 7987 & 9046 & 0.002122 & $1.40 \sim 1.11$ & $9.50 \mathrm{E}-07$ & $1.11 \mathrm{E}-06$ \\
\hline 2 & $\mathrm{~N} 2$ & 0.5 & 1.2 & 13940 & 14359 & 0.002446 & $1.52 \sim 1.16$ & $7.72 \mathrm{E}-07$ & $7.67 \mathrm{E}-07$ \\
\hline 3 & $\mathrm{~N} 2$ & 0.53 & 1.2 & 7485 & 15232 & 0.001277 & $1.29 \sim 1.11$ & $3.80 \mathrm{E}-07$ & $3.66 \mathrm{E}-07$ \\
\hline 4 & $\mathrm{~N} 2$ & 0.14 & 2.2 & 12121 & 7197 & 0.003581 & $1.64 \sim 1.08$ & $5.07 \mathrm{E}-07$ & $2.64 \mathrm{E}-06$ \\
\hline 5 & $\mathrm{~N} 2$ & 0.14 & 2.2 & 7855 & 7175 & 0.002509 & $1.44 \sim 1.08$ & $3.46 \mathrm{E}-07$ & $1.73 \mathrm{E}-06$ \\
\hline 6 & $\mathrm{~N} 2$ & 0.13 & 1.8 & 8220 & 5538 & 0.002889 & $1.52 \sim 1.14$ & $8.07 \mathrm{E}-07$ & $3.01 \mathrm{E}-06$ \\
\hline 7 & $\mathrm{~N} 2$ & 0.13 & 1.8 & 5919 & 5334 & 0.002347 & $1.40 \sim 1.06$ & $6.22 \mathrm{E}-07$ & $2.34 \mathrm{E}-06$ \\
\hline 8 & $\mathrm{~N} 2$ & 0.13 & 6.0 & 5095 & 18470 & 0.000736 & $1.17 \sim 1.07$ & $8.00 \mathrm{E}-09$ & $1.68 \mathrm{E}-07$ \\
\hline \hline
\end{tabular}


Table 5-1 Operating Conditions (continued)

\begin{tabular}{|c|c|c|c|c|c|c|c|c|c|}
\hline & Gas & $\mathrm{P}(\mathrm{MPa})$ & CFM & $"\left(\mathrm{~W} / \mathrm{m}^{2}\right)$ & nlet $\mathrm{Re}$ & Inlet $q^{+}$ & $\mathrm{Tw} / \mathrm{Tb}$ & $\max B o^{*}$ & $\max K$ \\
\hline 9 & N2 & 0.13 & 2.2 & 10640 & 7025 & 0.003245 & $1.58 \sim 1.07$ & 4.88E-07 & $2.43 \mathrm{E}-06$ \\
\hline 10 & $\mathrm{~N} 2$ & & 6.1 & 3051 & 619 & & & & \\
\hline 11 & $\mathrm{~N} 2$ & & 3.1 & & & & & & $2.28 \mathrm{E}-07$ \\
\hline 12 & $\mathrm{~N} 2$ & & 5.5 & & 21704 & \begin{tabular}{|l|}
0.0006 \\
\end{tabular} & & & $1.16 \mathrm{E}-07$ \\
\hline 13 & $\mathrm{~N} 2$ & & 4.8 & & 18768 & 0.000693 & 1.07 & $1.20 \mathrm{E}-08$ & $1.57 \mathrm{E}-07$ \\
\hline 14 & $\mathrm{~N} 2$ & & 1.2 & 11282 & 16895 & 0.001696 & 1.15 & $4.92 \mathrm{E}-07$ & $4.42 \mathrm{E}-07$ \\
\hline 15 & $\mathrm{~N} 2$ & & 1.2 & 15742 & 16767 & 0.002356 & 1.19 & $97 \mathrm{E}-07$ & $6.26 \mathrm{E}-07$ \\
\hline 16 & $\mathrm{~N} 2$ & & 1.2 & 1040 & 16806 & 0.003098 & 1.71 & $9.21 \mathrm{E}-07$ & $8.31 \mathrm{E}-07$ \\
\hline 17 & $\mathrm{~N} 2$ & & 0.9 & 17043 & 11887 & \begin{tabular}{|l|}
0.003271 \\
\end{tabular} & 1.78 & E-06 & $1.34 \mathrm{E}-06$ \\
\hline 18 & N2 & & 0.7 & 119 & 10023 & 0.00 & 1.73 & $\mathrm{E}-06$ & $1.35 \mathrm{E}-06$ \\
\hline 19 & N2 & & 0.5 & 41 & 7563 & 0.00 & 1.18 & $\overline{E-06}$ & $1.82 \mathrm{E}-06$ \\
\hline 20 & N2 & & 0.5 & & 534 & & 1.60 & $8-06$ & $1.29 \mathrm{E}-06$ \\
\hline 21 & $\mathrm{He}$ & & 6.1 & 41 & 3184 & 0.00 & 1.05 & $7.00 \mathrm{E}-09$ & $4.17 \mathrm{E}-07$ \\
\hline 22 & $\mathrm{He}$ & & 6.1 & & 3196 & & & & $8.11 \mathrm{E}-07$ \\
\hline 23 & $\mathrm{He}$ & & 6.1 & & 3235 & 0.00 & 1.1 & -08 & $1.57 \mathrm{E}-06$ \\
\hline 24 & $\mathrm{He}$ & & 6.2 & & 302 & & & -08 & $2.00 \mathrm{E}-06$ \\
\hline 25 & $\mathrm{He}$ & & 6.0 & 4401 & 3205 & 09 & 1.2 & -08 & $2.76 \mathrm{E}-06$ \\
\hline 26 & $\mathrm{He}$ & & & & 381 & & & & $3.52 \mathrm{E}-06$ \\
\hline 27 & $\mathrm{He}$ & & 6.2 & 814 & 3413 & $0.0 \mathrm{C}$ & 1.16 & $\mathrm{E}-08$ & $4.18 \mathrm{E}-06$ \\
\hline 28 & $\mathrm{He}$ & & 3.4 & & 894 & & & & $9.48 \mathrm{E}-06$ \\
\hline 29 & $\mathrm{He}$ & & 3.4 & 778 & 2001 & 0.0 & 1.31 & -07 & $5.79 \mathrm{E}-06$ \\
\hline 30 & $\mathrm{He}$ & & 3.4 & & 1817 & & & -07 & $4.54 \mathrm{E}-06$ \\
\hline 31 & $\mathrm{He}$ & & 3.7 & 02 & 4383 & & 1.1 & -08 & $7.68 \mathrm{E}-07$ \\
\hline 32 & $\mathrm{He}$ & & 3.7 & & 4434 & & & $8-07$ & $1.92 \mathrm{E}-06$ \\
\hline 33 & $\mathrm{He}$ & & 3.7 & 944 & 4516 & 87 & 1.44 & E-07 & $2.69 \mathrm{E}-06$ \\
\hline 34 & $\mathrm{He}$ & & 2.5 & & 2889 & & & -07 & $4.88 \mathrm{E}-06$ \\
\hline 35 & $\mathrm{He}$ & & 2.5 & 15741 & 2833 & 453 & 1.34 & E-07 & $3.85 \mathrm{E}-06$ \\
\hline 36 & $\mathrm{CO} 2$ & & 6.0 & & 461 & & & -08 & $8.30 \mathrm{E}-08$ \\
\hline 37 & $\mathrm{CO} 2$ & & 4.1 & 200 & 22916 & 0.00 & 1.40 & E-08 & $1.81 \mathrm{E}-07$ \\
\hline 38 & $\mathrm{CO} 2$ & & 5.3 & & 1282 & 0.00 & 1.32 & -08 & $9.60 \mathrm{E}-08$ \\
\hline 39 & $\mathrm{CO} 2$ & & 3.5 & 11300 & 21339 & 0.00 & $1.41 \sim 1.16$ & E-08 & $2.10 \mathrm{E}-07$ \\
\hline 40 & $\mathrm{CO} 2$ & & 2.8 & 526 & 17015 & & 1.48 & EE-07 & $3.37 \mathrm{E}-07$ \\
\hline 41 & $\mathrm{CO} 2$ & & 2.7 & 20776 & 16382 & 0.0 & 1.74 & $\mathrm{E}-07$ & $6.55 \mathrm{E}-07$ \\
\hline 42 & $\mathrm{CO} 2$ & & 2.1 & & 12930 & & 1.70 & E-07 & $8.25 \mathrm{E}-07$ \\
\hline 43 & $\mathrm{CO} 2$ & & 3.7 & & 42691 & & $1.49 \sim 1.22$ & $3.70 \mathrm{E}-08$ & $1.08 \mathrm{E}-07$ \\
\hline 44 & $\mathrm{CO} 2$ & & 2.1 & & 24316 & & $1.59 \sim 1.17$ & $2.18 \mathrm{E}-07$ & $2.83 \mathrm{E}-07$ \\
\hline 45 & $\mathrm{CO} 2$ & & 1.1 & & 12832 & 0.002 & $1.73 \sim 1.06$ & $1.73 \mathrm{E}-06$ & $8.47 \mathrm{E}-07$ \\
\hline 46 & $\mathrm{CO} 2$ & & 1.1 & & 28333 & 0.001887 & $1.76 \sim 1.15$ & $9.21 \mathrm{E}-07$ & $3.13 \mathrm{E}-07$ \\
\hline 47 & $\mathrm{CO} 2$ & & 1.2 & & 28518 & & $1.36 \sim 1.15$ & $3.57 \mathrm{E}-07$ & $1.17 \mathrm{E}-07$ \\
\hline 48 & $\mathrm{CO} 2$ & 0.52 & 1.2 & 20005 & 28075 & 0.001355 & $1.61 \sim 1.20$ & $6.62 \mathrm{E}-07$ & $2.26 \mathrm{E}-07$ \\
\hline
\end{tabular}


Table 5-1 Operating Conditions (continued)

\begin{tabular}{|l||c||c||c|c||c||c||c||c||l||}
\hline \hline case & Gas & $\mathrm{P}(\mathrm{MPa})$ & $\mathrm{CFM}$ & $q^{\prime \prime}\left(\mathrm{W} / \mathrm{m}^{2}\right)$ & Inlet Re & Inlet $q^{+}$ & Tw/Tb & $\max B o^{*}$ & $\max K_{v}$ \\
\hline \hline 49 & $\mathrm{CO} 2$ & 0.52 & 0.9 & 15840 & 20933 & 0.001395 & $1.61 \sim 1.18$ & $1.44 \mathrm{E}-06$ & $3.13 \mathrm{E}-07$ \\
\hline \hline 50 & $\mathrm{CO} 2$ & 0.52 & 0.9 & 20921 & 20757 & 0.001837 & $1.76 \sim 1.16$ & $1.77 \mathrm{E}-06$ & $4.18 \mathrm{E}-07$ \\
\hline \hline 51 & $\mathrm{CO} 2$ & 0.52 & 0.8 & 18372 & 19208 & 0.001731 & $1.72 \sim 1.16$ & $2.07 \mathrm{E}-06$ & $4.25 \mathrm{E}-07$ \\
\hline 52 & $\mathrm{CO} 2$ & 0.53 & 0.7 & 17593 & 17265 & 0.00178 & $1.78 \sim 1.15$ & $2.95 \mathrm{E}-06$ & $4.94 \mathrm{E}-07$ \\
\hline 53 & $\mathrm{CO} 2$ & 0.57 & 0.6 & 13789 & 16548 & 0.00146 & $1.69 \sim 1.16$ & $3.36 \mathrm{E}-06$ & $4.21 \mathrm{E}-07$ \\
\hline 54 & $\mathrm{CO} 2$ & 0.57 & 0.6 & 15248 & 16658 & 0.001528 & $1.75 \sim 1.25$ & $3.66 \mathrm{E}-06$ & $4.60 \mathrm{E}-07$ \\
\hline 55 & $\mathrm{CO} 2$ & 0.66 & 0.4 & 11379 & 12545 & 0.001408 & $1.85 \sim 1.23$ & $9.80 \mathrm{E}-06$ & $6.06 \mathrm{E}-07$ \\
\hline 56 & $\mathrm{CO} 2$ & 0.65 & 0.4 & 7642 & 12089 & 0.00098 & $1.68 \sim 1.16$ & $7.22 \mathrm{E}-06$ & $4.36 \mathrm{E}-07$ \\
\hline 57 & $\mathrm{CO} 2$ & 0.67 & 0.4 & 11614 & 12249 & 0.001436 & $1.88 \sim 1.25$ & $1.11 \mathrm{E}-05$ & $6.49 \mathrm{E}-07$ \\
\hline 58 & $\mathrm{CO} 2$ & 0.58 & 0.6 & 12816 & 14791 & 0.001372 & $1.83 \sim 1.22$ & $4.71 \mathrm{E}-06$ & $4.91 \mathrm{E}-07$ \\
\hline \hline
\end{tabular}

\subsection{Nitrogen Data (Cases 1 through 20)}

Table 5-2 briefly summarizes the operating condition for the nitrogen data.

Table 5-2 Summary of Nitrogen Runs

\begin{tabular}{|c|c|}
\hline Total number of Runs & 20 \\
\hline Operating Pressure Range & $0.13-0.59 \mathrm{MPa}$ \\
\hline Operating Power Range & $291-1989$ Watts \\
\hline Operating Volumetric Flow Rate Range & $0.5-6.1 \mathrm{CFM}$ \\
\hline Inlet Temperature & $\sim 302 \mathrm{~K}$ \\
\hline Inlet Reynolds Number Range & $5,300-23,600$ \\
\hline Inlet $q^{+}$Range & $0.0003-0.0036$ \\
\hline Inlet $B o^{*}$ Range & $3 \times 10^{-9}-6 \times 10^{-6}$ \\
\hline Inlet $K_{v}$ Range & $6 \times 10^{-8}-3 \times 10^{-6}$ \\
\hline
\end{tabular}

From Table 5-1, it can be observed that the nitrogen runs are all in the turbulent flow heat transfer regime and some high heat fluxes cases are in the DTHT regime, since the buoyancy and acceleration parameters were clearly above or near the threshold indicated by McEligot \& Jackson's work (2004).

Figure 5-1 plots the measured Nusselt number versus the Reynolds number for all the nitrogen runs. It can be observed that most of the nitrogen data agreed well with the Nusselt number predicted by the Gnielinski correlation. However, significant heat transfer deterioration began at the Reynolds number of about 10,000. Runs number 4, 6, $17,18,19$ and 20 showed significant deterioration from the forced turbulent convection heat transfer. 
As it was described in Chapter 3, the Reynolds number, buoyancy parameter and acceleration parameter all decrease from inlet to outlet for the gas flow due to its thermal properties dependence on the temperature change. Figure 5-1 shows the inlet to outlet direction.

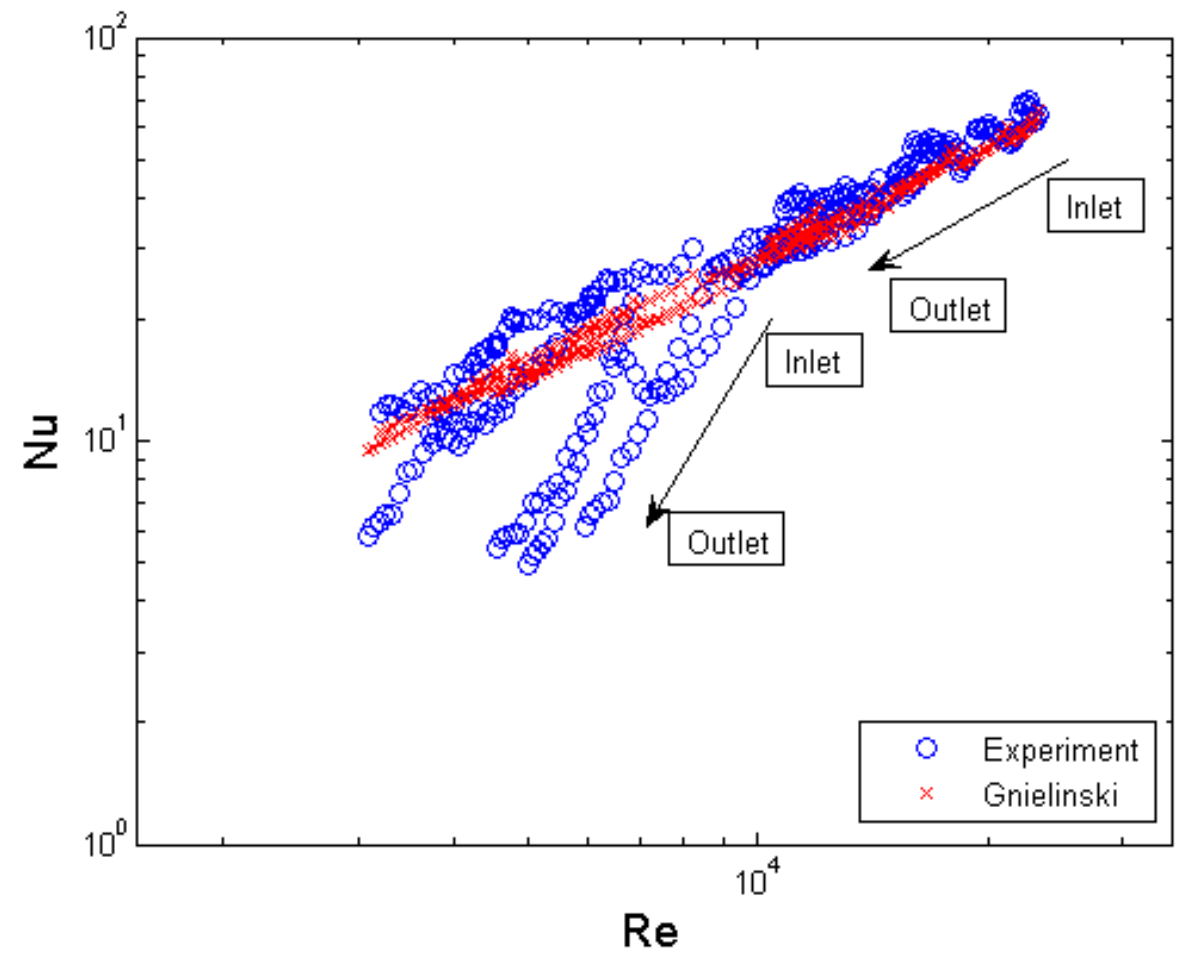

Figure 5-1 Nitrogen Nusselt Number vs. Reynolds Number

Figures 5-2 and 5-3 plot the temperature profile of the DTHT case along with the normal forced turbulent heat transfer case. Selected runs for the comparison purposes have numbers $6,7,16$ and 17 per Table 5-1, and were chosen so that the operating pressure be nearly the same between Case 6,7 and 16, 17 .

In Figure 5-2, black dotted line is the predicted wall temperature using the Gnielinski correlation for the turbulent heat transfer. . It can be clearly seen that the measured wall temperature of Case 6 is significantly higher than the estimated wall temperature (maximum $164^{\circ} \mathrm{C}$ difference). If we compare measured and predicted wall temperatures for Run 6 to those of Run 7, it is obvious that Case 6 was operating at the DTHT regime and significant reduction in the heat transfer coefficient occurred. Since Case 6 was operated at $K_{v_{i n}} \approx 3 \times 10^{-6}$, it can be deduced that the deterioration of heat transfer occurred because of significant acceleration effect due to high heating. 


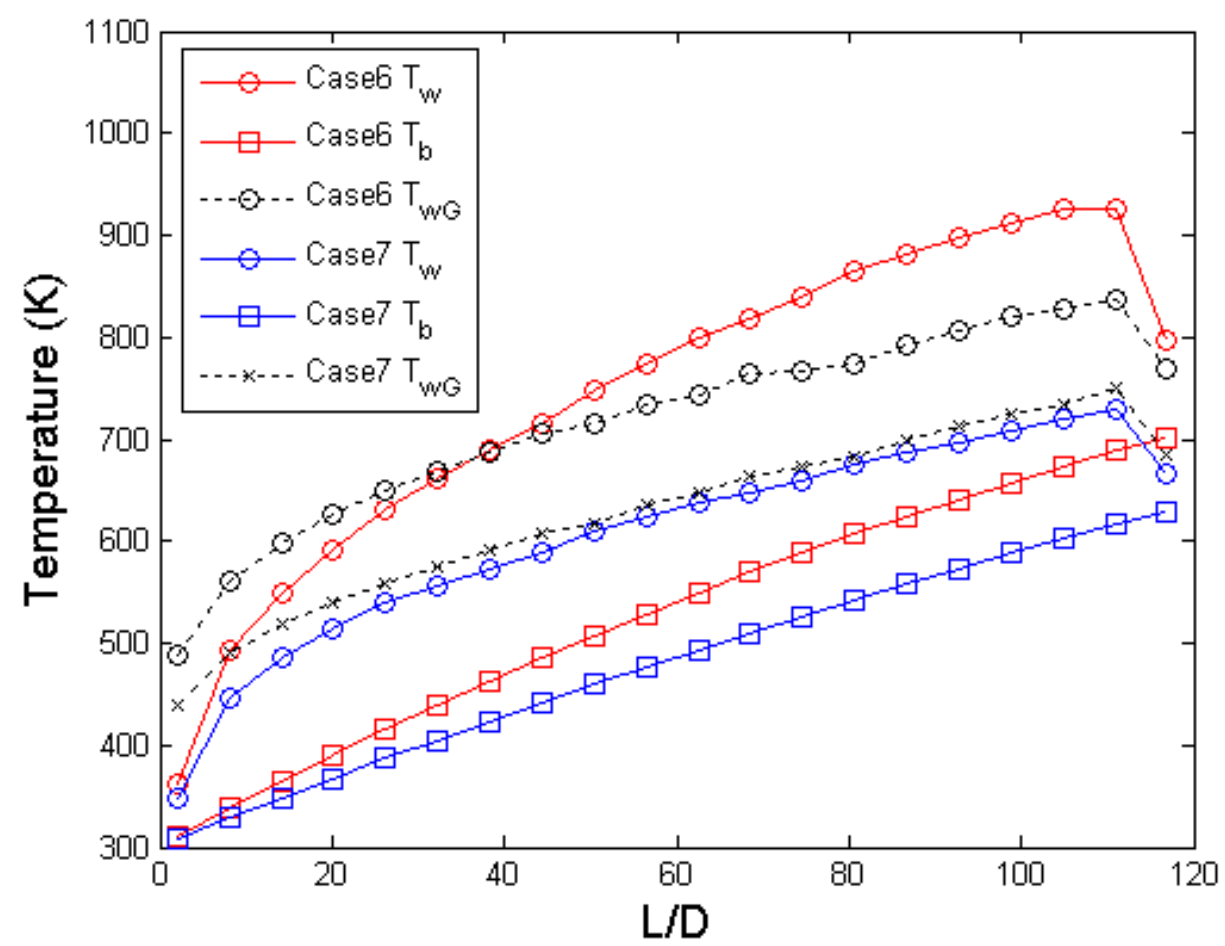

Figure 5-2 Case 6 and 7 Wall, Bulk and Predicted Temperature Profiles

In Figure 5-3, again black dotted line is the predicted wall temperature using the Gnielinski correlation. The measured wall temperature of Run 17 is significantly higher than the predicted wall temperature (maximum $165^{\circ} \mathrm{C}$ difference). Such a large difference between measured and predicted wall temperatures of Run 17 in comparison to Run 16 is a consequence of significant reduction in the heat transfer coefficient of Run 17. Thus, Run 17 was operating at the DTHT regime. Since Run 17 had large Bo number, $B o_{\text {in }}^{*} \approx 2 \times 10^{-6}$, this deterioration is likely caused by buoyancy effect due to high heat flux in this test.

Interesting observation that can be made from Figures 5-2 and 5-3 is that the temperature profile differs between the acceleration induced DTHT and the buoyancy induced DTHT. The acceleration effect driven DTHT seems like a steady laminarization of the turbulent flow toward the down stream, while the buoyancy effect driven DTHT has a maximum in the middle of the channel. Since, the buoyancy parameter decreases along the channel for heated gas flow, the buoyancy effect is decreasing along the channel. After certain reduction of the buoyancy force occurs, the turbulent flow seems to regain its turbulent intensity to return back to the normal turbulent flow, "re-turbulization" so to speak. This phenomenon will be addressed in more detail when carbon dioxide data are discussed. 


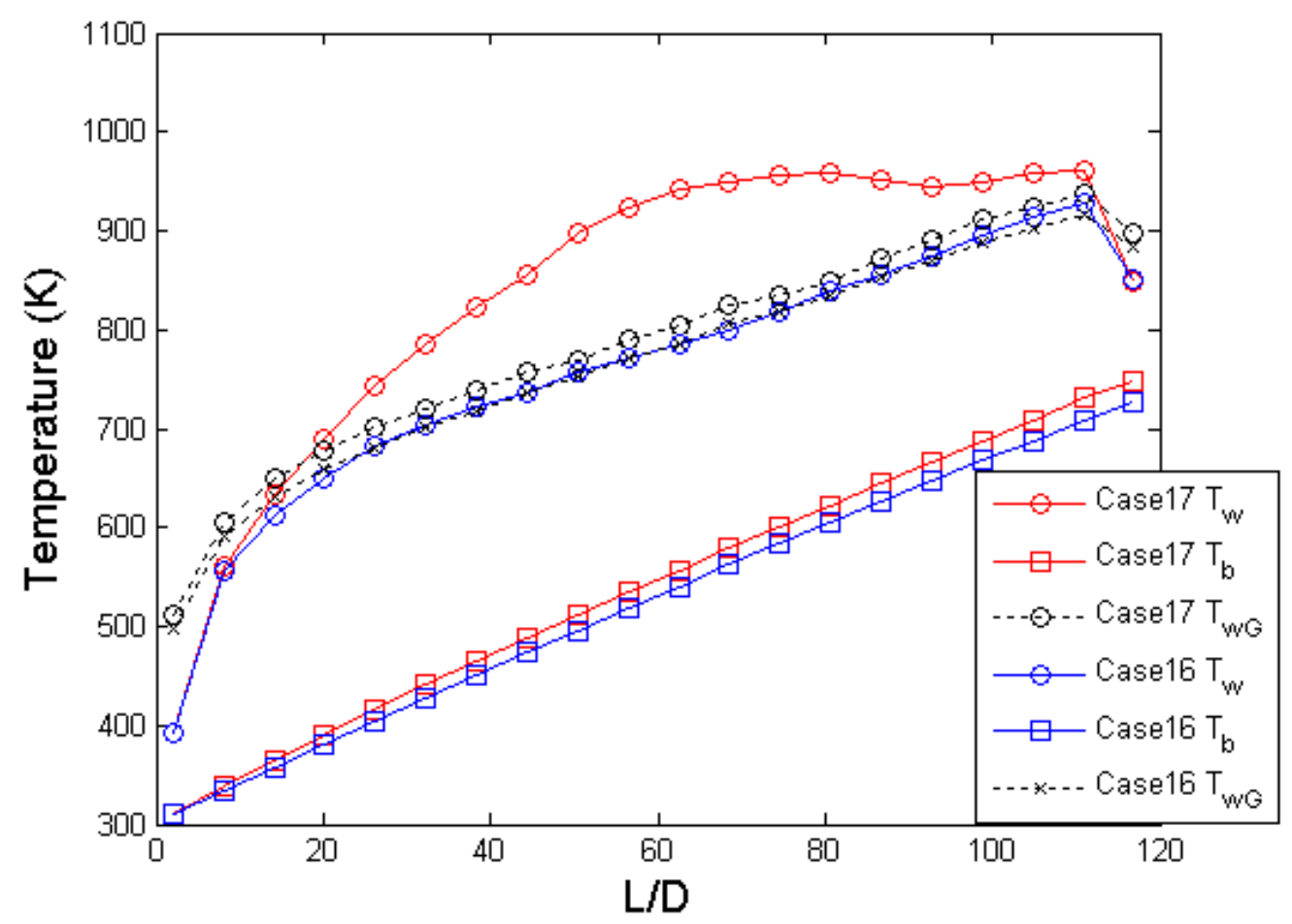

Figure 5-3 Case 16 and 17 Wall, Bulk and Predicted Temperature Profiles

Figures 5-4 and 5-5 plot the ratio of measured $\mathrm{Nu}$ over predicted $\mathrm{Nu}$ using Gnielinski correlation versus buoyancy and acceleration parameters, respectively. These figures confirm that six DTHT cases either operated in the DTHT regime due to the high acceleration effect or the buoyancy effect. The maximum reduction in the local heat transfer coefficient, compared to the Gnielinski correlation is $76 \%$ (Run 20). This is a substantial reduction of heat transfer capability. 


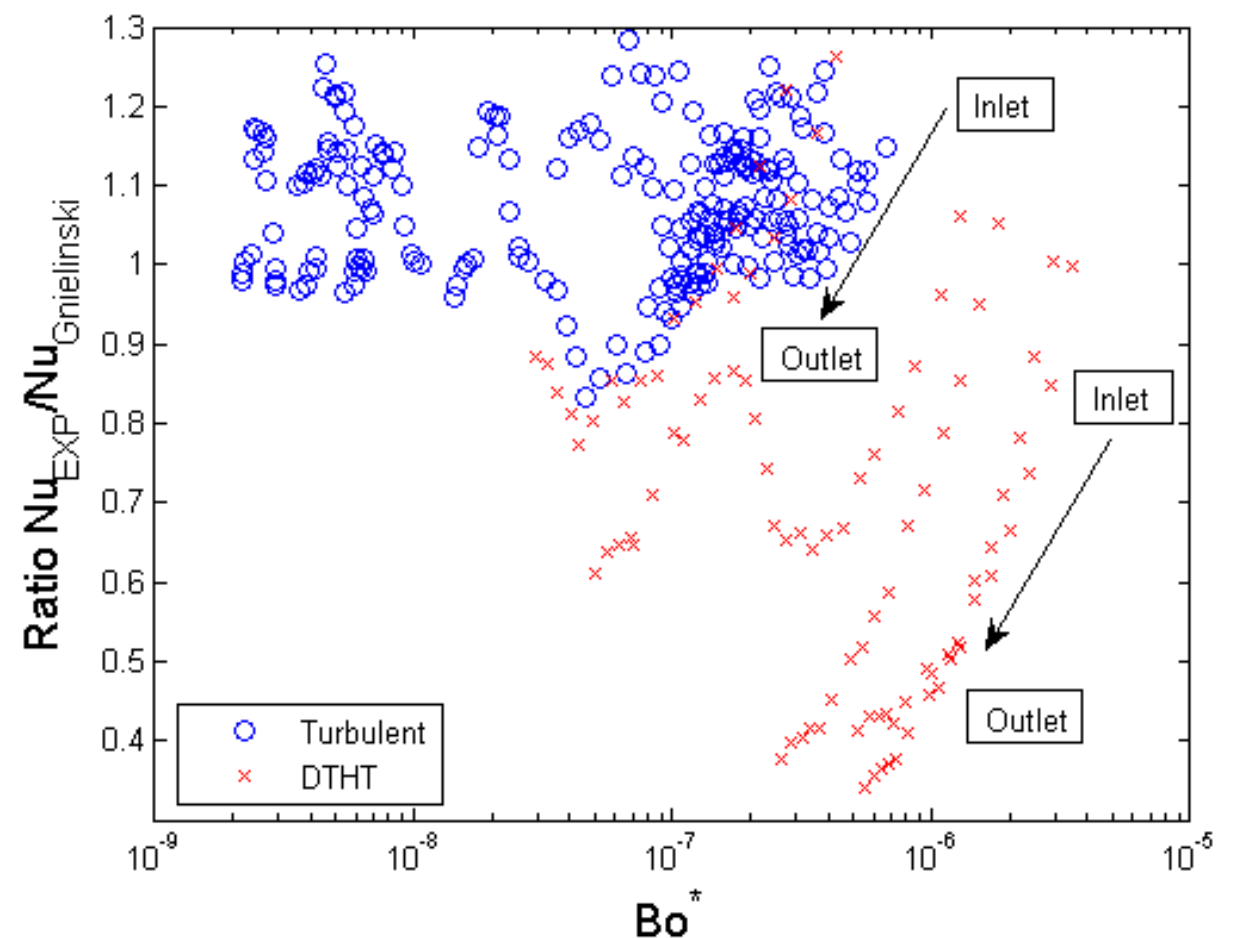

Figure 5-4 Nitrogen Nusselt Number Ratio vs. Buoyancy Parameter

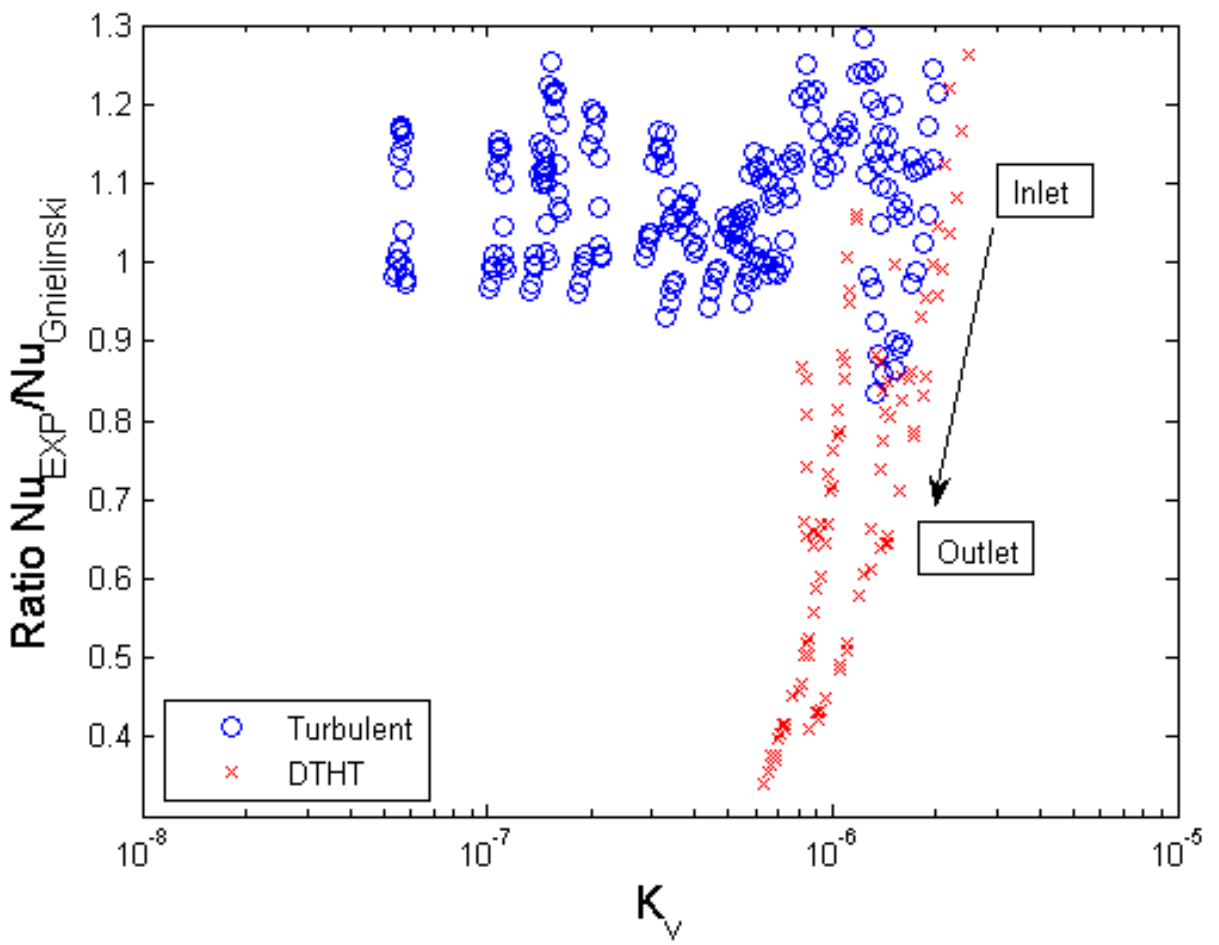

Figure 5-5 Nitrogen Nusselt Number Ratio vs. Acceleration Parameter

Before we start the discussion on which effect is responsible for inducing the DTHT 
regime, one point needs to be thought through thoroughly. The acceleration parameter threshold is set when the turbulent flow becomes fully laminarized and the heat transfer coefficient decreases from the turbulent flow value to the laminar flow value. The minimum reduction in the fully acceleration driven DTHT can be calculated for this case by taking the ratio of the laminar Nusselt number $(\mathrm{Nu}=4.364)$ to the turbulent Nusselt number at the Reynolds number 2,300 $(\mathrm{Nu}=7.211$ at $\operatorname{Pr}=0.7$ with Eq. 2-20), which yields $4.364 / 7.211=0.605$. Therefore, the minimum reduction, when the acceleration parameter exceeded the threshold, is $40 \%$.

In contrast, the buoyancy parameter threshold is set when the measured Nusselt number is reduced from the forced convection turbulent flow Nusselt number by $5 \%$ [Jackson et al., 1989 \& McEligot \& Jackson, 2004]. Therefore, it is unreasonable to treat both thresholds equally, since the acceleration parameter threshold impact is much greater than the buoyancy parameter threshold and either one of the parameter thresholds needs to be modified.

To have a comparable buoyancy parameter threshold to the acceleration threshold, the buoyancy threshold is redefined as the buoyancy number that gives the same fractional reduction of the Nusselt number compared to the acceleration threshold. This can be calculated using Jackson's heat transfer correlation (Eq. 2-23). Thus, the equivalent buoyancy parameter threshold per Eq. $(2-23)$ is $B o^{*} \approx 3 \times 10^{-6}$, which gives $40 \%$ reduction in the Nusselt number. Therefore, $B o^{*} \approx 3 \times 10^{-6}$ will be used as a measure for the buoyancy threshold and this threshold value will be used for comparing the relative strength of the buoyancy effect versus the acceleration effect.

Figure 5-6 plots inlet buoyancy parameter and the acceleration parameter for all the DTHT runs with nitrogen and compares them with the threshold value for each parameter. It can be concluded that Runs 4 and 6 were operating at the DTHT regime driven primarily by flow acceleration, while the remaining cases were primarily buoyancy driven, as Runs 4 and 6 are closer to the acceleration effect threshold than to the buoyancy effect threshold and other runs have opposite trend.

By comparing the operating conditions between Case 4, 6 and Case 17 to 20, this observation can be validated further. Case 17 to 20 were operating at relatively high system pressure compared to Cases 4 and 6 . The discussion in Chapter 3 has shown that when the gas is pressurized the buoyancy effect becomes stronger than the acceleration effect. Thus, our observation seems reasonable. In other words, no interference between the buoyancy effect and the acceleration effect was observed.

To summarize the nitrogen runs, the following can be stated:

1. Nitrogen forced convection turbulent heat transfer data confirmed that the facility could produce reliable forced convection having very close agreement with wellestablished Gnielinski correlation. This validated correct operation of the facility and instrumentation.

2. Six cases operated at the DTHT regime, 2 cases operated at the DTHT regime due to the acceleration effect and 4 cases were due to the buoyancy effect. 


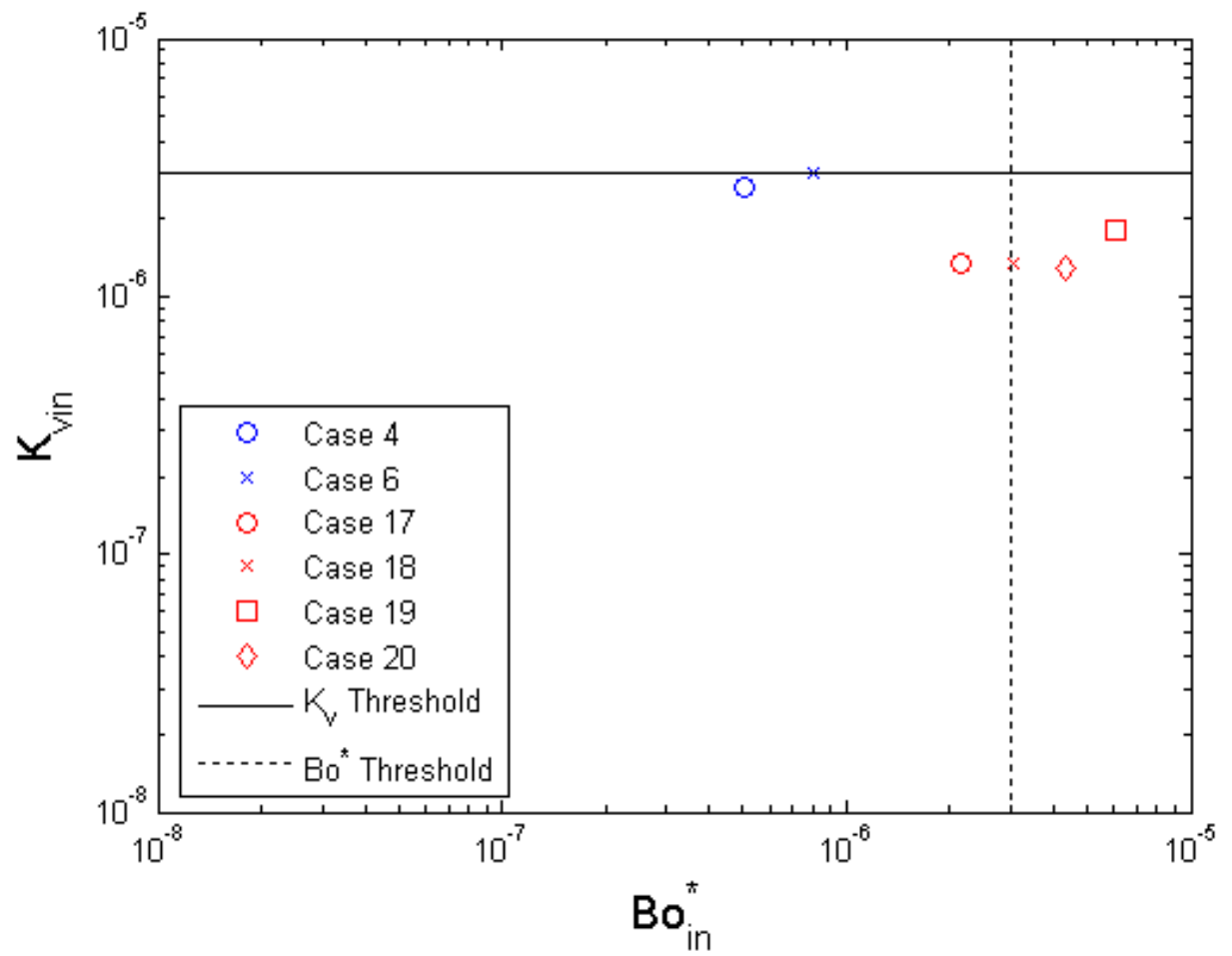

Figure 5-6 Map of DTHT Runs: Inlet Acceleration Parameter vs. Inlet Buoyancy

Parameter

\subsection{Helium Data (Cases 21 through 35)}

Table 5-3 briefly summarizes the operating condition for the helium data.

Table 5-3 Summary of Helium Runs

\begin{tabular}{|c|c|}
\hline Total number of Runs & 15 \\
\hline Operating Pressure Range & $0.17-0.40 \mathrm{MPa}$ \\
\hline Operating Power Range & $200-2630 \mathrm{Watts}$ \\
\hline Operating Volumetric Flow Rate Range & $2.5-6.1 \mathrm{CFM}$ \\
\hline Inlet Temperature & $\sim 304 \mathrm{~K}$ \\
\hline Inlet Reynolds Number Range & $1,800-4,500$ \\
\hline Inlet $q^{+}$Range & $0.0003-0.0039$ \\
\hline Inlet $B o^{*}$ Range & $7 \times 10^{-9}-4 \times 10^{-7}$ \\
\hline Inlet $K_{v}$ Range & $4 \times 10^{-7}-5 \times 10^{-6}$ \\
\hline
\end{tabular}

From the table, it is shown that the helium runs have potential of covering the laminar, mixed convection laminar, transition between laminar and turbulent, turbulent and the acceleration driven DTHT regime. 
Figure 5-7 presents the helium runs in the Nusselt number vs. Reynolds number plot.. The Gnielinski correlation (Eq. 2-7) and the forced convection laminar correlation (Eq.22 ) were used to show the predicted forced Nusselt number in both regimes. However, it is important to note that the Gnielinski correlation range does not extent below local Reynolds number of 2,300, but even if the local Reynolds number is lower than 2,300 still the Gnielinski correlation was used to predict the Nusselt number if the inlet local Reynolds number is higher than 2,300. This scheme is applied to have continuous heat transfer coefficient in single channel.

The figure clearly shows that helium operated in the mixed convection laminar, transition, turbulent and DTHT regime. Runs 28 through 30 are in the mixed convection laminar flow regime, Runs 25 through 27 and 34 through 35 are in the transition regime, Runs 21 through 24 and 31 through 32 are in the turbulent regime and finally Case 33 is in the DTHT regime.

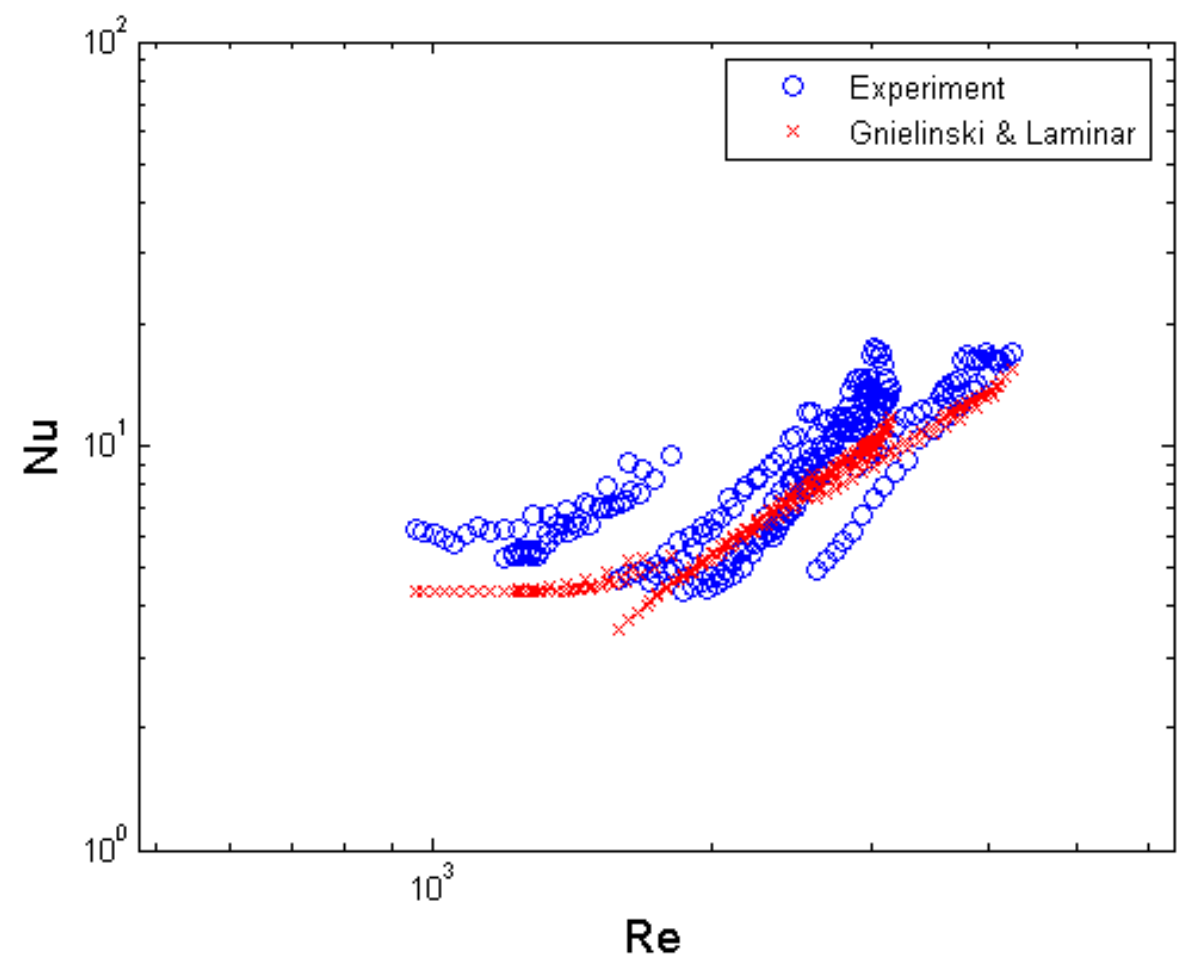

Figure 5-7 Helium Nusselt Number vs. Reynolds Number.

Figure 5-8 and 5-9 shows the ratio of the measured Nusselt number to the Nusselt number predicted by Gnielinski correlation versus the buoyancy parameter and the acceleration parameter. Three regimes presented in the figures involve turbulent, DTHT and transition regimes. 


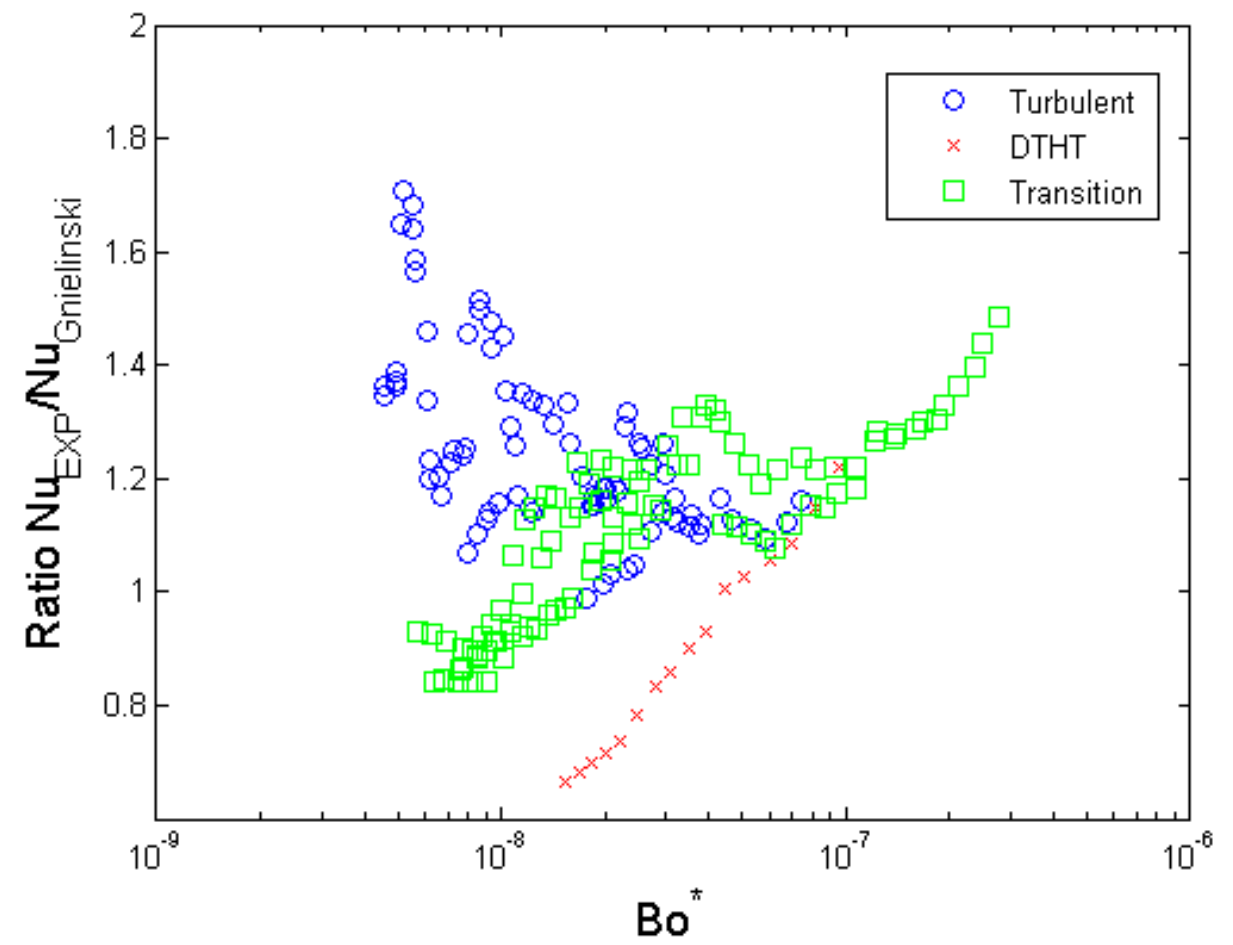

Figure 5-8 Nusselt Number Ratio vs. Helium Buoyancy Parameter

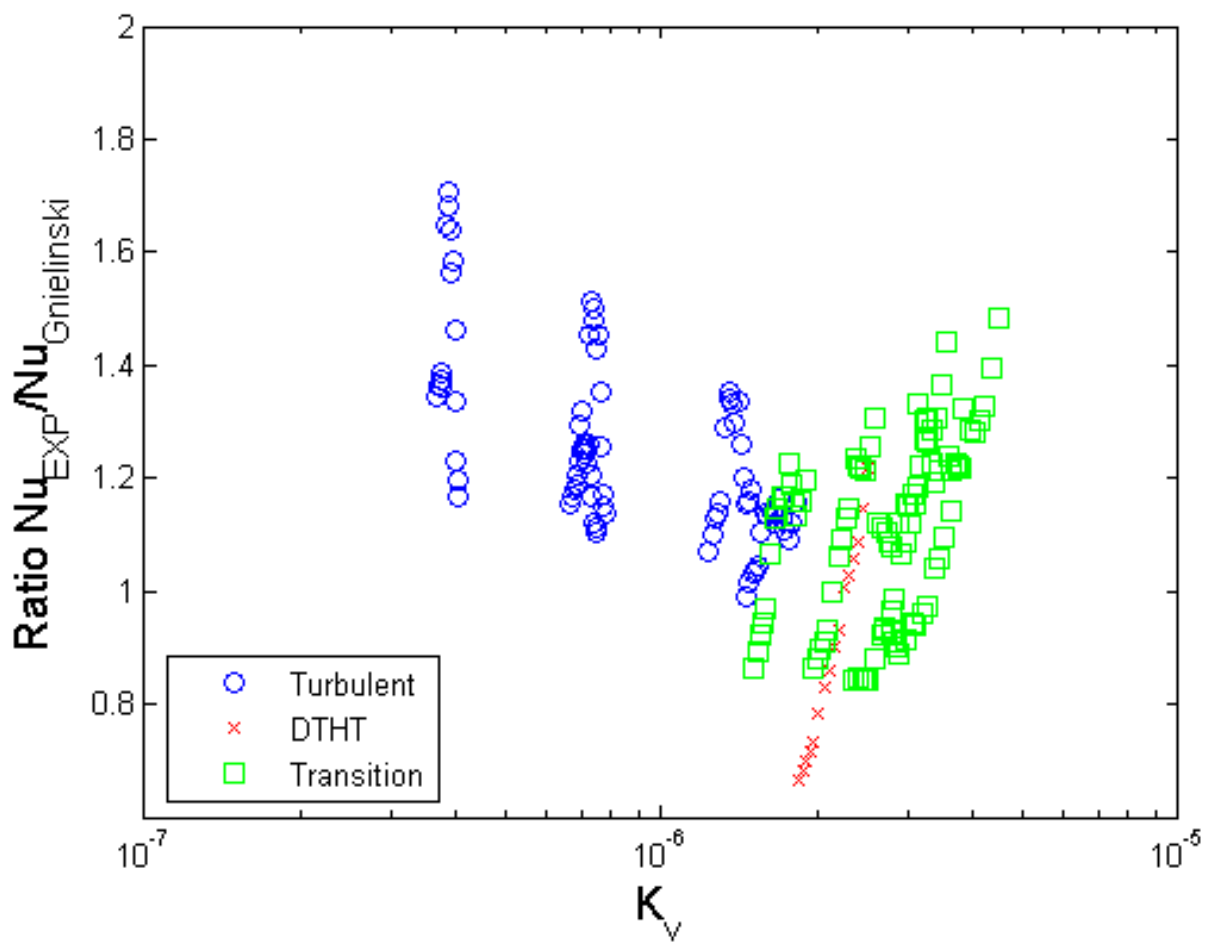

Figure 5-9 Helium Nusselt Number Ratio vs. Acceleration Parameter 
It is obvious from the figures that the DTHT run was due to the acceleration effect, since the $K_{v_{\text {in }}} \approx 3 \times 10^{-6}$ while $B o_{i n}^{*} \approx 1 \times 10^{-7}$. Interesting phenomenon is that even though the several runs in the transition flow category have stronger acceleration effect than the DTHT run the heat transfer coefficient is not deteriorated. One possible explanation is in the application of the transition criteria that were discussed in $2^{\text {nd }}$ annual report. Since there is a possibility of having laminar to turbulent transition earlier than at the Reynolds number of 2,300 in the heated flow where the heating causes instability of the stable flow [Lee et al., 2005 a-c], this "turbulization" process can become stronger than the acceleration-driven "laminarization" process. From Figure 2-3, since the turbulence is generated from the velocity gradient, steeper gradient at the wall and two maximum points of the velocity profile due to the high heating can create flow instability strong enough to maintain the turbulence even though the acceleration effect is strong. On Figure 5-8, the enhancement of the transition regime heat transfer coefficient compared to the Gnielinski correlation follows the increase of the buoyancy parameter, which supports this hypothesis.

Another unexpected behavior from the figures is that the helium turbulent forced convection heat transfer is significantly enhanced compared to the Gnielinski correlation prediction when both the buoyancy effect and the acceleration effect are small. For a logical explanation, more data to check the reproducibility of the data and theoretical development should be followed in this regime.

Figure 5-10 shows the correlation between the non-dimensional number that we have selected from Chapter 2 and ratio of the measured Nusselt number to the Nusselt number predicted by laminar flow heat transfer correlation. Figure 5-10 indicates that the nondimensional number that was chosen seems to correlate the enhancement of the laminar convection. The enhancement is due to the buoyancy force, which drives a steeper wall velocity gradient and enhances the heat transfer process. However, to verify if the flow is truly laminar or not, the information of velocity and temperature profiles of the flow are required.

To summarize the helium runs:

1. Helium runs covered mixed laminar, transition, turbulent and DTHT regimes together.

2. The DTHT regime was due to the acceleration effect.

3. Some unexpected behavior in the transition regime was observed and more investigation will be needed to understand the physics behind it.

4. Laminar heat transfer was enhanced due to the buoyancy effect and the selected non-dimensional number seems to correlate the enhancement up to certain degree. 


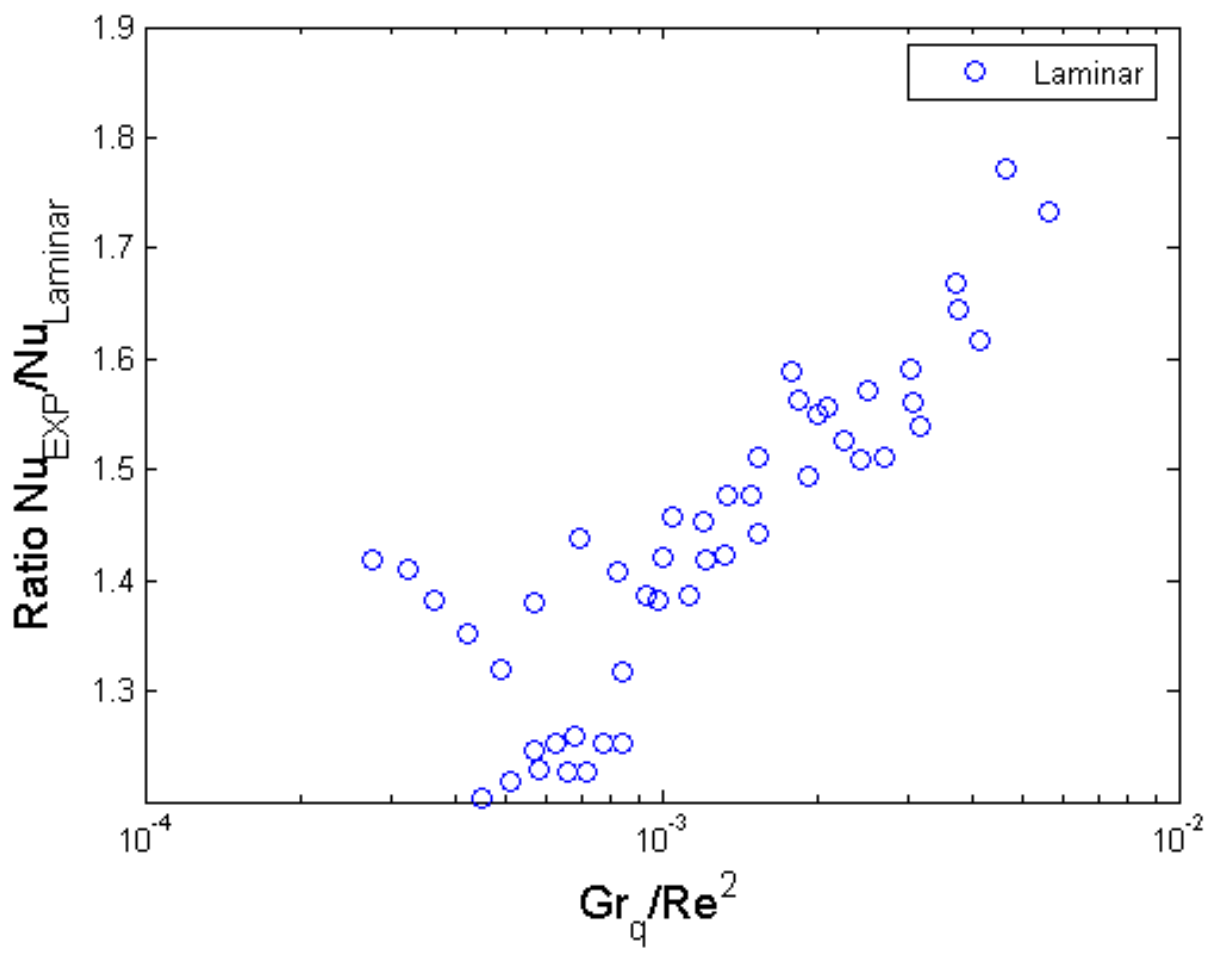

Figure 5-10 Helium Laminar Nusselt Number Ratio vs. Laminar Buoyancy Parameter

\subsection{Carbon Dioxide Data (Runs 36 through 58)}

Table 5-4 briefly summarizes the operating condition for the carbon dioxide data.

Table 5-4 Summary of Carbon Dioxide Runs

\begin{tabular}{|c|c|}
\hline Total number of Runs & 23 \\
\hline Operating Pressure Range & $0.13-0.67 \mathrm{MPa}$ \\
\hline Operating Power Range & $720-2690 \mathrm{Watts}$ \\
\hline Operating Volumetric Flow Rate Range & $0.4-6.0 \mathrm{CFM}$ \\
\hline Inlet Temperature & $\sim 303 \mathrm{~K}$ \\
\hline Inlet Reynolds Number Range & $12,000-42,700$ \\
\hline Inlet $q^{+}$Range & $0.0006-0.0022$ \\
\hline Inlet $B o^{*}$ Range & $1 \times 10^{-8}-1 \times 10^{-5}$ \\
\hline Inlet $K_{v}$ Range & $8 \times 10^{-8}-8.5 \times 10^{-7}$ \\
\hline
\end{tabular}

From the table, it is shown that the carbon dioxide runs operated in the turbulent and buoyancy induced DTHT regime.

Figure 5-11 shows the carbon dioxide data versus the Reynolds number. Runs 52 through 58 operated in the DTHT regime and rest of the runs operated in the turbulent regime. 


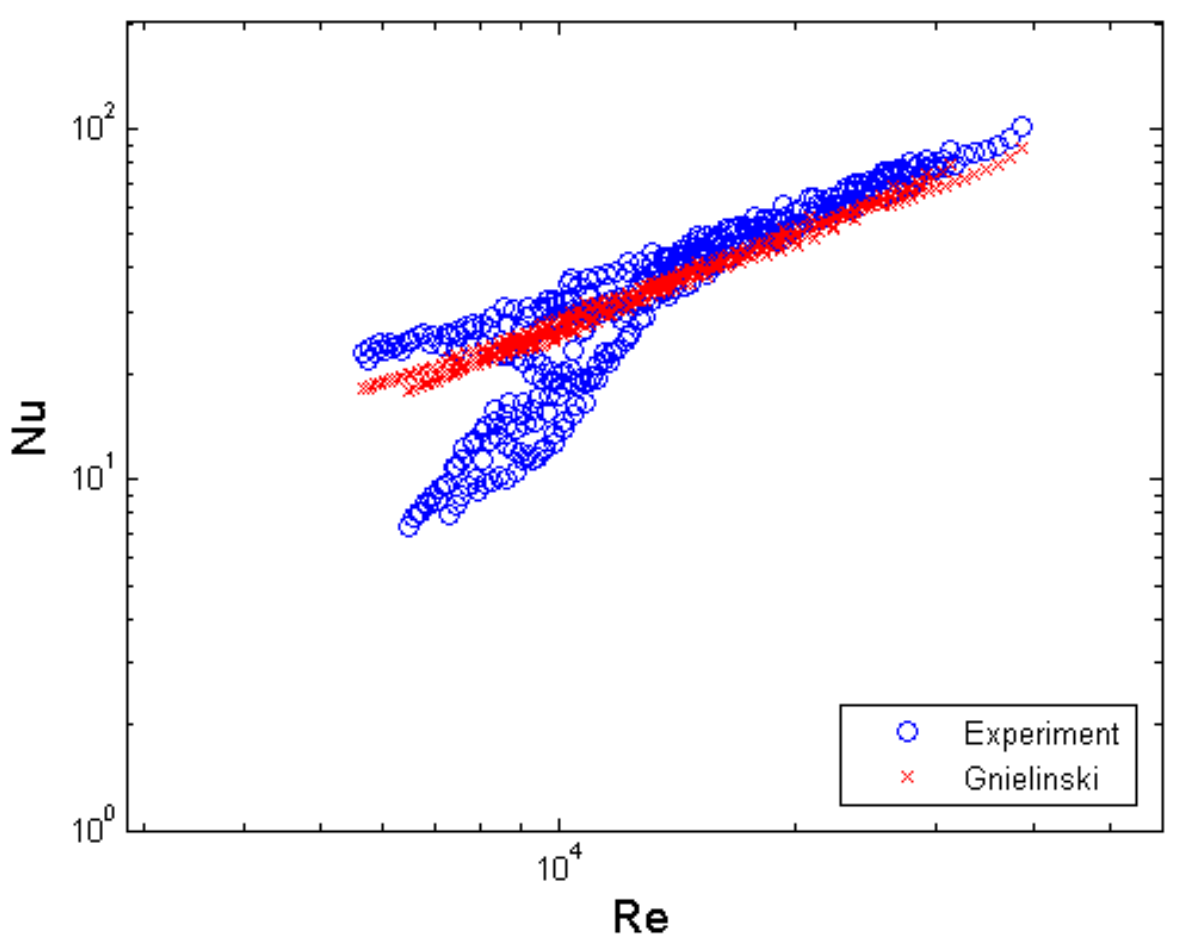

Figure 5-11 Carbon Dioxide Nusselt Number vs. Reynolds Number

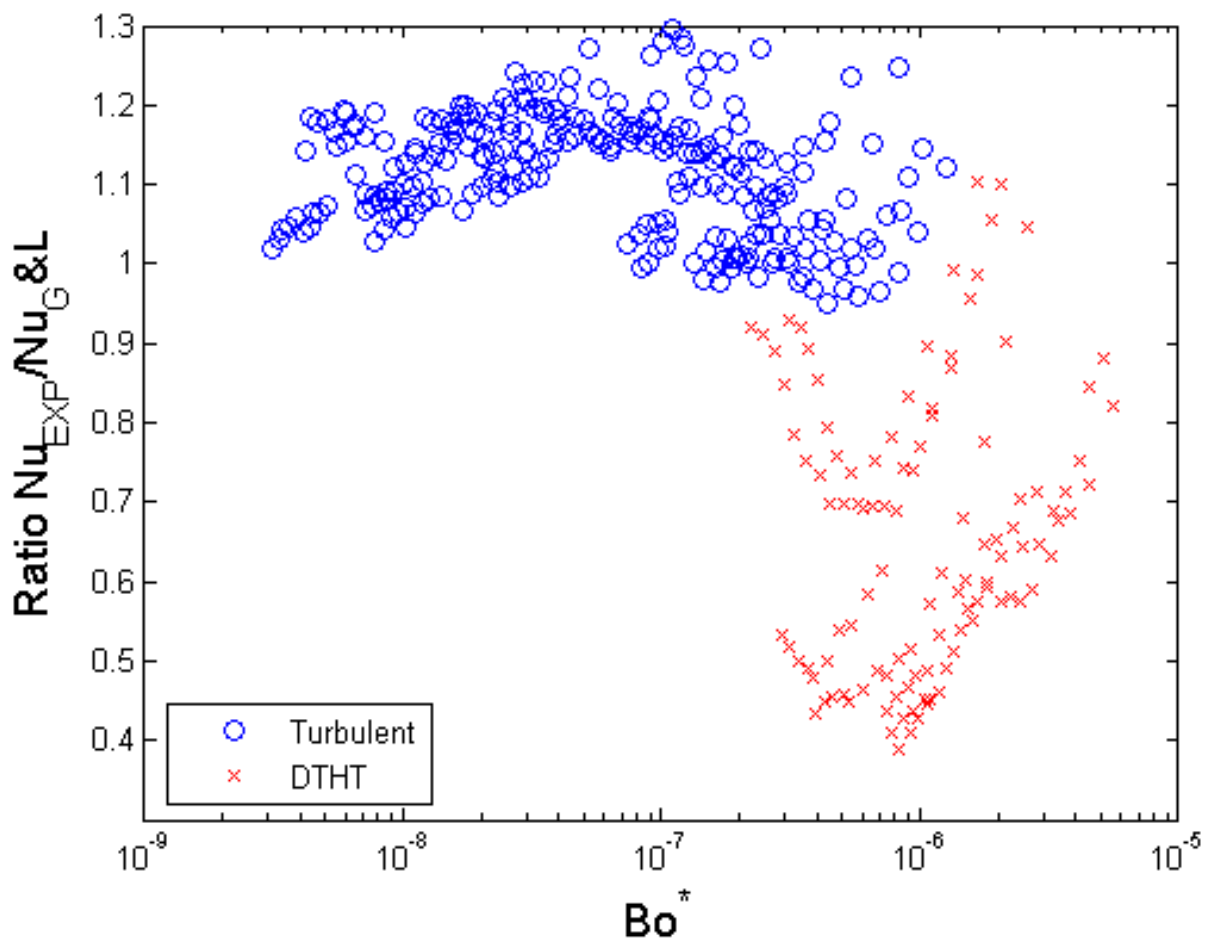

Figure 5-12 Carbon Dioxide Nusselt Number Ratio vs. Buoyancy Parameter 


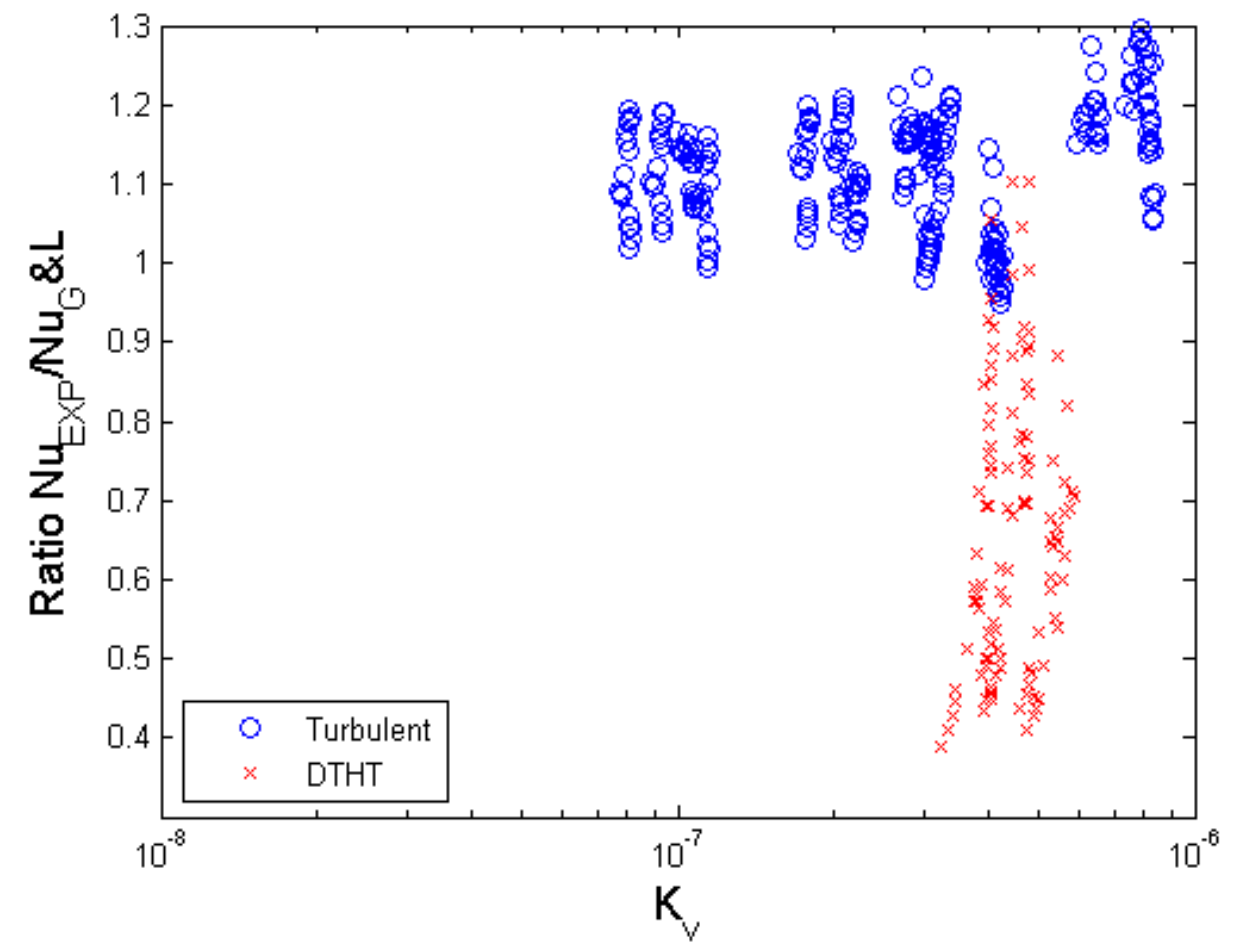

Figure 5-13 Carbon Dioxide Nusselt Number Ratio vs. Acceleration Parameter

Figures 5-12 and 5-13 clearly show that the all the DTHT regime runs with the carbon dioxide is due to the buoyancy effect. However, new phenomenon that was not fully covered in the nitrogen runs was found in the carbon dioxide runs. The temperature profile of "re-turbulization" and the non re-turbulizing flow are shown in Figure 5-14 and 5-15 respectively. Runs 50 (inlet $B o^{*}=1.77 \times 10^{-6}$ ) and 52 (inlet $B o^{*}=2.95 \times 10^{-6}$ ) are compared to show the re-turbulizing flow and Runs 50 and 57 (inlet $B o^{*}=1.11 \times 10^{-5}$ ) are compared to show the non re-turbulizing flow. By comparing Figure 5-14 and 5-15, it can be concluded that as the buoyancy parameter increases the wall temperature maximum moves toward down stream of the channel.

To summarize the carbon dioxide runs:

1. Carbon dioxide runs were operated at the turbulent and the buoyancy induced DTHT regime.

2. Seven carbon dioxide runs were operated at the DTHT regime.

3. New regimes were observed in the buoyancy induced DTHT, which is "returbulizing" regime and "non re-turbulizing" regime. 


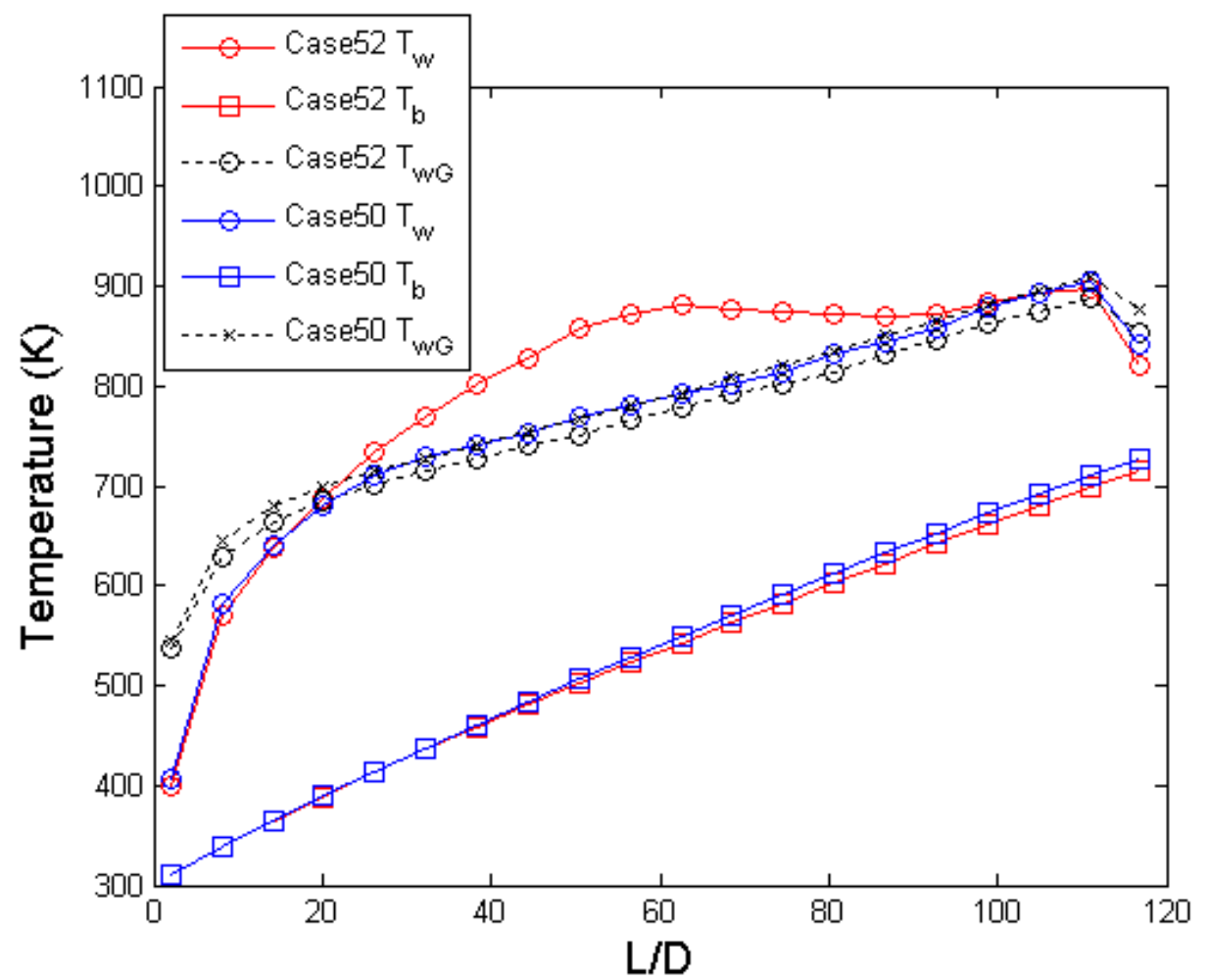

Figure 5-14 Temperature Profile of Re-turbulizing Flow (Run 50 and 52)

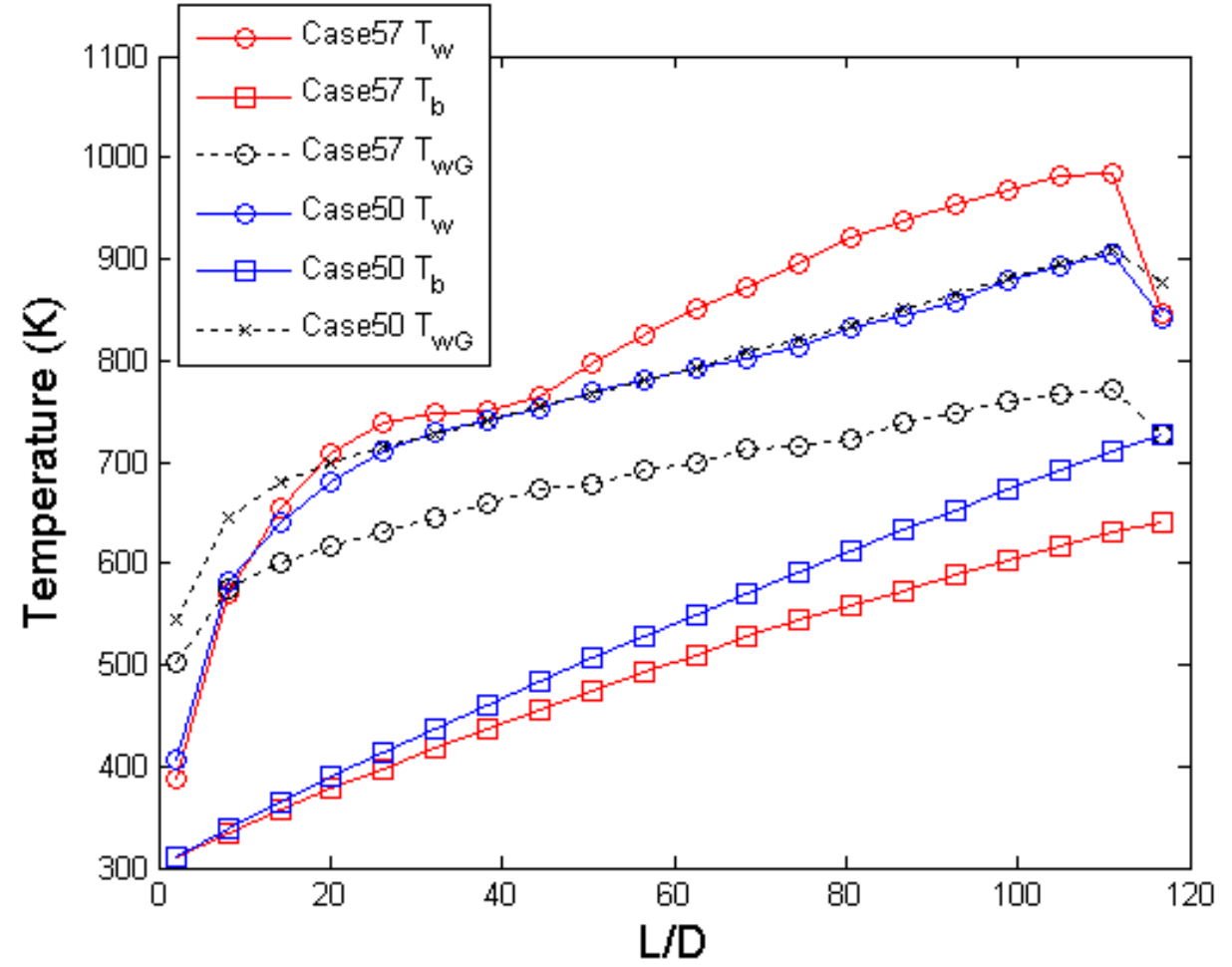

Figure 5-15 Temperature Profile of non Re-turbulizing Flow (Run 50 and 57) 


\subsection{Data Summary}

This section will summarize all the data. Table 5-5 summarizes all the experimental runs

Table 5-5 Summary of All the Experimental Runs

\begin{tabular}{|c|c|}
\hline Total number of Runs & $58\left(\mathrm{~N}_{2}, \mathrm{He}, \mathrm{CO}_{2}\right)$ \\
\hline Operating Pressure Range & $0.13-0.67 \mathrm{MPa}$ \\
\hline Operating Power Range & $200-2690$ Watts \\
\hline Operating Volumetric Flow Rate Range & $0.4-6.1 \mathrm{CFM}$ \\
\hline Inlet Temperature & $300-305 \mathrm{~K}$ \\
\hline Inlet Reynolds Number Range & $1,800-42,700$ \\
\hline Inlet $q^{+}$Range & $0.0003-0.0039$ \\
\hline Inlet $B o^{*}$ Range & $3 \times 10^{-9}-1 \times 10^{-5}$ \\
\hline Inlet $K_{v}$ Range & $6 \times 10^{-8}-5 \times 10^{-6}$ \\
\hline
\end{tabular}

The operating flow regimes were mixed convection laminar, transition from laminar to turbulent, turbulent, the buoyancy induced DTHT and the acceleration induced DTHT regimes. Table 5-6 summarizes the case number for each regime. This can be also found in more detail in Table 5-1.

Table 5-6 Cases for Each Regime

\begin{tabular}{||c||c||c||}
\hline Heat Transfer Regime & Case \# & Total \\
\hline \hline Mixed Convection Laminar & $28-30$ & 3 \\
\hline Transition from Laminar to Turbulent & $25-27,34,35$ & 5 \\
\hline Buoyancy Induced DTHT & $17-20,52-58$ & 11 \\
\hline Acceleration Induced DTHT & $4,6,33$ & 3 \\
\hline Turbulent Heat Transfer & Remaining Cases & 36 \\
\hline
\end{tabular}

Figure 5-16 depicts the local Nusselt number versus Reynolds number along with the Gnielinski correlation (Eq. 2-7) and the laminar correlation (Eq. 2-2) predictions. As it was mentioned in the nitrogen experimental data summary, even though the Gnielinski correlation is valid above the local Reynolds number of 2,300, the correlation is used for the case when the inlet Reynolds number is higher than 2,300 regardless of the local Reynolds number.

Figures 5-17 and 5-18 show the ratio of the measured Nusselt number to the predicted Nusselt number by the Gnielinski correlation versus the buoyancy parameter and acceleration parameter. Four regimes are marked differently in each figure to show the different behavior of each regime. 


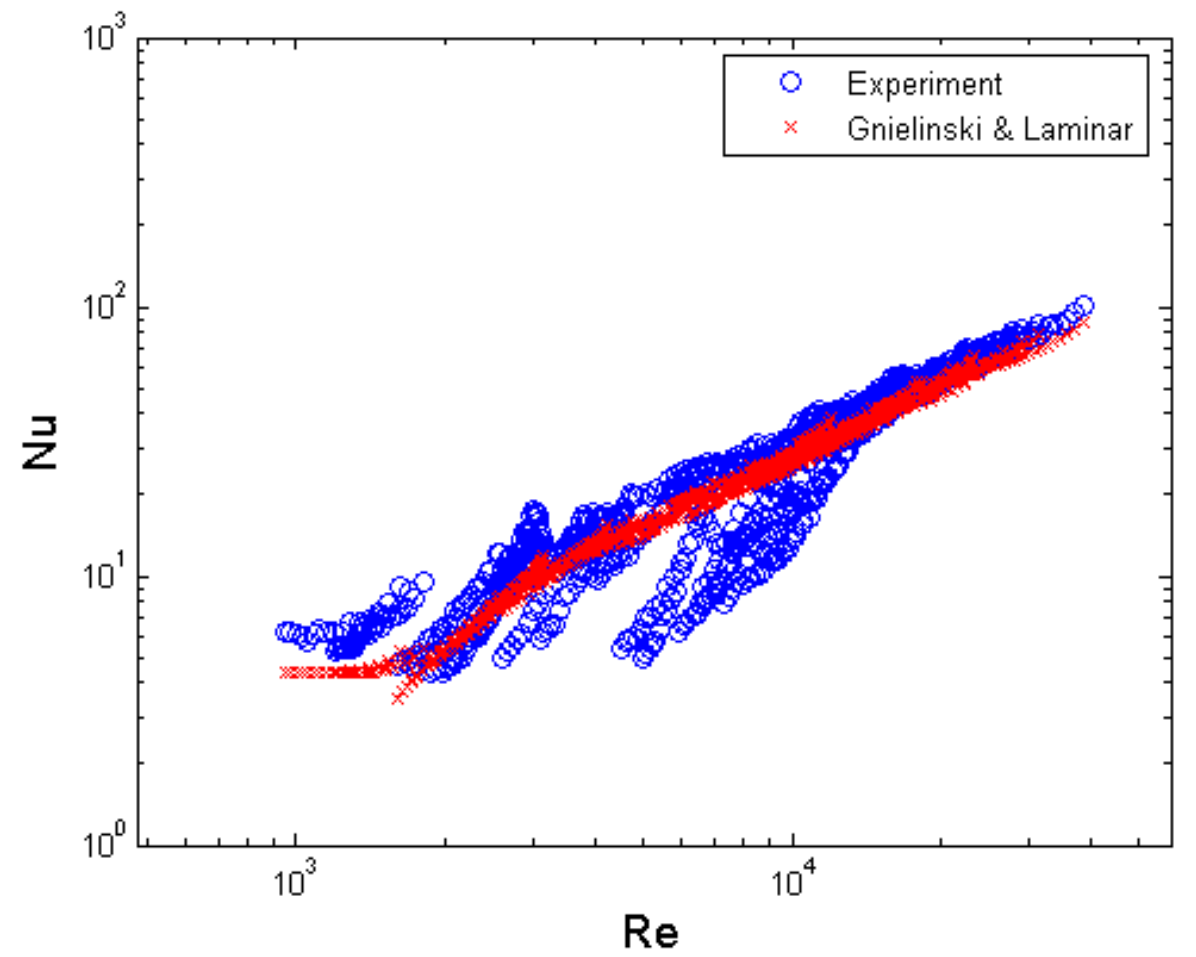

Figure 5-16 Reynolds Number vs. Nusselt Number

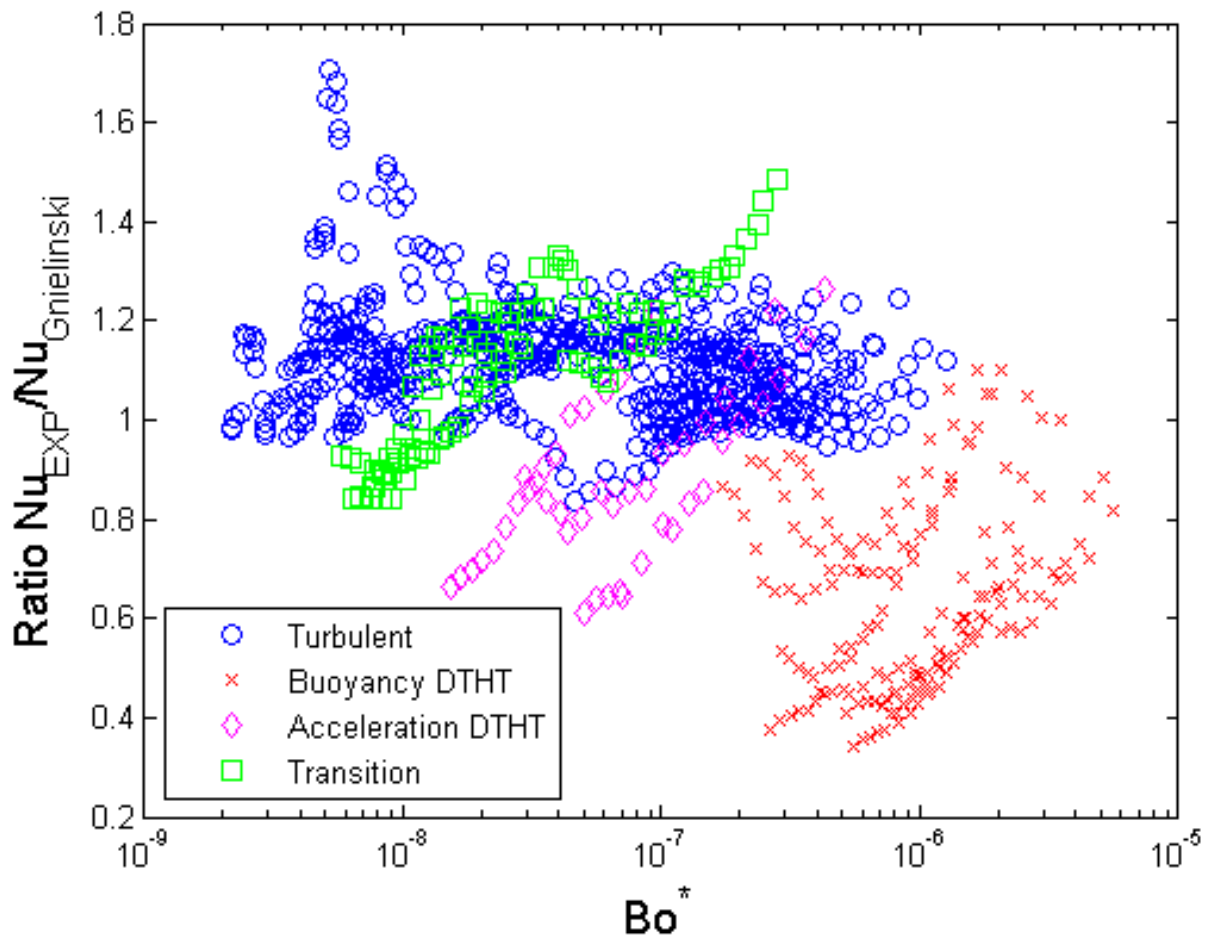

Figure 5-17 Nusselt Number Ratio vs. Buoyancy Parameter 


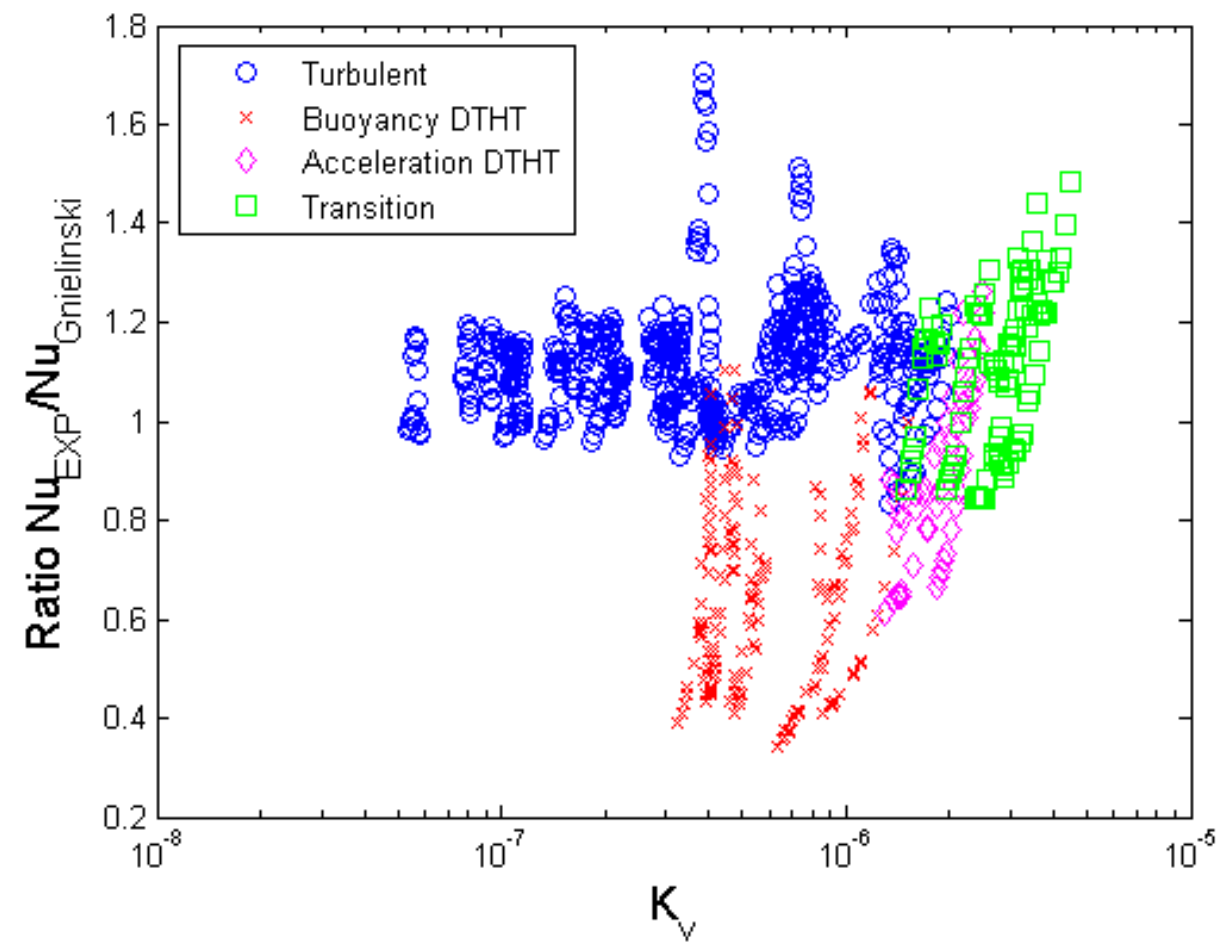

Figure 5-18 Nusselt Number Ratio vs. Acceleration Parameter

From the nitrogen data and carbon dioxide data, it was shown that the buoyancy induced DTHT regime needs to be further divided into "re-turbulizing" and "non re-turbulizing" regimes. If we further divide the buoyancy induced DTHT into two sub-regimes, which is re-turbulizing flow and non re-turbulizing flow, the boundary between the two can be found from Figure 5-19. Figure 5-19 shows the inlet buoyancy number of DTHT runs with the location of maximum heat transfer reduction. From Figure 5-19, $B o^{*} \approx 3.5 \times 10^{-6}$ is identified as a boundary between the re-turbulizing flow and non returbulizing flow. Blue markers are re-turbulized runs, where the maximum reduction occurred at the middle of the test section, while red markers are non re-turbulized flow towards the outlet of the test section.

Figure 5-20 is the plot of DTHT cases for the inlet buoyancy number and the acceleration number. Figure also shows the original threshold value indicated in [McEligot \& Jackson, 2004] and the new thresholds identified from the MIT experimental data. Table 5-7 summarizes thresholds for the DTHT regime.

Table 5-7 Threshold Values for Each Regime

\begin{tabular}{|c||c||c||}
\hline Heat Transfer Regime & Original & MIT \\
\hline \hline Acceleration Induced DTHT & $K_{v}=3 \times 10^{-6}$ & $K_{v_{i n}}=2 \times 10^{-6}$ \\
\hline Buoyancy Induced DTHT & $B o^{*}=6 \times 10^{-7}$ & $B o_{i n}^{*}=2 \times 10^{-6}$ \\
\hline Re-turbulization Boundary & N/A & $B o_{i n}^{*}=3.5 \times 10^{-6}$ \\
\hline
\end{tabular}




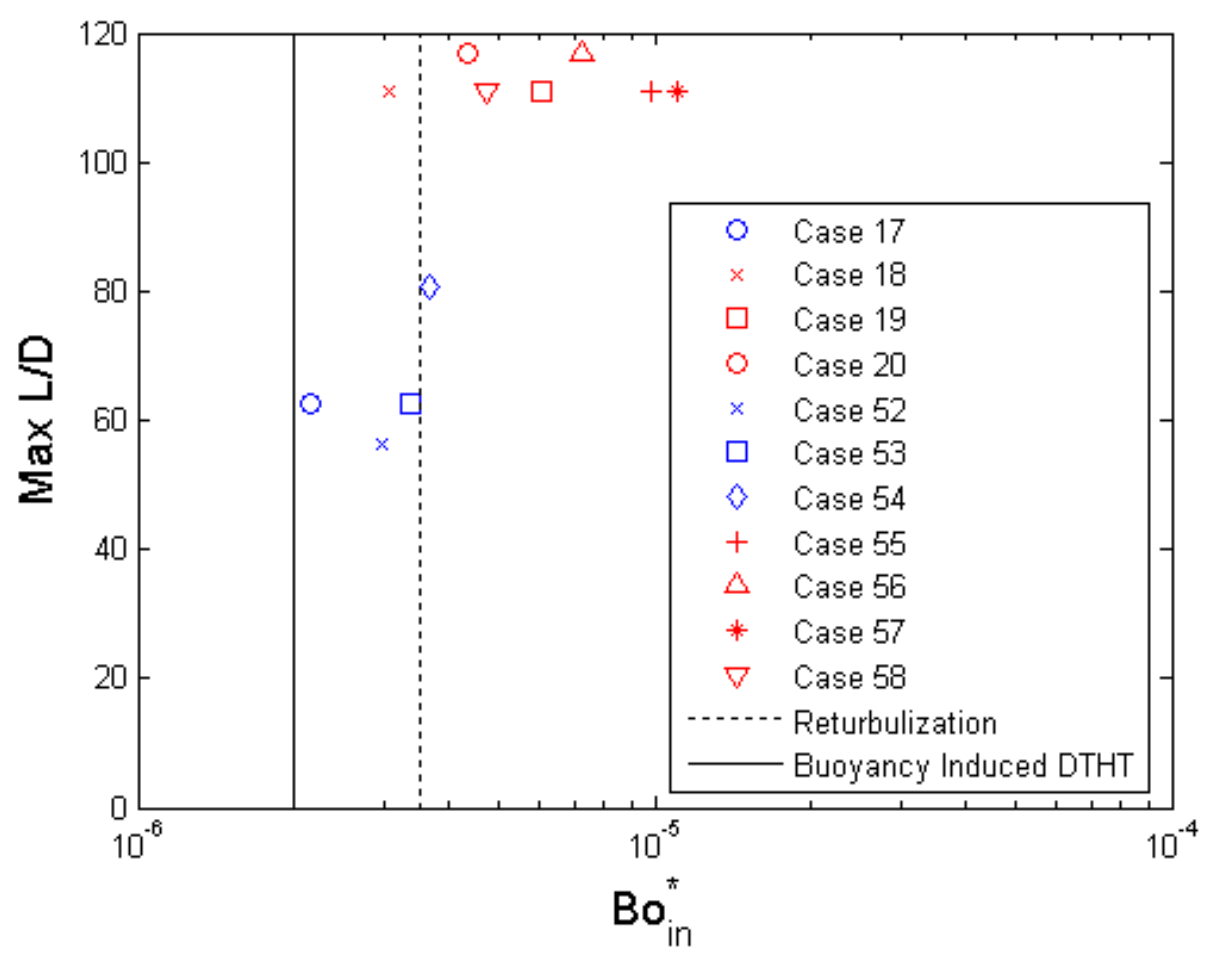

Figure 5-19 Location of Maximum Reduction vs. Inlet Buoyancy Parameter

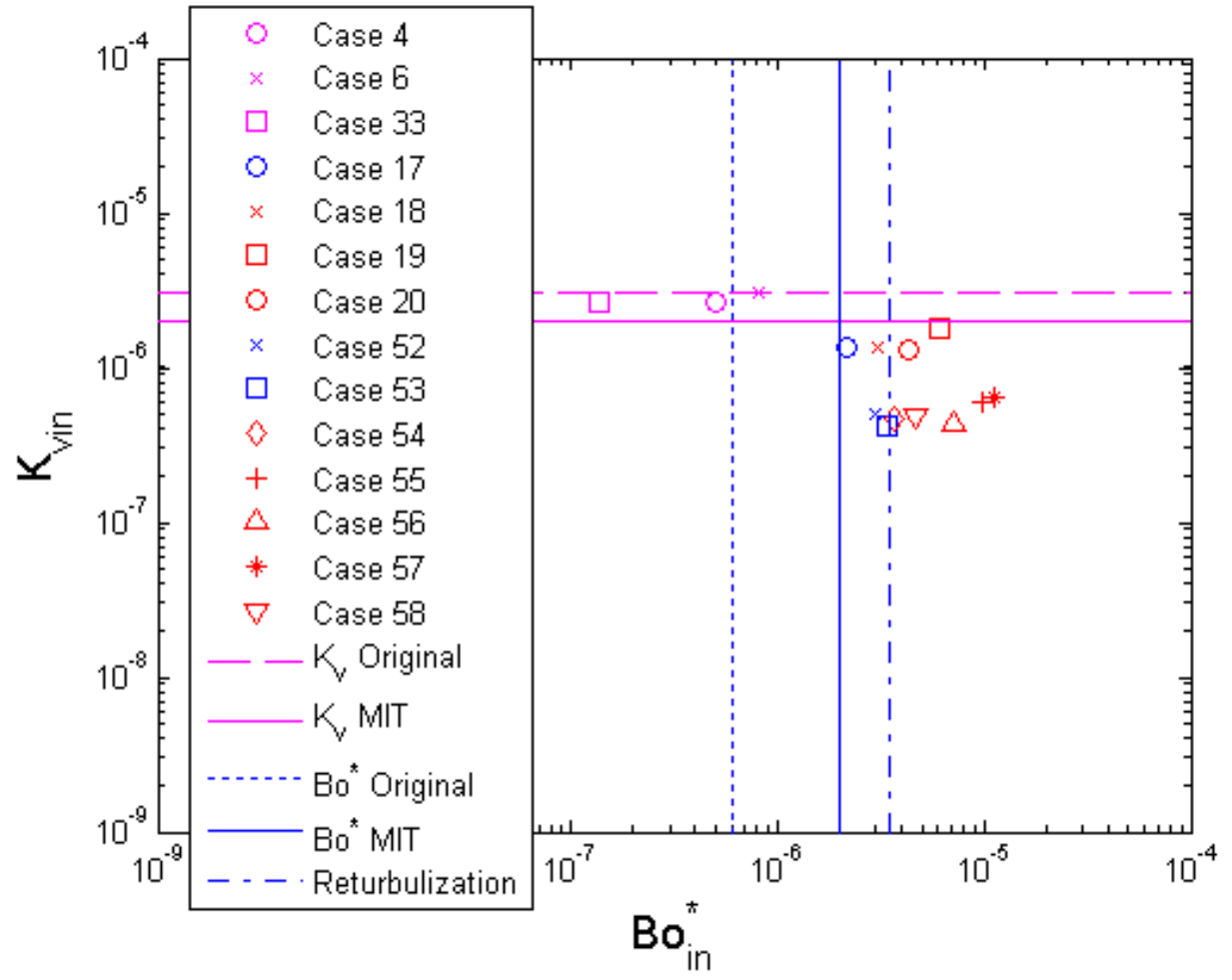

Figure 5-20 Inlet Acceleration Parameter vs. Inlet Buoyancy Parameter 


\subsection{Validity of Experimental Data and Uncertainty Analysis}

It is essential to check the validity of the data and quantify their uncertainty before comparing the data to the existing correlations. One of the key validity checks can be made from the heat balance between added heat input and gas enthalpy rise. This chapter will discuss the heat balance error and present the results of uncertainty analysis.

Figure 5-21 shows the power error and the maximum reduction in the Nusselt number in terms of the inlet Reynolds number. The power error is defined as the normalized difference between the heat input to the gas flow, which is calculated from the power input and the heat losses measurement, and the enthalpy difference between the inlet and outlet temperatures measured by the inlet and outlet flow thermocouples. The maximum reduction on the figure is defined as the percentage reduction in the measured Nusselt number when it is compared to the Gnielinksi correlation or the laminar correlation for turbulent and laminar flow, respectively. As shown in the last section, the mixed convection laminar and some cases of low Reynolds number turbulent flow showed enhancement over the correlation. In these cases, the maximum reduction is not shown on Figure 5-21.

Since, the Gnielinski correlation claims to have agreement with the experimental data within $20 \%$, the reduction below $20 \%$ can not be seen as the turbulent heat transfer reduction. Figure 5-21 shows that when the maximum reduction is significant the power error is also large. This is because the outlet flow thermocouple measures the temperature near the centerline, so when the flow laminarizes the centerline temperature is much smaller than the bulk temperature due to its inverse parabolic profile.

This could be also confirmed by observing some of the DTHT cases that had higher heat exchanger inlet temperature than the test section outlet temperature even though there is no heat provided in the chimney section and heat loss in the chimney occurs. When the gas flow is laminarized, the test section outlet thermocouple measures the centerline temperature of the inverse parabolic temperature profile, which is smaller than the bulk temperature and since the heat exchanger thermocouple measures fully mixed temperature, the heat exchanger inlet temperature is measured higher than the test section outlet temperature.

This indicates that the power error in the DTHT cases is not only due to the measurement device uncertainties but it is also due to the characteristic of the DTHT regime. Also, when the forced convection has the same inlet Reynolds number with a DTHT case the power error is below $10 \%$, which reconfirms that the flow measurement and power measurement devices do not have problem.

Therefore, the heat balance error is not only due to the uncertainties in the facility heat losses and instrumentation, but also due to the characteristic of the DTHT regime. In fact, the comparison of heat exchanger outlet temperature with heated section outlet temperature was another indicator of entrance into DTHT regime. An approach to reduce the increase in power error when operation falls within DTHT regime is to install an effective mixer after the test section outlet and to measure the temperature when the gas 
is fully mixed. However, this would lead to higher pressure drop in the loop and impair the natural circulation operation. It is noted that all the data presented here were taken with nominally forced flow by using the compressor but preservation of natural circulation capability of the loop is important for code benchmarking and investigation of the effects of natural circulation coupling to the DTHT regime.

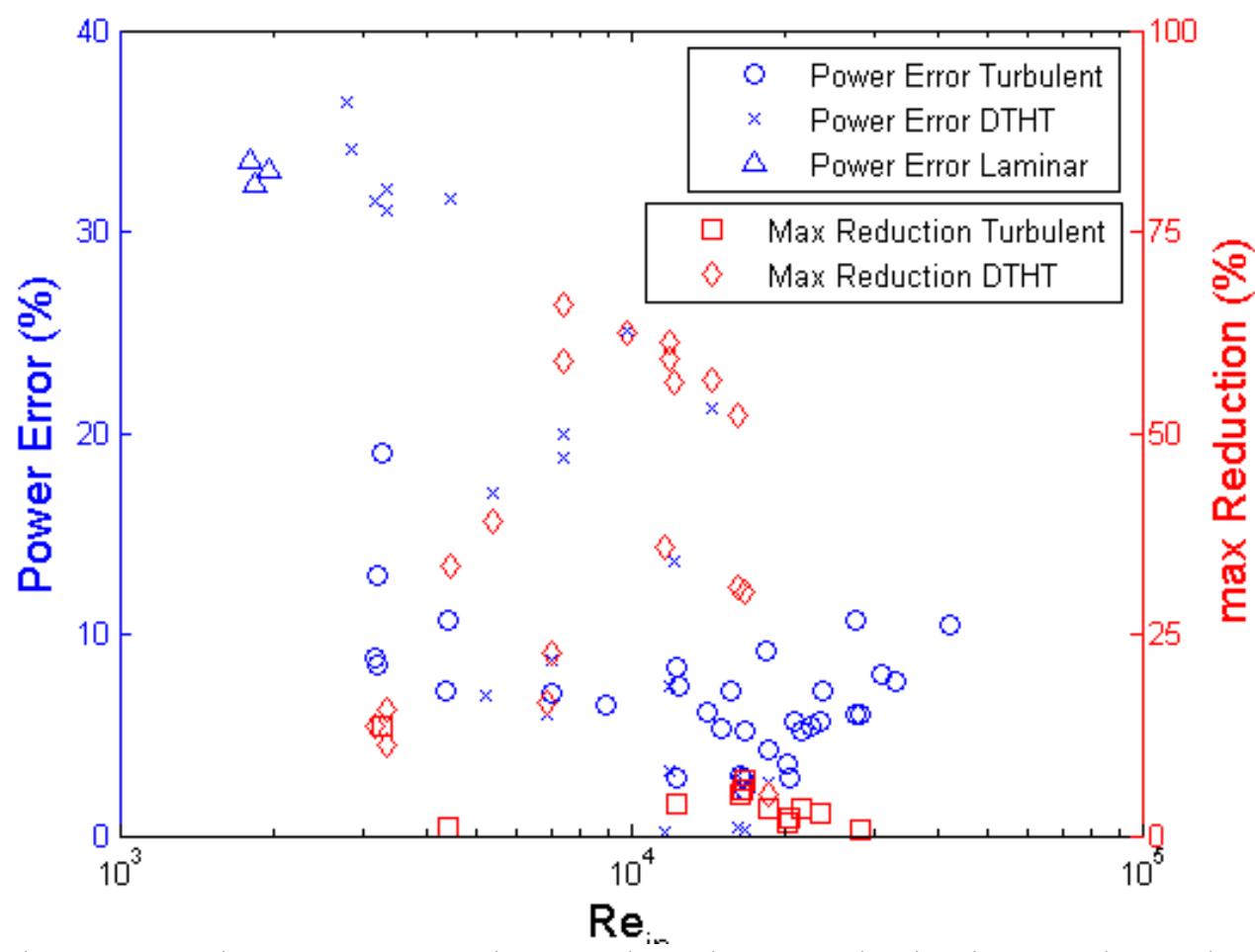

Figure 5-21 Inlet Re vs. Heat Balance and maximum Reduction in Nusselt Number Observed

Methodology of uncertainty analysis was presented in the $2^{\text {nd }}$ Annual report [Lee at al., $2005 \mathrm{c}$ ]. Therefore, only the results of uncertainty analysis will be reported here. Figure 522 shows the maximum uncertainty of the measured Nusselt number for different inlet Reynolds number runs for two cases: one with properties uncertainties accounted for and one without considering properties uncertainties. Typically, uncertainties on gas properties are not included, but since the bulk temperature is calculated using heat balance, the uncertainties of gas thermo-physical properties, in particular on specific heat, can build up and become an important uncertainty source. Thus, the overall uncertainty was calculated both including and excluding the thermo-physical properties uncertainties to identify their impact on the overall uncertainty.

The low Reynolds number runs, which are performed with helium, have higher uncertainty (up to $25 \%$ ) than other runs and the thermo-physical properties consideration does make some differences due to the specific heat uncertainties build up. This means that the measurement device uncertainty is the measure source of the total uncertainty most of time, but some low Reynolds number runs uncertainty are affected by the gas properties uncertainties. 
The reason why some runs have higher uncertainty than the others is because the wall to bulk temperature difference is small for the high uncertainty runs along with the gas properties uncertainties. Small temperature difference means the relative uncertainty of the temperature rise since the thermocouple uncertainty is given as $\pm 1 \mathrm{~K}$. Eq.4-15 in $2^{\text {nd }}$ year report is presented again to show the major uncertainty source (Eq. 5-1). As the temperature difference wall to bulk decreases the highlighted terms in Eq. 5-1 increases. Therefore, the total uncertainty in the heat transfer coefficient measurement increases. To mitigate this problem, T/Cs with smaller uncertainty (less than $\pm 1 \mathrm{~K}$ ) will be necessary to lower the total uncertainty for high uncertainty runs. Figure 5-23 shows the inverse temperature difference versus inlet Reynolds number, which clearly shows that the trend is similar with Figure 5-22.

$$
\frac{\Delta h}{h}=\left[\left(\frac{\Delta q_{w}^{\prime \prime}}{q_{w}^{\prime \prime}}\right)^{2}+\left(\frac{\Delta T_{w}}{\left(T_{w}-T_{b}\right)}\right)^{2}+\left(\frac{\Delta T_{b}}{\left(T_{w}-T_{b}\right)}\right)^{2}\right]^{\frac{1}{2}}
$$

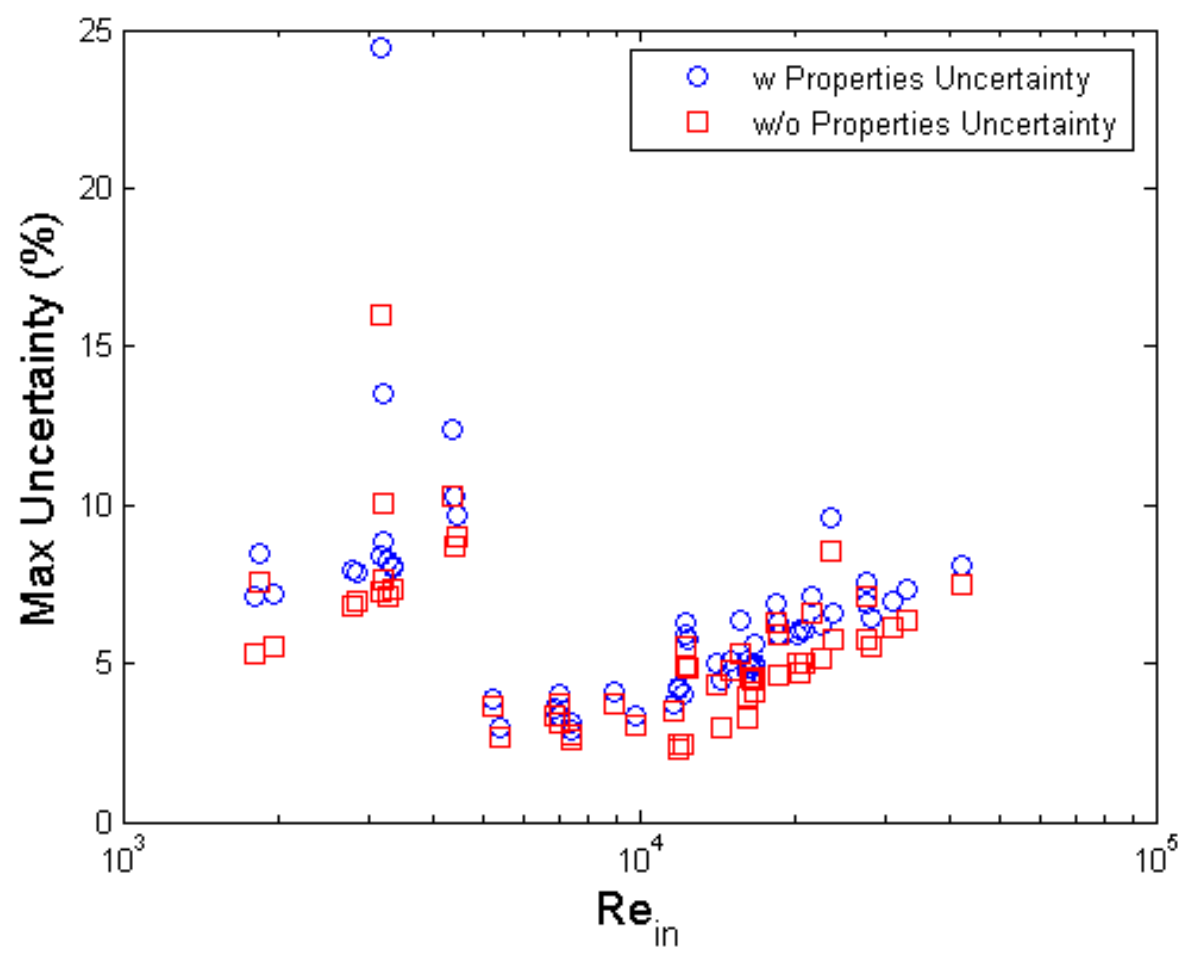

Figure 5-22 Uncertainty of Experimental Measurement considering the properties uncertainty and without considering the properties uncertainty vs. Inlet Re 


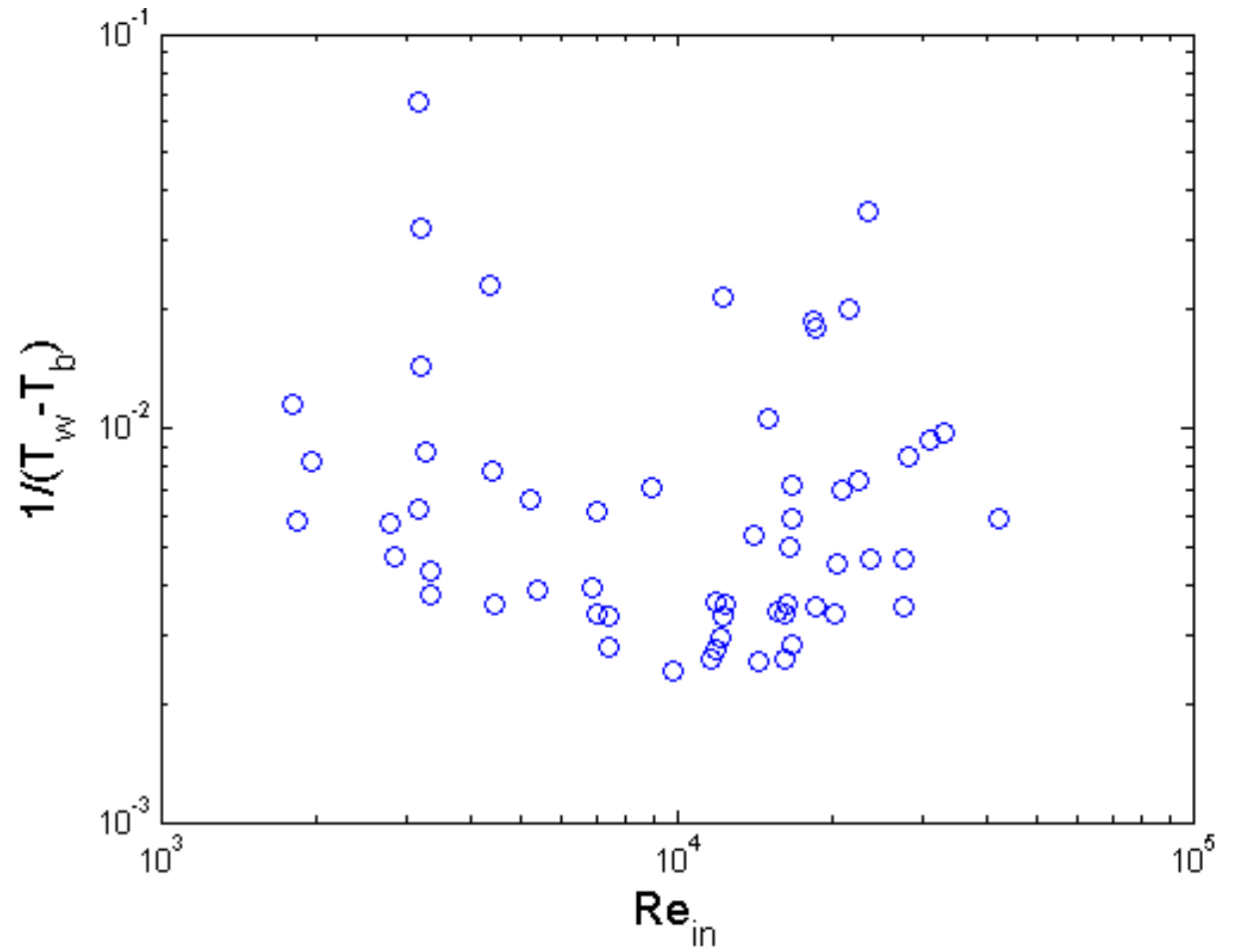

Figure 5-23 Inverse Temperature Difference of Wall and Bulk vs. Inlet Re

In conclusion, most nitrogen and carbon dioxide runs have low uncertainty (below 10\%) and are thus reliable data. But even helium data, which are difficult to obtain with high accuracy due to small wall to bulk temperature difference (due to high helium conductivity) and small flow rates (due to low density) have relatively low uncertainty with most of runs below $15 \%$ and only one run reaching $25 \%$. The individual measurement device uncertainty, which was used for calculating the uncertainty propagation, was all tabulated in $2^{\text {nd }}$ annual report [Lee et al., 2005c]. Table 5-8 shows the thermo-physical properties uncertainties per NIST properties database, which was used to calculated properties of all gases in this experiment. However, it is noted that this NIST reference gives 5\% uncertainty for helium specific heat. Since helium properties in NIST database [Lemmon et al., 2002] are available for a large temperature range from almost absolute zero to $1500^{\circ} \mathrm{C}$, and variation of $c_{p}$ uncertainty over various ranges of temperatures and pressures is not given, we contacted NIST experts regarding helium $c_{p}$ uncertainty for the range of temperatures and pressures in our experiment. The value of much smaller uncertainty of $0.5 \%$ for the range between $300 \mathrm{~K}$ and $1000 \mathrm{~K}$ and pressure between 0.1 and $1 \mathrm{MPa}$ was confirmed [Arp, 2006].

Table 5-8 Thermo-Physical Properties Uncertainty (from Lemmon et al., 2002)

\begin{tabular}{||c||c||c|c||}
\hline Thermo-Physical Properties & Nitrogen & Helium & Carbon Dioxide \\
\hline \hline Thermal Conductivity & $2.0 \%$ & $5.0 \%$ & $5.0 \%$ \\
\hline Density & $0.02 \%$ & $0.1 \%$ & $0.05 \%$ \\
\hline Viscosity & $2.0 \%$ & $10 \%$ & $0.3 \%$ \\
\hline Specific Heat & $0.3 \%$ & $0.5 \%$ & $0.15 \%$ \\
\hline
\end{tabular}




\subsection{Newly Proposed Heat Transfer Map}

The heat transfer regime map was continuously under development by various researchers starting from work by Metais \& Eckert [1964]. This section will update the heat transfer regime map with newly proposed non-dimensional numbers and thresholds for the gas heat transfer. The basic non-dimensional number that will be used for our project will be the non-dimensional heat flux $q^{+}$and the Reynolds number.

The thresholds for acceleration driven DTHT and the buoyancy driven DTHT were tabulated in Table 5-7 and these thresholds can be translated into the relationship between $q^{+}$and $\operatorname{Re}$ using Equations 5-2 and 5-3.

$$
\begin{aligned}
& K_{v_{t h}}=\frac{4 q^{+}}{\mathrm{Re}}=2 \times 10^{-6} \Rightarrow q^{+}=5 \times 10^{-7} \mathrm{Re} \\
& B o_{t h}{ }^{*}=\frac{\mathrm{Gr}_{q}}{\operatorname{Re}^{3.425} \operatorname{Pr}^{0.8}}=2 \times 10^{-6} \Rightarrow q^{+}=2 \times 10^{-6}\left(\frac{g D^{3}}{\alpha^{0.2} v^{1.8}}\right) \operatorname{Re}^{2.425} \\
& \text { since, } \mathrm{Gr}_{q}=\frac{g \beta q_{w}^{\prime \prime} D^{4}}{k v^{2}}=\frac{q^{\prime \prime}}{G c_{p} T_{b}} \frac{G D}{\mu} \frac{\alpha}{v} \frac{\rho c_{p}}{k} \frac{g \beta D^{3}}{\alpha}=\frac{q^{+} \operatorname{Re}}{\operatorname{Pr}} \frac{g \beta D^{3}}{\alpha^{2}}
\end{aligned}
$$

It should be noted that there are different versions of non-dimensional heat flux $q^{+}$such as $q_{w}^{\prime \prime} / G H_{b}, q_{w}^{\prime \prime} / G c_{p} T_{b}$ and $\beta q_{w}^{\prime \prime} / G c_{p}$. For the ideal gas situation, these definitions are all the same but for real gas such as nitrogen or carbon dioxide, different definitions can result in small differences in calculated values. In the data reduction process and the correlation development process, the first definition was always used, since it is directly the ratio of the wall heat flux to the flow enthalpy flux, which has a physical meaning while the others are the approximation to the first definition. Also, when performing numerical calculations, generally the bulk temperature is estimated from the mixing cup enthalpy, use of the first definition allows one to skip one step making calculations easier and faster. However, during the transformation of the equation, the second or third definition will be also used since it is a reasonable approximation to the first definition and can be useful when transforming one non-dimensional number to the other.

The buoyancy effect threshold has another variable other than $q^{+}$and $\mathrm{Re}$, which is a new non-dimensional number $\left(g D^{3} / \alpha^{0.2} v^{1.8}\right)$. This new non-dimensional number is a function of temperature and pressure, since the thermal diffusivity and the kinematic viscosity are gas properties and they vary with temperature and pressure. Since gas properties can generally be approximated with $\rho \sim P T^{-1}, k \sim T^{0.8}, c_{p} \sim T^{0.1}$ and $\mu \sim T^{0.7}$, a rough estimate of the non-dimensional number can be derived and the following equation shows the result (Eq. 5-4). This indicates that the buoyancy number threshold on $q^{+}$and $\operatorname{Re}$ map will have larger slope when the pressure increases and temperature decreases. Within the MIT experimental data the $\left(g D^{3} / \alpha^{0.2} v^{1.8}\right)$ value at inlet varies from order of 6,000 to $2 \times 10^{7}$ depending on the operating pressure. The temperature 
influence is not shown in MIT experiment, since the inlet temperature was kept nearly constant.

$$
\left(\frac{g D^{3}}{\alpha^{0.2} v^{1.8}}\right)=C\left(\frac{\rho c_{p}}{k}\right)^{0.2}\left(\frac{\rho}{\mu}\right)^{1.8} \sim C P T^{-3.4}
$$

Sample heat transfer map is generated and shown in Figure 5-24. The "ND" in the legend depicts the new non-dimensional number $\left(g D^{3} / \alpha^{0.2} v^{1.8}\right)$ value. Three different values are shown in the figure to show the buoyancy threshold changes with $\left(g D^{3} / \alpha^{0.2} v^{1.8}\right)$ number variation. This heat transfer regime map should be used with the inlet condition, since all the thresholds are proposed with the inlet buoyancy number and the inlet acceleration number and there is only one map when the operating pressure, temperature and working fluid are kept the same.

If we compare Figure 5-24 to Figure 1-2, there are some significant differences between these two maps. First of all, the original map (Figure 1-2) covers only the buoyancy effect of the turbulent heat transfer regime while the new map (Figure 5-24) covers the acceleration effect as well. This is because the Rayleigh number, which is the major variable on the original map, cannot reflect the acceleration effect directly. To cover the acceleration effect on the original map would require transformation of the Rayleigh number in a similar manner as the buoyancy parameter was transformed (Eq. 5-3).

Secondly, the original map demarcates the boundary with the local Reynolds number and Rayleigh number while the new map evaluates the boundary with the inlet Reynolds number and inlet non-dimensional heat flux. The reason why we shifted from the local parameters to inlet parameters is due to the experimental observation. As it can be clearly seen from Figures 5-17 and 5-18, various Nusselt numbers can co-exist for the same local buoyancy or acceleration parameter. However, if one selects the inlet condition for the boundary, the boundary between the turbulent flow and DTHT regime can be clearly demarcated throughout the channel (Figure 5-20). Therefore, our experiments seem to suggest that using inlet conditions as boundaries between different heat transfer regimes are more reasonable approach than the use of non-dimensional numbers evaluated at local conditions. We expect this to hold not only within the MIT experimental data set but also for broader range of gas flow heat transfer experiments since this is the nature of the gas DTHT, which not just based on the specific characteristics of the MIT experimental facility.

Lastly, the free convection regime for either laminar or turbulent flow from the original map is missing in the new map. In a laminar flow case, the buoyancy effect does not induce abrupt change in the heat transfer coefficient compared to the DTHT regime, which can be also observed from the work of other researchers [Hallman, 1961]. Thus, drawing a clear line between the laminar and the mixed-laminar regimes did not seem reasonable when comparing the characteristics of the DTHT boundaries to the laminar mixed convection boundaries. Also the free convection turbulent boundary is not shown in the new map due to the lack of gas experimental data. 
The limitation of the new heat transfer map is: (1) it can be used only for a gas flow (2) it should be applied only when the flow orientation is upward heated flow, and (3) if the non-dimensional heat flux is outside the MIT experimental data set then a great caution is needed for evaluating the heat transfer coefficient based on the new heat transfer map.

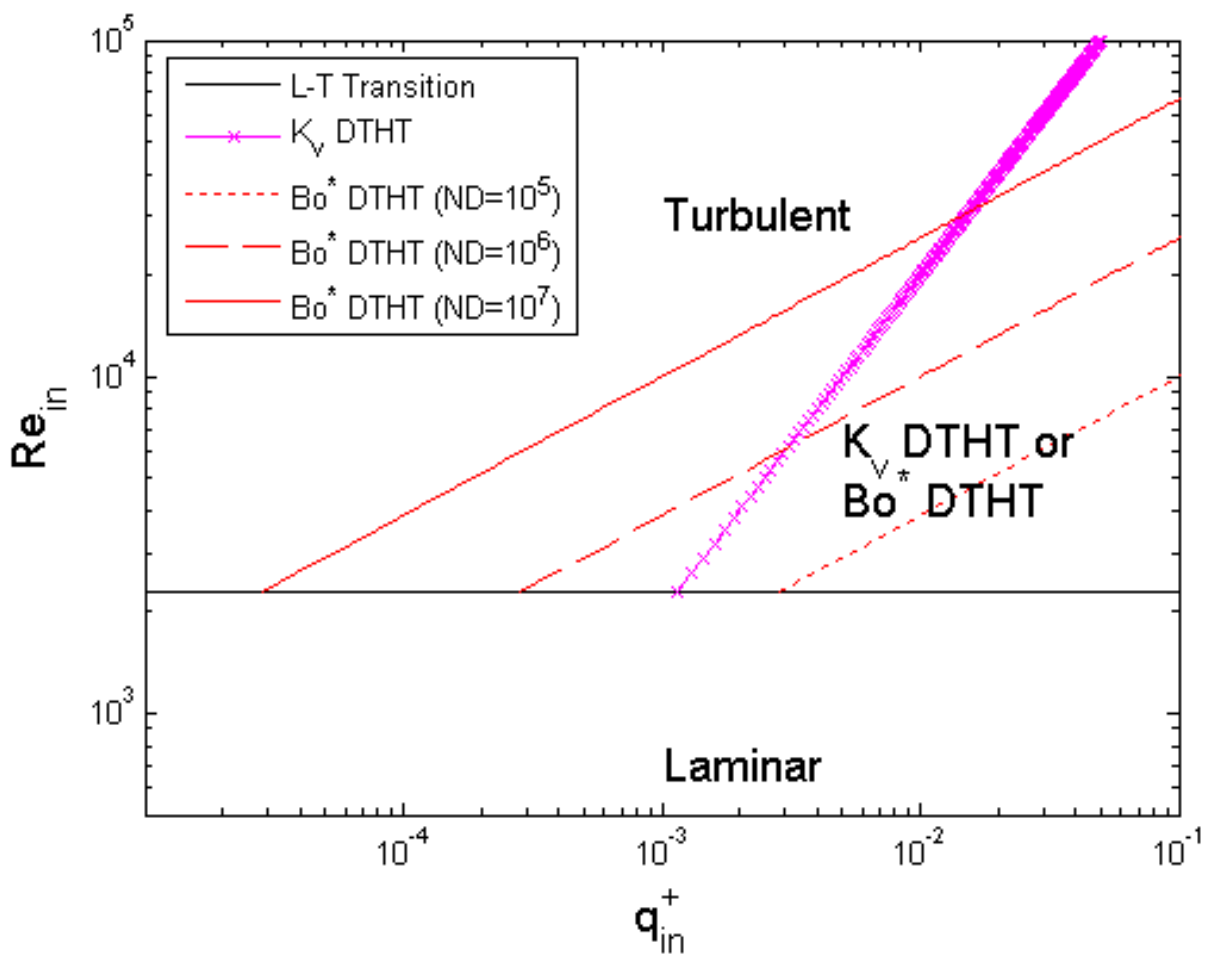

Figure 5-24 Newly Proposed Heat Transfer Map

Figure 5-25 shows the experimental conditions on the proposed heat transfer map. "NDmax" and "NDmin" are the maximum and minimum values of $N D=\left(g D^{3} / \alpha^{0.2} v^{1.8}\right)$ in the MIT experimental data set. To avoid confusion, it is noted that even though some data are taken in between the maximum and minimum buoyancy threshold, the data did not fall in the DTHT regime, since the operating pressure and gas were different for each case. 


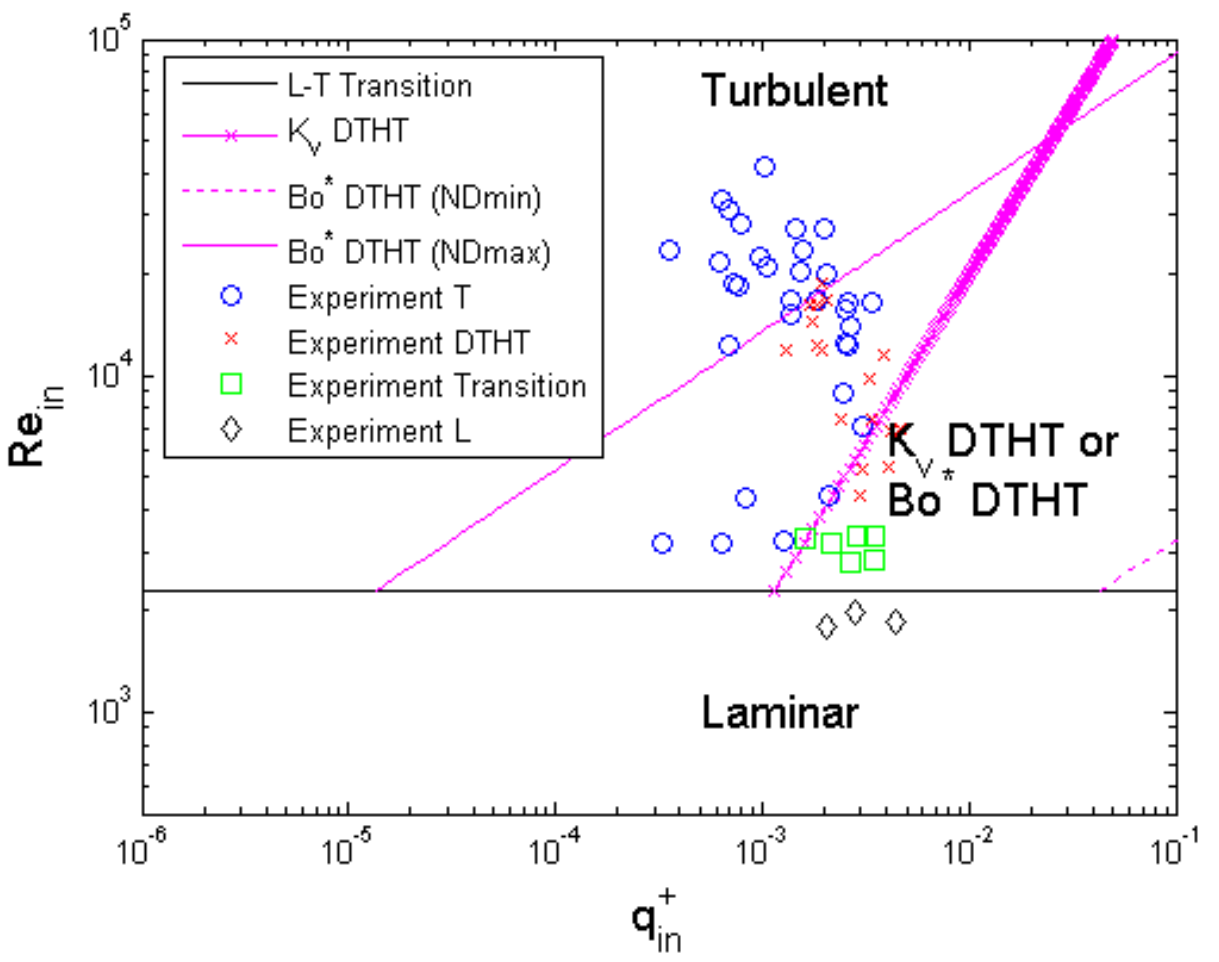

Figure 5-25 Experimental data plotted on the Newly Proposed Map 


\section{Data Comparison with Earlier Correlations}

This chapter is separated into two sections. First, the mixed convection laminar correlations will be compared against the helium mixed convection data. Next, the DTHT correlations for the buoyancy effect and the acceleration effect will be compared to our experimental data.

Before presenting the comparison of the correlations to the experimental data, two indicators to measure how well a correlation can fit the experimental data will be defined. One is the R-square and the other is the $20 \%$ indicator. The R-square is defined by Eq. 61.

$$
\mathrm{R}^{2}=1-\frac{\sum\left(\mathrm{Nu}_{\mathrm{EXP}}-\mathrm{Nu}_{\text {Correlation }}\right)^{2}}{\sum\left(\mathrm{Nu}_{\mathrm{EXP}}-\overline{\mathrm{Nu}_{\mathrm{EXP}}}\right)^{2}}
$$

The $20 \%$ indicator is simply defined as a fraction of the experimental data included in $\pm 20 \%$ band of the correlation. Therefore, good fitting correlation will show both Rsquare and $20 \%$ indicator values close to unity. Any negative value for R-square indicates that all the predicted Nusselt numbers are either higher or lower than the measured Nusselt numbers.

All the comparison result will be depicted on a plot that has $\mathrm{X}$-axis as the correlation predicted Nusselt number and Y-axis as the measured Nusselt number. Therefore, the more data are concentrated near $Y=X$ line, the better the performance of a correlation, making the value of the two indicators closer to unity. Also when most of the points are on the upper side of $Y=X$ line the correlation under predicts the Nusselt number while most points being on the lower side will be the opposite case.

\subsection{Mixed Convection Laminar Correlations Comparison}

This section will only compare the mixed convection laminar data to the correlation for mixed convection laminar case (Eq. 2-12, 13, 14 and 16). Equation 2-15 is not compared since there is no carbon dioxide laminar heat transfer data. Subscript, "Laminar" is Equation 2-2, "Hallman" Equation 2-12, "Worsoe-schmidt1" Equation 2-13, "Worsoeschmidt2" Equation 2-14 and "Churchill" Equation 2-16.

Figure 6-1 to 6-5 plot the comparison of these correlations predictions with data. 


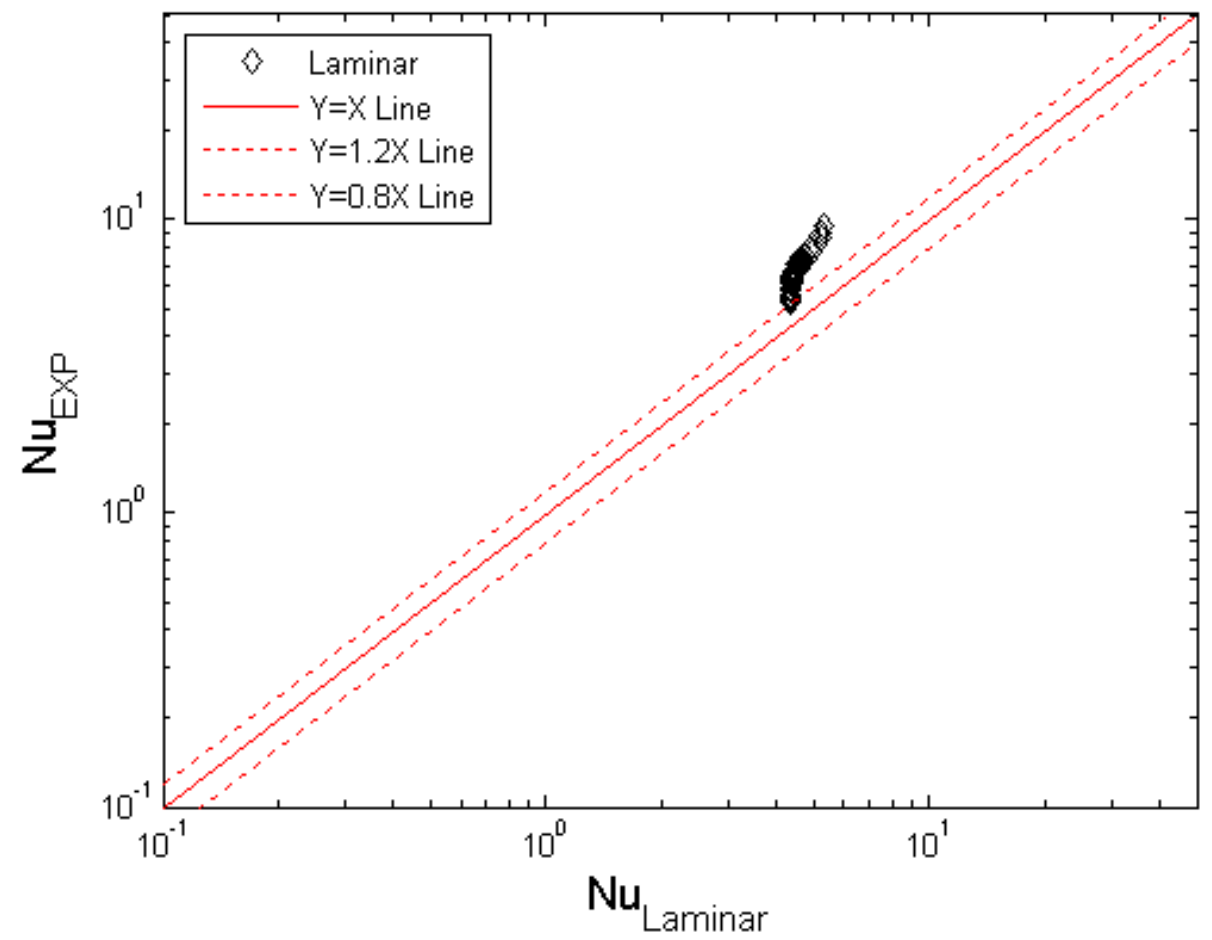

Figure 6-1 Experimental Nu vs. Laminar Correlation Predicted Nu

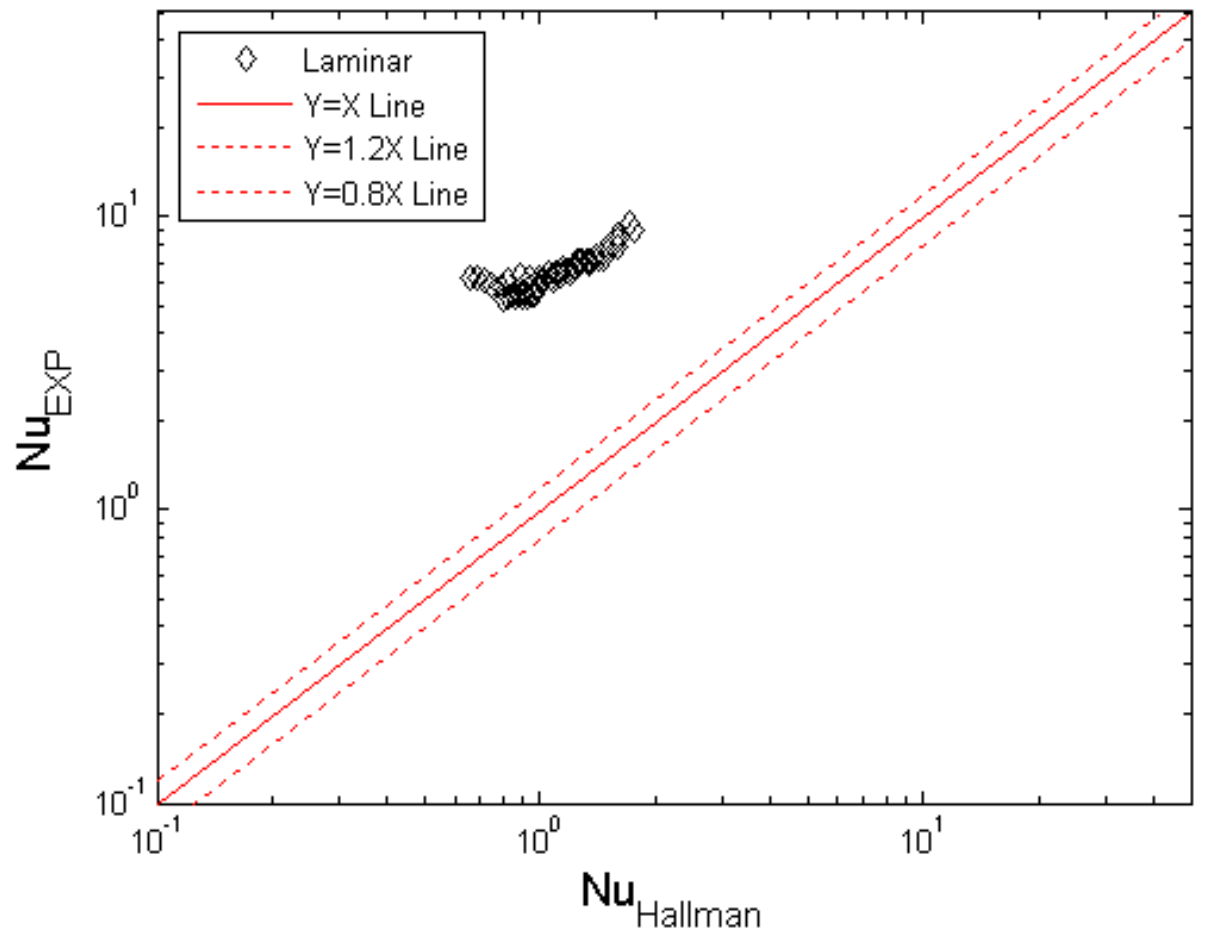

Figure 6-2 Experimental $\mathrm{Nu}$ vs. Hallman Correlation Predicted $\mathrm{Nu}$ 


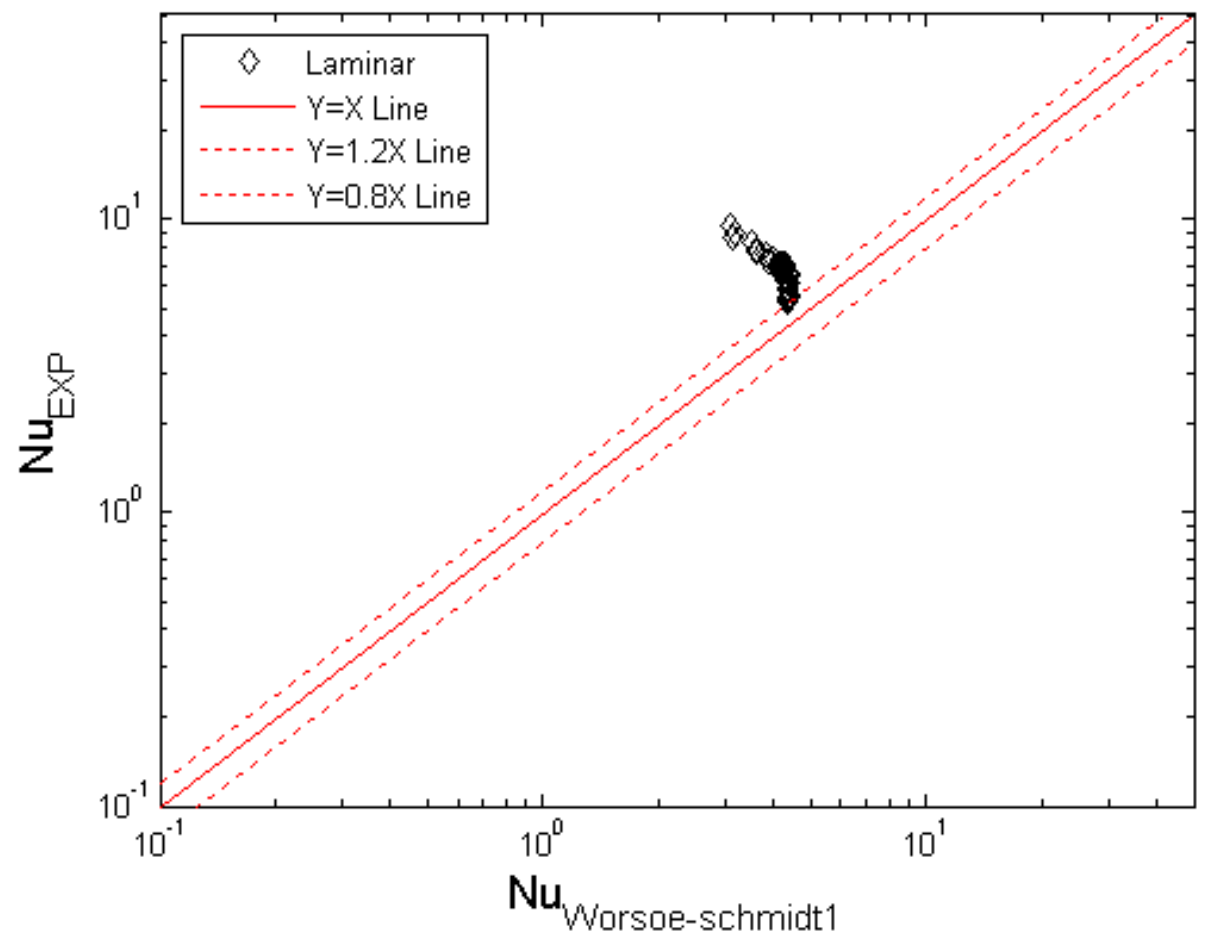

Figure 6-3 Experimental Nu vs. Worsøe-schmidt \& Leppert Correlation Predicted Nu

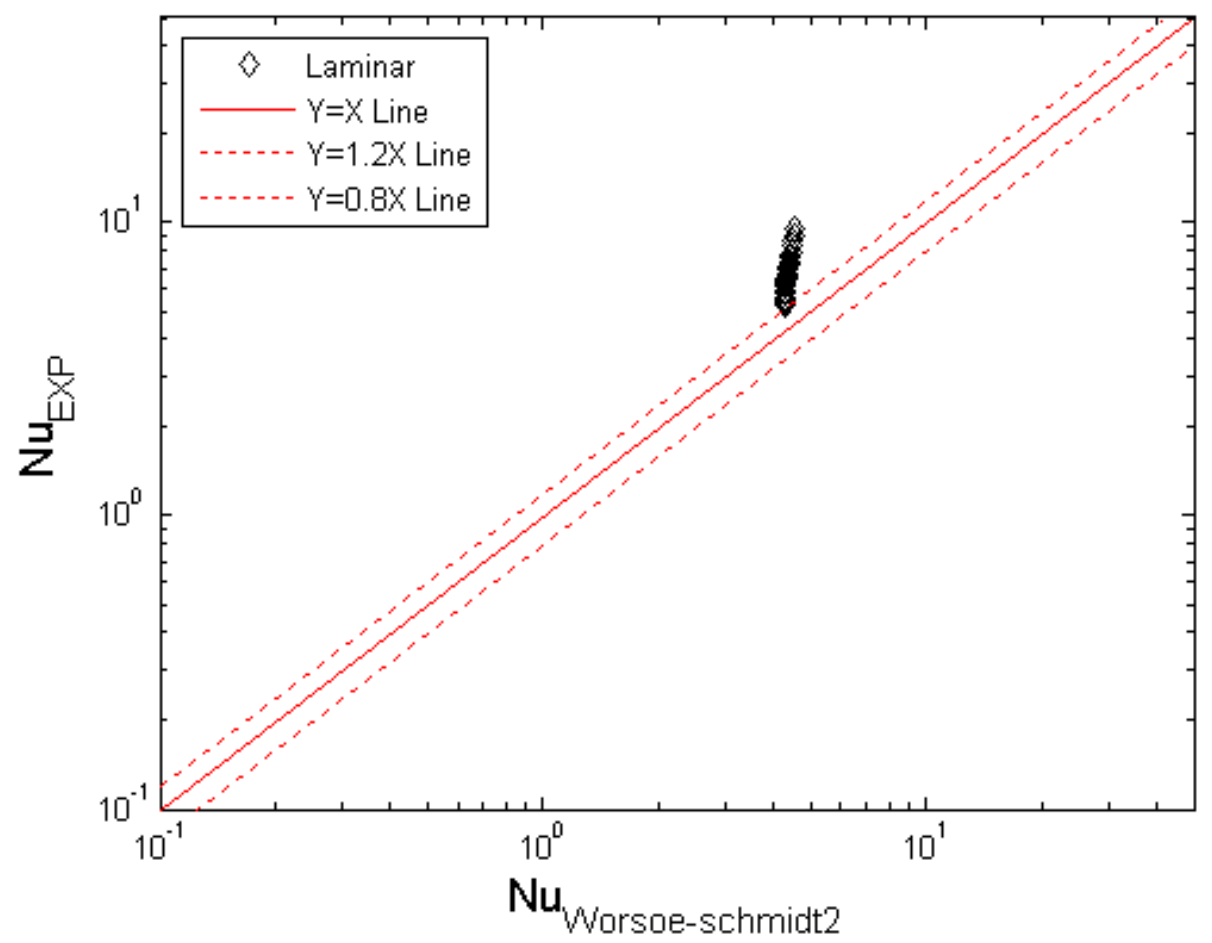


Figure 6-4 Experimental Nu vs. Worsøe-schmidt Correlation Predicted Nu

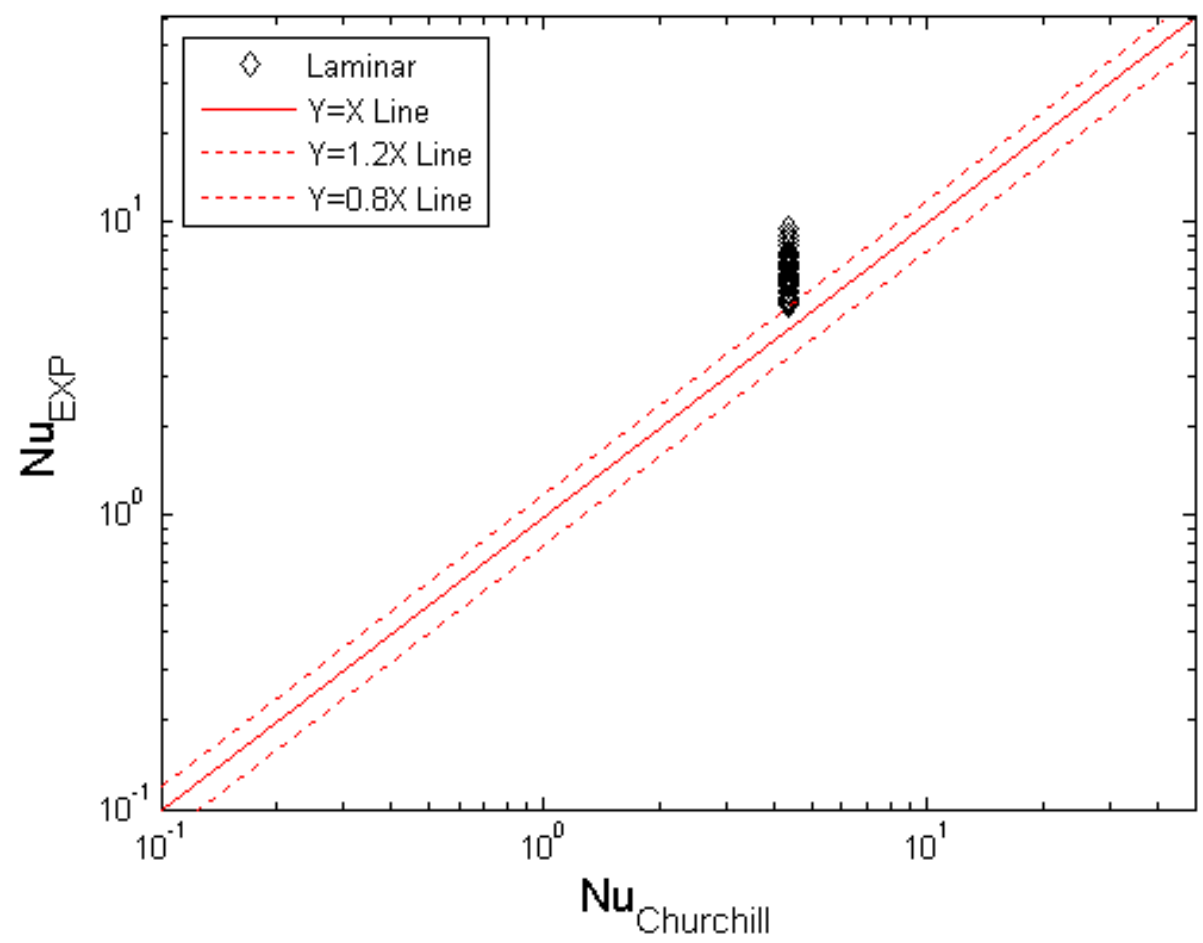

Figure 6-5 Experimental Nu vs. Churchill Correlation Predicted $\mathrm{Nu}$

Table 6-1 summarizes the two indicators for the above five correlations.

Table 6-1 Mixed Convection Laminar Correlation Fitness Indicators

\begin{tabular}{|c|c|c|}
\hline Correlation & R-square & $20 \%$ fraction \\
\hline \hline Laminar & -3.7577 & 0.0000 \\
\hline Hallman & -29.7585 & 0.0000 \\
\hline Worsoeschmidt1 & -6.5282 & 0.0000 \\
\hline Worsoeschmidt2 & -4.8404 & 0.0000 \\
\hline Churchill & -4.8489 & 0.0000 \\
\hline
\end{tabular}

The figures and Table 6-1 show that the predictions using mixed convection laminar correlations available in the literatures are not satisfactory in comparison to the gas experimental data obtained at MIT loop. This is mainly because selected correlations were either based on the water experiments, which did not necessarily aspire to fit the mixed convection laminar 'gas' flow. However, it is surprising that the correlations of Worsøe-schmidt do not fit the data even though the numerical analyses were performed with the gas properties and the data were within the numerical analyses range. This may be due to high uncertainty in the helium measurement or the effect of surface rougness that could not be captured by numerical simulations. Any final conclusion would be too early to make at this point, since these data sets are small and this is the first attempt to 
compare the mixed convection laminar correlation to gas data. Moreover, because power error of these runs was relatively large, the reproducibility of the helium data needs to be checked.

\subsection{DTHT Correlations Comparison}

This section presents the comparison of the DTHT correlations against the MIT data. Table 6-2 summarizes the correlations tested together with equation numbers in this report. Correlation names in the first column will be used as Nusselt number subscripts.

Table 6-2 Tested Correlations with Equation Numbers

\begin{tabular}{||c||c||}
\hline Subscripts & Equation Number \\
\hline \hline "Gnielinski" & $2-7$ \\
"Petukhov1" & $2-19$ \\
"Herbert" & $2-21$ \\
"Petukhov2" & $2-22$ \\
"Jackson" & $2-23$ \\
"Parlatan" & $2-24$ \\
"Vilemas" & $2-26$ \\
"Celeta" & $2-27$ \\
"Symolon" & $2-29$ \\
"McEligot" & $2-30$ \\
"Perkins" & $2-31$ \\
"Taylor" & $2-32$ \\
\hline
\end{tabular}

Figures 6-6 through 6-17 present the comparisons. The Gnielinski correlation was just selected to show how forced convection correlation performs in the turbulent, DTHT and transition regimes compared to the other correlations. 


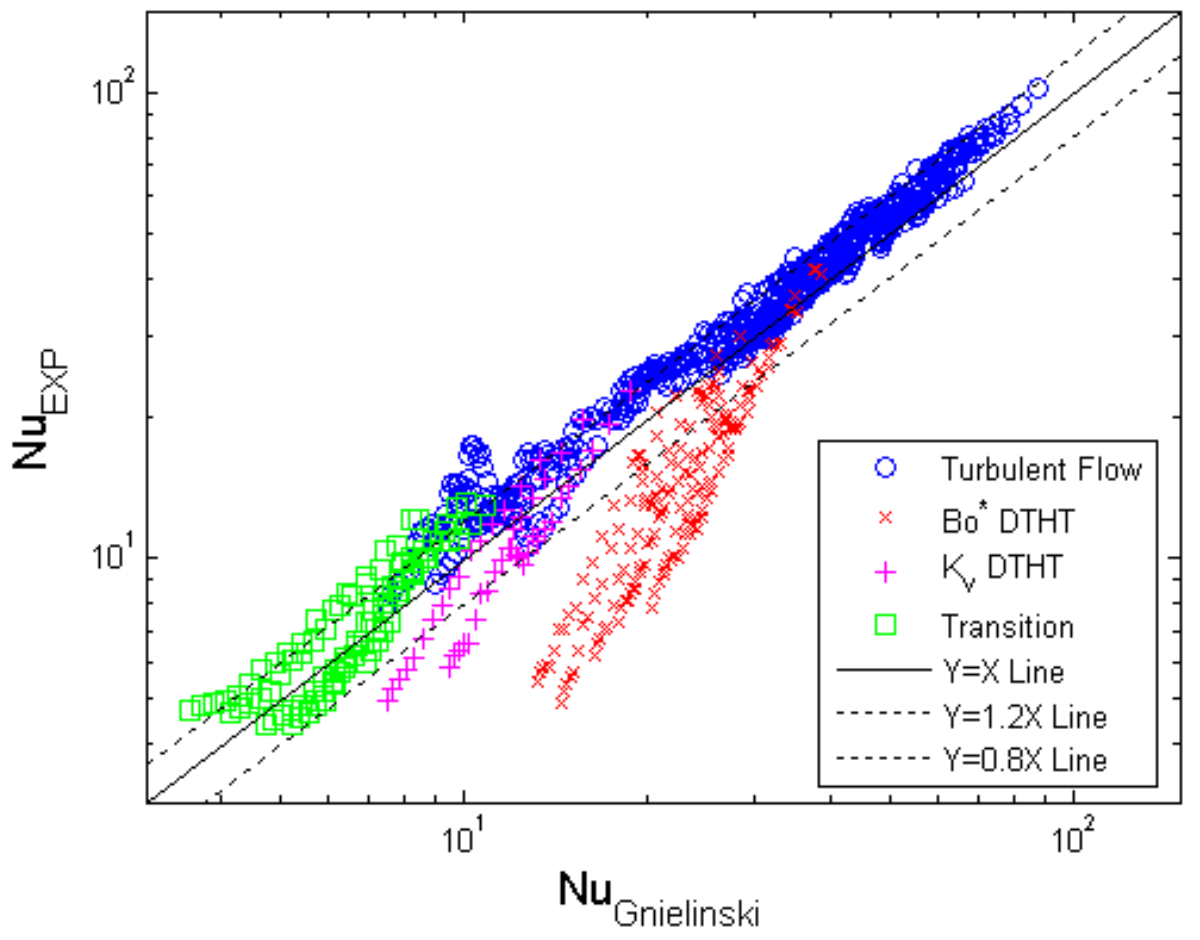

Figure 6-6 Experimental Nu vs. Gnielinski \& Laminar Correlation Predicted Nu

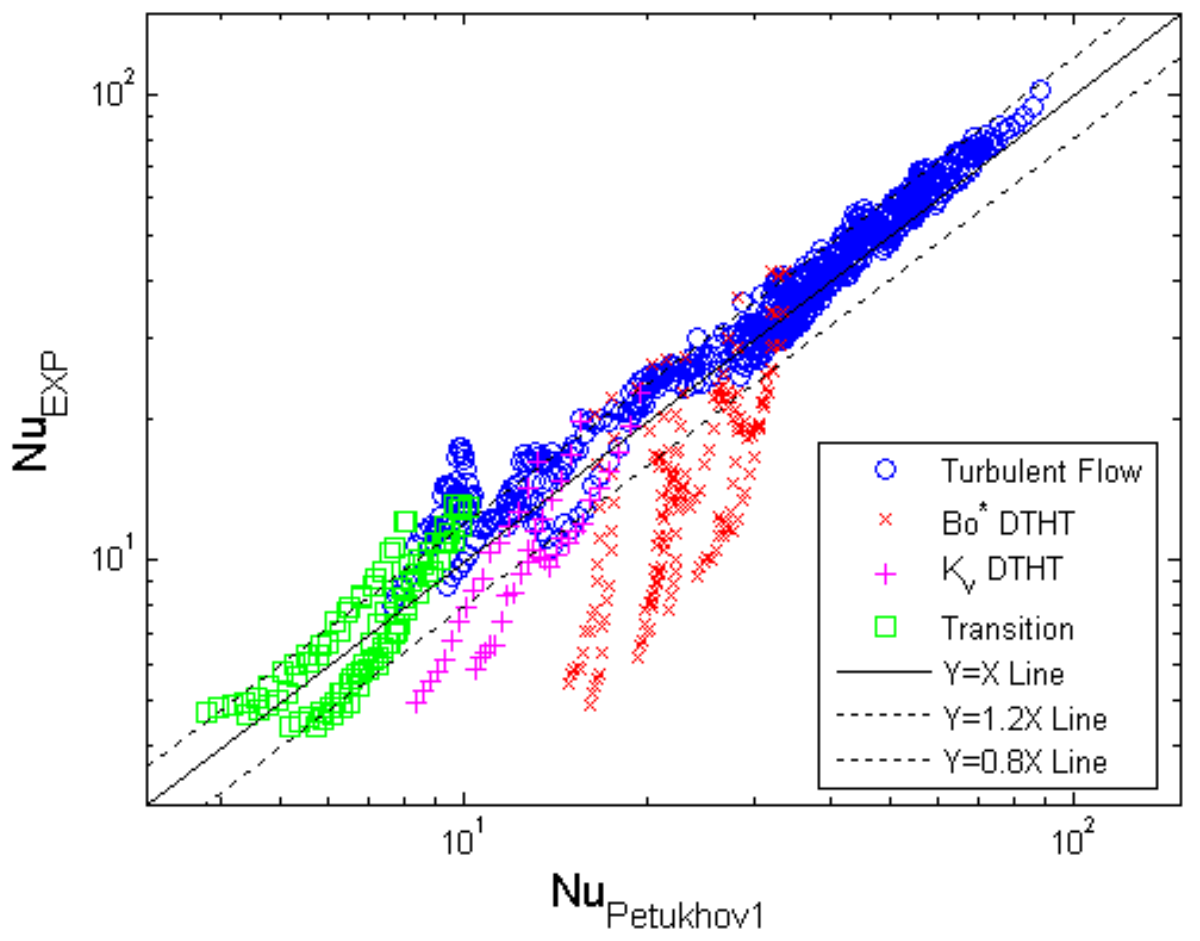

Figure 6-7 Experimental Nu vs. Petukhov \& Strigin Correlation Predicted $\mathrm{Nu}$ 


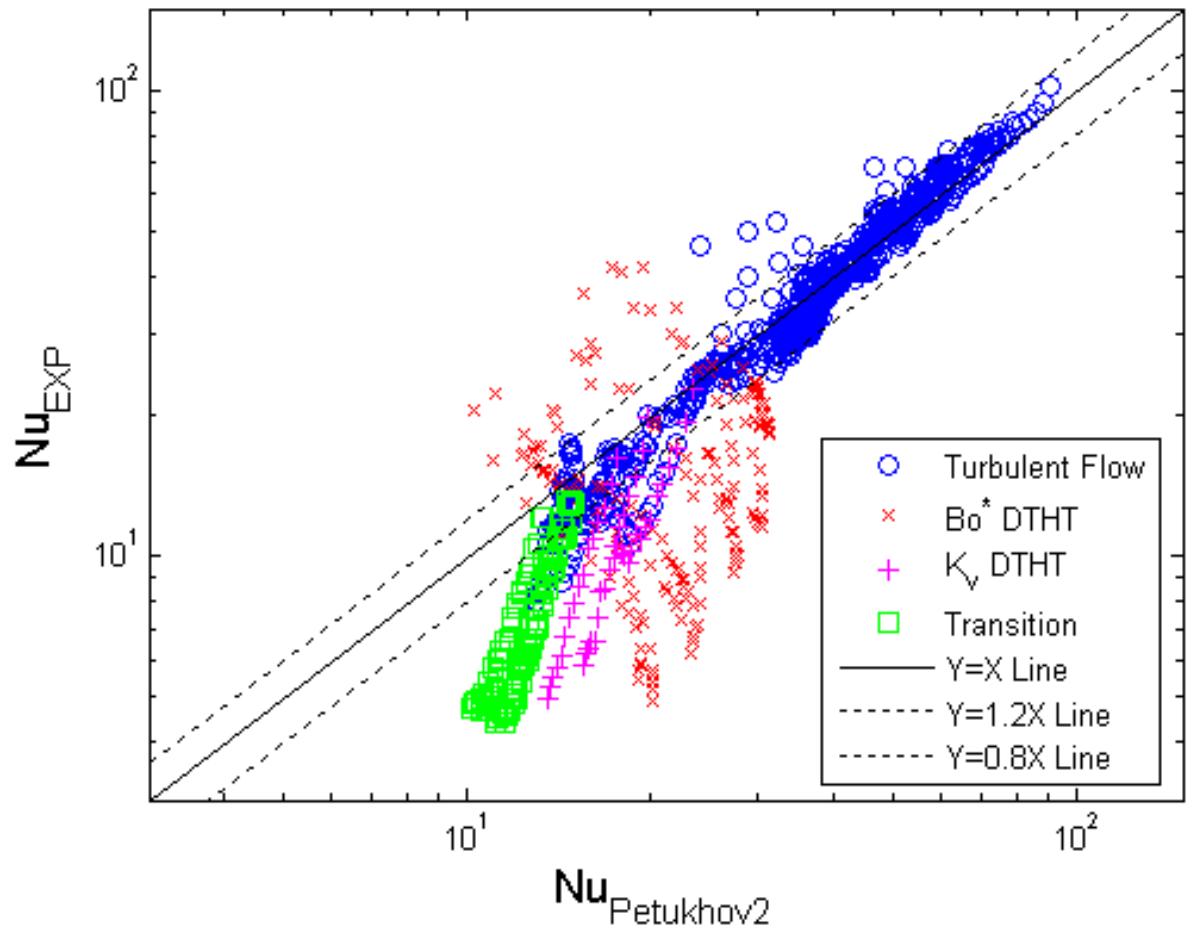

Figure 6-8 Experimental Nu vs. Petukhov \& Polyakov Correlation Predicted Nu

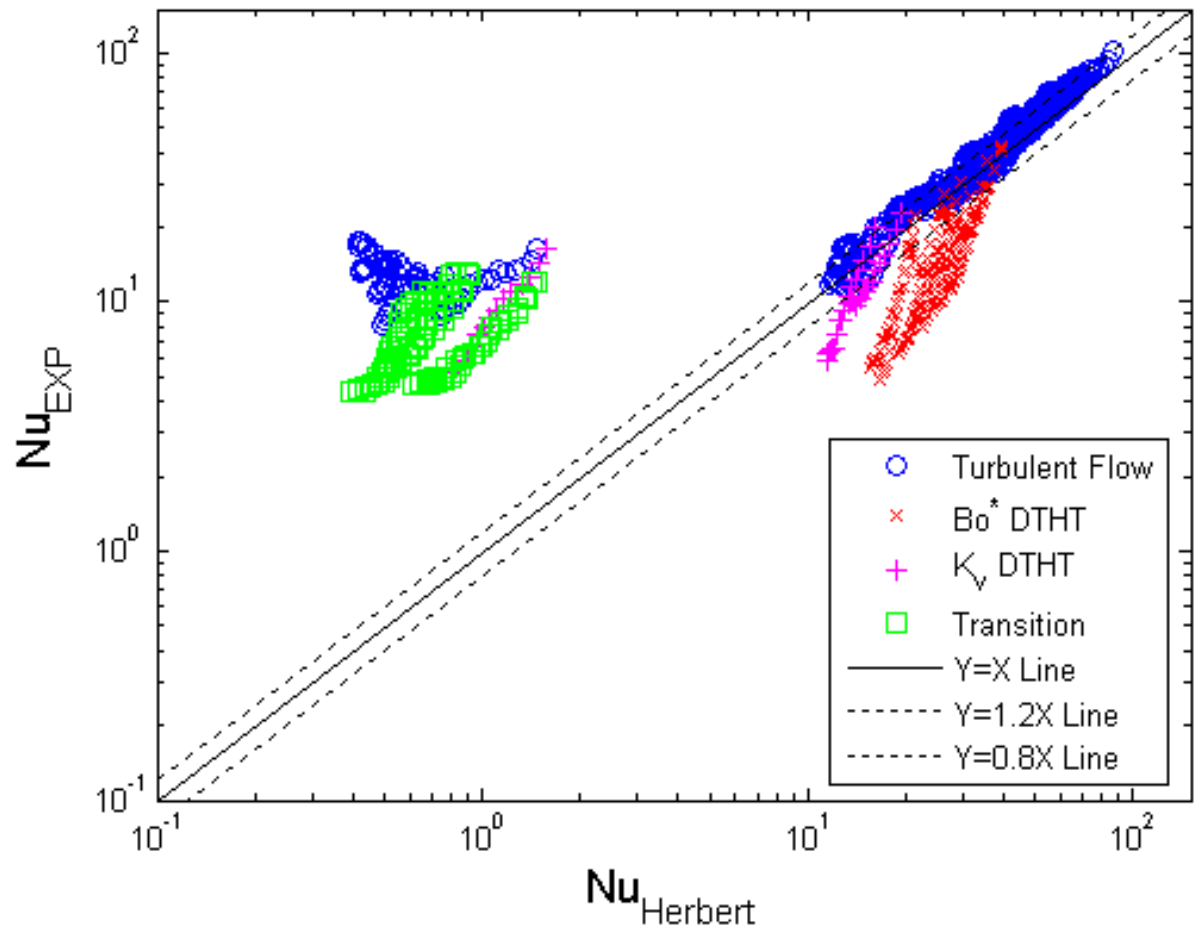

Figure 6-9Experimental Nu vs. Herbert \& Sterns Correlation Predicted Nu 


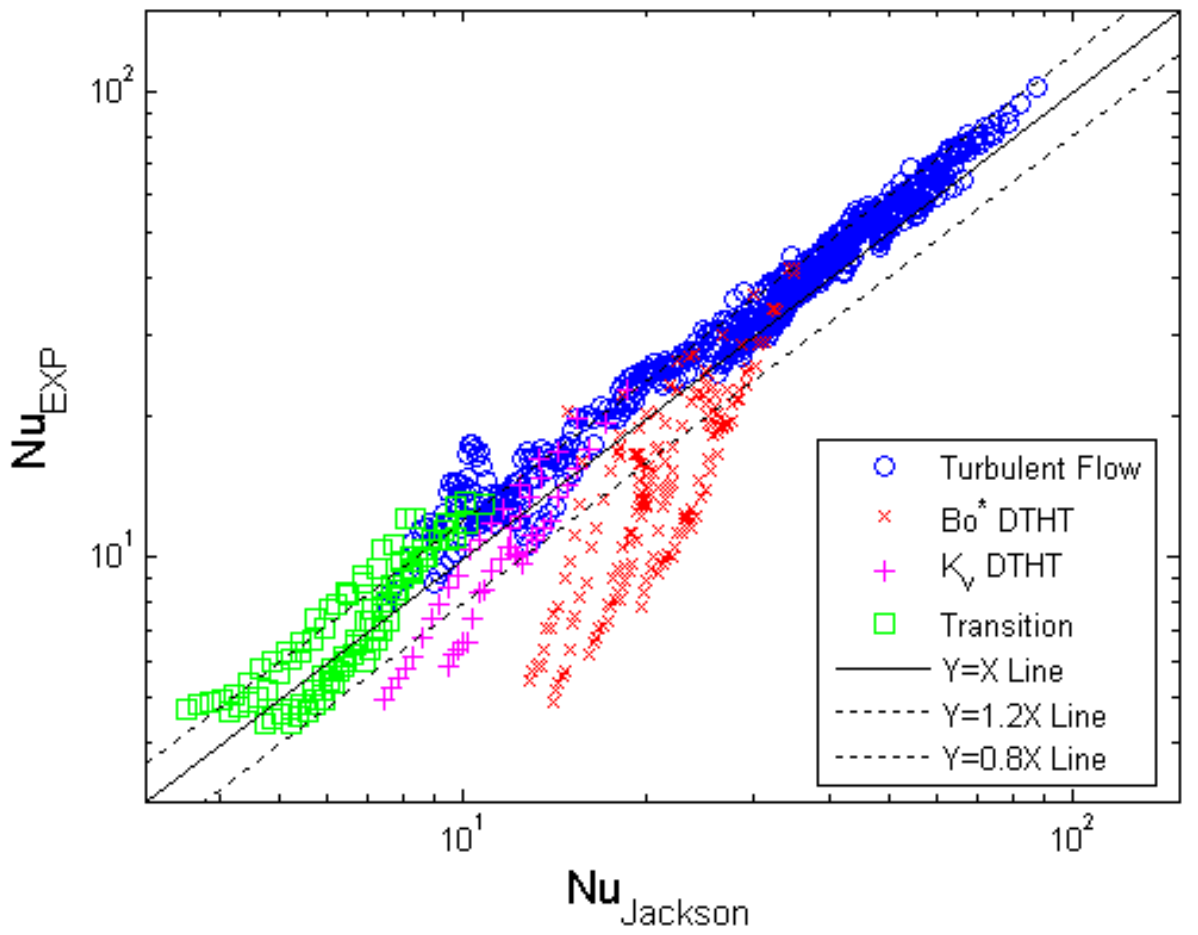

Figure 6-10 Experimental Nu vs. Jackson et al. Correlation Predicted Nu

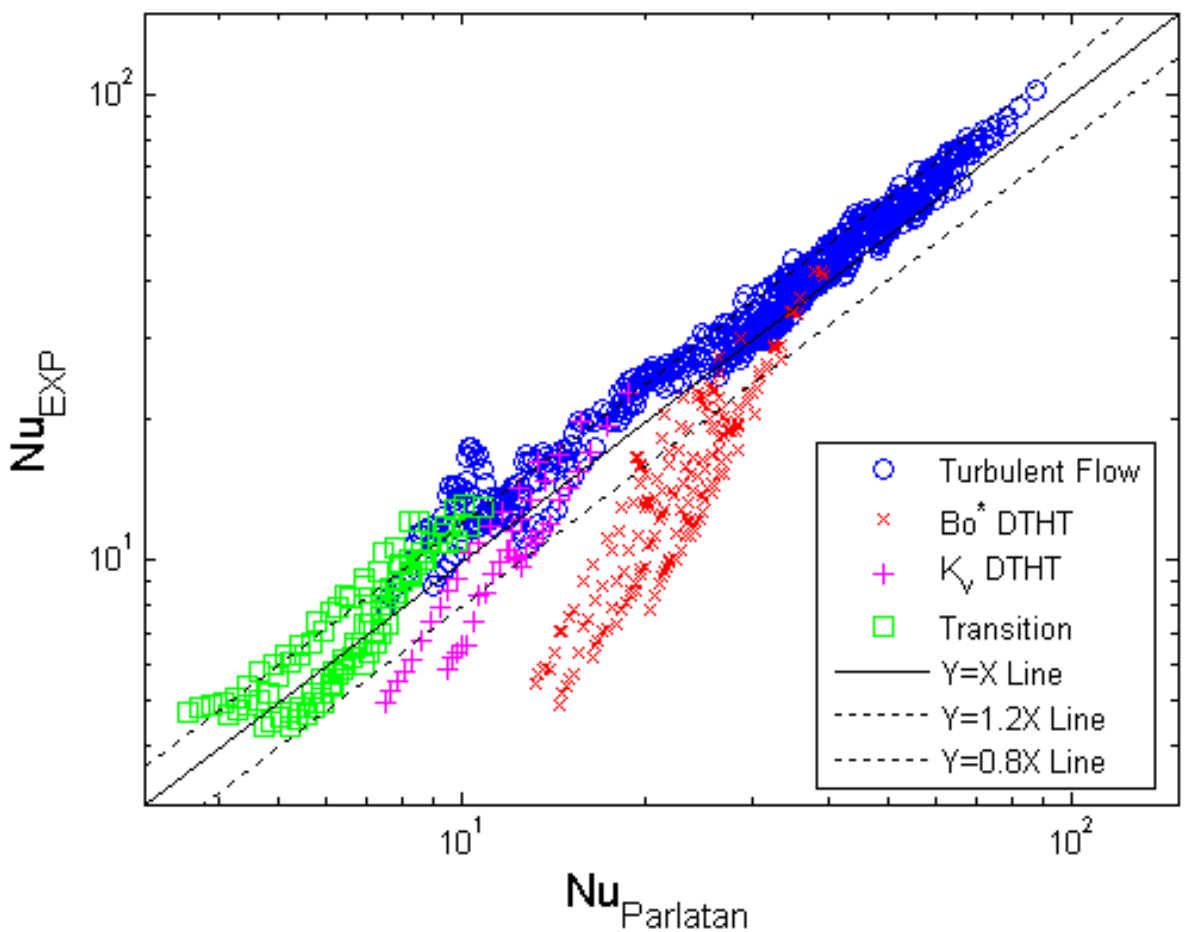

Figure 6-11 Experimental Nu vs. Parlatan Correlation Predicted Nu 


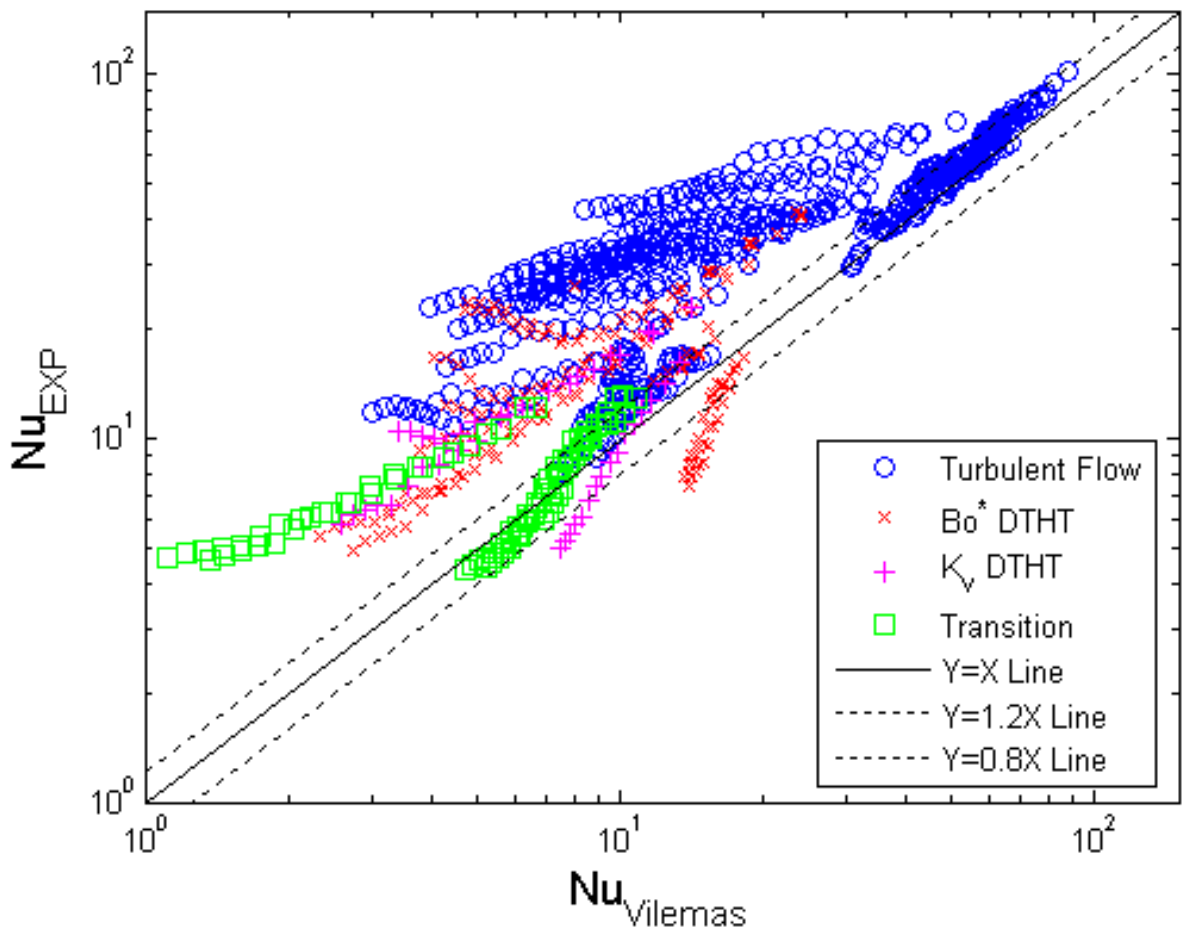

Figure 6-12 Experimental Nu vs. Vilemas et al. Correlation Predicted Nu

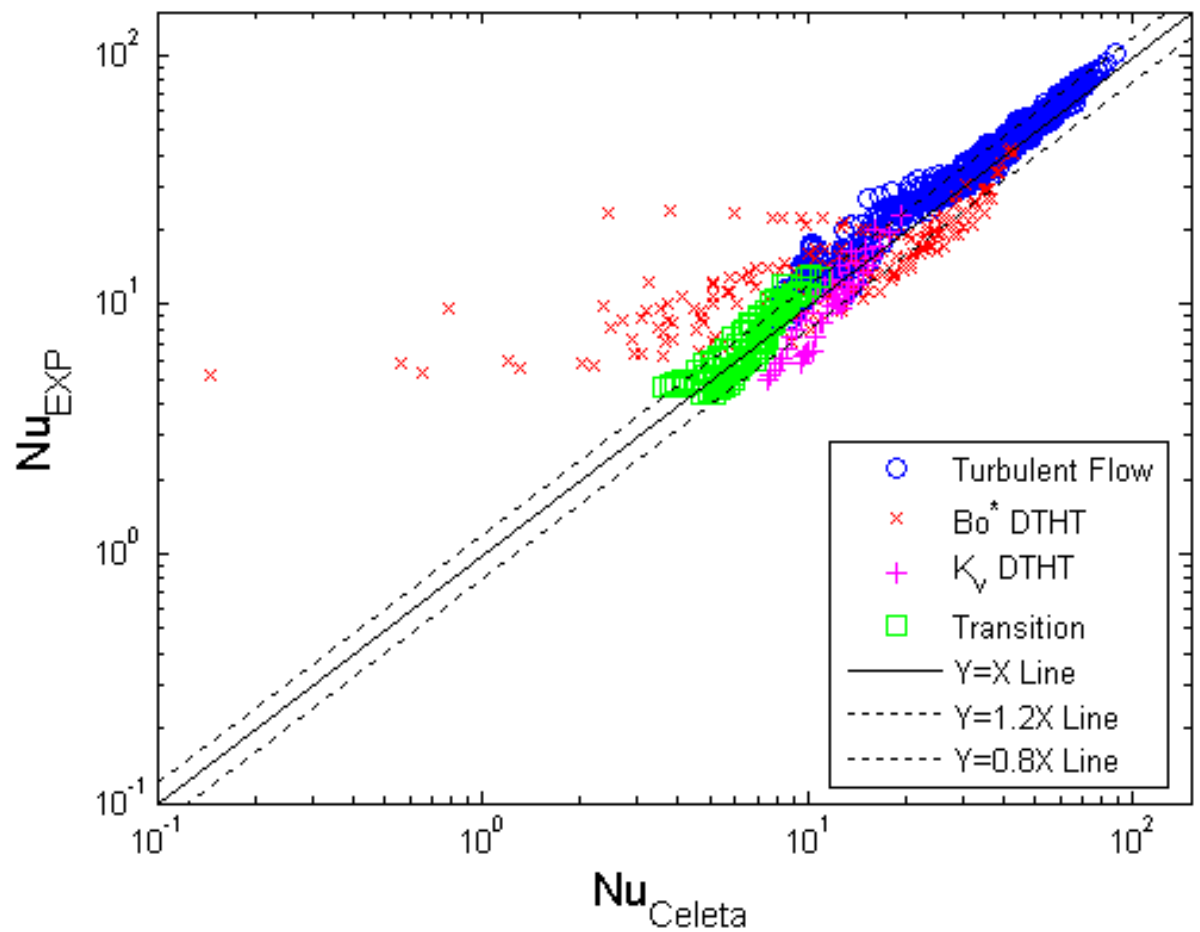

Figure 6-13 Experimental Nu vs. Celeta et al. Correlation Predicted Nu 


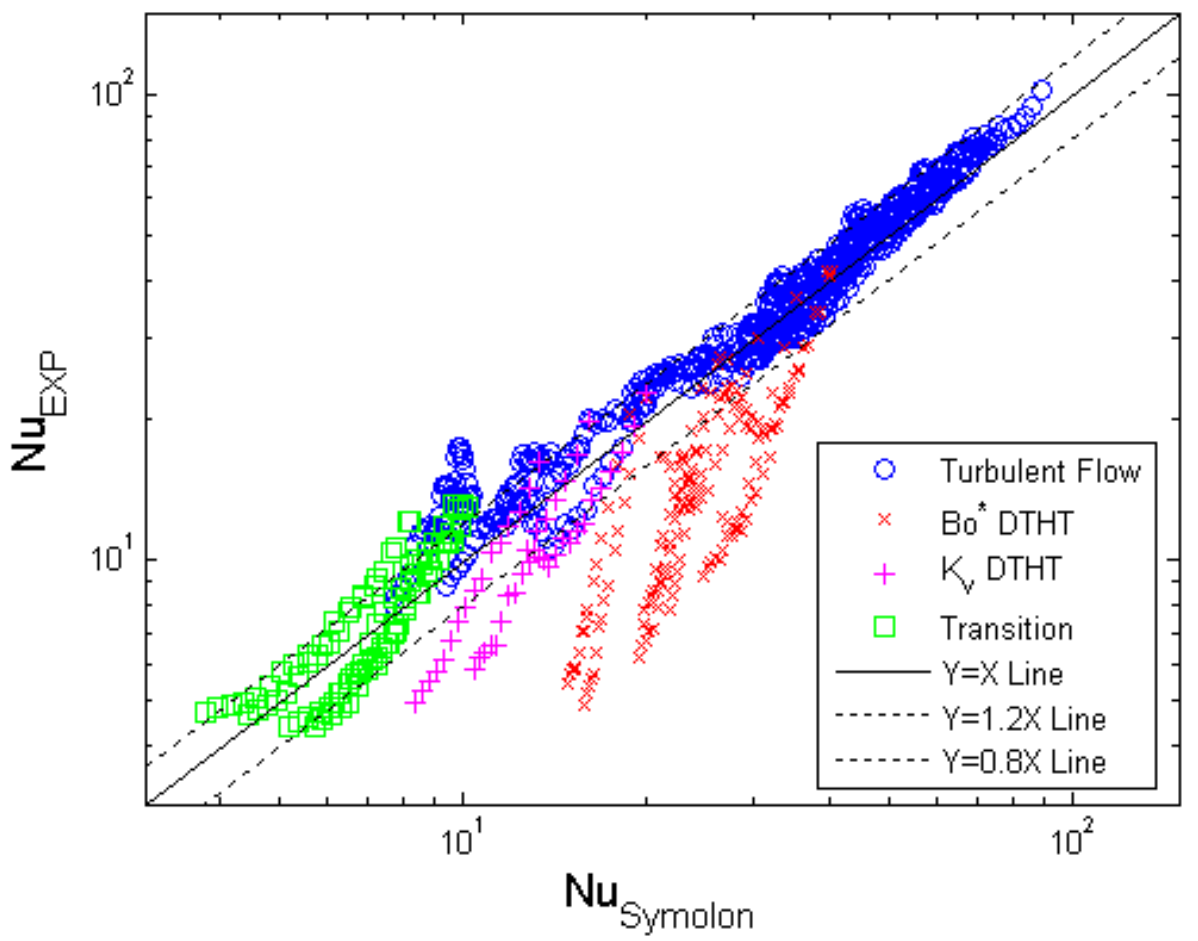

Figure 6-14 Experimental Nu vs. Symolon et al. Correlation Predicted Nu

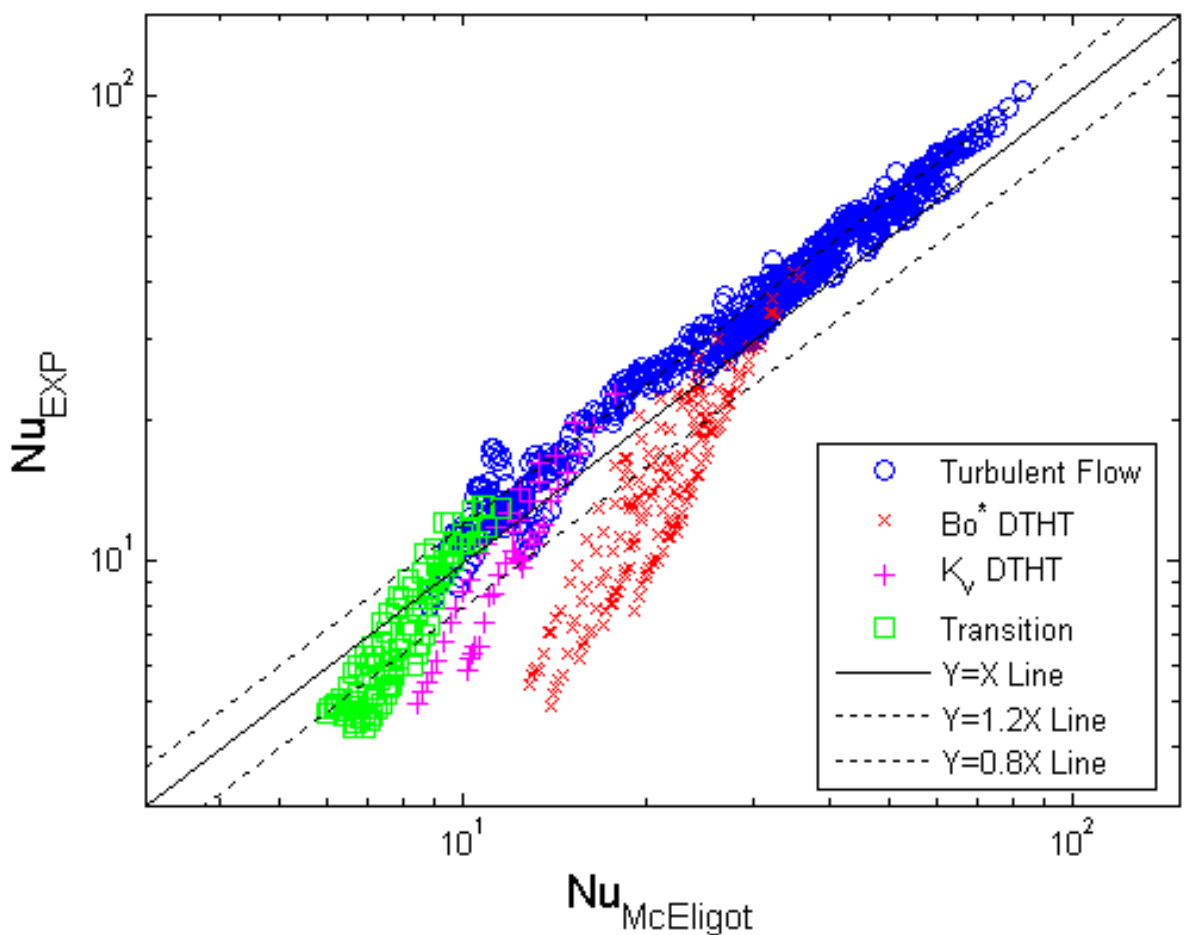

Figure 6-15 Experimental Nu vs. McEligot Correlation Predicted Nu 


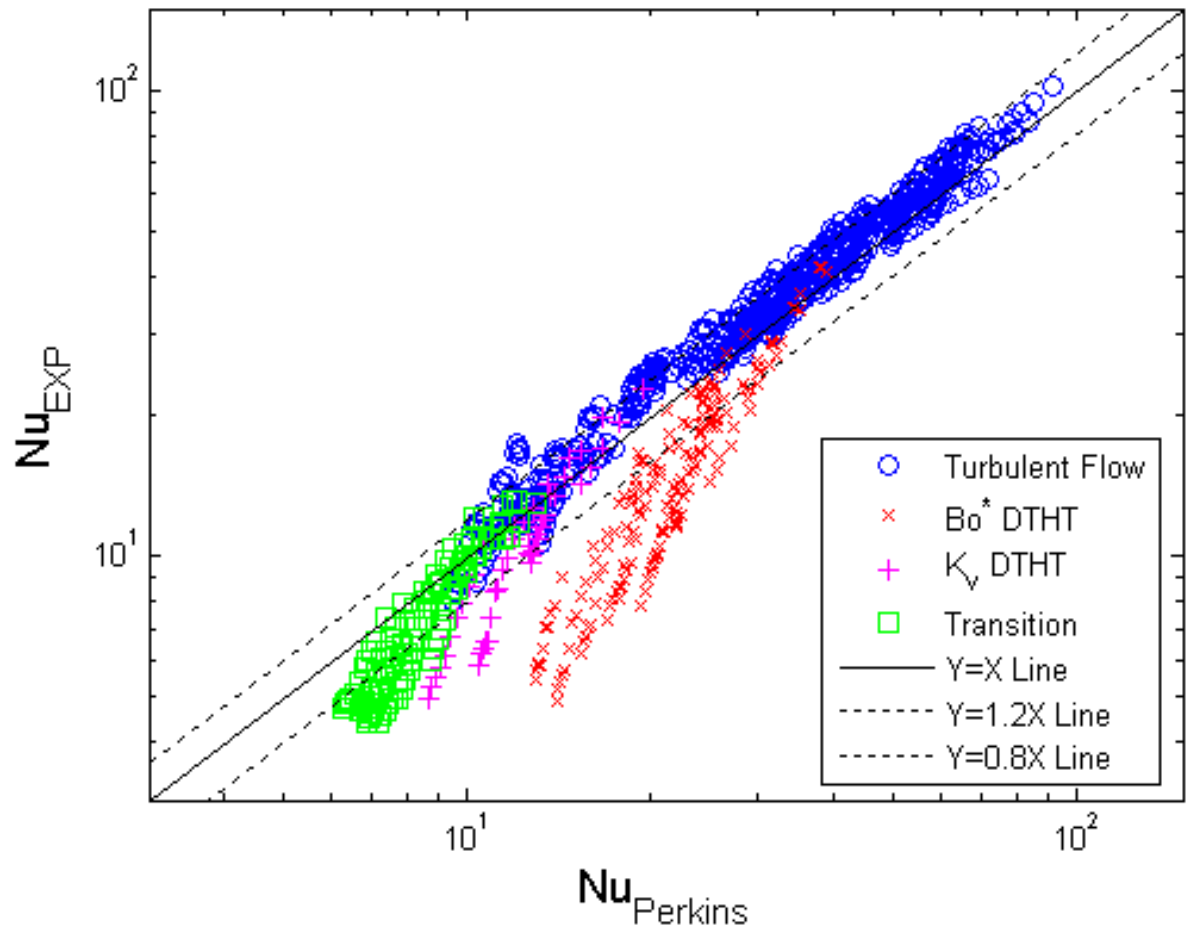

Figure 6-16 Experimental Nu vs. Perkins \& Worsøe-schmidt Correlation Predicted Nu

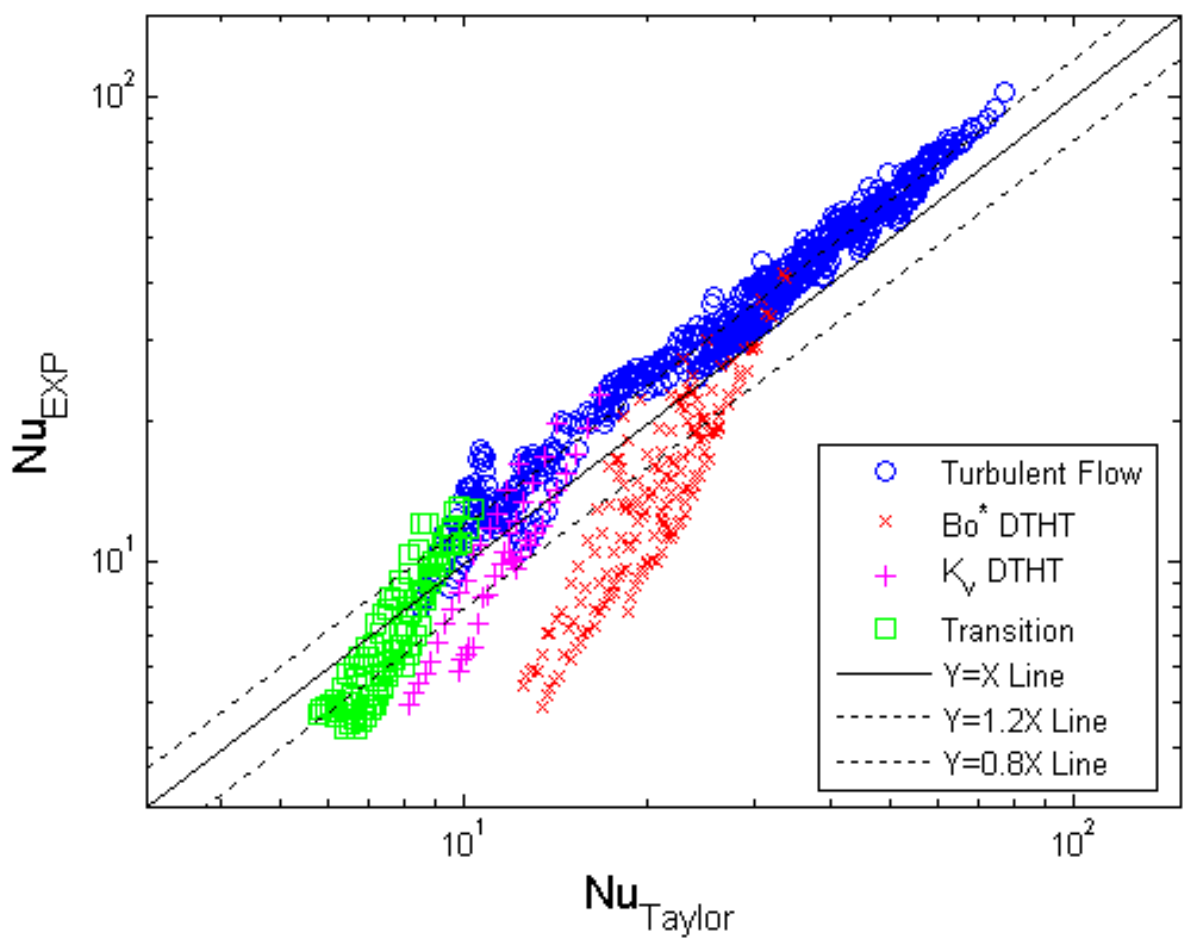

Figure 6-17al Experiment $\mathrm{Nu}$ vs. Taylor Correlation Predicted $\mathrm{Nu}$ 
Tables 6-3 and 6-4 summarize the comparison to the experimental data in terms of Rsquare and $\pm 20 \%$ band for all the data and each heat transfer regime separately, respectively. In the R-square table, values over $90 \%$ are highlighted with yellow and the best fitting correlation for each regime is highlighted with light blue color. Similarly in the $20 \%$ band table, values over $75 \%$ are highlighted with yellow and the highest correlation for each regime is highlighted with light blue color.

Table 6-3 R-square Value for Various Correlations

\begin{tabular}{|c||c|c|c||c|c||}
\hline Correlation & All & Turbulent & $B^{*}{ }^{*}$ DTHT & $K_{v}$ DTHT & Transition \\
\hline \hline Gnielinski & 0.9275 & 0.9357 & -0.1649 & 0.7138 & 0.6785 \\
\hline Petukhov1 & 0.9207 & 0.9469 & -0.5950 & 0.4710 & 0.6623 \\
\hline Petukhov2 & 0.8873 & 0.9489 & -1.3321 & -2.0029 & -3.3808 \\
\hline Herbert & 0.8527 & 0.8801 & -1.0010 & -0.9451 & -7.4225 \\
\hline Jackson & 0.9330 & 0.9326 & 0.0858 & 0.7098 & 0.6711 \\
\hline Parlatan & 0.9260 & 0.9361 & -0.2230 & 0.7144 & 0.6797 \\
\hline Vilemas & 0.4917 & 0.2009 & -0.4386 & -0.4416 & -0.0787 \\
\hline Celeta & 0.9405 & 0.9323 & 0.3425 & 0.7097 & 0.7059 \\
\hline Symolon & 0.9155 & 0.9512 & -0.8558 & 0.4652 & 0.6718 \\
\hline McEligot & 0.9082 & 0.8872 & 0.1416 & 0.6136 & 0.6434 \\
\hline Perkins & 0.9376 & 0.9385 & 0.1333 & 0.6376 & 0.6336 \\
\hline Taylor & 0.8780 & 0.8328 & 0.1828 & 0.6112 & 0.6089 \\
\hline
\end{tabular}

Table 6-4 20\% Band Value for Various Correlations

\begin{tabular}{||c||c||c||c||c||c||}
\hline \hline Correlation & All & Turbulent & $B^{*}$ DTHT & $K_{v}$ DTHT & Transition \\
\hline \hline Gnielinski & 0.6909 & 0.8482 & 0.2216 & 0.6250 & 0.6667 \\
\hline Petukhov1 & 0.6739 & 0.8464 & 0.1705 & 0.4167 & 0.7188 \\
\hline Petukhov2 & 0.6114 & 0.8946 & 0.1250 & 0.1250 & 0.0938 \\
\hline Herbert & 0.5261 & 0.7554 & 0.1648 & 0.2292 & 0.0000 \\
\hline Jackson & 0.7011 & 0.8429 & 0.2898 & 0.6250 & 0.6667 \\
\hline Parlatan & 0.6898 & 0.8482 & 0.2159 & 0.6250 & 0.6667 \\
\hline Vilemas & 0.3080 & 0.3429 & 0.0966 & 0.1875 & 0.5521 \\
\hline Celeta & 0.6898 & 0.8196 & 0.2841 & 0.6250 & 0.7083 \\
\hline Symolon & 0.6830 & 0.8625 & 0.1534 & 0.4167 & 0.7396 \\
\hline McEligot & 0.5739 & 0.6589 & 0.2898 & 0.6042 & 0.5833 \\
\hline Perkins & 0.6886 & 0.8464 & 0.2500 & 0.6250 & 0.6042 \\
\hline Taylor & 0.4489 & 0.4661 & 0.2784 & 0.5417 & 0.6146 \\
\hline \hline
\end{tabular}

Examining the figures and tables, the best correlation in terms of R-square is Celeta et al. correlation followed by Jackson et al. correlation which is the best correlation in terms of $20 \%$ band indicator. Surprisingly, both Celeta et al. and Jackson et al. correlations perform better in the acceleration driven DTHT regime (see Table 6-3 and 6-4). However, 
the problem with Celeta et al. correlation is that since the correlation was developed with a smaller maximum $L / D$ test section than the MIT facility, the predicted Nusselt number is much smaller (even smaller than the forced convection laminar Nusselt number 4.364) than the measured value at the buoyancy induced DTHT regime when $L / D$ value is large (see Figure 6-12). The other reason is due to the characteristic of the gas flow; the re-turbulizing cases cannot be captured with the water experiment. Therefore, Celeta et al. correlation can under predict the heat transfer coefficient in certain situations when it is applied to the gas heat transfer.

Most of the correlations over predict the Nusselt number in both acceleration and buoyancy driven DTHT except for Vilemas et al. and Celeta et al. correlations. This indicates that those correlations cannot be applied for designing a gas heat transfer system, since it gives non-conservative value of heat transfer coefficient. However, Vilemas et al. correlation tends to under predict the heat transfer coefficient too much, even for the turbulent heat transfer regime and also Vilemas et al. correlation poorly fits data compared to the other correlations.

Key points from the correlation comparison with the data is that since gases have different property behavior with the temperature compared to liquids or super critical fluids, most of the DTHT correlations that were fitted with liquids or super critical fluids tend to over predict the Nusselt number, except for Celeta et al. correlation. However, Celeta et al. correlation under predicts the heat transfer coefficient for "re-turbulizing flow" due to the same reason why other correlations over predicts. In addition, since Celeta et al. correlation involves wall temperature based properties when it is estimating the free convection Nusselt number (Eq. 2-27), it is not an easy correlation to utilize for estimating the wall temperature.

Therefore, a new correlation that can successfully fit the gas DTHT regime with a reasonable agreement to the turbulent, transition and laminar heat transfer data is necessary. In addition, required wall temperature information should be minimized to simplify calculation, even though the power law of wall to bulk temperature ratio can capture the radial properties variation has been well known and was used extensively among various investigators. 


\section{MIT Correlation Development}

This section will discuss the new correlations that were developed for each regime. However, to enhance the user's flexibility in the choice of the correlation sets depending on the computational power and specific engineering application, three correlation sets were developed. The first section will discuss the basic ideas for developing each correlation. The second to fourth section will discuss three correlation sets that were developed at MIT. Next section will show the correlation performance compared to the experimental data and the correlations described in Chapter 2. The last two sections will discuss the limitation of the correlation application and the smoothness of the developed correlation.

\subsection{Correlation Development Criteria and Basic Ideas}

The new correlation should include the following characteristics:

1. It should reflect the physical phenomena that are occurring in the physical world.

2. It should be simple and easy to apply in engineering calculations.

A. Most of the physical properties should be evaluated at bulk temperature.

B. Any kind of iteration should be minimized.

3. It should cover all heat transfer regimes including turbulent, laminar and deteriorated turbulent as well as mixed convection laminar regimes with good accuracy.

4. It should be relatively smooth between different flow regimes in order to minimize numerical treatment between the flow regimes if the real experimental data behaves smoothly, when crossing the heat transfer regime boundary.

Beginning with the laminar flow correlation development, one can see from Figure 5-6 that the normal laminar correlation under predicts the Nusselt number. When the heat flux is high and the flow is laminar, the major effect that changes the laminar heat transfer is the buoyancy effect. As explained in the previous section, the buoyancy effect tends to make a steeper velocity gradient near the wall and increase the laminar heat transfer. This is called laminar mixed-convection, since the forced and natural convections are "mixed" together in the laminar flow.

It is noted that the acceleration effect only laminarizes the turbulent flow and has no effect on the laminar flow, thus the acceleration effect is not considered for the laminar flow. From the non-dimensional form of Navier- Stokes equation the main nondimensional number that measures the strength of the buoyancy effect is $\mathrm{Gr}_{q} / \operatorname{Re}^{2}$ (it is also called the Richardson number) for heat flux boundary condition (refer to Eq. 2-10). Also, from Figure 5-10, $\mathrm{Gr}_{q} / \mathrm{Re}^{2}$ successfully shows a correlation with the ratio of the measured Nusselt number to the Nusselt number predicted by laminar forced convection correlation. Therefore, $\mathrm{Gr}_{q} / \mathrm{Re}^{2}$ will be selected as the governing non-dimensional parameter for the laminar mixed-convection regime. 
For the DTHT regime correlation development, one can observe on Figure 5-6 that the pattern for the DTHT correlation should be branching out from the Gnielinski correlation or the forced convection correlation when certain criteria are met. The functional form for the DTHT regime with such characteristics can be found by changing the constant in the Gnielinski correlation to functional form (Eq. 7-1). This technique is following the Gnielinski approach to fit the data better, near the laminar to turbulent flow transition.

$$
\begin{aligned}
& \mathrm{Nu}_{\text {Gnielinsky }}=\frac{(f / 8)(\mathrm{Re}-1000) \operatorname{Pr}}{1+12.7 \sqrt{f / 8}\left(\operatorname{Pr}^{2 / 3}-1\right)}\left(\frac{T_{w}}{T_{b}}\right)^{-0.45}\left(1+\frac{x^{-\frac{2}{3}}}{D}\right) \\
& \rightarrow \mathrm{Nu}_{M I T}=\frac{(f / 8)(\operatorname{Re}-F(x)) \operatorname{Pr}}{1+12.7 \sqrt{f / 8}\left(\operatorname{Pr}^{2 / 3}-1\right)}\left(\frac{T_{w}}{T_{b}}\right)^{-0.45}\left(1+\frac{x^{-\frac{2}{3}}}{D}\right)
\end{aligned}
$$

Figure 7-1 depicts the example form of the modified Gnielinski correlation. If $F(x)$ is different from 1,000 and can be correlated to a non-dimensional number that is relevant to a particular case for capturing the key physical phenomena, then one can expect that the predicted Nusselt number will behave similarly to the experimental data. Since, major turbulent heat transport deterioration in the current MIT data is due to the acceleration effect and buoyancy effect, the relevant non-dimensional parameters for $\mathrm{F}(\mathrm{x})$ are selected to be the acceleration parameter and buoyancy number.

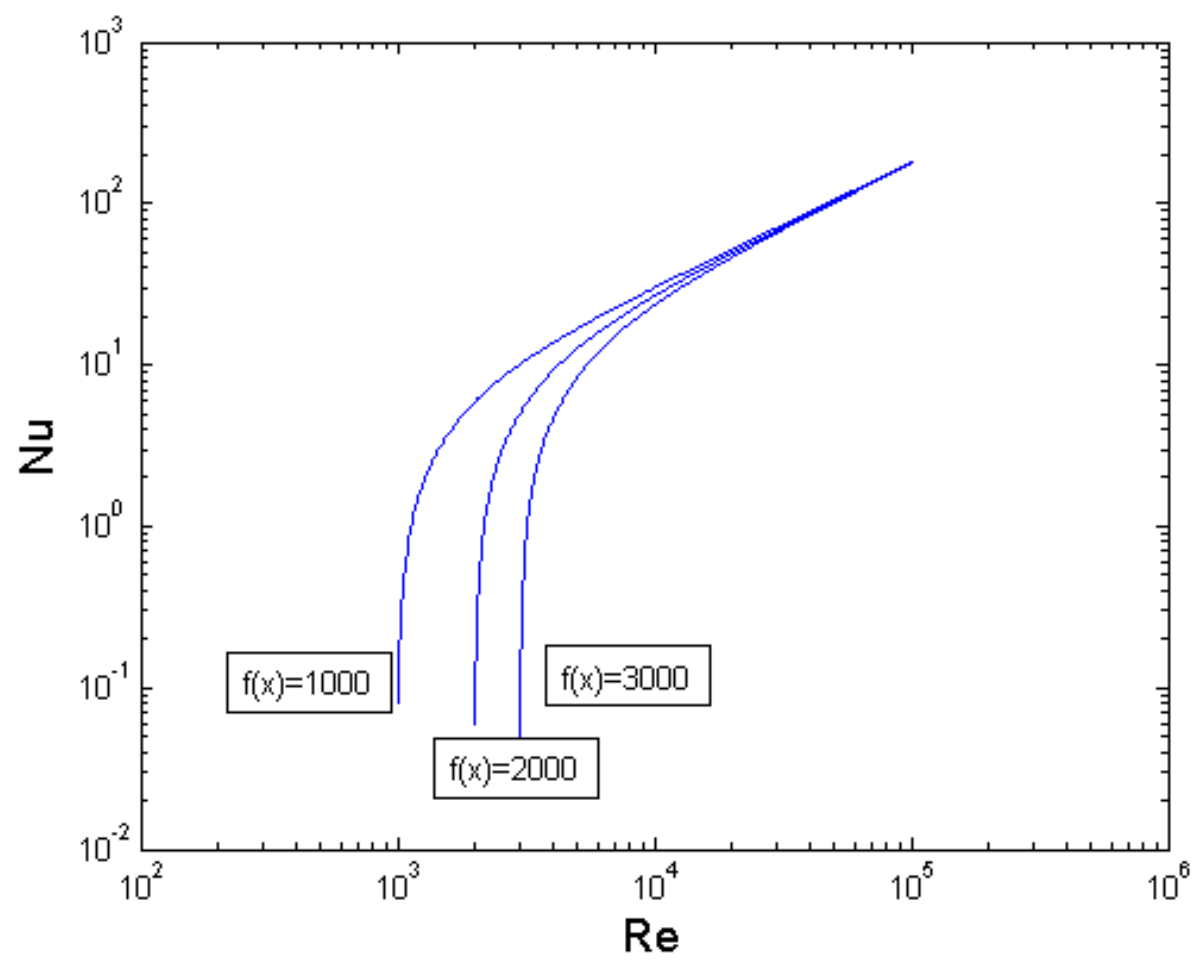

Figure 7-1 Functional Form for the New Correlation 
The other important characteristic for the new correlation is that the condition for determining each regime (laminar, turbulent, DTHT and so forth) should be based on the inlet non-dimensional numbers such as the Reynolds number or the buoyancy parameter. This kind of correlation selection based on the inlet condition can be found from Vilemas et al. (Eq. 2-26). For example the turbulent and laminar flows are primarily determined first in the developing length where there is no heating. The decrease in the Reynolds number due to heating (this is true only for the gas flow since the viscosity of the gas increases with the temperature rise while water decreases which leads to increased Reynolds number) and the deterioration of the turbulence due to the buoyancy and acceleration effects are responsible for reducing the turbulent heat transport in the turbulent flow if the flow enters the heated test section as turbulent flow. Therefore, the new correlation is designed to select the correlation from a correlation set based on the inlet non-dimensional numbers.

Basic procedure for developing a correlation starts from correlating $\mathrm{F}(\mathrm{x})$ to a nondimensional number for each regime with a test function. Two functional forms are usually tested to minimize the number of coefficients to fit (Eq. 7-2).

$$
F(x)=C_{1}(N o n D)^{C_{2}} \quad \text { or } \quad F(x)=C_{1} \log _{10}\left(C_{2} \text { NonD }\right)
$$

Since the governing non-dimensional numbers (the buoyancy parameter and acceleration parameter) vary by on order of magnitude while the function response is much slower than the changes in non-dimensional numbers, the power law form and the logarithmic form were chosen. These two functional forms have an advantage over other polynomial functional forms as they accommodate large variation of a variable to yield a small functional response.

Before proceeding to the correlation sections, the second re-turbulization threshold has to be also decided. Figure 7-2 shows the re-turbulizing cases only. From the figure, most of the cases begin to recover to the normal turbulent heat transfer after exceeding the local buoyancy parameter of $B o^{*} \approx 6 \times 10^{-7}$. It should be noted that the channel inlet is on the right in the figure and the outlet is on the left, since the gas buoyancy number decreases as the flow proceeds to the down stream of the channel.

This condition will be used for developing the correlation and further dividing the heat transfer regime. 


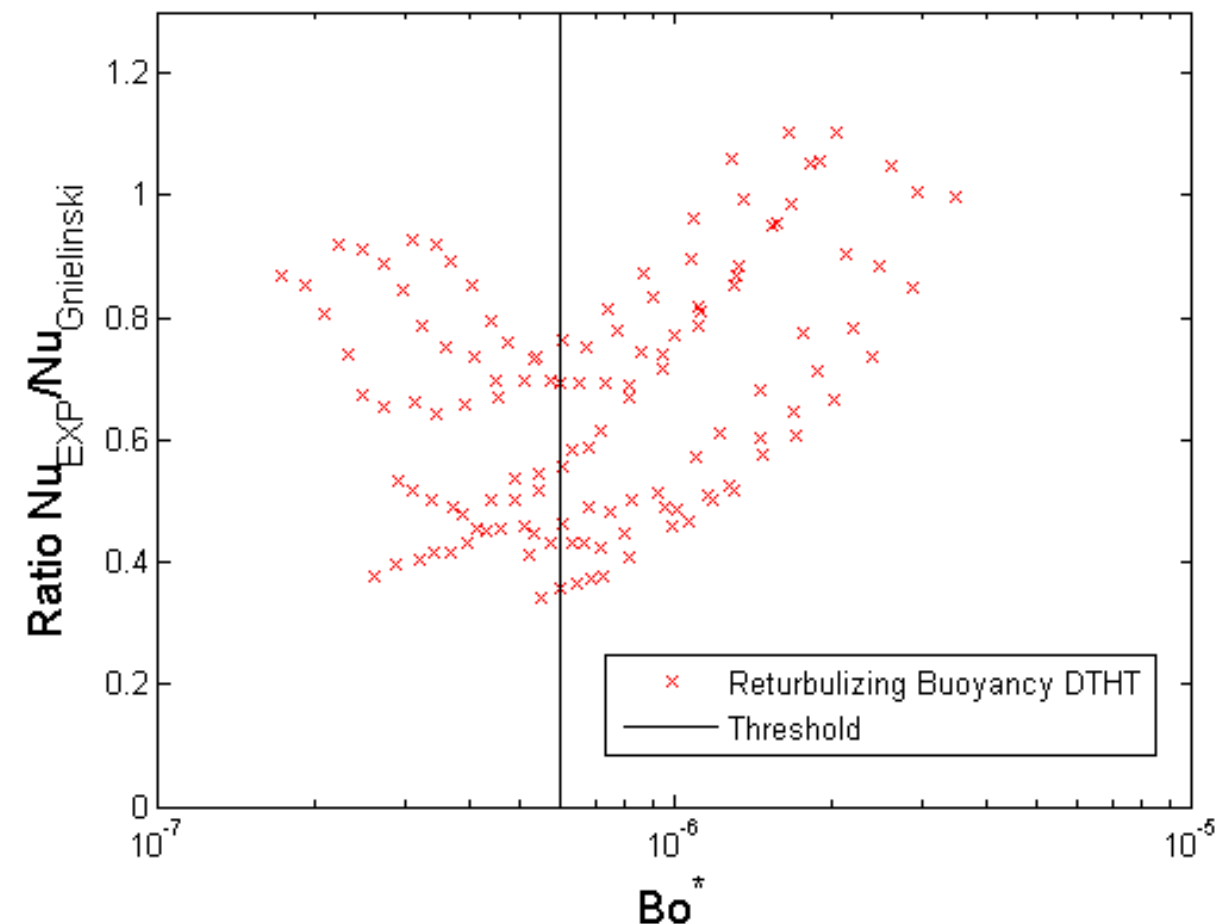

Figure 7-2 $2^{\text {nd }}$ Threshold of Re-turbulization

\subsection{Type-1 MIT Correlation}

After extensive exploration of correlation forms having incorporated the underlying phenomenological effects discussed above and data fitting, the form of the correlation covering turbulent, laminar, the DTHT and laminar mixed-convection regimes was developed. The correlation form is shown next.

\section{Type-1 MIT Correlation}

If $K_{v}$ inlet $<2.0 \times 10^{-6}, B o^{*}{ }_{\text {inlet }}<2.0 \times 10^{-6}$ and $\mathrm{Re}_{\text {inlet }}>2300$ (Turbulent)

$$
\mathrm{Nu}_{M I T 1}=\frac{(f / 8)(\operatorname{Re}-1000) \operatorname{Pr}}{1+12.7 \sqrt{f / 8}\left(\operatorname{Pr}^{2 / 3}-1\right)}\left(\frac{T_{w}}{T_{b}}\right)^{-0.45}\left(1+\frac{x^{-\frac{2}{3}}}{D}\right)=\mathrm{Nu}_{\text {Gnielinsky }}
$$

$$
\begin{gathered}
\text { If } K_{v \text { inlet }} \geq 2.0 \times 10^{-6}, B o^{*}{ }_{\text {inlet }}<2.0 \times 10^{-6} \text { and } \mathrm{Re}_{\text {inlet }}>2300 \text { ( } K_{v} \text { DTHT) } \\
\mathrm{Nu}_{\text {MIT1-temp }}=\frac{(f / 8)\left(\mathrm{Re}-0.185 K_{v}^{-2 / 3}\right) \operatorname{Pr}}{1+12.7 \sqrt{f / 8}\left(\operatorname{Pr}^{2 / 3}-1\right)}\left(\frac{T_{w}}{T_{b}}\right)^{-0.45}\left(1+\frac{x}{D}{ }^{-\frac{2}{3}}\right)
\end{gathered}
$$




$$
\begin{gathered}
\mathrm{Nu}_{\text {MIT1 }}=\max \left(\mathrm{Nu}_{\text {MIT1-temp }}, \mathrm{Nu}_{\text {Laminar }}\right) \\
\text { If } K_{v \text { inlet }}<2.0 \times 10^{-6}, 3.5 \times 10^{-6}>B o^{*}{ }_{\text {inlet }} \geq 2.0 \times 10^{-6} \text { and } \mathrm{Re}_{\text {inlet }}>2300 \\
\text { If } B o^{*} \geq 6.0 \times 10^{-7}\left(\text { Re-turbulizing } B o^{*} \text { DTHT }\right) \\
\mathrm{Nu}_{\text {MIT1-temp }}=\frac{(f / 8)\left(\mathrm{Re}-1.45 \times 10^{-7} B o^{*-1.7}\right) \operatorname{Pr}}{1+12.7 \sqrt{f / 8}\left(\operatorname{Pr}^{2 / 3}-1\right)}\left(\frac{T_{w}}{T_{b}}\right)^{-0.45}\left(1+\frac{x}{D}\right) \\
\mathrm{Nu}_{\text {MIT1 }}^{-\frac{2}{3}}=\max \left(\mathrm{Nu}_{\text {MIT1-temp }}, \mathrm{Nu}_{\text {Laminar }}\right)
\end{gathered}
$$

else $B o^{*}<6.0 \times 10^{-7}$ (Re-turbulizing $B o^{*}$ DTHT)

$$
\begin{aligned}
& \mathrm{Nu}_{\text {MIT1-temp }}=\frac{(f / 8)\left(\operatorname{Re}-8.34 \times 10^{7} B o^{* 0.69}\right) \operatorname{Pr}}{1+12.7 \sqrt{f / 8}\left(\operatorname{Pr}^{2 / 3}-1\right)}\left(\frac{T_{w}}{T_{b}}\right)^{-0.45}\left(1+\frac{x^{-\frac{2}{3}}}{D}\right) \\
& \mathrm{Nu}_{\text {MIT1 }}=\max \left(\mathrm{Nu}_{\text {MIT1-temp }}, \mathrm{Nu}_{\text {Laminar }}\right)
\end{aligned}
$$

If $K_{v \text { inlet }}<2.0 \times 10^{-6}, B o^{*}{ }_{\text {inlet }} \geq 3.5 \times 10^{-6}$ and $\mathrm{Re}_{\text {inlet }}>2300$ (Bo DTHT)

$$
\begin{aligned}
& \mathrm{Nu}_{\text {MIT1-temp }}=\frac{(f / 8)\left(\operatorname{Re}-79.4 B o^{*-0.28}\right) \operatorname{Pr}}{1+12.7 \sqrt{f / 8}\left(\operatorname{Pr}^{2 / 3}-1\right)}\left(\frac{T_{w}}{T_{b}}\right)^{-0.45}\left(1+\frac{x^{-\frac{2}{3}}}{D}\right) \\
& \mathrm{Nu}_{M I T 1}=\max \left(\mathrm{Nu}_{M I T 1-\text { temp }}, \mathrm{Nu}_{\text {Laminar }}\right)
\end{aligned}
$$

If $\mathrm{Re}_{\text {inlet }} \leq 2300$ (Mixed Convection Laminar and Forced Convection Laminar)

$$
\mathrm{Nu}_{\text {MIT-Laminar }}=\max \left(1,3.0\left(\frac{\mathrm{Gr}_{\mathrm{q}}}{\mathrm{Re}^{2}}\right)^{0.11}\right) \mathrm{Nu}_{\text {Laminar }}
$$

$f=\left(1.82 \log _{10} \operatorname{Re}-1.64\right)^{-2}$

$\mathrm{Nu}_{\text {Laminar }}=\left(\frac{1}{\mathrm{Nu}_{\infty}}-\frac{1}{2} \sum_{m=1}^{10} \frac{\exp \left(-\gamma_{m}^{2} x^{+}\right)}{A_{m} \gamma_{m}^{4}}\right)^{-1}$

where $\mathrm{Nu}_{\infty}=4.364, \quad x^{+}=\frac{2 x / D}{\operatorname{Re} \operatorname{Pr}}, \quad \gamma_{m}=4 m+\frac{4}{3}, \quad A_{m}=0.4165 \gamma_{m}^{-7 / 3}$

In the DTHT and laminar convection regimes a few characteristics of the correlation should be discussed. First, since the deteriorated turbulent heat transfer coefficient cannot be lower than the laminar convection, the DTHT regime heat transfer coefficient will be the maximum between the modified Gnielinski correlation and the laminar convection 
correlation. This will provide a lower bound for the correlation.

The heat transfer correlation for the transition regime is not separately developed. This is because the Gnielinski correlation fits the transitional regime data reasonably well (see Tables 6-3 and 6-4). Also since the governing parameter is not well defined in the laminar to turbulent transition regime the correlation for this regime is not separately developed.

For the laminar mixed-convection, the multiplication factor to forced convection laminar correlation was developed with the governing non-dimensional number of $\mathrm{Gr}_{q} / \mathrm{Re}^{2}$. The power and the leading coefficient are also determined from the empirical curve fitting. To give a lower bound to the correlation, the multiplication factor is a maximum value between unity, which means it can cover both the normal forced laminar convection and the mixed-convection laminar. All properties and non-dimensional numbers are evaluated at local bulk temperature.

Figure 7-3 shows the performance of the Type-1 MIT correlation set. The discussion of the two indicators will be presented in section 7.5.

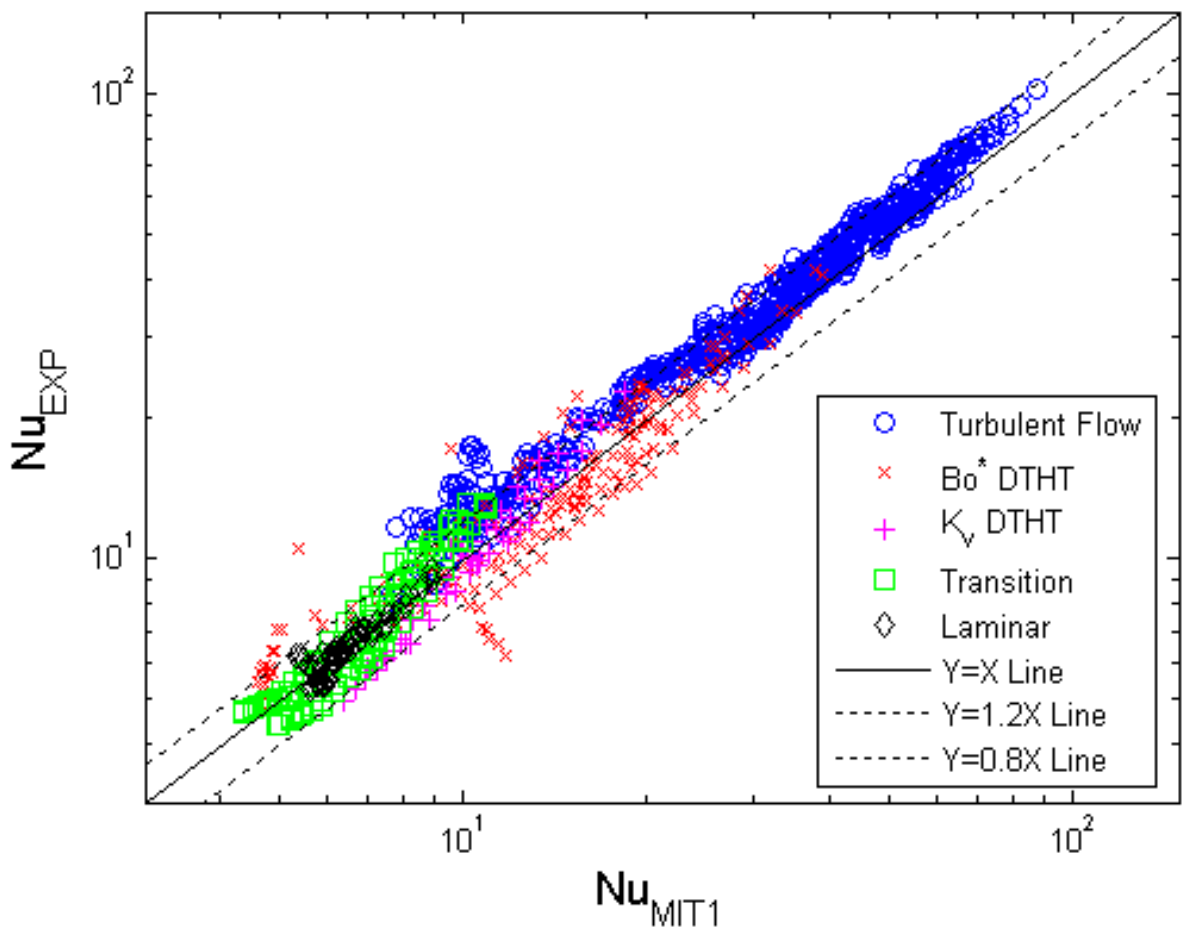

Figure 7-3 Experimental Nu vs. First Kind MIT Correlation Predicted Nu

The Type-1 MIT correlation requires iteration on wall temperature due to the Gnielinski temperature modification factor of $\left(T_{w} / T_{b}\right)^{-0.45}$ to predicting a heat transfer coefficient. Therefore, Type-2 correlation set will be developed that has $\left(T_{w} / T_{b}\right)^{-0.5}$ as the modification factor to minimize the iteration. This will be discussed in the next section. 


\subsection{Type-2 MIT Correlation}

The motivation for developing $2^{\text {nd }}$ kind correlations is to consider the following points.

1. Term $\left(T_{w} / T_{b}\right)^{-0.45}$ in the original Gnielinski form makes the prediction of heat transfer coefficient more difficult, since it requires iteration on unknown wall temperature. To avoid iterations, correlations with $\left(T_{w} / T_{b}\right)^{-0.5}$ were developed. (Eq. 7-3) shows how to even eliminate any iteration process for predicting the wall temperature.

$$
\begin{aligned}
& h_{P V}=h_{C P}\left(\frac{T_{w}}{T_{b}}\right)^{-0.5} \\
& T_{w}=T_{b}+\frac{q^{\prime \prime}}{h_{P V}}=T_{b}+\frac{q^{\prime \prime}}{h_{C P}}\left(\frac{T_{w}}{T_{b}}\right)^{0.5} \\
& \Leftrightarrow T_{w}^{2}-\left(2 T_{b}+\frac{q^{\prime \prime 2}}{h_{C P}{ }^{2} T_{b}}\right) T_{w}+T_{b}^{2}=0 \\
& \Leftrightarrow T_{w}=\frac{1}{2}\left[2 T_{b}+\frac{q^{\prime \prime 2}}{h_{C P}{ }^{2} T_{b}}+\sqrt{\left(2 T_{b}+\frac{q^{\prime \prime 2}}{h_{C P}{ }^{2} T_{b}}\right)^{2}-4 T_{b}^{2}}\right] \\
& \Leftrightarrow T_{w}=T_{b}+\frac{1}{2}\left[\frac{q^{\prime \prime 2}}{h_{C P}{ }^{2} T_{b}}+\sqrt{\left(\frac{q^{\prime \prime 2}}{h_{C P}{ }^{2} T_{b}}\right)^{2}+\frac{2 q^{\prime \prime 2}}{h_{C P}{ }^{2}}}\right]
\end{aligned}
$$

2. The friction factor that is used for the Gnielinski correlation is valid only for $\operatorname{Re}>10,000$ (Filonenko correlation). Therefore, the friction factor needs to be reconsidered when the Gnielinski correlation is applied to $\operatorname{Re}<10,000$. The Blasius friction factor is commonly used for lower Reynolds number turbulent flow friction factor, which is valid for $\operatorname{Re}>4,000$. Between Reynolds number 4,000 and 2,300 the friction factor will be estimated from the interpolation between the friction factor at 4,000 with Blasius correlation and the laminar friction factor at 2,300.

The following set of correlations is the Type- 2 correlation developed at MIT. It should be noted that power law of the governing non-dimensional number fitted better in the Type-1 correlation while the logarithmic function of the governing non-dimensional number performed better in the Type- 2 correlation. Again the transition regime was not separated and the laminar correlation is the same, since it is not affected by the temperature modification factor change. 


\section{Type-2 MIT Correlation}

If $K_{v \text { inlet }}<2.0 \times 10^{-6}, B o^{*}{ }_{\text {inlet }}<2.0 \times 10^{-6}$ and $\mathrm{Re}_{\text {inlet }}>2300$ (Turbulent)

$$
\mathrm{Nu}_{\text {MIT } 2}=\frac{(f / 8)(\mathrm{Re}+500) \operatorname{Pr}}{1+12.7 \sqrt{f / 8}\left(\operatorname{Pr}^{2 / 3}-1\right)}\left(\frac{T_{w}}{T_{b}}\right)^{-0.5}\left(1+\frac{x^{-\frac{2}{3}}}{D}\right)
$$

If $K_{v}$ inlet $\geq 2.0 \times 10^{-6}, B o^{*}{ }_{\text {inlet }}<2.0 \times 10^{-6}$ and $\mathrm{Re}_{\text {inlet }}>2300\left(K_{v}\right.$ DTHT)

$$
\begin{aligned}
& \mathrm{Nu}_{\text {MIT 2-temp }}=\frac{(f / 8)\left[\mathrm{Re}-3,500 \log _{10}\left(3.8 \times 10^{5} K_{v}\right)\right] \operatorname{Pr}}{1+12.7 \sqrt{f / 8}\left(\operatorname{Pr}^{2 / 3}-1\right)}\left(\frac{T_{w}}{T_{b}}\right)^{-0.5}\left(1+\frac{x^{-\frac{2}{3}}}{D}\right) \\
& \mathrm{Nu}_{\text {MIT2 }}=\max \left(\mathrm{Nu}_{\text {MIT 2-temp }}, \mathrm{Nu}_{\text {Laminar }}\right)
\end{aligned}
$$

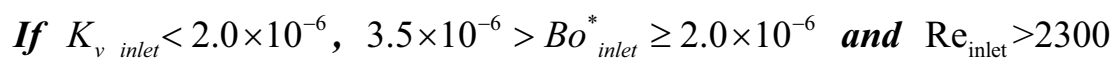

$$
\begin{aligned}
& \text { If } B o^{*} \geq 6.0 \times 10^{-7} \quad \text { (Re-turbulizing } B o^{*} \text { DTHT) } \\
& \mathrm{Nu}_{\text {MIT2-temp }}=\frac{(f / 8)\left[\operatorname{Re}+6,500 \log _{10}\left(4.3 \times 10^{5} B o^{*}\right)\right] \operatorname{Pr}}{1+12.7 \sqrt{f / 8}\left(\operatorname{Pr}^{2 / 3}-1\right)}\left(\frac{T_{w}}{T_{b}}\right)^{-0.5}\left(1+\frac{x}{D}^{-\frac{2}{3}}\right) \\
& \mathrm{Nu}_{\text {MIT2 }}=\max \left(\mathrm{Nu}_{\text {MIT2-temp }}, \mathrm{Nu}_{\text {Laminar }}\right)
\end{aligned}
$$

else $B o^{*}<6.0 \times 10^{-7} \quad$ (Re-turbulizing $B o^{*}$ DTHT)

$$
\begin{aligned}
& \mathrm{Nu}_{\text {MIT2-temp }}=\frac{(f / 8)\left[\mathrm{Re}-3,900 \log _{10}\left(2 \times 10^{7} B o^{*}\right)\right] \operatorname{Pr}}{1+12.7 \sqrt{f / 8}\left(\operatorname{Pr}^{2 / 3}-1\right)}\left(\frac{T_{w}}{T_{b}}\right)^{-0.5}\left(1+\frac{x^{-\frac{2}{3}}}{D}\right) \\
& \mathrm{Nu}_{\text {MIT2 }}=\max \left(\mathrm{Nu}_{\text {MIT2-temp }}, \mathrm{Nu}_{\text {Laminar }}\right)
\end{aligned}
$$

If $K_{v}$ inlet $<2.0 \times 10^{-6}, B o^{*}{ }_{\text {inlet }} \geq 3.5 \times 10^{-6}$ and $\mathrm{Re}_{\text {inlet }}>2300\left(B o^{*}\right.$ DTHT $)$

$$
\begin{aligned}
& \mathrm{Nu}_{\text {MIT2-temp }}=\frac{(f / 8)\left[\mathrm{Re}+2,000 \log _{10}\left(1.6 \times 10^{4} B o^{*}\right)\right] \operatorname{Pr}}{1+12.7 \sqrt{f / 8}\left(\operatorname{Pr}^{2 / 3}-1\right)}\left(\frac{T_{w}}{T_{b}}\right)^{-0.5}\left(1+\frac{x^{-\frac{2}{3}}}{D}\right) \\
& \mathrm{Nu}_{\text {MIT2 }}=\max \left(\mathrm{Nu}_{\text {MIT 2-temp }}, \mathrm{Nu}_{\text {Laminar }}\right)
\end{aligned}
$$


If $\mathrm{Re}_{\text {inlet }} \leq 2300$ (Mixed Convection Laminar and Forced Convection Laminar)

$$
\mathrm{Nu}_{\text {MIT-Laminar }}=\max \left(1,3.0\left(\frac{\mathrm{Gr}_{\mathrm{q}}}{\mathrm{Re}^{2}}\right)^{0.11}\right) \mathrm{Nu}_{\text {Laminar }}
$$

If $\operatorname{Re} \geq 10,000$

$$
f=\left(1.82 \log _{10} \operatorname{Re}-1.64\right)^{-2} \quad \text { (Filonenko) }
$$

If $10,000>\operatorname{Re} \geq 4,000$

$$
f=0.314 / \operatorname{Re}^{0.25} \quad \text { (Blasius) }
$$

If $4,000>\operatorname{Re} \geq 2,300$

$$
f=0.012+6.86 \times 10^{-6} \mathrm{Re}
$$

(Interpolation between Laminar Friction Factor at 2,300 and Blasius at 4,000)

If $2,300>\mathrm{Re}$

$f=64 / \mathrm{Re}$
$\mathrm{Nu}_{\text {Laminar }}=\left(\frac{1}{\mathrm{Nu}_{\infty}}-\frac{1}{2} \sum_{m=1}^{10} \frac{\exp \left(-\gamma_{m}^{2} x^{+}\right)}{A_{m} \gamma_{m}^{4}}\right)^{-1}$

where $\mathrm{Nu}_{\infty}=4.364, \quad x^{+}=\frac{2 x / D}{\operatorname{Re} \operatorname{Pr}}, \quad \gamma_{m}=4 m+\frac{4}{3}, \quad A_{m}=0.4165 \gamma_{m}^{-7 / 3}$

The Type -2 MIT correlation performance is shown in Figure 7-4.

Dividing many heat transfer regimes and having separate correlation for each regime can easily induce frequent jumps in the Nusselt number when the correlation is applied for actual engineering problems, due to a frequent regime change. To reduce this problem and simplify the correlation form as much as possible, the Type-3 MIT correlation is developed, which is presented in the next section. 


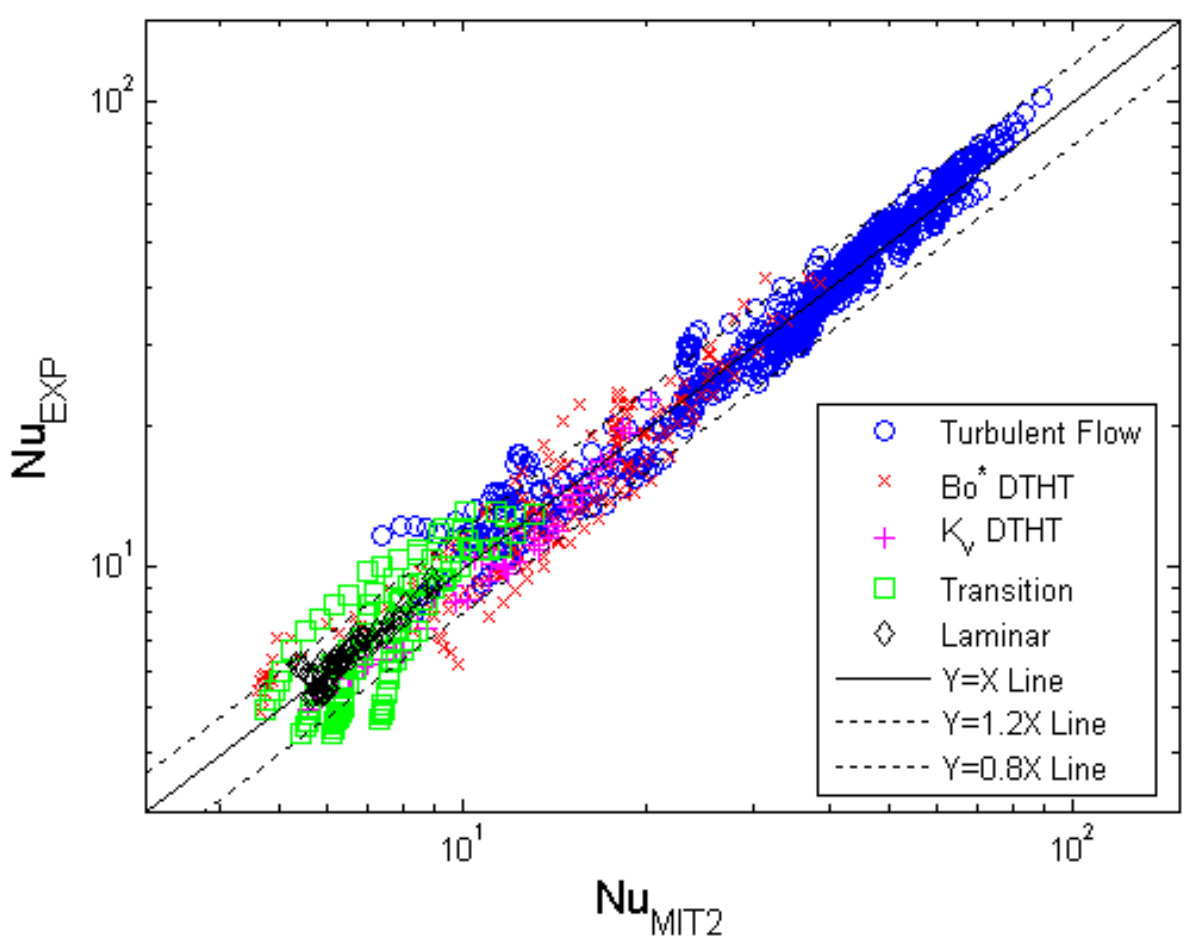

Figure 7-4 Experimental Nu vs. Second Kind MIT Correlation Predicted Nu

\subsection{Type-3 MIT Correlation}

The Type-3 MIT correlation utilizes a new non-dimensional number that can combine the acceleration effect and the buoyancy effect. The new non-dimensional number is developed from a purely empirical standpoint. The basic idea of new non-dimensional number is finding the best ratio between non-dimensional heat flux $\left(q^{+}\right)$and the Reynolds number, since the acceleration effect and the buoyancy effect both have the ratio of the heat flux to bulk flow velocity. The resulting new non-dimensional number is $q^{+} / \mathrm{Re}^{0.44}$. Figure 7-5 shows the experimental Nusselt number over predicted Nusselt ratio versus the new non-dimensional number. The new non-dimensional number pushes the buoyancy induced DTHT and the acceleration induced DTHT data to overlap with each other. This makes possible to correlate both effects with one non-dimensional number and derive a simplified correlation.

Another advantage of the Type-3 MIT correlation is that the interference between the acceleration effect and the buoyancy effect is automatically addressed by using only one non-dimensional number. The flow regime that overlaps over the buoyancy induced DTHT and the acceleration effect was not covered in our experiments, thus the regime is still unknown. Since most of the literature deal with only one effect at a time, the DTHT regime with overlapping acceleration and buoyancy effects is a new regime that requires further attention. However, the new non-dimensional number bypasses this problem, as both effects are combined in one non-dimensional number. 


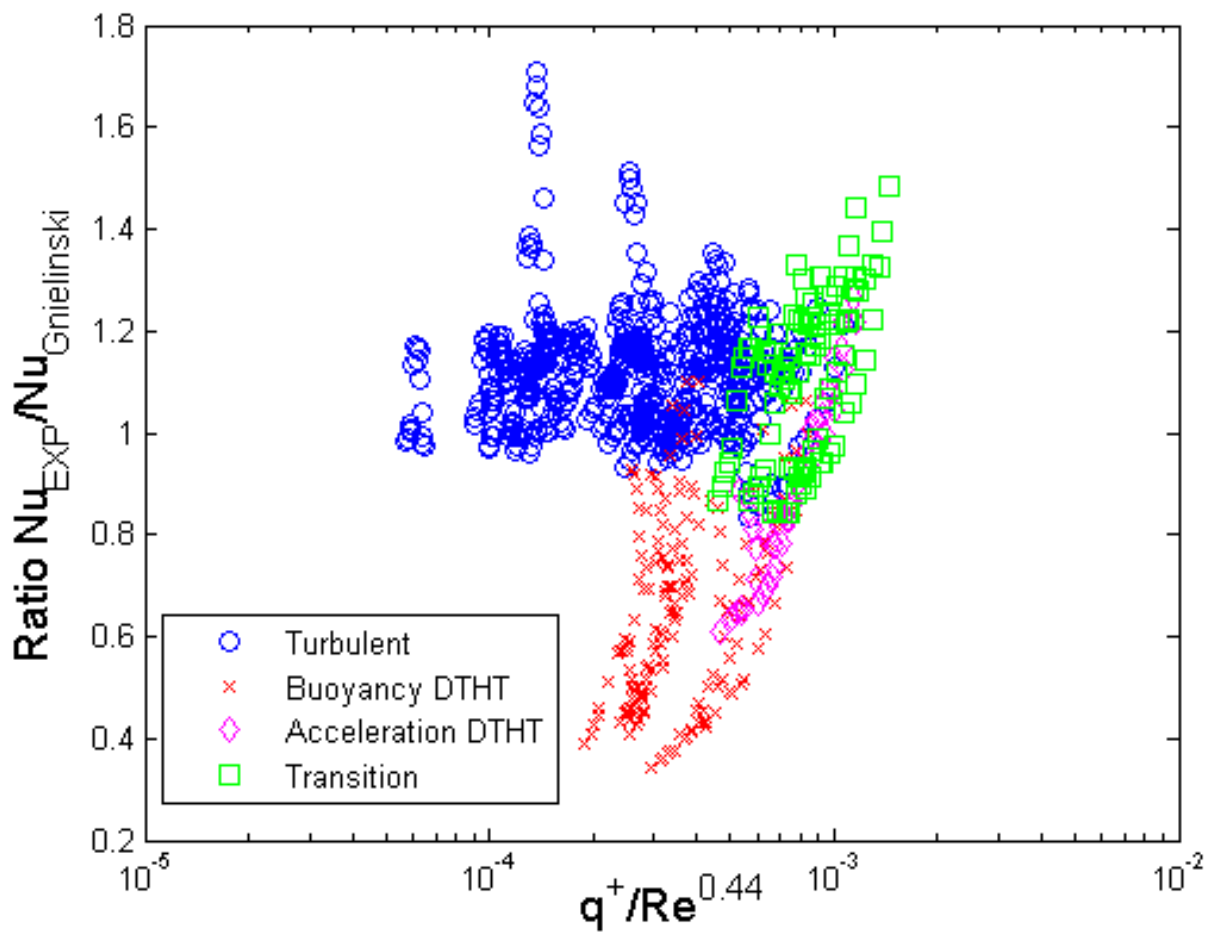

Figure 7-5 Nu Ratio vs. New Non-Dimensional Number

Following set of equation is the Type-3 MIT correlation. The comparison of the correlation to the experimental data is shown in Figure 7-6. It should be noted that the friction factor was calculated by Filonenko's correlation only for simplicity purpose and for the normal turbulent heat transfer, the Gnielinski correlation with $\left(T_{w} / T_{b}\right)^{-0.5}$ is used.

\section{Type-3 MIT Correlation}

If $K_{v \text { inlet }}<2.0 \times 10^{-6}, B o^{*}{ }_{\text {inlet }}<2.0 \times 10^{-6}$ and $\mathrm{Re}_{\text {inlet }}>2300$ (Turbulent)

$$
\mathrm{Nu}_{\text {MIT3 }}=\frac{(f / 8)(\operatorname{Re}-1000) \operatorname{Pr}}{1+12.7 \sqrt{f / 8}\left(\operatorname{Pr}^{2 / 3}-1\right)}\left(\frac{T_{w}}{T_{b}}\right)^{-0.5}\left(1+\frac{x}{D}^{-\frac{2}{3}}\right)
$$

$$
\text { If } K_{v \text { inlet }} \geq 2.0 \times 10^{-6} \text { or } B o^{*}{ }_{\text {inlet }} \geq 2.0 \times 10^{-6} \text { and } \mathrm{Re}_{\text {inlet }}>2300 \text { (DTHT) }
$$

$$
\begin{aligned}
& \mathrm{Nu}_{\text {MIT3-temp }}=\frac{(f / 8)\left(\operatorname{Re}-0.011\left(\frac{q^{+}}{\operatorname{Re}^{0.44}}\right)^{-1.16}\right) \operatorname{Pr}}{1+12.7 \sqrt{f / 8}\left(\operatorname{Pr}^{2 / 3}-1\right)}\left(\frac{T_{w}}{T_{b}}\right)^{-0.5}\left(1+\frac{x}{D}\right) \\
& \mathrm{Nu}_{\text {MIT3 }}=\max \left(\mathrm{Nu}_{\text {MIT3-temp }}, \mathrm{Nu}_{\text {Laminar }}\right)
\end{aligned}
$$


If $\mathrm{Re}_{\text {inlet }} \leq 2300$ (Mixed Convection Laminar and Forced Convection Laminar)

$$
\mathrm{Nu}_{\text {MIT-Laminar }}=\max \left(1,3.0\left(\frac{\mathrm{Gr}_{\mathrm{q}}}{\mathrm{Re}^{2}}\right)^{0.11}\right) \mathrm{Nu}_{\text {Laminar }}
$$

$f=\left(1.82 \log _{10} \operatorname{Re}-1.64\right)^{-2}$

$\mathrm{Nu}_{\text {Laminar }}=\left(\frac{1}{\mathrm{Nu}_{\infty}}-\frac{1}{2} \sum_{m=1}^{10} \frac{\exp \left(-\gamma_{m}^{2} x^{+}\right)}{A_{m} \gamma_{m}^{4}}\right)^{-1}$

where $\mathrm{Nu}_{\infty}=4.364, \quad x^{+}=\frac{2 x / D}{\operatorname{Re} \operatorname{Pr}}, \quad \gamma_{m}=4 m+\frac{4}{3}, \quad A_{m}=0.4165 \gamma_{m}^{-7 / 3}$

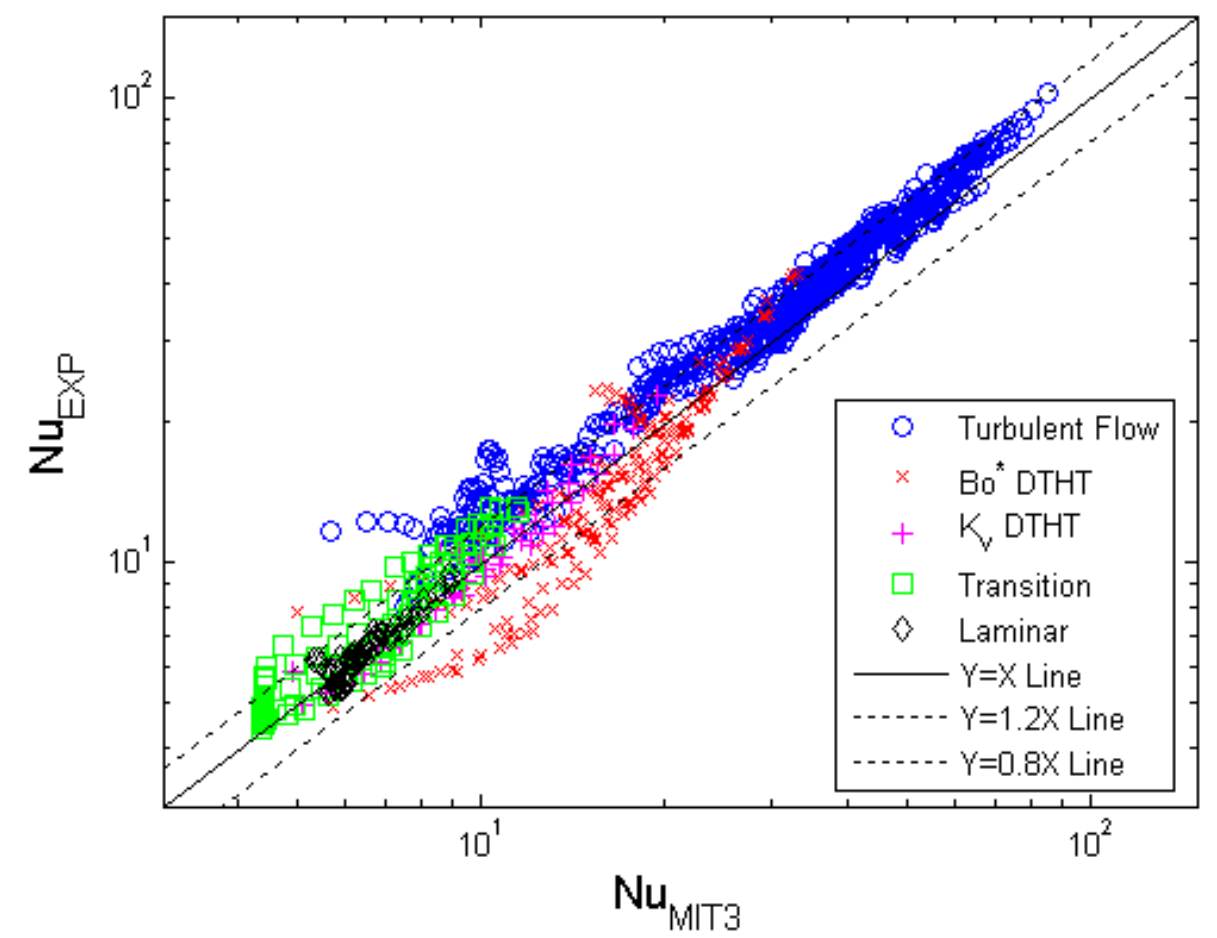

Figure 7-6 Experimental Nu vs. Third Kind MIT Correlation Predicted Nu

\subsection{MIT Correlations Comparison}

Table 7-1 summarizes the MIT-Laminar correlation performance in terms of the two indicators. Compared to the other four mixed convection laminar correlation, the MITLaminar correlation fits the experimental data successfully. All the mixed convection laminar experimental data are within $\pm 20 \%$ band. However, as mentioned in Chapter 6 , 
more experimental data for the mixed convection laminar regime will be required to make final conclusion. The future experiment should be performed with other gases such as nitrogen or carbon dioxide at low flow rate.

Table 7-1 Summary of MIT-Laminar Correlation Performance

\begin{tabular}{|c||c||c||}
\hline \hline Correlation & R-square & $20 \%$ Fraction \\
\hline \hline Laminar & -3.7577 & 0.0000 \\
\hline Hallman & -29.7585 & 0.0000 \\
\hline Worsoeschmidt1 & -6.5282 & 0.0000 \\
\hline Worsoeschmidt2 & -4.8404 & 0.0000 \\
\hline Churchill & -4.8489 & 0.0000 \\
\hline MIT & 0.8911 & 1.0000 \\
\hline
\end{tabular}

Tables 7-2 and Table 7-3 summarize the performance of the all three types of MIT correlations and compare them to other DTHT correlations. Again, correlation's value is highlighted with yellow when the R-square value is over $90 \%$ and $20 \%$ band value over $75 \%$, and light blue is used to highlight the best result in each regime for both indicators.

Table 7-2 Summary of MIT Correlations Performance (R-square)

\begin{tabular}{|c|c|c|c||c|c|}
\hline Correlation & All & Turbulent & $B^{*}{ }^{*}$ DTHT & $K_{v}$ DTHT & Transition \\
\hline \hline Gnielinski & 0.9275 & 0.9357 & -0.1649 & 0.7138 & 0.6785 \\
\hline Petukhov1 & 0.9207 & 0.9469 & -0.5950 & 0.4710 & 0.6623 \\
\hline Petukhov2 & 0.8873 & 0.9489 & -1.3321 & -2.0029 & -3.3808 \\
\hline Herbert & 0.8527 & 0.8801 & -1.001 & -0.9451 & -7.4225 \\
\hline Jackson & 0.9330 & 0.9326 & 0.0858 & 0.7098 & 0.6711 \\
\hline Parlatan & 0.9260 & 0.9361 & -0.2230 & 0.7144 & 0.6797 \\
\hline Vilemas & 0.4917 & 0.2009 & -0.4386 & -0.4416 & -0.0787 \\
\hline Celeta & 0.9405 & 0.9323 & 0.3425 & 0.7097 & 0.7059 \\
\hline Symolon & 0.9155 & 0.9512 & -0.8558 & 0.4652 & 0.6718 \\
\hline McEligot & 0.9082 & 0.8872 & 0.1416 & 0.6136 & 0.6434 \\
\hline Perkins & 0.9376 & 0.9385 & 0.1333 & 0.6376 & 0.6336 \\
\hline Taylor & 0.8780 & 0.8328 & 0.1828 & 0.6112 & 0.6089 \\
\hline MIT1 & 0.9573 & 0.9331 & 0.8810 & 0.8710 & 0.8096 \\
\hline MIT2 & 0.9761 & 0.9659 & 0.8813 & 0.9032 & 0.6864 \\
\hline MIT3 & 0.9475 & 0.9193 & 0.8151 & 0.9319 & 0.7873 \\
\hline
\end{tabular}


Table 7-3 Summary of MIT Correlations Performance (20\% band)

\begin{tabular}{|c|c|c|c||c|c||}
\hline \hline Correlation & All & Turbulent & $B^{*}{ }^{*}$ DTHT & $K_{v}$ DTHT & Transition \\
\hline \hline Gnielinski & 0.6909 & 0.8482 & 0.2216 & 0.6250 & 0.6667 \\
\hline Petukhov1 & 0.6739 & 0.8464 & 0.1705 & 0.4167 & 0.7188 \\
\hline Petukhov2 & 0.6114 & 0.8946 & 0.1250 & 0.1250 & 0.0938 \\
\hline Herbert & 0.5261 & 0.7554 & 0.1648 & 0.2292 & 0.0000 \\
\hline Jackson & 0.7011 & 0.8429 & 0.2898 & 0.6250 & 0.6667 \\
\hline Parlatan & 0.6898 & 0.8482 & 0.2159 & 0.6250 & 0.6667 \\
\hline Vilemas & 0.3080 & 0.3429 & 0.0966 & 0.1875 & 0.5521 \\
\hline Celeta & 0.6898 & 0.8196 & 0.2841 & 0.6250 & 0.7083 \\
\hline Symolon & 0.6830 & 0.8625 & 0.1534 & 0.4167 & 0.7396 \\
\hline McEligot & 0.5739 & 0.6589 & 0.2898 & 0.6042 & 0.5833 \\
\hline Perkins & 0.6886 & 0.8464 & 0.2500 & 0.6250 & 0.6042 \\
\hline Taylor & 0.4489 & 0.4661 & 0.2784 & 0.5417 & 0.6146 \\
\hline MIT1 & 0.8045 & 0.8089 & 0.7273 & 0.8750 & 0.8854 \\
\hline MIT2 & 0.8682 & 0.9393 & 0.7273 & 1.000 & 0.6458 \\
\hline MIT3 & 0.7420 & 0.7571 & 0.6023 & 0.9792 & 0.7917 \\
\hline
\end{tabular}

Overall MIT1 and MIT2 correlations perform the best in the entire heat transfer regime set and MIT3 shows satisfying performance as well. Comparing the three MIT correlations against the best correlation from Chapter 6, i.e. Celeta et al. correlation, they all perform better than or are equivalent to Celeta et al. correlation in all regimes. Therefore, it can be concluded that all three correlations can be applied for gas heat transfer engineering calculation. One can choose a correlation set among the three sets by considering the available computational resources, the character of the problem and the desired accuracy of the calculations in view of the application requirements.

\subsection{Limitation of MIT correlations}

The limitation of MIT correlation is the range of covered area thorough the experiments, since the correlation is fitted with MIT data only. Table 7-4 shows the non-dimensional number range of all the experimental data. However, one should keep in mind that the upper limit of the Reynolds number could always be extended up to the Gnielinski correlation, which is $\operatorname{Re}<10^{6}$ for the forced normal turbulent heat transfer regime. This is because only slight modification or no change was made to the Gnielinski correlation when developing three types of MIT correlation for the normal turbulent heat transfer regime.

The other restrictions for applying the developed correlation are:

1. Since liquids and super critical fluids behave very differently from the gas (Chapter 3), the developed correlation is applicable only to the gas heat transfer cases. 
2. The interference between the acceleration effect and the buoyancy effect is not fully covered, where the operating conditions are above both the acceleration threshold and the buoyancy threshold at the same time. MIT1 and MIT2 are not capable of dealing with this regime since the experimental data did not cover this overlapping regime and the conditions for choosing the flow regime does not cover the mixture regime at all. MIT3 also have a validity problem due to the same reason but does not have a problem with obtaining a heat transfer coefficient value for this regime. In short, the relative influence of the buoyancy effect and acceleration effect on the turbulent flow are not fully covered yet. More experimental data and theoretical development should be followed to cover this regime. Some theories, such as one that was presented by Petukhov \& Polyakov (1988) covers both effect at the same time but authors agree that the theory for this regime is not fully capable of understanding regime.

3. Since there is a potential of having different governing physical phenomena depending on the geometrical conditions, applying a correlation to a problem, which has large discrepancy of geometrical features from the experimental facility, is not recommended (such as smaller or larger diameter, larger $L / D$ value etc.). This is because the gas turbulent heat transfer for the DTHT regime cannot be determined with the local parameter solely, which indicates that the channel geometry and the inlet conditions are very important. Therefore, when applying a developed correlation to a sample problem, the geometrical similarity and inlet condition similarity should be close to the actual experimental setup to have a high confidence in the calculation result.

It is obvious that to expand the correlation application limits, more data on these regimes with gases as working fluids is necessary.

Table 7-4 Summary of All the Experimental Runs

\begin{tabular}{|c|c|}
\hline Inlet Re Range & $1,800-42,700$ \\
\hline Inlet $q^{+}$Range & $0.0003-0.0039$ \\
\hline Inlet $B o^{*}$ Range & $3 \times 10^{-9}-1 \times 10^{-5}$ \\
\hline Inlet $K_{v}$ Range & $6 \times 10^{-8}-5 \times 10^{-6}$ \\
\hline Maximum $L / D$ & 116 \\
\hline Maximum $T_{w} / T_{b}$ & 1.88 \\
\hline Inner Diameter & $15.7 \mathrm{~mm}$ \\
\hline
\end{tabular}




\subsection{Smoothness of Developed Correlations}

In the last section, it was shown that MIT correlations satisfy the first three objectives for a 'good' correlation. The non-dimensional numbers that were chosen for the correlation reflected the physical phenomena, all the physical properties are evaluated at bulk temperature and the only necessary iteration required (for MIT1 only) is the original iteration for the properties variation term in the Gnielinski correlation. However, the last objective requires some discussion for testing the MIT1, MIT2 and MIT3 correlations, respectively.

The main problem with validating the last objective is that there is no general relationship between $K_{v}, B o^{*}, q^{+}$and $\mathrm{Gr}_{q}$. In other words, it is impossible to analytically differentiate the laminar correlation, turbulent correlation and the DTHT correlation with respect to the Reynolds number and match both slopes and the values at the each boundary to check the smoothness of the correlation, when the operating conditions are passing through the flow regime boundary.

However, the physical phenomena, which were observed through the experiment, showed that the reduction in the turbulent heat transfer coefficient when it is crossing the threshold is very abrupt phenomena. Figure 5-17 and 5-18 are presented here again as Figure 7-7 and Figure 7-8 to show the acute change in the Nusselt number when the threshold is crossed along the channel.

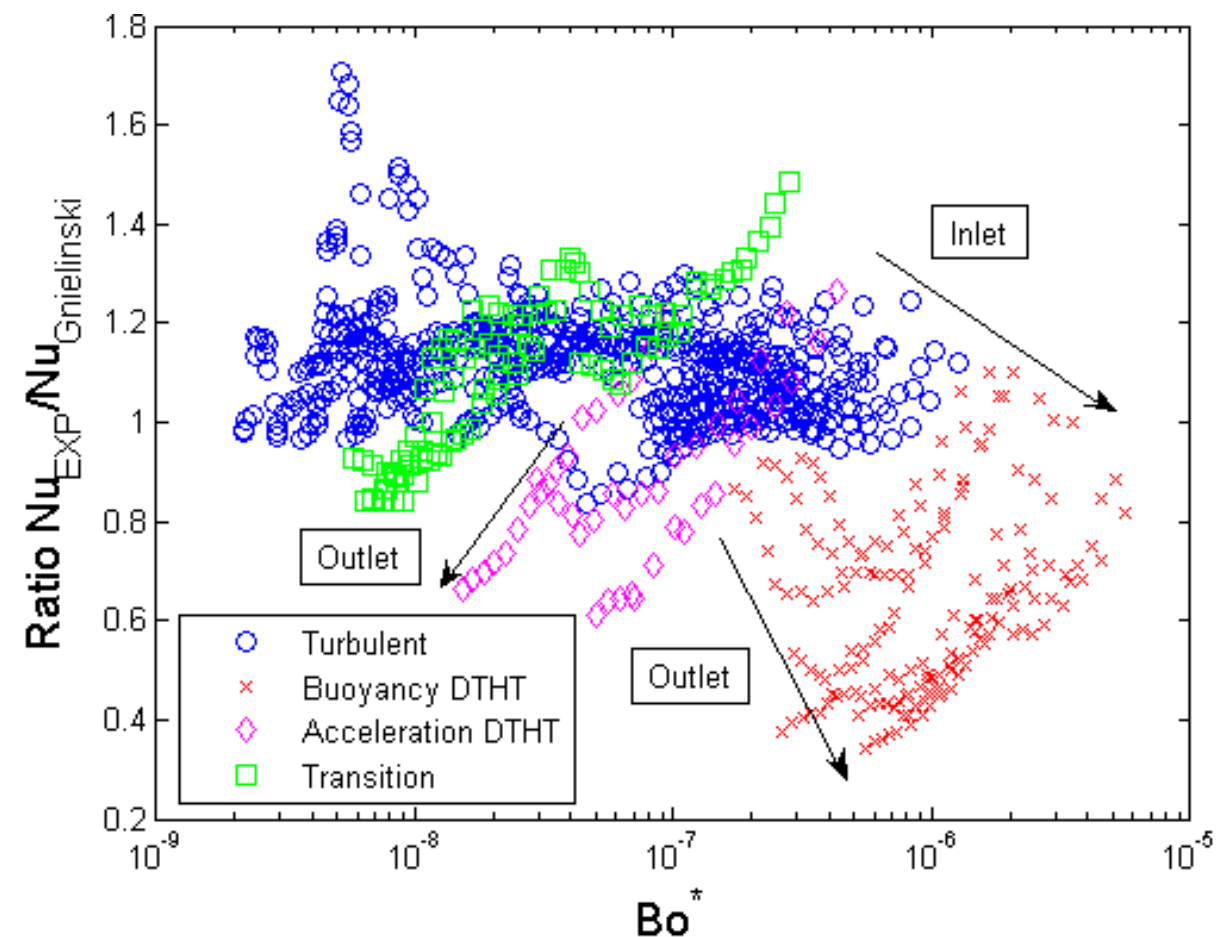

Figure 7-7 Nusselt Number Ratio vs. Buoyancy Parameter 


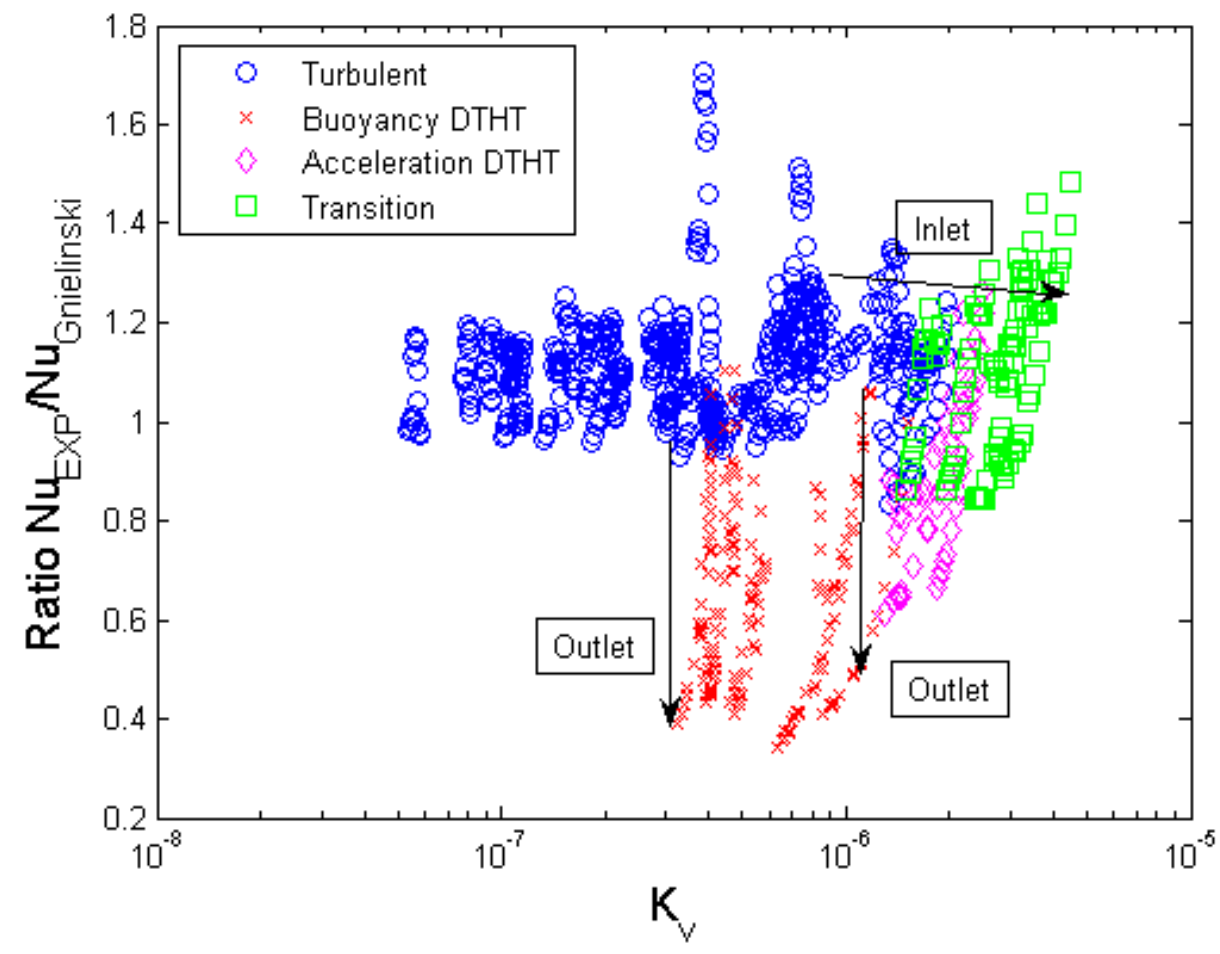

Figure 7-8 Nusselt Number Ratio vs. Acceleration Parameter

Both figures clearly show that when the operating condition is crossing the buoyancy induced DTHT boundary or the acceleration induced DTHT boundary the Nusselt number on the down stream changes steeply while the heat transfer reduction of the inlet is smooth. This indicates that the inlet Nusselt number variation with the flow regime should be smooth, but forcing the correlation to perform smoothly even at the downstream does not reflect the real physical world and it would fail to predict actual experimental data. Therefore, correlations will be checked for smoothness only in terms of inlet parameters.

Figures 7-9 through 7-11 show the calculated inlet Nusselt number of three MIT correlations for:

1. Three gases: nitrogen, helium and carbon dioxide

2. Heat flux from 2,000 to $30,000 \mathrm{~W} / \mathrm{m}^{2} \mathrm{~K}$

3. Pressure from 0.1 to $0.9 \mathrm{MPa}$

4. Volumetric flow rate from 0.2 to $3.0 \mathrm{CFM}$

5. Maximum Buoyancy Parameter: $3.36 \times 10^{-5}$

6. Maximum Acceleration Parameter: $5.95 \times 10^{-5}$

Figures show that all three correlations have a smooth inlet Nusselt number transition from the normal turbulent flow to the acceleration driven DTHT. MIT1 and MIT3 exhibit rapid transition from the mixed convection laminar to turbulent or mixed convection 
laminar to DTHT compared to the MIT2 correlation. Therefore, it can be concluded that MIT2 has the best performance in terms of the smoothness of correlation over the regime changes. However, the figures also show that the mixed convection laminar heat transfer regime and laminar to turbulent heat transfer regime requires more attention to understand the physical phenomenon of the transition thoroughly. At this point it is not clear if the sudden change of heat transfer coefficient is a real phenomenon. More transition data will have to be obtained to resolve this transition region and update the proposed correlations to predict the experimental Nusselt number in this region more accurately.

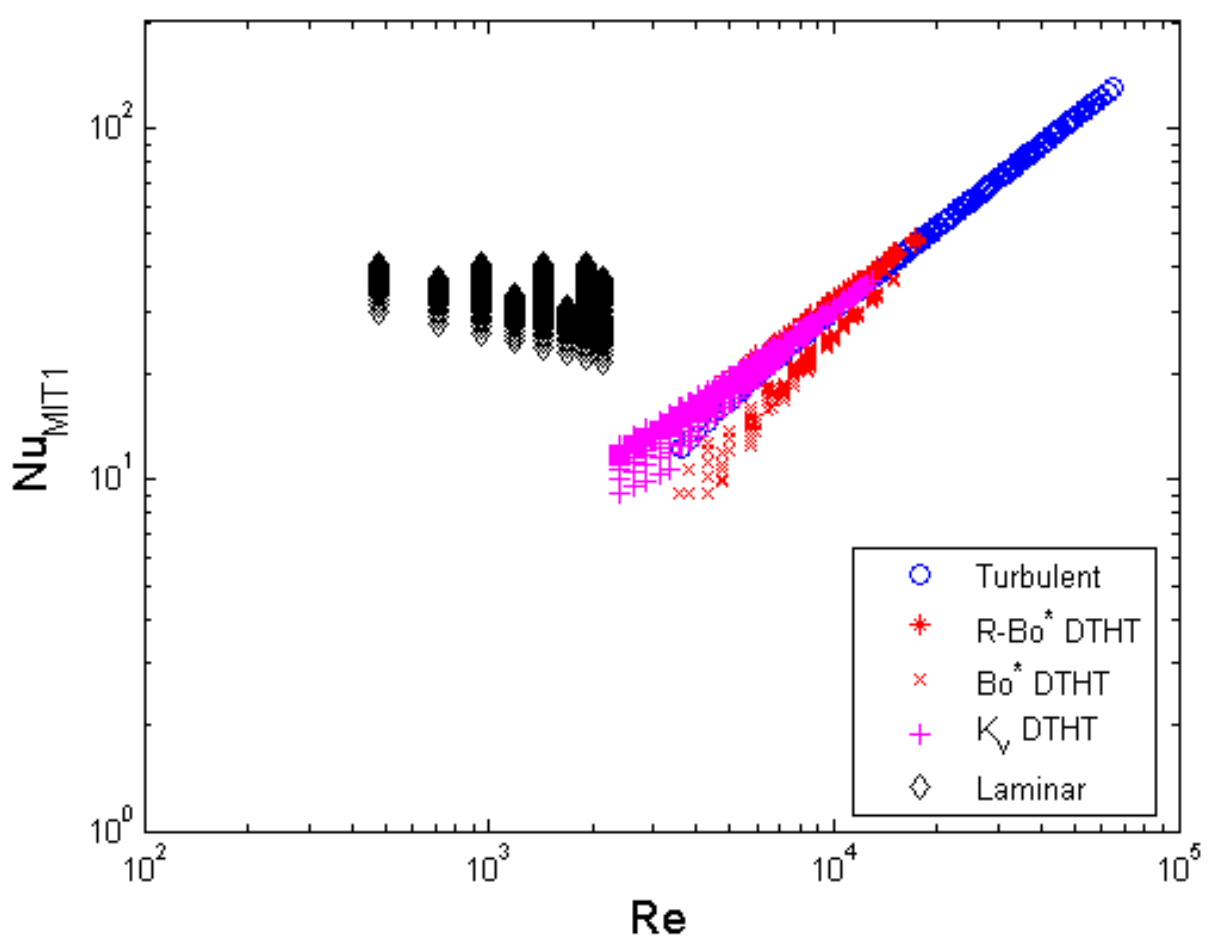

Figure 7-9 MIT1 Inlet Nusselt Number vs. Reynolds Number 


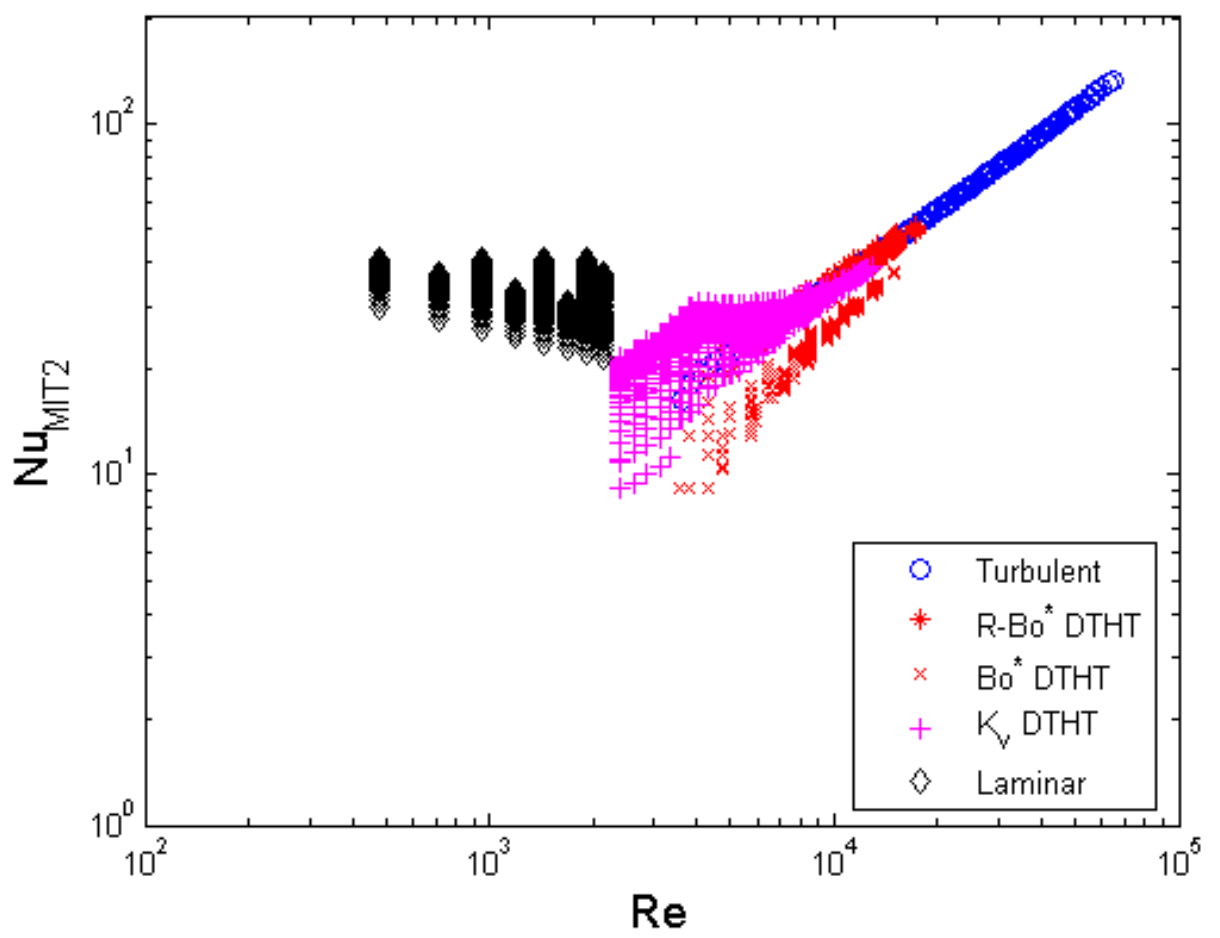

Figure 7-10 MIT2 Inlet Nusselt Number vs. Reynolds Number

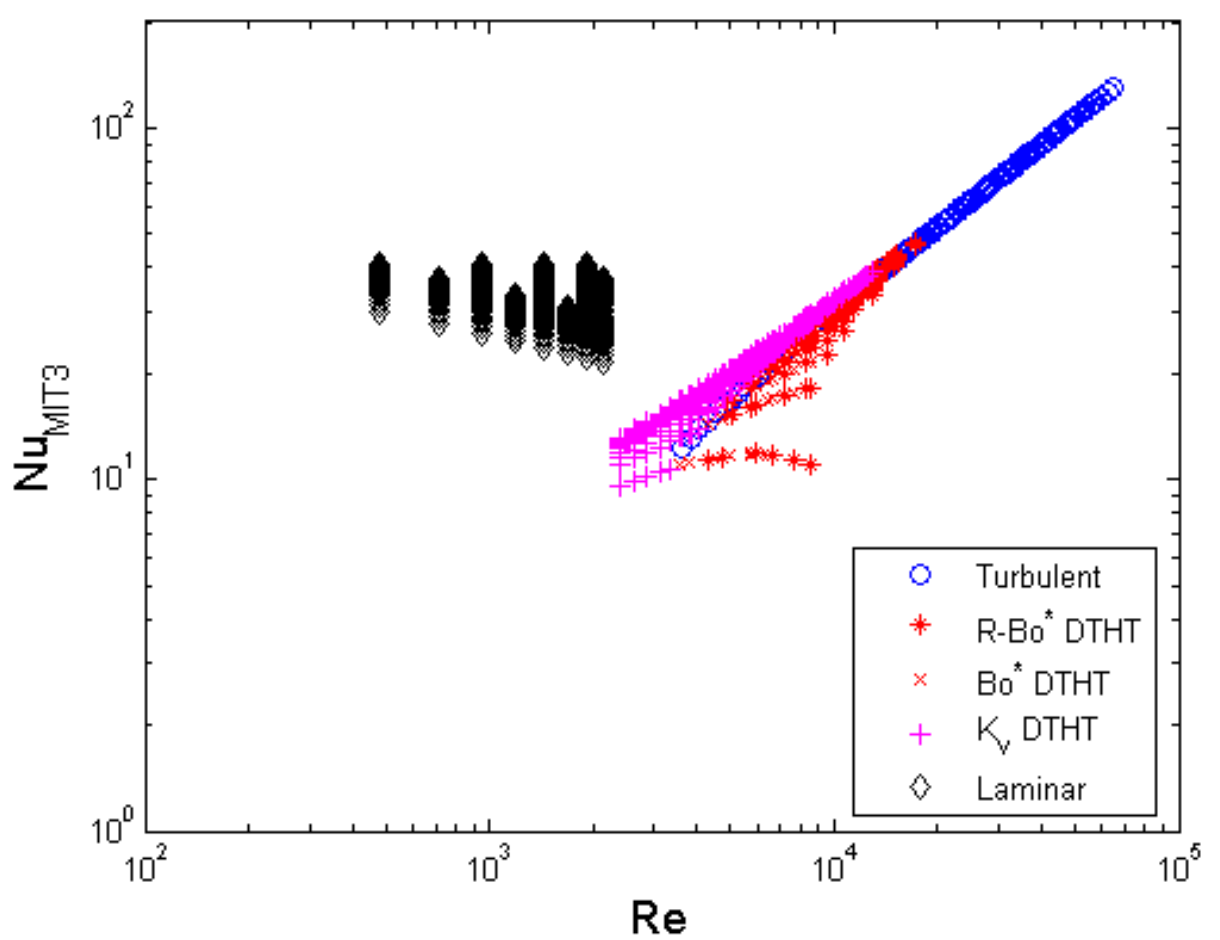

Figure 7-11 MIT3 Inlet Nusselt Number vs. Reynolds Number 


\section{Summary and Conclusions}

This chapter will summarize the main activities performed during the third year of the project and the results achieved, as documented throughout this report and main conclusions. To summarize:

1. The gas-cooled fast reactor (GFR) for Generation IV has generated considerable interest and is under development in the U.S., France, and Japan. One of the key candidates is a block-core configuration first proposed by MIT, has the potential to operate in Deteriorated Turbulent Heat Transfer (DTHT) regime or in the transition between the DTHT and normal forced or laminar convection regime during post-loss-of-coolant accident (LOCA) conditions. This is contrary to most industrial applications where operation is in a well-defined and well-known turbulent forced convection regime. As a result, important new need emerged to develop heat transfer correlations that make possible rigorous and accurate predictions of Decay Heat Removal (DHR) during post LOCA in these regimes.

2. Additional literature review was performed and documented in Chapter 2. It summarizes most of the available correlations in: (1) forced laminar, (2) forced turbulent, (3) mixed convection laminar, (4) buoyancy driven DTHT and (5) acceleration driven DTHT regimes. The physics behind each regime was explained and the gap of experimental work was identified also.

3. In Chapter 3, preliminary analysis on the GFR DHR system was performed and using the literature review results and GFR conditions. It confirmed that the GFR block type core has a potential to operate in the DTHT regime. Also, the difference in the performance in the DTHT regime between various working fluids was identified using a new approach. The newly proposed approach separates the non-dimensional number into a properties dependent group and an operator controlled group. This approach proved that gas, liquid and super critical phase fluids all behave differently in single channel under DTHT regime conditions. Therefore, the water and super critical fluids data cannot be directly applied to the gas heat transfer case due to this characteristic.

4. Description of upgraded parts in the experimental facility and of the control and data reduction software were presented in Chapter 4 along with the procedure, data reduction program and the thermal characteristics of the insulation material. Upgraded parts were successfully installed and a substantial amount of programming and controlling techniques were developed and incorporated into the control and data reduction software. A few problems with the measurement of the thermal resistance of the insulation material were identified and resolved with a reasonable effort.

5. Experimental data were presented in Chapter 5. Each gas data namely: (1) nitrogen, (2) helium and (3) carbon dioxide were presented and each gas covered different physical phenomena. All data basically covered the forced turbulent heat 
transfer regime, nitrogen data covered the acceleration driven DTHT and buoyancy driven DTHT, helium data covered the mixed convection laminar, acceleration driven DTHT and the laminar to turbulent transition regimes and carbon dioxide data covered the returbulizing buoyancy driven DTHT and nonreturbulizing buoyancy induced DTHT. The validity of the data was established using the heat balance and the uncertainty analysis. Based on experimental data, the traditional threshold for the DTHT regime was updated to account for phenomena observed in the facility and a new heat transfer regime map was proposed. Overall, it can be stated that substantial reduction of heat transfer coefficient was observed in DTHT regime, which will have significant impact on the core and DHR design of passive GFR. Figure 8-1 and 8-2 show the inlet conditions for all experimental data on a newly proposed heat transfer regime map. Figure 8-1 markers are different for different operating gases while Figure 8-2 markers distinguish between different flow regimes.

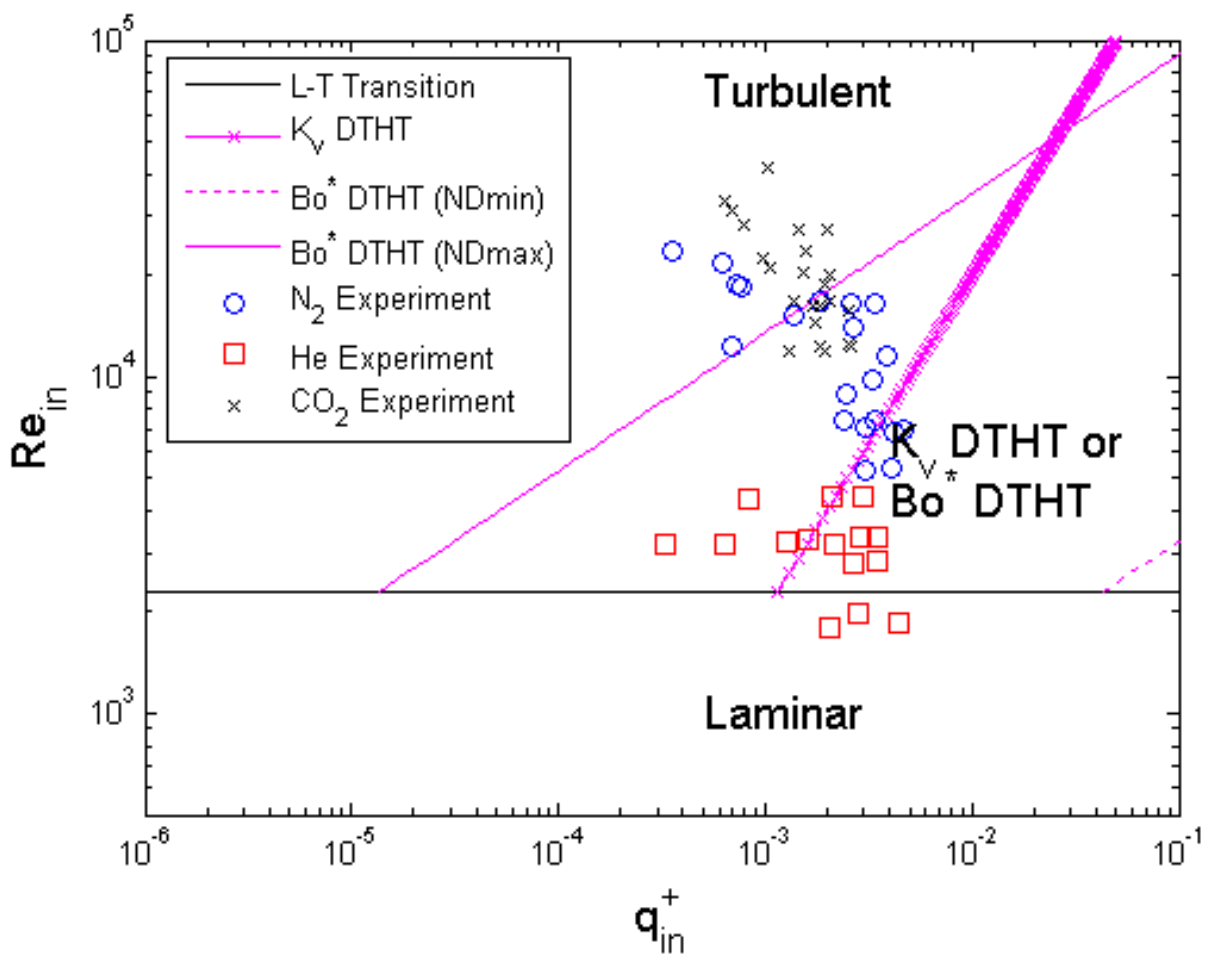

Figure 8-1 Inlet Experimental Conditions (Markers Depict Different Gases) 


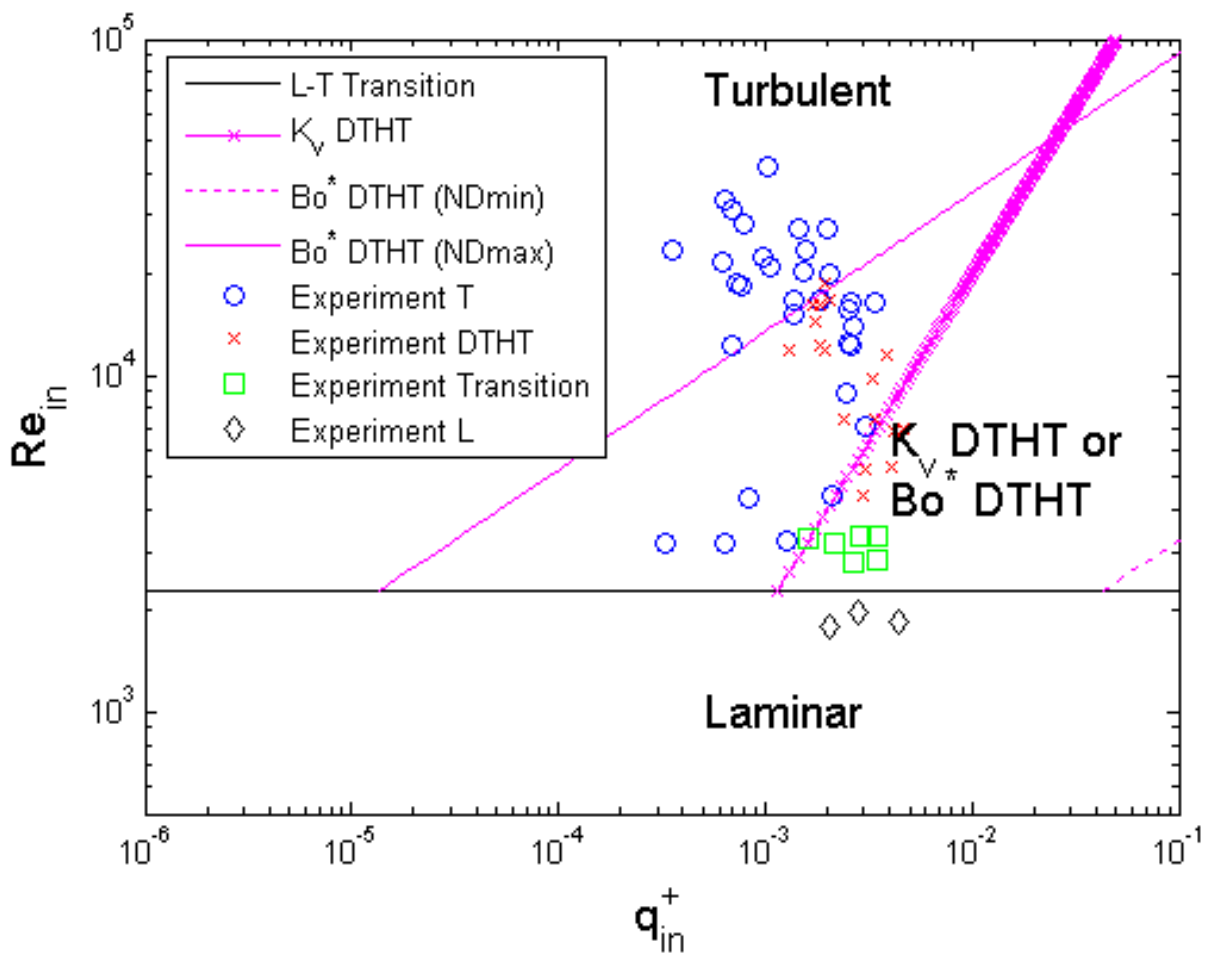

Figure 8-2 Inlet Experimental Conditions (Markers Depict Different Flow Regimes)

6. The MIT data were compared to the existing correlations in Chapter 6, which were presented in Chapter 2. None of the mixed convection laminar correlation agreed with the data and some correlations exhibited large discrepancies. For the forced turbulent and the DTHT regime correlation, Celeta et al. correlation showed the best fit with the data. However, due to larger $L / D$ ratio of MIT facility compared to Celeta et al. facility and the returbuliziation due to the gas characteristics, the correlation sometimes under predicts the heat transfer coefficient. Also, since Celeta et al. correlation requires the information of the wall temperature to evaluate the heat transfer coefficient, it is difficult to apply this correlation directly for predicting the wall temperature.

7. Three new sets of correlation that cover all heat transfer regimes were developed. The basic idea is to use the best available and most widely recommended Gnielinski correlation for forced turbulent convection and modify its empirically fitted constant (the Re-1000 part) by empirically fitted function that accounts for physical phenomena driving the DTHT regimes. Thus, the function is dependent on the acceleration parameter or the buoyancy parameter depending on the heat transfer regime. Type- 1 correlation set is the most accurate fit suitable for scientific applications, Type- 2 simplifies the calculations by eliminating iteration process on wall temperature and Type-3 is the simplest form suitable for industrial applications. The first two types show very good fit with the data, while the third type also exhibits slightly less accuracy, which was traded off for simplicity. Smoothness of the correlation when correlation is transitioning from 
one regime to another was evaluated for each correlation. The turbulent to DTHT regime transition is relatively smoother than the turbulent to the mixed convection laminar heat transfer regime. More data on the laminar-turbulent transition are required to understand the flow phenomena more thoroughly.

In conclusion, the Gas-cooled Fast Reactor Decay Heat Removal system have a potential to operate in (1) forced laminar, (2) forced turbulent, (3) mixed convection laminar, (4) buoyancy induced deteriorated turbulent heat transfer and (5) acceleration induced deteriorated turbulent heat transfer regimes during a transient. However, due to the lack of knowledge with gas heat transfer in (3) to (5) regimes, an experimental facility was built to uncover theses gaps and enhance understanding of gas heat transfer in these regimes. Obtained experimental data show in some cases substantial reduction in heat transfer capability of down to $30 \%$ of the forced turbulent convection values, which will impact core and decay heat removal system design of passive GFRs. The existing correlations were found to fit these new experimental data poorly and confirmed the need for better correlation. Thus, three new correlation sets were developed to cover (1) - (5) regimes and provide flexibility of choice to the user between in terms of accuracy versus simplicity of use and they all showed a good agreement with all the data and to have good characteristics of a desirable correlation. 


\section{Future Work}

This section will briefly discuss the potential of the MIT facility to explore the next interesting topics in the gas heat transfer area.

\section{Test Section Modification}

Unfortunately, the data that were presented in this report were only obtained from one test section diameter size due to the time limit. The larger or smaller size test section diameter will change the governing physical phenomena significantly. Figure 10-1 shows the effect of the test section size variation. This calculation is performed with: operating pressure of $1 \mathrm{~atm}$, nitrogen as a working fluid, inlet temperature at $300 \mathrm{~K}$, heat flux at $1 \mathrm{~kW} / \mathrm{m}^{2}$ and volumetric flow rate at 1.0 CFM. Y-axis shows the acceleration and Jackson's buoyancy parameters normalized to the threshold value of $2 \times 10^{-6}$ for both cases.

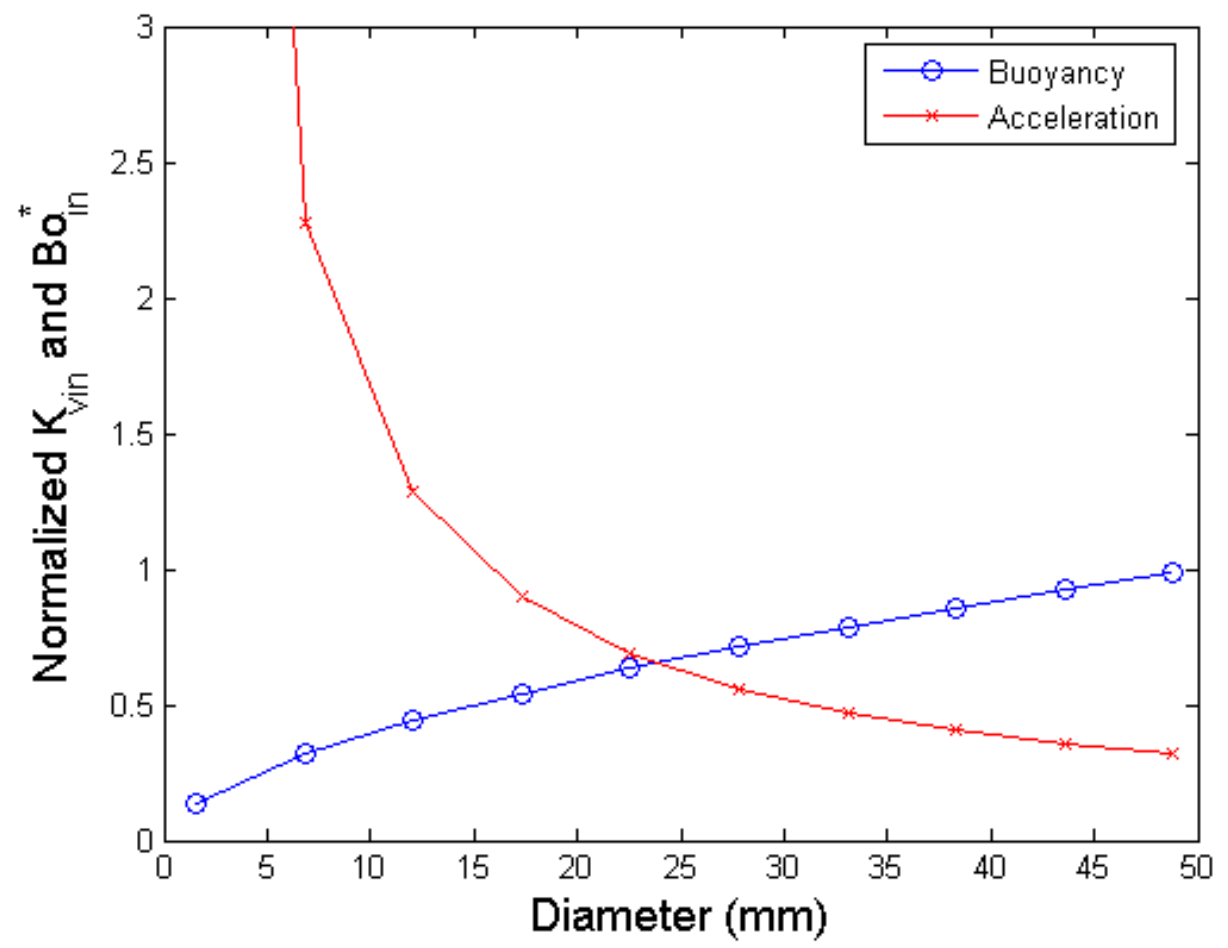

Figure 9-1 Governing Physical Phenomena Variation with the Test Section Diameter Size

Figure 9-1 clearly shows that when the test section size gets smaller the acceleration effect is pronounced and when the situation is opposite, the buoyancy effect is higher than the acceleration effect. The reason is obvious, since the acceleration parameter is inversely proportional to the diameter while the buoyancy parameter is proportional to the diameter. This figure also explains the reason why most of the acceleration effect experiments, which were summarized in $2^{\text {nd }}$ annual report, were all done with a small size 
test section $(\sim 3 \mathrm{~mm})$ while the buoyancy effect studies usually used large diameter test section $(\sim 10 \mathrm{~cm})$. Since, the current test section size is in between the two extremes, it will be interesting to reproduce two extreme cases in the MIT experiment by changing the test section diameter size. Changing the shape of test section will be also an interesting topic to investigate, especially in view of the fact that the GFR under development in France at CEA has currently plate type geometry.

\section{Different Heat Flux Shape and Flow Orientation}

Another proposed future work will be studying the effect of the applied heat flux shape. Since, the results from this report clearly show that the gas heat transfer depends more on the inlet condition than the local conditions, the heat flux shape will clearly change the single channel heat transfer characteristic. This is because the buoyancy parameter and the acceleration parameter will start to behave differently from the uniform heat flux cases.

The downward heated flow is another topic of interest since there are designs with heated down flow (for example GT-MHR and older GCFR). Generally it is accepted that the downward heated flow heat transfer coefficient is enhanced due to the buoyancy effect, but Figure 10-1 shows that when the test section is small enough the acceleration effect will prevail over the buoyancy effect. Since the acceleration is not affected by the orientation of the flow (no gravitational acceleration in the acceleration parameter) the deterioration due to acceleration can be also predicted. However, because buoyancy effect is now contributing to increased heat transfer, the interference between buoyancyenhanced heat transfer in the down flow and acceleration driven deterioration is not clear. Thus, a small size test section with the downward heating experiment can provide surprising results.

\section{Differential Pressure Measurement and Friction Factor Correlation Development}

From the Reynolds analogy, the friction factor and the heat transfer coefficient tend to vary together. There is a large uncertainty on the effects of buoyancy forces on friction factor, in particular in the DTHT regime. A modest data set and some publications that were identified suggest that friction factor can either be increased or decreased by buoyancy assisting mixed convection. Petukhov and Strigin [Petukhov and Strigin, 1968], [Petukhov, 1977] developed the correlation that predicts significant increase of friction factor with Rayleigh number. Other data were found to exhibit decrease in friction factor as buoyancy forces increase, followed by an increase with Grashof number or buoyancy force [Carr, et. al., 1973]. These correlations and data only pertain to the turbulent mixed convection regime, at the current time no data or correlations have been found that pertains to laminar mixed convection effects. Therefore, friction factor correlation that can reliably predict friction factor in buoyancy affected flows needs to be developed. This is particularly important for GFR decay heat removal applications where deterioration of friction factor can substantially impair decay heat removal under natural 
circulation and its impact is expected to be higher than that of heat transfer coefficient. .

Thus, measuring a friction factor for DTHT regime is key future work to be performed. This activity was originally planned in the initial stages of the proposal for this project, but was removed from the work scope because of funding limitations.

\section{Natural Circulation}

After implementing a newly developed correlation to a system analysis code, such as RELAP5-3D, an experimental validation will be necessary to check the code performance. Up to date, all of the data presented in this report were obtained using the forced circulation situation. Since the GDR DHR operates under natural circulation, it is of high interest to test natural circulation operation and benchmark RELAP5-3D code under natural circulation regime. The loop, which was built with natural circulation capability and prototypical heated channel dimensions provides good opportunity for such tests. Furthermore, it is of interest to investigate how the transition to DTHT regime affects natural circulation and validate if the loop operation maintains stable mode of operation. The current loop facility setup is a valuable source of information to the numerical analysis community.

\section{Hot-wire Measurement}

Due to the time constraint, the issues of hot-wire facility operation could not be resolved during the project. Few problems were identified with the current design in this report and this is mainly due to lack of experience of operating hotwire in the pressurized system. After fixing the identified problems and installing a few more upgrades to the facility, the hotwire flow transducer could be made fully operational. By having a reliable flow measurement with the hotwire, a lower flow rate than the low limit of turbine meter could be measured allowing the data sets to extend lower flow rates.

Since the DTHT regime and mixed convection laminar regime heat transfer characteristic changes due to the local flow structure, it will provide an insight for measuring the velocity profile, temperature profile and turbulent kinetic energy profile in the test section. The hotwire technology can be applied to measure these quantities after accumulating the operating knowledge with the flow transducer hotwire. 


\section{References}

Aicher T. and Martin H., "New Correlation for Mixed Turbulent Natural and Forced Convection Heat Transfer in Vertical Tubes", International Journal of Heat \& Mass Transfer, Vol. 40, No. 15, pp. 3617-3626, 1997

Arp V., Cryodata Inc., Private Communication, September 2006.

Bankston C. A., "The Transition from Turbulent to Laminar Gas Flow in a Heated Pipe", Journal of Heat Transfer, pp. 569-579, November 1970

Burmeister, L.C., "Convective Heat Transfer", $2^{\text {nd }}$ ed., John Wiley \& Sons, Inc., 1993

Carr A.D., Connor M.A., and Buhr H.O., "Velocity, Temperature and Turbulence Measurements in Air for Pipe Flow with Combined Free and Forced Convection," Journal of Heat Transfer, pp. 445-452, November 1973

Celata Gian Piero, D’Annibale Francesco, Chiaradia Andrea and Cumo Maurizio, "Upflow Turbulent Mixed Convection Heat Transfer in Vertical Pipes", International Journal of Heat \& Mass Transfer, Vol. 41, pp. 4037-4054, 1998

Churchill S. W., "Combined Free and Forced Convection in Channels", Heat Exchanger Design Handbook, Chap. 2.5.10, Begell House, Inc., 1998

Cochran P., Saha P., Hejzlar P., McEligot D. M., McCreery G. E. and Schultz R. R., "Scaling Analysis and Selection of Test Facility for Fundamental Thermal-Hydraulic Studies related to Advanced Gas-Cooled Reactor", INL/EXT-05-00158, Idaho National Laboratory and Massachusetts Institute of Technology, Department of Nuclear Science and Engineering, June 2004a

Cochran P., Lee J. I., Saha P., Hejzlar P., McEligot D. M., McCreery G. E. and Schultz R. R., "Design of Thermal-Hydraulic Loop Related to Advanced Gas-Cooled Reactor, First Annual Report", MIT-GFR-020, INL/EXT, Idaho National Laboratory and Massachusetts Institute of Technology, Department of Nuclear Science and Engineering, September $2004 b$

Garnier J.C., "Decay Heat Removal - Some Performance Evaluations," 2nd CEA-France /ANL-US Technical Workshop Meeting on I-NERI GFR Project, Argonne National Laboratory, Argonne, IL, March 31-April 3, 2003

Gnielinski V., "New Equations for Heat and Mass Transfer in Turbulent Pipe and Channel flow", International Chemical Engineering, Vol. 16, No. 2, pp. 359-387, April 1976

Hall W. B. and Jackson J. D., "Laminarization of a Turbulent Pipe Flow by Buoyancy Forces", ASME 69-HT-55, 1969 
Hallman T. M., "Combined Forced and Free-Laminar Heat Transfer in Vertical Tubes with Uniform Internal Heat Generation", Transaction of American Society of Mechanical Engineers, Vol. 78, pp. 1831-1841, 1955

Hallman T. M., "Experimental Study of Combined Forced and Free Laminar Convection in a Vertical Tube", NASA Technical Note, TN D-1104, December 1961

Hejzlar, P., "A modular, gas turbine fast reactor concept (MFGR-GT)", Transactions of the American Nuclear Society, Vol. 84, Milwaukee, Wisconsin, June 17-21, 2001

Hejzlar P., Driscoll M.J., and Todreas N.E., "The Long-Life Modular Gas Turbine Fast Reactor Concept," International Congress on Advanced Nuclear Power Plants, Hollywood, Florida, June 9-13, 2002

Jackson J. D., Cotton M. A. and Axcell B. P., "Studies of Mixed Convection in Vertical Tubes”, International Journal of Heat \& Fluid Flow, Vol. 10, pp. 2-15, March 1989

Kakaç Sadık, Shah Ramesh K. and Aung Win, "Handbook of Single-Phase Convective Heat Transfer”, Chp. 15, John Wiley \& Sons, 1987

Kaupas V. E., Poškas P. S. and Vilemas J. V., "Heat Transfer to a Transition-Range Gas Flow in a Pipe at High Heat Fluxes (2.Heat Transfer in Laminar to Turbulent Flow Transition)", 1989 Scripta Techinca, Inc. pp.340-351, 1989

Kays W. M., Crawford M. E., "Convective Heat and Mass Transfer", $3^{\text {rd }}$ edition, McGraw-Hill, Inc., 1993

Koppius A. M. and Trines G. R. M., "The dependence of hot-wire calibration on gas temperature at low Reynolds number", International Journal of Heat and Mass Transfer Vol. 19 pp.967-974, 1998

Lee J. I., "The Flow Structure under Mixed Convection in a Uniformly Heated Vertical Pipe", Master Thesis, Nuclear Science and Engineering Department, Massachusetts Institute of Technology, May 2005a

Lee J. I., P. Saha, P. Hejzlar and M. S. Kazimi, "Transitional Flow from Laminar to Turbulent Mixed Convection in a Heated Vertical Pipe", International Congress on Advances in Nuclear Power Plants ICAPP '05, Paper 5320, Seoul, Korea, May 15-19, $2005 b$

Lee J. I., Hejzlar P., Stahle P., Saha P., McEligot D. M., McCreery G. E. and Schultz R. R., "Design of Thermal-Hydraulic Loop Related to Advanced Gas-Cooled Reactor, Second Annual Report", MIT-GFR-033, INL/EXT, Idaho National Laboratory and Massachusetts Institute of Technology, Department of Nuclear Science and Engineering, September $2005 c$

Lemmon E.V., McLinden M.O., and Huber M.L., "REFPROP, Reference Fluid Thermodynamic and Transport Properties", NIST Standard Reference Database 23, 
Version 7.0, 2002.

McEligot D. M., "Effect of Large Temperature Gradients on Turbulent Flow of Gases in the Downstream Region of Tubes", Technical Report 247-5, Mechanical Engineering Department, Stanford University, March 1963

McEligot, D.M., Magee, P.M., and Leppert, G., "Effect of large temperature gradients on heat transfer: the downstream region", J. Heat Transfer, Vol. 87, Issue 1, pp. 67-76, 1965

McEligot D. M., Ormand L. W. and Perkins H. C., "Internal Low Reynolds Number Turbulent and Transitional Gas Flow with Heat Transfer", Journal of Heat Transfer, Vol. 88, pp. 239-245, 1966.

McEligot, D. M., "Internal gas flow heat transfer with slight property variation", Bulletin of Mechanical Engineering Education, Vol. 6, pp. 251-263, 1967

McEligot D. M. and Coon C. W. and Perkins H. C., "Relaminarization in Tubes", International Journal of Heat \& Mass Transfer: Shorter Communications, Vol.13, pp. 431433, 1969

McEligot D. M. and Jackson J. D., "Deterioration Criteria for Convective Heat Transfer in Gas Flow through Non-Circular Ducts", Nuclear Engineering and Design (Short Communication), Vol. 232, pp. 327-333, 2004

Metais B. and Eckert E. R. G., "Forced, Mixed and Free Convection Regimes", Journal of Heat Transfer, pp. 295-297, May 1964

Monin A. S. and Yaglom A. M., "Statistical Fluid Mechanics Vol. 1", MIT Press, 1979

Parlatan Yüksel, "Friction Factor and Nusselt Number Behavior in Trubulent Mixed Convection in Vertical Pipes", Ph. D Thesis, Nuclear Engineering Department, Massachusetts Institute of Technology, June 1993

Perkins H. C. and Worsøe-schmidt P. M., "Turbulent Heat and Momentum Transfer for Gases in a Circular Tube at Wall to Bulk Temperature Ratios to Seven", International Journal of Heat and Mass Transfer, Vol. 8, pp. 1011-1031, 1965

Petukhov B. S. and B. K. Strigin, "Experimental Investigation of Heat Transfer with Viscous-Inertial-Gravitational Flow of a Liquid in Vertical Tubes", High Temperatures, Vol. 6, pp.896-899, 1968

Petukhov B. S. Kurganov V. A. and Gladuntsov A. I., "Heat Transfer in Turbulent Pipe Flow of Gases with Variable Properties", Heat Transfer Soviet Research, Vol. 5, No. 4, pp. 109-116, July-August 1973

Petukhov, B.S., "Turbulent Flow and Heat Transfer in Pipes Under Considerable Effect of Thermogravitational Forces," from Heat Transfer and Turbulent Buoyant Convection, Vol. 2, edited by Spalding, D.B., and Afgan, N., Hemisphere Publishing Corp., 1977 
Petukhov B. S. and Polyakov A. F., "Heat Transfer in Turbulent Mixed Convection", Hemisphere Publishing Corporation, 1988

Polyakov A. F. and Shindin S. A., "Development of Turbulent Heat Transfer over the Length of Vertical Tubes in the Presence of Mixed Air Convection", International Journal of Heat \& Mass Transfer, Vol. 31, No. 5, pp. 987-992, 1988

Poškas P. S., Kaupas V. E. and Vilemas J. V., "Heat Transfer to a Transition-Range Gas Flow in a Pipe at High Heat Fluxes (3.Effect of Buoyancy on Local Heat Transfer in Forced Turbulent Flow)", 1989 Scripta Techinca, Inc. pp.352-361, 1989

Seigel, R., E. M. Sparrow, and T. M. Hallman, "Steady Laminar Heat Transfer in a Circular Tube with Prescribed Wall Heat Flux," Applied Scientific Research, A7, pp. 386-392, 1958

Symolon P., Neuhaus W. and Odell R., "Mixed Convection Heat Transfer Experiments in Smooth and Rouch Vertical Tubes", Transaction of American Nuclear Society, Vol. 92, pp. 387-390, June 2005

Tanaka Hiroaki, Maruyama Shigeo and Hatano Shunichi, "Combined Forced and Natural Convection Heat Transfer for Upward Flow in a Uniformly Heated, Vertical Pipe", International Journal of Heat \& Mass Transfer, Vol. 30, No. 1, pp. 165-174, 1987

Taylor M. F., "Experimental Local Heat-Trnasfer Data for Precooled Hydrogen and Helium at Surface Temperatures up to $5300^{\circ}$ R", NASA Technical Note TN D-2595, January 1965

Vilemas J., Ušpuras E. and Šimonis V., "Turbulent Momentum and Heat Transfer in Channel Gas Flow at High Heat Loads", Experimental Thermal and Fluid Science, Vol. 4, pp. 375-398, 1991

Vilemas J. V., Poškas P. S., and Kaupas V. E., "Local Heat Transfer in a Vertical GasCooled Tube with Turbulent Mixed Convection and Different Heat Fluxes", International Journal of Heat \& Mass Transfer, Vol. 35, No. 10, pp. 2421-2428, 1992

Williams W., Hejzlar P., Driscoll M. J., Lee W-J., and Saha P., "Analysis of a Convection Loop for GFR Post-LOCA Decay Heat Removal from a Block-Type Core", MIT-ANPTR-095, March 2003

Williams W. C., Hejzlar P. and Driscoll M. J., "Decay Heat Removal from GFR Core by Natural Convection", International Congress on Advances in Nuclear Power Plants ICAPP '04, Paper 4166, Pittsburgh, USA, June 13-17, 2004

Worsøe-schmidt P. M. and Leppert G., "Heat Transfer and Friction for Laminar Flow of Gas in a Circular Tube at High Heating Rate", International Journal of Heat \& Mass Transfer, Vol. 8, pp. 1281-1301, 1965

Worsøe-schmidt P. M., "Heat Transfer and Friction for Laminar Flow of Helium and 
Carbon Dioxide in a Circular Tube at High Heating Rate", International Journal of Heat \& Mass Transfer, Vol. 9, pp. 1291-1295, 1966 



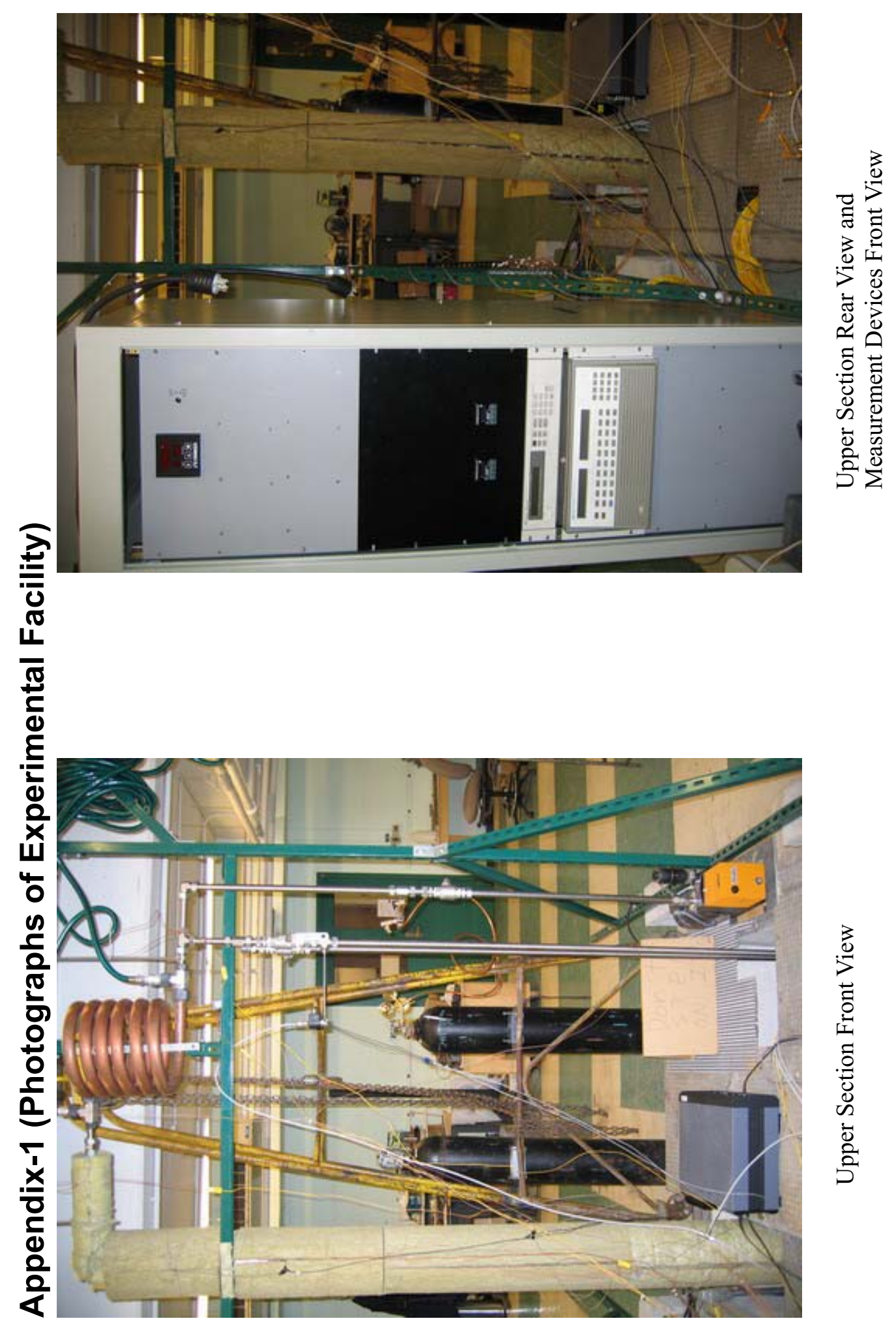




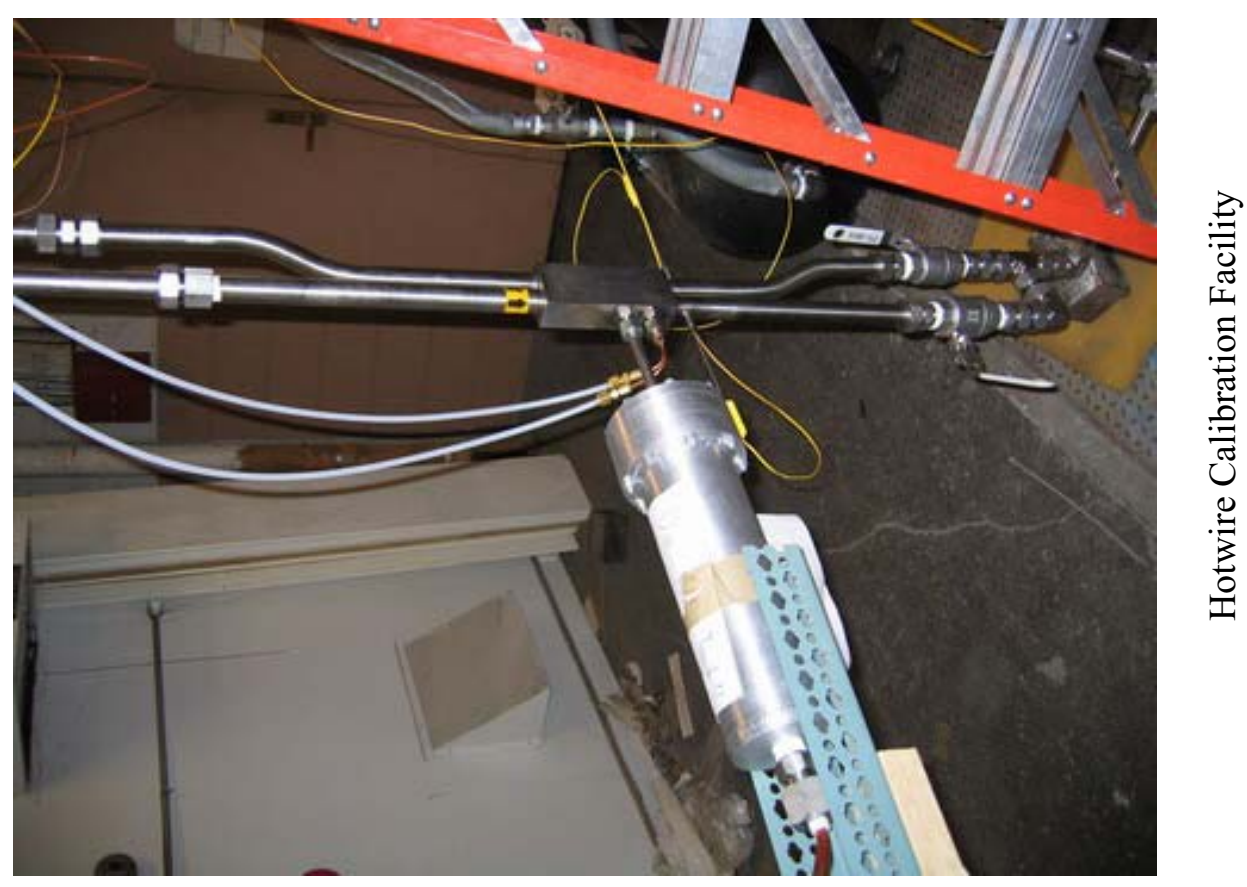

q

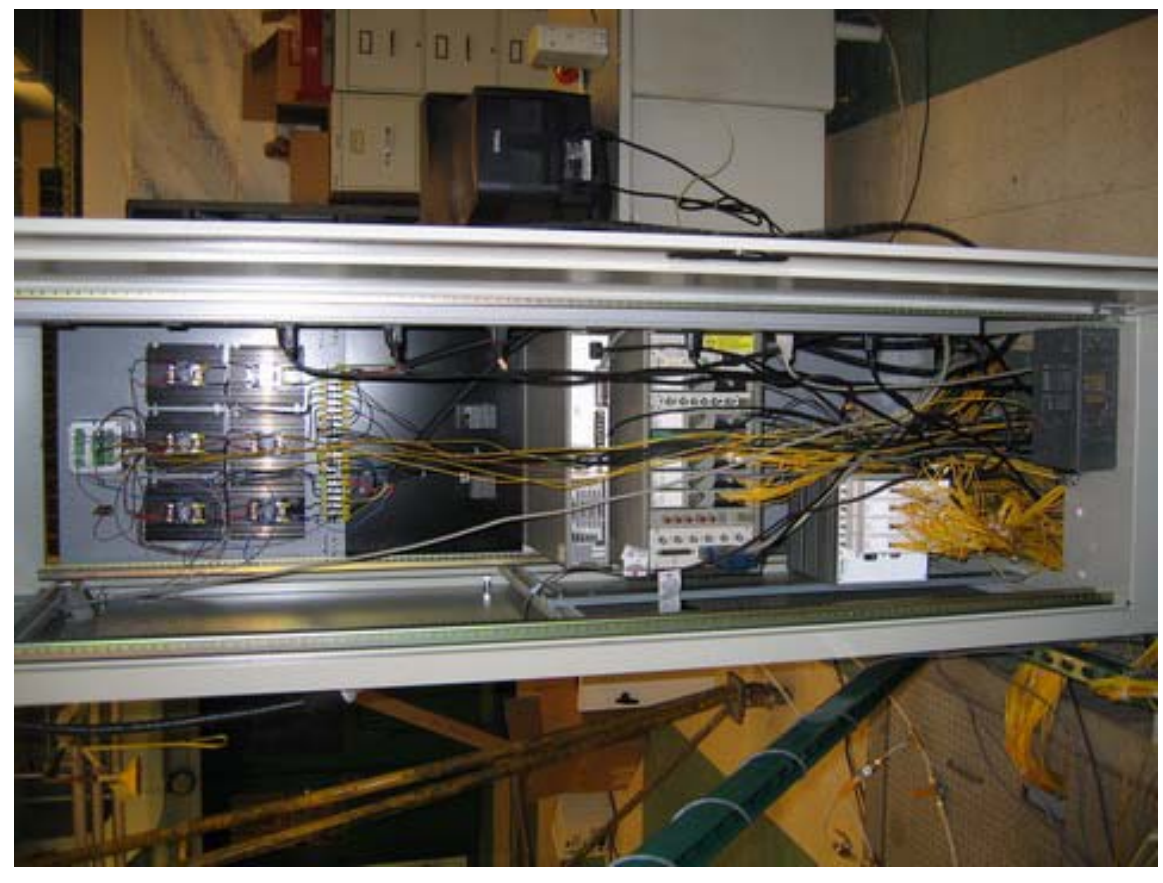

 


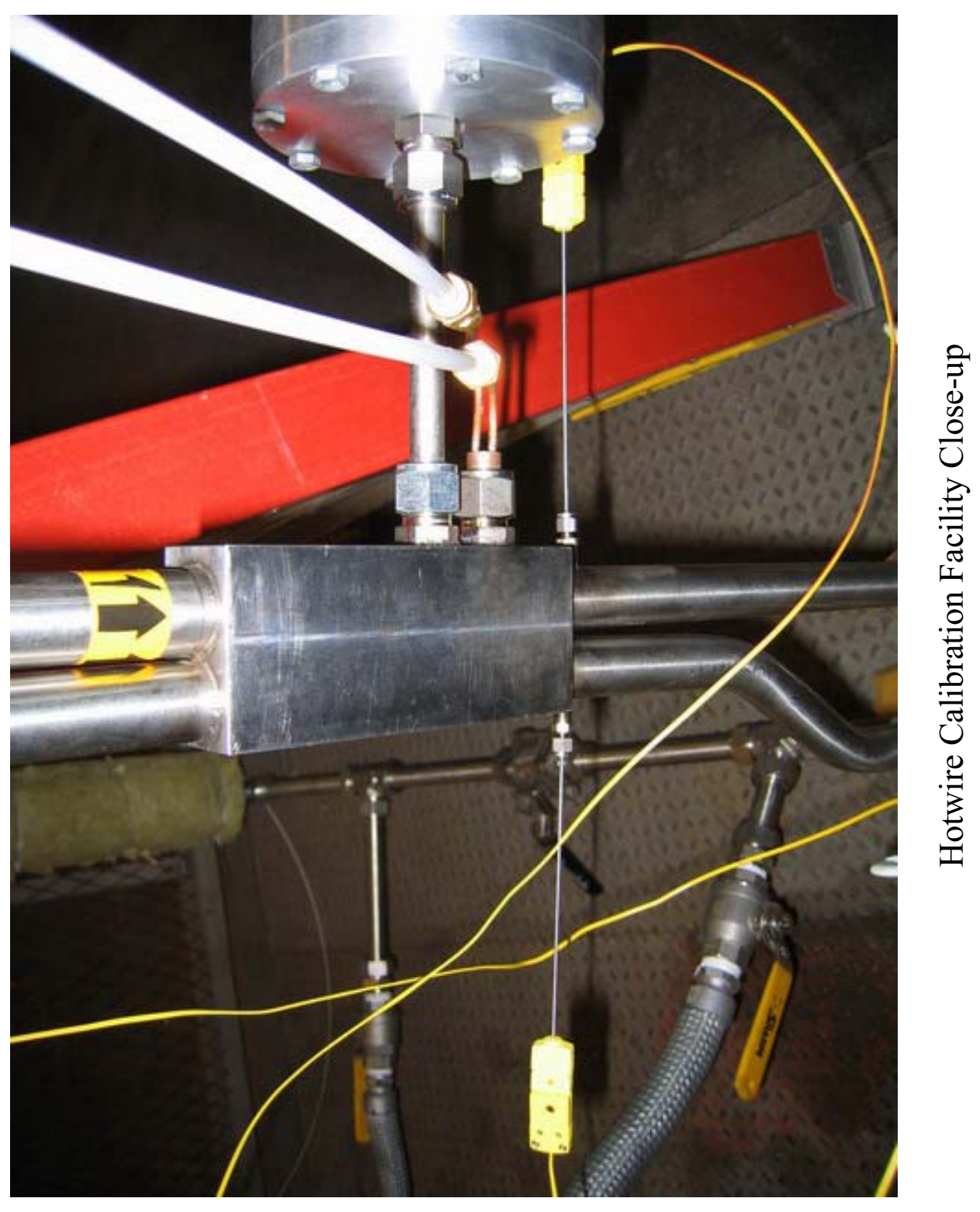

‡ 


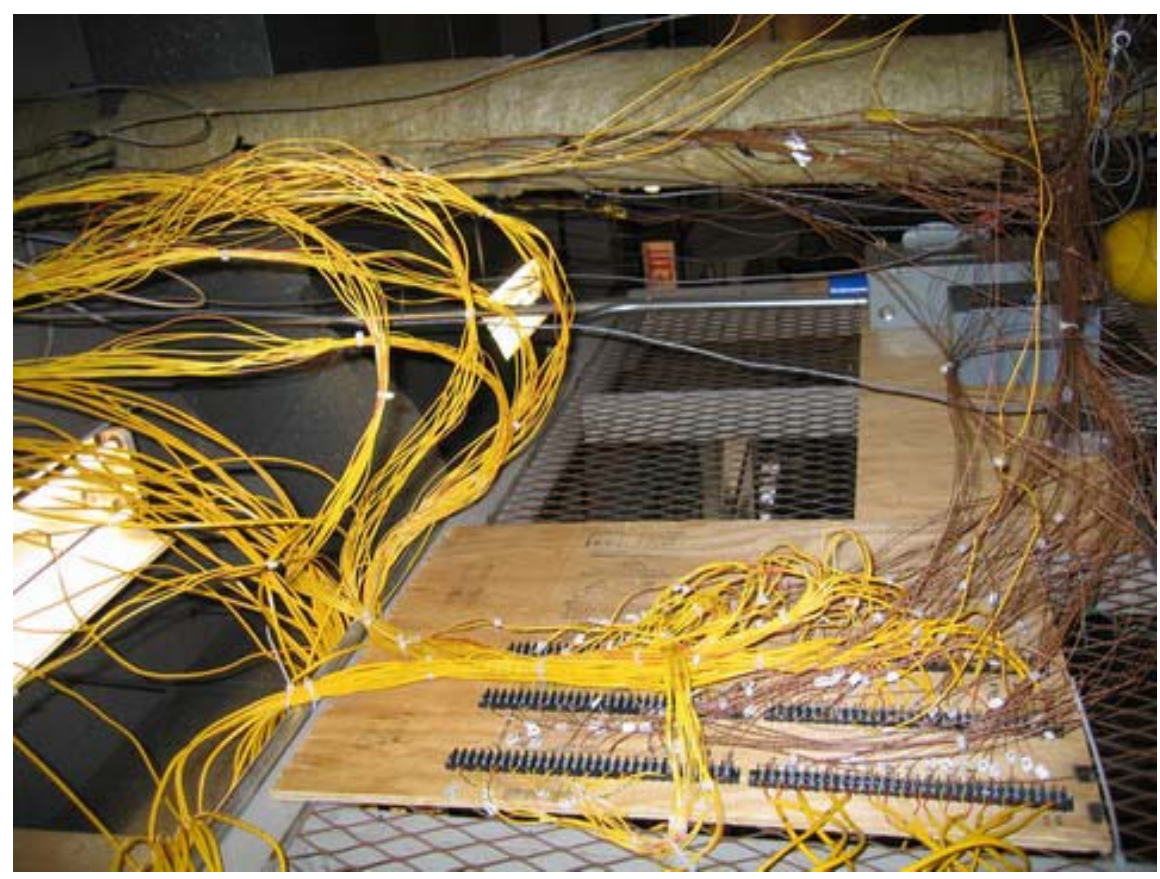

ט

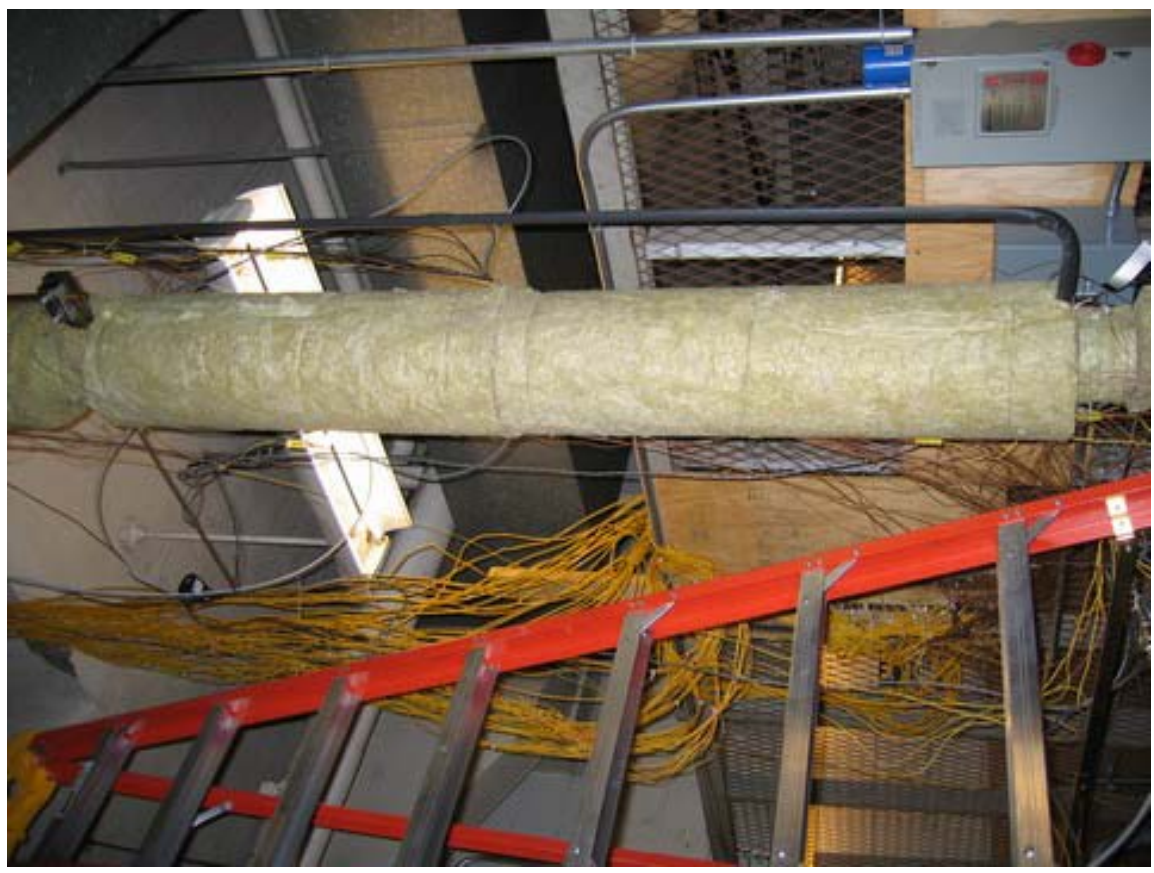

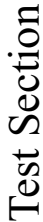




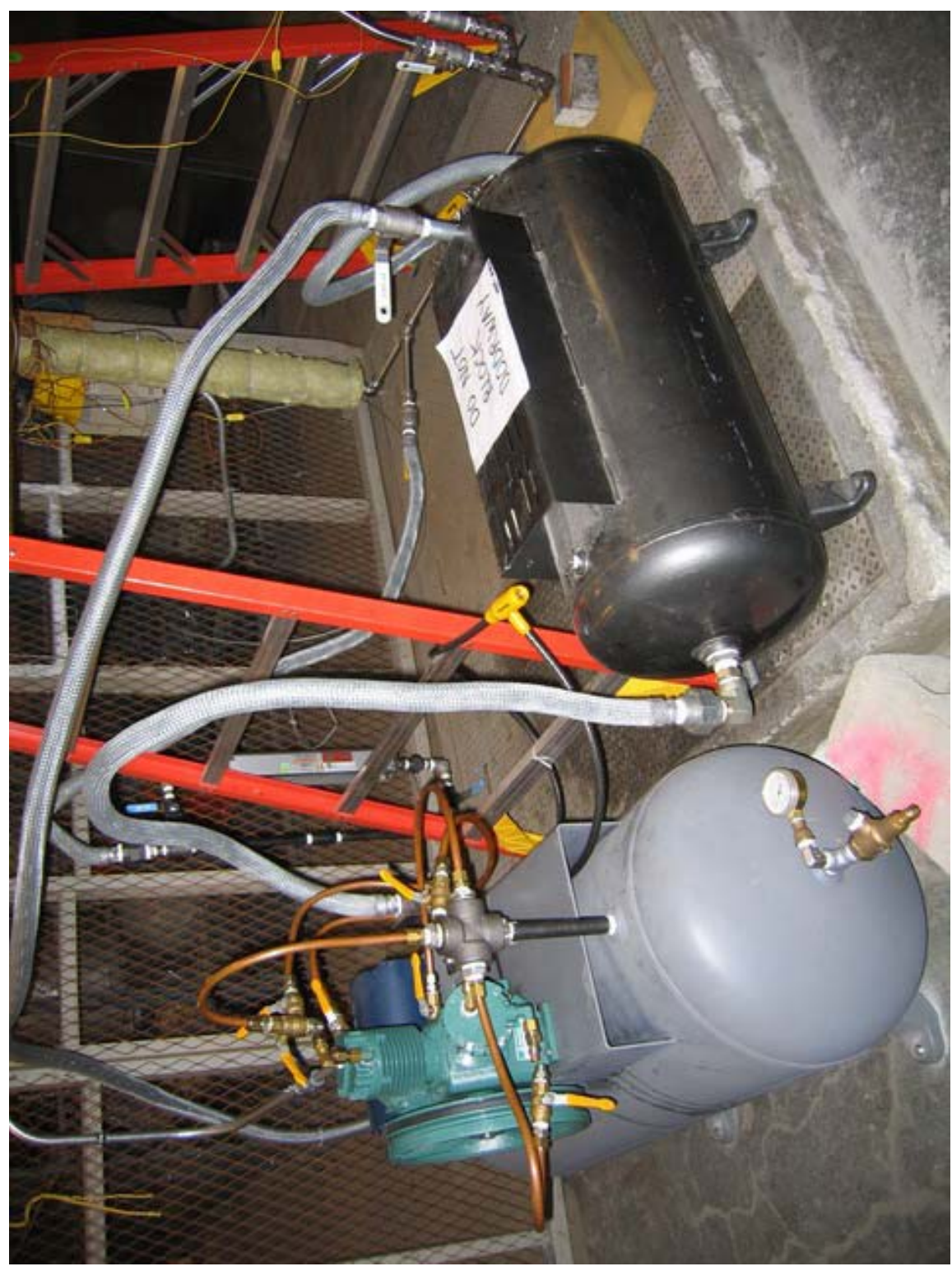

ह 


\section{Appendix-2 (Table of Data)}

This appendix documents data for all 58 runs in the form of tables. These data range is shown in Table A2-1.

Table A2-1. Summary of All the Experimental Runs

\begin{tabular}{|c|c|}
\hline Total number of Runs & $58\left(\mathrm{~N}_{2}, \mathrm{He}, \mathrm{CO}_{2}\right)$ \\
\hline Operating Pressure Range & $0.13-0.67 \mathrm{MPa}$ \\
\hline Operating Power Range & $200-2690$ Watts \\
\hline Operating Volumetric Flow Rate Range & $0.4-6.1 \mathrm{CFM}$ \\
\hline Inlet Temperature & $300-305 \mathrm{~K}$ \\
\hline Inlet Reynolds Number Range & $1,800-42,700$ \\
\hline Inlet $q^{+}$Range & $0.0003-0.0039$ \\
\hline Inlet $B o^{*}$ Range & $3 \times 10^{-9}-1 \times 10^{-5}$ \\
\hline Inlet $K_{v}$ Range & $6 \times 10^{-8}-5 \times 10^{-6}$ \\
\hline
\end{tabular}

The first two rows in the following tables summarize experimental conditions for each run. Next 21 rows show $\mathrm{L} / \mathrm{D}$, wall temperature, bulk temperature, heat flux, heat transfer coefficient, uncertainty on the heat transfer coefficient, the Nusselt number, uncertainty on the Nusselt number, the Reynolds number, non-dimensional heat flux (based on the enthalpy), the heat flux based Grashof number, the Prandtl number, buoyancy number and acceleration number from left to right column respectively. All the non-dimensional numbers are evaluated at the fluid bulk temperature including the inlet non-dimensional numbers. Temperature unit is Kelvin (K). Symbols used in the data tables are summarized on the next page. 
Appendix-2 Nomenclature

$c_{p}$ : specific heat at constant pressure $(\mathrm{J} / \mathrm{kg} K)$

$g$ : gravitational acceleration $\left(\mathrm{m}^{2} / \mathrm{sec}\right)$

$h$ : heat transfer coefficient $\left(\mathrm{W} / \mathrm{m}^{2} K\right)$

$k$ : thermal conductivity of gas $(\mathrm{W} / \mathrm{m} K)$

$q^{\prime \prime}:$ heat flux $\left(\mathrm{W} / \mathrm{m}^{2}\right)$

$D$ : pipe diameter $(\mathrm{m})$

$G$ : mass flux $\left(\mathrm{kg} / \mathrm{m}^{2} \mathrm{sec}\right)$

$H$ : enthalpy $(\mathrm{J} / \mathrm{kg})$

$L$ : length (m)

$P$ : system pressure $(\mathrm{MPa})$

$T_{W}$ : wall temperature $(K)$

$T_{b}$ : bulk temperature $(K)$

$\alpha:$ thermal diffusivity $=\frac{k}{\rho c_{p}}\left(\mathrm{~m}^{2} / \mathrm{sec}\right)$

$\beta$ : thermal expansion coefficient $=-\frac{1}{\rho}\left(\frac{\partial \rho}{\partial T}\right)_{P}\left(K^{-1}\right)$

$\mu$ : dynamic viscosity $(\mathrm{kg} / \mathrm{m} \mathrm{sec})$

$v:$ kinematic viscosity $\left(\mathrm{m}^{2} / \mathrm{sec}\right)$

$\rho$ : density $\left(\mathrm{kg} / \mathrm{m}^{3}\right)$

$\mathrm{Gr}_{\mathrm{q}}:$ Grashof number based on heat flux $=\frac{g \beta q_{w}^{\prime \prime} D^{4}}{k v^{2}}$

$\mathrm{Bo}^{*}$ : buoyancy parameter $=\frac{\mathrm{Gr}_{\mathrm{q}}}{\operatorname{Re}^{3.425} \operatorname{Pr}^{0.8}}$

$\mathrm{K}_{\mathrm{v}}$ : acceleration parameter $=\frac{v}{U_{b}^{2}} \frac{d U_{b}}{d x} \approx \frac{4 q^{+}}{\operatorname{Re}}$

$\mathrm{Nu}:$ Nusselt number $=\frac{h D}{k}$

Pr: Prandtl number $=\frac{v}{\alpha}$

Re: Reynolds number $=\frac{U D}{v}$

$q^{+}:$nondimensional heat flux $=\frac{q_{w}^{\prime \prime}}{G H_{b}}$ 


\begin{tabular}{|c|c|c|c|c|c|c|c|c|c|c|c|c|c|c|c|c|c|c|c|c|c|c|c|}
\hline & & $\vec{v}$ & | & $\begin{array}{l}8 \\
0 \\
1 \\
=1 \\
-1\end{array}$ & 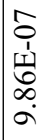 & 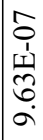 & 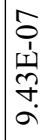 & 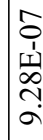 & 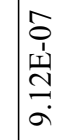 & $\mid \begin{array}{l}1 \\
1 \\
11 \\
0 \\
0 \\
0\end{array}$ & & 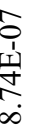 & 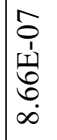 & 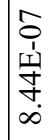 & 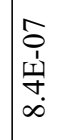 & 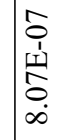 & 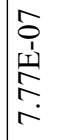 & 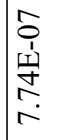 & $\begin{array}{l}\hat{a} \\
\hat{1} \\
\\
\mathfrak{n} \\
r\end{array}$ & 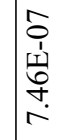 & 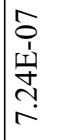 & 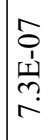 & 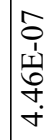 \\
\hline & & $\stackrel{\oplus}{\infty}$ & $\mid \begin{array}{c}\hat{a} \\
1 \\
1 \\
n \\
a \\
a\end{array}$ & $\begin{array}{l}\hat{o} \\
1 \\
1 \\
\\
\\
r\end{array}$ & 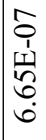 & $\mid \begin{array}{l}\hat{0} \\
1 \\
1 \\
0 \\
0 \\
i \\
i\end{array}$ & 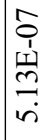 & 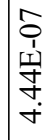 & 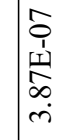 & 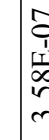 & & 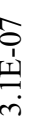 & 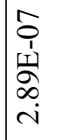 & 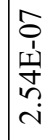 & 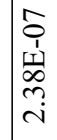 & 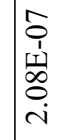 & 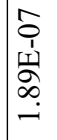 & 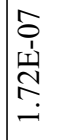 & 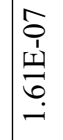 & 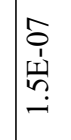 & 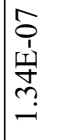 & $\mid \begin{array}{c}\hat{0} \\
1 \\
\\
\stackrel{1}{2} \\
\end{array}$ & $\begin{array}{c}\infty \\
0 \\
0 \\
1 \\
\tilde{n} \\
\tilde{n} \\
r\end{array}$ \\
\hline & & 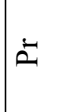 & $\begin{array}{l}\stackrel{0}{\mathbb{N}} \\
\underset{N}{0}\end{array}$ & $\underset{\stackrel{N}{N}}{\stackrel{0}{0}}$ & $\begin{array}{l}\stackrel{\mathbb{N}}{0} \\
\stackrel{0}{0}\end{array}$ & $\underset{\vec{\Omega}}{\stackrel{a}{\Omega}}$ & $\frac{\infty}{\mathbb{N}}$ & $\stackrel{\infty}{\stackrel{\infty}{\mathbb{d}}}$ & $\underset{\stackrel{\infty}{\mathbb{N}}}{\stackrel{\infty}{\sigma}}$ & $\frac{\infty}{a}$ & & $\frac{a}{\stackrel{N}{N}} \stackrel{0}{0}$ & त્ & 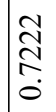 & 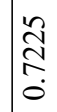 & 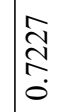 & तે & $\begin{array}{l}\stackrel{\sim}{\hat{N}} \\
\stackrel{0}{0}\end{array}$ & $\begin{array}{l}\tilde{n} \\
\stackrel{N}{N} \\
\dot{0}\end{array}$ & 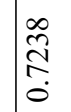 & 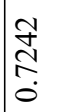 & 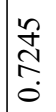 & 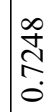 \\
\hline & & $\widetilde{J}$ & 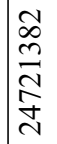 & $\begin{array}{l}1 \\
6 \\
0 \\
0 \\
0 \\
6 \\
6 \\
-1\end{array}$ & $\begin{array}{l}\infty \\
\infty \\
\infty \\
\infty \\
\cdots \\
m \\
\end{array}$ & $\begin{array}{l}\hat{y} \\
\infty \\
o \\
\hat{\delta} \\
\alpha\end{array}$ & $\begin{array}{l}\hat{2} \\
\hat{2} \\
\stackrel{2}{\infty} \\
\infty\end{array}$ & 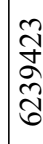 & 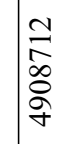 & $\begin{array}{l}8 \\
\frac{8}{7}\end{array}$ & & 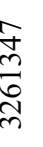 & $\begin{array}{l}3 \\
0 \\
0 \\
0 \\
\stackrel{0}{0} \\
\stackrel{\sim}{N}\end{array}$ & 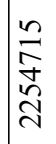 & 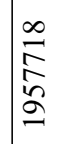 & 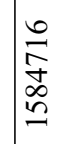 & $\begin{array}{l}\hat{\alpha} \\
\stackrel{\alpha}{\tilde{y}} \\
\underline{\underline{v}}\end{array}$ & 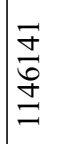 & 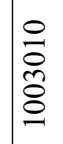 & $\begin{array}{l}\infty \\
\stackrel{\infty}{\sim} \\
\stackrel{\sim}{\infty} \\
\infty\end{array}$ & $\begin{array}{l}\stackrel{g}{\mathrm{~J}} \\
\text { İ } \\
\text { ㄱ }\end{array}$ & 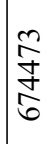 & 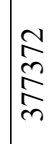 \\
\hline & & $t$ & 突 & 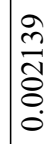 & 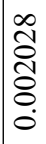 & $\frac{\frac{1}{2}}{\frac{2}{8}}$ & $\begin{array}{l}\bar{\infty} \\
\frac{\infty}{8} \\
0 \\
0\end{array}$ & $\begin{array}{l}\text { Nิ } \\
\frac{8}{8} \\
0\end{array}$ & $\begin{array}{l}\text { fo } \\
\stackrel{0}{8} \\
\stackrel{0}{\circ}\end{array}$ & 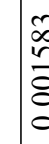 & & $\begin{array}{l}\frac{1}{\sigma} \\
\frac{d}{8} \\
\\
0\end{array}$ & 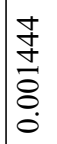 & 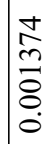 & $\begin{array}{l}0 \\
\text { ñ } \\
\stackrel{2}{8} \\
\dot{0} \\
0\end{array}$ & 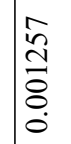 & 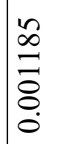 & $\frac{\bar{n}}{\stackrel{n}{*}}$ & $\underset{\Xi}{\Xi}$ & $\mid$\begin{tabular}{l}
0 \\
$\stackrel{0}{0}$ \\
\hdashline \\
0 \\
0
\end{tabular} & ְ̋ & $\frac{}{1}$ & 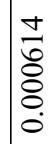 \\
\hline & & $\mathscr{\sim}$ & $\mid \begin{array}{l}\infty \\
\stackrel{2}{\infty} \\
\infty\end{array}$ & $\underset{\infty}{\stackrel{f}{\infty}}$ & \begin{tabular}{l}
$\sim$ \\
\multirow{\infty}{\infty}{} \\
$\infty$
\end{tabular} & $\hat{\curvearrowright}$ & શิ) & $\underset{\mathcal{Z}}{\mathcal{F}}$ & ָָ & 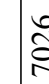 & & ‡ & $\sqrt{6}$ & $\frac{7}{6}$ & $\begin{array}{l}\text { J } \\
\text { ర్ర }\end{array}$ & त్రิ & $\frac{\sigma}{6}$ & $\begin{array}{l} \pm \\
\approx \\
\text { in }\end{array}$ & $\underset{\substack{\mathbb{N} \\
\infty \\
\infty}}{n}$ & $\mid \begin{array}{l}\infty \\
0 \\
i n\end{array}$ & 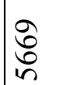 & $\begin{array}{l}0 \\
i n \\
i n\end{array}$ & 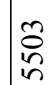 \\
\hline లే & $\tilde{z}$ & 兽 & $\underset{\dot{\sigma}}{\stackrel{\theta}{0}}$ & $\stackrel{\nabla}{-}$ & $\stackrel{n}{2}$ & aे & $\grave{o}$ & $\stackrel{\infty}{\infty}$ & $\hat{\sigma}$ & $\begin{array}{l}\infty \\
0 \\
c\end{array}$ & & ڤ̊ & ڤ̊. & જે & - & $\hat{a}$ & $\begin{array}{c}\infty \\
\infty \\
0\end{array}$ & $\mid \begin{array}{c}\partial \\
0 \\
0\end{array}$ & $\begin{array}{c}\infty \\
\infty \\
0\end{array}$ & $\mid \begin{array}{l}+ \\
\infty \\
0\end{array}$ & $\vec{\infty}$ & 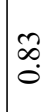 & $\stackrel{\leftrightarrow}{-}$ \\
\hline $\begin{array}{l}\frac{ \pm}{\sigma} \\
\frac{\vec{\omega}}{\Xi} \\
\Xi\end{array}$ & $\begin{array}{l}\tilde{\Xi} \\
\widetilde{\widetilde{\delta}} \\
0 \\
0\end{array}$ & $\vec{z}$ & $\begin{array}{l}0 \\
0 \\
\dot{0} \\
0\end{array}$ & $\begin{array}{l}\vec{N} \\
\sim \\
n\end{array}$ & $\begin{array}{l}\infty \\
\infty \\
\grave{\lambda}\end{array}$ & $\frac{\mathcal{I}}{\stackrel{\sim}{N}}$ & $\begin{array}{l}\hat{\gamma} \\
\hat{\sim}\end{array}$ & ì & $\begin{array}{l}\text { مे } \\
\text { ते }\end{array}$ & $\begin{array}{l}n \\
y \\
0 \\
0\end{array}$ & & $\begin{array}{l}n \\
a \\
a\end{array}$ & \begin{tabular}{l}
0 \\
\hdashline \\
\end{tabular} & $\begin{array}{l}\infty \\
\infty \\
\dot{\sim}\end{array}$ & $\begin{array}{l}\tilde{c} \\
\tilde{r}\end{array}$ & $\begin{array}{l}\vec{\lambda} \\
\vec{\lambda}\end{array}$ & $\begin{array}{l}\widetilde{\sigma} \\
\stackrel{\sim}{N}\end{array}$ & $\frac{i}{n}$ & $\frac{8}{\dot{\lambda}}$ & $\mid \begin{array}{l}\dot{0} \\
\stackrel{i}{1}\end{array}$ & $\begin{array}{l}\infty \\
\infty \\
\stackrel{\infty}{-}\end{array}$ & ले & त̂̃ \\
\hline $\begin{array}{l}\frac{\mathscr{a}}{\underline{0}} \\
\frac{\overrightarrow{0}}{\Xi}\end{array}$ & 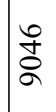 & 鴶 & $\vec{ָ}$ & $\stackrel{\bar{N}}{i}$ & $\stackrel{゚}{\stackrel{2}{ت}}$ & . & nn & 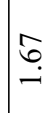 & $\stackrel{\ddagger}{\infty}$ & 8 & & $\hat{a}$ & 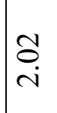 & $\stackrel{n}{i}$ & $\stackrel{\overbrace{}}{\sim}$ & $\stackrel{\widetilde{\lambda}}{\mathrm{i}}$ & $\stackrel{a}{a}$ & $\vec{i}$ & $\underset{i}{\stackrel{J}{i}}$ & $\underset{i}{\stackrel{t}{i}}$ & $\stackrel{\vec{a}}{i}$ & $\stackrel{i}{i}$ & $\underset{f}{\mathscr{f}}$ \\
\hline$\underset{\Xi}{\stackrel{\Xi}{\Xi}}$ & $\stackrel{\infty}{\stackrel{\Upsilon}{2}}$ & 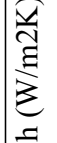 & $\stackrel{\partial}{\stackrel{\infty}{\infty}}$ & $\underset{\square}{ \pm}$ & $\begin{array}{l}\bar{\sigma} \\
\hat{n}\end{array}$ & 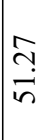 & $\frac{n}{n}$ & $\frac{\bar{n}}{n}$ & $\begin{array}{l}\text { m. } \\
\text { ñ. } \\
\text { nn }\end{array}$ & $\begin{array}{l}\vec{c} \\
\text { c } \\
\text { in }\end{array}$ & & $\begin{array}{l}\infty \\
\dot{\infty} \\
\dot{n}\end{array}$ & 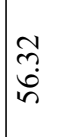 & in & $\begin{array}{l}2 \\
\infty \\
\infty \\
\infty\end{array}$ & $\underset{n}{\stackrel{\infty}{n}}$ & $\begin{array}{l}\stackrel{+}{n} \\
\tilde{n}\end{array}$ & $\begin{array}{l}\stackrel{+}{N} \\
\stackrel{\sim}{n}\end{array}$ & $\begin{array}{l}8 \\
\text {. } \\
\text { in }\end{array}$ & $\begin{array}{l}\tilde{N} \\
\text { in }\end{array}$ & $\vec{n}$ & $\begin{array}{l}q \\
i \\
i n\end{array}$ & F্ট \\
\hline$\sum_{\text {至 }}$ & $\exists$ & $\begin{array}{l}\text { है } \\
\vdots \\
=\end{array}$ & $\begin{array}{l}\frac{1}{2} \\
\stackrel{2}{\infty} \\
\stackrel{2}{2}\end{array}$ & $\begin{array}{l}\hat{\infty} \\
\stackrel{2}{0} \\
\stackrel{0}{2}\end{array}$ & $\begin{array}{l}\overline{0} \\
\text { ते } \\
\text { ते }\end{array}$ & i. & $\stackrel{0}{\vec{J}}$ & 年 & 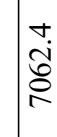 & $\begin{array}{l}0 \\
0 \\
2 \\
2 \\
1 \\
1\end{array}$ & & $\begin{array}{l}\underset{j}{i} \\
\text { d }\end{array}$ & 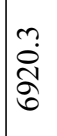 & 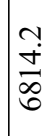 & 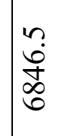 & $\mid$\begin{tabular}{l}
$\hat{n}$ \\
\multirow{8}{b}{} \\
0
\end{tabular} & $\begin{array}{l}\infty \\
\tilde{n} \\
\tilde{f} \\
\tilde{\sigma}\end{array}$ & 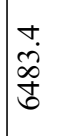 & $\begin{array}{l}n \\
\infty \\
\\
\end{array}$ & $\begin{array}{l}n \\
\tilde{b} \\
\delta \\
\delta\end{array}$ & 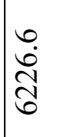 & 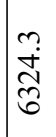 & $\underset{\partial}{\alpha}$ \\
\hline 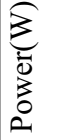 & $\stackrel{2}{n}$ & 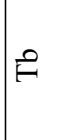 & $\begin{array}{l}\vec{m} \\
\vec{d} \\
\vec{m}\end{array}$ & 文 & $\begin{array}{l}\stackrel{8}{0} \\
\dot{\infty} \\
m \\
m\end{array}$ & $\begin{array}{l}\text { f } \\
\text { ind } \\
\text { m }\end{array}$ & î̉ & $\mid \begin{array}{l}0 \\
\dot{0} \\
\infty \\
m\end{array}$ & $\begin{array}{l}8 \\
\stackrel{8}{0} \\
\dot{j} \\
+\end{array}$ & $\begin{array}{l}i n \\
\infty \\
z\end{array}$ & & 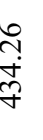 & $\begin{array}{l}\infty \\
\dot{q} \\
\dot{q}\end{array}$ & 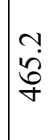 & 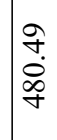 & $\begin{array}{l}n \\
n \\
n \\
a \\
g\end{array}$ & $\frac{9}{0}$ & 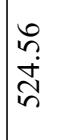 & $\begin{array}{l}\infty \\
\infty \\
\infty \\
\infty \\
\infty \\
n\end{array}$ & $\begin{array}{l}\tilde{\sigma} \\
\tilde{n} \\
\tilde{n} \\
\tilde{n}\end{array}$ & 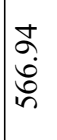 & $\begin{array}{l}\hat{2} \\
\dot{0} \\
\infty \\
i n\end{array}$ & ๙ิ \\
\hline$\sum_{0}^{\pi}$ & $\stackrel{m}{0}$ & 3 & 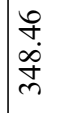 & 三 & 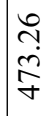 & $\stackrel{\mathfrak{q}}{q}$ & $\begin{array}{l}n \\
\hat{m} \\
i n\end{array}$ & $\begin{array}{l}\stackrel{8}{0} \\
\stackrel{ \pm}{\hbar}\end{array}$ & $\tilde{n}$ & $\begin{array}{l}\vec{J} \\
\overrightarrow{7}\end{array}$ & 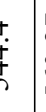 & 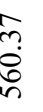 & $\begin{array}{l}\infty \\
0 \\
i \\
i \\
i n\end{array}$ & $\begin{array}{l}\stackrel{+}{I} \\
\dot{0} \\
i \\
i n\end{array}$ & \begin{tabular}{l}
$n$ \\
\multirow{2}{0}{} \\
2
\end{tabular} & $\frac{2}{\vec{E}}$ & $\frac{\infty}{\infty}$ & $\begin{array}{l}\infty \\
0 \\
\dot{j} \\
\dot{J}\end{array}$ & $\begin{array}{l}n \\
\tilde{n} \\
\tilde{n}\end{array}$ & 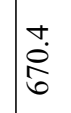 & $\begin{array}{l}9 \\
\dot{d} \\
\infty \\
\infty\end{array}$ & 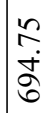 & $\begin{array}{l}\infty \\
\tilde{n} \\
\tilde{n} \\
\hat{b}\end{array}$ \\
\hline $\begin{array}{l}\mathscr{y} \\
\tilde{z} \\
\tilde{z}\end{array}$ & - & $\stackrel{9}{9}$ & $\sim$ & $\sigma_{\infty}$ & $\exists$ & ָั. & $\stackrel{\sim}{\sim}$ & 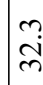 & $\underset{m}{\infty}$ & $\vec{\nabla}$ & & $\begin{array}{l}\text { t. } \\
\dot{8}\end{array}$ & $\begin{array}{l}n \\
\varnothing \\
i\end{array}$ & $\begin{array}{l}n \\
\text { in }\end{array}$ & $\mid \begin{array}{l}n \\
\infty \\
0\end{array}$ & $\begin{array}{l}\stackrel{\circ}{I} \\
\stackrel{+}{I}\end{array}$ & $\begin{array}{l}0 \\
\dot{\infty}\end{array}$ & $\hat{\infty}$ & $\hat{i}$ & $\mid \begin{array}{l}\infty \\
\infty \\
\alpha\end{array}$ & $\begin{array}{l}\infty \\
\dot{0} \\
\stackrel{0}{0}\end{array}$ & 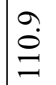 & \\
\hline
\end{tabular}




\begin{tabular}{|c|c|c|c|c|c|c|c|c|c|c|c|c|c|c|c|c|c|c|c|c|c|c|c|c|}
\hline & & $\vec{\Delta}$ & 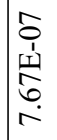 & 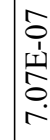 & $\begin{array}{l}\frac{1}{1} \\
\frac{1}{5} \\
\frac{1}{5}\end{array}$ & & 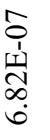 & 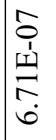 & $\begin{array}{l}\hat{0} \\
\hat{1} \\
1 \\
\hat{n} \\
\hat{n} \\
b\end{array}$ & $\begin{array}{l}\hat{0} \\
\hat{1} \\
1 \\
\infty \\
\infty \\
0 \\
0\end{array}$ & 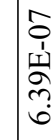 & 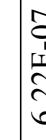 & & $\begin{array}{l}5 \\
0 \\
1 \\
11 \\
\\
0 \\
0 \\
0\end{array}$ & 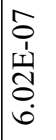 & 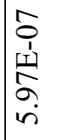 & 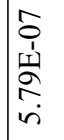 & 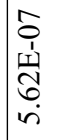 & 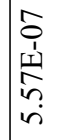 & \begin{tabular}{l}
$\hat{O}$ \\
$\hat{1}$ \\
\\
\multirow{+}{*}{} \\
$\dot{n}$
\end{tabular} & $\begin{array}{c}\hat{y} \\
\mathbf{1} \\
\underline{1} \\
\infty \\
\tilde{n} \\
i\end{array}$ & 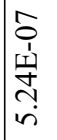 & 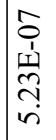 & 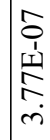 \\
\hline & & $\stackrel{\oplus}{\infty}$ & 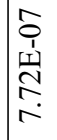 & 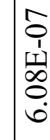 & $\begin{array}{l}\frac{a}{1} \\
\frac{1}{5} \\
\text { na }\end{array}$ & & 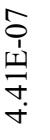 & $\begin{array}{l}\hat{o} \\
\dot{1} \\
\dot{1} \\
\vdots \\
\stackrel{+}{+}\end{array}$ & \begin{tabular}{l}
$\hat{O}$ \\
$\hat{1}$ \\
\\
\multirow{+}{\gamma}{} \\
$\dot{n}$
\end{tabular} & $\begin{array}{l}\hat{\sigma} \\
\hat{1} \\
\underline{1} \\
\hat{o} \\
\dot{r}\end{array}$ & 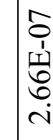 & $\begin{array}{l}0 \\
0 \\
y \\
y \\
y \\
y \\
a\end{array}$ & & 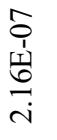 & 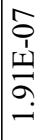 & 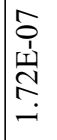 & 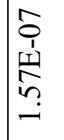 & 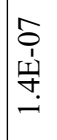 & 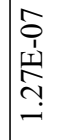 & \begin{tabular}{l}
$\hat{0}$ \\
1 \\
$\underline{1}$ \\
$\infty$ \\
\hdashline \\
\end{tabular} & 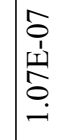 & $\begin{array}{l}\infty \\
0 \\
0 \\
1 \\
\vdots \\
0 \\
0\end{array}$ & 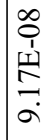 & 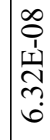 \\
\hline & & 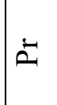 & $\begin{array}{l}\stackrel{\curvearrowright}{N} \\
\stackrel{0}{0}\end{array}$ & 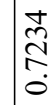 & ڤ̃ & & 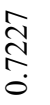 & $\begin{array}{l}\stackrel{2}{N} \\
\stackrel{N}{0} \\
\dot{0}\end{array}$ & 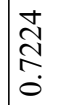 & $\underset{\mathbb{Z}}{\stackrel{J}{0}}$ & 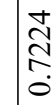 & ڤ̊ & & 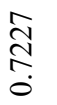 & 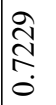 & $\underset{\stackrel{N}{N}}{\stackrel{N}{0}}$ & 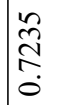 & $\begin{array}{l}\infty \\
\stackrel{N}{N} \\
\stackrel{0}{0}\end{array}$ & 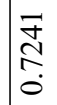 & 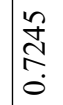 & 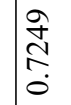 & 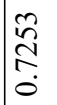 & 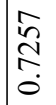 & $\begin{array}{l}\overrightarrow{0} \\
\stackrel{0}{N} \\
0\end{array}$ \\
\hline & & $\sigma$ & $\frac{\mathfrak{z}}{2}$ & 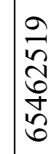 & $\frac{\tilde{\sigma}}{\tilde{z}}$ & & 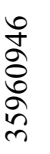 & $\begin{array}{l}\tilde{\hat{N}} \\
\tilde{n} \\
\infty \\
\infty \\
\infty \\
\sim\end{array}$ & 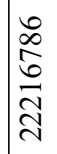 & 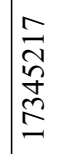 & $\underset{\infty}{\infty}$ & $\begin{array}{l}z \\
\partial \\
\alpha \\
0 \\
v \\
\underline{z} \\
=\end{array}$ & & 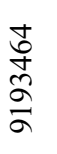 & 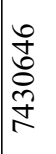 & 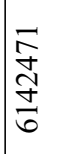 & 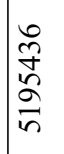 & \begin{tabular}{l} 
त్ \\
ర్ర \\
\multirow{\gamma}{*}{}
\end{tabular} & 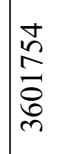 & $\begin{array}{l}a \\
\partial \\
0 \\
\infty \\
\infty \\
m\end{array}$ & $\mid \begin{array}{l}n \\
0 \\
0 \\
0 \\
0 \\
2\end{array}$ & 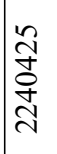 & 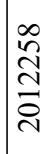 & 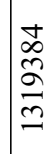 \\
\hline & & t & 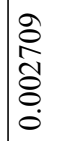 & $\begin{array}{l}n \\
\infty \\
\tilde{\delta} \\
\delta \\
\stackrel{0}{0}\end{array}$ & 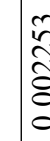 & & 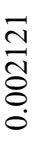 & 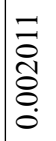 & $\begin{array}{l}\infty \\
\stackrel{0}{0} \\
\stackrel{0}{8} \\
0\end{array}$ & $\frac{n}{\infty}$ & 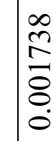 & Jृ & & 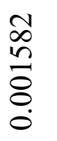 & $\begin{array}{l}\infty \\
0 \\
0 \\
\\
8 \\
0 \\
0\end{array}$ & $\frac{0}{\stackrel{0}{0}}$ & $\begin{array}{l}\vec{\infty} \\
\stackrel{\infty}{0} \\
\stackrel{8}{8} \\
\dot{0}\end{array}$ & 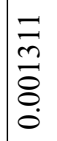 & 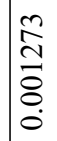 & 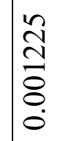 & $\mid \begin{array}{l}\vec{\infty} \\
\stackrel{\vec{\sigma}}{\bar{O}} \\
\stackrel{0}{0}\end{array}$ & సे & 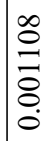 & 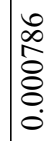 \\
\hline & & $\mathscr{\simeq}$ & 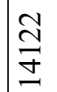 & $\begin{array}{l}n \\
\infty \\
0 \\
\tilde{c}\end{array}$ & స్ & & ঙે & ڤ̆ & $\begin{array}{l}\infty \\
\stackrel{\infty}{n} \\
\cong\end{array}$ & 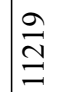 & $\begin{array}{l}\infty \\
\infty \\
\infty \\
0\end{array}$ & $\mid \begin{array}{l}\mathfrak{1} \\
\vdots \\
0 \\
0\end{array}$ & & 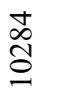 & ઠิ & 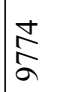 & 芒 & & $\underset{\sigma}{\exists}$ & $\hat{\aleph}$ & $\mid \begin{array}{l} \pm \\
\infty \\
\infty \\
\infty\end{array}$ & $\begin{array}{l}\text { त్రి } \\
\infty\end{array}$ & 产 & $\underset{\infty}{\mathcal{m}}$ \\
\hline లే & $\tilde{z}$ & 莺 & $\begin{array}{l}0 \\
2 \\
2\end{array}$ & $\begin{array}{l}\check{N} \\
i\end{array}$ & $\stackrel{\infty}{2}$ & & לֶ. & $\stackrel{0}{\stackrel{0}{*}}$ & $\stackrel{\mathcal{F}}{\stackrel{乛}{-}}$ & $\stackrel{0}{+}$ & $n$ & 7 & & 导 & $\stackrel{n}{\text { If }}$ & $\stackrel{n}{n}$ & $\stackrel{?}{\stackrel{f}{\sim}}$ & సે & $\stackrel{+}{m}$ & $\stackrel{\infty}{\stackrel{\infty}{\sim}}$ & 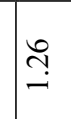 & $\stackrel{?}{-}$ & $\stackrel{\sim}{\underset{I}{J}}$ & $\hat{\tilde{i}}$ \\
\hline $\begin{array}{l}\frac{+}{\sigma} \\
\frac{\vec{\omega}}{\Xi} \\
\Xi\end{array}$ & 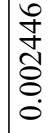 & $\vec{z}$ & 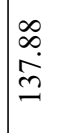 & $\stackrel{n}{\stackrel{n}{f}}$ & 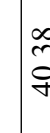 & & ڤ్రి & $\begin{array}{l}\hat{\alpha} \\
\dot{m}\end{array}$ & 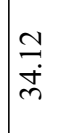 & $\begin{array}{l}\stackrel{2}{2} \\
\stackrel{m}{m}\end{array}$ & $\underset{\mathrm{m}}{\overrightarrow{\mathrm{d}}}$ & ?) & & $\underset{\sim}{\stackrel{n}{n}}$ & $\begin{array}{l}\vec{b} \\
\dot{m}\end{array}$ & $\frac{\hat{b}}{\dot{m}}$ & 志. & $\begin{array}{l}\hat{n} \\
\infty \\
\infty \\
\sim\end{array}$ & $\begin{array}{l}\infty \\
\infty \\
\infty \\
\infty \\
\sim\end{array}$ & $\underset{\stackrel{\sim}{\sim}}{\stackrel{F}{n}}$ & 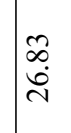 & $\begin{array}{l}\infty \\
\infty \\
i j\end{array}$ & 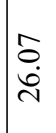 & $\begin{array}{l}\underset{\sim}{\sim} \\
\infty \\
\sim\end{array}$ \\
\hline $\begin{array}{l}\mathscr{\mathscr { I }} \\
\frac{\overrightarrow{0}}{\Xi}\end{array}$ & 衽 & 駕 & 芩 & ì & c & & $\stackrel{\circ}{i}$ & $\begin{array}{l}\tilde{S} \\
\text { ì }\end{array}$ & $\hat{i}$ & $\mid \begin{array}{l}\infty \\
\infty \\
i \\
i\end{array}$ & $\frac{m}{m}$ & 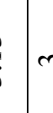 & & $\stackrel{m}{m}$ & ஸे & 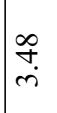 & $\stackrel{m}{m}$ & $\frac{ \pm}{m}$ & 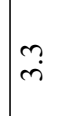 & $\stackrel{\sim}{\infty}$ & $\begin{array}{l}\infty \\
N \\
m\end{array}$ & $\begin{array}{l}\tilde{n} \\
m \\
m\end{array}$ & $\underset{m}{m}$ & $\vec{i}$ \\
\hline 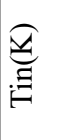 & $\stackrel{\curvearrowright}{\curvearrowright}$ & 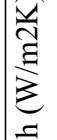 & 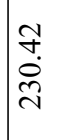 & $\begin{array}{l}\infty \\
\infty \\
\infty \\
\infty\end{array}$ & $\begin{array}{l}\hat{2} \\
\approx\end{array}$ & & $\begin{array}{l}\infty \\
\stackrel{0}{\circ} \\
\stackrel{2}{0}\end{array}$ & $\begin{array}{l}n \\
\infty \\
\infty \\
\infty \\
0\end{array}$ & $\begin{array}{l}\infty \\
0 \\
0 \\
0\end{array}$ & $\stackrel{\text { S }}{i}$ & \begin{tabular}{l}
0 \\
$\stackrel{2}{N}$ \\
\multirow{I}{*}{}
\end{tabular} & $\begin{array}{l}a \\
\tilde{f} \\
\tilde{f}\end{array}$ & & $\begin{array}{l}\stackrel{g}{+} \\
\text { i }\end{array}$ & $\begin{array}{l}\infty \\
i \\
i \\
n \\
n\end{array}$ & 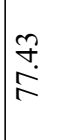 & 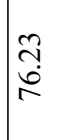 & $\begin{array}{l}\infty \\
\infty \\
i \\
\end{array}$ & $\begin{array}{l}\infty \\
\infty \\
\stackrel{n}{n}\end{array}$ & $\stackrel{\hat{n}}{\stackrel{i}{r}}$ & $\mid \infty$ & $\begin{array}{l}\hat{\sigma} \\
i \\
i\end{array}$ & $\stackrel{\stackrel{ }{i}}{\dot{T}}$ & $\begin{array}{l}\varkappa \\
\hat{\Sigma} \\
\infty\end{array}$ \\
\hline$\sum_{\text {至 }}$ & $\stackrel{\sim}{\longrightarrow}$ & $\begin{array}{l}\text { శ્ } \\
\text { है } \\
= \\
=\end{array}$ & $\begin{array}{l}\hat{\sigma} \\
\hat{\sigma}\end{array}$ & 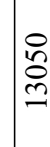 & 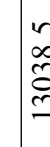 & & 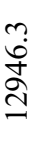 & 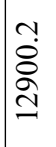 & 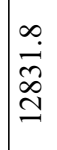 & 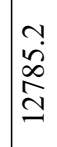 & $\begin{array}{l}\infty \\
\stackrel{0}{\beth} \\
\end{array}$ & $\begin{array}{l}2 \\
\alpha \\
2 \\
z \\
z\end{array}$ & & 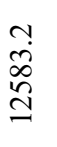 & 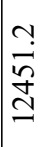 & 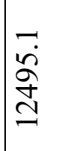 & 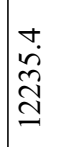 & 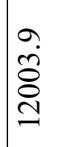 & તี & $\stackrel{\grave{\Omega}}{\grave{\Xi}}$ & $\begin{array}{l}\infty \\
\stackrel{\infty}{\infty} \\
\infty \\
ٍ\end{array}$ & $\begin{array}{l}\infty \\
\infty \\
\hat{\sigma} \\
\underline{\Xi} \\
=\end{array}$ & 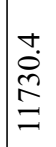 & $\frac{\partial}{\approx}$ \\
\hline $\begin{array}{l}\sum_{\pi}^{0} \\
0 \\
0 \\
0 \\
0\end{array}$ & ્ָ & 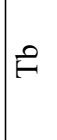 & 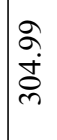 & $\underset{\sim}{\stackrel{\sim}{\sim}}$ & in & & $\overrightarrow{\tilde{D}}$ & 㐫. & 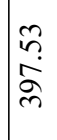 & $\begin{array}{l}n \\
\dot{b} \\
\dot{\gamma} \\
\gamma\end{array}$ & & is & & $\vec{m}$ & $\begin{array}{l}\hat{\sigma} \\
\dot{\infty} \\
\stackrel{+}{+}\end{array}$ & 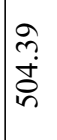 & $\begin{array}{l}b \\
\vec{j} \\
\text { in }\end{array}$ & $\begin{array}{l}0 \\
\infty \\
\infty \\
i n\end{array}$ & $\begin{array}{l}n \\
n \\
n \\
n \\
n\end{array}$ & $\frac{d}{a}$ & $\begin{array}{l}\hat{n} \\
\infty \\
\infty \\
\infty\end{array}$ & 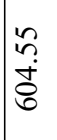 & $\begin{array}{l}\sigma 0 \\
\stackrel{0}{0} \\
\text { ర్ }\end{array}$ & $\begin{array}{l}\hat{+} \\
\dot{\tilde{f}} \\
\text { }\end{array}$ \\
\hline$\sum_{0}^{\pi}$ & $\tilde{a}$ & 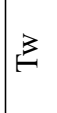 & \begin{tabular}{l}
$q$ \\
\multirow{\sigma}{*}{} \\
$\dot{b}$ \\
$\tilde{e}$
\end{tabular} & 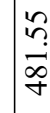 & $\begin{array}{l}\frac{f}{5} \\
\frac{5}{4}\end{array}$ & if & $\begin{array}{l}\stackrel{+}{0} \\
\dot{j} \\
\text { in }\end{array}$ & 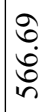 & $\begin{array}{l}\hat{\sigma} \\
\dot{\infty} \\
\infty \\
i n\end{array}$ & 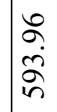 & 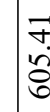 & $\begin{array}{l}2 \\
\alpha \\
\tilde{a} \\
0\end{array}$ & & 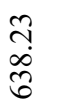 & $\begin{array}{l}\hat{0} \\
\text { in } \\
\hat{\sigma}\end{array}$ & $\begin{array}{l}\hat{\imath} \\
\hat{0} \\
0\end{array}$ & 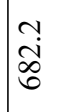 & $\stackrel{m}{\mathfrak{n}}$ & $\begin{array}{l}\hat{0} \\
\infty \\
\cdots\end{array}$ & 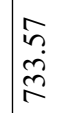 & 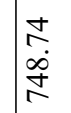 & 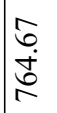 & 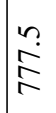 & 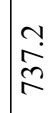 \\
\hline $\begin{array}{l}\mathscr{y} \\
\tilde{z} \\
\tilde{z}\end{array}$ & $N$ & $\stackrel{\rho}{a}$ & $N$ & $\vec{\infty}$ & $\bar{J}$ & & ָุ' & సֶ. & 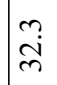 & $\begin{array}{l}m \\
\infty \\
m\end{array}$ & $\nabla$ & $\sum_{i}$ & $\dot{\hat{r}}$ & $\begin{array}{l}n \\
0 \\
i n\end{array}$ & 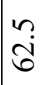 & $\mid \begin{array}{l}n \\
\infty \\
0\end{array}$ & $\begin{array}{l}0 \\
\stackrel{+}{i}\end{array}$ & $\begin{array}{l}0 \\
\dot{\infty}\end{array}$ & $\hat{\infty}$ & $\hat{i}$ & $\mid \begin{array}{l}\infty \\
\infty \\
\alpha\end{array}$ & $\stackrel{\infty}{\dot{0}}$ & 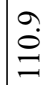 & \\
\hline
\end{tabular}




\begin{tabular}{|c|c|c|c|c|c|c|c|c|c|c|c|c|c|c|c|c|c|c|c|c|c|c|c|c|}
\hline & & $\vec{\Delta}$ & 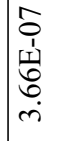 & 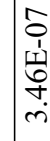 & 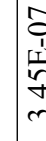 & & 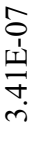 & 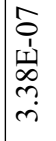 & 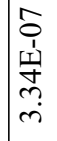 & 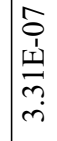 & 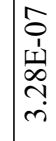 & 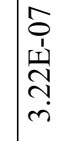 & \begin{tabular}{l}
2 \\
\multirow{1}{1}{} \\
\\
\end{tabular} & 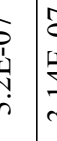 & $\begin{array}{l}\hat{S} \\
\dot{1} \\
\stackrel{1}{+} \\
\dot{m} \\
\dot{m}\end{array}$ & \begin{tabular}{|l}
$\hat{0}$ \\
$\hat{1}$ \\
$\hat{1}$ \\
\\
\\
$\dot{m}$
\end{tabular} & $\mid \begin{array}{l}\hat{0} \\
\hat{1} \\
1 \\
\hat{y} \\
0 \\
\dot{r}\end{array}$ & $\begin{array}{l}\hat{o} \\
\hat{1} \\
\mathbf{1} \\
\hat{a} \\
\text { i }\end{array}$ & 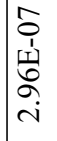 & $\begin{array}{l}\hat{o} \\
\hat{1} \\
\tilde{\omega} \\
\hat{\alpha} \\
\hat{i}\end{array}$ & $\begin{array}{l}\hat{o} \\
\hat{i} \\
\hat{1} \\
\hat{i} \\
\hat{i}\end{array}$ & 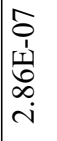 & 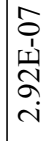 & 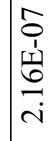 \\
\hline & & $\stackrel{\oplus}{\infty}$ & 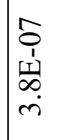 & 官 & 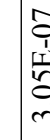 & & 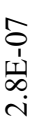 & 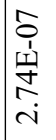 & $\begin{array}{l}\hat{o} \\
\hat{1} \\
\\
\\
\text { in }\end{array}$ & $\begin{array}{l}\hat{i} \\
\hat{1} \\
\hat{1} \\
\hat{n} \\
\end{array}$ & 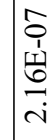 & 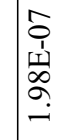 & 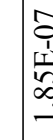 & & 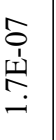 & 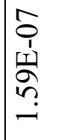 & 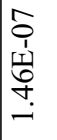 & $\begin{array}{c}\hat{s} \\
\hat{1} \\
1 \\
\\
\\
-1\end{array}$ & 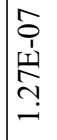 & \begin{tabular}{c}
$\hat{S}$ \\
$\mathbf{1}$ \\
1 \\
\multirow{1}{+}{} \\
\end{tabular} & 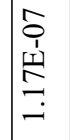 & 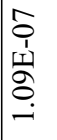 & $\begin{array}{l}0 \\
0 \\
1 \\
11 \\
\\
0 \\
-1\end{array}$ & 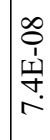 \\
\hline & & $\tilde{\Sigma}$ & 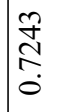 & సิ & 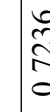 & & 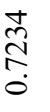 & 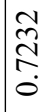 & 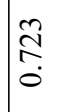 & 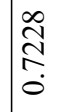 & & 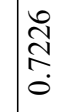 & Î & & 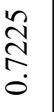 & 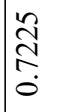 & 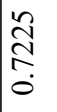 & $\underset{\mathbb{N}}{\stackrel{\Xi}{0}}$ & 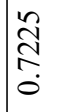 & 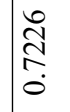 & 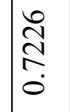 & $\begin{array}{l}\hat{N} \\
\stackrel{N}{0}\end{array}$ & $\begin{array}{l}\infty \\
\underset{N}{N} \\
\dot{0}\end{array}$ & 文 \\
\hline & & $\bar{\Xi}$ & 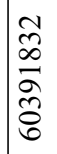 & 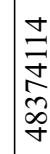 & $\begin{array}{l}\frac{1}{7} \\
\frac{2}{7}\end{array}$ & & 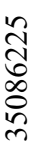 & 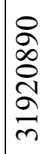 & 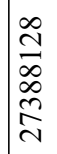 & 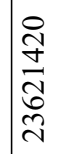 & 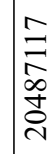 & 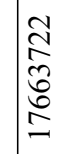 & 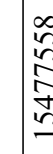 & & 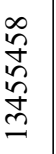 & $\begin{array}{l}\vec{Z} \\
\widetilde{\approx} \\
\infty \\
\infty \\
=\end{array}$ & 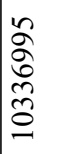 & 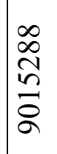 & 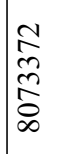 & 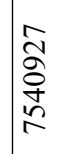 & 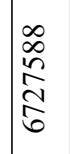 & $\begin{array}{l}\tilde{n} \\
\tilde{\alpha} \\
\approx \\
\tilde{n} \\
i n\end{array}$ & 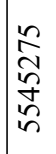 & 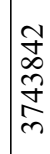 \\
\hline & & t & 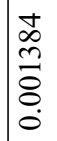 & 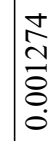 & 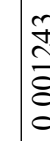 & & 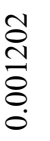 & 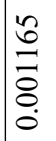 & 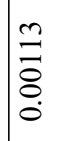 & $\begin{array}{l}\hat{\sigma} \\
\overline{0} \\
\overline{8} \\
0 \\
0\end{array}$ & $\begin{array}{l}\hat{8} \\
\frac{8}{8} \\
0 \\
0\end{array}$ & $\mid \begin{array}{l}\infty \\
\tilde{\delta} \\
\stackrel{8}{8} \\
\stackrel{0}{0}\end{array}$ & 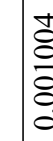 & & 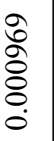 & 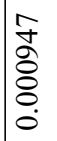 & 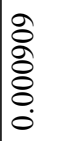 & 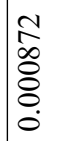 & 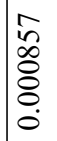 & $\begin{array}{l}\hat{\tilde{O}} \\
\hat{0} \\
0 \\
0 \\
0 \\
0\end{array}$ & $\mid \begin{array}{l}0 \\
0 \\
0 \\
0 \\
0 \\
0 \\
0 \\
0\end{array}$ & $\begin{array}{l}\hat{\alpha} \\
\hat{\hat{\sigma}} \\
\vdots \\
0 \\
\dot{0}\end{array}$ & & $\begin{array}{l}+ \\
\infty \\
0 \\
o \\
0 \\
0 \\
0 \\
0\end{array}$ \\
\hline & & $\mathscr{\approx}$ & $\stackrel{\tilde{\sigma}}{\underline{n}}$ & 胥 & $\stackrel{\circ}{\exists}$ & & $\begin{array}{l}\text { ¿̊ } \\
\text { oे }\end{array}$ & 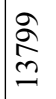 & $\mid \begin{array}{l}\stackrel{2}{n} \\
\cong\end{array}$ & $\begin{array}{l}\stackrel{+}{\approx} \\
\triangleq \\
\cong\end{array}$ & 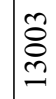 & $\begin{array}{l}\mathfrak{d} \\
\stackrel{1}{\beth}\end{array}$ & 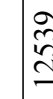 & & $\begin{array}{l}\underset{\Xi}{\Xi} \\
\widetilde{\Xi}\end{array}$ & 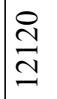 & $\stackrel{\widehat{\Omega}}{\Xi}$ & $\stackrel{\substack{9 \\
\Xi}}{\Xi}$ & 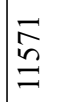 & 景 & $\underset{\Xi}{\stackrel{\Xi}{\Xi}}$ & 。े & fo & $\underset{\infty}{\infty}$ \\
\hline తే & $\tilde{z}$ & 莺 & $\vec{a}$ & 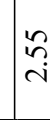 & $\frac{m}{n}$ & & $\stackrel{\infty}{\leftrightarrow}$ & $\stackrel{\infty}{\infty}$ & $\stackrel{9}{-}$ & $\stackrel{\infty}{\rightarrow}$ & $\stackrel{\infty}{\stackrel{\infty}{i}}$ & $\widehat{a}$ & 3 & & $\hat{\mathfrak{S}}$ & $\stackrel{\tilde{o}}{i}$ & $\stackrel{\sigma}{-}$ & $\stackrel{\bullet}{\stackrel{0}{\circ}}$ & $\stackrel{\infty}{\infty}$ & $\stackrel{\infty}{\stackrel{n}{\rightarrow}}$ & $\stackrel{n}{?}$ & $\stackrel{\infty}{\stackrel{\infty}{.}}$ & กֶ. & $\stackrel{+}{\circ}$ \\
\hline $\begin{array}{l}\frac{+}{\sigma} \\
\frac{\vec{U}}{\Xi} \\
\Xi\end{array}$ & 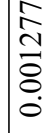 & $\vec{z}$ & $\begin{array}{l}\stackrel{+}{N} \\
\tilde{n} \\
\end{array}$ & $\frac{n}{\partial}$ & $\begin{array}{l}\infty \\
\nexists\end{array}$ & & 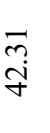 & 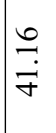 & $\frac{\vec{\jmath}}{\dot{\gamma}}$ & $\begin{array}{l}\infty \\
\infty \\
\dot{\sigma} \\
\vec{\forall}\end{array}$ & 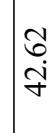 & $\begin{array}{l}\mathscr{D} \\
\infty \\
\dot{q}\end{array}$ & $\begin{array}{l}\text { ś } \\
\text { fo }\end{array}$ & 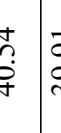 & $\bar{\sigma}$ & $\stackrel{\text { I }}{\vec{q}}$ & 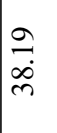 & $\begin{array}{l}\tilde{n} \\
\tilde{m}\end{array}$ & $\begin{array}{l}\infty \\
\stackrel{\infty}{0} \\
\dot{m}\end{array}$ & 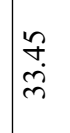 & $\begin{array}{l}\infty \\
0 \\
i \\
\tilde{n}\end{array}$ & $\frac{\sigma}{m}$ & 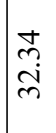 & ठ̂. \\
\hline $\begin{array}{l}\stackrel{\mathscr{Z}}{\stackrel{4}{0}} \\
\frac{0}{\Xi}\end{array}$ & तิ & 㝕 & 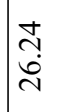 & $\stackrel{亏}{f}$ & $\begin{array}{l}n \\
n \\
c\end{array}$ & & ָे & $\frac{\partial}{\dot{m}}$ & $\underset{m}{m}$ & in & $\begin{array}{l}\hat{\infty} \\
\dot{m}\end{array}$ & $\underset{\sim}{\stackrel{+}{\sim}}$ & $\tilde{\alpha}$ & : & ले & $\exists$ & $\hat{\sigma}$ & $\stackrel{\text { for }}{m}$ & 守. & $\vec{m}$ & $\mid \begin{array}{l}\infty \\
\stackrel{n}{n}\end{array}$ & 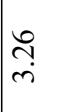 & $\underset{\dot{m}}{F}$ & $\underset{\forall}{\stackrel{f}{*}}$ \\
\hline$\underset{\Xi}{\Xi}$ & ৯े & 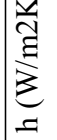 & $\underset{\overrightarrow{\vec{\lambda}}}{\Xi}$ & $\begin{array}{l}\delta \\
\dot{D}\end{array}$ & 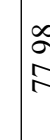 & & 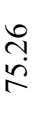 & $\begin{array}{l}\stackrel{2}{2} \\
\dot{T}\end{array}$ & 吕 & $\hat{ָ}$ & 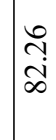 & $\begin{array}{l}\text { సे. } \\
\stackrel{\infty}{ }\end{array}$ & $\bar{\alpha}$ & & 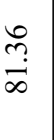 & $\begin{array}{l}\tilde{m} \\
\tilde{\infty}\end{array}$ & 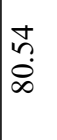 & $\hat{\text { ळे }}$ & $\stackrel{\infty}{\stackrel{\infty}{\mathbb{I}}}$ & $\begin{array}{c}\alpha \\
\tilde{\Omega}\end{array}$ & $\begin{array}{l}\bar{m} \\
\tilde{n}\end{array}$ & $\begin{array}{l}\stackrel{\mathfrak{n}}{\mathrm{i}} \\
\text { in }\end{array}$ & $\begin{array}{l}\hat{\sigma} \\
\dot{+}\end{array}$ & $\mid \begin{array}{l}\infty \\
\stackrel{N}{N} \\
\end{array}$ \\
\hline 泣 & 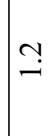 & $\begin{array}{l}\text { है } \\
\vdots \\
=\end{array}$ & 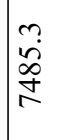 & i্ & $\begin{array}{l}\text { है } \\
\text { है } \\
\frac{1}{7}\end{array}$ & & $\begin{array}{l}\infty \\
\infty \\
\stackrel{\infty}{\mathbb{N}} \\
\end{array}$ & $\underset{ }{\stackrel{N}{\Xi}}$ & 穴 & $\underset{⿱ 亠 䒑}{\stackrel{2}{R}}$ & 害 & $\begin{array}{l}\text { â } \\
\infty \\
0 \\
\delta\end{array}$ & 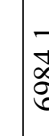 & $\begin{array}{l}\text { 苟 } \\
0 \\
0 \\
0 \\
0\end{array}$ & ठั & 年 & $\frac{9}{\infty}$ & 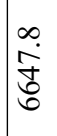 & 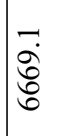 & $\begin{array}{l}\infty \\
\dot{f} \\
\dot{b}\end{array}$ & $\frac{n}{\bar{\Xi}}$ & $\left\{\begin{array}{l}n \\
0 \\
n \\
n \\
0\end{array}\right.$ & 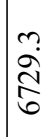 & $\begin{array}{l}\hat{\infty} \\
\stackrel{\alpha}{\alpha}\end{array}$ \\
\hline $\begin{array}{l}\sum_{\pi} \\
0 \\
0 \\
0 \\
2\end{array}$ & $\stackrel{0}{ }$ & $\vec{F}$ & 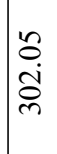 & $\stackrel{\vec{\vartheta}}{\overrightarrow{\bar{m}}}$ & $\bar{\pi}$ & & 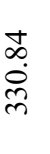 & mे. & $\begin{array}{l}\tilde{\infty} \\
\stackrel{\alpha}{\sigma} \\
\tilde{m}\end{array}$ & $\begin{array}{l}\hat{N} \\
\hat{n} \\
\end{array}$ & $\mid \begin{array}{r}\hat{0} \\
\dot{\infty} \\
\dot{m}\end{array}$ & $\begin{array}{l}8 \\
\infty \\
\infty \\
\infty \\
\infty\end{array}$ & 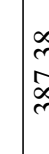 & $\begin{array}{l}\infty \\
? \\
? \\
\infty \\
n \\
n\end{array}$ & $\begin{array}{l}\hat{\sigma} \\
\dot{0} \\
\text { mे }\end{array}$ & $\mid \begin{array}{l}\infty \\
\infty \\
\tilde{o} \\
\dot{q}\end{array}$ & $\begin{array}{l}\curvearrowleft \\
\dot{J} \\
\dot{\gamma}\end{array}$ & 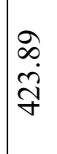 & 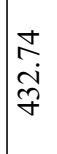 & 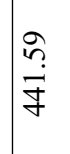 & 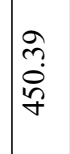 & $\begin{array}{l}m \\
\vec{a} \\
\frac{8}{f}\end{array}$ & 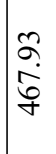 & 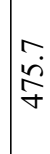 \\
\hline$\sum_{2}^{\pi}$ & חֶ? & 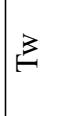 & & 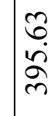 & $\frac{m}{\frac{m}{7}}$ & 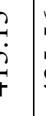 & 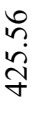 & 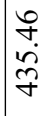 & $\begin{array}{l}\tilde{n} \\
\stackrel{y}{y} \\
\tilde{y}\end{array}$ & \begin{tabular}{l}
$\vec{n}$ \\
$\infty$ \\
\multirow{f}{f}{}
\end{tabular} & 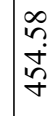 & $\begin{array}{l}\tilde{b} \\
\dot{b} \\
\dot{q}\end{array}$ & 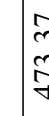 & $\begin{array}{l}n \\
\vdots \\
y \\
j\end{array}$ & $\begin{array}{c}\stackrel{\infty}{+} \\
\stackrel{\infty}{+} \\
+\end{array}$ & $\begin{array}{l}\curvearrowleft \\
\infty \\
\infty \\
\infty \\
\dot{\sigma}\end{array}$ & $\frac{0}{a}$ & $\begin{array}{l}\stackrel{0}{2} \\
\dot{n} \\
i n\end{array}$ & $\begin{array}{l}n \\
\tilde{U} \\
\tilde{N} \\
\hat{n}\end{array}$ & $\underset{\substack{\infty \\
\dot{n}}}{\stackrel{\infty}{n}}$ & $\begin{array}{l}\infty \\
n \\
0 \\
0 \\
n \\
n\end{array}$ & $\begin{array}{l}n \\
f \\
g \\
\dot{f} \\
\text { in }\end{array}$ & $\begin{array}{l}n \\
\infty \\
\infty \\
n \\
n\end{array}$ & $\begin{array}{l}n \\
\infty \\
\dot{J} \\
n\end{array}$ \\
\hline $\begin{array}{l}\tilde{y} \\
\tilde{z} \\
\tilde{z}\end{array}$ & $m$ & $\stackrel{\rho}{3}$ & $N$ & $\infty$ & $\bar{J}$ & 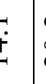 & $\begin{array}{l}\text { ָे } \\
\text { ते }\end{array}$ & ָ̊. & 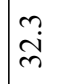 & $\begin{array}{l}n \\
\infty \\
m\end{array}$ & $\stackrel{+}{+}$ & $\stackrel{+}{\circ}$ & है & ?ֶ. & in & $\mid \begin{array}{l}n \\
\infty \\
0\end{array}$ & $\begin{array}{l}\stackrel{0}{ } \\
\stackrel{+}{I}\end{array}$ & $\begin{array}{l}0 \\
\dot{\infty}\end{array}$ & $\hat{\infty}$ & $\hat{\check{a}}$ & $\mid \begin{array}{l}\infty \\
\infty \\
\infty\end{array}$ & $\begin{array}{l}\infty \\
\dot{d} \\
=\end{array}$ & 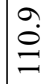 & לे. \\
\hline
\end{tabular}




\begin{tabular}{|c|c|c|c|c|c|c|c|c|c|c|c|c|c|c|c|c|c|c|c|c|c|c|c|c|}
\hline & & $\vec{\Delta}$ & $\begin{array}{l}0 \\
0 \\
1 \\
\text { I⿱ } \\
\text { co } \\
\text { i }\end{array}$ & 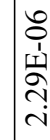 & 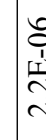 & & 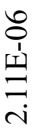 & 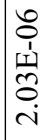 & 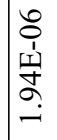 & $\begin{array}{l}8 \\
\vdots \\
1 \\
0 \\
0 \\
\infty \\
-\end{array}$ & 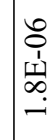 & 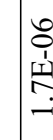 & 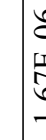 & . & 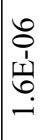 & 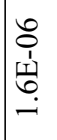 & 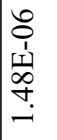 & 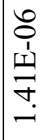 & 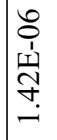 & 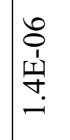 & $\mid \begin{array}{l}8 \\
0 \\
1 \\
1 \\
\infty \\
m \\
\\
-1\end{array}$ & 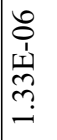 & 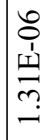 & 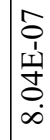 \\
\hline & & $\stackrel{\oplus}{\infty}$ & 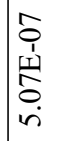 & 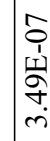 & 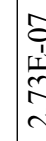 & & 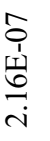 & 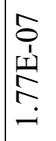 & 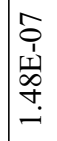 & 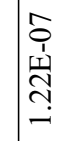 & 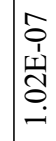 & $\mid \begin{array}{l}\infty \\
0 \\
1 \\
\\
\mathbf{1} \\
\infty \\
\infty\end{array}$ & r & 番 & $\begin{array}{l}\infty \\
0 \\
0 \\
1 \\
\\
n \\
0 \\
0\end{array}$ & $\begin{array}{l}\infty \\
0 \\
0 \\
1 \\
\infty \\
\infty \\
i n\end{array}$ & 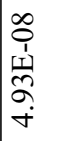 & 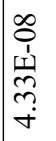 & 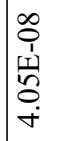 & 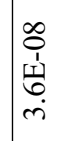 & 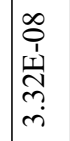 & $\begin{array}{l}\infty \\
o \\
o \\
1 \\
\\
\\
\grave{i}\end{array}$ & 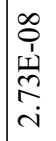 & 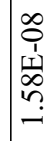 \\
\hline & & 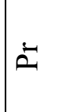 & $\overrightarrow{\mathbb{N}}$ & 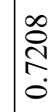 & $\begin{array}{l}\stackrel{2}{c} \\
\text { a }\end{array}$ & & 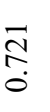 & 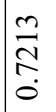 & 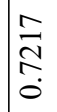 & 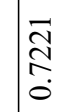 & 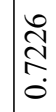 & 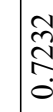 & مَ & 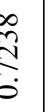 & 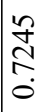 & $\begin{array}{l}\tilde{N} \\
\stackrel{N}{N} \\
\dot{0}\end{array}$ & $\begin{array}{l}\infty \\
\stackrel{n}{a} \\
\stackrel{0}{0}\end{array}$ & $\begin{array}{l}\tilde{n} \\
\stackrel{1}{N} \\
\dot{0}\end{array}$ & $\underset{\stackrel{N}{N}}{\stackrel{ }{0}}$ & 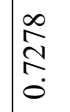 & $\begin{array}{l}\tilde{n} \\
\infty \\
\mathbb{N} \\
\tilde{0} \\
0\end{array}$ & 令 & & 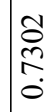 \\
\hline & & $\widetilde{J}$ & 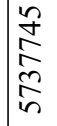 & 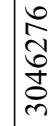 & $\frac{\infty}{2}$ & & 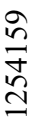 & 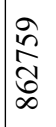 & $\frac{\mathbf{n}}{2}$ & $\begin{array}{l}\hat{\lambda} \\
\infty \\
o \\
q\end{array}$ & 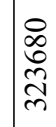 & 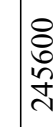 & $\stackrel{一}{\nexists}$ & $\frac{\partial}{2}$ & $\begin{array}{l}\infty \\
\stackrel{\infty}{0} \\
\stackrel{0}{n} \\
\end{array}$ & 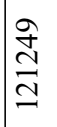 & $\begin{array}{l}\text { Oे } \\
\text { 安 } \\
\text { a }\end{array}$ & స్ర్రి & $\begin{array}{l}0 \\
\hat{0} \\
0 \\
0\end{array}$ & $\begin{array}{l}\tilde{y} \\
\infty \\
\dot{\alpha} \\
\omega\end{array}$ & $\underset{\underset{f}{f}}{\underset{f}{f}}$ & \begin{tabular}{l}
8 \\
\hdashline \\
2 \\
m.
\end{tabular} & $\underset{m}{\stackrel{m}{F}}$ & $\mid \begin{array}{c}\boldsymbol{D} \\
\\
\end{array}$ \\
\hline & & $t$ & 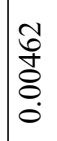 & $\begin{array}{l}\infty \\
\stackrel{2}{0} \\
\hat{\delta} \\
\stackrel{8}{0}\end{array}$ & 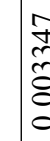 & & 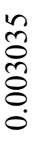 & $\begin{array}{l}\stackrel{ }{\hat{~}} \\
\hat{\widehat{\delta}} \\
\stackrel{0}{0}\end{array}$ & 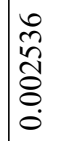 & 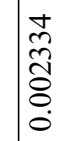 & 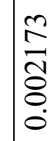 & $\frac{2}{2}$ & 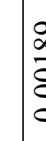 & $\begin{array}{l}0 \\
\\
0 \\
0 \\
0\end{array}$ & $\mid \begin{array}{l}\frac{N}{2} \\
\frac{2}{8} \\
0 \\
0\end{array}$ & $\mid \begin{array}{l}n \\
\frac{2}{2} \\
\frac{1}{8} \\
0 \\
0\end{array}$ & $\mid \begin{array}{l}0 \\
n \\
n \\
n \\
8 \\
0 \\
0\end{array}$ & $\begin{array}{l}0 \\
\stackrel{1}{+} \\
\stackrel{8}{8} \\
0\end{array}$ & 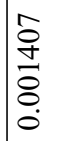 & $\begin{array}{l}i n \\
0 \\
\frac{3}{8} \\
0 \\
0\end{array}$ & $\begin{array}{l}0 \\
\ddot{m} \\
\stackrel{0}{8} \\
0 \\
0\end{array}$ & 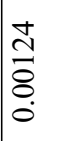 & 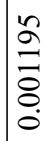 & 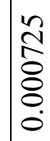 \\
\hline & & $\mathscr{\sim}$ & बें & 我 & $\tilde{\widetilde{a}}$ & & 点 & $\begin{array}{l}8 \\
\vdots \\
\vdots \\
n\end{array}$ & $\begin{array}{l}\text { तิ } \\
\text { in }\end{array}$ & $\overline{\overrightarrow{8}}$ & $\begin{array}{l}\tilde{a} \\
\infty \\
\alpha\end{array}$ & ర్ & & $\frac{0}{8}$ & 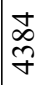 & \begin{tabular}{l}
\multirow{J}{0}{} \\
Y
\end{tabular} & $\frac{n}{\frac{n}{\gamma}}$ & 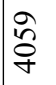 & 尽 & $\begin{array}{l}n \\
\infty \\
\infty \\
n \\
n\end{array}$ & $\mid \begin{array}{l}n \\
\infty \\
n \\
n\end{array}$ & $\vec{m}$ & 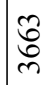 & $\underset{\substack{0 \\
0}}{0}$ \\
\hline లే & $\tilde{z}$ & 莺 & 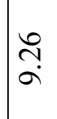 & : & $\bar{a}$ & & $\begin{array}{l}\infty \\
n \\
n \\
0\end{array}$ & $\tilde{n}$ & $\stackrel{m}{\stackrel{0}{0}}$ & ڤे) & के & $\stackrel{m}{3}$ & & ? & 3 & $\stackrel{m}{?}$ & 3 & \begin{tabular}{l}
$\infty$ \\
\multirow{1}{0}{} \\
0
\end{tabular} & 3 & $\stackrel{m}{m}$ & ?n? & 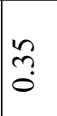 & $\stackrel{m}{\stackrel{0}{0}}$ & $\begin{array}{l}6 \\
i\end{array}$ \\
\hline $\begin{array}{l}\frac{+}{\sigma} \\
\frac{\vec{\omega}}{\Xi}\end{array}$ & $\begin{array}{l}\vec{\infty} \\
\hat{n} \\
\hat{\delta} \\
0 \\
0\end{array}$ & $\vec{z}$ & $\begin{array}{l}\vec{\sigma} \\
\dot{\delta} \\
\dot{0}\end{array}$ & İ & $\begin{array}{l}\stackrel{2}{2} \\
\check{\lambda}\end{array}$ & & $\stackrel{\overbrace{}}{\stackrel{f}{2}}$ & $=$ & $\begin{array}{l}0 \\
+ \\
\\
n\end{array}$ & $\begin{array}{l}\text { Î } \\
\stackrel{\Xi}{\Xi}\end{array}$ & $\underset{\dot{\vartheta}}{\stackrel{\gamma}{2}}$ & $\begin{array}{l}\bar{\Xi} \\
\text { ב. }\end{array}$ & is & है & $\begin{array}{l}\stackrel{2}{\varrho} \\
\stackrel{0}{0}\end{array}$ & $\stackrel{\cong}{\Xi}$ & 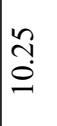 & $\begin{array}{l}\infty \\
0 \\
0\end{array}$ & बे. & $\frac{\partial}{0}$ & $\because$ & $\stackrel{\infty}{\stackrel{\infty}{0}} \stackrel{0}{\circ}$ & $\stackrel{n}{\Xi}$ & $\mid \begin{array}{l} \pm \\
\vec{\lambda}\end{array}$ \\
\hline $\begin{array}{l}\mathscr{\mathscr { I }} \\
\frac{\vec{\Delta}}{\Xi}\end{array}$ & $\hat{\sigma}$ & 當 & ले & $\stackrel{\circ}{\stackrel{2}{2}}$ & $\tilde{z}$ & & فِ & $\hat{\delta}$ & $\mid \begin{array}{l}\infty \\
\infty \\
\infty\end{array}$ & {$\left[\begin{array}{l}n \\
\infty \\
\infty \\
0\end{array}\right.$} & 車 & $\hat{a}$ & & $\frac{\infty}{0}$ & 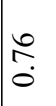 & 雍 & $\stackrel{\infty}{\infty}$ & $\hat{\hat{o}}$ & $\mid \begin{array}{l}\infty \\
\infty \\
0 \\
0\end{array}$ & $\stackrel{1}{2}$ & $\underset{-}{0}$ & $\exists$ & ले & กู \\
\hline$\underset{\Xi}{\stackrel{\Xi}{\Xi}}$ & ळे & 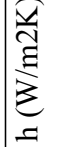 & 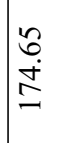 & $\begin{array}{l}\stackrel{\infty}{\circ} \\
\dot{+} \\
\text { in }\end{array}$ & $\begin{array}{l}\tilde{z} \\
\vec{z}\end{array}$ & & $\begin{array}{l}2 \\
\stackrel{2}{\circ} \\
\dot{q}\end{array}$ & $\begin{array}{l}\vec{J} \\
\infty \\
\infty \\
\infty \\
n\end{array}$ & $\begin{array}{l}0 \\
\cdots \\
m \\
m\end{array}$ & $\begin{array}{l}\tilde{\infty} \\
\tilde{m} \\
\tilde{m}\end{array}$ & $\begin{array}{l}\stackrel{0}{2} \\
m \\
m \\
m\end{array}$ & $\begin{array}{l}2 \\
\infty \\
0\end{array}$ & & '. & $\begin{array}{l}0 \\
\stackrel{0}{0} \\
\dot{0} \\
\end{array}$ & $\frac{\sigma}{\sigma}$ & $\begin{array}{l}\hat{\theta} \\
\dot{\rho} \\
\dot{m}\end{array}$ & $\frac{ \pm}{\partial}$ & $\mid \begin{array}{l}2 \\
\infty \\
\dot{n}\end{array}$ & $\stackrel{m}{\stackrel{n}{n}}$ & $\begin{array}{l}\mathcal{I} \\
\stackrel{\vec{m}}{m}\end{array}$ & $\begin{array}{l}\infty \\
\infty \\
\dot{m}\end{array}$ & $\begin{array}{l}n \\
\text { mे? } \\
\text { mे }\end{array}$ & $\frac{ \pm}{\dot{\infty}}$ \\
\hline$\sum_{\text {至 }}$ & $\tilde{Z}$ & $\begin{array}{l}\text { đิ } \\
\vdots \\
\vdots \\
=\end{array}$ & $\frac{\stackrel{N}{\Xi}}{\stackrel{\Xi}{\beth}}$ & 点 & 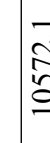 & & 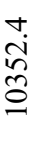 & 芦 & $\begin{array}{l}\hat{\dot{m}} \\
\stackrel{\alpha}{\alpha}\end{array}$ & : & $\begin{array}{l}\text { Oे } \\
\text { ơ } \\
\text { वे }\end{array}$ & वे & 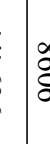 & مٌ & $\begin{array}{l}\sim \\
m \\
\infty \\
\infty \\
\infty\end{array}$ & $\frac{\sim}{\stackrel{1}{*}}$ & $\begin{array}{l}- \\
\dot{\infty} \\
\infty \\
\infty\end{array}$ & $\begin{array}{l}\infty \\
\dot{\delta} \\
\stackrel{0}{0}\end{array}$ & $\underset{\sim}{\approx}$ & $\begin{array}{l}\tilde{N} \\
\tilde{N} \\
\infty\end{array}$ & 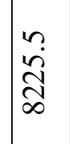 & $\begin{array}{l}\infty \\
\dot{+} \\
2 \\
2\end{array}$ & $\stackrel{0}{\curvearrowright}$ & $\begin{array}{l}\infty \\
\stackrel{0}{0} \\
\sigma \\
\sigma\end{array}$ \\
\hline 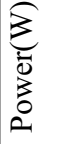 & $\stackrel{\text { O }}{=}$ & 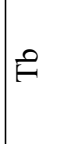 & $\frac{n}{\infty}$ & $\begin{array}{l}\stackrel{゚}{a} \\
\stackrel{\text { I }}{m}\end{array}$ & $\begin{array}{l}8 \\
\text { ñ } \\
\text { m. }\end{array}$ & & $\begin{array}{l}\vec{\infty} \\
\dot{\sigma} \\
\text { }\end{array}$ & 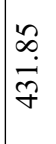 & \begin{tabular}{l}
1 \\
\hdashline \\
$\dot{8}$ \\
+
\end{tabular} & 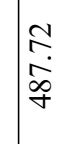 & $\begin{array}{l}\overrightarrow{6} \\
\dot{ \pm} \\
n\end{array}$ & \begin{tabular}{l}
0 \\
0 \\
\multirow{1}{*}{}
\end{tabular} & $\mid \begin{array}{l}\delta \\
0 \\
y \\
\vdots \\
y \\
5\end{array}$ & $\begin{array}{l}\sigma \\
6 \\
0 \\
0 \\
n\end{array}$ & 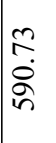 & $\mid \begin{array}{l}\tilde{N} \\
\tilde{n} \\
\sigma\end{array}$ & $\mid \begin{array}{l}2 \\
\infty \\
\infty \\
\hat{\sigma}\end{array}$ & 年 & $\begin{array}{l}0 \\
\infty \\
\infty \\
0\end{array}$ & 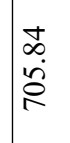 & 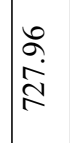 & $\mid \begin{array}{l}0 \\
\dot{9} \\
\frac{1}{1}\end{array}$ & فे & $\underset{\infty}{\infty}$ \\
\hline$\sum_{0}^{\pi}$ & $\stackrel{ \pm}{ \pm}$ & 3 & $\begin{array}{l}0 \\
\stackrel{0}{0} \\
0\end{array}$ & & $\frac{a}{a}$ & 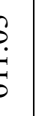 & ?ै & $\frac{ \pm}{\overrightarrow{0}}$ & 吕 & ते & $\begin{array}{l}\text { I } \\
\stackrel{2}{2} \\
2\end{array}$ & $\begin{array}{l}n \\
2 \\
\tilde{n} \\
\infty\end{array}$ & $\begin{array}{ll}4 \\
4 \\
5\end{array}$ & $\begin{array}{l}n \\
? \\
0 \\
0 \\
\infty\end{array}$ & $\begin{array}{l}\stackrel{2}{2} \\
\infty \\
\infty \\
\infty\end{array}$ & $\begin{array}{l}\stackrel{2}{\infty} \\
\stackrel{\infty}{\infty}\end{array}$ & $\mid \begin{array}{l}\hat{0} \\
\infty \\
a \\
\sigma\end{array}$ & $\begin{array}{l}\tilde{N} \\
\infty \\
\infty \\
\sigma \\
\tilde{n}\end{array}$ & 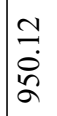 & - & \& & 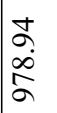 & $\frac{\hat{N}}{\stackrel{i}{a}}$ & 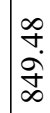 \\
\hline $\begin{array}{l}\mathscr{y} \\
\tilde{z} \\
\tilde{z}\end{array}$ & $\nabla$ & $\stackrel{\rho}{a}$ & $N$ & $\vec{\infty}$ & $\bar{I}$ & $t$ & $\begin{array}{l}\text { ָे } \\
\text { ஸे }\end{array}$ & तֶ. & 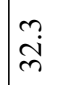 & $\begin{array}{c}m \\
\infty \\
m\end{array}$ & $\stackrel{+}{\stackrel{f}{+}}$ & $\bar{n}$ & $y$ & ?ֶ. & $\begin{array}{l}n \\
\tilde{J}\end{array}$ & $\mid \begin{array}{l}n \\
\infty \\
0\end{array}$ & $\begin{array}{l}\stackrel{\bullet}{0} \\
\dot{I}\end{array}$ & $\begin{array}{l}0 \\
\dot{\infty}\end{array}$ & $\hat{\infty}$ & $\hat{\check{a}}$ & $\begin{array}{l}\infty \\
\infty \\
\infty\end{array}$ & $\begin{array}{l}\infty \\
\dot{0} \\
\stackrel{0}{0}\end{array}$ & $\stackrel{\grave{O}}{\Xi}$ & $\stackrel{a}{\sigma}$ \\
\hline
\end{tabular}




\begin{tabular}{|c|c|c|c|c|c|c|c|c|c|c|c|c|c|c|c|c|c|c|c|c|c|c|c|c|}
\hline & & $\vec{v}$ & 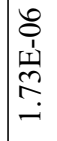 & $\begin{array}{l}8 \\
0 \\
1 \\
n \\
n \\
n \\
-1\end{array}$ & $\begin{array}{l}0 \\
\vdots \\
\frac{1}{a} \\
\frac{1}{9}\end{array}$ & & 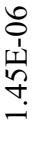 & 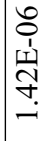 & 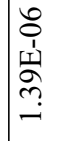 & 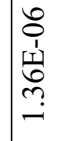 & 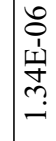 & 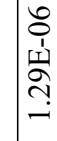 & 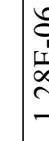 & 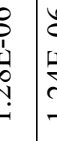 & 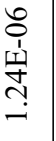 & $\begin{array}{l}8 \\
0 \\
1 \\
\\
\\
\\
\end{array}$ & 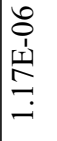 & 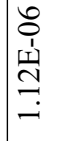 & 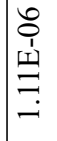 & $\begin{array}{l}0 \\
0 \\
1 \\
1 \\
\infty \\
\infty \\
- \\
-\end{array}$ & $\begin{array}{c}0 \\
0 \\
1 \\
11 \\
0 \\
0 \\
-\end{array}$ & 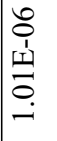 & 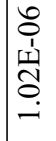 & 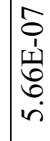 \\
\hline & & ஜீ & 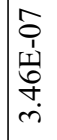 & $\begin{array}{l}\hat{s} \\
1 \\
\text { 11 } \\
\text { i } \\
\text { in }\end{array}$ & 空 & & 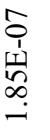 & 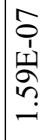 & 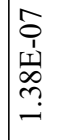 & $\begin{array}{c}\hat{S} \\
1 \\
\\
\\
-\end{array}$ & 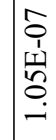 & $\begin{array}{c}\infty \\
0 \\
1 \\
\underline{1} \\
\cdots \\
a\end{array}$ & $\mid \begin{array}{l}\alpha \\
\alpha \\
\alpha \\
\alpha \\
\alpha \\
\alpha\end{array}$ & $\begin{array}{l}0 \\
1 \\
1 \\
0 \\
0 \\
0 \\
0\end{array}$ & 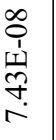 & 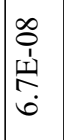 & $\begin{array}{l}\infty \\
0 \\
1 \\
i \\
\infty \\
\infty \\
i n \\
i\end{array}$ & 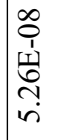 & 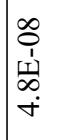 & $\begin{array}{l}\infty \\
0 \\
1 \\
1 \\
m \\
\sim \\
+\end{array}$ & $\mid \begin{array}{c}\infty \\
0 \\
0 \\
1 \\
\dot{1} \\
0 \\
\dot{+} \\
\dot{+}\end{array}$ & $\begin{array}{l}\infty \\
o \\
1 \\
n \\
n \\
n \\
n \\
n \\
n\end{array}$ & 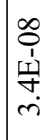 & 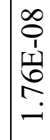 \\
\hline & & 2 & $\stackrel{\vec{N}}{\stackrel{\mathbb{0}}{0}}$ & હे & 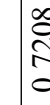 & & 总 & $\begin{array}{l}\stackrel{O}{\stackrel{N}{N}} \\
\stackrel{N}{0}\end{array}$ & $\overrightarrow{\mathbb{N}}$ & 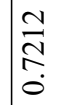 & 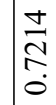 & 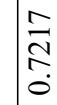 & $\grave{\S}$ & $\begin{array}{l}N \\
N \\
\dot{D}\end{array}$ & $\begin{array}{l}\underset{\mathbb{Z}}{\mathbb{S}} \\
\dot{0}\end{array}$ & 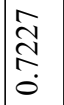 & 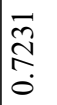 & 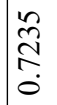 & ڤે & 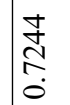 & 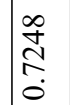 & 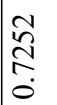 & $\mid \begin{array}{c}\hat{n} \\
\stackrel{i}{0} \\
\dot{0}\end{array}$ & 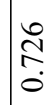 \\
\hline & & অ্் & 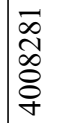 & \begin{tabular}{l}
\multirow{N}{n}{} \\
$\hat{n}$ \\
$\tilde{n}$
\end{tabular} & 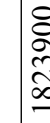 & & 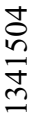 & 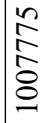 & $\frac{\infty}{\infty}$ & $\begin{array}{l}\overline{\hat{n}} \\
\hat{\alpha} \\
\hat{n}\end{array}$ & 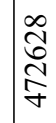 & $\frac{8}{0}$ & $\mid \begin{array}{l}\vec{\alpha} \\
\bar{c} \\
\bar{c}\end{array}$ & 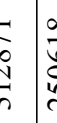 & 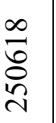 & 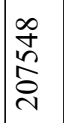 & 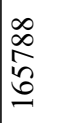 & 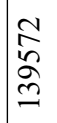 & $\begin{array}{l}\hat{\sigma} \\
\infty \\
=\end{array}$ & $\begin{array}{l}\bar{n} \\
\tilde{\alpha} \\
\sigma\end{array}$ & $\mid \begin{array}{l}n \\
0 \\
0 \\
\infty\end{array}$ & 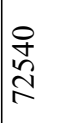 & 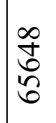 & $\begin{array}{l}0 \\
\text { N} \\
\sim \\
\sim \\
\tilde{n}\end{array}$ \\
\hline & & t & 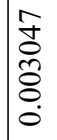 & 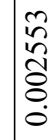 & 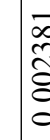 & & 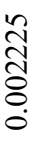 & 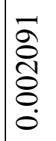 & $\mid \begin{array}{l}\infty \\
\stackrel{\infty}{2} \\
\stackrel{8}{8} \\
0 \\
0\end{array}$ & $\begin{array}{c}\hat{\hat{\alpha}} \\
\frac{\infty}{0} \\
\stackrel{8}{0} \\
0\end{array}$ & $\begin{array}{l}\infty \\
\infty \\
0 \\
\\
0 \\
0 \\
0\end{array}$ & $\begin{array}{l}\overline{\widehat{\sigma}} \\
\stackrel{0}{8} \\
0 \\
0\end{array}$ & go & 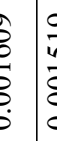 & $\begin{array}{l}\frac{a}{n} \\
\frac{n}{8} \\
\dot{0} \\
\dot{0}\end{array}$ & 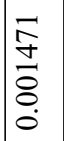 & $\mid \begin{array}{l}n \\
0 \\
0 \\
0 \\
8 \\
0 \\
0\end{array}$ & 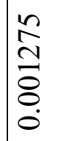 & 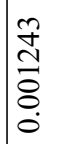 & \begin{tabular}{l}
$\infty$ \\
$\infty$ \\
\hdashline \\
$\vec{\Xi}$ \\
0 \\
0
\end{tabular} & $\mid \begin{array}{c}\stackrel{\partial}{ٍ} \\
\vec{\Xi} \\
\ddot{0} \\
\dot{0}\end{array}$ & 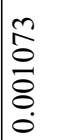 & $\frac{\infty}{2}$ & 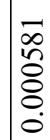 \\
\hline & & $\mathscr{\sim}$ & 웅 & $\begin{array}{l}\hat{\infty} \\
0 \\
0\end{array}$ & 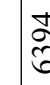 & & $\frac{n}{n}$ & $\begin{array}{l}+ \\
0 \\
\text { in }\end{array}$ & 兽 & $\begin{array}{l}\infty \\
\stackrel{\circ}{n} \\
n\end{array}$ & $\begin{array}{l}0 \\
\tilde{n} \\
i n\end{array}$ & $\frac{\infty}{i n}$ & ֻૃ & & 命 & $\mid \begin{array}{l}\infty \\
\stackrel{\infty}{\gamma} \\
\gamma\end{array}$ & $\frac{d}{0}$ & 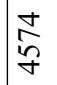 & 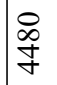 & $\begin{array}{l}\tilde{F} \\
\text { के }\end{array}$ & $\vec{F}$ & $\tilde{\widetilde{y}}$ & $\frac{3}{7}$ & $\stackrel{\varrho}{\ni}$ \\
\hline తే & $\tilde{z}$ & 莺 & $\underset{\hat{\imath}}{\infty}$ & $\stackrel{8}{\circ}$ & ? & & $\tilde{o}$ & : & : & oे & $\stackrel{t}{\stackrel{0}{0}}$ & $\stackrel{\overbrace{}}{\stackrel{2}{0}}$ & ก & $\begin{array}{l}\frac{1}{0} \\
\dot{0}\end{array}$ & $\stackrel{i}{0}$ & $\stackrel{n}{0}$ & $\stackrel{t}{\stackrel{0}{0}}$ & 尚 & 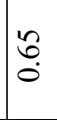 & $\begin{array}{l}\infty \\
0 \\
0 \\
0\end{array}$ & 焉 & 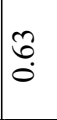 & $\stackrel{\infty}{0}$ & ते \\
\hline $\begin{array}{l}\frac{+}{\sigma} \\
\frac{\vec{U}}{\Xi} \\
\Xi\end{array}$ & 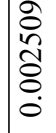 & $\vec{z}$ & $\frac{ \pm}{2}$ & $\begin{array}{l}n \\
\tilde{e} \\
\tilde{n}\end{array}$ & $\begin{array}{l}\stackrel{2}{ } \\
\vec{\tau}\end{array}$ & & 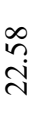 & $\overrightarrow{\bar{N}}$ & 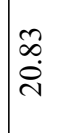 & 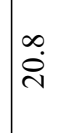 & $\stackrel{\mathscr{N}}{\stackrel{\sim}{N}}$ & $\hat{\sigma}$ & $\bar{c}$ & $\vec{c}$ & Sָ & $\bar{\sigma}$ & $\begin{array}{l}\infty \\
\infty \\
\infty \\
\infty\end{array}$ & $\vec{ָ}$ & $\stackrel{2}{\stackrel{7}{ }}$ & $\stackrel{\hat{\imath}}{\hat{\sigma}}$ & ભે & $\begin{array}{l}\hat{n} \\
n \\
n\end{array}$ & $\stackrel{\sim}{\sim}$ & $\frac{\sigma}{\vec{v}}$ \\
\hline $\begin{array}{l}\stackrel{\mathscr{U}}{\simeq} \\
\frac{\tilde{O}}{\Xi}\end{array}$ & $\stackrel{n}{\stackrel{n}{r}}$ & 悹 & $\hat{n}$ & $\stackrel{2}{\stackrel{2}{I}}$ & 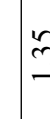 & & $\stackrel{\imath}{\beth}$ & $\stackrel{\vec{I}}{\unlhd}$ & $\stackrel{\infty}{\stackrel{\infty}{\sim}}$ & {$\left[\begin{array}{l}\infty \\
m \\
\rightarrow\end{array}\right.$} & 華 & $\tilde{n}$ & is & $n$ & کُ & $\stackrel{\vartheta}{-}$ & $\stackrel{શ}{\stackrel{-}{-}}$ & in & $\stackrel{\infty}{\infty}$ & $\stackrel{\circ}{\stackrel{2}{\prime}}$ & $\underline{F}$ & $\stackrel{\mathbb{Z}}{\stackrel{2}{*}}$ & $\mid \begin{array}{l}0 \\
\infty \\
-\end{array}$ & $\hat{\sigma}$ \\
\hline$\underset{\Xi}{\Xi}$ & $\stackrel{\infty}{\grave{\lambda}}$ & 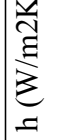 & 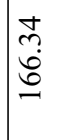 & $\frac{m}{n}$ & $\begin{array}{l}n \\
z \\
z\end{array}$ & & 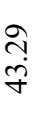 & $\begin{array}{l}8 \\
\text { ì } \\
\text { I }\end{array}$ & $\begin{array}{l}\partial \\
\dot{y} \\
\dot{f}\end{array}$ & $\begin{array}{l}n \\
\tilde{f} \\
\dot{f}\end{array}$ & $\begin{array}{l}\stackrel{2}{6} \\
\stackrel{6}{+}\end{array}$ & $\begin{array}{l}n \\
\tilde{n} \\
y\end{array}$ & 7 & & $\frac{\vec{n}}{\tilde{f}}$ & $\mid \begin{array}{l}n \\
\ddot{g} \\
g\end{array}$ & $\begin{array}{c}\stackrel{8}{o} \\
\dot{\infty} \\
\stackrel{+}{f}\end{array}$ & \begin{tabular}{l}
\multirow{0}{0}{} \\
$\dot{y}$ \\
$\forall$
\end{tabular} & $\mid \begin{array}{l}3 \\
\dot{\sigma} \\
\dot{\sigma}\end{array}$ & $\begin{array}{l}\tilde{\gamma} \\
\tilde{\sigma}\end{array}$ & $\begin{array}{l}\infty \\
\infty \\
\dot{y}\end{array}$ & $\overrightarrow{\tilde{n}}$ & 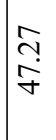 & $\stackrel{\substack{f \\
f}}{f}$ \\
\hline 泣 & $\stackrel{ָ}{i}$ & $\begin{array}{l}\text { वै } \\
\text { है } \\
=-\end{array}$ & $\begin{array}{l}+ \\
\ddot{n} \\
\infty \\
\infty \\
\curvearrowright\end{array}$ & 菨 & ڤ్ & & $\begin{array}{l}0 \\
\dot{0} \\
\infty \\
\infty \\
0\end{array}$ & $\begin{array}{l}n \\
\stackrel{\sim}{1} \\
\infty \\
\infty\end{array}$ & 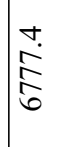 & $\begin{array}{l}+ \\
\stackrel{n}{\hat{n}} \\
\hat{\sigma}\end{array}$ & $\frac{1}{2}$ & $\begin{array}{l}n \\
\tilde{n} \\
\tilde{n}\end{array}$ & $\begin{array}{l}6 \\
6 \\
6 \\
6\end{array}$ & $\begin{array}{l}0 \\
0 \\
2 \\
\hat{0} \\
0\end{array}$ & 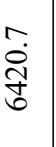 & 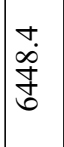 & $\mid \begin{array}{c}n \\
\infty \\
\infty\end{array}$ & $\begin{array}{l}\bar{i} \\
\hat{i} \\
\text { in }\end{array}$ & 官 & $\begin{array}{l}\hat{\infty} \\
\infty \\
0 \\
0 \\
i n\end{array}$ & $\begin{array}{l}\hat{\partial} \\
\hat{\lambda} \\
\infty \\
i n\end{array}$ & $\begin{array}{l}n \\
n \\
\tilde{b} \\
i \\
n\end{array}$ & 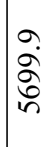 & $\begin{array}{l}n \\
\check{a} \\
\check{m}\end{array}$ \\
\hline $\begin{array}{l}\sum_{\pi}^{2} \\
0 \\
0 \\
0 \\
2\end{array}$ & 곤 & $\vec{F}$ & 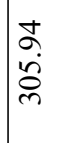 & 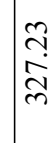 & 先 & & $\frac{\tilde{c}}{\tilde{\delta}}$ & $\begin{array}{l}\infty \\
n \\
\infty \\
\infty \\
n \\
n\end{array}$ & $\begin{array}{l}2 \\
\hat{\alpha} \\
\hat{\sigma}\end{array}$ & $\begin{array}{l}\infty \\
\stackrel{\sim}{\mathfrak{y}} \\
\underset{y}{4}\end{array}$ & $\begin{array}{l}\hat{A} \\
\stackrel{+}{+}\end{array}$ & $\begin{array}{l}2 \\
0 \\
\dot{b} \\
\dot{q}\end{array}$ & $\frac{5}{\alpha}$ & 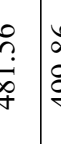 & $\begin{array}{l}\stackrel{2}{\infty} \\
\stackrel{a}{\sigma}\end{array}$ & $\frac{\hat{a}}{\stackrel{n}{n}}$ & 㝕 & $\begin{array}{l}\hat{N} \\
\text { in } \\
\tilde{n}\end{array}$ & 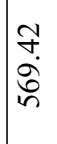 & $\begin{array}{l}\infty \\
\hat{n} \\
\sim \infty \\
\infty\end{array}$ & 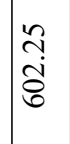 & $\begin{array}{l}\infty \\
0 \\
\infty \\
0 \\
\sigma\end{array}$ & $\stackrel{\overrightarrow{0}}{\widehat{\sigma}}$ & $\begin{array}{l}\bar{\sigma} \\
\tilde{\sigma} \\
\sigma\end{array}$ \\
\hline$\sum_{2}^{\pi}$ & $\stackrel{ \pm}{0}$ & 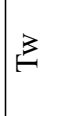 & $\begin{array}{l}\text { In } \\
\tilde{n} \\
\tilde{n}\end{array}$ & $\begin{array}{l}\hat{2} \\
8 \\
8\end{array}$ & $\begin{array}{l}8 \\
8 \\
8\end{array}$ & t. & 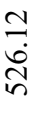 & $\begin{array}{l}m \\
\infty \\
\infty \\
\infty \\
n\end{array}$ & $\begin{array}{l}\tilde{N} \\
\tilde{0} \\
\stackrel{n}{n}\end{array}$ & 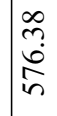 & $\begin{array}{l}\vec{b} \\
\dot{\infty} \\
i \\
i\end{array}$ & $\begin{array}{l}\text { nે } \\
\stackrel{8}{0} \\
\text { d }\end{array}$ & है & : & ్ֶర & $\begin{array}{l}= \\
\vec{\infty} \\
\dot{0}\end{array}$ & 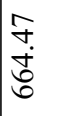 & 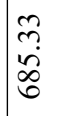 & $\begin{array}{l}n \\
\stackrel{2}{\sigma}\end{array}$ & $\begin{array}{l}\mathbb{7} \\
\dot{J} \\
\dot{T}\end{array}$ & 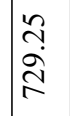 & \begin{tabular}{l}
$\infty$ \\
0 \\
\multirow{J}{*}{} \\
\multirow{2}{*}{}
\end{tabular} & $\begin{array}{r}\stackrel{N}{*} \\
\stackrel{n}{r}\end{array}$ & 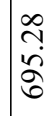 \\
\hline 苋 & in & $\stackrel{\theta}{a}$ & $N$ & $\infty$ & $\exists$ & & 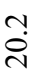 & ֶָ. & $\stackrel{m}{i}$ & $\begin{array}{c}m \\
\infty \\
m\end{array}$ & $\stackrel{+}{\forall}$ & 官 & vै & ?? & הֶ & $\mid \begin{array}{l}n \\
\infty \\
\infty \\
0\end{array}$ & $\stackrel{\bullet}{\stackrel{0}{*}}$ & $\begin{array}{l}0 \\
\dot{\infty}\end{array}$ & $\hat{\infty}$ & $\hat{\check{a}}$ & $\mid \begin{array}{l}\infty \\
\infty \\
\infty\end{array}$ & $\begin{array}{l}\infty \\
\dot{d} \\
=\end{array}$ & $\stackrel{\grave{o}}{\varrho}$ & bे \\
\hline
\end{tabular}




\begin{tabular}{|c|c|c|c|c|c|c|c|c|c|c|c|c|c|c|c|c|c|c|c|c|c|c|c|c|}
\hline & & $\vec{v}$ & $\begin{array}{l}0 \\
0 \\
1 \\
1 \\
\vdots \\
0 \\
\dot{m} \\
\dot{m}\end{array}$ & \begin{tabular}{l}
8 \\
0 \\
1 \\
\cline { 1 - 1 } \\
$i n$ \\
$i$
\end{tabular} & $\begin{array}{l}\frac{8}{4} \\
\frac{1}{5} \\
\frac{1}{7}\end{array}$ & & 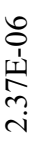 & 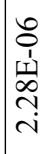 & 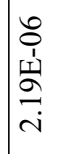 & 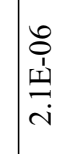 & 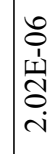 & 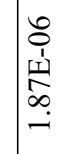 & 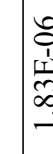 & in & 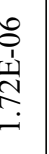 & 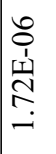 & 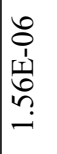 & 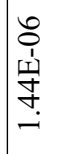 & 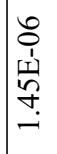 & 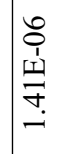 & 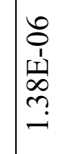 & 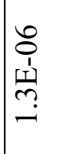 & $\begin{array}{l}8 \\
1 \\
1 \\
\\
\\
\end{array}$ & 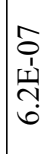 \\
\hline & & $\stackrel{\oplus}{\infty}$ & $\begin{array}{l}0 \\
0 \\
1 \\
\text { 卒 } \\
0 \\
\infty \\
\infty\end{array}$ & 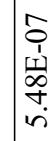 & $\begin{array}{l}\vec{a} \\
1 \\
\frac{1}{5} \\
7 \\
7\end{array}$ & & 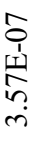 & $\begin{array}{l}\hat{o} \\
\hat{1} \\
\hat{W} \\
\infty \\
\infty \\
\end{array}$ & 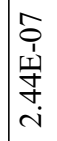 & $\begin{array}{l}\hat{o} \\
\hat{1} \\
\text { İ }\end{array}$ & 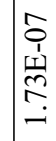 & 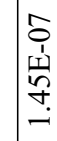 & 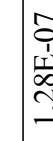 & 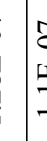 & 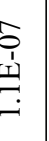 & $\begin{array}{l}\hat{o} \\
1 \\
=\end{array}$ & $\begin{array}{l}\infty \\
0 \\
0 \\
1 \\
1 \\
⿱ ⺊ 1 \\
m \\
\infty \\
\infty \\
\infty\end{array}$ & 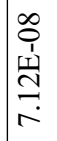 & 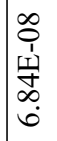 & 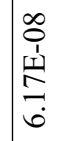 & 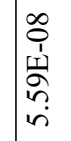 & $\begin{array}{l}\infty \\
0 \\
1 \\
1 \\
1 \\
2 \\
0 \\
i \\
n\end{array}$ & 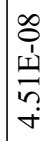 & 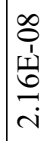 \\
\hline & & $\vec{D}$ & 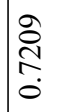 & 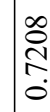 & $\begin{array}{l}\stackrel{c}{c} \\
\stackrel{a}{a}\end{array}$ & & હેे & 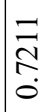 & 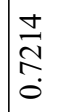 & $\underset{\vec{N}}{\stackrel{N}{0}}$ & 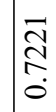 & 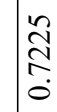 & $\hat{\mathfrak{N}}$ & & $\begin{array}{l}\stackrel{+}{N} \\
\stackrel{0}{0}\end{array}$ & 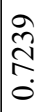 & 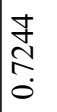 & 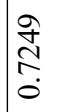 & 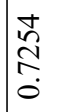 & $\begin{array}{l}\hat{\tilde{y}} \\
\stackrel{\hat{\sigma}}{0}\end{array}$ & 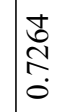 & 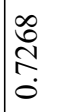 & $\underset{\substack{\hat{N} \\
\stackrel{0}{0}}}{ }$ & \\
\hline & & $\widetilde{J}$ & 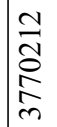 & 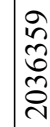 & $\frac{\vec{J}}{\stackrel{\Xi}{\pi}}$ & & $\begin{array}{l}\underset{+}{\sigma} \\
\underset{\sigma}{\sigma}\end{array}$ & 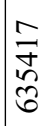 & $\begin{array}{l}0 \\
2 \\
\infty \\
8 \\
b \\
q\end{array}$ & 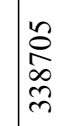 & 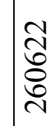 & $\begin{array}{l}8 \\
2 \\
2 \\
2\end{array}$ & $\mid \begin{array}{l}0 \\
n \\
n\end{array}$ & ذ̇ & $\frac{0}{\stackrel{0}{c}}$ & 悆 & 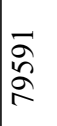 & 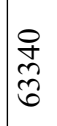 & $\begin{array}{l}\hat{8} \\
\stackrel{8}{2} \\
\text { in }\end{array}$ & 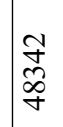 & 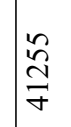 & 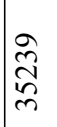 & $\begin{array}{l}\hat{\text { a }} \\
\stackrel{\text { సे }}{ }\end{array}$ & $\begin{array}{l}\stackrel{0}{\infty} \\
\stackrel{\infty}{m} \\
\end{array}$ \\
\hline & & $t$ & 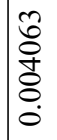 & 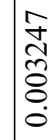 & 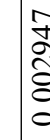 & & 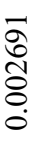 & 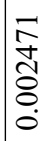 & 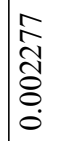 & $\begin{array}{l}\hat{\sigma} \\
\stackrel{\vec{\sigma}}{\delta} \\
\stackrel{0}{0}\end{array}$ & 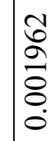 & $\frac{a}{\frac{2}{2}}$ & $\mid \frac{\mathfrak{\sigma}}{8}$ & & $\begin{array}{l}\frac{1}{n} \\
\frac{n}{8} \\
0 \\
0\end{array}$ & $\begin{array}{l}\infty \\
\stackrel{\infty}{\infty} \\
\stackrel{\infty}{8} \\
\\
0\end{array}$ & 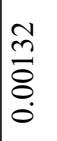 & 六 & \begin{tabular}{l}
$\infty$ \\
$\infty$ \\
\hdashline \\
\\
0 \\
0
\end{tabular} & 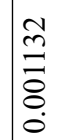 & $\mid \begin{array}{l}\stackrel{\bullet}{0} \\
\stackrel{8}{0} \\
\stackrel{0}{8} \\
\stackrel{0}{0}\end{array}$ & $\frac{\overline{8}}{\grave{8}}$ & $\mid \begin{array}{l}n \\
\hat{\delta} \\
0 \\
0 \\
0\end{array}$ & $\begin{array}{l}0 \\
0 \\
0 \\
8 \\
0 \\
0 \\
0\end{array}$ \\
\hline & & $\mathscr{\sim}$ & $\stackrel{\infty}{\hat{n}}$ & $\begin{array}{l}\bar{n} \\
\tilde{n}\end{array}$ & مิ & & 筞 & Fै & $\frac{\hat{\sigma}}{7}$ & $\frac{m}{a}$ & $\underset{\substack{\hat{\infty} \\
\text { 足 }}}{ }$ & 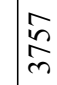 & $\tilde{b}$ & & $\begin{array}{l}n^{\circ} \\
m \\
m\end{array}$ & 㝘 & ब্ল & సి & $\begin{array}{l}8 \\
\text { लn } \\
\text { m. }\end{array}$ & $\begin{array}{l}\vec{\sim} \\
\text { m }\end{array}$ & $\underset{m}{m}$ & ठे & 害 & $\stackrel{m}{\vec{D}}$ \\
\hline లే & $\tilde{z}$ & 莺 & nิ? & 吕 & $\begin{array}{l}\infty \\
a \\
a\end{array}$ & & & $\stackrel{t}{0}$ & ?ִ? & ?. & חै & $\hat{\jmath}$ & $\tilde{n}$ & & 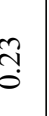 & $\stackrel{\overbrace{}}{\hat{0}}$ & $\overrightarrow{\tilde{N}}$ & $\frac{9}{0}$ & $\frac{9}{0}$ & $\stackrel{\infty}{0}$ & $\stackrel{\infty}{\frac{\infty}{0}}$ & $\stackrel{1}{0}$ & $\frac{\infty}{0}$ & 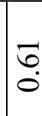 \\
\hline $\begin{array}{l}\frac{+}{\sigma} \\
\frac{\vec{U}}{\Xi} \\
\Xi \Xi\end{array}$ & $\begin{array}{l}\infty \\
\infty \\
\infty \\
\tilde{\delta} \\
\dot{\delta} \\
\dot{0}\end{array}$ & $\vec{z}$ & $\frac{\infty}{\stackrel{a}{a}}$ & $\stackrel{\Re}{\tilde{n}}$ & $\begin{array}{l}+ \\
0 \\
0 \\
\end{array}$ & & $\begin{array}{l}\text { ळे } \\
\text { ఏ. }\end{array}$ & $\stackrel{n}{\stackrel{n}{ \pm}}$ & $\begin{array}{l}J \\
\stackrel{g}{2}\end{array}$ & 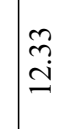 & $\stackrel{n}{n}$ & $\bar{\alpha}$ & 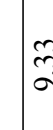 & & $\vec{n}$ & के & $\stackrel{+}{r}$ & ñ & 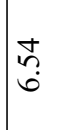 & 节 & $\frac{7}{6}$ & $\begin{array}{l}\vec{\infty} \\
\dot{n}\end{array}$ & $\begin{array}{l}n \\
\infty \\
i \\
i\end{array}$ & O. \\
\hline $\begin{array}{l}\frac{\mathscr{a}}{\underline{0}} \\
\frac{\overrightarrow{0}}{\Xi}\end{array}$ & $\begin{array}{l}\infty \\
\tilde{n} \\
n \\
n\end{array}$ & 駕 & $\begin{array}{l}0 \\
\\
\end{array}$ & $\stackrel{+}{-}$ & $\bar{c}$ & & $\begin{array}{l}n \\
\infty \\
0 \\
0\end{array}$ & $\stackrel{\overbrace{}}{\circ}$ & 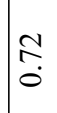 & I. & . & है & $\mid \begin{array}{l}x \\
i \\
c\end{array}$ & & 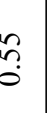 & $\begin{array}{l}0 \\
n \\
0 \\
0\end{array}$ & $\tilde{n}$ & वे. & $\stackrel{\infty}{\stackrel{\infty}{0}}$ & $\stackrel{\infty}{\stackrel{\infty}{0}}$ & $\ddot{0}$ & $\stackrel{\infty}{+}$ & $\hat{n}$ & $\bar{\Omega}$ \\
\hline$\underset{\Xi}{\stackrel{\Xi}{\Xi}}$ & \&্ল & 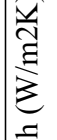 & 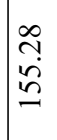 & $\begin{array}{l}0 \\
\text { rె? } \\
\dot{f}\end{array}$ & $\begin{array}{l}\tilde{\infty} \\
\tilde{m}\end{array}$ & & ले & $\frac{\tilde{\sigma}}{\dot{m}}$ & $\begin{array}{l}\tilde{n} \\
\grave{\lambda}\end{array}$ & 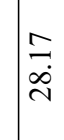 & 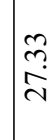 & $\begin{array}{l}\text { సे } \\
\text { సं }\end{array}$ & శ̊ & & $\begin{array}{l}0 \\
\text { in } \\
\text { in }\end{array}$ & 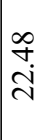 & 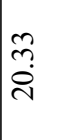 & $\begin{array}{l}\infty \\
\infty \\
\infty \\
\infty\end{array}$ & $\begin{array}{l}\hat{2} \\
\infty \\
\infty\end{array}$ & $\begin{array}{l}0 \\
\infty \\
\infty\end{array}$ & $\mid \begin{array}{l}\infty \\
+ \\
\infty \\
-\end{array}$ & $\stackrel{ \pm}{\stackrel{ \pm}{I}}$ & $\begin{array}{l}\tilde{N} \\
\infty \\
\infty \\
-\end{array}$ & $\begin{array}{l}\widehat{\hat{त}} \\
\text { }\end{array}$ \\
\hline$\sum_{\text {忘 }}$ & $\stackrel{\infty}{-}$ & $\begin{array}{l}\text { है } \\
\vdots \\
=\end{array}$ & $\begin{array}{l}\infty \\
\stackrel{\hat{\vec{J}}}{\delta}\end{array}$ & 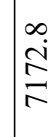 & 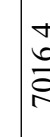 & & $\begin{array}{l}1 \\
\infty \\
\infty \\
\infty \\
0 \\
0\end{array}$ & 홍 & 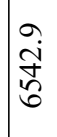 & $\begin{array}{l}\hat{\lambda} \\
\hat{\delta} \\
\tilde{\sigma}\end{array}$ & $\underset{\text { 离 }}{\text { J }}$ & $\begin{array}{l}\infty \\
\tilde{n} \\
\infty \\
\infty \\
n\end{array}$ & $\mid$\begin{tabular}{l}
7 \\
$\stackrel{8}{i}$ \\
\hdashline
\end{tabular} & & $\begin{array}{l}\infty \\
\dot{0} \\
\tilde{n} \\
n \\
n\end{array}$ & $\begin{array}{l}\nabla \\
\stackrel{d}{0} \\
i n \\
i n\end{array}$ & $\begin{array}{l}n \\
\infty \\
o \\
0 \\
i n\end{array}$ & 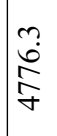 & $\begin{array}{l}\stackrel{y}{r} \\
\tilde{n} \\
\infty \\
\infty \\
+\end{array}$ & 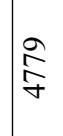 & $\mid \begin{array}{l}\infty \\
\stackrel{0}{\rho} \\
\stackrel{f}{f}\end{array}$ & $\stackrel{\infty}{\stackrel{\infty}{\stackrel{f}{f}}}$ & 趈 & 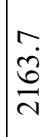 \\
\hline 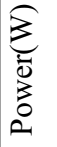 & $\stackrel{\Re}{\vDash}$ & 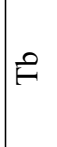 & 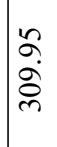 & $\begin{array}{l}\tilde{m} \\
\infty \\
\infty \\
m\end{array}$ & $\begin{array}{l}n \\
\text { d } \\
\text { d }\end{array}$ & & $\begin{array}{l}8 \\
\dot{8} \\
\text { ळे }\end{array}$ & $\begin{array}{l}\partial \\
\dot{\nabla} \\
\vec{\gamma}\end{array}$ & $\stackrel{m}{\stackrel{m}{\sigma}}$ & 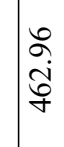 & $\begin{array}{l}\overline{0} \\
\dot{\infty} \\
\dot{\infty}\end{array}$ & $\begin{array}{l}\text { ô } \\
\infty \\
\infty \\
i n\end{array}$ & $\begin{array}{l}3 \\
0 \\
i\end{array}$ & $\begin{array}{l}5 \\
0 \\
5 \\
4\end{array}$ & $\begin{array}{l}6 \\
6 \\
6 \\
6 \\
n\end{array}$ & $\begin{array}{l}\overrightarrow{6} \\
0 \\
0 \\
n\end{array}$ & $\begin{array}{l}\hat{N} \\
\infty \\
\infty \\
\infty \\
i\end{array}$ & \begin{tabular}{l}
0 \\
\multirow{2}{*}{} \\
$\dot{0}$ \\
8
\end{tabular} & 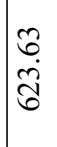 & $\frac{d}{\stackrel{d}{0}}$ & 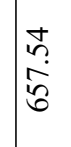 & 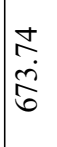 & $\begin{array}{l}\overrightarrow{\hat{d}} \\
\stackrel{\alpha}{0}\end{array}$ & 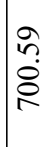 \\
\hline$\sum_{0}^{\pi}$ & $\stackrel{m}{0}$ & 3 & $\begin{array}{l}\infty \\
\infty \\
i \\
\text { id } \\
ల\end{array}$ & $\begin{array}{l}\infty \\
\stackrel{0}{\sigma} \\
\tilde{g}\end{array}$ & \begin{tabular}{l}
$\infty$ \\
\multirow{2}{*}{} \\
$\dot{\sigma}$
\end{tabular} & ? & $\begin{array}{l}\text { in } \\
\text { in } \\
\text { in }\end{array}$ & $\frac{\hat{0}}{\sqrt{0}}$ & $\begin{array}{l}\circ \\
\circ \\
0 \\
0 \\
0\end{array}$ & 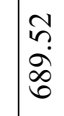 & $\underset{⿱}{\stackrel{f}{+}}$ & 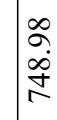 & 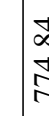 & f & $\frac{m}{2}$ & 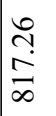 & $\begin{array}{l}\dot{J} \\
0 \\
\dot{q} \\
+ \\
\infty\end{array}$ & 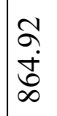 & 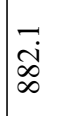 & $\begin{array}{l}\vec{b} \\
\hat{\infty} \\
\infty\end{array}$ & $\begin{array}{l}\stackrel{\sim}{m} \\
\stackrel{a}{a}\end{array}$ & 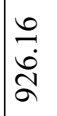 & 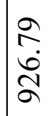 & $\begin{array}{l}\approx \\
6 \\
2 \\
2\end{array}$ \\
\hline $\begin{array}{l}\mathscr{y} \\
\tilde{z} \\
\tilde{z}\end{array}$ & 6 & $\stackrel{9}{9}$ & $N$ & $\vec{\infty}$ & $\exists$ & $t$ & ָุ' & సֶ. & 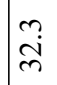 & $\begin{array}{c}m \\
\infty \\
m\end{array}$ & $\underset{f}{\stackrel{f}{f}}$ & में & & 4 & గn & $\mid \begin{array}{l}n \\
\infty \\
0\end{array}$ & \begin{tabular}{l}
$\bullet$ \\
$\dot{I}$ \\
\multirow{I}{*}{}
\end{tabular} & $\begin{array}{l}0 \\
\dot{\infty}\end{array}$ & $\hat{\infty}$ & $\hat{i}$ & $\mid \begin{array}{l}\infty \\
\infty \\
\alpha\end{array}$ & $\stackrel{\infty}{\dot{0}}$ & $\grave{\varrho}$ & \\
\hline
\end{tabular}




\begin{tabular}{|c|c|c|c|c|c|c|c|c|c|c|c|c|c|c|c|c|c|c|c|c|c|c|c|}
\hline & & $\vec{\Delta}$ & 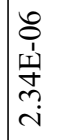 & 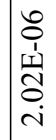 & $\begin{array}{l}8 \\
0 \\
1 \\
1 \\
0 \\
ٌ \\
-1\end{array}$ & 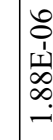 & ס & 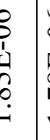 & \begin{tabular}{l}
8 \\
0 \\
1 \\
1 \\
$\infty$ \\
\multirow{2}{*}{} \\
-
\end{tabular} & 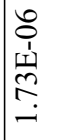 & 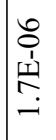 & $\begin{array}{l}8 \\
1 \\
1 \\
1 \\
0 \\
-1 \\
-1\end{array}$ & 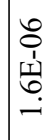 & 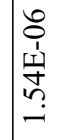 & 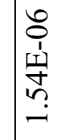 & 总 & 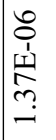 & 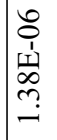 & $\begin{array}{l}0 \\
0 \\
1 \\
1 \\
2 \\
\dddot{n} \\
-1\end{array}$ & 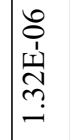 & 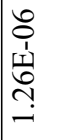 & 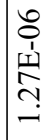 & \begin{tabular}{l} 
o \\
$\mathbf{1}$ \\
ț \\
\multirow{2}{*}{} \\
in
\end{tabular} \\
\hline & & $\stackrel{\oplus}{\infty}$ & 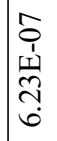 & $\begin{array}{l}\hat{o} \\
\hat{1} \\
\underline{1} \\
\infty \\
\mathfrak{r} \\
\gamma\end{array}$ & \begin{tabular}{l}
$\hat{o}$ \\
$\hat{1}$ \\
\multicolumn{1}{c}{} \\
$\tilde{\infty}$ \\
$\tilde{n}$ \\
$\dot{n}$
\end{tabular} & $\mid \begin{array}{c}\hat{0} \\
\dot{1} \\
\stackrel{\omega}{N} \\
\tilde{n}\end{array}$ & s & : & 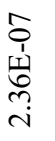 & 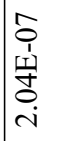 & 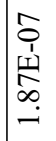 & 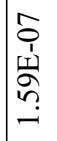 & 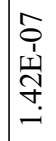 & 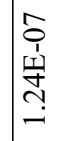 & 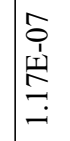 & $\begin{array}{l}\hat{a} \\
\hat{1} \\
\underline{y}\end{array}$ & 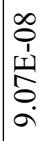 & $\mid$\begin{tabular}{c}
$\infty$ \\
0 \\
1 \\
1 \\
\multirow{1}{+}{} \\
$m$ \\
$\infty$ \\
$\infty$
\end{tabular} & $\begin{array}{l}\infty \\
0 \\
0 \\
1 \\
\infty \\
\infty \\
\\
\end{array}$ & $\mid \begin{array}{c}\infty \\
0 \\
0 \\
1 \\
\\
0 \\
\\
\end{array}$ & 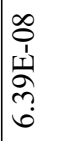 & 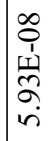 & 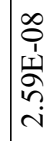 \\
\hline & & $\vec{D}$ & 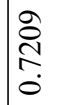 & 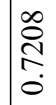 & 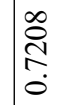 & $\mid \begin{array}{l}\infty \\
\stackrel{N}{N} \\
\dot{0}\end{array}$ & ફ & $\frac{b_{0}}{b_{0}}$ & $\underset{\stackrel{\mathbb{S}}{0}}{\overrightarrow{0}}$ & $\frac{\stackrel{N}{N}}{\stackrel{N}{0}}$ & 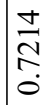 & 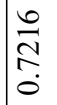 & 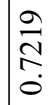 & 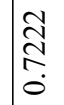 & 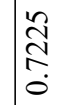 & 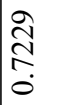 & 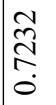 & \begin{tabular}{l}
0 \\
\multirow{N}{N}{} \\
$\stackrel{0}{0}$
\end{tabular} & 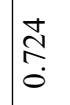 & 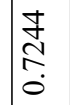 & 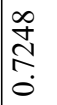 & 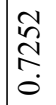 & 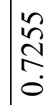 \\
\hline & & $\widetilde{J}$ & 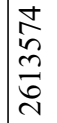 & $\frac{\sigma}{\sigma}$ & $\begin{array}{l}n \\
\infty \\
0 \\
0 \\
= \\
=\end{array}$ & $\begin{array}{l}\mathcal{F} \\
\mathscr{D} \\
\infty \\
\infty \\
\infty\end{array}$ & $\begin{array}{l}\delta \\
\vdots \\
\vdots \\
\text { ja }\end{array}$ & है & $\begin{array}{l}\text { oे } \\
\underset{\text { N }}{\infty} \\
\stackrel{+}{+}\end{array}$ & 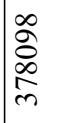 & 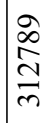 & 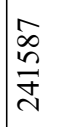 & 气̂ & $\begin{array}{l}\overrightarrow{\widehat{D}} \\
\stackrel{\infty}{\Omega} \\
\stackrel{2}{n}\end{array}$ & $\begin{array}{l}\vec{\sigma} \\
\tilde{D} \\
\infty \\
\sim\end{array}$ & $\begin{array}{l}0 \\
i n \\
o \\
0\end{array}$ & 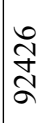 & $\begin{array}{l}0 \\
\tilde{n} \\
2 \\
2\end{array}$ & $\begin{array}{l}\infty \\
0 \\
0 \\
0 \\
0\end{array}$ & $\begin{array}{l}\overrightarrow{\widehat{\delta}} \\
\text { in }\end{array}$ & $\begin{array}{l}0 \\
\infty \\
0 \\
\text { in }\end{array}$ & 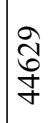 & 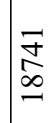 \\
\hline & & $t$ & 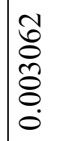 & 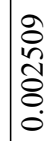 & 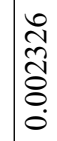 & $\mid \frac{\tilde{n}}{\widehat{\delta}}$ & 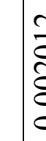 & : & $\begin{array}{l}\stackrel{n}{\infty} \\
\vec{\alpha} \\
\stackrel{0}{0}\end{array}$ & $\begin{array}{l}\hat{\infty} \\
\stackrel{\infty}{a} \\
\stackrel{8}{0} \\
0\end{array}$ & 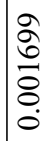 & $\begin{array}{l}n \\
\frac{0}{0} \\
\frac{n}{8} \\
0 \\
0\end{array}$ & $\frac{1}{2}$ & 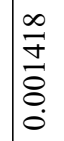 & $\mid \begin{array}{c}2 \\
\infty \\
\stackrel{m}{8} \\
\stackrel{8}{0}\end{array}$ & 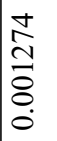 & 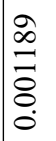 & $\stackrel{\bar{\theta}}{\bar{\sigma}}$ & 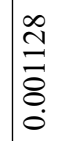 & $\begin{array}{l}\vec{\infty} \\
0 \\
0 \\
0 \\
0 \\
\dot{0}\end{array}$ & $\frac{n}{0}$ & 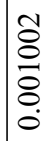 & 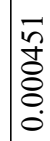 \\
\hline & & $\mathscr{\simeq}$ & $\underset{\widetilde{\sim}}{\tilde{n}}$ & 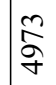 & $\frac{\hat{r}}{\hat{\gamma}}$ & $\stackrel{\substack{f \\
f}}{a}$ & 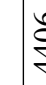 & 8 & 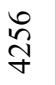 & $\frac{\tilde{J}}{\sigma}$ & बे & $\begin{array}{l}\infty \\
\infty \\
m \\
m\end{array}$ & $\mid \begin{array}{l}\infty \\
\infty \\
\infty\end{array}$ & 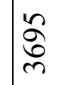 & 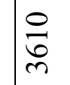 & 扂 & 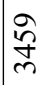 & ले & ల్లి & 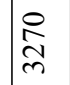 & $\stackrel{n}{\underset{n}{n}}$ & $\frac{d}{m}$ & $\frac{\hat{\jmath}}{\bar{m}}$ \\
\hline తే & $\tilde{z}$ & 莺 & $?$ & $\begin{array}{l}n \\
\infty \\
0 \\
0\end{array}$ & $\begin{array}{l}\widetilde{\sigma} \\
0 \\
0\end{array}$ & กี่ & 5 & f. & $\stackrel{0}{+}$ & f̊. & fo & $\vec{t}$ & $\stackrel{f}{\circ}$ & 导 & İ & $\underset{⿱}{*}$ & F. & 导 & to. & f̊. & $\stackrel{0}{\stackrel{0}{0}}$ & กิ & 9 \\
\hline $\begin{array}{l}\frac{+}{\sigma} \\
\frac{\Delta}{\Xi} \\
\Xi\end{array}$ & 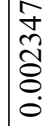 & $\vec{z}$ & $\frac{n}{\infty}$ & $\begin{array}{l}\tilde{y} \\
\tilde{z}\end{array}$ & $\begin{array}{l}\bar{N} \\
\stackrel{i}{1}\end{array}$ & $\stackrel{\text { I }}{\text { I }}$ & 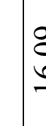 & : & 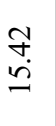 & $\begin{array}{l}\partial \\
\dot{\Xi}\end{array}$ & $\begin{array}{l}\hat{\sigma} \\
\dot{ \pm}\end{array}$ & $\begin{array}{l}\stackrel{0}{\sim} \\
\stackrel{n}{\sim}\end{array}$ & $\begin{array}{l}0 \\
\stackrel{0}{2}\end{array}$ & 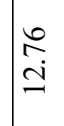 & $\begin{array}{l}\stackrel{⿱}{N} \\
\end{array}$ & 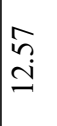 & $\begin{array}{l}\infty \\
\doteq \\
=\end{array}$ & $\stackrel{n}{\stackrel{\sim}{c}}$ & $\begin{array}{l}\stackrel{\searrow}{\mathcal{I}} \\
\end{array}$ & 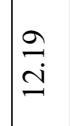 & $\stackrel{\cong}{\Xi}$ & 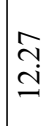 & $\underset{\sim}{\mathbb{n}}$ \\
\hline 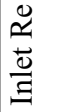 & $\begin{array}{l}\dot{m} \\
\text { ñ } \\
n\end{array}$ & 駕 & 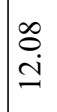 & 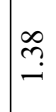 & ô. & 2 & 2 & 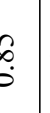 & $\begin{array}{c}\hat{\infty} \\
0 \\
0\end{array}$ & $\hat{o}$ & $\bar{\alpha}$ & $\begin{array}{l}\infty \\
\infty \\
0\end{array}$ & gे & ڤ̊ & $\underset{-}{\sigma}$ & $\underbrace{-}_{0}$ & 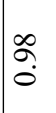 & $\exists$ & $\underset{:}{\Xi}$ & $\stackrel{\mathbb{Z}}{\stackrel{I}{I}}$ & $\stackrel{\sim}{\longrightarrow}$ & $\stackrel{\sim}{\stackrel{f}{*}}$ & $\begin{array}{l}n \\
\text { in }\end{array}$ \\
\hline$\underset{\Xi}{\stackrel{\Xi}{\Xi}}$ & $\underset{m}{8}$ & 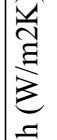 & 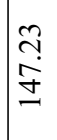 & $\underset{f}{F}$ & $\stackrel{\tilde{n}}{\dot{m}}$ & $\mid \begin{array}{l}2 \\
\vdots \\
j \\
m\end{array}$ & $\begin{array}{l}\alpha \\
0 \\
c\end{array}$ & 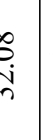 & $\begin{array}{l}n \\
\frac{\infty}{m} \\
m\end{array}$ & $\frac{\hat{\vartheta}}{\dot{m}}$ & (ָ) & $\overrightarrow{\dot{e}}$ & 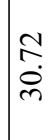 & 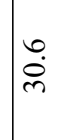 & 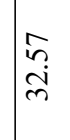 & $\begin{array}{l}\dot{b} \\
\dot{m}\end{array}$ & f̊. & m & $\begin{array}{l}\stackrel{2}{2} \\
\text { ì }\end{array}$ & $\begin{array}{l}\hat{f} \\
\dot{m}\end{array}$ & $\begin{array}{l}\tilde{J} \\
\infty \\
\infty \\
\infty\end{array}$ & $\begin{array}{l}\infty \\
\stackrel{\infty}{0} \\
\dot{m}\end{array}$ & 籴 \\
\hline$\sum_{\text {至 }}$ & $\stackrel{\infty}{-}$ & $\begin{array}{l}\text { है } \\
\vdots \\
=\end{array}$ & $\frac{\dot{\sigma}}{\partial}$ & $\begin{array}{l}\infty \\
\dot{0} \\
\infty \\
i n\end{array}$ & 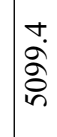 & $\begin{array}{l}= \\
\dot{\sigma} \\
\dot{\sigma}\end{array}$ & $\begin{array}{l}1 \\
y \\
g \\
2\end{array}$ & d. & $\begin{array}{l}\infty \\
\dot{m} \\
\dot{\infty} \\
\infty \\
\dot{+}\end{array}$ & $\frac{\hat{\sigma}}{\stackrel{+}{*}}$ & \begin{tabular}{l}
$\infty$ \\
$i$ \\
$\stackrel{\infty}{f}$ \\
\multirow{\gamma}{*}{}
\end{tabular} & $\begin{array}{l}\tilde{y} \\
\infty \\
\infty \\
\tilde{\sigma}\end{array}$ & $\begin{array}{l}\infty \\
\dot{9} \\
\stackrel{+}{y} \\
f\end{array}$ & 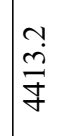 & 总 & $\mid$\begin{tabular}{l}
$n$ \\
$\stackrel{0}{0}$ \\
\\
\multirow{\gamma}{*}{}
\end{tabular} & $\frac{9}{\vec{i}}$ & 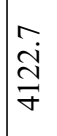 & $\begin{array}{l}\vec{\forall} \\
\dot{0} \\
\infty \\
\dot{O} \\
\dot{+}\end{array}$ & 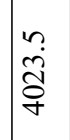 & $\underset{\substack{+\infty \\
\infty}}{\infty}$ & $\frac{m}{a}$ & $\begin{array}{l}0 \\
\stackrel{2}{2} \\
\end{array}$ \\
\hline $\begin{array}{l}\sum_{\pi}^{0} \\
0 \\
0 \\
0 \\
0\end{array}$ & $\begin{array}{l}\infty \\
n \\
n \\
n\end{array}$ & 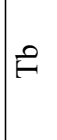 & 年 & $\begin{array}{c}\mathbb{N} \\
\infty \\
\infty \\
\sim\end{array}$ & $\begin{array}{l}\vec{F} \\
\dot{\infty} \\
\dot{m}\end{array}$ & ָे & y & 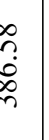 & $\begin{array}{l}0 \\
\qquad \\
\dot{o} \\
\dot{q}\end{array}$ & 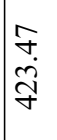 & $\frac{n}{n}$ & 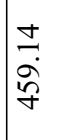 & $\begin{array}{l}\stackrel{+}{0} \\
\stackrel{0}{0} \\
\stackrel{f}{f}\end{array}$ & $\begin{array}{l}\text { సे } \\
\text { } \\
\dot{q}\end{array}$ & $\mid \begin{array}{l}8 \\
\stackrel{0}{0} \\
\text { in }\end{array}$ & 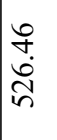 & $\begin{array}{l}\hat{o} \\
\text { i } \\
\text { in }\end{array}$ & 先 & $\begin{array}{l}\stackrel{2}{i} \\
i \underset{i}{i n}\end{array}$ & $\begin{array}{l}\infty \\
\infty \\
\infty \\
\infty \\
\infty\end{array}$ & \begin{tabular}{l}
\multirow{2}{*}{} \\
ç \\
$\delta$ \\
$\delta$
\end{tabular} & 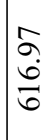 & 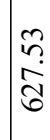 \\
\hline$\sum_{0}^{\pi}$ & $\stackrel{m}{0}$ & 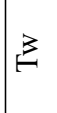 & $\mid \begin{array}{l}0 \\
b \\
\dot{f} \\
\tilde{m}\end{array}$ & $\begin{array}{l}\vec{n} \\
\dot{q} \\
q\end{array}$ & $\begin{array}{l}\vec{\forall} \\
\dot{\infty} \\
\dot{\sigma}\end{array}$ & $\begin{array}{l}\bar{a} \\
\text { jo } \\
\text { in }\end{array}$ & $\delta$ & हैं & $\begin{array}{l}\vec{a} \\
\text { हैं } \\
i n\end{array}$ & $\begin{array}{l}\vec{\forall} \\
\stackrel{n}{n} \\
i n\end{array}$ & 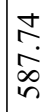 & 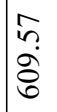 & 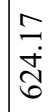 & $\frac{n}{n}$ & $\overrightarrow{\tilde{r}}$ & \begin{tabular}{l}
0 \\
\hdashline \\
0 \\
0 \\
0
\end{tabular} & $\begin{array}{l}2 \\
\infty \\
i \\
\hat{\sigma}\end{array}$ & $\begin{array}{l}\hat{N} \\
\stackrel{0}{0} \\
\otimes \\
0\end{array}$ & $\begin{array}{l}\text { Na } \\
\stackrel{8}{0} \\
8 \\
8\end{array}$ & $\mid \begin{array}{l}\infty \\
\stackrel{0}{0} \\
\infty \\
\stackrel{0}{R}\end{array}$ & 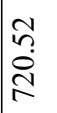 & 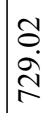 & $\begin{array}{l}\overrightarrow{0} \\
\text { बें } \\
\end{array}$ \\
\hline 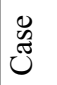 & $r$ & $\stackrel{9}{\Omega}$ & $N$ & $\bar{\infty}$ & $\ddot{ \pm}$ & $\overline{7}$ & & $\begin{array}{l}y \\
\\
\end{array}$ & 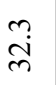 & $\begin{array}{c}n \\
\infty \\
m\end{array}$ & $\underset{f}{\forall}$ & $\stackrel{0}{8}$ & $\begin{array}{l}n \\
0 \\
n\end{array}$ & 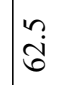 & $\begin{array}{l}n \\
\infty \\
0 \\
0\end{array}$ & $\begin{array}{l}\stackrel{\bullet}{0} \\
\dot{I}\end{array}$ & $\begin{array}{l}0 \\
\dot{\infty}\end{array}$ & $\vec{\infty}$ & $\hat{\tilde{a}}$ & $\mid \begin{array}{l}\infty \\
\dot{\alpha} \\
\propto\end{array}$ & $\begin{array}{l}\infty \\
\dot{0} \\
\stackrel{0}{0}\end{array}$ & $\stackrel{\grave{O}}{\Xi}$ & $\stackrel{a}{\sigma}$ \\
\hline
\end{tabular}




\begin{tabular}{|c|c|c|c|c|c|c|c|c|c|c|c|c|c|c|c|c|c|c|c|c|c|c|c|c|}
\hline & & $\vec{\Delta}$ & \begin{tabular}{|l}
$\hat{0}$ \\
$\hat{1}$ \\
1 \\
$\underline{1}$ \\
0 \\
0 \\
-
\end{tabular} & 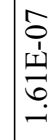 & $\begin{array}{l}\frac{1}{1} \\
\frac{1}{6} \\
\frac{1}{2}\end{array}$ & & 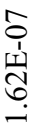 & 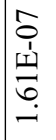 & $\begin{array}{l}\hat{S} \\
1 \\
1 \\
\text { 11 } \\
\\
-\end{array}$ & 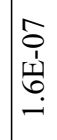 & 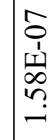 & $\begin{array}{c}\hat{o} \\
1 \\
1 \\
\\
\\
-1\end{array}$ & 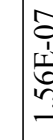 & 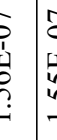 & 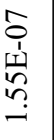 & 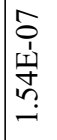 & 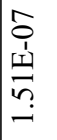 & 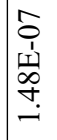 & 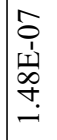 & 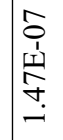 & 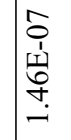 & 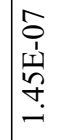 & 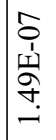 & $\begin{array}{c}\hat{0} \\
\mathbf{1} \\
\text { I1 } \\
\infty \\
\end{array}$ \\
\hline & & $\stackrel{\oplus}{\infty}$ & 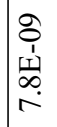 & $\begin{array}{c}2 \\
0 \\
n \\
n \\
n \\
r \\
r\end{array}$ & $\stackrel{2}{1}$ & & 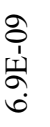 & 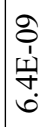 & 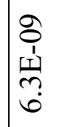 & 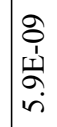 & 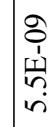 & 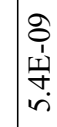 & $\begin{array}{l}8 \\
\text { 咅 } \\
\text { in }\end{array}$ & & 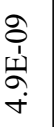 & 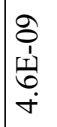 & 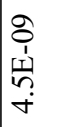 & 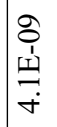 & 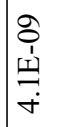 & $\begin{array}{l}\stackrel{\partial}{c} \\
\text { II } \\
\dot{1} \\
\dot{m}\end{array}$ & 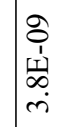 & $\begin{array}{l}o \\
0 \\
1 \\
\underline{1} \\
\dot{1} \\
\dot{r}\end{array}$ & 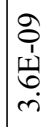 & $\begin{array}{l}\partial \\
\hat{o} \\
\overrightarrow{1} \\
\vec{i} \\
\vec{i}\end{array}$ \\
\hline & & 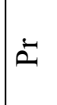 & 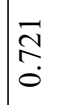 & 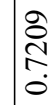 & $\begin{array}{l}\stackrel{2}{\tilde{I}} \\
\text { a }\end{array}$ & bे & ڤ્̀े & 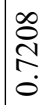 & 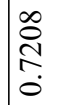 & 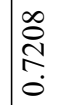 & 畩 & $\begin{array}{l}\infty \\
\stackrel{N}{N} \\
0\end{array}$ & 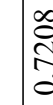 & $\begin{array}{l}\frac{8}{2} \\
\frac{2}{0} \\
\dot{0}\end{array}$ & $\begin{array}{l}\infty \\
\stackrel{0}{\mathbb{S}} \\
\dot{0}\end{array}$ & 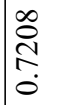 & 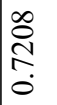 & 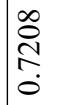 & 옹 & 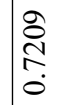 & 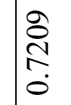 & 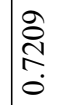 & $\stackrel{\vec{d}}{\stackrel{\Xi}{0}}$ & $\vec{N}$ \\
\hline & & $\widetilde{J}$ & $\begin{array}{l}\hat{\tilde{\Omega}} \\
\stackrel{\tilde{O}}{\tilde{O}} \\
\stackrel{\sim}{\sim}\end{array}$ & 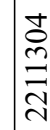 & $\begin{array}{l}5 \\
\text { है } \\
\text { s }\end{array}$ & & $\begin{array}{l}\infty \\
\stackrel{0}{\circ} \\
\stackrel{0}{0} \\
\infty\end{array}$ & 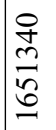 & 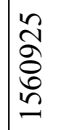 & 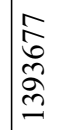 & 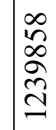 & $\begin{array}{l}\tilde{2} \\
\alpha \\
\infty \\
0 \\
= \\
=\end{array}$ & 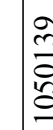 & 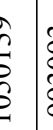 & ڤু & $\begin{array}{l}\infty \\
\stackrel{\infty}{n} \\
\stackrel{\infty}{\infty}\end{array}$ & 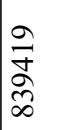 & 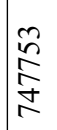 & $\begin{array}{l}\infty \\
\infty \\
i n \\
\end{array}$ & $\begin{array}{l}\tilde{\infty} \\
\tilde{D} \\
\tilde{f} \\
0\end{array}$ & 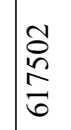 & $\begin{array}{l}n \\
\varnothing \\
\vdots \\
i n\end{array}$ & 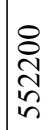 & 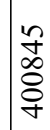 \\
\hline & & $t$ & 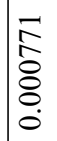 & 代 & ڤ્ & & $\begin{array}{l}\frac{n}{8} \\
\stackrel{8}{8} \\
0 \\
0\end{array}$ & $\begin{array}{l}\hat{8} \\
8 \\
0 \\
0\end{array}$ & $\begin{array}{l}\infty \\
\infty \\
0 \\
0 \\
\delta \\
0 \\
0\end{array}$ & \begin{tabular}{l}
$\infty$ \\
\multirow{2}{0}{} \\
$\delta$ \\
$\delta$ \\
0 \\
0
\end{tabular} & $\begin{array}{l}n \\
0 \\
8 \\
8 \\
8 \\
0\end{array}$ & 売 & वृ & م. & 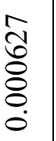 & \begin{tabular}{l}
0 \\
\hdashline \\
0 \\
8 \\
0 \\
0
\end{tabular} & $\begin{array}{l}2 \\
\hat{\sim} \\
\hat{\delta} \\
0 \\
0 \\
0\end{array}$ & 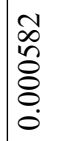 & $\begin{array}{l}n \\
i n \\
8 \\
8 \\
0 \\
0\end{array}$ & 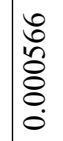 & $\mid \begin{array}{l}0 \\
n \\
n \\
\delta \\
\delta \\
0 \\
0\end{array}$ & 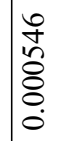 & $\begin{array}{l}n \\
n \\
2 \\
8 \\
8 \\
0 \\
0\end{array}$ & 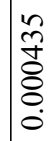 \\
\hline & & $\mathscr{\simeq}$ & $\begin{array}{l}\infty \\
\infty \\
\tilde{\infty}\end{array}$ & 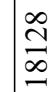 & $\begin{array}{l}0 \\
\infty \\
ٌ \\
\end{array}$ & & $\stackrel{\sqrt{2}}{\varrho}$ & $\stackrel{\mathbb{J}}{\stackrel{I}{I}}$ & 壳 & જू & 年 & $\begin{array}{l}\infty \\
\infty \\
0 \\
0\end{array}$ & है & $\hat{6}$ & С్ర్రి & ટ્ర్రి & $\begin{array}{l}0 \\
2 \\
\infty \\
n \\
n \\
2\end{array}$ & 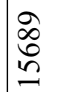 & 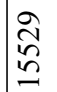 & $\begin{array}{l}\mathbb{N} \\
\tilde{n} \\
\Omega\end{array}$ & 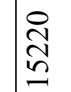 & 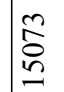 & $\widehat{\widehat{a}}$ & 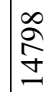 \\
\hline లే & $\tilde{z}$ & 兽 & $\tilde{\sim}$ & $\stackrel{\sim}{\hat{n}}$ & $\hat{m}$ & & $\begin{array}{l}\infty \\
\stackrel{\infty}{0} \\
\dot{r}\end{array}$ & $\hat{j}$ & $\frac{\Xi}{m}$ & ले & 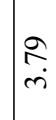 & $\vec{\sigma}$. & $\begin{array}{l}2 \\
0 \\
0\end{array}$ & b. & 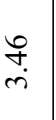 & $\stackrel{\infty}{\dot{m}}$ & $\underset{\dot{m}}{\stackrel{f}{f}}$ & $\begin{array}{l}n \\
\tilde{m} \\
\dot{n}\end{array}$ & $\begin{array}{l}\infty \\
i \\
i\end{array}$ & $\stackrel{\circ}{\stackrel{\text { in }}{ }}$ & $\stackrel{\vec{i}}{i}$ & $\begin{array}{l}\infty \\
\infty \\
i\end{array}$ & $\begin{array}{l}+ \\
\stackrel{\infty}{0} \\
i\end{array}$ & 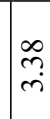 \\
\hline $\begin{array}{l}\frac{+}{\sigma} \\
\frac{\vec{U}}{\Xi} \\
\Xi \Xi\end{array}$ & 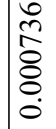 & $\vec{z}$ & 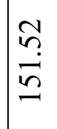 & $\begin{array}{l}\text { to } \\
\text { in }\end{array}$ & $\begin{array}{l}\text { b } \\
\text { in }\end{array}$ & & $\begin{array}{l}\text { : } \\
\text { nं }\end{array}$ & $\begin{array}{l}\approx \\
\hat{i} \\
i\end{array}$ & $\begin{array}{l}30 \\
\tilde{n} \\
n\end{array}$ & $\begin{array}{l}2 \\
\text { in }\end{array}$ & $\begin{array}{l}7 \\
f \\
i \\
i n\end{array}$ & f & $\begin{array}{l}0 \\
\text { in }\end{array}$ & : & $\begin{array}{l}m \\
\dot{+} \\
\dot{n}\end{array}$ & 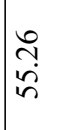 & $\begin{array}{l}\partial े \\
\text { ते } \\
\text { in }\end{array}$ & 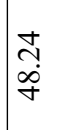 & 告 & 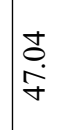 & $\frac{1}{\stackrel{5}{6}}$ & $\begin{array}{l}\tilde{n} \\
y \\
y\end{array}$ & $\mid$\begin{tabular}{l}
$\infty$ \\
\multirow{6}{+}{} \\
$\dot{\sigma}$
\end{tabular} & $\stackrel{2}{\stackrel{f}{+}}$ \\
\hline $\begin{array}{l}\stackrel{\mathscr{I}}{\tilde{U}} \\
\frac{\overrightarrow{0}}{\Xi}\end{array}$ & $\begin{array}{l}\stackrel{P}{f} \\
\text { 足 }\end{array}$ & 离 & 商 & $\overrightarrow{6}$ & $\begin{array}{l}q \\
i n\end{array}$ & & $\underset{\dot{n}}{\dot{n}}$ & $\begin{array}{l}\stackrel{J}{N} \\
\text { in }\end{array}$ & f & $\begin{array}{l}2 \\
\text { in } \\
\end{array}$ & i. & $\frac{7}{6}$ & $\mid \begin{array}{l}x \\
b \\
b \\
b\end{array}$ & $\begin{array}{l}8 \\
? \\
?\end{array}$ & 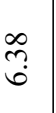 & $\stackrel{\infty}{\circ}$ & 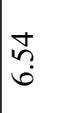 & $\begin{array}{l}\infty \\
i \\
i\end{array}$ & $\vec{n}$ & $\begin{array}{l}\stackrel{0}{2} \\
\text { in }\end{array}$ & $\begin{array}{l}f \\
\dot{\forall} \\
\text { in }\end{array}$ & $\begin{array}{l}\infty \\
0 \\
i \\
i\end{array}$ & $\begin{array}{l}8 \\
: \\
\text { in }\end{array}$ & $\mid \begin{array}{l}\infty \\
\infty \\
0\end{array}$ \\
\hline$\underset{\Xi}{\stackrel{\Xi}{\Xi}}$ & 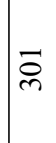 & 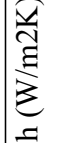 & $\begin{array}{l}\text { đo } \\
\stackrel{0}{0} \\
\stackrel{2}{2}\end{array}$ & $\begin{array}{l}n \\
\tilde{8} \\
\tilde{8}\end{array}$ & $\begin{array}{l}\text { in } \\
\text { Jै }\end{array}$ & & $\begin{array}{l}\stackrel{\circ}{+} \\
\text { ă }\end{array}$ & 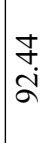 & $\begin{array}{l}+ \\
\infty \\
\dot{J}\end{array}$ & $\begin{array}{l}\infty \\
\infty \\
\infty \\
\alpha\end{array}$ & 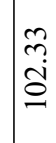 & $\begin{array}{l}+ \\
2 \\
2\end{array}$ & $\frac{1}{a}$ & $\frac{5}{2}$ & $\begin{array}{l}\bar{b} \\
\dot{\sigma} \\
\end{array}$ & $\begin{array}{l}\hat{a} \\
\text { dे }\end{array}$ & $\begin{array}{l}\stackrel{b}{0} \\
\stackrel{d}{\delta} \\
\delta\end{array}$ & $\begin{array}{l}\dot{0} \\
\dot{\alpha}\end{array}$ & $\frac{ \pm}{\sigma}$ & $\begin{array}{l}\hat{\alpha} \\
\tilde{\alpha}\end{array}$ & $\begin{array}{l}\text { f̀ } \\
\text { ¿̇ }\end{array}$ & $\frac{\partial}{\vec{\alpha}}$ & $\begin{array}{l}\hat{\alpha} \\
\dot{\alpha}\end{array}$ & $\frac{\tilde{\alpha}}{\dot{a}}$ \\
\hline$\sum_{\text {至 }}$ & 6 & $\begin{array}{l}\text { है } \\
\vdots \\
=\end{array}$ & $\begin{array}{l}3 \\
\stackrel{2}{2} \\
\stackrel{2}{n}\end{array}$ & $\frac{\text { ㄱ. }}{\stackrel{\alpha}{\sigma}}$ & 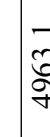 & t. & 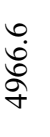 & $\begin{array}{l}\stackrel{g}{\dot{\delta}} \\
\text { ğ }\end{array}$ & $\begin{array}{l}\hat{\alpha} \\
\hat{\alpha}\end{array}$ & 弪 & $\frac{\text { Na }}{\grave{a}}$ & 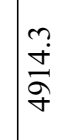 & §̊ & 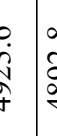 & $\begin{array}{l}\infty \\
\dot{\alpha} \\
\stackrel{\alpha}{\alpha} \\
\dot{\sigma}\end{array}$ & $\begin{array}{l}\overrightarrow{\dot{d}} \\
\infty \\
\infty \\
+\end{array}$ & 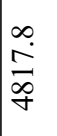 & 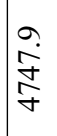 & 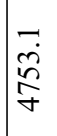 & 竎 & 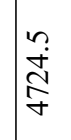 & 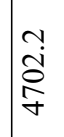 & 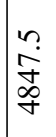 & 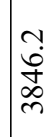 \\
\hline 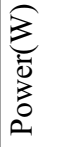 & $\begin{array}{l}n \\
\infty \\
+\infty\end{array}$ & 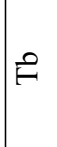 & 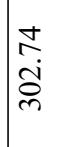 & $\begin{array}{l}\hat{y} \\
\infty \\
0 \\
0 \\
0\end{array}$ & $\frac{\tilde{A}}{\frac{m}{m}}$ & & $\frac{\tilde{a}}{m}$ & 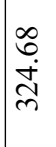 & $\begin{array}{l}0 \\
0 \\
0 \\
0 \\
m\end{array}$ & $\begin{array}{l}\hat{\sigma} \\
\dot{n} \\
\tilde{m}\end{array}$ & $\begin{array}{l}\exists \\
\dot{F} \\
\dot{m}\end{array}$ & $\begin{array}{l}n \\
n \\
0 \\
0 \\
m \\
m\end{array}$ & $\frac{8}{r}$ & 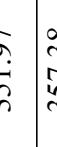 & $\begin{array}{l}\infty \\
\stackrel{\infty}{n} \\
\stackrel{n}{m} \\
m\end{array}$ & $\mid \begin{array}{l}\hat{A} \\
\text { id } \\
\text { din }\end{array}$ & $\begin{array}{l}= \\
= \\
0 \\
0 \\
0 \\
0\end{array}$ & $\begin{array}{l}\infty \\
m \\
\stackrel{n}{m} \\
m\end{array}$ & $\begin{array}{l}\sigma \\
\infty \\
\infty \\
\infty \\
m\end{array}$ & $\begin{array}{l}\infty \\
\infty \\
\dot{\infty} \\
\infty\end{array}$ & $\mid \begin{array}{l}\mathscr{8} \\
\ddot{\alpha} \\
\infty \\
\infty\end{array}$ & 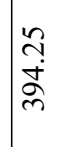 & 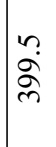 & 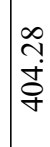 \\
\hline$\sum_{0}^{\pi}$ & $\stackrel{m}{0}$ & 3 & $\begin{array}{l}\hat{0} \\
\tilde{n} \\
\tilde{n}\end{array}$ & N̦ & 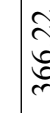 & & 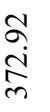 & 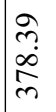 & 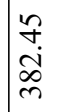 & $\begin{array}{l}\hat{\gamma} \\
\dot{\infty} \\
\infty\end{array}$ & 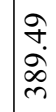 & $\begin{array}{l}\text { N } \\
\text { à } \\
\text { mे }\end{array}$ & $\begin{array}{l}\vec{v} \\
8\end{array}$ & 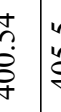 & \begin{tabular}{l}
$n$ \\
\hdashline \\
$\vdots$ \\
$\vdots$
\end{tabular} & 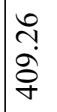 & $\begin{array}{l}\infty \\
\stackrel{n}{n} \\
\forall\end{array}$ & $\begin{array}{l}\infty \\
0 \\
\stackrel{+}{+} \\
\text { f }\end{array}$ & 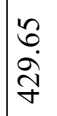 & $\begin{array}{l}\vec{\nabla} \\
\dot{\vec{f}} \\
\stackrel{+}{*}\end{array}$ & $\frac{n}{2}$ & 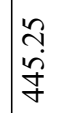 & 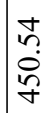 & \begin{tabular}{l}
$n$ \\
$n$ \\
0 \\
\multirow{7}{7}{} \\
\end{tabular} \\
\hline $\begin{array}{l}\mathscr{y} \\
\tilde{z} \\
\tilde{z}\end{array}$ & $\infty$ & $\stackrel{\rho}{a}$ & $N$ & $\vec{\infty}$ & $\bar{I}$ & $t$ & ָุ' & సֶ. & 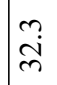 & $\begin{array}{l}m \\
\infty \\
m\end{array}$ & $\underset{f}{\stackrel{f}{f}}$ & में & $\begin{array}{l}\text { s } \\
\text { है }\end{array}$ & $\begin{array}{r}? \\
: \\
:\end{array}$ & הֶ & $\mid \begin{array}{l}n \\
\infty \\
0\end{array}$ & $\begin{array}{l}\stackrel{\bullet}{0} \\
\stackrel{I}{I}\end{array}$ & $\begin{array}{l}0 \\
\dot{\infty}\end{array}$ & $\hat{\infty}$ & $\hat{i}$ & $\mid \begin{array}{l}\infty \\
\infty \\
\alpha\end{array}$ & $\begin{array}{l}\infty \\
\stackrel{0}{0}\end{array}$ & 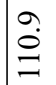 & \\
\hline
\end{tabular}




\begin{tabular}{|c|c|c|c|c|c|c|c|c|c|c|c|c|c|c|c|c|c|c|c|c|c|c|c|}
\hline & & $\vec{v}$ & 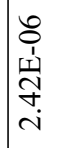 & $\begin{array}{l}8 \\
1 \\
1 \\
1 \\
1 \\
i\end{array}$ & 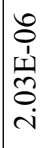 & 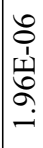 & 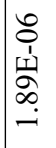 & 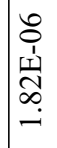 & 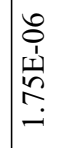 & 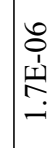 & 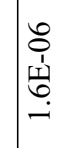 & 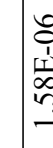 & 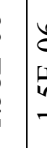 & 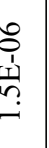 & 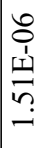 & 总 & 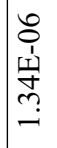 & 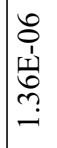 & 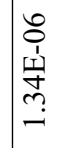 & 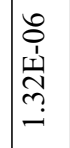 & 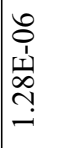 & 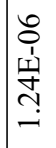 & $\begin{array}{r}\hat{0} \\
\mathbf{1} \\
\text { 11 } \\
0 \\
0 \\
\end{array}$ \\
\hline & & $\stackrel{\oplus}{\infty}$ & \begin{tabular}{|l}
$\hat{0}$ \\
$\hat{1}$ \\
\\
$\infty$ \\
$\infty$ \\
$\dot{\gamma}$
\end{tabular} & $\begin{array}{l}\hat{0} \\
\hat{1} \\
\omega \\
\\
\tilde{n} \\
\tilde{c}\end{array}$ & $\begin{array}{l}\hat{o} \\
\text { 1े } \\
\hat{n} \\
\text { in } \\
\text { in }\end{array}$ & 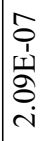 & 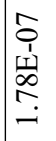 & 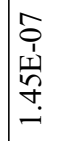 & $\begin{array}{l}\hat{0} \\
\hat{1} \\
\underline{1} \\
\\
\end{array}$ & 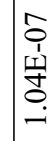 & $\begin{array}{c}\infty \\
0 \\
1 \\
1 \\
5 \\
\infty \\
\infty \\
\infty\end{array}$ & 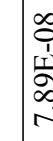 & & 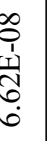 & $\begin{array}{l}0 \\
0 \\
0 \\
1 \\
\text { t1 } \\
0 \\
0 \\
0\end{array}$ & 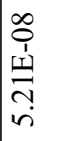 & $\begin{array}{l}\infty \\
0 \\
1 \\
11 \\
\infty \\
i n \\
\forall\end{array}$ & 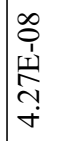 & $\begin{array}{l}\infty \\
\infty \\
\dot{1} \\
\omega \\
\sigma \\
\sigma \\
\dot{m}\end{array}$ & 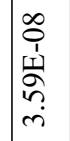 & 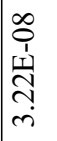 & 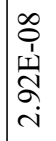 & $\begin{array}{c}\infty \\
0 \\
0 \\
\underline{1} \\
\\
\\
\end{array}$ \\
\hline & & 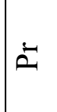 & $\overrightarrow{\widetilde{N}}$ & 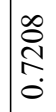 & 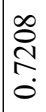 & 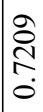 & $\underset{\stackrel{N}{\mathbb{N}}}{\stackrel{N}{0}}$ & 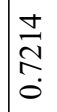 & 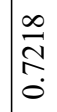 & 离 & 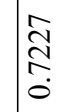 & $\tilde{\approx}$ & مَ & & 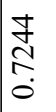 & 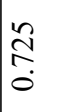 & $\begin{array}{l}\stackrel{n}{2} \\
\stackrel{\mathbb{N}}{0} \\
\dot{0}\end{array}$ & $\begin{array}{l}\overrightarrow{0} \\
\stackrel{1}{0} \\
\dot{0}\end{array}$ & $\begin{array}{l}\hat{\sigma} \\
\stackrel{2}{\hat{O}} \\
0\end{array}$ & $\mid \begin{array}{l}\stackrel{n}{N} \\
\stackrel{N}{0}\end{array}$ & 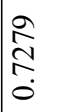 & $\begin{array}{l}n \\
\infty \\
\stackrel{N}{N} \\
\dot{0}\end{array}$ & 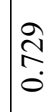 \\
\hline & & $\widetilde{J}$ & 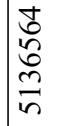 & 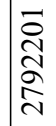 & 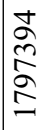 & $\begin{array}{l}\text { on } \\
\stackrel{n}{2} \\
\hat{\sigma} \\
=\end{array}$ & 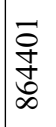 & ¿े & 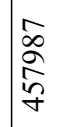 & $\begin{array}{l}\stackrel{g}{o ̂} \\
D \\
\infty \\
\tilde{n}\end{array}$ & 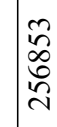 & $\mid \begin{array}{l}\infty \\
\infty \\
0 \\
0 \\
0\end{array}$ & 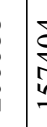 & $\begin{array}{l}\text { do } \\
\text { a } \\
n \\
n\end{array}$ & 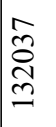 & 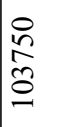 & 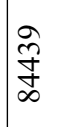 & $\frac{\vec{N}}{\stackrel{N}{r}}$ & $\begin{array}{l}\vec{D} \\
\mathbb{Z}\end{array}$ & 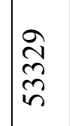 & 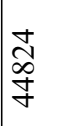 & $\begin{array}{l}\hat{े} \\
\infty \\
\infty\end{array}$ & $\frac{\vec{\sigma}}{2}$ \\
\hline & & t & 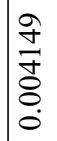 & $\begin{array}{l}n \\
\tilde{n} \\
\tilde{\delta} \\
\delta \\
0 \\
0\end{array}$ & 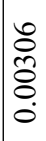 & 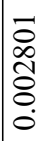 & 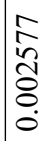 & 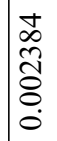 & $\begin{array}{l}\tilde{\tilde{I}} \\
\widetilde{\Xi} \\
\tilde{\Xi} \\
0 \\
0\end{array}$ & 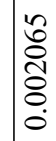 & $\begin{array}{l}\infty \\
\infty \\
\varnothing \\
0 \\
0\end{array}$ & 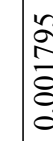 & & $\begin{array}{l}0 \\
0 \\
0 \\
0 \\
8 \\
0 \\
0\end{array}$ & \begin{tabular}{l}
$\infty$ \\
\multirow{0}{0}{} \\
$\stackrel{8}{8}$ \\
0 \\
0
\end{tabular} & 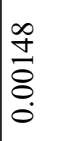 & $\begin{array}{l}\vec{\infty} \\
\infty \\
\tilde{8} \\
\dot{0} \\
\dot{0}\end{array}$ & 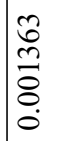 & $\begin{array}{l}\frac{\infty}{1} \\
\frac{m}{8} \\
\stackrel{8}{0} \\
0\end{array}$ & 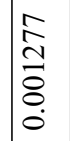 & 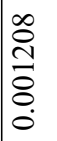 & 苦 & 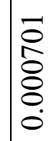 \\
\hline & & $\mathscr{\sim}$ & 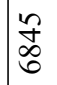 & ठิ & 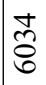 & $\underset{\substack{\infty \\
i n \\
i n}}{ }$ & \begin{tabular}{l}
\multirow{0}{0}{} \\
市
\end{tabular} & $\begin{array}{l}\text { } \\
\hat{\imath} \\
\tilde{n}\end{array}$ & 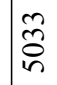 & 志 & ôे & 资 & & \begin{tabular}{l}
$\infty$ \\
\multirow{y}{*}{}
\end{tabular} & $\vec{F}$ & 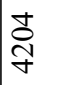 & $\frac{\partial}{\partial}$ & $\overrightarrow{\widetilde{\sigma}}$ & $\begin{array}{l}\mathscr{D} \\
2 \\
\tilde{m}\end{array}$ & $\begin{array}{l}\hat{n} \\
\infty \\
\infty\end{array}$ & $\frac{\tilde{\infty}}{\infty}$ & $\frac{n}{\frac{n}{m}}$ & 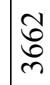 \\
\hline లే & $\tilde{z}$ & 莺 & $\stackrel{7}{a}$ & $\hat{o}$ & $\ddot{o}$ & $\stackrel{0}{\circ}$ & $\tilde{o}$ & 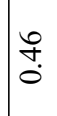 & $\stackrel{?}{f}$ & $\stackrel{?}{\stackrel{2}{\circ}}$ & ְి & ra & & 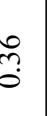 & 吕 & 节 & $\stackrel{m}{\dddot{0}}$ & 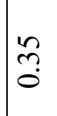 & $\begin{array}{l}\infty \\
\tilde{0} \\
0\end{array}$ & F. & fo & 守. & $\begin{array}{l}\mathbf{b} \\
\text { i }\end{array}$ \\
\hline $\begin{array}{l}\frac{+}{\sigma} \\
\frac{\vec{\omega}}{\Xi} \\
\Xi\end{array}$ & 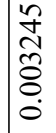 & $\vec{z}$ & $\stackrel{\infty}{\stackrel{\infty}{\infty}}$ & $\begin{array}{l}f \\
\text { ते }\end{array}$ & $\begin{array}{l}\overline{0} \\
\vec{\lambda}\end{array}$ & \begin{tabular}{l}
$\infty$ \\
$\infty$ \\
$\infty$ \\
\hdashline
\end{tabular} & $\stackrel{n}{n}$ & $\underset{\sim}{\tilde{\sigma}}$ & $\begin{array}{l}\dot{\partial} \\
\ddot{g}\end{array}$ & $\stackrel{\text { mे }}{\stackrel{-}{ \pm}}$ & $\begin{array}{l}\tilde{O} \\
\stackrel{\simeq}{\simeq}\end{array}$ & \begin{tabular}{c}
$\Delta$ \\
\multicolumn{7}{c}{}
\end{tabular} & & $\stackrel{n}{E}$ & $\stackrel{\text { }}{\Xi}$ & 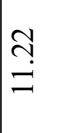 & $\stackrel{ }{0}$ & $\stackrel{\vartheta}{\exists}$ & $\stackrel{n}{n}$ & $\stackrel{\circ}{\varrho}$ & $\stackrel{\circ}{\stackrel{2}{=}}$ & 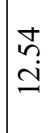 & $\begin{array}{l}\hat{\alpha} \\
\hat{\lambda}\end{array}$ \\
\hline $\begin{array}{l}\frac{\mathscr{a}}{\underline{0}} \\
\frac{\overrightarrow{0}}{\Xi}\end{array}$ & ָૂ & 泀 & $\begin{array}{l}\tilde{N} \\
\text { in }\end{array}$ & $\stackrel{ }{ت}$ & $\stackrel{\Xi}{\stackrel{\Xi}{ }}$ & : & $\stackrel{8}{0}$ & Oे. & $\hat{o}$ & $\hat{\delta}$ & م્ & $\tilde{\alpha}$ & & ס. & oे & $\begin{array}{l}\infty \\
\stackrel{0}{0}\end{array}$ & $\begin{array}{l}\text { के } \\
0 \\
0\end{array}$ & $\stackrel{\infty}{\infty}$ & $\stackrel{g}{\circ}$ & $\vec{\beth}$ & $\stackrel{?}{-}$ & กี & $\mid \begin{array}{l}\infty \\
\infty \\
\infty \\
\infty\end{array}$ \\
\hline 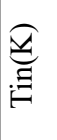 & \& & 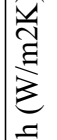 & 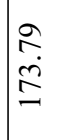 & $\frac{m}{n}$ & $\begin{array}{l}\dot{J} \\
\dot{f}\end{array}$ & $\begin{array}{l}\hat{0} \\
\dot{9}\end{array}$ & $\frac{a}{\frac{1}{m}}$ & $\begin{array}{l}2 \\
\hat{n} \\
\tilde{n}\end{array}$ & $\begin{array}{l}\infty \\
\infty \\
\dot{m}\end{array}$ & 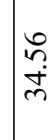 & $\frac{\hat{\sigma}}{m}$ & $\frac{\delta}{\rho}$ & & $\frac{a}{m}$ & $\begin{array}{l}\infty \\
\infty \\
i \\
i \\
n\end{array}$ & $\frac{\vec{b}}{\dot{m}}$ & $\bar{m}$ & $\begin{array}{l}\hat{N} \\
\tilde{n}\end{array}$ & $\begin{array}{l}n \\
n \\
n\end{array}$ & 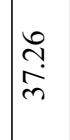 & $\underset{\substack{ \pm \infty \\
\infty}}{ \pm}$ & $\mid \begin{array}{l}\infty \\
\infty \\
\stackrel{+}{+} \\
\end{array}$ & 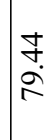 \\
\hline$\sum_{\text {忘 }}$ & $\tilde{Z}$ & $\begin{array}{l}\text { đิ } \\
\vdots \\
\vdots \\
=\end{array}$ & ? & 妾 & $\begin{array}{l}0 \\
\dot{0} \\
\infty \\
\alpha \\
\sigma\end{array}$ & 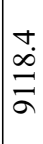 & $\begin{array}{l}\vec{\infty} \\
\tilde{2} \\
\stackrel{2}{2}\end{array}$ & $\begin{array}{l}0 \\
\stackrel{+}{\hat{D}} \\
\infty\end{array}$ & $\begin{array}{l}0 \\
\dot{8} \\
0 \\
\infty \\
\infty\end{array}$ & $\mid \begin{array}{l}n \\
\infty \\
\infty \\
\infty \\
\infty\end{array}$ & $\begin{array}{l}0 \\
\infty \\
\dot{0} \\
\dot{\infty}\end{array}$ & $\frac{z}{\alpha}$ & $\begin{array}{l}5 \\
\vdots \\
\vdots \\
\vdots \\
r\end{array}$ & $\begin{array}{l}0 \\
\dot{0} \\
0 \\
\infty \\
\\
\end{array}$ & $\begin{array}{l}\hat{n} \\
2 \\
2\end{array}$ & $\begin{array}{l}0 \\
\stackrel{0}{0} \\
\stackrel{0}{2} \\
\end{array}$ & ڤ̊ & 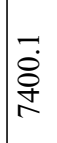 & $\begin{array}{l}\hat{i} \\
\hat{n}\end{array}$ & $\mid \begin{array}{l}\tilde{a} \\
\infty \\
\check{n} \\
\infty\end{array}$ & 离 & $\begin{array}{l}\vec{n} \\
\stackrel{\infty}{2} \\
\stackrel{8}{r}\end{array}$ & $\begin{array}{l}\tilde{n} \\
\stackrel{\alpha}{\xi}\end{array}$ \\
\hline 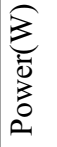 & $\stackrel{8}{\circ}$ & 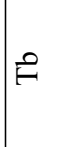 & 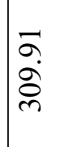 & $\begin{array}{l}\partial \\
\text { aे } \\
\text { ले }\end{array}$ & 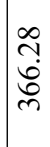 & $\tilde{\text { }}$ & $\frac{\partial}{\partial}$ & \begin{tabular}{l}
$\infty$ \\
$\infty$ \\
$\dot{f}$ \\
\multirow{f}{f}{}
\end{tabular} & $\begin{array}{l}\sigma \\
\hat{\sigma} \\
\dot{q}\end{array}$ & $\begin{array}{l}\hat{n} \\
\text { gं } \\
\text { f }\end{array}$ & $\begin{array}{l}\stackrel{w}{m} \\
\infty \\
\bar{n}\end{array}$ & $\begin{array}{l}4 \\
7 \\
5\end{array}$ & 8 & $\begin{array}{l}\hat{b} \\
\text { ț } \\
n\end{array}$ & $\begin{array}{l}\tilde{f} \\
\dot{8} \\
\infty \\
\infty \\
n\end{array}$ & $\begin{array}{l}\stackrel{J}{A} \\
\infty \\
0 \\
0 \\
0\end{array}$ & 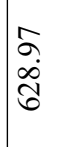 & f̊ & $\begin{array}{l}5 \\
\dot{0} \\
\dot{8}\end{array}$ & $\begin{array}{l}n \\
\hat{n} \\
0 \\
0\end{array}$ & $\frac{\pi}{0}$ & $\begin{array}{l}\tilde{n} \\
\stackrel{n}{2} \\
\stackrel{n}{r}\end{array}$ & 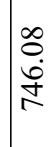 \\
\hline$\sum_{0}^{\pi}$ & $\stackrel{m}{0}$ & 3 & $\frac{m}{\stackrel{n}{n}}$ & $\begin{array}{l}n \\
\tilde{n} \\
\tilde{0} \\
\tilde{n}\end{array}$ & 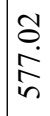 & 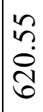 & $\begin{array}{l}+2 \\
2 \\
\tilde{\sigma} \\
0\end{array}$ & $\begin{array}{l}\stackrel{2}{1} \\
\stackrel{1}{8} \\
\stackrel{0}{0}\end{array}$ & $\frac{a}{\circ}$ & $\begin{array}{l}\hat{\infty} \\
0 \\
\hat{n} \\
\hat{n}\end{array}$ & $\stackrel{\mathbb{N}}{\mathbb{N}}$ & $\begin{array}{l}0 \\
\text { ? }\end{array}$ & $\frac{\delta}{\delta}$ & $\begin{array}{l}\dot{\Phi} \\
\dot{\Phi} \\
\dot{\infty}\end{array}$ & $\begin{array}{l}0 \\
n \\
\infty \\
\infty \\
\infty \\
\infty\end{array}$ & $\mid \begin{array}{l}n \\
n \\
n \\
\infty \\
\infty\end{array}$ & 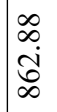 & 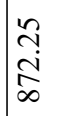 & $\begin{array}{l}\mathcal{I} \\
\stackrel{\circ}{\circ} \\
\infty \\
\infty\end{array}$ & $\begin{array}{l}\infty \\
\infty \\
\infty \\
\infty \\
\infty\end{array}$ & $\begin{array}{l}0 \\
2 \\
2 \\
\infty\end{array}$ & $\begin{array}{l}\hat{\sigma} \\
\text { ஸे } \\
\alpha\end{array}$ & 离. \\
\hline $\begin{array}{l}\mathscr{y} \\
\tilde{z} \\
\tilde{z}\end{array}$ & $a$ & $\stackrel{\rho}{a}$ & $N$ & $\vec{\infty}^{\infty}$ & 王 & $\dot{\sim}$ & సֶ. & 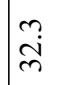 & $\begin{array}{l}m \\
\infty \\
m\end{array}$ & $\stackrel{+}{\stackrel{f}{+}}$ & 苛 & & 4 & חֶ & $\mid \begin{array}{l}n \\
\infty \\
0\end{array}$ & $\begin{array}{l}\stackrel{\bullet}{0} \\
\dot{I}\end{array}$ & $\begin{array}{l}0 \\
\dot{\infty}\end{array}$ & $\hat{\infty}$ & $\hat{\check{a}}$ & $\begin{array}{l}\infty \\
\infty \\
\infty\end{array}$ & $\begin{array}{l}\infty \\
\dot{0} \\
\stackrel{0}{0}\end{array}$ & $\stackrel{\grave{O}}{\Xi}$ & $\stackrel{a}{\sigma}$ \\
\hline
\end{tabular}




\begin{tabular}{|c|c|c|c|c|c|c|c|c|c|c|c|c|c|c|c|c|c|c|c|c|c|c|c|}
\hline & & $\gtrsim$ & 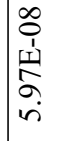 & 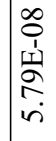 & 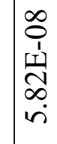 & $\begin{array}{l}\infty \\
0 \\
1 \\
\frac{1}{2} \\
2 \\
i\end{array}$ & & $\begin{array}{l}\infty \\
0 \\
\text { 11 } \\
\text { ț } \\
i n \\
i n\end{array}$ & $\begin{array}{l}\infty \\
0 \\
0 \\
1 \\
\text { 采 } \\
\text { in } \\
\text { in }\end{array}$ & $\begin{array}{l}\infty \\
0 \\
o \\
1 \\
\\
\\
i \\
i n\end{array}$ & 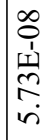 & 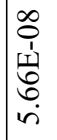 & $\begin{array}{l}\infty \\
0 \\
\hat{1} \\
1 \\
\hat{1} \\
0 \\
\text { in } \\
\text { n. }\end{array}$ & $\begin{array}{c}\infty \\
0 \\
0 \\
1 \\
1 \\
⿱ 1 \\
0 \\
i n \\
i n\end{array}$ & 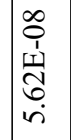 & 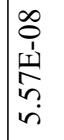 & 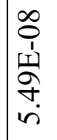 & \begin{tabular}{|l}
$\infty$ \\
0 \\
0 \\
1 \\
1 \\
$n$ \\
$n$ \\
$n$
\end{tabular} & $\begin{array}{l}\infty \\
0 \\
1 \\
1 \\
\infty \\
\infty \\
1 \\
\dot{1} \\
\dot{n}\end{array}$ & 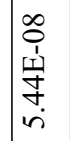 & 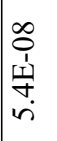 & 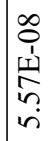 & $\begin{array}{l}\infty \\
0 \\
1 \\
1 \\
\\
0 \\
\dot{+}\end{array}$ \\
\hline & & $\stackrel{\oplus}{\infty}$ & 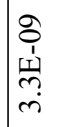 & $\begin{array}{l}2 \\
\hat{1} \\
\underline{1} \\
\dot{\mu} \\
\dot{m}\end{array}$ & $\begin{array}{l}\hat{\partial} \\
\hat{1} \\
\text { 12 } \\
\hat{i}\end{array}$ & $\begin{array}{l}2 \\
\frac{1}{1} \\
\frac{1}{2} \\
2\end{array}$ & & $\begin{array}{l}\hat{a} \\
\hat{1} \\
\text { İ } \\
\text { ì }\end{array}$ & 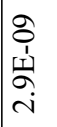 & $\begin{array}{l}\stackrel{\partial}{o} \\
\stackrel{1}{\omega} \\
\stackrel{1}{i} \\
i\end{array}$ & 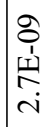 & 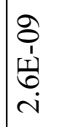 & 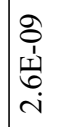 & 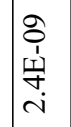 & 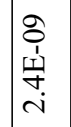 & 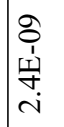 & 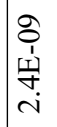 & 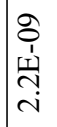 & 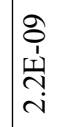 & $\begin{array}{l}\text { oे } \\
\hat{1} \\
\text { N1 } \\
\text { in }\end{array}$ & 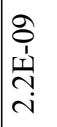 & 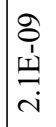 & 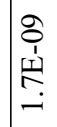 \\
\hline & & 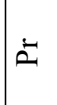 & 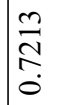 & $\underset{\mathbb{N}}{\stackrel{N}{0}}$ & 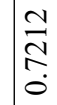 & İ & & $\begin{array}{l}\frac{N}{N} \\
\stackrel{N}{0}\end{array}$ & 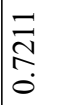 & $\underset{\overrightarrow{\mathbb{N}}}{\stackrel{\vec{N}}{0}}$ & 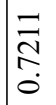 & 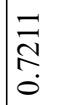 & 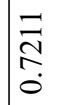 & $\underset{\vec{N}}{\vec{N}}$ & $\begin{array}{l}\overrightarrow{\bar{N}} \\
\stackrel{\mathbb{0}}{0}\end{array}$ & 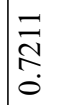 & $\stackrel{\vec{N}}{\stackrel{0}{0}}$ & $\overrightarrow{\mathbb{N}}$ & $\underset{\stackrel{\vec{N}}{0}}{\stackrel{0}{0}}$ & $\underset{\mathbb{N}}{\stackrel{\overrightarrow{0}}{0}}$ & $\overrightarrow{\mathbb{N}}$ & $\overrightarrow{\mathbb{N}}$ & $\overrightarrow{\stackrel{d}{o}}$ \\
\hline & & $\widetilde{J}$ & 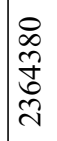 & $\begin{array}{l}\widetilde{\Omega} \\
\tilde{N} \\
\tilde{స}\end{array}$ & 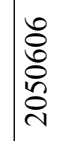 & 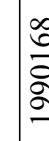 & & $\frac{\wp}{\sigma}$ & 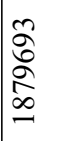 & 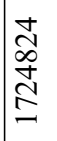 & $\begin{array}{l}\overrightarrow{1} \\
\stackrel{\hat{\Omega}}{\hat{\sigma}} \\
\underline{\sigma}\end{array}$ & $\begin{array}{l}\tilde{2} \\
\hat{\sigma} \\
\sigma \\
\sigma\end{array}$ & 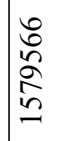 & $\begin{array}{l}\frac{m}{m} \\
\stackrel{2}{f} \\
\text { J }\end{array}$ & 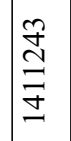 & 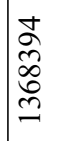 & 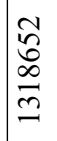 & 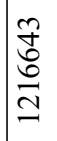 & $\begin{array}{l}\text { f } \\
\infty \\
0 \\
0 \\
0 \\
=\end{array}$ & $\begin{array}{l}\infty \\
\infty \\
\tilde{n} \\
\\
\equiv\end{array}$ & 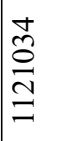 & 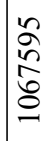 & $\underset{\substack{n \\
\infty}}{\stackrel{\vec{n}}{\infty}}$ \\
\hline & & t & 设 & 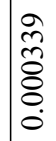 & $\begin{array}{l}\infty \\
\tilde{m} \\
\tilde{\delta} \\
\delta \\
0\end{array}$ & 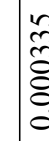 & & $\begin{array}{l}\vec{m} \\
\tilde{\hat{\delta}} \\
\delta \\
\dot{0}\end{array}$ & 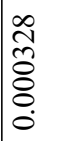 & 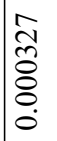 & 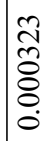 & 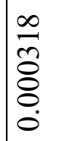 & $\begin{array}{l}\frac{n}{0} \\
\frac{\hat{\delta}}{8} \\
0 \\
0\end{array}$ & $\begin{array}{l}m \\
\frac{m}{m} \\
\delta \\
\delta \\
0\end{array}$ & $\begin{array}{l}\overrightarrow{\tilde{\delta}} \\
\bar{\delta} \\
\dot{0}\end{array}$ & 总 & ڤ్̊ి & 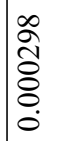 & 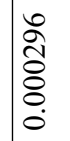 & 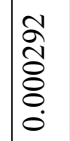 & 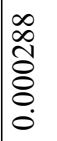 & 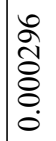 & 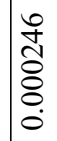 \\
\hline & & $\mathscr{\simeq}$ & $\begin{array}{l}\hat{0} \\
\hat{n} \\
\hat{n}\end{array}$ & $\underset{\widetilde{\sigma}}{\stackrel{\Xi}{\sigma}}$ & $\begin{array}{l}\tilde{N} \\
\tilde{N} \\
\tilde{N}\end{array}$ & 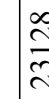 & & 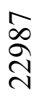 & 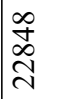 & 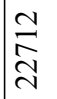 & 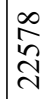 & 导 & $\underset{\widetilde{\pi}}{\stackrel{0}{\widetilde{N}}}$ & $\begin{array}{l}\infty \\
\stackrel{\infty}{\vec{N}}\end{array}$ & 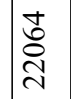 & $\overrightarrow{\vec{N}}$ & 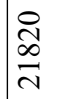 & $\frac{\widetilde{\Omega}}{\stackrel{\widetilde{N}}{\sim}}$ & $\begin{array}{l}\infty \\
\infty \\
n \\
i \\
\end{array}$ & 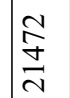 & $\begin{array}{l}\dot{8} \\
\frac{0}{2}\end{array}$ & $\underset{\underset{\sim}{\sim}}{\stackrel{f}{二}}$ & $\underset{ন}{J}$ \\
\hline$\tilde{\Xi}$ & $\tilde{z}$ & 莺 & $\mid \begin{array}{c}\tilde{1} \\
\infty \\
\infty \\
m\end{array}$ & $\stackrel{\hat{r}}{i}$ & $\begin{array}{l}\underset{+}{*} \\
i\end{array}$ & in & & $\begin{array}{l}\underset{+}{+} \\
+ \\
+\end{array}$ & $\frac{O}{i n}$ & . & तె & $\begin{array}{l}\approx \\
\text { in }\end{array}$ & o. & 芯 & へֶ. & $\begin{array}{l}\infty \\
\dot{b} \\
\dot{n}\end{array}$ & ก̂ & $\stackrel{\infty}{\stackrel{\infty}{\stackrel{f}{*}}}$ & $\begin{array}{l}\mathscr{n} \\
\sim \\
+\end{array}$ & 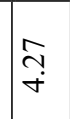 & $\stackrel{亏}{\vec{f}}$ & $\stackrel{\infty}{\stackrel{\infty}{+}}$ & $\stackrel{\hat{q}}{\dot{q}}$ \\
\hline $\begin{array}{l}\frac{+}{\sigma} \\
\frac{\vec{U}}{\Xi} \\
\Xi\end{array}$ & 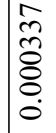 & $\vec{z}$ & 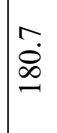 & $\begin{array}{l}n \\
n \\
\infty \\
0\end{array}$ & $\begin{array}{l}\text { f } \\
\dot{f}\end{array}$ & $\mid \begin{array}{l}0 \\
1 \\
1 \\
0\end{array}$ & & $\begin{array}{l}0 \\
\frac{0}{6}\end{array}$ & 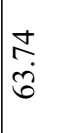 & aे & $\begin{array}{l}n \\
\text { กิ } \\
8\end{array}$ & $\underset{i}{i}$ & $\begin{array}{l}\not{n} \\
\infty \\
\infty \\
0\end{array}$ & 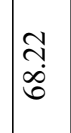 & 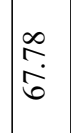 & $\begin{array}{l}0 \\
0 \\
\dot{b}\end{array}$ & $\begin{array}{l}\text { Ș } \\
\text { in }\end{array}$ & $\begin{array}{l}8 \\
\dot{0} \\
i\end{array}$ & 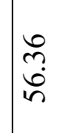 & $\begin{array}{l}8 \\
\text { in } \\
\text { in }\end{array}$ & 离 & $\begin{array}{l}3 \\
0 \\
i n\end{array}$ & \begin{tabular}{l}
$\infty$ \\
\multirow{+}{*}{} \\
$\tilde{n}$ \\
$\tilde{n}$
\end{tabular} \\
\hline $\begin{array}{l}\mathscr{\mathscr { I }} \\
\frac{\overrightarrow{0}}{\Xi}\end{array}$ & $\begin{array}{l}\overrightarrow{\vec{b}} \\
\underset{\sim}{\sim}\end{array}$ & 离 & $\infty$ & $\begin{array}{l}0 \\
2 \\
a\end{array}$ & $\vec{a}$ & $\underset{\infty}{\infty}$ & & $\underset{\infty}{\vec{\infty}}$ & $\vec{\infty}$ & $\stackrel{t}{\stackrel{0}{0}}$ & $\stackrel{\varrho}{\varrho}$ & $\begin{array}{l}0 \\
\\
0\end{array}$ & $\begin{array}{l}\text { S. } \\
\bigcirc \\
0\end{array}$ & $\bar{n}$ & $\mid \begin{array}{l}\infty \\
0 \\
= \\
=\end{array}$ & $=$ & $\underset{\infty}{\Delta}$ & $\begin{array}{l}0 \\
\infty \\
\infty\end{array}$ & $\underset{\substack{J \\
\infty}}{\mathbb{N}}$ & $\stackrel{0}{\stackrel{2}{r}}$ & 文 & $\mid \begin{array}{l}n \\
\infty \\
\infty\end{array}$ & ๙a \\
\hline$\underset{\Xi}{\stackrel{\Xi}{\Xi}}$ & $\underset{m}{\stackrel{2}{0}}$ & 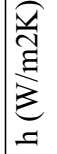 & 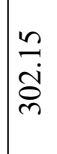 & $\begin{array}{l}\tilde{m} \\
ٍ \\
=\end{array}$ & $\frac{ \pm}{\partial}$ & $\begin{array}{l}0 \\
0 \\
0 \\
0\end{array}$ & & 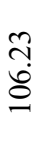 & ஜ̊. & సֶ. & 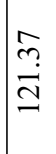 & $\begin{array}{l}\bar{\sigma} \\
\infty \\
=\end{array}$ & 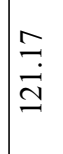 & 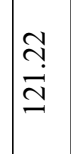 & $\underset{\Xi}{\Xi}$ & $\begin{array}{l}\hat{\infty} \\
ٍ \\
ٍ \\
=\end{array}$ & $\begin{array}{l}m \\
\stackrel{0}{0}\end{array}$ & $\begin{array}{l}\sigma \\
\hat{\sigma} \\
\hat{\sigma}\end{array}$ & $\begin{array}{l}\stackrel{2}{ } \\
\stackrel{0}{0} \\
0\end{array}$ & $\underset{\dot{\Xi}}{\stackrel{\Xi}{0}}$ & fr & 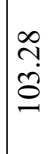 & 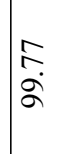 \\
\hline$\sum_{\text {至 }}$ & $\overrightarrow{6}$ & $\begin{array}{l}\text { वै } \\
\text { है } \\
=-\end{array}$ & $\begin{array}{l}\infty \\
\dot{0} \\
0 \\
0 \\
0\end{array}$ & $\begin{array}{l}n \\
\hat{0} \\
\stackrel{2}{2}\end{array}$ & $\begin{array}{l}\stackrel{\infty}{2} \\
\grave{2}\end{array}$ & \begin{tabular}{l}
$\bar{n}$ \\
\multirow{2}{n}{}
\end{tabular} & & $\begin{array}{l}n \\
\vdots \\
0 \\
\grave{v}\end{array}$ & 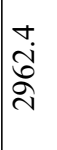 & $\begin{array}{l}n \\
\stackrel{n}{a} \\
\stackrel{2}{2}\end{array}$ & $\mid$\begin{tabular}{l}
0 \\
$\dot{0}$ \\
\multirow{2}{1}{}
\end{tabular} & $\begin{array}{l}\text { ָे } \\
\infty \\
\check{\hat{N}}\end{array}$ & $\begin{array}{l}0 \\
\infty \\
\check{N} \\
\check{\hat{N}}\end{array}$ & $\begin{array}{l}\overrightarrow{\dot{0}} \\
\grave{2} \\
\grave{\hat{n}}\end{array}$ & $\begin{array}{l}\hat{\dot{m}} \\
\hat{\tilde{\nu}}\end{array}$ & $\begin{array}{l}0 \\
\stackrel{+}{\mathbf{N}}\end{array}$ & $\begin{array}{l}\hat{\sigma} \\
\stackrel{0}{0} \\
\infty \\
\text { v }\end{array}$ & $\begin{array}{l}n \\
\sim \\
\infty \\
\infty \\
\infty\end{array}$ & $\begin{array}{l}\hat{\sigma} \\
\dot{\infty} \\
\infty \\
\infty \\
\sim\end{array}$ & $\begin{array}{l}0 \\
\dot{\otimes} \\
\dot{0} \\
\infty \\
\infty \\
\sim\end{array}$ & $\begin{array}{l}+ \\
\stackrel{\sigma}{\sigma} \\
\underset{\sim}{\infty}\end{array}$ & $\begin{array}{l}\vec{m} \\
\stackrel{2}{2}\end{array}$ & $\begin{array}{l}a \\
\hat{0} \\
\stackrel{+}{\sim}\end{array}$ \\
\hline $\begin{array}{l}\sum_{\pi}^{2} \\
0 \\
0 \\
0 \\
0\end{array}$ & $\overrightarrow{\grave{\lambda}}$ & 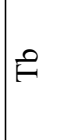 & $\mid \begin{array}{c}0 \\
\infty \\
\dot{\infty} \\
\dot{m}\end{array}$ & 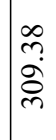 & $\overrightarrow{\bar{\rho}}$ & $\frac{m}{a}$ & & \begin{tabular}{l}
$\infty$ \\
\multirow{0}{0}{} \\
m.
\end{tabular} & $\frac{n}{a}$ & $\begin{array}{l}\tilde{\delta} \\
\underset{\sim}{n} \\
\tilde{m}\end{array}$ & $\begin{array}{l}\tilde{n} \\
\stackrel{n}{*} \\
\tilde{m}\end{array}$ & 官 & $\begin{array}{l}\hat{n} \\
\hat{\alpha} \\
\tilde{m}\end{array}$ & $\begin{array}{l}\hat{o} \\
i \\
\tilde{m} \\
m\end{array}$ & $\begin{array}{l}\stackrel{\wp}{n} \\
m \\
m \\
m\end{array}$ & $\mid \begin{array}{l}0 \\
0 \\
\hat{m} \\
m\end{array}$ & సै & $\frac{\hat{a}}{\dot{j}}$ & $\begin{array}{l}\mathcal{F} \\
\stackrel{J}{f} \\
\text { f }\end{array}$ & 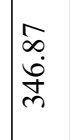 & mà & $\frac{0}{\stackrel{2}{n}}$ & $\begin{array}{l}8 \\
\dot{0} \\
\dot{n} \\
m\end{array}$ \\
\hline$\sum_{0}^{\pi}$ & $\frac{1}{0}$ & 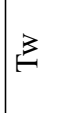 & 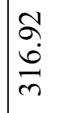 & 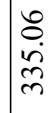 & 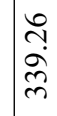 & 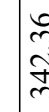 & & $\begin{array}{l}\stackrel{\infty}{ } \\
\dot{j}\end{array}$ & 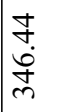 & $\begin{array}{l}\vec{b} \\
\text { f } \\
\text { m }\end{array}$ & 旁 & $\frac{\infty}{\stackrel{\infty}{\dot{n}}}$ & $\begin{array}{l}\tilde{D} \\
\infty \\
\tilde{n} \\
\tilde{m}\end{array}$ & 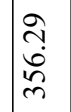 & $\begin{array}{l}\Re \\
\dot{0} \\
\infty \\
\infty \\
\infty\end{array}$ & 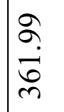 & $\frac{n}{\pi}$ & $\begin{array}{l}\infty \\
\alpha \\
\hat{\sigma} \\
\tilde{m}\end{array}$ & $\underset{m}{\stackrel{i}{i}}$ & $\begin{array}{l}\vec{N} \\
\stackrel{n}{n} \\
\text { nn }\end{array}$ & \begin{tabular}{l}
0 \\
0 \\
\multirow{2}{*}{} \\
$m$
\end{tabular} & 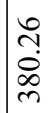 & $\begin{array}{l}\stackrel{0}{2} \\
\infty \\
\infty \\
\infty\end{array}$ \\
\hline 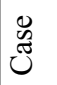 & 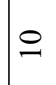 & $\stackrel{9}{9}$ & $N$ & $\bar{\infty}$ & $\ddot{ \pm}$ & है & & તֶ. & $\stackrel{n}{n}$ & $\begin{array}{c}n \\
\infty \\
\infty\end{array}$ & $\underset{f}{\dot{f}}$ & ì. & $\begin{array}{l}n \\
0 \\
i n\end{array}$ & $\begin{array}{l}n \\
i \\
\text { Ju}\end{array}$ & $\begin{array}{l}n \\
\infty \\
0 \\
0\end{array}$ & $\begin{array}{l}\stackrel{0}{0} \\
\dot{I}\end{array}$ & $\begin{array}{l}0 \\
\dot{\infty}\end{array}$ & $\hat{\infty}$ & $\hat{\check{a}}$ & $\mid \begin{array}{l}\infty \\
\infty \\
\propto\end{array}$ & $\begin{array}{l}\infty \\
\dot{+} \\
\stackrel{0}{0}\end{array}$ & 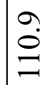 & \\
\hline
\end{tabular}




\begin{tabular}{|c|c|c|c|c|c|c|c|c|c|c|c|c|c|c|c|c|c|c|c|c|c|c|c|c|}
\hline & & $\vec{v}$ & 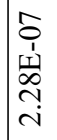 & 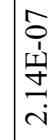 & $\begin{array}{l}\frac{a}{1} \\
\frac{1}{n} \\
\frac{1}{n}\end{array}$ & & 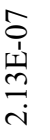 & 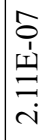 & $\begin{array}{l}\hat{0} \\
1 \\
\underline{1} \\
\bar{\lambda}\end{array}$ & $\begin{array}{l}\hat{s} \\
1 \\
\underline{1} \\
\\
\text { in }\end{array}$ & 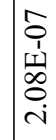 & 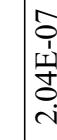 & 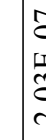 & & 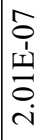 & $\begin{array}{l}\hat{o} \\
\hat{1} \\
\text { ⿳亠丷⿵冂丶 }\end{array}$ & 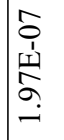 & $\begin{array}{l}\hat{a} \\
\hat{1} \\
\underline{\omega} \\
\bar{\sigma} \\
\underline{-}\end{array}$ & 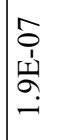 & 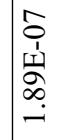 & 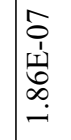 & 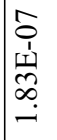 & $\begin{array}{l}\hat{\sigma} \\
\hat{1} \\
\hat{1} \\
\hat{\sigma} \\
\underline{-}\end{array}$ & 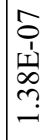 \\
\hline & & $\stackrel{\oplus}{\infty}$ & 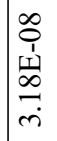 & 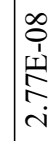 & 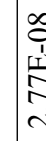 & & 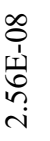 & 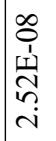 & 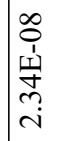 & $\begin{array}{l}\infty \\
\stackrel{\infty}{0} \\
1 \\
\stackrel{1}{N} \\
\sim \\
i \\
i\end{array}$ & 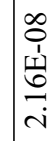 & 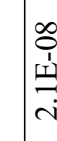 & 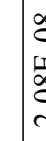 & 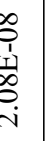 & 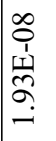 & 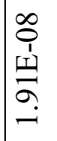 & 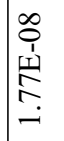 & 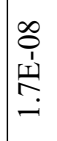 & $\begin{array}{l}\infty \\
0 \\
0 \\
\text { 亗 } \\
\stackrel{0}{0} \\
-\end{array}$ & 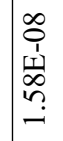 & 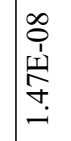 & 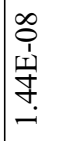 & 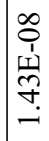 & 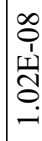 \\
\hline & & $\vec{D}$ & 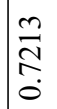 & $\frac{N}{N}$ & $\frac{\pi}{2}$ & & 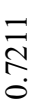 & 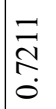 & $\underset{\vec{\Xi}}{\stackrel{\Xi}{\Xi}}$ & $\underset{\vec{N}}{\stackrel{\vec{N}}{0}}$ & $\stackrel{\vec{N}}{\stackrel{\vec{O}}{0}}$ & $\stackrel{\bar{\Omega}}{\tilde{\sigma}}$ & F & $\frac{\vec{E}}{\dot{b}}$ & $\stackrel{\vec{N}}{\hat{\sigma}}$ & $\stackrel{\vec{N}}{\stackrel{\mathbb{O}}{0}}$ & $\overrightarrow{\mathbb{N}}$ & $\stackrel{\vec{N}}{\stackrel{0}{0}}$ & $\stackrel{\vec{N}}{\stackrel{\mathbb{O}}{0}}$ & $\overrightarrow{\mathbb{N}}$ & $\overrightarrow{\widetilde{N}}$ & $\underset{\overrightarrow{\mathbb{N}}}{\overrightarrow{\mathrm{N}}}$ & $\underset{\substack{\vec{N} \\
\stackrel{\vec{O}}{0}}}{ }$ & 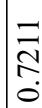 \\
\hline & & $\widetilde{J}$ & 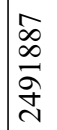 & 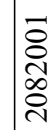 & $\begin{array}{l}\text { bे } \\
\frac{1}{5} \\
\frac{2}{2}\end{array}$ & & בે & $\begin{array}{l}\overrightarrow{\hat{\sigma}} \\
\hat{2} \\
\underline{6}\end{array}$ & 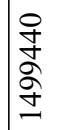 & $\begin{array}{l}\widetilde{\sigma} \\
\tilde{n} \\
\tilde{J} \\
\tilde{\Xi}\end{array}$ & 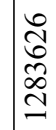 & 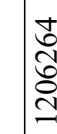 & $\frac{5}{7}$ & $\frac{a}{a}$ & 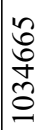 & $\begin{array}{l}\stackrel{\sim}{\widetilde{\alpha}} \\
\stackrel{\alpha}{\alpha}\end{array}$ & $\begin{array}{l}\underset{N}{J} \\
\stackrel{\sim}{\infty} \\
\infty \\
\infty\end{array}$ & 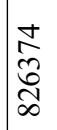 & $\begin{array}{l}\frac{\partial}{2} \\
\stackrel{2}{n}\end{array}$ & $\frac{8}{2}$ & 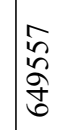 & $\frac{8}{\frac{8}{\sigma}}$ & $\begin{array}{l}\stackrel{a}{a} \\
\infty \\
\ddot{n} \\
i n\end{array}$ & $\begin{array}{l}n \\
\tilde{w} \\
z \\
\gamma\end{array}$ \\
\hline & & $t$ & 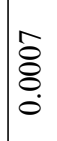 & $\begin{array}{l}\infty \\
0 \\
0 \\
0 \\
0 \\
0 \\
0\end{array}$ & $\begin{array}{l}\text { J } \\
\text { d } \\
\text { gे } \\
0\end{array}$ & & 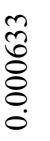 & $\begin{array}{l}\frac{0}{0} \\
8 \\
8 \\
0 \\
0\end{array}$ & 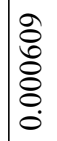 & $\begin{array}{l}\bar{\Xi} \\
0 \\
\delta \\
0 \\
0 \\
0\end{array}$ & $\begin{array}{l}\bar{\sigma} \\
\hat{\tilde{o}} \\
8 \\
0 \\
0\end{array}$ & 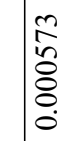 & . & 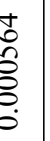 & 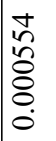 & $\begin{array}{l}n \\
\text { in } \\
\text { ôे } \\
8 \\
0 \\
0\end{array}$ & 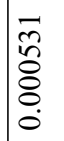 & 䓟 & 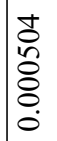 & 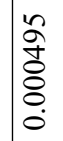 & $\mid \begin{array}{l}n \\
\infty \\
\vdots \\
0 \\
0 \\
0 \\
0 \\
0\end{array}$ & 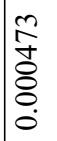 & 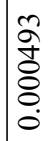 & 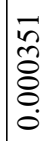 \\
\hline & & $\mathscr{\simeq}$ & 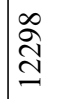 & 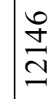 & ¿̊ & & $\begin{array}{l}\stackrel{8}{\circ} \\
\stackrel{\infty}{=}\end{array}$ & $\stackrel{\infty}{\stackrel{N}{\Xi}}$ & $\hat{a}$ & 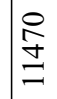 & 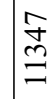 & $\stackrel{\infty}{\Xi}$ & $\exists$ & $\underline{E}$ & $\stackrel{\check{\sigma}}{\Xi}$ & $\begin{array}{l}\stackrel{ \pm}{\circ} \\
\stackrel{8}{\varrho}\end{array}$ & $\begin{array}{l}\stackrel{2}{2} \\
\hat{\vdots}\end{array}$ & ஓे & $\hat{\tilde{\varepsilon}}$ & $\begin{array}{l}8 \\
0 \\
0\end{array}$ & 重 & $\overrightarrow{\widetilde{\sigma}}$ & $\tilde{\widehat{\delta}}$ & $\frac{\tilde{n}}{\sigma}$ \\
\hline లే & $\tilde{z}$ & 竞 & 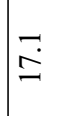 & 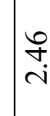 & 2 & & 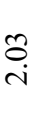 & $\stackrel{\infty}{\infty}$ & $\frac{n}{i}$ & $\stackrel{\overrightarrow{\vec{i}}}{\vec{i}}$ & in & $\stackrel{\infty}{i}$ & & $\tilde{i}$ & 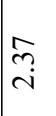 & ָे & $\overrightarrow{\tilde{N}}$ & $\stackrel{\infty}{0}$ & $\stackrel{+}{\stackrel{2}{*}}$ & ర్ర & $\cong$ & $\stackrel{n}{n}$ & $\stackrel{\infty}{-}$ & $\begin{array}{l}8 \\
\text { i }\end{array}$ \\
\hline $\begin{array}{l}\frac{+}{\sigma} \\
\frac{\vec{\omega}}{\Xi} \\
\Xi\end{array}$ & $\begin{array}{l}0 \\
0 \\
0 \\
0 \\
0 \\
0 \\
0 \\
0\end{array}$ & $\vec{z}$ & $\underset{\vec{\Xi}}{\vec{I}}$ & $\underset{\mathcal{J}}{\vec{J}}$ & $\begin{array}{l}\alpha \\
\infty \\
\alpha^{2}\end{array}$ & & $\underset{n}{\stackrel{J}{n}}$ & $\begin{array}{l}0 \\
\infty \\
0 \\
\infty \\
n\end{array}$ & 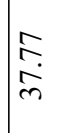 & $\begin{array}{l}\bar{n} \\
\dot{n}\end{array}$ & $\mid \begin{array}{l}0 \\
\infty \\
\infty \\
\dot{f}\end{array}$ & 市. & & 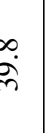 & $\begin{array}{l}n \\
\tilde{n} \\
\tilde{n}\end{array}$ & $=$ & $\frac{\mathfrak{m}}{m}$ & $\begin{array}{l}\vec{i} \\
\text { } \\
\text {. }\end{array}$ & $\frac{\vec{m}}{m}$ & $\stackrel{\vartheta}{\stackrel{m}{m}}$ & 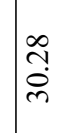 & $\begin{array}{l}\vec{b} \\
\grave{\lambda}\end{array}$ & $\begin{array}{l}\hat{\alpha} \\
\hat{\delta} \\
\hat{n}\end{array}$ & $\begin{array}{l}\circ \\
\stackrel{0}{0} \\
\stackrel{2}{*}\end{array}$ \\
\hline $\begin{array}{l}\mathscr{\mathscr { I }} \\
\frac{\overrightarrow{0}}{\Xi}\end{array}$ & 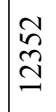 & 駕 & $\begin{array}{l}0 \\
\substack{0 \\
\infty \\
\infty}\end{array}$ & $\begin{array}{l}\stackrel{J}{+} \\
\dot{+}\end{array}$ & $\vec{m}$ & ? & ભે & $\frac{\partial}{m}$ & $\begin{array}{l}\infty \\
\dot{0} \\
\dot{m}\end{array}$ & $\begin{array}{l}\tilde{\alpha} \\
\dot{m} \\
\tilde{r}\end{array}$ & ir & F & & $\stackrel{8}{+}$ & חֶ? & $\stackrel{\vartheta}{\ni}$ & $\underset{+}{\nexists}$ & $\frac{\sim}{m}$ & $\stackrel{m}{m}$ & $\begin{array}{l}\infty \\
\stackrel{\infty}{\infty}\end{array}$ & 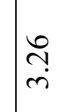 & बे & $\vec{n}$ & $\stackrel{7}{\sim}$ \\
\hline$\underset{\Xi}{\stackrel{\Xi}{\Xi}}$ & 占 & 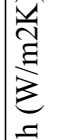 & $\frac{\infty}{\dot{\hat{\imath}}}$ & $\stackrel{i}{\stackrel{n}{\pi}}$ & $\begin{array}{l}\vec{J} \\
\qquad 8 \\
0\end{array}$ & & $\begin{array}{l}\circ \\
\infty \\
\dot{b}\end{array}$ & $\begin{array}{l}\vec{\sigma} \\
\dot{f}\end{array}$ & 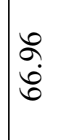 & $\begin{array}{l}\infty \\
\infty \\
\stackrel{R}{2}\end{array}$ & \begin{tabular}{|l} 
\\
$\dot{T}$ \\
$\dot{T}$
\end{tabular} & $\stackrel{2}{i}$ & & 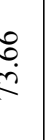 & \begin{tabular}{r}
\multirow{2}{*}{} \\
$\stackrel{2}{2}$
\end{tabular} & $\begin{array}{l}\infty \\
\infty \\
\end{array}$ & $\begin{array}{l}n \\
2 \\
\grave{2}\end{array}$ & $\begin{array}{l}\tilde{O} \\
\text { î }\end{array}$ & $\frac{\tilde{n}}{6}$ & $\stackrel{n}{\longrightarrow}$ & $\begin{array}{l}\bar{\alpha} \\
\hat{n}\end{array}$ & $\begin{array}{l}\text { oे } \\
\text { in }\end{array}$ & 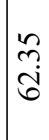 & $\begin{array}{l}\circ \\
\infty \\
\infty \\
i n\end{array}$ \\
\hline$\sum_{\text {至 }}$ & $\vec{m}$ & $\begin{array}{l}\text { है } \\
\vdots \\
=\end{array}$ & $\frac{\vec{\sigma}}{\dot{\sigma}}$ & 今ે & $\mathfrak{c}$ & & $\begin{array}{l}\stackrel{0}{\dot{\alpha}} \\
\text { ते }\end{array}$ & $\begin{array}{l}n \\
\stackrel{n}{\hat{\alpha}} \\
\grave{2}\end{array}$ & तิ & $\begin{array}{l}m \\
\stackrel{2}{2} \\
\hat{2}\end{array}$ & $\mid \begin{array}{l}\infty \\
\dot{0} \\
\dot{0} \\
\grave{\nu}\end{array}$ & $\vec{a}$ & $\begin{array}{l}0 \\
\vdots \\
\vdots \\
c\end{array}$ & à & $\begin{array}{l}\text { नें } \\
\text { ¿े }\end{array}$ & $\begin{array}{l}\vec{\sim} \\
\hat{\alpha} \\
\dot{\alpha}\end{array}$ & $\mid \begin{array}{l}0 \\
\infty \\
\sim \\
\sim\end{array}$ & 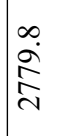 & $\begin{array}{l}m \\
\stackrel{\infty}{\infty} \\
\stackrel{\infty}{N} \\
\end{array}$ & $\begin{array}{l}n \\
\stackrel{d}{d} \\
\stackrel{i}{v}\end{array}$ & $\begin{array}{l}\hat{\sigma} \\
\stackrel{\sim}{\sim} \\
\hat{\sim}\end{array}$ & 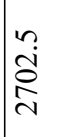 & $\underset{\stackrel{\sigma}{\alpha}}{\stackrel{+}{\infty}}$ & 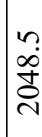 \\
\hline 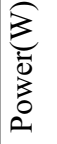 & స్ల & 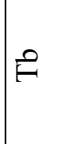 & 勇 & $\frac{n}{\bar{m}}$ & $\frac{\substack{\infty \\
m}}{m}$ & & $\begin{array}{l}\stackrel{\infty}{\grave{\pi}} \\
\stackrel{\sim}{n}\end{array}$ & $\begin{array}{l}0 \\
0 \\
0 \\
\tilde{m}\end{array}$ & $\frac{\tilde{\sigma}}{\dot{m}}$ & $\begin{array}{l}\hat{\infty} \\
\dot{n} \\
\tilde{m}\end{array}$ & 疍 & $\begin{array}{l}n \\
\hat{n} \\
\tilde{m}\end{array}$ & $\begin{array}{l}0 \\
\vdots \\
j \\
\vdots \\
\vdots\end{array}$ & 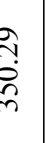 & $\begin{array}{l}n \\
0 \\
i n \\
n \\
m\end{array}$ & $\begin{array}{l}0 \\
\stackrel{2}{0} \\
\tilde{n}\end{array}$ & 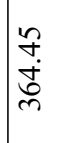 & $\begin{array}{l}0 \\
0 \\
0 \\
0 \\
\text { D. }\end{array}$ & 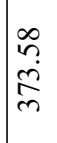 & $\begin{array}{l}8 \\
\infty \\
\infty \\
\infty \\
m\end{array}$ & $\begin{array}{l}\infty \\
\sim \\
\sim \\
\infty \\
\infty\end{array}$ & 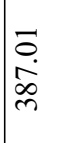 & 欮 & $\begin{array}{l}\tilde{n} \\
\sim \\
2 \\
\text { mे }\end{array}$ \\
\hline$\sum_{0}^{\pi}$ & $\stackrel{7}{0}$ & $\exists$ & $\begin{array}{l}\sim \\
\text { âd } \\
\text { } \\
\tilde{m}\end{array}$ & $\begin{array}{l}\text { సे } \\
\tilde{n} \\
\text { ले }\end{array}$ & $\begin{array}{l}8 \\
0 \\
0\end{array}$ & ? & 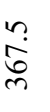 & 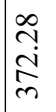 & 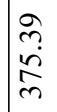 & $\underset{m}{\stackrel{a}{\sim}}$ & $\mid \begin{array}{c}\tilde{D} \\
\infty \\
\dot{\infty} \\
\infty \\
\infty\end{array}$ & $\begin{array}{l}\bar{\alpha} \\
\infty \\
\infty \\
\infty\end{array}$ & $\begin{array}{l}5 \\
\delta \\
2 \\
\alpha\end{array}$ & $\begin{array}{l}\infty \\
\infty \\
\infty \\
\infty \\
\infty \\
m\end{array}$ & 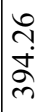 & $\begin{array}{l}\mathfrak{2} \\
\infty \\
\infty \\
\tilde{m}\end{array}$ & 字 & $\begin{array}{l}\infty \\
\infty \\
\dot{\gamma} \\
\dot{\gamma}\end{array}$ & $\mid \begin{array}{l}\stackrel{N}{\infty} \\
\frac{\infty}{\sigma}\end{array}$ & $\stackrel{m}{\stackrel{m}{F}}$ & 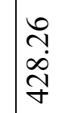 & 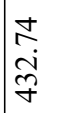 & 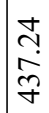 & 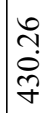 \\
\hline $\begin{array}{l}0 \\
0 \\
\tilde{z} \\
\tilde{z}\end{array}$ & $=$ & $\stackrel{9}{9}$ & $\sim$ & $\vec{\infty}$ & $\bar{J}$ & 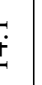 & ָุ' & సֶ. & 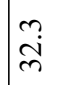 & $\begin{array}{l}m \\
\infty \\
m\end{array}$ & $\stackrel{+}{\dot{f}}$ & $n$ & $y$ & ?ֶ. & $\begin{array}{l}n \\
\tilde{J}\end{array}$ & $\mid \begin{array}{l}n \\
\infty \\
0\end{array}$ & 占 & $\begin{array}{l}0 \\
\dot{\infty}\end{array}$ & $\hat{\infty}$ & $\hat{i}$ & $\mid \begin{array}{l}\infty \\
\infty \\
\alpha\end{array}$ & $\begin{array}{l}\infty \\
\dot{0} \\
\stackrel{0}{0}\end{array}$ & $\grave{\varrho}$ & \\
\hline
\end{tabular}




\begin{tabular}{|c|c|c|c|c|c|c|c|c|c|c|c|c|c|c|c|c|c|c|c|c|c|c|c|}
\hline & & $\vec{\Delta}$ & 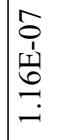 & 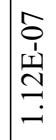 & 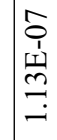 & 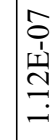 & & 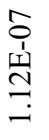 & 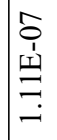 & 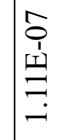 & 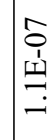 & 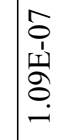 & 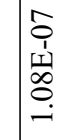 & 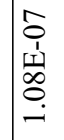 & 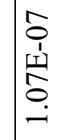 & 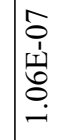 & 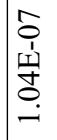 & 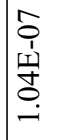 & 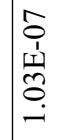 & 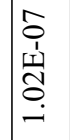 & 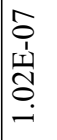 & 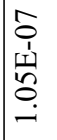 & $\begin{array}{l}\infty \\
0 \\
1 \\
1 \\
n \\
\infty \\
\infty \\
\infty\end{array}$ \\
\hline & & $\stackrel{\oplus}{\infty}$ & 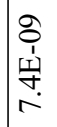 & $\begin{array}{l}2 \\
0 \\
1 \\
01 \\
0 \\
0\end{array}$ & $\begin{array}{l}2 \\
\text { oे } \\
1 \\
\underline{1} \\
0 \\
0\end{array}$ & 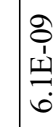 & & $\begin{array}{l}\text { oे } \\
\text { 11 } \\
\text { İ }\end{array}$ & 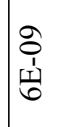 & 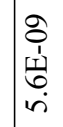 & $\begin{array}{l}0 \\
0 \\
1 \\
1 \\
n \\
i n \\
\text { in }\end{array}$ & 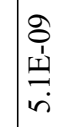 & 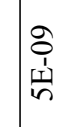 & 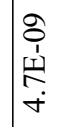 & 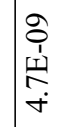 & 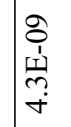 & 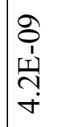 & 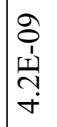 & $\begin{array}{l}\text { oे } \\
\text { के } \\
\text { I' } \\
\text { ले } \\
\text { r) }\end{array}$ & 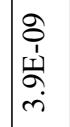 & 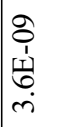 & 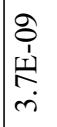 & 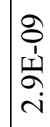 \\
\hline & & 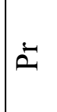 & 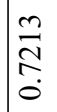 & 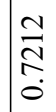 & 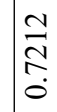 & $\frac{\stackrel{N}{N}}{\stackrel{N}{0}}$ & & 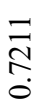 & $\underset{\vec{\Xi}}{\stackrel{\Xi}{\sigma}}$ & $\underset{\overrightarrow{\mathbb{N}}}{\stackrel{\Xi}{0}}$ & 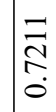 & $\stackrel{\vec{N}}{\stackrel{\delta}{\delta}}$ & $\overrightarrow{\mathbb{N}}$ & $\underset{\mathbb{N}}{\vec{N}}$ & $\stackrel{\vec{N}}{\stackrel{\mathbb{0}}{0}}$ & $\overrightarrow{\stackrel{N}{\hat{O}}}$ & $\overrightarrow{\mathbb{N}}$ & $\underset{\mathbb{N}}{\stackrel{\mathbb{0}}{0}}$ & $\underset{\stackrel{\vec{N}}{0}}{\stackrel{0}{0}}$ & $\underset{\stackrel{\mathbb{N}}{0}}{0}$ & $\begin{array}{l}\overline{\vec{N}} \\
\stackrel{ }{0}\end{array}$ & $\underset{\vec{\sim}}{\stackrel{\vec{N}}{0}}$ & $\underset{\vec{Z}}{\stackrel{Z}{0}}$ \\
\hline & & $\widetilde{J}$ & 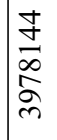 & 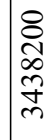 & $\begin{array}{c}\hat{\infty} \\
\tilde{f} \\
\tilde{D} \\
\tilde{m}\end{array}$ & $\begin{array}{l}0 \\
0 \\
\tilde{n} \\
\tilde{N} \\
\text { సે }\end{array}$ & & 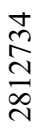 & 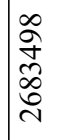 & 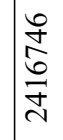 & 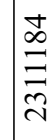 & 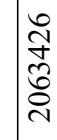 & $\mid \begin{array}{l}\hat{\circ} \\
\stackrel{1}{\hat{2}} \\
2\end{array}$ & $\begin{array}{l}\Re \\
\infty \\
\infty \\
\infty \\
\cong\end{array}$ & 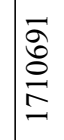 & $\begin{array}{l}\stackrel{D}{n} \\
\alpha \\
\infty \\
n \\
n\end{array}$ & 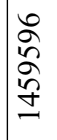 & ले & 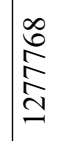 & $\mid \begin{array}{l}\overline{\widetilde{I}} \\
\stackrel{\infty}{\mathbb{N}} \\
\mathbb{\Xi}\end{array}$ & $\stackrel{\text { }}{\stackrel{\text { }}{\Xi}}$ & 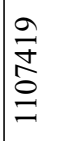 & 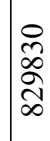 \\
\hline & & $t$ & 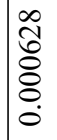 & ¿̊. & $\begin{array}{l}0 \\
2 \\
2 \\
\delta \\
0 \\
0\end{array}$ & \begin{tabular}{l}
$\infty$ \\
$\infty$ \\
\hdashline \\
$\varnothing$ \\
0 \\
0 \\
0
\end{tabular} & & $\begin{array}{l}\hat{\text { s. }} \\
\text { ô } \\
\vdots \\
0 \\
0\end{array}$ & 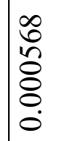 & $\begin{array}{l}\overrightarrow{0} \\
\stackrel{\circ}{0} \\
\vdots \\
0 \\
0\end{array}$ & $\begin{array}{l}\tilde{n} \\
\hat{\delta} \\
\delta \\
0 \\
0 \\
0\end{array}$ & 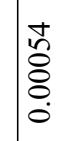 & 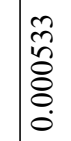 & 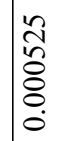 & $\mid \begin{array}{l}\infty \\
\grave{n} \\
\grave{\delta} \\
0 \\
0\end{array}$ & 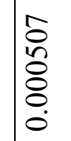 & 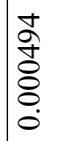 & \begin{tabular}{l}
$\infty$ \\
$\infty$ \\
\multirow{8}{\infty}{} \\
8 \\
0 \\
0
\end{tabular} & 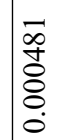 & 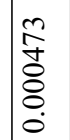 & $\begin{array}{l}n \\
0 \\
0 \\
0 \\
8 \\
0 \\
0\end{array}$ & 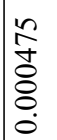 & $\begin{array}{l}\infty \\
\tilde{e} \\
\tilde{\delta} \\
\delta \\
0 \\
0\end{array}$ \\
\hline & & $\approx$ & 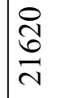 & $\frac{0}{\stackrel{0}{n}}$ & $\underset{\vec{v}}{\stackrel{\tilde{v}}{v}}$ & 离 & & 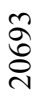 & 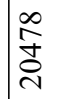 & $\begin{array}{l}\text { రे } \\
\stackrel{\text { సे }}{ }\end{array}$ & 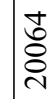 & $\begin{array}{l}\sqrt[6]{0} \\
\stackrel{0}{\sigma}\end{array}$ & 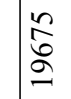 & 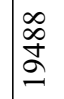 & 今ે & 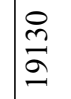 & $\begin{array}{l}8 \\
\stackrel{0}{0} \\
-1\end{array}$ & 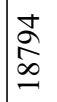 & $\begin{array}{l}\mathscr{\hat { \delta }} \\
\infty \\
\mathscr{\infty}\end{array}$ & 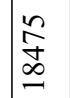 & $\begin{array}{l}\tilde{N} \\
\stackrel{\infty}{\infty}\end{array}$ & $\frac{\stackrel{R}{2}}{\underline{\infty}}$ & $\tilde{\mathscr{\infty}}$ \\
\hline లే & $\tilde{z}$ & 兽 & 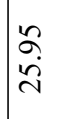 & 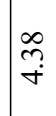 & فे & ¿े. & & $\underset{r}{\stackrel{7}{*}}$ & ñ. & $\underset{\dot{m}}{\alpha}$ & $\stackrel{\overbrace{}}{\rightleftharpoons}$ & $\begin{array}{l}\tilde{r} \\
\dot{f}\end{array}$ & $\underset{+}{\sigma}$ & $\stackrel{\partial}{\stackrel{\overrightarrow{+}}{+}}$ & \begin{tabular}{|l}
$\dot{\theta}$ \\
$\dot{+}$
\end{tabular} & $\nabla$ & $\frac{\sim}{m}$ & 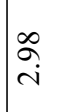 & $\hat{\tilde{\omega}}$ & $\begin{array}{l}\tilde{\infty} \\
\dot{i}\end{array}$ & $\stackrel{\hat{\gamma}}{i}$ & $\begin{array}{l}\infty \\
\infty \\
i \\
i\end{array}$ & 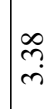 \\
\hline $\begin{array}{l}\frac{t}{\sigma} \\
\frac{\vec{\omega}}{\Xi} \\
\Xi \Xi\end{array}$ & | & $\vec{z}$ & $\stackrel{n}{\stackrel{0}{0}}$ & $\begin{array}{l}n \\
\hat{\sigma} \\
\hat{\sigma}\end{array}$ & f̊. & 永 & & 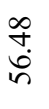 & 竎 & 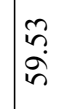 & 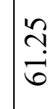 & \begin{tabular}{l}
$\infty$ \\
\multirow{n}{n}{} \\
$i n$
\end{tabular} & $\begin{array}{l}8 \\
0 \\
\text { in }\end{array}$ & in & $\begin{array}{l}\text { t } \\
\infty \\
\infty \\
i\end{array}$ & 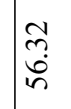 & $\begin{array}{l}m \\
\dddot{n} \\
\stackrel{n}{n}\end{array}$ & $\frac{\partial}{\vec{q}}$ & $\begin{array}{l}\infty \\
0 \\
\infty \\
\infty \\
\alpha\end{array}$ & ふָ? & $\begin{array}{l}\hat{\sigma} \\
\dot{6} \\
+\end{array}$ & 菊 & $\stackrel{0}{\stackrel{0}{f}}$ \\
\hline $\begin{array}{l}\mathscr{\mathscr { I }} \\
\frac{\overrightarrow{0}}{\Xi}\end{array}$ & 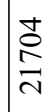 & 㝕 & $\overrightarrow{\tilde{p}}$ & $\stackrel{\grave{N}}{r}$ & గુ & $\frac{7}{6}$ & & $\begin{array}{l}\bar{\sigma} \\
\dot{n}\end{array}$ & $\frac{0}{6}$ & के & $\stackrel{\infty}{\substack{n\\
}}$ & 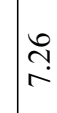 & $\stackrel{\infty}{\stackrel{\infty}{\sim}}$ & & 守 & $\underset{7}{\stackrel{7}{r}}$ & $\begin{array}{l}0 \\
\infty \\
i n\end{array}$ & $\begin{array}{l}\infty \\
n \\
i n\end{array}$ & $\begin{array}{l}\infty \\
i \\
i\end{array}$ & $\underset{f}{f}$ & $\begin{array}{l}\hat{\sigma} \\
i\end{array}$ & nิ & 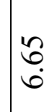 \\
\hline$\underset{\Xi}{\stackrel{\Xi}{\Xi}}$ & ஓ্ల & 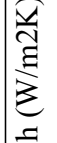 & $\begin{array}{l}\infty \\
\stackrel{+}{\sim} \\
\stackrel{\sim}{N}\end{array}$ & $\begin{array}{l}m \\
\infty \\
\infty \\
0\end{array}$ & 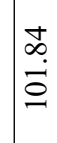 & $\begin{array}{l}\infty \\
\approx \\
\delta\end{array}$ & & $\begin{array}{l}\hat{\infty} \\
\infty \\
\alpha\end{array}$ & : & $\begin{array}{l}\infty \\
\tilde{0} \\
\stackrel{0}{0} \\
\varrho\end{array}$ & 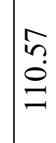 & $\begin{array}{l}\infty \\
0 \\
\infty \\
0 \\
0\end{array}$ & $\mid \begin{array}{l}\infty \\
\infty \\
\infty \\
0\end{array}$ & $\mid \begin{array}{l}\infty \\
\infty \\
0 \\
0\end{array}$ & 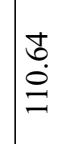 & $\vec{\nabla}$ & $\begin{array}{l}\hat{\hat{~}} \\
\mathscr{\swarrow}\end{array}$ & Oें & 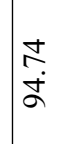 & $\begin{array}{l}n \\
\tilde{\sigma} \\
\tilde{\alpha}\end{array}$ & $\begin{array}{l}\vec{J} \\
\text { à }\end{array}$ & $\begin{array}{l}\dot{2} \\
\dot{\sigma} \\
\dot{\alpha}\end{array}$ & के \\
\hline$\sum_{\text {忘 }}$ & n & $\begin{array}{l}\text { ठิ } \\
\text { है } \\
= \\
=\end{array}$ & 离 & 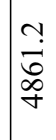 & $\begin{array}{l}\hat{\infty} \\
\infty \\
\infty \\
\dot{\alpha}\end{array}$ & $\underset{\partial}{\partial}$ & & $\underset{\substack{\infty \\
\infty}}{\infty}$ & $\underset{\substack{\infty \\
\stackrel{\infty}{\infty} \\
\infty \\
+}}{ }$ & 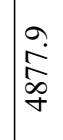 & $\underset{\substack{n \\
\infty \\
\infty}}{|c|}$ & 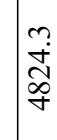 & $\begin{array}{l}\infty \\
\ddot{\sim} \\
\infty \\
\dot{\infty}\end{array}$ & 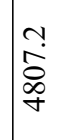 & 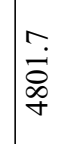 & 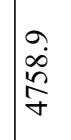 & $\begin{array}{c}3 \\
\text { a } \\
\stackrel{+}{+}\end{array}$ & 穴 & $\begin{array}{l}\infty \\
0 \\
o \\
\sigma\end{array}$ & 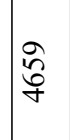 & $\begin{array}{l}2 \\
\text { तें } \\
\text { के }\end{array}$ & 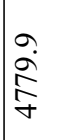 & $\frac{a}{2}$ \\
\hline $\begin{array}{l}\sum_{\pi} \\
0 \\
0 \\
0 \\
2\end{array}$ & $\stackrel{\infty}{\underset{f}{f}}$ & 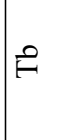 & 离 & $\frac{\Omega}{\sigma}$ & 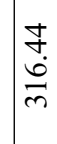 & $\begin{array}{l}\infty \\
0 \\
0 \\
0\end{array}$ & & $\begin{array}{l}n \\
\ddot{n} \\
n \\
m\end{array}$ & $\begin{array}{l}\tilde{\sigma} \\
\dot{0} \\
\tilde{m} \\
\text { m. }\end{array}$ & 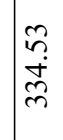 & $\begin{array}{l}n \\
\text { ले. } \\
\text { mे }\end{array}$ & $\begin{array}{l}\stackrel{+}{n} \\
\stackrel{m}{f} \\
\stackrel{m}{m}\end{array}$ & $\begin{array}{l}\tilde{\sigma} \\
\dot{\infty} \\
\stackrel{+}{\infty} \\
\dot{m}\end{array}$ & $\begin{array}{l}\text { f } \\
\text { i } \\
\text { rn }\end{array}$ & 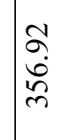 & $\mid \begin{array}{l}n \\
m \\
\dot{b} \\
m \\
m\end{array}$ & $\begin{array}{l}\mathbb{N} \\
\hat{0} \\
0 \\
0\end{array}$ & 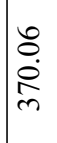 & $\underset{m}{\stackrel{+}{+}}$ & $\mid \begin{array}{l}\mathbb{N} \\
\infty \\
\infty \\
m\end{array}$ & $\begin{array}{l}\sigma \\
\tilde{\sigma} \\
\infty \\
\infty \\
\approx\end{array}$ & $\underset{\substack{n \\
\infty \\
\infty}}{\infty}$ & 文 \\
\hline$\sum_{0}^{\pi}$ & $\frac{7}{0}$ & 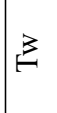 & 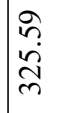 & $\begin{array}{l}\infty \\
\infty \\
\dot{0} \\
\infty \\
m\end{array}$ & 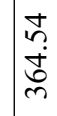 & 公 & & $\begin{array}{l}\stackrel{D}{\infty} \\
\dot{\mathbb{d}} \\
m\end{array}$ & 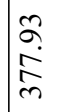 & $\begin{array}{l}\hat{m} \\
\hat{0} \\
\infty \\
\tilde{m}\end{array}$ & 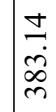 & $\begin{array}{l}\infty \\
\infty \\
\infty \\
\infty \\
\infty\end{array}$ & $\frac{\alpha}{\alpha}$ & $\begin{array}{l}\text { స̃ } \\
\text { } \\
\text { ळे }\end{array}$ & 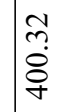 & 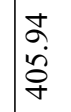 & $\underset{\stackrel{\infty}{+}}{\stackrel{\infty}{+}}$ & $\frac{n}{\sigma}$ & 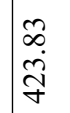 & 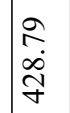 & $\begin{array}{l}= \\
\ddot{\sim} \\
\ddot{\sigma}\end{array}$ & $\stackrel{\vec{r}}{\stackrel{\sim}{\tilde{\sigma}}}$ & $\begin{array}{l}8 \\
0 \\
+ \\
+\end{array}$ \\
\hline $\begin{array}{l}\mathscr{y} \\
\tilde{z} \\
\tilde{z}\end{array}$ & $\simeq$ & $\stackrel{9}{9}$ & $N$ & $\vec{\infty}$ & $\overrightarrow{\dot{I}}$ & है & & તֶ. & 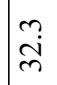 & $\begin{array}{l}n \\
\infty \\
\infty \\
m\end{array}$ & $\stackrel{+}{\dot{f}}$ & 芦 & $\begin{array}{l}n \\
0 \\
i n\end{array}$ & $\begin{array}{l}n \\
i \\
\text { d }\end{array}$ & $\begin{array}{l}n \\
\infty \\
\infty \\
\infty\end{array}$ & $\begin{array}{l}\stackrel{\circ}{I} \\
\stackrel{+}{I}\end{array}$ & $\begin{array}{l}0 \\
\dot{\infty}\end{array}$ & $\hat{\infty}$ & $\hat{\check{a}}$ & $\begin{array}{l}\infty \\
\infty \\
\infty\end{array}$ & $\begin{array}{l}\infty \\
\dot{+} \\
\stackrel{0}{0}\end{array}$ & 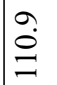 & \\
\hline
\end{tabular}




\begin{tabular}{|c|c|c|c|c|c|c|c|c|c|c|c|c|c|c|c|c|c|c|c|c|c|c|c|c|}
\hline & & $\vec{\Delta}$ & 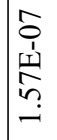 & $\begin{array}{l}\hat{2} \\
1 \\
1 \\
\\
\\
-1\end{array}$ & 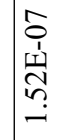 & 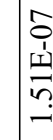 & & 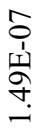 & 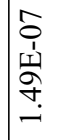 & 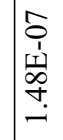 & 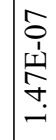 & 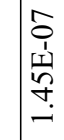 & 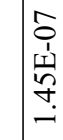 & 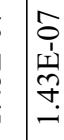 & 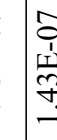 & 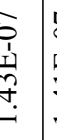 & 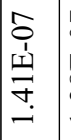 & $\begin{array}{l}\hat{s} \\
\hat{1} \\
1 \\
\infty \\
\infty \\
\\
-\end{array}$ & 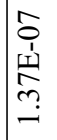 & 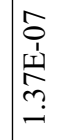 & $\begin{array}{c}\hat{s} \\
\hat{1} \\
\underline{1} \\
\tilde{n} \\
\mathfrak{n} \\
-1\end{array}$ & 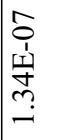 & $\begin{array}{c}\hat{0} \\
1 \\
1 \\
\infty \\
\infty \\
n \\
-1\end{array}$ & $\begin{array}{c}\hat{0} \\
\mathbf{1} \\
\text { I⿱ } \\
\text { o } \\
-1\end{array}$ \\
\hline & & $\stackrel{\oplus}{\infty}$ & 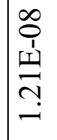 & $\begin{array}{l}\infty \\
0 \\
1 \\
1 \\
\infty \\
\infty \\
0 \\
-1\end{array}$ & $\begin{array}{l}\infty \\
0 \\
1 \\
1 \\
\infty \\
0 \\
0 \\
-1\end{array}$ & 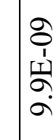 & & $\begin{array}{l}\text { oे } \\
\text { 11 } \\
\infty \\
\text { o. }\end{array}$ & 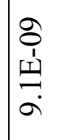 & $\begin{array}{l}\text { oे } \\
1 \\
\text { I⿱ } \\
\alpha\end{array}$ & 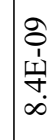 & 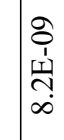 & 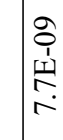 & 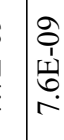 & $\begin{array}{l}8 \\
9 \\
1 \\
1\end{array}$ & 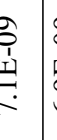 & $\mid \begin{array}{c}0 \\
0 \\
1 \\
\text { I⿱ } \\
\hat{b} \\
0\end{array}$ & 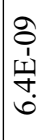 & 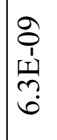 & $\begin{array}{l}o \\
\hat{1} \\
\underline{1} \\
\hat{\sigma} \\
\text { in }\end{array}$ & $\begin{array}{l}o \\
\hat{1} \\
1 \\
\infty \\
\infty \\
\dot{n} \\
\text { in }\end{array}$ & 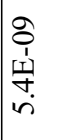 & 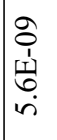 & $\begin{array}{l}\text { o } \\
1 \\
\text { In } \\
\text { f } \\
\dot{f}\end{array}$ \\
\hline & & 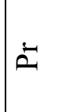 & 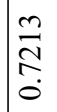 & 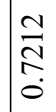 & $\frac{\mathfrak{I}}{\mathbb{N}} \underset{0}{0}$ & $\underset{\vec{N}}{\stackrel{\vec{N}}{0}}$ & & 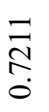 & $\underset{\vec{\Xi}}{\stackrel{\Xi}{\sigma}}$ & $\underset{\overrightarrow{\mathbb{S}}}{\overrightarrow{0}}$ & $\stackrel{\vec{N}}{\stackrel{\Delta}{0}}$ & $\overrightarrow{\mathbb{N}}$ & $\underset{\mathbb{N}}{\mathbb{N}}$ & $\stackrel{\vec{N}}{\stackrel{\Omega}{\delta}}$ & $\widehat{\mathbb{Z}}$ & $\vec{c}$ & $\overrightarrow{\stackrel{N}{\hat{O}}}$ & $\overrightarrow{\mathbb{N}}$ & $\underset{\mathbb{N}}{\overrightarrow{0}}$ & $\underset{\overrightarrow{\vec{V}}}{\stackrel{\overrightarrow{\mathrm{d}}}{\mathrm{o}}}$ & $\underset{\vec{Z}}{\stackrel{\Xi}{0}}$ & $\begin{array}{l}\overrightarrow{\vec{N}} \\
\stackrel{0}{0}\end{array}$ & $\underset{\vec{N}}{\stackrel{\vec{N}}{0}}$ & 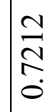 \\
\hline & & $\widetilde{J}$ & 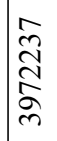 & $\begin{array}{l}\hat{\delta} \\
0 \\
\infty \\
\tilde{m} \\
\tilde{m}\end{array}$ & 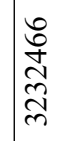 & 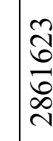 & & 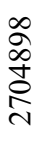 & 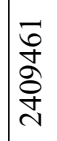 & 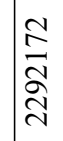 & 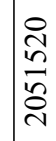 & $\begin{array}{l}2 \\
\stackrel{0}{\sigma} \\
2 \\
2\end{array}$ & 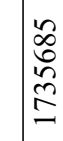 & $\begin{array}{l}0 \\
0 \\
0 \\
0 \\
0 \\
0 \\
-\end{array}$ & 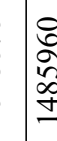 & 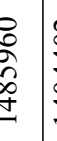 & 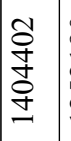 & 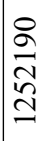 & 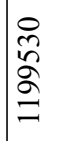 & 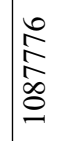 & 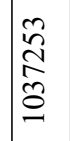 & 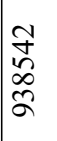 & $\begin{array}{l}m \\
\infty \\
\tilde{N} \\
\sigma\end{array}$ & $\begin{array}{l}\tilde{0} \\
0 \\
0 \\
\hat{0} \\
0\end{array}$ \\
\hline & & $t$ & 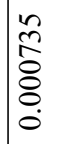 & 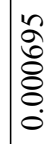 & $\begin{array}{l}0 \\
\dot{0} \\
\vdots \\
0 \\
0\end{array}$ & 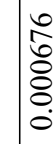 & & $\begin{array}{l}\hat{8} \\
\vdots \\
\vdots \\
\vdots \\
0\end{array}$ & $\begin{array}{l}1 \\
2 \\
0 \\
8 \\
8 \\
0 \\
0\end{array}$ & 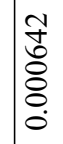 & 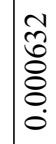 & 旾 & $\mid \begin{array}{l}8 \\
0 \\
8 \\
8 \\
0\end{array}$ & 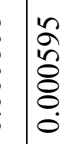 & $\begin{array}{l}n \\
\infty \\
2 \\
\delta \\
8 \\
0\end{array}$ & 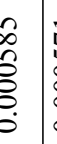 & $\mid$ & 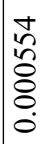 & 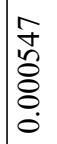 & $\begin{array}{l}\infty \\
\tilde{n} \\
\hat{n} \\
\delta \\
\delta \\
0 \\
0\end{array}$ & $\begin{array}{l}\infty \\
\tilde{N} \\
\tilde{\delta} \\
\delta \\
0 \\
0\end{array}$ & $\begin{array}{l}\infty \\
\frac{\infty}{n} \\
8 \\
0 \\
0 \\
0\end{array}$ & 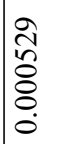 & 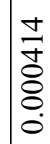 \\
\hline & & $\mathscr{\simeq}$ & \begin{tabular}{l}
$\tilde{\infty}$ \\
0 \\
$\infty$ \\
\hdashline
\end{tabular} & 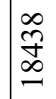 & $\begin{array}{l}n \\
\stackrel{1}{1} \\
\infty \\
-1\end{array}$ & $\mid \begin{array}{l}\infty \\
\vdots \\
\vdots \\
\end{array}$ & & 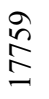 & 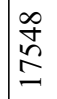 & $\stackrel{\Re}{\stackrel{\overbrace{}}{\Xi}}$ & $\underset{I}{\stackrel{g}{E}}$ & బิ & $\begin{array}{l}\mathbf{6} \\
6 \\
6\end{array}$ & $\mid \begin{array}{l}\infty \\
6 \\
6 \\
6\end{array}$ & $\frac{9}{5}$ & $\frac{c}{d}$ & 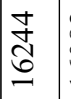 & 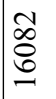 & $\begin{array}{l}\varkappa \\
\widehat{\Omega} \\
\end{array}$ & $\stackrel{\substack{\hat{n} \\
\underline{n}}}{2}$ & 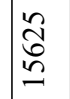 & 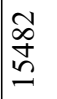 & ळે & $\stackrel{m}{\tilde{N}}$ \\
\hline$\tilde{\Xi}$ & $\tilde{z}$ & 兽 & & $\begin{array}{l}\tilde{n} \\
\tilde{n}\end{array}$ & $\hat{\hat{i}}$ & $\begin{array}{l}\infty \\
\infty \\
i\end{array}$ & & $\underset{t}{i}$ & $\widehat{\alpha}$ & ô. & $\stackrel{m}{m}$ & $\frac{\sim}{m}$ & 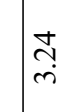 & $\frac{亏}{m}$ & $\stackrel{2}{\sim}$ & $\begin{array}{l}\hat{n} \\
\vec{n}\end{array}$ & $\hat{\hat{i}}$ & $\vec{n}$ & 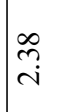 & $\stackrel{\vec{i}}{\mathrm{i}}$ & $\begin{array}{l}\stackrel{0}{N} \\
i\end{array}$ & $\underset{\sim}{\sim}$ & 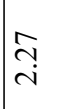 & $\stackrel{\bullet}{i}$ \\
\hline $\begin{array}{l}\frac{+}{\sigma} \\
\frac{\vec{\omega}}{\Xi} \\
\Xi\end{array}$ & 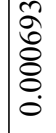 & $\vec{z}$ & $\begin{array}{l}\infty \\
\dot{\infty} \\
\dot{J}\end{array}$ & $\vec{N}$ & $\begin{array}{l}\hat{a} \\
\tilde{n} \\
i n\end{array}$ & à. & & $\begin{array}{l}\infty \\
\stackrel{0}{0} \\
\stackrel{8}{n}\end{array}$ & $\begin{array}{l}\infty \\
\infty \\
\infty \\
\infty\end{array}$ & $\begin{array}{l}0 \\
+ \\
i \\
i\end{array}$ & $\begin{array}{l}\infty \\
\infty \\
\tilde{n} \\
\hat{n}\end{array}$ & $\frac{\partial}{\grave{n}}$ & $\begin{array}{l}\infty \\
\tilde{N} \\
i\end{array}$ & $\begin{array}{l}\stackrel{0}{\circ} \\
\text { in }\end{array}$ & $\frac{5}{n}$ & $\begin{array}{c}0 \\
\tilde{i} \\
\end{array}$ & $\begin{array}{l}\tilde{N} \\
\hat{q}\end{array}$ & $\begin{array}{l}\because \\
\stackrel{y}{f} \\
\dot{f}\end{array}$ & $\mid \begin{array}{c}\hat{\sigma} \\
\dot{\gamma}\end{array}$ & 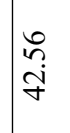 & 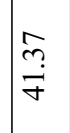 & $\begin{array}{l}\hat{b} \\
\dot{q}\end{array}$ & $\frac{\tilde{n}}{\vec{\gamma}}$ & $\begin{array}{l}0 \\
\infty \\
\infty \\
m\end{array}$ \\
\hline 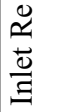 & $\begin{array}{l}\infty \\
\stackrel{0}{0} \\
\infty \\
-1\end{array}$ & 駕 & $\begin{array}{l}\sigma \\
\tilde{n} \\
\tilde{n} \\
m\end{array}$ & $\begin{array}{l} \pm \\
\infty \\
\text { in }\end{array}$ & \begin{tabular}{l}
$\infty$ \\
\multirow{+}{+}{}
\end{tabular} & $\mid \begin{array}{l}\infty \\
+ \\
+\end{array}$ & & $\stackrel{\sim}{+}$ & $\begin{array}{l}8 \\
\text { in }\end{array}$ & ले & $\begin{array}{l}\text { के } \\
\text { in }\end{array}$ & ir. & $\begin{array}{l}\approx \\
\tilde{n} \\
\tilde{n}\end{array}$ & $\begin{array}{l}\infty \\
i \\
i n\end{array}$ & & bे. & $\begin{array}{l}n \\
n \\
n\end{array}$ & $\stackrel{\infty}{\stackrel{\infty}{f}}$ & 节 & $\begin{array}{l}\hat{\sigma} \\
+\end{array}$ & $\underset{+}{\stackrel{+}{*}}$ & مै & $\vec{n}$ & $\begin{array}{l}\hat{y} \\
\text { in }\end{array}$ \\
\hline$\underset{\Xi}{\stackrel{\Xi}{\Xi}}$ & ஓ্ల & 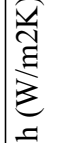 & 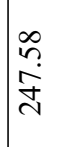 & 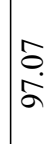 & $\overrightarrow{\bar{\alpha}}$ & $\begin{array}{l}\overrightarrow{2} \\
\infty \\
\infty\end{array}$ & & 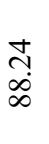 & $\begin{array}{l}30 \\
\stackrel{8}{8}\end{array}$ & $\begin{array}{l}\infty \\
0 \\
\dot{d}\end{array}$ & $\begin{array}{c}m \\
\infty \\
\infty\end{array}$ & $\begin{array}{l}\hat{\sigma} \\
\dot{0} \\
\sigma\end{array}$ & $\begin{array}{l}\hat{b} \\
\tilde{\alpha}\end{array}$ & 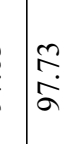 & $\begin{array}{l}\bar{n} \\
\alpha \\
\sigma\end{array}$ & $\begin{array}{c}n \\
\infty \\
\infty \\
\infty\end{array}$ & $\mid$ & $\begin{array}{l}\overrightarrow{1} \\
\infty \\
\infty \\
\infty\end{array}$ & $\stackrel{\hat{~}}{\dot{\infty}}$ & $\begin{array}{l}\vec{b} \\
\dot{0} \\
\infty\end{array}$ & $\begin{array}{l}n \\
\tilde{\infty} \\
\infty \\
\infty\end{array}$ & $\begin{array}{l}\vec{J} \\
i \\
\infty\end{array}$ & $\begin{array}{l}\approx \\
\stackrel{\infty}{\infty} \\
\dot{\infty}\end{array}$ & $\begin{array}{l}n \\
6 \\
2 \\
2\end{array}$ \\
\hline 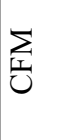 & $\stackrel{\infty}{+}$ & $\begin{array}{l}\text { ठิ } \\
\text { है } \\
= \\
=\end{array}$ & $\begin{array}{l}\hat{8} \\
\dot{8} \\
\stackrel{8}{\circ}\end{array}$ & $\underset{\substack{+\stackrel{\alpha}{\infty}}}{\stackrel{\alpha}{\infty}}$ & 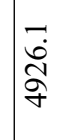 & ई̊) & & 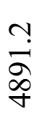 & 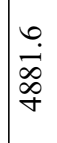 & 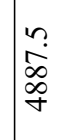 & 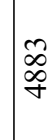 & 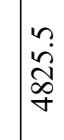 & 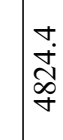 & $\mid \begin{array}{l}\infty \\
\tilde{\delta} \\
\dot{\infty} \\
\dot{\alpha}\end{array}$ & $\hat{2}$ & $\frac{\gamma}{f}$ & $\begin{array}{l}\infty \\
\dot{g} \\
\stackrel{g}{f} \\
\end{array}$ & $\begin{array}{l}1 \\
\infty \\
0 \\
0 \\
o \\
+\end{array}$ & $\begin{array}{l}m \\
\infty \\
0 \\
0 \\
0 \\
+\end{array}$ & $\begin{array}{l}\hat{i} \\
\hat{n} \\
\dot{\sigma}\end{array}$ & $\begin{array}{l}\hat{\widehat{C}} \\
\text { Oें }\end{array}$ & 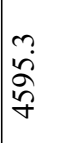 & $\begin{array}{l}\stackrel{0}{0} \\
\stackrel{0}{\circ} \\
\stackrel{2}{f}\end{array}$ & $\begin{array}{l}0 \\
\dot{J} \\
\stackrel{0}{n}\end{array}$ \\
\hline $\begin{array}{l}\sum_{\pi}^{0} \\
0 \\
0 \\
0 \\
0\end{array}$ & $\stackrel{\mathscr{\infty}}{\mathscr{\alpha}}$ & 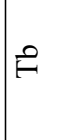 & 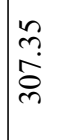 & 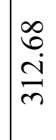 & $\frac{d}{\vec{d}}$ & $\begin{array}{l}\vec{n} \\
\text { ñर }\end{array}$ & & 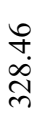 & $\stackrel{\vec{m}}{\stackrel{m}{m}}$ & $\begin{array}{l}t \\
\infty \\
\infty \\
\infty \\
m\end{array}$ & 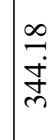 & 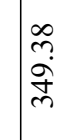 & $\begin{array}{l}n \\
n \\
\tilde{n} \\
m \\
m\end{array}$ & 离 & $\mid \begin{array}{l}\infty \\
\infty \\
0 \\
0 \\
0\end{array}$ & 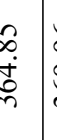 & 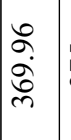 & $\stackrel{n}{m}$ & $\underset{\infty}{\infty}$ & $\begin{array}{l}\stackrel{\partial}{\alpha} \\
\dot{+} \\
\infty \\
\infty\end{array}$ & $\begin{array}{l}\stackrel{\circ}{2} \\
2 \\
\infty \\
\infty\end{array}$ & $\begin{array}{l}\infty \\
\text { में } \\
\text { ले }\end{array}$ & $\begin{array}{l}\partial े \\
\dot{\alpha} \\
\hat{\alpha}\end{array}$ & 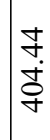 \\
\hline$\sum_{0}^{\pi}$ & $\stackrel{0}{0}$ & 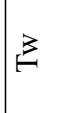 & $\begin{array}{l}\tilde{D} \\
\tilde{\sim} \\
\tilde{n}\end{array}$ & 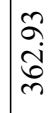 & 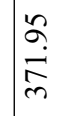 & $\mid \begin{array}{l}n \\
\vdots \\
\infty \\
n \\
m\end{array}$ & & $\begin{array}{c}\stackrel{\partial}{\infty} \\
\infty \\
\infty\end{array}$ & $\begin{array}{c}n \\
\infty \\
\infty \\
n \\
n\end{array}$ & 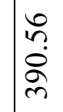 & $\begin{array}{l}\mathscr{\infty} \\
\dot{\alpha} \\
\hat{m}\end{array}$ & $\begin{array}{l}\hat{b} \\
\hat{2} \\
\tilde{m}\end{array}$ & 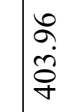 & $\mid \begin{array}{l}\infty \\
\infty \\
\dot{\rho} \\
\dot{q}\end{array}$ & $\left\{\begin{array}{l}n \\
m \\
7\end{array}\right.$ & $\begin{array}{l}\hat{n} \\
\frac{n}{\sigma} \\
z\end{array}$ & $\mid$ & \begin{tabular}{l}
$\infty$ \\
\\
సें \\
\multirow{\gamma}{*}{}
\end{tabular} & 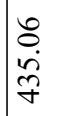 & 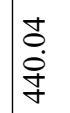 & 守 & $\begin{array}{l}n \\
0 \\
8 \\
7 \\
7\end{array}$ & $\begin{array}{l}\infty \\
\infty \\
\infty \\
n \\
\gamma \\
\gamma\end{array}$ & 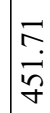 \\
\hline $\begin{array}{l}0 \\
0 \\
\tilde{z} \\
\tilde{z}\end{array}$ & 9 & $\stackrel{9}{9}$ & $N$ & $\vec{\infty}$ & $\overrightarrow{\dot{I}}$ & है & & તֶ. & 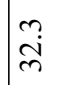 & $\begin{array}{l}n \\
\infty \\
\infty \\
m\end{array}$ & $\stackrel{+}{\dot{f}}$ & ì. & $\begin{array}{l}n \\
i \\
i n\end{array}$ & $\begin{array}{l}n \\
\text { }\end{array}$ & $\begin{array}{l}n \\
\infty \\
\infty\end{array}$ & م. & $\mid \begin{array}{l}0 \\
\dot{I}\end{array}$ & $\begin{array}{l}0 \\
\dot{\infty}\end{array}$ & $\stackrel{1}{\infty}$ & $\hat{\check{a}}$ & $\begin{array}{l}\infty \\
\infty \\
\infty\end{array}$ & $\stackrel{\infty}{\dot{0}}$ & 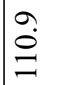 & \\
\hline
\end{tabular}




\begin{tabular}{|c|c|c|c|c|c|c|c|c|c|c|c|c|c|c|c|c|c|c|c|c|c|c|c|c|}
\hline & & 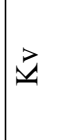 & 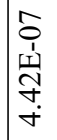 & 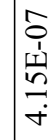 & $\begin{array}{l}\frac{a}{1} \\
1 \\
\frac{1}{7} \\
7\end{array}$ & & 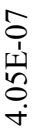 & $\begin{array}{l}\hat{s} \\
\hat{1} \\
\text { 竞 } \\
\alpha \\
\text { m. }\end{array}$ & $\begin{array}{l}\hat{\sigma} \\
\hat{1} \\
\underline{1} \\
\hat{\alpha} \\
\dot{m}\end{array}$ & $\begin{array}{l}\hat{s} \\
\hat{1} \\
1 \\
\infty \\
\infty \\
\dot{r}\end{array}$ & $\begin{array}{l}\hat{y} \\
\hat{1} \\
1 \\
\hat{1} \\
\infty \\
\infty \\
\end{array}$ & 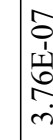 & 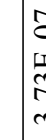 & & $\begin{array}{l}\hat{s} \\
0 \\
1 \\
\infty \\
0 \\
\dot{r} \\
\dot{r}\end{array}$ & 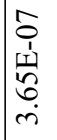 & 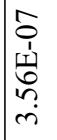 & $\begin{array}{l}\hat{s} \\
\hat{1} \\
\underline{1} \\
\infty \\
\infty \\
\dot{m}\end{array}$ & 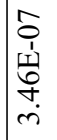 & 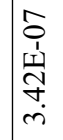 & $\begin{array}{c}\hat{y} \\
\hat{1} \\
\underline{y} \\
\tilde{n} \\
\dot{n}\end{array}$ & $\begin{array}{l}\hat{s} \\
\stackrel{1}{1} \\
1 \\
\\
\\
\end{array}$ & $\begin{array}{l}\hat{O} \\
\hat{1} \\
\\
\\
\end{array}$ & 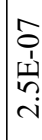 \\
\hline & & $\stackrel{\oplus}{\infty}$ & 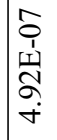 & 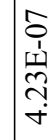 & $\begin{array}{l}3 \\
1 \\
d \\
c\end{array}$ & & 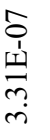 & $\begin{array}{l}\hat{o} \\
\hat{1} \\
\underline{1} \\
\tilde{\sigma} \\
\hat{r} \\
\dot{r}\end{array}$ & $\begin{array}{l}\hat{o} \\
1 \\
\hat{1} \\
\infty \\
\hat{i} \\
\hat{i}\end{array}$ & 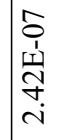 & 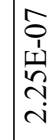 & 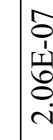 & 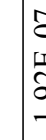 & & 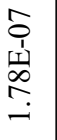 & 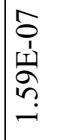 & 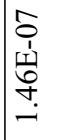 & $\begin{array}{l}\hat{0} \\
1 \\
\underline{1} \\
\\
\\
\end{array}$ & 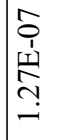 & 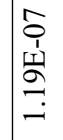 & 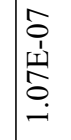 & 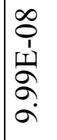 & $\mid \begin{array}{c}\infty \\
0 \\
0 \\
1 \\
1 \\
\\
\\
\alpha\end{array}$ & 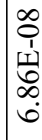 \\
\hline & & $\Xi$ & 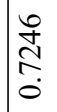 & $\underset{\stackrel{J}{\mathbb{N}}}{\stackrel{F}{0}}$ & $\begin{array}{l}\hat{\imath} \\
\tilde{a}\end{array}$ & & 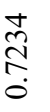 & 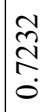 & 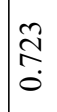 & 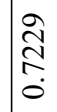 & 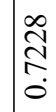 & 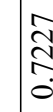 & $\hat{\mathfrak{t}}$ & & $\begin{array}{l}\stackrel{\hat{N}}{\mathbb{N}} \\
\dot{0}\end{array}$ & $\begin{array}{l}\hat{\mathbb{N}} \\
\stackrel{\mathbb{N}}{0}\end{array}$ & $\begin{array}{l}\infty \\
\mathbb{N} \\
\stackrel{N}{0}\end{array}$ & तે & 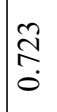 & $\underset{\widetilde{N}}{\stackrel{N}{0}}$ & 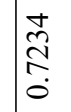 & $\begin{array}{l}\tilde{N} \\
\stackrel{N}{N} \\
\dot{0}\end{array}$ & 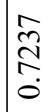 & సิ \\
\hline & & $\stackrel{\sigma}{3}$ & 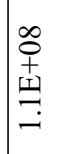 & \begin{tabular}{l}
$\hat{\sigma}$ \\
\multirow{d}{\sigma}{} \\
$\frac{n}{\infty}$ \\
$\infty$
\end{tabular} & $\begin{array}{l}\partial \\
\partial \\
0 \\
0 \\
0 \\
0 \\
0 \\
0\end{array}$ & & 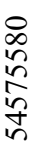 & 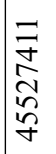 & 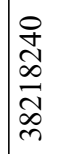 & 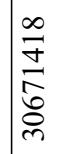 & 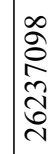 & 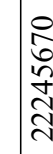 & $\mid \begin{array}{l}5 \\
5 \\
z \\
\delta \\
2\end{array}$ & 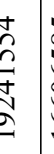 & $\begin{array}{l}n \\
\infty \\
0 \\
0 \\
0 \\
0 \\
0 \\
-1\end{array}$ & $\begin{array}{l}\vec{\infty} \\
\tilde{n} \\
\tilde{N} \\
\infty \\
\cdots \\
=\end{array}$ & 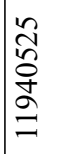 & $\begin{array}{l}n \\
\hat{0} \\
\infty \\
n \\
\tilde{0} \\
0\end{array}$ & $\begin{array}{l}\frac{\pi}{6} \\
\frac{0}{ \pm} \\
\frac{1}{\sigma}\end{array}$ & $\begin{array}{l}\infty \\
\frac{\infty}{0} \\
\underset{0}{0} \\
\infty\end{array}$ & $\begin{array}{l}\vec{m} \\
\vec{\infty} \\
\infty \\
\infty \\
0\end{array}$ & $\begin{array}{l}\vec{f} \\
\stackrel{0}{0} \\
\stackrel{8}{0}\end{array}$ & 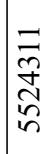 & $\begin{array}{l}\infty \\
\stackrel{\infty}{d} \\
\infty \\
\infty \\
m\end{array}$ \\
\hline & & $t$ & 告 & $\begin{array}{l}\hat{\hat{b}} \\
\stackrel{0}{8} \\
8 \\
0 \\
0\end{array}$ & $\begin{array}{l}\frac{1}{0} \\
\frac{0}{8} \\
8 \\
0\end{array}$ & & 苦 & 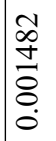 & 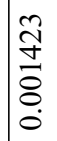 & 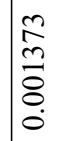 & वे & $\stackrel{\widehat{I}}{\stackrel{\Xi}{8}}$ & ปી & & $\begin{array}{l}\stackrel{\partial}{\vec{\sigma}} \\
\dot{\sigma} \\
\dot{0}\end{array}$ & 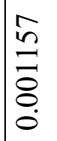 & $\stackrel{\infty}{\stackrel{\infty}{0}}$ & 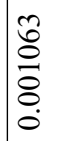 & 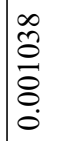 & \begin{tabular}{l}
$\infty$ \\
$\stackrel{8}{0}$ \\
$\stackrel{8}{8}$ \\
\hdashline
\end{tabular} & $\mid \begin{array}{c}\hat{\hat{a}} \\
\hat{\hat{O}} \\
\dot{8} \\
\dot{0}\end{array}$ & 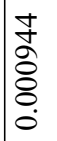 & $\begin{array}{l}\tilde{n} \\
\hat{\alpha} \\
\dot{8} \\
0 \\
0\end{array}$ & 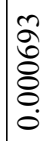 \\
\hline & & $\mathscr{\simeq}$ & $\stackrel{8}{2}$ & $\frac{\infty}{\sigma}$ & $\begin{array}{l}\stackrel{2}{5} \\
\text { in }\end{array}$ & & 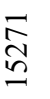 & 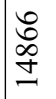 & $\begin{array}{l}\infty \\
\infty \\
⿱ 亠 乂 \\
\Xi\end{array}$ & $\begin{array}{l}0 \\
\\
\exists \\
\Xi\end{array}$ & 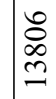 & 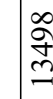 & §ુ & 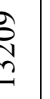 & 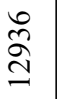 & 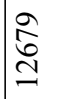 & 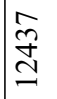 & 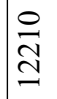 & $\stackrel{2}{\precsim}$ & $\bar{\Xi}$ & $\mid \begin{array}{l}\infty \\
\vdots \\
ٍ\end{array}$ & $\underset{\Xi}{\vec{J}}$ & $\stackrel{\infty}{\cong}$ & $\begin{array}{l}\infty \\
\stackrel{0}{0} \\
ٍ\end{array}$ \\
\hline లే & $\tilde{z}$ & 莺 & 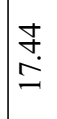 & $\begin{array}{l}3 \\
i \\
i\end{array}$ & $\frac{\infty}{c}$ & & 9 & $\stackrel{\infty}{\stackrel{\infty}{\sim}}$ & $\underset{\infty}{\infty}$ & $\underline{\underline{-}}$ & $\stackrel{\sim}{2}$ & $\stackrel{\infty}{\infty}$ & 2 & . & & 邑 & $\stackrel{N}{\stackrel{2}{*}}$ & $\stackrel{n}{n}$ & 守 & f. & $\stackrel{g}{\stackrel{g}{g}}$ & $\stackrel{\nexists}{-}$ & $\stackrel{\infty}{\infty}$ & $\hat{\imath}$ \\
\hline $\begin{array}{l}\frac{+}{\sigma} \\
\frac{\vec{\omega}}{\Xi} \\
\Xi\end{array}$ & 造 & $\vec{z}$ & $\stackrel{\partial}{\mathcal{I}}$ & $\frac{n}{n}$ & $\begin{array}{l}\tilde{z} \\
\tilde{z}\end{array}$ & & $\begin{array}{l}n \\
\stackrel{n}{g} \\
\stackrel{f}{q}\end{array}$ & 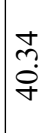 & $\begin{array}{l}\stackrel{0}{2} \\
\stackrel{n}{m}\end{array}$ & $\begin{array}{l}\hat{0} \\
\dot{q}\end{array}$ & $\begin{array}{l}\dot{0} \\
\stackrel{+}{+} \\
\dot{q}\end{array}$ & $\begin{array}{l}\overline{0} \\
\infty \\
\infty\end{array}$ & $\frac{1}{2}$ & $\begin{array}{l}f \\
\dot{0} \\
i \\
n\end{array}$ & $\begin{array}{l}\stackrel{0}{0} \\
i \\
i\end{array}$ & $\underset{m}{\stackrel{ \pm}{n}}$ & $\begin{array}{l}f \\
\dot{y} \\
m\end{array}$ & 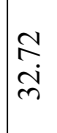 & $\overrightarrow{\vec{d}}$ & $\underset{\dot{m}}{\dot{m}}$ & 占. & $\begin{array}{l}0 \\
\sim \\
\end{array}$ & $\ddot{2}$ & $\begin{array}{l}\hat{\sigma} \\
\infty \\
\sim\end{array}$ \\
\hline 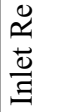 & 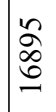 & 駕 & 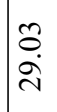 & $\vec{q}$ & శ్ & & $\stackrel{m}{m}$ & $\frac{\partial}{m}$ & 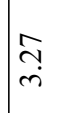 & $\begin{array}{l}\infty \\
n \\
n \\
n\end{array}$ & $\stackrel{\infty}{\stackrel{\infty}{m}}$ & $\sqrt{6}$ & f & $\frac{n}{n}$ & $\stackrel{n}{\stackrel{n}{m}}$ & $\bar{\sigma}$ & $\stackrel{m}{\stackrel{n}{n}}$ & $\stackrel{m}{m}$ & $\vec{m}$ & సે & $\stackrel{\infty}{m}$ & సે & $\stackrel{m}{m}$ & $\stackrel{\text { I }}{+}$ \\
\hline 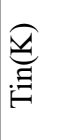 & ర్ల & 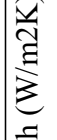 & $\begin{array}{l}\stackrel{\Im}{a} \\
\stackrel{\sim}{\sim}\end{array}$ & $\frac{0}{\infty}$ & 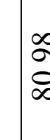 & & $\stackrel{?}{\gtrless}$ & $\begin{array}{l}\sigma \\
0 \\
i\end{array}$ & बे & 文. & 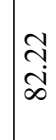 & $\underset{\infty}{\infty}$ & $\frac{i}{2}$ & & $\begin{array}{l}\hat{\infty} \\
\dot{\infty} \\
\dot{\infty}\end{array}$ & $\mid \begin{array}{l}\mathfrak{r} \\
\text { i } \\
\infty\end{array}$ & \begin{tabular}{l}
$\infty$ \\
\hdashline \\
$\infty$ \\
$\infty$
\end{tabular} & $\begin{array}{l}\infty \\
n \\
n \\
n\end{array}$ & $\begin{array}{l}\infty \\
2 \\
n \\
i n \\
n\end{array}$ & $\vec{m}$ & $\begin{array}{l}\hat{n} \\
\dot{I}\end{array}$ & $\stackrel{\mathscr{f}}{\stackrel{2}{r}}$ & 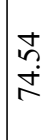 & $\frac{\infty}{\dot{I}}$ \\
\hline$\sum_{\text {至 }}$ & $\stackrel{\sim}{\longrightarrow}$ & $\begin{array}{l}\text { శ્ } \\
\text { है } \\
= \\
=\end{array}$ & 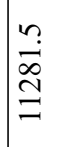 & $\begin{array}{l}\infty \\
\stackrel{8}{0} \\
\stackrel{8}{0}\end{array}$ & ڤิ & ț & 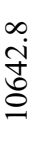 & $\begin{array}{l}\infty \\
\text { â } \\
\tilde{o} \\
ٍ\end{array}$ & $\mid \begin{array}{l}0 \\
\infty \\
\tilde{\infty} \\
\hat{O} \\
0\end{array}$ & 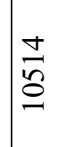 & $\overrightarrow{\frac{\pi}{5}}$ & $\begin{array}{l}\infty \\
0 \\
0 \\
0 \\
0\end{array}$ & 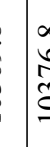 & $\begin{array}{l}\infty \\
\dot{0} \\
0 \\
0\end{array}$ & $\begin{array}{l}\vec{n} \\
\vec{n} \\
0 \\
0\end{array}$ & 穴 & 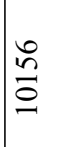 & $\begin{array}{l}\hat{\infty} \\
\infty \\
\alpha \\
\alpha\end{array}$ & 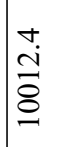 & \begin{tabular}{l}
1 \\
\multirow{0}{0}{} \\
$\alpha$ \\
$\alpha$
\end{tabular} & 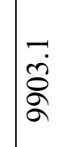 & $\begin{array}{l}n \\
n \\
\infty \\
\infty \\
a\end{array}$ & $\begin{array}{l}\text { तु } \\
\frac{\sigma}{\sigma}\end{array}$ & 察 \\
\hline 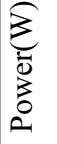 & 宅 & $\stackrel{\theta}{F}$ & 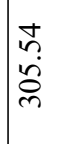 & $\begin{array}{l}n \\
0 \\
\infty \\
m\end{array}$ & $\frac{\tilde{F}}{m}$ & & $\begin{array}{l}\stackrel{\vartheta}{\vec{J}} \\
\stackrel{\vec{J}}{m}\end{array}$ & $\begin{array}{l}\infty \\
\infty \\
\dot{0} \\
\infty \\
m\end{array}$ & 綿 & $\begin{array}{l}n \\
\dot{\delta} \\
\dot{d} \\
\infty \\
\infty\end{array}$ & $\begin{array}{l}0 \\
\dot{+} \\
\text { iे }\end{array}$ & $\begin{array}{l}u \\
2 \\
0 \\
q\end{array}$ & $\frac{2}{2}$ & $\frac{y}{y}$ & 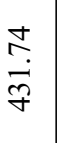 & \begin{tabular}{l}
$\overrightarrow{0}$ \\
$\dot{J}$ \\
\multirow{J}{*}{}
\end{tabular} & 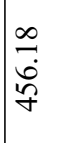 & 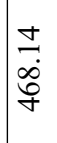 & $\stackrel{\infty}{\infty}$ & $\frac{\tilde{\infty}}{\dot{a}}$ & $\begin{array}{l}\infty \\
n \\
\tilde{n} \\
\tilde{n} \\
n\end{array}$ & $\begin{array}{l}i \\
i n \\
i n\end{array}$ & $\begin{array}{l}\infty \\
\dot{\delta} \\
i \\
i\end{array}$ & $\frac{d}{0}$ \\
\hline$\sum_{0}^{\pi}$ & กิ & 3 & $\begin{array}{l}0 \\
0 \\
i \\
\tilde{n} \\
m\end{array}$ & 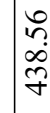 & $\begin{array}{l}\vec{\alpha} \\
\qquad z \\
\sigma\end{array}$ & $\begin{array}{l}t_{0} \\
\vdots \\
\vdots \\
+\end{array}$ & $\begin{array}{c}\hat{\infty} \\
\dot{\infty} \\
\dot{+} \\
\dot{\sigma}\end{array}$ & 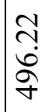 & $\begin{array}{l}\vec{n} \\
\stackrel{8}{0} \\
\tilde{n}\end{array}$ & 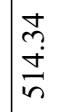 & $\begin{array}{l}\vec{n} \\
\tilde{N} \\
\text { n }\end{array}$ & $\begin{array}{l}\infty \\
0 \\
i \\
\text { in }\end{array}$ & I & 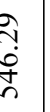 & $\begin{array}{l}\stackrel{n}{\dot{r}} \\
\stackrel{i}{n}\end{array}$ & 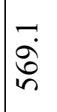 & $\begin{array}{l}\infty \\
n \\
i \\
\infty \\
\infty\end{array}$ & 导 & 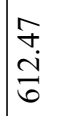 & ণ্ं & 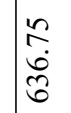 & $\begin{array}{l}0 \\
+ \\
+ \\
0 \\
0 \\
0\end{array}$ & 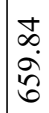 & $\begin{array}{l}= \\
\infty \\
\widetilde{6}\end{array}$ \\
\hline $\begin{array}{l}\mathscr{y} \\
\tilde{z} \\
\tilde{z}\end{array}$ & \pm & $\stackrel{9}{9}$ & $N$ & $\vec{\infty}$ & $\bar{J}$ & $t$ & 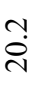 & ֶָ. & 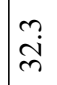 & $\begin{array}{l}m \\
\infty \\
m\end{array}$ & $\stackrel{+}{\dot{f}}$ & & $y$ & ?ֶ. & $\begin{array}{l}n \\
i \\
\text { d }\end{array}$ & $\mid \begin{array}{l}n \\
\infty \\
0\end{array}$ & $\begin{array}{l}\stackrel{0}{0} \\
\stackrel{+}{I}\end{array}$ & $\begin{array}{l}0 \\
\dot{\infty}\end{array}$ & $\hat{\infty}$ & $\hat{i}$ & $\mid \begin{array}{l}\infty \\
\infty \\
\alpha\end{array}$ & $\begin{array}{l}\infty \\
\dot{0} \\
\stackrel{0}{0}\end{array}$ & $\grave{\varrho}$ & \\
\hline
\end{tabular}




\begin{tabular}{|c|c|c|c|c|c|c|c|c|c|c|c|c|c|c|c|c|c|c|c|c|c|c|c|c|}
\hline & & 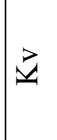 & 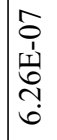 & $\begin{array}{l}\hat{p} \\
\mathbf{1} \\
1 \\
\infty \\
\dot{n} \\
\dot{n}\end{array}$ & 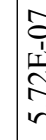 & & 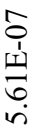 & 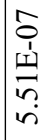 & 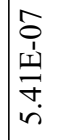 & 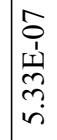 & $\begin{array}{l}\hat{o} \\
\hat{1} \\
\mathbf{y} \\
\hat{i} \\
i \\
i\end{array}$ & 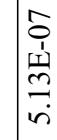 & 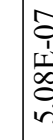 & 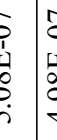 & 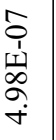 & $\begin{array}{l}\hat{o} \\
\hat{1} \\
\underline{1} \\
\hat{\alpha} \\
\dot{\gamma}\end{array}$ & 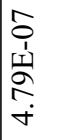 & 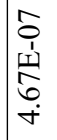 & 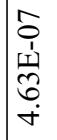 & 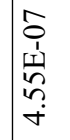 & $\begin{array}{c}\hat{s} \\
\dot{1} \\
\dot{1} \\
\infty \\
\dot{+} \\
\dot{+}\end{array}$ & 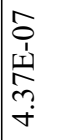 & 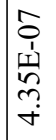 & 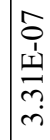 \\
\hline & & $\stackrel{\oplus}{\infty}$ & 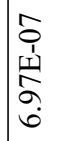 & 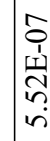 & $\begin{array}{l}\overrightarrow{1} \\
\frac{1}{7} \\
\overrightarrow{7}\end{array}$ & & 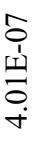 & $\begin{array}{l}\hat{0} \\
\hat{1} \\
\hat{1} \\
0 \\
0 \\
0 \\
\dot{n}\end{array}$ & 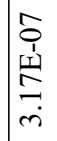 & $\begin{array}{l}\hat{i} \\
\hat{i} \\
\stackrel{1}{\hat{N}} \\
\stackrel{i}{i}\end{array}$ & 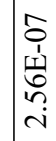 & $\begin{array}{c}\hat{o} \\
\hat{1} \\
\\
\\
\end{array}$ & 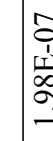 & $\begin{array}{c}0 \\
1 \\
0 \\
0 \\
0 \\
2 \\
2\end{array}$ & 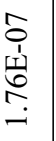 & 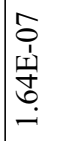 & 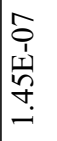 & 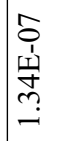 & 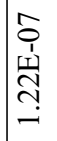 & 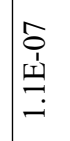 & $\begin{array}{c}\hat{0} \\
\hat{1} \\
\underline{1} \\
\hat{0} \\
\underline{-}\end{array}$ & 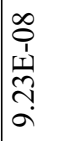 & 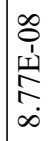 & $\begin{array}{c}\infty \\
0 \\
1 \\
\omega \\
\infty \\
\sigma \\
0\end{array}$ \\
\hline & & 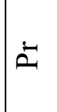 & 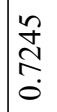 & 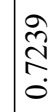 & 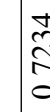 & & $\stackrel{\overrightarrow{\tilde{\Omega}}}{\stackrel{\Delta}{0}}$ & 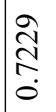 & 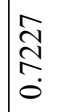 & 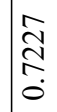 & 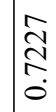 & 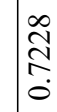 & $\mid \stackrel{\Xi}{\overparen{I}}$ & vै & $\begin{array}{l}\overrightarrow{\tilde{N}} \\
\stackrel{0}{0}\end{array}$ & 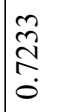 & 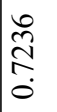 & 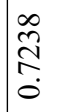 & $\underset{\stackrel{\mathcal{T}}{\mathbb{N}}}{\stackrel{\sim}{0}}$ & $\begin{array}{l}\stackrel{n}{d} \\
\stackrel{2}{d} \\
0\end{array}$ & 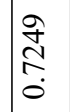 & 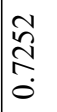 & \begin{tabular}{l}
0 \\
\multirow{2}{N}{} \\
$\vdots$ \\
0
\end{tabular} & $\mid \begin{array}{c}0 \\
0 \\
0 \\
0\end{array}$ \\
\hline & & $\stackrel{\sigma}{3}$ & 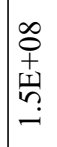 & 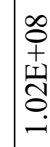 & 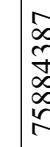 & & 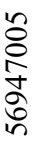 & 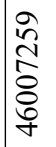 & $\begin{array}{l}\frac{n}{m} \\
\frac{n}{n} \\
n \\
m \\
m\end{array}$ & $\begin{array}{l}\hat{\alpha} \\
\stackrel{\alpha}{\alpha} \\
\stackrel{\partial}{\hat{\alpha}}\end{array}$ & 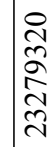 & 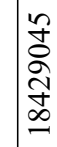 & 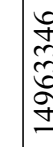 & 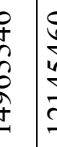 & 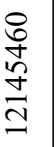 & $\begin{array}{l}0 \\
\tilde{\alpha} \\
\infty \\
0 \\
0 \\
0 \\
0\end{array}$ & $\begin{array}{l}2 \\
\hat{f} \\
\dot{y} \\
\dot{y} \\
\dot{b} \\
\infty\end{array}$ & 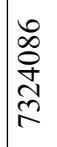 & 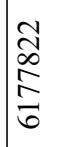 & 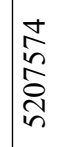 & 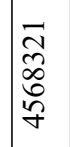 & $\begin{array}{l}0 \\
\hat{n} \\
0 \\
\infty \\
\infty \\
\infty\end{array}$ & 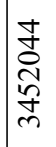 & 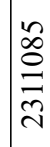 \\
\hline & & む & 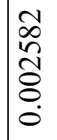 & స్ & $\bar{\Xi}$ & & 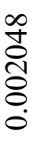 & $\frac{1}{2}$ & 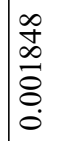 & 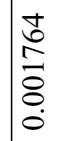 & $\begin{array}{l}\overrightarrow{\bar{b}} \\
\stackrel{8}{8} \\
\dot{0}\end{array}$ & 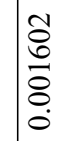 & 告 & 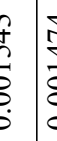 & $\begin{array}{l}\frac{+}{+} \\
\frac{+}{8} \\
\dot{0}\end{array}$ & $\mid \begin{array}{c}\hat{y} \\
\stackrel{\mathcal{O}}{8} \\
\stackrel{0}{0}\end{array}$ & $\mid \begin{array}{l}n \\
\tilde{n} \\
\\
8 \\
0 \\
0\end{array}$ & $\begin{array}{l}\overrightarrow{\widehat{A}} \\
\overline{8} \\
\stackrel{8}{0}\end{array}$ & 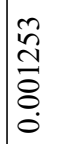 & 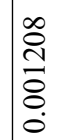 & $\mid$\begin{tabular}{l}
0 \\
0 \\
\hdashline \\
\hdashline \\
0 \\
0
\end{tabular} & 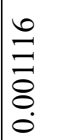 & & 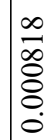 \\
\hline & & $\mathscr{\simeq}$ & 总 & $\begin{array}{l}\varkappa \\
\hat{n} \\
n\end{array}$ & $\frac{0}{n}$ & & $\begin{array}{l}\bar{\sigma} \\
\overline{1}\end{array}$ & $\stackrel{\Xi}{\exists}$ & $\begin{array}{l}\widetilde{2} \\
0 \\
\end{array}$ & $\begin{array}{l}\curvearrowright \\
\approx \\
\approx\end{array}$ & 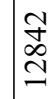 & 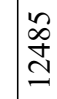 & $\stackrel{0}{\simeq}$ & 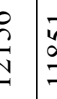 & $\begin{array}{l}\vec{\sim} \\
\Xi \\
\Xi\end{array}$ & $\begin{array}{l}\hat{0} \\
\stackrel{2}{\Xi}\end{array}$ & 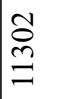 & $\stackrel{\infty}{\varrho}$ & 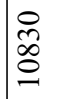 & $\frac{\Xi}{\sigma}$ & 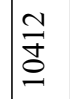 & ָิ & $\stackrel{m}{8}$ & \\
\hline$\tilde{\Xi}$ & $\tilde{z}$ & 莺 & $\begin{array}{l} \pm \\
\stackrel{\infty}{\Xi} \\
\beth\end{array}$ & $\tilde{i}$ & 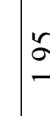 & & $\stackrel{尺}{\nexists}$ & ڤิ & 華 & nิ & ن. & $\stackrel{\leftrightarrow}{n}$ & in & $\tilde{r}$ & & 華 & $\stackrel{\vec{n}}{-}$ & $\stackrel{?}{\stackrel{F}{-}}$ & $\hat{n}$ & ले & $\vec{m}$ & 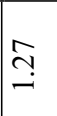 & 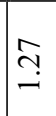 & $\underset{\mathrm{i}}{\mathrm{i}}$ \\
\hline $\begin{array}{l}\frac{+}{\sigma} \\
\frac{\vec{\omega}}{\Xi} \\
\Xi\end{array}$ & 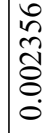 & $\vec{z}$ & 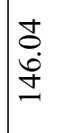 & $\begin{array}{l}5 \\
\text { in }\end{array}$ & $\begin{array}{c}\approx \\
\vec{z}\end{array}$ & & $\begin{array}{l}\bar{n} \\
\text { ñ }\end{array}$ & 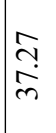 & ֶָ. & $\begin{array}{l}n \\
\hat{n} \\
n\end{array}$ & $\begin{array}{l}\infty \\
\ddot{n} \\
\ddot{n}\end{array}$ & $\begin{array}{l}\stackrel{0}{2} \\
\stackrel{m}{m}\end{array}$ & 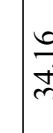 & 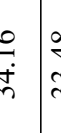 & $\stackrel{\substack{\infty \\
m}}{m}$ & $\begin{array}{l}\infty \\
\sim \\
\infty \\
n\end{array}$ & $\frac{\dot{d}}{\dot{m}}$ & $\begin{array}{l}\infty \\
0 \\
0 \\
\dot{m}\end{array}$ & $\begin{array}{l}\overrightarrow{0} \\
\stackrel{2}{2}\end{array}$ & 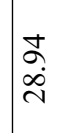 & $\vec{\infty}$ & $\begin{array}{l}8 \\
\stackrel{\sim}{N}\end{array}$ & 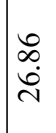 & 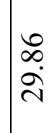 \\
\hline 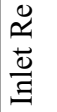 & $\begin{array}{l}6 \\
6 \\
6\end{array}$ & 駕 & $\stackrel{\infty}{\stackrel{\lambda}{0}}$ & $\stackrel{\underset{\sim}{+}}{\sim}$ & ले & & $\stackrel{\partial}{\vec{r}}$ & $\hat{\widehat{\lambda}}$ & $\stackrel{\infty}{\stackrel{\infty}{i}}$ & $\frac{b}{m}$ & त̂. & $\hat{\sim}$ & 7 & d & rn & $\begin{array}{l}\sigma \\
\text { r. }\end{array}$ & 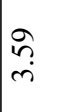 & $\stackrel{n}{m}$ & $\stackrel{\mathscr{f}}{\stackrel{f}{~}}$ & $\stackrel{n}{n}$ & ले & $\begin{array}{l}\infty \\
m \\
m\end{array}$ & $\ddot{m}$ & $\begin{array}{l}\infty \\
\infty \\
i \\
\text { in }\end{array}$ \\
\hline$\underset{\Xi}{\stackrel{\Xi}{\Xi}}$ & D্ & 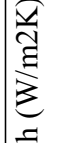 & 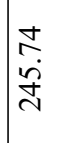 & $=$ & ते & & $\begin{array}{l}m \\
i \\
i\end{array}$ & f̊. & $\begin{array}{l}\infty \\
\infty \\
\end{array}$ & $\stackrel{2}{i n}$ & $\stackrel{5}{\frac{\infty}{\infty}}$ & 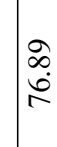 & 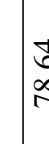 & $\begin{array}{l}0 \\
0 \\
0 \\
0\end{array}$ & $\frac{\text { ra }}{2}$ & $\mid \begin{array}{l}\infty \\
\dot{\infty}\end{array}$ & $\begin{array}{l}\bar{n} \\
\frac{n}{2}\end{array}$ & $\grave{\circ}$ & $\underset{\substack{\infty\\
}}{\stackrel{\infty}{N}}$ & $\underset{\substack{\mathbb{N} \\
\stackrel{N}{R}}}{ }$ & 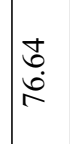 & 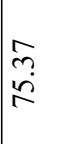 & 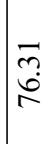 & $\begin{array}{l}n \\
\tilde{n} \\
\infty \\
\infty\end{array}$ \\
\hline$\sum_{\text {至 }}$ & $\stackrel{\sim}{\longrightarrow}$ & $\begin{array}{l}\text { శ્ } \\
\text { है } \\
= \\
=\end{array}$ & 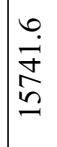 & 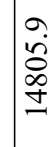 & $\begin{array}{l}0 \\
\infty \\
\infty \\
\infty \\
d \\
\end{array}$ & & 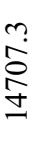 & $\begin{array}{l}7 \\
2 \\
2 \\
0 \\
\vdots \\
1\end{array}$ & $\begin{array}{l}n \\
\infty \\
\infty \\
n \\
\pm \\
\end{array}$ & 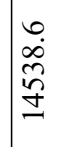 & 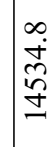 & 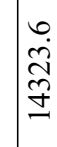 & $\mid \begin{array}{l}\alpha \\
\mathcal{E} \\
\tilde{g}\end{array}$ & 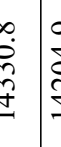 & 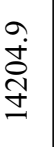 & 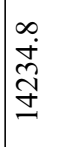 & $\underset{\hat{\sigma}}{\stackrel{2}{2}}$ & 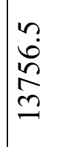 & 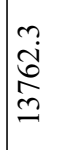 & $\begin{array}{l}a \\
0 \\
0 \\
0 \\
0\end{array}$ & 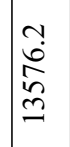 & 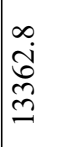 & 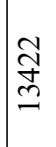 & 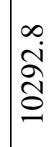 \\
\hline $\begin{array}{l}\sum_{\pi}^{0} \\
0 \\
0 \\
0 \\
0\end{array}$ & ஓ & $\stackrel{\theta}{F}$ & $\mid \begin{array}{c}\hat{y} \\
\hat{\sigma} \\
\bar{n}\end{array}$ & $\begin{array}{l}\vec{b} \\
\text { aे } \\
\sim\end{array}$ & 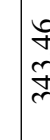 & & $\begin{array}{l}\tilde{n} \\
\dot{0} \\
\end{array}$ & $\begin{array}{l}2 \\
\infty \\
\infty \\
\infty \\
\infty\end{array}$ & $\begin{array}{l}\text { fं } \\
\text { ळे } \\
\text { ले }\end{array}$ & $\begin{array}{l}\hat{\alpha} \\
\dot{\gamma} \\
\gamma\end{array}$ & $\frac{\mathcal{F}}{\stackrel{f}{f}}$ & 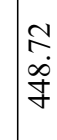 & $\mid \begin{array}{l}2 \\
\alpha \\
b \\
a\end{array}$ & 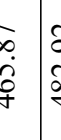 & 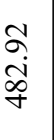 & $\begin{array}{l}\infty \\
\infty \\
\stackrel{a}{\sigma}\end{array}$ & $\begin{array}{l}\stackrel{8}{0} \\
\stackrel{0}{0} \\
i n\end{array}$ & $\begin{array}{l}\cong \\
\stackrel{2}{m} \\
\tilde{n}\end{array}$ & 守 & $\begin{array}{l}0 \\
\ddot{b} \\
\dot{b} \\
i n\end{array}$ & $\begin{array}{l}\dot{t} \\
\dot{\infty} \\
i n \\
\text { in }\end{array}$ & $\begin{array}{l}\frac{y}{f} \\
\frac{1}{2} \\
i \\
n\end{array}$ & $\frac{1}{\infty}$ & 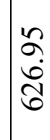 \\
\hline$\sum_{0}^{\pi}$ & ถุ & 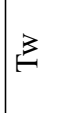 & $\stackrel{m}{n}$ & $\frac{\vec{\tau}}{\vec{g}}$ & $\begin{array}{l}\vec{J} \\
\text { हn } \\
\text { in }\end{array}$ & 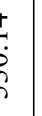 & 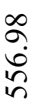 & $\begin{array}{l}\infty \\
m \\
\infty \\
\infty \\
i n\end{array}$ & $\begin{array}{l}n \\
\hat{n} \\
\tilde{n}\end{array}$ & $\begin{array}{l}n \\
\alpha \\
\ddot{\delta} \\
\delta\end{array}$ & $\begin{array}{l}0 \\
\stackrel{0}{r} \\
\sigma \\
\end{array}$ & $\begin{array}{l}\overline{0} \\
\ddot{n} \\
\tilde{\sigma}\end{array}$ & 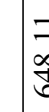 & & 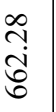 & 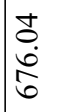 & 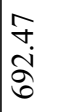 & 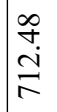 & 导 & 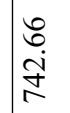 & $\begin{array}{l}\hat{\imath} \\
\infty \\
\infty \\
\curvearrowright\end{array}$ & 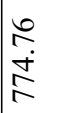 & $\begin{array}{l}\overrightarrow{0} \\
\stackrel{\alpha}{\infty}\end{array}$ & $\begin{array}{l}n \\
\vdots \\
0 \\
0 \\
1\end{array}$ \\
\hline $\begin{array}{l}\mathscr{y} \\
\tilde{z} \\
\tilde{z}\end{array}$ & $n$ & $\stackrel{9}{9}$ & $N$ & $\infty$ & $\bar{J}$ & 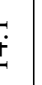 & 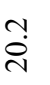 & సֶ. & 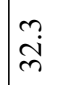 & $\begin{array}{l}n \\
\infty \\
\infty\end{array}$ & $\stackrel{+}{\dot{f}}$ & 芦 & $\begin{array}{l}\text { s } \\
\text { है }\end{array}$ & & הֶ & $\mid \begin{array}{l}n \\
\infty \\
0\end{array}$ & $\begin{array}{l}\stackrel{\bullet}{0} \\
\stackrel{I}{I}\end{array}$ & $\begin{array}{l}0 \\
\dot{\infty}\end{array}$ & $\hat{\infty}$ & $\hat{\check{a}}$ & $\begin{array}{l}\infty \\
\infty \\
\infty\end{array}$ & $\stackrel{\infty}{\dot{0}}$ & 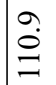 & \\
\hline
\end{tabular}




\begin{tabular}{|c|c|c|c|c|c|c|c|c|c|c|c|c|c|c|c|c|c|c|c|c|c|c|c|c|}
\hline & & 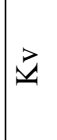 & 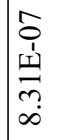 & $\begin{array}{l}\hat{0} \\
1 \\
1 \\
0 \\
0 \\
r \\
r\end{array}$ & 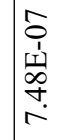 & 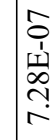 & & 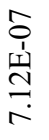 & 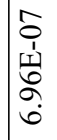 & $\begin{array}{l}\hat{0} \\
1 \\
1 \\
\omega \\
\infty \\
0 \\
0\end{array}$ & 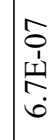 & 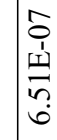 & 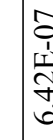 & $\begin{array}{l}0 \\
\vdots \\
\vdots \\
\vdots \\
\vdots \\
0\end{array}$ & 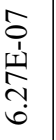 & 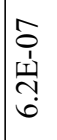 & 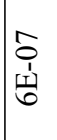 & $\begin{array}{l}\hat{0} \\
\hat{1} \\
\underline{1} \\
\tilde{n} \\
\infty \\
\dot{n}\end{array}$ & $\begin{array}{l}\hat{y} \\
\text { 1 } \\
\text { 章 } \\
\text { in } \\
\text { in }\end{array}$ & $\begin{array}{l}\hat{i} \\
1 \\
\underline{1} \\
\hat{b} \\
i \\
\dot{n}\end{array}$ & $\begin{array}{l}\hat{y} \\
\hat{1} \\
\hat{H} \\
\underline{0} \\
\dot{i n}\end{array}$ & 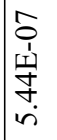 & 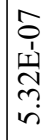 & 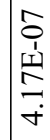 \\
\hline & & $\stackrel{\oplus}{\infty}$ & 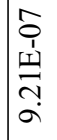 & 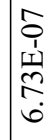 & \begin{tabular}{l}
$\hat{0}$ \\
$\hat{1}$ \\
\multicolumn{1}{|c}{} \\
$\hat{0}$ \\
$\dot{n}$
\end{tabular} & 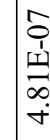 & & 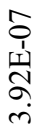 & $\begin{array}{l}\hat{o} \\
\hat{1} \\
\underline{1} \\
\tilde{m} \\
\dot{n}\end{array}$ & $\begin{array}{l}\hat{o} \\
\hat{1} \\
1 \\
\hat{2} \\
\hat{i} \\
\text { in }\end{array}$ & 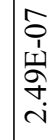 & 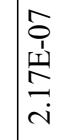 & $\mid$\begin{tabular}{c}
0 \\
1 \\
1 \\
\multirow{1}{1}{} \\
0
\end{tabular} & 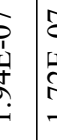 & 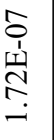 & 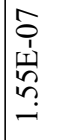 & 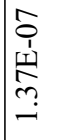 & 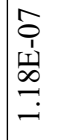 & 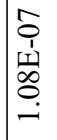 & $\begin{array}{l}\infty \\
0 \\
1 \\
1 \\
\infty \\
\infty \\
\sigma \\
\alpha\end{array}$ & $\begin{array}{l}\infty \\
0 \\
0 \\
1 \\
⿱ 1 \\
2 \\
\infty \\
\infty\end{array}$ & 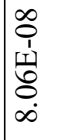 & 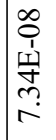 & \begin{tabular}{c}
$\infty$ \\
0 \\
0 \\
11 \\
\multirow{2}{+}{} \\
$\dot{v}$
\end{tabular} \\
\hline & & $\ddot{2}$ & 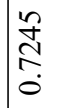 & $\underset{\tilde{N}}{\stackrel{N}{0}}$ & 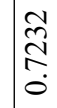 & 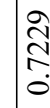 & & $\underset{\stackrel{N}{N}}{\stackrel{N}{0}}$ & 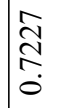 & 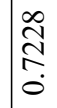 & 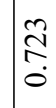 & 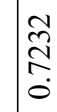 & 尺̃ & 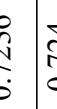 & 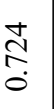 & 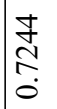 & 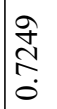 & 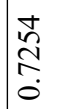 & & 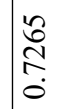 & 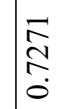 & 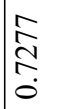 & $\begin{array}{l}\stackrel{0}{\infty} \\
\stackrel{N}{N} \\
0\end{array}$ & 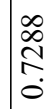 \\
\hline & & 灾 & 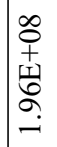 & 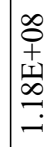 & $\begin{array}{l}\tilde{N} \\
\tilde{2} \\
\infty \\
\tilde{N} \\
\tilde{\infty} \\
\infty\end{array}$ & $\mid \begin{array}{c}\hat{m} \\
\tilde{f} \\
\tilde{8}\end{array}$ & & 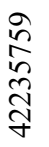 & $\begin{array}{l}\vec{a} \\
\stackrel{\vec{\infty}}{\underline{m}} \\
\stackrel{m}{n}\end{array}$ & 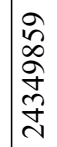 & $\mid \begin{array}{l}n \\
\sim \\
\infty \\
\infty \\
\infty \\
\infty \\
\infty \\
-1\end{array}$ & 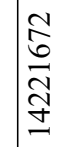 & 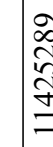 & 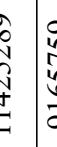 & $\begin{array}{l}\frac{2}{2} \\
\hat{n} \\
\vdots \\
a\end{array}$ & 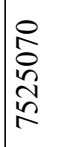 & 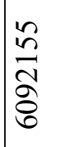 & 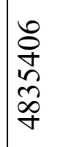 & 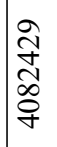 & 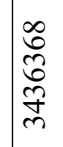 & 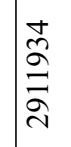 & 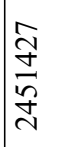 & 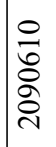 & 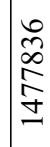 \\
\hline & & む & 疍 & 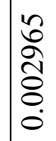 & $\begin{array}{l}\overline{0} \\
\hat{\widehat{\sigma}} \\
\delta \\
0\end{array}$ & 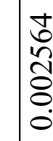 & & 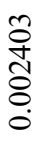 & 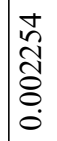 & 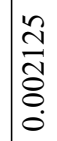 & 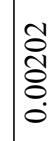 & & $\frac{n}{\infty}$ & 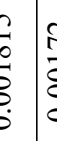 & $\begin{array}{l}\frac{1}{c} \\
\bar{\sigma} \\
0 \\
0\end{array}$ & 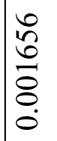 & 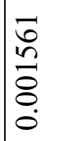 & $\begin{array}{l}\vec{\infty} \\
\stackrel{\infty}{+} \\
\stackrel{8}{8} \\
\dot{0}\end{array}$ & 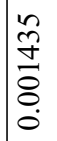 & $\frac{\infty}{m}$ & $\mid \begin{array}{c}\text { ते } \\
\stackrel{0}{8} \\
\stackrel{8}{0}\end{array}$ & $\begin{array}{l}n \\
0 \\
0 \\
0 \\
0 \\
0\end{array}$ & $\frac{⿱}{2}$ & 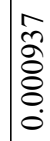 \\
\hline & & $\mathscr{\simeq}$ & 吾 & $\begin{array}{l}\infty \\
\tilde{n} \\
n \\
n\end{array}$ & $\begin{array}{l}\mathfrak{2} \\
\stackrel{8}{ \pm}\end{array}$ & $\mid \begin{array}{l}\infty \\
\infty \\
O \\
\Xi\end{array}$ & & 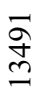 & ڤે̀ & 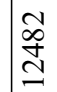 & 离 & 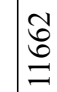 & 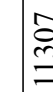 & 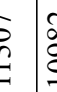 & $\begin{array}{l}0 \\
\infty \\
0 \\
0\end{array}$ & $\begin{array}{l}\mathscr{0} \\
\ddot{O} \\
\mathscr{O}\end{array}$ & 文 & $\frac{n}{0}$ & $\tilde{\widehat{\alpha}}$ & 趸 & 总 & Oे & $\frac{\widetilde{a}}{\sigma}$ & $\begin{array}{l}\tilde{\infty} \\
\infty \\
\infty\end{array}$ \\
\hline$\tilde{\Xi}$ & $\tilde{z}$ & 兽 & 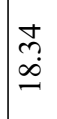 & $\underset{i}{\stackrel{J}{i}}$ & $\stackrel{?}{i}$ & in & & $\hat{m}$ & $\stackrel{?}{-}$ & $\vec{m}$ & $\ddot{n}$ & $\stackrel{\infty}{\stackrel{\infty}{\sim}}$ & 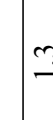 & $?$ & $\bar{g}$ & $\hat{n}$ & ஜூ & $\bar{m}$ & $\vec{m}$ & సે & 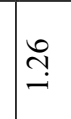 & $\vec{ָ}$ & 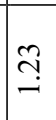 & $\begin{array}{l}\mathbf{D} \\
\infty \\
i\end{array}$ \\
\hline $\begin{array}{l}\frac{+}{\sigma} \\
\frac{\vec{\omega}}{\Xi} \\
\Xi\end{array}$ & 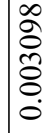 & $\vec{z}$ & $\begin{array}{l}+ \\
\infty \\
\dot{\infty} \\
\dot{0}\end{array}$ & $\begin{array}{l}\dot{b} \\
\dot{q} \\
\dot{q}\end{array}$ & $\underset{\vec{\gamma}}{\bar{\sigma}}$ & 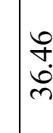 & & $\begin{array}{l}\hat{\sigma} \\
\stackrel{m}{m}\end{array}$ & $\begin{array}{l}n \\
\stackrel{n}{i} \\
\sim\end{array}$ & $\frac{\partial}{\dot{m}}$ & $\frac{\vec{N}}{m}$ & $\begin{array}{l}\delta \\
\text { নे }\end{array}$ & $\mid \begin{array}{l}\infty \\
2 \\
2\end{array}$ & b. & $\begin{array}{l}\text { ले } \\
\text { ते }\end{array}$ & $\begin{array}{l}\tilde{n} \\
\stackrel{2}{2} \\
\text { ते }\end{array}$ & $\begin{array}{l}\hat{n} \\
\infty \\
\infty \\
\sim\end{array}$ & 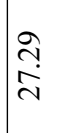 & $\stackrel{\infty}{\stackrel{\infty}{\sim}}$ & 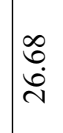 & 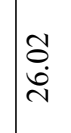 & $\begin{array}{l}\overrightarrow{0} \\
\ddot{i}\end{array}$ & 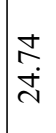 & $\begin{array}{l}+ \\
\vec{m} \\
m\end{array}$ \\
\hline 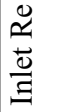 & $\begin{array}{l}0 \\
\stackrel{D}{0} \\
0 \\
\end{array}$ & 离 & $\vec{i}$ & $\stackrel{\partial}{\overrightarrow{+}}$ & $\frac{\vec{m}}{m}$ & $\infty$ & & $\begin{array}{l}n \\
i \\
i\end{array}$ & $\underset{\substack{t \\
i}}{i}$ & 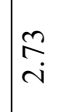 & $\hat{\hat{i}}$ & $\overline{\mathrm{i}}$ & $\stackrel{\alpha}{c}$ & $\stackrel{0}{0}$ & ゙ָ & $\vec{\gamma}$ & 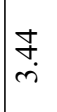 & $\stackrel{\vartheta}{\stackrel{\vartheta}{r}}$ & $\hat{n}$ & $\begin{array}{l}\hat{\sigma} \\
\dot{m}\end{array}$ & 它 & $\begin{array}{l}\infty \\
n \\
n\end{array}$ & $\stackrel{m}{\stackrel{m}{m}}$ & $\bar{\sigma}$ \\
\hline$\underset{\Xi}{\stackrel{\Xi}{\Xi}}$ & D্ & 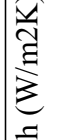 & & $\begin{array}{l}\hat{\alpha} \\
\infty \\
\infty\end{array}$ & $\stackrel{\substack{n \\
i}}{i}$ & $\frac{7}{2}$ & & $\begin{array}{l}5 \\
8 \\
8\end{array}$ & 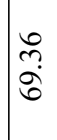 & 足 & $\begin{array}{l}\hat{6} \\
\text { i }\end{array}$ & $\frac{\partial}{i}$ & $\underset{7}{7}$ & & $i$ & $\underset{\substack{+\infty}}{\infty}$ & $\begin{array}{l}\tilde{N} \\
\infty \\
\infty\end{array}$ & $\stackrel{n}{2}$ & $\mid \begin{array}{l}n \\
\infty \\
\infty \\
\infty\end{array}$ & $\begin{array}{l}\infty \\
0 \\
2\end{array}$ & $\begin{array}{l}\stackrel{2}{2} \\
2 \\
2\end{array}$ & $\begin{array}{l}\infty \\
\infty \\
i \\
i\end{array}$ & 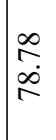 & $\stackrel{\sim}{\Im}$ \\
\hline$\sum_{\text {至 }}$ & $\stackrel{\sim}{\longrightarrow}$ & $\begin{array}{l}\text { శ્ } \\
\text { है } \\
= \\
=\end{array}$ & $\frac{\hat{\sigma}}{\stackrel{\tilde{\sigma}}{\vec{N}}}$ & $\frac{a}{\stackrel{a}{2}}$ & $\begin{array}{l}\hat{8} \\
8 \\
0 \\
0\end{array}$ & \begin{tabular}{l}
$n$ \\
2 \\
\multirow{2}{*}{} \\
s
\end{tabular} & & $\begin{array}{l}n \\
\infty \\
\infty \\
\tilde{\sigma}\end{array}$ & $\begin{array}{l}\overrightarrow{\widehat{I}} \\
\text { ปิ }\end{array}$ & $\begin{array}{l}\hat{\sigma} \\
\vec{\sigma} \\
\bar{\sigma}\end{array}$ & 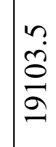 & $\begin{array}{l}0 \\
\stackrel{0}{0} \\
\infty \\
\infty \\
-1\end{array}$ & $\mid \begin{array}{l}\alpha \\
\hat{\alpha} \\
\alpha\end{array}$ & 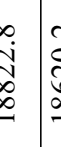 & 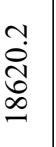 & $\begin{array}{l}\stackrel{2}{2} \\
\hat{\infty} \\
\infty \\
\infty\end{array}$ & $\begin{array}{l}\stackrel{0}{\infty} \\
\hat{\delta} \\
\infty \\
\infty \\
\infty\end{array}$ & $\frac{n}{\stackrel{n}{r}}$ & 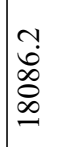 & 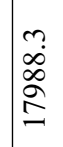 & 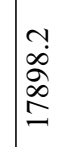 & 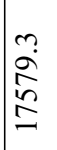 & 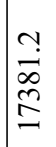 & $\begin{array}{l}n \\
\infty \\
0 \\
0 \\
0 \\
n\end{array}$ \\
\hline $\begin{array}{l}\sum_{\pi}^{0} \\
0 \\
0 \\
0 \\
0\end{array}$ & $\stackrel{\mathscr{\partial}}{\stackrel{\sigma}{\sigma}}$ & $\stackrel{\theta}{F}$ & 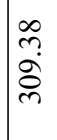 & $\begin{array}{l}m \\
\infty \\
m \\
m \\
m\end{array}$ & $\begin{array}{l}0 \\
\vdots \\
\dot{n} \\
\text { m } \\
m\end{array}$ & 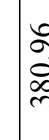 & & $\begin{array}{l}\hat{y} \\
\dot{d} \\
\dot{q}\end{array}$ & 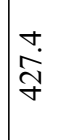 & 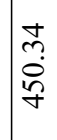 & 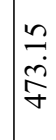 & $\begin{array}{l}\stackrel{N}{a} \\
\ddot{g} \\
\sigma \\
q\end{array}$ & $\begin{array}{l}\qquad \\
\alpha \\
\alpha \\
i\end{array}$ & 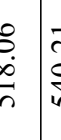 & $\begin{array}{l}\overrightarrow{\widehat{c}} \\
\stackrel{9}{+} \\
\dot{n}\end{array}$ & 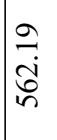 & $\begin{array}{l}\bar{\sigma} \\
\ddot{\infty} \\
\infty \\
\infty\end{array}$ & $\begin{array}{l}n \\
\vdots \\
0 \\
8 \\
0\end{array}$ & 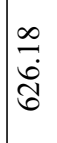 & $=$ & 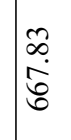 & $\begin{array}{l}\hat{N} \\
\infty \\
\infty \\
\infty\end{array}$ & 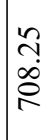 & 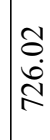 \\
\hline$\sum_{0}^{\pi}$ & ถุ & 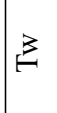 & $\begin{array}{l}\stackrel{2}{m} \\
\text { ले }\end{array}$ & $\begin{array}{l}\hat{n} \\
\tilde{n} \\
\tilde{n} \\
n\end{array}$ & $\frac{\tilde{n}}{\bar{\sigma}}$ & 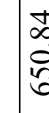 & & 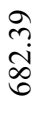 & 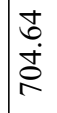 & 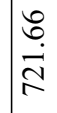 & 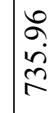 & 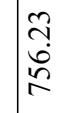 & \begin{tabular}{|l}
$\alpha$ \\
0 \\
0 \\
$\vdots$ \\
1 \\
1
\end{tabular} & \begin{tabular}{l|l}
0 \\
2 \\
2
\end{tabular} & $\begin{array}{l}\infty \\
\stackrel{\infty}{\circ} \\
\stackrel{\infty}{\infty}\end{array}$ & $\frac{9}{\stackrel{8}{8}}$ & $\frac{a}{\infty}$ & बे. & 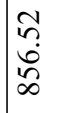 & $\begin{array}{l}\infty \\
n \\
\stackrel{+}{+} \\
\infty\end{array}$ & $\begin{array}{l}\tilde{N} \\
\dot{\alpha} \\
\infty\end{array}$ & $\begin{array}{l}n \\
\dot{d} \\
\sigma \\
\sigma\end{array}$ & 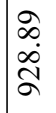 & $\begin{array}{l}n \\
\hat{\sigma} \\
\infty \\
\infty\end{array}$ \\
\hline $\begin{array}{l}\mathscr{y} \\
\tilde{z} \\
\tilde{z}\end{array}$ & $\stackrel{0}{0}$ & $\stackrel{9}{9}$ & $N$ & $\vec{\infty}$ & $\ddot{ \pm}$ & है & & તֶ. & 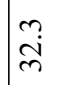 & $\begin{array}{l}n \\
\infty \\
\infty\end{array}$ & $\stackrel{+}{\stackrel{f}{+}}$ & 芦 & $\mid \begin{array}{l}n \\
\text { b } \\
\text { in }\end{array}$ & \begin{tabular}{l|l}
$n$ \\
$:$ \\
\end{tabular} & הֶ & $\mid \begin{array}{l}n \\
\infty \\
0\end{array}$ & $\begin{array}{l}\stackrel{0}{0} \\
\stackrel{+}{I}\end{array}$ & $\begin{array}{l}0 \\
\dot{\infty}\end{array}$ & $\hat{\infty}$ & $\hat{i}$ & $\begin{array}{l}\infty \\
\infty \\
\infty\end{array}$ & $\begin{array}{l}\infty \\
\stackrel{0}{0} \\
\mathbb{0}\end{array}$ & 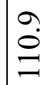 & \\
\hline
\end{tabular}




\begin{tabular}{|c|c|c|c|c|c|c|c|c|c|c|c|c|c|c|c|c|c|c|c|c|c|c|}
\hline & & $\vec{v}$ & 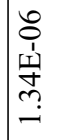 & 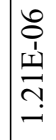 & 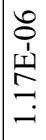 & 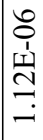 & 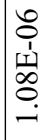 & 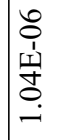 & 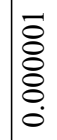 & 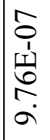 & 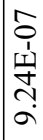 & 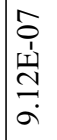 & $\begin{array}{l}\hat{0} \\
\hat{1} \\
\hat{y} \\
\infty \\
\infty \\
\infty \\
\infty\end{array}$ & \begin{tabular}{c}
$\hat{0}$ \\
$\hat{1}$ \\
\multicolumn{1}{|c}{} \\
$\infty$ \\
$\infty$ \\
$\infty$
\end{tabular} & 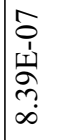 & 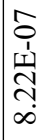 & $\begin{array}{l}\hat{0} \\
\hat{1} \\
\underline{1} \\
\infty \\
\infty \\
\infty \\
\infty\end{array}$ & \begin{tabular}{l}
$\hat{0}$ \\
$\hat{1}$ \\
\multirow{1}{1}{} \\
$\infty$ \\
$\infty$
\end{tabular} & 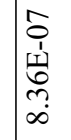 & 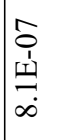 & $\begin{array}{l}\hat{a} \\
\hat{1} \\
1 \\
\infty \\
\infty \\
\infty \\
\end{array}$ & 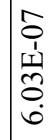 \\
\hline & & $\stackrel{\oplus}{\infty}$ & 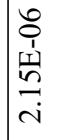 & $\begin{array}{l}0 \\
0 \\
1 \\
\text { İ } \\
\text { to } \\
-1\end{array}$ & 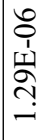 & $\mid \begin{array}{c}8 \\
0 \\
1 \\
1 \\
0 \\
0 \\
0 \\
-1\end{array}$ & $\begin{array}{l}0 \\
0 \\
\hat{1} \\
\hat{1} \\
\hat{6} \\
\infty \\
\infty \\
\infty\end{array}$ & $\begin{array}{l}\hat{O} \\
\hat{1} \\
\\
\\
\end{array}$ & \begin{tabular}{l}
$\hat{0}$ \\
$\hat{1}$ \\
1 \\
\multirow{1}{0}{} \\
0 \\
0
\end{tabular} & 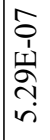 & 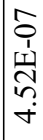 & 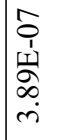 & 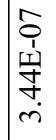 & 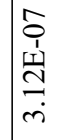 & $\mid \begin{array}{c}\hat{o} \\
\hat{1} \\
\hat{n} \\
\hat{n} \\
i\end{array}$ & 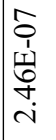 & 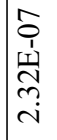 & 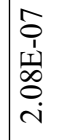 & 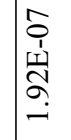 & 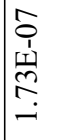 & 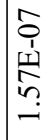 & 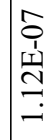 \\
\hline & & 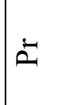 & $\underset{\stackrel{O}{\mathbb{N}}}{\stackrel{O}{0}}$ & 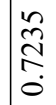 & 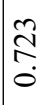 & 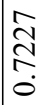 & $\begin{array}{l}\stackrel{0}{\mathbb{N}} \\
\stackrel{N}{0}\end{array}$ & 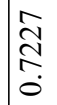 & 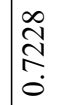 & 䒔 & $\begin{array}{l}\stackrel{n}{2} \\
\stackrel{N}{N} \\
\stackrel{0}{0}\end{array}$ & $\begin{array}{l}\stackrel{\widehat{N}}{\widehat{N}} \\
\stackrel{0}{0}\end{array}$ & 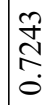 & 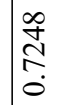 & $\begin{array}{l}\hat{n} \\
\stackrel{N}{N} \\
\dot{0}\end{array}$ & 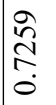 & $\begin{array}{l}\mathbb{U} \\
\stackrel{N}{N} \\
\dot{0}\end{array}$ & $\stackrel{\widehat{N}}{\hat{\sigma}}$ & $\mid \begin{array}{l}\frac{0}{N} \\
\stackrel{N}{0}\end{array}$ & $\begin{array}{l}\infty \\
\infty \\
\stackrel{\infty}{N} \\
\dot{0}\end{array}$ & $\begin{array}{l}\stackrel{\infty}{\infty} \\
\stackrel{N}{0} \\
\dot{0}\end{array}$ & ते \\
\hline & & $\widetilde{J}$ & 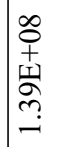 & 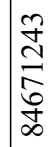 & $\begin{array}{l}o \\
+ \\
\infty \\
\infty \\
\infty \\
\infty \\
\dot{0} \\
n\end{array}$ & 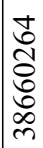 & 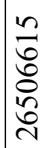 & 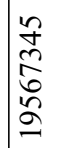 & $\begin{array}{l}0 \\
0 \\
0 \\
0 \\
0 \\
0 \\
0 \\
\end{array}$ & $\stackrel{n}{\stackrel{n}{z}}$ & $\begin{array}{l}\text { ర్d } \\
\stackrel{0}{n} \\
\tilde{n} \\
\infty\end{array}$ & 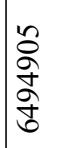 & 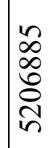 & $\begin{array}{l}\infty \\
\tilde{n} \\
\tilde{\sigma} \\
\tilde{\gamma}\end{array}$ & $\begin{array}{l}\infty \\
\infty \\
\infty \\
\infty \\
0 \\
0 \\
m\end{array}$ & 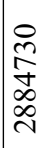 & 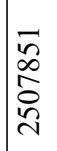 & 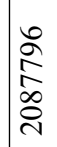 & 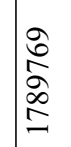 & 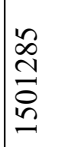 & 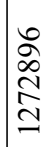 & $\begin{array}{c}\widetilde{\delta} \\
\stackrel{n}{\infty} \\
\infty \\
\infty\end{array}$ \\
\hline & & $t$ & $\begin{array}{l}\stackrel{\delta}{2} \\
\infty \\
\tilde{\delta} \\
\dot{\delta} \\
\dot{0}\end{array}$ & $\begin{array}{l}\tilde{\infty} \\
\tilde{N} \\
\tilde{\delta} \\
0 \\
0\end{array}$ & 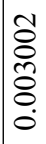 & $\begin{array}{l}\bar{n} \\
\hat{\widehat{\alpha}} \\
\stackrel{0}{0} \\
\dot{0}\end{array}$ & 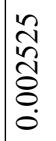 & $\begin{array}{l}\text { సे } \\
\widehat{\Xi} \\
\tilde{\delta} \\
\dot{0}\end{array}$ & $\begin{array}{l}\hat{n} \\
\tilde{\sigma} \\
\tilde{\delta} \\
\dot{0}\end{array}$ & 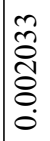 & $\begin{array}{l}\tilde{b} \\
\infty \\
\stackrel{\infty}{8} \\
0 \\
0\end{array}$ & 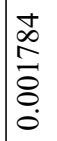 & $\begin{array}{l}\vec{\infty} \\
\stackrel{\infty}{0} \\
\stackrel{8}{8} \\
0\end{array}$ & 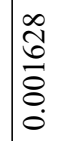 & $\frac{n}{\frac{n}{n}}$ & 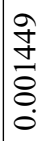 & 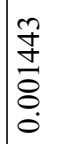 & $\underset{8}{\stackrel{7}{8}}$ & $\mid \begin{array}{c}\hat{0} \\
0 \\
\frac{0}{8} \\
0 \\
0\end{array}$ & 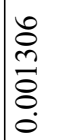 & 号 & $\mid \begin{array}{l}\infty \\
\tilde{\hat{\sigma}} \\
\grave{\delta} \\
\dot{0} \\
\dot{0}\end{array}$ \\
\hline & & $\mathscr{\sim}$ & 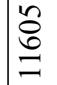 & $\begin{array}{l}\infty \\
\infty \\
\infty\end{array}$ & $\begin{array}{l}\stackrel{2}{0} \\
\text { ठิ }\end{array}$ & $\begin{array}{l}m \\
\infty \\
\alpha \\
\alpha\end{array}$ & 年 & 命 & $\widehat{\widehat{D}}$ & 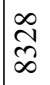 & ర్ & 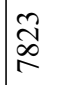 & $\stackrel{0}{0}$ & 舒 & $\stackrel{\overrightarrow{\mathrm{N}}}{\mathrm{i}}$ & $\stackrel{\infty}{\stackrel{0}{2}}$ & $\mid \begin{array}{l}\infty \\
\infty \\
\infty \\
0\end{array}$ & $\underset{n}{n}$ & ฉે & 苦 & 文 & స్ర \\
\hline లే & $\tilde{z}$ & 兽 & $\begin{array}{l}0 \\
\dot{0} \\
\end{array}$ & & $\stackrel{n}{2}$ & 声 & $\stackrel{6}{6}$ & $\begin{array}{l}\infty \\
n \\
0 \\
0\end{array}$ & 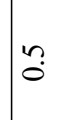 & If & ఫे & ले & ?ִ & $\hat{n}$ & $\stackrel{n}{n}$ & $\Xi_{0}^{+}$ & f̊. & $\hat{n}$ & 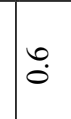 & : & $\mid \begin{array}{l}\infty \\
0 \\
0\end{array}$ & $\begin{array}{l}\hat{\sigma} \\
i\end{array}$ \\
\hline $\begin{array}{l}\frac{+}{\sigma} \\
\frac{\vec{\omega}}{\Xi}\end{array}$ & $\begin{array}{l}\overrightarrow{\hat{N}} \\
\tilde{\delta} \\
0 \\
0 \\
0\end{array}$ & $\vec{z}$ & 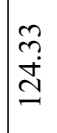 & $\begin{array}{l} \pm \\
0 \\
\text { iे }\end{array}$ & $\begin{array}{l}\infty \\
\stackrel{\infty}{0} \\
\dot{m}\end{array}$ & 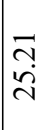 & $\stackrel{\infty}{\stackrel{\infty}{\sim}}$ & $\underset{\Xi}{\Xi}$ & 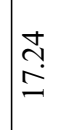 & 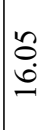 & $\stackrel{\vartheta}{\stackrel{ \pm}{ \pm}}$ & $\begin{array}{l}n \\
0 \\
2 \\
2\end{array}$ & $\begin{array}{l}\stackrel{2}{2} \\
\ddot{2}\end{array}$ & $\stackrel{m}{2}$ & 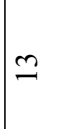 & $\begin{array}{l}\hat{N} \\
\ddot{n}\end{array}$ & $\begin{array}{l}\overrightarrow{0} \\
\dot{ \pm}\end{array}$ & $\begin{array}{l}0 \\
\infty \\
\end{array}$ & $\begin{array}{l}\dot{b} \\
\stackrel{0}{0}\end{array}$ & $\frac{2}{2}$ & ?ִ & 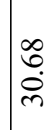 \\
\hline $\begin{array}{l}\stackrel{\mathscr{a}}{\simeq} \\
\frac{\tilde{\omega}}{\Xi} \\
\Xi\end{array}$ & 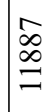 & 㝕 & $\frac{\tilde{\alpha}}{\bar{\lambda}}$ & $\underset{i}{\stackrel{t}{i}}$ & 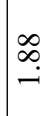 & $\stackrel{n}{n}$ & 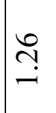 & $\stackrel{\cong}{=}$ & $\underset{\sigma}{\sigma}$ & $\hat{a}$ & $\begin{array}{l}0 \\
\infty \\
0 \\
0\end{array}$ & $\begin{array}{l}\mathscr{0} \\
\stackrel{0}{0} \\
0\end{array}$ & $\mid \begin{array}{l}n \\
\infty \\
0 \\
0\end{array}$ & $\bar{\sigma}$ & $\stackrel{n}{o}$ & $\hat{\theta}$ & $\stackrel{\overbrace{}}{\stackrel{1}{a}}$ & $\stackrel{n}{n}$ & $\stackrel{n}{\stackrel{2}{=}}$ & $\mid \begin{array}{l}\infty \\
\infty \\
\infty \\
-\end{array}$ & $\stackrel{m}{i}$ & $\mid \begin{array}{c}\infty \\
\infty \\
\infty\end{array}$ \\
\hline$\underset{\Xi}{\stackrel{\Xi}{\Xi}}$ & $\bar{\rho}$ & 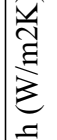 & 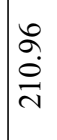 & 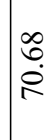 & $\begin{array}{l}\text { bִ } \\
\text { in }\end{array}$ & $\begin{array}{l}0 \\
\infty \\
\infty \\
i \\
i n\end{array}$ & 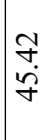 & $\underset{\stackrel{\infty}{N}}{\stackrel{\sim}{\mathcal{F}}}$ & $\begin{array}{l}\text { ț } \\
\dot{m}\end{array}$ & $\begin{array}{c}m \\
\infty \\
\infty \\
m\end{array}$ & $\begin{array}{l}\text { oे } \\
\text { in } \\
\text { n. }\end{array}$ & $\begin{array}{l}\dot{\infty} \\
\dot{m}\end{array}$ & 吕 & $\frac{n}{\dot{0}}$ & 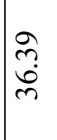 & $\begin{array}{l}0 \\
\stackrel{0}{\infty} \\
\infty \\
\infty\end{array}$ & 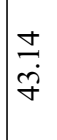 & $\begin{array}{l}\hat{0} \\
\infty \\
\infty \\
\dot{+}\end{array}$ & $\underset{⿱ 亠 䒑}{\stackrel{ \pm}{n}}$ & $\begin{array}{l}\vec{y} \\
\tilde{n}\end{array}$ & $\begin{array}{l}0 \\
0 \\
6 \\
\bullet\end{array}$ & 긍 \\
\hline$\sum_{\text {至 }}$ & $\hat{o}$ & $\begin{array}{l}\text { ठี } \\
\text { है } \\
= \\
\sigma\end{array}$ & $\stackrel{?}{\stackrel{?}{\&}}$ & 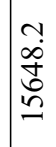 & \begin{tabular}{l}
0 \\
$\infty$ \\
$\infty$ \\
\multirow{2}{n}{} \\
\end{tabular} & $\begin{array}{l}\text { fó } \\
\text { 吾 }\end{array}$ & 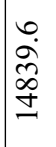 & 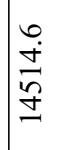 & $\begin{array}{l}\vec{\infty} \\
\infty \\
\vec{J}\end{array}$ & $\begin{array}{l}0 \\
\dot{0} \\
\dot{o} \\
\dot{+}\end{array}$ & $\begin{array}{l}\infty \\
\ddot{\delta} \\
n \\
n \\
n\end{array}$ & 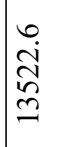 & 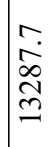 & $\begin{array}{l}\hat{\partial} \\
\grave{\hat{\alpha}} \\
\hat{2}\end{array}$ & 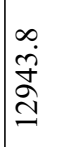 & 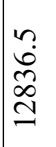 & 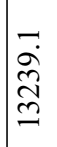 & $\underset{\mathscr{N}}{\stackrel{丶}{2}}$ & 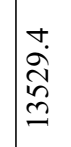 & $\begin{array}{l}3 \\
n \\
n \\
\approx \\
n \\
n\end{array}$ & $\begin{array}{l}\hat{\sigma} \\
\infty \\
\tilde{\infty} \\
\hat{\infty} \\
\end{array}$ & $\begin{array}{l}\sim \\
\tilde{\infty} \\
\infty \\
\tilde{O} \\
0\end{array}$ \\
\hline 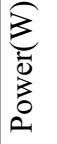 & : & $\stackrel{\theta}{F}$ & $\frac{\hat{\sigma}}{m}$ & \begin{tabular}{l}
\multirow{J}{*}{} \\
$\infty$ \\
$\infty$ \\
$m$
\end{tabular} & 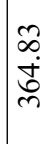 & $\begin{array}{l}\infty \\
\dot{2} \\
\dot{m}\end{array}$ & $\begin{array}{l}\tilde{\hat{\jmath}} \\
\underset{\sigma}{\sigma}\end{array}$ & 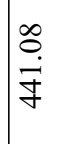 & $\begin{array}{l}1 \\
\tilde{y} \\
\vdots \\
b \\
b\end{array}$ & $\begin{array}{l}\text { I } \\
\dot{\infty} \\
\dot{+}\end{array}$ & $\begin{array}{l}\infty \\
\stackrel{1}{3} \\
i \\
\text { In }\end{array}$ & 商 & $\begin{array}{l}\stackrel{0}{1} \\
\stackrel{n}{n} \\
i n\end{array}$ & 寺. & $\frac{\text { đ̃ }}{3}$ & 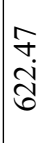 & $\begin{array}{l}\infty \\
\infty \\
\tilde{J}\end{array}$ & $\begin{array}{l}2 \\
0 \\
0 \\
0 \\
0\end{array}$ & $\begin{array}{l}n \\
\tilde{n} \\
\infty \\
0 \\
0\end{array}$ & 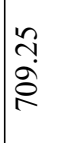 & 官 & $\begin{array}{l}\infty \\
\infty \\
\infty \\
\infty \\
\\
\end{array}$ \\
\hline$\sum_{0}^{\pi}$ & $\hat{n}$ & 3 & & $\begin{array}{l}\infty \\
\infty \\
\tilde{n} \\
n \\
n\end{array}$ & $\begin{array}{l}\vec{\nabla} \\
\text { ָे }\end{array}$ & $\mid \begin{array}{l}\partial \\
\infty \\
\infty \\
0\end{array}$ & $\begin{array}{l}\tilde{\partial} \\
\stackrel{\mathcal{I}}{\tau}\end{array}$ & 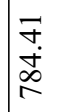 & $\begin{array}{l}\tilde{y} \\
\ddot{n} \\
\infty \\
\infty\end{array}$ & $\begin{array}{l}8 \\
0 \\
\infty \\
\infty \\
\infty\end{array}$ & $\begin{array}{l}\stackrel{1}{\vec{\alpha}} \\
\dot{\alpha}\end{array}$ & $\begin{array}{l}\text { J } \\
\text { নू. } \\
\text { S }\end{array}$ & 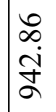 & $\begin{array}{l}\tilde{a} \\
\infty \\
\dot{a} \\
\alpha\end{array}$ & $\begin{array}{l}\hat{\alpha} \\
\dot{0} \\
\swarrow \alpha\end{array}$ & $\begin{array}{l}\infty \\
\infty \\
\infty \\
\infty \\
\infty \\
\alpha\end{array}$ & 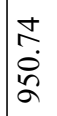 & $\begin{array}{l}n \\
0 \\
i \mathfrak{a} \\
\sigma\end{array}$ & $\mid \begin{array}{l}n \\
0 \\
g \\
g \\
g\end{array}$ & 先 & $\begin{array}{l}0 \\
6 \\
0 \\
0 \\
0\end{array}$ & 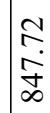 \\
\hline $\begin{array}{l}\mathscr{y} \\
\tilde{z} \\
\tilde{z}\end{array}$ & $=$ & $\stackrel{9}{9}$ & $\sim$ & $\vec{\infty}^{\infty}$ & 无 & 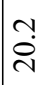 & సֶ. & 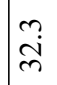 & $\begin{array}{l}m \\
\infty \\
m\end{array}$ & \& & in & $\begin{array}{l}n \\
\varnothing \\
i\end{array}$ & $\begin{array}{l}n \\
\text { in }\end{array}$ & $\mid \begin{array}{l}n \\
\infty \\
0\end{array}$ & $\begin{array}{l}0 \\
\stackrel{+}{\sim}\end{array}$ & $\begin{array}{l}0 \\
\dot{\infty}\end{array}$ & $\hat{\infty}$ & $\hat{\check{a}}$ & $\mid \begin{array}{l}\infty \\
\infty \\
\alpha\end{array}$ & $\begin{array}{l}\infty \\
\dot{0} \\
\stackrel{0}{0}\end{array}$ & 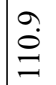 & \\
\hline
\end{tabular}




\begin{tabular}{|c|c|c|c|c|c|c|c|c|c|c|c|c|c|c|c|c|c|c|c|c|c|c|c|}
\hline & & $\vec{\Delta}$ & 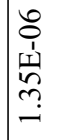 & 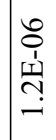 & 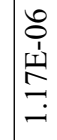 & 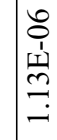 & $\begin{array}{l}0 \\
1 \\
\frac{1}{1} \\
8 \\
\end{array}$ & 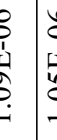 & 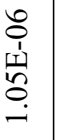 & 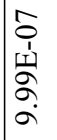 & $\begin{array}{l}0 \\
0 \\
\hat{1} \\
\hat{t} \\
0 \\
0 \\
0 \\
0\end{array}$ & $\begin{array}{l}\hat{0} \\
\hat{1} \\
\underline{1} \\
\vdots \\
0 \\
0\end{array}$ & \begin{tabular}{|l}
$\hat{0}$ \\
$\hat{1}$ \\
$\underline{1}$ \\
$\infty$ \\
$\infty$ \\
$\infty$ \\
$\infty$
\end{tabular} & 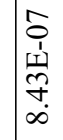 & $\begin{array}{l}\hat{0} \\
\hat{1} \\
\\
\tilde{n} \\
\infty \\
\infty\end{array}$ & $\mid \begin{array}{c}0 \\
0 \\
1 \\
1 \\
0 \\
0 \\
r \\
r\end{array}$ & 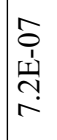 & 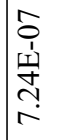 & $\begin{array}{c}5 \\
0 \\
1 \\
1 \\
\infty \\
0 \\
0 \\
r\end{array}$ & $\begin{array}{c}\hat{s} \\
\hat{1} \\
\text { 1े } \\
\hat{\sigma} \\
\hat{0}\end{array}$ & 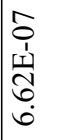 & 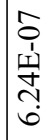 & 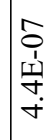 \\
\hline & & $\stackrel{\oplus}{\infty}$ & 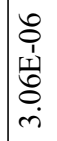 & 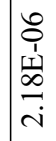 & 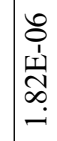 & 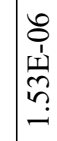 & $\begin{array}{c}0 \\
1 \\
\frac{1}{m} \\
-1 \\
-1\end{array}$ & 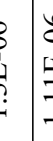 & 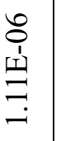 & 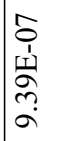 & 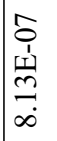 & 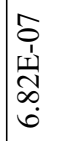 & 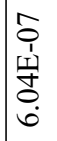 & 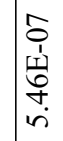 & $\begin{array}{l}\hat{o} \\
\hat{1} \\
\text { İ } \\
\hat{\sigma}\end{array}$ & 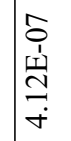 & 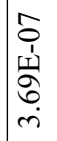 & 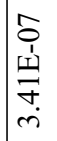 & $\begin{array}{l}\hat{0} \\
\hat{1} \\
\underline{1} \\
\infty \\
\vec{m} \\
\dot{m}\end{array}$ & 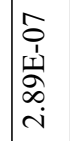 & $\begin{array}{l}\hat{0} \\
\hat{1} \\
\underline{1} \\
\hat{6} \\
\text { in }\end{array}$ & $\begin{array}{l}\hat{O} \\
\mathbf{1} \\
\underline{1} \\
\hat{N} \\
\end{array}$ & $\begin{array}{c}n \\
0 \\
1 \\
n \\
n \\
n \\
-1\end{array}$ \\
\hline & & $\vec{D}$ & 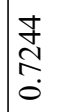 & $\underset{\tilde{N}}{\stackrel{N}{0}}$ & 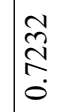 & 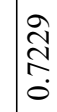 & $\hat{\curvearrowright}$ & & 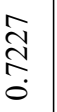 & 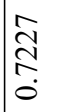 & \begin{tabular}{l}
$\infty$ \\
\multirow{N}{N}{} \\
$\dot{0}$
\end{tabular} & 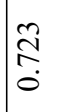 & 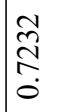 & 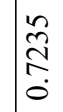 & $\begin{array}{l}\infty \\
\stackrel{N}{N} \\
\stackrel{0}{0}\end{array}$ & 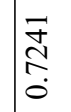 & 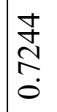 & 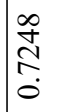 & $\begin{array}{l}\overrightarrow{\widetilde{a}} \\
\stackrel{2}{0} \\
0\end{array}$ & 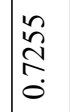 & $\begin{array}{l}\stackrel{2}{n} \\
\stackrel{1}{0} \\
\dot{0}\end{array}$ & 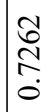 & 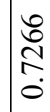 \\
\hline & & $\widetilde{J}$ & 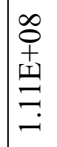 & $\begin{array}{l}n \\
\hat{\tilde{D}} \\
\infty \\
\hat{\tilde{n}} \\
\hat{\delta}\end{array}$ & 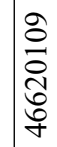 & $\begin{array}{l}\hat{n} \\
\frac{n}{n} \\
n \\
n \\
n\end{array}$ & 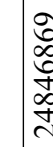 & & $\begin{array}{l}\stackrel{P}{\infty} \\
\infty \\
\stackrel{+}{+} \\
\infty \\
\infty\end{array}$ & 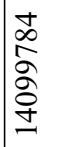 & $\mid \begin{array}{l}\hat{8} \\
8 \\
8 \\
8 \\
0\end{array}$ & $\begin{array}{l}\hat{\hat{~}} \\
\tilde{y} \\
\tilde{\infty} \\
\infty\end{array}$ & 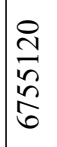 & $\mid \begin{array}{l}\hat{0} \\
\tilde{n} \\
0 \\
i \\
n\end{array}$ & 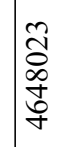 & 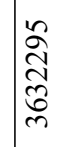 & 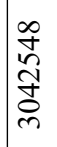 & 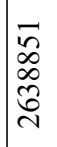 & 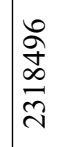 & 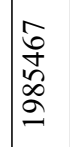 & 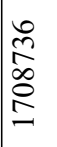 & $\mid$\begin{tabular}{|}
$\vec{\Xi}$ \\
$\infty$ \\
$\underset{\infty}{\Xi}$
\end{tabular} & $\begin{array}{l}\overrightarrow{\vec{N}} \\
\vec{\Omega}\end{array}$ \\
\hline & & $t$ & 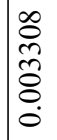 & 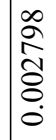 & $\begin{array}{l}n \\
\infty \\
n \\
\hat{\delta} \\
\delta \\
0\end{array}$ & ळેे & $\frac{\mathfrak{I}}{\tilde{\varepsilon}}$ & 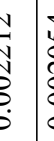 & 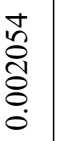 & 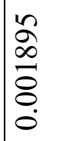 & $\mid \begin{array}{l}0 \\
\stackrel{0}{i} \\
\frac{1}{8} \\
0 \\
0\end{array}$ & $\begin{array}{l}8 \\
8 \\
0 \\
8 \\
8 \\
0\end{array}$ & $\begin{array}{l}0 \\
\tilde{n} \\
\\
8 \\
0 \\
0\end{array}$ & 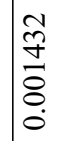 & 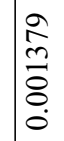 & 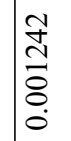 & $\begin{array}{l}\text { 守 } \\
\bar{\Xi} \\
\stackrel{8}{0}\end{array}$ & 㐫 & $\mid \begin{array}{l}0 \\
0 \\
0 \\
0 \\
8 \\
0 \\
0\end{array}$ & $\mid \begin{array}{l}\bar{n} \\
0 \\
0 \\
0 \\
0\end{array}$ & 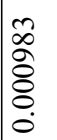 & 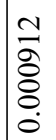 & 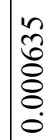 \\
\hline & & $\mathscr{\simeq}$ & $\begin{array}{l}\tilde{N} \\
\alpha \\
\alpha\end{array}$ & సે & $\begin{array}{l}\vec{D} \\
\infty \\
\infty\end{array}$ & 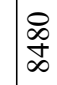 & $\frac{1}{\infty}$ & & $\begin{array}{l}\infty \\
\substack{\infty \\
\infty}\end{array}$ & 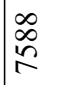 & $\stackrel{+}{\cong}$ & $\underset{⿱}{\stackrel{9}{r}}$ & $\overline{8}$ & $\overline{\widehat{\widehat{\sigma}}}$ & 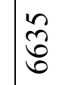 & भ̂े & $\begin{array}{l}\infty \\
0 \\
\delta \\
6\end{array}$ & స్ & $\frac{9}{6}$ & 咚 & ळิ & $\begin{array}{l}8 \\
\infty \\
\infty \\
n\end{array}$ & 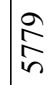 \\
\hline$\tilde{\Xi}$ & $\tilde{z}$ & 兽 & $\stackrel{\overbrace{}}{=}$ & $\underset{-}{\exists}$ & مै & $\hat{\sigma}$ & బֶ. & & f̊ & $\stackrel{t}{\circ}$ & $\mid \begin{array}{l}n \\
n \\
0 \\
0\end{array}$ & సิ & $\hat{ָ}$ & గit & กี & กุ & $\frac{9}{0}$ & $\stackrel{\infty}{\stackrel{\infty}{0}}$ & $\stackrel{\infty}{\longrightarrow}$ & $\stackrel{0}{0}$ & $\stackrel{0}{0}$ & $\stackrel{7}{0}$ & 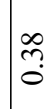 \\
\hline $\begin{array}{l}\frac{+}{\sigma} \\
\frac{\vec{\omega}}{\Xi} \\
\Xi\end{array}$ & 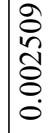 & $\vec{z}$ & $\frac{\partial}{\stackrel{\vec{E}}{\Xi}}$ & $\begin{array}{l}3 \\
\dot{n} \\
n\end{array}$ & 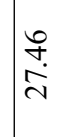 & $\begin{array}{l}+ \\
\text { ते } \\
\text { in }\end{array}$ & $\underset{\infty}{\infty}$ & 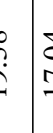 & $\begin{array}{l}\underset{I}{J} \\
\stackrel{1}{2}\end{array}$ & $\stackrel{g}{\dot{ \pm}}$ & $\begin{array}{l}\stackrel{n}{\sim} \\
\stackrel{0}{\sim}\end{array}$ & 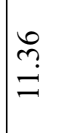 & $\stackrel{\Im}{\stackrel{f}{\varrho}}$ & f̊. & $\hat{\sigma}$ & $\stackrel{2}{2}$ & $\cong$ & $\underset{r}{0}$ & $\frac{0}{6}$ & ñ & $\frac{0}{6}$ & 命 & $\stackrel{\overbrace{}}{\stackrel{2}{r}}$ \\
\hline 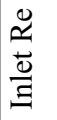 & ฮิ & 蔤 & $\begin{array}{l}\stackrel{0}{n} \\
\stackrel{2}{2}\end{array}$ & $\stackrel{m}{i}$ & $\stackrel{\infty}{n}$ & $\stackrel{\text { }}{\stackrel{2}{n}}$ & $\bar{a}$ & 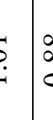 & $\begin{array}{c}\infty \\
\infty \\
0 \\
0\end{array}$ & $\stackrel{\hat{f}}{\hat{0}}$ & $\hat{o}$ & 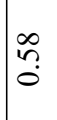 & ñn & f̊. & $\stackrel{\infty}{\stackrel{\infty}{0}}$ & 守 & J. & ‡. & t. & $\ddot{0}$ & 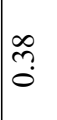 & f. & $\stackrel{\infty}{\stackrel{\infty}{-}}$ \\
\hline$\underset{\Xi}{\stackrel{\Xi}{\Xi}}$ & 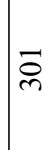 & 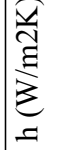 & $\begin{array}{c}0 \\
\infty \\
\stackrel{\infty}{2}\end{array}$ & $\begin{array}{l}3 \\
\tilde{\sigma}\end{array}$ & 守 & $\mid \begin{array}{l}\stackrel{\varkappa}{\sigma} \\
\dot{f}\end{array}$ & $\begin{array}{l}\infty \\
i s \\
i \\
j \\
j\end{array}$ & $\begin{array}{c}0 \\
? \\
:\end{array}$ & $\begin{array}{l}\overrightarrow{7} \\
\dot{0} \\
\dot{n}\end{array}$ & $\underset{\hat{i}}{\hat{n}}$ & 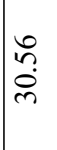 & $\begin{array}{l}0 \\
\dot{0} \\
\sim\end{array}$ & $\begin{array}{l}\tilde{i} \\
\ddot{v} \\
\end{array}$ & 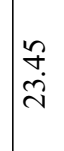 & $\begin{array}{l}\text { ț } \\
\text { ते }\end{array}$ & $\hat{i}$ & 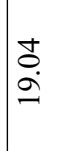 & 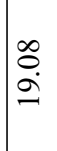 & $\begin{array}{l}\mathbb{N} \\
\infty \\
\infty\end{array}$ & $\begin{array}{l}n \\
\infty \\
\infty\end{array}$ & 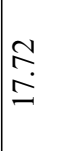 & $\stackrel{\infty}{\stackrel{\infty}{\sim}}$ & $\mid \begin{array}{l} \pm \\
\hat{A} \\
\text { a }\end{array}$ \\
\hline$\sum_{\text {至 }}$ & $\tilde{o}$ & $\begin{array}{l}\text { đิ } \\
\text { है } \\
= \\
=\end{array}$ & $\underset{\stackrel{亠}{\Xi}}{\stackrel{\Xi}{\Xi}}$ & 竎 & 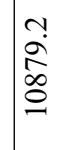 & 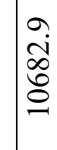 & E & & 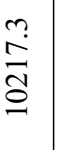 & $\begin{array}{l}\infty \\
\infty \\
\infty\end{array}$ & $\begin{array}{l}n \\
\text { à } \\
0 \\
0 \\
\alpha\end{array}$ & $\frac{n}{\underset{f}{J}}$ & 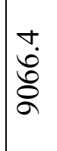 & 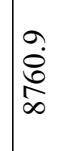 & $\underset{\infty}{\infty} \underset{\infty}{\infty}$ & $\underset{\infty}{\stackrel{0}{\Xi}}$ & $\stackrel{+}{\stackrel{亠}{\rightleftarrows}}$ & $\begin{array}{l}\infty \\
\infty \\
\infty \\
\infty\end{array}$ & $\overrightarrow{\stackrel{N}{\mathbb{N}}}$ & $\begin{array}{l}3 \\
8 \\
8 \\
0 \\
2\end{array}$ & \begin{tabular}{l}
$\infty$ \\
\multirow{J}{J}{} \\
$\stackrel{\infty}{r}$
\end{tabular} & ลิ & 守 \\
\hline 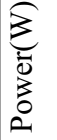 & $\stackrel{\mathcal{J}}{\Xi}$ & $\stackrel{\theta}{F}$ & $\mid \begin{array}{l}\infty \\
\infty \\
\infty \\
0 \\
0\end{array}$ & $\begin{array}{l}\stackrel{\text { సे }}{\text { in }} \\
\text { }\end{array}$ & 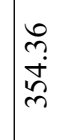 & $\begin{array}{l}\hat{0} \\
\dot{0} \\
\text { in }\end{array}$ & 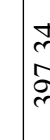 & & $\begin{array}{l}\mathcal{I} \\
\underset{\infty}{\forall} \\
\frac{\sigma}{\sigma}\end{array}$ & $\begin{array}{l}\vec{n} \\
\infty \\
\tilde{\sigma}\end{array}$ & 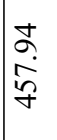 & $\begin{array}{l}\infty \\
\stackrel{\infty}{\sigma} \\
\stackrel{f}{\gamma}\end{array}$ & $\begin{array}{l}\mathscr{2} \\
\stackrel{+}{+} \\
\dot{q}\end{array}$ & $\begin{array}{l}\stackrel{i}{a} \\
\stackrel{i}{n}\end{array}$ & $\begin{array}{l}\overrightarrow{0} \\
\text { in }\end{array}$ & $\begin{array}{l}2 \\
\hat{\imath} \\
\dot{6} \\
\dot{1}\end{array}$ & 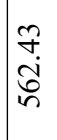 & 站 & $\begin{array}{l}\overrightarrow{0} \\
\ddot{j} \\
\tilde{n}\end{array}$ & $\begin{array}{l}8 \\
0 \\
\infty \\
0 \\
0\end{array}$ & 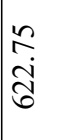 & $\hat{\sigma}$ & 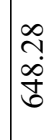 \\
\hline$\sum_{0}^{\pi}$ & $\begin{array}{l}\infty \\
n \\
n\end{array}$ & 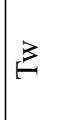 & $\frac{a}{\stackrel{0}{0}}$ & ㄱ. & $\begin{array}{l}\overrightarrow{7} \\
\dot{b} \\
i\end{array}$ & 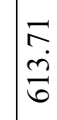 & $\frac{5}{7}$ & $\begin{array}{l}\stackrel{0}{?} \\
? \\
0 \\
0\end{array}$ & 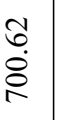 & $\begin{array}{l}0 \\
0 \\
\dot{9} \\
\end{array}$ & 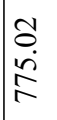 & 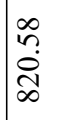 & \begin{tabular}{l}
$\vec{m}$ \\
\multirow{2}{*}{} \\
$\infty$
\end{tabular} & $\begin{array}{l}\infty \\
\infty \\
\infty \\
\infty \\
\infty \\
\infty\end{array}$ & $\begin{array}{l}\stackrel{゚}{2} \\
\stackrel{0}{\sigma} \\
\sigma\end{array}$ & $\begin{array}{l}\infty \\
\infty \\
\infty \\
\infty \\
\alpha \\
\alpha\end{array}$ & 竎 & $\begin{array}{l}n \\
\tilde{n} \\
\infty \\
\alpha\end{array}$ & $\begin{array}{l}\bar{n} \\
n \\
\tilde{\delta} \\
0\end{array}$ & 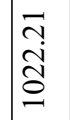 & 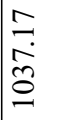 & $\begin{array}{l}n \\
0 \\
\tilde{n} \\
\tilde{0}\end{array}$ & $\begin{array}{l}\triangleright \\
\infty \\
\tilde{\delta} \\
\infty\end{array}$ \\
\hline $\begin{array}{l}\mathscr{y} \\
\tilde{z} \\
\tilde{z}\end{array}$ & $\infty$ & $\stackrel{9}{9}$ & $N$ & $\vec{\infty}$ & $\ddot{ \pm}$ & त्. & $\hat{\imath}$ & 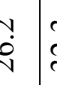 & 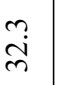 & $\begin{array}{c}n \\
\infty \\
\infty\end{array}$ & $\underset{f}{\dot{f}}$ & ì. & \begin{tabular}{l}
$n$ \\
\hdashline \\
$i n$
\end{tabular} & $\begin{array}{l}n \\
\text { in }\end{array}$ & $\begin{array}{l}n \\
\infty \\
\infty \\
\infty\end{array}$ & $\begin{array}{l}\stackrel{0}{+} \\
\stackrel{+}{I}\end{array}$ & $\begin{array}{l}0 \\
\dot{\infty}\end{array}$ & $\hat{\infty}$ & $\hat{\check{a}}$ & $\begin{array}{l}\infty \\
\infty \\
\infty\end{array}$ & $\begin{array}{l}\infty \\
\dot{0} \\
\stackrel{\sigma}{0}\end{array}$ & 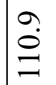 & \\
\hline
\end{tabular}




\begin{tabular}{|c|c|c|c|c|c|c|c|c|c|c|c|c|c|c|c|c|c|c|c|c|c|c|c|c|}
\hline & & $\vec{\Delta}$ & 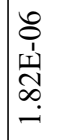 & 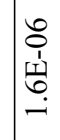 & $\begin{array}{l}8 \\
\vdots \\
1 \\
\text { N } \\
\\
-1\end{array}$ & 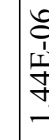 & & 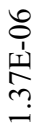 & 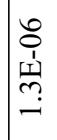 & $\begin{array}{l}8 \\
\vdots \\
1 \\
\\
\\
\end{array}$ & 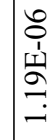 & 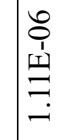 & ב & 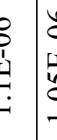 & 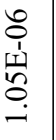 & 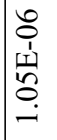 & 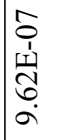 & 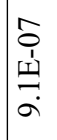 & 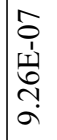 & 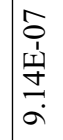 & $\begin{array}{c}\hat{y} \\
\hat{1} \\
\underline{1} \\
\tilde{o} \\
0 \\
\alpha\end{array}$ & 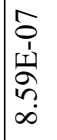 & 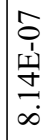 & $\begin{array}{l}\hat{0} \\
1 \\
1 \\
\infty \\
0 \\
0 \\
\text { in }\end{array}$ \\
\hline & & $\stackrel{\oplus}{\infty}$ & $\begin{array}{l}0 \\
0 \\
1 \\
\text { 11 } \\
0 \\
0 \\
0\end{array}$ & 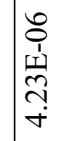 & $\begin{array}{c}8 \\
0 \\
1 \\
1 \\
\infty \\
+ \\
\dot{+} \\
\end{array}$ & $\mid \begin{array}{c}0 \\
1 \\
1 \\
\infty \\
\infty \\
\infty \\
c\end{array}$ & & 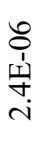 & 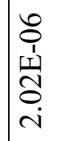 & 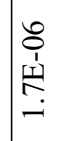 & 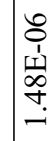 & 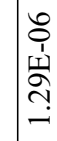 & 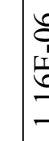 & 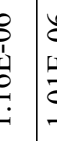 & $\begin{array}{l}0 \\
0 \\
1 \\
1 \\
1 \\
0 \\
. \\
-\end{array}$ & 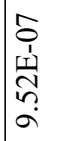 & $\begin{array}{l}\hat{0} \\
\hat{1} \\
\hat{1} \\
\hat{2} \\
\end{array}$ & $\begin{array}{l}\hat{0} \\
\hat{1} \\
\\
\\
\end{array}$ & 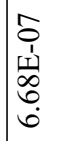 & 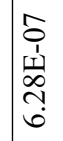 & $\mid \begin{array}{c}\hat{o} \\
\hat{1} \\
\\
\\
\hat{n} \\
i n\end{array}$ & 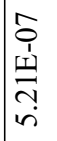 & 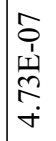 & 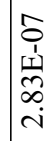 \\
\hline & & 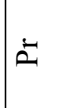 & 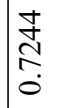 & 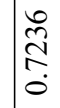 & 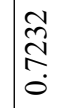 & ָิ & & 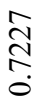 & $\underset{\mathbb{N}}{\stackrel{\Xi}{0}}$ & 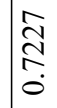 & 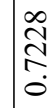 & 勇 & $\overline{\tilde{n}}$ & $\begin{array}{l}\vec{b} \\
\dot{b}\end{array}$ & $\begin{array}{l}\stackrel{2}{\hat{N}} \\
\stackrel{N}{\circ}\end{array}$ & 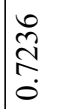 & 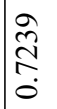 & 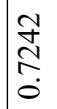 & 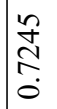 & 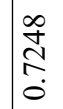 & 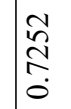 & $\begin{array}{l}\tilde{n} \\
\stackrel{2}{N} \\
\dot{0}\end{array}$ & 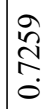 & 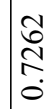 \\
\hline & & $\widetilde{J}$ & 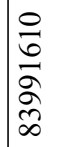 & 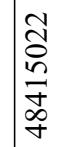 & $\begin{array}{l}\hat{\delta} \\
\hat{2} \\
\hat{\sigma} \\
\infty \\
\tilde{m}\end{array}$ & İ & & 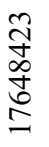 & $\begin{array}{l}2 \\
\hat{0} \\
2 \\
m \\
2 \\
2\end{array}$ & $\begin{array}{l}\tilde{n} \\
\tilde{n} \\
\infty \\
\tilde{\alpha} \\
\alpha\end{array}$ & 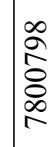 & 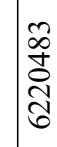 & $\mid \begin{array}{l}\overline{8} \\
\bar{y} \\
\bar{y} \\
\bar{r}\end{array}$ & $\begin{array}{l}5 \\
\hat{5} \\
6 \\
5\end{array}$ & $\begin{array}{l}\vec{f} \\
\stackrel{心}{m} \\
\stackrel{F}{F}\end{array}$ & 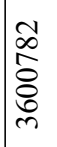 & 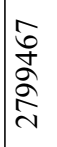 & 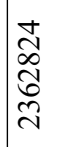 & 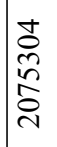 & 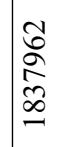 & $\mid \begin{array}{l}0 \\
\substack{0 \\
n \\
\infty \\
n \\
n}\end{array}$ & $\begin{array}{l}\infty \\
\infty \\
\infty \\
0 \\
0 \\
\end{array}$ & 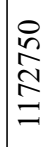 & 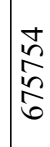 \\
\hline & & $t$ & 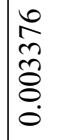 & 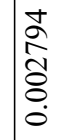 & 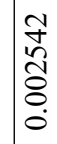 & $\mid \begin{array}{l}\infty \\
\tilde{\Xi} \\
\tilde{\delta}\end{array}$ & & 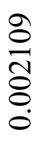 & $\begin{array}{l}\overrightarrow{2} \\
\stackrel{\sigma}{8} \\
\dot{0}\end{array}$ & $\frac{0}{8}$ & $\mid \begin{array}{l}0 \\
0 \\
0 \\
8 \\
8 \\
0 \\
0\end{array}$ & $\mid \begin{array}{l}\hat{2} \\
\stackrel{n}{8} \\
8 \\
0 \\
0\end{array}$ & zo & 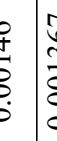 & $\begin{array}{l}\overline{0} \\
\frac{1}{2} \\
\overline{8} \\
0 \\
0\end{array}$ & $\begin{array}{l}\stackrel{+}{m} \\
\stackrel{2}{\circ} \\
\stackrel{8}{8} \\
0\end{array}$ & 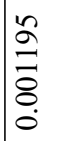 & & 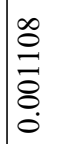 & 咅 & $\mid$ & 令 & 商 & 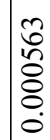 \\
\hline & & $\mathscr{\sim}$ & 总 & 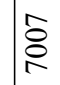 & 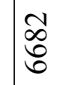 & f̊t & & $\frac{\hat{n}}{6}$ & 年 & $\begin{array}{l}8 \\
\text { in }\end{array}$ & $\begin{array}{l}\text { ta } \\
\text { in }\end{array}$ & 㗭 & $\overline{\bar{s}}$ & $\bar{n}$ & $\frac{\infty}{n}$ & 硧 & $\begin{array}{l}\hat{\grave{o}} \\
\stackrel{q}{q}\end{array}$ & $\underset{\substack{+\stackrel{\infty}{q}}}{ }$ & 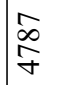 & 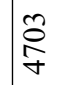 & & $\begin{array}{l}\text { 守 } \\
\text { f }\end{array}$ & $\vec{\infty}$ & নे \\
\hline లే & $\tilde{z}$ & 莺 & $\stackrel{n}{\infty}$ & જे & ñ? & f & & חై & $\stackrel{\overbrace{}}{\hat{o}}$ & $\stackrel{J}{0}$ & İ & $\frac{2}{0}$ & $\frac{\alpha}{c}$ & $\stackrel{0}{0}$ & $\stackrel{\infty}{\stackrel{\infty}{0}}$ & $\stackrel{7}{0}$ & $\stackrel{0}{\circ}$ & $\stackrel{n}{0}$ & $\stackrel{n}{0}$ & $\stackrel{n}{0}$ & $\frac{0}{0}$ & $\stackrel{ \pm}{0}$ & $\stackrel{n}{0}$ & ?ֶ? \\
\hline $\begin{array}{l}\frac{+}{\sigma} \\
\frac{\vec{\omega}}{\Xi} \\
\Xi\end{array}$ & $\begin{array}{l}\infty \\
\widetilde{\Xi} \\
\widehat{్} \\
0 \\
0\end{array}$ & $\vec{z}$ & बे. & $\begin{array}{l}0 \\
\infty \\
\infty\end{array}$ & $\begin{array}{l}\bar{b} \\
\dot{i}\end{array}$ & $\begin{array}{l}0 \\
0 \\
0\end{array}$ & & $\begin{array}{l}\stackrel{8}{0} \\
\ddot{g}\end{array}$ & $\stackrel{\sim}{\Xi}$ & $\begin{array}{l}\text { D } \\
\text { a } \\
\sigma\end{array}$ & $\hat{\sigma}^{\circ}$ & م & $i$ & $\hat{n}$ & $\stackrel{\circ}{\stackrel{2}{r}}$ & $\stackrel{\overbrace{}}{r}$ & $\overrightarrow{\tilde{\sigma}}$ & $\begin{array}{l}\infty \\
\infty \\
i n\end{array}$ & $\vec{\sigma}$ & $\begin{array}{l}\infty \\
\infty \\
i n\end{array}$ & $\stackrel{n}{\hat{n}}$ & fr. & ते & ?ु. \\
\hline 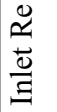 & $\begin{array}{l}\Re \\
\stackrel{2}{n}\end{array}$ & 駕 & $\begin{array}{l}\stackrel{0}{n} \\
\stackrel{n}{ \pm}\end{array}$ & & - & $\stackrel{2}{a}$ & & $\hat{n}$ & 守. & ț & $\stackrel{\text { If }}{0}$ & $\hat{\jmath}$ & ع & $?$ & $\begin{array}{l}0 \\
\text { חె? } \\
0\end{array}$ & ?ִ. & $\tilde{n}$ & $\tilde{\dddot{m}}$ & $\stackrel{n}{\tilde{c}}$ & ले & $\tilde{m}$ & $\stackrel{\tilde{m}}{3}$ & $\begin{array}{l}0 \\
\text { חִ } \\
0\end{array}$ & ơ \\
\hline 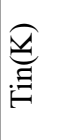 & $\bar{\rho}$ & 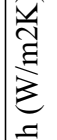 & $\mid \begin{array}{l}n \\
\alpha \\
0 \\
0 \\
0\end{array}$ & $\stackrel{m}{\stackrel{n}{n}}$ & 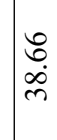 & $\frac{\infty}{\infty}$ & & 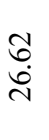 & $\begin{array}{l}\infty \\
0 \\
\tilde{\lambda}\end{array}$ & $\frac{\mathfrak{g}}{\dot{\sim}}$ & İ. & $\begin{array}{l}\hat{\lambda} \\
\infty \\
\infty\end{array}$ & $\mid \begin{array}{l}a \\
0 \\
\Omega\end{array}$ & : & 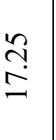 & 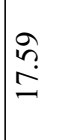 & $\frac{0}{6}$ & $\begin{array}{l}\text { ते } \\
\text { in }\end{array}$ & $\begin{array}{l}\infty \\
\stackrel{\infty}{\sim} \\
\end{array}$ & $\begin{array}{l}\infty \\
\infty \\
\end{array}$ & $\ddot{g}$ & $\begin{array}{l}\vec{n} \\
\ddot{n}\end{array}$ & $\begin{array}{l}\tilde{N} \\
\text { in }\end{array}$ & $\mid \begin{array}{l}\infty \\
\tilde{\infty} \\
\infty\end{array}$ \\
\hline$\sum_{\text {至 }}$ & ? & $\begin{array}{l}\text { है } \\
\vdots \\
=\end{array}$ & 啇 & $\overrightarrow{\tilde{N}}$ & 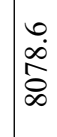 & $\mid \begin{array}{l}n \\
\infty \\
0 \\
7 \\
7\end{array}$ & & 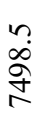 & 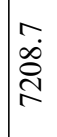 & $\frac{0}{6}$ & 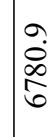 & $\underset{n}{2}$ & $\begin{array}{l}7 \\
8 \\
8 \\
8\end{array}$ & ك̧ & $\begin{array}{l}\infty \\
\dot{\vec{D}} \\
\text { ָु. }\end{array}$ & 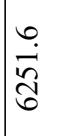 & $\underset{\substack{i \\
i n}}{\stackrel{i}{i}}$ & $\stackrel{\overrightarrow{\bar{n}}}{i n}$ & $\begin{array}{l}0 \\
2 \\
\hat{2} \\
i \\
n\end{array}$ & $\begin{array}{l}\infty \\
\stackrel{\infty}{\hat{\delta}} \\
i\end{array}$ & $\begin{array}{l}n \\
\stackrel{n}{0} \\
i\end{array}$ & $\begin{array}{l}\infty \\
\infty \\
n \\
n\end{array}$ & $\begin{array}{l}0 \\
\stackrel{0}{\infty} \\
\tilde{n}\end{array}$ & ڤે \\
\hline 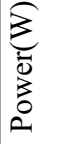 & $\frac{\infty}{\infty}$ & 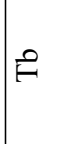 & 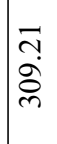 & $\begin{array}{l}\infty \\
\stackrel{\infty}{i} \\
\stackrel{m}{m}\end{array}$ & $\begin{array}{l}\infty \\
0 \\
\dot{+} \\
\dot{m} \\
m\end{array}$ & $\begin{array}{l}\vec{D} \\
\infty \\
n \\
m\end{array}$ & & $\begin{array}{l}\text { İ } \\
\text { ڤू } \\
\text { ले }\end{array}$ & $\begin{array}{l}\tilde{\sigma} \\
\infty \\
\\
\forall \\
\forall\end{array}$ & $\begin{array}{l}\mathcal{G} \\
\dot{+} \\
\dot{q} \\
\stackrel{+}{*}\end{array}$ & $\begin{array}{l}\infty \\
\infty \\
i \\
\tilde{f} \\
\forall\end{array}$ & $\begin{array}{l}\infty \\
\stackrel{2}{?} \\
\stackrel{f}{f}\end{array}$ & $\hat{a}$ & 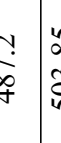 & $\begin{array}{l}\infty \\
\infty \\
\tilde{n} \\
\tilde{n}\end{array}$ & $\begin{array}{l}\hat{\lambda} \\
\stackrel{i}{0} \\
\tilde{n}\end{array}$ & $\begin{array}{l}8 \\
0 \\
\dot{0} \\
\tilde{n} \\
\text { n. }\end{array}$ & $\begin{array}{l}\infty \\
\dot{0} \\
\hat{n} \\
\text { n. }\end{array}$ & $\begin{array}{l}\bar{n} \\
n \\
0 \\
n\end{array}$ & $\begin{array}{l}\stackrel{̃}{~} \\
\stackrel{\infty}{\infty} \\
\infty \\
i n\end{array}$ & $\begin{array}{l}\infty \\
\infty \\
\dot{j} \\
i n\end{array}$ & $\frac{ \pm}{8}$ & 총 & $\begin{array}{l}\infty \\
\tilde{n} \\
\tilde{\sigma}\end{array}$ \\
\hline$\sum_{0}^{\pi}$ & ?ֶ. & 3 & \begin{tabular}{l}
$n$ \\
\multirow{d}{*}{} \\
\end{tabular} & $\mid \begin{array}{l}\sigma \\
b \\
\alpha \\
\alpha \\
\sigma\end{array}$ & $\begin{array}{l}n \\
\dot{6} \\
\hat{\delta} \\
n\end{array}$ & $\mid$ & & 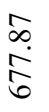 & $\mid \begin{array}{l}\text { ते } \\
\text { ָे }\end{array}$ & \begin{tabular}{l}
0 \\
\multirow{1}{*}{} \\
$\stackrel{0}{2}$ \\
$\stackrel{2}{2}$
\end{tabular} & 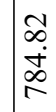 & $\begin{array}{l}\hat{\alpha} \\
\hat{\alpha}\end{array}$ & $\mid \begin{array}{l}0 \\
0 \\
y \\
\alpha\end{array}$ & $\begin{array}{l}b \\
\dot{y} \\
\dot{b} \\
0\end{array}$ & $\begin{array}{l}\hat{n} \\
\tilde{3} \\
\infty \\
\infty\end{array}$ & 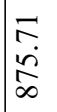 & $\frac{ \pm}{\stackrel{ \pm}{\infty}}$ & 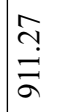 & $\begin{array}{l}\hat{\alpha} \\
\text { } ૂ \\
\text { à }\end{array}$ & $\begin{array}{l}\infty \\
\stackrel{\infty}{\infty} \\
\tilde{n}\end{array}$ & 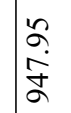 & $\begin{array}{l}\text { to } \\
\dot{8} \\
8\end{array}$ & 寺 & ले \\
\hline $\begin{array}{l}\mathscr{y} \\
\tilde{z} \\
\tilde{z}\end{array}$ & 9 & $\stackrel{9}{9}$ & $N$ & $\vec{\infty}^{\infty}$ & $\ddot{ \pm}$ & $\tilde{\tilde{z}}$ & & તֶ. & 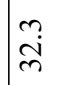 & $\begin{array}{l}m \\
\infty \\
m\end{array}$ & 导 & 芦 & $\begin{array}{l}\text { s } \\
\text { है }\end{array}$ & $\begin{array}{r}? \\
: \\
:\end{array}$ & הֶ & $\mid \begin{array}{l}n \\
\infty \\
0\end{array}$ & $\begin{array}{l}\stackrel{0}{0} \\
\stackrel{+}{I}\end{array}$ & $\begin{array}{l}0 \\
\dot{\infty}\end{array}$ & $\hat{\infty}$ & $\hat{i}$ & $\mid \begin{array}{l}\infty \\
\infty \\
\alpha\end{array}$ & $\begin{array}{l}\infty \\
\dot{0} \\
\stackrel{0}{0}\end{array}$ & 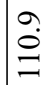 & \\
\hline
\end{tabular}




\begin{tabular}{|c|c|c|c|c|c|c|c|c|c|c|c|c|c|c|c|c|c|c|c|c|c|c|c|c|}
\hline & & $\vec{v}$ & 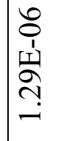 & 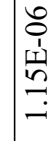 & 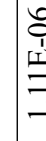 & & 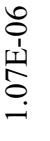 & 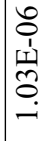 & $\begin{array}{l}\hat{o} \\
\hat{1} \\
\text { yà } \\
\alpha \\
\alpha\end{array}$ & $\begin{array}{l}\hat{o} \\
\hat{1} \\
1 \\
\hat{n} \\
\hat{\sigma} \\
\sigma\end{array}$ & 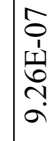 & 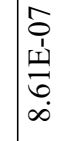 & $\mid \begin{array}{c}\hat{y} \\
\vdots \\
w \\
n \\
\infty \\
\infty\end{array}$ & $\mid \begin{array}{c}0 \\
1 \\
\underline{\omega} \\
\infty \\
\infty\end{array}$ & 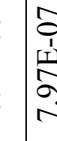 & $\begin{array}{l}0 \\
1 \\
1 \\
\vdots \\
\vdots\end{array}$ & $\mid \begin{array}{c}0 \\
0 \\
1 \\
\\
\\
r\end{array}$ & 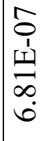 & $\mid \begin{array}{c}0 \\
1 \\
1 \\
1 \\
0 \\
0 \\
0\end{array}$ & $\begin{array}{l}5 \\
0 \\
1 \\
1 \\
0 \\
6 \\
0 \\
0\end{array}$ & 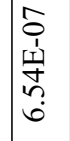 & 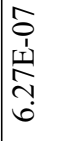 & 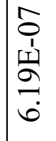 & 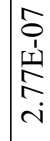 \\
\hline & & ஜீ & 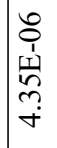 & 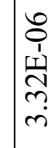 & $\begin{array}{l}0 \\
\vdots \\
\frac{1}{1} \\
\vdots \\
2 \\
2\end{array}$ & & $\begin{array}{l}\stackrel{8}{8} \\
\stackrel{1}{1} \\
\stackrel{1}{+} \\
i\end{array}$ & 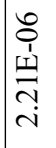 & $\begin{array}{l}8 \\
0 \\
1 \\
1 \\
\infty \\
\infty \\
- \\
-\end{array}$ & 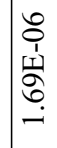 & $\mid \begin{array}{l}8 \\
0 \\
1 \\
1 \\
0 \\
\vdots \\
-1 \\
-1\end{array}$ & 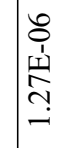 & 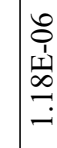 & $\begin{array}{l}0 \\
0 \\
1 \\
11 \\
0 \\
0 \\
-1\end{array}$ & $\begin{array}{l}2 \\
1 \\
1 \\
\frac{1}{2} \\
0 \\
0\end{array}$ & $\frac{b}{c}$ & $\mid \begin{array}{c}\hat{0} \\
1 \\
\underline{1} \\
\frac{1}{\infty} \\
\infty\end{array}$ & 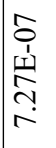 & $\begin{array}{l}\hat{0} \\
1 \\
\text { 告 } \\
\text { bे }\end{array}$ & \begin{tabular}{c}
$\hat{s}$ \\
9 \\
1 \\
I \\
\multirow{7}{0}{}
\end{tabular} & $\begin{array}{c}\hat{0} \\
\mathbf{9} \\
1 \\
0 \\
0 \\
0 \\
0\end{array}$ & 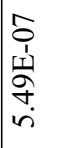 & 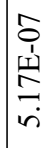 & $\begin{array}{l}\hat{i} \\
\hat{1} \\
\hat{H} \\
\hat{n} \\
\hat{v}\end{array}$ \\
\hline & & 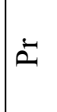 & 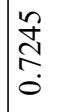 & సิ & $\stackrel{n}{2}$ & & 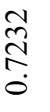 & 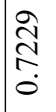 & 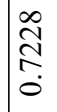 & $\underset{\mathbb{N}}{\stackrel{\Xi}{0}}$ & 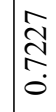 & 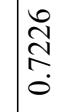 & $\underset{\mathbb{N}}{\mathbb{N}}$ & ה્ત & 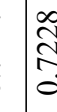 & 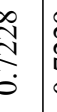 & $\mid$ & $\begin{array}{l}\overrightarrow{\widehat{N}} \\
\stackrel{0}{0}\end{array}$ & 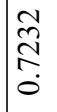 & 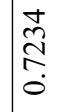 & $\mid \begin{array}{l}\tilde{n} \\
\stackrel{\mathbb{N}}{\cong} \\
\dot{0}\end{array}$ & $\begin{array}{l}\hat{\curvearrowright} \\
\stackrel{N}{0}\end{array}$ & సે & 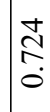 \\
\hline & & $\mathscr{J}$ & $\begin{array}{l}8 \\
0 \\
\stackrel{2}{2} \\
\hat{2} \\
0 \\
0\end{array}$ & 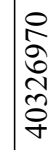 & 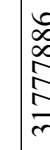 & & 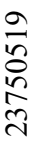 & 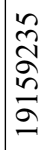 & 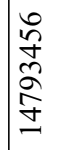 & 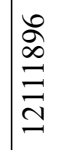 & 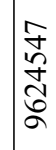 & $\begin{array}{l}\hat{\infty} \\
\stackrel{\delta}{\infty} \\
\stackrel{\infty}{\approx}\end{array}$ & 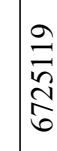 & $\begin{array}{l}m \\
0 \\
8 \\
0 \\
0 \\
n \\
n\end{array}$ & 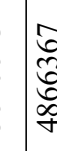 & 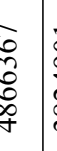 & 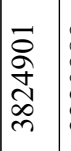 & 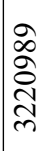 & 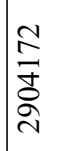 & 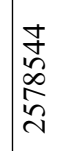 & $\mid \begin{array}{l}\tilde{\infty} \\
\tilde{D} \\
\tilde{\hat{~}} \\
\tilde{N}\end{array}$ & 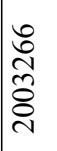 & 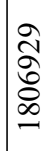 & $\begin{array}{l}\frac{1}{0} \\
\stackrel{0}{i}\end{array}$ \\
\hline & & t & 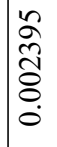 & 華 & $\frac{\sigma}{8}$ & & 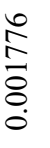 & $\begin{array}{l}\hat{0} \\
0 \\
8 \\
8 \\
0 \\
0\end{array}$ & 年 & 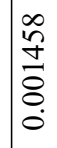 & $\mid$\begin{tabular}{l}
$\vec{\infty}$ \\
0 \\
\hdashline \\
0 \\
0 \\
0
\end{tabular} & $\begin{array}{l}\stackrel{0}{2} \\
\cong \\
8 \\
0 \\
0\end{array}$ & 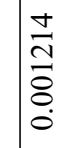 & $\mid \begin{array}{l}m \\
\stackrel{m}{\Xi} \\
\stackrel{8}{0}\end{array}$ & $\frac{2}{8}$ & $\frac{6}{0}$ & $\mid$ & $\begin{array}{l}0 \\
0 \\
0 \\
8 \\
8 \\
0 \\
0\end{array}$ & $\begin{array}{l}a \\
\stackrel{े}{0} \\
\stackrel{0}{0}\end{array}$ & $\begin{array}{l}0 \\
0 \\
0 \\
0 \\
0 \\
0 \\
0\end{array}$ & $\begin{array}{l}\overrightarrow{\tilde{D}} \\
0 \\
0 \\
0 \\
0 \\
\dot{0}\end{array}$ & $\begin{array}{l}\frac{1}{\infty} \\
\frac{1}{8} \\
8 \\
0 \\
0\end{array}$ & $\begin{array}{l}0 \\
0 \\
8 \\
8 \\
0\end{array}$ & ले \\
\hline & & $\mathscr{\sim}$ & $\underset{I}{\stackrel{ \pm}{I}}$ & $\stackrel{m}{\stackrel{2}{R}}$ & $\begin{array}{l}\not{\infty} \\
\underset{\alpha}{\sigma}\end{array}$ & & $\bar{ర}$ & 蓶 & $\vec{\infty}$ & $\frac{\infty}{\sigma}$ & $\begin{array}{l}\text { के } \\
\text { in }\end{array}$ & $\begin{array}{l}n \\
\infty \\
n \\
n\end{array}$ & $\frac{\pi}{\sqrt{n}}$ & 命 & 章 & 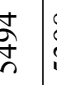 & 文 & $\frac{m}{n}$ & 离 & $\begin{array}{l}\infty \\
n \\
n \\
n\end{array}$ & $\begin{array}{l}0 \\
\infty \\
: \\
i n\end{array}$ & 官 & $\tilde{n}$ & $\frac{\circ}{\frac{\sigma}{q}}$ \\
\hline లే & $\tilde{z}$ & 莺 & $\hat{\alpha}$ & : & a & & $\tilde{n}$ & $\stackrel{\text { f }}{0}$ & 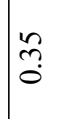 & ָे & సे & กิ & $\stackrel{1}{\circ}$ & $\frac{9}{0}$ & $\frac{9}{0}$ & $\frac{a}{3}$ & $\stackrel{5}{0}$ & $\stackrel{2}{0}$ & $\stackrel{n}{0}$ & $\stackrel{ \pm}{0}$ & $\stackrel{ \pm}{0}$ & $\stackrel{m}{0}$ & 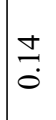 & กิ \\
\hline $\begin{array}{l}\frac{+}{\sigma} \\
\frac{\vec{U}}{\Xi} \\
\Xi\end{array}$ & 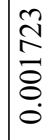 & $\vec{z}$ & $\begin{array}{l}\frac{J}{2} \\
\infty \\
\infty\end{array}$ & ป̀ & 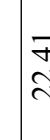 & & $\begin{array}{c}\vec{n} \\
\infty \\
\infty\end{array}$ & $\stackrel{m}{n}$ & $\stackrel{m}{m}$ & $\stackrel{\vec{\sigma}}{=}$ & 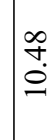 & $\begin{array}{l}\vec{\infty} \\
\infty \\
\infty\end{array}$ & $\underset{\infty}{\tilde{\infty}}$ & $\stackrel{\infty}{\stackrel{\infty}{\sim}}$ & & ָै & 苞 & $\hat{i n}$ & $\begin{array}{l}n \\
n \\
n\end{array}$ & $\begin{array}{l}0 \\
n \\
n \\
n\end{array}$ & $\frac{\infty}{i n}$ & $\vec{\gamma}$ & 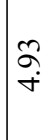 & $\underset{f}{q}$ \\
\hline $\begin{array}{l}\stackrel{\mathscr{U}}{\simeq} \\
\frac{\tilde{O}}{\Xi}\end{array}$ & 恚 & 悹 & $\hat{\sigma}$ & $\stackrel{\Re}{\cong}$ & ح & & $\begin{array}{c}\infty \\
0 \\
0\end{array}$ & 禹 & $\stackrel{0}{\circ}$ & $\bar{n}$ & $\stackrel{\infty}{+}$ & $\stackrel{\nabla}{0}$ & $\hat{n}$ & $\tilde{m}$ & $\stackrel{7}{0}$ & 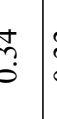 & $\hat{n}$ & $\bar{m}$ & $\tilde{o}$ & ? & $\ddot{0}$ & ते & $\stackrel{3}{0}$ & b. \\
\hline$\underset{\Xi}{\Xi}$ & $\overrightarrow{\mathrm{m}}$ & 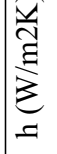 & 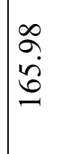 & $\hat{n}$ & $\begin{array}{l}v \\
b \\
f\end{array}$ & & ঙ্ণ & 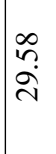 & 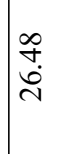 & $\underset{\vec{\lambda}}{\stackrel{+}{\tilde{\lambda}}}$ & $\frac{\partial}{\vec{N}}$ & $\begin{array}{l}\hat{\alpha} \\
\infty \\
\underline{\alpha}\end{array}$ & $\mid \begin{array}{l}\hat{0} \\
\infty \\
-\infty\end{array}$ & 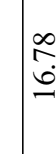 & & f. & $\begin{array}{l}\stackrel{F}{F} \\
\dot{ \pm}\end{array}$ & $\begin{array}{l}\tilde{n} \\
\tilde{n}\end{array}$ & $\mid \begin{array}{l}\infty \\
m \\
m \\
m\end{array}$ & $\stackrel{m}{\stackrel{m}{m}}$ & $\begin{array}{l}\hat{D} \\
i \\
\end{array}$ & $\stackrel{\stackrel{\Xi}{I}}{\leftrightarrows}$ & $\begin{array}{l}\vec{b} \\
\text { I }\end{array}$ & $\vec{\sigma}$ \\
\hline 泣 & ? & 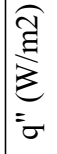 & 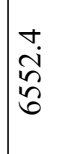 & 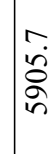 & 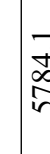 & & 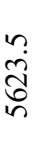 & $\begin{array}{l}n \\
\infty \\
\infty \\
\infty \\
i\end{array}$ & {$\left[\begin{array}{l}\tilde{n} \\
\tilde{n}\end{array}\right.$} & $\begin{array}{l}\infty \\
\dot{\infty} \\
\infty \\
\dot{n}\end{array}$ & $\mid \begin{array}{l}\vec{j} \\
\infty \\
\infty \\
\stackrel{0}{n} \\
i n\end{array}$ & $\begin{array}{l}\infty \\
\dot{0} \\
\stackrel{\hat{\sigma}}{f} \\
\dot{f}\end{array}$ & 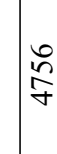 & 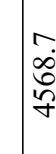 & $\left\{\begin{array}{l}n \\
\tilde{n} \\
\tilde{z}\end{array}\right.$ & $\begin{array}{l}n \\
\hat{n} \\
b \\
y\end{array}$ & $\begin{array}{l}\infty \\
\dot{J} \\
\forall \\
\forall\end{array}$ & 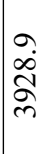 & $\begin{array}{l}\infty \\
\stackrel{0}{0} \\
\stackrel{2}{n} \\
\text { m. }\end{array}$ & $\begin{array}{l}\infty \\
\dot{\vec{a}} \\
\dot{m}\end{array}$ & $\begin{array}{l}n \\
\infty \\
\infty \\
\infty \\
\infty \\
n\end{array}$ & $\frac{n}{n}$ & $\begin{array}{l}n \\
\infty \\
\infty \\
\infty \\
\infty \\
n\end{array}$ & 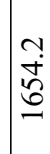 \\
\hline $\begin{array}{l}\sum_{\pi} \\
0 \\
0 \\
0 \\
2\end{array}$ & $\vec{\sigma}$ & $\vec{F}$ & 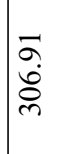 & $\begin{array}{l}\infty \\
\sim \\
\sim \\
\sim\end{array}$ & $\begin{array}{l}\grave{n} \\
\text { ले }\end{array}$ & & $\begin{array}{l}\vec{n} \\
\dot{\sim} \\
\stackrel{n}{m}\end{array}$ & वे. & 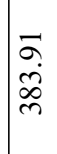 & $\begin{array}{l}\tilde{\sigma} \\
\infty \\
\infty \\
\tilde{m}\end{array}$ & $\stackrel{\overbrace{}}{\stackrel{F}{\vec{F}}}$ & $\begin{array}{l}\varkappa \\
\stackrel{+}{q} \\
\gamma\end{array}$ & $\begin{array}{l}\infty \\
\dot{\sigma} \\
\tilde{\gamma}\end{array}$ & 胥 & 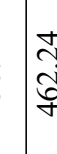 & 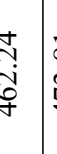 & $\begin{array}{l}\infty \\
\dot{\rho} \\
\dot{\gamma} \\
\end{array}$ & $\begin{array}{l}\hat{n} \\
\dot{\infty} \\
\dot{\infty} \\
\dot{+}\end{array}$ & $\begin{array}{l}\delta \\
\stackrel{\sigma}{a} \\
\stackrel{q}{q}\end{array}$ & $\begin{array}{l}\tilde{f} \\
\ddot{\theta} \\
\ddot{n}\end{array}$ & $\begin{array}{l}\hat{\theta} \\
i n \\
i n\end{array}$ & $\begin{array}{l}\infty \\
0 \\
\ddot{n} \\
i \\
i\end{array}$ & $\begin{array}{l}\nexists \\
\sim \\
n \\
n \\
n\end{array}$ & 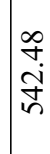 \\
\hline$\sum_{2}^{\pi}$ & $\overbrace{}^{\infty}$ & 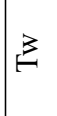 & 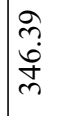 & $\begin{array}{l}\stackrel{\vartheta}{a} \\
\infty \\
\stackrel{+}{\sigma}\end{array}$ & $\bar{n}$ & 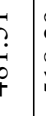 & $\begin{array}{c}\text { ते } \\
\stackrel{\infty}{n}\end{array}$ & $\begin{array}{l}m \\
\hat{\alpha} \\
\tilde{n} \\
\tilde{n}\end{array}$ & 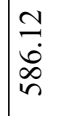 & 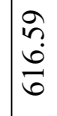 & $\mid \begin{array}{l}n \\
\stackrel{0}{f} \\
\text { fon }\end{array}$ & $\underset{\hat{N}}{\hat{N}}$ & 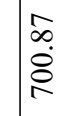 & 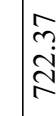 & 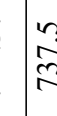 & $\frac{?}{n}$ & $\mid$ & $\begin{array}{l}\stackrel{0}{2} \\
\sim \\
\stackrel{2}{\Sigma} \\
i\end{array}$ & 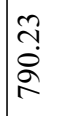 & $\begin{array}{l}n \\
\hat{⿵} \\
\infty \\
\infty\end{array}$ & $\begin{array}{l}m \\
\underset{\Xi}{ \pm}\end{array}$ & 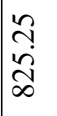 & $\begin{array}{l}\infty \\
\infty \\
\stackrel{\sim}{\sim} \\
\infty\end{array}$ & $\begin{array}{l}\tilde{0} \\
\dot{0} \\
\tilde{N}\end{array}$ \\
\hline 苋 & 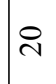 & $\stackrel{\rho}{a}$ & $N$ & $\vec{\infty}$ & $\bar{J}$ & & 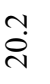 & ָ̊. & 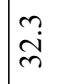 & $\begin{array}{c}m \\
\infty \\
m\end{array}$ & $\stackrel{+}{\forall}$ & 官 & $\begin{array}{l}n \\
\ddot{8} \\
i n\end{array}$ & in & $\ddot{n}$ & $\overbrace{\infty}^{\infty}$ & $\begin{array}{l}\bullet \\
\stackrel{0}{T}\end{array}$ & : & $\hat{\infty}$ & $\hat{\check{a}}$ & $\mid \begin{array}{l}\infty \\
\infty \\
\infty\end{array}$ & $\begin{array}{l}\infty \\
\dot{d} \\
=\end{array}$ & $\stackrel{\grave{o}}{\varrho}$ & $\stackrel{a}{\stackrel{b}{G}}$ \\
\hline
\end{tabular}




\begin{tabular}{|c|c|c|c|c|c|c|c|c|c|c|c|c|c|c|c|c|c|c|c|c|c|c|c|c|}
\hline & & $\vec{\Delta}$ & 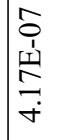 & 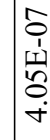 & 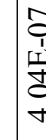 & & 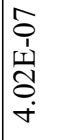 & 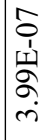 & 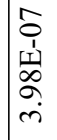 & 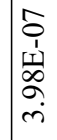 & 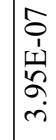 & 辛 & 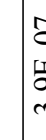 & & 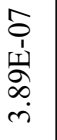 & \begin{tabular}{|l}
$\hat{0}$ \\
$\hat{1}$ \\
$\underline{1}$ \\
$\infty$ \\
$\infty$ \\
\\
\end{tabular} & \begin{tabular}{|l}
$\hat{0}$ \\
$\hat{1}$ \\
$\hat{1}$ \\
$\hat{\infty}$ \\
$\hat{n}$
\end{tabular} & $\begin{array}{l}\hat{a} \\
\hat{1} \\
\\
\hat{1} \\
\dot{m}\end{array}$ & $\begin{array}{l}\hat{0} \\
\hat{1} \\
\hat{1} \\
\\
\hat{n} \\
\dot{m}\end{array}$ & 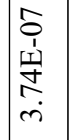 & 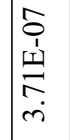 & 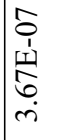 & 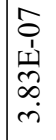 & 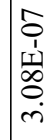 \\
\hline & & $\stackrel{\circ}{\circ}$ & 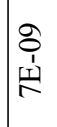 & \begin{tabular}{c}
8 \\
1 \\
1 \\
\multirow{1}{*}{} \\
0
\end{tabular} & $\begin{array}{l}\frac{8}{1} \\
\frac{1}{2} \\
\text { bo }\end{array}$ & & 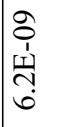 & 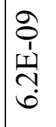 & 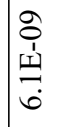 & $\begin{array}{l}2 \\
\hat{1} \\
1 \\
0 \\
0 \\
0\end{array}$ & $\begin{array}{l}\partial \\
\hat{1} \\
\underline{y} \\
\text { in } \\
\text { in }\end{array}$ & $\begin{array}{l}2 \\
\frac{1}{1} \\
\frac{1}{2} \\
n\end{array}$ & 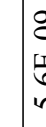 & 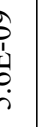 & 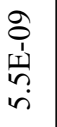 & 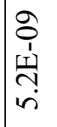 & 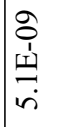 & $\begin{array}{l}8 \\
8 \\
1 \\
\text { 离 }\end{array}$ & 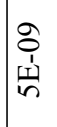 & 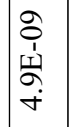 & $\begin{array}{l}2 \\
\text { oे } \\
\text { I1 } \\
\text { to } \\
\dot{+}\end{array}$ & 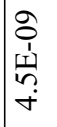 & 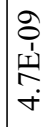 & $\begin{array}{l}\hat{o} \\
\hat{O} \\
1 \\
\infty \\
\infty \\
\dot{m}\end{array}$ \\
\hline & & 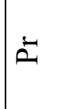 & $\begin{array}{l}m \\
\hat{\mathscr{\delta}} \\
\stackrel{0}{0} \\
0\end{array}$ & $\begin{array}{l}\text { శூ } \\
\stackrel{0}{0} \\
0\end{array}$ & $\begin{array}{l}\approx \\
\text { है } \\
0\end{array}$ & & 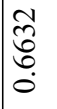 & $\begin{array}{c}2 \\
\widetilde{0} \\
0 \\
0 \\
0\end{array}$ & $\begin{array}{l}\overrightarrow{3} \\
\dot{0} \\
0 \\
0\end{array}$ & $\begin{array}{l}\overrightarrow{\widehat{\delta}} \\
\dot{0} \\
\dot{0}\end{array}$ & 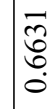 & శ̊ & हु & b. & $\begin{array}{l}3 \\
0 \\
0 \\
0 \\
0\end{array}$ & 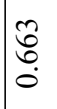 & 莕 & 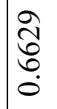 & त्ठ઼े & $\begin{array}{l}\text { ते } \\
\text { ర్ } \\
0\end{array}$ & $\begin{array}{l}\text { ते } \\
\text { ठ̊ } \\
\stackrel{0}{0}\end{array}$ & $\begin{array}{l}\text { ते } \\
\text { ర్ర } \\
\dot{0}\end{array}$ & $\begin{array}{l}\infty \\
\text { đ్ర } \\
\stackrel{0}{0} \\
\dot{0}\end{array}$ & 足 \\
\hline & & $\mathscr{J}$ & 紊 & âे & ช̊ & & $\frac{\partial}{\partial}$ & $\stackrel{0}{0}$ & 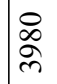 & 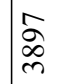 & 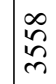 & $\underset{\text { 守 }}{\text { 足 }}$ & ָિ & v & సे & 志 & অ & 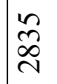 & 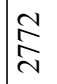 & 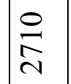 & $\mid \begin{array}{l}\infty \\
\stackrel{\infty}{+} \\
\stackrel{\sim}{\sim}\end{array}$ & $\underset{\stackrel{ }{\sim}}{\stackrel{\sim}{\sim}}$ & $\stackrel{\triangleright}{\stackrel{\infty}{\sim}}$ & $\stackrel{8}{\circ}$ \\
\hline & & t & 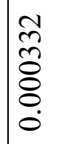 & 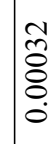 & $\frac{\infty}{\tilde{\delta}}$ & & $\begin{array}{l}\frac{n}{2} \\
\tilde{n} \\
\vdots \\
0 \\
0\end{array}$ & 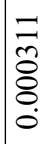 & 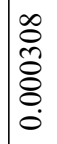 & $\begin{array}{l}0 \\
0 \\
\tilde{\delta} \\
\delta \\
0 \\
0\end{array}$ & $\begin{array}{l}\text { ôे } \\
\tilde{\delta} \\
0 \\
0 \\
0\end{array}$ & ஜ̊ & 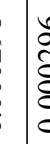 & : & 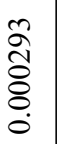 & 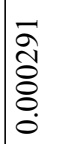 & 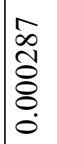 & 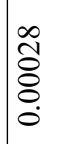 & 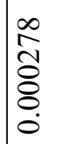 & 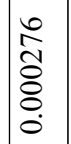 & $\begin{array}{l}\mathcal{N} \\
\hat{\delta} \\
\delta \\
0 \\
0\end{array}$ & 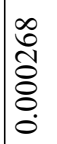 & 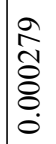 & 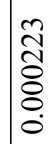 \\
\hline & & $\ddot{\sim}$ & $\frac{\infty}{\frac{\infty}{m}}$ & $\frac{\vec{b}}{m}$ & $\frac{\vec{y}}{m}$ & & 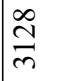 & $\frac{\cong}{m}$ & 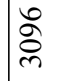 & 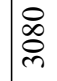 & 壱 & త্ & ¿ & 管 & $\stackrel{\vec{\rho}}{\vec{m}}$ & 离 & ஓे & ڤે & $\begin{array}{l}\text { రू } \\
\stackrel{\text { సे }}{ }\end{array}$ & वे & $\tilde{\tilde{\lambda}}$ & તิ & ठे̀े & 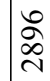 \\
\hline שే & $\stackrel{0}{1}$ & 总 & $\stackrel{+}{\stackrel{5}{\leftrightarrows}}$ & $\hat{\sigma}$ & 7 & & 䓦 & & $\widehat{\Omega}$ & $\stackrel{2}{n}$ & $\vec{\sigma}$ & $\tilde{n}$ & 7 & i & $\begin{array}{l}\vec{\infty} \\
i \\
i\end{array}$ & $\vec{i}$ & $\begin{array}{l}\stackrel{0}{0} \\
\text { in }\end{array}$ & 足 & $\vec{\infty}$ & $\mid \begin{array}{l}\infty \\
\infty \\
- \\
-1\end{array}$ & $\stackrel{\infty}{\infty}$ & $\stackrel{ \pm}{\stackrel{2}{*}}$ & 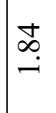 & $\stackrel{\infty}{-}$ \\
\hline $\begin{array}{l}\frac{ \pm}{\sigma} \\
\frac{ \pm}{ \pm} \\
\Xi\end{array}$ & 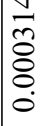 & $\vec{z}$ & 莳 & 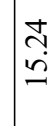 & $\begin{array}{l}\infty \\
\mathcal{m}\end{array}$ & & $\begin{array}{l}\ddot{0} \\
\ddot{2} \\
\ddot{2}\end{array}$ & $\overrightarrow{\hat{n}}$ & 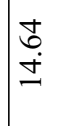 & $\begin{array}{l}\hat{\imath} \\
\stackrel{n}{2}\end{array}$ & فㅇ. & g & $\frac{5}{7}$ & 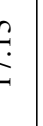 & $\underset{\Xi}{\stackrel{J}{\Xi}}$ & $\stackrel{n}{\stackrel{n}{=}}$ & $\stackrel{2}{\approx}$ & $\begin{array}{l}\infty \\
\ddot{\sim}\end{array}$ & $\begin{array}{l}\infty \\
n \\
n \\
n\end{array}$ & $\begin{array}{l}\stackrel{\nabla}{\sim} \\
\dot{\sim}\end{array}$ & $\stackrel{\mathscr{f}}{\stackrel{9}{g}}$ & $\frac{n}{n}$ & 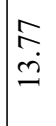 & $\stackrel{\infty}{\stackrel{\infty}{\mathcal{I}}}$ \\
\hline $\begin{array}{l}\stackrel{\mathscr{a}}{\sharp} \\
\frac{\ddot{\theta}}{\Xi}\end{array}$ & $\frac{\vec{\infty}}{m}$ & 衰 & 定 & פे & 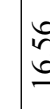 & & 莳 & $\stackrel{\hat{\sigma}}{\sigma}$ & $\stackrel{m}{2}$ & $\begin{array}{l}\hat{n} \\
\tilde{N}\end{array}$ & के & $\begin{array}{l}\infty \\
n \\
n\end{array}$ & $\left\{\begin{array}{l}\tilde{\alpha} \\
\tilde{\gamma}\end{array}\right.$ & & $\begin{array}{l} \pm \\
\ddot{2} \\
\text { iे }\end{array}$ & $\begin{array}{l}\infty \\
\infty \\
\hat{\lambda}\end{array}$ & $\stackrel{n}{\stackrel{\sim}{\sim}}$ & $\hat{m}$ & $\begin{array}{l}\tilde{\alpha} \\
\infty \\
\infty\end{array}$ & $\begin{array}{l}\infty \\
2 \\
2 \\
2\end{array} \mid$ & 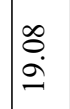 & $\begin{array}{l}\hat{n} \\
\infty \\
\infty\end{array}$ & $\vec{a}$ & $\begin{array}{l}0 \\
\text { ?2} \\
\Omega\end{array}$ \\
\hline$\underset{\Xi}{\stackrel{\Xi}{\Xi}}$ & 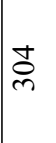 & 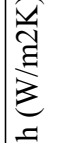 & 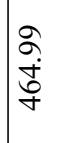 & ભે & $\begin{array}{l}\bar{a} \\
\text { f }\end{array}$ & & $\begin{array}{l}\tilde{\delta} \\
\dot{\sigma} \\
\ddot{\theta}\end{array}$ & 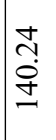 & $\begin{array}{l}n \\
\tilde{n} \\
n\end{array}$ & $\begin{array}{l}\hat{\alpha} \\
\hat{\sigma}\end{array}$ & $\mid \begin{array}{l}\infty \\
n \\
n \\
n \\
= \\
=\end{array}$ & $\mathfrak{I}$ & $\begin{array}{l}7 \\
8 \\
0 \\
0\end{array}$ & 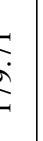 & $\begin{array}{l}\infty \\
n \\
\infty \\
\infty \\
\infty \\
-\end{array}$ & \begin{tabular}{l}
$n$ \\
\multirow{2}{*}{} \\
$\infty$ \\
$\infty$
\end{tabular} & $\begin{array}{l}\tilde{n} \\
\tilde{\infty} \\
\infty \\
\underline{\Xi}\end{array}$ & $\begin{array}{l}\infty \\
\stackrel{\dot{\Xi}}{\text { In }}\end{array}$ & $\begin{array}{l}\Xi \\
0 \\
\dot{0} \\
\pm\end{array}$ & $\mid \begin{array}{l}\nexists \\
+ \\
\infty \\
\pm \\
\pm\end{array}$ & 吾 & $\begin{array}{l}\tilde{n} \\
\tilde{y} \\
\tilde{J}\end{array}$ & $\begin{array}{l}\infty \\
\stackrel{\infty}{\circ} \\
-\end{array}$ & $\begin{array}{l}\stackrel{n}{\alpha} \\
\tilde{m}\end{array}$ \\
\hline$\sum_{\text {至 }}$ & $\overrightarrow{6}$ & $\begin{array}{l}\text { త્ } \\
\text { ఏ } \\
= \\
=\end{array}$ & 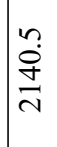 & 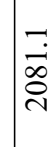 & 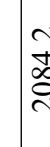 & & 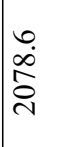 & $\begin{array}{l}n \\
\infty \\
\infty \\
0 \\
\varnothing \\
\end{array}$ & $\begin{array}{l}0 \\
\dot{0} \\
\stackrel{8}{0} \\
\stackrel{2}{2}\end{array}$ & 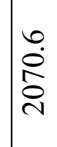 & 官 & Zु & ? & ty & 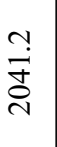 & 柋 & $\begin{array}{l}\infty \\
\text { ते } \\
\text { ¿े }\end{array}$ & $\begin{array}{l}\infty \\
\stackrel{0}{\circ} \\
\varrho\end{array}$ & $\begin{array}{l}\infty \\
\dot{0} \\
\infty \\
\infty\end{array}$ & $\begin{array}{l}m \\
\tilde{\infty} \\
\infty \\
2\end{array}$ & 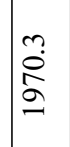 & $\begin{array}{l}0 \\
\ddot{n} \\
\approx \\
\approx\end{array}$ & 莳 & \begin{tabular}{l}
$\hat{\sigma}$ \\
\multirow{0}{0}{} \\
-
\end{tabular} \\
\hline 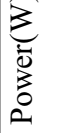 & હે & $\vec{F}$ & 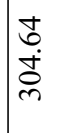 & 离 & 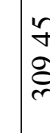 & & 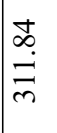 & $\begin{array}{l}\vec{\sim} \\
\dot{v} \\
m\end{array}$ & $\begin{array}{l}\infty \\
n \\
\stackrel{n}{0} \\
m\end{array}$ & $\begin{array}{l}n \\
\alpha \\
\infty \\
m\end{array}$ & 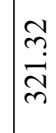 & $\begin{array}{l}3 \\
\tilde{\pi}\end{array}$ & 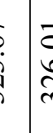 & $\begin{array}{l}5 \\
\dot{b} \\
\vdots \\
2\end{array}$ & $\begin{array}{l}\tilde{n} \\
\infty \\
\infty \\
\infty \\
m \\
m\end{array}$ & $\begin{array}{l}\text { aे } \\
\stackrel{0}{0} \\
\text { m. }\end{array}$ & $\begin{array}{l}\overrightarrow{0} \\
\infty \\
\tilde{m}\end{array}$ & $\begin{array}{l}\vec{m} \\
\cdots \\
m \\
m\end{array}$ & $\underset{\substack{n \\
m}}{m}$ & $\begin{array}{l}\infty \\
\infty \\
\dot{a} \\
\tilde{m}\end{array}$ & $\begin{array}{l}m \\
\stackrel{m}{\dot{H}} \\
\stackrel{m}{m}\end{array}$ & \begin{tabular}{l}
$\infty$ \\
$m$ \\
\multirow{j}{*}{} \\
$\tilde{m}$
\end{tabular} & $\begin{array}{l}\hat{\sigma} \\
\dot{0} \\
\dot{+} \\
m\end{array}$ & $\begin{array}{l}\stackrel{2}{2} \\
\infty \\
\dot{+} \\
m\end{array}$ \\
\hline 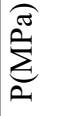 & $\frac{1}{0}$ & 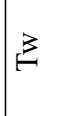 & 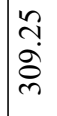 & 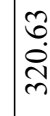 & $\begin{array}{l}\overrightarrow{7} \\
\stackrel{7}{\pi}\end{array}$ & & 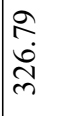 & $\begin{array}{l}0 \\
\stackrel{2}{0} \\
\infty \\
\stackrel{1}{m}\end{array}$ & $\begin{array}{l}\vec{n} \\
\stackrel{0}{m} \\
\tilde{m}\end{array}$ & 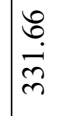 & 离 & $\begin{array}{l}\tilde{n} \\
\tilde{m} \\
\tilde{m}\end{array}$ & 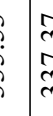 & $\frac{5}{2}$ & $\begin{array}{l}\text { f. } \\
\text { ले } \\
\text { mे }\end{array}$ & & $\begin{array}{l}\tilde{n} \\
\tilde{f} \\
\stackrel{f}{f}\end{array}$ & $\begin{array}{l}\hat{N} \\
\infty \\
\infty \\
\dot{m}\end{array}$ & $\frac{a}{\frac{a}{n}}$ & $\begin{array}{l}\tilde{n} \\
\tilde{n} \\
\tilde{n}\end{array}$ & $\begin{array}{l}\tilde{n} \\
\tilde{n} \\
\tilde{n}\end{array}$ & $\begin{array}{l}\sigma \\
o \\
\infty \\
\infty \\
n \\
m\end{array}$ & 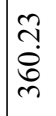 & 立 \\
\hline 离 & $\vec{\sim}$ & 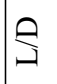 & $N$ & $\vec{\infty}$ & $\exists$ & & तิ & ָั & $\stackrel{m}{i}$ & $\begin{array}{c}m \\
\infty \\
\infty\end{array}$ & $\stackrel{+}{+}$ & in & $y$ & $\dot{\ell}$ & $\begin{array}{l}\tilde{n} \\
\text { Uु }\end{array}$ & $\begin{array}{l}n \\
\infty \\
\infty\end{array}$ & $\begin{array}{l}0 \\
\dot{I} \\
\end{array}$ & $\ddot{\infty}$ & $\hat{\infty}$ & $\hat{i}$ & $\mid \begin{array}{l}\infty \\
\infty \\
\infty\end{array}$ & 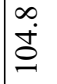 & $\stackrel{\varrho}{\varrho}$ & $\underset{b}{\sigma}$ \\
\hline
\end{tabular}




\begin{tabular}{|c|c|c|c|c|c|c|c|c|c|c|c|c|c|c|c|c|c|c|c|c|c|c|c|c|}
\hline & & $\vec{\Delta}$ & 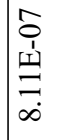 & 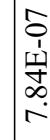 & 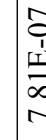 & & 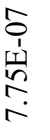 & $\begin{array}{l}\hat{1} \\
0 \\
1 \\
1 \\
0 \\
0 \\
0 \\
r\end{array}$ & 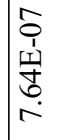 & 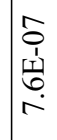 & $\mid \begin{array}{l}\hat{0} \\
1 \\
1 \\
\\
n \\
r \\
r\end{array}$ & 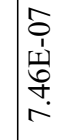 & 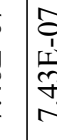 & 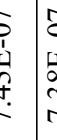 & 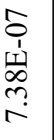 & 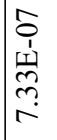 & 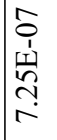 & 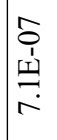 & 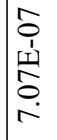 & 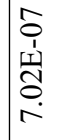 & 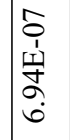 & 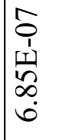 & 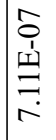 & 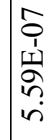 \\
\hline & & $\stackrel{\oplus}{\infty}$ & 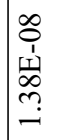 & 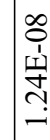 & 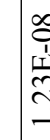 & & 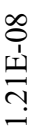 & 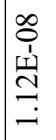 & 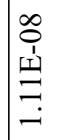 & 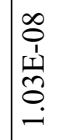 & 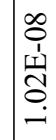 & 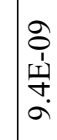 & $\mid \begin{array}{l}2 \\
0 \\
\frac{1}{2} \\
\sigma\end{array}$ & 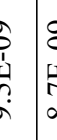 & 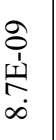 & 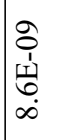 & 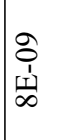 & $\begin{array}{l}\text { oे } \\
\text { 11 } \\
\infty \\
\infty \\
r\end{array}$ & 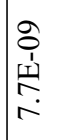 & 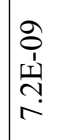 & 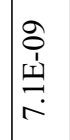 & 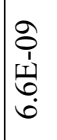 & 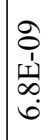 & $\begin{array}{l}\text { o } \\
\hat{1} \\
1 \\
\text { in } \\
\text { in }\end{array}$ \\
\hline & & $\ddot{2}$ & $\begin{array}{l}m \\
\widetilde{0} \\
0 \\
0 \\
0\end{array}$ & 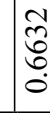 & $\begin{array}{l}\text { శ్ } \\
\text { c } \\
0\end{array}$ & $\begin{array}{l}1 \\
b \\
\\
\end{array}$ & 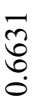 & $\begin{array}{l}\overrightarrow{\widehat{n}} \\
\stackrel{0}{0} \\
\ddot{0}\end{array}$ & $\begin{array}{l}0 \\
0 \\
0 \\
0\end{array}$ & $\begin{array}{l}0 \\
0 \\
0 \\
0\end{array}$ & 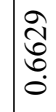 & : & $\mid \begin{array}{l}\infty \\
\tilde{\Xi} \\
0\end{array}$ & \begin{tabular}{l|l}
0 \\
0 \\
0 \\
0 \\
0
\end{tabular} & 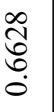 & $\begin{array}{l}\infty \\
\widetilde{\Xi} \\
\stackrel{0}{0} \\
\stackrel{0}{0}\end{array}$ & $\mid \begin{array}{l}\hat{\widehat{O}} \\
0 \\
0 \\
0\end{array}$ & $\begin{array}{l}\hat{\widehat{U}} \\
\stackrel{0}{0} \\
\stackrel{0}{0}\end{array}$ & $\begin{array}{l}\hat{\widehat{D}} \\
\stackrel{0}{0} \\
\stackrel{0}{0}\end{array}$ & $\begin{array}{l}0 \\
\mathbb{Z} \\
0 \\
0 \\
0\end{array}$ & 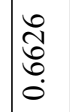 & $\begin{array}{l}0 \\
\text { तु } \\
0 \\
0 \\
0\end{array}$ & \begin{tabular}{l}
0 \\
\multirow{్}{0}{} \\
0 \\
0 \\
0
\end{tabular} & 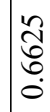 \\
\hline & & $\mathscr{J}$ & $\begin{array}{l}\overrightarrow{0} \\
\approx\end{array}$ & 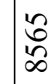 & $\frac{\sigma}{\infty}$ & & 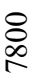 & ? & : & 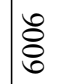 & 吕 & $\frac{n}{n}$ & $\bar{z}$ & $\begin{array}{r}\hat{\gamma} \\
\hat{\alpha}\end{array}$ & 竎 & 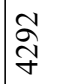 & 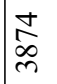 & 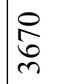 & 忩 & $\begin{array}{l}\text { } \\
\text { ले }\end{array}$ & 令 & 尽 & 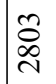 & 串 \\
\hline & & t & | & 文 & $\begin{array}{l}8 \\
\text { d } \\
\text { छे } \\
c\end{array}$ & 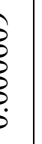 & $\begin{array}{l}0 \\
\vdots \\
\hat{े} \\
\vdots \\
0 \\
0\end{array}$ & $\begin{array}{l}\hat{\infty} \\
\vdots \\
o \\
\vdots \\
0 \\
0\end{array}$ & $\begin{array}{l}\infty \\
\vdots \\
\hat{n} \\
\varnothing \\
0 \\
0 \\
0\end{array}$ & $\begin{array}{l}\tilde{n} \\
\vdots \\
0 \\
0 \\
0\end{array}$ & 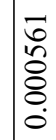 & 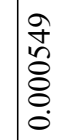 & 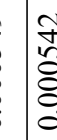 & : & $\begin{array}{l}\tilde{n} \\
\hat{n} \\
\vdots \\
0 \\
0 \\
0\end{array}$ & 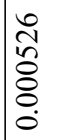 & 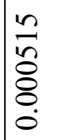 & 号 & 旁 & 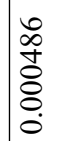 & 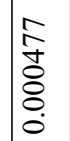 & 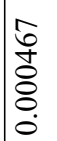 & 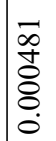 & 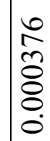 \\
\hline & & $\ddot{\sim}$ & $\frac{\infty}{m}$ & $\frac{\tilde{n}}{m}$ & $\frac{\AA}{m}$ & & $\begin{array}{l}\infty \\
\infty \\
0 \\
\text { d) }\end{array}$ & 文 & 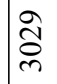 & ఫ্ৰ & त̂̀ & $\frac{\mathbb{Z}}{\stackrel{\mathbb{N}}{ }}$ & $\frac{\infty}{2}$ & $\frac{\infty}{\sqrt{2}}$ & ब्ञ & 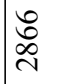 & 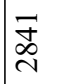 & $\underset{\sim}{\stackrel{\sim}{\sim}}$ & 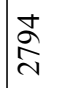 & 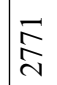 & 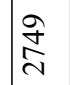 & $\stackrel{\widehat{N}}{\stackrel{N}{N}}$ & 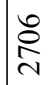 & 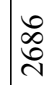 \\
\hline تే & $\stackrel{0}{1}$ & 竞 & $\stackrel{1}{0}$ & $\stackrel{n}{f}$ & $\frac{9}{7}$ & t. & $\stackrel{\simeq}{=}$ & $\stackrel{0}{-}$ & $\vec{\beth}$ & $\stackrel{+}{m}$ & $\sqrt{n}$ & 导 & $n$ & $?$ & nn & $\tilde{n}$ & $\stackrel{f}{\stackrel{f}{-}}$ & $\stackrel{+}{0}$ & $\stackrel{\Xi}{\stackrel{\Delta}{-}}$ & $\vec{\sigma}$ & $\stackrel{\infty}{0}$ & $\hat{a}$ & $\stackrel{\circ}{\circ}$ & $\hat{\delta}$ \\
\hline $\begin{array}{l}\frac{t}{\sigma} \\
\frac{ \pm}{\Delta} \\
\frac{\Xi}{\Xi}\end{array}$ & $\begin{array}{l}\overrightarrow{0} \\
0 \\
0 \\
0 \\
0\end{array}$ & $\vec{z}$ & ले & $\underset{\Xi}{ \pm}$ & $\bar{c}$ & & $\begin{array}{l}\stackrel{8}{0} \\
\text { i }\end{array}$ & în & $\begin{array}{l}0 \\
\stackrel{0}{=}\end{array}$ & $\begin{array}{l}\hat{\Omega} \\
\tilde{\Omega}\end{array}$ & $\stackrel{\Re}{\stackrel{\overbrace{}}{ \pm}}$ & $\begin{array}{l}\stackrel{ \pm}{I} \\
\stackrel{+}{ \pm}\end{array}$ & $\mid \begin{array}{l}5 \\
\vdots \\
\exists\end{array}$ & 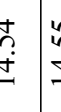 & 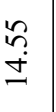 & 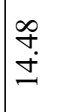 & 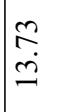 & $\begin{array}{l}\hat{6} \\
=\end{array}$ & $\stackrel{+}{=}$ & $\stackrel{\Xi}{m}$ & $\underset{\Xi}{\sigma}$ & $\begin{array}{l}\hat{b} \\
0 \\
0\end{array}$ & 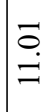 & 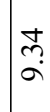 \\
\hline $\begin{array}{l}\stackrel{\mathscr{a}}{\sharp} \\
\frac{\ddot{\theta}}{\Xi}\end{array}$ & $\frac{\curvearrowright}{m}$ & 衰 & 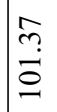 & in & o & & ְ̊. & $\tilde{n}$ & 㑒 & $\begin{array}{l}n \\
n \\
n\end{array}$ & $\begin{array}{l}f \\
\dot{v} \\
i\end{array}$ & $\begin{array}{l}\infty \\
\dot{\Xi} \\
\dot{ \pm}\end{array}$ & $\left\{\begin{array}{l}n \\
n \\
n\end{array}\right.$ & $\begin{array}{l}c \\
\dot{a}\end{array}$ & $\begin{array}{l}m \\
\stackrel{2}{0} \\
-\end{array}$ & \begin{tabular}{l}
$\infty$ \\
\multirow{\sim}{0}{} \\
$ِ$
\end{tabular} & $\begin{array}{l}n \\
\ddot{n} \\
\ddot{n}\end{array}$ & $\stackrel{\infty}{\exists}$ & $\underline{\alpha}$ & $\underset{\Xi}{\stackrel{\Xi}{\Xi}}$ & $\begin{array}{l}1 \\
0 \\
0\end{array}$ & $\vec{n}$ & $\stackrel{ \pm}{\stackrel{0}{\varrho}}$ & $\underset{O}{0}$ \\
\hline$\underset{\Xi}{\stackrel{\Xi}{\Xi}}$ & 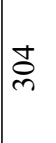 & 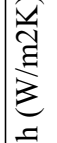 & $\begin{array}{l}\stackrel{\vartheta}{+} \\
\stackrel{\leftrightarrow}{\sigma} \\
\forall\end{array}$ & $\begin{array}{l}\stackrel{l}{2} \\
\stackrel{g}{g} \\
g\end{array}$ & 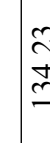 & & $\frac{\hat{\sigma}}{\dot{m}}$ & 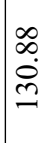 & $\begin{array}{l}\overrightarrow{0} \\
\ddot{0} \\
\grave{2}\end{array}$ & $\begin{array}{l}\vec{n} \\
\infty \\
\stackrel{\infty}{ \pm}\end{array}$ & $\mid \begin{array}{l}\mathbf{1} \\
n \\
\infty \\
n \\
n \\
n\end{array}$ & $\begin{array}{l}\text { ㄱ. } \\
\stackrel{+}{n}\end{array}$ & $\begin{array}{l}7 \\
\text { in }\end{array}$ & 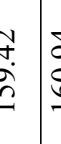 & 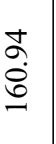 & 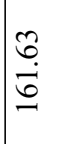 & $\begin{array}{l}\tilde{n} \\
\tilde{n} \\
\end{array}$ & $\frac{n}{\stackrel{n}{\sim}}$ & $\begin{array}{l}0 \\
n \\
0 \\
\infty \\
n \\
-\end{array}$ & $\begin{array}{l}0 \\
ٌ \\
0 \\
\end{array}$ & 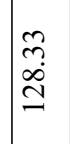 & $\begin{array}{l}0 \\
+ \\
\check{1} \\
\end{array}$ & 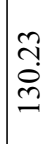 & $\stackrel{\vartheta}{\beth}$ \\
\hline$\sum_{\text {至 }}$ & $\overrightarrow{6}$ & 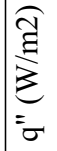 & $\begin{array}{l}\partial े \\
\dot{\gamma}\end{array}$ & ஷి & $\begin{array}{l}n \\
z \\
z \\
z \\
y \\
y\end{array}$ & $\begin{array}{l}? \\
b \\
t \\
t\end{array}$ & $\begin{array}{l}m \\
\stackrel{2}{a} \\
\dot{a}\end{array}$ & $\overline{\tilde{\sigma}}$ & $\begin{array}{l}0 \\
0 \\
0 \\
+ \\
+\end{array}$ & $\mid \begin{array}{l}n \\
n \\
\vdots \\
o \\
q\end{array}$ & 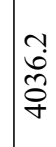 & 㝘 & ڤ̂̀ & $\frac{?}{b}$ & $\begin{array}{l}\stackrel{\sigma}{\sigma} \\
\text { ळे }\end{array}$ & 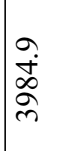 & $\begin{array}{l}\tilde{i} \\
\tilde{n} \\
\tilde{m}\end{array}$ & 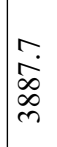 & $\begin{array}{l}\tilde{n} \\
\infty \\
\infty \\
\infty \\
m\end{array}$ & $\begin{array}{l}n \\
0 \\
\infty \\
\infty \\
m\end{array}$ & 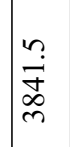 & $\begin{array}{l}0 \\
\dot{0} \\
\infty \\
\infty \\
m\end{array}$ & సִ & $\begin{array}{l}\vec{\infty} \\
\stackrel{0}{N} \\
\vec{m}\end{array}$ \\
\hline 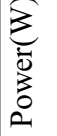 & ঝ̊ & $\vec{F}$ & 离 & $\frac{\text { fै. }}{\stackrel{0}{m}}$ & $\begin{array}{l}\infty \\
a \\
\frac{n}{m}\end{array}$ & & $\frac{m}{\stackrel{m}{m}}$ & 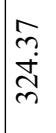 & 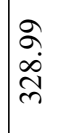 & 占 & $\begin{array}{l}\vec{N} \\
\infty \\
\tilde{m}\end{array}$ & $\begin{array}{l}\infty \\
\stackrel{j}{j} \\
\tilde{m}\end{array}$ & ב̂ & $?$ & $\begin{array}{c}\tilde{\sigma} \\
\dot{\vec{n}} \\
\tilde{m}\end{array}$ & $\begin{array}{l}\infty \\
\stackrel{\infty}{0} \\
\infty \\
n \\
n\end{array}$ & 立 & $\begin{array}{l}\mathfrak{a} \\
\hat{j} \\
0 \\
0\end{array}$ & $\begin{array}{l}\tilde{\alpha} \\
\hat{\sigma} \\
\hat{\sigma}\end{array}$ & $\underset{m}{\stackrel{+}{m}}$ & $\begin{array}{l}\text { フ } \\
\stackrel{\infty}{\infty} \\
\stackrel{\infty}{\infty}\end{array}$ & 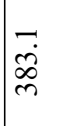 & $\begin{array}{l}n \\
\tilde{r} \\
\infty \\
\infty\end{array}$ & $\begin{array}{l}\infty \\
\stackrel{n}{a} \\
\tilde{m}\end{array}$ \\
\hline$\sum_{0=1}^{\pi}$ & $\frac{\infty}{0}$ & 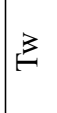 & $\begin{array}{l}\vec{\infty} \\
i \\
\infty \\
m\end{array}$ & $\stackrel{\infty}{\stackrel{\infty}{\sim}}$ & is & ? & $\begin{array}{l}\infty \\
\stackrel{\infty}{\circ} \\
\stackrel{n}{m}\end{array}$ & $\begin{array}{l}n \\
m \\
n \\
n \\
n\end{array}$ & $\begin{array}{l}\vec{\infty} \\
\infty \\
m\end{array}$ & $\begin{array}{l}\text { क } \\
0 \\
0 \\
0 \\
0\end{array}$ & $\begin{array}{l}\hat{\sigma} \\
\dot{d} \\
\text { d. }\end{array}$ & $\begin{array}{l}\hat{b} \\
\dot{0} \\
0 \\
\text { లె }\end{array}$ & 文 & 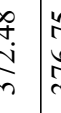 & 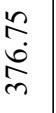 & 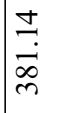 & $\begin{array}{l}\infty \\
n \\
\infty \\
\infty \\
\infty \\
\infty\end{array}$ & वे & $\begin{array}{l}\tilde{6} \\
\stackrel{2}{2} \\
\hat{2}\end{array}$ & $\begin{array}{l}\infty \\
\infty \\
\dot{\sigma}\end{array}$ & $\begin{array}{l}\hat{b} \\
\infty \\
\dot{q} \\
\dot{q}\end{array}$ & 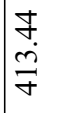 & $\begin{array}{l}n \\
\frac{n}{\gamma} \\
\frac{\gamma}{\gamma}\end{array}$ & $\hat{\partial}$ \\
\hline 苋 & $\tilde{N}$ & 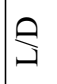 & $N$ & $\vec{\infty}$ & $\exists$ & & ָ̃. & ָั & $\stackrel{m}{i}$ & $\begin{array}{c}n \\
\infty \\
\infty\end{array}$ & $\stackrel{+}{+}$ & $\stackrel{+}{\circ}$ & $\begin{array}{l}n \\
n \\
n\end{array}$ & $\begin{array}{l}? \\
? \\
?\end{array}$ & $\begin{array}{l}n \\
\text { a }\end{array}$ & $\begin{array}{l}n \\
\infty \\
\infty \\
\infty\end{array}$ & $\begin{array}{l}\dot{0} \\
\dot{I}\end{array}$ & $\ddot{\infty}$ & $\hat{\infty}$ & $\hat{\check{a}}$ & $\mid \begin{array}{l}\infty \\
\infty \\
\infty\end{array}$ & $\begin{array}{l}\infty \\
\dot{d} \\
=\end{array}$ & 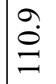 & $\underset{b}{\sigma}$ \\
\hline
\end{tabular}




\begin{tabular}{|c|c|c|c|c|c|c|c|c|c|c|c|c|c|c|c|c|c|c|c|c|c|c|c|}
\hline & & $\vec{\Delta}$ & 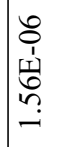 & $\begin{array}{l}8 \\
0 \\
1 \\
1 \\
\\
\\
-1\end{array}$ & 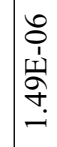 & 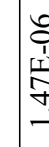 & & 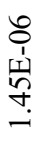 & 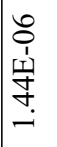 & 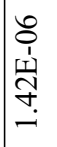 & 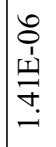 & 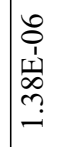 & $\begin{array}{l}8 \\
0 \\
1 \\
\\
\\
\\
-1\end{array}$ & 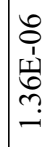 & 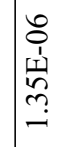 & 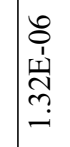 & 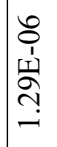 & 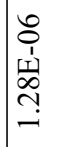 & $\begin{array}{c}8 \\
0 \\
1 \\
1 \\
\\
\\
-1\end{array}$ & 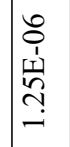 & $\begin{array}{l}8 \\
0 \\
1 \\
\\
\\
\\
-\end{array}$ & 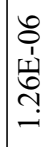 & $\begin{array}{l}\hat{o} \\
1 \\
\mu \\
\bar{n} \\
\sigma \\
\sigma\end{array}$ \\
\hline & & $\stackrel{\oplus}{\infty}$ & 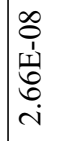 & 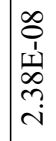 & $\mid \begin{array}{c}\infty \\
0 \\
1 \\
\omega \\
\infty \\
\\
\end{array}$ & 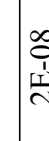 & & $\begin{array}{l}\infty \\
0 \\
0 \\
1 \\
+1 \\
\infty \\
- \\
-\end{array}$ & 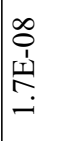 & 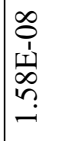 & 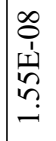 & 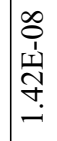 & 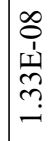 & 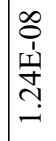 & 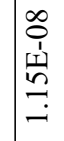 & 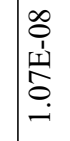 & 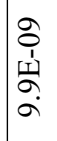 & 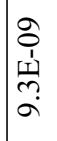 & 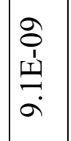 & 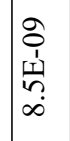 & $\begin{array}{l}\text { Oे } \\
\text { 1 } \\
\text { 山ل }\end{array}$ & 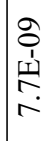 & $\begin{array}{l}\text { o } \\
\hat{1} \\
\text { 11 } \\
0 \\
\text { in }\end{array}$ \\
\hline & & $\ddot{2}$ & 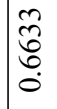 & $\begin{array}{l}\overrightarrow{\widehat{\sigma}} \\
0 \\
0 \\
0\end{array}$ & 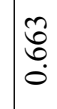 & 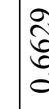 & & $\begin{array}{l}\text { त्रे } \\
\text { ठ্. } \\
\stackrel{0}{0}\end{array}$ & 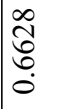 & $\begin{array}{l}\hat{\widehat{J}} \\
\stackrel{0}{0} \\
\stackrel{0}{0}\end{array}$ & $\begin{array}{l}\hat{\widehat{V}} \\
\text { ర్ర } \\
\dot{0}\end{array}$ & 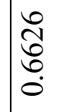 & 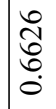 & 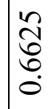 & 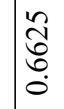 & $\mid \begin{array}{l}\widetilde{Z} \\
\text { ర్ } \\
\stackrel{0}{0}\end{array}$ & 志 & $\begin{array}{l}\underset{\mathbb{J}}{0} \\
\stackrel{0}{0} \\
\dot{0}\end{array}$ & $\begin{array}{l}\mathbb{Z} \\
\mathbb{Z} \\
0 \\
0\end{array}$ & 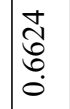 & $\begin{array}{l}\underset{\mathbb{U}}{0} \\
\stackrel{0}{0} \\
\dot{0}\end{array}$ & $\begin{array}{l}\underset{\mathbb{Z}}{\sigma} \\
\stackrel{0}{0} \\
\dot{0}\end{array}$ & 志 \\
\hline & & $\overline{0}$ & \begin{tabular}{l}
\multirow{2}{0}{} \\
$\stackrel{2}{2}$ \\
2
\end{tabular} & 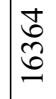 & 余 & 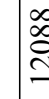 & & \begin{tabular}{l}
0 \\
\multirow{2}{*}{} \\
0
\end{tabular} & 咅 & & $\stackrel{n}{\sim}$ & $\begin{array}{l}\infty \\
\infty \\
\end{array}$ & $\hat{\tilde{D}}$ & $\begin{array}{l}\text { ठे } \\
\text { वे }\end{array}$ & 过 & $\mid \begin{array}{l}\infty \\
\infty \\
\infty\end{array}$ & $\overrightarrow{\bar{f}}$ & 恶 & $\begin{array}{l}\hat{\infty} \\
\infty \\
\sim\end{array}$ & $\mid \begin{array}{l}8 \\
\vdots \\
\end{array}$ & స̊ & 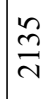 & 紗 \\
\hline & & $t$ & 党 & $\underset{\infty}{\infty}$ & 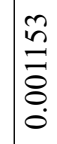 & $\begin{array}{l}0 \\
\\
0 \\
0 \\
0\end{array}$ & & $\begin{array}{l}\bar{\infty} \\
\stackrel{0}{\circ} \\
\stackrel{0}{\circ} \\
\dot{0}\end{array}$ & 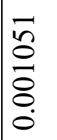 & 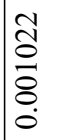 & 商 & 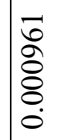 & 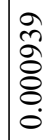 & 夰 & 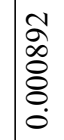 & 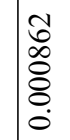 & 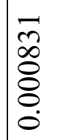 & 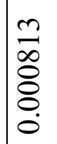 & 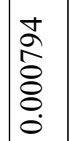 & 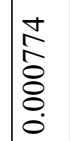 & $\begin{array}{l}\tilde{N} \\
\hat{\sigma} \\
\vdots \\
0 \\
0\end{array}$ & $\begin{array}{l}\overrightarrow{0} \\
\vdots \\
0 \\
0 \\
0\end{array}$ & 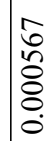 \\
\hline & & $\ddot{\sim}$ & $\underset{\sim}{\stackrel{\sim}{m}}$ & 守 & 㐫 & ڤેे & & ते & $\overrightarrow{\widetilde{ন}}$ & 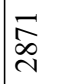 & $\begin{array}{l}\tilde{N} \\
\tilde{N} \\
\stackrel{\sim}{N}\end{array}$ & $\stackrel{\overbrace{}}{\stackrel{\overbrace{}}{*}}$ & $\stackrel{\overbrace{}}{\curvearrowright}$ & $\overrightarrow{\mathrm{D}}$ & $\overrightarrow{\widetilde{d}}$ & $\frac{\sim}{\sim}$ & $\begin{array}{l}n \\
\tilde{n}\end{array}$ & 早 & $\begin{array}{l}8 \\
\\
\end{array}$ & $\underset{\stackrel{\overbrace{}}{\mathcal{~}}}{\stackrel{\Re}{f}}$ & $\underset{\mathrm{d}}{\vec{J}}$ & $\underset{\text { }}{\stackrel{\bigcirc}{\sim}}$ & 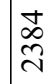 \\
\hline تే & $\stackrel{0}{1}$ & 总 & $\begin{array}{l}\infty \\
\infty \\
\infty \\
\infty\end{array}$ & $\stackrel{m}{-}$ & - & 2 & & $\begin{array}{l}\infty \\
\infty \\
0 \\
0\end{array}$ & $\hat{o}$ & $\hat{a}$ & . & : & $\stackrel{\circ}{\circ}$ & $\frac{n}{3}$ & à & $\mid \begin{array}{l}n \\
\infty \\
0 \\
0\end{array}$ & $\tilde{0}$ & ôे & $\begin{array}{l}t \\
0 \\
0\end{array}$ & $\hat{n}$ & $\begin{array}{l}n \\
n \\
0\end{array}$ & n & f̊ \\
\hline $\begin{array}{l}\frac{t}{\sigma} \\
\frac{\overrightarrow{0}}{\Xi}\end{array}$ & 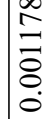 & $\vec{z}$ & $\hat{q}$ & $\begin{array}{l}\hat{o} \\
\underline{\omega} \\
\underline{2}\end{array}$ & ڤ̊ & $\begin{array}{l}\infty \\
0 \\
\end{array}$ & & 艺 & $\begin{array}{l}\infty \\
\stackrel{\infty}{=} \\
=\end{array}$ & ڤ̊ & în & $\stackrel{N}{=}$ & $\stackrel{\infty}{\stackrel{\infty}{\Xi}}$ & $\stackrel{\vec{\sigma}}{=}$ & $\stackrel{\infty}{\stackrel{\infty}{=}}$ & $\stackrel{\Re}{\stackrel{\varrho}{\varrho}}$ & ஸे & $\stackrel{n}{a}$ & $\begin{array}{l}\infty \\
\infty\end{array}$ & $\underset{\infty}{\stackrel{m}{\infty}}$ & $\underset{\infty}{J}$ & $\mid \begin{array}{c}0 \\
\infty \\
\infty\end{array}$ & चै \\
\hline $\begin{array}{l}\stackrel{\mathscr{a}}{\sharp} \\
\frac{\ddot{\theta}}{\Xi}\end{array}$ & 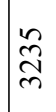 & 䔍 & $\begin{array}{l}\bar{n} \\
\vdots \\
\infty\end{array}$ & $\begin{array}{l}n \\
\\
\end{array}$ & $\frac{\partial}{a}$ & $\mid \begin{array}{l}+ \\
\infty \\
\infty\end{array}$ & & $\underset{\infty}{q}$ & $\hat{a}$ & $\stackrel{t}{\hat{a}}$ & $\stackrel{\overbrace{}}{\stackrel{\vartheta}{0}}$ & $\stackrel{\sim}{\stackrel{0}{0}}$ & 足 & $\begin{array}{l}0 \\
0 \\
0\end{array}$ & 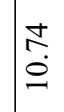 & $\frac{\infty}{2}$ & $\begin{array}{l}\infty \\
\infty \\
\end{array}$ & $\stackrel{n}{n}$ & $\stackrel{m}{r}$ & 6̊. & ñ & బู้ & $\begin{array}{l}\stackrel{0}{2} \\
\text { in }\end{array}$ \\
\hline$\underset{\Xi}{\stackrel{\Xi}{\Xi}}$ & 总 & 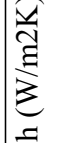 & 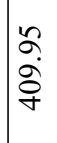 & \begin{tabular}{l}
$n$ \\
\multirow{n}{n}{} \\
\end{tabular} & $\begin{array}{l}\tilde{\sigma} \\
\tilde{n} \\
\tilde{n}\end{array}$ & $\begin{array}{l}1 \\
0 \\
0 \\
3\end{array}$ & & $\begin{array}{l}\text { d̦ } \\
\text { ఏ }\end{array}$ & 守 & $\begin{array}{l}0 \\
\infty \\
\dot{\sim} \\
-\end{array}$ & $\begin{array}{l}\hat{\infty} \\
\dot{\vec{J}} \\
\overrightarrow{\dot{J}}\end{array}$ & $\begin{array}{l}0 \\
0 \\
\dot{0} \\
-2\end{array}$ & $\begin{array}{l}\stackrel{0}{n} \\
\stackrel{n}{n} \\
\end{array}$ & $\begin{array}{l}\hat{b} \\
\stackrel{0}{0} \\
-\end{array}$ & ป̂ & $\begin{array}{l}n \\
\ddot{n} \\
\cong \\
n\end{array}$ & $\begin{array}{l}n \\
\infty \\
ٍ \\
=\end{array}$ & 文 & 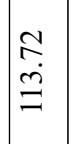 & $\stackrel{t}{ٍ}$ & $\begin{array}{l}\infty \\
0 \\
\dot{0} \\
\varrho \\
0\end{array}$ & $\begin{array}{l}\infty \\
\stackrel{\leftrightarrow}{0} \\
\stackrel{0}{0}\end{array}$ & $\begin{array}{l}\hat{\sigma} \\
\dot{\infty}\end{array}$ \\
\hline$\sum_{\text {至 }}$ & $\overrightarrow{6}$ & 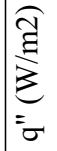 & $\begin{array}{l}n \\
\stackrel{n}{\infty} \\
\infty\end{array}$ & ஓి & $\begin{array}{l}\vec{i} \\
\tilde{\infty} \\
\stackrel{\infty}{\infty}\end{array}$ & $\underset{\infty}{\stackrel{c}{a}}$ & & $\begin{array}{l}\text { ळे } \\
\stackrel{2}{\circ}\end{array}$ & 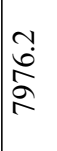 & ڤa. & $\begin{array}{l}n \\
\vdots \\
\alpha \\
\alpha \\
\kappa\end{array}$ & $\begin{array}{l}\overrightarrow{0} \\
\approx \\
\infty \\
\infty\end{array}$ & 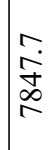 & 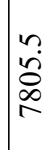 & 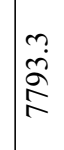 & 吕 & 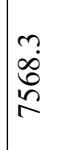 & $\begin{array}{l}0 \\
i n \\
n \\
n\end{array}$ & $\begin{array}{l}\vec{v} \\
\tilde{N} \\
\tilde{n} \\
r\end{array}$ & 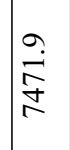 & 告 & 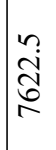 & 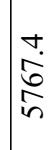 \\
\hline 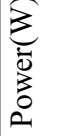 & $\stackrel{\infty}{\infty}$ & $\vec{F}$ & $\mid \overrightarrow{\hat{~}}$ & 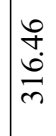 & $\begin{array}{l}\hat{n} \\
n \\
n \\
n \\
n\end{array}$ & 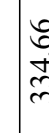 & & $\begin{array}{l}\vec{\sim} \\
\dot{m}\end{array}$ & $\begin{array}{l}\vec{i} \\
\tilde{i} \\
\tilde{n}\end{array}$ & के & 它 & $\begin{array}{l}\infty \\
\\
\stackrel{n}{n}\end{array}$ & $\begin{array}{l}\mathscr{f} \\
\infty \\
\infty \\
\infty \\
\infty\end{array}$ & i̊ & $\begin{array}{l}n \\
0 \\
\dot{0} \\
\dot{q}\end{array}$ & 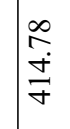 & 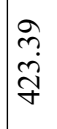 & $\frac{\bar{\sigma}}{\dot{\sigma}}$ & \begin{tabular}{l}
$\mathcal{F}$ \\
$\stackrel{+}{J}$ \\
\multirow{g}{*}{}
\end{tabular} & $\begin{array}{l}\infty \\
\infty \\
\infty \\
\dot{f}\end{array}$ & 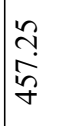 & 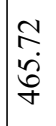 & $\begin{array}{l}\hat{N} \\
\\
\vartheta\end{array}$ \\
\hline$\sum_{0=1}^{\pi}$ & $\frac{\infty}{0}$ & 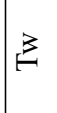 & 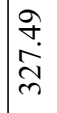 & $\begin{array}{l}\mathbb{1} \\
\infty \\
\infty \\
\infty \\
ల \\
ల\end{array}$ & $\begin{array}{l}\hat{0} \\
\dot{\alpha} \\
\infty \\
m\end{array}$ & $\begin{array}{l}\infty \\
0 \\
0 \\
m\end{array}$ & & 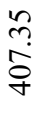 & 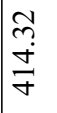 & 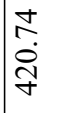 & 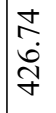 & 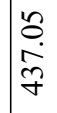 & $\begin{array}{l}\stackrel{0}{0} \\
\dot{J} \\
\text { J }\end{array}$ & 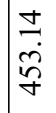 & 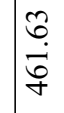 & $\begin{array}{l}\vec{b} \\
\stackrel{i}{F} \\
\vec{f}\end{array}$ & $\frac{n}{\infty}$ & $\begin{array}{l}0 \\
\stackrel{n}{a} \\
\stackrel{q}{q}\end{array}$ & $\begin{array}{l}0 \\
n \\
0 \\
0 \\
n\end{array}$ & $\begin{array}{l}n \\
n \\
0 \\
n \\
n\end{array}$ & $\begin{array}{l}\hat{n} \\
\underline{0} \\
\tilde{n} \\
n\end{array}$ & $\begin{array}{l}\vec{n} \\
\infty \\
\tilde{n} \\
n\end{array}$ & $\begin{array}{l}n \\
\tilde{m} \\
\tilde{n}\end{array}$ \\
\hline 离 & $\ddot{\imath}$ & 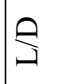 & $N$ & $\vec{\infty}$ & 王 & ๙ี & & ֶָ. & $\stackrel{m}{\stackrel{n}{n}}$ & $\begin{array}{c}m \\
\infty \\
\infty\end{array}$ & $\begin{array}{l}\dot{J} \\
\dot{f}\end{array}$ & ti. & $\begin{array}{l}n \\
0 \\
0\end{array}$ & 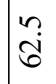 & $\begin{array}{l}n \\
\infty \\
\infty \\
\infty\end{array}$ & $\begin{array}{l}0 \\
\stackrel{+}{T}\end{array}$ & $\ddot{\infty}$ & $\hat{\infty}$ & $\hat{\check{a}}$ & $\mid \begin{array}{l}\infty \\
\infty \\
\infty\end{array}$ & 㐫 & $\stackrel{\varrho}{\varrho}$ & $\stackrel{\vec{b}}{=}$ \\
\hline
\end{tabular}




\begin{tabular}{|c|c|c|c|c|c|c|c|c|c|c|c|c|c|c|c|c|c|c|c|c|c|c|c|}
\hline & & $\vec{\Delta}$ & 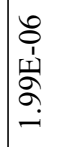 & 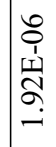 & 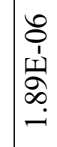 & 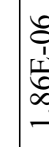 & 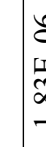 & 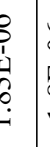 & 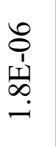 & 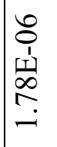 & 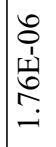 & 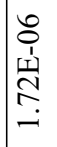 & 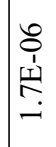 & 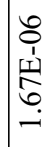 & 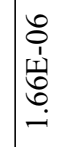 & 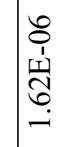 & 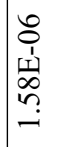 & 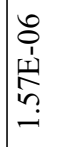 & $\begin{array}{c}8 \\
0 \\
1 \\
11 \\
n \\
n \\
-1\end{array}$ & $\begin{array}{c}8 \\
0 \\
1 \\
11 \\
n \\
n \\
-1\end{array}$ & 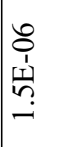 & $\begin{array}{l}0 \\
0 \\
1 \\
\\
\text { N } \\
\\
-1\end{array}$ & | \\
\hline & & $\stackrel{\oplus}{\infty}$ & 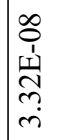 & \begin{tabular}{l}
$\infty$ \\
0 \\
1 \\
1 \\
\multirow{1}{+}{} \\
$\grave{\lambda}$
\end{tabular} & 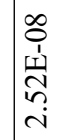 & 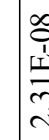 & 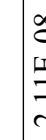 & 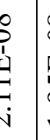 & 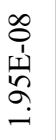 & 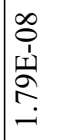 & 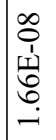 & 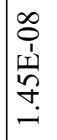 & 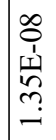 & $\begin{array}{l}\infty \\
0 \\
1 \\
1 \\
\\
\\
\end{array}$ & 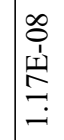 & $\begin{array}{l}\infty \\
0 \\
0 \\
1 \\
1 \\
\infty \\
0 \\
-\end{array}$ & $\begin{array}{l}\infty \\
0 \\
1 \\
\underline{1}\end{array}$ & 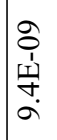 & $\begin{array}{l}2 \\
0 \\
1 \\
\omega \\
\infty \\
\infty \\
\infty\end{array}$ & $\begin{array}{l}2 \\
\partial \\
1 \\
1 \\
m \\
\infty \\
\infty\end{array}$ & 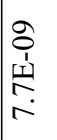 & 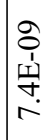 & $\begin{array}{l}\text { o } \\
\hat{1} \\
1 \\
\text { in } \\
\text { in }\end{array}$ \\
\hline & & $\ddot{2}$ & $\begin{array}{l}\tilde{\widehat{O}} \\
\stackrel{0}{0} \\
\stackrel{0}{0}\end{array}$ & $\begin{array}{l}\overrightarrow{\tilde{O}} \\
0 \\
0\end{array}$ & 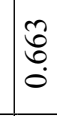 & 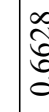 & a & $\begin{array}{l}0 \\
0 \\
0 \\
0 \\
0\end{array}$ & $\begin{array}{l}\hat{\widehat{O}} \\
\stackrel{0}{0} \\
\stackrel{0}{0}\end{array}$ & 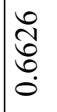 & 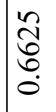 & 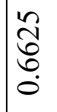 & 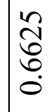 & 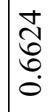 & 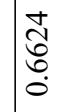 & $\mid \begin{array}{l}\mathbb{Z} \\
\stackrel{J}{0} \\
\stackrel{0}{0}\end{array}$ & 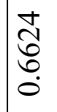 & 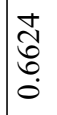 & $\begin{array}{l}\mathbb{Z} \\
\mathbb{J} \\
0 \\
0\end{array}$ & $\mid \begin{array}{l}\mathbb{Z} \\
\mathbb{ర} \\
0 \\
0\end{array}$ & \begin{tabular}{l}
\multirow{2}{\mathbb{ర}}{} \\
$\stackrel{0}{0}$ \\
0
\end{tabular} & 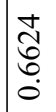 & 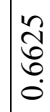 \\
\hline & & $\overline{0}$ & 命 & 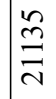 & 胥 & $\mathfrak{a}$ & $\frac{1}{z}$ & 卓 & $\stackrel{n}{\exists}$ & $\overrightarrow{0}$ & 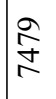 & $\frac{0}{8}$ & 令 & $\frac{1}{0}$ & 守 & $\begin{array}{l}\tilde{N} \\
\tilde{n}\end{array}$ & & 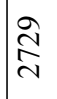 & $\stackrel{\bar{y}}{\underset{d}{d}}$ & $\stackrel{\bullet}{\sim}$ & $\frac{m}{2}$ & $\stackrel{N}{\Sigma}$ & $\stackrel{\infty}{=}$ \\
\hline & & t & $\begin{array}{l}\overrightarrow{\tilde{0}} \\
\stackrel{8}{\circ} \\
\dot{0}\end{array}$ & 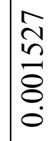 & 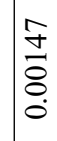 & $\frac{\bar{z}}{\bar{g}}$ & $\begin{array}{l}y \\
y \\
c \\
z \\
c \\
c\end{array}$ & & 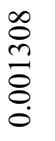 & 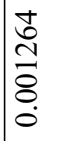 & 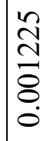 & $\stackrel{ }{\Xi}$ & 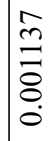 & $\mid \begin{array}{l}\infty \\
\stackrel{0}{0} \\
\stackrel{0}{8} \\
\stackrel{0}{0}\end{array}$ & 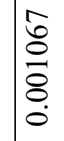 & $\mid \begin{array}{l}2 \\
o \\
o \\
o \\
0 \\
0\end{array}$ & $\begin{array}{l}0 \\
\infty \\
\stackrel{8}{8} \\
\varnothing \\
\dot{0}\end{array}$ & $\begin{array}{l}\overline{0} \\
8 \\
8 \\
\varnothing \\
0\end{array}$ & $\begin{array}{l}\tilde{D} \\
\tilde{O} \\
\dot{\delta} \\
\dot{0} \\
\dot{0}\end{array}$ & $\begin{array}{l}\hat{o} \\
\delta \\
\delta \\
0 \\
0 \\
\dot{0}\end{array}$ & 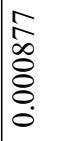 & 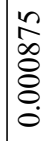 & 离 \\
\hline & & $\ddot{\sim}$ & 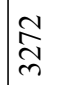 & $\frac{\infty}{m}$ & $\frac{\infty}{0}$ & ह̂ & 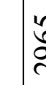 & לֶ. & ڤે & $\begin{array}{l}\infty \\
\infty \\
\infty \\
\sim\end{array}$ & $\stackrel{\infty}{\stackrel{\infty}{\sim}}$ & $\underset{\mathbb{N}}{\stackrel{\mathbb{N}}{\mathbf{N}}}$ & 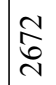 & స్ర్రి & n & 尽 & $\begin{array}{l}\infty \\
\stackrel{\infty}{+} \\
\stackrel{+}{\sim}\end{array}$ & $\underset{\text { 采 }}{ }$ & 文 & $\underset{\tilde{\hat{v}}}{\overrightarrow{\hat{v}}}$ & $\begin{array}{l}\infty \\
\tilde{\sim} \\
\end{array}$ & \begin{tabular}{|c}
$\widetilde{\sigma}$ \\
ते
\end{tabular} & 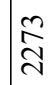 \\
\hline تే & $\stackrel{0}{1}$ & 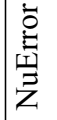 & $a$ & ले & $\overbrace{-}$ & $\infty$ & r & : & $\stackrel{2}{\hat{0}}$ & $\vec{\infty}$ & {$\left[\begin{array}{l}n \\
\infty \\
0 \\
0\end{array}\right.$} & $\stackrel{0}{i}$ & $\stackrel{\mathbb{2}}{\stackrel{0}{0}}$ & 它 & ర్ర & ñ & $\stackrel{n}{0}$ & Iั & $\dot{0}$ & $\mid \begin{array}{l}\infty \\
? \\
0 \\
0\end{array}$ & 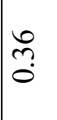 & חִ & ते \\
\hline $\begin{array}{l}\frac{ \pm}{\sigma} \\
\frac{ \pm}{ \pm} \\
\Xi\end{array}$ & $\frac{0}{n}$ & $\vec{z}$ & $\underset{f}{\stackrel{J}{f}}$ & 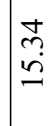 & $\begin{array}{l}\infty \\
\grave{j} \\
\text { In }\end{array}$ & $\begin{array}{l}+ \\
\infty \\
=\end{array}$ & $g$ & 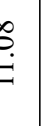 & 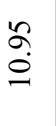 & $\grave{\varrho}$ & 苟 & $\hat{0}$ & $\begin{array}{l}\infty \\
\infty \\
\alpha\end{array}$ & å. & $\hat{\sigma}$ & ते & กै? & ڤ్ & $\begin{array}{l}2 \\
6 \\
0 \\
6\end{array}$ & n̊ & $\begin{array}{l}0 \\
2 \\
i n\end{array}$ & $\begin{array}{l}0 \\
\infty \\
\text { in }\end{array}$ & $\stackrel{\vec{r}}{\stackrel{\sim}{*}}$ \\
\hline 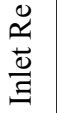 & 周 & 寄 & $\stackrel{\Re}{\stackrel{2}{\infty}}$ & $\begin{array}{l}\varkappa \\
\hat{\jmath} \\
\end{array}$ & $\stackrel{\infty}{\sigma^{\circ}}$ & $\begin{array}{l}\overline{0} \\
\infty\end{array}$ & $\begin{array}{ll}4 \\
3 \\
7\end{array}$ & $\hat{~}$ & $\underset{\infty}{ \pm}$ & $\begin{array}{l}n \\
\infty \\
\infty\end{array}$ & $\begin{array}{c}\infty \\
\infty \\
\infty\end{array}$ & à & $\overline{\widehat{a}}$ & $i \frac{n}{n}$ & 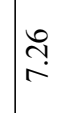 & f̊ & ते & $\stackrel{8}{\circ}$ & $\begin{array}{l}0 \\
+ \\
+\end{array}$ & ঙे & F & $\underset{\dot{\sigma}}{\tilde{\sigma}}$ & $\tilde{n}$ \\
\hline$\underset{\Xi}{\stackrel{\Xi}{\Xi}}$ & 总 & 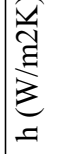 & 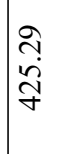 & $\begin{array}{l}\infty \\
2 \\
\infty \\
\infty \\
2\end{array}$ & $\begin{array}{l}\infty \\
\vdots \\
m \\
m\end{array}$ & 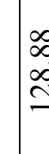 & 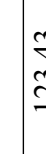 & t. & $\begin{array}{l}\stackrel{\Re}{\sim} \\
\stackrel{\Xi}{\beth}\end{array}$ & 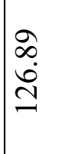 & $\begin{array}{l}8 \\
\dot{0} \\
\dot{0} \\
-\end{array}$ & $\begin{array}{l}\tilde{N} \\
\text { İ } \\
\text { In }\end{array}$ & 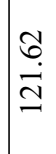 & $\begin{array}{l}n \\
n \\
\infty \\
=\end{array}$ & $\stackrel{a}{=}$ & $\mid \begin{array}{l}\hat{\jmath} \\
\infty \\
\stackrel{0}{O}\end{array}$ & $\frac{m}{\alpha}$ & $\begin{array}{l}\dot{\Xi} \\
\dot{d}\end{array}$ & $\underset{\tilde{a}}{\tilde{\sigma}}$ & $\mid \begin{array}{c}\infty \\
\infty \\
\infty \\
\infty\end{array}$ & $\begin{array}{l}\vec{n} \\
\dot{\infty}\end{array}$ & $\underset{\dot{\infty}}{\stackrel{一}{\infty}}$ & 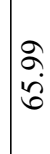 \\
\hline$\sum_{\text {至 }}$ & ָู & $\begin{array}{l}\text { శี } \\
\text { ఏ } \\
= \\
=\end{array}$ & 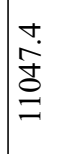 & 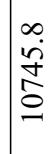 & $\begin{array}{l}0 \\
\stackrel{\mathbb{N}}{0} \\
\stackrel{0}{0}\end{array}$ & \begin{tabular}{l}
7 \\
\multirow{2}{*}{} \\
है
\end{tabular} & $\begin{array}{l}\alpha \\
\alpha \\
z \\
z\end{array}$ & 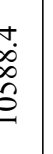 & $\begin{array}{l}\hat{0} \\
\text { ñ } \\
\tilde{o}\end{array}$ & $\begin{array}{l}\hat{\sigma} \\
\tilde{\sigma} \\
\tilde{0}\end{array}$ & 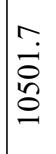 & $\begin{array}{l}\hat{o} \\
\dot{q} \\
\text { ô } \\
0\end{array}$ & $\frac{\hat{\sigma}}{\tilde{\sigma}}$ & 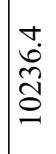 & $\begin{array}{l}n \\
\stackrel{n}{\sigma} \\
\widetilde{\sigma}\end{array}$ & $\mid \begin{array}{l}\stackrel{f}{0} \\
\stackrel{8}{0}\end{array}$ & $\begin{array}{l}\hat{\ddot{n}} \\
\text { ळे }\end{array}$ & $\begin{array}{l}\ddot{2} \\
\ddot{\alpha}\end{array}$ & $\begin{array}{l}\infty \\
\stackrel{\infty}{~} \\
\infty \\
\sigma \\
\sigma\end{array}$ & 茫 & 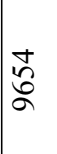 & $\begin{array}{l}\vec{a} \\
\ddot{\alpha} \\
\alpha\end{array}$ & $\underset{F}{\stackrel{P}{F}}$ \\
\hline $\begin{array}{l}3 \\
0 \\
0 \\
0 \\
0 \\
0 \\
0\end{array}$ & छ & $\vec{F}$ & 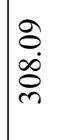 & $\begin{array}{l}m \\
\stackrel{0}{0} \\
\sim\end{array}$ & $\begin{array}{l}\stackrel{\sigma}{\vec{j}} \\
\text { m}\end{array}$ & 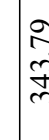 & $\begin{array}{l}5 \\
4 \\
y \\
5\end{array}$ & $\begin{array}{l}n \\
\hat{n} \\
n \\
n\end{array}$ & 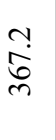 & $\begin{array}{l}+ \\
\infty \\
\infty \\
\infty \\
\infty\end{array}$ & 告 & $\frac{\hat{\alpha}}{\dot{\alpha}}$ & $\begin{array}{l}\infty \\
\sim \\
\ddot{z} \\
\forall\end{array}$ & 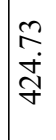 & $\begin{array}{l}\tilde{\delta} \\
\dot{\delta} \\
\tilde{\gamma}\end{array}$ & $\stackrel{\stackrel{\sim}{N}}{\stackrel{\sim}{f}}$ & 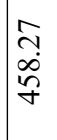 & 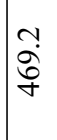 & $\begin{array}{l}= \\
\dot{0} \\
\stackrel{+}{+}\end{array}$ & $\begin{array}{l}\text { वे } \\
\text { @ें } \\
\dot{q}\end{array}$ & $\begin{array}{l}\infty \\
0 \\
\dot{0} \\
\dot{0}\end{array}$ & $\begin{array}{l}\underset{+}{d} \\
\stackrel{i}{i n}\end{array}$ & $\begin{array}{l}\tilde{\infty} \\
\dot{\sim} \\
\tilde{N}\end{array}$ \\
\hline$\sum_{0=1}^{\pi}$ & $\frac{\infty}{0}$ & 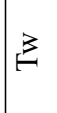 & $\begin{array}{l}\hat{\sigma} \\
\dot{\sim} \\
\text { mे }\end{array}$ & $\underset{\substack{N \\
\infty}}{\stackrel{N}{n}}$ & 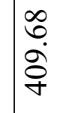 & $\begin{array}{l}0 \\
1 \\
0 \\
7 \\
7\end{array}$ & 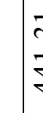 & $\frac{\vec{?}}{8}$ & $\frac{\infty}{\dot{\square}}$ & $\frac{\mathfrak{i}}{\stackrel{\delta}{+}}$ & $\stackrel{\infty}{\stackrel{\infty}{\vec{r}}}$ & $\begin{array}{l}\hat{n} \\
\dot{0} \\
\infty \\
+\end{array}$ & $\begin{array}{l}\tilde{n} \\
\infty \\
\dot{\sigma}\end{array}$ & 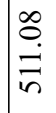 & 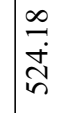 & 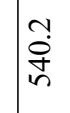 & $\begin{array}{l}\infty \\
0 \\
0 \\
0 \\
0 \\
n\end{array}$ & $\begin{array}{l}\vec{J} \\
\stackrel{+}{i} \\
i n\end{array}$ & $\begin{array}{l}\overrightarrow{0} \\
\infty \\
\infty \\
\infty \\
n\end{array}$ & 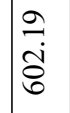 & $\frac{\infty}{6}$ & 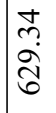 & $\begin{array}{l}\hat{0} \\
\dot{0} \\
\text { రై }\end{array}$ \\
\hline 离 & $\stackrel{\Xi}{\sim}$ & 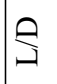 & $N$ & $\vec{\infty}$ & 王 & ণ & 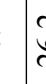 & ָै. & $\stackrel{\text { }}{2}$ & $\begin{array}{c}m \\
\infty \\
\infty\end{array}$ & $\underset{f}{\dot{f}}$ & $\ddot{i}$ & $\begin{array}{l}n \\
0 \\
0\end{array}$ & 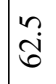 & $\begin{array}{l}n \\
\infty \\
\infty \\
\infty\end{array}$ & $\begin{array}{l}0 \\
\stackrel{+}{T}\end{array}$ & $\ddot{\infty}$ & $\hat{\infty}$ & $\hat{a}$ & $\mid \begin{array}{l}\infty \\
\infty \\
\infty\end{array}$ & 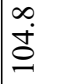 & $\stackrel{\varrho}{\varrho}$ & $\stackrel{\vec{b}}{=}$ \\
\hline
\end{tabular}




\begin{tabular}{|c|c|c|c|c|c|c|c|c|c|c|c|c|c|c|c|c|c|c|c|c|c|c|c|}
\hline & & $\vec{\Delta}$ & 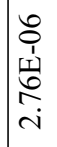 & 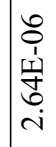 & $\begin{array}{c}8 \\
o \\
1 \\
\\
\vdots \\
\\
\text { r. }\end{array}$ & $\mid \begin{array}{l}0 \\
1 \\
1 \\
n \\
n \\
n \\
n\end{array}$ & & 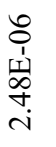 & 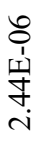 & 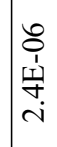 & 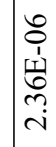 & $\begin{array}{c}\stackrel{8}{1} \\
1 \\
\text { In } \\
\\
\text { in }\end{array}$ & 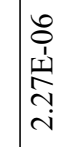 & 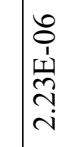 & 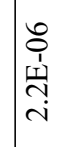 & 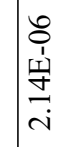 & $\begin{array}{l}8 \\
0 \\
1 \\
1 \\
0 \\
0 \\
i \\
i\end{array}$ & 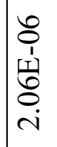 & $\begin{array}{c}0 \\
0 \\
1 \\
1 \\
\tilde{1} \\
0 \\
i \\
i\end{array}$ & \begin{tabular}{l}
$\stackrel{8}{0}$ \\
\multicolumn{1}{c}{} \\
\multicolumn{1}{c}{}
\end{tabular} & 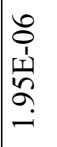 & 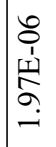 & 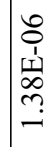 \\
\hline & & $\stackrel{\oplus}{\infty}$ & 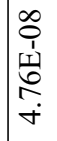 & 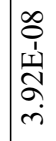 & $\begin{array}{c}\infty \\
\infty \\
0 \\
1 \\
\omega \\
n \\
m \\
m \\
m\end{array}$ & 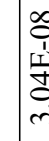 & & 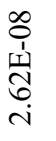 & 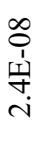 & $\begin{array}{l}\infty \\
0 \\
0 \\
1 \\
\text { w1 } \\
\text { o } \\
\dot{i}\end{array}$ & 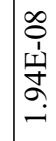 & \begin{tabular}{c}
$\infty$ \\
0 \\
0 \\
1 \\
\multicolumn{1}{|c}{} \\
- \\
-1
\end{tabular} & 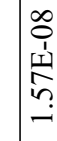 & 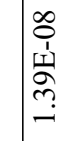 & 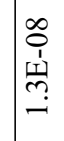 & 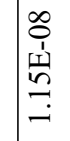 & 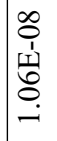 & 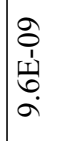 & 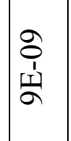 & 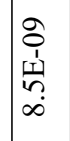 & 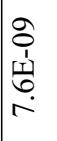 & 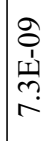 & 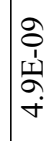 \\
\hline & & $\ddot{2}$ & 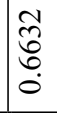 & $\begin{array}{l}\hat{0} \\
0 \\
0 \\
0\end{array}$ & $\begin{array}{l}\text { ते } \\
\text { ర్} \\
0 \\
0\end{array}$ & శ̊ & & 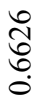 & $\begin{array}{l}0 \\
\text { đ్ర } \\
0 \\
0 \\
0\end{array}$ & 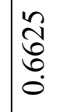 & 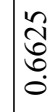 & 芯 & 芯 & $\mid \begin{array}{l}\mathbb{Z} \\
\text { d } \\
0 \\
0\end{array}$ & 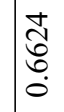 & $\mid \begin{array}{l}\mathbb{Z} \\
\stackrel{J}{0} \\
\stackrel{0}{0}\end{array}$ & 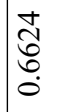 & $\begin{array}{l}2 \\
\hat{\sigma} \\
0 \\
0 \\
0\end{array}$ & 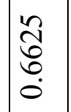 & 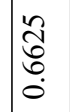 & $\begin{array}{l}0 \\
\text { đ్ర } \\
0 \\
0\end{array}$ & 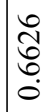 & $\begin{array}{l}\hat{\widehat{V}} \\
\stackrel{0}{0} \\
\stackrel{0}{0}\end{array}$ \\
\hline & & $\mathscr{J}$ & 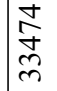 & 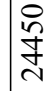 & $\begin{array}{l}\text { ț } \\
0 \\
\infty \\
-1\end{array}$ & $\mid \begin{array}{l}2 \\
i \\
i\end{array}$ & & $\begin{array}{l}\infty \\
\infty \\
\infty \\
ٍ \\
=\end{array}$ & $\underset{a}{a}$ & \begin{tabular}{l}
$\hat{\infty}$ \\
$\infty$ \\
\hdashline
\end{tabular} & F & $\frac{\pi}{n}$ & 点 & 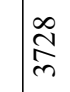 & $\underset{\approx}{\approx}$ & ڤ્ટે & तે & $\underset{2}{2}$ & $\underline{2}$ & 竞 & 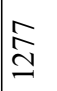 & $\stackrel{n}{\exists}$ & $\vec{I}$ \\
\hline & & $t$ & 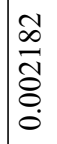 & 离 & 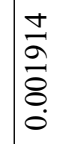 & $\frac{0}{\infty}$ & & $\begin{array}{l}\frac{\hat{N}}{\mathbb{a}} \\
\stackrel{8}{0} \\
\dot{0}\end{array}$ & $\begin{array}{l}\stackrel{0}{0} \\
\stackrel{0}{8} \\
\stackrel{0}{0}\end{array}$ & $\begin{array}{l}\infty \\
\hat{n} \\
\vdots \\
\varnothing \\
0 \\
0\end{array}$ & $\begin{array}{l}\frac{1}{n} \\
\frac{n}{8} \\
8 \\
0 \\
0\end{array}$ & $\underset{\stackrel{\tilde{f}}{\sigma}}{\stackrel{8}{8}}$ & $\mid \begin{array}{l}\infty \\
\infty \\
\stackrel{\infty}{8} \\
\stackrel{8}{0} \\
0\end{array}$ & $\mid \begin{array}{l}\vec{m} \\
\stackrel{0}{8} \\
\overline{8} \\
\dot{0}\end{array}$ & $\mid \begin{array}{l}0 \\
\stackrel{\infty}{1} \\
\stackrel{1}{8} \\
\dot{0} \\
0\end{array}$ & $\mid \begin{array}{c}\overparen{3} \\
\stackrel{2}{8} \\
0 \\
0\end{array}$ & 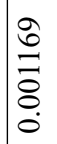 & 兽 & $\begin{array}{l}\hat{a} \\
\dot{0} \\
\dot{0} \\
\dot{0} \\
\dot{0}\end{array}$ & 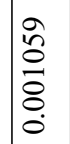 & $\begin{array}{l}\frac{0}{0} \\
\stackrel{0}{8} \\
0 \\
0\end{array}$ & $\frac{\infty}{\stackrel{0}{0}}$ & $\mid \begin{array}{l}\hat{\alpha} \\
\dot{0} \\
\dot{0} \\
\dot{0}\end{array}$ \\
\hline & & $\approx$ & $\frac{8}{m}$ & 文 & ڤ̆̀े & $\mid \begin{array}{l}8 \\
\infty \\
0\end{array}$ & & $\stackrel{2}{\infty}$ & 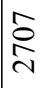 & 该 & $\begin{array}{l}\hat{b} \\
\grave{n}\end{array}$ & $\begin{array}{l} \pm \\
\stackrel{0}{n} \\
\end{array}$ & $\underset{\sim}{\stackrel{J}{\sim}}$ & ঐे & $\underset{\tilde{\lambda}}{\infty}$ & 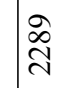 & $\underset{\mathbb{Z}}{\stackrel{\sim}{N}}$ & ¿্ণి & $\frac{\grave{n}}{\sim}$ & $\frac{i}{\pi}$ & 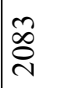 & 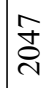 & $\stackrel{\infty}{\stackrel{\sim}{\sim}}$ \\
\hline تే & $\stackrel{0}{1}$ & 总 & aे. & $\stackrel{+}{-}$ & $\stackrel{\infty}{\circ}$ & $\mid \begin{array}{l}\infty \\
\infty \\
0\end{array}$ & & $\stackrel{2}{\circ}$ & $\frac{\pi}{0}$ & $\stackrel{\mathbb{2}}{0}$ & $\tilde{o}$ & $\hat{n}$ & ñ & $n$ & $\stackrel{0}{1}$ & F. & $\stackrel{+}{m}$ & 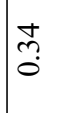 & 3 & ?3. & $\tilde{\widehat{~}}$ & $\hat{ָ}$ & ڤn \\
\hline $\begin{array}{l}\frac{ \pm}{\sigma} \\
\frac{ \pm}{ \pm} \\
\Xi\end{array}$ & 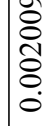 & $\vec{z}$ & $\hat{\check{\gamma}}$ & $\begin{array}{l}\vec{\infty} \\
\ddot{n}\end{array}$ & $\overrightarrow{\widetilde{d}}$ & 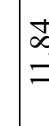 & & $\begin{array}{l}+ \\
\infty \\
0 \\
0\end{array}$ & $\stackrel{+}{\stackrel{0}{\circ}}$ & $\begin{array}{l}t \\
0 \\
0 \\
0\end{array}$ & $\stackrel{\vartheta}{a}$ & $\underset{\infty}{\stackrel{n}{\infty}}$ & $\underset{\infty}{\infty}$ & $\stackrel{0}{\stackrel{2}{r}}$ & $\stackrel{?}{r}$ & ț & $\hat{\hat{n}}$ & $\begin{array}{l}\infty \\
0 \\
i \\
i\end{array}$ & $\begin{array}{l}m \\
\dot{v} \\
n\end{array}$ & in & \% & $\mid \begin{array}{r}\varkappa \\
\dot{\gamma}\end{array}$ & $\stackrel{\varkappa}{\varkappa}$ \\
\hline $\begin{array}{l}\stackrel{\mathscr{a}}{\sharp} \\
\frac{\ddot{\theta}}{\Xi}\end{array}$ & 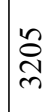 & 莺 & 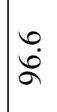 & $\begin{array}{l}\infty \\
\infty \\
\end{array}$ & ले & $\mid \begin{array}{l}0 \\
\infty \\
\infty\end{array}$ & & $\underset{\infty}{+}$ & $\begin{array}{l}\infty \\
\infty \\
\sim \\
\sim\end{array}$ & $\stackrel{\vartheta}{\stackrel{2}{r}}$ & 市. & : & ஸे & $\mid \begin{array}{l}n \\
\infty \\
i n\end{array}$ & $\underset{i}{\stackrel{f}{*}}$ & $\stackrel{\infty}{\stackrel{\infty}{\sim}}$ & 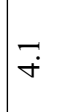 & $\begin{array}{l}\infty \\
\infty \\
\infty \\
n\end{array}$ & $\vec{r}$ & 吕 & $\vec{\sigma}$. & $\underset{\sim}{\tilde{f}}$ & $\stackrel{m}{m}$ \\
\hline$\underset{\Xi}{\stackrel{\Xi}{\Xi}}$ & 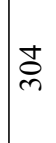 & 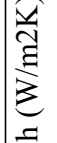 & $\begin{array}{l}\hat{6} \\
\dot{J} \\
\text { J }\end{array}$ & $\begin{array}{l}0 \\
2 \\
0 \\
0\end{array}$ & $\overrightarrow{\dot{g}}$ & ma & & $\begin{array}{l} \pm \\
\stackrel{ \pm}{+} \\
\stackrel{ \pm}{\Xi}\end{array}$ & $\begin{array}{l}\hat{\tilde{y}} \\
\tilde{\Xi}\end{array}$ & 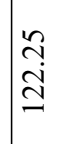 & 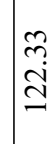 & $\begin{array}{l}\infty \\
\stackrel{0}{I} \\
ٍ\end{array}$ & $\frac{n}{8}$ & 志 & $\stackrel{n}{\stackrel{0}{8}}$ & 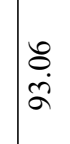 & 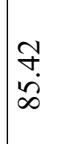 & $\begin{array}{l}2 \\
\infty \\
\infty \\
\infty\end{array}$ & $\begin{array}{l}2 \\
\stackrel{8}{\infty} \\
\infty\end{array}$ & $\underset{\substack{\infty \\
\infty}}{\vec{\infty}}$ & 导 & $\stackrel{n}{n}$ & $\begin{array}{l}\stackrel{\bullet}{\grave{d}} \\
\text { d. }\end{array}$ \\
\hline$\sum_{\text {至 }}$ & 6 & 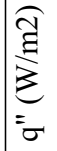 & 享 & 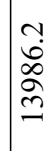 & $\begin{array}{l}0 \\
\ddot{n} \\
\tilde{n} \\
\tilde{n}\end{array}$ & $\begin{array}{l}n \\
n \\
n \\
n\end{array}$ & & $\begin{array}{l}\vec{m} \\
\stackrel{m}{n} \\
\stackrel{m}{n}\end{array}$ & 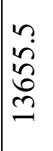 & 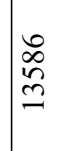 & $\begin{array}{l}n \\
0 \\
0 \\
n \\
n \\
n\end{array}$ & $\begin{array}{c}\tilde{\rho} \\
\tilde{m} \\
\tilde{n}\end{array}$ & 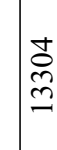 & $\begin{array}{l}\vec{n} \\
\infty \\
\underline{m}\end{array}$ & 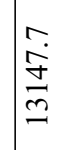 & $\mid \begin{array}{l}\infty \\
\dot{0} \\
\stackrel{0}{0} \\
\stackrel{\bigcup}{0}\end{array}$ & 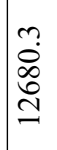 & 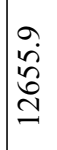 & 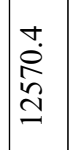 & 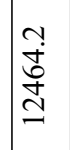 & 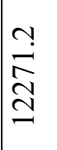 & 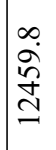 & $\begin{array}{l}\tilde{n} \\
\stackrel{\hat{\sigma}}{\infty} \\
\stackrel{\infty}{0}\end{array}$ \\
\hline 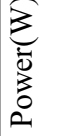 & ñ & $\theta$ & $\begin{array}{l}\infty \\
\text { } \\
\stackrel{\rho}{\rho} \\
\dot{m}\end{array}$ & 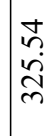 & 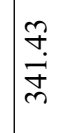 & \begin{tabular}{l}
$\stackrel{J}{n}$ \\
\multirow{n}{n}{}
\end{tabular} & & $\underset{\hat{N}}{\stackrel{n}{n}}$ & $\begin{array}{l}\vec{n} \\
\infty \\
\infty \\
n\end{array}$ & $\begin{array}{l}\tilde{O} \\
\dot{\sigma} \\
\dot{+}\end{array}$ & $\begin{array}{l}\text { fे } \\
\stackrel{\partial}{\vec{\gamma}}\end{array}$ & 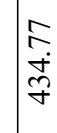 & $\mid \begin{array}{l}\hat{\sigma} \\
\hat{\sigma}\end{array}$ & $\mid \begin{array}{l}\sigma \\
\dot{0} \\
\dot{\sigma}\end{array}$ & $\begin{array}{l}\hat{\partial} \\
\grave{\partial} \\
\stackrel{f}{ }\end{array}$ & $\mid \begin{array}{l}\infty \\
\dot{d} \\
\dot{q}\end{array}$ & $\begin{array}{l}\hat{n} \\
\stackrel{2}{0} \\
\ddot{n}\end{array}$ & $\begin{array}{l}\stackrel{2}{\hat{\lambda}} \\
\hat{\sim}\end{array}$ & $\begin{array}{l}n \\
\infty \\
\infty \\
n \\
n\end{array}$ & $\mid \begin{array}{l}\vec{i} \\
\tilde{n} \\
i n\end{array}$ & $\begin{array}{l}\text { fo. } \\
\stackrel{0}{0} \\
\stackrel{0}{0}\end{array}$ & $\begin{array}{l}n \\
n \\
0 \\
\infty \\
i n\end{array}$ & $\begin{array}{l}\sigma \\
i \\
\tilde{\sigma} \\
i\end{array}$ \\
\hline$\sum_{0=1}^{\pi}$ & $\frac{\infty}{0}$ & 3 & 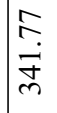 & $\begin{array}{l}\alpha \\
\text { ò } \\
\stackrel{+}{+}\end{array}$ & 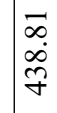 & $\frac{1}{7}$ & & $\begin{array}{l}\hat{\alpha} \\
\hat{i} \\
\dot{\alpha}\end{array}$ & $\begin{array}{l}\hat{\widehat{त}} \\
\text { } \\
\vec{\sigma}\end{array}$ & 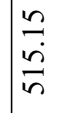 & 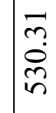 & $\begin{array}{l}\text { ㄱ. } \\
\tilde{n} \\
\tilde{n}\end{array}$ & $\mid \begin{array}{l}\infty \\
\infty \\
i \\
i n\end{array}$ & 守 & $\stackrel{\hat{\Upsilon}}{\bar{\sigma}}$ & $\underset{\widetilde{\sigma}}{\stackrel{\nabla}{0}}$ & $\begin{array}{l}\infty \\
\infty \\
\hat{\sigma}\end{array}$ & $\begin{array}{l}\hat{f} \\
\stackrel{0}{0} \\
\text { S. }\end{array}$ & $\begin{array}{l}\infty \\
\tilde{\sigma} \\
\hat{\sigma}\end{array}$ & $\begin{array}{l}\frac{n}{\mathfrak{c}} \\
\stackrel{0}{r}\end{array}$ & $\begin{array}{l}\sigma \\
\stackrel{\sigma}{N} \\
\text { in }\end{array}$ & 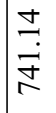 & $\begin{array}{l}\vec{\infty} \\
\ddot{N} \\
\approx\end{array}$ \\
\hline 离 & in & 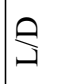 & $N$ & $\vec{\infty}$ & ت্ & ๙ี & & ֶָ. & $\stackrel{m}{\tilde{d}}$ & $\begin{array}{c}m \\
\infty \\
\infty\end{array}$ & $\stackrel{+}{+}$ & $\stackrel{+}{\circ}$ & 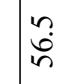 & 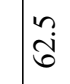 & $\begin{array}{l}n \\
\infty \\
\infty \\
\infty\end{array}$ & $\begin{array}{l}0 \\
\dot{+}\end{array}$ & $\ddot{\infty}$ & $\hat{\infty}$ & $\hat{\check{a}}$ & $\mid \begin{array}{l}\infty \\
\infty \\
\infty\end{array}$ & 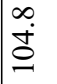 & $\stackrel{\varrho}{\varrho}$ & $\underset{b}{\sigma}$ \\
\hline
\end{tabular}




\begin{tabular}{|c|c|c|c|c|c|c|c|c|c|c|c|c|c|c|c|c|c|c|c|c|c|c|c|}
\hline & & $\vec{\Delta}$ & 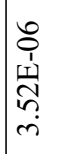 & $\begin{array}{l}8 \\
0 \\
1 \\
1 \\
n \\
n \\
m \\
m\end{array}$ & 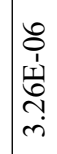 & 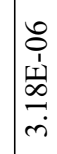 & 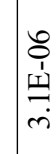 & 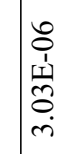 & 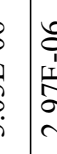 & 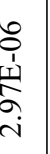 & 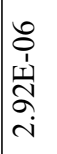 & 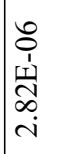 & 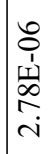 & 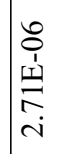 & $\mid \begin{array}{l}8 \\
0 \\
1 \\
1 \\
0 \\
0 \\
i\end{array}$ & 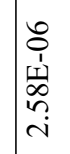 & 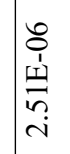 & $\begin{array}{l}0 \\
0 \\
1 \\
1 \\
\infty \\
+ \\
\dot{1} \\
i\end{array}$ & \begin{tabular}{l}
8 \\
0 \\
1 \\
1 \\
\multirow{1}{5}{} \\
\multirow{2}{*}{} \\
$i$
\end{tabular} & 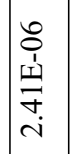 & $\begin{array}{c}0 \\
o \\
o \\
11 \\
n \\
n \\
i \\
i\end{array}$ & 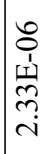 & $\begin{array}{c}0 \\
0 \\
1 \\
11 \\
0 \\
0 \\
-1 \\
-1\end{array}$ \\
\hline & & $\stackrel{\circ}{\infty}$ & 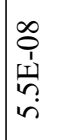 & 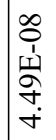 & $\begin{array}{c}\infty \\
0 \\
1 \\
1 \\
\infty \\
\dot{m} \\
\dot{n}\end{array}$ & 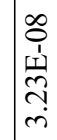 & 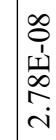 & 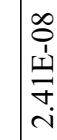 & i & 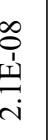 & 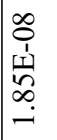 & 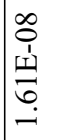 & 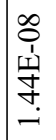 & $\begin{array}{l}\infty \\
0 \\
0 \\
1 \\
\infty \\
\infty \\
\\
-1\end{array}$ & 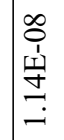 & 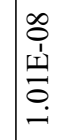 & $\begin{array}{l}\text { oे } \\
\text { İ } \\
\text { İ }\end{array}$ & $\begin{array}{l}\text { ô } \\
\text { 1 } \\
\text { 年 } \\
\infty \\
\infty\end{array}$ & 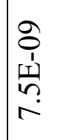 & $\mid \begin{array}{c}2 \\
\hat{1} \\
\omega \\
\infty \\
0 \\
0 \\
0\end{array}$ & 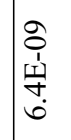 & 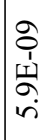 & 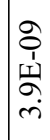 \\
\hline & & $\dot{D}$ & 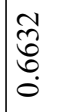 & $\begin{array}{l}\text { ते } \\
\text { ర્ટ } \\
0\end{array}$ & 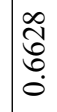 & 兽 & 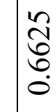 & స్ & ?ְ & 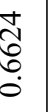 & 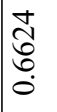 & 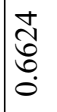 & 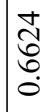 & 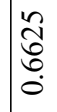 & 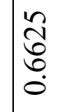 & 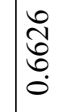 & $\begin{array}{l}\text { İं } \\
\text { ర్ } \\
\stackrel{0}{0}\end{array}$ & $\begin{array}{l}\hat{\widehat{J}} \\
0 \\
0 \\
0\end{array}$ & $\begin{array}{l}\hat{\widehat{V}} \\
\stackrel{0}{0} \\
\stackrel{0}{0}\end{array}$ & $\mid \begin{array}{l}\infty \\
\mathbb{U} \\
0 \\
0 \\
0\end{array}$ & त्ञ్రి & $\underset{\mathscr{0}}{0}$ & 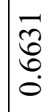 \\
\hline & & $\mathscr{J}$ & $\begin{array}{l}\overrightarrow{0} \\
\hat{n}\end{array}$ & $\frac{\widetilde{N}}{\frac{\infty}{m}}$ & $\begin{array}{l}\infty \\
\tilde{\lambda} \\
\tilde{\lambda}\end{array}$ & 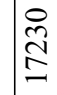 & 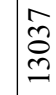 & 离 & & 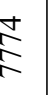 & $\frac{F}{\sigma}$ & 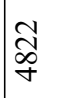 & 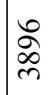 & $\frac{0}{m}$ & & ڤे & $\stackrel{\cong}{\Xi}$ & 它 & 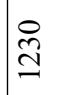 & $\underset{O}{\mathbb{O}}$ & $\frac{0}{a}$ & $\stackrel{\partial े}{\stackrel{D}{~}}$ & $\overrightarrow{\vec{q}}$ \\
\hline & & t & 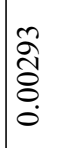 & 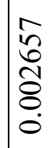 & 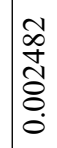 & 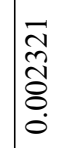 & $\begin{array}{c}\tilde{\infty} \\
\widetilde{\widetilde{\delta}} \\
0 \\
0\end{array}$ & 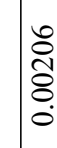 & $\frac{9}{1}$ & $\begin{array}{l}\frac{a}{\sigma} \\
\frac{a}{0} \\
0 \\
0\end{array}$ & $\mid$\begin{tabular}{l}
4 \\
$\infty$ \\
$\infty$ \\
\hdashline \\
0 \\
0 \\
0
\end{tabular} & $\frac{t}{\stackrel{8}{8}}$ & 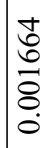 & $\mid$\begin{tabular}{l}
2 \\
$\hat{n}$ \\
\hdashline \\
8 \\
0 \\
0
\end{tabular} & 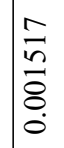 & $\mid \begin{array}{l}f \\
\mathscr{8} \\
\dot{8}\end{array}$ & \begin{tabular}{l}
0 \\
2 \\
$n$ \\
\hdashline \\
8 \\
0 \\
0
\end{tabular} & $\mid \begin{array}{l}\Delta \\
\stackrel{0}{m} \\
\stackrel{8}{8} \\
\dot{0}\end{array}$ & $\begin{array}{l}0 \\
\stackrel{0}{0} \\
\stackrel{1}{0} \\
8 \\
0 \\
0\end{array}$ & 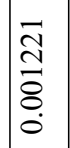 & 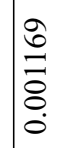 & 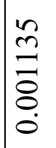 & $\begin{array}{l}\text { } \\
\hat{2} \\
8 \\
8 \\
0 \\
0\end{array}$ \\
\hline & & $\approx$ & స్ & $\frac{n}{m}$ & ఫิ & ते & $\underset{\sim}{\Delta}$ & $\stackrel{0}{\stackrel{0}{*}}$ & & $\begin{array}{l}\text { त्र } \\
\text { ते }\end{array}$ & 守 & 官 & సે & $\tilde{\overbrace{}}$ & $\underset{\mathbb{N}}{\stackrel{N}{N}}$ & 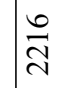 & $\frac{a}{d}$ & $\stackrel{\varrho}{\underset{\sim}{二}}$ & 尺े & $\widehat{\widehat{\sim}}$ & 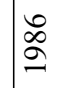 & $\stackrel{\infty}{2}$ & $\stackrel{6}{2}$ \\
\hline שै & $\stackrel{\oplus}{I}$ & 总 & $\hat{\circ}$ & nִ & ڤo & $\begin{array}{l}\infty \\
\infty \\
0 \\
0\end{array}$ & 宬 & $\ddot{0}$ & & "n & $\bar{n}$ & $\stackrel{\text { fof }}{0}$ & సి & חొ & חె? & 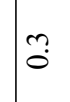 & $\tilde{\hat{o}}$ & 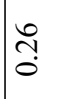 & ִָ & $\hat{\tilde{O}}$ & $\stackrel{n}{\tilde{o}}$ & $\stackrel{+}{\tilde{O}}$ & ڤ్ \\
\hline $\begin{array}{l} \pm \\
\frac{\vec{\omega}}{\omega} \\
\frac{\Xi}{\Xi}\end{array}$ & 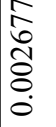 & $\vec{z}$ & $\begin{array}{l}\hat{n} \\
6 \\
\dot{q}\end{array}$ & $\overrightarrow{\tilde{\sigma}}$ & $\frac{\infty}{m}$ & $\stackrel{\oplus}{=}$ & 音 & & & $\begin{array}{c}n \\
n \\
\infty \\
\infty\end{array}$ & $\underset{\infty}{\infty}$ & O̊. & 触 & $\stackrel{1}{6}$ & $\begin{array}{l}\infty \\
\infty \\
i n \\
\text { in }\end{array}$ & $\begin{array}{l}\vec{\forall} \\
\dot{n}\end{array}$ & $\mid \begin{array}{l}\stackrel{\infty}{\stackrel{f}{+}} \\
\end{array}$ & $\begin{array}{l}\tilde{D} \\
\dot{\sim}\end{array}$ & $\underset{+}{\hat{\sigma}}$ & $\begin{array}{l}n \\
n \\
+\end{array}$ & $\stackrel{+}{+}$ & ले & $\begin{array}{l}\hat{\infty} \\
\dot{n}\end{array}$ \\
\hline $\begin{array}{l}\stackrel{\ddot{q}}{\Delta} \\
\frac{\overrightarrow{0}}{\Xi}\end{array}$ & $\vec{\infty}$ & 薑 & 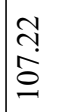 & $\begin{array}{l}n \\
0 \\
n \\
-1\end{array}$ & $\stackrel{2}{0}$ & $\underset{\infty}{\infty}$ & 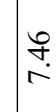 & 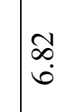 & & $\hat{\widehat{\sigma}}$ & $\begin{array}{l}\text { ta } \\
\text { in }\end{array}$ & $\vec{\sigma}$ & $\stackrel{\sim}{\dot{f}}$ & 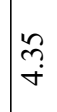 & $\stackrel{n}{\stackrel{r}{*}}$ & $\stackrel{શ}{\hat{r}}$ & $\underset{\dot{r}}{\stackrel{J}{*}}$ & $\hat{m}$ & $\stackrel{m}{m}$ & $\vec{m}$ & $\hat{ָ}$ & ले & $\underset{\sim}{\sigma}$ \\
\hline$\underset{\Xi}{\underline{G}}$ & 志 & 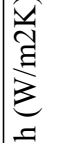 & 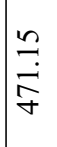 & $\begin{array}{l}\infty \\
\infty \\
\stackrel{2}{\beth}\end{array}$ & 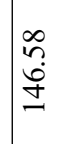 & $\mid \begin{array}{l}\infty \\
\stackrel{n}{m} \\
\end{array}$ & 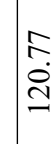 & $\cong$ & & $\begin{array}{l}\text { के } \\
\text { oे }\end{array}$ & 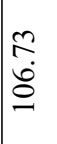 & 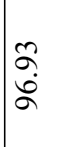 & 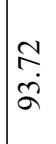 & $\ddot{\infty}$ & $\vec{m}$ & $\begin{array}{l}\bar{n} \\
\check{\infty} \\
\infty\end{array}$ & 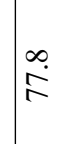 & 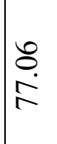 & în. & $\begin{array}{l}\alpha \\
\alpha \\
\alpha\end{array}$ & $\begin{array}{l}\infty \\
\infty \\
\dot{T}\end{array}$ & $\stackrel{1}{2}$ & $\begin{array}{l}\hat{y} \\
\infty \\
\infty \\
0\end{array}$ \\
\hline$\sum_{\text {贡 }}$ & ชై & 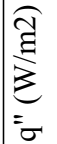 & 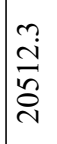 & $\begin{array}{l}n \\
\swarrow \\
2 \\
2 \\
2\end{array}$ & 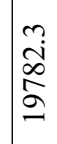 & $\begin{array}{l}\overrightarrow{4} \\
0 \\
0 \\
0\end{array}$ & 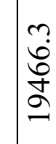 & ને & $\begin{array}{l}\grave{a} \\
\grave{a}\end{array}$ & 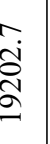 & 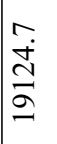 & 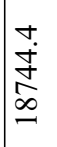 & $\begin{array}{l}\infty \\
0 \\
\infty \\
\infty \\
\infty \\
\infty\end{array}$ & 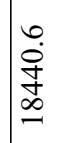 & $\begin{array}{l}n \\
\tilde{2} \\
\infty \\
\infty \\
\infty\end{array}$ & $\mid \begin{array}{l}n \\
\overline{0} \\
2 \\
\end{array}$ & 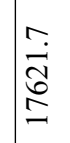 & $\begin{array}{l}n \\
\widehat{\widehat{\delta}} \\
\stackrel{0}{=}\end{array}$ & 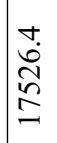 & $\begin{array}{l}n \\
\tilde{I} \\
\underline{I} \\
-\underline{-}\end{array}$ & $\begin{array}{l}\mathbf{0} \\
\underset{J}{\mathbf{C}} \\
\underline{I}\end{array}$ & $\underset{\stackrel{\vec{I}}{\stackrel{I}{I}}}{\vec{I}}$ & 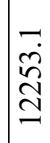 \\
\hline 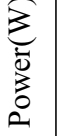 & $\stackrel{\infty}{\stackrel{\sim}{\Omega}}$ & $\stackrel{0}{F}$ & 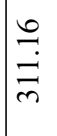 & $\begin{array}{l}\hat{a} \\
\text { in } \\
\tilde{m}\end{array}$ & $\begin{array}{l}\infty \\
m \\
\dot{\sim} \\
n \\
m\end{array}$ & $\begin{array}{l}\dot{d} \\
\stackrel{i}{n} \\
m\end{array}$ & 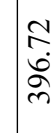 & $\frac{8}{\frac{8}{7}}$ & $\begin{array}{l}y \\
y \\
\alpha \\
y \\
y\end{array}$ & 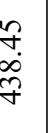 & 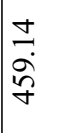 & $\begin{array}{l}\hat{n} \\
\stackrel{\gamma}{\gamma}\end{array}$ & $\stackrel{\hat{a}}{\stackrel{\alpha}{\alpha}}$ & $\begin{array}{l}\bar{\infty} \\
\dot{\vec{n}}\end{array}$ & 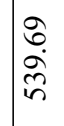 & $\begin{array}{l}\bar{n} \\
\ddot{n} \\
i n\end{array}$ & $\begin{array}{l}\bar{n} \\
\infty \\
\infty \\
i n\end{array}$ & 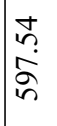 & $\begin{array}{l}\bar{n} \\
\varnothing \\
6\end{array}$ & $\mid \begin{array}{l}\infty \\
\tilde{n} \\
\tilde{n} \\
\tilde{\sigma}\end{array}$ & \begin{tabular}{l} 
t \\
$\dot{+}$ \\
\multirow{6}{6}{}
\end{tabular} & 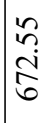 & \begin{tabular}{l}
$\mathcal{F}$ \\
\multirow{2}{*}{} \\
$\infty$ \\
$\infty$ \\
0
\end{tabular} \\
\hline 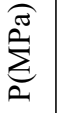 & $\stackrel{\infty}{\stackrel{\infty}{0}}$ & $\underline{3}$ & 芦 & $\begin{array}{l}n \\
\tilde{f} \\
f \\
f\end{array}$ & $\begin{array}{l}\tilde{m} \\
\stackrel{m}{a} \\
\dot{b}\end{array}$ & $\begin{array}{l}\hat{\sigma} \\
\dot{\mathbb{t}} \\
\tilde{n}\end{array}$ & $\begin{array}{l}\bar{a} \\
\bar{n} \\
\tilde{n}\end{array}$ & $\begin{array}{l}\hat{-} \\
\text { in } \\
\infty \\
n\end{array}$ & $\frac{2}{6}$ & $\begin{array}{l}n \\
\stackrel{n}{3} \\
0\end{array}$ & 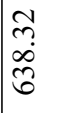 & \begin{tabular}{l}
$n$ \\
\multirow{i}{\delta}{} \\
$\sigma$
\end{tabular} & $\frac{n}{\operatorname{d}}$ & $\begin{array}{l}0 \\
\infty \\
\\
\end{array}$ & $\begin{array}{l}\hat{n} \\
\hat{n} \\
\hat{n}\end{array}$ & 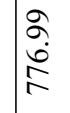 & $\begin{array}{l}\overrightarrow{0} \\
\ddot{\hat{n}} \\
\infty\end{array}$ & 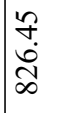 & 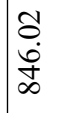 & $\begin{array}{l}\tilde{\alpha} \\
\dot{d} \\
\infty\end{array}$ & $\begin{array}{l}\stackrel{J}{\sim} \\
\infty \\
\infty \\
\infty\end{array}$ & $\begin{array}{l}\vec{n} \\
\stackrel{2}{\infty} \\
\infty\end{array}$ & $\begin{array}{l}2 \\
\bar{\delta} \\
\infty\end{array}$ \\
\hline Ü & $i$ & $\stackrel{2}{a}$ & $\sim$ & $\vec{\infty}$ & $\overline{ \pm}$ & กิ. & तु. & 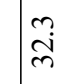 & $\underset{\alpha}{\alpha}$ & ma & $\stackrel{\nabla}{\dot{f}}$ & ti. & $\begin{array}{l}n \\
0 \\
0\end{array}$ & in & $\begin{array}{l}n \\
\infty \\
\infty \\
\infty\end{array}$ & $\begin{array}{l}\bullet \\
\stackrel{0}{+}\end{array}$ & $\ddot{\infty}$ & $\hat{\varnothing}$ & & $\mid \begin{array}{l}\infty \\
\infty \\
\infty \\
\sigma\end{array}$ & 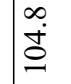 & $\stackrel{\partial}{\varrho}$ & ف․ \\
\hline
\end{tabular}




\begin{tabular}{|c|c|c|c|c|c|c|c|c|c|c|c|c|c|c|c|c|c|c|c|c|c|c|c|c|}
\hline & & $\vec{\Delta}$ & 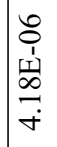 & $\begin{array}{c}0 \\
o \\
1 \\
1 \\
n \\
2 \\
m \\
m\end{array}$ & 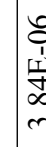 & & 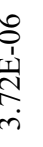 & 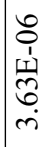 & $\begin{array}{l}8 \\
0 \\
1 \\
1 \\
n \\
n \\
n \\
n\end{array}$ & 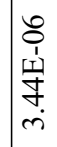 & $\begin{array}{l}\stackrel{8}{8} \\
1 \\
1 \\
\\
\\
\end{array}$ & 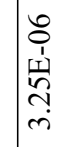 & $\begin{array}{l}0 \\
\frac{1}{1} \\
\frac{1}{2} \\
\\
\end{array}$ & 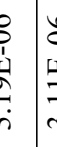 & 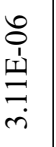 & 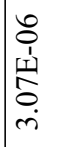 & 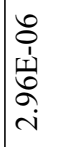 & $\begin{array}{l}0 \\
0 \\
1 \\
1 \\
\infty \\
\infty \\
i \\
i\end{array}$ & 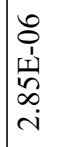 & $\begin{array}{c}0 \\
0 \\
1 \\
1 \\
\infty \\
\infty \\
i\end{array}$ & 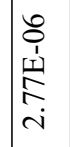 & 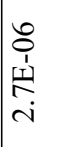 & \begin{tabular}{l}
0 \\
0 \\
1 \\
1 \\
\multirow{1}{0}{} \\
0 \\
0 \\
$i$
\end{tabular} & 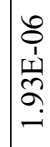 \\
\hline & & $\stackrel{\oplus}{\infty}$ & 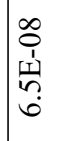 & 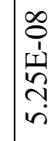 & 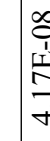 & & 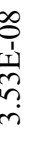 & $\begin{array}{l}\infty \\
0 \\
1 \\
1 \\
\infty \\
\infty \\
i \\
ن\end{array}$ & 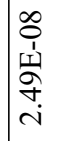 & $\begin{array}{l}\infty \\
0 \\
0 \\
1 \\
\\
0 \\
0 \\
i\end{array}$ & \begin{tabular}{l}
$\infty$ \\
0 \\
0 \\
\multicolumn{1}{|c}{} \\
$\infty$ \\
$\infty$ \\
-1 \\
-1
\end{tabular} & 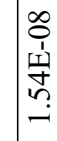 & 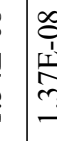 & 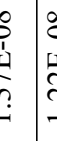 & $\begin{array}{l}\infty \\
0 \\
0 \\
1 \\
\\
\\
-\end{array}$ & 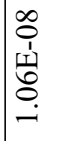 & 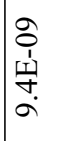 & 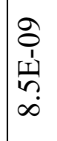 & 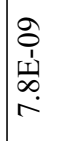 & $\begin{array}{c}2 \\
\hat{1} \\
1 \\
\underline{1} \\
\hat{0}\end{array} \mid$ & 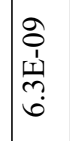 & $\begin{array}{l}2 \\
\hat{1} \\
i \\
\infty \\
\infty \\
\dot{n} \\
\text { in }\end{array}$ & $\begin{array}{l}\text { Oे } \\
\text { 离 } \\
\text { m. } \\
\text { in }\end{array}$ & 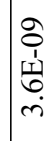 \\
\hline & & $\ddot{2}$ & \begin{tabular}{l} 
त̂ \\
\multirow{6}{0}{} \\
0 \\
0
\end{tabular} & 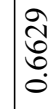 & $\begin{array}{l}\text { है } \\
\text { है } \\
c\end{array}$ & : & 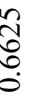 & $\begin{array}{l}\text { îे } \\
\text { ర్ర } \\
\stackrel{0}{0}\end{array}$ & 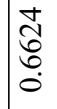 & 志 & 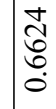 & 芯 & 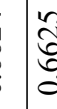 & $\begin{array}{l}0 \\
0 \\
: \\
:\end{array}$ & 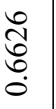 & 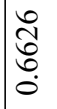 & $\begin{array}{l}\hat{\widehat{J}} \\
\stackrel{0}{0} \\
\stackrel{0}{0}\end{array}$ & 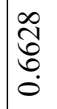 & त्ठ઼े & $\begin{array}{l}n \\
0 \\
0 \\
0\end{array}$ & $\begin{array}{l}\overrightarrow{\tilde{\delta}} \\
\dot{0} \\
\dot{0}\end{array}$ & $\begin{array}{l}\text { त̂ } \\
\widehat{0} \\
0 \\
0 \\
0\end{array}$ & $\begin{array}{l}m \\
\widehat{\delta} \\
0 \\
0 \\
0\end{array}$ & 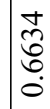 \\
\hline & & $\overline{0}$ & $\frac{\bar{\pi}}{\tilde{n}}$ & $\frac{7}{8}$ & 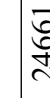 & & 必 & ڤે & ఫू & $\tilde{\partial}$ & $\underset{\substack{n \\
n}}{n}$ & §ิ & 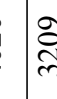 & Sh & $\stackrel{\substack{n \\
i}}{n}$ & @ & ஜூర్ర & 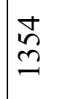 & $\stackrel{\stackrel{n}{q}}{=}$ & $\tilde{\sigma}$ & $\bar{\Omega}$ & $\begin{array}{l}\infty \\
0 \\
0\end{array}$ & in & 勇 \\
\hline & & $t$ & 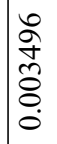 & 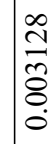 & $\begin{array}{l}\infty \\
\infty \\
\infty \\
\check{c} \\
c \\
c\end{array}$ & & 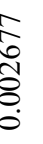 & 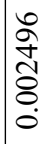 & 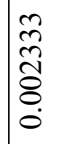 & 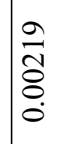 & 离 & సे & $\mid \begin{array}{l}n \\
\infty \\
\delta \\
\vdots\end{array}$ & 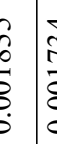 & 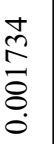 & 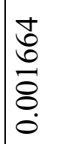 & 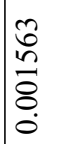 & $\frac{\infty}{+}$ & 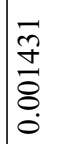 & $\begin{array}{l}0 \\
2 \\
0 \\
0 \\
8 \\
0 \\
0\end{array}$ & 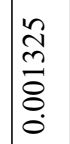 & 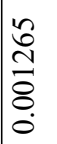 & $\begin{array}{l}\vec{\Xi} \\
\stackrel{\Xi}{\Xi} \\
\stackrel{8}{0}\end{array}$ & $\begin{array}{l}\hat{0} \\
\infty \\
8 \\
0 \\
0 \\
0\end{array}$ \\
\hline & & $\ddot{\sim}$ & 离 & $\frac{\infty}{\infty}$ & $\underset{ल}{\Xi}$ & & $\begin{array}{c}n \\
\stackrel{n}{\infty} \\
\sim\end{array}$ & 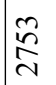 & 胥 & 染 & $\underset{\sim}{\sim}$ & $\underset{\sim}{\stackrel{\sim}{\sim}}$ & હे & & $\overrightarrow{\widetilde{\Omega}}$ & $\stackrel{\infty}{\underset{\sim}{\sim}}$ & $\underset{\vec{v}}{\stackrel{\partial}{v}}$ & $\tilde{\overbrace{}}$ & 陔 & $\stackrel{\infty}{2}$ & $\stackrel{+}{2}$ & $\underset{\infty}{\mathbb{\infty}}$ & $\stackrel{\widetilde{\infty}}{\infty}$ & $\stackrel{8}{\infty}$ \\
\hline تే & $\stackrel{0}{1}$ & 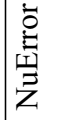 & $\exists$ & $\stackrel{n}{n}$ & $\tilde{\varepsilon}$ & & $\begin{array}{l}1 \\
\infty \\
0 \\
0\end{array}$ & 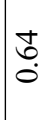 & $\hat{n}$ & $\tilde{n}$ & $\stackrel{n}{f}$ & I. & â & $?$ & m? & के & సิ & $\hat{\widehat{n}}$ & 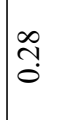 & $\begin{array}{l}\tilde{y} \\
\tilde{o}\end{array}$ & $\stackrel{n}{n}$ & İ & ָ̊. & $\overline{0}$ \\
\hline $\begin{array}{l}\frac{ \pm}{\sigma} \\
\frac{ \pm}{ \pm} \\
\Xi\end{array}$ & $\frac{\tilde{2}}{2}$ & $\vec{z}$ & 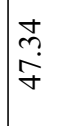 & ల్రి & ๙ & & S. & f̊. & $\mid \begin{array}{l}\stackrel{+}{ } \\
\infty \\
\infty\end{array}$ & $\stackrel{\infty}{\sim}$ & $?$ & $\tilde{6}$ & $\frac{9}{6}$ & $\begin{array}{c}n \\
0 \\
0\end{array}$ & $\begin{array}{l}n \\
\stackrel{n}{n} \\
i n\end{array}$ & 苟 & $\begin{array}{l}0 \\
\dot{n}\end{array}$ & $\begin{array}{l}\mathscr{D} \\
\dot{+} \\
+\end{array}$ & $\stackrel{\vec{\gamma}}{\vec{r}}$ & $\begin{array}{l}0 \\
\dot{+}\end{array}$ & $\vec{n}$ & $\begin{array}{l}\infty \\
m \\
\sim \\
+\end{array}$ & 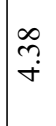 & 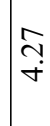 \\
\hline 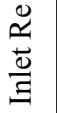 & $\frac{m}{\tilde{m}}$ & 寄 & $\begin{array}{l}\hat{o} \\
\text { i } \\
ٍ\end{array}$ & $\begin{array}{l}0 \\
\stackrel{1}{n} \\
\end{array}$ & 용 & & $\begin{array}{l}\vec{n} \\
\infty\end{array}$ & $\stackrel{8}{\circ}$ & 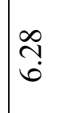 & $\vec{n}$ & $\vec{\forall}$ & $\stackrel{5}{+}$ & $\stackrel{0}{+}$ & $\begin{array}{l}\text { f } \\
\text { f }\end{array}$ & $\underset{+}{\widetilde{\sim}}$ & $\stackrel{n}{\stackrel{\sim}{+}}$ & $\begin{array}{l}\infty \\
\infty \\
\text { nे }\end{array}$ & $\begin{array}{r}3 \\
\dot{m}\end{array}$ & 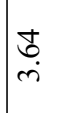 & $\begin{array}{c}\hat{6} \\
\dot{m}\end{array}$ & $\ddot{r}$ & $\begin{array}{l}\hat{b} \\
\dot{r}\end{array}$ & $\begin{array}{l}\infty \\
\infty \\
\infty \\
\end{array}$ & तु \\
\hline$\underset{\Xi}{\stackrel{\Xi}{\Xi}}$ & 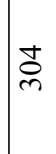 & 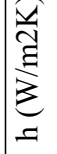 & $\mid \begin{array}{l}n \\
\tilde{n} \\
\infty \\
\dot{\infty}\end{array}$ & ڤ̂̃ & 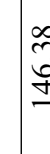 & & 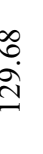 & $\stackrel{n}{\stackrel{2}{\Xi}}$ & సે & $\begin{array}{l}\overrightarrow{0} \\
\dot{0} \\
\underline{0}\end{array}$ & 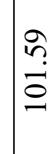 & $\begin{array}{l}\stackrel{f}{+} \\
\text { ๙ু }\end{array}$ & $\frac{\bar{\sigma}}{\sigma}$ & Ș & $\begin{array}{l}\stackrel{0}{\infty} \\
\stackrel{\infty}{\infty}\end{array}$ & $\underset{\infty}{\infty}$ & $\begin{array}{l}\hat{n} \\
\infty \\
\infty\end{array}$ & $\begin{array}{l}\overrightarrow{0} \\
\dot{\infty}\end{array}$ & $\underset{\infty}{\vec{\infty}}$ & $\begin{array}{l}n \\
\tilde{s} \\
\infty\end{array}$ & 宫. & $\begin{array}{l}\infty \\
\infty \\
2\end{array}$ & $\frac{\infty}{\infty}$ & $\begin{array}{l}\infty \\
\infty \\
\infty \\
\infty\end{array}$ \\
\hline$\sum_{\text {至 }}$ & ָู & $\begin{array}{l}\text { đี } \\
\text { है } \\
= \\
=\end{array}$ & 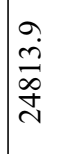 & $\begin{array}{l}0 \\
0 \\
o \\
d \\
d \\
d\end{array}$ & $\begin{array}{l}\infty \\
\approx \\
\approx \\
\approx\end{array}$ & & 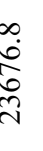 & 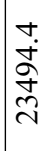 & $\begin{array}{l}-\underset{\dot{D}}{\infty} \\
\tilde{N} \\
\widetilde{\sim}\end{array}$ & $\begin{array}{l}\overrightarrow{\dot{D}} \\
\infty \\
\stackrel{0}{0}\end{array}$ & 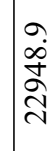 & 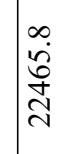 & $\mid \begin{array}{l}\hat{\imath} \\
\tilde{z} \\
\tilde{z}\end{array}$ & 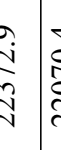 & 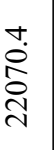 & 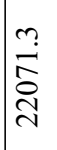 & $\begin{array}{l}0 \\
\stackrel{+}{4} \\
\stackrel{\sim}{\sim} \\
\sim\end{array}$ & $\begin{array}{l}0 \\
\dot{0} \\
\stackrel{\bar{\sigma}}{二}\end{array}$ & 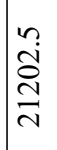 & $\begin{array}{l}\sim \\
\tilde{D} \\
\infty \\
\stackrel{0}{\sim} \\
\sim\end{array}$ & $\begin{array}{l}0 \\
\tilde{\alpha} \\
\hat{\sigma}\end{array}$ & 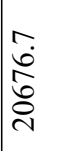 & 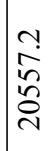 & $\mid \begin{array}{l}+ \\
\stackrel{8}{2} \\
\stackrel{a}{I}\end{array}$ \\
\hline $\begin{array}{l}3 \\
0 \\
0 \\
0 \\
0 \\
0 \\
0\end{array}$ & సે & 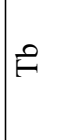 & $\begin{array}{l}\infty \\
\sim \\
i \\
n\end{array}$ & $\begin{array}{l}\hat{\sigma} \\
\infty \\
m \\
m\end{array}$ & लె & & 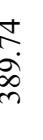 & 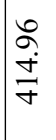 & $\begin{array}{l}\hat{\sigma} \\
\hat{\sigma} \\
\stackrel{\gamma}{\gamma}\end{array}$ & 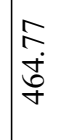 & $\begin{array}{l}\infty \\
\text { ?. } \\
\stackrel{\infty}{\infty} \\
\infty\end{array}$ & $\begin{array}{l}\stackrel{0}{0} \\
\stackrel{n}{n} \\
i n\end{array}$ & $\begin{array}{l}3 \\
0 \\
0 \\
n\end{array}$ & $\begin{array}{l}b \\
b \\
n\end{array}$ & $\frac{\dot{\vec{D}}}{\stackrel{\vec{D}}{n}}$ & 象 & $\mid \begin{array}{l}\tilde{2} \\
\infty \\
\infty \\
0 \\
0\end{array}$ & $\stackrel{0}{\stackrel{0}{3}}$ & $\begin{array}{l}\infty \\
\infty \\
\tilde{\sigma}\end{array}$ & \begin{tabular}{l}
7 \\
\multirow{6}{0}{} \\
6 \\
6
\end{tabular} & $\begin{array}{l}\sigma \\
\alpha \\
\alpha \\
\sigma\end{array}$ & $\frac{\sim}{\mathbb{N}}$ & $\begin{array}{l}\Re \\
\\
\stackrel{\sim}{\sim}\end{array}$ & ָ̊ \\
\hline$\sum_{0=1}^{\pi}$ & $\frac{\infty}{0}$ & 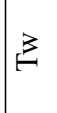 & 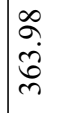 & 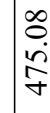 & $\begin{array}{l}\text { 辛 } \\
\text { in }\end{array}$ & & $\begin{array}{c}\text { N } \\
\text { in } \\
\text { in }\end{array}$ & $\begin{array}{l}\bar{\sigma} \\
\dot{ \pm} \\
\sigma\end{array}$ & $\stackrel{\infty}{\stackrel{0}{0}}$ & \begin{tabular}{l}
$n$ \\
\multirow{r}{*}{} \\
$\ddot{\infty}$ \\
$\tilde{\delta}$
\end{tabular} & $\begin{array}{l}\text { ते } \\
\text { in } \\
\text { ॠ }\end{array}$ & $\begin{array}{l}\infty \\
\stackrel{0}{+} \\
\dot{0} \\
i\end{array}$ & 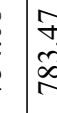 & 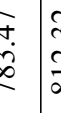 & $\begin{array}{l}\underset{N}{\tilde{N}} \\
\underset{\infty}{\infty}\end{array}$ & $\begin{array}{l}= \\
\infty \\
\infty \\
\infty\end{array}$ & 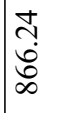 & 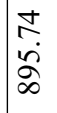 & $\begin{array}{l}\tilde{m} \\
\infty \\
\alpha\end{array}$ & $\begin{array}{l}\tilde{a} \\
\tilde{a} \\
\tilde{\sigma}\end{array}$ & $\begin{array}{l}\infty \\
\infty \\
\alpha \\
\alpha\end{array}$ & $\begin{array}{l}\infty \\
\stackrel{\infty}{0} \\
\stackrel{0}{\infty} \\
2\end{array}$ & $\begin{array}{l}\text { ते } \\
\text { బे }\end{array}$ & $\begin{array}{l}\tilde{m} \\
\stackrel{+}{*} \\
\sigma\end{array}$ \\
\hline 离 & $\hat{\imath}$ & 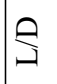 & $N$ & $\infty$ & $\exists$ & & ֶָ. & సֶ. & $\stackrel{m}{i}$ & $\begin{array}{c}m \\
\infty \\
\infty\end{array}$ & $\stackrel{+}{+}$ & 芦 & $\begin{array}{l}n \\
\text { ?n } \\
\text { ñ }\end{array}$ & $\begin{array}{l}? \\
? \\
?\end{array}$ & הֶ & $\begin{array}{l}n \\
\infty \\
\infty\end{array}$ & $\begin{array}{l}\stackrel{0}{*} \\
\stackrel{+}{I}\end{array}$ & $\ddot{\infty}$ & $\hat{\infty}$ & $\hat{a}$ & $\mid \begin{array}{l}\infty \\
\infty \\
\infty\end{array}$ & 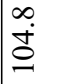 & 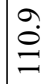 & $\stackrel{\vec{b}}{=}$ \\
\hline
\end{tabular}




\begin{tabular}{|c|c|c|c|c|c|c|c|c|c|c|c|c|c|c|c|c|c|c|c|c|c|c|c|c|}
\hline & & $\vec{\Delta}$ & 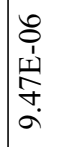 & & 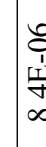 & & 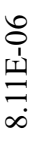 & 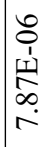 & 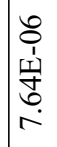 & 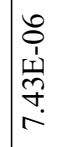 & $\mid \begin{array}{l}\mathscr{8} \\
0 \\
1 \\
1 \\
\\
\end{array}$ & 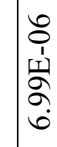 & $\mid \begin{array}{l}0 \\
0 \\
1 \\
1 \\
0 \\
0 \\
0 \\
0\end{array}$ & $\begin{array}{l}0 \\
0 \\
\vdots \\
0 \\
0 \\
0 \\
0\end{array}$ & $\begin{array}{l}0 \\
0 \\
1 \\
1 \\
\\
0 \\
0 \\
0\end{array}$ & $\mid \begin{array}{l}0 \\
0 \\
1 \\
1 \\
\\
\\
0 \\
0\end{array}$ & 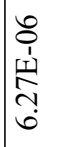 & $\begin{array}{l}8 \\
0 \\
1 \\
1 \\
0 \\
0 \\
0 \\
6\end{array}$ & $\begin{array}{c}0 \\
0 \\
\text { t1 } \\
\text { th }\end{array}$ & \begin{tabular}{l}
0 \\
0 \\
1 \\
1 \\
\multicolumn{1}{|c}{} \\
$\infty$ \\
$\dot{r}$ \\
$\dot{r}$
\end{tabular} & 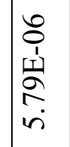 & 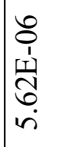 & $\begin{array}{l}0 \\
0 \\
1 \\
1 \\
n \\
n \\
n \\
n\end{array}$ & 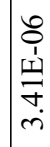 \\
\hline & & $\stackrel{\oplus}{\infty}$ & $\mid \begin{array}{l}\hat{0} \\
\hat{1} \\
\underline{1} \\
\tilde{n} \\
\tilde{n}\end{array}$ & \begin{tabular}{l}
$\hat{\sigma}$ \\
$\hat{1}$ \\
\multicolumn{1}{c}{} \\
$\hat{6}$ \\
in
\end{tabular} & 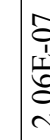 & & 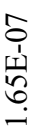 & $\begin{array}{l}\hat{9} \\
\hat{1} \\
\text { Wh} \\
\\
\end{array}$ & 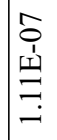 & 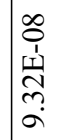 & 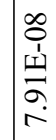 & $\begin{array}{l}\infty \\
0 \\
0 \\
1 \\
\\
6 \\
0 \\
0\end{array}$ & 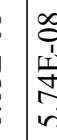 & $\begin{array}{l}0 \\
1 \\
\vdots \\
\vdots \\
\dot{0} \\
\dot{0}\end{array}$ & \begin{tabular}{l}
$\infty$ \\
0 \\
0 \\
1 \\
+ \\
\multirow{2}{1}{} \\
$\dot{+}$
\end{tabular} & 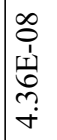 & 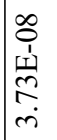 & 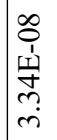 & \begin{tabular}{|l}
$\infty$ \\
0 \\
1 \\
离
\end{tabular} & \begin{tabular}{|c|}
$\infty$ \\
0 \\
0 \\
1 \\
\\
0 \\
0 \\
$i$
\end{tabular} & 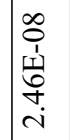 & $\begin{array}{c}\infty \\
0 \\
1 \\
1 \\
\infty \\
\infty \\
\\
\end{array}$ & 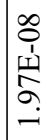 & 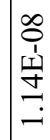 \\
\hline & & 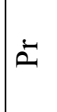 & $\begin{array}{l}\overrightarrow{\widehat{\sigma}} \\
\stackrel{0}{0} \\
\stackrel{0}{0}\end{array}$ & 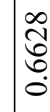 & $\begin{array}{l}\text { है } \\
\text { है } \\
c\end{array}$ & : & 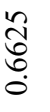 & 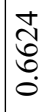 & 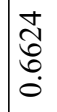 & 志 & 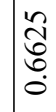 & : & 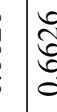 & \begin{tabular}{l|l}
0 \\
0 \\
0 \\
0 \\
0
\end{tabular} & 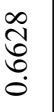 & 空 & $\begin{array}{l}\widetilde{0} \\
0 \\
0 \\
0\end{array}$ & $\begin{array}{l}\overrightarrow{\widehat{0}} \\
\stackrel{0}{0} \\
0\end{array}$ & $\begin{array}{l}m \\
\widehat{6} \\
0 \\
0 \\
0\end{array}$ & $\begin{array}{l} \pm \\
0 \\
0 \\
0 \\
0\end{array}$ & $\begin{array}{l}n \\
\tilde{\delta} \\
0 \\
0 \\
0\end{array}$ & $\begin{array}{l}\hat{\tilde{b}} \\
\mathbb{0} \\
0 \\
0\end{array}$ & $\begin{array}{l}\infty \\
\widetilde{0} \\
0 \\
0 \\
0\end{array}$ & $\begin{array}{l}\text { ते } \\
\text { ర్ } \\
\stackrel{0}{0}\end{array}$ \\
\hline & & $\mathscr{J}$ & $\begin{array}{l}2 \\
\approx \\
0 \\
0 \\
0\end{array}$ & 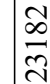 & જી & & $\frac{\infty}{\infty}$ & $\frac{9}{6}$ & 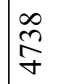 & $\underset{\mathcal{N}}{\tilde{y}}$ & त̂ & $\begin{array}{l}\overline{0} \\
\infty\end{array}$ & $\stackrel{\overbrace{}}{7}$ & $\begin{array}{l}0 \\
\text { D } \\
\text { y }\end{array}$ & ઠ̊ & $\stackrel{\wp}{\infty}$ & $\underbrace{\infty}_{0}$ & 怘 & 得 & 令 & 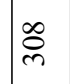 & $\vec{\curvearrowright}$ & $\overline{\vec{\lambda}}$ & $\cong$ \\
\hline & & t & 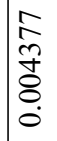 & $\begin{array}{l}0 \\
\text { ô } \\
\hat{\delta} \\
0 \\
0\end{array}$ & 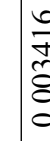 & & 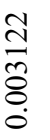 & $\begin{array}{l}\vec{\infty} \\
\infty \\
\widetilde{o} \\
\tilde{O} \\
\dot{0}\end{array}$ & 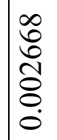 & $\begin{array}{l}n \\
\infty \\
\vdots \\
\tilde{\delta} \\
\vdots \\
0 \\
0\end{array}$ & 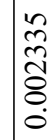 & 官 & 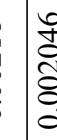 & 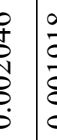 & $\begin{array}{l}\frac{\infty}{a} \\
\frac{a}{a} \\
\dot{a} \\
\dot{0}\end{array}$ & $\frac{\infty}{\infty}$ & 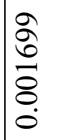 & $\begin{array}{l}\hat{a} \\
\hat{n} \\
\overline{8} \\
0\end{array}$ & $\frac{4}{n}$ & 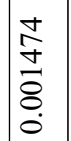 & 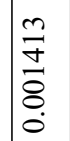 & $\begin{array}{l}\tilde{y} \\
\stackrel{0}{8} \\
0 \\
0\end{array}$ & $\frac{\grave{1}}{\grave{3}}$ & $\mid \begin{array}{l}\bar{\infty} \\
\stackrel{8}{\delta} \\
\stackrel{\delta}{0} \\
\dot{0}\end{array}$ \\
\hline & & $\ddot{\approx}$ & 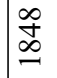 & 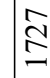 & है & & 号 & d & હે & $\begin{array}{l}\infty \\
ٍ \\
\end{array}$ & 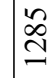 & $\widehat{\widetilde{\Xi}}$ & ळ & & 莺 & $\stackrel{\infty}{\Xi}$ & 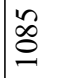 & $\approx$ & $\widehat{\widehat{\Xi}}$ & $\bar{\Xi}$ & $\hat{\widehat{a}}$ & 芯 & & $\frac{\sigma}{\alpha}$ \\
\hline تُ & 蒫 & 竞 & 7 & $\begin{array}{l}\text { Oో } \\
0 \\
0\end{array}$ & $\stackrel{0}{c}$ & & $\ddot{n}$ & $\stackrel{0}{\stackrel{0}{0}}$ & $\stackrel{n}{n}$ & $\begin{array}{l}J \\
0\end{array}$ & $\stackrel{n}{f}$ & F. & J & & $\vec{\nabla}$ & $\stackrel{?}{f}$ & $\stackrel{?}{\stackrel{m}{0}}$ & $\stackrel{?}{\stackrel{f}{0}}$ & f̊. & $\begin{array}{l}0 \\
0 \\
0 \\
0\end{array}$ & $\tilde{n}$ & $\bar{n}$ & nุ? & 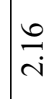 \\
\hline $\begin{array}{l}\frac{t}{\sigma} \\
\frac{\overrightarrow{0}}{\Xi}\end{array}$ & $\begin{array}{l}0 \\
\infty \\
\infty \\
\tilde{\delta} \\
0 \\
0\end{array}$ & $\vec{z}$ & $\begin{array}{l}\infty \\
\dot{m}\end{array}$ & $\stackrel{g}{g}$ & $\stackrel{\infty}{c}$ & & 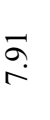 & 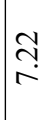 & $\begin{array}{l}2 \\
0 \\
6\end{array}$ & $\tilde{\sigma}$ & $\tilde{\sigma}$ & तु & $\stackrel{t}{\tilde{\sigma}}$ & 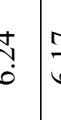 & $\frac{1}{6}$ & సิ & ô. & $\begin{array}{l}\stackrel{0}{2} \\
\text { in }\end{array}$ & in & $\begin{array}{l}\tilde{\theta} \\
\hat{\theta}\end{array}$ & $\frac{6}{6}$ & $\frac{9}{6}$ & مُ & $\stackrel{\square}{\varrho}$ \\
\hline $\begin{array}{l}\stackrel{\mathscr{a}}{\sharp} \\
\frac{\ddot{\theta}}{\Xi}\end{array}$ & 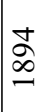 & 衰 & $\frac{\text { f. }}{\dot{\sigma}}$ & $\stackrel{n}{\infty}$ & $\begin{array}{l}\infty \\
i n\end{array}$ & & $\begin{array}{l}\tilde{\sigma} \\
\dot{r}\end{array}$ & $\begin{array}{l}\hat{6} \\
+ \\
+\end{array}$ & $\stackrel{\mathbb{T}}{\stackrel{\sim}{+}}$ & $\overrightarrow{\dot{+}}$ & 离 & $\frac{9}{n}$ & in & $?$ & 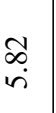 & 告 & $\stackrel{\infty}{+}$ & ભે & $\underset{r}{\stackrel{J}{r}}$ & $\vec{\infty}$ & $\mid \begin{array}{l}\infty \\
n \\
\infty \\
\infty\end{array}$ & $\stackrel{\text { İ }}{2}$ & ک̊ & $\mid \begin{array}{l}n \\
\dot{j} \\
\dot{f}\end{array}$ \\
\hline$\underset{\Xi}{\stackrel{\Xi}{\Xi}}$ & ồ & 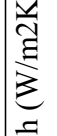 & $\begin{array}{l}0 \\
\tilde{n} \\
0 \\
0 \\
n \\
m\end{array}$ & $\begin{array}{l}2 \\
\check{\jmath} \\
\vdots\end{array}$ & 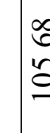 & & 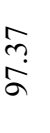 & $\underset{\sim}{*}$ & 文. & $\begin{array}{l}\hat{\alpha} \\
\dot{\alpha}\end{array}$ & $\begin{array}{l}\infty \\
\infty \\
\infty \\
\infty\end{array}$ & $\begin{array}{l}\infty \\
\sim \\
\sim\end{array}$ & $\begin{array}{l}\bar{a} \\
\text { oे }\end{array}$ & م. & $\begin{array}{l}\frac{\Omega}{\dot{a}} \\
\end{array}$ & 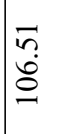 & $\begin{array}{l}\tilde{y} \\
\tilde{o} \\
0\end{array}$ & \begin{tabular}{l}
\multirow{8}{*}{} \\
$\stackrel{\delta}{0}$
\end{tabular} & $\begin{array}{l}n \\
\tilde{0} \\
\infty \\
0 \\
0\end{array}$ & $\begin{array}{l}\Xi \\
\dot{\Xi} \\
\beth\end{array}$ & 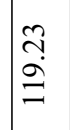 & 竎 & $\begin{array}{l}\tilde{\sigma} \\
i \\
\\
-\end{array}$ & $\begin{array}{l}\tilde{\infty} \\
\ddot{\delta} \\
\ddot{\sigma}\end{array}$ \\
\hline$\sum_{\text {兊 }}$ & $\stackrel{+}{r}$ & $\begin{array}{l}\text { త્ } \\
\text { ఏ } \\
= \\
=\end{array}$ & 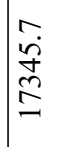 & 恣 & 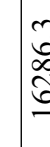 & & $\begin{array}{l}\vec{i} \\
\overrightarrow{0} \\
-\end{array}$ & $\begin{array}{l}\vec{\infty} \\
\infty \\
\hat{n} \\
\tilde{n}\end{array}$ & 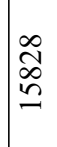 & $\begin{array}{l}n \\
\tilde{O} \\
\tilde{D} \\
n \\
n\end{array}$ & 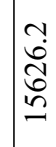 & 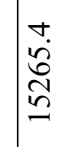 & 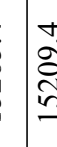 & 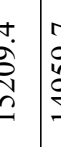 & 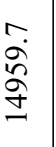 & 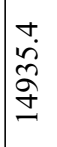 & $\stackrel{n}{\frac{n}{J}}$ & $\begin{array}{l}0 \\
\tilde{i} \\
\tilde{\sigma} \\
\exists \\
ت\end{array}$ & $\begin{array}{l}\hat{\sigma} \\
\stackrel{\sigma}{\Xi}\end{array}$ & 足 & $\begin{array}{l}\stackrel{0}{2} \\
\stackrel{2}{2}\end{array}$ & 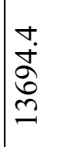 & 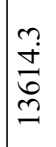 & $\frac{n}{\square}$ \\
\hline 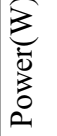 & $\widehat{\widehat{\sigma}}$ & $\vec{F}$ & $\begin{array}{l}\hat{\sigma} \\
\stackrel{\sigma}{ \pm} \\
m\end{array}$ & 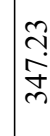 & $\begin{array}{l}\infty \\
\infty \\
\infty \\
\end{array}$ & & $\begin{array}{l}\stackrel{+}{0} \\
\dot{\sigma} \\
\forall\end{array}$ & $\begin{array}{l}\hat{a} \\
\dot{y} \\
\dot{y}\end{array}$ & 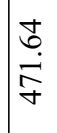 & $\begin{array}{l}\tilde{\sigma} \\
\text { î } \\
\tilde{S} \\
i n\end{array}$ & 枀 & $\begin{array}{l}\frac{\sigma}{0} \\
\overline{0} \\
i\end{array}$ & $\frac{3}{2}$ & 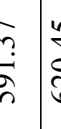 & 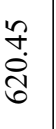 & $\begin{array}{l}\hat{N} \\
\stackrel{y}{\sigma}\end{array}$ & 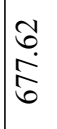 & 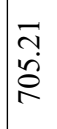 & $\begin{array}{l}\tilde{N} \\
\stackrel{2}{n} \\
\sim\end{array}$ & $\begin{array}{l}n \\
\mathfrak{n} \\
n \\
n\end{array}$ & $\begin{array}{l}2 \\
\dot{0} \\
\infty \\
\propto 0\end{array}$ & $\begin{array}{l}f \\
\stackrel{0}{\infty} \\
\infty \\
\infty\end{array}$ & $\begin{array}{l}\infty \\
\infty \\
\infty \\
\infty \\
\infty\end{array}$ & 占 \\
\hline$\sum_{0=1}^{\pi}$ & $\frac{2}{0}$ & 3 & $\begin{array}{l}m \\
\tilde{b} \\
\tilde{n}\end{array}$ & $\begin{array}{l}\mathcal{d} \\
\infty \\
\stackrel{d}{f}\end{array}$ & $\begin{array}{l}\text { б. } \\
\text { in }\end{array}$ & & 守 & 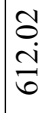 & 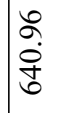 & ? & त̂ & $\frac{\bar{m}}{\bar{i}}$ & 吾 & 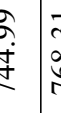 & $\begin{array}{l}\vec{n} \\
\infty \\
\infty \\
i\end{array}$ & $\begin{array}{l}\stackrel{q}{+} \\
\stackrel{\infty}{\curvearrowright}\end{array}$ & $\begin{array}{l}= \\
\frac{n}{\infty}\end{array}$ & $\begin{array}{l}\tilde{O} \\
\stackrel{i}{j} \\
\tilde{\infty}\end{array}$ & గ్ర & $\begin{array}{l}\sim \\
\dot{\infty} \\
\infty \\
\infty\end{array}$ & $\begin{array}{l}\sigma \\
\dot{0} \\
\dot{0}\end{array}$ & 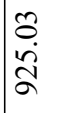 & $\underset{\stackrel{\mathcal{I}}{\mathcal{J}}}{\mathcal{J}}$ & 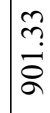 \\
\hline 离 & $\stackrel{\infty}{\sim}$ & 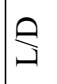 & $N$ & $\vec{\infty}$ & $\exists$ & $t$ & ָ̃. & సֶ. & $\stackrel{m}{i}$ & $\begin{array}{c}m \\
\infty \\
\infty\end{array}$ & $\stackrel{+}{+}$ & $\stackrel{+}{\circ}$ & $\begin{array}{l}n \\
n \\
n\end{array}$ & $\begin{array}{l}? \\
? \\
?\end{array}$ & $\begin{array}{l}n \\
\text { a }\end{array}$ & $\begin{array}{l}n \\
\infty \\
\infty\end{array}$ & $\begin{array}{l}0 \\
\dot{I} \\
\end{array}$ & $\ddot{\infty}$ & $\hat{\infty}$ & $\hat{\check{a}}$ & $\mid \begin{array}{l}\infty \\
\infty \\
\infty\end{array}$ & 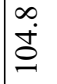 & 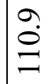 & $\stackrel{a}{\sigma}$ \\
\hline
\end{tabular}




\begin{tabular}{|c|c|c|c|c|c|c|c|c|c|c|c|c|c|c|c|c|c|c|c|c|c|c|c|c|}
\hline & & $\vec{\Delta}$ & $\begin{array}{l}0 \\
0 \\
1 \\
\text { ț } \\
2 \\
\text { in }\end{array}$ & 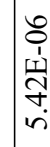 & 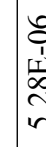 & & 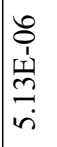 & 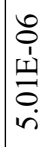 & $\begin{array}{l}8 \\
\stackrel{1}{1} \\
\text { 章 } \\
\dot{+}\end{array}$ & $\begin{array}{l}0 \\
⿱ \\
1 \\
1 \\
\infty \\
\dot{+} \\
+\end{array}$ & 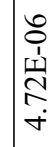 & 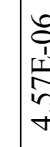 & 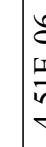 & 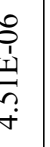 & 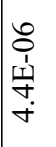 & 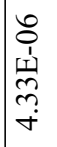 & 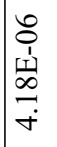 & 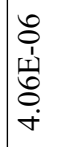 & 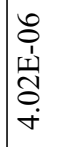 & \begin{tabular}{l}
8 \\
0 \\
1 \\
11 \\
\multirow{1}{0}{} \\
2 \\
$\dot{m}$
\end{tabular} & \begin{tabular}{l}
8 \\
0 \\
1 \\
\multicolumn{1}{c}{} \\
$\infty$ \\
$\dot{n}$ \\
$\dot{m}$
\end{tabular} & 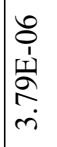 & $\begin{array}{l}0 \\
0 \\
1 \\
\omega \\
\infty \\
\infty \\
\\
\dot{n}\end{array}$ & 总 \\
\hline & & $\stackrel{\oplus}{\infty}$ & $\begin{array}{l}\hat{o} \\
\hat{1} \\
\underline{\omega} \\
\\
\end{array}$ & 旁 & $\begin{array}{l}\overrightarrow{9} \\
\frac{1}{5} \\
\frac{1}{7}\end{array}$ & & 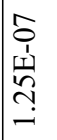 & 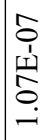 & 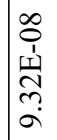 & $\begin{array}{l}\infty \\
0 \\
1 \\
1 \\
\\
-1 \\
\infty \\
\infty\end{array}$ & $\mid \begin{array}{c}\infty \\
0 \\
1 \\
1 \\
\\
\\
r\end{array}$ & $\begin{array}{l}\infty \\
0 \\
1 \\
1 \\
2 \\
0 \\
0\end{array}$ & d & 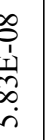 & $\begin{array}{l}\infty \\
0 \\
0 \\
1 \\
1 \\
0 \\
-1 \\
\text { in }\end{array}$ & 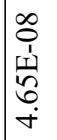 & 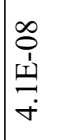 & 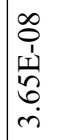 & 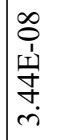 & $\begin{array}{l}\infty \\
0 \\
0 \\
1 \\
m \\
m \\
m \\
m\end{array}$ & 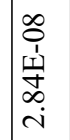 & $\begin{array}{l}\infty \\
0 \\
1 \\
1 \\
\\
0 \\
\sim \\
\sim \\
\sim\end{array}$ & 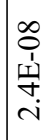 & \begin{tabular}{l}
$\infty$ \\
\multirow{2}{1}{} \\
$\stackrel{1}{\sim}$ \\
\multirow{\sim}{\sim}{} \\
$\stackrel{-}{-}$
\end{tabular} \\
\hline & & $\ddot{2}$ & 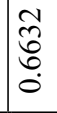 & त्ञ్రి & $\begin{array}{l}\hat{\sigma} \\
\text { ç } \\
c\end{array}$ & b. & 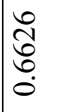 & $\begin{array}{l}\text { îे } \\
\text { ర్ర } \\
\stackrel{0}{0}\end{array}$ & 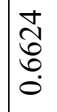 & 志 & 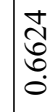 & 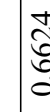 & 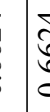 & 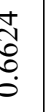 & 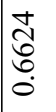 & 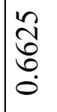 & 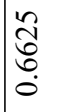 & 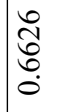 & 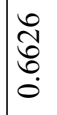 & $\begin{array}{l}\tilde{\hat{\mathcal{D}}} \\
\dot{0} \\
\dot{0}\end{array}$ & 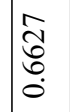 & $\begin{array}{l}\infty \\
\text { đ్ర } \\
\stackrel{0}{0} \\
\dot{0}\end{array}$ & $\begin{array}{l}\text { ते } \\
\text { ర్రి } \\
\dot{0}\end{array}$ & 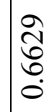 \\
\hline & & $\mathscr{J}$ & $\begin{array}{l}\stackrel{a}{\hat{N}} \\
\hat{ָ}\end{array}$ & $\begin{array}{l}\bar{\sim} \\
\tilde{N} \\
\end{array}$ & 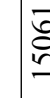 & & I & $\begin{array}{l}\tilde{\omega} \\
\tilde{\infty} \\
\infty\end{array}$ & $\begin{array}{l}\infty \\
\infty \\
0 \\
0\end{array}$ & $\frac{n}{n}$ & 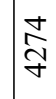 & లై & 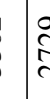 & हे & ¿̊. & $\stackrel{\infty}{\infty}$ & $\stackrel{\infty}{\stackrel{\infty}{\Xi}}$ & $\stackrel{\varrho}{\beth}$ & 韋 & $\stackrel{\infty}{\infty}$ & $\stackrel{8}{\circ}$ & Iี & b & $\frac{0}{m}$ \\
\hline & & $t$ & 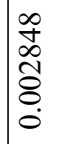 & 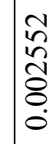 & $\begin{array}{l}n \\
\approx \\
\check{\Xi} \\
\Xi \\
c\end{array}$ & & $\begin{array}{l}\overrightarrow{\widetilde{Z}} \\
\tilde{\Xi} \\
\vdots \\
0 \\
0\end{array}$ & 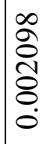 & 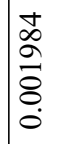 & $\begin{array}{l}\vec{\infty} \\
\infty \\
\ddot{\delta} \\
\dot{0}\end{array}$ & 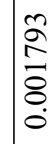 & 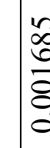 & $\frac{\alpha}{5}$ & . & 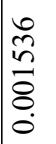 & 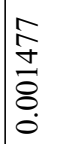 & ڤ్ & $\frac{\infty}{\frac{\infty}{m}}$ & 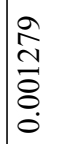 & 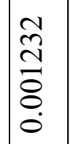 & $\begin{array}{l}\infty \\
\infty \\
\bar{\Xi} \\
\dot{8} \\
0\end{array}$ & $\begin{array}{l}\stackrel{+}{m} \\
\stackrel{3}{8} \\
\stackrel{8}{0}\end{array}$ & 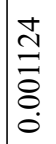 & $\begin{array}{l}n \\
\hat{\delta} \\
8 \\
8 \\
0 \\
0\end{array}$ \\
\hline & & $\ddot{\sim}$ & $\stackrel{\partial}{2}$ & $\begin{array}{l}\mathcal{D} \\
\infty \\
\infty\end{array}$ & 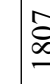 & & 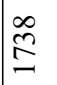 & $\frac{1}{6}$ & ర్రి & $\begin{array}{l}\infty \\
0 \\
n \\
n\end{array}$ & ڤ્ & 号 & & ஜ & $\stackrel{\infty}{2}$ & $\begin{array}{l}\hat{6} \\
\end{array}$ & 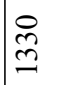 & 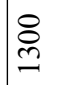 & 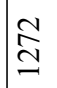 & $\stackrel{\sim}{\stackrel{\sim}{I}}$ & స్ & $\stackrel{\circ}{ٍ}$ & $\stackrel{\cong}{\Xi}$ & 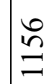 \\
\hline تే & $\stackrel{0}{1}$ & 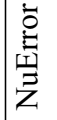 & . & อे & $\begin{array}{l}v \\
b \\
c\end{array}$ & & 苟 & $\stackrel{g}{0}$ & f̊ & f̊. & 市 & I & & $\hat{b}$ & $\hat{3}$ & $\hat{0}$ & 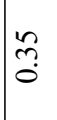 & mे & $\hat{n}$ & $\begin{array}{l}+ \\
\tilde{O} \\
0\end{array}$ & $\hat{n}$ & 施 & t. & के \\
\hline $\begin{array}{l}\frac{ \pm}{\sigma} \\
\frac{ \pm}{ \pm} \\
\Xi\end{array}$ & 年 & $\vec{z}$ & $\begin{array}{l}\hat{\partial} \\
\dot{m} \\
\text { m. }\end{array}$ & 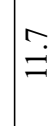 & 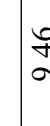 & & $\tilde{\infty}$ & $\stackrel{0}{r}$ & ָे & $\stackrel{\infty}{\stackrel{0}{r}}$ & $\stackrel{\sigma}{\circ}$ & ఫु & & ch & ठे. & @o & $\begin{array}{l}0 \\
i \\
i n\end{array}$ & $\hat{n}$ & $\begin{array}{l}0 \\
i \\
i n \\
n\end{array}$ & $\left|\begin{array}{l}0 \\
n \\
n \\
n\end{array}\right|$ & 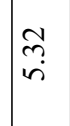 & 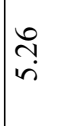 & \begin{tabular}{l}
0 \\
\multirow{1}{*}{} \\
in
\end{tabular} & $\vec{\sim}$ \\
\hline $\begin{array}{l}\stackrel{\mathscr{a}}{\sharp} \\
\frac{\ddot{\theta}}{\Xi}\end{array}$ & 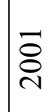 & 衰 & $\begin{array}{l}\tilde{\sigma} \\
\text { î }\end{array}$ & $\underset{\infty}{0}$ & $\begin{array}{l}\infty \\
\vdots \\
\text { in }\end{array}$ & & $\begin{array}{l}\bar{\sigma} \\
\dot{+}\end{array}$ & 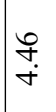 & $\stackrel{\vartheta}{\stackrel{f}{*}}$ & $\begin{array}{l}\stackrel{a}{+} \\
+\end{array}$ & iे & $\underset{7}{\stackrel{7}{7}}$ & & $\begin{array}{l}1 \\
r \\
\dot{f}\end{array}$ & $\stackrel{m}{m} \underset{+}{m}$ & $\stackrel{n}{n}$ & $\overrightarrow{+}$ & $F$ & $\hat{\sim}$ & $\underset{+}{\stackrel{f}{+}}$ & $\underset{f}{t}$ & $\stackrel{\infty}{\stackrel{\infty}{\leftarrow}}$ & ते & थู \\
\hline$\underset{\Xi}{\stackrel{\Xi}{\Xi}}$ & 总 & 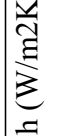 & 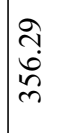 & 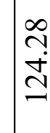 & $\begin{array}{l}\text { f } \\
\text { I }\end{array}$ & & $\mid \begin{array}{l}\infty \\
\dot{\alpha} \\
\alpha\end{array}$ & $\frac{ \pm}{\dot{8}}$ & 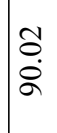 & $\bar{m}$ & 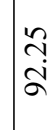 & $\underset{\infty}{\infty}$ & & & $\frac{1}{\infty}$ & $\begin{array}{l}\infty \\
\infty \\
\infty \\
\infty\end{array}$ & $\begin{array}{l}6 \\
\dot{0} \\
\infty\end{array}$ & \begin{tabular}{l}
$\tilde{n}$ \\
\multirow{\infty}{\infty}{}
\end{tabular} & $\begin{array}{l}\hat{m} \\
\dot{\infty}\end{array}$ & $\begin{array}{l}\tilde{D} \\
\infty \\
\infty\end{array}$ & $\underset{\substack{\infty \\
\infty}}{\stackrel{2}{2}}$ & $\frac{a}{\infty}$ & 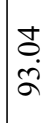 & 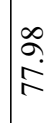 \\
\hline$\sum_{\text {兊 }}$ & $\stackrel{\dot{m}}{\dot{m}}$ & 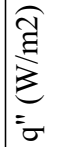 & 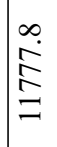 & $\begin{array}{l}\stackrel{\sim}{0} \\
\stackrel{\Xi}{=}\end{array}$ & \begin{tabular}{l}
$\infty$ \\
\multirow{I}{I}{} \\
$\Xi$
\end{tabular} & & 号 & 守 & 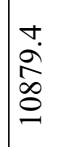 & $\underset{\hat{i}}{\stackrel{a}{a}}$ & $\underset{⿱}{\stackrel{t}{5}}$ & है & 告 & o. & $\begin{array}{l}\infty \\
\infty \\
\infty \\
0 \\
0 \\
0 \\
0\end{array}$ & $\mid \begin{array}{c}\infty \\
\hat{\delta} \\
ٍ\end{array}$ & $\underset{\stackrel{\sim}{\Xi}}{\stackrel{\Xi}{\Xi}}$ & ì. & $\begin{array}{l}+\dot{\infty} \\
\stackrel{\infty}{\sigma} \\
\stackrel{\sigma}{\alpha}\end{array}$ & $\begin{array}{l}n \\
\infty \\
\infty \\
\infty \\
\sigma\end{array} \mid$ & 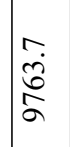 & 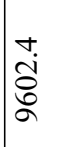 & $\begin{array}{l}+ \\
\dot{+} \\
\stackrel{\infty}{\hat{\sigma}} \\
\end{array}$ & ㄱ. \\
\hline 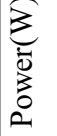 & 气 & 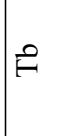 & $\frac{\infty}{\stackrel{\infty}{\infty}}$ & 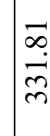 & 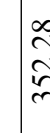 & & 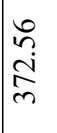 & $\begin{array}{l}\tilde{\sigma} \\
\text { ì } \\
\text { mे }\end{array}$ & $\begin{array}{l}\stackrel{0}{n} \\
\stackrel{\sim}{\gamma} \\
\gamma\end{array}$ & $\begin{array}{l}\tilde{n} \\
\tilde{n} \\
\tilde{\sigma} \\
\tilde{\gamma}\end{array}$ & $\begin{array}{l}2 \\
i \\
i \\
\gamma \\
\gamma\end{array}$ & $\frac{n}{7}$ & 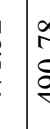 & 递 & $\begin{array}{l}\text { aें } \\
\text { in }\end{array}$ & $\begin{array}{l}\infty \\
\infty \\
\infty \\
\infty \\
n\end{array}$ & $\begin{array}{l}\stackrel{0}{n} \\
\stackrel{5}{*} \\
\dot{v}\end{array}$ & $\begin{array}{l}0 \\
\infty \\
0 \\
0 \\
n\end{array}$ & $\begin{array}{l}\tilde{\alpha} \\
\tilde{\infty} \\
\infty \\
\infty\end{array}$ & $\frac{8}{\stackrel{8}{2}}$ & $\begin{array}{l}\infty \\
\infty \\
\ddot{\sigma}\end{array}$ & 令 & $\begin{array}{l}\vec{N} \\
\tilde{n} \\
\hat{\sigma}\end{array}$ & $\begin{array}{l}\sigma \\
\dot{0} \\
\hat{\sigma}\end{array}$ \\
\hline$\sum_{0=1}^{\pi}$ & $\frac{2}{0}$ & 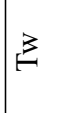 & $\begin{array}{l}\Phi \\
\infty \\
\tilde{f} \\
\tilde{m}\end{array}$ & $\begin{array}{l}\text { In } \\
\text { İ } \\
\text { y }\end{array}$ & $\begin{array}{l}\infty \\
2 \\
\infty \\
y \\
y\end{array}$ & $\begin{array}{l}0 \\
0 \\
0\end{array}$ & $\mid$\begin{tabular}{|}
$\vec{\alpha}$ \\
$\dot{\alpha}$ \\
$\dot{\alpha}$ \\
$\dot{\alpha}$
\end{tabular} & $\begin{array}{l}\infty \\
m \\
m \\
m \\
n\end{array}$ & $\begin{array}{l}\tilde{F} \\
\tilde{n} \\
\tilde{n}\end{array}$ & $\begin{array}{l}\hat{\partial} \\
\text { i } \\
i n \\
i n\end{array}$ & $\begin{array}{l}+ \\
\infty \\
\infty \\
0 \\
0 \\
\infty\end{array}$ & $\bar{z}$ & $\frac{7}{5}$ & $\frac{3}{6}$ & $\begin{array}{l}\text { तิ } \\
\text { तु }\end{array}$ & $\mid \begin{array}{l}\tilde{b} \\
\stackrel{0}{0} \\
\tilde{b}\end{array}$ & $\begin{array}{l}\bar{m} \\
\dot{8} \\
\delta\end{array}$ & $\begin{array}{l}\infty \\
\infty \\
\infty \\
0\end{array}$ & $\stackrel{\infty}{\stackrel{\infty}{\circ}}$ & $\begin{array}{l}\vec{r} \\
\underline{\sigma} \\
\stackrel{r}{r}\end{array}$ & $\frac{\vec{p}}{\stackrel{2}{2}}$ & $\begin{array}{l}2 \\
\stackrel{0}{0} \\
\frac{1}{2}\end{array}$ & $\stackrel{n}{n}$ & \begin{tabular}{l}
$n$ \\
on \\
\multirow{1}{1}{}
\end{tabular} \\
\hline 离 & নे & 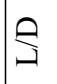 & $N$ & $\vec{\infty}$ & $\exists$ & & तิ & సֶ. & $\stackrel{m}{i}$ & $\begin{array}{c}m \\
\infty \\
\infty\end{array}$ & $\stackrel{+}{+}$ & in & v & $\begin{array}{l}n \\
\text { no } \\
n\end{array}$ & $\begin{array}{l}\tilde{n} \\
\text { Uु }\end{array}$ & $\begin{array}{l}n \\
\infty \\
\infty \\
\infty\end{array}$ & $\begin{array}{l}0 \\
\dot{I} \\
\end{array}$ & $\ddot{\infty}$ & $\hat{\infty}$ & $\hat{i}$ & $\mid \begin{array}{l}\infty \\
\infty \\
\infty\end{array}$ & 㐫 & 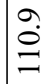 & $\stackrel{a}{\sigma}$ \\
\hline
\end{tabular}




\begin{tabular}{|c|c|c|c|c|c|c|c|c|c|c|c|c|c|c|c|c|c|c|c|c|c|c|c|c|}
\hline & & $\vec{\Delta}$ & 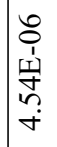 & 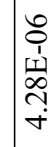 & 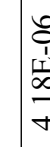 & & $\begin{array}{l}8 \\
0 \\
1 \\
1 \\
1 \\
0 \\
0 \\
\dot{+}\end{array}$ & 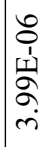 & 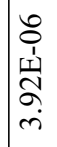 & $\begin{array}{l}0 \\
0 \\
1 \\
1 \\
0 \\
0 \\
\infty \\
\dot{r}\end{array}$ & 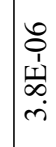 & 竜 & (5) & 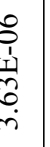 & $\begin{array}{l}8 \\
0 \\
0 \\
1 \\
1 \\
0 \\
n \\
n \\
n\end{array}$ & 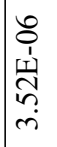 & 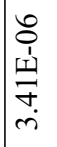 & $\begin{array}{l}8 \\
0 \\
1 \\
\\
m \\
m \\
m\end{array}$ & 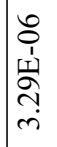 & $\begin{array}{l}8 \\
0 \\
1 \\
11 \\
\\
\\
\end{array}$ & 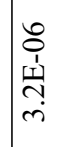 & 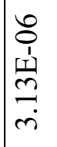 & 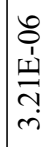 & 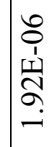 \\
\hline & & $\stackrel{\oplus}{\infty}$ & 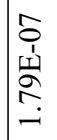 & 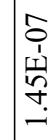 & 全 & & 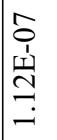 & 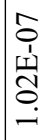 & $\begin{array}{l}\infty \\
0 \\
1 \\
1 \\
1 \\
\infty \\
\infty \\
\infty\end{array}$ & \begin{tabular}{l}
$\infty$ \\
0 \\
1 \\
\multicolumn{1}{|c}{} \\
- \\
$\infty$ \\
$\infty$
\end{tabular} & $\mid \begin{array}{c}\infty \\
0 \\
1 \\
1 \\
\\
\\
r\end{array}$ & $\begin{array}{l}\infty \\
0 \\
1 \\
1 \\
\\
0\end{array}$ & 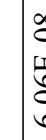 & . & $\begin{array}{l}\infty \\
0 \\
0 \\
\text { I1 } \\
0 \\
\text { in }\end{array}$ & \begin{tabular}{|l}
$\infty$ \\
0 \\
1 \\
站
\end{tabular} & 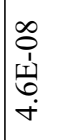 & 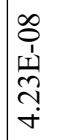 & \begin{tabular}{|l}
$\infty$ \\
0 \\
1 \\
岁 \\
$\gamma$
\end{tabular} & 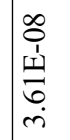 & 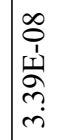 & $\begin{array}{l}\infty \\
0 \\
1 \\
1 \\
⿱ n \\
\\
\\
\end{array}$ & $\begin{array}{c}\infty \\
0 \\
1 \\
\text { I⿱ } \\
m\end{array}$ & 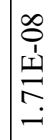 \\
\hline & & $\ddot{2}$ & 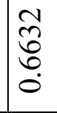 & $\begin{array}{l}\overrightarrow{\widehat{\sigma}} \\
\stackrel{0}{0} \\
0\end{array}$ & $\begin{array}{l}\text { ¿े } \\
\text { cे } \\
c\end{array}$ & S. & $\begin{array}{l}\infty \\
\mathbb{1} \\
0 \\
0 \\
0 \\
0\end{array}$ & $\begin{array}{l}\hat{\widehat{N}} \\
\stackrel{0}{0} \\
\stackrel{0}{0}\end{array}$ & $\begin{array}{l}0 \\
\mathbb{Z} \\
0 \\
0 \\
0\end{array}$ & 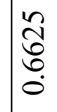 & مि & 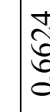 & 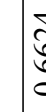 & 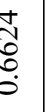 & 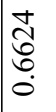 & 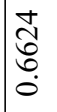 & 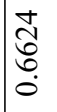 & 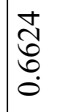 & 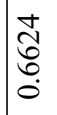 & 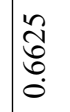 & 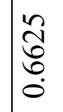 & 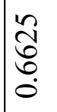 & 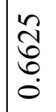 & 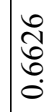 \\
\hline & & $\overline{0}$ & $\begin{array}{l}\overrightarrow{8} \\
\infty \\
\stackrel{\infty}{*}\end{array}$ & 离 & $\begin{array}{l}n \\
z \\
z\end{array}$ & & $\underset{\substack{\mathcal{I} \\
\text { I }}}{ }$ & $\vec{f}$ & 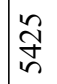 & 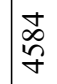 & 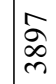 & $\overline{\mathrm{m}}$ & 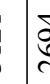 & $\begin{array}{c}+ \\
\text { C) } \\
\text { N }\end{array}$ & $\frac{\sim}{\tilde{\lambda}}$ & $\stackrel{\circ}{2}$ & 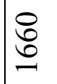 & $\overrightarrow{\tilde{J}}$ & $\overrightarrow{\widehat{I}}$ & 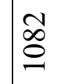 & $\hat{n}$ & 离 & $i$ & $\frac{\mathcal{I}}{F}$ \\
\hline & & t & 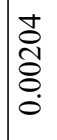 & $\begin{array}{l}\tilde{\hat{D}} \\
\infty \\
\stackrel{0}{8} \\
\dot{0}\end{array}$ & 范 & & 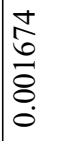 & 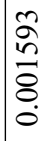 & 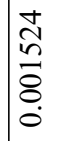 & $\begin{array}{l}\overrightarrow{0} \\
\stackrel{0}{0} \\
\dot{8} \\
0\end{array}$ & $\mid \begin{array}{l}8 \\
\stackrel{9}{9} \\
8 \\
0 \\
0\end{array}$ & $\frac{\infty}{\approx}$ & $\frac{\delta}{\partial}$ & 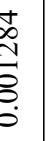 & 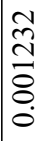 & $\mid \begin{array}{l}n \\
\stackrel{2}{8} \\
\vdots \\
0\end{array}$ & 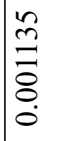 & 文 & 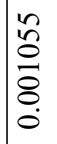 & $\begin{array}{l}\tilde{d} \\
0 \\
0 \\
0 \\
0 \\
0\end{array}$ & 会 & 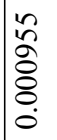 & 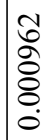 & $\begin{array}{l}\infty \\
0 \\
\swarrow \\
\vdots \\
\vdots \\
0 \\
0 \\
0\end{array}$ \\
\hline & & $\ddot{\sim}$ & $\stackrel{2}{\beth}$ & હે & $\underline{\mathscr{\alpha}}$ & & 串 & $\tilde{n}$ & 芯 & $\frac{6}{n}$ & 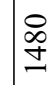 & 过 & & $\frac{n}{ \pm}$ & 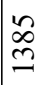 & $\mid \begin{array}{l}\hat{n} \\
\underline{n}\end{array}$ & $\vec{m}$ & 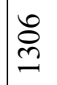 & 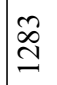 & $\begin{array}{l}\vec{\Xi} \\
\beth\end{array}$ & $\grave{\Xi ે}$ & $\stackrel{\vec{\beth}}{\beth}$ & $\stackrel{\partial}{=}$ & $\underset{\infty}{\infty}$ \\
\hline تే & 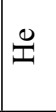 & 氙 & $\begin{array}{l}\bar{\sigma} \\
\text { in }\end{array}$ & $\stackrel{m}{0}$ & $\tilde{n}$ & & $\stackrel{n}{\circ}$ & $\stackrel{\nabla}{0}$ & $\underset{0}{+}$ & $\stackrel{t}{0}$ & $\stackrel{m}{\tilde{0}}$ & व & & 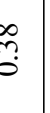 & ڤે & $\ddot{0}$ & ?̊. & ๙̃? & $\tilde{n}$ & $\stackrel{m}{0}$ & శె? & 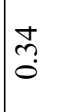 & సे? & ?ִ \\
\hline $\begin{array}{l}\frac{ \pm}{\sigma} \\
\frac{ \pm}{ \pm} \\
\Xi\end{array}$ & 垈 & $\vec{z}$ & $\begin{array}{l}\vec{q} \\
\text { in } \\
\text { nd }\end{array}$ & 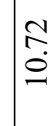 & $\begin{array}{l}b \\
\infty\end{array}$ & & 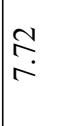 & $\stackrel{\Xi}{r}$ & $\underset{r}{\stackrel{t}{0}}$ & $\underset{r}{\sigma}$ & $\Rightarrow$ & n & & $\tilde{b}$ & ભું & స్ & ڤ. & 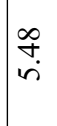 & 尔 & $\begin{array}{l}n \\
n \\
n\end{array}$ & 宇 & $\begin{array}{l}f \\
⿱ 乛 \\
i n\end{array}$ & $\stackrel{m}{i n}$ & $\vec{m}$ \\
\hline $\begin{array}{l}\stackrel{\mathscr{a}}{\sharp} \\
\frac{\ddot{\theta}}{\Xi}\end{array}$ & $\underline{\infty}$ & 衰 & $\begin{array}{l}\vec{\exists} \\
\ddot{n}\end{array}$ & 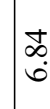 & $\stackrel{\infty}{\propto}$ & & $\stackrel{M}{\vec{r}}$ & 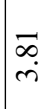 & $\overrightarrow{\vec{m}}$ & $\ddot{\nabla}$ & $\underset{f}{\stackrel{m}{f}}$ & $\stackrel{2}{a}$ & & $\frac{\infty}{+}$ & 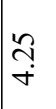 & $\underset{\forall}{\stackrel{\forall}{+}}$ & $\begin{array}{l}\stackrel{\sim}{\sim} \\
\dot{\forall}\end{array}$ & $\vec{i}$ & $\begin{array}{l}\infty \\
\infty \\
n\end{array}$ & $\stackrel{\tilde{r}}{\dot{f}}$ & $\stackrel{n}{r}$ & సे & $\stackrel{\infty}{+}$ & $\begin{array}{l}\infty \\
\dot{+}\end{array}$ \\
\hline$\underset{\Xi}{\stackrel{\Xi}{\Xi}}$ & 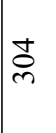 & 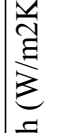 & 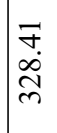 & $\stackrel{n}{\Xi}$ & $\begin{array}{c}\widehat{\sigma} \\
\sigma\end{array}$ & & {$\left[\begin{array}{l}n \\
n \\
\infty \\
\infty\end{array}\right.$} & $\frac{\hat{b}}{\infty}$ & $\begin{array}{l}0 \\
\\
\infty \\
\infty\end{array}$ & $\stackrel{\vartheta}{\overrightarrow{+}}$ & $\stackrel{m}{\stackrel{m}{\infty}}$ & $\hat{\infty}$ & & $\begin{array}{l}6 \\
6 \\
6 \\
\infty\end{array}$ & $\begin{array}{l}\infty \\
\infty \\
\infty\end{array}$ & $\begin{array}{l}n \\
n \\
\infty \\
\infty\end{array}$ & $\begin{array}{l}\infty \\
\infty \\
\infty \\
\infty\end{array}$ & సิ & 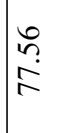 & $\begin{array}{l}\tilde{2} \\
\tilde{R}\end{array}$ & $\begin{array}{l}\infty \\
\stackrel{\infty}{\infty} \\
\infty\end{array}$ & $\underset{\dot{\infty}}{\exists}$ & $\begin{array}{l}1 \\
\infty \\
\infty \\
\infty \\
\infty\end{array}$ & 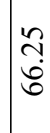 \\
\hline$\sum_{\text {至 }}$ & $\stackrel{+}{r}$ & $\begin{array}{l}\text { త્ } \\
\text { ఏ } \\
= \\
=\end{array}$ & $\begin{array}{l}6 \\
6 \\
0 \\
0 \\
1\end{array}$ & 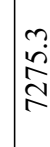 & \begin{tabular}{l}
$\infty$ \\
\multirow{1}{\infty}{} \\
\multirow{2}{*}{}
\end{tabular} & & 离 & 룽 & 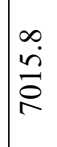 & $\begin{array}{l}0 \\
\dot{+} \\
\text { ô }\end{array}$ & 亲 & $\begin{array}{l}\vec{\infty} \\
\tilde{\sigma}\end{array}$ & م & & 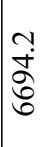 & 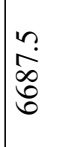 & $\begin{array}{l}\infty \\
\tilde{n} \\
\tilde{\sigma}\end{array}$ & 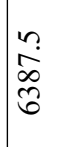 & đ̊̊ & 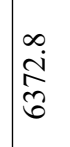 & 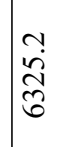 & 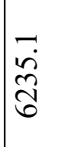 & $\frac{n}{\stackrel{\sim}{*}}$ & $\begin{array}{l}\tilde{m} \\
\tilde{3} \\
\infty \\
\infty\end{array}$ \\
\hline 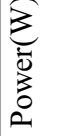 & $\stackrel{0}{ }$ & $\vec{F}$ & $\mid \begin{array}{l}\infty \\
\infty \\
\infty \\
0 \\
\infty \\
\infty\end{array}$ & $\begin{array}{l}\bar{\infty} \\
\tilde{d} \\
\sim\end{array}$ & $\begin{array}{l}c \\
\infty \\
\infty \\
m\end{array}$ & & 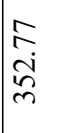 & $\begin{array}{l}0 \\
\hat{0} \\
\hat{n} \\
\end{array}$ & 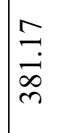 & $\begin{array}{l}\tilde{n} \\
\ddot{n} \\
\tilde{m}\end{array}$ & $\begin{array}{l}\overrightarrow{\hat{a}} \\
\stackrel{\partial}{\sigma} \\
\dot{q}\end{array}$ & $\tilde{7}$ & 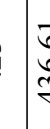 & $\begin{array}{l}0 \\
\dot{0} \\
\dot{y}\end{array}$ & 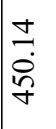 & $\begin{array}{l}\infty \\
n \\
\widehat{\delta} \\
\dot{b}\end{array}$ & $\begin{array}{l}\infty \\
\infty \\
\stackrel{0}{\circ} \\
\stackrel{\gamma}{+}\end{array}$ & $\begin{array}{l}+ \\
\infty \\
\infty \\
\infty \\
\dot{\sigma}\end{array}$ & $\begin{array}{l}\hat{\sigma} \\
\text { is } \\
\text { in }\end{array}$ & $\begin{array}{l}n \\
n \\
i n \\
n\end{array}$ & $\begin{array}{l}\hat{y} \\
\infty \\
\tilde{N} \\
\tilde{n}\end{array}$ & $\begin{array}{l}\infty \\
\infty \\
0 \\
\dot{j} \\
\text { in }\end{array}$ & $\begin{array}{l}0 \\
\tilde{n} \\
\tilde{n}\end{array}$ & 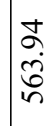 \\
\hline$\sum_{0=1}^{\pi}$ & $\frac{\infty}{0}$ & 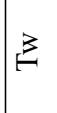 & 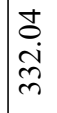 & $\begin{array}{l}\infty \\
\infty \\
\infty \\
\infty\end{array}$ & $\left\{\begin{array}{l}8 \\
\qquad \\
7\end{array}\right.$ & 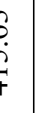 & $\begin{array}{l}-7 \\
0 \\
\varnothing \\
\%\end{array}$ & 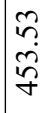 & 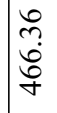 & 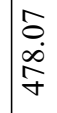 & $\mid \begin{array}{l}\stackrel{2}{2} \\
\infty \\
\infty \\
\infty \\
+\infty\end{array}$ & $\begin{array}{l}n \\
0 \\
u \\
n \\
n\end{array}$ & $\mid \begin{array}{l}8 \\
y \\
7 \\
78\end{array}$ & $\frac{n}{n}$ & in & $\frac{⿱ 亠 䒑}{\stackrel{*}{*}}$ & $\begin{array}{l}\stackrel{ }{A} \\
\tilde{n} \\
\tilde{n} \\
i n\end{array}$ & $\begin{array}{l}n \\
\tilde{n} \\
i n\end{array}$ & $\begin{array}{l}\tilde{N} \\
\sim \\
\infty \\
\infty\end{array}$ & $\begin{array}{l}\infty \\
\infty \\
\ddot{n} \\
i n\end{array}$ & 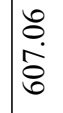 & $\frac{0}{\frac{2}{\sigma}}$ & 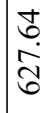 & 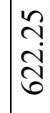 \\
\hline 离 & లి & $\stackrel{3}{2}$ & $N$ & $\vec{\infty}$ & $\bar{J}$ & $t$ & तิ & ָे & $\stackrel{m}{i}$ & $\begin{array}{c}m \\
\infty \\
\infty\end{array}$ & $\stackrel{+}{+}$ & in & $y$ & ?n & 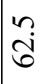 & $\begin{array}{l}n \\
\infty \\
\infty \\
0\end{array}$ & $\begin{array}{l}\stackrel{\bullet}{ \pm} \\
\stackrel{+}{I}\end{array}$ & $\begin{array}{l}0 \\
\dot{\infty}\end{array}$ & $\hat{\infty}$ & $\hat{\check{a}}$ & $\mid \begin{array}{l}\infty \\
\infty \\
\infty\end{array}$ & 㐫 & $\stackrel{\grave{\theta}}{\exists}$ & $\underset{b}{\sigma}$ \\
\hline
\end{tabular}




\begin{tabular}{|c|c|c|c|c|c|c|c|c|c|c|c|c|c|c|c|c|c|c|c|c|c|c|c|c|}
\hline & & $\vec{\Delta}$ & 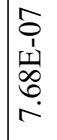 & 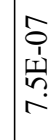 & 告 & & 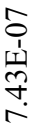 & 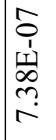 & 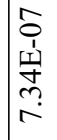 & $\begin{array}{l}\hat{2} \\
\hat{1} \\
\text { In } \\
\\
r\end{array}$ & 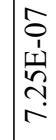 & $\begin{array}{l}0 \\
\mathbf{1} \\
\frac{1}{0} \\
\end{array}$ & & 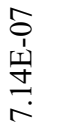 & $\mid \begin{array}{l}0 \\
0 \\
1 \\
\\
0 \\
0 \\
r\end{array}$ & 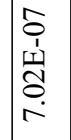 & 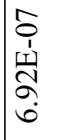 & 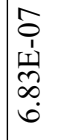 & $\mid \begin{array}{l}0 \\
0 \\
1 \\
\underline{1} \\
0 \\
0 \\
0 \\
0\end{array}$ & 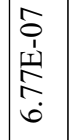 & 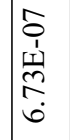 & 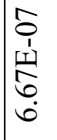 & 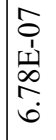 & \begin{tabular}{l}
$\hat{0}$ \\
$\hat{1}$ \\
\multicolumn{1}{|c}{} \\
$\hat{\sigma}$ \\
$\dot{r}$ \\
$\dot{r}$
\end{tabular} \\
\hline & & $\stackrel{\oplus}{\infty}$ & 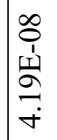 & $\begin{array}{l}\infty \\
0 \\
1 \\
\omega \\
\infty \\
\dot{n} \\
\dot{n}\end{array}$ & 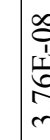 & & 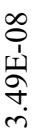 & 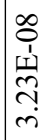 & 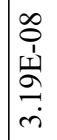 & 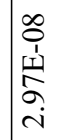 & 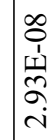 & $\begin{array}{l}\infty \\
0 \\
1 \\
\underline{1} \\
\\
\end{array}$ & & 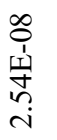 & 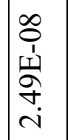 & 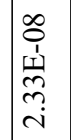 & 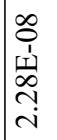 & 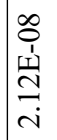 & 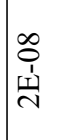 & $\begin{array}{l}\infty \\
0 \\
0 \\
1 \\
1 \\
\infty \\
\infty \\
- \\
-\end{array}$ & 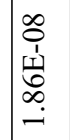 & $\begin{array}{l}\infty \\
0 \\
1 \\
\text { 11 } \\
\\
\infty \\
-\infty \\
-1\end{array}$ & 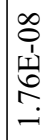 & 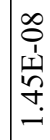 \\
\hline & & 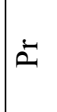 & $\begin{array}{l}\infty \\
\text { ్ָర } \\
0 \\
0 \\
0\end{array}$ & $\begin{array}{l}\hat{\imath} \\
\text { ठ } \\
0\end{array}$ & $\begin{array}{l}\text { है } \\
\text { है } \\
c\end{array}$ & : & 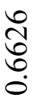 & 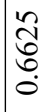 & 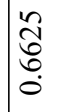 & 志 & 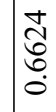 & $\hat{\overbrace{}}$ & & $\begin{array}{l}\tilde{\widehat{U}} \\
\stackrel{0}{0} \\
\stackrel{0}{0}\end{array}$ & 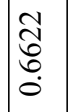 & 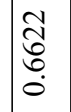 & 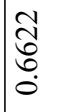 & $\begin{array}{l}\overrightarrow{\widehat{V}} \\
\stackrel{0}{0} \\
\stackrel{0}{0}\end{array}$ & $\begin{array}{l}\overrightarrow{\widetilde{\sigma}} \\
\stackrel{0}{0} \\
\stackrel{0}{0}\end{array}$ & $\begin{array}{l}\overrightarrow{\widetilde{V}} \\
\dot{0} \\
0\end{array}$ & $\begin{array}{l}\overrightarrow{\widetilde{b}} \\
\stackrel{0}{0} \\
\dot{0}\end{array}$ & $\begin{array}{l}\overrightarrow{\widetilde{V}} \\
\stackrel{0}{0} \\
\dot{0}\end{array}$ & 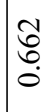 & 莡 \\
\hline & & $\mathscr{J}$ & $\begin{array}{l}\approx \\
\hat{\Omega} \\
\infty \\
\infty\end{array}$ & 尺े & $\frac{\infty}{\pi}$ & & ठू' & $\frac{9}{i}$ & 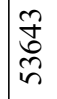 & $\begin{array}{l}\tilde{Z} \\
\infty \\
\stackrel{D}{f}\end{array}$ & 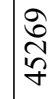 & 竎 & & 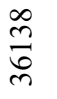 & 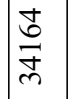 & 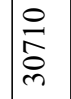 & $\begin{array}{l}0 \\
\infty \\
\infty \\
\stackrel{\infty}{\sim}\end{array}$ & $\begin{array}{l}\hat{\hat{ิ}} \\
\hat{\widehat{N}}\end{array}$ & $\begin{array}{l}\infty \\
\infty \\
\infty \\
\end{array}$ & 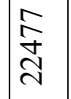 & $\begin{array}{l}\tilde{\infty} \\
\tilde{N} \\
\hat{N}\end{array}$ & 京 & 令 & 导 \\
\hline & & t & 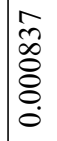 & 离 & 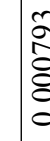 & & 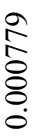 & $\begin{array}{l}\text { to } \\
\stackrel{0}{0} \\
\vdots \\
0 \\
0\end{array}$ & 麁 & 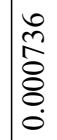 & 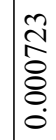 & ఏ & & 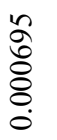 & $\mid \begin{array}{l}\infty \\
0 \\
0 \\
0 \\
0 \\
0\end{array}$ & $\mid \begin{array}{l}1 \\
0 \\
0 \\
\vdots \\
0 \\
0 \\
0\end{array}$ & $\mid \begin{array}{l}\vec{n} \\
\vdots \\
0 \\
0 \\
0 \\
0\end{array}$ & 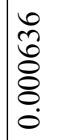 & 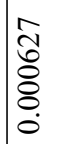 & $\begin{array}{l}\frac{1}{0} \\
\overline{8} \\
8 \\
0 \\
0\end{array}$ & $\mid \begin{array}{l}\hat{\delta} \\
0 \\
0 \\
0 \\
0 \\
0\end{array}$ & 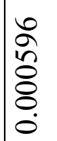 & 送 & 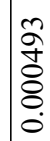 \\
\hline & & $\ddot{\sim}$ & $\begin{array}{l}\widetilde{d} \\
\stackrel{\sigma}{\gamma}\end{array}$ & & \begin{tabular}{l}
0 \\
\multirow{y}{y}{}
\end{tabular} & & $\bar{\partial}$ & $\stackrel{\infty}{\stackrel{\infty}{\xi}}$ & $\begin{array}{l}\circ \\
\& \\
\stackrel{+}{q}\end{array}$ & $\begin{array}{l}0 \\
\delta \\
\delta \\
\sigma\end{array}$ & $\begin{array}{l}\hat{\infty} \\
\stackrel{m}{m}\end{array}$ & ळે & & $\mid \begin{array}{c} \pm \\
\infty \\
m \\
m\end{array}$ & $\underset{\substack{a \\
\infty \\
m}}{a}$ & $\mid \begin{array}{l}0 \\
\infty \\
\infty \\
\text { m }\end{array}$ & $\begin{array}{l}\mathbb{d} \\
\text { m }\end{array}$ & 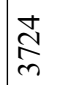 & 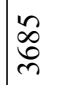 & 足 & 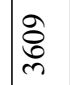 & $\stackrel{\substack{n \\
\tilde{n}}}{n}$ & 命 & $\mid \begin{array}{l}n \\
n \\
n \\
m\end{array}$ \\
\hline రే & 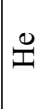 & 总 & $\stackrel{\text { N̦}}{=}$ & ¿ & t & & $\stackrel{\circ}{\circ}$ & $\underset{\sim}{\widetilde{\sigma}}$ & $\underset{-}{3}$ & 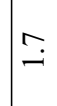 & . & $\stackrel{0}{0}$ & & 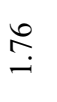 & $\stackrel{ }{-}$ & $\stackrel{+}{\infty}$ & $\stackrel{\vartheta}{-}$ & $\stackrel{0}{\stackrel{0}{+}}$ & Iે & $\stackrel{\mathcal{I}}{\stackrel{\sim}{-}}$ & ले & ְొ & $\stackrel{m}{m}$ & $\stackrel{\text { }}{\overbrace{}}$ \\
\hline $\begin{array}{l}\frac{t}{\sigma} \\
\frac{\overrightarrow{0}}{\Xi}\end{array}$ & 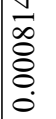 & $\vec{z}$ & 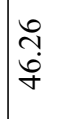 & $\begin{array}{l}\hat{\alpha} \\
\infty \\
-\infty\end{array}$ & 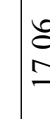 & & $n$ & 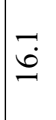 & 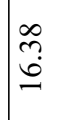 & $\begin{array}{l}\overrightarrow{0} \\
\dot{0}\end{array}$ & : & $\underline{\widehat{z}}$ & & लై & 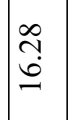 & $\begin{array}{l}\vec{\infty} \\
\dot{\varphi} \\
-\end{array}$ & సֶ. & $\begin{array}{l}\hat{b} \\
\pm \\
\pm\end{array}$ & $\stackrel{f}{\stackrel{f}{\ddagger}}$ & $\begin{array}{l}\mathfrak{a} \\
\pm \\
\pm\end{array} \mid$ & $\begin{array}{l} \pm \\
\text { mे }\end{array}$ & $\begin{array}{l}\vec{\sigma} \\
\ddot{\sigma}\end{array}$ & $\begin{array}{l}\hat{\sigma} \\
\dot{2}\end{array}$ & 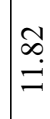 \\
\hline $\begin{array}{l}\stackrel{\mathscr{a}}{\sharp} \\
\frac{\ddot{\theta}}{\Xi}\end{array}$ & $\begin{array}{l}\infty \\
⿱ ㇒ \\
\mathscr{f}\end{array}$ & 䔍 & $\stackrel{尺}{\stackrel{\Xi}{\Xi}}$ & $\begin{array}{l}+ \\
\stackrel{2}{2}\end{array}$ & 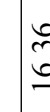 & & $\underset{n}{\vec{n}}$ & $\begin{array}{l}\infty \\
m \\
n \\
n\end{array}$ & $\overrightarrow{\tilde{\sigma}}$ & $\vec{ָ}$ & $\begin{array}{l}\infty \\
n \\
\infty \\
\infty \\
-1\end{array}$ & $\stackrel{\overbrace{}}{\mathcal{7}}$ & & $\begin{array}{l}m \\
\infty \\
\infty\end{array}$ & $\left|\begin{array}{c} \pm \\
m \\
\infty \\
-\infty\end{array}\right|$ & ô. & $\stackrel{m}{a}$ & స్+ & $\underset{0}{0}$ & $\begin{array}{l}\tilde{\hat{n}} \\
\underline{n}\end{array}$ & $\begin{array}{l}\vec{\sigma} \\
\ddot{n}\end{array}$ & $\begin{array}{l}\hat{i} \\
\ddot{n}\end{array}$ & $\begin{array}{l}n \\
n \\
n\end{array}$ & $\stackrel{\mathscr{m}}{\underset{ \pm}{ \pm}}$ \\
\hline$\underset{\Xi}{\stackrel{\Xi}{\Xi}}$ & 㒸 & 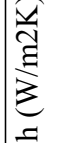 & 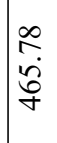 & $\begin{array}{l}\hat{\sigma} \\
\tilde{\sigma}\end{array}$ & $\begin{array}{l}\text { in } \\
\text { š } \\
\end{array}$ & & $\cong$ & ¿े & $\stackrel{ \pm}{ \pm}$ & $\begin{array}{l}\infty \\
\infty \\
\infty \\
\infty\end{array}$ & $\mid \begin{array}{l}0 \\
\infty \\
\infty \\
\infty \\
-1\end{array}$ & $\frac{n}{\infty}$ & & $\begin{array}{l}8 \\
\dot{\partial} \\
\dot{\infty} \\
\infty\end{array}$ & $\begin{array}{l}\hat{\infty} \\
\infty \\
\infty \\
\infty\end{array}$ & 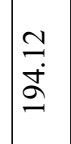 & $\begin{array}{l}\infty \\
\stackrel{\infty}{\alpha} \\
\infty\end{array}$ & 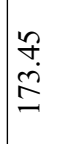 & 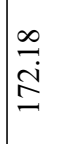 & $\mid \begin{array}{l}\infty \\
\dot{I} \\
\Xi\end{array}$ & $\begin{array}{l}0 \\
\infty \\
0 \\
0 \\
0\end{array}$ & 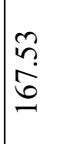 & $\stackrel{\Xi}{\stackrel{2}{2}}$ & $\begin{array}{l}m \\
\tilde{n} \\
\infty \\
\dot{0}\end{array}$ \\
\hline$\sum_{\text {至 }}$ & $\dot{m}$ & $\begin{array}{l}\text { త્ } \\
\text { ఏ } \\
= \\
=\end{array}$ & $\stackrel{\infty}{\stackrel{\infty}{r}}$ & $\underset{\substack{n\\
}}{\sim}$ & $\begin{array}{l}\infty \\
\infty \\
\infty \\
\sim \\
\end{array}$ & & ָ̊. & ঔ̊ & $\mid \begin{array}{l}0 \\
\infty \\
\infty \\
\infty \\
\sim\end{array}$ & $\begin{array}{l}\vec{\infty} \\
\infty \\
\infty \\
\infty\end{array}$ & $\begin{array}{l}\infty \\
\dot{\infty} \\
\stackrel{\infty}{\sim} \\
\stackrel{n}{n}\end{array}$ & तै & & $\stackrel{m}{\stackrel{N}{\approx}}$ & 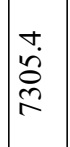 & $\overline{\grave{\Sigma}}$ & ते & 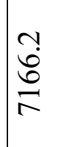 & $\begin{array}{l}n \\
\infty \\
\frac{\infty}{n}\end{array}$ & $\frac{\vec{Q}}{i}$ & $\stackrel{\infty}{\stackrel{2}{\gtrless}}$ & 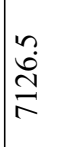 & $\stackrel{\curvearrowright}{\stackrel{\curvearrowright}{\curvearrowright}}$ & 영 \\
\hline 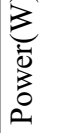 & $\hat{\imath}$ & $\vec{F}$ & 命 & $\frac{\hat{i}}{\mathrm{i}}$ & $\frac{\infty}{m}$ & & $\begin{array}{l}\text { है } \\
\text { ते } \\
\text { n. }\end{array}$ & $\frac{\partial}{\stackrel{m}{m}}$ & $\underset{m}{\stackrel{m}{n}}$ & $\begin{array}{l}\text { f } \\
\dot{m} \\
\tilde{m}\end{array}$ & $\begin{array}{l}\bar{b} \\
\dot{d} \\
\dot{\sigma}\end{array}$ & $\begin{array}{l}n \\
2 \\
n \\
m\end{array}$ & & $\mid \begin{array}{l}\infty \\
\infty \\
\dot{0} \\
\dot{0} \\
m\end{array}$ & $\begin{array}{l}\bar{a} \\
\overline{0} \\
\tilde{n}\end{array}$ & $\begin{array}{l}\infty \\
\hat{n} \\
\tilde{n}\end{array}$ & $\mid \begin{array}{l}\tilde{0} \\
\dot{\infty} \\
\infty \\
m\end{array}$ & $\begin{array}{l}\partial \\
\alpha \\
\infty \\
\infty\end{array}$ & 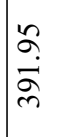 & $\begin{array}{l}\alpha \\
\alpha \\
\alpha \\
\tilde{m}\end{array}$ & $\begin{array}{l}\infty \\
\infty \\
\dot{\sigma} \\
\dot{\sigma}\end{array}$ & $\begin{array}{l}\infty \\
\infty \\
\dot{\sigma} \\
\dot{q}\end{array}$ & $\begin{array}{l}2 \\
i n \\
\frac{7}{\gamma}\end{array}$ & $\stackrel{\stackrel{+}{?}}{\stackrel{\text { f }}{+}}$ \\
\hline$\sum_{0}^{\pi}$ & $\stackrel{t}{0}$ & 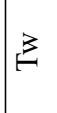 & $\underset{\sim}{\stackrel{\sim}{N}}$ & $\begin{array}{l}+ \\
\infty \\
0 \\
\qquad \\
n\end{array}$ & $\begin{array}{l}0 \\
\text { s } \\
\text { d }\end{array}$ & & 㐫 & 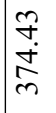 & 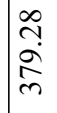 & $\begin{array}{l}\tilde{N} \\
\stackrel{\infty}{+} \\
\tilde{m}\end{array}$ & $\begin{array}{l}\infty \\
\infty \\
\infty \\
\infty \\
\infty \\
\infty\end{array}$ & $\frac{9}{6}$ & & $\begin{array}{l}n \\
\stackrel{n}{+} \\
\dot{+}\end{array}$ & $\begin{array}{l}\vec{N} \\
\vec{o} \\
\dot{q}\end{array}$ & $\begin{array}{l}\stackrel{\vec{n}}{\Xi} \\
\vec{F}\end{array}$ & $\begin{array}{l}\varnothing \\
\infty \\
\infty \\
\forall \\
\forall\end{array}$ & $\stackrel{\bar{m}}{\stackrel{\vartheta}{\gamma}}$ & $\begin{array}{l}\tilde{\sigma} \\
\dot{m} \\
\tilde{\gamma}\end{array}$ & $\begin{array}{l}\tilde{0} \\
\dot{0} \\
\tilde{\gamma}\end{array}$ & 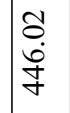 & $\begin{array}{l}n \\
\tilde{n} \\
\tilde{y} \\
y\end{array}$ & 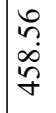 & 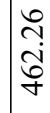 \\
\hline 离 & $\bar{m}$ & 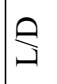 & $N$ & $\vec{\infty}$ & $\bar{J}$ & & ָ̃. & ָั & $\stackrel{m}{i}$ & $\begin{array}{c}m \\
\infty \\
m\end{array}$ & $\stackrel{+}{+}$ & $\begin{array}{l}7 \\
8\end{array}$ & & $\begin{array}{l}n \\
0 \\
i n\end{array}$ & 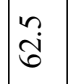 & $\mid \begin{array}{l}n \\
\infty \\
\infty \\
0\end{array}$ & $\begin{array}{l}0 \\
\dot{I} \\
\end{array}$ & $\ddot{\infty}$ & $\hat{\infty}$ & $\hat{i}$ & $\mid \begin{array}{l}\infty \\
\infty \\
\infty\end{array}$ & 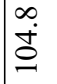 & $\stackrel{\varrho}{\varrho}$ & $\stackrel{\vec{b}}{=}$ \\
\hline
\end{tabular}




\begin{tabular}{|c|c|c|c|c|c|c|c|c|c|c|c|c|c|c|c|c|c|c|c|c|c|c|c|}
\hline & & $\vec{\Delta}$ & 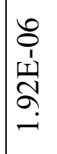 & $\begin{array}{l}8 \\
0 \\
1 \\
1 \\
\\
\infty \\
- \\
-1\end{array}$ & 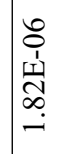 & 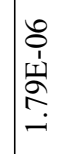 & 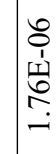 & $\mid \begin{array}{c}0 \\
0 \\
\frac{1}{1} \\
\\
7 \\
7\end{array}$ & 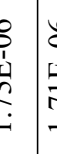 & 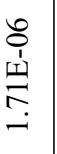 & 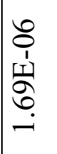 & 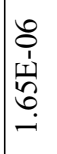 & 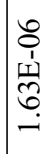 & $\begin{array}{l}0 \\
0 \\
1 \\
1 \\
6 \\
-1 \\
-1\end{array}$ & 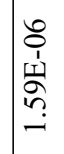 & 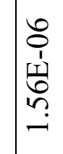 & $\begin{array}{c}8 \\
o \\
1 \\
1 \\
n \\
n \\
-\end{array}$ & 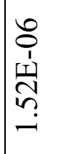 & 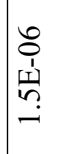 & $\mid \begin{array}{c}0 \\
0 \\
1 \\
1 \\
\infty \\
\infty \\
-i \\
-1\end{array}$ & 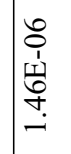 & 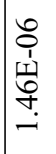 & 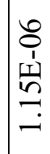 \\
\hline & & $\ddot{~}$ & 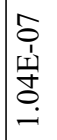 & $\begin{array}{l}\infty \\
0 \\
1 \\
1 \\
1 \\
0 \\
\infty \\
\infty\end{array}$ & 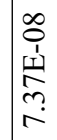 & 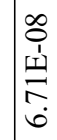 & $\begin{array}{l}\infty \\
0 \\
0 \\
1 \\
\infty \\
\infty \\
n\end{array}$ & 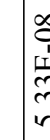 & 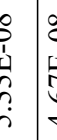 & 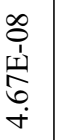 & 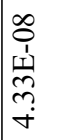 & $\begin{array}{l}\infty \\
0 \\
0 \\
1 \\
\infty \\
\infty \\
\end{array}$ & 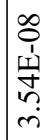 & 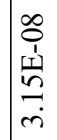 & 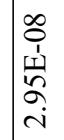 & 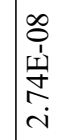 & 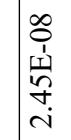 & 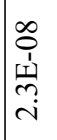 & 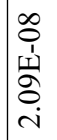 & 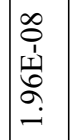 & 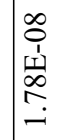 & 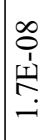 & 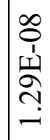 \\
\hline & & $\ddot{\sim}$ & 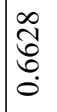 & $\begin{array}{l}0 \\
\text { ర్రి } \\
\stackrel{0}{0} \\
0\end{array}$ & 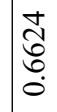 & त्र్రి & 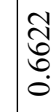 & $\bar{a}$ & $\begin{array}{l}\bar{y} \\
\dot{b} \\
\dot{0}\end{array}$ & $\begin{array}{l}\overrightarrow{\widetilde{d}} \\
\stackrel{0}{0} \\
\dot{0}\end{array}$ & ठ․ & ర్రి & $\begin{array}{l}1 \\
0 \\
0 \\
0 \\
0\end{array}$ & 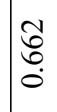 & ర్ర & 它 & $\begin{array}{l}\overrightarrow{\widetilde{d}} \\
\stackrel{0}{0}\end{array}$ & $\begin{array}{l}\overrightarrow{\widetilde{J}} \\
\stackrel{0}{0} \\
\stackrel{0}{0}\end{array}$ & $\begin{array}{l}\overrightarrow{\widehat{V}} \\
\stackrel{0}{0} \\
\dot{0}\end{array}$ & 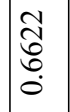 & $\begin{array}{l}\text { तु } \\
\text { ర̊: } \\
0\end{array}$ & 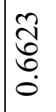 & त్ర్రి \\
\hline & & $\mathscr{\Xi}$ & 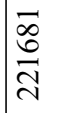 & $\begin{array}{l}\overrightarrow{\widehat{\sigma}} \\
\hat{\sigma}\end{array}$ & $\begin{array}{l}\vec{\infty} \\
\infty \\
ٍ \\
ٍ\end{array}$ & $\frac{\grave{c}}{\tilde{c}}$ & $\begin{array}{l}\tilde{\hat{b}} \\
\stackrel{0}{0}\end{array}$ & 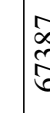 & \begin{tabular}{l|l}
0 \\
$n$ \\
0 \\
0 & $\vdots$ \\
\end{tabular} & 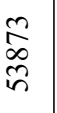 & $\mid \begin{array}{l}\infty \\
\infty \\
i \\
\forall\end{array}$ & 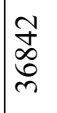 & $\frac{8}{m}$ & 离 & 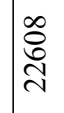 & $\underset{\tilde{N}}{\tilde{\approx}}$ & 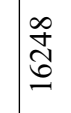 & 离 & $\stackrel{\widehat{\beth}}{\stackrel{\beth}{\beth}}$ & $\mid \begin{array}{l}n \\
2 \\
0 \\
0\end{array}$ & $\frac{\hat{\sigma}}{a}$ & $\underset{\infty}{\stackrel{N}{N}}$ & $\begin{array}{l}+ \\
2 \\
\infty \\
n\end{array}$ \\
\hline & & む & 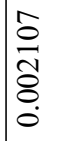 & 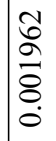 & $\begin{array}{l}0 \\
0 \\
\infty \\
\\
0 \\
0\end{array}$ & $\mid$\begin{tabular}{l}
$\infty$ \\
$\stackrel{\infty}{\sigma}$ \\
$\stackrel{\sigma}{0}$ \\
\hdashline
\end{tabular} & $\mid \begin{array}{c}\infty \\
0 \\
0 \\
8 \\
0 \\
0\end{array}$ & b & 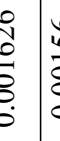 & 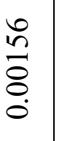 & $\mid \begin{array}{l}4 \\
\stackrel{0}{n} \\
0 \\
0 \\
0 \\
0\end{array}$ & 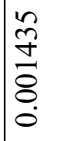 & 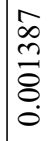 & 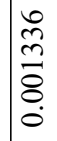 & & $\mid \begin{array}{c}\text { I } \\
\stackrel{\Xi}{8} \\
\stackrel{0}{0}\end{array}$ & $\frac{\sigma}{\sigma}$ & $\mid \begin{array}{l}\infty \\
\stackrel{n}{a} \\
\overline{8} \\
0 \\
0\end{array}$ & 光 & $\mid \begin{array}{l}\stackrel{\partial}{o} \\
\stackrel{0}{0} \\
\stackrel{0}{0} \\
\dot{0}\end{array}$ & $\begin{array}{l}\vec{n} \\
\vdots \\
\vdots \\
0 \\
0\end{array}$ & 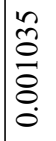 & $\begin{array}{l}8 \\
0 \\
0 \\
0 \\
0 \\
0 \\
0 \\
0\end{array}$ \\
\hline & & $\approx$ & $\begin{array}{l}\tilde{\infty} \\
\tilde{\gamma}\end{array}$ & 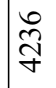 & $\frac{\hat{\sigma}}{\gamma}$ & $\begin{array}{l}\infty \\
\infty \\
\text { ले }\end{array}$ & $\begin{array}{l}\text { ठ } \\
\infty \\
\infty\end{array}$ & 政 & \begin{tabular}{l|l}
0 & 5 \\
$n$ & $y$
\end{tabular} & 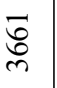 & 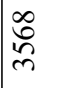 & $\vec{\infty}$ & సે & స్ల & 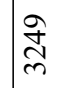 & $\frac{\bar{\infty}}{m}$ & $\stackrel{\varrho}{=}$ & 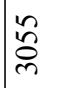 & ঐু & $\vec{F}$ & $\begin{array}{l}\infty \\
\infty \\
\infty \\
\sim\end{array}$ & $\mid \begin{array}{c}\widehat{\infty} \\
\stackrel{\sim}{\sim}\end{array}$ & 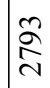 \\
\hline రే & $\ddot{I}$ & 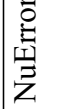 & $\hat{\mathrm{I}}$ & $\hat{\tilde{c}}$ & . & $\stackrel{\overbrace{}}{\dddot{\vartheta}}$ & 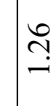 & 7 & $=$ & $\stackrel{ \pm}{\underset{I}{S}}$ & $\stackrel{\sim}{\longrightarrow}$ & $\stackrel{0}{\longrightarrow}$ & $\stackrel{\text { aे }}{\text {. }}$ & $\stackrel{m}{\rightleftarrows}$ & $\stackrel{\Xi}{\Delta}$ & $\underset{\text { Ca }}{\widehat{d}}$ & {$\left[\begin{array}{l}n \\
\infty \\
0\end{array}\right.$} & $\mid \begin{array}{l}\infty \\
\infty \\
0 \\
0\end{array}$ & $\stackrel{\infty}{\infty}$ & $\stackrel{尺}{\hat{0}}$ & $\frac{\pi}{\dot{0}}$ & 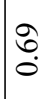 & : \\
\hline 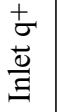 & 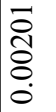 & $\vec{z}$ & 苗 & ָָ & $\underset{\widetilde{\sigma}}{\tilde{\sigma}}$ & $\begin{array}{l}\stackrel{n}{\sim} \\
\dot{I}\end{array}$ & $\overrightarrow{\dot{d}}$ & $\stackrel{f}{f}$ & $\stackrel{f}{c}$ & 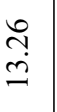 & $\vec{m}$ & 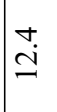 & $\stackrel{\infty}{\stackrel{\infty}{\beth}}$ & $\hat{\varrho}$ & 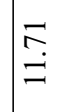 & 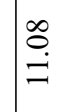 & 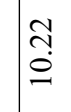 & $\partial$ & $\begin{array}{l}\hat{b} \\
\sigma\end{array}$ & 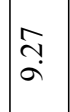 & $\begin{array}{l}\infty \\
\infty \\
\infty \\
\infty\end{array}$ & $\begin{array}{l}\hat{b} \\
\infty\end{array}$ & ले \\
\hline 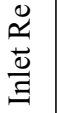 & 袋 & 薑 & 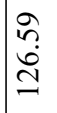 & $\stackrel{\sim}{\stackrel{\sim}{*}}$ & 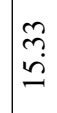 & $\begin{array}{l}\tilde{n} \\
m \\
m\end{array}$ & 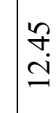 & 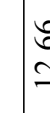 & 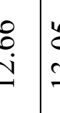 & $\begin{array}{l}n \\
\ddot{n} \\
\ddot{n}\end{array}$ & $\begin{array}{l}\overrightarrow{+} \\
\infty \\
\end{array}$ & $\stackrel{゚}{\stackrel{i}{a}}$ & $\vec{m}$ & $\cong$ & $\vec{\varrho}$ & 芯 & of & $\stackrel{\infty}{\stackrel{0}{\varrho}}$ & $\begin{array}{l}\text { ț } \\
\stackrel{0}{0}\end{array}$ & $\mid \begin{array}{l}\infty \\
1 \\
0 \\
0\end{array}$ & 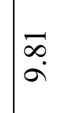 & $\begin{array}{l}6 \\
0 \\
0\end{array}$ & $\frac{n}{a}$ \\
\hline$\underset{\Xi}{\underline{G}}$ & 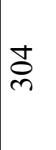 & 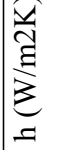 & $\begin{array}{l}\stackrel{ \pm}{n} \\
\bar{n}\end{array}$ & $\frac{\vec{n}}{\dot{\sim}}$ & $\stackrel{i}{\stackrel{2}{I}}$ & $\begin{array}{l}a \\
\dot{0} \\
+ \\
-1\end{array}$ & 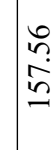 & $\mid \begin{array}{l}1 \\
2 \\
6 \\
6\end{array}$ & & $\begin{array}{l}\vec{\sigma} \\
\overrightarrow{0} \\
-\end{array}$ & $\begin{array}{l}\infty \\
\infty \\
\vdots \\
ٍ \\
0\end{array}$ & $\begin{array}{l}\stackrel{?}{+} \\
\infty \\
\infty \\
\sim\end{array}$ & $\begin{array}{l}0 \\
0 \\
0 \\
0\end{array}$ & $\begin{array}{l}\tilde{b} \\
\dot{0} \\
\tilde{n}\end{array}$ & 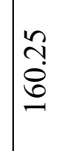 & 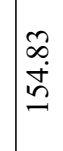 & $\begin{array}{l}\tilde{\infty} \\
\dot{y} \\
\tilde{y}\end{array}$ & 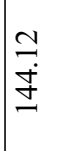 & $\begin{array}{l}\stackrel{g}{i} \\
\stackrel{y}{I}\end{array}$ & $\begin{array}{l} \pm \\
\dot{g} \\
\end{array}$ & 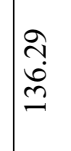 & 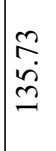 & 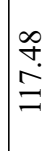 \\
\hline 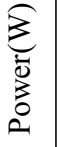 & $\underset{\infty}{\stackrel{\infty}{\infty}}$ & 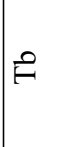 & $\begin{array}{l}\infty \\
\stackrel{\infty}{+} \\
\stackrel{0}{0} \\
\stackrel{n}{n}\end{array}$ & $\begin{array}{l}n \\
\stackrel{n}{n} \\
\sim\end{array}$ & $\begin{array}{l}\hat{b} \\
\dot{+} \\
\text { के }\end{array}$ & $\mid \begin{array}{l}n \\
\dot{0} \\
\dot{b} \\
m \\
m\end{array}$ & ले & $\begin{array}{l}\alpha \\
0 \\
d \\
c\end{array}$ & 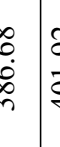 & $\frac{\text { S̆ }}{\dot{\sigma}}$ & $\frac{\Xi}{\frac{J}{\sigma}}$ & $\begin{array}{l}\stackrel{\infty}{\sim} \\
\underset{\sim}{\sim} \\
\stackrel{\gamma}{+}\end{array}$ & 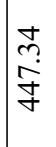 & 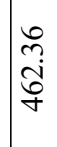 & 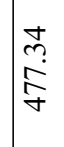 & $\mid \begin{array}{l}\tilde{\hat{N}} \\
\tilde{\alpha} \\
\tilde{\sigma}\end{array}$ & $\begin{array}{l}\circ \\
\circ \\
\check{0} \\
\stackrel{2}{n}\end{array}$ & $\begin{array}{l}\tilde{\sigma} \\
\stackrel{\vec{\pi}}{\tilde{N}} \\
\text { n. }\end{array}$ & 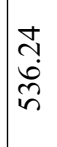 & $\mid \begin{array}{l}\tilde{D} \\
0 \\
0 \\
n \\
n\end{array}$ & 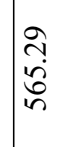 & $\begin{array}{l}\frac{0}{2} \\
\stackrel{a}{i n} \\
\text { in }\end{array}$ & $\begin{array}{l}\infty \\
\alpha \\
\alpha \\
\tilde{n}\end{array}$ \\
\hline 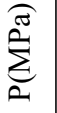 & $\stackrel{t}{0}$ & 3 & 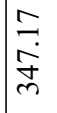 & $\begin{array}{l}\infty \\
\infty \\
\infty \\
\gamma \\
\gamma\end{array}$ & 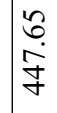 & $\begin{array}{l}\infty \\
0 \\
0 \\
\dot{\sigma} \\
+ \\
\end{array}$ & 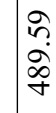 & 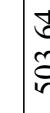 & $\begin{array}{l}\dot{d} \\
\dot{b} \\
\dot{n} \\
\end{array}$ & $\begin{array}{l}\hat{\sigma} \\
\hat{\sigma} \\
\tilde{n}\end{array}$ & $\begin{array}{l}2 \\
\infty \\
\infty \\
i \\
n\end{array}$ & $\begin{array}{l}\infty \\
\infty \\
\infty \\
\sim \\
\dot{n}\end{array}$ & ले & $\begin{array}{l}n \\
n \\
0 \\
i n \\
n\end{array}$ & 官 & $\begin{array}{l}\hat{n} \\
\infty \\
0 \\
0\end{array}$ & $\begin{array}{l}\stackrel{̃}{\sim} \\
\stackrel{్}{\sigma}\end{array}$ & 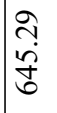 & $\begin{array}{l}0 \\
6 \\
8 \\
8 \\
0\end{array}$ & $\left|\begin{array}{c}0 \\
\hat{i} \\
\hat{\sigma}\end{array}\right|$ & $\begin{array}{l}\dot{0} \\
\infty \\
\tilde{\sigma} \\
0\end{array}$ & $\frac{\infty}{\frac{\infty}{0}}$ & $\begin{array}{l}\mathcal{O} \\
\mathbb{N} \\
\end{array}$ \\
\hline Ü & $\tilde{m}$ & $\stackrel{2}{a}$ & $\sim$ & $\vec{\infty}$ & $\exists$ & ֻั. & तु. & $\overbrace{}^{2}$ & nู. & $\begin{array}{c}m \\
\infty \\
\infty \\
m\end{array}$ & $\stackrel{\nabla}{\dot{f}}$ & ti. & $\begin{array}{l}n \\
0 \\
0 \\
n\end{array}$ & in & $\begin{array}{l}n \\
\infty \\
\infty \\
\infty\end{array}$ & $\begin{array}{l}0 \\
\dot{I}\end{array}$ & $\ddot{\infty}$ & $\hat{\varnothing}$ & & $\left|\begin{array}{l}\infty \\
\infty \\
\infty\end{array}\right|$ & 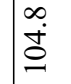 & $\stackrel{\partial}{\varrho}$ & 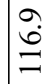 \\
\hline
\end{tabular}




\begin{tabular}{|c|c|c|c|c|c|c|c|c|c|c|c|c|c|c|c|c|c|c|c|c|c|c|c|}
\hline & & $\vec{\Delta}$ & $\begin{array}{l}0 \\
\vdots \\
1 \\
\text { ț } \\
\text { b } \\
\text { i }\end{array}$ & $\begin{array}{l}0 \\
0 \\
1 \\
1 \\
\\
i n \\
i\end{array}$ & 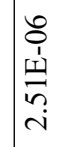 & 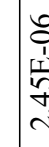 & & 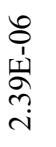 & 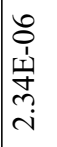 & 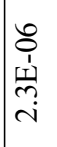 & 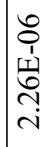 & 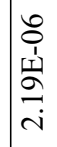 & 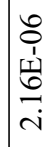 & 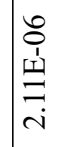 & 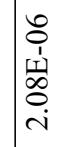 & $\mid \begin{array}{c}0 \\
0 \\
1 \\
\omega \\
0 \\
i \\
i\end{array}$ & 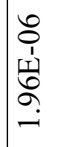 & 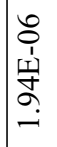 & $\begin{array}{c}8 \\
0 \\
1 \\
1 \\
\vdots \\
2 \\
-1 \\
-1\end{array}$ & $\begin{array}{l}8 \\
0 \\
1 \\
1 \\
\infty \\
\infty \\
- \\
-\end{array}$ & 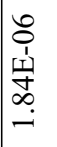 & $\begin{array}{l}0 \\
0 \\
1 \\
1 \\
\\
\infty \\
-1 \\
-1\end{array}$ & 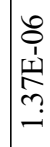 \\
\hline & & $\stackrel{\oplus}{\infty}$ & 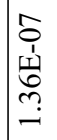 & 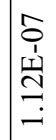 & $\begin{array}{l}\infty \\
0 \\
0 \\
1 \\
\alpha \\
\sigma \\
\sigma \\
\sigma\end{array}$ & $\mid \begin{array}{l}\infty \\
0 \\
1 \\
\frac{1}{2} \\
\infty \\
\infty\end{array}$ & & 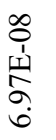 & $\mid \begin{array}{l}\infty \\
0 \\
0 \\
1 \\
11 \\
0 \\
0 \\
0\end{array}$ & $\begin{array}{l}\infty \\
0 \\
0 \\
1 \\
1 \\
0 \\
0 \\
i n \\
i n\end{array}$ & 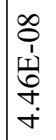 & $\begin{array}{l}\infty \\
0 \\
1 \\
1 \\
\\
\sigma \\
\dot{m}\end{array}$ & 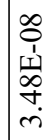 & 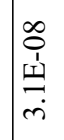 & $\mid \begin{array}{l}\infty \\
0 \\
\dot{1} \\
\infty \\
\stackrel{\infty}{0} \\
i \\
i\end{array}$ & 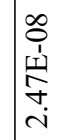 & 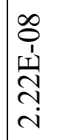 & 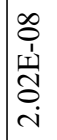 & $\begin{array}{c}\infty \\
0 \\
0 \\
1 \\
1 \\
\infty \\
\infty \\
-i\end{array}$ & 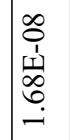 & 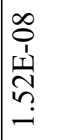 & 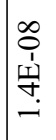 & 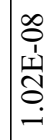 \\
\hline & & 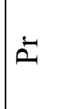 & $\begin{array}{l}\hat{\widehat{V}} \\
\stackrel{0}{0} \\
\stackrel{0}{0}\end{array}$ & 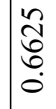 & 芯 & శ్ర & & $\begin{array}{l}\overrightarrow{\widehat{C}} \\
\stackrel{0}{0} \\
\stackrel{0}{0}\end{array}$ & : & ర్ర & ర్ & ర్ర & $\begin{array}{l}\overrightarrow{\widetilde{J}} \\
0 \\
0 \\
0\end{array}$ & $\overrightarrow{\widetilde{d}}$ & $\begin{array}{l}\tilde{Z} \\
\text { ర్ర } \\
\stackrel{0}{0}\end{array}$ & $\mid \begin{array}{l}\tilde{\delta} \\
0 \\
0 \\
0\end{array}$ & 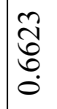 & 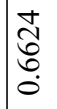 & $\begin{array}{l}2 \\
\hat{C} \\
0 \\
0 \\
0\end{array}$ & $\begin{array}{l}0 \\
\mathbb{Z} \\
0 \\
0 \\
0\end{array}$ & 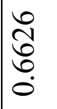 & 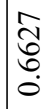 & 芯 \\
\hline & & $\mathscr{J}$ & 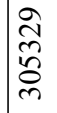 & $\begin{array}{l}\hat{n} \\
\tilde{n} \\
\vec{n}\end{array}$ & $\begin{array}{l}\hat{\sigma} \\
\tilde{N} \\
i n \\
0\end{array}$ & 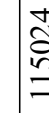 & & 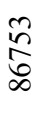 & 莒 & 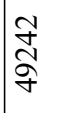 & $\mid \begin{array}{c}\mathcal{N} \\
\infty \\
\infty \\
\infty \\
\infty\end{array}$ & $\frac{n}{2}$ & 悉 & $\bar{\sigma}$ & $\begin{array}{l}2 \\
\tilde{\sigma} \\
\underline{6}\end{array}$ & 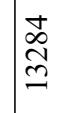 & $\underset{\sigma}{\stackrel{a}{o}}$ & ஓू. & $\stackrel{2}{\stackrel{2}{r}}$ & $\begin{array}{l}\vec{\infty} \\
\hat{\sigma}\end{array}$ & $\begin{array}{l}\text { } \\
\tilde{n} \\
n\end{array}$ & 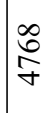 & $\underset{\sim}{\stackrel{\hat{N}}{m}}$ \\
\hline & & $t$ & 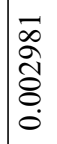 & 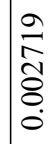 & 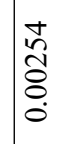 & $\mid \begin{array}{l}\Re \\
\tilde{I} \\
\varrho \\
\varrho\end{array}$ & & $\begin{array}{l}\text { ते } \\
\text { ते } \\
\stackrel{0}{0} \\
\dot{0}\end{array}$ & 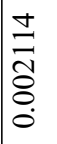 & 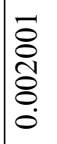 & 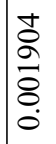 & 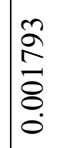 & 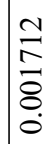 & $\begin{array}{l}\overline{\hat{\sigma}} \\
\stackrel{0}{0} \\
\stackrel{0}{0} \\
\dot{0}\end{array}$ & $\begin{array}{l}0 \\
\stackrel{2}{n} \\
\overline{8} \\
0 \\
0\end{array}$ & $\mid \begin{array}{c}\stackrel{n}{+} \\
\stackrel{8}{8} \\
\stackrel{8}{0}\end{array}$ & 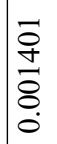 & 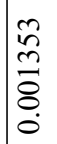 & $\begin{array}{l}\tilde{D} \\
\bar{n} \\
\stackrel{0}{0} \\
0 \\
0\end{array}$ & 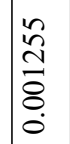 & $\stackrel{1}{\dddot{8}}$ & $\underset{\square}{\overrightarrow{0}}$ & $\begin{array}{l}0 \\
0 \\
\infty \\
8 \\
0 \\
0 \\
0\end{array}$ \\
\hline & & $\ddot{\sim}$ & 斈 & 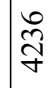 & 吉 & $\stackrel{\bar{\alpha}}{2}$ & & $\frac{m}{m}$ & 总 & 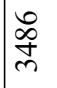 & 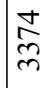 & 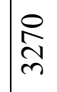 & $\stackrel{n}{\frac{n}{n}}$ & 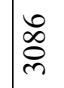 & 离 & $\mid \begin{array}{c}\infty \\
\stackrel{\sim}{N}\end{array}$ & $\begin{array}{l}\infty \\
\infty \\
\infty \\
\sim\end{array}$ & 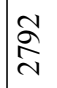 & $\underset{\grave{N}}{\stackrel{N}{ }}$ & $\vec{i}$ & 节 & 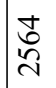 & त्ञ \\
\hline تే & $\stackrel{0}{1}$ & 总 & $\begin{array}{l}\stackrel{+}{n} \\
\dot{ \pm}\end{array}$ & 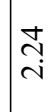 & $\hat{n}$ & $\grave{\lambda}$ & & oे & $\underset{\sigma}{\sigma}$ & $\hat{\alpha}$ & 声 & oे & 尚 & $\tilde{n}$ & $\because n$ & 龸 & 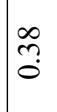 & ñ & $\stackrel{m}{\tilde{0}}$ & $\overline{\tilde{o}}$ & 3 & 3 & $\tilde{n}$ \\
\hline $\begin{array}{l}\frac{t}{\sigma} \\
\frac{\overrightarrow{0}}{\Xi}\end{array}$ & $\begin{array}{l}\hat{\infty} \\
\hat{\alpha} \\
\delta \\
0 \\
0 \\
0\end{array}$ & $\vec{z}$ & 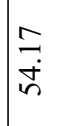 & oे & సु. & İ & & $\begin{array}{l}\infty \\
\stackrel{0}{ } \\
\stackrel{j}{J}\end{array}$ & $\stackrel{\stackrel{F}{.}}{=}$ & ڤ̊ & @ֶ) & $\stackrel{1}{a}$ & $\mid \begin{array}{l}\hat{n} \\
\infty\end{array}$ & $\stackrel{\infty}{\stackrel{\infty}{r}}$ & 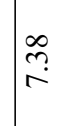 & $\overrightarrow{6}$ & $\overrightarrow{6}$ & $\begin{array}{l}\infty \\
i \\
i n\end{array}$ & $\begin{array}{l}a \\
\dot{v}\end{array} \mid$ & 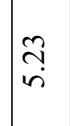 & \% & $\begin{array}{l}\infty \\
\infty \\
+\end{array}$ & $\underset{f}{\stackrel{f}{*}}$ \\
\hline $\begin{array}{l}\stackrel{\mathscr{a}}{\sharp} \\
\frac{\ddot{\theta}}{\Xi}\end{array}$ & $\frac{6}{8}$ & 衰 & 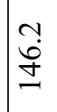 & 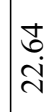 & ڤై & $\mid \begin{array}{l}\infty \\
\infty \\
m \\
0\end{array}$ & & $\stackrel{+}{\stackrel{t}{=}}$ & ¿̊. & $\stackrel{\text { ִֻ }}{\varrho}$ & $\stackrel{n}{a}$ & $\begin{array}{l}\hat{y} \\
\infty\end{array}$ & $\underset{r}{\infty}$ & ô & ְ̊. & $\underset{i n}{\mathbb{N}}$ & $\begin{array}{l}\hat{\partial} \\
\dot{i n}\end{array}$ & $\stackrel{⿱ 亠}{\stackrel{+}{+}}$ & $\vec{n}$ & $\stackrel{\Re}{+}$ & $\begin{array}{l}\infty \\
\stackrel{+}{+} \\
\dot{0}\end{array}$ & 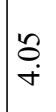 & 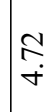 \\
\hline$\underset{\Xi}{\stackrel{\Xi}{\Xi}}$ & 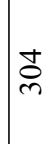 & 点 & 告 & 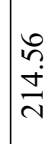 & 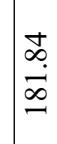 & $\begin{array}{l}\infty \\
\cdots \\
1 \\
0 \\
-1\end{array}$ & & $\begin{array}{l}\text { ते } \\
\text { ñ }\end{array}$ & $\begin{array}{l}\stackrel{0}{0} \\
\stackrel{+}{\Xi}\end{array}$ & \begin{tabular}{|l}
$\stackrel{U}{J}$ \\
$\stackrel{J}{I}$
\end{tabular} & $\begin{array}{l}\bar{a} \\
\infty \\
\tilde{n}\end{array}$ & $\begin{array}{l}\infty \\
2 \\
\vdots \\
ٍ \\
ٍ\end{array}$ & $\begin{array}{l}\stackrel{\Xi}{\Xi} \\
\cong\end{array}$ & $\begin{array}{l}\stackrel{0}{0} \\
\stackrel{d}{]} \\
=\end{array}$ & $\stackrel{0}{\stackrel{0}{\Xi}}$ & & $\begin{array}{l}0 \\
\stackrel{0}{\circ}\end{array}$ & $\begin{array}{l}0 \\
\alpha \\
\alpha\end{array}$ & $\begin{array}{l}2 \\
\dot{\alpha}\end{array}$ & 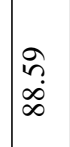 & $\vec{\nabla}$ & $\frac{1}{\infty}$ & $\begin{array}{l}\infty \\
0 \\
2 \\
2\end{array}$ \\
\hline$\sum_{\text {至 }}$ & $\dot{m}$ & $\begin{array}{l}\text { శี } \\
\text { ఏ } \\
= \\
=\end{array}$ & 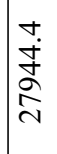 & 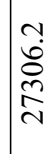 & $\frac{\stackrel{m}{I}}{\stackrel{\sim}{N}}$ & 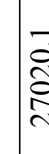 & & 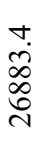 & $\begin{array}{l}\overrightarrow{1} \\
\text { in } \\
\hat{c} \\
\text { in }\end{array}$ & $\begin{array}{l}\text { aे } \\
\text { त్ర్ర } \\
\text { ָे }\end{array}$ & 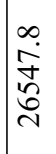 & $\begin{array}{l}\infty \\
\tilde{f} \\
\dot{\sigma} \\
\tilde{N}\end{array}$ & 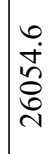 & $\begin{array}{l}0 \\
\frac{2}{2} \\
i\end{array}$ & 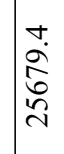 & $\begin{array}{l}n \\
\tilde{n} \\
\tilde{n}\end{array}$ & 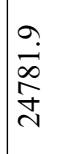 & $\begin{array}{l}\stackrel{+}{\dot{+}} \\
\stackrel{+}{\stackrel{+}{+}}\end{array}$ & 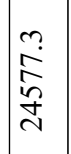 & $\begin{array}{l}\vec{m} \\
\stackrel{\tilde{J}}{\sim}\end{array}$ & 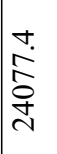 & 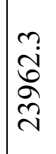 & $\begin{array}{l}n \\
\dot{0} \\
\tilde{\infty} \\
\infty \\
-1\end{array}$ \\
\hline 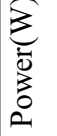 & $\begin{array}{l}\widehat{ָ} \\
\text { }\end{array}$ & 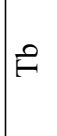 & 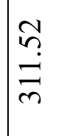 & $\begin{array}{l}\hat{\infty} \\
\hat{m} \\
\hat{m}\end{array}$ & $\begin{array}{l}\hat{\sigma} \\
\infty \\
\tilde{n} \\
m \\
n\end{array}$ & הิ & & 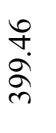 & $\begin{array}{l}\exists \\
\overrightarrow{\vec{I}} \\
\stackrel{f}{*}\end{array}$ & $\begin{array}{l}8 \\
\stackrel{0}{0} \\
\stackrel{y}{f}\end{array}$ & \begin{tabular}{l}
$m$ \\
\hdashline \\
$\dot{0}$ \\
+
\end{tabular} & $\begin{array}{l}\dot{a} \\
\infty \\
\infty \\
\dot{\sigma}\end{array}$ & $\begin{array}{l}\text { fo } \\
\dot{8} \\
\stackrel{8}{n}\end{array}$ & $\begin{array}{l}\stackrel{2}{\hat{n}} \\
\hat{n}\end{array}$ & 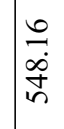 & $\begin{array}{l}\infty \\
0 \\
\infty \\
0 \\
0 \\
\infty\end{array}$ & $\begin{array}{l}n \\
\infty \\
\infty \\
\infty \\
\infty\end{array}$ & $\begin{array}{l}+ \\
\infty \\
\infty \\
\infty \\
0 \\
0\end{array}$ & $\begin{array}{l}n \\
\infty \\
\infty \\
\tilde{d}\end{array}$ & $\mid \begin{array}{l}n \\
\infty \\
\infty \\
0\end{array}$ & I & $\begin{array}{l}\sqrt[n]{n} \\
\infty \\
\infty \\
0\end{array}$ & in \\
\hline$\sum_{0}^{\pi}$ & $\stackrel{+}{0}$ & 3 & 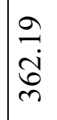 & 官 & 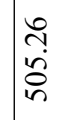 & $\begin{array}{l}\infty \\
0 \\
\vdots \\
i n\end{array}$ & & 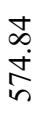 & గ̂̃ & $\begin{array}{l}\sigma \\
\infty \\
\stackrel{\sigma}{0}\end{array}$ & $\begin{array}{l}\hat{n} \\
i n \\
\tilde{b}\end{array}$ & ิㅗ & $\stackrel{\vec{\alpha}}{\stackrel{2}{\gtrless}}$ & $\begin{array}{l}\stackrel{9}{\overrightarrow{0}} \\
\stackrel{n}{n}\end{array}$ & $\frac{7}{2}$ & $\underset{\infty}{\stackrel{0}{\vec{\prime}}}$ & 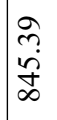 & $\frac{n}{\infty}$ & $\begin{array}{l}\infty \\
\infty \\
\infty \\
\infty \\
\infty\end{array}$ & $\begin{array}{l}\tilde{n} \\
\tilde{m} \\
\stackrel{\sim}{\alpha}\end{array}$ & $\begin{array}{l}0 \\
o \\
\partial \\
\sigma\end{array}$ & $\begin{array}{l}0 \\
\hat{0} \\
0\end{array}$ & શे \\
\hline 苋 & $m$ & 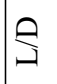 & $N$ & $\vec{\infty}$ & 王 & ปे & & ֶָ. & $\stackrel{m}{\stackrel{n}{n}}$ & $\begin{array}{c}m \\
\infty \\
\infty\end{array}$ & $\begin{array}{l}\dot{J} \\
\dot{f}\end{array}$ & ti. & $\begin{array}{l}n \\
0 \\
0\end{array}$ & 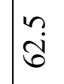 & $\begin{array}{l}n \\
\infty \\
\infty \\
\infty\end{array}$ & $\begin{array}{l}0 \\
\stackrel{+}{T}\end{array}$ & $\ddot{\infty}$ & $\hat{\infty}$ & $\hat{\check{a}}$ & $\mid \begin{array}{l}\infty \\
\infty \\
\infty\end{array}$ & 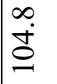 & $\stackrel{\varrho}{\varrho}$ & $\stackrel{\vec{b}}{=}$ \\
\hline
\end{tabular}




\begin{tabular}{|c|c|c|c|c|c|c|c|c|c|c|c|c|c|c|c|c|c|c|c|c|c|c|c|}
\hline & & $\vec{\Delta}$ & $\begin{array}{l}8 \\
\stackrel{1}{1} \\
+1 \\
\infty \\
\infty \\
\infty \\
\dot{\forall}\end{array}$ & 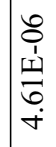 & 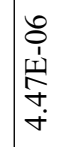 & 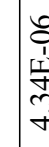 & & 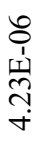 & 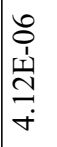 & 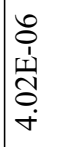 & 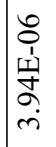 & 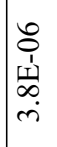 & 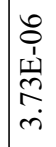 & 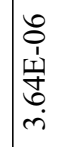 & 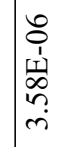 & 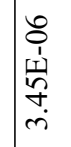 & $\begin{array}{l}8 \\
0 \\
1 \\
1 \\
\\
m \\
m \\
m\end{array}$ & 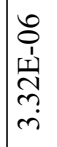 & $\begin{array}{l}8 \\
0 \\
1 \\
1 \\
\\
\\
\end{array}$ & 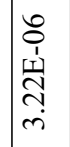 & 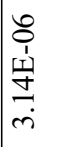 & 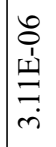 & 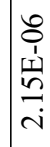 \\
\hline & & $\stackrel{\oplus}{\infty}$ & 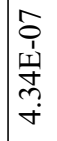 & 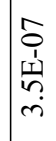 & $\begin{array}{c}\hat{o} \\
\hat{1} \\
\dot{1} \\
\infty \\
\hat{i} \\
\hat{i}\end{array}$ & $\mid \begin{array}{l}0 \\
0 \\
1 \\
1 \\
0 \\
2 \\
n\end{array}$ & & $\begin{array}{c}\hat{o} \\
\text { 1̀ } \\
\hat{\sigma} \\
\hat{-}\end{array}$ & 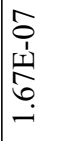 & 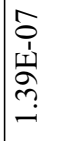 & 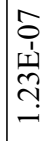 & 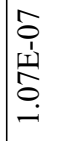 & 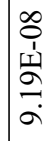 & 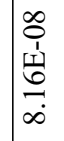 & 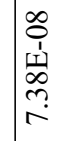 & 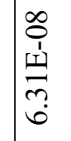 & $\begin{array}{l}\infty \\
0 \\
0 \\
1 \\
\\
6 \\
\text { in } \\
\dot{1}\end{array}$ & 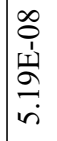 & 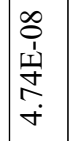 & 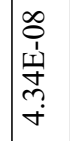 & 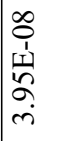 & 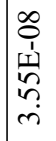 & 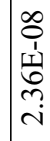 \\
\hline & & $\ddot{2}$ & $\begin{array}{l}\hat{i} \\
0 \\
0 \\
0\end{array}$ & 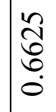 & त्ञ్రి & శ్ర & & $\begin{array}{l}\overrightarrow{\widetilde{d}} \\
\stackrel{0}{0} \\
\stackrel{0}{0}\end{array}$ & ర్ర & ర్ర & ర్ర & $\begin{array}{l}\overrightarrow{\widetilde{J}} \\
\stackrel{0}{0} \\
\dot{0}\end{array}$ & $\begin{array}{l}\overrightarrow{\widetilde{\sigma}} \\
\stackrel{0}{0} \\
\dot{0}\end{array}$ & 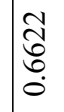 & त्ञ & $\mid$\begin{tabular}{l}
\multirow{Z}{0}{} \\
$\stackrel{0}{0}$ \\
0
\end{tabular} & 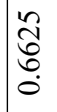 & \begin{tabular}{l}
0 \\
\multirow{2}{0}{} \\
0 \\
0 \\
0
\end{tabular} & $\begin{array}{l}\hat{\hat{T}} \\
\dot{0} \\
\dot{0}\end{array}$ & 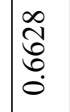 & 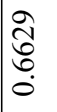 & 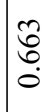 & $\begin{array}{l}\overrightarrow{\widetilde{b}} \\
\dot{0} \\
\dot{0}\end{array}$ \\
\hline & & $\mathscr{J}$ & 疍 & $\begin{array}{l}\infty \\
\stackrel{2}{0} \\
\stackrel{2}{2}\end{array}$ & $\begin{array}{l}\text { సे } \\
\text { }\end{array}$ & 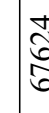 & & $\begin{array}{l}\overrightarrow{8} \\
\stackrel{8}{f}\end{array}$ & 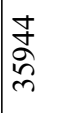 & $\begin{array}{l}\hat{2} \\
\hat{\tilde{N}} \\
\text { N. }\end{array}$ & ڤิ & ठి & $\begin{array}{l}\tilde{\Omega} \\
\bumpeq \\
\bumpeq\end{array}$ & $\tilde{\alpha}$ & $\underset{\infty}{\stackrel{\infty}{\Xi}}$ & 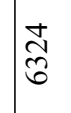 & 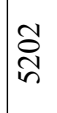 & $\begin{array}{l}\odot \\
\infty \\
\stackrel{\sim}{+}\end{array}$ & $\overrightarrow{\hat{D}}$ & $\frac{\vec{i}}{m}$ & 守 & $\begin{array}{l}\infty \\
\stackrel{\sim}{\sim} \\
\text { N }\end{array}$ & $\underset{\sim}{ \pm}$ \\
\hline & & t & 号 & 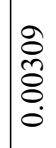 & $\begin{array}{l}\tilde{n} \\
\infty \\
\tilde{N} \\
\delta \\
0 \\
0\end{array}$ & $\mid \begin{array}{l}0 \\
0 \\
0 \\
0 \\
0 \\
0\end{array}$ & & $\begin{array}{l}\stackrel{\partial}{0} \\
\stackrel{1}{\Delta} \\
\stackrel{0}{0} \\
\dot{0}\end{array}$ & 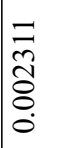 & 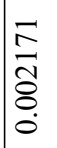 & 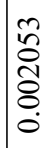 & $\begin{array}{l}0 \\
\frac{1}{\sigma} \\
\frac{8}{8} \\
0 \\
0\end{array}$ & 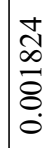 & 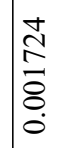 & $\begin{array}{l}\vec{n} \\
\stackrel{0}{0} \\
\stackrel{0}{0} \\
0\end{array}$ & $\mid \begin{array}{c}\infty \\
\stackrel{\infty}{+} \\
\stackrel{5}{8} \\
0 \\
0\end{array}$ & 官 & 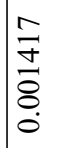 & $\begin{array}{l}\hat{D} \\
\tilde{n} \\
\tilde{\sigma} \\
\dot{0}\end{array}$ & $\begin{array}{l}\frac{1}{m} \\
\stackrel{m}{8} \\
\stackrel{0}{0}\end{array}$ & 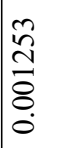 & 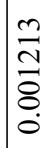 & $\begin{array}{l}\tilde{2} \\
\infty \\
0 \\
\delta \\
0 \\
0\end{array}$ \\
\hline & & $\ddot{\sim}$ & $\mid \begin{array}{c}\hat{\infty} \\
\tilde{N}\end{array}$ & 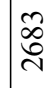 & $\begin{array}{l}n \\
\tilde{n} \\
n\end{array}$ & $\stackrel{\overbrace{}}{\sim}$ & & ֶֻ. & $\underset{ন}{\stackrel{J}{ন}}$ & $\overrightarrow{\vec{d}}$ & $\begin{array}{l}0 \\
\stackrel{2}{\circ} \\
\stackrel{\sim}{2}\end{array}$ & $\stackrel{ }{\stackrel{\overbrace{}}{\circ}}$ & 䒬 & $\stackrel{2}{\infty}$ & $\underset{\infty}{\infty}$ & $\mid \begin{array}{l} \pm \\
\stackrel{\Xi}{\Xi}\end{array}$ & $\underset{\substack{I \\
I}}{\stackrel{\infty}{+}}$ & $\stackrel{๑}{\unrhd}$ & 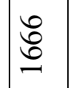 & స్రి & 离 & \begin{tabular}{l}
8 \\
\hdashline \\
$n$
\end{tabular} & 芯 \\
\hline שే & $\stackrel{\mathscr{I}}{\mathbf{I}}$ & 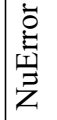 & $\hat{a}$ & $\stackrel{m}{9}$ & ఫ̆ & $\frac{n}{a}$ & & ڤ్రి & $\begin{array}{l}n \\
n \\
0\end{array}$ & 菅 & to & ปี. & $\stackrel{t}{0}$ & $\tilde{n}$ & 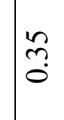 & ?n & 3 & ஸి & ते & ?3. & $\vec{m}$ & $\bar{m}$ & $\begin{array}{c}\infty \\
\tilde{0} \\
0\end{array}$ \\
\hline $\begin{array}{l}\frac{ \pm}{\sigma} \\
\frac{ \pm}{ \pm} \\
\Xi\end{array}$ & 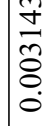 & $\vec{z}$ & $\begin{array}{l}\infty \\
\stackrel{+}{+} \\
\dot{f}\end{array}$ & $\begin{array}{l}\hat{\imath} \\
\ddot{n}\end{array}$ & $\begin{array}{l}\vec{I} \\
\stackrel{I}{I}\end{array}$ & 2 & & $\stackrel{ \pm}{a}$ & $\begin{array}{l}\infty \\
\infty \\
\infty \\
\infty\end{array}$ & $\stackrel{\curvearrowright}{\stackrel{2}{r}}$ & $\stackrel{m}{r}$ & : & ஸి & $\begin{array}{l}n \\
2 \\
i n\end{array}$ & $\underset{i n}{\stackrel{f}{n}}$ & it & $\begin{array}{l}0 \\
\text { in } \\
\text { in }\end{array}$ & 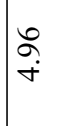 & $\begin{array}{l}\infty \\
\infty \\
+ \\
+\end{array} \mid$ & $\begin{array}{l}\vec{\infty} \\
\dot{+}\end{array}$ & $\stackrel{\sim}{\dot{r}}$ & 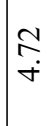 & $\underset{+}{\stackrel{f}{f}}$ \\
\hline 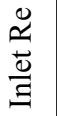 & $\begin{array}{l}\stackrel{\partial}{\infty} \\
\stackrel{\infty}{N}\end{array}$ & 衰 & $\begin{array}{l}0 \\
b \\
\vdots \\
\vdots\end{array}$ & $\underset{\dot{q}}{\stackrel{q}{2}}$ & $\bar{n}$ & $\frac{2}{2}$ & & : & : & $\begin{array}{l}\hat{6} \\
\dot{r}\end{array}$ & 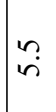 & $\begin{array}{l}\bar{\infty} \\
+ \\
+\end{array}$ & $\begin{array}{l}\hat{6} \\
+\end{array}$ & $\underset{+}{\stackrel{+}{+}}$ & $\underset{+}{\stackrel{+}{+}}$ & $\underset{\sim}{\stackrel{\sim}{+}}$ & ळे & $\overrightarrow{+}$ & 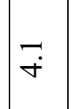 & $\underset{f}{\stackrel{\infty}{+}}$ & $\vec{\sim}$ & $\underset{f}{\stackrel{f}{f}}$ & $=$ \\
\hline 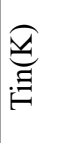 & ర్లి & 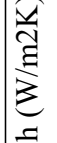 & 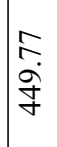 & $\begin{array}{l}\infty \\
\underset{\mathbb{J}}{0}\end{array}$ & $\begin{array}{l}0 \\
\substack{0 \\
m \\
m}\end{array}$ & $\overrightarrow{\tilde{z}}$ & & $\begin{array}{l}\stackrel{\text { İ }}{ٍ} \\
\stackrel{\Xi}{=}\end{array}$ & $\begin{array}{l}\hat{0} \\
\infty \\
0 \\
0\end{array}$ & $\begin{array}{l}\delta \\
\dot{d} \\
\dot{d}\end{array}$ & ầ & $\begin{array}{l}\hat{\sigma} \\
\dot{+}\end{array}$ & 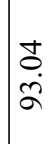 & $\overrightarrow{\hat{\Omega}}$ & 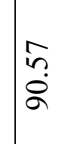 & 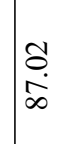 & $\underset{\infty}{\tilde{\infty}}$ & $\Phi$ & $\stackrel{\hat{Z}}{\dot{\Phi}}$ & $\begin{array}{l}2 \\
\tilde{\infty} \\
\infty \\
\infty\end{array}$ & $\frac{\infty}{\infty}$ & $\frac{n}{\infty}$ & $\mid \begin{array}{l}\mathbb{J} \\
\dot{\infty}\end{array}$ \\
\hline$\sum_{\text {至 }}$ & $n$ & $\begin{array}{l}\text { đี } \\
\text { है } \\
= \\
=\end{array}$ & 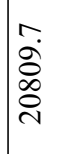 & 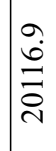 & 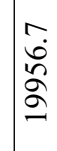 & $\mid \begin{array}{l}\mathcal{2} \\
\infty \\
\vdots \\
0\end{array}$ & & $\begin{array}{l}\infty \\
\text { i } \\
\widehat{\delta} \\
\stackrel{2}{2}\end{array}$ & \begin{tabular}{l}
\multirow{2}{*}{} \\
Oें \\
ఫे
\end{tabular} & 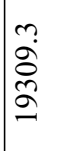 & $\begin{array}{l}0 \\
\stackrel{0}{ } \\
\widetilde{\Omega} \\
\sigma\end{array}$ & $\begin{array}{l}\infty \\
\infty \\
\infty \\
\infty \\
\infty\end{array}$ & 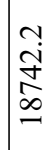 & $\begin{array}{l}0 \\
\dot{\nu} \\
\infty \\
\infty \\
\infty \\
\infty\end{array}$ & $\begin{array}{l}n \\
0 \\
0 \\
+ \\
\infty \\
\infty \\
-1\end{array}$ & 站 & 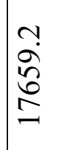 & 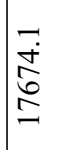 & $\begin{array}{l}\underset{+}{ \pm} \\
\stackrel{N}{\Sigma}\end{array}$ & $\begin{array}{l}n \\
\stackrel{2}{ \pm} \\
\stackrel{+}{ \pm}\end{array}$ & 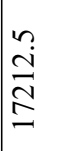 & $\frac{ \pm}{I}$ & 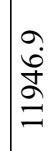 \\
\hline 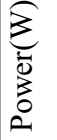 & $\stackrel{2}{\varrho}$ & $\vec{F}$ & $\begin{array}{l}\mathcal{W} \\
\stackrel{\sim}{m} \\
\tilde{m}\end{array}$ & $\begin{array}{l}0 \\
+ \\
\infty \\
\infty \\
m \\
m\end{array}$ & $\begin{array}{l}\stackrel{2}{2} \\
\dot{0} \\
i \\
\text { no }\end{array}$ & $\begin{array}{l}n \\
\infty \\
\infty \\
\infty \\
m\end{array}$ & & $\begin{array}{l}\stackrel{\rho}{\dot{g}} \\
\vec{\sigma}\end{array}$ & $\begin{array}{l}\vec{\sigma} \\
\infty \\
⿱ 亠 䒑 \\
⿱ 亠 乂\end{array}$ & $\begin{array}{l}2 \\
\infty \\
\text { రु } \\
+\end{array}$ & $\mid \begin{array}{c}\vec{N} \\
\stackrel{\infty}{\infty} \\
\dot{\alpha}\end{array}$ & $\frac{\tilde{N}}{\bar{\hbar}}$ & 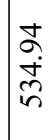 & $\begin{array}{l}\text { 辛 } \\
\infty \\
0 \\
n \\
n\end{array}$ & 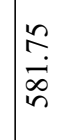 & 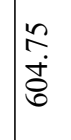 & 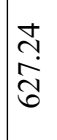 & $\begin{array}{l}n \\
\tilde{a} \\
q \\
\sigma\end{array}$ & $\frac{\infty}{\vec{T}}$ & $\begin{array}{l}\hat{\sigma} \\
\hat{\sigma}\end{array}$ & $\begin{array}{l}\infty \\
\infty \\
\ddot{n} \\
\end{array}$ & $\underset{\substack{n\\
}}{\stackrel{0}{n}}$ & $\begin{array}{l}\alpha \\
\alpha \\
\kappa \\
\kappa\end{array}$ \\
\hline$\sum_{0=1}^{\pi}$ & ले & 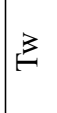 & $\begin{array}{l}\infty \\
\infty \\
\infty \\
\infty \\
\infty\end{array}$ & $\begin{array}{l}2 \\
\hat{\sigma} \\
\stackrel{8}{+}\end{array}$ & $\frac{n}{2}$ & $\mid \begin{array}{l}n \\
\vdots \\
a \\
i \\
n\end{array}$ & & $\begin{array}{l}\hat{0} \\
\dot{\infty} \\
i \\
i\end{array}$ & $\mid \begin{array}{l}n \\
\infty \\
6\end{array}$ & \begin{tabular}{l}
$\tilde{n}$ \\
$\infty$ \\
\multirow{\delta}{0}{}
\end{tabular} & 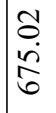 & 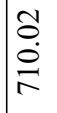 & $\begin{array}{l}\hat{n} \\
\hat{0} \\
\approx \\
n\end{array}$ & તָ̃ & $\begin{array}{l}\underset{f}{*} \\
\dot{\infty} \\
\stackrel{\infty}{\propto}\end{array}$ & $\underset{\infty}{\stackrel{\infty}{\stackrel{\infty}{\Xi}}}$ & $\begin{array}{l}\bar{\alpha} \\
\infty \\
\infty \\
\infty\end{array}$ & $\begin{array}{l} \pm \\
\partial \\
\ddot{\infty}\end{array}$ & $\begin{array}{l}m \\
\vec{a} \\
\infty \\
\infty\end{array}$ & $\begin{array}{l}\nabla \\
\dot{0} \\
\infty \\
\infty\end{array}$ & $\begin{array}{l}\infty \\
\frac{\partial}{\sigma} \\
\sigma\end{array}$ & $\begin{array}{l}\infty \\
\tilde{m} \\
\sigma\end{array}$ & 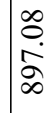 \\
\hline 苋 & m & 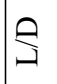 & $N$ & $\vec{\infty}$ & 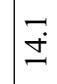 & ๙ี & & ֶָ. & $\stackrel{m}{\stackrel{n}{n}}$ & $\begin{array}{c}m \\
\infty \\
\infty\end{array}$ & $\underset{f}{\dot{f}}$ & $\stackrel{i}{\dot{s}}$ & $\begin{array}{l}n \\
0 \\
0\end{array}$ & 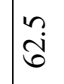 & $\begin{array}{l}n \\
\infty \\
\infty \\
\infty\end{array}$ & $\begin{array}{l}0 \\
\stackrel{+}{T}\end{array}$ & $\ddot{\infty}$ & $\hat{\infty}$ & $\hat{i}$ & $\mid \begin{array}{l}\infty \\
\infty \\
\infty\end{array}$ & 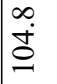 & 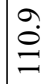 & $\stackrel{\vec{b}}{=}$ \\
\hline
\end{tabular}




\begin{tabular}{|c|c|c|c|c|c|c|c|c|c|c|c|c|c|c|c|c|c|c|c|c|c|c|c|}
\hline & & $\vec{\Delta}$ & $\begin{array}{l}0 \\
0 \\
1 \\
1 \\
\\
\infty \\
\infty \\
\dot{n}\end{array}$ & $\begin{array}{l}0 \\
0 \\
1 \\
1 \\
6 \\
0 \\
\dot{n} \\
\dot{1}\end{array}$ & $\begin{array}{l}8 \\
0 \\
1 \\
1 \\
\\
n \\
m\end{array}$ & 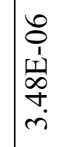 & 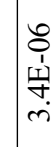 & 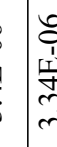 & 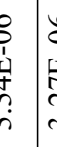 & 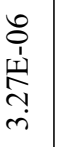 & $\begin{array}{l}8 \\
0 \\
1 \\
\\
\\
\end{array}$ & 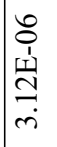 & 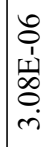 & $\begin{array}{l}0 \\
0 \\
1 \\
1 \\
m \\
m\end{array}$ & 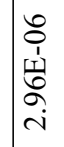 & 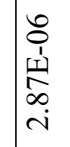 & 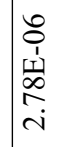 & 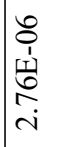 & 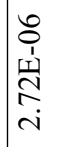 & $\mid \begin{array}{c}0 \\
0 \\
1 \\
1 \\
0 \\
0 \\
0 \\
i\end{array}$ & 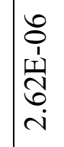 & 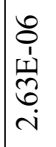 & 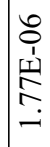 \\
\hline & & $\ddot{~}$ & 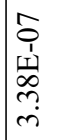 & $\begin{array}{l}\hat{i} \\
\hat{1} \\
\underline{1} \\
\hat{i} \\
\hat{i}\end{array}$ & \begin{tabular}{l}
$\hat{o}$ \\
$\hat{1}$ \\
\multicolumn{1}{c}{} \\
$\hat{\sigma}$ \\
$\mathbf{i}$
\end{tabular} & 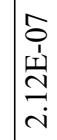 & 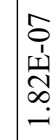 & 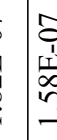 & 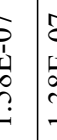 & 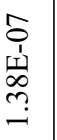 & 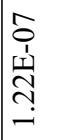 & 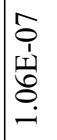 & 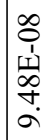 & $\begin{array}{l}\infty \\
0 \\
1 \\
1 \\
\\
\infty \\
\infty \\
\infty\end{array}$ & 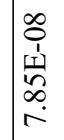 & 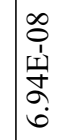 & 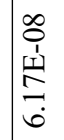 & $\begin{array}{l}\infty \\
0 \\
0 \\
1 \\
\\
\infty \\
\infty \\
i \\
n\end{array}$ & 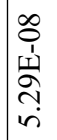 & $\mid \begin{array}{l}\infty \\
0 \\
1 \\
1 \\
\infty \\
\dot{+} \\
\dot{+}\end{array}$ & 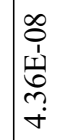 & 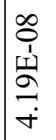 & $\begin{array}{c}\infty \\
0 \\
1 \\
\omega \\
0 \\
0 \\
\\
\end{array}$ \\
\hline & & $\ddot{\sim}$ & $\begin{array}{l}\infty \\
\text { đ్ర } \\
0 \\
0 \\
0\end{array}$ & 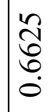 & 志 & त्र & $\begin{array}{l}\text { तु } \\
0 \\
0 \\
0\end{array}$ & శ్ర & $\begin{array}{l}\bar{v} \\
\stackrel{0}{0} \\
\stackrel{0}{\circ}\end{array}$ & $\begin{array}{l}\overrightarrow{\widetilde{C}} \\
\stackrel{0}{0} \\
\dot{0}\end{array}$ & ठ․ & ర్రి & $\begin{array}{l}\overrightarrow{\widetilde{J}} \\
\stackrel{0}{0} \\
\dot{0}\end{array}$ & $\begin{array}{l}\overrightarrow{\widetilde{J}} \\
\dot{0} \\
\dot{0}\end{array}$ & $\overrightarrow{\widetilde{d}}$ & $\begin{array}{l}\tilde{T} \\
\stackrel{0}{0} \\
\ddot{0}\end{array}$ & 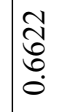 & $\begin{array}{l}\tilde{\widehat{U}} \\
\stackrel{0}{0} \\
\stackrel{0}{0}\end{array}$ & $\begin{array}{l}\tilde{\hat{U}} \\
\stackrel{0}{0} \\
\stackrel{0}{0}\end{array}$ & $\mid \begin{array}{l}\mathbb{Z} \\
\mathbb{Z} \\
0 \\
0\end{array}$ & î. & 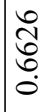 & 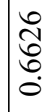 \\
\hline & & $\mathscr{\Xi}$ & $\mid \begin{array}{l}\infty \\
\tilde{n} \\
\tilde{n}\end{array}$ & $\begin{array}{l}\mathscr{n} \\
\infty \\
\infty \\
\infty \\
0\end{array}$ & $\begin{array}{l}n \\
n \\
n \\
\infty \\
\infty\end{array}$ & 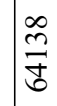 & 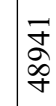 & $\begin{array}{l}\vec{d} \\
\infty \\
\stackrel{\Delta}{\sigma}\end{array}$ & 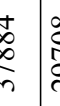 & 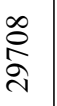 & $\begin{array}{l}\infty \\
\infty \\
\infty \\
\end{array}$ & $\begin{array}{l}\tilde{\delta} \\
\infty \\
\approx\end{array}$ & $\frac{\infty}{n}$ & $\mid \begin{array}{l}\widetilde{N} \\
\mathbb{D} \\
\mathcal{I}\end{array}$ & $\begin{array}{l}0 \\
\tilde{n} \\
\varrho\end{array}$ & 离 & $\stackrel{\infty}{\Re}$ & $\overrightarrow{\widehat{\sigma}}$ & $\begin{array}{l}\text { in } \\
\text { in }\end{array}$ & 韋 & $\underset{\infty}{\infty}$ & 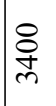 & $\hat{\widetilde{N}}$ \\
\hline & & む & 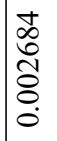 & 导 & 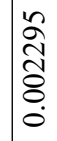 & 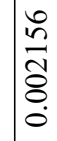 & $\begin{array}{l}0 \\
\tilde{\delta} \\
\overparen{\delta} \\
\delta \\
0\end{array}$ & ई̊ & 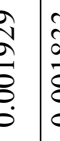 & $\begin{array}{l}\tilde{n} \\
\infty \\
\tilde{\delta} \\
\dot{0} \\
\dot{0}\end{array}$ & 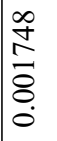 & \begin{tabular}{l}
$\infty$ \\
\multirow{0}{0}{} \\
$\stackrel{0}{8}$ \\
0 \\
0
\end{tabular} & $\mid \begin{array}{l}\infty \\
\infty \\
\stackrel{\infty}{n} \\
\stackrel{8}{8} \\
\dot{0}\end{array}$ & 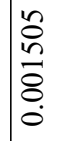 & 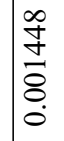 & 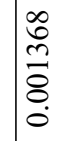 & 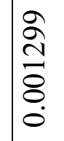 & 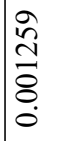 & $\begin{array}{l}\frac{n}{1} \\
\stackrel{a}{8} \\
\stackrel{8}{0}\end{array}$ & $\mid \begin{array}{l}\mathbb{N} \\
\Xi \bar{\Xi} \\
\overline{8} \\
\dot{0}\end{array}$ & 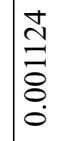 & 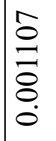 & 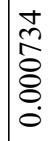 \\
\hline & & $\cong$ & 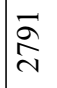 & 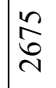 & $\vec{i}$ & 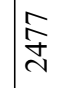 & ๙ิ & $\frac{\partial}{\approx}$ & 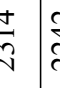 & 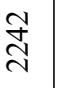 & $\frac{n}{\sim}$ & $\underset{\vec{\sim}}{\Xi}$ & 芩 & 总 & 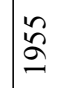 & ڤे & $\begin{array}{l}0 \\
\infty \\
\infty\end{array}$ & $\begin{array}{l}0 \\
\infty \\
\infty\end{array}$ & 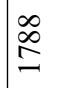 & $\stackrel{\bumpeq}{\bumpeq}$ & $\stackrel{2}{\Xi}$ & $\begin{array}{l}0 \\
0 \\
0\end{array}$ & : \\
\hline తే & $\ddot{I}$ & 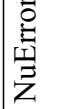 & $\mid \begin{array}{l}0 \\
\infty \\
\infty \\
\infty\end{array}$ & $\stackrel{\oplus}{\stackrel{m}{-}}$ & o. & $\stackrel{乏}{o}$ & : & in & $\frac{n}{6}$ & ñ & 守 & İ. & Iั & 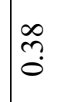 & m & $\bar{n}$ & సे & 3 & $\stackrel{\grave{\jmath}}{\delta}$ & $\mid \begin{array}{l}\stackrel{0}{0} \\
0\end{array}$ & $\stackrel{\widehat{N}}{0}$ & ஸ̊ & ڤ̊ \\
\hline $\begin{array}{l}\frac{t}{\sigma} \\
\frac{\overrightarrow{0}}{\Xi} \\
\Xi\end{array}$ & 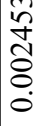 & $\vec{z}$ & 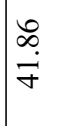 & $\begin{array}{l}\hat{\alpha} \\
\dot{J}\end{array}$ & $\stackrel{I}{\mathcal{I}}$ & $\begin{array}{l}\tilde{\sigma} \\
\stackrel{0}{0}\end{array}$ & $\bar{n}$ & $\mid \begin{array}{l}0 \\
\infty \\
\infty\end{array}$ & 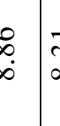 & $\bar{\infty}$ & $\bar{\sigma}$ & 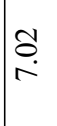 & $\begin{array}{l}0 \\
6 \\
6 \\
0\end{array}$ & సֶ. & $\hat{\sigma}$ & $\begin{array}{l}\vec{\sigma} \\
\dot{n}\end{array}$ & $\frac{\infty}{\dot{n}}$ & $\begin{array}{l}\hat{\sigma} \\
\dot{i n}\end{array}$ & $\begin{array}{l}\alpha \\
\dot{\gamma}\end{array}$ & $\underset{\nabla}{\stackrel{\nabla}{+}}$ & $\underset{+}{\mathbb{t}}$ & $\begin{array}{l}\hat{\sigma} \\
\dot{+}\end{array}$ & $\begin{array}{l}\tilde{D} \\
\infty \\
\sim\end{array}$ \\
\hline 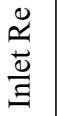 & $\widehat{\infty}_{\hat{N}}^{\infty}$ & 薑 & $\mid \begin{array}{l} \pm \\
\infty \\
\infty \\
\infty\end{array}$ & $\begin{array}{l}\mathbb{J} \\
\stackrel{I}{J}\end{array}$ & $\frac{n}{a}$ & ڤे & ڤึ) & $\frac{n}{6}$ & $\frac{0}{0}$ & $\begin{array}{l}\infty \\
\text { in }\end{array}$ & $\begin{array}{l}\vec{b} \\
\dot{n}\end{array}$ & $\stackrel{尺}{\stackrel{F}{+}}$ & $\stackrel{\sim}{\stackrel{*}{*}}$ & 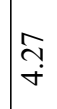 & $\stackrel{\partial}{\vec{\sigma}}$ & $\mid \begin{array}{l}\infty \\
\infty\end{array}$ & $\hat{n}$ & 守. & ণे. & \begin{tabular}{l}
0 \\
\multirow{2}{*}{} \\
$\dot{r}$
\end{tabular} & $\stackrel{q}{\stackrel{f}{r}}$ & $\stackrel{\infty}{\infty}$ & $\stackrel{\vartheta}{\hat{m}}$ \\
\hline$\underset{\Xi}{\underline{\Xi}}$ & 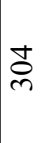 & 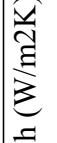 & 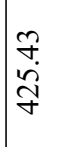 & $\begin{array}{l}0 \\
\infty \\
\infty \\
n \\
n\end{array}$ & $\stackrel{m}{\stackrel{m}{m}}$ & $\stackrel{\vec{I}}{\stackrel{\vec{I}}{.}}$ & $\begin{array}{l}\hat{\infty} \\
\stackrel{\Xi}{\Xi} \\
\Xi\end{array}$ & $\begin{array}{l}0 \\
2 \\
0 \\
0 \\
0\end{array}$ & : & 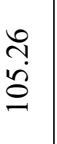 & $\stackrel{m}{\tilde{o}}$ & 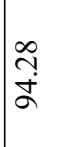 & $\underset{\dot{a}}{\infty}$ & $\mid \begin{array}{l}n \\
n \\
\infty \\
\infty\end{array}$ & $\frac{\vec{n}}{\frac{\infty}{\infty}}$ & $\begin{array}{l}0 \\
\stackrel{0}{\infty} \\
\infty\end{array}$ & $\begin{array}{l}n \\
\infty \\
\infty \\
\infty\end{array}$ & 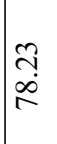 & $\begin{array}{l}\infty \\
\infty \\
\infty \\
\infty \\
\infty\end{array}$ & 守 & 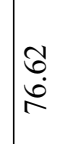 & $\begin{array}{l}\sigma \\
\sigma \\
\infty \\
\infty \\
\end{array}$ & $\begin{array}{l}\infty \\
1 \\
ن \\
ن\end{array}$ \\
\hline$\sum_{\text {贡 }}$ & $\stackrel{n}{i}$ & $\begin{array}{l}\text { તี } \\
\text { है } \\
= \\
=\end{array}$ & 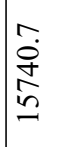 & 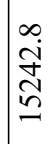 & $\begin{array}{l}\tilde{Z} \\
\tilde{U} \\
\end{array}$ & $\begin{array}{l}a \\
0 \\
0 \\
0 \\
n \\
n\end{array}$ & $\begin{array}{l}\infty \\
\dot{j} \\
\stackrel{g}{d}\end{array}$ & 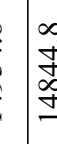 & 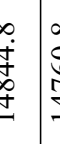 & $\begin{array}{l}\infty \\
\dot{0} \\
\stackrel{0}{+} \\
\text { I }\end{array}$ & 每 & 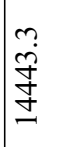 & $\begin{array}{l}\vec{\infty} \\
\dot{J}\end{array}$ & 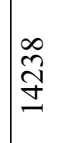 & 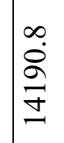 & $\mid \begin{array}{l}尺 \\
\infty \\
\\
\end{array}$ & $\begin{array}{l}\overrightarrow{0} \\
\stackrel{0}{0} \\
\stackrel{0}{0}\end{array}$ & $\begin{array}{l}m \\
2 \\
\tilde{n} \\
\end{array}$ & $\begin{array}{l}\infty \\
\stackrel{\sim}{1} \\
\tilde{n} \\
\end{array}$ & $\begin{array}{l}n \\
\bar{m} \\
\tilde{m} \\
m\end{array}$ & \begin{tabular}{l}
$n$ \\
$n$ \\
\multirow{n}{n}{} \\
$n$ \\
$n$
\end{tabular} & 糵 & $\hat{\hat{\alpha}}$ \\
\hline 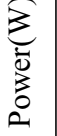 & 宔 & 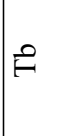 & $\begin{array}{l}\frac{n}{2} \\
\dot{0} \\
m\end{array}$ & $\begin{array}{l}\hat{\sigma} \\
\stackrel{0}{0} \\
\text { m}\end{array}$ & 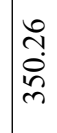 & $\begin{array}{l}\hat{0} \\
\dot{0} \\
0 \\
\text { n. }\end{array}$ & $\begin{array}{l}\infty \\
2 \\
\infty \\
\infty \\
\infty\end{array}$ & $\mid \begin{array}{l}\vec{y} \\
\alpha \\
\dot{a} \\
\dot{q}\end{array}$ & 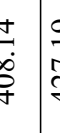 & $\frac{\vartheta}{\stackrel{\leftrightarrow}{\Im}}$ & \begin{tabular}{l}
$n$ \\
\hdashline \\
$\dot{f}$
\end{tabular} & $\begin{array}{l}\bar{a} \\
\dot{\sigma} \\
\dot{+}\end{array}$ & $\begin{array}{l}\infty \\
\dot{\infty} \\
\dot{\infty} \\
\infty \\
+\end{array}$ & $\frac{\alpha}{\alpha}$ & $\begin{array}{l}\tilde{N} \\
\tilde{ก} \\
\tilde{n}\end{array}$ & 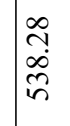 & 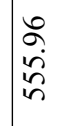 & $\begin{array}{l}0 \\
\stackrel{2}{2} \\
\stackrel{n}{n}\end{array}$ & 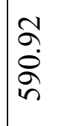 & $\mid \begin{array}{l}0 \\
1 \\
\infty \\
0 \\
0\end{array}$ & 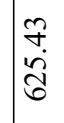 & \begin{tabular}{l}
$\infty$ \\
$\sim$ \\
\multirow{J}{*}{} \\
\multirow{J}{*}{}
\end{tabular} & $\begin{array}{l} \pm \\
0 \\
\tilde{n} \\
0\end{array}$ \\
\hline 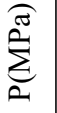 & 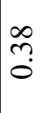 & $\underline{3}$ & 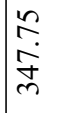 & 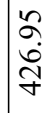 & 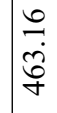 & $\mid \begin{array}{l}\tilde{y} \\
\tilde{q} \\
\tilde{q}\end{array}$ & ते & $\begin{array}{l}3 \\
8 \\
7 \\
\text { in }\end{array}$ & 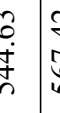 & 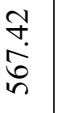 & $\begin{array}{l}f \\
\infty \\
\infty \\
\infty \\
n\end{array}$ & $\begin{array}{l}= \\
\frac{\infty}{\sigma} \\
\end{array}$ & 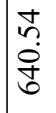 & ㄱ. & $\begin{array}{l}\infty \\
\tilde{\infty} \\
\infty \\
\infty \\
\infty\end{array}$ & |ro & 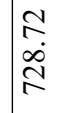 & $\underset{2}{\frac{2}{2}}$ & 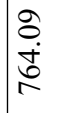 & 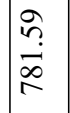 & $\begin{array}{l}m \\
\infty \\
\infty\end{array}$ & $\begin{array}{l}\hat{0} \\
\dot{m} \\
\infty\end{array}$ & $\begin{array}{l}\infty \\
0 \\
0 \\
\stackrel{2}{2}\end{array}$ \\
\hline Ü & $m$ & $\stackrel{2}{a}$ & $\sim$ & $\vec{\infty}$ & $\exists$ & กิ. & तु. & $\hat{\imath}$ & nู. & $\begin{array}{c}m \\
\infty \\
\infty \\
m\end{array}$ & $\stackrel{\nabla}{\dot{f}}$ & ti. & $\begin{array}{l}n \\
0 \\
0 \\
n\end{array}$ & in & $\begin{array}{l}n \\
\infty \\
\infty \\
\infty\end{array}$ & $\begin{array}{l}0 \\
\stackrel{+}{I}\end{array}$ & $\begin{array}{l}0 \\
\dot{\infty}\end{array}$ & $\hat{\varnothing}$ & & $\left|\begin{array}{l}\infty \\
\infty \\
\infty\end{array}\right|$ & 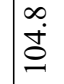 & $\stackrel{\partial}{\varrho}$ & 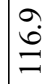 \\
\hline
\end{tabular}




\begin{tabular}{|c|c|c|c|c|c|c|c|c|c|c|c|c|c|c|c|c|c|c|c|c|c|c|}
\hline & & $\vec{z}$ & 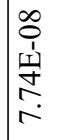 & $\begin{array}{l}\infty \\
0 \\
0 \\
1 \\
\infty \\
n \\
r \\
r\end{array}$ & 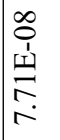 & 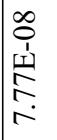 & 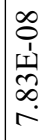 & $\begin{array}{l}\infty \\
0 \\
1 \\
1 \\
\infty \\
\infty \\
\infty \\
r \\
\end{array}$ & 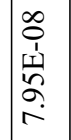 & 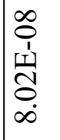 & $\begin{array}{l}\infty \\
0 \\
1 \\
1 \\
\infty\end{array}$ & 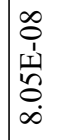 & $\left|\begin{array}{c}\infty \\
0 \\
1 \\
1 \\
\\
0 \\
\infty \\
\infty\end{array}\right|$ & $\mid \begin{array}{c}\infty \\
0 \\
1 \\
1 \\
\stackrel{1}{J} \\
\underset{\infty}{\infty}\end{array}$ & $\mid \begin{array}{c}\infty \\
0 \\
1 \\
1 \\
\infty \\
0 \\
0 \\
\infty\end{array}$ & 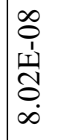 & $\mid \begin{array}{c}\infty \\
0 \\
1 \\
1 \\
\text { 닝 } \\
0 \\
\infty \\
\infty\end{array}$ & $\mid \begin{array}{c}\infty \\
0 \\
1 \\
1 \\
2 \\
0 \\
\infty \\
\infty\end{array}$ & \begin{tabular}{l}
$\infty$ \\
0 \\
1 \\
1 \\
\multicolumn{1}{|c}{} \\
0 \\
$\infty$ \\
$\infty$
\end{tabular} & $\mid \begin{array}{c}\infty \\
0 \\
0 \\
1 \\
1 \\
0 \\
\infty \\
\infty\end{array}$ & 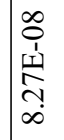 & 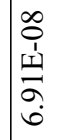 \\
\hline & & $\stackrel{\oplus}{\infty}$ & 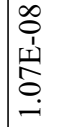 & 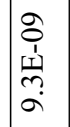 & 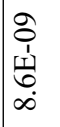 & 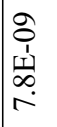 & 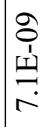 & $\begin{array}{l}2 \\
0 \\
1 \\
1 \\
01 \\
0 \\
0\end{array}$ & $\begin{array}{l}\text { oे } \\
1 \\
\text { t1 }\end{array}$ & $\begin{array}{l}8 \\
0 \\
1 \\
\text { I⿱ } \\
\text { in } \\
\text { in }\end{array}$ & 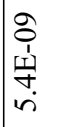 & $\begin{array}{l}\text { ô } \\
\text { 音 } \\
\text { in }\end{array}$ & 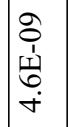 & 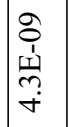 & 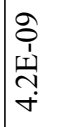 & 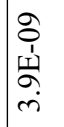 & 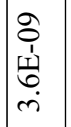 & $\begin{array}{c}2 \\
\hat{1} \\
\dot{1} \\
\dot{\sigma} \\
\dot{r}\end{array}$ & $\left|\begin{array}{c}2 \\
0 \\
1 \\
n \\
m \\
n \\
n\end{array}\right|$ & 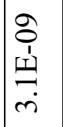 & $\begin{array}{l}\text { Oे } \\
\text { 离 } \\
\text { m }\end{array}$ & 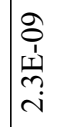 \\
\hline & & $\vec{D}$ & $\mid \begin{array}{l}\infty \\
0 \\
0 \\
0 \\
0\end{array}$ & $\begin{array}{l}0 \\
i \\
i n \\
0 \\
0\end{array}$ & $\begin{array}{l}\text { 京 } \\
\\
\stackrel{0}{0}\end{array}$ & 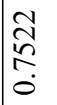 & $\frac{\partial}{\hat{d}}$ & $\mid \begin{array}{l}n \\
\stackrel{f}{f} \\
\stackrel{0}{0}\end{array}$ & 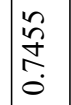 & 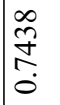 & 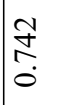 & $\mid \frac{0}{0}$ & $\mid \begin{array}{c}2 \\
\stackrel{\infty}{0} \\
\stackrel{0}{0}\end{array}$ & $\mid \begin{array}{l}0 \\
\stackrel{2}{2} \\
0 \\
0\end{array}$ & $\begin{array}{l}n \\
\stackrel{n}{0} \\
\\
0\end{array}$ & 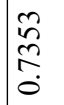 & $\mid \begin{array}{c}m \\
\stackrel{f}{\tilde{c}} \\
\stackrel{0}{0}\end{array}$ & 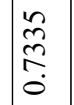 & 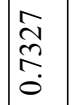 & $\frac{a}{\stackrel{2}{0}}$ & $\frac{m}{m}$ & 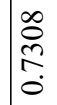 \\
\hline & & $\mathscr{J}$ & 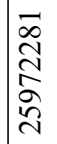 & 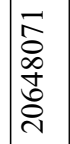 & 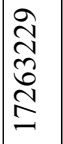 & 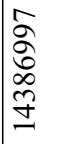 & 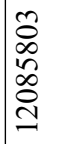 & 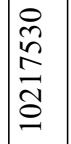 & 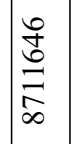 & 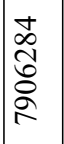 & $\begin{array}{l}\text { సे } \\
\text { ते } \\
\text { तु }\end{array}$ & 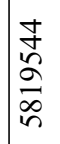 & $\begin{array}{l}\infty \\
\stackrel{\infty}{\hat{N}} \\
\tilde{\tilde{O}} \\
i n\end{array}$ & $\begin{array}{l}\tilde{\infty} \\
\stackrel{\infty}{\sigma} \\
\tilde{\sigma} \\
\tilde{\sigma}\end{array}$ & $\begin{array}{l}\infty \\
\infty \\
\infty \\
o \\
o \\
o \\
\rightarrow\end{array}$ & $\begin{array}{l}\hat{n} \\
\frac{\hat{\sigma}}{\infty} \\
\dot{m}\end{array}$ & $\mid \begin{array}{l}0 \\
0 \\
0 \\
0 \\
0 \\
m\end{array}$ & $\mid \begin{array}{l}\infty \\
\mathbb{N} \\
\mathbb{N} \\
\mathbb{N}\end{array}$ & $\begin{array}{l}\hat{\hat{n}} \\
\hat{n} \\
\hat{\lambda} \\
\tilde{\lambda}\end{array}$ & $\begin{array}{l}n \\
\hat{n} \\
\tilde{n} \\
\tilde{d} \\
\tilde{d}\end{array}$ & 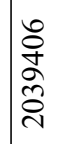 & 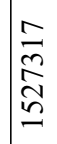 \\
\hline & & t & 触 & $\begin{array}{l}\overrightarrow{8} \\
\dot{0} \\
0 \\
\dot{0}\end{array}$ & $\mid \begin{array}{l}0 \\
0 \\
0 \\
8 \\
0 \\
0 \\
0\end{array}$ & 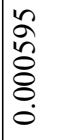 & $\begin{array}{l}n \\
\infty \\
\infty \\
o \\
0 \\
0 \\
0\end{array}$ & $\left|\begin{array}{l}n \\
\hat{n} \\
\tilde{\delta} \\
0 \\
0 \\
0\end{array}\right|$ & $\begin{array}{l}\infty \\
0 \\
0 \\
0 \\
0 \\
0\end{array}$ & 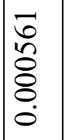 & $\begin{array}{l}\hat{f} \\
\text { ô } \\
0 \\
0 \\
0 \\
0\end{array}$ & 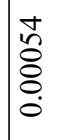 & $\left|\begin{array}{l}\vec{n} \\
\hat{o} \\
\delta \\
0 \\
\dot{0}\end{array}\right|$ & $\mid \begin{array}{l}2 \\
\tilde{1} \\
\tilde{\delta} \\
\delta \\
0 \\
0\end{array}$ & $\mid \begin{array}{c}n \\
\hat{n} \\
0 \\
0 \\
0 \\
0\end{array}$ & 苂 & 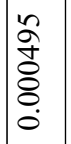 & $\mid \begin{array}{l}\infty \\
\infty \\
0 \\
0 \\
0 \\
0 \\
0\end{array}$ & $\mid$\begin{tabular}{l}
$\vec{\infty}$ \\
\multirow{0}{0}{} \\
0 \\
0 \\
0
\end{tabular} & 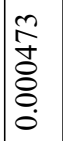 & 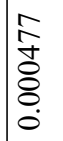 & \\
\hline & & $\approx$ & $\frac{m}{m}$ & $\begin{array}{l}\overrightarrow{\widetilde{Z}} \\
\tilde{m}\end{array}$ & $\frac{\infty}{m}$ & $\overrightarrow{\vec{\sigma}}$ & $\begin{array}{l}\infty \\
\infty \\
\stackrel{\infty}{\text { సे }}\end{array}$ & $\begin{array}{l}\overrightarrow{\grave{\aleph}} \\
\stackrel{\mathrm{N}}{ }\end{array}$ & $\begin{array}{l}0 \\
\varkappa \\
\infty \\
\sim\end{array}$ & 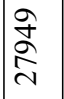 & 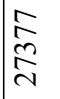 & 令 & 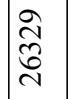 & $\left|\begin{array}{l}n \\
\infty \\
\infty \\
n \\
\end{array}\right|$ & $\begin{array}{l}n \\
\infty \\
\tilde{n} \\
\end{array}$ & $\begin{array}{l}\stackrel{゚}{2} \\
\stackrel{2}{d} \\
\end{array}$ & $\mid \begin{array}{l}0 \\
\stackrel{+}{+} \\
\stackrel{+}{\sim}\end{array}$ & 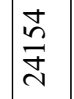 & 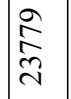 & 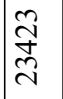 & 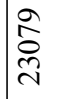 & 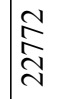 \\
\hline లే & ठิ & 窻 & $\frac{\partial}{m}$ & તู & $\vec{n}$ & $\tilde{\sigma}$ & 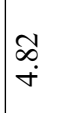 & $\mid \begin{array}{l}0 \\
\dot{1} \\
\text { in }\end{array}$ & 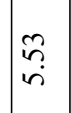 & $\begin{array}{l}\hat{6} \\
i \\
i\end{array}$ & $\frac{\partial}{\vec{n}}$ & in & $\begin{array}{l}\text { त̂ } \\
\text { in }\end{array}$ & $\mid \begin{array}{c}J \\
\dot{r} \\
i n\end{array}$ & $\begin{array}{c}0 \\
\text { in } \\
\text { in }\end{array}$ & $\stackrel{\sim}{\sim}$ & $\begin{array}{l}\stackrel{\Xi}{ } \\
\dot{+}\end{array}$ & $\stackrel{\Xi}{F}$ & $\begin{array}{l}n \\
\dot{+}\end{array}$ & $\begin{array}{c}\hat{\infty} \\
\infty \\
n\end{array}$ & $\begin{array}{l}\infty \\
\infty \\
\infty \\
\infty\end{array}$ & $\stackrel{m}{\vec{r}}$ \\
\hline $\begin{array}{l} \pm \\
\sigma \\
\frac{\overrightarrow{0}}{\Xi}\end{array}$ & 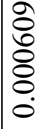 & $\vec{z}$ & 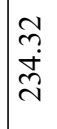 & $\begin{array}{l}\vec{J} \\
\dot{J} \\
\dot{J}\end{array}$ & 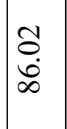 & $\frac{\tilde{c}}{\infty}$ & $\begin{array}{l}\dot{y} \\
\infty \\
\infty \\
i\end{array}$ & $\mid \begin{array}{l}\infty \\
\infty \\
\infty\end{array}$ & $\begin{array}{l}\infty \\
2 \\
2 \\
2\end{array}$ & $\tilde{n}$ & 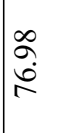 & 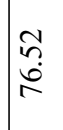 & $\begin{array}{l}8 \\
i \\
i\end{array}$ & $\begin{array}{l}\stackrel{\infty}{\sim} \\
\stackrel{+}{+}\end{array}$ & 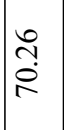 & $\frac{\tilde{\sigma}}{\tilde{\sigma}}$ & $\mid \begin{array}{l}\underset{\mathbb{Z}}{\mathrm{J}} \\
\text { | }\end{array}$ & $\tilde{n}$ & $\begin{array}{l}\text { tọ } \\
\text { in }\end{array}$ & $\begin{array}{l}\hat{n} \\
\infty \\
i n\end{array}$ & $\begin{array}{l}0 \\
+ \\
\infty \\
\infty\end{array}$ & 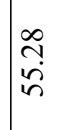 \\
\hline $\begin{array}{l}\stackrel{\mathscr{I}}{\simeq} \\
\frac{\vec{\omega}}{\Xi}\end{array}$ & 官 & 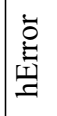 & $\begin{array}{l}\stackrel{n}{m} \\
\dot{m}\end{array}$ & ले & $\begin{array}{l}\hat{6} \\
\dot{n}\end{array}$ & $\begin{array}{l}\infty \\
\vdots \\
i \\
\text { in }\end{array}$ & 蒿 & in & กู & $\hat{\sigma}$ & छे & $\underset{\sim}{\stackrel{ \pm}{N}}$ & $\stackrel{?}{\stackrel{?}{\sim}}$ & $\stackrel{r}{\stackrel{N}{r}}$ & 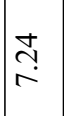 & ఫु & స్తి & 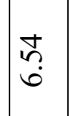 & f. & 守 & (6) & $\stackrel{0}{?}$ \\
\hline$\underset{\Xi}{\stackrel{્}{\Xi}}$ & 怘 & $\underbrace{\frac{\partial}{2}}_{=}$ & 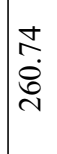 & $\begin{array}{l}\tilde{N} \\
\stackrel{2}{0} \\
\stackrel{0}{0}\end{array}$ & 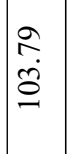 & 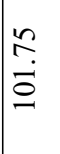 & $\begin{array}{l}\tilde{\sigma} \\
\text { di } \\
0\end{array}$ & $\mid \begin{array}{l}n \\
\mathfrak{c} \\
0\end{array}$ & $\begin{array}{l}n \\
\stackrel{n}{0} \\
ٍ\end{array}$ & $\mid \begin{array}{l}n \\
n \\
n \\
=\end{array}$ & $\begin{array}{l}\bar{\sigma} \\
\triangleq \\
\Xi\end{array}$ & 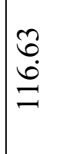 & $\begin{array}{l}9 \\
\mathbf{0} \\
\equiv \\
\Xi\end{array}$ & $\begin{array}{l}\infty \\
0 \\
0 \\
0 \\
=\end{array}$ & $\begin{array}{l}\stackrel{\vartheta}{\sigma} \\
= \\
=\end{array}$ & $\begin{array}{l}\vec{\sigma} \\
\infty \\
\stackrel{0}{0}\end{array}$ & 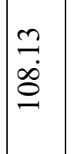 & $\begin{array}{l}0 \\
0 \\
0 \\
0\end{array}$ & $\begin{array}{l}n \\
\infty \\
\infty \\
0\end{array}$ & $\begin{array}{l}\tilde{n} \\
\tilde{\infty} \\
\stackrel{\infty}{0} \\
=\end{array}$ & $\stackrel{\Xi}{\Xi}$ & $\begin{array}{l}\widetilde{O} \\
\text { S. } \\
0\end{array}$ \\
\hline 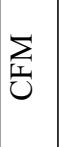 & 6 & $\begin{array}{l}\text { đิ } \\
\text { है } \\
= \\
=\end{array}$ & 商 & $\begin{array}{l}\infty \\
\stackrel{\infty}{i} \\
\hat{n} \\
0 \\
\underline{0}\end{array}$ & 守 & 㝘 & $\begin{array}{l}0 \\
\infty \\
\infty \\
\hat{\infty} \\
0\end{array}$ & $\mid \begin{array}{c}n \\
\infty \\
\infty \\
\tilde{0} \\
0\end{array}$ & $\begin{array}{l}\dot{t} \\
\dot{\overrightarrow{0}} \\
\tilde{\sigma} \\
\hat{\sigma}\end{array}$ & $\stackrel{\tilde{\rho}}{\tilde{\hat{\sigma}}}$ & 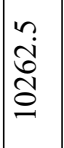 & $\begin{array}{l}\infty \\
\infty \\
0 \\
0 \\
0 \\
0\end{array}$ & $\begin{array}{l}\vec{a} \\
\vec{\sigma} \\
\underline{\sigma}\end{array}$ & 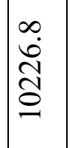 & 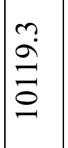 & ఏి & $\begin{array}{l}\hat{0} \\
\dot{8} \\
\stackrel{8}{0}\end{array}$ & $\begin{array}{l}0 \\
\infty \\
2 \\
\sigma \\
\sigma\end{array}$ & $\begin{array}{l}+ \\
\text { ḋ } \\
\text { مे }\end{array}$ & 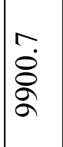 & $\mid \begin{array}{l}n \\
i n \\
a \\
a \\
0\end{array}$ & 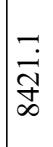 \\
\hline $\begin{array}{l}\sum_{\pi}^{2} \\
0 \\
0 \\
0 \\
0\end{array}$ & હે & $\vec{F}$ & $\begin{array}{l}a \\
\infty \\
\infty \\
\dot{\rho}\end{array}$ & 弚 & $\begin{array}{l}\vec{\infty} \\
\stackrel{\infty}{d} \\
\tilde{m}\end{array}$ & $\begin{array}{l}\infty \\
n \\
\infty \\
m \\
m\end{array}$ & \begin{tabular}{l}
0 \\
c \\
\multirow{J}{*}{} \\
m
\end{tabular} & $\left|\begin{array}{l}n \\
\infty \\
i \\
n \\
n\end{array}\right|$ & 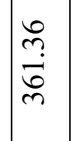 & $\begin{array}{l}\hat{\imath} \\
\stackrel{0}{0} \\
\text { D. }\end{array}$ & $\mid \begin{array}{c}m \\
\infty \\
\infty \\
\infty \\
m\end{array}$ & $\mid \begin{array}{l}n \\
\tilde{\infty} \\
\infty \\
\infty \\
\infty\end{array}$ & $\begin{array}{l}\vec{n} \\
\dot{\nabla} \\
\dot{m}\end{array}$ & $\begin{array}{l}\hat{n} \\
i \\
\tilde{q} \\
\end{array}$ & $\left|\begin{array}{l}\infty \\
n \\
0 \\
⿱ 亠 \\
\gamma\end{array}\right|$ & $\begin{array}{l}\mathcal{F} \\
\stackrel{\infty}{\sigma} \\
\vec{\gamma}\end{array}$ & 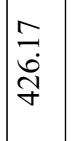 & $\begin{array}{l}\infty \\
\infty \\
\dot{m} \\
\tilde{\gamma}\end{array}$ & $\begin{array}{l}\tilde{n} \\
\dot{g} \\
\dot{f}\end{array}$ & $\begin{array}{l}\stackrel{\infty}{\circ} \\
\stackrel{+}{+}\end{array}$ & 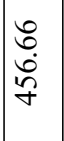 & 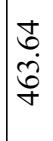 \\
\hline 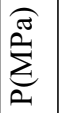 & $\stackrel{m}{0}$ & 3 & 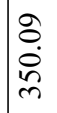 & $\begin{array}{l}\hat{\sigma} \\
\text { ì } \\
\ni\end{array}$ & $\underset{\stackrel{f}{f}}{\stackrel{\Re}{f}}$ & $\begin{array}{l}\hat{\infty} \\
\hat{\infty} \\
\tilde{\gamma}\end{array}$ & $\stackrel{0}{f}$ & 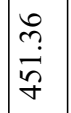 & 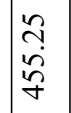 & 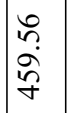 & 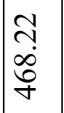 & $\mid \begin{array}{l}\infty \\
\stackrel{\infty}{f} \\
\stackrel{+}{f}\end{array}$ & $\begin{array}{l}\dot{+} \\
\stackrel{m}{a} \\
\dot{\infty} \\
\dot{+}\end{array}$ & $\mid \begin{array}{l}\dot{J} \\
\dot{\infty} \\
\infty \\
\dot{+}\end{array}$ & $\mid \begin{array}{l}\hat{\sigma} \\
\hat{\alpha} \\
\dot{q}\end{array}$ & $\begin{array}{l}\bullet \\
\stackrel{0}{0} \\
i \\
i n\end{array}$ & $\begin{array}{l}m \\
\infty \\
\infty \\
\infty\end{array}$ & $\begin{array}{l}n \\
n \\
n \\
n\end{array}$ & $\begin{array}{l}\hat{m} \\
\tilde{n} \\
\tilde{n}\end{array}$ & \begin{tabular}{l}
0 \\
\multirow{2}{*}{} \\
$\dot{0}$ \\
$\dot{d}$
\end{tabular} & $\stackrel{n}{\stackrel{n}{*}}$ & חָ \\
\hline 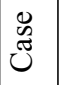 & in & $\stackrel{9}{9}$ & $N$ & $\vec{\infty}$ & $\overrightarrow{ \pm}$ & ָิ & ֶֶ. & $\begin{array}{l}n \\
\tilde{n}\end{array}$ & $\begin{array}{l}n \\
\infty \\
\infty \\
m\end{array}$ & $\mid \begin{array}{l}+ \\
\dot{J}\end{array}$ & $\begin{array}{l}\text { tr } \\
\text { in }\end{array}$ & \begin{tabular}{l}
$n$ \\
\hdashline \\
0 \\
$n$
\end{tabular} & $\mid \begin{array}{l}n \\
i \\
b\end{array}$ & $\left|\begin{array}{l}n \\
\infty \\
\infty \\
0\end{array}\right|$ & 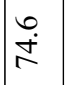 & $\begin{array}{l}0 \\
\dot{0} \\
\infty\end{array}$ & $\begin{array}{l}\hat{\infty} \\
\infty\end{array}$ & $\hat{i}$ & $\mid \begin{array}{l}\infty \\
\infty \\
\infty\end{array}$ & $\begin{array}{l}\infty \\
\dot{0} \\
\stackrel{0}{0}\end{array}$ & $\stackrel{\varrho}{\grave{O}}$ & \\
\hline
\end{tabular}




\begin{tabular}{|c|c|c|c|c|c|c|c|c|c|c|c|c|c|c|c|c|c|c|c|c|c|c|}
\hline & & $\vec{z}$ & 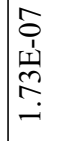 & 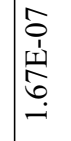 & 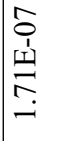 & 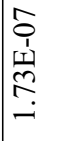 & 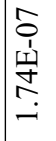 & 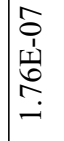 & 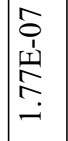 & 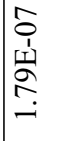 & 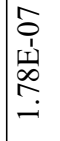 & 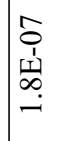 & 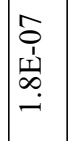 & 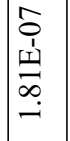 & 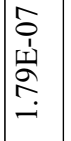 & 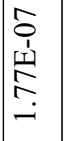 & $\mid \begin{array}{l}5 \\
0 \\
1 \\
\underline{1} \\
2 \\
-1\end{array}$ & 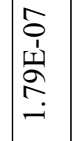 & 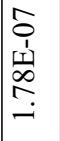 & 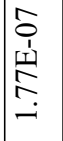 & 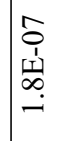 & 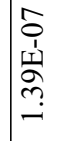 \\
\hline & & $\stackrel{\oplus}{\infty}$ & 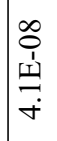 & $\begin{array}{l}\infty \\
0 \\
1 \\
1 \\
n \\
n \\
n \\
n\end{array}$ & 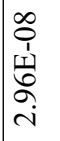 & 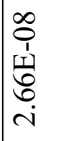 & 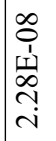 & 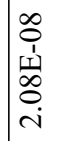 & 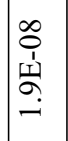 & 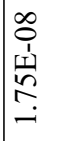 & 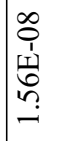 & 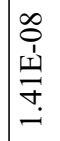 & 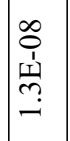 & $\mid \begin{array}{c}\infty \\
0 \\
⿱ 1 \\
\omega \\
\\
\\
-1 \\
\end{array}$ & 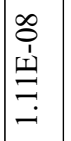 & 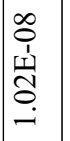 & $\mid \begin{array}{c}0 \\
\hat{1} \\
1 \\
\underline{1} \\
0 \\
0\end{array}$ & $\begin{array}{l}2 \\
\text { o } \\
\text { İ } \\
\alpha\end{array}$ & 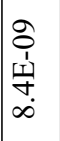 & 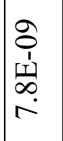 & 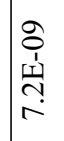 & 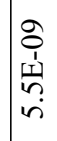 \\
\hline & & $\vec{D}$ & $\begin{array}{l}0 \\
8 \\
0 \\
0\end{array}$ & $\begin{array}{l}\infty \\
n \\
n \\
\tilde{n} \\
0\end{array}$ & 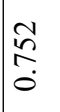 & $\begin{array}{l}\stackrel{+}{\infty} \\
\stackrel{+}{+} \\
\stackrel{0}{0}\end{array}$ & $\frac{n}{\frac{n}{5}}$ & 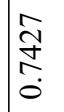 & $\frac{\widetilde{o}}{\stackrel{+}{+}}$ & $\begin{array}{l}\vec{\infty} \\
\stackrel{\infty}{\hat{0}} \\
\tilde{0}\end{array}$ & $\begin{array}{l}\frac{d}{0} \\
\stackrel{n}{0} \\
0\end{array}$ & 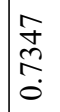 & $\mid \begin{array}{c}\vec{m} \\
\stackrel{\overbrace{}}{2} \\
\stackrel{0}{0}\end{array}$ & $\begin{array}{l}\vec{\sim} \\
\tilde{2} \\
\tilde{0}\end{array}$ & $\frac{\mathfrak{N}}{\mathfrak{m}}$ & $\begin{array}{l}\tilde{e} \\
\tilde{e} \\
\tilde{0}\end{array}$ & 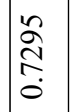 & $\begin{array}{l}\stackrel{\partial}{\mathbb{D}} \\
\stackrel{\mathbb{S}}{0}\end{array}$ & $\begin{array}{l}\stackrel{0}{\infty} \\
\stackrel{N}{0} \\
\tilde{0}\end{array}$ & 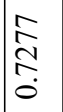 & 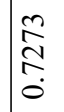 & î \\
\hline & & $\mathscr{J}$ & 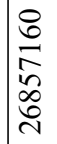 & $\begin{array}{l}\tilde{D} \\
\infty \\
\infty \\
\infty \\
\infty \\
\infty \\
-\end{array}$ & \begin{tabular}{|l|}
$\overrightarrow{0}$ \\
0 \\
0 \\
0 \\
0 \\
0 \\
$=$
\end{tabular} & 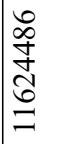 & $\begin{array}{l}\curvearrowright \\
\infty \\
\approx \\
\infty \\
\infty \\
\infty\end{array}$ & 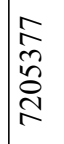 & $\begin{array}{l}\text { 守 } \\
\underset{7}{F} \\
\text { in }\end{array}$ & 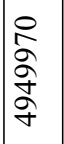 & $\begin{array}{l}\tilde{\lambda} \\
\tilde{2} \\
\stackrel{+}{\sigma}\end{array}$ & 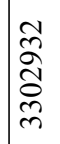 & $\begin{array}{l}\overrightarrow{\widehat{a}} \\
\hat{\widehat{\alpha}} \\
\hat{\imath}\end{array}$ & 令 & 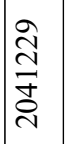 & 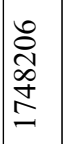 & $\mid \begin{array}{l}+ \\
\tilde{n} \\
2 \\
\tilde{N} \\
\end{array}$ & $\begin{array}{l}m \\
\infty \\
\tilde{n} \\
\tilde{n}\end{array}$ & 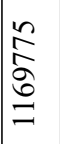 & $\begin{array}{l}\overline{\widetilde{\alpha}} \\
\widetilde{\widehat{\sigma}} \\
\text { }\end{array}$ & 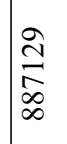 & 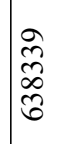 \\
\hline & & t & $\begin{array}{l}\infty \\
\frac{\infty}{0} \\
\stackrel{8}{8} \\
0 \\
0\end{array}$ & $\begin{array}{l}2 \\
\delta \\
\delta \\
\delta \\
0 \\
0\end{array}$ & 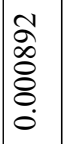 & 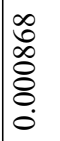 & 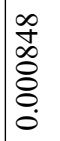 & 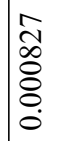 & $\begin{array}{l}\overrightarrow{\overrightarrow{0}} \\
\dot{\infty} \\
\dot{8} \\
\dot{0}\end{array}$ & 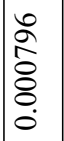 & \begin{tabular}{|l}
$\vec{E}$ \\
$\hat{\mathbf{O}}$ \\
$\mathbf{8}$ \\
0 \\
0
\end{tabular} & $\begin{array}{l}\frac{n}{2} \\
\frac{2}{8} \\
8 \\
0 \\
0\end{array}$ & 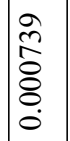 & $\left|\begin{array}{l}\hat{\mathbb{N}} \\
\hat{\delta} \\
\delta \\
\vdots \\
0\end{array}\right|$ & 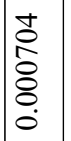 & $\begin{array}{l}0 \\
0 \\
0 \\
\delta \\
\delta \\
0 \\
0\end{array}$ & 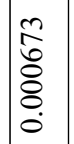 & $\begin{array}{l}\overrightarrow{0} \\
0 \\
0 \\
0 \\
0 \\
0\end{array}$ & 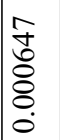 & 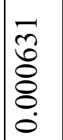 & 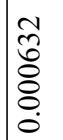 & \begin{tabular}{l}
$\vec{\infty}$ \\
\multirow{8}{0}{} \\
$\stackrel{8}{0}$ \\
0
\end{tabular} \\
\hline & & $\approx$ & 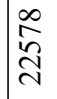 & 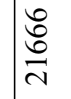 & $\begin{array}{l}\vec{b} \\
\infty \\
\stackrel{\sim}{0}\end{array}$ & 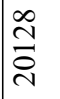 & 志 & $\mid \begin{array}{l}\tilde{\infty} \\
\infty \\
\infty\end{array}$ & $\begin{array}{l}\stackrel{\text { }}{\infty} \\
\infty\end{array}$ & $\mid \begin{array}{l}\infty \\
\substack{\infty \\
\Sigma \\
=}\end{array}$ & $\begin{array}{l}\infty \\
\stackrel{\infty}{\beth} \\
\end{array}$ & $\begin{array}{l}\hat{0} \\
0 \\
0 \\
0\end{array}$ & $\begin{array}{l}\overrightarrow{0} \\
\mathbb{0} \\
0\end{array}$ & 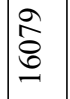 & $\mid \begin{array}{c}\mathbb{N} \\
\stackrel{\mathbb{n}}{n}\end{array}$ & 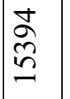 & $\mid \begin{array}{l}n \\
0 \\
0 \\
n \\
n\end{array}$ & $\begin{array}{l}\tilde{\hat{\Omega}} \\
\dot{\Xi}\end{array}$ & 窎 & $\begin{array}{l}\infty \\
\dddot{J} \\
\beth\end{array}$ & $\stackrel{\circ}{\circ}$ & $\mid \begin{array}{l}\stackrel{8}{\infty} \\
\stackrel{2}{2}\end{array}$ \\
\hline שै & ठิ & 窻 & 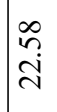 & $\hat{\tilde{m}}$ & $\stackrel{\widehat{r}}{\stackrel{n}{n}}$ & $\begin{array}{l}\tilde{N} \\
\tilde{r} \\
\tilde{m}\end{array}$ & 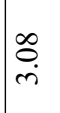 & $\hat{i}$ & बे & $\underset{\tilde{n}}{\tilde{n}}$ & 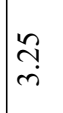 & $\frac{m}{m}$ & बे & $\left|\begin{array}{l}n \\
\hat{i}\end{array}\right|$ & $\begin{array}{l}\stackrel{0}{i} \\
i\end{array}$ & $\begin{array}{l}\stackrel{\infty}{+} \\
\stackrel{i}{i}\end{array}$ & $\mid \begin{array}{l}\tilde{f} \\
i \\
\end{array}$ & $\mid \begin{array}{l}\mathfrak{J} \\
\mathbf{i}\end{array}$ & 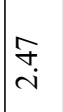 & 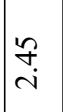 & 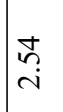 & 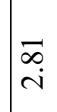 \\
\hline $\begin{array}{l} \pm \\
\sigma \\
\frac{\overrightarrow{0}}{\Xi}\end{array}$ & 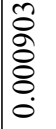 & $\vec{z}$ & 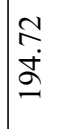 & $\frac{\mathfrak{r}}{\stackrel{n}{r}}$ & ৫ே. & $\begin{array}{l}\infty \\
\stackrel{\infty}{\infty} \\
\infty \\
i n\end{array}$ & 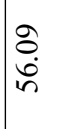 & $\begin{array}{l}8 \\
\text { in } \\
\text { in }\end{array}$ & 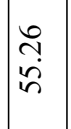 & $\begin{array}{l}n \\
n \\
n \\
n \\
n\end{array}$ & $\begin{array}{l}\vec{\infty} \\
\tilde{i} \\
i\end{array}$ & $\begin{array}{l}\tilde{N} \\
\hat{i} \\
\text { n. }\end{array}$ & $\mid \begin{array}{l}8 \\
\dot{\vec{x}} \\
\end{array}$ & $\begin{array}{l}7 \\
\dot{8} \\
i n\end{array}$ & $\begin{array}{l}\vec{b} \\
\vec{f}\end{array}$ & $\begin{array}{c}\mathbb{N} \\
\dot{g} \\
\dot{q}\end{array}$ & $\vec{i}$ & $\begin{array}{l}\infty \\
\infty \\
\dot{\forall} \\
\end{array}$ & $\mid \begin{array}{l}\mathbb{N} \\
\dot{q} \\
\dot{q}\end{array}$ & $\begin{array}{l}\hat{n} \\
\hat{n}\end{array}$ & 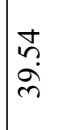 & $\underset{\bar{n}}{\bar{n}}$ \\
\hline $\begin{array}{l}\stackrel{\mathscr{x}}{\simeq} \\
\frac{\vec{\omega}}{\Xi}\end{array}$ & 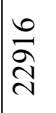 & 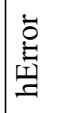 & $\begin{array}{l}\infty \\
\infty \\
\\
\dot{\lambda}\end{array}$ & 衣 & $\stackrel{n}{r}$ & $\vec{m}$ & $\stackrel{\infty}{\infty}$ & $\stackrel{n}{n}$ & $\dot{m}$ & 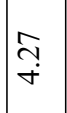 & 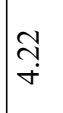 & $\stackrel{n}{+}$ & $\underset{\sim}{\tilde{\sigma}}$ & $\stackrel{\infty}{\stackrel{+}{+}}$ & $\underset{\leftarrow}{\tilde{T}}$ & $\stackrel{\partial}{\vec{F}}$ & $\stackrel{\overbrace{}}{\stackrel{\sim}{+}}$ & $\vec{m}$ & $\vec{m}$ & $\stackrel{\sim}{\sim}$ & $\stackrel{i}{r}$ & $\begin{array}{l}3 \\
\dot{n} \\
\dot{n}\end{array}$ \\
\hline$\underset{\Xi}{\stackrel{\Xi}{\Xi}}$ & 占 & $\underbrace{\stackrel{y}{\Xi}}_{\beth}$ & 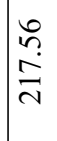 & $\begin{array}{l}\vec{J} \\
\dot{\infty} \\
\dot{\infty}\end{array}$ & $\stackrel{n}{\stackrel{n}{2}}$ & $\infty$ & $\begin{array}{l}\stackrel{0}{0} \\
\infty \\
\infty \\
i\end{array}$ & $\mid \begin{array}{l}n \\
n \\
\infty \\
\infty\end{array}$ & $\begin{array}{l}\text { 于 } \\
\dot{\infty}\end{array}$ & $\mid \begin{array}{l}\infty \\
n \\
\infty \\
\infty \\
\infty\end{array}$ & $\begin{array}{l}\vec{b} \\
\dot{\infty}\end{array}$ & $\begin{array}{l}\infty \\
2 \\
\infty\end{array}$ & $\begin{array}{l} \pm \\
\dot{a} \\
\sigma\end{array}$ & $\frac{\Xi}{\dot{\sigma}}$ & $\mid \begin{array}{l}\infty \\
\stackrel{2}{a} \\
\dot{a}\end{array}$ & $\begin{array}{l} \pm \\
\ddot{\infty} \\
\infty\end{array}$ & $\mid \begin{array}{l}f \\
\dot{0} \\
\infty\end{array}$ & $\begin{array}{l}n \\
\vdots \\
\infty \\
\infty\end{array}$ & $\mid \begin{array}{l}0 \\
\dot{\infty} \\
\infty\end{array}$ & 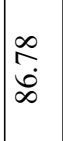 & $\begin{array}{l}\infty \\
\infty \\
\infty \\
\infty\end{array}$ & 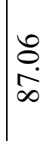 \\
\hline$\sum_{\text {至 }}^{\Sigma}$ & F & $\begin{array}{l}\text { वै } \\
\text { है } \\
=-\end{array}$ & 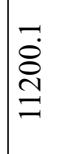 & 帒 & 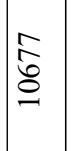 & $\frac{\overrightarrow{0}}{\overrightarrow{0}}$ & 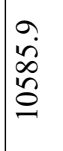 & 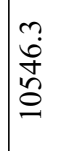 & $\begin{array}{l}\stackrel{2}{0} \\
\hat{n} \\
\hat{o}\end{array}$ & 离 & 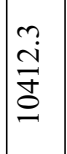 & $\underset{\widetilde{I}}{\stackrel{\Xi}{\Xi}}$ & $\left|\begin{array}{l}\hat{n} \\
\hat{i} \\
\tilde{n} \\
\hat{\theta}\end{array}\right|$ & \begin{tabular}{|l}
$\infty$ \\
0 \\
0 \\
0 \\
$ల$ \\
0 \\
0
\end{tabular} & $\mid \begin{array}{c}m \\
\stackrel{m}{\vec{z}} \\
\underline{\sigma}\end{array}$ & $\begin{array}{l}\overrightarrow{1} \\
\hat{1} \\
8 \\
0 \\
-\end{array}$ & $\mid \begin{array}{l}3 \\
6 \\
8 \\
8 \\
0\end{array}$ & $\begin{array}{l}0 \\
\dot{i} \\
\stackrel{\delta}{0}\end{array}$ & 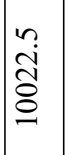 & 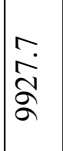 & $\mid \begin{array}{l}\infty \\
0 \\
\infty \\
\infty \\
\varrho \\
0\end{array}$ & 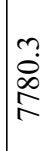 \\
\hline $\begin{array}{l}\sum_{\pi}^{2} \\
0 \\
0 \\
0 \\
0\end{array}$ & $\begin{array}{l}\infty \\
\stackrel{\leftrightarrow}{\circ}\end{array}$ & $\vec{F}$ & 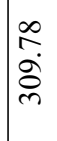 & 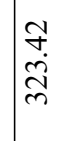 & $\begin{array}{l}n \\
n \\
0 \\
\infty \\
n \\
n\end{array}$ & 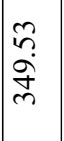 & 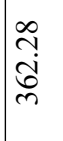 & $\mid \begin{array}{c}\infty \\
\stackrel{\infty}{+} \\
\dot{m}\end{array}$ & 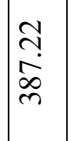 & $\begin{array}{l}\infty \\
\text { ळे. } \\
\text { ले }\end{array}$ & $\begin{array}{l}\stackrel{+}{n} \\
\vec{F} \\
\vec{y}\end{array}$ & $\mid \begin{array}{l}\hat{\tilde{j}} \\
\tilde{\hat{\gamma}} \\
\hat{\gamma}\end{array}$ & $\begin{array}{l}\exists \\
\tilde{n} \\
\tilde{v}\end{array}$ & $\begin{array}{l}\hat{b} \\
\dot{0} \\
\dot{f}\end{array}$ & $\left|\begin{array}{l}8 \\
\infty \\
\infty \\
⿱ ㇒ \\
+\end{array}\right|$ & 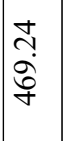 & $\mid$\begin{tabular}{c}
\multirow{2}{*}{} \\
$\stackrel{a}{+}$ \\
$\stackrel{+}{+}$
\end{tabular} & $\begin{array}{l}\underset{0}{-} \\
\stackrel{\vec{\sigma}}{\sigma}\end{array}$ & 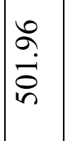 & $\begin{array}{l}0 \\
i \\
i n \\
i n\end{array}$ & $\begin{array}{l}\vec{N} \\
\tilde{N} \\
\tilde{n}\end{array}$ & $\begin{array}{l}\tilde{\sigma} \\
i \\
\tilde{n} \\
\tilde{n}\end{array}$ \\
\hline 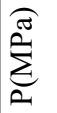 & $\stackrel{m}{0}$ & 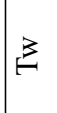 & 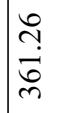 & $\begin{array}{l}\stackrel{\text { ते }}{\infty} \\
\stackrel{+}{+}\end{array}$ & $\underset{\stackrel{f}{\stackrel{f}{f}}}{\stackrel{P}{f}}$ & $\begin{array}{l}8 \\
0 \\
\ddot{\infty} \\
\infty \\
\dot{\sigma}\end{array}$ & 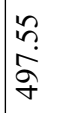 & 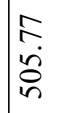 & $\frac{\hat{a}}{\bar{n}}$ & 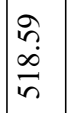 & $\begin{array}{l}\infty \\
n \\
0 \\
0 \\
n \\
n\end{array}$ & $\begin{array}{l}\overrightarrow{\hat{n}} \\
\stackrel{\vec{n}}{n}\end{array}$ & $\begin{array}{l}0 \\
0 \\
\infty \\
\dot{0} \\
n\end{array}$ & $\begin{array}{l}2 \\
\hat{n} \\
\tilde{n}\end{array}$ & $\mid \begin{array}{l}\mathfrak{n} \\
\stackrel{2}{n} \\
\hat{n}\end{array}$ & $\begin{array}{l}n \\
0 \\
\infty \\
\infty \\
n\end{array}$ & $\mid \begin{array}{l}0 \\
0 \\
\hat{\sigma} \\
\hat{n}\end{array}$ & 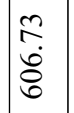 & $\frac{2}{\frac{1}{6}}$ & $\frac{\overrightarrow{\dot{\sigma}}}{\text { তু }}$ & $\begin{array}{l}\hat{\sigma} \\
\dot{0} \\
\text { రై }\end{array}$ & $\frac{\widehat{\sigma}}{\widehat{\sigma}}$ \\
\hline $\begin{array}{l}0 \\
0 \\
0 \\
0 \\
0\end{array}$ & $\hat{n}$ & $\stackrel{9}{9}$ & $\sim$ & $\vec{\infty}$ & $\overrightarrow{ \pm}$ & ¿̂. & ¿ֶ. & $\underset{m}{m}$ & $\mid \begin{array}{c}m \\
\infty \\
m \\
m\end{array}$ & $\mid \begin{array}{l}\dot{J} \\
\dot{f}\end{array}$ & $\begin{array}{l}t \\
\dot{B}\end{array}$ & in & $\begin{array}{l}n \\
i \\
\delta\end{array}$ & $\left|\begin{array}{l}n \\
\infty \\
0 \\
0\end{array}\right|$ & $\begin{array}{l}0 \\
\stackrel{+}{i}\end{array}$ & $\begin{array}{l}0 \\
\dot{0} \\
\infty\end{array}$ & $\hat{\infty}$ & $\overline{\check{a}}$ & $\left|\begin{array}{l}\infty \\
\infty \\
\infty \\
\alpha\end{array}\right|$ & $\begin{array}{l}\infty \\
\dot{0} \\
\dot{0}\end{array}$ & $\stackrel{\partial}{\varrho}$ & ڤे \\
\hline
\end{tabular}




\begin{tabular}{|c|c|c|c|c|c|c|c|c|c|c|c|c|c|c|c|c|c|c|c|c|c|c|}
\hline & & $\vec{z}$ & 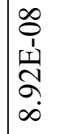 & 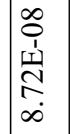 & $\begin{array}{l}\infty \\
0 \\
1 \\
1 \\
2 \\
\infty \\
\infty \\
\infty\end{array}$ & 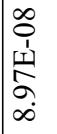 & 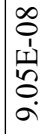 & 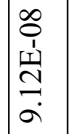 & 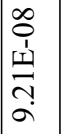 & $\begin{array}{l}\infty \\
0 \\
1 \\
1 \\
\\
\\
\\
\alpha\end{array}$ & 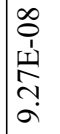 & 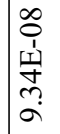 & $\left|\begin{array}{l}\infty \\
0 \\
1 \\
1 \\
2 \\
2 \\
2 \\
a\end{array}\right|$ & 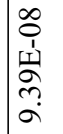 & 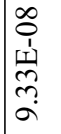 & $\begin{array}{l}\infty \\
0 \\
\hat{1} \\
\stackrel{1}{\Lambda} \\
\hat{\sim} \\
\alpha\end{array}$ & 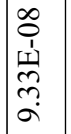 & $\left|\begin{array}{c}\infty \\
0 \\
1 \\
N \\
n \\
n \\
\sigma\end{array}\right|$ & 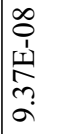 & 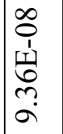 & $\begin{array}{l}\infty \\
0 \\
1 \\
1 \\
\\
n \\
\alpha \\
\alpha\end{array}$ & 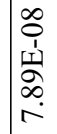 \\
\hline & & $\stackrel{\oplus}{\infty}$ & 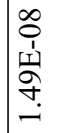 & $\begin{array}{l}\infty \\
0 \\
1 \\
1 \\
m \\
\\
\end{array}$ & 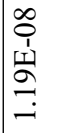 & 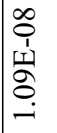 & 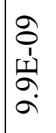 & 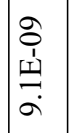 & 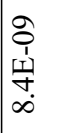 & 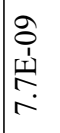 & 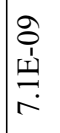 & 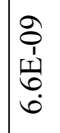 & $\mid \begin{array}{c}2 \\
\hat{1} \\
1 \\
\dot{1} \\
\dot{0}\end{array}$ & $\begin{array}{l}\text { oे } \\
\text { 1 } \\
\text { In } \\
\text { in }\end{array}$ & 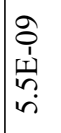 & 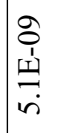 & 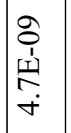 & 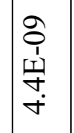 & 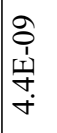 & 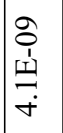 & 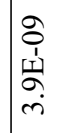 & $\begin{array}{l}\text { oे } \\
\text { 岌 } \\
\text { m }\end{array}$ \\
\hline & & $\vec{D}$ & $\stackrel{\overrightarrow{0}}{\stackrel{5}{0}}$ & $\mid \begin{array}{c}n \\
i n \\
0 \\
0\end{array}$ & 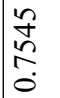 & 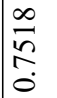 & 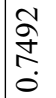 & $\mid \begin{array}{l}\hat{0} \\
\stackrel{+}{0} \\
\dot{0}\end{array}$ & 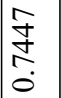 & 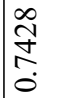 & $\underset{\vec{J}}{\tilde{O}}$ & 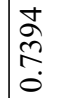 & $\mid \begin{array}{c}\stackrel{a}{2} \\
\stackrel{2}{0} \\
\stackrel{0}{0}\end{array}$ & 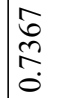 & 离 & 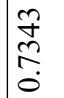 & $\mid \begin{array}{c}+ \\
\stackrel{2}{2} \\
\stackrel{2}{0}\end{array}$ & $\mid \begin{array}{c}0 \\
\tilde{N} \\
\tilde{R} \\
0\end{array}$ & 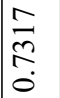 & $\stackrel{\vec{n}}{\stackrel{\vec{n}}{0}}$ & & ì \\
\hline & & $\mathscr{J}$ & $\begin{array}{l}\text { Jे } \\
8 \\
0 \\
0 \\
\infty \\
\infty \\
\text { N }\end{array}$ & 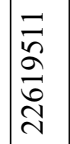 & $\mid \begin{array}{l}n \\
n \\
n \\
o \\
\infty \\
\infty \\
-\end{array}$ & 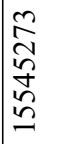 & 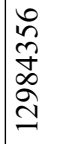 & 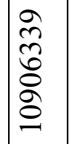 & 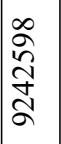 & \begin{tabular}{l}
0 \\
\multirow{2}{*}{} \\
$\infty$ \\
$\infty$ \\
$\infty$ \\
$\infty$
\end{tabular} & 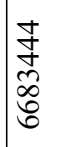 & 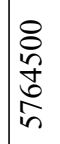 & $\begin{array}{l}\infty \\
\tilde{B} \\
\tilde{Z} \\
n \\
n\end{array}$ & 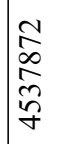 & 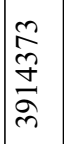 & 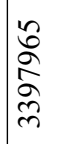 & 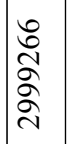 & $\begin{array}{l}\tilde{f} \\
\mathfrak{a} \\
\dot{d} \\
d\end{array}$ & 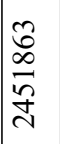 & $\begin{array}{l}\vec{b} \\
\vdots \\
\grave{2}\end{array}$ & 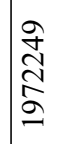 & 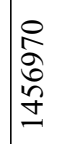 \\
\hline & & t & 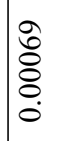 & $\begin{array}{l}n \\
2 \\
0 \\
0 \\
0 \\
0 \\
0\end{array}$ & 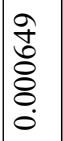 & 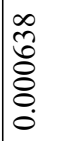 & 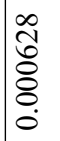 & $\begin{array}{l}\tilde{0} \\
\overline{0} \\
8 \\
0 \\
0\end{array}$ & $\begin{array}{l}\infty \\
0 \\
0 \\
0 \\
0 \\
0 \\
0 \\
0\end{array}$ & 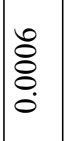 & $\begin{array}{l}n \\
2 \\
0 \\
o \\
0 \\
0 \\
0\end{array}$ & $\begin{array}{l}\infty \\
0 \\
\hat{n} \\
\varnothing \\
\vdots \\
0 \\
0\end{array}$ & $\begin{array}{l}1 \\
0 \\
0 \\
0 \\
0 \\
0 \\
0\end{array}$ & $\begin{array}{l}\infty \\
0 \\
\vdots \\
o \\
\vdots \\
0 \\
0 \\
0\end{array}$ & 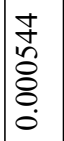 & $\begin{array}{l}\widetilde{N} \\
\tilde{o} \\
\vdots \\
\vdots \\
0\end{array}$ & $\mid \begin{array}{l}0 \\
N \\
\tilde{\delta} \\
\delta \\
0 \\
0\end{array}$ & $\begin{array}{l}\frac{\partial}{n} \\
\tilde{\delta} \\
\delta \\
\dot{0}\end{array}$ & 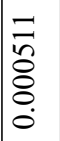 & 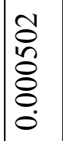 & 造 & 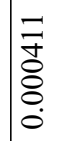 \\
\hline & & $\approx$ & 会 & 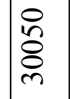 & $\begin{array}{l}\overrightarrow{\widetilde{Z}} \\
\text { নे }\end{array}$ & 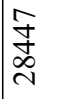 & 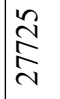 & $\begin{array}{l}\vec{n} \\
\grave{2}\end{array}$ & 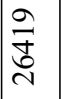 & 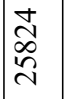 & $\begin{array}{l}\hat{\sigma} \\
\tilde{\sim} \\
\end{array}$ & $\underset{\stackrel{f}{\mathcal{J}}}{\stackrel{f}{\sim}}$ & $\mid$\begin{tabular}{l}
$\infty$ \\
\multirow{d}{J}{} \\
$\underset{\sim}{\sim}$
\end{tabular} & $\mid \begin{array}{c}\tilde{\infty} \\
\stackrel{\infty}{\tilde{N}} \\
\tilde{N}\end{array}$ & 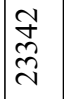 & $\begin{array}{l}\text { సे } \\
\text { సे }\end{array}$ & 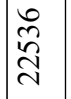 & $\begin{array}{l}\vec{\sigma} \\
\vec{N}\end{array}$ & $\begin{array}{l}\stackrel{ \pm}{D} \\
\frac{\infty}{\sim}\end{array}$ & $\begin{array}{l}\mathbb{J} \\
\stackrel{d}{d} \\
\stackrel{N}{N}\end{array}$ & 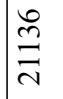 & 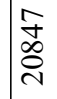 \\
\hline שै & ठิ & 苫 & $\hat{n}$ & $\begin{array}{l}\qquad 0 \\
\infty \\
i \\
i\end{array}$ & ஓे & के & ले & $\vec{F}$ & भे & $\mid \begin{array}{c}\tilde{\infty} \\
\stackrel{+}{+}\end{array}$ & $\begin{array}{l}\stackrel{0}{0} \\
\stackrel{+}{*}\end{array}$ & $\begin{array}{l}\infty \\
\infty \\
\dot{\sigma}\end{array}$ & $\begin{array}{l}\infty \\
\dot{\sigma}\end{array}$ & 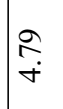 & ले & $\begin{array}{l}+ \\
\infty \\
\dot{m}\end{array}$ & $\begin{array}{l}\infty \\
\dot{m}\end{array}$ & $\begin{array}{l}\tilde{\sigma} \\
\dot{m}\end{array}$ & $\begin{array}{l}\infty \\
\infty \\
\dot{n}\end{array}$ & $\begin{array}{l}\vec{b} \\
\dot{r}\end{array}$ & 官 & बे \\
\hline $\begin{array}{l} \pm \\
\sigma \\
\frac{\overrightarrow{0}}{\Xi}\end{array}$ & 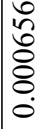 & $\vec{z}$ & $\underset{\sim}{\stackrel{n}{\sim}}$ & 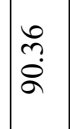 & $\frac{n}{\infty}$ & 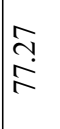 & $\begin{array}{l}0 \\
\stackrel{+}{i}\end{array}$ & $\begin{array}{l}\infty \\
\infty \\
\sim\end{array}$ & $\begin{array}{l}\vec{J} \\
\dot{I}\end{array}$ & $\begin{array}{l}0 \\
\text { ô. } \\
\text { in }\end{array}$ & $\begin{array}{l}\ddot{n} \\
\stackrel{i}{\pi}\end{array}$ & $\underset{⿱ 亠 䒑}{ \pm}$ & $\left|\begin{array}{l}1 \\
\infty \\
0 \\
0\end{array}\right|$ & $\begin{array}{l}80 \\
80\end{array}$ & $=$ & 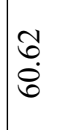 & $\begin{array}{l}7 \\
\text { in }\end{array}$ & $\begin{array}{l}\hat{n} \\
\infty \\
\infty\end{array}$ & $\begin{array}{l}3 \\
0 \\
\dot{0} \\
i n\end{array}$ & $\begin{array}{l}\tilde{n} \\
\tilde{n} \\
n\end{array}$ & $\begin{array}{l}0 \\
n \\
n \\
n \\
n\end{array}$ & $\begin{array}{l}\tilde{n} \\
\tilde{i}\end{array}$ \\
\hline $\begin{array}{l}\stackrel{\mathscr{z}}{\underline{\Delta}} \\
\frac{\vec{\omega}}{\Xi}\end{array}$ & 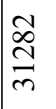 & 营 & $\frac{\vec{\sigma}}{\bar{m}}$ & $\begin{array}{l}\hat{\infty} \\
i \\
\dot{n}\end{array}$ & $\underset{n}{\tilde{n}}$ & $\begin{array}{l}n \\
\dot{n} \\
i n\end{array}$ & ô & $\begin{array}{l}\infty \\
i \\
i n \\
i n\end{array}$ & $\begin{array}{l}n \\
\infty \\
n \\
n\end{array}$ & 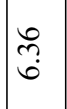 & స్ర & nֶ. & 占 & 志 & $\underset{\hat{\sigma}}{\stackrel{尺}{0}}$ & $\stackrel{\infty}{0}$ & o. & 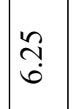 & $\frac{7}{6}$ & $\stackrel{\infty}{6}$ & ఫु. & 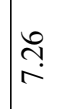 \\
\hline$\underset{\Xi}{\stackrel{્}{\Xi}}$ & ஓे & $\underbrace{\stackrel{y}{\Xi}}_{\beth}$ & 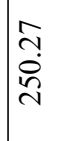 & $\begin{array}{l}\tilde{m} \\
\mathfrak{\delta} \\
\varrho\end{array}$ & $\begin{array}{l}\text { bे } \\
2 \\
2\end{array}$ & 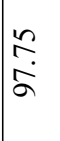 & $\begin{array}{l}\partial \\
o \\
\infty \\
o\end{array}$ & $\begin{array}{l}\mathfrak{N} \\
\stackrel{8}{0}\end{array}$ & $\stackrel{1}{a}$ & $\begin{array}{l}\tilde{6} \\
\stackrel{0}{0} \\
0\end{array}$ & $\frac{n}{\infty}$ & 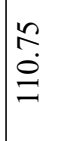 & $\stackrel{\partial}{\stackrel{\partial}{\Xi}}$ & $\begin{array}{l}\infty \\
\stackrel{\infty}{\Xi} \\
=\end{array}$ & $\begin{array}{l}\overrightarrow{\bar{\Delta}} \\
\stackrel{\Xi}{\Xi}\end{array}$ & $\begin{array}{l}\vec{n} \\
\mathfrak{o} \\
0\end{array}$ & $\mid \begin{array}{r}n \\
\tilde{o} \\
\varrho\end{array}$ & $\begin{array}{l}\stackrel{0}{0} \\
\stackrel{0}{\circ} \\
0\end{array}$ & 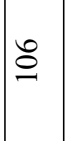 & Әิ & $\begin{array}{l}0 \\
\stackrel{0}{0} \\
\stackrel{\infty}{0} \\
0\end{array}$ & $\begin{array}{l}\text { f } \\
\dot{\Xi} \\
\stackrel{0}{0}\end{array}$ \\
\hline$\sum_{\text {I }}$ & $\stackrel{m}{n}$ & $\begin{array}{l}\text { ฮี } \\
\text { है } \\
= \\
=\end{array}$ & 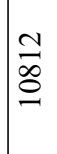 & 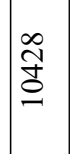 & 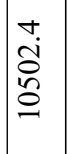 & $\mid \begin{array}{l}0 \\
0 \\
0 \\
0 \\
0 \\
0\end{array}$ & $\begin{array}{l}r \\
\hat{0} \\
0 \\
0 \\
0\end{array}$ & 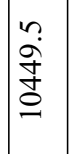 & ป̂ & 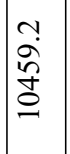 & 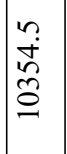 & $\begin{array}{l}a \\
\hat{\delta} \\
\tilde{0} \\
0\end{array}$ & $\begin{array}{l}\overrightarrow{1} \\
\tilde{0} \\
\tilde{O} \\
0\end{array}$ & 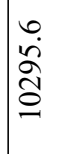 & 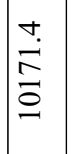 & \begin{tabular}{l} 
?a \\
\multirow{H}{0}{} \\
8 \\
0
\end{tabular} & $\begin{array}{l}n \\
\tilde{i} \\
\mathscr{O} \\
\stackrel{\Xi}{O}\end{array}$ & $\stackrel{\hat{s}}{8}$ & \begin{tabular}{l}
$n$ \\
\multirow{8}{*}{} \\
8 \\
0
\end{tabular} & $\begin{array}{l}n \\
\text { à } \\
\text { ă }\end{array}$ & $\begin{array}{l}0 \\
\dot{2} \\
\stackrel{2}{0}\end{array}$ & $\begin{array}{l}0 \\
\dot{0} \\
\hat{\infty} \\
\infty\end{array}$ \\
\hline $\begin{array}{l}\sum_{\pi}^{2} \\
0 \\
0 \\
0 \\
0\end{array}$ & હ̊ & $\vec{F}$ & 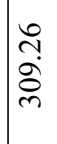 & $\begin{array}{l}\partial \\
\infty \\
\frac{\infty}{m}\end{array}$ & 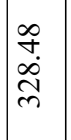 & $\underset{\hat{m}}{\stackrel{a}{m}}$ & 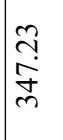 & 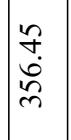 & $\begin{array}{l}\infty \\
n \\
n \\
b \\
n \\
n\end{array}$ & $\begin{array}{l}\mathfrak{n} \\
+ \\
\tilde{n} \\
m\end{array}$ & $\begin{array}{l}0 \\
\infty \\
\infty \\
\infty\end{array}$ & 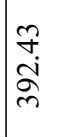 & $\mid \begin{array}{l}\infty \\
\stackrel{+}{\dot{\sigma}} \\
\dot{q}\end{array}$ & 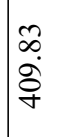 & $\begin{array}{l}0 \\
\tilde{n} \\
\infty \\
\forall \\
\forall\end{array}$ & 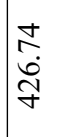 & $\mid \begin{array}{c}\tilde{o} \\
\dot{d} \\
\tilde{\sigma} \\
\end{array}$ & $\begin{array}{l}\infty \\
1 \\
\tilde{f} \\
\tilde{f}\end{array}$ & 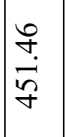 & $\begin{array}{l}n \\
\tilde{a} \\
\tilde{y} \\
\gamma\end{array}$ & 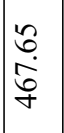 & $\begin{array}{l}\hat{o} \\
\stackrel{i}{f} \\
\hat{f}\end{array}$ \\
\hline 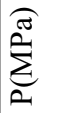 & $\frac{\Delta}{0}$ & 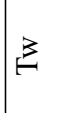 & 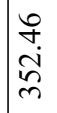 & $\frac{\infty}{\not}$ & $\begin{array}{l}\bar{\sigma} \\
\tilde{\gamma}\end{array}$ & $\vec{y}$ & 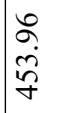 & $\mid$\begin{tabular}{c}
1 \\
\hdashline \\
8 \\
$\dot{8}$
\end{tabular} & $\begin{array}{l}\vec{b} \\
\dot{b} \\
\dot{b}\end{array}$ & $\begin{array}{l} \\
\stackrel{0}{9} \\
\stackrel{9}{f}\end{array}$ & 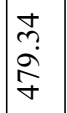 & $\mid \begin{array}{l}\overrightarrow{0} \\
\dot{0} \\
\dot{\infty}\end{array}$ & $\begin{array}{l}\tilde{N} \\
\tilde{g} \\
\dot{\sigma}\end{array}$ & $\mid \begin{array}{l}\infty \\
\stackrel{\infty}{2} \\
\stackrel{\alpha}{q} \\
\sigma\end{array}$ & $\begin{array}{l}\dot{\partial} \\
\dot{a} \\
\dot{n}\end{array}$ & $\begin{array}{l}m \\
\stackrel{2}{i} \\
\text { กิ }\end{array}$ & $\begin{array}{l}0 \\
\stackrel{0}{0} \\
\tilde{n}\end{array}$ & $\begin{array}{l}n \\
\infty \\
\tilde{n} \\
\tilde{n}\end{array}$ & 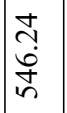 & $\begin{array}{l}0 \\
\hat{n} \\
n \\
n\end{array}$ & 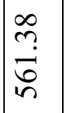 & 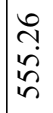 \\
\hline $\begin{array}{l}0 \\
\mathscr{Z} \\
\tilde{z} \\
\tilde{z}\end{array}$ & 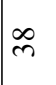 & $\stackrel{9}{9}$ & $N$ & $\vec{\infty}$ & $\overrightarrow{ \pm}$ & 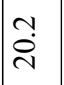 & ֶֶ. & $\begin{array}{l}n \\
\tilde{n}\end{array}$ & $\left|\begin{array}{c}m \\
\infty \\
\infty \\
m\end{array}\right|$ & $\mid \begin{array}{l}+ \\
\dot{J}\end{array}$ & $\begin{array}{l}t \\
\dot{8}\end{array}$ & $\begin{array}{l}n \\
0 \\
i \\
n\end{array}$ & $\mid \begin{array}{l}n \\
i \\
0\end{array}$ & $\begin{array}{l}n \\
\infty \\
\infty \\
0\end{array}$ & $\begin{array}{l}0 \\
\stackrel{+}{T}\end{array}$ & $\begin{array}{l}0 \\
\dot{0} \\
\infty\end{array}$ & $\begin{array}{l}\hat{\infty} \\
\infty\end{array}$ & $\hat{a}$ & $\left|\begin{array}{l}\infty \\
\infty \\
\infty\end{array}\right|$ & $\begin{array}{l}\infty \\
\dot{0} \\
\stackrel{0}{0}\end{array}$ & $\stackrel{\varrho}{\grave{O}}$ & \\
\hline
\end{tabular}




\begin{tabular}{|c|c|c|c|c|c|c|c|c|c|c|c|c|c|c|c|c|c|c|c|c|c|c|}
\hline & & $\vec{\Delta}$ & 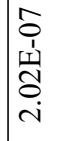 & 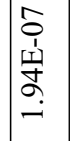 & $\begin{array}{l}\hat{0} \\
\hat{1} \\
1 \\
\propto \\
o \\
-1\end{array}$ & 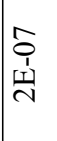 & 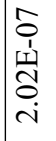 & 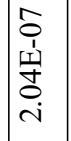 & 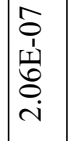 & 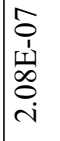 & 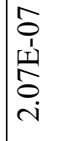 & 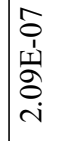 & 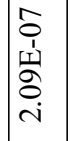 & 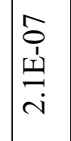 & 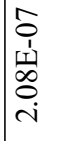 & 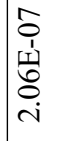 & 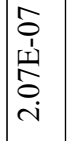 & $\begin{array}{c}\hat{s} \\
\hat{1} \\
1 \\
\vdots \\
0 \\
i\end{array}$ & 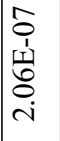 & 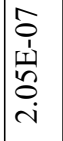 & 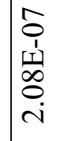 & 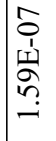 \\
\hline & & $\stackrel{\oplus}{\infty}$ & 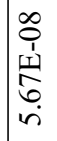 & \begin{tabular}{l}
$\infty$ \\
0 \\
1 \\
1 \\
$\infty$ \\
\multirow{1}{*}{} \\
$\dot{\sigma}$
\end{tabular} & $\begin{array}{l}\infty \\
0 \\
\stackrel{1}{1} \\
\stackrel{1}{m} \\
m \\
\forall \\
\forall\end{array}$ & $\begin{array}{l}\infty \\
0 \\
0 \\
1 \\
\text { ஸ્j } \\
0 \\
\dot{0} \\
\dot{r}\end{array}$ & 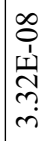 & $\begin{array}{l}\infty \\
0 \\
0 \\
1 \\
\\
0 \\
\dot{r} \\
\dot{r}\end{array}$ & 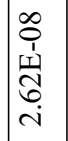 & 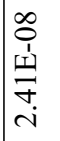 & $\begin{array}{l}\infty \\
\stackrel{\infty}{1} \\
\stackrel{1}{\sim} \\
\underset{\sim}{\sim}\end{array}$ & 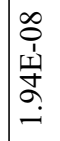 & 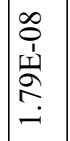 & $\mid \begin{array}{c}\infty \\
0 \\
0 \\
1 \\
0 \\
0 \\
0 \\
- \\
-\end{array}$ & $\mid$\begin{tabular}{c}
$\infty$ \\
0 \\
$i$ \\
\multicolumn{1}{c}{} \\
$\tilde{n}$ \\
\\
-1
\end{tabular} & 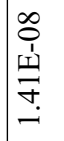 & 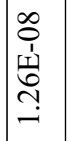 & $\begin{array}{c}\infty \\
0 \\
1 \\
1 \\
\infty \\
-1 \\
-1\end{array}$ & 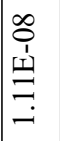 & 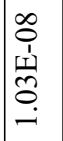 & $\begin{array}{l}\hat{o} \\
\hat{1} \\
\text { Wh} \\
\text { aे }\end{array}$ & $\begin{array}{l}\stackrel{\partial}{c} \\
\text { II } \\
\stackrel{1}{r}\end{array}$ \\
\hline & & $\vec{D}$ & $\begin{array}{l}\infty \\
0 \\
0 \\
0 \\
0 \\
0\end{array}$ & 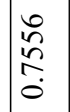 & $\frac{0}{\frac{\pi}{n}}$ & 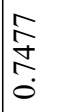 & $\frac{\mathfrak{y}}{\stackrel{y}{0}}$ & 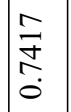 & 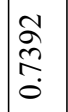 & $\underset{\stackrel{n}{n}}{\stackrel{n}{0}}$ & $\begin{array}{l}\hat{n} \\
\approx \\
0\end{array}$ & 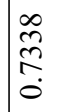 & $\mid \begin{array}{l}\mathbb{A} \\
\stackrel{2}{2} \\
\stackrel{2}{0}\end{array}$ & $\frac{m}{m}$ & 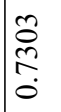 & $\begin{array}{l}\stackrel{n}{\hat{N}} \\
\dot{0}\end{array}$ & $\mid \begin{array}{c}2 \\
\stackrel{2}{N} \\
\dot{0}\end{array}$ & $\begin{array}{l}\tilde{D} \\
\mathbb{N} \\
\stackrel{0}{0}\end{array}$ & $\underset{\substack{\stackrel{0}{N} \\
\stackrel{0}{0}}}{ }$ & 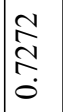 & $\begin{array}{l}\hat{0} \\
\stackrel{N}{0} \\
\dot{0}\end{array}$ & 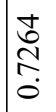 \\
\hline & & $\mathscr{J}$ & 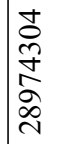 & $\begin{array}{l}0 \\
0 \\
0 \\
0 \\
\infty \\
0 \\
0\end{array}$ & 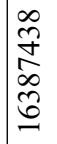 & 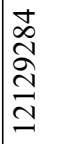 & 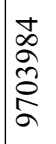 & 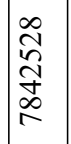 & $\begin{array}{l} \pm \\
\tilde{m} \\
\hat{\delta} \\
\delta\end{array}$ & $\begin{array}{l}m \\
\cdots \\
o \\
i \\
i n \\
i n\end{array}$ & $\begin{array}{l}\hat{0} \\
8 \\
0 \\
0 \\
\Rightarrow\end{array}$ & 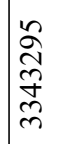 & 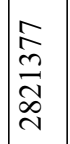 & 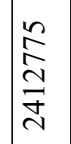 & 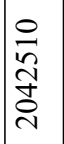 & 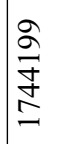 & 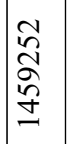 & 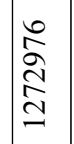 & 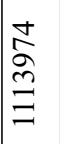 & $\begin{array}{l}\hat{0} \\
\tilde{n} \\
\hat{\sigma}\end{array}$ & 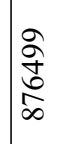 & $\begin{array}{l}8 \\
2 \\
2 \\
\text { in }\end{array}$ \\
\hline & & t & 命 & $\begin{array}{l}n \\
\hat{\alpha} \\
\delta \\
0 \\
0 \\
0\end{array}$ & 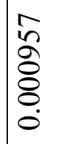 & 合 & $\begin{array}{l}\hat{2} \\
\text { oे } \\
\vdots \\
0 \\
0\end{array}$ & $\left|\begin{array}{l}+ \\
\infty \\
0 \\
0 \\
0 \\
0 \\
0\end{array}\right|$ & 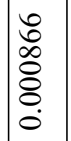 & 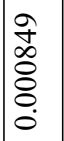 & $\begin{array}{l}0 \\
0 \\
0 \\
0 \\
0 \\
0\end{array}$ & 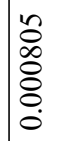 & $\left|\begin{array}{l}n \\
\infty \\
0 \\
0 \\
0 \\
0 \\
0 \\
0\end{array}\right|$ & $\mid \begin{array}{c}\overrightarrow{\hat{s}} \\
\hat{\sigma} \\
0 \\
0 \\
\dot{0}\end{array}$ & $\mid \begin{array}{l}\frac{n}{1} \\
\frac{1}{8} \\
8 \\
0 \\
0 \\
0\end{array}$ & $\begin{array}{l}\overrightarrow{\widehat{N}} \\
\stackrel{\hat{\Xi}}{8} \\
\vdots \\
0\end{array}$ & 章 & $\begin{array}{l}0 \\
0 \\
0 \\
8 \\
0 \\
0\end{array}$ & 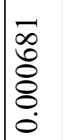 & 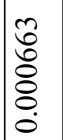 & 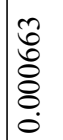 & 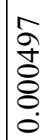 \\
\hline & & $\approx$ & 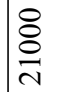 & $\begin{array}{l}\hat{\partial} \\
\dot{0} \\
\dot{i}\end{array}$ & $\begin{array}{l}\widetilde{\Xi} \\
\text { ડે }\end{array}$ & 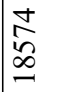 & $\begin{array}{l}\text { İ } \\
\text { İ }\end{array}$ & $\mid \begin{array}{c}n \\
\tilde{\Xi} \\
\Xi\end{array}$ & \begin{tabular}{l}
0 \\
\multirow{6}{6}{}
\end{tabular} & 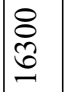 & $\begin{array}{l}n \\
1 \\
\infty \\
n \\
n\end{array}$ & \begin{tabular}{l}
$\cong$ \\
\multirow{y}{*}{} \\
\end{tabular} & $\mid \begin{array}{l}0 \\
\tilde{2} \\
n \\
n\end{array}$ & 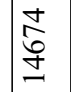 & 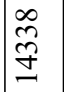 & $\widehat{\widehat{O}}$ & $\mid \begin{array}{c}\hat{n} \\
\stackrel{n}{n}\end{array}$ & $\begin{array}{l}3 \\
0 \\
0 \\
m\end{array}$ & 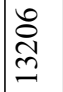 & $\begin{array}{l}\hat{0} \\
\grave{\Xi}\end{array}$ & 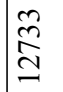 & 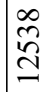 \\
\hline שै & ठี & 窻 & $\underset{\vec{\lambda}}{\vec{N}}$ & $\hat{n}$ & $\stackrel{\Delta}{r}$ & $\mid \begin{array}{l}\alpha \\
\infty \\
\end{array}$ & ڤે & 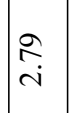 & $\begin{array}{l}\infty \\
i\end{array}$ & $\stackrel{\overrightarrow{\hat{i}}}{i}$ & $\underset{⿱ 亠}{i}$ & $\stackrel{n}{a}$ & $\stackrel{+}{i}$ & $\mid \begin{array}{l}\infty \\
0 \\
i \\
\text { in }\end{array}$ & 总 & $\begin{array}{l}\infty \\
\\
i\end{array}$ & $\mid \begin{array}{l}n \\
\dot{n} \\
i\end{array}$ & $\ddot{r}$ & $\stackrel{\infty}{\stackrel{\infty}{i}}$ & $\begin{array}{l}\partial \\
\text { i }\end{array}$ & $\overrightarrow{\vec{i}}$ & $\stackrel{\infty}{\stackrel{\infty}{i}}$ \\
\hline $\begin{array}{l}\frac{+}{\sigma} \\
\frac{\vec{U}}{\Xi}\end{array}$ & 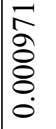 & $\vec{z}$ & $\begin{array}{l}\tilde{N} \\
\stackrel{\infty}{\infty} \\
\stackrel{\infty}{2}\end{array}$ & $\underset{\overrightarrow{0}}{=}$ & $\begin{array}{l}2 \\
0 \\
8 \\
8\end{array}$ & 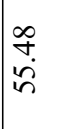 & $\begin{array}{l}\mathbf{D} \\
\infty \\
i \\
i n\end{array}$ & $\mid \begin{array}{l}\infty \\
\stackrel{\vec{n}}{n}\end{array}$ & $\frac{\alpha}{\hat{n}}$ & $\frac{n}{\sim}$ & $\begin{array}{l}0 \\
\stackrel{2}{a} \\
a \\
q\end{array}$ & $\begin{array}{l}\mathscr{o} \\
\dot{q} \\
\dot{q}\end{array}$ & $\begin{array}{l}\stackrel{\partial}{\sigma} \\
\vec{\gamma}\end{array}$ & $\begin{array}{l}\mathcal{f} \\
\mathcal{f} \\
\dot{f}\end{array}$ & $\begin{array}{l}\vec{\infty} \\
\dot{J}\end{array}$ & $\stackrel{\infty}{\stackrel{一}{\vec{F}}}$ & 志 & $\begin{array}{l}\vec{n} \\
\dot{m}\end{array}$ & $\begin{array}{l}\hat{f} \\
\infty \\
\infty \\
m\end{array}$ & 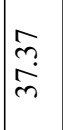 & $\underset{m}{\infty}$ & 节 \\
\hline $\begin{array}{l}\stackrel{\mathscr{z}}{\underline{\Delta}} \\
\frac{\vec{\omega}}{\Xi}\end{array}$ & $\underset{\text { }}{\stackrel{\curvearrowright}{N}}$ & 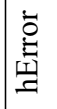 & $\begin{array}{l}\hat{\tilde{N}} \\
\hat{\sim}\end{array}$ & $\begin{array}{l}\mathbb{b} \\
\dot{m}\end{array}$ & $\stackrel{\bar{m}}{m}$ & $\begin{array}{l}\infty \\
\dot{0} \\
\dot{m}\end{array}$ & $\vec{m}$ & $\underset{\substack{\infty \\
m}}{ }$ & ț & $\hat{\sigma}$ & ঐे & $\overrightarrow{\widetilde{r}}$ & $\stackrel{+}{\stackrel{m}{+}}$ & & 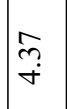 & $\stackrel{\infty}{\stackrel{\infty}{m}}$ & $\exists$ & $\stackrel{\Xi}{\nexists}$ & $\stackrel{\infty}{\stackrel{\infty}{+}}$ & $\stackrel{\partial}{\vec{r}}$ & 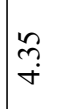 & 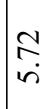 \\
\hline$\underset{\Xi}{\stackrel{\widehat{a}}{\Xi}}$ & 裉 & $\underbrace{\stackrel{y}{\Xi}}_{\beth}$ & $\begin{array}{l}\overrightarrow{\vec{d}} \\
\overrightarrow{\mathrm{v}}\end{array}$ & $\frac{n}{\stackrel{\infty}{\infty}}$ & ঙ্ৰ? & $\mid \begin{array}{l}0 \\
\dot{b} \\
i \\
1\end{array}$ & $\begin{array}{l}\stackrel{0}{2} \\
\stackrel{+}{+}\end{array}$ & $\stackrel{\vec{N}}{\stackrel{N}{R}}$ & $\overrightarrow{\dot{\infty}}$ & $\frac{m}{\infty}$ & 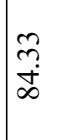 & $\begin{array}{l}\stackrel{n}{\hat{\infty}} \\
\infty \\
\infty\end{array}$ & $\begin{array}{c}\simeq \\
\infty \\
\infty \\
\infty\end{array} \mid$ & $\frac{\bar{z}}{\dot{8}}$ & $\mid \begin{array}{l}\infty \\
0 \\
\infty \\
\infty \\
\infty\end{array}$ & \begin{tabular}{l}
\multirow{n}{n}{} \\
$\infty$ \\
$\infty$
\end{tabular} & $\stackrel{m}{\infty}$ & $\begin{array}{l}\hat{\alpha} \\
\dot{\infty}\end{array}$ & $\begin{array}{l}0 \\
0 \\
\infty \\
\infty \\
\infty\end{array}$ & $\begin{array}{l}\infty \\
\dot{\infty} \\
\dot{\infty}\end{array}$ & 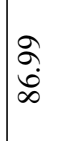 & $\mid \begin{array}{l}n \\
n \\
\infty \\
\infty\end{array}$ \\
\hline$\sum_{\text {至 }}^{\Sigma}$ & $\stackrel{n}{n}$ & $\begin{array}{l}\text { đิ } \\
\text { है } \\
= \\
=\end{array}$ & 官 & \begin{tabular}{l}
$\infty$ \\
0 \\
\multirow{0}{0}{} \\
$\varrho$ \\
0
\end{tabular} & $\frac{a}{\hat{n}}$ & $\begin{array}{l}7 \\
\dot{0} \\
0 \\
0 \\
0\end{array}$ & त్రి & $\mid \begin{array}{l}0 \\
\dot{0} \\
\infty \\
0 \\
0 \\
0\end{array}$ & $\begin{array}{l}n \\
\tilde{\infty} \\
\infty \\
ٍ \\
\varrho \\
0\end{array}$ & $\begin{array}{l}n \\
\tilde{\hat{\sigma}} \\
\hat{o} \\
0\end{array}$ & 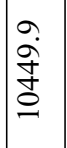 & $\begin{array}{l}\hat{0} \\
\infty \\
o \\
0 \\
0 \\
0\end{array}$ & $\mid \begin{array}{l}\hat{\tilde{\sigma}} \\
\tilde{\tilde{O}}\end{array}$ & 文 & $\begin{array}{l}\overrightarrow{\text { ले }} \\
\text { तิ } \\
\underline{0}\end{array}$ & $\begin{array}{l}n \\
\infty \\
\infty \\
\infty \\
o \\
0 \\
0\end{array}$ & $\mid \begin{array}{c}+ \\
\dot{0} \\
\stackrel{0}{0} \\
0\end{array}$ & $\begin{array}{l}0 \\
\tilde{2} \\
\hat{8} \\
0\end{array}$ & $\widehat{\widehat{\Xi}}$ & 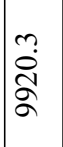 & $\begin{array}{l}0 \\
\stackrel{+}{+} \\
\stackrel{8}{8}\end{array}$ & $\frac{n}{8}$ \\
\hline $\begin{array}{l}\sum_{\pi}^{2} \\
0 \\
0 \\
0 \\
0\end{array}$ & 合 & $\vec{F}$ & $\begin{array}{l}\text { N̦ } \\
\stackrel{n}{m}\end{array}$ & 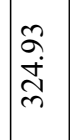 & $\begin{array}{l}8 \\
\stackrel{0}{0} \\
\text { ले }\end{array}$ & $\begin{array}{l}\infty \\
\hat{\sim} \\
\tilde{n} \\
m\end{array}$ & $\begin{array}{l}0 \\
0 \\
0 \\
0 \\
0 \\
\text { ". }\end{array}$ & $\mid \begin{array}{l} \pm \\
\dot{0} \\
\infty \\
m\end{array}$ & $\begin{array}{l}\vec{\sigma} \\
\stackrel{\sim}{2}\end{array}$ & 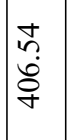 & 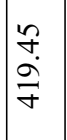 & $\mid \begin{array}{l}\stackrel{n}{a} \\
\underset{\gamma}{\sim}\end{array}$ & $\begin{array}{l}\infty \\
0 \\
\dot{J} \\
\dot{f}\end{array}$ & $\mid \begin{array}{l}8 \\
\dot{1} \\
\frac{5}{8}\end{array}$ & 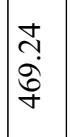 & 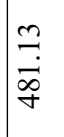 & $\mid \begin{array}{l}\circ \\
\infty \\
i \\
\stackrel{\sigma}{\sigma}\end{array}$ & \begin{tabular}{l}
$\infty$ \\
+ \\
\multirow{5}{+}{} \\
$i n$
\end{tabular} & $\begin{array}{l}2 \\
2 \\
i n \\
n\end{array}$ & $\begin{array}{l}\infty \\
\stackrel{1}{a} \\
\stackrel{i}{n} \\
i n\end{array}$ & $\left|\begin{array}{l}n \\
n \\
\infty \\
0 \\
n \\
n\end{array}\right|$ & $\begin{array}{l}q \\
q \\
\infty \\
\dot{+} \\
\dot{n}\end{array}$ \\
\hline 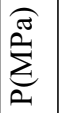 & $\frac{\Delta}{0}$ & 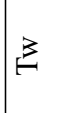 & 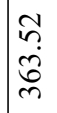 & $\begin{array}{l}0 \\
n \\
n \\
n \\
\gamma\end{array}$ & 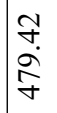 & 落 & $\begin{array}{l}\infty \\
m \\
\infty \\
\infty \\
\infty \\
\infty\end{array}$ & 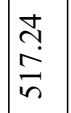 & $\begin{array}{l}\bar{\alpha} \\
\tilde{\sim} \\
\tilde{n}\end{array}$ & \begin{tabular}{|l|}
$\infty$ \\
$a$ \\
0 \\
$\tilde{n}$ \\
$n$
\end{tabular} & $\begin{array}{l}\tilde{n} \\
\tilde{y} \\
\tilde{n}\end{array}$ & $\mid \begin{array}{l}\stackrel{N}{a} \\
i \\
i n\end{array}$ & $\begin{array}{l}\mathbb{U} \\
i \\
i \\
i \\
n\end{array}$ & $\mid \begin{array}{l}\infty \\
\tilde{n} \\
i \\
i n\end{array}$ & $\begin{array}{l}F \\
\forall \\
\infty \\
\infty \\
n\end{array}$ & 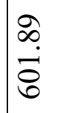 & 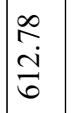 & $\begin{array}{l}\tilde{\sigma} \\
\tilde{\hat{\sigma}}\end{array}$ & $\begin{array}{c}\infty \\
\infty \\
\tilde{\sigma} \\
\hat{\sigma}\end{array}$ & $\begin{array}{l}\hat{N} \\
\dot{J} \\
\text { J }\end{array}$ & $\begin{array}{l}\tilde{n} \\
\tilde{\sigma} \\
\tilde{\sigma}\end{array}$ & $\begin{array}{l}\overline{0} \\
\hat{\sigma} \\
\text { on }\end{array}$ \\
\hline 岕 & ले & $\stackrel{9}{9}$ & $\sim$ & $\vec{\infty}$ & 亣 & กิ & ֶָ. & 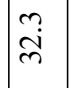 & $\mid \begin{array}{c}m \\
\infty \\
m \\
m\end{array}$ & $\mid \begin{array}{l}\dot{J} \\
\dot{f}\end{array}$ & $\begin{array}{l}t \\
\dot{B}\end{array}$ & \begin{tabular}{l}
$n$ \\
\hdashline \\
\hdashline \\
$n$
\end{tabular} & $\begin{array}{l}n \\
i \\
\delta\end{array}$ & $\mid \begin{array}{l}n \\
\infty \\
\infty \\
0\end{array}$ & $\mid \begin{array}{l}0 \\
\stackrel{+}{i}\end{array}$ & $\begin{array}{l}0 \\
\dot{\infty} \\
\infty\end{array}$ & $\hat{\infty}$ & $\overline{\check{a}}$ & $\left|\begin{array}{l}\infty \\
\infty \\
\infty \\
\alpha\end{array}\right|$ & $\begin{array}{l}\infty \\
\dot{0} \\
\dot{0}\end{array}$ & $\stackrel{\partial}{\varrho}$ & ڤे \\
\hline
\end{tabular}




\begin{tabular}{|c|c|c|c|c|c|c|c|c|c|c|c|c|c|c|c|c|c|c|c|c|c|c|}
\hline & & $\vec{z}$ & 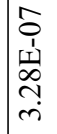 & 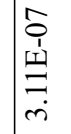 & 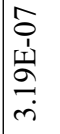 & \begin{tabular}{|l}
$\hat{0}$ \\
$\hat{1}$ \\
$\hat{1}$ \\
$\tilde{n}$ \\
\end{tabular} & 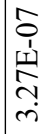 & 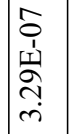 & \begin{tabular}{|l}
$\hat{0}$ \\
$\hat{1}$ \\
$\underline{1}$ \\
$\tilde{m}$ \\
\end{tabular} & 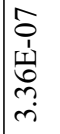 & \begin{tabular}{l}
$\hat{o}$ \\
$\hat{1}$ \\
\multicolumn{1}{|c}{} \\
$\tilde{m}$ \\
\end{tabular} & 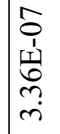 & $\mid \begin{array}{l}\hat{o} \\
1 \\
1 \\
\\
\tilde{n} \\
\end{array}$ & 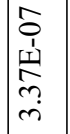 & 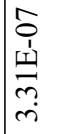 & $\begin{array}{l}\hat{0} \\
\hat{1} \\
\underline{1} \\
\\
\text { ry }\end{array}$ & 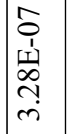 & 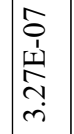 & $\mid \begin{array}{l}\hat{0} \\
1 \\
\underline{1} \\
\\
\end{array}$ & 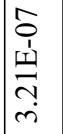 & $\begin{array}{l}\hat{0} \\
\hat{1} \\
\\
\\
\end{array}$ & $\begin{array}{l}\hat{o} \\
\hat{1} \\
\text { ñ } \\
\tilde{n} \\
\end{array}$ \\
\hline & & $\stackrel{\oplus}{\infty}$ & 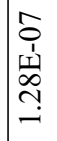 & 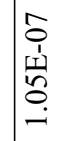 & \begin{tabular}{l}
$\infty$ \\
0 \\
0 \\
1 \\
\multicolumn{1}{|c}{} \\
$\infty$ \\
$\infty$ \\
$\infty$ \\
$\infty$
\end{tabular} & 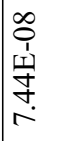 & 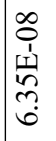 & 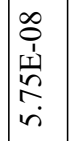 & \begin{tabular}{l}
$\infty$ \\
0 \\
$\hat{1}$ \\
1 \\
$\infty$ \\
$\infty$ \\
\multirow{r}{*}{}
\end{tabular} & 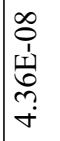 & $\begin{array}{l}\infty \\
0 \\
\hat{1} \\
\hat{1} \\
\alpha \\
\alpha \\
\dot{n}\end{array}$ & 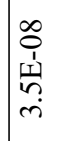 & 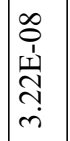 & $\begin{array}{l}\infty \\
0 \\
1 \\
1 \\
\\
\infty \\
i \\
\sim\end{array}$ & 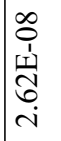 & 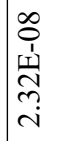 & 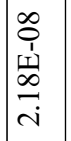 & $\begin{array}{l}\infty \\
0 \\
0 \\
1 \\
\tilde{1} \\
0 \\
\dot{i} \\
\sim\end{array}$ & $\mid \begin{array}{c}\infty \\
0 \\
0 \\
1 \\
1 \\
\infty \\
\infty \\
-i\end{array}$ & 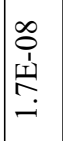 & 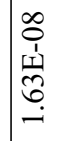 & 年 \\
\hline & & $\vec{D}$ & $\begin{array}{l}0 \\
0 \\
0 \\
0\end{array}$ & $\begin{array}{l}\vec{f} \\
\stackrel{n}{2} \\
0\end{array}$ & $\frac{\vec{a}}{\stackrel{\vec{J}}{\hat{O}}}$ & $\frac{\infty}{\frac{0}{2}}$ & $\underset{\frac{m}{7}}{\stackrel{m}{0}}$ & $\mid \begin{array}{c}\tilde{\infty} \\
\widetilde{N} \\
\hat{0}\end{array}$ & 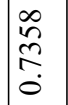 & 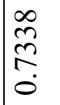 & $\stackrel{\overrightarrow{\widetilde{N}}}{\stackrel{\tilde{0}}{0}}$ & 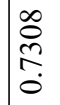 & 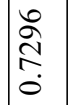 & $\mid \begin{array}{l}\infty \\
\infty \\
\stackrel{\infty}{N} \\
0\end{array}$ & 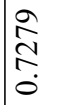 & 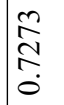 & 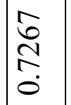 & 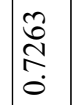 & $\mid \begin{array}{l}0 \\
\stackrel{N}{0}\end{array}$ & 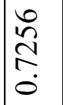 & 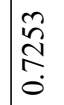 & 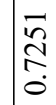 \\
\hline & & $\mathscr{J}$ & 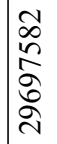 & $\begin{array}{l}\infty \\
n \\
\tilde{n} \\
\bar{n} \\
\sigma\end{array}$ & 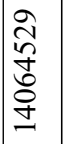 & 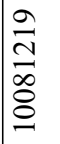 & 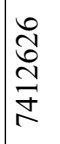 & 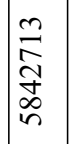 & \begin{tabular}{l}
$\mathcal{J}$ \\
$\widetilde{J}$ \\
$\tilde{Z}$ \\
\multirow{Z}{Z}{}
\end{tabular} & 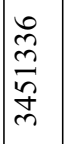 & 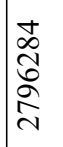 & 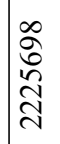 & $\mid \begin{array}{l}0 \\
0 \\
0 \\
\tilde{n} \\
\infty \\
-1\end{array}$ & 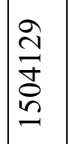 & 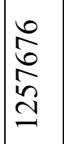 & 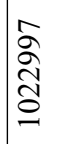 & $\begin{array}{l}\stackrel{n}{\vec{\sigma}} \\
\infty \\
\infty\end{array}$ & 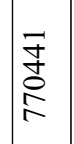 & 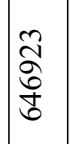 & $\begin{array}{l}\vec{D} \\
\stackrel{m}{0} \\
\vec{D} \\
i n\end{array}$ & $\begin{array}{l}\overrightarrow{\widetilde{\Xi}} \\
\text { ָे } \\
\text { in }\end{array}$ & $\frac{\vec{J}}{\stackrel{g}{g}}$ \\
\hline & & t & 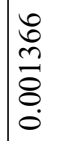 & 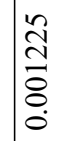 & 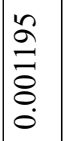 & $\begin{array}{l}n \\
\frac{n}{3} \\
\overline{8} \\
0 \\
0\end{array}$ & $\begin{array}{l}\overline{\widetilde{I}} \\
\bar{\Xi} \\
\dot{0} \\
0\end{array}$ & $\mid \begin{array}{l}\infty \\
\stackrel{\infty}{0} \\
\stackrel{0}{0} \\
0 \\
0\end{array}$ & \begin{tabular}{l}
$\infty$ \\
\hdashline \\
\hdashline \\
\hdashline \\
0 \\
0
\end{tabular} & $\mid \begin{array}{c}\tilde{D} \\
\hat{\delta} \\
\frac{0}{8} \\
0 \\
0\end{array}$ & 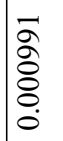 & 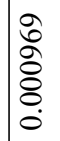 & $\begin{array}{c}\hat{2} \\
\hat{\delta} \\
\stackrel{0}{0} \\
\dot{0}\end{array}$ & 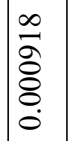 & $\begin{array}{l}\infty \\
\infty \\
0 \\
0 \\
0 \\
0 \\
0\end{array}$ & 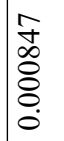 & 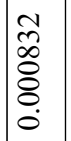 & $\mid \begin{array}{c}1 \\
0 \\
0 \\
0 \\
0 \\
0 \\
0\end{array}$ & $\mid \begin{array}{l}\tilde{\hat{S}} \\
\hat{\delta} \\
0 \\
0 \\
0\end{array}$ & $\begin{array}{l}n \\
0 \\
\vdots \\
\vdots \\
0 \\
0\end{array}$ & 递 & 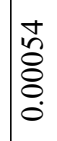 \\
\hline & & $\approx$ & 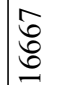 & $\begin{array}{l}\infty \\
\\
i n \\
-1\end{array}$ & $\mid \begin{array}{r}\text { ڤે } \\
\text { gे }\end{array}$ & $\begin{array}{l}\tilde{N} \\
\tilde{J}\end{array}$ & 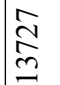 & $\frac{2}{2}$ & 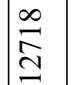 & 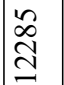 & $\begin{array}{l}\stackrel{+}{\infty} \\
\stackrel{\partial}{=}\end{array}$ & $\underline{\hat{n}}$ & $\vec{\Xi}$ & $\frac{0}{2}$ & $\begin{array}{l}n \\
\tilde{\delta} \\
0 \\
0\end{array}$ & 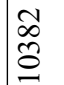 & $\stackrel{\infty}{\stackrel{\infty}{\sigma}}$ & ลे & 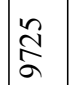 & 芯 & $\begin{array}{l}\hat{n} \\
\hat{\alpha}\end{array}$ & 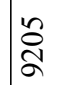 \\
\hline లే & రิ & 窻 & $\stackrel{\overbrace{}}{\stackrel{2}{\Sigma}}$ & $\stackrel{n}{a}$ & ते & $\underset{\tilde{i}}{\tilde{i}}$ & Oे & $\stackrel{\infty}{\stackrel{\infty}{-}}$ & $\stackrel{\circ}{2}$ & त̂ & $\stackrel{\Omega}{-}$ & 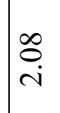 & $\mid \begin{array}{l}n \\
g \\
-\end{array}$ & $\stackrel{\hat{\sigma}}{i}$ & 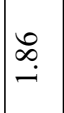 & $\stackrel{\infty}{\infty}$ & $\underset{-}{\tilde{C}}$ & $\stackrel{\Re}{\stackrel{q}{-}}$ & $\stackrel{\infty}{\stackrel{\infty}{0}}$ & nn & $\cong$ & 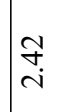 \\
\hline $\begin{array}{l} \pm \\
\sigma \\
\frac{\overrightarrow{0}}{\Xi}\end{array}$ & 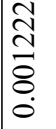 & $\vec{z}$ & $\frac{n}{n}$ & $\begin{array}{l}\hat{b} \\
\text { in }\end{array}$ & 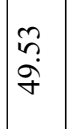 & $\begin{array}{l}\overrightarrow{\ddot{y}} \\
\dot{q}\end{array}$ & 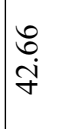 & $\underset{f}{\vec{f}}$ & $\frac{\grave{j}}{\vec{\gamma}}$ & $\stackrel{\sim}{\vec{\gamma}}$ & $\frac{\partial}{\vec{m}}$ & $\begin{array}{l}\stackrel{n}{o} \\
\infty \\
m\end{array}$ & $\begin{array}{l}a \\
\infty \\
\hat{m}\end{array}$ & $\frac{n}{n}$ & 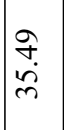 & $\begin{array}{l}\infty \\
\infty \\
\infty \\
\infty\end{array}$ & $\overrightarrow{\tilde{i}}$ & $\frac{\infty}{n}$ & $\begin{array}{l}\vec{\infty} \\
0 \\
\dot{\infty}\end{array}$ & $\begin{array}{l}\stackrel{n}{a} \\
\grave{i}\end{array}$ & $\begin{array}{l}\hat{2} \\
\text { ते }\end{array}$ & $\begin{array}{l}\hat{\infty} \\
0 \\
\infty \\
\infty\end{array}$ \\
\hline 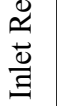 & $\frac{n}{2}$ & 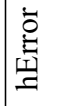 & $\begin{array}{l}\vec{\alpha} \\
\infty\end{array}$ & 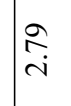 & సે & $\overrightarrow{\tilde{n}}$ & $\stackrel{\sim}{\tilde{r}}$ & $\stackrel{\infty}{\stackrel{\infty}{\sim}}$ & $\stackrel{d}{i}$ & 志 & $\underset{\sigma}{\tilde{r}}$ & 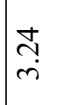 & $\begin{array}{l}m \\
m \\
m\end{array}$ & $\mid \begin{array}{l}\infty \\
n \\
m\end{array}$ & 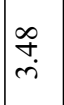 & ते & ma & $\stackrel{\dot{m}}{\dot{m}}$ & $\underset{\dot{r}}{\stackrel{q}{r}}$ & $\begin{array}{l}\stackrel{l}{0} \\
\stackrel{1}{m}\end{array}$ & 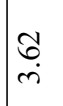 & $\begin{array}{l}\stackrel{2}{a} \\
\text { in }\end{array}$ \\
\hline$\underset{\Xi}{\stackrel{\Xi}{\Xi}}$ & 占 & $\underbrace{\stackrel{y}{\Xi}}_{\beth}$ & $\underset{\tilde{\sigma}}{\tilde{\sigma}}$ & $\begin{array}{l}\infty \\
n \\
? \\
?\end{array}$ & $\begin{array}{l}\text { ते } \\
\text { है }\end{array}$ & $\stackrel{\infty}{\stackrel{\infty}{6}}$ & $\underset{\text { I }}{\stackrel{7}{f}}$ & $\mid \begin{array}{l}\infty \\
0 \\
0 \\
0\end{array}$ & $\stackrel{m}{\stackrel{9}{0}}$ & م. & $\begin{array}{l}\mathbb{J} \\
\stackrel{i}{i} \\
\mathbb{i}\end{array}$ & $\begin{array}{l}\sigma \\
\delta \\
i \\
i\end{array}$ & $\mid \begin{array}{l}0 \\
0 \\
6 \\
0\end{array}$ & $\left|\begin{array}{l}n \\
0 \\
\infty \\
\infty \\
i\end{array}\right|$ & $\stackrel{2}{\stackrel{2}{R}}$ & \begin{tabular}{l}
\multirow{2}{*}{} \\
ले
\end{tabular} & $\begin{array}{l}\infty \\
0 \\
i \\
i\end{array}$ & $\begin{array}{l}0 \\
i \\
i \\
i\end{array}$ & 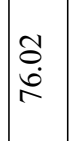 & \begin{tabular}{l}
8 \\
\multirow{2}{*}{} \\
$i$ \\
$i$
\end{tabular} & 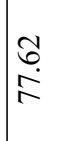 & 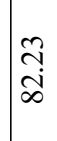 \\
\hline$\sum_{\text {至 }}$ & $\stackrel{\infty}{i}$ & $\begin{array}{l}\text { đิ } \\
\text { है } \\
= \\
=\end{array}$ & $\begin{array}{l}n \\
\stackrel{0}{0} \\
ٍ \\
=\end{array}$ & $\begin{array}{l}\bar{i} \\
\tilde{b} \\
\hat{0}\end{array}$ & $\begin{array}{l}\hat{\circ} \\
\stackrel{8}{0} \\
\stackrel{0}{\circ}\end{array}$ & $\mid \begin{array}{l}3 \\
6 \\
\vdots \\
\vdots \\
0 \\
0\end{array}$ & 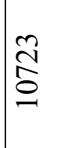 & $\begin{array}{l}0 \\
i \\
0 \\
0\end{array}$ & $\begin{array}{l}\infty \\
i \\
\mathbb{ర} \\
\stackrel{\delta}{0} \\
-\end{array}$ & $\begin{array}{l}n \\
8 \\
0 \\
0 \\
0\end{array}$ & $\begin{array}{l}\infty \\
\tilde{0} \\
\tilde{0} \\
0 \\
0\end{array}$ & 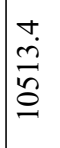 & $\begin{array}{l}\overrightarrow{+} \\
\dot{J} \\
\stackrel{+}{0}\end{array}$ & 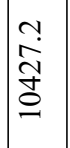 & $\mid \begin{array}{l}\overrightarrow{ \pm} \\
\widehat{\Xi} \\
\underline{O}\end{array}$ & ळें & $\mid \begin{array}{l}0 \\
0 \\
0 \\
8 \\
0 \\
0\end{array}$ & $\begin{array}{l}\hat{m} \\
\dot{m} \\
\stackrel{\Xi}{0}\end{array}$ & $\begin{array}{l}\stackrel{\Upsilon}{\Omega} \\
\hat{\alpha} \\
\alpha\end{array}$ & $\begin{array}{l}3 \\
0 \\
\infty \\
\infty \\
\alpha \\
\alpha\end{array}$ & 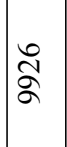 & 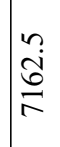 \\
\hline $\begin{array}{l}\sum_{\pi} \\
0 \\
0 \\
0 \\
0\end{array}$ & 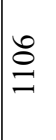 & $\vec{F}$ & 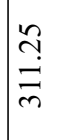 & $\begin{array}{l}\stackrel{2}{0} \\
\stackrel{\tilde{D}}{\text { m }}\end{array}$ & $\begin{array}{l}\tilde{\infty} \\
\stackrel{+}{+} \\
\tilde{m}\end{array}$ & 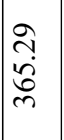 & 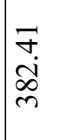 & तै & $\begin{array}{l}\stackrel{\mathbb{N}}{i} \\
\stackrel{\gamma}{\gamma}\end{array}$ & $\begin{array}{l}\tilde{\sigma} \\
\text { id } \\
\tilde{\gamma}\end{array}$ & \begin{tabular}{l}
$\hat{\sigma}$ \\
\multirow{f}{f}{}
\end{tabular} & $\begin{array}{l}\dot{\sigma} \\
\ddot{0} \\
\dot{\sigma}\end{array}$ & \begin{tabular}{l}
$\stackrel{8}{0}$ \\
$\stackrel{\nabla}{\sigma}$ \\
\multirow{\sigma}{*}{}
\end{tabular} & $\begin{array}{l}\hat{y} \\
\dot{q} \\
\dot{q}\end{array}$ & 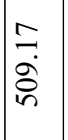 & $\begin{array}{l}8 \\
\stackrel{1}{0} \\
i n\end{array}$ & $\begin{array}{l}\bar{a} \\
\hat{n} \\
\tilde{n}\end{array}$ & $\begin{array}{c}\tilde{\sigma} \\
i \\
\tilde{n} \\
n \\
\end{array}$ & $\begin{array}{l}2 \\
2 \\
\tilde{0} \\
n \\
n\end{array}$ & $\begin{array}{l}\text { రె } \\
\stackrel{2}{0} \\
\hat{n}\end{array}$ & $\mid$\begin{tabular}{l}
$\infty$ \\
\hdashline \\
$i$ \\
$i n$
\end{tabular} & $\begin{array}{l}+ \\
\infty \\
\dot{0} \\
\dot{0}\end{array}$ \\
\hline 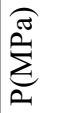 & $\stackrel{\Delta}{0}$ & 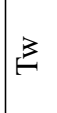 & 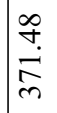 & 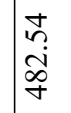 & $\begin{array}{l}\stackrel{+}{m} \\
\stackrel{m}{n}\end{array}$ & $\begin{array}{l}2 \\
\hat{n} \\
\hat{n}\end{array}$ & 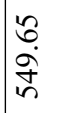 & $\begin{array}{l}\hat{R} \\
\dot{8} \\
\stackrel{8}{n}\end{array}$ & 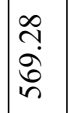 & $\left|\begin{array}{c}0 \\
0 \\
\infty \\
i n \\
i n\end{array}\right|$ & $\begin{array}{l}\hat{n} \\
i \\
\tilde{n}\end{array}$ & 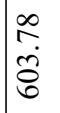 & 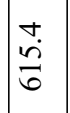 & 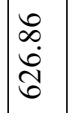 & $\frac{n}{\dot{g}}$ & $\begin{array}{l}\text { fे. } \\
\text { லิ่ }\end{array}$ & $\frac{5}{\hat{\sigma}}$ & $\begin{array}{l}\hat{n} \\
\dot{+} \\
\dot{0}\end{array}$ & 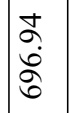 & $\begin{array}{l}0 \\
\dot{2} \\
\stackrel{2}{2}\end{array}$ & $\frac{\hat{a}}{\grave{N}}$ & $\frac{\mathfrak{a}}{\hat{\alpha}}$ \\
\hline $\begin{array}{l}0 \\
\mathscr{Z} \\
\tilde{z} \\
\tilde{z}\end{array}$ & q & $\stackrel{9}{9}$ & $\sim$ & $\vec{\infty}$ & $\overrightarrow{ \pm}$ & ָิ & 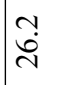 & $\begin{array}{l}n \\
\tilde{n}\end{array}$ & $\mid \begin{array}{c}m \\
\infty \\
m\end{array}$ & $\mid \begin{array}{l}+ \\
\dot{J}\end{array}$ & $\begin{array}{l}t \\
\dot{8}\end{array}$ & $\begin{array}{l}n \\
0 \\
i \\
n\end{array}$ & $\mid \begin{array}{l}n \\
i \\
0\end{array}$ & $\left|\begin{array}{l}n \\
\infty \\
\infty \\
0\end{array}\right|$ & 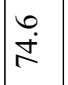 & $\begin{array}{l}0 \\
\dot{0} \\
\infty\end{array}$ & $\begin{array}{l}\hat{\infty} \\
\infty\end{array}$ & $\hat{i}$ & $\mid \begin{array}{l}\infty \\
\infty \\
\infty\end{array}$ & $\begin{array}{l}\infty \\
\dot{0} \\
\stackrel{0}{0}\end{array}$ & $\stackrel{\varrho}{\grave{O}}$ & \\
\hline
\end{tabular}




\begin{tabular}{|c|c|c|c|c|c|c|c|c|c|c|c|c|c|c|c|c|c|c|c|c|c|c|}
\hline & & $\vec{z}$ & 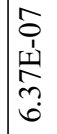 & 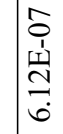 & 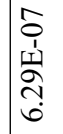 & 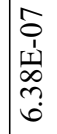 & 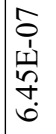 & 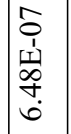 & 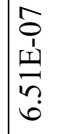 & 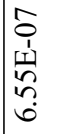 & 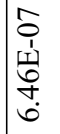 & 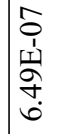 & 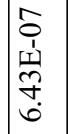 & 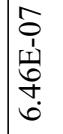 & 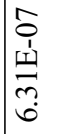 & 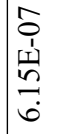 & 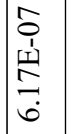 & 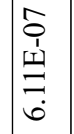 & 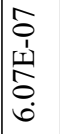 & \begin{tabular}{|l}
$\hat{0}$ \\
$\hat{1}$ \\
\multicolumn{1}{|c}{} \\
$\hat{\sigma}$ \\
in
\end{tabular} & $\begin{array}{l}\hat{o} \\
\hat{1} \\
\hat{1} \\
\infty \\
\dot{\infty} \\
\dot{n}\end{array}$ & 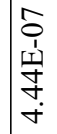 \\
\hline & & $\stackrel{\oplus}{\infty}$ & 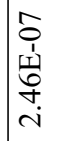 & $\begin{array}{l}\hat{0} \\
1 \\
1 \\
\\
\\
-1\end{array}$ & 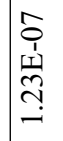 & 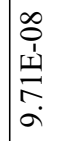 & 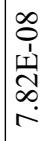 & \begin{tabular}{|c|}
$\infty$ \\
0 \\
0 \\
1 \\
$\infty$ \\
$\infty$ \\
0 \\
0
\end{tabular} & 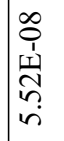 & 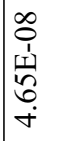 & 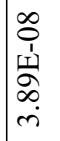 & 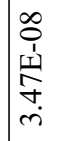 & $\mid \begin{array}{l}\infty \\
0 \\
1 \\
\underline{1} \\
\hat{\alpha} \\
\grave{i}\end{array}$ & 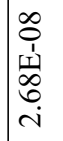 & \begin{tabular}{l}
$\infty$ \\
0 \\
0 \\
\multicolumn{1}{|c}{} \\
\\
\end{tabular} & 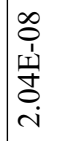 & $\mid \begin{array}{c}\infty \\
0 \\
1 \\
1 \\
\sim \\
\infty \\
\infty \\
-1 \\
-1\end{array}$ & \begin{tabular}{l}
$\infty$ \\
0 \\
1 \\
1 \\
0 \\
0 \\
\hdashline \\
$-i$
\end{tabular} & $\begin{array}{l}\infty \\
0 \\
o \\
1 \\
\\
\\
\\
-\end{array}$ & 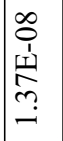 & 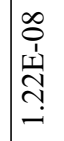 & 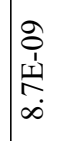 \\
\hline & & $\vec{D}$ & 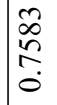 & $\frac{\vec{\infty}}{\stackrel{\sigma}{+}}$ & $\underset{⿱ 亠 乂}{\stackrel{\Xi}{0}}$ & $\begin{array}{l}\overrightarrow{0} \\
\stackrel{n}{0} \\
\dot{0}\end{array}$ & $\frac{n}{\approx}$ & $\stackrel{\Re}{\stackrel{0}{0}}$ & 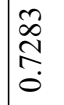 & 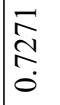 & $\begin{array}{l}\widetilde{J} \\
\stackrel{1}{N} \\
0\end{array}$ & 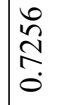 & 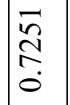 & 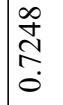 & 点 & 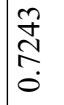 & $\mid \begin{array}{l}\vec{J} \\
\stackrel{\mathbb{N}}{a} \\
\dot{0}\end{array}$ & 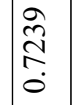 & 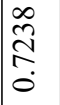 & $\begin{array}{l}0 \\
\stackrel{N}{N} \\
\dot{0}\end{array}$ & 总 & $\underset{\tilde{N}}{\stackrel{N}{0}}$ \\
\hline & & $\mathscr{J}$ & $\begin{array}{l}\hat{0} \\
\stackrel{0}{0} \\
\stackrel{2}{2} \\
\stackrel{2}{f} \\
f\end{array}$ & $\begin{array}{l}\infty \\
\infty \\
i n \\
i n \\
i n\end{array}$ & 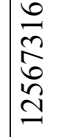 & 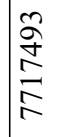 & 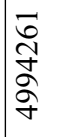 & $\begin{array}{l}2 \\
2 \\
\infty \\
n \\
n \\
n\end{array}$ & 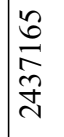 & 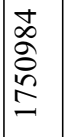 & 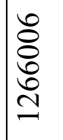 & $\begin{array}{l}\overrightarrow{2} \\
\mathscr{\infty} \\
\approx \\
\propto\end{array}$ & 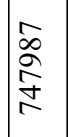 & $\frac{\tilde{n}}{\text { 点 }}$ & 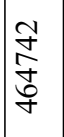 & 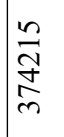 & $\begin{array}{l}\infty \\
\infty \\
\vec{f} \\
\stackrel{\infty}{0} \\
\end{array}$ & $\begin{array}{l}\vec{F} \\
\hat{\tilde{n}} \\
\hat{\sim}\end{array}$ & 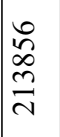 & 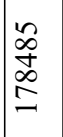 & 宂 & $\frac{\mathfrak{z}}{8}$ \\
\hline & & t & 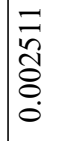 & 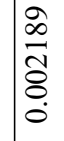 & 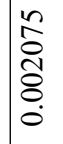 & 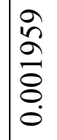 & $\begin{array}{l}\tilde{\delta} \\
\infty \\
\infty \\
0 \\
0 \\
0\end{array}$ & $\mid \begin{array}{l}\infty \\
\stackrel{0}{0} \\
\stackrel{0}{0} \\
0 \\
0 \\
0\end{array}$ & \begin{tabular}{l}
$\infty$ \\
$\infty$ \\
0 \\
\hdashline \\
\hdashline \\
0 \\
0
\end{tabular} & $\mid \begin{array}{c}\tilde{\hat{\sigma}} \\
\stackrel{0}{8} \\
0 \\
0\end{array}$ & $\begin{array}{l}n \\
\tilde{n} \\
\tilde{a} \\
\vdots \\
0 \\
0\end{array}$ & 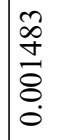 & 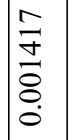 & 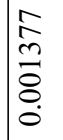 & 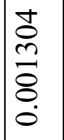 & 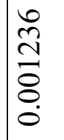 & 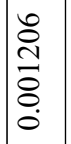 & $\begin{array}{c}0 \\
0 \\
0 \\
0 \\
0 \\
0\end{array}$ & 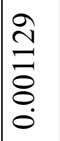 & $\begin{array}{l}0 \\
\stackrel{0}{0} \\
0 \\
0 \\
0 \\
0\end{array}$ & 柋 & $\begin{array}{l}\hat{乏} \\
\hat{\delta} \\
\delta \\
0 \\
0\end{array}$ \\
\hline & & $\approx$ & 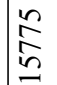 & $\begin{array}{l}n \\
\tilde{O} \\
\Xi \\
\Xi\end{array}$ & $\frac{\widetilde{\partial}}{\Omega}$ & ঐิ & $\stackrel{\mathfrak{f}}{\mathfrak{H}}$ & $\frac{ \pm}{2}$ & 命 & ڤे & ฉ্ & $\underset{\sigma}{\sigma}$ & $\left|\begin{array}{l}\vec{\infty} \\
\infty \\
\infty\end{array}\right|$ & 夙 & 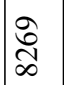 & $\begin{array}{l}\infty \\
\infty \\
\infty \\
\infty\end{array}$ & $\underset{\substack{\Delta \\
\infty}}{\infty}$ & 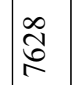 & $\underset{I}{\stackrel{f}{I}}$ & 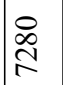 & $\stackrel{\widehat{N}}{\curvearrowright}$ & $\stackrel{8}{\circ}$ \\
\hline లే & రิ & 窻 & 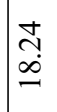 & $\vec{\sigma}$. & $\underset{i}{i}$ & $\vec{\infty}$. & $\stackrel{\infty}{\stackrel{\infty}{\circ}}$ & $\stackrel{N}{\stackrel{N}{-}}$ & $\underset{-}{\tilde{6}}$ & in & $\stackrel{\text { nิ }}{=}$ & $\stackrel{\infty}{\infty}$ & $\stackrel{3}{6}$ & $\stackrel{\infty}{\infty}$ & $\stackrel{\vec{b}}{-}$ & $\stackrel{\infty}{n}$ & $\mid \begin{array}{l}\infty \\
\stackrel{0}{-}\end{array}$ & ڤू & $\stackrel{\infty}{\dddot{n}}$ & 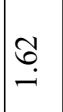 & $\stackrel{8}{\stackrel{0}{\circ}}$ & ชู \\
\hline $\begin{array}{l} \pm \\
\sigma \\
\frac{\overrightarrow{0}}{\Xi}\end{array}$ & 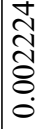 & $\vec{z}$ & 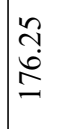 & $\begin{array}{l}\infty \\
\stackrel{\infty}{n} \\
i n\end{array}$ & $\begin{array}{l}n \\
\dot{f}\end{array}$ & 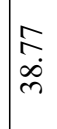 & 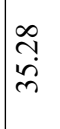 & $\mid \begin{array}{l}\mathcal{F} \\
\tilde{m} \\
\tilde{m}\end{array}$ & $\begin{array}{l}0 \\
i \\
i\end{array}$ & $\begin{array}{l}0 \\
\\
\stackrel{n}{n}\end{array}$ & $\begin{array}{l}2 \\
\infty \\
\dot{\infty} \\
\dot{m}\end{array}$ & $\begin{array}{l}\infty \\
\infty \\
\infty \\
\infty \\
m\end{array}$ & $\mid \begin{array}{l}n \\
n \\
0 \\
n \\
n\end{array}$ & î & $\begin{array}{l}n \\
\alpha \\
\infty \\
\sim\end{array}$ & $\begin{array}{l}\infty \\
\stackrel{\infty}{\sim} \\
\sim\end{array}$ & $\stackrel{\vec{i}}{\stackrel{\vec{\lambda}}{*}}$ & 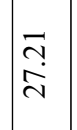 & 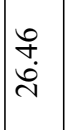 & $\begin{array}{l}f \\
\ddot{i}\end{array}$ & $\mid \begin{array}{l}0 \\
0 \\
\end{array}$ & ڤે \\
\hline$\frac{\mathscr{\mathscr { U }}}{\stackrel{\tilde{\omega}}{\Xi}}$ & $\begin{array}{l}\infty \\
\infty \\
\widetilde{\delta} \\
-\end{array}$ & 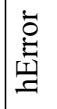 & $\mid \begin{array}{l}\infty \\
\stackrel{\sim}{\sim} \\
\stackrel{\sim}{~}\end{array}$ & $\stackrel{\tilde{\rho}}{\dot{m}}$ & 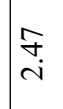 & 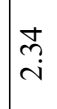 & 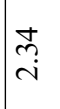 & $\stackrel{\infty}{\stackrel{\infty}{\sim}}$ & $\vec{i}$ & $\hat{\tilde{m}}$ & $\frac{b}{m}$ & $\stackrel{\infty}{\stackrel{\infty}{\infty}}$ & $\begin{array}{l}\infty \\
\dot{r}\end{array}$ & $\stackrel{\sim}{\sim}$ & $\underset{+}{ \pm}$ & $\stackrel{m}{\Rightarrow}$ & $\underset{+}{\stackrel{q}{+}}$ & $\begin{array}{l}\mathbb{U} \\
\stackrel{+}{+}\end{array}$ & $\stackrel{\sim}{+}$ & $\underset{F}{F}$ & $\underset{\infty}{\infty}$ & 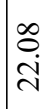 \\
\hline$\underset{\Xi}{\stackrel{્}{\Xi}}$ & 裉 & 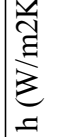 & 离 & $\begin{array}{l}\tilde{n} \\
\stackrel{+}{N}\end{array}$ & $\begin{array}{l}n \\
0 \\
0\end{array}$ & $\mid \begin{array}{l}0 \\
\infty \\
\dot{J}\end{array}$ & $\begin{array}{l}\hat{\sigma} \\
\dot{0}\end{array}$ & 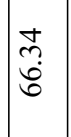 & $\begin{array}{l}0 \\
n \\
0 \\
0\end{array}$ & 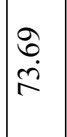 & $\begin{array}{l}3 \\
\dot{0} \\
\dot{1}\end{array}$ & $\begin{array}{l}8 \\
0 \\
\infty \\
\infty\end{array}$ & $\left|\begin{array}{l}\infty \\
\infty \\
\dot{\infty} \\
\dot{\infty}\end{array}\right|$ & 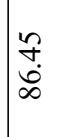 & $\begin{array}{l}\infty \\
\infty \\
\dot{\infty}\end{array}$ & $\underset{\infty}{\stackrel{\sim}{\infty}}$ & $\mid \begin{array}{l}0 \\
0 \\
\infty\end{array}$ & $\begin{array}{l}0 \\
\ddot{\infty} \\
\infty\end{array}$ & $\begin{array}{l}0 \\
\infty \\
\infty\end{array}$ & $\begin{array}{l}J \\
\sigma \\
\infty \\
\infty\end{array}$ & 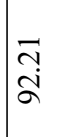 & 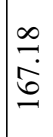 \\
\hline$\sum_{\text {至 }}^{\Sigma}$ & $\hat{i}$ & $\begin{array}{l}\text { đิ } \\
\text { है } \\
= \\
=\end{array}$ & $\begin{array}{l}\overrightarrow{0} \\
\stackrel{0}{\hat{\sigma}} \\
\stackrel{2}{2}\end{array}$ & 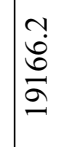 & $\begin{array}{l}\vec{\infty} \\
\stackrel{\overbrace{}}{2} \\
\stackrel{2}{\sigma}\end{array}$ & $\begin{array}{l}\infty \\
\dot{0} \\
\stackrel{0}{\infty} \\
\infty \\
-1\end{array}$ & $\begin{array}{l}\stackrel{i}{\vec{\infty}} \\
\infty \\
\infty \\
\infty\end{array}$ & $\left|\begin{array}{l}n \\
\mathbb{N} \\
\mathbb{N} \\
\infty \\
-1\end{array}\right|$ & 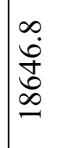 & 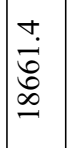 & $\begin{array}{l}\tilde{3} \\
\tilde{\infty} \\
\infty \\
\infty\end{array}$ & $\begin{array}{l}\hat{0} \\
\infty \\
\infty \\
\infty\end{array}$ & $\begin{array}{c}0 \\
\dot{+} \\
\infty \\
\infty \\
-\infty\end{array}$ & $\begin{array}{l}\tilde{N} \\
\stackrel{2}{\hat{~}} \\
\underline{\infty} \\
\underline{\infty}\end{array}$ & $\begin{array}{l}n \\
\infty \\
\infty \\
\infty \\
\cong \\
\Xi\end{array}$ & 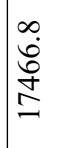 & $\mid \begin{array}{l}n \\
\stackrel{+}{0} \\
\stackrel{n}{\Xi} \\
=\end{array}$ & $\begin{array}{l}\hat{\widehat{i}} \\
\mathbf{0} \\
\underline{+} \\
-\end{array}$ & $\mid \begin{array}{l}n \\
\tilde{a} \\
\tilde{N} \\
\end{array}$ & 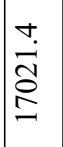 & $\mid \begin{array}{c}\tilde{N} \\
\infty \\
0 \\
0 \\
-1\end{array}$ & 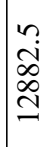 \\
\hline $\begin{array}{l}\sum_{\pi} \\
0 \\
0 \\
0 \\
0\end{array}$ & $\stackrel{ \pm}{\stackrel{5}{S}}$ & $\vec{F}$ & $\frac{\infty}{\dot{0}}$ & $\frac{\partial}{\overrightarrow{\dot{n}}}$ & $\overrightarrow{\dot{\infty}}$ & $\begin{array}{l}\hat{\alpha} \\
\dot{\vec{\sigma}} \\
\vec{\gamma}\end{array}$ & $\mid \begin{array}{l}\infty \\
\dot{y} \\
\dot{f}\end{array}$ & $\begin{array}{l}\widetilde{\hat{~}} \\
\stackrel{\mathrm{J}}{\sigma}\end{array}$ & ָี & $\mid \begin{array}{l}2 \\
\infty \\
\infty \\
i \\
n\end{array}$ & $\begin{array}{l}\overrightarrow{0} \\
\dot{0} \\
\tilde{n} \\
n\end{array}$ & $\begin{array}{l}n \\
\stackrel{n}{i} \\
\infty \\
\infty \\
i n\end{array}$ & $\begin{array}{l}\nabla \\
\dot{0} \\
\dot{0} \\
0\end{array}$ & 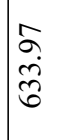 & $\begin{array}{l}\overline{0} \\
0 \\
\hat{\sigma}\end{array}$ & $\begin{array}{l}\tilde{\vartheta} \\
\ddot{\infty} \\
\tilde{\infty}\end{array}$ & $\mid \begin{array}{l}\infty \\
2 \\
0 \\
0 \\
2\end{array}$ & $\begin{array}{l}\vec{n} \\
\tilde{n} \\
\tilde{n}\end{array}$ & $\begin{array}{l}\vec{i} \\
\tilde{n} \\
\tilde{n}\end{array}$ & $\begin{array}{l}f \\
\stackrel{f}{0} \\
\stackrel{2}{*}\end{array}$ & $\begin{array}{l}\infty \\
n \\
\infty \\
\infty \\
\Re\end{array}$ & $\begin{array}{l}\infty \\
\infty \\
\stackrel{\infty}{+} \\
\infty\end{array}$ \\
\hline$\sum_{0}^{\pi}$ & $\frac{\Delta}{0}$ & 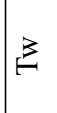 & $\begin{array}{l}\hat{\sigma} \\
\infty \\
\sigma\end{array}$ & 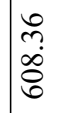 & $\begin{array}{l}\text { f. } \\
\infty \\
0 \\
0 \\
\end{array}$ & 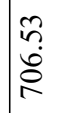 & $\begin{array}{l}\infty \\
\infty \\
\infty \\
\approx\end{array}$ & $\begin{array}{l}\cong \\
\cdots \\
i n \\
n\end{array}$ & 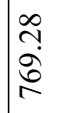 & 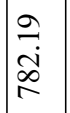 & $\begin{array}{l}\stackrel{\circ}{0} \\
\dot{d} \\
\dot{\infty}\end{array}$ & $\mid \begin{array}{l}\infty \\
\infty \\
\infty \\
\infty \\
\infty\end{array}$ & $\begin{array}{l}n \\
n \\
0 \\
\infty \\
\infty \\
\infty\end{array}$ & $\begin{array}{l}n \\
m \\
w \\
\infty \\
\infty\end{array}$ & $\begin{array}{l}0 \\
0 \\
0 \\
\infty \\
\infty\end{array}$ & $\begin{array}{l}\hat{\infty} \\
\stackrel{0}{0} \\
\infty\end{array}$ & 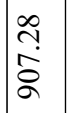 & $\begin{array}{l}\hat{n} \\
\tilde{0} \\
\alpha \\
\alpha\end{array}$ & $\left|\begin{array}{l}\approx \\
\infty \\
\dot{\alpha} \\
\tilde{\alpha}\end{array}\right|$ & 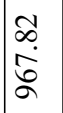 & $\begin{array}{l}\hat{n} \\
\stackrel{0}{0} \\
\infty\end{array}$ & $\begin{array}{l}\dot{\$} \\
\dot{+} \\
\infty\end{array}$ \\
\hline $\begin{array}{l}0 \\
\mathscr{Z} \\
\tilde{z} \\
\tilde{z}\end{array}$ & $F$ & $\stackrel{\rho}{a}$ & $N$ & $\vec{\infty}$ & 市 & 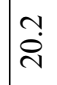 & ֶֶ. & $\begin{array}{l}n \\
\tilde{n}\end{array}$ & $\begin{array}{c}m \\
\infty \\
\infty \\
m\end{array}$ & $\mid \begin{array}{l}+ \\
\dot{J}\end{array}$ & $\begin{array}{l}t \\
\dot{8}\end{array}$ & 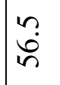 & $\mid \begin{array}{l}n \\
i \\
0\end{array}$ & $\begin{array}{l}n \\
\infty \\
\infty \\
0\end{array}$ & $\begin{array}{l}0 \\
\stackrel{+}{T}\end{array}$ & $\begin{array}{l}0 \\
\dot{0} \\
\infty\end{array}$ & $\begin{array}{l}\hat{\infty} \\
\infty\end{array}$ & $\hat{a}$ & $\mid \begin{array}{l}\infty \\
\infty \\
\infty \\
\sigma\end{array}$ & $\begin{array}{l}\infty \\
\dot{0} \\
\stackrel{0}{0}\end{array}$ & $\stackrel{\varrho}{\grave{O}}$ & \\
\hline
\end{tabular}




\begin{tabular}{|c|c|c|c|c|c|c|c|c|c|c|c|c|c|c|c|c|c|c|c|c|c|c|}
\hline & & $\vec{\Delta}$ & 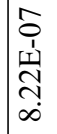 & 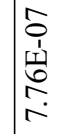 & 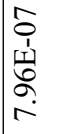 & $\mid \begin{array}{c}0 \\
0 \\
1 \\
\text { th } \\
0 \\
0 \\
\infty\end{array}$ & 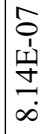 & $\begin{array}{l}\hat{a} \\
\hat{1} \\
\underline{1} \\
\stackrel{+}{\infty} \\
\infty\end{array}$ & $\begin{array}{c}\hat{a} \\
\hat{1} \\
\underline{1} \\
\stackrel{5}{\infty} \\
\infty\end{array}$ & $\begin{array}{l}\hat{y} \\
\mathbf{1} \\
1 \\
\\
\\
\infty \\
\infty\end{array}$ & 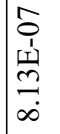 & 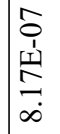 & $\mid \begin{array}{c}0 \\
0 \\
1 \\
1 \\
0 \\
0 \\
\infty \\
\infty\end{array}$ & $\mid \begin{array}{l}0 \\
\hat{1} \\
\text { İ } \\
0 \\
0 \\
\infty \\
\infty\end{array}$ & 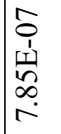 & $\begin{array}{l}\hat{0} \\
\hat{1} \\
\underline{1} \\
\tilde{b} \\
\end{array}$ & 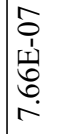 & $\mid \begin{array}{c}\mathfrak{o} \\
1 \\
1 \\
\infty \\
n \\
r \\
r\end{array}$ & 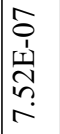 & 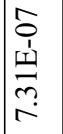 & 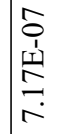 & 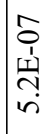 \\
\hline & & $\stackrel{\oplus}{\infty}$ & 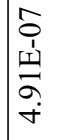 & $\begin{array}{c}\hat{s} \\
\hat{1} \\
\text { In } \\
\\
\dot{m} \\
\end{array}$ & 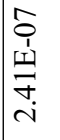 & 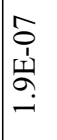 & $\begin{array}{c}\hat{o} \\
\hat{1} \\
1 \\
\tilde{n} \\
\\
-\end{array}$ & $\begin{array}{l}\hat{0} \\
\hat{1} \\
\\
\\
\end{array}$ & 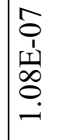 & $\begin{array}{l}\infty \\
0 \\
0 \\
1 \\
\text { th } \\
0 \\
0 \\
a \\
a\end{array}$ & 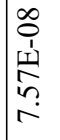 & 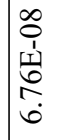 & $\begin{array}{l}\infty \\
0 \\
0 \\
1 \\
0 \\
0 \\
i \\
i\end{array}$ & 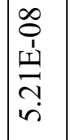 & 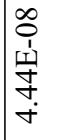 & \begin{tabular}{l}
$\infty$ \\
0 \\
$\vdots$ \\
\multicolumn{1}{|c}{} \\
$\hat{\sigma}$ \\
$\dot{m}$
\end{tabular} & 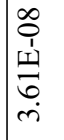 & \begin{tabular}{c}
$\infty$ \\
0 \\
0 \\
\multicolumn{1}{c}{} \\
2 \\
$\dot{n}$ \\
$\dot{m}$
\end{tabular} & 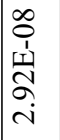 & $\begin{array}{l}\infty \\
0 \\
0 \\
1 \\
\\
\\
i \\
i\end{array}$ & $\begin{array}{l}\infty \\
\text { o } \\
\text { 乑 } \\
\text { ల) } \\
\text { i }\end{array}$ & 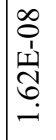 \\
\hline & & $\vec{D}$ & 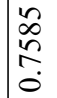 & 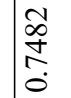 & $\frac{\mathfrak{I}}{\stackrel{7}{0}}$ & 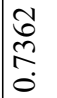 & 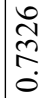 & 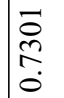 & $\begin{array}{l}\mathbf{0} \\
\stackrel{\infty}{\mathbb{N}} \\
\stackrel{0}{0}\end{array}$ & 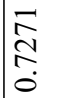 & 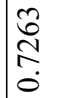 & 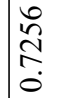 & $\mid \begin{array}{l}\vec{n} \\
\stackrel{a}{0} \\
\dot{0}\end{array}$ & 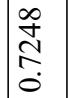 & 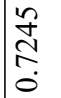 & 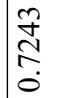 & 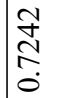 & 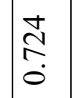 & 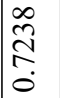 & 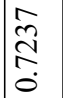 & 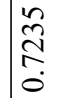 & 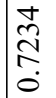 \\
\hline & & $\mathscr{J}$ & 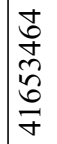 & 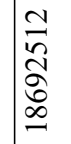 & $\mid \begin{array}{l}0 \\
\stackrel{2}{2} \\
0 \\
0 \\
0 \\
0\end{array}$ & 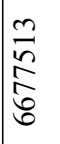 & 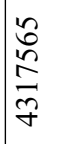 & 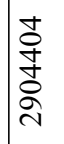 & 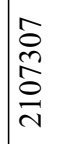 & 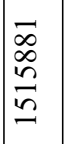 & $\begin{array}{l}\tilde{2} \\
\tilde{\hat{\alpha}} \\
\delta \\
\delta\end{array}$ & 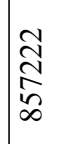 & 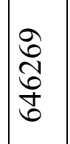 & $\begin{array}{l}0 \\
\infty \\
\infty \\
\tilde{N} \\
n\end{array} \mid$ & $\mid \begin{array}{l}\alpha \\
\infty \\
\alpha \\
\alpha \\
\tilde{m}\end{array}$ & 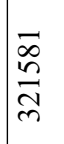 & 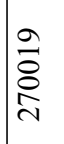 & $\begin{array}{l}\hat{\widehat{\delta}} \\
\grave{\Delta}\end{array}$ & $\begin{array}{l}\text { त्र } \\
\vdots \\
0 \\
0 \\
-\end{array}$ & $\begin{array}{l}\infty \\
\widetilde{N} \\
\tilde{\tilde{n}} \\
\end{array}$ & 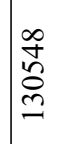 & $\begin{array}{l}\infty \\
\infty \\
\infty \\
\infty\end{array}$ \\
\hline & & t & 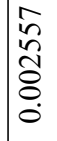 & $\begin{array}{l}\infty \\
\infty \\
\widetilde{1} \\
\delta \\
\dot{0}\end{array}$ & 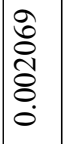 & $\frac{n}{2}$ & $\begin{array}{l}\tilde{N} \\
\infty \\
\infty \\
\vdots \\
0 \\
0\end{array}$ & 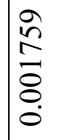 & $\begin{array}{l}\hat{\widehat{A}} \\
\frac{0}{8} \\
0 \\
0 \\
0\end{array}$ & $\mid \begin{array}{c}0 \\
b \\
0 \\
0 \\
0 \\
0\end{array}$ & 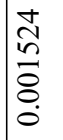 & 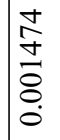 & $\mid \begin{array}{l}\tilde{o} \\
\dot{+} \\
\tilde{\sigma} \\
\dot{0} \\
\dot{0}\end{array}$ & $\left|\begin{array}{l}\tilde{0} \\
\tilde{D} \\
\tilde{\Xi} \\
0 \\
0\end{array}\right|$ & 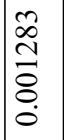 & $\begin{array}{l}\stackrel{1}{a} \\
\stackrel{\Xi}{\Xi} \\
0 \\
0\end{array}$ & $\mid \begin{array}{l}\infty \\
\stackrel{\infty}{二} \\
\overrightarrow{8} \\
0 \\
0\end{array}$ & 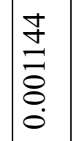 & 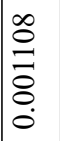 & 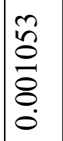 & 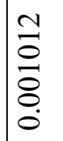 & 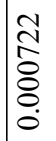 \\
\hline & & $\approx$ & $\underset{\Xi}{\stackrel{J}{\Xi}}$ & 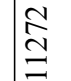 & 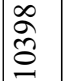 & 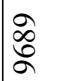 & $\frac{\delta}{\sigma}$ & 总 & $\frac{\infty}{\infty}$ & 旅 & 离 & $\stackrel{\bar{N}}{\mathbb{N}}$ & $\mid \begin{array}{l}0 \\
0 \\
0 \\
0\end{array}$ & $\mid \begin{array}{l}\infty \\
\approx \\
\delta\end{array}$ & 点 & $\hat{n}$ & $\begin{array}{l}\infty \\
\infty \\
0\end{array}$ & $\begin{array}{l}n \\
\hat{\delta} \\
\delta\end{array}$ & $\begin{array}{l}+ \\
2 \\
\text { D }\end{array}$ & 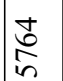 & 帒 & 量 \\
\hline है & రీ & 章 & $\ddot{\vartheta}$ & $\stackrel{m}{\sim}$ & 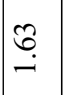 & $\stackrel{\infty}{\stackrel{\infty}{?}}$ & 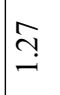 & $\tilde{?}$ & $\stackrel{\sim}{-}$ & $\stackrel{\infty}{=}$ & $\stackrel{\mathbb{I}}{\stackrel{I}{I}}$ & $\stackrel{\mathbb{J}}{=}$ & 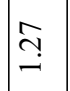 & $\mid \begin{array}{l}0 \\
\\
-1\end{array}$ & $\tilde{\overbrace{}}$ & $\stackrel{\Upsilon}{-}$ & $\stackrel{?}{-}$ & $\stackrel{\vec{m}}{-}$ & 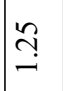 & $\stackrel{\sim}{-}$ & సે & $\stackrel{2}{r}$ \\
\hline $\begin{array}{l} \pm \\
\sigma \\
\frac{\overrightarrow{0}}{\Xi}\end{array}$ & $\begin{array}{l}\hat{\sigma} \\
\widehat{\widehat{\widehat{\sigma}}} \\
\vdots \\
0\end{array}$ & $\vec{z}$ & $\begin{array}{l}\infty \\
i \\
\\
\end{array}$ & $\frac{n}{\mathfrak{r}}$ & $\underset{m}{\stackrel{1}{n}}$ & $\underset{\infty}{\stackrel{\infty}{\sim}}$ & $\begin{array}{l}\hat{\sigma} \\
\ddot{i}\end{array}$ & $\begin{array}{l}0 \\
\stackrel{n}{\sim} \\
\end{array}$ & 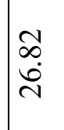 & 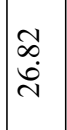 & $\begin{array}{l}3 \\
\mathfrak{b} \\
i\end{array}$ & $\begin{array}{l}0 \\
\infty \\
\\
\end{array}$ & $\begin{array}{l}0 \\
i \\
i \\
i \\
\end{array}$ & 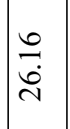 & \begin{tabular}{|l|} 
足 \\
$\stackrel{+}{\sim}$
\end{tabular} & $\begin{array}{l}\text { f. } \\
\ddot{\sim}\end{array}$ & $\hat{\vec{v}}$ & $\begin{array}{l}\vec{m} \\
\vec{n}\end{array}$ & $\begin{array}{l}\stackrel{\infty}{\hat{i}} \\
\stackrel{i}{N}\end{array}$ & $\begin{array}{l}\infty \\
\vec{\sim}\end{array}$ & तે & $\underset{\sim}{\stackrel{\gamma}{\sigma}}$ \\
\hline $\begin{array}{l}\stackrel{\mathscr{z}}{\underline{\Delta}} \\
\frac{\vec{\omega}}{\Xi}\end{array}$ & 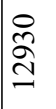 & 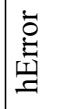 & $\begin{array}{l}m \\
m \\
n \\
n\end{array}$ & ָ̊ & $\stackrel{\infty}{\rightarrow}$ & 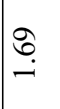 & $\underset{-}{\sigma}$ & $\stackrel{શ}{I}$ & $\hat{\sigma}$ & $\stackrel{\infty}{\vec{i}}$ & ते & $\begin{array}{l}n \\
\tilde{n} \\
i\end{array}$ & $\begin{array}{l}\vec{\infty} \\
\stackrel{i}{i}\end{array}$ & $\stackrel{\equiv}{m}$ & $\stackrel{\sim}{\sim}$ & $\frac{n}{m}$ & $\underset{\sim}{\stackrel{f}{r}}$ & $\begin{array}{l}\dot{0} \\
\dot{m}\end{array}$ & $\begin{array}{l}\hat{\sigma} \\
\dot{m}\end{array}$ & 它 & $\underset{+}{\partial}$ & $\frac{m}{\stackrel{2}{c}}$ \\
\hline$\underset{\Xi}{\stackrel{\widehat{a}}{\Xi}}$ & $\underset{\text { d }}{ \pm}$ & 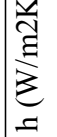 & 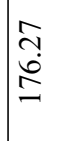 & 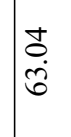 & $\begin{array}{l}n \\
\tilde{n} \\
n \\
n\end{array}$ & 免 & $\frac{n}{n}$ & $\begin{array}{l}\hat{6} \\
\stackrel{1}{\circ} \\
\stackrel{n}{n}\end{array}$ & $\underset{i}{\stackrel{D}{n}}$ & बे. & $\begin{array}{l}\infty \\
\infty \\
\dot{\sigma} \\
\end{array}$ & $\begin{array}{l}n \\
\hat{6} \\
\dot{b}\end{array}$ & $\left|\begin{array}{l}a \\
\dot{0} \\
\dot{0}\end{array}\right|$ & $\mid \begin{array}{l}0 \\
i \\
\\
\end{array}$ & $\mid \begin{array}{c}\hat{f} \\
\text { i } \\
\text { in }\end{array}$ & 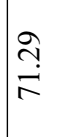 & 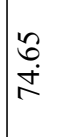 & $\begin{array}{l}\infty \\
2 \\
n \\
n\end{array}$ & 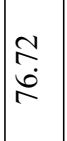 & $\begin{array}{l}\infty \\
\approx \\
i \\
i\end{array}$ & $\begin{array}{l}0 \\
9 \\
9\end{array}$ & \\
\hline$\sum_{\text {至 }}$ & $\overrightarrow{\sim i}$ & $\begin{array}{l}\text { đิ } \\
\text { है } \\
= \\
=\end{array}$ & $\begin{array}{l}0 \\
0 \\
0 \\
0 \\
0\end{array}$ & $\begin{array}{l}\overrightarrow{\tilde{i}} \\
\frac{\delta}{\underline{n}}\end{array}$ & 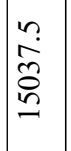 & 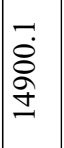 & 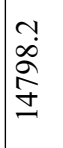 & $\begin{array}{l}\hat{i} \\
\dot{\infty} \\
\dot{0} \\
\dot{J}\end{array}$ & $\begin{array}{l}\exists \\
\dot{a} \\
\dot{a} \\
\dot{y}\end{array}$ & $\begin{array}{l}0 \\
+ \\
0 \\
0 \\
0 \\
\end{array}$ & $\mid \begin{array}{l}\tilde{J} \\
\tilde{J} \\
\tilde{\Xi} \\
\Xi\end{array}$ & $\begin{array}{l}0 \\
\stackrel{0}{0} \\
\tilde{m} \\
\dot{\Xi}\end{array}$ & $\begin{array}{l}\vec{Z} \\
\overrightarrow{0} \\
\vec{\Xi} \\
\end{array}$ & ָ̃ & $\mid \begin{array}{l}2 \\
\hat{\tilde{N}} \\
\infty \\
\stackrel{-}{-}\end{array}$ & 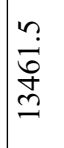 & $\begin{array}{l}0 \\
\dot{0} \\
\tilde{n} \\
n \\
n\end{array}$ & $\begin{array}{l}n \\
\infty \\
\infty \\
0 \\
\infty \\
n\end{array}$ & $\begin{array}{l}\overrightarrow{\hat{2}} \\
\stackrel{\tilde{\Omega}}{\Omega}\end{array}$ & 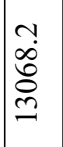 & $\begin{array}{l}\stackrel{3}{i} \\
\stackrel{N}{\infty} \\
\stackrel{N}{N}\end{array}$ & $\begin{array}{l}\infty \\
\dot{0} \\
\hat{\sigma} \\
\sigma\end{array}$ \\
\hline $\begin{array}{l}\sum_{\pi}^{2} \\
0 \\
0 \\
0 \\
0\end{array}$ & 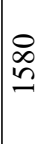 & $\vec{F}$ & $\begin{array}{l}0 \\
0 \\
0 \\
0 \\
m\end{array}$ & $\stackrel{m}{m}$ & $\begin{array}{l}\infty \\
\stackrel{\infty}{\infty} \\
\infty \\
\infty\end{array}$ & \begin{tabular}{l}
\multirow{\sigma}{*}{} \\
$\stackrel{\dot{\gamma}}{\sigma}$
\end{tabular} & $\begin{array}{l}\vec{P} \\
\stackrel{g}{q}\end{array}$ & $\begin{array}{l}\vec{\sigma} \\
\stackrel{i}{\hat{\sigma}} \\
\vec{f}\end{array}$ & 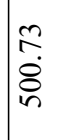 & $\begin{array}{l}\hat{N} \\
\infty \\
\sim \\
\tilde{N}\end{array}$ & $\begin{array}{l}m \\
\ddot{n} \\
n \\
n\end{array}$ & 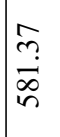 & $\mid \begin{array}{l}8 \\
\dot{0} \\
8 \\
8\end{array}$ & $\mid \begin{array}{l}0 \\
\tilde{n} \\
\tilde{\sigma} \\
\tilde{\sigma}\end{array}$ & $\mid \begin{array}{l} \pm \\
\hat{n} \\
\hat{6}\end{array}$ & $\begin{array}{l}\hat{0} \\
\stackrel{0}{0} \\
\infty \\
0\end{array}$ & $\mid \begin{array}{l}\dot{J} \\
\dot{0} \\
\dot{d} \\
R\end{array}$ & $\begin{array}{l}8 \\
\stackrel{\dot{1}}{1} \\
\end{array}$ & 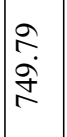 & $\frac{\infty}{\stackrel{\curvearrowright}{\gtrless}}$ & 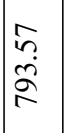 & $\underset{\infty}{\stackrel{\infty}{\Xi}}$ \\
\hline 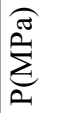 & $\frac{\Delta}{0}$ & 3 & 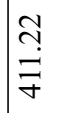 & $\begin{array}{l}\hat{\theta} \\
\dot{8}\end{array}$ & $\frac{\hat{\sigma}}{\tilde{\gamma}}$ & $\begin{array}{l}\infty \\
\infty \\
\dot{0} \\
0\end{array}$ & $\stackrel{n}{\stackrel{n}{N}}$ & 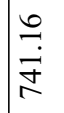 & $\begin{array}{l}5 \\
0 \\
0 \\
\end{array}$ & $\frac{m}{i}$ & 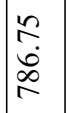 & $\mid \begin{array}{l}0 \\
0 \\
\dot{\infty} \\
\dot{\infty}\end{array}$ & $\mid \begin{array}{l}n \\
\infty \\
\infty \\
\infty\end{array}$ & $\begin{array}{l}\tilde{b} \\
\dot{0} \\
0 \\
\infty \\
\delta\end{array}$ & $\mid \begin{array}{l}0 \\
\infty \\
\dot{+} \\
\infty\end{array}$ & $\begin{array}{l}\vec{b} \\
\ddot{D} \\
\infty\end{array}$ & $\mid \begin{array}{l}\vec{b} \\
\ddot{\infty} \\
\infty \\
\infty \\
\infty\end{array}$ & $\begin{array}{l}\vec{N} \\
\dot{\Xi} \\
\&\end{array}$ & $\mid \begin{array}{l}\tilde{n} \\
\tilde{J} \\
\stackrel{+}{\sigma}\end{array}$ & $\begin{array}{l}\hat{\alpha} \\
\text { ga } \\
\text { a }\end{array}$ & 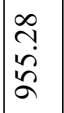 & గñ \\
\hline 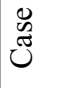 & F & $\stackrel{\rho}{a}$ & $N$ & $\vec{\infty}$ & $\overrightarrow{ \pm}$ & กิ & तु. & $\underset{m}{m}$ & 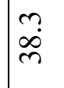 & $\mid \begin{array}{l}\dot{J} \\
\dot{f}\end{array}$ & $\begin{array}{l}t \\
\dot{B}\end{array}$ & \begin{tabular}{l}
$n$ \\
\hdashline \\
\hdashline \\
$n$
\end{tabular} & $\begin{array}{l}n \\
i \\
\delta\end{array}$ & $\left|\begin{array}{l}n \\
\infty \\
0 \\
0\end{array}\right|$ & $\begin{array}{l}0 \\
\stackrel{+}{i}\end{array}$ & $\begin{array}{l}0 \\
\dot{\infty} \\
\infty\end{array}$ & $\hat{\infty}$ & $\overline{\check{a}}$ & $\left|\begin{array}{l}\infty \\
\infty \\
\infty \\
\alpha\end{array}\right|$ & $\begin{array}{l}\infty \\
\dot{0} \\
\dot{0}\end{array}$ & $\stackrel{\partial}{\varrho}$ & $\hat{\sigma}$ \\
\hline
\end{tabular}




\begin{tabular}{|c|c|c|c|c|c|c|c|c|c|c|c|c|c|c|c|c|c|c|c|c|c|c|}
\hline & & $\vec{z}$ & $\begin{array}{l}\infty \\
0 \\
1 \\
\text { 章 } \\
\hat{\sigma} \\
\alpha\end{array}$ & $\begin{array}{l}0 \\
0 \\
1 \\
1 \\
\vdots \\
0 \\
\sigma \\
\sigma\end{array}$ & $\begin{array}{l}\infty \\
0 \\
1 \\
\\
\\
\alpha\end{array}$ & $\mid \begin{array}{l}\hat{o} \\
\hat{1} \\
\underline{\underline{y}}\end{array}$ & 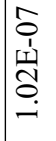 & $\mid \begin{array}{c}\hat{o} \\
\hat{1} \\
\underline{1} \\
\hat{\sigma} \\
- \\
-i\end{array}$ & 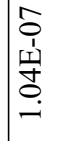 & 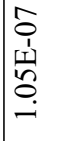 & 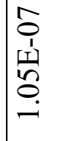 & 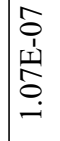 & 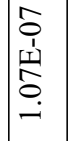 & $\mid \begin{array}{c}\hat{0} \\
\mathbf{1} \\
1 \\
\\
\mathbf{0} \\
- \\
-i\end{array}$ & 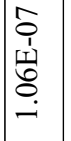 & 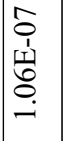 & 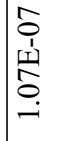 & 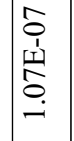 & 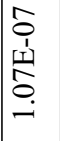 & 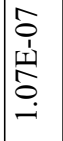 & 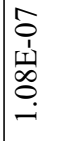 & $\begin{array}{l}\infty \\
0 \\
0 \\
\text { I } \\
\text { N } \\
\sim \\
\sigma \\
\sigma\end{array}$ \\
\hline & & $\stackrel{\oplus}{\infty}$ & 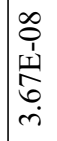 & 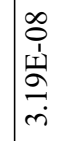 & $\begin{array}{l}\infty \\
0 \\
1 \\
1 \\
\infty \\
\infty \\
i \\
i\end{array}$ & 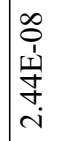 & 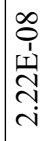 & $\mid \begin{array}{l}\infty \\
0 \\
0 \\
1 \\
\\
0 \\
i \\
\end{array}$ & 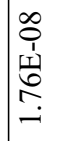 & $\mid \begin{array}{c}\infty \\
0 \\
1 \\
1 \\
\vdots \\
0 \\
- \\
-1\end{array}$ & 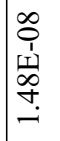 & 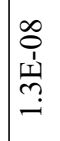 & $\begin{array}{l}\infty \\
0 \\
1 \\
\\
\\
\\
-\end{array}$ & $\mid \begin{array}{c}\infty \\
0 \\
⿱ 1 \\
\underline{1} \\
ٍ \\
\beth \\
=\end{array}$ & $\mid \begin{array}{c}\infty \\
0 \\
0 \\
1 \\
\text { ஸे } \\
0 \\
- \\
-\end{array}$ & 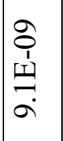 & 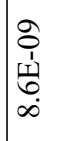 & $\begin{array}{l}2 \\
\hat{1} \\
1 \\
\infty\end{array}$ & 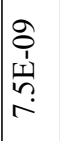 & 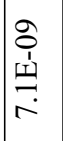 & 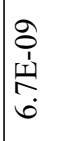 & 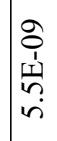 \\
\hline & & $\vec{D}$ & 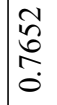 & 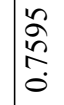 & $\begin{array}{l}\frac{\hat{f}}{2} \\
\stackrel{0}{0}\end{array}$ & 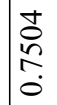 & $\frac{5}{\mathfrak{o}}$ & $\mid \begin{array}{l}0 \\
\stackrel{D}{+} \\
\dot{0}\end{array}$ & 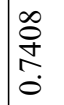 & 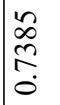 & $\begin{array}{l}n \\
\stackrel{2}{2} \\
\\
0\end{array}$ & 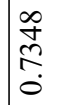 & 色 & $\mid \begin{array}{r}\overrightarrow{\widetilde{\Omega}} \\
\stackrel{0}{0}\end{array}$ & $\underset{\vec{m}}{\stackrel{\vec{m}}{0}}$ & 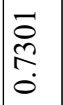 & 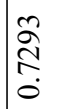 & $\begin{array}{l}\infty \\
\mathbb{0} \\
\mathbb{N} \\
\dot{0}\end{array}$ & 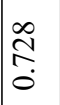 & 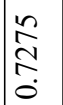 & 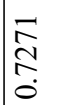 & 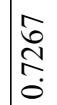 \\
\hline & & $\mathscr{J}$ & 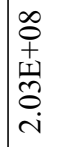 & 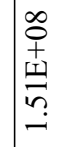 & \begin{tabular}{l}
$\infty$ \\
0 \\
+ \\
ज1 \\
$\infty$ \\
\hdashline \\
$=$
\end{tabular} & $\begin{array}{l}\hat{0} \\
\frac{+}{\alpha} \\
2 \\
\stackrel{\infty}{\infty}\end{array}$ & 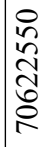 & 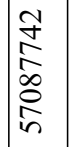 & 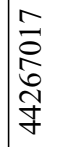 & 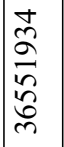 & 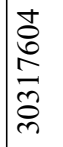 & $\begin{array}{l}\stackrel{\infty}{\sigma} \\
\stackrel{\sim}{m} \\
\stackrel{\sim}{\sim}\end{array}$ & 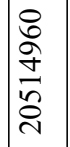 & 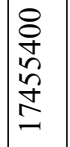 & 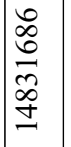 & 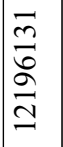 & 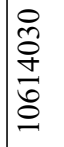 & 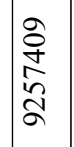 & $\mid \begin{array}{l}\mathcal{\delta} \\
\tilde{n} \\
\hat{\delta} \\
\infty \\
\infty\end{array}$ & 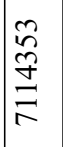 & $\begin{array}{l}\overrightarrow{\widehat{\alpha}} \\
\hat{\infty} \\
\widehat{b}\end{array}$ & 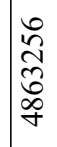 \\
\hline & & t & 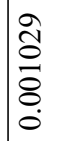 & 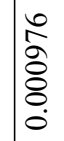 & 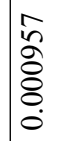 & 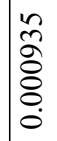 & 命 & $\mid \begin{array}{l}0 \\
0 \\
0 \\
0 \\
0 \\
0 \\
0\end{array}$ & 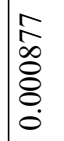 & $\mid \begin{array}{l}\infty \\
2 \\
\infty \\
0 \\
0 \\
0 \\
0 \\
0\end{array}$ & $\begin{array}{l}\hat{\tilde{O}} \\
\hat{0} \\
\vdots \\
0 \\
0\end{array}$ & 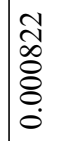 & 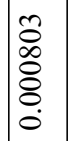 & 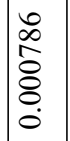 & 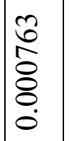 & $\mid \begin{array}{l}\frac{n}{1} \\
\frac{1}{8} \\
0 \\
0 \\
0\end{array}$ & 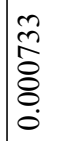 & $\mid \begin{array}{l}\frac{\partial}{\hat{\delta}} \\
\delta \\
\dot{\delta} \\
\dot{0}\end{array}$ & $\mid \begin{array}{l}8 \\
8 \\
8 \\
8 \\
0 \\
0\end{array}$ & 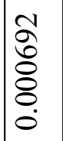 & $\mid \begin{array}{l}0 \\
0 \\
0 \\
0 \\
0 \\
0 \\
0 \\
0\end{array}$ & $\begin{array}{l}\tilde{1} \\
\infty \\
\tilde{\delta} \\
\delta \\
0 \\
0 \\
0\end{array}$ \\
\hline & & $\approx$ & 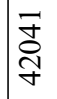 & $\begin{array}{l}\tilde{0} \\
\tilde{O} \\
\sigma\end{array}$ & $\begin{array}{l}\vec{\infty} \\
\infty \\
\infty \\
\infty\end{array}$ & 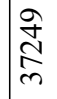 & 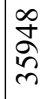 & $\left|\begin{array}{l}\infty \\
\stackrel{n}{f} \\
\tilde{N}\end{array}\right|$ & $\begin{array}{l}\stackrel{P}{6} \\
\text { m } \\
m\end{array}$ & 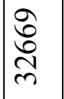 & 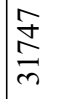 & 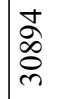 & 命 & 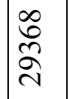 & $\mid \begin{array}{l}+ \\
0 \\
0 \\
\infty \\
\stackrel{\infty}{\sim}\end{array}$ & $\begin{array}{l}\underset{d}{0} \\
\infty \\
\sim \\
\sim\end{array}$ & 站 & $\begin{array}{l}\tilde{\partial} \\
\hat{\alpha} \\
\tilde{N}\end{array}$ & 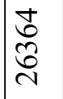 & 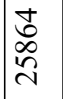 & $\begin{array}{l}\stackrel{2}{2} \\
\hat{n}\end{array}$ & $\mid \begin{array}{l}\stackrel{a}{2} \\
\stackrel{+}{d}\end{array}$ \\
\hline שै & ठิ & 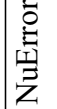 & 将 & $\begin{array}{l}\tilde{n} \\
\infty\end{array}$ & $\underset{\gamma}{\vec{\gamma}}$ & | & ชู & ஸి. & ชู & $\frac{9}{6}$ & $\begin{array}{l}\stackrel{2}{2} \\
\stackrel{2}{n}\end{array}$ & 要 & $\mid \begin{array}{l}\Delta \\
\text { in } \\
\end{array}$ & $\begin{array}{l}\overrightarrow{\hat{n}} \\
\text { n' }\end{array}$ & $\left|\begin{array}{l}n \\
\infty \\
i n\end{array}\right|$ & $\begin{array}{l}\tilde{b} \\
i \\
i\end{array}$ & $\begin{array}{l}n \\
m \\
n\end{array}$ & 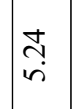 & $\begin{array}{l}0 \\
\vec{n} \\
\dot{n}\end{array}$ & $\begin{array}{l}\vec{F} \\
i n\end{array}$ & $\begin{array}{l}\text { D } \\
\text { in } \\
\text { in }\end{array}$ & 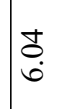 \\
\hline $\begin{array}{l} \pm \\
\sigma \\
\frac{\overrightarrow{0}}{\Xi}\end{array}$ & ڤे & $\vec{z}$ & $\stackrel{1}{\stackrel{\hat{~}}{2}}$ & $\begin{array}{l}\stackrel{0}{1} \\
\stackrel{n}{n} \\
=\end{array}$ & $\stackrel{+}{\stackrel{+}{0}}$ & $\mid$\begin{tabular}{l}
$\infty$ \\
\multirow{2}{*}{} \\
$\dot{\alpha}$
\end{tabular} & $\begin{array}{l}\infty \\
\sim \\
\infty \\
\infty\end{array}$ & $\left|\begin{array}{l}n \\
3 \\
0 \\
\infty\end{array}\right|$ & $\begin{array}{l}\tilde{\partial} \\
\dot{\infty} \\
\infty\end{array}$ & $\hat{\text { ळे }}$ & $\begin{array}{l}\bar{n} \\
2 \\
2\end{array}$ & 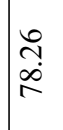 & $\stackrel{2}{2}$ & 2 & $\mid \begin{array}{l}0 \\
n \\
n \\
n\end{array}$ & $\begin{array}{l}\infty \\
0 \\
0 \\
0\end{array}$ & $\begin{array}{l}\tilde{O} \\
\dot{0} \\
\dot{\theta}\end{array}$ & $\mid \begin{array}{l}0 \\
0 \\
0 \\
0\end{array}$ & $\left|\begin{array}{l}7 \\
+ \\
6 \\
6\end{array}\right|$ & $\begin{array}{l}\stackrel{0}{1} \\
\stackrel{f}{0}\end{array}$ & $\hat{n}$ & $\begin{array}{l}\widetilde{D} \\
\mathbb{S} \\
\mathbb{U}\end{array}$ \\
\hline $\begin{array}{l}\stackrel{\mathscr{z}}{\underline{\Delta}} \\
\frac{\vec{\omega}}{\Xi}\end{array}$ & $\begin{array}{l}\overline{\mathscr{O}} \\
\stackrel{\gamma}{f}\end{array}$ & 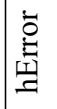 & 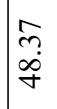 & $\hat{\sigma}$ & $\begin{array}{l}\infty \\
\infty \\
\infty\end{array}$ & $\underset{\infty}{\infty}$ & $\underset{\infty}{ \pm}$ & ले & $\vec{\sigma}$ & f̆ & $\hat{\widehat{\alpha}}$ & $\stackrel{\mathbb{2}}{\stackrel{N}{a}}$ & aे & $\mid \begin{array}{l}0 \\
0 \\
0\end{array}$ & $\mid \begin{array}{l}n \\
0 \\
0 \\
0\end{array}$ & $\stackrel{ \pm}{\stackrel{m}{0}}$ & 告 & $\mid \begin{array}{l}\infty \\
0 \\
0 \\
0\end{array}$ & $\begin{array}{l}\mathbf{D} \\
\infty \\
\stackrel{0}{0}\end{array}$ & $\stackrel{n}{a}$ & $\stackrel{\overbrace{}}{=}$ & $\underset{\vartheta}{\tilde{f}}$ \\
\hline$\underset{\Xi}{\stackrel{\Xi}{\Xi}}$ & ఫ్లి & $\underbrace{\stackrel{y}{\Xi}}_{\beth}$ & $\begin{array}{l}\bar{n} \\
\dot{v}\end{array}$ & $\begin{array}{l}\infty \\
\infty \\
\infty \\
\infty \\
\end{array}$ & $\stackrel{\ominus}{=}$ & 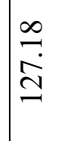 & I․ & $\mid \begin{array}{l}m \\
\grave{\Xi} \\
\grave{I}\end{array}$ & $\begin{array}{l}\vec{\sim} \\
\stackrel{m}{\Xi}\end{array}$ & 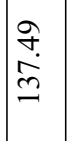 & $\begin{array}{l}2 \\
i n \\
2 \\
2\end{array}$ & ले & $\mid \begin{array}{l}n \\
\dot{9} \\
\dot{q} \\
\end{array}$ & 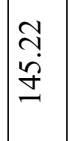 & $\begin{array}{l}\hat{a} \\
\dot{J}\end{array}$ & $\frac{\partial}{\vec{I}}$ & $\begin{array}{l}\exists \\
\vec{g} \\
\dot{g}\end{array}$ & $\begin{array}{l}\stackrel{0}{2} \\
\dot{J} \\
\end{array}$ & $\begin{array}{l}\infty \\
\dot{q} \\
\dot{q} \\
\end{array}$ & $\begin{array}{l}\partial \\
\dot{\theta} \\
\dot{I}\end{array}$ & ầ & $\overrightarrow{\dot{n}}$ \\
\hline$\sum_{\text {至 }}$ & $\vec{m}$ & $\begin{array}{l}\text { đิ } \\
\text { है } \\
= \\
=\end{array}$ & 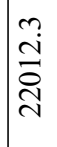 & 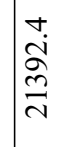 & $\begin{array}{l}n \\
\stackrel{n}{\infty} \\
\stackrel{\infty}{\sim} \\
\sim\end{array}$ & 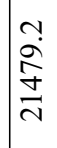 & 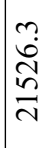 & 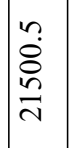 & 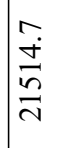 & $\mid \begin{array}{l}m \\
\stackrel{+}{+} \\
\stackrel{+}{v}\end{array}$ & $\begin{array}{l}\bar{i} \\
\grave{z} \\
\bar{\lambda}\end{array}$ & $\underset{⿱ 亠 㠯}{\stackrel{n}{7}}$ & $\left|\begin{array}{l}\hat{\vdots} \\
\tilde{n} \\
\tilde{n} \\
\tilde{N}\end{array}\right|$ & 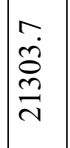 & $\begin{array}{l}\overrightarrow{\dot{0}} \\
\stackrel{8}{0} \\
\overrightarrow{2}\end{array}$ & 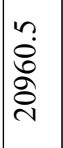 & 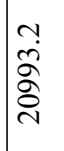 & 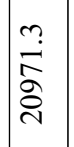 & mà & 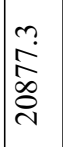 & $\begin{array}{l}\stackrel{a}{\sigma} \\
\stackrel{\vec{d}}{\stackrel{v}{v}}\end{array}$ & $\begin{array}{l}n \\
n \\
\\
\infty \\
-\end{array}$ \\
\hline $\begin{array}{l}\sum_{\pi}^{2} \\
0 \\
0 \\
0 \\
0\end{array}$ & 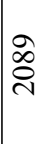 & $\vec{F}$ & $\begin{array}{l}\infty \\
\infty \\
\infty \\
\infty \\
m\end{array}$ & 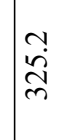 & $\begin{array}{l}\hat{2} \\
\hat{m} \\
\hat{n}\end{array}$ & $\vec{m}$ & $\begin{array}{l}\vec{\infty} \\
\dot{0} \\
\dot{0} \\
\text { n. }\end{array}$ & $\left|\begin{array}{c}\hat{n} \\
\dot{0} \\
\infty \\
m\end{array}\right|$ & $\begin{array}{l}\stackrel{n}{c} \\
\stackrel{m}{m}\end{array}$ & $\begin{array}{l}\text { बे } \\
\dot{8} \\
\text { के }\end{array}$ & 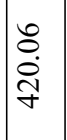 & 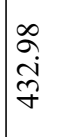 & $\begin{array}{l}\mathfrak{n} \\
\stackrel{n}{g} \\
\dot{y}\end{array}$ & $\left|\begin{array}{l}\hat{n} \\
\infty \\
n \\
\tilde{f}\end{array}\right|$ & 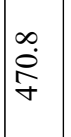 & $\begin{array}{c}\tilde{o} \\
\dot{\alpha} \\
\infty \\
\dot{\sigma}\end{array}$ & $\begin{array}{l}\stackrel{7}{二} \\
\ddot{q} \\
\vec{q}\end{array}$ & 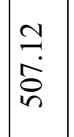 & 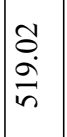 & $\begin{array}{l}\vec{\infty} \\
0 \\
0 \\
i n \\
\text { in }\end{array}$ & 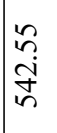 & $\begin{array}{l}\forall \\
\dot{n} \\
i n \\
n\end{array}$ \\
\hline 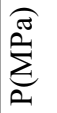 & ָ̊? & 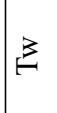 & $\mid \begin{array}{l}\infty \\
\dot{\infty} \\
\infty \\
m\end{array}$ & \begin{tabular}{l}
$\infty$ \\
$\infty$ \\
$\stackrel{0}{\gamma}$ \\
\multirow{\gamma}{*}{}
\end{tabular} & $\begin{array}{l}\tilde{n} \\
\tilde{8} \\
\tilde{n}\end{array}$ & $\frac{\stackrel{\Omega}{\vec{\Sigma}}}{\hat{n}}$ & $\begin{array}{l}n \\
i n \\
\tilde{n}\end{array}$ & $\begin{array}{l}\vec{b} \\
\dot{0} \\
\dot{+} \\
n\end{array}$ & $\begin{array}{l}\hat{\hat{y}} \\
\hat{n} \\
\hat{n}\end{array}$ & 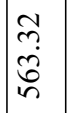 & 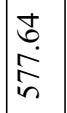 & $\mid \begin{array}{c}\vec{\lambda} \\
\stackrel{\infty}{\infty} \\
\infty \\
i n\end{array}$ & $\begin{array}{l}\vec{\infty} \\
\infty \\
i \\
i n\end{array}$ & $\begin{array}{l}\hat{0} \\
\dot{8} \\
\delta\end{array}$ & $\mid$\begin{tabular}{l}
$\infty$ \\
\multirow{1}{0}{} \\
0 \\
$\sigma$
\end{tabular} & $\mid \begin{array}{l}n \\
f \\
0 \\
0 \\
0 \\
0\end{array}$ & $\begin{array}{l}\vec{\infty} \\
\dot{\vec{j}}\end{array}$ & $\tilde{6}$ & $\mid \begin{array}{l}\vec{J} \\
i \\
\mathbb{i} \\
0\end{array}$ & $\frac{n}{i}$ & $\mid \begin{array}{l}\tilde{n} \\
\tilde{\infty} \\
\infty\end{array}$ & $\stackrel{n}{\pi}$ \\
\hline $\begin{array}{l}0 \\
\mathscr{Z} \\
\tilde{z} \\
\tilde{z}\end{array}$ & $\mathscr{F}$ & $\stackrel{\rho}{a}$ & $N$ & $\vec{\infty}$ & 市 & 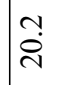 & సֶ. & $\begin{array}{l}n \\
\tilde{n}\end{array}$ & $\begin{array}{c}m \\
\infty \\
\infty \\
m\end{array}$ & $\mid \begin{array}{l}+ \\
\dot{J}\end{array}$ & $\begin{array}{l}t \\
\dot{8}\end{array}$ & $\ddot{i n}$ & $\mid \begin{array}{l}n \\
i \\
0\end{array}$ & $\left|\begin{array}{l}n \\
\infty \\
\infty \\
0\end{array}\right|$ & $\begin{array}{l}0 \\
\stackrel{+}{\mp}\end{array}$ & $\begin{array}{l}0 \\
\dot{\infty} \\
\infty\end{array}$ & $\hat{\infty}$ & $\hat{a}$ & $\left|\begin{array}{l}\infty \\
\infty \\
\infty\end{array}\right|$ & $\begin{array}{l}\infty \\
\dot{0} \\
\stackrel{0}{0}\end{array}$ & $\stackrel{\varrho}{\varrho}$ & \\
\hline
\end{tabular}




\begin{tabular}{|c|c|c|c|c|c|c|c|c|c|c|c|c|c|c|c|c|c|c|c|c|c|c|}
\hline & & $\vec{\Delta}$ & 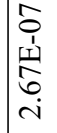 & $\begin{array}{l}\hat{0} \\
\mathbf{1} \\
1 \\
\\
\hat{n} \\
i\end{array}$ & $\begin{array}{l}0 \\
0 \\
1 \\
01 \\
0 \\
0 \\
i \\
i\end{array}$ & 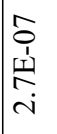 & 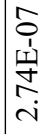 & 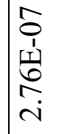 & 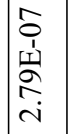 & 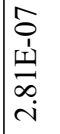 & 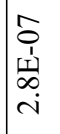 & 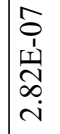 & $\begin{array}{l}\hat{0} \\
1 \\
1 \\
\mathbb{1} \\
\infty \\
i \\
i\end{array}$ & 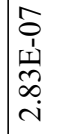 & 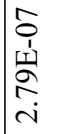 & 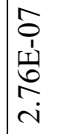 & 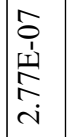 & $\begin{array}{c}\hat{s} \\
1 \\
i \\
i \\
i \\
i\end{array}$ & 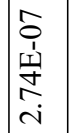 & $\mid \begin{array}{c}\hat{0} \\
1 \\
1 \\
\\
i \\
i \\
i\end{array}$ & 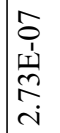 & 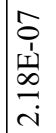 \\
\hline & & $\stackrel{\oplus}{\infty}$ & \begin{tabular}{|l}
$\hat{o}$ \\
$\hat{1}$ \\
$\underline{1}$ \\
$\infty$ \\
$\vec{i}$
\end{tabular} & 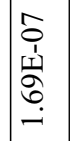 & 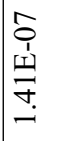 & 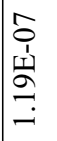 & 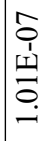 & 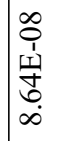 & 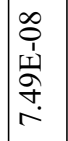 & $\mid \begin{array}{c}\infty \\
0 \\
0 \\
1 \\
\tilde{n} \\
\\
0\end{array}$ & 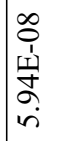 & 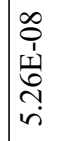 & $\begin{array}{l}\infty \\
0 \\
⿱ 1 \\
1 \\
\tilde{1} \\
0 \\
\dot{+} \\
\dot{\gamma}\end{array}$ & 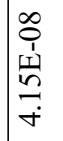 & 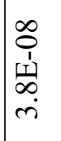 & $\begin{array}{l}\infty \\
0 \\
0 \\
1 \\
\infty \\
\infty \\
m \\
m\end{array}$ & \begin{tabular}{|c|}
0 \\
0 \\
0 \\
1 \\
th \\
0 \\
$\dot{0}$ \\
$\dot{m}$
\end{tabular} & $\begin{array}{l}\infty \\
0 \\
1 \\
1 \\
0 \\
\infty \\
\infty \\
i\end{array}$ & 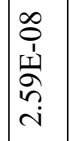 & 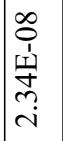 & 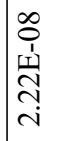 & 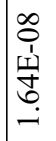 \\
\hline & & $\vec{D}$ & $\begin{array}{l}\frac{d}{0} \\
\stackrel{0}{0} \\
0\end{array}$ & $\begin{array}{l}\tilde{D} \\
\stackrel{n}{2} \\
0 \\
0\end{array}$ & 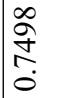 & 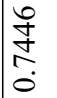 & $\frac{n}{o}$ & $\underset{\substack{n \\
\stackrel{2}{0}}}{\stackrel{0}{0}}$ & 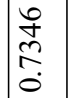 & $\begin{array}{l}\stackrel{2}{2} \\
\stackrel{2}{0} \\
\stackrel{0}{0}\end{array}$ & $\stackrel{\vec{n}}{\stackrel{0}{0}}$ & 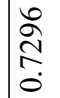 & 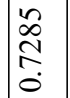 & 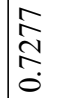 & 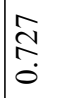 & 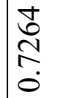 & $\mid \begin{array}{l}0 \\
\stackrel{N}{0} \\
0\end{array}$ & $\begin{array}{l}0 \\
\stackrel{0}{0} \\
\stackrel{0}{0}\end{array}$ & $\mid \begin{array}{l}\tilde{Z} \\
\stackrel{\tilde{N}}{0}\end{array}$ & 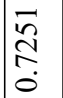 & 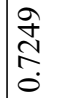 & 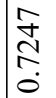 \\
\hline & & $\mathscr{J}$ & 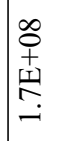 & $\begin{array}{l}\infty \\
0 \\
+ \\
\omega \\
n \\
0 \\
\vdots \\
-1\end{array}$ & $\mid \begin{array}{l}\underset{⿱}{+} \\
\stackrel{+}{+} \\
\stackrel{\infty}{R} \\
\stackrel{R}{R}\end{array}$ & 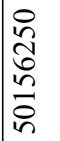 & 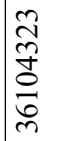 & 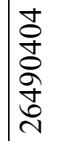 & 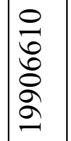 & 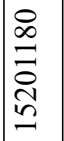 & 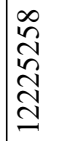 & 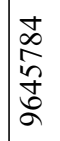 & 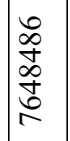 & $\underset{\Xi}{\stackrel{\Xi}{\Xi}}$ & 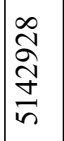 & 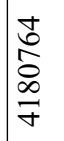 & 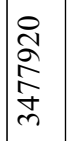 & $\mid \begin{array}{l}\mathcal{y} \\
\alpha \\
\alpha \\
\partial \\
\hat{~} \\
\end{array}$ & $\begin{array}{l}\hat{n} \\
\tilde{\widehat{n}} \\
\tilde{n}\end{array}$ & 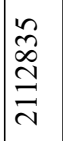 & 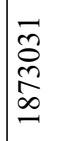 & 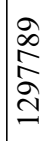 \\
\hline & & t & 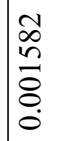 & 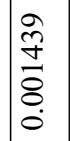 & 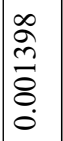 & 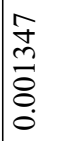 & 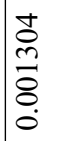 & 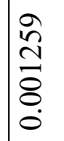 & 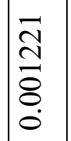 & 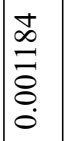 & 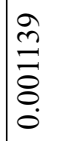 & 三. & $\left|\begin{array}{l}n \\
\hat{0} \\
0 \\
0 \\
0 \\
0 \\
0\end{array}\right|$ & $\frac{\infty}{0}$ & $\mid \begin{array}{l}n \\
0 \\
0 \\
0 \\
0 \\
0\end{array}$ & 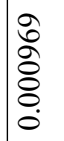 & 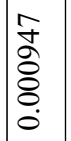 & $\mid \begin{array}{c}\tilde{Z} \\
\tilde{\delta} \\
\delta \\
0 \\
0\end{array}$ & $\mid \begin{array}{l}\hat{\alpha} \\
\dot{\alpha} \\
\dot{0} \\
0 \\
\dot{0}\end{array}$ & 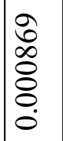 & 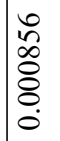 & 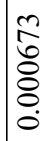 \\
\hline & & $\approx$ & 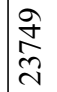 & $\underset{\mathbb{N}}{\mathbb{N}}$ & $\mid \begin{array}{l}\mathcal{J} \\
\stackrel{d}{d}\end{array}$ & $\begin{array}{l}\frac{+}{2} \\
\text { à }\end{array}$ & ‡̛ & $\begin{array}{l}\overrightarrow{\widetilde{J}} \\
\stackrel{\infty}{\Omega}\end{array}$ & 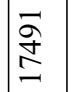 & $\underset{\tilde{D}}{\tilde{\infty}}$ & $\begin{array}{l}\overrightarrow{\widetilde{\Omega}} \\
\underline{\sigma}\end{array}$ & 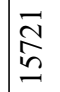 & సે & 全 & 冚 & 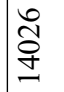 & $\mid$\begin{tabular}{l}
$\infty$ \\
$\infty$ \\
\hdashline \\
-2
\end{tabular} & $\begin{array}{l}\vec{N} \\
\tilde{m}\end{array}$ & 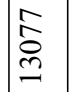 & $\begin{array}{l}\hat{0} \\
\infty \\
\stackrel{\Xi}{\Xi}\end{array}$ & 站 & $\underset{\widetilde{N}}{\approx}$ \\
\hline है & ठี & 窻 & 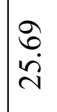 & $\stackrel{F}{F}$ & mे & $\stackrel{\infty}{\stackrel{i}{i}}$ & 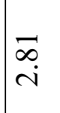 & $\stackrel{\circ}{\stackrel{i}{i}}$ & $\begin{array}{l}\infty \\
\infty \\
i\end{array}$ & $\vec{\rho}_{\dot{m}}$ & $\begin{array}{l}\infty \\
\infty \\
i \\
i\end{array}$ & $\stackrel{\widehat{i}}{i}$ & $\begin{array}{l}a \\
\dot{D} \\
i\end{array} \mid$ & $\stackrel{\infty}{\infty}$ & $\mid \begin{array}{c}\infty \\
\infty \\
i\end{array}$ & $\vec{n}$ & $\ddot{n}$ & $\begin{array}{l}\vec{b} \\
i \\
i\end{array}$ & 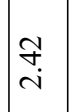 & $\underset{\substack{\infty \\
i \\
i}}{ }$ & $\stackrel{n}{i}$ & $\overrightarrow{\dot{\gamma}}$ \\
\hline $\begin{array}{l} \pm \\
\sigma \\
\frac{\overrightarrow{0}}{\Xi}\end{array}$ & 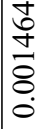 & $\vec{z}$ & 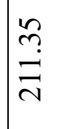 & $\begin{array}{l}\vec{\sigma} \\
\dot{T}\end{array}$ & $\underset{\hat{\sigma}}{\stackrel{2}{0}}$ & in & 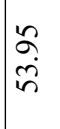 & 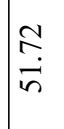 & $\begin{array}{l}\infty \\
\infty \\
\infty \\
\infty\end{array}$ & त̂̀ & $\begin{array}{l}3 \\
\dot{+} \\
\dot{f}\end{array}$ & $\begin{array}{l}\vec{\sigma} \\
\dot{\sigma}\end{array}$ & $\begin{array}{l}\infty \\
\dot{\sigma} \\
\dot{\sigma}\end{array}$ & $\begin{array}{l}n \\
\mathfrak{y} \\
\dot{y}\end{array}$ & $\begin{array}{l}n \\
\tilde{\sigma} \\
\dot{q}\end{array}$ & $\stackrel{n}{\stackrel{\vec{F}}{\vec{\gamma}}}$ & 吕 & $\begin{array}{l}3 \\
0 \\
\hat{n}\end{array}$ & $\mid \begin{array}{c} \pm \\
\infty \\
\infty \\
\infty\end{array}$ & $\stackrel{\hat{i}}{i}$ & $\frac{n}{n}$ & $\begin{array}{l}\stackrel{0}{0} \\
\stackrel{i}{f}\end{array}$ \\
\hline$\frac{\mathscr{\mathscr { U }}}{\stackrel{\tilde{\omega}}{\Xi}}$ & 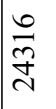 & 营 & $\mid \begin{array}{l}\hat{y} \\
\infty \\
\sim\end{array}$ & $\hat{n}$ & 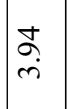 & $\stackrel{\overbrace{}}{\stackrel{r}{r}}$ & $\begin{array}{l}\vec{\infty} \\
\dot{m}\end{array}$ & $\begin{array}{l}\partial \\
\dot{r}\end{array}$ & 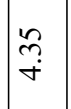 & 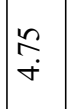 & 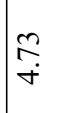 & $\begin{array}{l}n \\
\text { in } \\
\text { in }\end{array}$ & $\begin{array}{l}0 \\
\text { Ny } \\
\text { in }\end{array}$ & 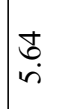 & $\begin{array}{l}6 \\
6 \\
\text { in }\end{array}$ & 文 & $\begin{array}{l}\infty \\
0 \\
i \\
i n\end{array}$ & $\left|\begin{array}{l}0 \\
\infty \\
i\end{array}\right|$ & 吉 & ڤ & 6 & $\stackrel{\mathbb{2}}{\stackrel{0}{0}}$ \\
\hline$\underset{\Xi}{\stackrel{\Xi}{\Xi}}$ & $\stackrel{n}{\tilde{n}}$ & $\underbrace{\stackrel{y}{\Xi}}_{\beth}$ & 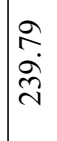 & $\frac{\sigma}{2}$ & 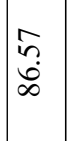 & $\mid$\begin{tabular}{l}
$\vec{H}$ \\
\multirow{2}{*}{} \\
$\dot{\infty}$
\end{tabular} & $\begin{array}{l}\hat{\alpha} \\
\dot{\infty}\end{array}$ & $\begin{array}{l}\infty \\
\infty \\
\infty \\
\infty\end{array}$ & $\stackrel{ }{\dot{8}}$ & $\begin{array}{l}\infty \\
\stackrel{\infty}{+} \\
\dot{\alpha}\end{array}$ & 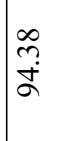 & 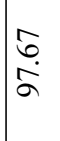 & $\begin{array}{l}0 \\
+ \\
a \\
2\end{array}$ & $\begin{array}{l}2 \\
\grave{\delta} \\
\hat{\sigma}\end{array}$ & 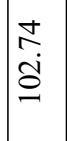 & Эั & 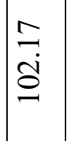 & $\begin{array}{c}\tilde{y} \\
\stackrel{8}{0} \\
\tilde{O}\end{array}$ & 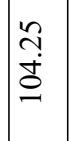 & $\begin{array}{l}\hat{\sigma} \\
\dot{0} \\
0\end{array}$ & $\begin{array}{l}1 \\
\text { o. } \\
0\end{array}$ & $\begin{array}{l}\infty \\
\stackrel{n}{+} \\
\stackrel{\Xi}{I}\end{array}$ \\
\hline$\sum_{\text {至 }}$ & $\vec{i}$ & $\begin{array}{l}\text { ฮี } \\
\text { है } \\
= \\
=\end{array}$ & $\begin{array}{l}\stackrel{n}{\stackrel{2}{2}} \\
\underset{2}{2}\end{array}$ & $\begin{array}{l}\infty \\
\infty \\
\infty \\
\infty \\
\infty\end{array}$ & $\begin{array}{l}\hat{i} \\
\infty \\
\infty \\
\infty \\
\infty \\
-\end{array}$ & 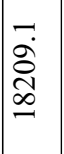 & $\begin{array}{l}\exists \\
\overrightarrow{\dot{D}} \\
\infty \\
\infty\end{array}$ & 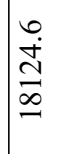 & $\stackrel{n}{\underset{i}{\leftrightarrows}}$ & 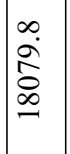 & 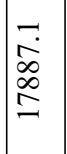 & $\frac{\sqrt{-}}{\frac{2}{\Omega}}$ & $\left|\begin{array}{l}0 \\
\dot{\bar{\Xi}} \\
\stackrel{\infty}{\Xi} \\
=\end{array}\right|$ & $\begin{array}{l}\infty \\
\infty \\
\infty \\
\infty \\
\end{array}$ & $\begin{array}{l}\vec{a} \\
\stackrel{a}{a} \\
\Xi\end{array}$ & 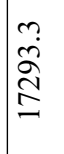 & 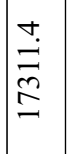 & 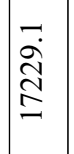 & 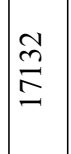 & ర్ & 蒿 & $\begin{array}{l}- \\
\infty \\
0 \\
0 \\
\\
-2\end{array}$ \\
\hline $\begin{array}{l}\sum_{\pi} \\
0 \\
0 \\
0 \\
0\end{array}$ & $\widehat{\infty}$ & $\vec{F}$ & $\begin{array}{l}\stackrel{2}{\tilde{m}} \\
\stackrel{m}{m}\end{array}$ & $\frac{n}{\stackrel{n}{m}}$ & $\begin{array}{l}\hat{\sigma} \\
\dot{n} \\
\tilde{n}\end{array}$ & $\begin{array}{c}m \\
\stackrel{n}{n} \\
\hat{n}\end{array}$ & $\begin{array}{l}\hat{n} \\
\ddot{n} \\
\tilde{m}\end{array}$ & 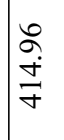 & 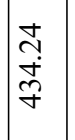 & $\begin{array}{l}\tilde{N} \\
\tilde{n} \\
\tilde{\gamma}\end{array}$ & 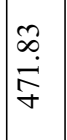 & $\begin{array}{l}= \\
\stackrel{\square}{\dot{q}} \\
\dot{q}\end{array}$ & $\begin{array}{l} \pm \\
\mathbb{\infty} \\
\infty \\
\infty \\
n\end{array}$ & $\begin{array}{l}\sigma \\
\alpha \\
\sim \\
\tilde{n}\end{array}$ & $\begin{array}{l}\hat{n} \\
\tilde{y} \\
\tilde{f}\end{array}$ & $\begin{array}{l}\hat{n} \\
\stackrel{0}{0} \\
i \\
i n\end{array}$ & $\mid \begin{array}{l}m \\
\stackrel{2}{*} \\
i n\end{array}$ & $\begin{array}{l}\vec{r} \\
\dot{n} \\
\tilde{n}\end{array}$ & $\begin{array}{l}8 \\
0 \\
0 \\
6\end{array}$ & 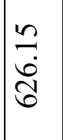 & $\begin{array}{l}\infty \\
\stackrel{\infty}{i} \\
\stackrel{\mathcal{G}}{0}\end{array}$ & $\begin{array}{l}\tilde{n} \\
\tilde{0} \\
\tilde{0}\end{array}$ \\
\hline 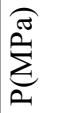 & ָ̊? & 3 & $\begin{array}{l}n \\
\stackrel{n}{a} \\
\text { ma }\end{array}$ & $\begin{array}{l}n \\
\tilde{n} \\
\hat{n}\end{array}$ & $\begin{array}{l}= \\
\dot{0} \\
\stackrel{0}{n}\end{array}$ & $\begin{array}{l}n \\
\stackrel{2}{8} \\
\dot{n}\end{array}$ & $\begin{array}{l}\hat{n} \\
\hat{2} \\
\hat{b}\end{array}$ & 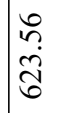 & 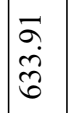 & $\begin{array}{l}\infty \\
\sigma \\
\tilde{j} \\
\tilde{\sigma}\end{array}$ & $\frac{n}{n}$ & $\mid \begin{array}{l}n \\
\tilde{n} \\
\tilde{\sigma}\end{array}$ & $\mid \begin{array}{c}\tilde{N} \\
\tilde{\infty} \\
\tilde{\delta}\end{array}$ & $\begin{array}{l}\infty \\
\infty \\
\infty \\
\infty \\
0\end{array}$ & $\begin{array}{l}\infty \\
\dot{2} \\
\stackrel{\gamma}{\gamma}\end{array}$ & $\begin{array}{l}\infty \\
n \\
\sim \\
\sim \\
n\end{array}$ & 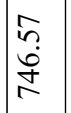 & $\begin{array}{l}m \\
\vdots \\
8\end{array}$ & 帝 & \begin{tabular}{l}
\multirow{2}{c}{} \\
$\infty$ \\
$\infty$ \\
$\infty$
\end{tabular} & 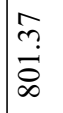 & 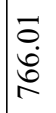 \\
\hline $\begin{array}{l}0 \\
\mathscr{Z} \\
\tilde{z} \\
\tilde{z}\end{array}$ & $\forall$ & $\stackrel{9}{9}$ & $N$ & $\vec{\infty}$ & $\overrightarrow{ \pm}$ & ָิ & ֶֶ. & $\underset{m}{m}$ & $\mid \begin{array}{c}m \\
\infty \\
m\end{array}$ & $\mid \begin{array}{l}+ \\
\dot{J}\end{array}$ & $\begin{array}{l}t \\
\dot{8}\end{array}$ & $\ddot{\circ}$ & $\mid \begin{array}{l}n \\
i \\
0\end{array}$ & $\begin{array}{l}n \\
\infty \\
\infty \\
0\end{array}$ & $\begin{array}{l}0 \\
\stackrel{+}{T}\end{array}$ & $\begin{array}{l}0 \\
\dot{0} \\
\infty\end{array}$ & $\begin{array}{l}\hat{\infty} \\
\infty\end{array}$ & $\hat{a}$ & $\left|\begin{array}{l}\infty \\
\infty \\
\infty\end{array}\right|$ & $\begin{array}{l}\infty \\
\dot{0} \\
\stackrel{0}{0}\end{array}$ & $\stackrel{\varrho}{\varrho}$ & \\
\hline
\end{tabular}




\begin{tabular}{|c|c|c|c|c|c|c|c|c|c|c|c|c|c|c|c|c|c|c|c|c|c|c|}
\hline & & $\vec{z}$ & 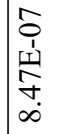 & $\begin{array}{l}\hat{o} \\
1 \\
1 \\
\infty \\
2 \\
\end{array}$ & 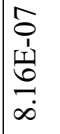 & 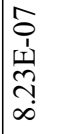 & \begin{tabular}{|l}
$\hat{0}$ \\
$\hat{1}$ \\
$\hat{1}$ \\
$\hat{N}$ \\
$\infty$ \\
$\infty$
\end{tabular} & 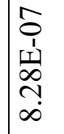 & 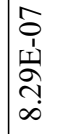 & 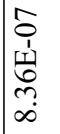 & $\begin{array}{l}\hat{0} \\
\hat{1} \\
\underline{1} \\
\\
\\
\infty\end{array}$ & 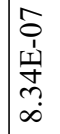 & $\begin{array}{c}\hat{0} \\
1 \\
1 \\
0 \\
\\
\infty \\
\infty\end{array}$ & $\begin{array}{c}\hat{o} \\
\hat{1} \\
\hat{1} \\
\hat{\infty} \\
\infty\end{array}$ & 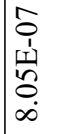 & $\begin{array}{l}\hat{0} \\
\hat{1} \\
\text { Wh} \\
\infty \\
\infty \\
\end{array}$ & 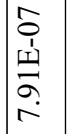 & 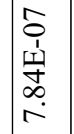 & 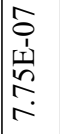 & \begin{tabular}{|l}
$\hat{0}$ \\
$\hat{1}$ \\
$\underline{1}$ \\
$\tilde{n}$ \\
\end{tabular} & 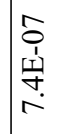 & 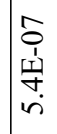 \\
\hline & & $\stackrel{\oplus}{\infty}$ & 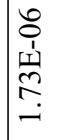 & $\begin{array}{c}0 \\
0 \\
1 \\
11 \\
0 \\
-1 \\
-1\end{array}$ & 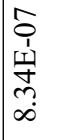 & 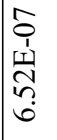 & 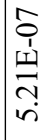 & 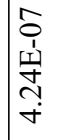 & $\begin{array}{l}\hat{0} \\
0 \\
1 \\
\hat{1} \\
0 \\
\dot{n}\end{array}$ & $\mid \begin{array}{c}\hat{0} \\
0 \\
1 \\
1 \\
0 \\
0 \\
\dot{r} \\
\dot{r}\end{array}$ & 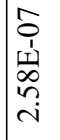 & $\begin{array}{l}\hat{s} \\
\hat{i} \\
\underline{1} \\
\hat{m} \\
\end{array}$ & $\mid \begin{array}{c}\hat{o} \\
\hat{1} \\
\underline{1} \\
\hat{\alpha} \\
- \\
-\end{array}$ & 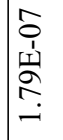 & 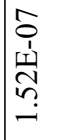 & $\begin{array}{c}\hat{s} \\
\hat{1} \\
\underline{1} \\
\\
-\end{array}$ & 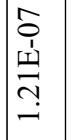 & $\begin{array}{c}s \\
\hat{1} \\
\underline{1} \\
\underline{-} \\
-1\end{array}$ & $\begin{array}{l}\hat{o} \\
\hat{o} \\
\underline{1} \\
\underline{y}\end{array}$ & 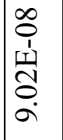 & 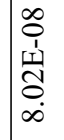 & $\begin{array}{l}\infty \\
0 \\
0 \\
1 \\
1 \\
0 \\
ن \\
\text { in }\end{array}$ \\
\hline & & $\vec{D}$ & 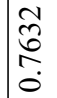 & 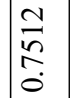 & $\stackrel{\mathscr{P}}{\stackrel{P}{O}}$ & 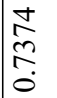 & 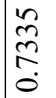 & 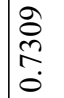 & 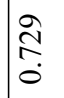 & 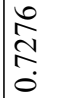 & \begin{tabular}{l}
$\stackrel{8}{0}$ \\
\multirow{N}{N}{} \\
$\dot{0}$
\end{tabular} & 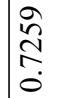 & 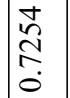 & 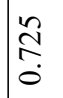 & 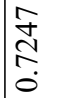 & 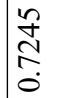 & $\mid \begin{array}{c}\mathfrak{+} \\
\mathbb{N} \\
\dot{0}\end{array}$ & 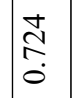 & $\underset{\stackrel{\overbrace{}}{\hat{~}}}{\stackrel{\overbrace{}}{0}}$ & 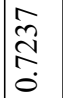 & 触 & 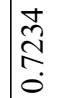 \\
\hline & & $\mathscr{J}$ & 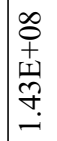 & 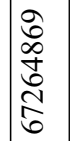 & 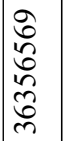 & 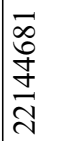 & 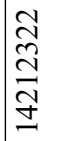 & $\frac{0}{\sigma}$ & 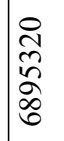 & 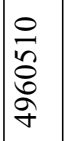 & 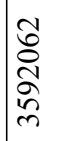 & 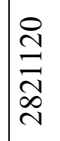 & 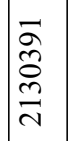 & 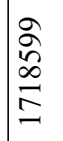 & 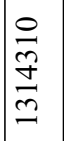 & 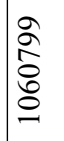 & 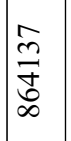 & $\begin{array}{l}\tilde{O} \\
\stackrel{0}{\mathbb{N}} \\
\mathbb{N}\end{array}$ & 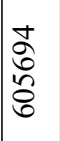 & 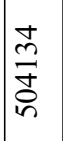 & 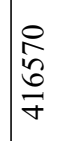 & 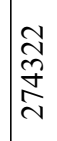 \\
\hline & & t & 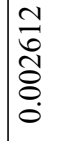 & 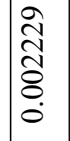 & 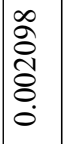 & $\frac{N}{\hat{\sigma}}$ & 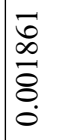 & 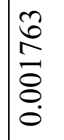 & 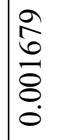 & $\mid \begin{array}{c}0 \\
0 \\
0 \\
0 \\
0 \\
0 \\
0\end{array}$ & $\begin{array}{l}\tilde{n} \\
\stackrel{2}{8} \\
0 \\
0\end{array}$ & $\mid \begin{array}{l}\infty \\
\stackrel{\infty}{\infty} \\
\stackrel{\infty}{8} \\
\stackrel{0}{0}\end{array}$ & $\begin{array}{l}\vec{\sim} \\
\stackrel{\mathcal{J}}{\sigma} \\
\dot{\delta} \\
\dot{0}\end{array}$ & 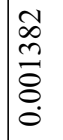 & 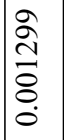 & 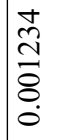 & 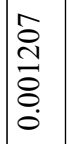 & $\begin{array}{c}0 \\
0 \\
0 \\
0 \\
0 \\
0 \\
0\end{array}$ & 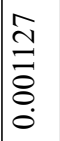 & $\frac{0}{0}$ & & $\mid \begin{array}{l}\infty \\
\tilde{1} \\
\hat{\delta} \\
0 \\
0 \\
0\end{array}$ \\
\hline & & $\approx$ & $\stackrel{\vec{\Xi}}{\stackrel{\Xi}{\Xi}}$ & $\stackrel{\bullet}{\Xi}$ & ঐे & $\mid \begin{array}{l} \pm \\
\infty \\
\alpha\end{array}$ & ठิ & $\frac{m}{\infty}$ & 命 & 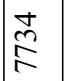 & $\underset{⿱ 亠}{\stackrel{I}{I}}$ & 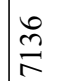 & \begin{tabular}{|l|l} 
\\
$\infty$ \\
$\infty$ \\
0 \\
0
\end{tabular} & $\begin{array}{l}\infty \\
0 \\
0 \\
0\end{array}$ & 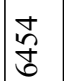 & $\stackrel{\overbrace{}}{\widehat{\sigma}}$ & $\frac{\hat{0}}{0}$ & 䔩 & $\underset{m}{\infty}$ & $\begin{array}{l}0 \\
\infty \\
0 \\
i \\
n\end{array}$ & $\begin{array}{l}t \\
0 \\
n \\
n\end{array}$ & $\begin{array}{l}\infty \\
0 \\
i n\end{array}$ \\
\hline שै & రิ & 窻 & $\begin{array}{l}\vec{\sigma} \\
\dot{\Xi}\end{array}$ & $\stackrel{\substack{n \\
i}}{i}$ & $\stackrel{\text { ڤn }}{\rightarrow}$ & 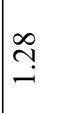 & $\stackrel{n}{=}$ & $\Xi$ & $\hat{\sigma}$ & $\vec{\sigma}$ & $\stackrel{8}{\circ}$ & $\exists$ & $\stackrel{0}{=}$ & $\tilde{\overbrace{}}$ & $\stackrel{?}{-}$ & 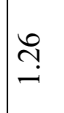 & $\mid \begin{array}{l}0 \\
\stackrel{0}{-} \\
-1\end{array}$ & $\begin{array}{l}n \\
\dddot{r} \\
-\end{array}$ & $\stackrel{m}{-}$ & $\stackrel{\oplus}{m}$ & 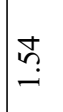 & 吕 \\
\hline $\begin{array}{l} \pm \\
\sigma \\
\frac{\overrightarrow{0}}{\Xi}\end{array}$ & 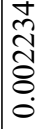 & $\vec{z}$ & 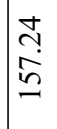 & $\begin{array}{l}\stackrel{0}{0} \\
\dot{b} \\
+\end{array}$ & $\begin{array}{l}\hat{\infty} \\
\dot{m} \\
\dot{m}\end{array}$ & $\vec{f}$ & $\begin{array}{l}0 \\
\stackrel{0}{0} \\
\sim\end{array}$ & 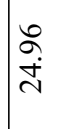 & $\begin{array}{l}\text { Õ } \\
\stackrel{+}{\sim}\end{array}$ & $\stackrel{m}{\stackrel{\sim}{\sim}}$ & $\begin{array}{l}\infty \\
\ddot{n} \\
\tilde{n}\end{array}$ & $\begin{array}{l}\partial \\
\stackrel{\partial}{+} \\
\text { i }\end{array}$ & $\mid \begin{array}{c}\stackrel{m}{p} \\
\stackrel{\sim}{\sim}\end{array}$ & 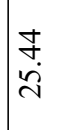 & $\begin{array}{l}2 \\
\dot{\tau} \\
+\end{array}$ & $\begin{array}{l}\stackrel{\Xi}{ } \\
\dot{\sim}\end{array}$ & 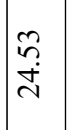 & $\begin{array}{l}0 \\
\stackrel{0}{+} \\
\stackrel{\sim}{\sim}\end{array}$ & $\begin{array}{l}\stackrel{0}{n} \\
\end{array}$ & 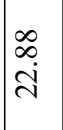 & $\begin{array}{l}\hat{\sigma} \\
\tilde{\lambda}\end{array}$ & $\underset{i}{\pi}$ \\
\hline $\begin{array}{l}\stackrel{\mathscr{z}}{\underline{\Delta}} \\
\frac{\vec{\omega}}{\Xi}\end{array}$ & 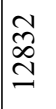 & 营 & $\stackrel{0}{0}$ & $\stackrel{\vec{\lambda}}{i}$ & $\stackrel{g}{\circ}$ & $\stackrel{n}{n}$ & $\stackrel{\sim}{\stackrel{q}{q}}$ & $\stackrel{g}{\stackrel{g}{-}}$ & $\stackrel{\infty}{\dddot{n}}$ & $\infty$ & $\underline{\Omega}$ & $\stackrel{\tilde{\lambda}}{\stackrel{\sim}{\lambda}}$ & $\tilde{n}$ & ì & $\begin{array}{l}\tilde{N} \\
\tilde{n}\end{array}$ & $\stackrel{\infty}{\substack{n \\
m}}$ & $\begin{array}{l}0 \\
\stackrel{0}{0} \\
\end{array}$ & $\begin{array}{l}\infty \\
\infty \\
\dot{r}\end{array}$ & $\begin{array}{l}\infty \\
\infty \\
\infty \\
\dot{m}\end{array}$ & \begin{tabular}{l}
\multirow{2}{*}{} \\
$\dot{m}$
\end{tabular} & $\begin{array}{l}\vec{\sigma} \\
\dot{+}\end{array}$ & $\begin{array}{l}\infty \\
\infty \\
\infty \\
\tilde{m}\end{array}$ \\
\hline$\underset{\Xi}{\stackrel{\widehat{a}}{\Xi}}$ & ల్లి & $\underbrace{\stackrel{y}{\Xi}}_{\beth}$ & $\begin{array}{l}\tilde{\alpha} \\
\stackrel{\infty}{\infty} \\
\infty\end{array}$ & त̂̃ & $\begin{array}{l}\infty \\
\stackrel{+}{\circ} \\
\dot{H}\end{array}$ & in & 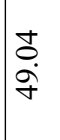 & $\mid \begin{array}{l}n \\
\hat{q} \\
\sigma\end{array}$ & 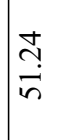 & $\begin{array}{l}\vec{\sigma} \\
\dot{\vec{n}} \\
\dot{n}\end{array}$ & $\begin{array}{l}7 \\
\stackrel{5}{0} \\
i n\end{array}$ & $\frac{\hat{n}}{\overrightarrow{0}}$ & \begin{tabular}{l}
$n$ \\
\hdashline \\
0 \\
0
\end{tabular} & $\frac{\hat{n}}{i}$ & $\frac{2}{2}$ & ते & 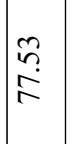 & $\vec{a}$ & $\begin{array}{l}\infty \\
0 \\
0 \\
2\end{array}$ & $\begin{array}{l}\vec{\infty} \\
\alpha \\
\alpha\end{array}$ & $\hat{\widehat{+}}$ & 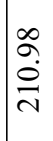 \\
\hline$\sum_{\text {至 }}$ & $\exists$ & $\begin{array}{l}\text { đิ } \\
\text { है } \\
= \\
=\end{array}$ & $\frac{1}{\frac{1}{\infty}}$ & $\begin{array}{l}\infty \\
\stackrel{\infty}{\underline{n}} \\
\stackrel{n}{n}\end{array}$ & $\begin{array}{l}\hat{n} \\
\tilde{n} \\
0 \\
n \\
n\end{array}$ & $\mid \begin{array}{l}\hat{0} \\
\infty \\
\vdots \\
\end{array}$ & 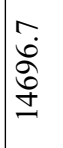 & $\begin{array}{l}\dot{m} \\
\dot{m} \\
\dot{J}\end{array}$ & 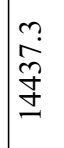 & 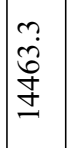 & 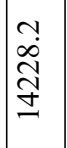 & $\mid \begin{array}{c}n \\
\stackrel{n}{n} \\
\tilde{\Xi}\end{array}$ & $\left|\begin{array}{l}\infty \\
\infty \\
\infty \\
\infty \\
\exists\end{array}\right|$ & 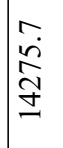 & \begin{tabular}{|l|}
0 \\
0 \\
0 \\
0 \\
0 \\
-2
\end{tabular} & 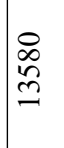 & 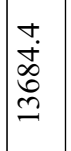 & $\begin{array}{l}n \\
0 \\
0 \\
0 \\
0 \\
-1\end{array}$ & $\mid \begin{array}{l}\hat{0} \\
\tilde{n} \\
\tilde{n}\end{array}$ & $\begin{array}{l}\infty \\
a \\
a \\
\underline{n} \\
\end{array}$ & 高 & $\begin{array}{l}\vec{\infty} \\
\tilde{N} \\
\tilde{n}\end{array}$ \\
\hline $\begin{array}{l}\sum_{\pi}^{2} \\
0 \\
0 \\
0 \\
0\end{array}$ & $\begin{array}{l}\stackrel{2}{\circ} \\
\stackrel{2}{2}\end{array}$ & $\vec{F}$ & $\begin{array}{l}\vec{n} \\
n \\
m \\
m\end{array}$ & $\begin{array}{l}0 \\
\dot{0} \\
\ddot{n}\end{array}$ & 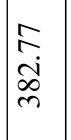 & $\mid \begin{array}{l}\stackrel{0}{2} \\
\dot{\vec{\sigma}} \\
\vec{\sigma}\end{array}$ & $\mid \begin{array}{l}\mathfrak{b} \\
\dot{f} \\
\dot{f}\end{array}$ & $\mid \begin{array}{l}\infty \\
\\
\stackrel{N}{F} \\
f\end{array}$ & $\begin{array}{l}\infty \\
0 \\
\stackrel{8}{0} \\
i n\end{array}$ & $\begin{array}{l}\vec{\sim} \\
\infty \\
\sim \\
\sim \\
\sim\end{array}$ & $\begin{array}{l}\vec{n} \\
\text { nn } \\
n\end{array}$ & 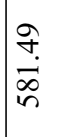 & 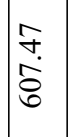 & $\begin{array}{l}8 \\
\infty \\
\widehat{్}\end{array}$ & $\mid \begin{array}{l}2 \\
\infty \\
\infty \\
0 \\
0\end{array}$ & 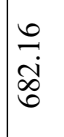 & $\mid \begin{array}{l}\infty \\
\infty \\
\hat{\delta} \\
\hat{n}\end{array}$ & $\begin{array}{l}\hat{n} \\
\grave{N}\end{array}$ & $\mid \begin{array}{l}\tilde{n} \\
\sim \\
n \\
n\end{array}$ & 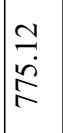 & $\frac{2}{2}$ & $\begin{array}{l}\Omega \\
\check{\Omega} \\
\infty \\
\infty\end{array}$ \\
\hline$\sum_{0}^{\pi}$ & ָ̊? & 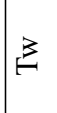 & $\begin{array}{l}\hat{\imath} \\
\infty \\
o \\
o \\
q\end{array}$ & $\begin{array}{l}n \\
\dot{d} \\
\text { in }\end{array}$ & $\frac{9}{\overrightarrow{0}}$ & 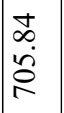 & 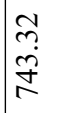 & $\begin{array}{l}8 \\
0 \\
\dot{b} \\
\circ\end{array}$ & 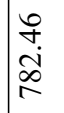 & $\mid \begin{array}{l}\hat{b} \\
\dot{a} \\
\hat{2}\end{array}$ & $\frac{\hat{N}}{\hat{\infty}}$ & $\mid \begin{array}{l}n \\
m \\
n \\
\infty \\
\infty\end{array}$ & $\begin{array}{l}0 \\
1 \\
\\
\infty\end{array}$ & $\begin{array}{l}\partial \\
\infty \\
\tilde{\infty}\end{array}$ & \begin{tabular}{l}
$n$ \\
\multirow{+}{*}{} \\
$\infty$
\end{tabular} & 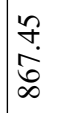 & $\left|\begin{array}{c}\hat{n} \\
i \\
\infty \\
\infty\end{array}\right|$ & $\begin{array}{l}8 \\
8 \\
\dot{8} \\
8\end{array}$ & $\mid \begin{array}{l}\infty \\
0 \\
\tilde{Z} \\
\tilde{\sigma}\end{array}$ & 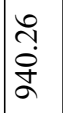 & $\begin{array}{l}m \\
\ddot{\dot{d}} \\
\sigma\end{array}$ & 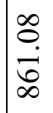 \\
\hline $\begin{array}{l}0 \\
\mathscr{Z} \\
\tilde{z} \\
\tilde{z}\end{array}$ & \& & $\stackrel{9}{9}$ & $\sim$ & $\vec{\infty}$ & $\overrightarrow{ \pm}$ & ָิ & ֶֶ. & $\underset{m}{m}$ & $\begin{array}{c}m \\
\infty \\
\infty \\
m\end{array}$ & $\mid \begin{array}{l}+ \\
\dot{J}\end{array}$ & $\begin{array}{l}t \\
\dot{8}\end{array}$ & 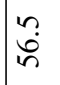 & $\mid \begin{array}{l}n \\
i \\
b\end{array}$ & $\begin{array}{l}n \\
\infty \\
\infty \\
0\end{array}$ & $\begin{array}{l}0 \\
\stackrel{+}{T}\end{array}$ & $\begin{array}{l}0 \\
\dot{0} \\
\infty\end{array}$ & $\begin{array}{l}\hat{\infty} \\
\infty\end{array}$ & $\hat{a}$ & $\left|\begin{array}{l}\infty \\
\infty \\
\infty\end{array}\right|$ & $\begin{array}{l}\infty \\
\dot{0} \\
\stackrel{0}{0}\end{array}$ & $\stackrel{\varrho}{\grave{O}}$ & \\
\hline
\end{tabular}




\begin{tabular}{|c|c|c|c|c|c|c|c|c|c|c|c|c|c|c|c|c|c|c|c|c|c|c|}
\hline & & $\vec{z}$ & 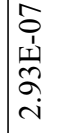 & $\begin{array}{l}\hat{o} \\
\hat{1} \\
\underline{1} \\
\infty \\
\infty \\
i\end{array}$ & 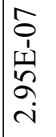 & 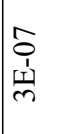 & \begin{tabular}{|l}
$\hat{0}$ \\
$\hat{9}$ \\
$\underline{1}$ \\
$\underline{1}$ \\
0 \\
\\
$\dot{n}$
\end{tabular} & 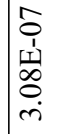 & $\begin{array}{l}\hat{\sigma} \\
\hat{1} \\
\underline{\omega} \\
\stackrel{m}{m}\end{array}$ & 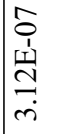 & 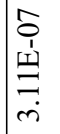 & 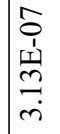 & $\mid \begin{array}{c}\hat{O} \\
1 \\
\stackrel{1}{1} \\
\\
\end{array}$ & $\begin{array}{l}\hat{a} \\
\hat{1} \\
\underline{m} \\
\\
\end{array}$ & \begin{tabular}{|l}
$\hat{0}$ \\
$\hat{1}$ \\
$\hat{1}$ \\
$\infty$ \\
0 \\
$\dot{m}$
\end{tabular} & \begin{tabular}{|l}
$\hat{o}$ \\
$\hat{1}$ \\
$\underline{1}$ \\
$\hat{o}$ \\
$\dot{n}$ \\
$\dot{n}$
\end{tabular} & 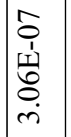 & $\begin{array}{c}\hat{o} \\
1 \\
1 \\
n \\
0 \\
\dot{n}\end{array}$ & $\mid$\begin{tabular}{c}
$\hat{0}$ \\
1 \\
1 \\
\multirow{1}{+}{} \\
$\dot{m}$ \\
$\dot{m}$
\end{tabular} & 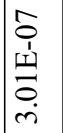 & 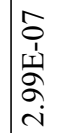 & 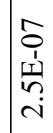 \\
\hline & & $\stackrel{\oplus}{\infty}$ & 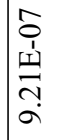 & $\begin{array}{c}\hat{0} \\
\mathbf{1} \\
\text { 11 } \\
\end{array}$ & 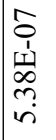 & 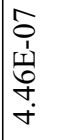 & $\begin{array}{l}\hat{s} \\
0 \\
1 \\
\\
\hat{n} \\
\dot{n}\end{array}$ & 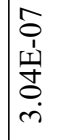 & $\begin{array}{c}\hat{0} \\
\hat{1} \\
\hat{\omega} \\
\\
\end{array}$ & 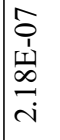 & 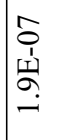 & 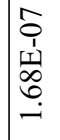 & 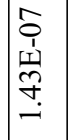 & 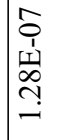 & 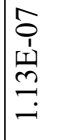 & 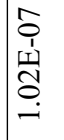 & 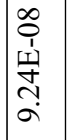 & $\begin{array}{c}\infty \\
0 \\
0 \\
1 \\
2 \\
\infty \\
\infty \\
\infty\end{array}$ & $\mid \begin{array}{l}\infty \\
0 \\
0 \\
1 \\
0 \\
0 \\
r \\
r\end{array}$ & 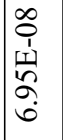 & 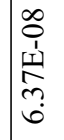 & 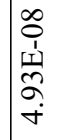 \\
\hline & & $\vec{D}$ & $\begin{array}{l}\infty \\
\stackrel{\infty}{n} \\
\stackrel{2}{0} \\
\dot{0}\end{array}$ & $\begin{array}{l}\overrightarrow{\widehat{D}} \\
\stackrel{0}{0}\end{array}$ & 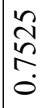 & $\frac{n}{\frac{n}{5}}$ & $\frac{\substack{o \\
⿱ 亠 䒑 \\
0}}{0}$ & $\mid \begin{array}{c}0 \\
\stackrel{2}{2} \\
\\
0\end{array}$ & 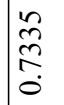 & 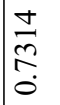 & $\begin{array}{l}\infty \\
\stackrel{2}{\hat{N}} \\
\stackrel{0}{0}\end{array}$ & 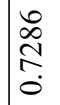 & 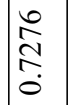 & $\begin{array}{l}\stackrel{0}{0} \\
\stackrel{N}{N} \\
0 \\
0\end{array}$ & 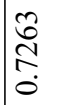 & 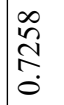 & 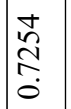 & $\mid \begin{array}{c}\vec{n} \\
\stackrel{a}{0} \\
\dot{0}\end{array}$ & 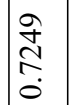 & 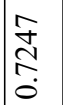 & 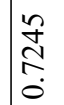 & 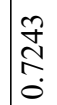 \\
\hline & & $\mathscr{J}$ & 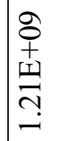 & 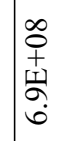 & 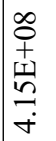 & $\begin{array}{l}\infty \\
0 \\
0 \\
+ \\
\text { Inj } \\
\stackrel{2}{0} \\
i \\
i\end{array}$ & $\begin{array}{l}\infty \\
0 \\
1 \\
\omega \\
\infty \\
\infty \\
-\infty \\
-1\end{array}$ & 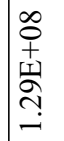 & 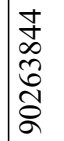 & $\mid \begin{array}{l}1 \\
2 \\
0 \\
0 \\
0 \\
0 \\
0\end{array}$ & $\begin{array}{l}\hat{n} \\
\hat{\sigma} \\
\tilde{m} \\
\tilde{n}\end{array}$ & 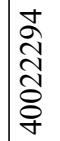 & $\mid \begin{array}{l}\hat{\tilde{n}} \\
\tilde{n} \\
\infty \\
\tilde{\delta} \\
\delta \\
\tilde{n}\end{array}$ & 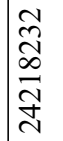 & 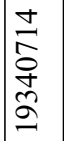 & $\begin{array}{l}\stackrel{m}{\approx} \\
\approx \\
\tilde{n} \\
\underline{n}\end{array}$ & $\mid \begin{array}{l}n \\
2 \\
0 \\
0 \\
0 \\
- \\
-\end{array}$ & $\begin{array}{l}\infty \\
\tilde{N} \\
\tilde{\Xi} \\
\infty \\
\infty \\
0\end{array}$ & $\begin{array}{l}\tilde{f} \\
\infty \\
\stackrel{0}{\sim} \\
\tilde{\sigma}\end{array}$ & 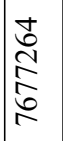 & $\begin{array}{l}\hat{2} \\
\hat{\sigma} \\
\hat{\sigma}\end{array}$ & 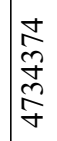 \\
\hline & & t & 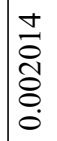 & 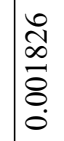 & 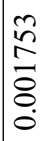 & $\mid \begin{array}{l}n \\
\hat{\sigma} \\
0 \\
0 \\
0 \\
0 \\
0\end{array}$ & $\stackrel{5}{\square}$ & 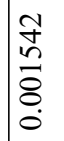 & 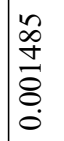 & \begin{tabular}{|l}
$\overrightarrow{\tilde{g}}$ \\
$\stackrel{\vec{\sigma}}{0}$ \\
$\dot{0}$
\end{tabular} & 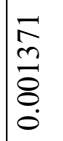 & 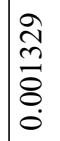 & 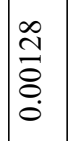 & 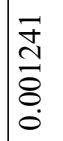 & $\mid \begin{array}{c}\infty \\
\stackrel{\infty}{二} \\
\stackrel{\overrightarrow{8}}{0} \\
0\end{array}$ & 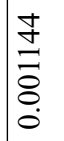 & 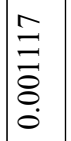 & $\mid \begin{array}{l}n \\
o \\
0 \\
0 \\
0 \\
0 \\
0\end{array}$ & $\mid \begin{array}{l}n \\
\tilde{o} \\
o \\
0 \\
0 \\
0\end{array}$ & $\begin{array}{l}\bar{\delta} \\
\stackrel{\delta}{\sigma} \\
\vdots \\
0\end{array}$ & 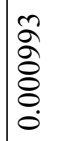 & 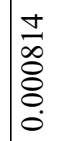 \\
\hline & & $\approx$ & 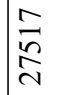 & 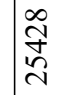 & $\underset{\hat{\curvearrowright}}{\stackrel{\sim}{\approx}}$ & $\frac{m}{\overrightarrow{\widetilde{N}}}$ & $\frac{\infty}{\stackrel{0}{\sigma}}$ & 赵 & $\frac{m}{2}$ & $\mid \begin{array}{c}\hat{N} \\
\infty \\
\infty\end{array}$ & $\begin{array}{l}m \\
\varpi \\
\Xi\end{array}$ & $\begin{array}{l}n \\
\hat{\sigma} \\
\underline{6}\end{array}$ & 定 & 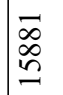 & 奈 & 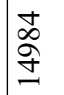 & $\begin{array}{l}\bar{\sigma} \\
\bar{\Xi}\end{array}$ & 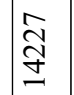 & $\mid \begin{array}{l}\stackrel{2}{2} \\
\stackrel{\infty}{2}\end{array}$ & 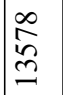 & $\begin{array}{l}\infty \\
\infty \\
\tilde{n}\end{array}$ & $\begin{array}{l}0 \\
\tilde{\delta} \\
\tilde{ల}\end{array}$ \\
\hline లే & రิ & 苫 & $\frac{\hat{\sigma}}{\dot{m}}$ & त̂ & $\begin{array}{l}\infty \\
\infty \\
r \\
r\end{array}$ & $\begin{array}{l}\tilde{\omega} \\
\dot{r}\end{array}$ & 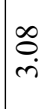 & $\begin{array}{l}\ddot{2} \\
\dot{m}\end{array}$ & $\begin{array}{l}n \\
\dot{n} \\
\dot{m}\end{array}$ & 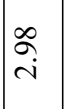 & à & $\frac{n}{m}$ & 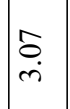 & $\frac{n}{m}$ & $\underset{m}{\vec{m}}$ & $\underset{\dot{r}}{\tilde{r}}$ & $\stackrel{\simeq}{\stackrel{m}{m}}$ & $\frac{\vec{m}}{\dot{m}}$ & $\stackrel{\sim}{n}$ & $\begin{array}{l}\text { त̃ } \\
\text { ri }\end{array}$ & $\tilde{m}$ & $\tilde{\omega}$ \\
\hline $\begin{array}{l} \pm \\
\sigma \\
\frac{\overrightarrow{0}}{\Xi}\end{array}$ & $\mid \begin{array}{l}\infty \\
\infty \\
\infty \\
\varnothing \\
0 \\
0\end{array}$ & $\vec{z}$ & $\begin{array}{l}\dot{v} \\
\tilde{n} \\
\hat{\sim}\end{array}$ & 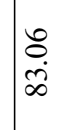 & $\begin{array}{l}\infty \\
\stackrel{\infty}{0} \\
\infty \\
\infty\end{array}$ & 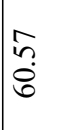 & $\begin{array}{l}0 \\
\infty \\
n \\
n \\
n\end{array}$ & $\begin{array}{l}n \\
\tilde{n} \\
i n\end{array}$ & $\frac{a}{i n}$ & $\frac{m}{8}$ & $\begin{array}{l}\vec{D} \\
\infty \\
\tilde{\sigma}\end{array}$ & $\begin{array}{l}\stackrel{n}{?} \\
\stackrel{f}{F}\end{array}$ & $\left|\begin{array}{l}n \\
0 \\
o \\
+\end{array}\right|$ & $\begin{array}{l}\infty \\
\infty \\
6 \\
\delta \\
+\end{array}$ & $\begin{array}{l}3 \\
\dot{b} \\
\dot{\sigma}\end{array}$ & $\begin{array}{l}8 \\
\dot{+} \\
\dot{7}\end{array}$ & $\mid \begin{array}{l}\dot{J} \\
\dot{J} \\
\dot{J}\end{array}$ & $\begin{array}{l}\stackrel{\vartheta}{\dot{q}} \\
\tilde{q}\end{array}$ & $\mid \begin{array}{l}\stackrel{\vec{r}}{\sigma} \\
\dot{\sigma}\end{array}$ & 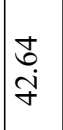 & $\begin{array}{l}\infty \\
\dot{j} \\
\mathcal{f}\end{array}$ & 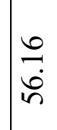 \\
\hline $\begin{array}{l}\stackrel{\mathscr{\Perp}}{ \pm} \\
\frac{\mathbb{\theta}}{\Xi}\end{array}$ & 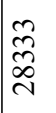 & 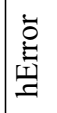 & $\begin{array}{l}n \\
b \\
\dot{m} \\
m\end{array}$ & $\begin{array}{l}\hat{\infty} \\
\dot{n}\end{array}$ & $\stackrel{\vartheta}{+}$ & $\underset{+}{0}$ & $\stackrel{0}{+}$ & $\stackrel{⿱ 亠}{\stackrel{+}{+}}$ & $\underset{n}{\stackrel{7}{n}}$ & in & $\vec{i}$ & $\stackrel{\infty}{\sigma}$ & ర్ & $\vec{m}$ & $\begin{array}{l}\tilde{\sigma} \\
\stackrel{6}{\circ}\end{array}$ & $\stackrel{+}{\stackrel{r}{r}}$ & \begin{tabular}{l}
$\infty$ \\
\multirow{\infty}{\infty}{} \\
$\infty$
\end{tabular} & $\left|\begin{array}{l}\infty \\
\infty \\
\infty\end{array}\right|$ & $\stackrel{ \pm}{a}$ & $\hat{n}$ & $\begin{array}{l}\infty \\
\stackrel{0}{0} \\
0\end{array}$ & $\frac{n}{\sqrt[n]{n}}$ \\
\hline$\underset{\Xi}{\underline{G}}$ & ల్లి & $\underbrace{\stackrel{y}{\Xi}}_{\beth}$ & $\underset{\sim}{\infty}$ & $\begin{array}{l}\hat{\imath} \\
\stackrel{\delta}{0} \\
0\end{array}$ & $\begin{array}{l}\hat{n} \\
\grave{a}\end{array}$ & $\begin{array}{l}\text { ले } \\
\text { से }\end{array}$ & 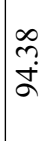 & $\begin{array}{l}\hat{b} \\
\ddot{a}\end{array}$ & $\begin{array}{l}\text { I. } \\
\text { à }\end{array}$ & 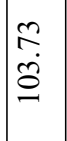 & $\begin{array}{l}\mathbb{N} \\
\stackrel{\Xi}{\Xi}\end{array}$ & $\frac{0}{0}$ & $\begin{array}{l}0 \\
\stackrel{0}{0} \\
\stackrel{j}{J} \\
\exists\end{array}$ & $\begin{array}{l}0 \\
\infty \\
\infty \\
= \\
=\end{array}$ & $\begin{array}{l}\underset{f}{c} \\
\stackrel{\tilde{I}}{\Xi}\end{array}$ & 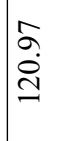 & 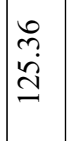 & 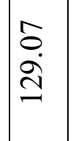 & $\frac{\infty}{\stackrel{\infty}{n}}$ & 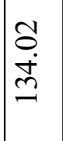 & 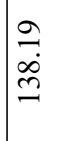 & $\begin{array}{l}6 \\
6 \\
\dot{0} \\
\infty\end{array}$ \\
\hline$\sum_{\text {至 }}$ & $\exists$ & $\begin{array}{l}\text { đิ } \\
\text { है } \\
= \\
=\end{array}$ & $\begin{array}{l}\infty \\
\infty \\
\infty \\
\hat{\infty} \\
\infty \\
\sim\end{array}$ & $\begin{array}{l}\infty \\
i \\
\tilde{N} \\
\hat{d} \\
\text { }\end{array}$ & 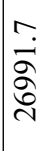 & 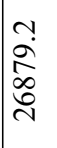 & $\begin{array}{l}\text { i. } \\
\infty \\
\infty \\
\infty \\
i \\
i\end{array}$ & 离 & 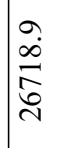 & 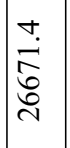 & 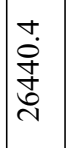 & 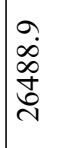 & 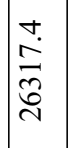 & 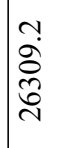 & 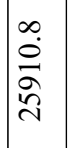 & $\begin{array}{l}\partial े \\
\dot{0} \\
\stackrel{0}{0} \\
i\end{array}$ & $\mid \begin{array}{l}\vec{n} \\
\stackrel{2}{\hat{n}} \\
\tilde{v}\end{array}$ & 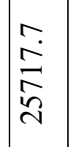 & 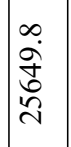 & 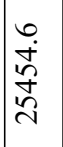 & $\mid \begin{array}{l}+ \\
+ \\
\infty \\
\tilde{n} \\
\sim \\
\sim\end{array}$ & 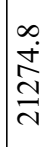 \\
\hline $\begin{array}{l}\sum_{\pi} \\
0 \\
0 \\
0 \\
0\end{array}$ & 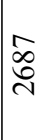 & $\vec{F}$ & $\begin{array}{l}n \\
n \\
n \\
n\end{array}$ & $\begin{array}{l}\hat{\sigma} \\
\dot{a} \\
\tilde{m}\end{array}$ & $\begin{array}{l}\hat{n} \\
\hat{b} \\
\infty \\
n\end{array}$ & $\begin{array}{l}\text { aे } \\
\text { ळे } \\
\text { ले }\end{array}$ & $\begin{array}{l}\dot{J} \\
\infty \\
\ddot{\gamma} \\
\forall\end{array}$ & $\frac{9}{\stackrel{9}{9}}$ & $\begin{array}{l}\hat{\sigma} \\
\text { ț } \\
\text { to }\end{array}$ & 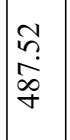 & $\begin{array}{l}n \\
n \\
0 \\
n \\
n\end{array}$ & $\begin{array}{l}m \\
\ddot{n} \\
\tilde{n}\end{array}$ & $\begin{array}{l}\hat{n} \\
n \\
n \\
n\end{array}$ & $\underset{\substack{n \\
i n}}{\stackrel{n}{i n}}$ & $\begin{array}{l}\vec{\infty} \\
\infty \\
\infty \\
\infty \\
n\end{array}$ & $\begin{array}{l}\tilde{\infty} \\
\stackrel{0}{\sigma}\end{array}$ & 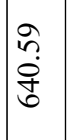 & $\begin{array}{l}\Xi \\
\dot{0} \\
\end{array}$ & $\frac{\bar{n}}{\bar{\infty}}$ & $\frac{\infty}{n}$ & 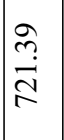 & $\begin{array}{l}\forall \\
\stackrel{2}{\curvearrowright}\end{array}$ \\
\hline 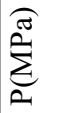 & ñn & 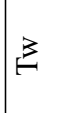 & $\begin{array}{l}\hat{\sigma} \\
\infty \\
\vec{\sigma}\end{array}$ & $\begin{array}{l}2 \\
\dot{\alpha} \\
\tilde{\alpha}\end{array}$ & $\begin{array}{l}\text { ה̃ } \\
\underset{G}{\mathbb{U}}\end{array}$ & $\begin{array}{l}\hat{\imath} \\
i \frac{1}{6} \\
\text { in }\end{array}$ & 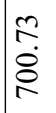 & $\begin{array}{l}\stackrel{2}{2} \\
\stackrel{2}{\gtrless}\end{array}$ & $\begin{array}{l}\infty \\
\infty \\
i \\
i \\
i\end{array}$ & $\mid \begin{array}{l}\hat{6} \\
\dot{j} \\
\stackrel{7}{\pi}\end{array}$ & $\begin{array}{l}\overrightarrow{0} \\
\dot{0} \\
\vec{R}\end{array}$ & $\begin{array}{l}\stackrel{2}{R} \\
\stackrel{i}{i} \\
i\end{array}$ & $\left|\begin{array}{l}\infty \\
\infty \\
\infty \\
\infty \\
\infty\end{array}\right|$ & $\begin{array}{l}\overrightarrow{\hat{y}} \\
\stackrel{2}{\Omega}\end{array}$ & $\left|\begin{array}{l}n \\
\alpha \\
\infty \\
\infty\end{array}\right|$ & $\begin{array}{c}\tilde{N} \\
\widetilde{\infty} \\
\infty\end{array}$ & $\mid$\begin{tabular}{l|}
$\infty$ \\
$\vdots$ \\
0 \\
$\infty$ \\
$\infty$
\end{tabular} & $\begin{array}{l}\vec{f} \\
\dot{0} \\
\dot{D}\end{array}$ & 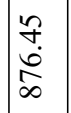 & $\frac{n}{\infty}$ & 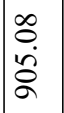 & $\begin{array}{l}\mathcal{Y} \\
\dot{n} \\
\tilde{\infty}\end{array}$ \\
\hline 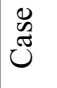 & \& & $\stackrel{9}{9}$ & $\sim$ & $\vec{\infty}$ & $\overline{ \pm}$ & กิ & ֶָ. & $\underset{m}{m}$ & 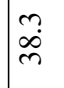 & $\mid \begin{array}{l}\dot{J} \\
\dot{f}\end{array}$ & $\begin{array}{l}t \\
\dot{B}\end{array}$ & in & $\left|\begin{array}{l}n \\
i \\
0\end{array}\right|$ & $\begin{array}{l}n \\
\infty \\
\infty \\
0\end{array}$ & $\mid \begin{array}{l}0 \\
\stackrel{+}{i}\end{array}$ & $\begin{array}{l}0 \\
\dot{\infty} \\
\infty\end{array}$ & $\hat{\infty}$ & $\overline{\check{a}}$ & $\begin{array}{l}\infty \\
\infty \\
\infty\end{array}$ & $\begin{array}{l}\infty \\
\dot{0} \\
\dot{0}\end{array}$ & $\stackrel{\varrho}{\hat{\varrho}}$ & $\hat{\sigma}$ \\
\hline
\end{tabular}




\begin{tabular}{|c|c|c|c|c|c|c|c|c|c|c|c|c|c|c|c|c|c|c|c|c|c|c|}
\hline & & $\vec{z}$ & 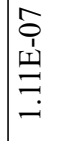 & 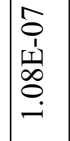 & $\begin{array}{l}\hat{0} \\
1 \\
\underline{1} \\
\underline{\underline{1}}\end{array}$ & 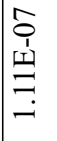 & 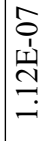 & 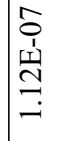 & 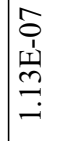 & 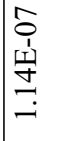 & 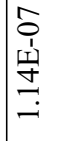 & $\begin{array}{l}\hat{0} \\
\hat{1} \\
\underline{1} \\
\\
\end{array}$ & 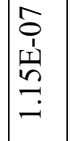 & 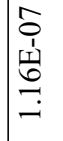 & $\begin{array}{l}0 \\
0 \\
i \\
\\
\\
= \\
=\end{array}$ & 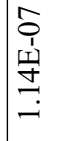 & 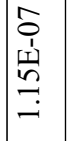 & 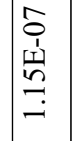 & 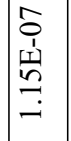 & 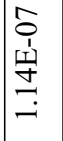 & 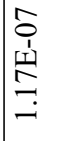 & 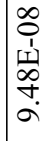 \\
\hline & & $\stackrel{\oplus}{\infty}$ & 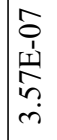 & $\begin{array}{c}\hat{0} \\
\mathbf{1} \\
\\
0 \\
0 \\
\dot{r}\end{array}$ & 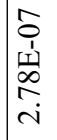 & 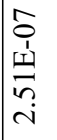 & 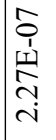 & 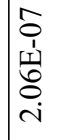 & 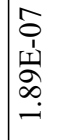 & 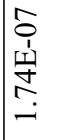 & 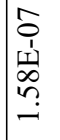 & 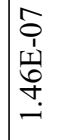 & 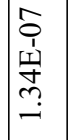 & $\begin{array}{l}\hat{0} \\
\hat{1} \\
\hat{1} \\
\\
\end{array}$ & 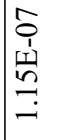 & 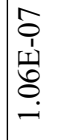 & 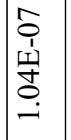 & $\begin{array}{l}\infty \\
0 \\
0 \\
1 \\
0 \\
0 \\
0 \\
\alpha\end{array}$ & $\mid$\begin{tabular}{l}
$\infty$ \\
0 \\
1 \\
\multicolumn{1}{|c}{} \\
2 \\
$\infty$ \\
$\infty$
\end{tabular} & 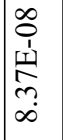 & $\begin{array}{l}\infty \\
0 \\
0 \\
1 \\
1 \\
0 \\
\infty \\
\infty\end{array}$ & $\begin{array}{l}\infty \\
0 \\
1 \\
\underline{1} \\
0 \\
0\end{array}$ \\
\hline & & $\vec{D}$ & 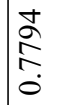 & $\stackrel{\tilde{N}}{\stackrel{\tilde{R}}{\hat{\sigma}}}$ & 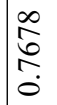 & $\stackrel{0}{0}$ & 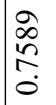 & $\begin{array}{l}\hat{n} \\
\hat{n} \\
\stackrel{0}{0}\end{array}$ & $\stackrel{\widetilde{n}}{\stackrel{\tilde{0}}{0}}$ & $\frac{\vec{a}}{\stackrel{\vec{f}}{\delta}}$ & 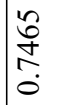 & $\stackrel{\frac{\pi}{5}}{\stackrel{f}{0}}$ & $\mid \begin{array}{l}\tilde{I} \\
\stackrel{Ð}{ث} \\
\dot{0}\end{array}$ & $\frac{\substack{0 \\
\stackrel{0}{0}}}{0}$ & $\begin{array}{l}\infty \\
\infty \\
\stackrel{\infty}{\curvearrowright} \\
\stackrel{0}{0}\end{array}$ & 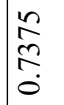 & $\begin{array}{l}\vec{b} \\
\stackrel{2}{0} \\
\dot{0}\end{array}$ & 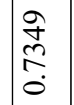 & 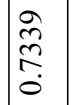 & $\stackrel{\tilde{2}}{\hat{0}}$ & 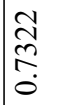 & $\frac{n}{\stackrel{n}{r}}$ \\
\hline & & $\mathscr{J}$ & 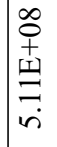 & \begin{tabular}{l}
$\infty$ \\
0 \\
+ \\
\multicolumn{1}{c}{} \\
$\dot{m}$ \\
$\dot{m}$
\end{tabular} & 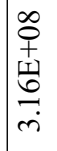 & $\mid \begin{array}{l}\infty \\
0 \\
+ \\
1 \\
\text { Tid } \\
n \\
n \\
i\end{array}$ & 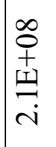 & 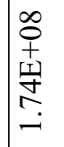 & 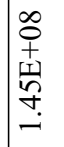 & 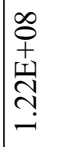 & 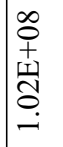 & 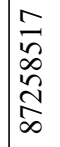 & 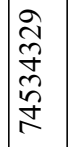 & 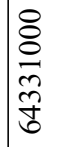 & 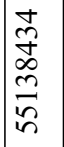 & 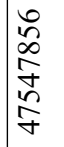 & 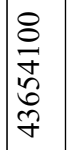 & $\begin{array}{c}\mathcal{N} \\
\mathbb{Z} \\
\mathbb{Z} \\
\mathbb{Z} \\
\infty \\
\sim \\
m\end{array}$ & $\begin{array}{l}\tilde{c} \\
0 \\
0 \\
0 \\
n \\
\tilde{m}\end{array}$ & 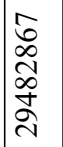 & 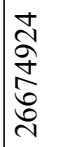 & $\begin{array}{l}\tilde{O} \\
\tilde{O} \\
\tilde{N} \\
\tilde{2}\end{array}$ \\
\hline & & t & 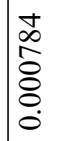 & $\begin{array}{l}0 \\
\tilde{E} \\
\vdots \\
0 \\
0 \\
0\end{array}$ & 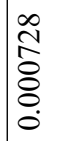 & 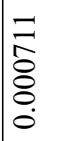 & 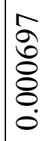 & 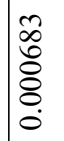 & 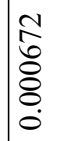 & 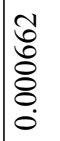 & 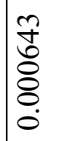 & 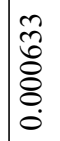 & $\begin{array}{l}\overrightarrow{\widetilde{\sigma}} \\
\delta \\
\delta \\
\dot{\delta}\end{array}$ & 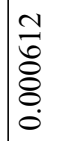 & 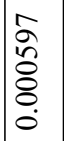 & $\begin{array}{l}\tilde{\infty} \\
\infty \\
o \\
\vdots \\
\vdots \\
0 \\
0\end{array}$ & $\mid \begin{array}{l}0 \\
\vdots \\
\tilde{o} \\
\vdots \\
0 \\
0 \\
0\end{array}$ & $\mid \begin{array}{l}n \\
0 \\
0 \\
0 \\
0 \\
0 \\
0\end{array}$ & 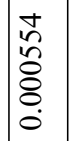 & 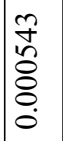 & 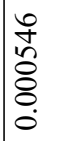 & 竎 \\
\hline & & $\approx$ & $\underset{\substack{\alpha \\
\sim}}{\stackrel{\alpha}{\sim}}$ & 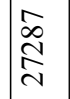 & 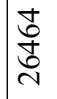 & 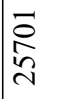 & 志 & 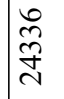 & 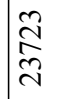 & 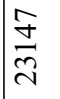 & \begin{tabular}{l}
$\overline{\vec{D}}$ \\
\multirow{\sim}{*}{}
\end{tabular} & 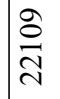 & $\mid \begin{array}{l}\hat{\tilde{b}} \\
\stackrel{\vec{N}}{1}\end{array}$ & $\overrightarrow{\vec{\nabla}}$ & 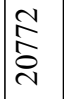 & 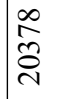 & 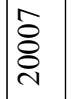 & 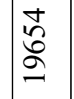 & $\frac{\partial}{2}$ & $\begin{array}{l}\tilde{\delta} \\
\vdots \\
\vdots\end{array}$ & 吕 & 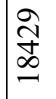 \\
\hline שै & రิ & 苫 & $\begin{array}{l}0 \\
\stackrel{0}{0} \\
i\end{array}$ & $\begin{array}{l}\tilde{D} \\
\stackrel{+}{+}\end{array}$ & $\stackrel{I}{\underset{f}{*}}$ & $\underset{\vec{r}}{\stackrel{\vartheta}{r}}$ & 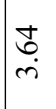 & $\begin{array}{l}\hat{\sigma} \\
m\end{array}$ & $\begin{array}{l}\infty \\
\infty \\
\dot{n}\end{array}$ & $\underset{+}{\sigma}$ & $\begin{array}{l}\vec{\infty} \\
\dot{r}\end{array}$ & $\begin{array}{l}\partial \\
\infty \\
\dot{n}\end{array}$ & $\left|\begin{array}{l}\infty \\
\infty \\
r\end{array}\right|$ & $\begin{array}{l}\infty \\
\infty \\
\infty \\
m\end{array}$ & $\underset{\sim}{\stackrel{N}{r}}$ & $\stackrel{9}{9}$ & $\mid \begin{array}{l}\tilde{c} \\
\tilde{n}\end{array}$ & $\begin{array}{l}\vec{m} \\
\dot{m}\end{array}$ & $\mid \begin{array}{l}n \\
\hat{i}\end{array}$ & $\begin{array}{l}\infty \\
\infty \\
i \\
i\end{array}$ & $\overrightarrow{\widehat{i}}$ & సె. \\
\hline $\begin{array}{l} \pm \\
\sigma \\
\frac{\overrightarrow{0}}{\Xi}\end{array}$ & 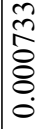 & $\vec{z}$ & $\stackrel{i}{i}$ & $\begin{array}{l}\tilde{n} \\
\tilde{w} \\
\infty\end{array}$ & $\underset{\stackrel{J}{I}}{\stackrel{I}{\mathbb{N}}}$ & m. & 守. & $\begin{array}{l}6 \\
6 \\
6 \\
0\end{array}$ & సે & $\begin{array}{l}\hat{\alpha} \\
\text { bे }\end{array}$ & $\begin{array}{l}\hat{b} \\
\text { bे }\end{array}$ & 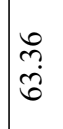 & $\left|\begin{array}{c}\tilde{D} \\
\infty \\
\dot{\sigma}\end{array}\right|$ & : & $\mid \begin{array}{l}\infty \\
0 \\
i \\
\text { in }\end{array}$ & $\stackrel{m}{n}$ & $\mid \begin{array}{l}f \\
\dot{\vec{n}}\end{array}$ & 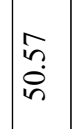 & $\left|\begin{array}{l}a \\
\dot{\sigma} \\
\dot{q}\end{array}\right|$ & $\begin{array}{l}\tilde{o} \\
\dot{\infty} \\
\dot{\phi}\end{array}$ & $\mid \begin{array}{c}\infty \\
\stackrel{\infty}{+} \\
\infty\end{array}$ & 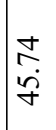 \\
\hline $\begin{array}{l}\stackrel{\mathscr{z}}{\underline{\Delta}} \\
\frac{\vec{\omega}}{\Xi}\end{array}$ & $\begin{array}{l}\infty \\
\sim \\
\infty \\
\sim\end{array}$ & 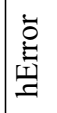 & $\begin{array}{l}\stackrel{\vartheta}{2} \\
\infty \\
\sim\end{array}$ & $\underset{\sigma}{\sigma}$ & $\stackrel{m}{+}$ & $\stackrel{\vec{t}}{\overrightarrow{+}}$ & $\stackrel{n}{\dot{r}}$ & 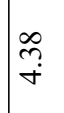 & $\stackrel{\infty}{\stackrel{\infty}{+}}$ & $\begin{array}{l}\text { ñ } \\
\text { in }\end{array}$ & $\begin{array}{l}n \\
\ddot{n}\end{array}$ & $\begin{array}{l}f \\
\dot{v} \\
i n\end{array}$ & 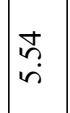 & $\begin{array}{l}\infty \\
0 \\
i \\
i n\end{array}$ & $\begin{array}{l}n \\
n \\
n\end{array}$ & $\begin{array}{l}n \\
\hat{n} \\
\dot{n}\end{array}$ & \begin{tabular}{|}
$\infty$ \\
$\dot{+}$ \\
$\dot{+}$
\end{tabular} & 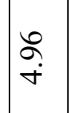 & $\underset{+}{\stackrel{+}{+}}$ & in & $\frac{\partial}{i n}$ & $\stackrel{7}{0}$ \\
\hline$\underset{\Xi}{\stackrel{\widehat{a}}{\Xi}}$ & 防 & $\underbrace{\stackrel{y}{\Xi}}_{\beth}$ & $\begin{array}{l}= \\
\infty \\
\stackrel{\sim}{*}\end{array}$ & $\begin{array}{l}3 \\
\dot{b} \\
2\end{array}$ & ڤั. & $\underset{\infty}{\infty}$ & \begin{tabular}{l}
$\mathbb{J}$ \\
\multirow{\infty}{\infty}{}
\end{tabular} & 官 & $\begin{array}{l}\vec{F} \\
\dot{J}\end{array}$ & $\begin{array}{l}\infty \\
\infty \\
\infty \\
\infty\end{array}$ & $\begin{array}{l}\stackrel{ \pm}{2} \\
\stackrel{2}{a}\end{array}$ & $\begin{array}{l}\infty \\
\stackrel{0}{0} \\
\stackrel{8}{0}\end{array}$ & $\mid \begin{array}{l}0 \\
\stackrel{0}{0} \\
\stackrel{8}{0}\end{array}$ & 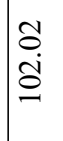 & $\begin{array}{l}+ \\
\stackrel{2}{\alpha}\end{array}$ & $\begin{array}{l}\stackrel{n}{\stackrel{2}{*}} \\
\dot{\alpha}\end{array}$ & $\mid \begin{array}{l}\sigma \\
\tilde{\sigma} \\
\sigma\end{array}$ & $\begin{array}{l}\stackrel{R}{\dot{\Phi}} \\
\dot{\sigma}\end{array}$ & $\begin{array}{l}\stackrel{J}{J} \\
\stackrel{\alpha}{ }\end{array}$ & $\underset{f}{\mathcal{F}}$ & $\frac{2}{8}$ & $\begin{array}{l}\alpha \\
\alpha \\
\alpha\end{array}$ \\
\hline$\sum_{\text {贡 }}$ & $\stackrel{\Upsilon}{\longrightarrow}$ & $\begin{array}{l}\text { đิ } \\
\text { है } \\
= \\
=\end{array}$ & 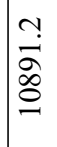 & 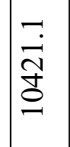 & 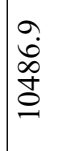 & 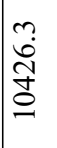 & $\begin{array}{l}\hat{\infty} \\
\infty \\
\infty \\
\hat{\infty}\end{array}$ & 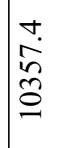 & $\begin{array}{l}\overrightarrow{0} \\
\hat{n} \\
\hat{0}\end{array}$ & 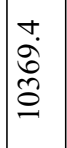 & 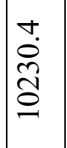 & 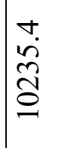 & 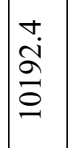 & 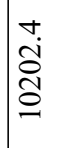 & $\mid \begin{array}{l}n \\
\infty \\
\infty \\
\stackrel{8}{\infty}\end{array}$ & $\begin{array}{l}0 \\
\dot{\infty} \\
2 \\
2\end{array}$ & $\begin{array}{l}0 \\
\stackrel{i}{\alpha} \\
\hat{\alpha}\end{array}$ & $\begin{array}{l}\hat{\sigma} \\
\stackrel{2}{\alpha}\end{array}$ & ஓু & $\begin{array}{l}\hat{\partial} \\
\infty \\
\infty \\
\alpha\end{array}$ & $\overrightarrow{\widehat{\delta}}$ & 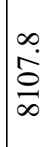 \\
\hline $\begin{array}{l}\sum_{\pi} \\
0 \\
0 \\
0 \\
0\end{array}$ & 㐫 & $\vec{F}$ & $\begin{array}{l}\infty \\
\infty \\
\dot{0} \\
\tilde{e}\end{array}$ & $\begin{array}{l}\mathcal{y} \\
\stackrel{i}{m} \\
m\end{array}$ & $\begin{array}{l}m \\
\sim \\
\sim \\
\tilde{n}\end{array}$ & $\begin{array}{l}0 \\
\stackrel{2}{0} \\
m \\
m\end{array}$ & 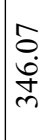 & $\begin{array}{l}8 \\
\varnothing \\
\varnothing \\
m \\
m\end{array}$ & $\begin{array}{l}\hat{\alpha} \\
\hat{0} \\
\text { r. }\end{array}$ & 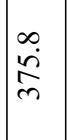 & $\begin{array}{l}q \\
\dot{q} \\
\ddot{\infty} \\
\infty \\
\sim\end{array}$ & $\begin{array}{l}8 \\
\ddot{d} \\
2 \\
\tilde{m}\end{array}$ & $\begin{array}{l}n \\
n \\
\dot{\delta} \\
\dot{q}\end{array}$ & $\mid \begin{array}{l}\sigma \\
\stackrel{\sigma}{\sigma} \\
\sigma\end{array}$ & $\begin{array}{l}\vec{\sim} \\
\ddot{\lambda} \\
\stackrel{+}{+}\end{array}$ & $\begin{array}{c}\tilde{N} \\
\stackrel{\sim}{\gamma} \\
\tilde{F}\end{array}$ & 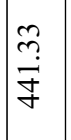 & $\begin{array}{l}\tilde{y} \\
\dot{y} \\
\check{y}\end{array}$ & 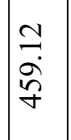 & 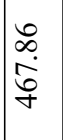 & $\begin{array}{l}\vec{\sigma} \\
\stackrel{0}{0} \\
\vec{\gamma}\end{array}$ & $\begin{array}{l}\stackrel{0}{n} \\
\stackrel{+}{+} \\
\dot{+}\end{array}$ \\
\hline 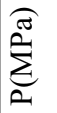 & $\hat{n}$ & 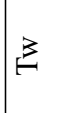 & 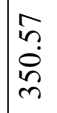 & 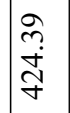 & $\stackrel{\substack{i \\
g}}{\mathfrak{g}}$ & $\begin{array}{l}\infty \\
\infty \\
\dot{\sim} \\
\stackrel{\sigma}{\sigma}\end{array}$ & $\begin{array}{l}\vec{\sigma} \\
\dot{\delta} \\
\dot{q}\end{array}$ & $\begin{array}{l} \pm \\
\underset{\dot{F}}{F}\end{array}$ & $\begin{array}{l}\hat{b} \\
\stackrel{2}{2} \\
\hat{f}\end{array}$ & $\begin{array}{l}0 \\
0 \\
\dot{\infty} \\
+ \\
+\end{array}$ & $\begin{array}{l}\hat{n} \\
\stackrel{2}{8} \\
\stackrel{+}{+}\end{array}$ & $\mid \begin{array}{c}\hat{m} \\
\stackrel{a}{q}\end{array}$ & $\begin{array}{l}\infty \\
0 \\
\dot{0} \\
\tilde{n}\end{array}$ & $\begin{array}{l}\tilde{n} \\
\tilde{n} \\
\tilde{n}\end{array}$ & 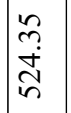 & $\begin{array}{l}\hat{b} \\
\hat{n} \\
\hat{n}\end{array}$ & 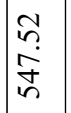 & $\begin{array}{l}n \\
n \\
n \\
n\end{array}$ & $\mid \begin{array}{l}\mathbb{J} \\
\dot{J} \\
\stackrel{D}{n}\end{array}$ & $\frac{\infty}{a}$ & $\begin{array}{l}\vec{\sim} \\
\stackrel{\infty}{\infty} \\
i n\end{array}$ & $\begin{array}{l}\infty \\
\infty \\
\dot{\rho} \\
i n \\
\text { in }\end{array}$ \\
\hline 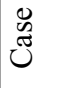 & $\hat{f}$ & $\stackrel{\rho}{a}$ & $\sim$ & $\vec{\infty}$ & $\bar{\Xi}$ & กิ & ֶָ. & $\underset{m}{m}$ & 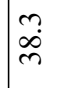 & $\underset{f}{\stackrel{+}{f}}$ & $\begin{array}{l}t \\
\dot{B}\end{array}$ & \begin{tabular}{l}
$n$ \\
\hdashline \\
\hdashline \\
$n$
\end{tabular} & $\left|\begin{array}{l}n \\
i \\
0\end{array}\right|$ & $\begin{array}{l}n \\
\infty \\
\infty \\
0\end{array}$ & $\mid \begin{array}{l}0 \\
\stackrel{+}{i}\end{array}$ & $\begin{array}{l}0 \\
\dot{\infty} \\
\infty\end{array}$ & $\hat{\infty}$ & $\bar{i}$ & $\begin{array}{l}\infty \\
\infty \\
\infty\end{array}$ & $\begin{array}{l}\infty \\
\dot{0} \\
\dot{0}\end{array}$ & $\stackrel{a}{\varrho}$ & $\hat{\sigma}$ \\
\hline
\end{tabular}




\begin{tabular}{|c|c|c|c|c|c|c|c|c|c|c|c|c|c|c|c|c|c|c|c|c|c|c|}
\hline & & $\vec{z}$ & 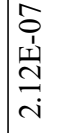 & 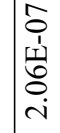 & 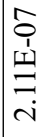 & 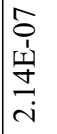 & 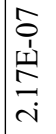 & $\mid \begin{array}{l}\hat{a} \\
\hat{1} \\
\underline{1} \\
\vec{\lambda} \\
\vec{i}\end{array}$ & 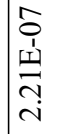 & \begin{tabular}{l}
$\hat{o}$ \\
$\hat{1}$ \\
\multicolumn{1}{c}{} \\
\\
\end{tabular} & 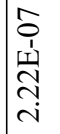 & 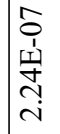 & 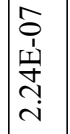 & 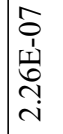 & 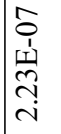 & 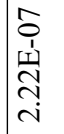 & 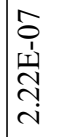 & 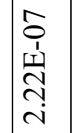 & $\mid \begin{array}{l}\hat{D} \\
1 \\
\underline{1} \\
\tilde{N} \\
\end{array}$ & 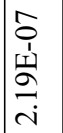 & 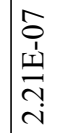 & 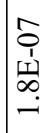 \\
\hline & & $\stackrel{\oplus}{\infty}$ & 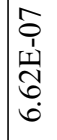 & $\begin{array}{l}\hat{s} \\
1 \\
\underline{1} \\
\bar{n} \\
\dot{n}\end{array}$ & 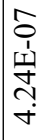 & 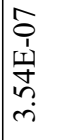 & $\begin{array}{l}\hat{0} \\
\hat{1} \\
\text { 离 }\end{array}$ & $\begin{array}{l}\hat{o} \\
\hat{1} \\
\hat{H} \\
\vec{i}\end{array}$ & 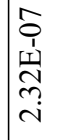 & 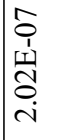 & 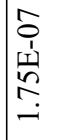 & 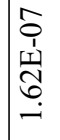 & 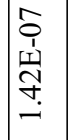 & 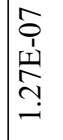 & 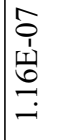 & 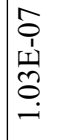 & $\mid \begin{array}{c}\infty \\
0 \\
0 \\
1 \\
1 \\
\infty \\
0 \\
0 \\
\alpha\end{array}$ & $\begin{array}{c}\infty \\
0 \\
0 \\
\mathfrak{1} \\
\mathbb{N} \\
\infty \\
\infty\end{array}$ & $\begin{array}{c}\infty \\
0 \\
0 \\
1 \\
1 \\
0 \\
\infty \\
\infty\end{array}$ & 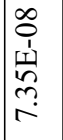 & 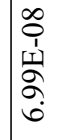 & 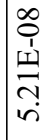 \\
\hline & & $\vec{D}$ & 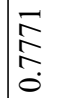 & $\begin{array}{l}8 \\
8 \\
0 \\
0 \\
0\end{array}$ & $\begin{array}{l}\stackrel{0}{\infty} \\
\stackrel{2}{2} \\
\stackrel{0}{0}\end{array}$ & $\mid \begin{array}{r}\vec{\pi} \\
\stackrel{n}{0}\end{array}$ & $\underset{f}{\stackrel{f}{0}}$ & $\mid \begin{array}{l}\infty \\
\stackrel{\mathbb{Z}}{\mathfrak{0}} \\
\dot{0}\end{array}$ & $\stackrel{n}{\stackrel{n}{2}}$ & 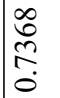 & 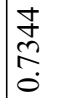 & 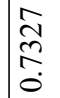 & $\mid \begin{array}{c}\frac{m}{m} \\
\stackrel{2}{0} \\
\dot{0}\end{array}$ & 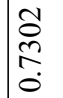 & & 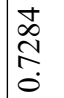 & $\underset{\substack{\hat{N} \\
\stackrel{N}{0}}}{ }$ & $\mid \begin{array}{l}\overrightarrow{\hat{N}} \\
\overrightarrow{0}\end{array}$ & 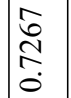 & \begin{tabular}{l}
$\hat{b}$ \\
\multirow{N}{N}{} \\
$\dot{0}$
\end{tabular} & ڤ્仓 & مُ \\
\hline & & $\mathscr{J}$ & \begin{tabular}{|l}
$\infty$ \\
0 \\
1 \\
1 \\
0 \\
0 \\
0 \\
$\infty$ \\
$\infty$
\end{tabular} & $\begin{array}{l}\infty \\
0 \\
+ \\
+ \\
+ \\
\dot{+} \\
\text { n. }\end{array}$ & 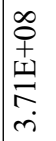 & \begin{tabular}{|l}
$\infty$ \\
0 \\
0 \\
1 \\
0 \\
0 \\
$i$ \\
$i$
\end{tabular} & $\begin{array}{l}\infty \\
0 \\
1 \\
1 \\
\infty \\
\infty \\
\infty \\
-1\end{array}$ & 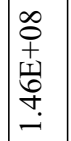 & 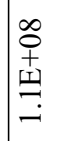 & $\mid \begin{array}{c}\tilde{N} \\
\tilde{\infty} \\
\tilde{N} \\
\infty \\
\infty \\
\infty\end{array}$ & 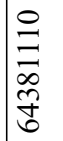 & 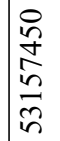 & $\mid \begin{array}{l}\underset{J}{J} \\
\underset{J}{\Xi} \\
\underset{\sim}{\sim}\end{array}$ & 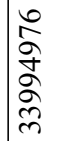 & 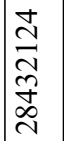 & 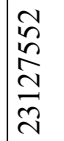 & 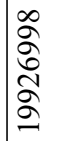 & $\begin{array}{l} \pm \\
\infty \\
\mathbb{Z} \\
\tilde{D} \\
\tilde{\sigma} \\
-\end{array}$ & 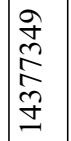 & 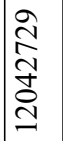 & $\begin{array}{l}\overrightarrow{0} \\
2 \\
\stackrel{0}{\delta} \\
0\end{array}$ & 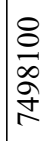 \\
\hline & & t & 萑 & $\begin{array}{l}\tilde{m} \\
\stackrel{3}{8} \\
\stackrel{8}{0} \\
0\end{array}$ & $\begin{array}{l}\hat{\hat{\jmath}} \\
\stackrel{8}{8} \\
\stackrel{0}{0}\end{array}$ & 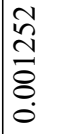 & 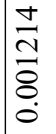 & 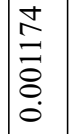 & $\begin{array}{l}\vec{\Xi} \\
\bar{\Xi} \\
\stackrel{\Xi}{0}\end{array}$ & 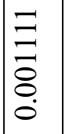 & $\begin{array}{l}0 \\
\stackrel{0}{a} \\
\stackrel{8}{0} \\
0\end{array}$ & 竎 & $\mid \begin{array}{l}m \\
\frac{a}{0} \\
\stackrel{0}{0} \\
0 \\
0\end{array}$ & 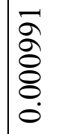 & $\mid \begin{array}{l}n \\
\hat{\alpha} \\
0 \\
0 \\
0 \\
0\end{array}$ & 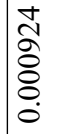 & \begin{tabular}{|l} 
\\
0 \\
0 \\
0 \\
0 \\
0 \\
0
\end{tabular} & $\mid \begin{array}{l}\tilde{D} \\
\infty \\
\infty \\
0 \\
0 \\
0 \\
0\end{array}$ & $\begin{array}{l}0 \\
0 \\
0 \\
0 \\
0 \\
0\end{array}$ & 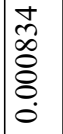 & 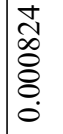 & 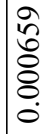 \\
\hline & & $\approx$ & 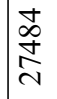 & है & $\begin{array}{l}0 \\
\infty \\
\infty \\
\stackrel{1}{\sim}\end{array}$ & $\underset{\sim}{\stackrel{\vec{f}}{\sim}}$ & 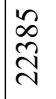 & 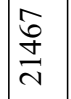 & 守 & 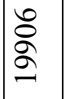 & $\stackrel{\widehat{\approx}}{\widehat{\Omega}}$ & 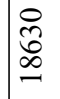 & 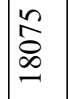 & 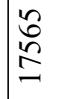 & 总 & $\begin{array}{l}0 \\
: \\
0 \\
0 \\
-\end{array}$ & \begin{tabular}{l}
$\infty$ \\
\multirow{0}{0}{} \\
$\underline{\sigma}$
\end{tabular} & $\begin{array}{l}\hat{a} \\
\hat{\infty} \\
\underline{a}\end{array}$ & $\mid \begin{array}{l}0 \\
n \\
n \\
n\end{array}$ & $\begin{array}{l}\hat{\tilde{N}} \\
\hat{\Omega}\end{array}$ & 离 & 勇 \\
\hline שै & రิ & 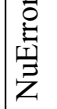 & $\stackrel{\infty}{\sim}$ & $\begin{array}{l}\infty \\
\dot{+}\end{array}$ & $\begin{array}{l}n \\
\infty \\
\dot{m} \\
\dot{n}\end{array}$ & $\begin{array}{l}\text { fr } \\
\dot{m}\end{array}$ & m. & $\begin{array}{l}\vec{F} \\
\dot{m}\end{array}$ & $\frac{n}{m}$ & $\frac{n}{m}$ & $\vec{N}$ & $\stackrel{\sim}{\stackrel{\sim}{m}}$ & $\left|\begin{array}{c}\sigma \\
\tilde{r} \\
\dot{r}\end{array}\right|$ & $\begin{array}{l}n \\
m \\
m\end{array}$ & $\begin{array}{l}\stackrel{2}{2} \\
\text { ì }\end{array}$ & $\underset{\hat{i}}{\tilde{i}}$ & $\begin{array}{l}\vec{\infty} \\
i\end{array}$ & $\left|\begin{array}{l} \pm \\
\infty \\
i\end{array}\right|$ & $\stackrel{+}{\stackrel{\Delta}{i}}$ & $\stackrel{\tilde{i}}{i}$ & $\begin{array}{l}\text { bे } \\
\text { i }\end{array}$ & $\begin{array}{l}\infty \\
\infty \\
\infty \\
r\end{array}$ \\
\hline $\begin{array}{l} \pm \\
\sigma \\
\frac{\overrightarrow{0}}{\Xi}\end{array}$ & $\mid \begin{array}{l}\tilde{n} \\
\stackrel{n}{a} \\
8 \\
0 \\
0\end{array}$ & $\vec{z}$ & 㐫 & $\begin{array}{l}\vec{\sigma} \\
\infty \\
\infty\end{array}$ & $\begin{array}{l}\infty \\
\infty \\
\infty \\
\infty \\
0\end{array}$ & 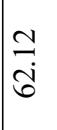 & $\begin{array}{l}n \\
0 \\
\infty \\
n \\
n\end{array}$ & \begin{tabular}{|l}
+ \\
$i n$ \\
$i n$
\end{tabular} & $\begin{array}{l}\hat{y} \\
\dot{H}\end{array}$ & $\begin{array}{l}8 \\
\ddot{n} \\
\hat{n}\end{array}$ & $\begin{array}{l}\text { to } \\
\text { Dे } \\
\text { in }\end{array}$ & $\begin{array}{l}m \\
\tilde{n} \\
\stackrel{n}{n}\end{array}$ & $\mid$\begin{tabular}{l}
0 \\
\hdashline \\
$\dot{g}$
\end{tabular} & $\begin{array}{l}\infty \\
\infty \\
\infty \\
\infty\end{array}$ & $\mid \begin{array}{l}0 \\
\infty \\
\vdots \\
\dot{+}\end{array}$ & $\begin{array}{l}\vec{g} \\
\dot{f}\end{array}$ & $\begin{array}{l}\hat{\sigma} \\
\dot{\gamma}\end{array}$ & 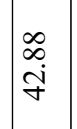 & 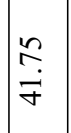 & $\begin{array}{l}\hat{b} \\
\dot{q} \\
+\end{array}$ & 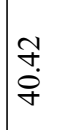 & $\stackrel{n}{\stackrel{n}{\sigma}}$ \\
\hline $\begin{array}{l}\stackrel{\mathscr{z}}{\underline{\Delta}} \\
\frac{\vec{\omega}}{\Xi}\end{array}$ & $\begin{array}{l}n \\
\hat{2} \\
\infty \\
i\end{array}$ & 营 & 永 & 号 & $\vec{m}$ & $\mid \begin{array}{l}\hat{\sigma} \\
\dot{+}\end{array}$ & $\begin{array}{l}\hat{O} \\
\dot{+}\end{array}$ & $\stackrel{\partial}{\vec{F}}$ & $\underset{f}{\stackrel{f}{f}}$ & $\mid \begin{array}{l}\infty \\
\infty \\
+ \\
+\end{array}$ & $\vec{\partial}$ & $\overrightarrow{\vec{n}}$ & $\begin{array}{l}n \\
q \\
i n \\
i n\end{array}$ & $\begin{array}{l}\infty \\
\infty \\
i n\end{array}$ & $\begin{array}{l}\infty \\
\infty \\
n \\
n\end{array}$ & $\vec{i}$ & $\begin{array}{l}0 \\
\infty \\
i n\end{array}$ & 号 & $\overrightarrow{6}$ & $\overrightarrow{\widetilde{\sigma}}$ & 龸 & $\begin{array}{l}n \\
n \\
a\end{array}$ \\
\hline$\underset{\Xi}{\stackrel{\widehat{a}}{\Xi}}$ & $\vec{\rho}$ & $\underbrace{\frac{1}{3}}_{\beth}$ & 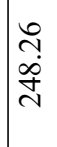 & 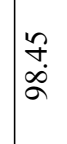 & $\begin{array}{l}\hat{\alpha} \\
\dot{\alpha}\end{array}$ & 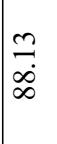 & $\begin{array}{l}2 \\
\frac{1}{\infty}\end{array}$ & $\begin{array}{l}\grave{\hat{~}} \\
\grave{\infty}\end{array}$ & 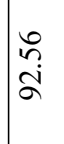 & fُ & 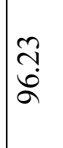 & $\begin{array}{l}\hat{n} \\
\hat{\alpha} \\
2\end{array}$ & $\mid \begin{array}{l}\stackrel{g}{\vec{f}} \\
\stackrel{\vec{\sigma}}{\varrho}\end{array}$ & $\begin{array}{l}\mathscr{2} \\
\dot{0} \\
\dot{0}\end{array}$ & $\begin{array}{l}\hat{n} \\
\dot{0} \\
0\end{array}$ & $\begin{array}{l}\text { } \\
\text { ठิ } \\
\hat{\sigma}\end{array}$ & $\begin{array}{l}\tilde{n} \\
\dot{d} \\
\varrho\end{array}$ & $\begin{array}{l}8 \\
\dot{0} \\
0\end{array}$ & 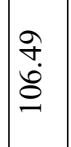 & $\begin{array}{l}\vec{\infty} \\
\dot{\delta} \\
\varrho\end{array}$ & $\frac{n}{2}$ & 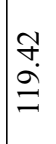 \\
\hline$\sum_{\text {至 }}^{\Sigma}$ & $\cong$ & $\begin{array}{l}\text { đิ } \\
\text { है } \\
= \\
=\end{array}$ & 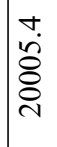 & $\begin{array}{l}\overrightarrow{0} \\
\stackrel{2}{2} \\
\infty \\
0\end{array}$ & $\begin{array}{l}\infty \\
\dot{0} \\
\text { ¿ें }\end{array}$ & 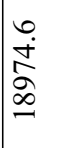 & $\begin{array}{l}\tilde{y} \\
\tilde{N} \\
\alpha \\
- \\
-\end{array}$ & $\mid \begin{array}{c}\vec{i} \\
\infty \\
\infty \\
\infty \\
-1\end{array}$ & $\begin{array}{l}\vec{\sim} \\
\infty \\
\infty \\
\infty\end{array}$ & $\mid \begin{array}{l}\infty \\
0 \\
0 \\
\infty \\
\infty \\
-1\end{array}$ & $\begin{array}{l}a \\
\infty \\
\infty \\
0 \\
\infty \\
-\infty\end{array}$ & 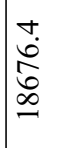 & $\left|\begin{array}{l}n \\
n \\
n \\
n \\
\infty \\
n\end{array}\right|$ & 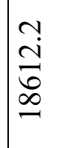 & $\mid \begin{array}{c}\tilde{Z} \\
\tilde{S} \\
\infty \\
\infty \\
-\end{array}$ & $\begin{array}{l}\vec{\infty} \\
\stackrel{\infty}{\infty} \\
\stackrel{\infty}{-}\end{array}$ & $\begin{array}{l}n \\
\bar{\infty} \\
\infty \\
\infty\end{array}$ & $\begin{array}{l}+\dot{n} \\
\sim \\
\infty \\
\infty \\
-\end{array}$ & \begin{tabular}{l}
$m$ \\
\multirow{2}{*}{} \\
$\tilde{O}$ \\
$\infty$ \\
$\infty$
\end{tabular} & $\begin{array}{l}2 \\
\infty \\
\infty \\
=\end{array}$ & $\begin{array}{l}\infty \\
\ddot{\vec{\Xi}} \\
\infty \\
\infty \\
-\end{array}$ & 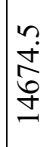 \\
\hline $\begin{array}{l}\sum_{\pi} \\
0 \\
0 \\
0 \\
0\end{array}$ & ఏ & $\vec{F}$ & 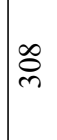 & $\begin{array}{l}\infty \\
n \\
\\
\tilde{n}\end{array}$ & 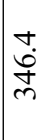 & |r & $\begin{array}{l}\infty \\
\dot{\infty} \\
\infty \\
\infty\end{array}$ & $\begin{array}{l}\text { I } \\
\dot{\dot{d}} \\
\dot{+}\end{array}$ & $\begin{array}{l}\stackrel{n}{\infty} \\
\infty \\
\gamma\end{array}$ & 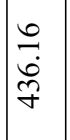 & $\begin{array}{l}\hat{\jmath} \\
\tilde{n} \\
y \\
y\end{array}$ & $\begin{array}{l}\stackrel{8}{0} \\
\stackrel{8}{+} \\
f\end{array}$ & $\begin{array}{l}\dot{b} \\
\dot{0} \\
\stackrel{0}{+}\end{array}$ & $\begin{array}{l}8 \\
\text { के } \\
\hat{\tilde{n}}\end{array}$ & $\begin{array}{l}\hat{\imath} \\
\stackrel{\vec{n}}{n}\end{array}$ & $\begin{array}{l}\dot{d} \\
\dot{w} \\
\tilde{n} \\
i n\end{array}$ & $\begin{array}{l}\text { Co } \\
\stackrel{8}{0} \\
i n\end{array}$ & $\begin{array}{l}8 \\
8 \\
8 \\
0 \\
n\end{array}$ & 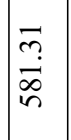 & $\begin{array}{l}\text { nె? } \\
\stackrel{0}{0} \\
\tilde{n}\end{array}$ & $\stackrel{\overbrace{}}{\stackrel{\overbrace{}}{\Xi}}$ & 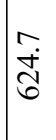 \\
\hline$\sum_{0}^{\pi}$ & กี่ & 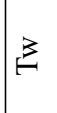 & $\begin{array}{l}\infty \\
\tilde{n} \\
\infty \\
\infty \\
m\end{array}$ & స̃. & $\begin{array}{l}n \\
0 \\
0 \\
n \\
n\end{array}$ & $\begin{array}{l}0 \\
\sim \\
\infty \\
\infty \\
i \\
i n\end{array}$ & $\begin{array}{l}\vec{b} \\
\infty \\
\infty \\
i n\end{array}$ & $\mid \begin{array}{l}\infty \\
\stackrel{+}{a} \\
\stackrel{i}{\sigma}\end{array}$ & $\begin{array}{l}\stackrel{\mathcal{J}}{\mathrm{J}} \\
\text { ปु }\end{array}$ & $\frac{\hat{\sigma}}{\overline{6}}$ & $\begin{array}{l}\hat{0} \\
\text { fo } \\
\end{array}$ & $\underset{\frac{D}{N}}{\stackrel{D}{0}}$ & $\begin{array}{l}\mathfrak{Z} \\
\hat{d} \\
\dot{b}\end{array}$ & $\begin{array}{l}\vec{b} \\
\dot{0} \\
0 \\
0\end{array}$ & $\begin{array}{l}\hat{\infty} \\
\dot{D} \\
\dot{\sigma}\end{array}$ & $\stackrel{n}{\stackrel{n}{ }}$ & 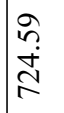 & $\begin{array}{l}n \\
0 \\
\approx \\
\approx\end{array}$ & 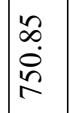 & 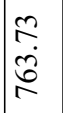 & 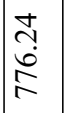 & 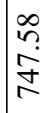 \\
\hline $\begin{array}{l}0 \\
\mathscr{Z} \\
\tilde{z} \\
\tilde{z}\end{array}$ & $\stackrel{\infty}{+}$ & $\stackrel{9}{9}$ & $\sim$ & $\vec{\infty}$ & $\overline{ \pm}$ & ָิ & ֶָ. & $\begin{array}{l}n \\
\tilde{n}\end{array}$ & $\begin{array}{c}m \\
\infty \\
\infty \\
m\end{array}$ & $\mid \begin{array}{l}+ \\
\dot{J}\end{array}$ & $\begin{array}{l}t \\
\dot{8}\end{array}$ & 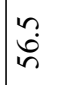 & $\mid \begin{array}{l}n \\
i \\
0\end{array}$ & $\begin{array}{l}n \\
\infty \\
\infty \\
0\end{array}$ & $\begin{array}{l}0 \\
\stackrel{+}{T}\end{array}$ & $\begin{array}{l}0 \\
\dot{0} \\
\infty\end{array}$ & $\hat{\infty}$ & $\hat{a}$ & $\left|\begin{array}{l}\infty \\
\infty \\
\infty\end{array}\right|$ & $\begin{array}{l}\infty \\
\dot{0} \\
\stackrel{0}{0}\end{array}$ & $\stackrel{\varrho}{\grave{O}}$ & \\
\hline
\end{tabular}




\begin{tabular}{|c|c|c|c|c|c|c|c|c|c|c|c|c|c|c|c|c|c|c|c|c|c|c|}
\hline & & $\vec{\Delta}$ & 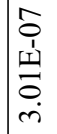 & $\begin{array}{l}\hat{O} \\
\hat{1} \\
1 \\
\infty \\
\infty \\
i \\
i\end{array}$ & 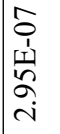 & 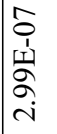 & \begin{tabular}{|l}
$\hat{0}$ \\
$\hat{1}$ \\
\multicolumn{1}{|c}{} \\
$\tilde{\delta}$ \\
$\dot{n}$ \\
$\dot{n}$
\end{tabular} & $\mid \begin{array}{l}\hat{o} \\
1 \\
1 \\
\hat{o} \\
\dot{r} \\
\dot{m}\end{array}$ & \begin{tabular}{|l}
$\hat{0}$ \\
$\hat{1}$ \\
$\underline{1}$ \\
$\ddot{2}$ \\
$\dot{m}$ \\
$\dot{m}$
\end{tabular} & 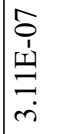 & 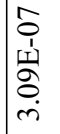 & 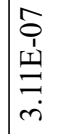 & 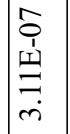 & $\begin{array}{l}\hat{a} \\
\hat{1} \\
\underline{m} \\
\\
\end{array}$ & \begin{tabular}{|l}
$\hat{0}$ \\
$\hat{1}$ \\
$\hat{1}$ \\
$\infty$ \\
0 \\
$\dot{m}$
\end{tabular} & \begin{tabular}{|l}
$\hat{o}$ \\
$\hat{1}$ \\
$\underline{1}$ \\
$\hat{o}$ \\
$\dot{n}$ \\
$\dot{n}$
\end{tabular} & 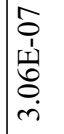 & 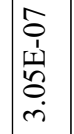 & $\mid \begin{array}{c}\hat{o} \\
1 \\
1 \\
\hat{1} \\
0 \\
\dot{r}\end{array}$ & $\begin{array}{c}\hat{0} \\
\text { 1 } \\
\text { ஸ⿱ }\end{array}$ & 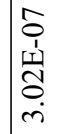 & 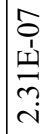 \\
\hline & & $\stackrel{\oplus}{\infty}$ & 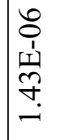 & $\begin{array}{c}0 \\
0 \\
1 \\
1 \\
\infty \\
0 \\
0 \\
-1\end{array}$ & $\begin{array}{l}\hat{y} \\
0 \\
1 \\
\\
\alpha \\
\infty \\
\infty\end{array}$ & 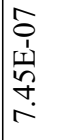 & 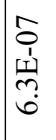 & 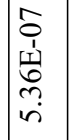 & 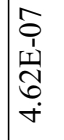 & 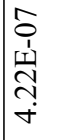 & $\begin{array}{l}\hat{s} \\
\hat{1} \\
1 \\
\hat{1} \\
\hat{\sigma} \\
\dot{n}\end{array}$ & 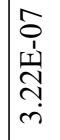 & $\left|\begin{array}{c}\hat{o} \\
\hat{1} \\
\omega \\
\tilde{N} \\
\infty \\
i\end{array}\right|$ & 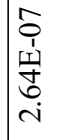 & $\mid \begin{array}{c}\hat{o} \\
\stackrel{1}{\omega} \\
\\
\\
i\end{array}$ & $\begin{array}{c}\hat{s} \\
\hat{1} \\
\underline{1} \\
\mathfrak{c} \\
\vec{i}\end{array}$ & 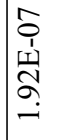 & $\begin{array}{c}\hat{s} \\
\hat{1} \\
1 \\
\tilde{n} \\
\mathfrak{r} \\
-\end{array}$ & 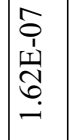 & 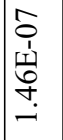 & 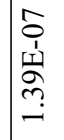 & $\begin{array}{l}\hat{0} \\
0 \\
1 \\
1 \\
0 \\
0 \\
-1\end{array}$ \\
\hline & & $\vec{D}$ & $\begin{array}{l}\infty \\
\infty \\
⿱ 乛 \\
0 \\
0\end{array}$ & $\begin{array}{l}\hat{\tilde{c}} \\
\stackrel{0}{0} \\
\dot{0}\end{array}$ & $\begin{array}{l}\frac{\infty}{2} \\
\hat{n} \\
\stackrel{0}{0}\end{array}$ & $\frac{m}{n}$ & $\frac{\vec{b}}{\stackrel{+}{5}}$ & 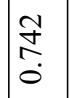 & 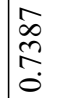 & 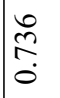 & 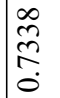 & 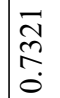 & $\mid \begin{array}{c}\infty \\
0 \\
\stackrel{2}{0} \\
0 \\
0\end{array}$ & $\stackrel{\hat{\mathrm{N}}}{\stackrel{\hat{N}}{0}}$ & $\begin{array}{l}\hat{\infty} \\
\stackrel{N}{N} \\
\stackrel{0}{0}\end{array}$ & 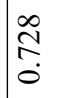 & $\begin{array}{l}\stackrel{+}{\mathbb{N}} \\
\stackrel{0}{\hat{N}}\end{array}$ & 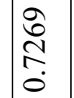 & 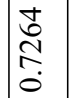 & & 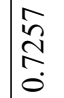 & 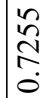 \\
\hline & & $\mathscr{J}$ & 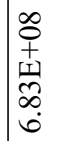 & $\begin{array}{l}\infty \\
+ \\
+ \\
\stackrel{1}{\sim} \\
\underset{+}{+} \\
\dot{+}\end{array}$ & 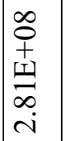 & 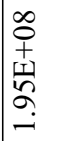 & 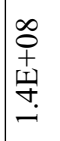 & $\mid \begin{array}{c}\infty \\
0 \\
+ \\
1 \\
\tilde{\omega} \\
\tilde{\sigma} \\
0 \\
-1\end{array}$ & 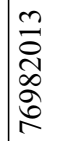 & 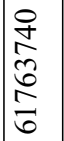 & 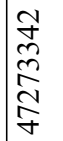 & 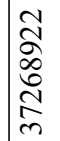 & 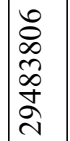 & 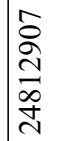 & 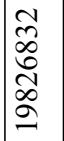 & 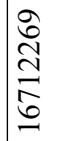 & 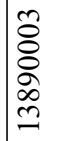 & $\begin{array}{l}\hat{\delta} \\
\tilde{N} \\
\tilde{n} \\
\Xi\end{array}$ & 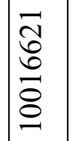 & $\begin{array}{l}\bar{n} \\
\hat{n} \\
\infty \\
\infty \\
\infty\end{array}$ & 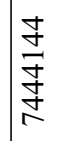 & $\begin{array}{l}\hat{\tilde{n}} \\
\hat{\text { a }} \\
\text { Oें } \\
\text { in }\end{array}$ \\
\hline & & t & 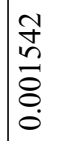 & 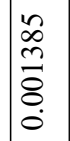 & 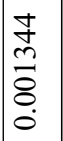 & 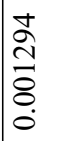 & 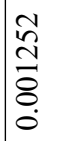 & 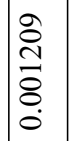 & 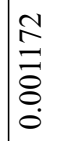 & 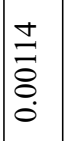 & $\begin{array}{l}n \\
o \\
o \\
o \\
0 \\
0\end{array}$ & 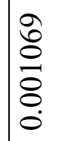 & $\mid \begin{array}{l}n \\
\tilde{0} \\
\tilde{0} \\
\tilde{0} \\
0 \\
0\end{array}$ & $\begin{array}{l}\frac{1}{0} \\
\stackrel{0}{0} \\
\stackrel{0}{0}\end{array}$ & $\begin{array}{l}\hat{\sigma} \\
\dot{8} \\
0 \\
\dot{0}\end{array}$ & 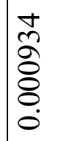 & $\begin{array}{l}0 \\
\\
0 \\
0 \\
0 \\
0 \\
0\end{array}$ & 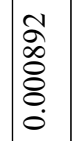 & $\mid \begin{array}{l}\infty \\
0 \\
0 \\
0 \\
0 \\
0 \\
0 \\
0\end{array}$ & 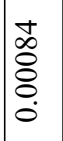 & 會 & | \\
\hline & & $\approx$ & 芳 & 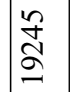 & 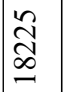 & $\stackrel{\sim}{\approx}$ & $\begin{array}{l}\tilde{2} \\
\tilde{2} \\
\underline{0}\end{array}$ & $\begin{array}{l}\overrightarrow{0} \\
2 \\
0\end{array}$ & $\begin{array}{l}\stackrel{f}{\sim} \\
\end{array}$ & 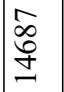 & $\begin{array}{l}\infty \\
\vec{\Xi} \\
\vec{\Xi}\end{array}$ & 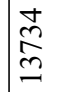 & 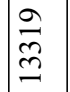 & 웅 & $\underset{\check{a}}{\cong}$ & 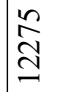 & $\begin{array}{l}\mathscr{O} \\
\stackrel{\Xi}{\Xi}\end{array}$ & $\stackrel{\stackrel{g}{g}}{\equiv}$ & 鴶 & $\stackrel{\Xi}{\Xi}$ & 吉 & $\mid \begin{array}{l}\hat{8} \\
\stackrel{\infty}{0}\end{array}$ \\
\hline تै & ठิ & 苫 & 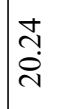 & $\underset{\dot{r}}{\vec{r}}$ & $\begin{array}{l}\stackrel{\bullet}{:} \\
i\end{array}$ & 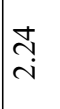 & $\overrightarrow{\mathrm{d}}$ & $\stackrel{n}{\mathscr{z}}$ & $\stackrel{8}{\stackrel{2}{2}}$ & $\stackrel{\infty}{\vec{i}}$ & के & 9 & $\vec{i}$ & $\stackrel{\text { gे }}{2}$ & 9 & $\hat{a}$ & $\stackrel{\infty}{\stackrel{\infty}{=}}$ & $\stackrel{n}{q}$ & $\stackrel{?}{\stackrel{\imath}{i}}$ & 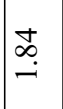 & $\stackrel{N}{\stackrel{N}{-}}$ & $\vec{b}$ \\
\hline $\begin{array}{l}\frac{t}{\sigma} \\
\frac{\overrightarrow{0}}{\Xi}\end{array}$ & 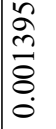 & $\vec{z}$ & $\begin{array}{l}\infty \\
\infty \\
\infty \\
\infty \\
\infty \\
\end{array}$ & 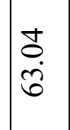 & $\begin{array}{l}\vec{F} \\
i \\
i n\end{array}$ & |c & $\mathscr{F}$ & $\mid \begin{array}{l}0 \\
\infty \\
\dot{q} \\
\end{array}$ & $\begin{array}{l}\infty \\
\infty \\
\infty \\
\infty\end{array}$ & $\begin{array}{l}\infty \\
\tilde{m} \\
\stackrel{m}{m}\end{array}$ & 吕 & $\underset{m}{\stackrel{f}{m}}$ & $\left|\begin{array}{l}n \\
n \\
0 \\
n\end{array}\right|$ & $\begin{array}{l}n \\
n \\
\infty \\
\infty\end{array}$ & $\begin{array}{l}0 \\
\dot{n} \\
n \\
n\end{array}$ & $\underset{m}{m}$ & $\begin{array}{l}\approx \\
\stackrel{n}{\sim} \\
\tilde{n}\end{array}$ & 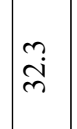 & $\frac{g}{\dot{g}}$ & 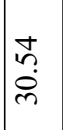 & $\begin{array}{l}\bar{n} \\
0 \\
\tilde{n}\end{array}$ & 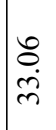 \\
\hline $\begin{array}{l}\stackrel{\mathscr{a}}{\underline{\Delta}} \\
\frac{\vec{\omega}}{\Xi}\end{array}$ & & 营 & $\frac{n}{\sim}$ & ஸि & $\begin{array}{l}n \\
b \\
i \\
i\end{array}$ & 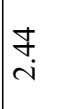 & $\stackrel{\vec{i}}{\mathrm{i}}$ & $\underset{\mathrm{f}}{\mathrm{i}}$ & $\begin{array}{l}\vec{U} \\
\text { i }\end{array}$ & $\mid \begin{array}{l} \pm \\
\infty \\
i\end{array}$ & 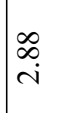 & $\underset{\dot{r}}{\partial}$ & ते & $\tilde{n}$ & $\begin{array}{l}\infty \\
n \\
n\end{array}$ & 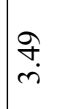 & $\begin{array}{l}\mathbb{U} \\
\stackrel{b}{r} \\
\dot{m}\end{array}$ & $\begin{array}{l}\infty \\
\dot{m} \\
\dot{m}\end{array}$ & $\stackrel{\infty}{\dot{m}}$ & $\begin{array}{l}\infty \\
\infty \\
\dot{m} \\
\dot{m}\end{array}$ & $\underset{+}{\sigma}$ & f. \\
\hline$\underset{\Xi}{\stackrel{\widehat{a}}{\Xi}}$ & ర্ & $\underbrace{\stackrel{y}{\Xi}}_{\beth}$ & $\begin{array}{l}\vec{b} \\
\dot{0} \\
\stackrel{\sim}{N}\end{array}$ & 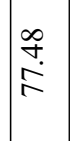 & $\begin{array}{l}\vec{\infty} \\
\text { هे }\end{array}$ & $\vec{\nabla}$ & $\begin{array}{l}2 \\
\hat{\sigma}\end{array}$ & $\overrightarrow{\hat{8}}$ & 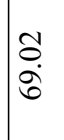 & $\frac{\bar{\sigma}}{\vec{r}}$ & $\frac{\infty}{\stackrel{\infty}{ }}$ & 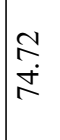 & $\begin{array}{l}0 \\
0 \\
0 \\
0\end{array}$ & $\begin{array}{l}\circ \\
\infty \\
2 \\
2\end{array}$ & $\frac{\partial}{2}$ & 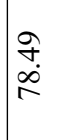 & 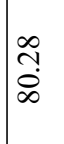 & 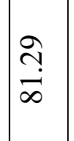 & $\underset{\infty}{\vec{\infty}}$ & $\underset{\infty}{\infty}$ & $\begin{array}{l}\vec{\infty} \\
\dot{\infty}\end{array}$ & $\tilde{\alpha}$ \\
\hline$\sum_{\text {至 }}$ & oे & $\begin{array}{l}\text { đิ } \\
\text { है } \\
= \\
=\end{array}$ & $\mid \begin{array}{l}n \\
\vdots \\
\infty \\
\infty \\
n\end{array}$ & $\begin{array}{l}0 \\
\stackrel{0}{0} \\
\stackrel{2}{ \pm}\end{array}$ & $\begin{array}{l}\stackrel{+}{\dot{\infty}} \\
\stackrel{\infty}{\Phi}\end{array}$ & 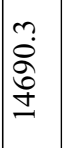 & $\begin{array}{l}\infty \\
0 \\
0 \\
0 \\
\vdots \\
1\end{array}$ & 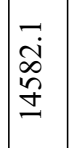 & 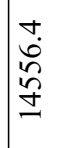 & 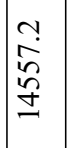 & 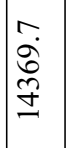 & $\begin{array}{l}2 \\
\partial \\
\tilde{\partial} \\
\tilde{\Xi}\end{array}$ & $\begin{array}{l}n \\
\stackrel{+}{+} \\
\stackrel{\Xi}{ \pm}\end{array}$ & $\begin{array}{l}\tilde{3} \\
\underset{J}{J} \\
\stackrel{\Xi}{\Xi}\end{array}$ & $\mid \begin{array}{l}n \\
0 \\
0 \\
0 \\
0 \\
0 \\
0\end{array}$ & 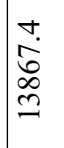 & 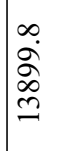 & $\begin{array}{l}0 \\
\infty \\
\infty \\
- \\
-\end{array}$ & $\begin{array}{l}\hat{0} \\
0 \\
0 \\
\tilde{0}\end{array}$ & $\begin{array}{l}n \\
\dot{0} \\
\infty \\
n \\
n \\
n\end{array}$ & 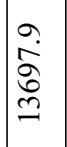 & $\begin{array}{l}\dot{y} \\
\tilde{\hat{\sigma}} \\
\ddot{n} \\
0\end{array}$ \\
\hline $\begin{array}{l}\sum_{\pi} \\
0 \\
0 \\
0 \\
0\end{array}$ & ڤ̊. & $\theta$ & $\begin{array}{l}\infty \\
+ \\
\infty \\
\infty \\
\infty \\
\infty\end{array}$ & $\begin{array}{l}n \\
\stackrel{0}{0} \\
\tilde{n}\end{array}$ & $\begin{array}{l}\vec{b} \\
\dot{\infty} \\
\dot{m}\end{array}$ & 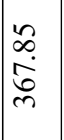 & $\begin{array}{l}\mathbb{N} \\
\dot{0} \\
\infty \\
\infty\end{array}$ & 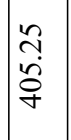 & $\begin{array}{l}\stackrel{f}{+} \\
\stackrel{\vartheta}{F}\end{array}$ & $\mid \frac{g}{\stackrel{f}{f}}$ & 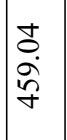 & 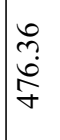 & $\begin{array}{l}\dot{f} \\
\dot{a} \\
\dot{q}\end{array}$ & 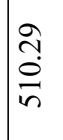 & $\begin{array}{l}\hat{\infty} \\
\dot{0} \\
\tilde{D} \\
\tilde{n}\end{array}$ & 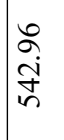 & $\begin{array}{l}\mathbf{1} \\
\infty \\
\infty \\
\infty \\
i n \\
i n\end{array}$ & $\begin{array}{l}\infty \\
n \\
+ \\
i n \\
i n\end{array}$ & $\begin{array}{l}\overrightarrow{0} \\
\text { in }\end{array}$ & $\begin{array}{l}\tilde{n} \\
\ddot{8} \\
\tilde{\delta}\end{array}$ & 六 & $\begin{array}{l}\infty \\
\dot{\gamma} \\
\hat{\sigma}\end{array}$ \\
\hline 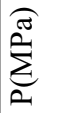 & กี่ & 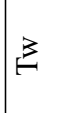 & 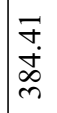 & $\begin{array}{l}\hat{\hat{\lambda}} \\
\stackrel{\vec{n}}{n}\end{array}$ & 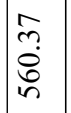 & $\begin{array}{l}n \\
\infty \\
\infty \\
\infty \\
\infty \\
n\end{array}$ & $\begin{array}{l}\stackrel{0}{2} \\
\infty \\
0 \\
0 \\
0\end{array}$ & $\begin{array}{l}n \\
\infty \\
\tilde{ర} \\
\tilde{\delta}\end{array}$ & 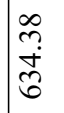 & $\mid \begin{array}{c}\infty \\
\infty \\
\tilde{\sigma}\end{array}$ & $\begin{array}{l}6 \\
\infty \\
0 \\
0\end{array}$ & 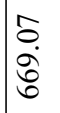 & 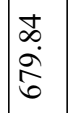 & $\begin{array}{l}n \\
0 \\
\infty \\
0 \\
0\end{array}$ & $\mid \begin{array}{l}\infty \\
\infty \\
i \\
尺 \\
尺\end{array}$ & $\begin{array}{l}\hat{6} \\
2 \\
2\end{array}$ & $\frac{\infty}{a}$ & $\begin{array}{l}\stackrel{2}{Z} \\
\dot{J} \\
\mathbb{I}\end{array}$ & $\begin{array}{l}\vec{n} \\
\infty \\
n \\
n\end{array}$ & $\frac{\hat{\infty}}{\stackrel{i}{N}}$ & $\begin{array}{l}\bar{\alpha} \\
\dot{\infty} \\
\alpha\end{array}$ & $\frac{n}{2}$ \\
\hline $\begin{array}{l}0 \\
\mathscr{Z} \\
\tilde{z} \\
\tilde{z}\end{array}$ & 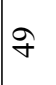 & $\stackrel{9}{9}$ & $\sim$ & $\vec{\infty}$ & $\overrightarrow{ \pm}$ & ָิ & ֶֶ. & $\begin{array}{l}n \\
\tilde{n}\end{array}$ & $\begin{array}{c}m \\
\infty \\
\infty \\
m\end{array}$ & $\mid \begin{array}{l}+ \\
\dot{J}\end{array}$ & $\begin{array}{l}t \\
\dot{8}\end{array}$ & $\begin{array}{l}n \\
0 \\
i \\
n\end{array}$ & $\mid \begin{array}{l}n \\
i \\
0\end{array}$ & $\begin{array}{l}n \\
\infty \\
\infty \\
0\end{array}$ & $\begin{array}{l}0 \\
\stackrel{+}{T}\end{array}$ & $\begin{array}{l}0 \\
\dot{0} \\
\infty\end{array}$ & $\hat{\infty}$ & $\hat{a}$ & $\mid \begin{array}{l}\infty \\
\infty \\
\infty\end{array}$ & $\begin{array}{l}\infty \\
\dot{0} \\
\stackrel{0}{0}\end{array}$ & $\stackrel{\varrho}{\grave{O}}$ & \\
\hline
\end{tabular}




\begin{tabular}{|c|c|c|c|c|c|c|c|c|c|c|c|c|c|c|c|c|c|c|c|c|c|c|}
\hline & & $\vec{\Delta}$ & 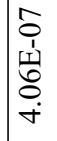 & 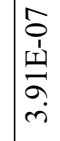 & $\begin{array}{l}\widehat{c} \\
\text { 1) } \\
\text { y. }\end{array}$ & 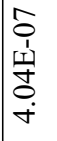 & 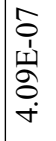 & 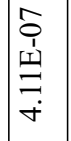 & 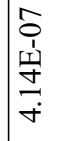 & 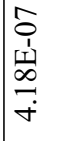 & 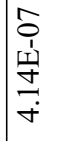 & 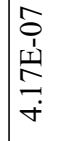 & 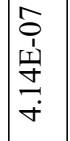 & 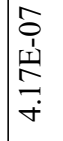 & 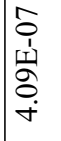 & 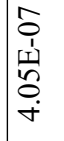 & 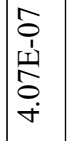 & $\begin{array}{c}\hat{0} \\
\hat{1} \\
1 \\
\hat{1} \\
o \\
\dot{r}\end{array}$ & 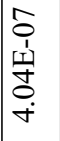 & 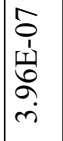 & 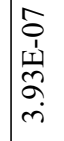 & $\begin{array}{l}\hat{o} \\
\hat{1} \\
\underline{\underline{H}} \\
\dot{m}\end{array}$ \\
\hline & & $\stackrel{\oplus}{\infty}$ & 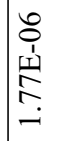 & 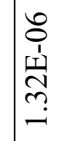 & 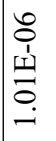 & $\begin{array}{c}\hat{0} \\
\hat{1} \\
\hat{1} \\
\\
0 \\
\infty \\
\infty\end{array}$ & 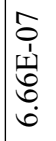 & 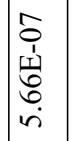 & $\begin{array}{l}\hat{0} \\
\hat{1} \\
\hat{1} \\
\infty \\
\infty \\
\dot{\gamma}\end{array}$ & 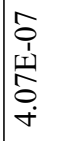 & $\begin{array}{l}\hat{0} \\
\hat{1} \\
\\
\tilde{n} \\
\end{array}$ & 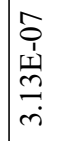 & $\mid \begin{array}{c}\hat{o} \\
\hat{1} \\
\underline{1} \\
\hat{\sigma} \\
\dot{i}\end{array}$ & 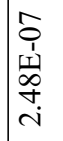 & 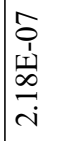 & $\begin{array}{l}\hat{o} \\
\hat{1} \\
\underline{1} \\
\hat{\alpha} \\
\underline{-}\end{array}$ & 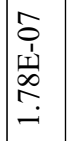 & $\begin{array}{c}\hat{s} \\
\hat{O} \\
1 \\
-1 \\
0 \\
-i\end{array}$ & 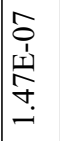 & 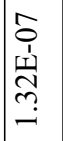 & 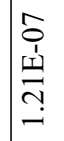 & $\begin{array}{l}\infty \\
0 \\
1 \\
1 \\
\mathbb{1} \\
\infty \\
\infty \\
\infty\end{array}$ \\
\hline & & $\vec{D}$ & 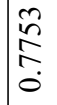 & 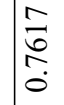 & $\begin{array}{l}\stackrel{̃}{\sim} \\
\stackrel{2}{\sim} \\
0\end{array}$ & 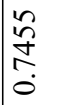 & $\frac{d}{\stackrel{0}{0}}$ & $\mid \begin{array}{l}8 \\
\stackrel{0}{2} \\
0 \\
0\end{array}$ & 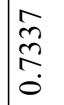 & $\frac{n}{\frac{n}{2}}$ & $\stackrel{\Re}{\stackrel{0}{0}}$ & $\mid \begin{array}{c}\stackrel{\infty}{\infty} \\
\stackrel{N}{N} \\
0\end{array}$ & 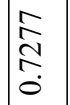 & $\stackrel{\widehat{N}}{\stackrel{\mathbb{0}}{0}}$ & 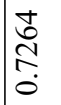 & ڤે & 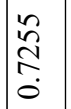 & $\begin{array}{l}\tilde{n} \\
\stackrel{2}{0} \\
\dot{0}\end{array}$ & $\underset{\substack{\stackrel{d}{\mathbb{N}} \\
\stackrel{0}{0}}}{ }$ & 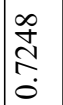 & 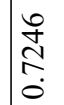 & 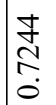 \\
\hline & & $\mathscr{J}$ & 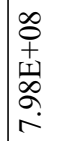 & $\begin{array}{l}\infty \\
0 \\
+ \\
1 \\
0 \\
0 \\
+ \\
\dot{+}\end{array}$ & 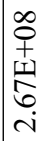 & 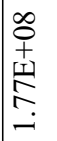 & 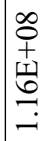 & $\mid \begin{array}{c}m \\
\tilde{m} \\
\tilde{H} \\
\infty \\
\tilde{J} \\
\infty\end{array}$ & 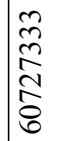 & 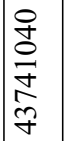 & $\begin{array}{l}0 \\
0 \\
+ \\
\infty \\
o \\
0 \\
m \\
m\end{array}$ & $\mid \begin{array}{l}0 \\
\infty \\
0 \\
0 \\
\infty \\
\end{array}$ & 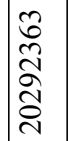 & 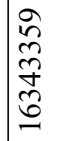 & 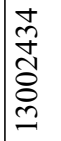 & 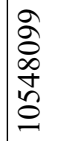 & $\mid \begin{array}{l}\vec{\infty} \\
\infty \\
\infty \\
\infty \\
\infty\end{array}$ & $\begin{array}{l}0 \\
\widetilde{m} \\
\stackrel{\sim}{\sim} \\
\approx\end{array}$ & $\begin{array}{l}\frac{a}{1} \\
\frac{n}{5} \\
\frac{5}{6}\end{array}$ & $\begin{array}{l}\tilde{n} \\
\tilde{\infty} \\
\stackrel{\infty}{\approx} \\
\tilde{n}\end{array}$ & 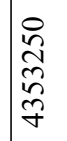 & 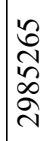 \\
\hline & & t & 㝘 & $\begin{array}{l}0 \\
\stackrel{0}{\infty} \\
\stackrel{0}{0} \\
0 \\
0\end{array}$ & $\begin{array}{l}0 \\
\frac{0}{\Sigma} \\
\frac{8}{0} \\
0 \\
0\end{array}$ & 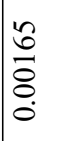 & $\begin{array}{l}\frac{a}{2} \\
\frac{n}{3} \\
8 \\
0 \\
0\end{array}$ & $\begin{array}{l}\vec{n} \\
\bar{\sigma} \\
0 \\
0\end{array}$ & $\begin{array}{l}\overline{5} \\
\frac{5}{8} \\
\vdots \\
0\end{array}$ & 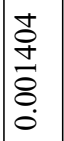 & $\begin{array}{l}\hat{\tilde{m}} \\
\hat{\tilde{z}} \\
\overline{8} \\
0\end{array}$ & बे. & 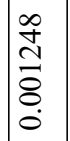 & 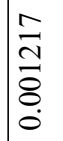 & $\underset{0}{0}$ & $\stackrel{n}{\Xi}$ & 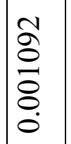 & $\begin{array}{l}\bar{b} \\
\stackrel{0}{0} \\
\dot{0} \\
\dot{0}\end{array}$ & 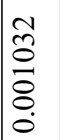 & 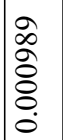 & 离 & 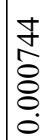 \\
\hline & & $\approx$ & 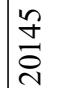 & $\begin{array}{l}\infty \\
\infty \\
\infty \\
\infty\end{array}$ & 壱 & $\underset{\hat{\sigma}}{\widehat{\sigma}}$ & 告 & $\mid \begin{array}{l}\infty \\
0 \\
0 \\
\vdots \\
\end{array}$ & 客 & 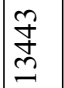 & $\begin{array}{l}\widehat{\widehat{~}} \\
\text { בิ }\end{array}$ & $\begin{array}{l}0 \\
\stackrel{0}{0} \\
\stackrel{1}{I}\end{array}$ & 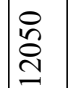 & $\underset{\hat{\sigma}}{=}$ & $\vec{心}$ & 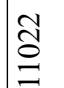 & 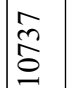 & $\underset{\mathbb{S}}{\mathbb{S}}$ & $\widehat{\widehat{\Xi}}$ & ஜ & 六 & $\frac{\pi}{0}$ \\
\hline שै & రิ & 苫 & 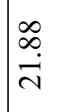 & $\underset{\dot{m}}{F}$ & $\stackrel{\infty}{\stackrel{\infty}{i}}$ & $\stackrel{m}{i}$ & $\stackrel{+}{a}$ & $\stackrel{-}{-}$ & $\stackrel{\infty}{\rightarrow}$ & $\mid \begin{array}{l}\infty \\
\infty \\
\infty \\
-\end{array}$ & $\stackrel{8}{\stackrel{\bullet}{:}}$ & $\stackrel{6}{6}$ & $\mid \begin{array}{l}\infty \\
\stackrel{\infty}{0} \\
-\end{array}$ & $\stackrel{\infty}{\stackrel{\infty}{\imath}}$ & $\stackrel{\vartheta}{\stackrel{2}{-}}$ & $\stackrel{n}{\stackrel{2}{\leftrightarrows}}$ & $\stackrel{શ}{I}$ & $\stackrel{\infty}{\stackrel{尺}{-}}$ & I. & $\mid$\begin{tabular}{l}
0 \\
\hdashline \\
-
\end{tabular} & $\stackrel{N}{\stackrel{ }{Z}}$ & 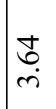 \\
\hline $\begin{array}{l} \pm \\
\sigma \\
\frac{\overrightarrow{0}}{\Xi}\end{array}$ & $\frac{\infty}{\infty}$ & $\vec{z}$ & $\mid \frac{\hat{\alpha}}{\partial}$ & $\begin{array}{l}\stackrel{+}{n} \\
\text { Ç }\end{array}$ & 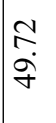 & $\begin{array}{l}\vec{\infty} \\
\dot{f} \\
\mathcal{f}\end{array}$ & $\begin{array}{l}n \\
n \\
\infty \\
m\end{array}$ & $\begin{array}{l}1 \\
\dot{0} \\
\tilde{n}\end{array}$ & $\begin{array}{l}\hat{\partial} \\
\dot{m}\end{array}$ & $\begin{array}{l}\stackrel{i}{1} \\
\stackrel{\text { m}}{m}\end{array}$ & 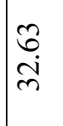 & 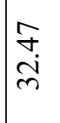 & $\left|\begin{array}{l}8 \\
\vdots \\
i \\
\tilde{n}\end{array}\right|$ & $\begin{array}{l}\vec{F} \\
\text { i }\end{array}$ & $\frac{\tilde{n}}{n}$ & 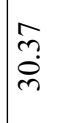 & $\begin{array}{l}n \\
n \\
0 \\
n \\
n\end{array}$ & $\begin{array}{l}\vec{n} \\
\dot{n} \\
\tilde{n}\end{array}$ & $\frac{n}{2}$ & $\begin{array}{l}\text { ते } \\
\text { क्र } \\
\text { in }\end{array}$ & 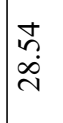 & 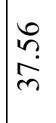 \\
\hline $\begin{array}{l}\stackrel{\mathscr{z}}{\underline{\Delta}} \\
\frac{\vec{\omega}}{\Xi}\end{array}$ & in & 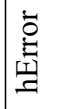 & $\begin{array}{l}\text { సे } \\
\text { సે }\end{array}$ & $\stackrel{\mathcal{F}}{\stackrel{f}{m}}$ & $\begin{array}{l}\infty \\
\stackrel{\infty}{0} \\
i\end{array}$ & $\stackrel{\vec{i}}{i}$ & $\overrightarrow{\tilde{i}}$ & $\stackrel{\sim}{\sim}$ & $\stackrel{\sim}{n}$ & $\stackrel{\mathbb{N}}{i}$ & $\stackrel{\grave{i}}{i}$ & $\underset{\dot{m}}{\Delta}$ & ஸे & $\begin{array}{l}\tilde{\sigma} \\
\dot{r}\end{array}$ & $\stackrel{\hat{i}}{\dot{m}}$ & $\begin{array}{l}\vec{\infty} \\
\dot{m}\end{array}$ & $\stackrel{\nearrow}{\stackrel{7}{+}}$ & 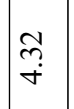 & $\vec{m}$ & $\stackrel{m}{\dot{m}}$ & 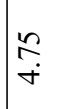 & $\stackrel{\infty}{\varrho}$ \\
\hline$\underset{\Xi}{\stackrel{\widehat{a}}{\Xi}}$ & 防 & 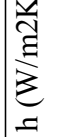 & $\begin{array}{l}\frac{\pi}{N} \\
\frac{N}{2}\end{array}$ & $\begin{array}{l}n \\
\infty \\
\infty \\
2\end{array}$ & $\begin{array}{l}\grave{n} \\
\stackrel{2}{2}\end{array}$ & 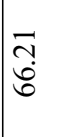 & $\begin{array}{l}\hat{\sigma} \\
\dot{0}\end{array}$ & $\begin{array}{l}n \\
n \\
n \\
0\end{array}$ & $\stackrel{\overbrace{}}{\stackrel{0}{6}}$ & 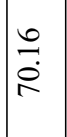 & $\begin{array}{l}\vec{b} \\
\dot{2}\end{array}$ & $\begin{array}{l}\infty \\
\curvearrowright \\
\end{array}$ & $\left|\begin{array}{l}\infty \\
n \\
0 \\
n \\
n\end{array}\right|$ & $\begin{array}{l}n \\
\stackrel{1}{0} \\
\infty\end{array}$ & $\begin{array}{l}\partial \\
\dot{c} \\
i \\
\infty\end{array}$ & 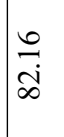 & $\mid \begin{array}{c}\mathbb{N} \\
\infty \\
\infty \\
\infty\end{array}$ & $\underset{\substack{0 \\
\infty \\
\infty}}{0}$ & 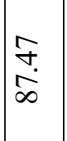 & $\underset{n}{n}$ & $\hat{\otimes}$ & 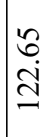 \\
\hline$\sum_{\text {至 }}$ & oे & $\begin{array}{l}\text { đิ } \\
\text { है } \\
= \\
=\end{array}$ & $\underset{\grave{d}}{\stackrel{\nabla}{\grave{~}}}$ & $\begin{array}{l}0 \\
8 \\
0 \\
0 \\
0 \\
0\end{array}$ & 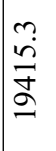 & $\begin{array}{l}\infty \\
\overrightarrow{\vec{a}} \\
\vec{a}\end{array}$ & $\frac{\vec{\pi}}{\frac{\sqrt{n}}{2}}$ & $\mid \begin{array}{l}\hat{\bar{a}} \\
\hat{\alpha} \\
\infty\end{array}$ & 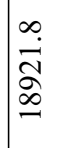 & $\mid \begin{array}{l}\hat{\tilde{N}} \\
\hat{\alpha} \\
\infty \\
\underline{\Delta}\end{array}$ & $\begin{array}{l}n \\
\tilde{r} \\
\hat{\delta} \\
\infty \\
-1 \\
\end{array}$ & 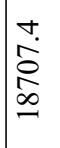 & $\left|\begin{array}{l}\infty \\
\infty \\
0 \\
\tilde{n} \\
\infty \\
-1\end{array}\right|$ & 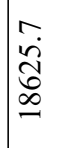 & $\mid \begin{array}{l}\hat{\vec{b}} \\
\tilde{1} \\
\infty \\
-\end{array}$ & $\begin{array}{l}3 \\
\infty \\
\infty \\
\infty \\
\infty \\
\infty\end{array}$ & $\left|\begin{array}{l}n \\
i \\
\tilde{n} \\
\infty \\
-\end{array}\right|$ & $\begin{array}{l}\hat{\vec{i}} \\
\stackrel{\Xi}{\Xi}\end{array}$ & $\mid \begin{array}{c}0 \\
\infty \\
\infty \\
\infty \\
\infty \\
0\end{array}$ & 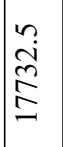 & 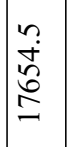 & $\begin{array}{l}\infty \\
\dot{\vec{n}} \\
\text { ભે }\end{array}$ \\
\hline $\begin{array}{l}\sum_{\pi} \\
0 \\
0 \\
0 \\
0\end{array}$ & $\stackrel{2}{2} \cong$ & $\vec{F}$ & $\begin{array}{l}\infty \\
\infty \\
\stackrel{\infty}{m} \\
\text { m. }\end{array}$ & $\begin{array}{l}n \\
\infty \\
\infty \\
m \\
m\end{array}$ & $\begin{array}{l}\infty \\
\infty \\
\infty \\
0 \\
\infty \\
\infty\end{array}$ & $\mid \begin{array}{l}2 \\
\infty \\
\infty \\
\infty \\
\infty \\
\infty\end{array}$ & $\begin{array}{l}\stackrel{\partial}{m} \\
\stackrel{2}{\gamma}\end{array}$ & 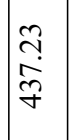 & 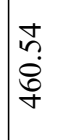 & $\begin{array}{l}\text { 于े } \\
\dot{\infty} \\
\stackrel{\infty}{+}\end{array}$ & 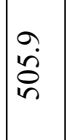 & $\begin{array}{l}\infty \\
\infty \\
\stackrel{\sim}{\sim} \\
\tilde{n}\end{array}$ & $\begin{array}{l}\tilde{n} \\
\tilde{a} \\
\tilde{y}\end{array}$ & $\begin{array}{l}\infty \\
\infty \\
\dot{0} \\
\text { in }\end{array}$ & $\frac{\infty}{\bar{\sigma}}$ & $\frac{a}{\stackrel{i}{\sigma}}$ & $\mid \begin{array}{c}\tilde{n} \\
\tilde{\sigma} \\
\widehat{\sigma}\end{array}$ & $\begin{array}{l}\vec{n} \\
i \\
\tilde{\sigma}\end{array}$ & $\mid \begin{array}{l}\hat{o} \\
\stackrel{i}{\delta}\end{array}$ & $\frac{\mathfrak{n}}{\stackrel{f}{8}}$ & 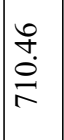 & 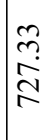 \\
\hline$\sum_{0}^{\pi}$ & ñ & 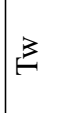 & $\begin{array}{l}n \\
\hat{o} \\
\dot{o} \\
q\end{array}$ & $\begin{array}{l}\infty \\
\dot{\infty} \\
\infty \\
\infty\end{array}$ & 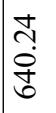 & 寺 & $\begin{array}{l}n \\
\stackrel{2}{2}\end{array}$ & $\mid \begin{array}{l}n \\
\stackrel{n}{0} \\
\infty \\
i \\
i\end{array}$ & I & $\begin{array}{l}\vec{b} \\
\dot{n} \\
i n \\
\end{array}$ & $\begin{array}{l} \pm \\
\stackrel{D}{R} \\
\stackrel{R}{R}\end{array}$ & 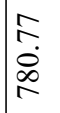 & $\begin{array}{l}\stackrel{2}{a} \\
\overline{2} \\
\end{array}$ & 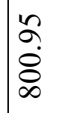 & $\begin{array}{l}\vec{n} \\
\dot{\infty}\end{array}$ & $\begin{array}{l}\infty \\
\infty \\
\dot{\infty} \\
\infty\end{array}$ & $\mid \begin{array}{c}\mathscr{\partial} \\
\dot{\square} \\
\dot{D}\end{array}$ & $\begin{array}{l}\infty \\
\infty \\
\infty \\
\infty \\
\infty\end{array}$ & $\left|\begin{array}{c}n \\
n \\
\infty \\
\infty\end{array}\right|$ & $\begin{array}{l}\stackrel{8}{0} \\
\dot{+} \\
\infty\end{array}$ & 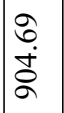 & 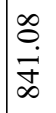 \\
\hline 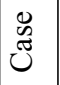 & in & $\stackrel{\rho}{a}$ & $\sim$ & $\vec{\infty}$ & $\ddot{ \pm}$ & ָิ & ֶָ. & $\begin{array}{l}n \\
\tilde{n}\end{array}$ & $\begin{array}{c}m \\
\infty \\
\infty \\
m\end{array}$ & $\mid \begin{array}{l}+ \\
\dot{J}\end{array}$ & $\begin{array}{l}t \\
\dot{8}\end{array}$ & 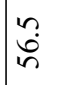 & $\mid \begin{array}{l}n \\
i \\
0\end{array}$ & $\begin{array}{l}n \\
\infty \\
\infty \\
0\end{array}$ & $\stackrel{0}{\stackrel{+}{I}}$ & $\begin{array}{l}0 \\
\dot{0} \\
\infty\end{array}$ & $\begin{array}{l}\hat{\infty} \\
\infty\end{array}$ & $\hat{a}$ & $\left|\begin{array}{l}\infty \\
\infty \\
\infty\end{array}\right|$ & $\begin{array}{l}\infty \\
\dot{0} \\
\stackrel{0}{0}\end{array}$ & $\stackrel{\varrho}{\grave{O}}$ & \\
\hline
\end{tabular}




\begin{tabular}{|c|c|c|c|c|c|c|c|c|c|c|c|c|c|c|c|c|c|c|c|c|c|c|}
\hline & & $\vec{z}$ & 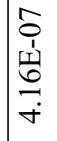 & $\begin{array}{l}\hat{o} \\
\hat{1} \\
1 \\
\approx \\
\alpha \\
\dot{n}\end{array}$ & 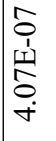 & 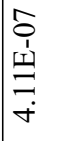 & 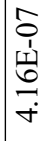 & $\left|\begin{array}{c}\hat{0} \\
1 \\
\underline{1} \\
\infty \\
\dot{+} \\
\dot{+}\end{array}\right|$ & 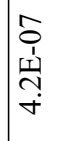 & 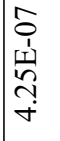 & 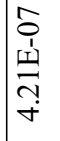 & $\mid \begin{array}{c}\hat{o} \\
\hat{1} \\
\hat{n} \\
\hat{a} \\
\dot{+}\end{array}$ & 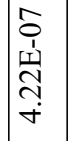 & 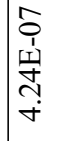 & 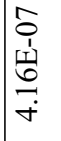 & 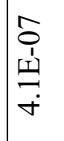 & 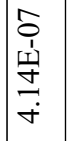 & 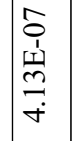 & 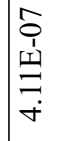 & 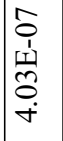 & 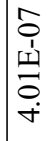 & $\begin{array}{l}\hat{s} \\
\mathbf{1} \\
\text { 됨 } \\
\text { o } \\
\dot{r}\end{array}$ \\
\hline & & $\stackrel{\oplus}{\infty}$ & 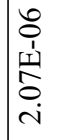 & 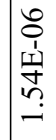 & 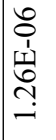 & 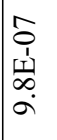 & 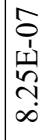 & 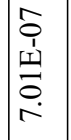 & 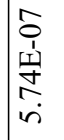 & 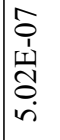 & $\begin{array}{l}\hat{o} \\
\hat{1} \\
\underline{1} \\
\tilde{n} \\
\stackrel{+}{*}\end{array}$ & $\begin{array}{l}\hat{0} \\
\hat{1} \\
\hat{1} \\
\infty \\
\infty \\
\dot{m}\end{array}$ & 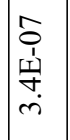 & \begin{tabular}{|l}
$\hat{0}$ \\
$\hat{1}$ \\
$\hat{1}$ \\
$\hat{o}$ \\
$\dot{n}$ \\
$\dot{n}$
\end{tabular} & 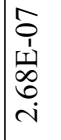 & 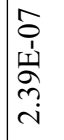 & 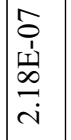 & $\begin{array}{l}\hat{o} \\
\hat{1} \\
\dot{1} \\
0 \\
i \\
i\end{array}$ & 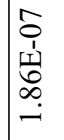 & 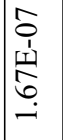 & 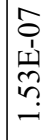 & $\begin{array}{l}\widehat{S} \\
1 \\
\text { II } \\
\text { I } \\
\end{array}$ \\
\hline & & $\vec{D}$ & $\stackrel{\substack{n \\
\stackrel{n}{i} \\
o}}{0}$ & 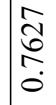 & $\begin{array}{l}0 \\
\tilde{n} \\
\\
0\end{array}$ & $\mid \begin{array}{c}\hat{\sigma} \\
\hat{j} \\
\hat{0}\end{array}$ & 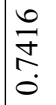 & $\underset{\stackrel{R}{尺}}{\stackrel{R}{0}}$ & 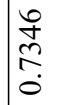 & 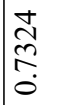 & 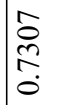 & 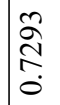 & 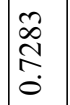 & & $\begin{array}{l}\infty \\
\stackrel{0}{\mathbb{N}} \\
\stackrel{N}{0}\end{array}$ & 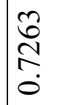 & 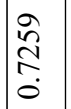 & $\mid \begin{array}{l}n \\
n \\
\\
0 \\
0\end{array}$ & 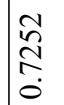 & 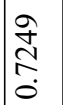 & 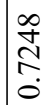 & 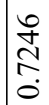 \\
\hline & & $\mathscr{J}$ & 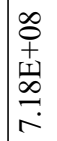 & $\begin{array}{l}\infty \\
0 \\
+ \\
+1 \\
0 \\
0 \\
+ \\
+\end{array}$ & 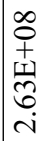 & 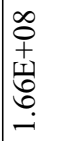 & 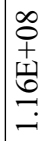 & $\mid \begin{array}{l}\stackrel{P}{D} \\
\tilde{n} \\
\infty \\
\infty \\
\infty \\
\infty \\
\infty\end{array}$ & $\begin{array}{l}\underset{1}{\infty} \\
\mathbb{N} \\
\underset{\sim}{\infty} \\
\infty \\
i\end{array}$ & 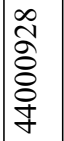 & $\begin{array}{l}\vec{b} \\
\overrightarrow{0} \\
\vec{n} \\
\infty \\
m\end{array}$ & 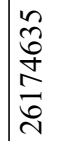 & $\begin{array}{l}\hat{\sigma} \\
\hat{\sigma} \\
\tilde{n} \\
\hat{\tilde{\nu}}\end{array}$ & 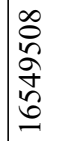 & 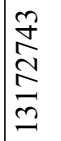 & $\begin{array}{l}\infty \\
\stackrel{\tilde{\gamma}}{+} \\
\stackrel{\Xi}{\Xi}\end{array}$ & 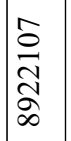 & 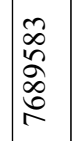 & 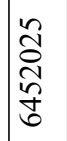 & 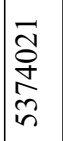 & 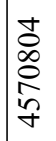 & $\begin{array}{l}\infty \\
\widetilde{్} \\
\tilde{0} \\
\\
m\end{array}$ \\
\hline & & t & 竎 & $\frac{0}{8}$ & $\begin{array}{l}\hat{f} \\
\frac{f}{8} \\
0 \\
0 \\
0\end{array}$ & 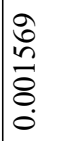 & 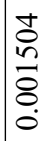 & $\mid \begin{array}{l}\vec{y} \\
\dot{8} \\
\dot{0}\end{array}$ & 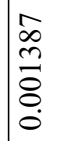 & 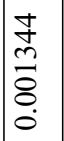 & 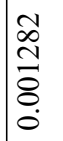 & 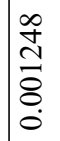 & 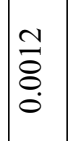 & 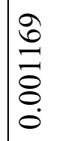 & 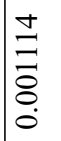 & $\frac{0}{0}$ & $\frac{n}{0}$ & 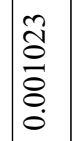 & 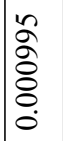 & 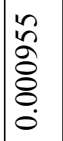 & 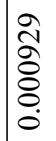 & 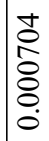 \\
\hline & & $\approx$ & $\begin{array}{l}\stackrel{8}{0} \\
\infty \\
\infty\end{array}$ & 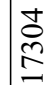 & 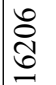 & $\begin{array}{l}\stackrel{a}{\hat{N}} \\
\hat{n}\end{array}$ & \begin{tabular}{l}
$n$ \\
$\infty$ \\
\multirow{J}{*}{}
\end{tabular} & $\begin{array}{l}2 \\
2 \\
2 \\
\end{array}$ & 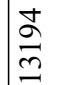 & $\begin{array}{l}8 \\
\mathbb{0} \\
\text { I }\end{array}$ & $\begin{array}{l}\infty \\
\stackrel{\infty}{\beth}\end{array}$ & $\overrightarrow{0}$ & $\underset{\stackrel{\hat{~}}{=}}{=}$ & $\underset{\substack{\infty \\
ٍ}}{=}$ & $\overrightarrow{\overrightarrow{\widehat{O}}}$ & 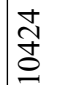 & $\stackrel{8}{8}$ & $\frac{n}{2}$ & $\mid \begin{array}{l}n \\
0 \\
0 \\
o\end{array}$ & $\frac{\Re}{f}$ & $\stackrel{\widehat{\Delta}}{\widehat{\alpha}}$ & $\underset{\sigma}{\vec{z}}$ \\
\hline שै & రీ & 章 & के & ì & $\stackrel{\substack{+i}}{\stackrel{+}{i}}$ & $\stackrel{\infty}{\infty}$ & $\stackrel{\infty}{\stackrel{n}{\longrightarrow}}$ & nृ & 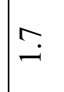 & in & 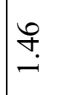 & 总 & f & $\stackrel{\sim}{n}$ & $\stackrel{n}{n}$ & $\stackrel{n}{n}$ & $\underset{-}{\sigma}$ & 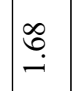 & $\stackrel{*}{n}$ & $\stackrel{f}{\stackrel{J}{-}}$ & $\vec{n}$ & $\vec{\sigma}$. \\
\hline $\begin{array}{l} \pm \\
\sigma \\
\frac{\overrightarrow{0}}{\Xi}\end{array}$ & $\begin{array}{l}\vec{n} \\
\stackrel{2}{8} \\
\vdots \\
0\end{array}$ & $\vec{z}$ & $\stackrel{0}{2}$ & $\begin{array}{l}0 \\
\infty \\
\infty \\
\infty \\
i n\end{array}$ & $\begin{array}{l}0 \\
\dot{\sigma} \\
\dot{+}\end{array}$ & 尚 & $\underset{i}{\stackrel{亠}{n}}$ & $\mid \begin{array}{l}\tilde{f} \\
\tilde{m} \\
\tilde{m}\end{array}$ & $\begin{array}{l}\stackrel{m}{\vec{i}} \\
\stackrel{n}{m}\end{array}$ & $\frac{\infty}{n}$ & $\overrightarrow{\dot{m}}$ & $\begin{array}{l}0 \\
0 \\
\dot{0}\end{array}$ & 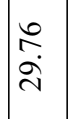 & 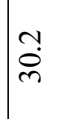 & 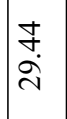 & $\begin{array}{l}\hat{n} \\
\infty \\
\infty\end{array}$ & $\begin{array}{l}\hat{n} \\
\infty \\
\infty\end{array}$ & $\begin{array}{l}\vec{i} \\
\infty \\
i\end{array} \mid$ & 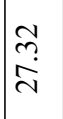 & f̧. & $\hat{\oplus}$ & $\begin{array}{l}\stackrel{n}{r} \\
\dot{m}\end{array}$ \\
\hline 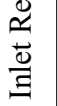 & 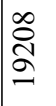 & 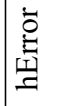 & 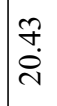 & $\underset{\tilde{r}}{\tilde{r}}$ & ָై & $\hat{i}$ & ta & $\hat{\mathrm{g}}$ & $\underset{i}{\stackrel{\infty}{c}}$ & 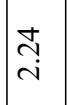 & $\stackrel{m}{i}$ & $\vec{n}$ & $\vec{i}$ & $\hat{\sigma}$ & $\frac{\bar{m}}{m}$ & $\overrightarrow{\widetilde{n}}$ & $\stackrel{\infty}{\stackrel{\infty}{\sim}}$ & $\begin{array}{l}\vec{b} \\
\dot{m}\end{array}$ & $\begin{array}{l}\vec{b} \\
\dot{m}\end{array}$ & 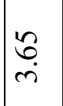 & $\hat{\hat{m}}$ & $\mid \begin{array}{l}\infty \\
n \\
\infty\end{array}$ \\
\hline$\underset{\Xi}{\stackrel{\widehat{a}}{\Xi}}$ & D্ & 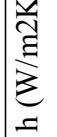 & 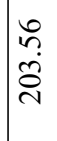 & $\begin{array}{l}\hat{n} \\
\stackrel{+}{T}\end{array}$ & 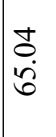 & $\mid \begin{array}{l}\vec{\infty} \\
\dot{8}\end{array}$ & $\begin{array}{l}\vec{\infty} \\
\infty \\
\infty\end{array}$ & $\mid \begin{array}{l}0 \\
0 \\
\text { in }\end{array}$ & $\begin{array}{l}\bar{n} \\
\tilde{8}\end{array}$ & $\begin{array}{l}\partial \\
\text { ते }\end{array}$ & $\begin{array}{l}\text { वे. } \\
\text { î. }\end{array}$ & $\begin{array}{l}\infty \\
0 \\
0 \\
8\end{array}$ & $\frac{6}{8}$ & $\vec{m}$ & $\begin{array}{l}\stackrel{J}{+} \\
\stackrel{+}{+}\end{array}$ & 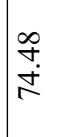 & $\stackrel{\infty}{\stackrel{\infty}{i}}$ & $\begin{array}{l}3 \\
2 \\
2\end{array}$ & Iั. & के. & $\mid \begin{array}{c}n \\
\\
\infty \\
\infty\end{array}$ & $\begin{array}{l}0 \\
\dot{b} \\
0 \\
0\end{array}$ \\
\hline 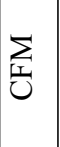 & $\stackrel{\infty}{\infty}$ & $\begin{array}{l}\text { đิ } \\
\text { है } \\
= \\
=\end{array}$ & 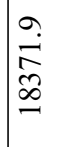 & ָ̃ & $\begin{array}{l}8 \\
\text { ठ․ } \\
-\end{array}$ & $\mid \begin{array}{l}2 \\
\stackrel{2}{a} \\
\hat{\sigma} \\
-1\end{array}$ & $\frac{7}{6}$ & $\mid \begin{array}{l}\infty \\
2 \\
2 \\
\hat{2} \\
0 \\
0\end{array}$ & $\frac{\infty}{2}$ & $\begin{array}{l}= \\
\bar{n} \\
\tilde{\sigma} \\
-\end{array}$ & \begin{tabular}{l}
$n$ \\
$\infty$ \\
\multirow{2}{\sigma}{} \\
-
\end{tabular} & $\begin{array}{l}\hat{\infty} \\
\infty \\
\hat{\infty} \\
\hat{\sigma}\end{array}$ & $\mid \begin{array}{l}n \\
\infty \\
0 \\
0 \\
0\end{array}$ & 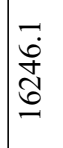 & $\begin{array}{l}\tilde{y} \\
\dot{ \pm} \\
\tilde{n}\end{array}$ & $\begin{array}{l}\infty \\
\infty \\
\infty \\
0 \\
0 \\
n \\
n\end{array}$ & $\begin{array}{l}+ \\
\tilde{O} \\
\infty \\
\stackrel{\infty}{n}\end{array}$ & 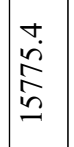 & 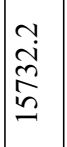 & $\begin{array}{l}\hat{n} \\
\stackrel{n}{n} \\
\stackrel{a}{n} \\
2\end{array}$ & 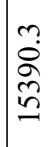 & $\begin{array}{l}\stackrel{\sim}{+} \\
\stackrel{\infty}{=}\end{array}$ \\
\hline 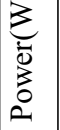 & $\stackrel{9}{I}$ & $\vec{F}$ & 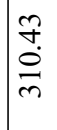 & 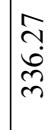 & 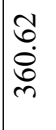 & 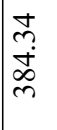 & 㝒 & 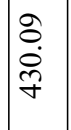 & $\begin{array}{l}\vec{N} \\
\tilde{i} \\
\vartheta\end{array}$ & $\begin{array}{l}\stackrel{0}{2} \\
\stackrel{2}{\gamma}\end{array}$ & $\begin{array}{l}\hat{i} \\
\tilde{a} \\
\hat{q}\end{array}$ & $\frac{5}{\overrightarrow{0}}$ & $\begin{array}{l}n \\
\tilde{0} \\
\tilde{0} \\
n\end{array}$ & $\begin{array}{l}\overrightarrow{0} \\
\hat{n} \\
i n\end{array}$ & $\begin{array}{l}2 \\
2 \\
0 \\
\hat{0} \\
i n\end{array}$ & $\begin{array}{l}\overrightarrow{\hat{n}} \\
\stackrel{2}{ } \\
i n\end{array}$ & 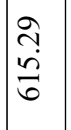 & 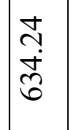 & $\begin{array}{l}\infty \\
\vdots \\
i \\
\hat{\sigma} \\
0\end{array}$ & 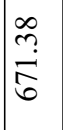 & $\begin{array}{l}\forall \\
\stackrel{8}{\circ} \\
\dot{0}\end{array}$ & $\vec{n}$ \\
\hline$\sum_{0}^{\pi}$ & ñ & 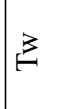 & $\mid \begin{array}{l}9 \\
\dot{0} \\
\dot{8} \\
+\end{array}$ & 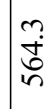 & 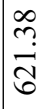 & \begin{tabular}{l}
$\infty$ \\
\\
\hdashline \\
0 \\
0
\end{tabular} & $\frac{\mathfrak{i}}{\dot{a}}$ & $\stackrel{\sim}{\stackrel{2}{\gtrless}}$ & 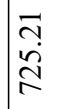 & 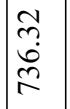 & $\stackrel{\infty}{\stackrel{一}{n}}$ & $\frac{n}{0}$ & 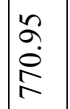 & $\begin{array}{l}\mathbb{W} \\
\infty \\
\infty \\
尺\end{array}$ & $\begin{array}{l}2 \\
\dot{2} \\
\grave{2}\end{array}$ & $\begin{array}{l}\tilde{D} \\
\infty \\
\varnothing \\
\infty \\
\infty\end{array}$ & $\mid \begin{array}{c}\infty \\
+ \\
\infty \\
\infty\end{array}$ & $\begin{array}{l}\tilde{n} \\
\tilde{R} \\
\tilde{N} \\
\infty\end{array}$ & $\mid \begin{array}{l}\hat{\sigma} \\
\dot{\vec{x}} \\
\dot{\infty}\end{array}$ & 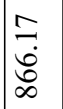 & 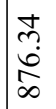 & 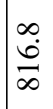 \\
\hline $\begin{array}{l}\mathscr{y} \\
\tilde{z} \\
\tilde{z}\end{array}$ & $\vec{n}$ & $\stackrel{\rho}{a}$ & 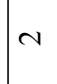 & $\vec{\infty}$ & $\overline{ \pm}$ & กิ & ֶָ. & in & 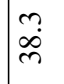 & $\mid \begin{array}{l}\dot{J} \\
\dot{f}\end{array}$ & $\begin{array}{l}t \\
\dot{B}\end{array}$ & in & $\left|\begin{array}{l}n \\
i \\
0\end{array}\right|$ & $\begin{array}{l}n \\
\infty \\
\infty \\
0\end{array}$ & $\begin{array}{l}0 \\
\stackrel{+}{i}\end{array}$ & $\begin{array}{l}0 \\
\dot{\infty} \\
\infty\end{array}$ & $\hat{\infty}$ & $\overline{\check{a}}$ & $\mid \begin{array}{l}\infty \\
\infty \\
\infty \\
\infty\end{array}$ & $\begin{array}{l}\infty \\
\dot{0} \\
\dot{0}\end{array}$ & 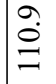 & $\hat{\sigma}$ \\
\hline
\end{tabular}




\begin{tabular}{|c|c|c|c|c|c|c|c|c|c|c|c|c|c|c|c|c|c|c|c|c|c|c|}
\hline & & $\vec{z}$ & 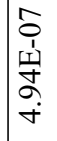 & $\begin{array}{l}\hat{o} \\
\hat{1} \\
\underline{1} \\
\hat{b} \\
\dot{+}\end{array}$ & 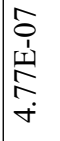 & $\begin{array}{l}\hat{0} \\
\hat{1} \\
\underline{1} \\
\infty \\
\dot{\gamma}\end{array}$ & 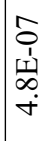 & 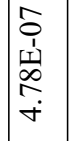 & 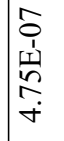 & 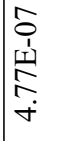 & 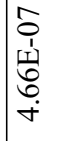 & $\begin{array}{c}\hat{O} \\
\hat{1} \\
\mathfrak{d} \\
\mathbb{N} \\
\dot{+}\end{array}$ & $\left|\begin{array}{c}\hat{o} \\
\dot{1} \\
\stackrel{H}{+} \\
\dot{+}\end{array}\right|$ & 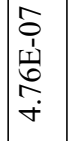 & 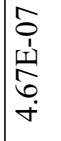 & 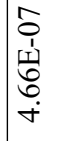 & 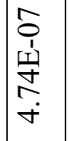 & 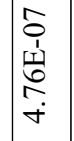 & $\mid \begin{array}{l}\hat{0} \\
\hat{1} \\
\text { to } \\
\stackrel{0}{0} \\
\stackrel{+}{+}\end{array}$ & 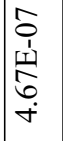 & 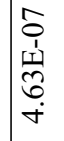 & 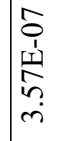 \\
\hline & & $\stackrel{\oplus}{\infty}$ & 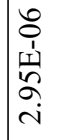 & 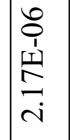 & 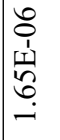 & 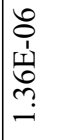 & 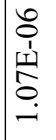 & 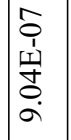 & 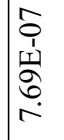 & 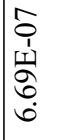 & $\begin{array}{l}\hat{s} \\
\hat{1} \\
\stackrel{1}{N} \\
\hat{N} \\
\text { in }\end{array}$ & 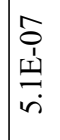 & $\begin{array}{l}\hat{o} \\
\hat{1} \\
\underline{1} \\
\mathfrak{r} \\
r\end{array}$ & $\begin{array}{l}\hat{0} \\
\hat{1} \\
\mathbf{1} \\
0 \\
\dot{+} \\
\dot{+}\end{array}$ & 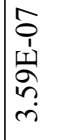 & $\begin{array}{l}\hat{s} \\
\hat{1} \\
\underline{1} \\
\hat{n} \\
\end{array}$ & 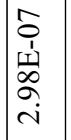 & $\begin{array}{c}\hat{s} \\
\hat{i} \\
\underline{1} \\
\mathbb{N} \\
i \\
i\end{array}$ & 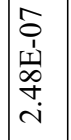 & 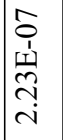 & 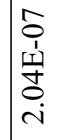 & 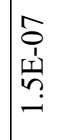 \\
\hline & & $\vec{D}$ & 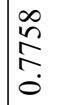 & $\begin{array}{l}\mathfrak{S} \\
\stackrel{0}{0}\end{array}$ & $\begin{array}{l}\hat{\tilde{N}} \\
\stackrel{n}{0} \\
0\end{array}$ & $\frac{\sqrt{n}}{\stackrel{y}{i}}$ & $\frac{1}{1}$ & $\mid \begin{array}{l}\hat{D} \\
\stackrel{2}{0} \\
0\end{array}$ & $\stackrel{t}{\stackrel{0}{0}}$ & $\frac{\infty}{\stackrel{\infty}{n}}$ & 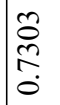 & 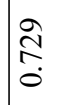 & 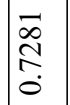 & $\mid \begin{array}{l}\tilde{\Xi} \\
\stackrel{\Xi}{\sigma}\end{array}$ & 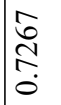 & $\begin{array}{l}\overrightarrow{0} \\
\stackrel{\mathbb{N}}{0} \\
\dot{0}\end{array}$ & 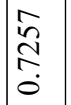 & 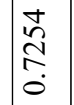 & 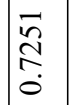 & 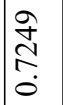 & 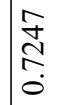 & 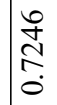 \\
\hline & & $\mathscr{J}$ & 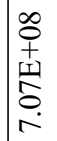 & $\begin{array}{l}\infty \\
0 \\
+ \\
\underline{1} \\
\infty \\
\infty \\
\dot{r}\end{array}$ & 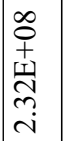 & 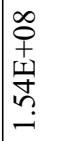 & $\begin{array}{l}\infty \\
0 \\
⿱ 亠 \\
\pm=1 \\
=\end{array}$ & 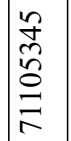 & 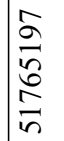 & 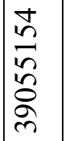 & 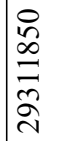 & 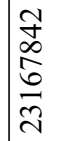 & 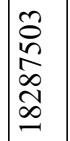 & 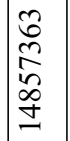 & $\mid \begin{array}{c}\hat{0} \\
\hat{\delta} \\
0 \\
\infty \\
= \\
=\end{array}$ & 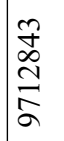 & 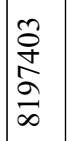 & $\begin{array}{l}\tilde{E} \\
\tilde{O} \\
0 \\
0 \\
0 \\
0\end{array}$ & $\begin{array}{l}\sqrt{2} \\
\widehat{\delta} \\
\infty \\
\infty \\
i n \\
\text { in }\end{array}$ & $\begin{array}{l}\stackrel{8}{\infty} \\
\stackrel{\infty}{\infty} \\
\stackrel{\infty}{\infty}\end{array}$ & 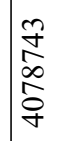 & 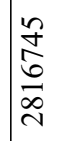 \\
\hline & & t & 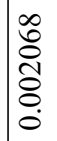 & 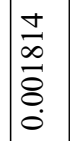 & 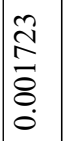 & $\begin{array}{l}\overline{\widehat{\sigma}} \\
\stackrel{0}{0} \\
0 \\
0\end{array}$ & 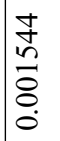 & $\mid \begin{array}{l}n \\
0 \\
0 \\
8 \\
8 \\
0 \\
0\end{array}$ & 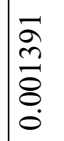 & \begin{tabular}{|l|}
$\vec{f}$ \\
$\stackrel{D}{0}$ \\
$\stackrel{8}{0}$ \\
$\dot{0}$
\end{tabular} & 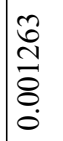 & 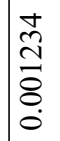 & 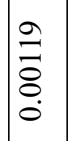 & 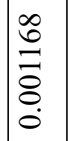 & 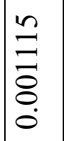 & $\begin{array}{l}\vec{\infty} \\
\stackrel{0}{0} \\
\overline{0} \\
0 \\
0\end{array}$ & $\mid \begin{array}{c}0 \\
\hat{0} \\
\stackrel{0}{0} \\
0 \\
\dot{0}\end{array}$ & $\begin{array}{l}\tilde{c} \\
\frac{0}{0} \\
\vdots \\
0\end{array}$ & 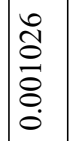 & 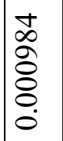 & 命 & 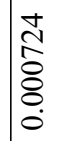 \\
\hline & & $\approx$ & $\sqrt{\frac{n}{6}}$ & $\begin{array}{l}8 \\
8 \\
\stackrel{1}{2}\end{array}$ & $\underset{J}{\stackrel{g}{J}}$ & $\begin{array}{l}\infty \\
\infty \\
心 \\
\end{array}$ & 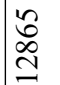 & 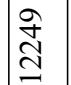 & $\stackrel{0}{\stackrel{\Xi}{\Xi}}$ & $\underset{\Xi}{\stackrel{\mathcal{I}}{\Xi}}$ & $\begin{array}{c}\hat{\infty} \\
\tilde{\infty} \\
\varrho\end{array}$ & 志 & 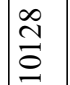 & $\begin{array}{l}\tilde{Z} \\
\approx \\
\alpha\end{array}$ & $\begin{array}{l}\text { F⿱ } \\
\text { 会 }\end{array}$ & $\begin{array}{c}\infty \\
\stackrel{\infty}{\mathbb{\alpha}}\end{array}$ & 冬 & $\begin{array}{l}\infty \\
\infty \\
\infty \\
\infty\end{array}$ & 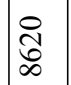 & \begin{tabular}{l}
$\infty$ \\
\multirow{\infty}{*}{}
\end{tabular} & 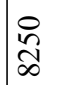 & $\frac{\widetilde{\sigma}}{\infty}$ \\
\hline లే & రิ & 苫 & $\begin{array}{l}0 \\
\infty \\
\infty \\
-\infty\end{array}$ & $\begin{array}{l}a \\
\text { i }\end{array}$ & 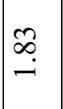 & $\stackrel{\Im}{f}$ & $\stackrel{\infty}{\stackrel{\infty}{\longrightarrow}}$ & - & $\begin{array}{l}\infty \\
\infty \\
0\end{array}$ & 合 & $\vec{\infty}$ & $\mid \begin{array}{c}0 \\
\infty \\
0 \\
0\end{array}$ & $\mid \begin{array}{c}0 \\
\infty \\
0 \\
0\end{array}$ & $\begin{array}{l}\infty \\
0 \\
0\end{array}$ & oे & $\hat{\delta}$ & 亏े & $\begin{array}{l}n \\
0 \\
-i\end{array}$ & $\mid \begin{array}{l}\infty \\
0 \\
- \\
-i\end{array}$ & $\exists$ & $\stackrel{\overbrace{}}{\leftrightarrows}$ & $\hat{\hat{i}}$ \\
\hline $\begin{array}{l}\stackrel{t}{\sigma} \\
\frac{\overrightarrow{0}}{\Xi}\end{array}$ & $\frac{\infty}{\frac{\infty}{1}}$ & $\vec{z}$ & 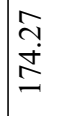 & $\begin{array}{l}\stackrel{0}{n} \\
\text { in } \\
\text { nn }\end{array}$ & $\frac{n}{\not}$ & $\begin{array}{l}\tilde{N} \\
\stackrel{m}{m}\end{array}$ & $\begin{array}{l} \pm \\
\infty \\
\infty \\
\infty \\
\sim\end{array}$ & $\mid \begin{array}{l}n \\
n \\
n\end{array}$ & $\begin{array}{l}\infty \\
\text { ते } \\
\text { ते }\end{array}$ & 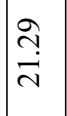 & $\vec{a}$ & $\begin{array}{l}0 \\
0 \\
\infty \\
-0\end{array}$ & $\begin{array}{l}\vec{\infty} \\
\infty \\
\infty\end{array}$ & 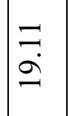 & $\stackrel{+}{2}$ & $\begin{array}{l}\tilde{d} \\
\stackrel{i}{d}\end{array}$ & $\mid \begin{array}{l}\nexists \\
\dot{\vec{\lambda}}\end{array}$ & $\left|\begin{array}{l}\infty \\
\mathbb{N} \\
\end{array}\right|$ & $\mid \begin{array}{l}n \\
\tilde{z} \\
a\end{array}$ & $\underset{\tilde{i}}{\tilde{i}}$ & $\mid \begin{array}{l}\hat{\imath} \\
\hat{\lambda}\end{array}$ & $\begin{array}{l}\stackrel{\sim}{\hat{n}} \\
\hat{n}\end{array}$ \\
\hline $\begin{array}{l}\stackrel{\mathscr{z}}{\underline{\Delta}} \\
\frac{\vec{\omega}}{\Xi}\end{array}$ & 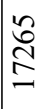 & 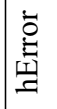 & ते & $\underset{\hat{\sigma}}{i}$ & s. & $\vec{\sigma}$ & ભે & 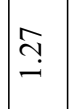 & $\stackrel{2}{=}$ & 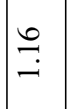 & $\stackrel{\infty}{\circ}$ & $\cong$ & $\stackrel{2}{\leftrightarrows}$ & $\tilde{n}$ & 点 & $\stackrel{n}{\stackrel{2}{r}}$ & $\vec{i}$ & $\stackrel{\vec{i}}{i}$ & $\begin{array}{l}\tilde{S} \\
\text { in }\end{array}$ & 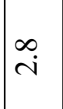 & $\frac{\partial}{m}$ & $\begin{array}{l}\mathbb{J} \\
\infty \\
\infty\end{array}$ \\
\hline$\underset{\Xi}{\stackrel{્}{\Xi}}$ & Бे & 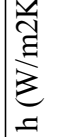 & $\mid \begin{array}{l}\infty \\
\infty \\
\vdots \\
\vdots \\
0\end{array}$ & $\frac{ \pm}{8}$ & 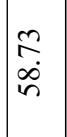 & बू. & $\begin{array}{l}\infty \\
\stackrel{\infty}{\infty} \\
\infty \\
+\end{array}$ & $\mid \begin{array}{l}n \\
\dot{y} \\
\ddot{f}\end{array}$ & $\begin{array}{l}\triangleright \\
\dot{\sigma} \\
\stackrel{\sim}{*}\end{array}$ & $\begin{array}{l}\varkappa \\
\tilde{f} \\
\tilde{f}\end{array}$ & $\vec{a}$ & $\overrightarrow{\vec{S}}$ & $\begin{array}{l}\bar{Z} \\
\dot{q}\end{array}$ & 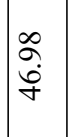 & $\mid \begin{array}{l}\stackrel{8}{0} \\
\ddot{q}\end{array}$ & N3 & $\mid \begin{array}{l}n \\
0 \\
i n \\
i n\end{array}$ & $\begin{array}{l}n \\
n \\
\tilde{b}\end{array} \mid$ & 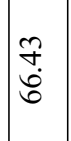 & 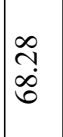 & $\mid \begin{array}{l}\infty \\
0 \\
i \\
i\end{array}$ & $\begin{array}{l}\text { ․ } \\
\stackrel{0}{0}\end{array}$ \\
\hline$\sum_{\text {至 }}^{\Sigma}$ & $\hat{\sigma}$ & $\begin{array}{l}\text { đิ } \\
\text { है } \\
= \\
=\end{array}$ & 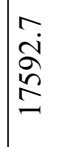 & 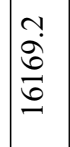 & 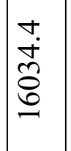 & 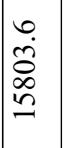 & $\begin{array}{l}a \\
\bar{n} \\
\tilde{n} \\
\end{array}$ & 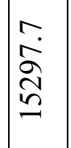 & 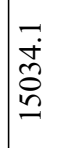 & $\mid \begin{array}{l}2 \\
\cdots \\
\infty \\
o \\
a \\
a\end{array}$ & 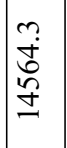 & $\begin{array}{l}\infty \\
0 \\
0 \\
0 \\
\dot{0} \\
\pm\end{array}$ & $\begin{array}{l}2 \\
8 \\
0 \\
\stackrel{n}{9} \\
-1\end{array}$ & $\stackrel{\circ}{\stackrel{9}{9}}$ & \begin{tabular}{|l}
$\infty$ \\
$\stackrel{\infty}{\oplus}$ \\
$\stackrel{\infty}{\Xi}$
\end{tabular} & 亲 & 会 & $\begin{array}{l}\vec{a} \\
\hat{Z} \\
\dot{Z} \\
\end{array}$ & 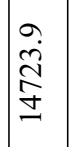 & 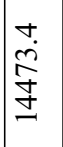 & $\mid \begin{array}{l}\hat{\mathrm{i}} \\
\stackrel{\hat{N}}{\tilde{\Xi}} \\
\stackrel{\Xi}{ \pm}\end{array}$ & $\stackrel{n}{\stackrel{n}{\Xi}}$ \\
\hline $\begin{array}{l}\sum_{\pi} \\
0 \\
0 \\
0 \\
0\end{array}$ & 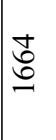 & $\vec{F}$ & 尚 & $\begin{array}{l}0 \\
m \\
\infty \\
\infty \\
m\end{array}$ & $\begin{array}{l}\hat{\alpha} \\
\hat{b} \\
\text { లె }\end{array}$ & $\mid \begin{array}{l}\infty \\
\infty \\
\infty \\
\infty \\
m\end{array}$ & $\begin{array}{l}\stackrel{\vartheta}{i} \\
\stackrel{\gamma}{\gamma}\end{array}$ & $\begin{array}{l}2 \\
\dot{n} \\
\tilde{\gamma}\end{array}$ & 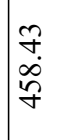 & $\begin{array}{l}\vec{n} \\
\stackrel{0}{\infty} \\
\infty \\
+\end{array}$ & 节 & 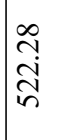 & $\begin{array}{l}\hat{z} \\
\stackrel{i}{\sim} \\
\stackrel{y}{n}\end{array}$ & $\begin{array}{l} \\
0 \\
0 \\
0 \\
n\end{array}$ & $\begin{array}{l}\sigma \\
\dot{\infty} \\
\infty \\
\infty\end{array}$ & 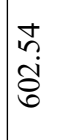 & $\begin{array}{l}\tilde{0} \\
\tilde{i} \\
\text { ర్ }\end{array}$ & $\begin{array}{l}\stackrel{+}{n} \\
\stackrel{+}{g}\end{array}$ & $\begin{array}{l}2 \\
\hat{o} \\
0 \\
0\end{array}$ & $\begin{array}{l}n \\
0 \\
\dot{d} \\
\infty \\
0\end{array}$ & 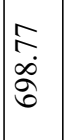 & 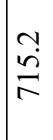 \\
\hline 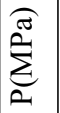 & $\hat{n}$ & 3 & $\begin{array}{l}\infty \\
\infty \\
\dot{\alpha} \\
\dot{m}\end{array}$ & $\begin{array}{l}\tilde{\lambda} \\
\stackrel{8}{i} \\
i n\end{array}$ & $\begin{array}{l}\hat{a} \\
\overline{0} \\
\widehat{\sigma}\end{array}$ & $\begin{array}{l}n \\
\tilde{\infty} \\
\infty\end{array}$ & 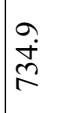 & $\mid \begin{array}{l}\infty \\
i \\
\mathfrak{d} \\
\end{array}$ & $\stackrel{\sim}{\stackrel{3}{\infty}}$ & $\mid \begin{array}{l}\hat{N} \\
\stackrel{0}{0} \\
\infty \\
\infty\end{array}$ & $\begin{array}{l}n \\
n \\
n \\
\infty\end{array}$ & $\mid \begin{array}{l}\infty \\
\underset{1}{N} \\
\stackrel{\infty}{\infty}\end{array}$ & $\mid \begin{array}{l}\stackrel{g}{+} \\
\dot{0} \\
\infty \\
\infty\end{array}$ & $\mid \begin{array}{l}8 \\
0 \\
0 \\
\infty \\
\infty\end{array}$ & $\mid \begin{array}{l}\tilde{n} \\
\infty \\
\infty \\
\infty\end{array}$ & $\begin{array}{l}\infty \\
\infty \\
i \\
i \infty \\
\infty\end{array}$ & $\begin{array}{l}+ \\
\infty \\
\infty \\
\infty \\
\infty\end{array}$ & $\begin{array}{l}\infty \\
\mathbb{i} \\
\mathbb{\infty} \\
\end{array}$ & $\mid \begin{array}{l}\infty \\
n \\
i \\
\infty \\
\infty \\
\infty\end{array}$ & $\begin{array}{l}\hat{\sigma} \\
\dot{i} \\
\grave{\alpha}\end{array}$ & $\begin{array}{l}\tilde{n} \\
\stackrel{0}{\circ} \\
\infty\end{array}$ & $\begin{array}{c}\text { సે } \\
\text { ֻे } \\
\infty\end{array}$ \\
\hline 怘 & in & $\stackrel{\rho}{a}$ & $N$ & $\vec{\infty}$ & $\overrightarrow{ \pm}$ & กิ & तु. & 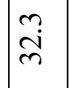 & 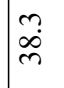 & $\mid \begin{array}{l}\dot{J} \\
\dot{f}\end{array}$ & $\begin{array}{l}t \\
\dot{B}\end{array}$ & \begin{tabular}{l}
$n$ \\
\hdashline \\
\hdashline \\
$n$
\end{tabular} & $\left|\begin{array}{l}n \\
i \\
0\end{array}\right|$ & $\left|\begin{array}{l}n \\
\infty \\
0 \\
0\end{array}\right|$ & $\begin{array}{l}0 \\
\stackrel{+}{i}\end{array}$ & $\begin{array}{l}0 \\
\dot{\infty} \\
\infty\end{array}$ & $\hat{\infty}$ & $\overline{\check{a}}$ & $\left|\begin{array}{l}\infty \\
\infty \\
\infty \\
\alpha\end{array}\right|$ & $\begin{array}{l}\infty \\
\dot{0} \\
\dot{0}\end{array}$ & $\stackrel{\partial}{\varrho}$ & $\hat{\sigma}$ \\
\hline
\end{tabular}




\begin{tabular}{|c|c|c|c|c|c|c|c|c|c|c|c|c|c|c|c|c|c|c|c|c|c|c|}
\hline & & $\vec{z}$ & 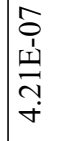 & 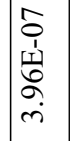 & 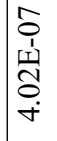 & 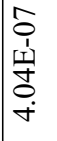 & 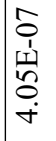 & 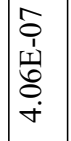 & $\begin{array}{l}\hat{o} \\
\hat{1} \\
\underline{1} \\
\tilde{\sigma} \\
\dot{+} \\
\dot{+}\end{array}$ & 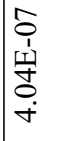 & 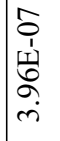 & $\mid \begin{array}{c}\hat{o} \\
\dot{1} \\
\underline{\underline{y}} \\
\bar{\sigma} \\
\dot{+} \\
\dot{+}\end{array}$ & $\left|\begin{array}{l}\hat{0} \\
\hat{1} \\
1 \\
\infty \\
\infty \\
\dot{m}\end{array}\right|$ & $\begin{array}{l}\hat{o} \\
\hat{1} \\
\hat{1} \\
\hat{o} \\
\dot{+} \\
\dot{+}\end{array}$ & 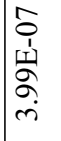 & $\begin{array}{l}\hat{\sigma} \\
\hat{1} \\
\text { tha } \\
\sigma \\
\dot{m}\end{array}$ & 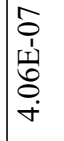 & $\begin{array}{c}\hat{S} \\
\hat{O} \\
1 \\
\\
0 \\
\dot{+}\end{array}$ & 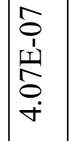 & 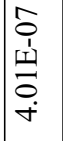 & 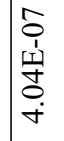 & 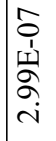 \\
\hline & & $\stackrel{\oplus}{\infty}$ & $\begin{array}{l}0 \\
0 \\
1 \\
\text { ț } \\
\text { m. } \\
m \\
m\end{array}$ & $\begin{array}{l}2 \\
0 \\
1 \\
n \\
2 \\
i \\
i\end{array}$ & 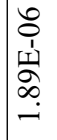 & 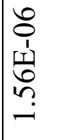 & 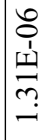 & 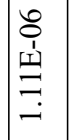 & $\begin{array}{l}\hat{o} \\
\hat{1} \\
\\
\alpha \\
\sigma\end{array}$ & $\mid \begin{array}{c}\hat{0} \\
0 \\
\omega \\
\omega \\
\infty \\
n \\
\infty \\
\infty\end{array}$ & 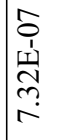 & 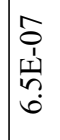 & 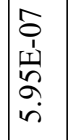 & \begin{tabular}{|l}
$\hat{0}$ \\
$\hat{1}$ \\
$\hat{1}$ \\
$\infty$ \\
\\
$i n$
\end{tabular} & 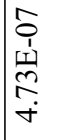 & 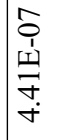 & 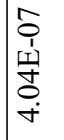 & 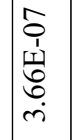 & 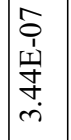 & 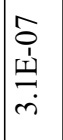 & 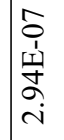 & $\begin{array}{l}\hat{2} \\
\stackrel{1}{1} \\
\text { N }\end{array}$ \\
\hline & & $\vec{D}$ & $\begin{array}{l}\stackrel{\infty}{\infty} \\
\stackrel{\infty}{i} \\
0\end{array}$ & $\begin{array}{l}\tilde{8} \\
0 \\
0 \\
0\end{array}$ & 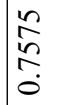 & $\begin{array}{l}\stackrel{2}{\circ} \\
\stackrel{2}{n} \\
\stackrel{0}{0}\end{array}$ & 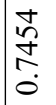 & $\mid \begin{array}{l}\vec{F} \\
\stackrel{0}{0}\end{array}$ & $\begin{array}{l}\vec{\infty} \\
\stackrel{2}{0} \\
\stackrel{0}{0}\end{array}$ & 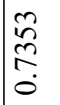 & 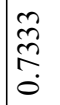 & 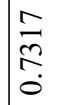 & 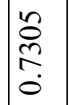 & 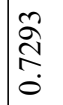 & 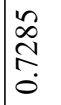 & 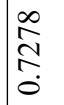 & 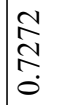 & $\begin{array}{l}\hat{E} \\
\mathbb{N} \\
\dot{0}\end{array}$ & 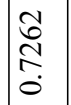 & 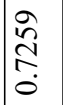 & 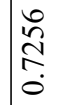 & 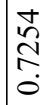 \\
\hline & & $\mathscr{J}$ & 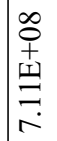 & $\begin{array}{l}\infty \\
0 \\
+ \\
\omega \\
\infty \\
\infty \\
\infty \\
\tilde{m}\end{array}$ & 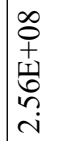 & 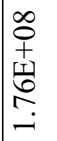 & 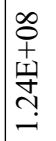 & 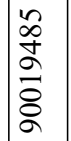 & $\begin{array}{l}\hat{n} \\
\hat{n} \\
\hat{\alpha} \\
\sigma\end{array}$ & 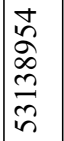 & 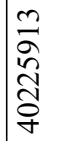 & $\begin{array}{l}\infty \\
\stackrel{\infty}{0} \\
\stackrel{N}{\hat{~}} \\
\frac{\mathrm{m}}{m}\end{array}$ & $\begin{array}{l}n \\
\tilde{n} \\
0 \\
n \\
\hat{0} \\
\sim\end{array}$ & 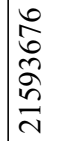 & 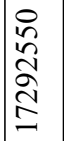 & 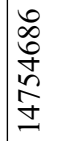 & 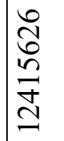 & $\mid \begin{array}{c}\hat{f} \\
\infty \\
\tilde{n} \\
\hat{\delta} \\
0\end{array}$ & $\begin{array}{l}\tilde{\Omega} \\
\stackrel{\Sigma}{\Sigma} \\
\Omega\end{array}$ & $\begin{array}{l}\overline{\hat{O}} \\
\tilde{n} \\
\hat{n} \\
\end{array}$ & 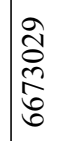 & 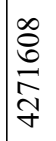 \\
\hline & & t & \begin{tabular}{l}
$\infty$ \\
\multirow{0}{0}{} \\
$\stackrel{8}{8}$ \\
0 \\
0
\end{tabular} & 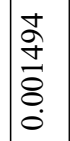 & 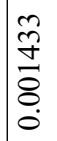 & 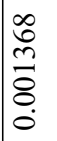 & 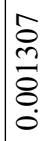 & 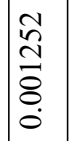 & 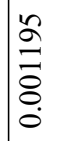 & $\mid \begin{array}{l}n \\
\stackrel{n}{a} \\
\bar{\Xi} \\
0 \\
0 \\
0\end{array}$ & 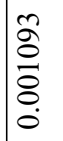 & 离 & 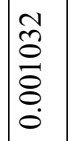 & 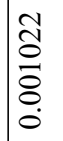 & \begin{tabular}{|l}
$\infty$ \\
0 \\
0 \\
0 \\
0 \\
0
\end{tabular} & $\begin{array}{l}\tilde{n} \\
\hat{o} \\
\vdots \\
\vdots \\
0\end{array}$ & 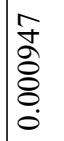 & 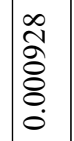 & 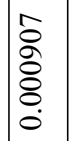 & $\begin{array}{l}0 \\
0 \\
\infty \\
\vdots \\
\vdots \\
0 \\
0\end{array}$ & 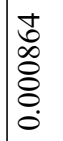 & 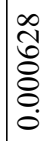 \\
\hline & & $\approx$ & $\underset{\sigma}{+}$ & $\stackrel{8}{\check{2}}$ & ڤે & $\begin{array}{l}\vec{n} \\
\tilde{n} \\
\sim\end{array}$ & ஓి & 文 & $\begin{array}{l}\mathscr{D} \\
ٍ \\
=\end{array}$ & $\stackrel{\vec{g}}{\Xi}$ & 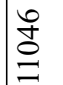 & 象 & $\mid \begin{array}{l}\infty \\
\infty \\
0 \\
0\end{array}$ & $\begin{array}{l}\infty \\
\infty \\
\stackrel{\Xi}{0}\end{array}$ & $\frac{\infty}{\infty}$ & $\sqrt{\tilde{n}}$ & స్ & $\vec{a}$ & $\vec{\infty}$ & $\underset{\substack{\mathbb{N} \\
\infty}}{\infty}$ & $\begin{array}{l}\mathscr{n} \\
\infty \\
\infty\end{array}$ & $\begin{array}{l}+ \\
⿱ \\
⿱ 亠 乂 \\
\infty\end{array}$ \\
\hline లే & రิ & 苫 & $\stackrel{+}{\stackrel{0}{\sigma}}$ & $\begin{array}{l}\mathbb{U} \\
i \\
i\end{array}$ & م & $\stackrel{\Im}{f}$ & $\stackrel{\overbrace{}}{\stackrel{2}{\leftrightarrows}}$ & $\exists$ & 훌. & $\mid \begin{array}{l}\infty \\
\infty \\
\infty \\
0\end{array}$ & $\stackrel{+}{\stackrel{0}{0}}$ & $\stackrel{\infty}{\infty}$ & $\begin{array}{ll}\infty \\
\infty \\
0 \\
0\end{array}$ & $\stackrel{\infty}{\stackrel{\infty}{0}}$ & $\mid \begin{array}{l}\infty \\
\infty \\
0 \\
0\end{array}$ & ڤ̊ & $\stackrel{\infty}{\Omega}$ & $\stackrel{\infty}{=}$ & $\stackrel{m}{=}$ & 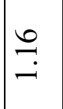 & 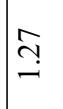 & $\vec{N}$ \\
\hline $\begin{array}{l}\stackrel{t}{\sigma} \\
\frac{\overrightarrow{0}}{\Xi}\end{array}$ & $\frac{1}{1}$ & $\vec{z}$ & $\begin{array}{l}3 \\
0 \\
6 \\
0\end{array}$ & $\stackrel{3}{i}$ & $\begin{array}{l}\infty \\
n \\
o \\
q\end{array}$ & $\begin{array}{l}\infty \\
\infty \\
\infty \\
m\end{array}$ & $\begin{array}{l}\infty \\
\infty \\
\infty \\
\infty \\
\sim\end{array}$ & $\left|\begin{array}{l}n \\
\infty \\
n \\
\end{array}\right|$ & $\begin{array}{l}\tilde{+} \\
\stackrel{\sim}{\sim}\end{array}$ & $\begin{array}{l}\infty \\
\stackrel{\infty}{\sim} \\
\dot{\sim}\end{array}$ & 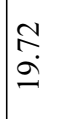 & $\begin{array}{l}\hat{\tilde{N}} \\
\text { à }\end{array}$ & $\left|\begin{array}{l}\hat{\alpha} \\
\infty \\
\infty\end{array}\right|$ & 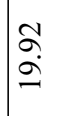 & 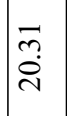 & $\stackrel{\hat{i}}{\bar{\lambda}}$ & $\overrightarrow{\tilde{i}}$ & $\frac{\partial}{\stackrel{\sim}{\sim}}$ & $\begin{array}{l}\vec{b} \\
\stackrel{\sim}{0}\end{array}$ & $\begin{array}{l}\tilde{n} \\
\tilde{n}\end{array}$ & $\stackrel{\sim}{\stackrel{\sim}{v}}$ & $\begin{array}{l}2 \\
\text { ते }\end{array}$ \\
\hline 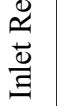 & 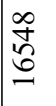 & 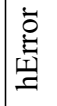 & 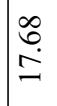 & సે. & $\stackrel{ \pm}{\stackrel{\Delta}{\longrightarrow}}$ & $\stackrel{\text { for }}{-}$ & $\stackrel{\sim}{\stackrel{\imath}{\leftrightarrows}}$ & $\stackrel{0}{=}$ & $\stackrel{\infty}{\circ}$ & . & ठे & $\stackrel{\tilde{\sigma}}{-}$ & $\stackrel{\infty}{\stackrel{\infty}{\longrightarrow}}$ & 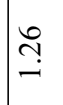 & $\stackrel{\text { I }}{-}$ & $\vec{\sigma}$ & $\bar{\sigma}$ & $\mid \begin{array}{l}\infty \\
\vec{i}\end{array}$ & $\hat{\tilde{i}}$ & 节 & $\stackrel{\infty}{i}$ & $\mid \begin{array}{l}\infty \\
i n\end{array}$ \\
\hline$\underset{\Xi}{\stackrel{\Xi}{\Xi}}$ & 客 & 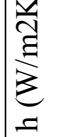 & $\begin{array}{l}\infty \\
\infty \\
\infty \\
\infty \\
\infty\end{array}$ & $\vec{b}$ & $\begin{array}{l}\widehat{\alpha} \\
\dot{H}\end{array}$ & 吕. & 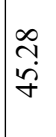 & $\begin{array}{l}\overrightarrow{\tilde{\gamma}} \\
\ddot{f}\end{array}$ & $\frac{\vec{f}}{\stackrel{m}{\vec{f}}}$ & $\begin{array}{l}\infty \\
\infty \\
\dot{q} \\
\dot{q}\end{array}$ & $\begin{array}{l}\hat{b} \\
\infty \\
\infty \\
m\end{array}$ & $\begin{array}{l}\sigma \\
\sigma \\
\text { ले }\end{array}$ & 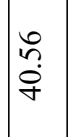 & $\underset{\forall}{\stackrel{\forall}{+}}$ & $\mid \begin{array}{c}\partial \\
\dot{f} \\
\dot{f}\end{array}$ & $\begin{array}{l}\vec{b} \\
\dot{n}\end{array}$ & 굴 & $\begin{array}{l}\hat{n} \\
\hat{n}\end{array}$ & 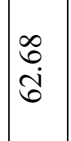 & 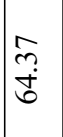 & $\mid \begin{array}{c}\widetilde{\sigma} \\
\dot{\delta} \\
\dot{\delta}\end{array}$ & $\underset{\infty}{\stackrel{+}{\infty}}$ \\
\hline$\sum_{\text {至 }}$ & $\stackrel{0}{\circ}$ & $\begin{array}{l}\text { đิ } \\
\text { है } \\
= \\
=\end{array}$ & $\begin{array}{l}n \\
\infty \\
\infty \\
\infty \\
\infty\end{array}$ & 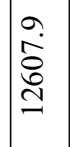 & 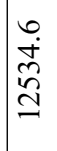 & 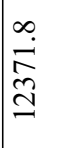 & 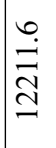 & 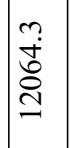 & 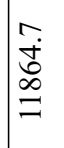 & 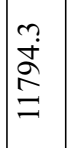 & $\begin{array}{l}n \\
\stackrel{n}{I} \\
\stackrel{\Xi}{\Xi}\end{array}$ & $\begin{array}{l}\stackrel{y}{*} \\
\stackrel{f}{\sim} \\
=\end{array}$ & 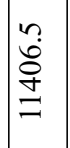 & $\begin{array}{l}n \\
\infty \\
\infty \\
\varrho \\
=\end{array}$ & 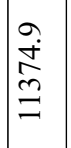 & $\begin{array}{l}\infty \\
\infty \\
\dot{w} \\
m \\
= \\
=\end{array}$ & $\begin{array}{l}n \\
\infty \\
\stackrel{\infty}{n} \\
=\end{array}$ & $\begin{array}{l}n \\
n \\
n \\
\cong \\
\Xi\end{array}$ & 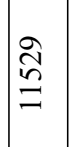 & $\begin{array}{l}\stackrel{n}{\vec{D}} \\
\infty \\
\stackrel{m}{\Xi} \\
\end{array}$ & 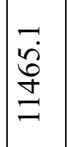 & $\begin{array}{l}\infty \\
\dot{\infty} \\
\infty \\
\infty \\
\infty\end{array}$ \\
\hline $\begin{array}{l}\sum_{\pi} \\
0 \\
0 \\
0 \\
0\end{array}$ & ְీ & $\vec{F}$ & $\begin{array}{l}\text { gे } \\
\text { oे } \\
\text { ô }\end{array}$ & 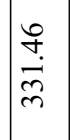 & $\begin{array}{l}\text { J } \\
\text { i } \\
m \\
m\end{array}$ & $\begin{array}{l}\infty \\
\infty \\
\stackrel{i}{m} \\
m\end{array}$ & $\begin{array}{l}\stackrel{0}{i} \\
\text { à } \\
\text { ma }\end{array}$ & $\overrightarrow{\vec{I}}$ & $\begin{array}{l}\infty \\
\dot{0} \\
\stackrel{\gamma}{+}\end{array}$ & $\mid \begin{array}{l}\hat{\vartheta} \\
\stackrel{g}{q}\end{array}$ & 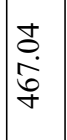 & $\begin{array}{l}\stackrel{0}{~} \\
\stackrel{+}{+} \\
+ \\
+\end{array}$ & $\begin{array}{l}\dot{d} \\
\dot{d} \\
\dot{\vec{n}} \\
\tilde{n}\end{array}$ & $\begin{array}{l}0 \\
\infty \\
\infty \\
\infty\end{array}$ & 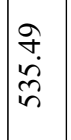 & 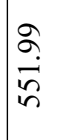 & 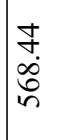 & $\begin{array}{l}\infty \\
\infty \\
\dot{\infty} \\
\infty \\
n\end{array}$ & $\overrightarrow{\widetilde{c}}$ & $\begin{array}{l}\infty \\
\\
\frac{1}{\sigma}\end{array}$ & $\begin{array}{l}\stackrel{\partial}{ल} \\
\hat{\vec{b}}\end{array}$ & $\begin{array}{l}2 \\
\hat{0} \\
\text { రे }\end{array}$ \\
\hline 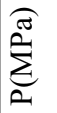 & $\hat{n}$ & 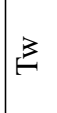 & $\underset{\substack{i \\
\infty \\
\infty}}{\vec{n}}$ & 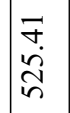 & $\begin{array}{l}\hat{6} \\
\dot{0} \\
0 \\
i\end{array}$ & $\begin{array}{l}\sigma \\
\text { तु } \\
\text { (n) }\end{array}$ & $\begin{array}{l}\stackrel{n}{f} \\
\text { ্ָర }\end{array}$ & $\overrightarrow{\tilde{n}}$ & $\underset{\widehat{\sigma}}{\stackrel{5}{\pi}}$ & $\mid \begin{array}{c}0 \\
0 \\
\infty \\
\Re \\
\Re\end{array}$ & $\begin{array}{l}\hat{n} \\
\hat{n} \\
\hat{n}\end{array}$ & $\begin{array}{l}\infty \\
\stackrel{\infty}{i} \\
\stackrel{2}{i}\end{array}$ & $\mid \begin{array}{l}\infty \\
\infty \\
i \\
\infty \\
尺\end{array}$ & 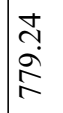 & $\left|\begin{array}{l}n \\
0 \\
i \\
i \\
i\end{array}\right|$ & $\begin{array}{l}0 \\
0 \\
0 \\
2\end{array}$ & $\begin{array}{l}\vec{N} \\
\stackrel{n}{i} \\
i\end{array}$ & 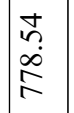 & $\mid \begin{array}{l}n \\
2 \\
\infty \\
\infty \\
2\end{array}$ & $\begin{array}{l}\text { oे } \\
\dot{2} \\
\text { I }\end{array}$ & 공 & $\begin{array}{l}\text { సे } \\
\infty \\
\stackrel{+}{+}\end{array}$ \\
\hline 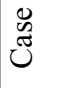 & $\tilde{n}$ & $\stackrel{\rho}{a}$ & $N$ & $\vec{\infty}$ & 亣 & ֻั & ֶָ. & 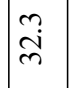 & 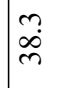 & $\mid \begin{array}{l}\dot{J} \\
\dot{f}\end{array}$ & $\begin{array}{l}t \\
\dot{B}\end{array}$ & \begin{tabular}{l}
$n$ \\
\hdashline \\
\hdashline \\
$n$
\end{tabular} & $\left|\begin{array}{l}n \\
i \\
0\end{array}\right|$ & $\begin{array}{l}n \\
\infty \\
\infty \\
0\end{array}$ & $\mid \begin{array}{l}0 \\
\stackrel{+}{i}\end{array}$ & $\begin{array}{l}0 \\
\dot{\infty} \\
\infty\end{array}$ & $\hat{\infty}$ & $\overline{\check{a}}$ & $\left|\begin{array}{l}\infty \\
\infty \\
\infty \\
\alpha\end{array}\right|$ & $\begin{array}{l}\infty \\
\dot{0} \\
\dot{0}\end{array}$ & $\stackrel{\partial}{\varrho}$ & ڤे \\
\hline
\end{tabular}




\begin{tabular}{|c|c|c|c|c|c|c|c|c|c|c|c|c|c|c|c|c|c|c|c|c|c|c|}
\hline & & $\vec{\Delta}$ & $\begin{array}{l}\hat{0} \\
+ \\
\text { ஸ⿱一土㇒ } \\
\stackrel{+}{+}\end{array}$ & $\begin{array}{l}0 \\
0 \\
1 \\
1 \\
0 \\
0 \\
n \\
+\end{array}$ & 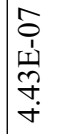 & 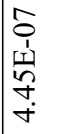 & 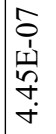 & 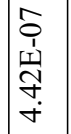 & 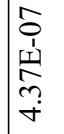 & 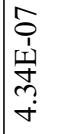 & 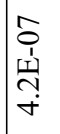 & 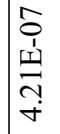 & 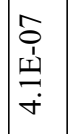 & 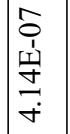 & 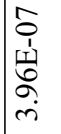 & $\begin{array}{l}\hat{0} \\
\hat{1} \\
\hat{1} \\
\infty \\
\infty \\
\dot{r}\end{array}$ & $\mid \begin{array}{l}\hat{o} \\
\hat{1} \\
\hat{1} \\
\hat{\alpha} \\
\dot{n}\end{array}$ & $\begin{array}{c}\hat{o} \\
1 \\
1 \\
\infty \\
2 \\
\dot{m}\end{array}$ & 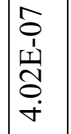 & 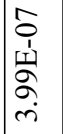 & 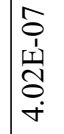 & $\begin{array}{l}\hat{o} \\
\hat{1} \\
\underline{1} \\
\infty \\
\dot{0} \\
\dot{m}\end{array}$ \\
\hline & & $\stackrel{\infty}{\infty}$ & $\begin{array}{l}0 \\
0 \\
1 \\
1 \\
\text { ஸे } \\
0 \\
\dot{c} \\
\dot{m}\end{array}$ & $\begin{array}{l}2 \\
0 \\
1 \\
\omega 1 \\
\vdots \\
i \\
i\end{array}$ & $\begin{array}{l}8 \\
0 \\
1 \\
11 \\
0 \\
0 \\
i\end{array}$ & 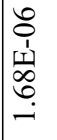 & 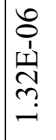 & 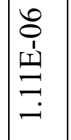 & 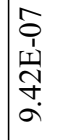 & 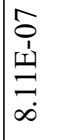 & 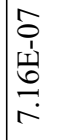 & 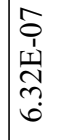 & 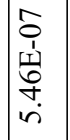 & $\mid \begin{array}{l}\hat{O} \\
\dot{1} \\
\dot{1} \\
\vec{\sigma} \\
\dot{+}\end{array}$ & 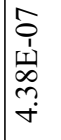 & $\begin{array}{l}\hat{1} \\
\hat{1} \\
\underline{1} \\
\infty \\
\infty \\
\dot{n}\end{array}$ & 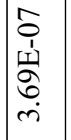 & $\begin{array}{c}\hat{s} \\
\hat{1} \\
\underline{1} \\
\sim \\
m \\
m\end{array}$ & 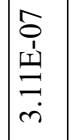 & 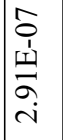 & 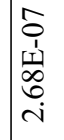 & 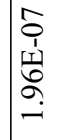 \\
\hline & & $\vec{D}$ & $\frac{\infty}{\stackrel{\infty}{\hat{~}}}$ & $\begin{array}{l}n \\
0 \\
0 \\
0\end{array}$ & $\begin{array}{l}\hat{n} \\
\hat{n} \\
0\end{array}$ & $\mid \begin{array}{c}\infty \\
\stackrel{\infty}{\hat{\alpha}} \\
\hat{\sigma}\end{array}$ & 畩 & $\underset{\tilde{N}}{\stackrel{2}{\sigma}}$ & 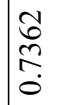 & 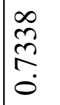 & $\frac{a}{\stackrel{2}{0}}$ & 总 & 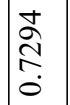 & 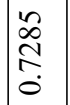 & 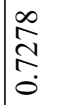 & 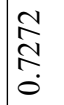 & 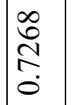 & $\left|\begin{array}{c}\tilde{0} \\
\mathbb{1} \\
0 \\
0\end{array}\right|$ & 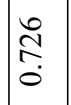 & 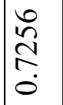 & 点 & 岕 \\
\hline & & $\mathscr{J}$ & 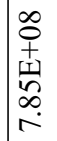 & 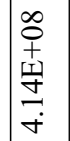 & 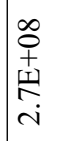 & 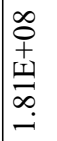 & 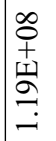 & 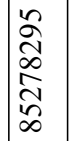 & 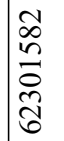 & $\mid \begin{array}{l}0 \\
\hat{\alpha} \\
o \\
\dot{+} \\
\dot{b} \\
\dot{f}\end{array}$ & 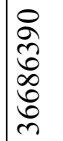 & 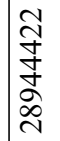 & 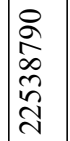 & 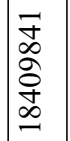 & 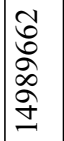 & $\begin{array}{l}\tilde{n} \\
\tilde{n} \\
\stackrel{n}{\beth} \\
\end{array}$ & $\mid \begin{array}{c}\infty \\
\stackrel{+}{+} \\
\stackrel{+}{+} \\
0 \\
0\end{array}$ & $\mid \begin{array}{c}\infty \\
\stackrel{+}{I} \\
\hat{0} \\
a \\
a\end{array}$ & 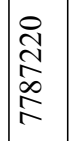 & 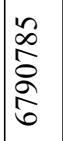 & 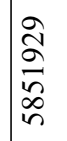 & 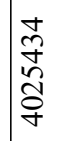 \\
\hline & & t & 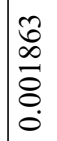 & $\begin{array}{l}\tilde{f} \\
\dot{0} \\
\stackrel{0}{0} \\
0 \\
0\end{array}$ & 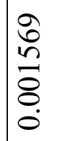 & 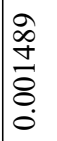 & $\begin{array}{l}0 \\
\stackrel{0}{+} \\
\stackrel{8}{8} \\
0 \\
0\end{array}$ & $\mid \begin{array}{l}\tilde{f} \\
\stackrel{0}{0} \\
\stackrel{8}{0} \\
0\end{array}$ & $\begin{array}{l}\overrightarrow{\widehat{\lambda}} \\
\stackrel{\vec{\Xi}}{0} \\
0\end{array}$ & 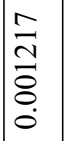 & 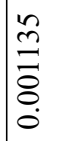 & 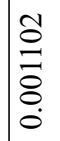 & 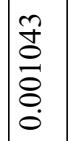 & 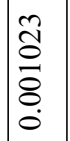 & $\mid \begin{array}{l}\tilde{n} \\
\hat{\alpha} \\
\grave{o} \\
0 \\
0\end{array}$ & 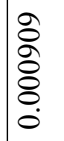 & 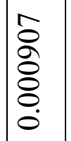 & $\mid \begin{array}{c} \pm \\
0 \\
0 \\
0 \\
0 \\
0 \\
0\end{array}$ & $\left|\begin{array}{l}2 \\
\infty \\
\infty \\
0 \\
0 \\
0 \\
0\end{array}\right|$ & 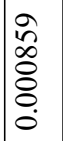 & 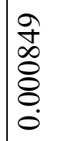 & 㕝 \\
\hline & & $\approx$ & $\underset{\widetilde{\sigma}}{\widetilde{\sigma}}$ & 今ิ & $\begin{array}{l}\vec{\sigma} \\
\underset{\Xi}{ \pm}\end{array}$ & 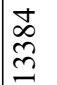 & 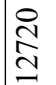 & 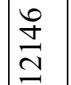 & $\underset{\substack{0 \\
=}}{\infty}$ & 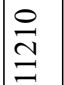 & $\begin{array}{l}\cong \\
\approx \\
\infty \\
\varrho\end{array}$ & 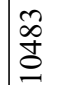 & $\frac{ \pm}{\frac{D}{0}}$ & $\stackrel{2}{\curvearrowright}$ & 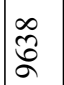 & के & $\frac{2}{a}$ & $\begin{array}{l}+ \\
\dot{\alpha} \\
\infty\end{array}$ & $\mid \begin{array}{l}\tilde{O} \\
\infty \\
\infty \\
\infty\end{array}$ & స్రి & 志 & $\overrightarrow{\tilde{\infty}}$ \\
\hline है & రీ & 总 & $\stackrel{\Xi}{\infty}$ & 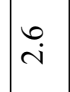 & 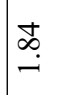 & $\stackrel{\infty}{\stackrel{n}{\longrightarrow}}$ & $\stackrel{n}{\longrightarrow}$ & $\exists$ & $\hat{\alpha}$ & $\mid \begin{array}{c}\infty \\
\infty \\
\infty \\
0\end{array}$ & $\hat{0}$ & $\begin{array}{l}\infty \\
n \\
0\end{array}$ & $\because$ & $\begin{array}{l}n \\
n \\
0\end{array}$ & 守. & I & ñ & $\stackrel{m}{\tilde{c}}$ & $\bar{n}$ & gे. & $\begin{array}{l}0 \\
? \\
0\end{array}$ & $\stackrel{8}{\circ}$ \\
\hline $\begin{array}{l} \pm \\
\sigma \\
\frac{\overrightarrow{0}}{\Xi}\end{array}$ & 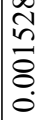 & $\vec{z}$ & 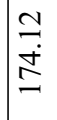 & 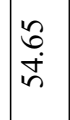 & $\begin{array}{l}\vec{\infty} \\
\vec{\forall}\end{array}$ & $\begin{array}{l}\stackrel{\infty}{\stackrel{1}{*}} \\
\stackrel{m}{m}\end{array}$ & $\begin{array}{l}8 \\
\infty \\
\infty \\
i \\
i\end{array}$ & $\begin{array}{l}\infty \\
\dot{+} \\
\stackrel{+}{+}\end{array}$ & 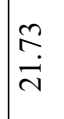 & $\stackrel{\infty}{\stackrel{\infty}{+}} \stackrel{\text {. }}{2}$ & $\begin{array}{l}\tilde{b} \\
\stackrel{0}{6}\end{array}$ & $\begin{array}{l}\tilde{n} \\
m \\
n \\
n\end{array}$ & 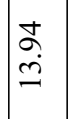 & $\begin{array}{l}0 \\
+ \\
\dot{2} \\
\end{array}$ & ્ָ & $\stackrel{n}{n}$ & $\stackrel{\infty}{\infty}$ & $\begin{array}{c}0 \\
\infty \\
= \\
=\end{array}$ & $\overrightarrow{\mathrm{I}}$ & $\stackrel{\mathfrak{I}}{\stackrel{\text { I }}{\mathcal{I}}}$ & $\stackrel{n}{m}$ & 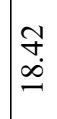 \\
\hline 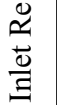 & 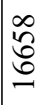 & 寄 & $\stackrel{ \pm}{2}$ & 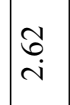 & 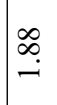 & $\stackrel{n}{\stackrel{n}{*}}$ & $\stackrel{?}{-}$ & $\stackrel{n}{=}$ & $\hat{\sigma}$ & a & $\stackrel{\infty}{0}$ & $\stackrel{0}{0}$ & $\stackrel{\vec{\sigma}}{\dot{0}}$ & $\stackrel{\mathbb{2}}{\stackrel{0}{0}}$ & $\begin{array}{l}\infty \\
0 \\
0\end{array}$ & $\stackrel{\bullet}{:}$ & bे & $\stackrel{+}{\stackrel{t}{0}}$ & $\vec{\infty}$ & 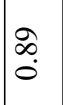 & $\stackrel{\infty}{\stackrel{\infty}{-}}$ & $\vec{i}$ \\
\hline$\underset{\Xi}{\stackrel{્}{\Xi}}$ & Бे & 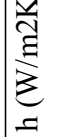 & $\begin{array}{l}\infty \\
\infty \\
0 \\
0 \\
0\end{array}$ & $\begin{array}{l}\hat{\theta} \\
\dot{0} \\
\dot{0}\end{array}$ & $\underset{i}{\stackrel{N}{n}}$ & 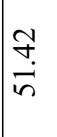 & $\overrightarrow{\tilde{j}}$ & $\left|\begin{array}{c}\tilde{O} \\
\dot{f} \\
\tilde{f}\end{array}\right|$ & $\begin{array}{l}\infty \\
\infty \\
\infty \\
\tilde{m}\end{array}$ & $\stackrel{\infty}{\stackrel{\infty}{\stackrel{m}{m}}}$ & $\begin{array}{l}\infty \\
\infty \\
\infty \\
\tilde{n}\end{array}$ & $\stackrel{\substack{\stackrel{n}{n} \\
i}}{i}$ & 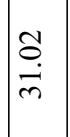 & $\mid$\begin{tabular}{l}
0 \\
\hdashline \\
$\dot{m}$
\end{tabular} & $\begin{array}{l}\vec{n} \\
\grave{\lambda} \\
\grave{\lambda}\end{array}$ & $\begin{array}{l}\infty \\
\infty \\
\infty \\
\infty \\
\sim\end{array}$ & $\mid \begin{array}{l}\hat{\alpha} \\
\hat{\lambda}\end{array}$ & 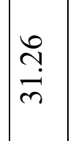 & & $\begin{array}{l}\vec{\infty} \\
\dot{m}\end{array}$ & $\mid \begin{array}{l}\partial \\
\infty \\
\infty \\
\infty\end{array}$ & $\vec{m}$ \\
\hline$\sum_{\text {至 }}$ & $\begin{array}{l}0 \\
\stackrel{0}{0}\end{array}$ & $\begin{array}{l}\text { đิ } \\
\text { है } \\
= \\
=\end{array}$ & 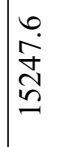 & 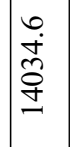 & 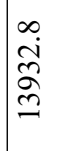 & 空 & 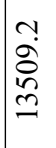 & 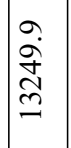 & 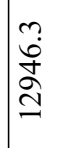 & 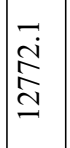 & 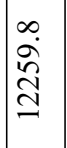 & 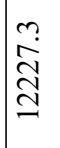 & $\underset{\substack{\stackrel{\infty}{\infty} \\
ٍ}}{=}$ & $\underset{⿱}{\stackrel{+}{*}}$ & $\mid \begin{array}{l}n \\
\stackrel{n}{a} \\
\tilde{\Xi} \\
=\end{array}$ & $\begin{array}{l}\tilde{\sigma} \\
\varrho \\
\Xi\end{array}$ & 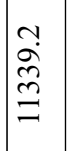 & 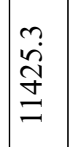 & 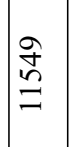 & 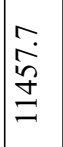 & $\begin{array}{l}\overrightarrow{0} \\
\stackrel{\infty}{0} \\
\stackrel{0}{6}\end{array}$ & $\begin{array}{l}0 \\
\hat{D} \\
\infty \\
\infty\end{array}$ \\
\hline $\begin{array}{l}\sum_{\pi} \\
0 \\
0 \\
0 \\
0\end{array}$ & 导 & $\vec{F}$ & 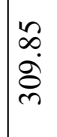 & $\begin{array}{l}0 \\
\stackrel{0}{0} \\
\stackrel{5}{+} \\
m\end{array}$ & $\begin{array}{l}\infty \\
n \\
i n \\
n \\
n\end{array}$ & $\mid \begin{array}{l}\overrightarrow{0} \\
\dot{\infty} \\
\infty \\
\infty\end{array}$ & $\frac{n}{\stackrel{n}{i}}$ & $\begin{array}{l}\hat{i} \\
\stackrel{\sim}{\sim}\end{array}$ & $\begin{array}{l}\stackrel{+}{0} \\
\dot{f} \\
\stackrel{f}{*}\end{array}$ & $\begin{array}{l}\hat{\sigma} \\
i \\
i \\
+\end{array}$ & $\frac{\tilde{n}}{\tilde{a}}$ & $\begin{array}{l}\stackrel{2}{2} \\
\stackrel{a}{a}\end{array}$ & $\begin{array}{l}\bar{n} \\
\stackrel{n}{n} \\
\tilde{n}\end{array}$ & $\mid \begin{array}{l}\infty \\
\dot{0} \\
\tilde{n}\end{array}$ & 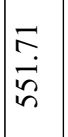 & $\begin{array}{l}\vec{\infty} \\
\vec{\infty} \\
i n\end{array}$ & $\mid \begin{array}{l}m \\
\tilde{m} \\
\infty \\
\infty\end{array}$ & $\begin{array}{l}\tilde{a} \\
\stackrel{2}{n}\end{array}$ & 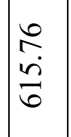 & $\frac{\vec{\sigma}}{\text { శై }}$ & $\begin{array}{l}n \\
? \\
\text { fon }\end{array}$ & 导. \\
\hline$\sum_{0}^{\pi}$ & $\hat{n}$ & 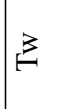 & $\mid \begin{array}{l}n \\
\infty \\
\infty \\
m\end{array}$ & $\begin{array}{l}\infty \\
\infty \\
\infty \\
\infty \\
n \\
n\end{array}$ & $\begin{array}{l}\stackrel{2}{2} \\
\infty \\
\infty \\
i n\end{array}$ & \begin{tabular}{|l}
$\vec{\infty}$ \\
$\dot{0}$ \\
$\dot{0}$
\end{tabular} & $\begin{array}{l}\tilde{y} \\
\tilde{\sigma} \\
\hat{\sigma}\end{array}$ & $\mid \begin{array}{l}\infty \\
\stackrel{2}{0} \\
\stackrel{2}{r}\end{array}$ & 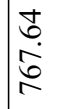 & $\begin{array}{l}\hat{i} \\
\dot{8} \\
\infty\end{array}$ & $\begin{array}{l}\tilde{n} \\
\dot{f} \\
\infty \\
\infty\end{array}$ & $\begin{array}{l}\infty \\
0 \\
\infty \\
\infty \\
\infty\end{array}$ & 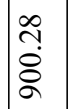 & $\left|\begin{array}{l}\frac{a}{2} \\
\infty \\
a\end{array}\right|$ & $\frac{m}{\alpha}$ & $\begin{array}{l}\hat{n} \\
n \\
n \\
\alpha\end{array}$ & $\mid \begin{array}{l}0 \\
\text { î } \\
\sigma\end{array}$ & $\begin{array}{l}\infty \\
\cdots \\
2 \\
2\end{array}$ & $\mid \begin{array}{l}\infty \\
0 \\
0 \\
0 \\
0\end{array}$ & $\begin{array}{l}0 \\
\infty \\
\dot{8} \\
\delta\end{array}$ & 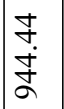 & $\begin{array}{l}\stackrel{\bullet}{2} \\
\underset{\sim}{ \pm}\end{array}$ \\
\hline $\begin{array}{l}0 \\
\mathscr{Z} \\
\tilde{z} \\
\tilde{z}\end{array}$ & in & $\stackrel{\rho}{a}$ & $N$ & $\vec{\infty}$ & $\exists$ & ָ̃. & ֶָ. & $\mid \begin{array}{c}n \\
i \\
n\end{array}$ & $\begin{array}{c}m \\
\infty \\
\infty \\
m\end{array}$ & $\mid \begin{array}{l}+ \\
\dot{J}\end{array}$ & $\begin{array}{l}t \\
\dot{8}\end{array}$ & $\ddot{\circ}$ & $\mid \begin{array}{l}n \\
i \\
0\end{array}$ & $\left|\begin{array}{l}n \\
\infty \\
\infty \\
0\end{array}\right|$ & 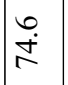 & $\begin{array}{l}0 \\
\dot{0} \\
\infty\end{array}$ & $\begin{array}{l}\hat{\infty} \\
\infty\end{array}$ & $\hat{a}$ & $\left|\begin{array}{l}\infty \\
\infty \\
\infty\end{array}\right|$ & $\begin{array}{l}\infty \\
\dot{0} \\
\stackrel{0}{0}\end{array}$ & 韮 & \\
\hline
\end{tabular}




\begin{tabular}{|c|c|c|c|c|c|c|c|c|c|c|c|c|c|c|c|c|c|c|c|c|c|c|}
\hline & & $\vec{\Delta}$ & \begin{tabular}{l}
0 \\
0 \\
1 \\
1 \\
\multirow{1}{0}{} \\
0 \\
0 \\
0
\end{tabular} & 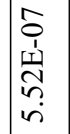 & 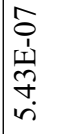 & 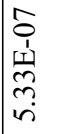 & 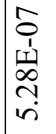 & 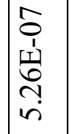 & $\begin{array}{l}\hat{0} \\
i \\
\text { w } \\
\text { in } \\
\text { in }\end{array}$ & 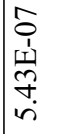 & 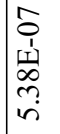 & 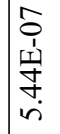 & 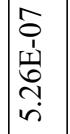 & 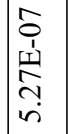 & 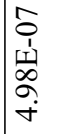 & 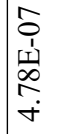 & \begin{tabular}{|l|}
$\hat{O}$ \\
$\hat{1}$ \\
$\hat{1}$ \\
$\infty$ \\
$\infty$ \\
$\dot{\gamma}$
\end{tabular} & 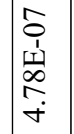 & 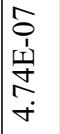 & $\begin{array}{l}\hat{0} \\
\hat{1} \\
\text { to } \\
\text { ro } \\
\dot{\gamma}\end{array}$ & 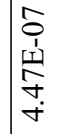 & $\begin{array}{l}\hat{i} \\
\hat{1} \\
\text { II } \\
2 \\
\text { in }\end{array}$ \\
\hline & & $\stackrel{\infty}{\infty}$ & $\begin{array}{l}8 \\
1 \\
1 \\
\infty \\
\infty \\
\sigma \\
\sigma\end{array}$ & $\begin{array}{l}8 \\
0 \\
1 \\
1 \\
0 \\
0 \\
0 \\
6\end{array}$ & 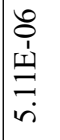 & 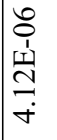 & 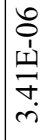 & $\mid \begin{array}{l}0 \\
0 \\
1 \\
1 \\
\infty \\
\infty \\
i \\
ن\end{array}$ & 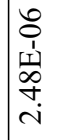 & 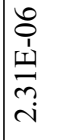 & 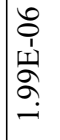 & 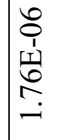 & $\mid \begin{array}{c}8 \\
1 \\
1 \\
\\
\\
-1 \\
-1\end{array}$ & 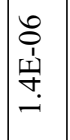 & $\mid \begin{array}{c}0 \\
0 \\
1 \\
1 \\
\infty \\
= \\
= \\
-1\end{array}$ & 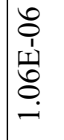 & 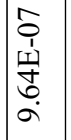 & $\begin{array}{c}\hat{s} \\
\hat{1} \\
\underline{1} \\
\hat{\alpha} \\
\infty \\
\infty\end{array}$ & 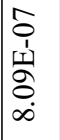 & 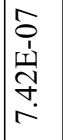 & 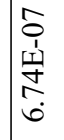 & $\begin{array}{l}\hat{o} \\
0 \\
1 \\
\text { ț } \\
0 \\
\dot{+}\end{array}$ \\
\hline & & $\vec{D}$ & 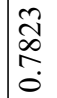 & $\begin{array}{l}\vec{o} \\
\stackrel{0}{o} \\
\dot{0}\end{array}$ & $\begin{array}{l}\infty \\
2 \\
\stackrel{2}{2} \\
\stackrel{0}{0}\end{array}$ & 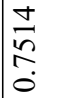 & 章 & 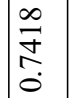 & $\begin{array}{l}\infty \\
\infty \\
\stackrel{\infty}{0} \\
\stackrel{0}{0}\end{array}$ & $\begin{array}{l}\stackrel{\infty}{\infty} \\
\stackrel{2}{0} \\
\stackrel{0}{0}\end{array}$ & 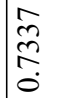 & $\underset{\widetilde{N}}{\stackrel{\vec{\Omega}}{0}}$ & $\mid \begin{array}{c}\hat{0} \\
\hat{n} \\
0 \\
0\end{array}$ & 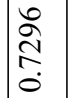 & $\begin{array}{l}\infty \\
\stackrel{\infty}{\mathbb{N}} \\
\stackrel{N}{0}\end{array}$ & $\begin{array}{l}\vec{\infty} \\
\stackrel{N}{N} \\
\dot{0}\end{array}$ & $\begin{array}{l}\stackrel{n}{\hat{N}} \\
\stackrel{N}{0}\end{array}$ & 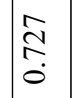 & 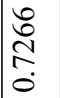 & 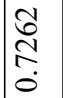 & & 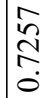 \\
\hline & & $\mathscr{J}$ & 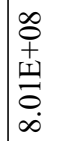 & 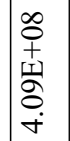 & 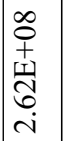 & 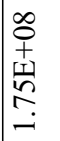 & 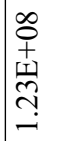 & 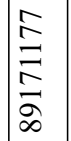 & 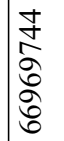 & $\mid \begin{array}{l}\text { t } \\
0 \\
0 \\
0 \\
0 \\
0 \\
1 \\
1\end{array}$ & 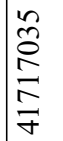 & $\begin{array}{l}\stackrel{9}{+} \\
\hat{\alpha} \\
\hat{\widehat{A}} \\
\text { }\end{array}$ & $\begin{array}{l}2 \\
2 \\
\tilde{a} \\
\tilde{n} \\
\approx\end{array}$ & 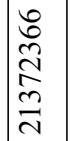 & 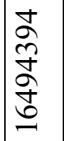 & $\begin{array}{l}0 \\
0 \\
0 \\
6 \\
0 \\
0 \\
\end{array}$ & $\mid \begin{array}{l}n \\
\infty \\
0 \\
0 \\
\infty \\
\\
=\end{array}$ & $\begin{array}{l}n \\
\tilde{\delta} \\
\delta \\
\delta \\
\sigma\end{array}$ & $\begin{array}{l}n \\
\text { an } \\
\infty \\
\infty \\
\infty \\
\infty\end{array}$ & 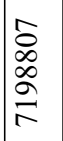 & 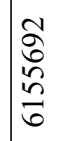 & 离 \\
\hline & & t & 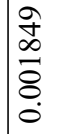 & $\begin{array}{l}\tilde{z} \\
\tilde{n} \\
\tilde{8} \\
0 \\
0\end{array}$ & 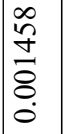 & 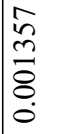 & 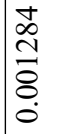 & $\mid \begin{array}{c}\hat{\tilde{J}} \\
\stackrel{\Xi}{\Xi} \\
0 \\
0\end{array}$ & $\begin{array}{l}\hat{\infty} \\
\stackrel{\infty}{\vec{\sigma}} \\
0 \\
0\end{array}$ & 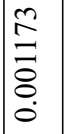 & $\begin{array}{l}\text { İ } \\
\stackrel{\Xi}{\Xi} \\
0 \\
0\end{array}$ & & $\begin{array}{l}\tilde{0} \\
\stackrel{0}{0} \\
0 \\
0 \\
0\end{array}$ & $\mid \begin{array}{l}0 \\
0 \\
0 \\
0 \\
0 \\
0 \\
0\end{array}$ & 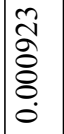 & $\begin{array}{l}0 \\
0 \\
0 \\
0 \\
0 \\
0 \\
0\end{array}$ & $\mid \begin{array}{l}0 \\
\infty \\
\infty \\
0 \\
0 \\
0 \\
0\end{array}$ & 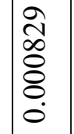 & $\begin{array}{l}0 \\
0 \\
0 \\
0 \\
0 \\
0 \\
0\end{array}$ & \begin{tabular}{l}
0 \\
\multirow{0}{0}{} \\
$\vdots$ \\
0 \\
0 \\
0
\end{tabular} & 告 & 它 \\
\hline & & $\approx$ & $\frac{n}{\vec{I}}$ & $\begin{array}{l}\infty \\
\infty \\
\cong\end{array}$ & 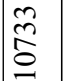 & $\stackrel{\infty}{\stackrel{\infty}{\sigma}}$ & ָু & $\frac{\partial}{\bar{\alpha}}$ & $\begin{array}{l}8 \\
\infty \\
\infty\end{array}$ & 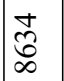 & ले & 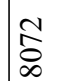 & $\mid \begin{array}{l}\infty \\
\infty \\
\infty \\
\infty\end{array}$ & $\frac{n}{2}$ & 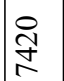 & $\underset{\mathbb{N}}{\stackrel{\Xi}{N}}$ & 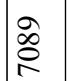 & ơ & $\vec{\otimes}$ & $\frac{2}{8}$ & 芯 & 䓟 \\
\hline שै & ठิ & 章 & $\begin{array}{l}n \\
\tilde{n} \\
\end{array}$ & ڤึ) & g. & $\hat{\circ}$ & กิ & ?ִ & $\begin{array}{l}\overrightarrow{0} \\
0\end{array}$ & 苟 & $\tilde{n}$ & $\stackrel{g}{\mathscr{f}}$ & $\because$ & $\overbrace{0}^{0}$ & $\hat{m}$ & ळे & ?ై & $\begin{array}{l}+ \\
3 \\
0\end{array}$ & $\overrightarrow{3}$ & $\stackrel{\infty}{\substack{n \\
0}}$ & 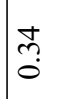 & $\stackrel{g}{\sigma}$ \\
\hline $\begin{array}{l} \pm \\
\sigma \\
\frac{\overrightarrow{0}}{\Xi}\end{array}$ & 客 & $\vec{z}$ & 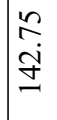 & $\begin{array}{l}\vec{\alpha} \\
\infty \\
\infty\end{array}$ & 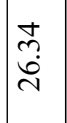 & $\hat{\tilde{\imath}}$ & $\stackrel{\vec{N}}{\beth}$ & 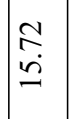 & $\begin{array}{l}\infty \\
\ddot{n} \\
n\end{array}$ & $\begin{array}{l}2 \\
\\
\end{array}$ & $\begin{array}{l}\vec{b} \\
\dot{ \pm}\end{array}$ & $\frac{\partial}{\dot{ \pm}}$ & $\begin{array}{l}\infty \\
\infty \\
\\
\mid\end{array} \mid$ & $\mid \begin{array}{l}\mathfrak{a} \\
\mathfrak{I} \\
\end{array}$ & $\mid \begin{array}{l}\infty \\
\infty \\
0\end{array}$ & $\stackrel{\mathbb{N}}{a}$ & $\ddot{\sigma}$ & $\mid \begin{array}{l} \pm \\
\infty \\
\infty\end{array}$ & $\begin{array}{l}\hat{n} \\
\infty\end{array}$ & $\underset{\infty}{=}$ & $\bar{\Omega}$ & $\vec{n}$ \\
\hline 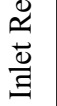 & 染 & 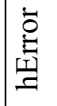 & 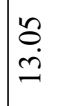 & $\stackrel{\text { qq }}{\stackrel{f}{-}}$ & $\begin{array}{l}\infty \\
0 \\
0\end{array}$ & : & $\hat{n}$ & 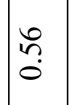 & $\begin{array}{l}\infty \\
n \\
0\end{array}$ & ô. & $\begin{array}{l}\mathbb{J} \\
\stackrel{0}{0}\end{array}$ & $\stackrel{\bullet}{0}$ & $\mid \begin{array}{l}0 \\
0 \\
0 \\
0\end{array}$ & $\overrightarrow{0}$ & 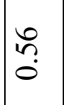 & $\bar{n}$ & $\bar{n}$ & 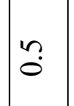 & 守. & 市. & $\because$ & $\exists$ \\
\hline 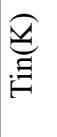 & Бे & 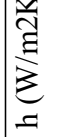 & 节 & $\begin{array}{l}\infty \\
\infty \\
\infty \\
\infty \\
+\end{array}$ & $\begin{array}{l}\infty \\
0 \\
\dot{0} \\
\dot{n}\end{array}$ & $\stackrel{7}{\stackrel{0}{0}}$ & 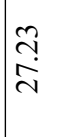 & 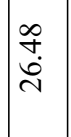 & 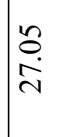 & $\begin{array}{l}\hat{\sigma} \\
\infty \\
\dot{\sim}\end{array}$ & 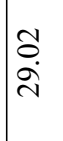 & $\begin{array}{l}\approx \\
\ddot{\lambda} \\
\stackrel{n}{2}\end{array}$ & $\mid \begin{array}{l}\vec{\infty} \\
\grave{\sim}\end{array}$ & $\left|\begin{array}{l}\tilde{n} \\
\tilde{\sim}\end{array}\right|$ & 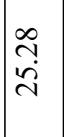 & $\underset{\sim}{\stackrel{\sim}{\sim}}$ & $\mid \begin{array}{l}\tilde{n} \\
\tilde{\lambda}\end{array}$ & $\begin{array}{l}\hat{\infty} \\
\ddot{N}\end{array} \mid$ & $\begin{array}{l}\tilde{n} \\
\underset{\sim}{\sim}\end{array}$ & $\frac{\sigma}{\vec{\lambda}}$ & 全 & $\begin{array}{l}\text { Oे } \\
\stackrel{+}{\sim}\end{array}$ \\
\hline$\sum_{\text {至 }}$ & $\stackrel{t}{0}$ & 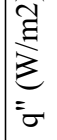 & 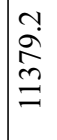 & $\begin{array}{l}\overrightarrow{\tilde{N}} \\
\infty \\
\stackrel{8}{0}\end{array}$ & $\begin{array}{l}0 \\
0 \\
\infty \\
0 \\
0 \\
a\end{array}$ & 吕 & $\begin{array}{l}\vec{\infty} \\
\stackrel{\infty}{\sim} \\
\sigma\end{array}$ & $\mid \begin{array}{l}0 \\
\dot{d} \\
\infty \\
\infty \\
\infty\end{array}$ & $\begin{array}{l}\hat{\tilde{n}} \\
\hat{\alpha} \\
\infty\end{array}$ & $\overrightarrow{+}$ & 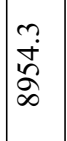 & 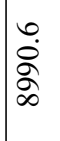 & $\mid \begin{array}{l}0 \\
0 \\
\infty \\
\infty\end{array}$ & $\mid \begin{array}{l}1 \\
0 \\
0 \\
0 \\
\infty \\
\infty\end{array}$ & 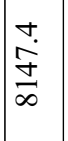 & $\begin{array}{l}\sim \\
\tilde{\infty} \\
\infty \\
\infty\end{array}$ & 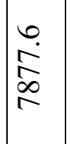 & 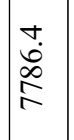 & 式 & 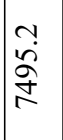 & $\begin{array}{l}0 \\
\stackrel{0}{2} \\
\stackrel{N}{N}\end{array}$ & 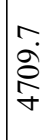 \\
\hline $\begin{array}{l}\sum_{\pi} \\
0 \\
0 \\
0 \\
0\end{array}$ & $\begin{array}{l}\infty \\
\stackrel{\leftrightarrow}{\circ}\end{array}$ & $\vec{F}$ & 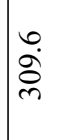 & 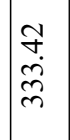 & $\begin{array}{l}\Delta \\
0 \\
n \\
n \\
n \\
m\end{array}$ & $\begin{array}{l}n \\
n \\
n \\
n \\
n \\
m\end{array}$ & $\frac{ \pm}{2}$ & $\begin{array}{l}\exists \\
\dot{\Xi} \\
F\end{array}$ & $\begin{array}{l}\tilde{\sigma} \\
i \\
\tilde{\gamma} \\
\tilde{\gamma}\end{array}$ & $\mid \begin{array}{c}\tilde{\delta} \\
\dot{\vec{s}} \\
\dot{q}\end{array}$ & đa. & $\mid \begin{array}{l}8 \\
\stackrel{\infty}{\infty} \\
\dot{\infty} \\
\dot{\alpha}\end{array}$ & $\begin{array}{l} \\
\dot{D} \\
\stackrel{5}{n}\end{array}$ & $\begin{array}{l}\vec{n} \\
\overrightarrow{\tilde{n}}\end{array}$ & 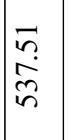 & $\begin{array}{l}\stackrel{2}{i} \\
i \tilde{n} \\
i n\end{array}$ & $\begin{array}{l}0 \\
0 \\
0 \\
0 \\
n\end{array}$ & $\begin{array}{l}\hat{n} \\
i \\
\infty \\
n\end{array}$ & $\mid \begin{array}{l}n \\
\infty \\
0 \\
0 \\
n \\
n\end{array}$ & $\begin{array}{l}\stackrel{0}{0} \\
\frac{0}{6}\end{array}$ & $\mid \begin{array}{l}\hat{n} \\
\stackrel{d}{d} \\
\text { d }\end{array}$ & 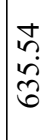 \\
\hline$\sum_{0}^{\pi}$ & $\begin{array}{l}\text { : } \\
:\end{array}$ & 3 & 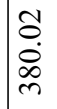 & $\begin{array}{l}\stackrel{2}{\hat{a}} \\
\hat{n} \\
\hat{n}\end{array}$ & 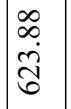 & $\begin{array}{l}\tilde{b} \\
\ddot{0} \\
\ddot{0}\end{array}$ & 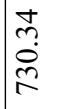 & $\mid \begin{array}{l}\infty \\
\stackrel{\infty}{+} \\
\stackrel{n}{n} \\
\end{array}$ & $\begin{array}{l}\stackrel{0}{c} \\
\stackrel{0}{2}\end{array}$ & \begin{tabular}{|l|}
$\infty$ \\
0 \\
$\dot{0}$ \\
0 \\
12
\end{tabular} \mid & $\begin{array}{l}\stackrel{2}{1} \\
\stackrel{2}{2}\end{array}$ & $\mid \begin{array}{l}\tilde{m} \\
\tilde{m} \\
\tilde{R}\end{array}$ & $\left|\begin{array}{c}0 \\
\dot{0} \\
\dot{0} \\
\infty\end{array}\right|$ & $\begin{array}{l}\vec{n} \\
n \\
\tilde{n}\end{array} \mid$ & $\begin{array}{l}2 \\
2 \\
\infty \\
\infty\end{array}$ & $\begin{array}{l}0 \\
0 \\
\infty \\
\infty \\
\infty \\
\infty\end{array}$ & $\mid \begin{array}{l}\tilde{N} \\
\tilde{n} \\
\delta\end{array}$ & $\begin{array}{l}\hat{\infty} \\
\tilde{Z} \\
\tilde{\alpha}\end{array}$ & ๙̃. & 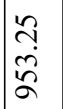 & $\begin{array}{l}\bar{a} \\
\hat{े} \\
\alpha\end{array}$ & $\frac{0}{+}$ \\
\hline 岕 & in & $\stackrel{\rho}{a}$ & $N$ & $\vec{\infty}$ & $\overrightarrow{ \pm}$ & กิ & तु. & 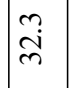 & 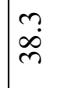 & $\mid \begin{array}{l}\dot{J} \\
\dot{f}\end{array}$ & $\begin{array}{l}t \\
\dot{B}\end{array}$ & \begin{tabular}{l}
$n$ \\
\hdashline \\
\hdashline \\
$n$
\end{tabular} & $\left|\begin{array}{l}n \\
i \\
0\end{array}\right|$ & $\left|\begin{array}{l}n \\
\infty \\
0 \\
0\end{array}\right|$ & $\begin{array}{l}0 \\
\stackrel{+}{i}\end{array}$ & $\begin{array}{l}0 \\
\dot{\infty} \\
\infty\end{array}$ & $\hat{\infty}$ & $\overline{\check{a}}$ & $\left|\begin{array}{l}\infty \\
\infty \\
\infty \\
\alpha\end{array}\right|$ & $\begin{array}{l}\infty \\
\dot{0} \\
\stackrel{0}{0}\end{array}$ & $\stackrel{\partial}{\varrho}$ & $\hat{\sigma}$ \\
\hline
\end{tabular}




\begin{tabular}{|c|c|c|c|c|c|c|c|c|c|c|c|c|c|c|c|c|c|c|c|c|c|c|}
\hline & & $\vec{\Delta}$ & 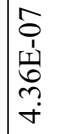 & $\begin{array}{l}\hat{0} \\
1 \\
1 \\
\approx \\
2 \\
\dot{m}\end{array}$ & 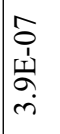 & 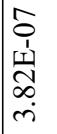 & \begin{tabular}{|l}
$\hat{0}$ \\
$\hat{1}$ \\
$\underline{1}$ \\
$\infty$ \\
$i$ \\
$\dot{m}$
\end{tabular} & 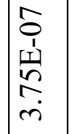 & 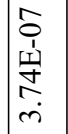 & $\begin{array}{c}\hat{0} \\
\hat{1} \\
1 \\
\infty \\
\infty \\
\dot{r}\end{array}$ & $\begin{array}{l}\hat{i} \\
\hat{1} \\
\hat{1} \\
\hat{N} \\
\stackrel{i}{n}\end{array}$ & \begin{tabular}{|l}
$\hat{o}$ \\
$\hat{1}$ \\
$\hat{\omega}$ \\
$\infty$ \\
$\infty$ \\
$\dot{m}$
\end{tabular} & $\begin{array}{l}\hat{o} \\
\hat{1} \\
\underline{1} \\
\infty \\
\dot{n}\end{array}$ & 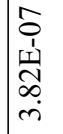 & 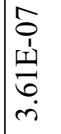 & 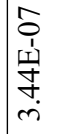 & 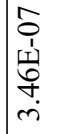 & $\begin{array}{c}\hat{o} \\
\dot{1} \\
\dot{1} \\
\dot{y} \\
\dot{r}\end{array}$ & $\mid \begin{array}{l}\hat{0} \\
1 \\
1 \\
\\
\tilde{n} \\
\end{array}$ & 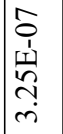 & 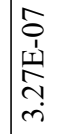 & 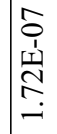 \\
\hline & & $\stackrel{\oplus}{\infty}$ & 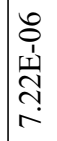 & 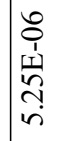 & 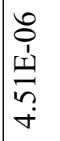 & $\begin{array}{l}8 \\
0 \\
1 \\
1 \\
\\
\\
\dot{n} \\
\dot{r}\end{array}$ & 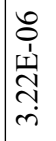 & 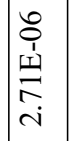 & 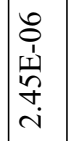 & 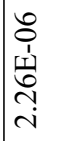 & $\begin{array}{l}8 \\
\varnothing \\
1 \\
1 \\
\tilde{1} \\
0 \\
i\end{array}$ & 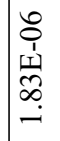 & $\mid$\begin{tabular}{l}
0 \\
0 \\
1 \\
1 \\
0 \\
0 \\
\hdashline \\
-1
\end{tabular} & 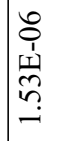 & 总 & 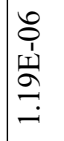 & 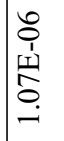 & 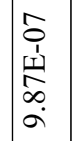 & 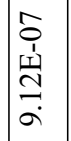 & 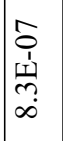 & 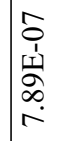 & $\begin{array}{c}\hat{0} \\
\mathbf{1} \\
1 \\
\infty \\
\infty \\
0 \\
\dot{\gamma}\end{array}$ \\
\hline & & $\vec{D}$ & 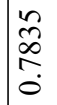 & 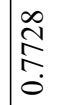 & $\begin{array}{l}n \\
\mathfrak{b} \\
0 \\
0\end{array}$ & $\begin{array}{l}\infty \\
\infty \\
\stackrel{n}{\curvearrowright} \\
i \\
0\end{array}$ & 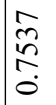 & $\left|\begin{array}{l}n \\
\frac{a}{d} \\
\stackrel{0}{0}\end{array}\right|$ & $\stackrel{0}{\stackrel{0}{0}}$ & $\stackrel{\overbrace{}}{\stackrel{f}{0}}$ & 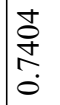 & 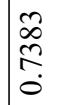 & 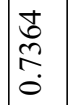 & 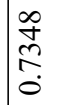 & 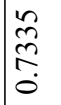 & 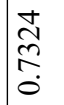 & $\frac{n}{m}$ & $\mid \begin{array}{c}\hat{2} \\
\stackrel{n}{0} \\
0\end{array}$ & $\stackrel{\Re}{\stackrel{0}{0}}$ & 离 & $\begin{array}{l}\stackrel{\infty}{\infty} \\
\stackrel{\text { N }}{0}\end{array}$ & 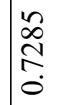 \\
\hline & & $\mathscr{J}$ & 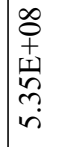 & $\begin{array}{l}\infty \\
0 \\
+ \\
\stackrel{+}{+} \\
\underset{j}{+} \\
\dot{m}\end{array}$ & 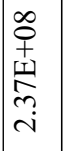 & 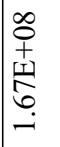 & 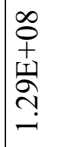 & $\begin{array}{l}\stackrel{0}{3} \\
\widehat{\delta} \\
0 \\
\mathbb{Z} \\
\sigma\end{array}$ & 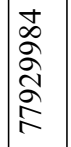 & 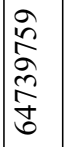 & 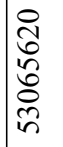 & 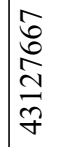 & $\mid \begin{array}{l}\tilde{n} \\
\tilde{n} \\
\approx \\
\overrightarrow{0} \\
n \\
m\end{array}$ & 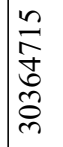 & 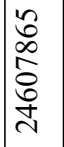 & 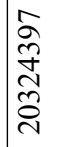 & 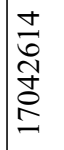 & 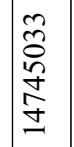 & 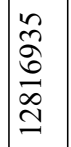 & \begin{tabular}{l}
$\hat{\sigma}$ \\
\multirow{2}{*}{} \\
$\vdots$ \\
$\vdots$ \\
$=$
\end{tabular} & 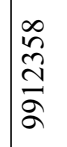 & 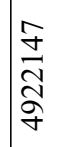 \\
\hline & & t & 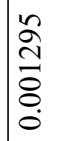 & ָ̃ & $\begin{array}{l}\tilde{n} \\
\hat{o} \\
0 \\
0 \\
0 \\
0\end{array}$ & 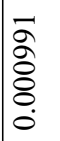 & $\begin{array}{l}\text { an } \\
\text { ôे } \\
8 \\
0 \\
0\end{array}$ & $\mid \begin{array}{l}0 \\
\vdots \\
0 \\
0 \\
0 \\
0 \\
0\end{array}$ & $\begin{array}{l}\infty \\
\infty \\
\infty \\
\varnothing \\
\vdots \\
0 \\
0\end{array}$ & $\mid \begin{array}{l}n \\
0 \\
\infty \\
0 \\
0 \\
0 \\
0\end{array}$ & $\begin{array}{l}n \\
\tilde{\infty} \\
o \\
o \\
0 \\
0\end{array}$ & $\begin{array}{l}\tilde{m} \\
\hat{\infty} \\
\stackrel{8}{8} \\
\stackrel{0}{0}\end{array}$ & $\begin{array}{l}\hat{\alpha} \\
\hat{\hat{o}} \\
0 \\
0 \\
0\end{array}$ & 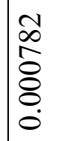 & $\mid \begin{array}{l}\hat{N} \\
\hat{\tilde{O}} \\
0 \\
0 \\
0\end{array}$ & 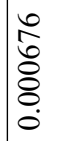 & 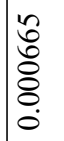 & $\mid \begin{array}{l}0 \\
0 \\
0 \\
0 \\
0 \\
0 \\
0\end{array}$ & $\mid \begin{array}{l}\tilde{Z} \\
\delta \\
\delta \\
0 \\
0\end{array}$ & 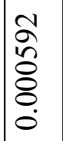 & \begin{tabular}{l}
$\infty$ \\
$\infty$ \\
$\infty$ \\
\hdashline \\
$\delta$ \\
0 \\
0
\end{tabular} & 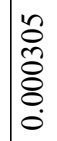 \\
\hline & & $\approx$ & $\mid$\begin{tabular}{l}
0 \\
$\infty$ \\
\hdashline \\
$=$
\end{tabular} & $\begin{array}{l}\stackrel{\infty}{\infty} \\
\stackrel{\Xi}{\Xi}\end{array}$ & 全 & 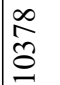 & ஓे & $\mid \begin{array}{l}\infty \\
6 \\
0 \\
2\end{array}$ & $\begin{array}{l}\infty \\
\infty \\
\sim\end{array}$ & $\frac{n}{a}$ & $\begin{array}{l}\vec{\nabla} \\
\infty \\
\infty \\
\infty\end{array}$ & $\frac{0}{0}$ & $\mid \begin{array}{c}\infty \\
\infty \\
\infty \\
\infty\end{array}$ & $\frac{\circ}{\infty}$ & 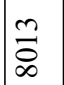 & 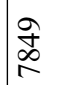 & Әे & $\begin{array}{l}\infty \\
n \\
n \\
n\end{array} \mid$ & $\mid \begin{array}{l}\tilde{I} \\
\stackrel{\pi}{2}\end{array}$ & 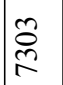 & $\underset{\infty}{\stackrel{\infty}{r}}$ & $\stackrel{\overbrace{}}{\gtrless}$ \\
\hline లే & రิ & 苫 & $\stackrel{\Xi}{=}$ & $\begin{array}{l}n \\
n \\
n \\
-1\end{array}$ & $\stackrel{\text { }}{\circ}$ & $\stackrel{n}{0}$ & กิ & 尚 & f̦. & $\stackrel{\infty}{+}$ & $\tilde{n}$ & $\hat{n}$ & $\stackrel{n}{q}$ & $\stackrel{f}{8}$ & I. & $\Xi_{0}^{+}$ & ț. & $\mid \begin{array}{l}0 \\
\tilde{n} \\
0\end{array}$ & $\begin{array}{l}m \\
\tilde{0}\end{array}$ & $\overrightarrow{3}$ & $\overrightarrow{3}$ & חొ \\
\hline $\begin{array}{l} \pm \\
\sigma \\
\frac{0}{U} \\
\Xi \Xi\end{array}$ & $\begin{array}{l}\infty \\
\stackrel{0}{0} \\
0 \\
0 \\
0 \\
0\end{array}$ & $\vec{z}$ & $\underset{\stackrel{m}{+}}{\stackrel{m}{m}}$ & $\begin{array}{l}\infty \\
\infty \\
\infty \\
\infty \\
m\end{array}$ & $\begin{array}{l}b \\
\infty \\
\dot{d} \\
\dot{\sim}\end{array}$ & $\overrightarrow{\tilde{d}}$ & 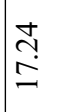 & 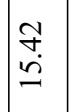 & $\begin{array}{l}\vec{n} \\
\dot{\Xi}\end{array}$ & $\begin{array}{l}0 \\
\\
\dot{J}\end{array}$ & $\begin{array}{l}\infty \\
\infty \\
\end{array}$ & $\exists$ & $\left|\begin{array}{l}n \\
\tilde{n} \\
n \\
n\end{array}\right|$ & $\begin{array}{l}\infty \\
\infty \\
\end{array}$ & $\stackrel{\Re}{=}$ & $\ddot{0}$ & $\vec{n}$ & $\begin{array}{l}\hat{\alpha} \\
\infty\end{array}$ & $\underset{\infty}{\mathcal{f}}$ & {$\left[\begin{array}{l}\infty \\
\infty \\
\\
\end{array}\right.$} & $\begin{array}{l}n \\
\infty \\
\end{array}$ & $\begin{array}{l}0 \\
\text { in }\end{array}$ \\
\hline $\begin{array}{l}\stackrel{\mathscr{z}}{\underline{\Delta}} \\
\frac{\vec{\omega}}{\Xi}\end{array}$ & 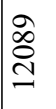 & 营 & $\stackrel{+}{\mathrm{I}}$ & ले & $\begin{array}{l}+ \\
\vdots \\
0\end{array}$ & . & $\tilde{n}$ & $\stackrel{\infty}{\stackrel{\infty}{0}}$ & f̊. & वे. & $\because$ & ñ & ñ & $\hat{n}$ & 守 & I & $\stackrel{?}{\stackrel{f}{0}}$ & 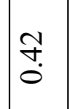 & $\stackrel{t}{0}$ & ले & t. & $\frac{0}{5}$ \\
\hline$\underset{\Xi}{\stackrel{\widehat{a}}{\Xi}}$ & 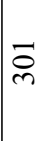 & $\underbrace{\stackrel{y}{\Xi}}_{\beth}$ & $\begin{array}{l}0 \\
\hat{0} \\
\hat{n}\end{array}$ & $\begin{array}{l}0 \\
\dot{0} \\
\dot{q}\end{array}$ & $\begin{array}{l}\vec{n} \\
\dot{m}\end{array}$ & 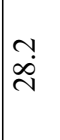 & $\begin{array}{l}\stackrel{0}{+} \\
\stackrel{\sim}{\sim}\end{array}$ & $\begin{array}{l}\hat{y} \\
\tilde{\lambda}\end{array}$ & $\begin{array}{l}\widehat{\widehat{~}} \\
\text { }\end{array}$ & तें & $\begin{array}{l}\infty \\
\infty \\
\tilde{\lambda}\end{array}$ & $\begin{array}{l}\overrightarrow{\widetilde{n}} \\
\ddot{\sim}\end{array}$ & $\mid \begin{array}{l}\stackrel{\sim}{\sim} \\
\stackrel{\dot{f}}{\sim} \\
\end{array}$ & $\underset{\stackrel{+}{+}}{\vec{d}}$ & $\begin{array}{l}\infty \\
n \\
i \\
\end{array}$ & 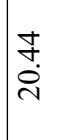 & ڤ̊ & 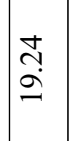 & $\begin{array}{l}0 \\
\infty \\
\infty\end{array}$ & 京 & $\underset{0}{0}$ & $\begin{array}{l}\widetilde{\sigma} \\
\dot{\Xi}\end{array}$ \\
\hline$\sum_{\text {至 }}$ & $\stackrel{t}{0}$ & $\begin{array}{l}\text { वै } \\
\text { है } \\
=-\end{array}$ & 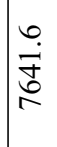 & 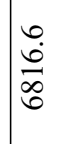 & 专 & $\begin{array}{l}0 \\
6 \\
\infty \\
\widehat{\sigma}\end{array}$ & $\begin{array}{l}n \\
\infty \\
\infty \\
\sigma \\
0\end{array}$ & $\mid \begin{array}{l}1 \\
8 \\
0 \\
0 \\
0\end{array}$ & $\begin{array}{l}n \\
0 \\
8 \\
8 \\
0\end{array}$ & 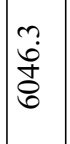 & $\begin{array}{l}b \\
\tilde{n} \\
\tilde{n} \\
\hat{n}\end{array}$ & 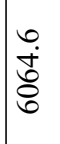 & 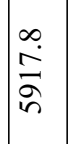 & $\frac{m}{n}$ & 咅 & 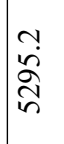 & $\begin{array}{l}\overrightarrow{0} \\
\stackrel{2}{2} \\
\text { in }\end{array}$ & $\begin{array}{l}+ \\
\stackrel{0}{0} \\
i \\
i n\end{array}$ & 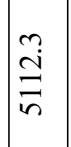 & $\frac{q}{q}$ & $\frac{a}{a}$ & $\begin{array}{l}n \\
\infty \\
\infty \\
0 \\
0 \\
\end{array}$ \\
\hline $\begin{array}{l}\sum_{\pi}^{2} \\
0 \\
0 \\
0 \\
0\end{array}$ & $\stackrel{i}{i}$ & $\theta$ & 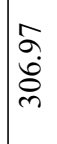 & $\begin{array}{l}\vec{N} \\
\text { } \\
\text { }\end{array}$ & $\begin{array}{l}n \\
0 \\
\infty \\
m \\
m\end{array}$ & $\begin{array}{l}\infty \\
\infty \\
\infty \\
\tilde{n} \\
m\end{array}$ & $\frac{+}{i}$ & 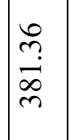 & $\begin{array}{l}\hat{\sigma} \\
\dot{+} \\
\tilde{m}\end{array}$ & $\stackrel{n}{\stackrel{2}{0}}$ & $\begin{array}{l}\vec{F} \\
\stackrel{\mathcal{O}}{\Im}\end{array}$ & $\mid \begin{array}{l}n \\
\tilde{n} \\
\tilde{\gamma}\end{array}$ & $\begin{array}{l}\tilde{n} \\
\tilde{o} \\
\dot{J} \\
y\end{array}$ & $\begin{array}{l}\infty \\
\infty \\
\infty \\
\infty \\
\wp\end{array}$ & $\begin{array}{l}\stackrel{8}{0} \\
\stackrel{8}{8} \\
\dot{f}\end{array}$ & $\frac{a}{\dot{\infty}}$ & $\begin{array}{l}\infty \\
\stackrel{\infty}{i} \\
\stackrel{\sigma}{\sigma}\end{array}$ & $\begin{array}{l}\hat{\sigma} \\
\dot{\hat{\sigma}} \\
\dot{n}\end{array}$ & $\begin{array}{l}2 \\
\tilde{n} \\
\tilde{n}\end{array}$ & $\begin{array}{l}\infty \\
\stackrel{+}{+} \\
\tilde{N}\end{array}$ & $\begin{array}{l}\overrightarrow{0} \\
\dot{f} \\
\tilde{n} \\
\end{array}$ & $\frac{0}{n}$ \\
\hline 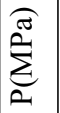 & $\begin{array}{l}n \\
\vdots \\
0\end{array}$ & 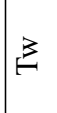 & 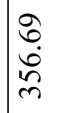 & 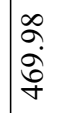 & $\begin{array}{l}\exists \\
\stackrel{7}{2} \\
\text { î }\end{array}$ & $\begin{array}{l} \pm \\
\infty \\
\infty \\
i n \\
i n\end{array}$ & 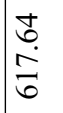 & $\begin{array}{l}n \\
\stackrel{n}{a} \\
\mathcal{G}\end{array}$ & 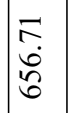 & 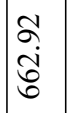 & $\begin{array}{l}n \\
n \\
\tilde{2} \\
6\end{array}$ & 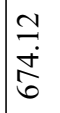 & $\begin{array}{l}\forall \\
\ddot{a} \\
\ddot{0} \\
0\end{array}$ & $\begin{array}{l}2 \\
\infty \\
\infty \\
0\end{array}$ & 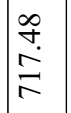 & $\begin{array}{l}\circ \\
\stackrel{2}{0} \\
\stackrel{+}{+}\end{array}$ & $\begin{array}{l}\stackrel{1}{2} \\
\infty \\
\stackrel{\infty}{i}\end{array}$ & $\begin{array}{l}m \\
\tilde{R} \\
\stackrel{N}{N}\end{array}$ & 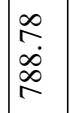 & $\stackrel{\infty}{\stackrel{\infty}{\dot{d}}}$ & & 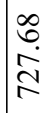 \\
\hline 怘 & $\because$ & $\stackrel{9}{9}$ & 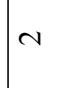 & $\vec{\infty}$ & $\overrightarrow{ \pm}$ & กิ & ¿ֶ. & 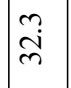 & $\mid \begin{array}{c}m \\
\infty \\
m \\
m\end{array}$ & $\mid \begin{array}{l}\dot{J} \\
\dot{f}\end{array}$ & $\begin{array}{l}t \\
\dot{B}\end{array}$ & \begin{tabular}{l}
$n$ \\
\hdashline \\
\hdashline \\
$n$
\end{tabular} & $\left|\begin{array}{l}n \\
i \\
0\end{array}\right|$ & $\begin{array}{l}n \\
\infty \\
\infty \\
0\end{array}$ & $\mid \begin{array}{l}0 \\
\stackrel{+}{i}\end{array}$ & $\begin{array}{l}0 \\
\dot{\infty} \\
\infty\end{array}$ & $\hat{\infty}$ & $\bar{i}$ & $\begin{array}{l}\infty \\
\infty \\
\infty\end{array}$ & $\begin{array}{l}\infty \\
\dot{0} \\
\dot{0}\end{array}$ & $\stackrel{\partial}{\varrho}$ & $\hat{\sigma}$ \\
\hline
\end{tabular}




\begin{tabular}{|c|c|c|c|c|c|c|c|c|c|c|c|c|c|c|c|c|c|c|c|c|c|c|}
\hline & & $\vec{\Delta}$ & 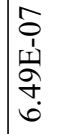 & 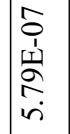 & 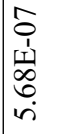 & 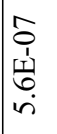 & 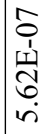 & 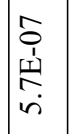 & 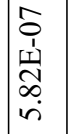 & 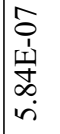 & 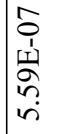 & 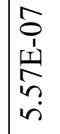 & 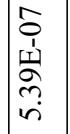 & 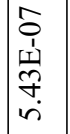 & 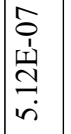 & 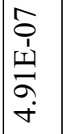 & 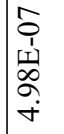 & $\mid \begin{array}{l}\hat{o} \\
\dot{1} \\
\hat{y} \\
\hat{\alpha} \\
\dot{\gamma}\end{array}$ & 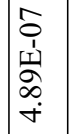 & 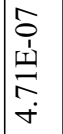 & $\mid \begin{array}{l}\hat{a} \\
\hat{1} \\
\tilde{n} \\
\hat{n} \\
\dot{\gamma}\end{array}$ & $\begin{array}{c}\hat{o} \\
\mathbf{1} \\
\text { m }\end{array}$ \\
\hline & & $\stackrel{\oplus}{\infty}$ & 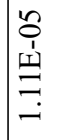 & \begin{tabular}{c}
8 \\
0 \\
1 \\
$=1$ \\
\hdashline \\
1
\end{tabular} & 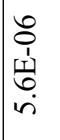 & 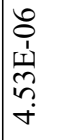 & $\begin{array}{l}8 \\
1 \\
1 \\
1 \\
\infty \\
\end{array}$ & 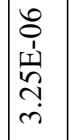 & 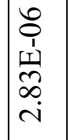 & 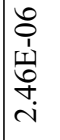 & 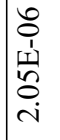 & 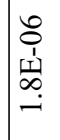 & 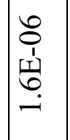 & 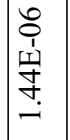 & 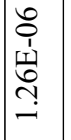 & 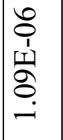 & 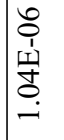 & $\begin{array}{c}\hat{s} \\
\hat{1} \\
\underline{\omega} \\
\tilde{n} \\
a\end{array}$ & 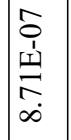 & 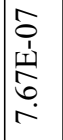 & 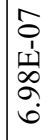 & 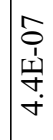 \\
\hline & & $\vec{D}$ & 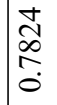 & $\begin{array}{l}\hat{\hat{0}} \\
\hat{0} \\
0\end{array}$ & $\begin{array}{l}\vec{D} \\
\stackrel{\infty}{2} \\
0 \\
0\end{array}$ & $\begin{array}{c}\stackrel{8}{\circ} \\
\stackrel{n}{2} \\
\dot{0}\end{array}$ & 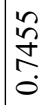 & $\mid \begin{array}{c}\mathbb{7} \\
\underset{0}{0}\end{array}$ & 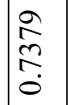 & 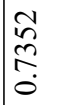 & $\stackrel{\widetilde{N}}{\stackrel{\overbrace{}}{0}}$ & $\underset{\substack{0 \\
\stackrel{n}{0}}}{\stackrel{0}{0}}$ & $\mid \begin{array}{c}\stackrel{m}{p} \\
\stackrel{p}{0} \\
\dot{0}\end{array}$ & 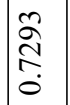 & $\left|\begin{array}{l}\mathscr{b} \\
\stackrel{\mathbb{N}}{N} \\
\dot{0}\end{array}\right|$ & $\begin{array}{l}\infty \\
\stackrel{N}{\mathbb{N}} \\
\dot{0}\end{array}$ & 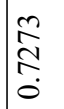 & 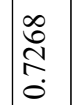 & 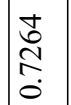 & $\begin{array}{l}\overrightarrow{0} \\
\stackrel{N}{0} \\
\dot{0}\end{array}$ & 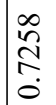 & مُ \\
\hline & & $\mathscr{J}$ & \begin{tabular}{|l}
$\infty$ \\
0 \\
0 \\
\pm \\
m. \\
$\infty$ \\
$\infty$
\end{tabular} & $\begin{array}{l}\infty \\
0 \\
+ \\
\text { In } \\
\vdots \\
\dot{\sigma} \\
\dot{+}\end{array}$ & 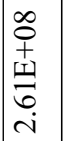 & 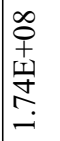 & 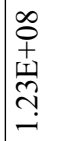 & $\mid \begin{array}{l}i \\
n \\
\tilde{n} \\
\tilde{n} \\
\tilde{\delta} \\
\alpha\end{array}$ & $\begin{array}{l}\widetilde{\alpha} \\
\stackrel{\infty}{\infty} \\
0 \\
\infty \\
\infty \\
0\end{array}$ & 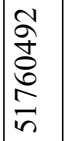 & 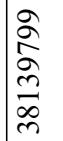 & 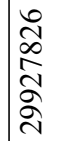 & 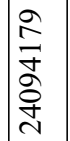 & 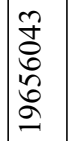 & $\mid \begin{array}{l}0 \\
0 \\
\infty \\
0 \\
\tilde{n} \\
n\end{array}$ & 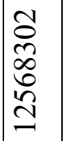 & $\mid \begin{array}{l}\hat{n} \\
\hat{\alpha} \\
o \\
0 \\
=\end{array}$ & $\begin{array}{l}\tilde{N} \\
\tilde{\widetilde{D}} \\
\tilde{N}\end{array}$ & 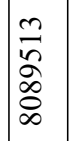 & $\begin{array}{l}7 \\
0 \\
0 \\
0 \\
0 \\
0\end{array}$ & 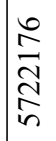 & 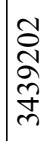 \\
\hline & & t & $\frac{\tilde{2}}{2}$ & $\begin{array}{l}\tilde{\delta} \\
0 \\
0 \\
8 \\
0 \\
0\end{array}$ & 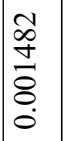 & 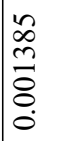 & $\begin{array}{l}\bar{\lambda} \\
\tilde{\sigma} \\
\overline{8} \\
0 \\
0\end{array}$ & 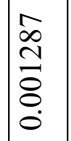 & 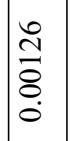 & 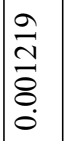 & $\begin{array}{l}\varkappa \\
ٍ \\
\bar{\Xi} \\
0 \\
0\end{array}$ & $\begin{array}{l}\hat{\alpha} \\
\stackrel{\circ}{\circ} \\
\stackrel{8}{\circ} \\
\stackrel{0}{\circ}\end{array}$ & $\mid \begin{array}{l}0 \\
0 \\
\\
0 \\
0 \\
0\end{array}$ & 官 & 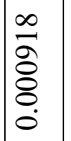 & $\begin{array}{l}0 \\
\infty \\
0 \\
0 \\
0 \\
0\end{array}$ & $\begin{array}{l}\text { 芯 } \\
\infty \\
0 \\
0 \\
0 \\
0 \\
0\end{array}$ & $\begin{array}{c}\tilde{\hat{A}} \\
\tilde{0} \\
\dot{0} \\
0 \\
0\end{array}$ & $\mid \begin{array}{l}n \\
0 \\
0 \\
0 \\
0 \\
0 \\
0\end{array}$ & 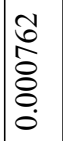 & 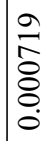 & 字 \\
\hline & & $\approx$ & $\frac{m}{a}$ & $\stackrel{\stackrel{\infty}{0}}{\stackrel{\Xi}{=}}$ & $\hat{\tilde{g}}$ & இু & वे & ڤ̂̀ & : & $\underset{\infty}{\vec{\Phi}}$ & $\begin{array}{l}n \\
\hat{\infty} \\
\infty\end{array}$ & $\stackrel{2}{\curvearrowright}$ & 点 & \begin{tabular}{l}
$n$ \\
0 \\
\hdashline
\end{tabular} & $\frac{\infty}{\stackrel{\infty}{r}}$ & $\frac{8}{2}$ & $\begin{array}{l}\widehat{W} \\
\infty \\
0 \\
0\end{array}$ & 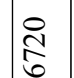 & $\begin{array}{l}\infty \\
\infty \\
6\end{array}$ & \begin{tabular}{l}
\multirow{U}{0}{} \\
ț
\end{tabular} & $\begin{array}{c}\sqrt{n} \\
\tilde{6}\end{array}$ & 孛 \\
\hline שี & ठิ & 䓂 & $\underline{\Xi}$ & $\vec{m}$ & $\begin{array}{l}\infty \\
\infty \\
\infty\end{array}$ & : & ڤn. & $\hat{n}$ & $\begin{array}{l}\infty \\
n \\
n \\
0\end{array}$ & ?n & $\bar{n}$ & $\stackrel{+}{n}$ & $\exists$ & $\mid \begin{array}{l}\infty \\
\tilde{n} \\
0\end{array}$ & J্ & 3 & $\tilde{m}$ & $\begin{array}{l}\infty \\
\\
0\end{array}$ & 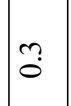 & กֶ. & $\stackrel{n}{a}$ & $\stackrel{?}{\mathscr{O}}$ \\
\hline $\begin{array}{l} \pm \\
\sigma \\
\frac{\overrightarrow{0}}{\Xi}\end{array}$ & 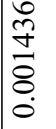 & $\vec{z}$ & 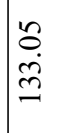 & ले & 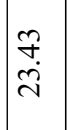 & $\mid \begin{array}{l}\alpha \\
\infty \\
\infty\end{array}$ & $\hat{\sigma}$ & 孚 & $\begin{array}{l}\infty \\
n \\
n \\
ٍ \\
n\end{array}$ & in & $\begin{array}{l}\mathbb{N} \\
\stackrel{2}{g}\end{array}$ & $\begin{array}{l}\tilde{J} \\
\stackrel{\text { Ij }}{ }\end{array}$ & 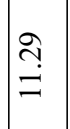 & $\underset{2}{\stackrel{2}{0}}$ & $\mid \begin{array}{l}\infty \\
n \\
a\end{array}$ & $\begin{array}{l}3 \\
\infty \\
\infty\end{array}$ & $\mid \begin{array}{l}n \\
q \\
\infty\end{array}$ & $\mid \begin{array}{l}\partial \\
\infty \\
\infty\end{array}$ & $\stackrel{\infty}{\sim}$ & $\stackrel{m}{?}$ & Oे. & 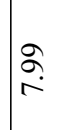 \\
\hline 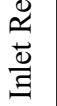 & 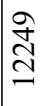 & 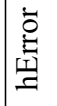 & $\stackrel{\infty}{\stackrel{\infty}{?}}$ & $\stackrel{6}{\Perp}$ & 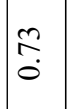 & $\stackrel{0}{0}$ & $\hat{n}$ & $\stackrel{0}{0}$ & $\stackrel{6}{0}$ & 文 & $\vec{\sigma}$ & $\stackrel{\infty}{\infty}$ & $\tilde{n}$ & $\hat{n}$ & 守. & $\stackrel{0}{+}$ & $\stackrel{0}{0}$ & 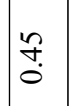 & 年 & $\stackrel{7}{0}$ & $\stackrel{n}{\stackrel{n}{0}}$ & $\stackrel{\hat{\theta}}{.}$ \\
\hline$\underset{\Xi}{\stackrel{્}{\Xi}}$ & Бे & 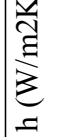 & $\begin{array}{l}\Phi \\
\infty \\
\dot{0} \\
\end{array}$ & $\underset{\hat{i}}{\stackrel{i}{f}}$ & $\begin{array}{l}\infty \\
\stackrel{N}{1} \\
i \\
\end{array}$ & $\mid \begin{array}{l}\infty \\
\infty \\
\infty \\
\stackrel{\sim}{\sim}\end{array}$ & 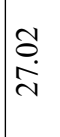 & $\left|\begin{array}{l}m \\
\dot{0} \\
\infty \\
\sim\end{array}\right|$ & $\begin{array}{l}\hat{\hat{\lambda}} \\
\hat{\lambda}\end{array}$ & 离 & $\begin{array}{l}\tilde{n} \\
\stackrel{\sim}{\sim}\end{array}$ & 吕 & $\begin{array}{l}\mathbb{N} \\
\dot{J}\end{array}$ & $\begin{array}{l}0 \\
\stackrel{n}{\sim} \\
\stackrel{+}{n}\end{array}$ & $\begin{array}{l}0 \\
\dot{i}\end{array}$ & $\frac{\partial}{\vec{i}}$ & $\underset{\stackrel{J}{N}}{\stackrel{N}{N}}$ & $\begin{array}{l}\alpha \\
\hat{\tilde{d}} \\
\end{array}$ & 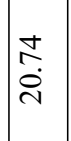 & ô & $\mid \begin{array}{l}\infty \\
\varrho \\
\varrho\end{array}$ & $\begin{array}{l}\infty \\
\grave{\lambda}\end{array}$ \\
\hline$\sum_{\text {至 }}$ & $\stackrel{t}{0}$ & $\begin{array}{l}\text { 己ै } \\
\vdots \\
= \\
\bar{\sigma}\end{array}$ & 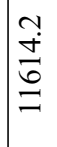 & $\tilde{\delta}$ & $\begin{array}{l}\infty \\
\stackrel{0}{0} \\
\stackrel{0}{\circ}\end{array}$ & {$\left[\begin{array}{c}n \\
\infty \\
\infty \\
\tilde{\alpha}\end{array}\right.$} & $\underset{\stackrel{J}{i}}{\stackrel{\sim}{\sim}}$ & $\begin{array}{l}\vec{n} \\
\tilde{n} \\
\sigma\end{array}$ & $\stackrel{m}{\tilde{f}}$ & $\overrightarrow{\overline{\vec{\sigma}}}$ & $\begin{array}{c}n \\
\infty \\
\infty \\
\infty \\
\infty \\
\infty\end{array}$ & $\underset{\infty}{\stackrel{N}{\stackrel{\infty}{\infty}}}$ & $\begin{array}{l}0 \\
\dot{J} \\
\dot{J}\end{array}$ & $\mid \begin{array}{l}- \\
\infty \\
\infty \\
\infty \\
\infty\end{array}$ & $\mid$\begin{tabular}{l}
$n$ \\
\multirow{2}{a}{} \\
$\stackrel{2}{r}$
\end{tabular} & $\frac{1}{\vec{\delta}}$ & $\frac{\mathfrak{i}}{\stackrel{2}{i}}$ & 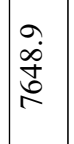 & 竞 & $\begin{array}{l}\infty \\
\stackrel{\infty}{1} \\
\underset{r}{1}\end{array}$ & îj & $\begin{array}{l}+ \\
\dot{0} \\
0 \\
0 \\
+\end{array}$ \\
\hline $\begin{array}{l}\sum_{\pi} \\
0 \\
0 \\
0 \\
0\end{array}$ & ఏ & $\vec{F}$ & के & $\begin{array}{l}\vec{n} \\
\stackrel{p}{m} \\
m\end{array}$ & $\begin{array}{l}n \\
\tilde{n} \\
b \\
n \\
n\end{array}$ & 吕. & $\begin{array}{l}\text { vo } \\
\stackrel{1}{2} \\
\text { ले }\end{array}$ & $\begin{array}{l}\vec{\sigma} \\
\vec{\sigma} \\
\vec{\sigma}\end{array}$ & 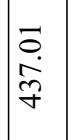 & 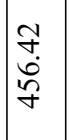 & 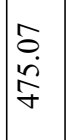 & 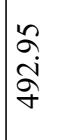 & $\begin{array}{l}\tilde{z} \\
\tilde{0} \\
\stackrel{n}{n}\end{array}$ & $\mid \begin{array}{l}\hat{0} \\
\hat{\tilde{n}} \\
\hat{n}\end{array}$ & 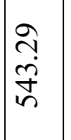 & $\begin{array}{l}n \\
n \\
\infty \\
\infty \\
n\end{array}$ & 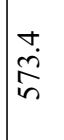 & $\begin{array}{l}\infty \\
\infty \\
\infty \\
\infty \\
\infty\end{array}$ & $\begin{array}{l}\mathbb{N} \\
\stackrel{i}{\delta} \\
\delta\end{array}$ & 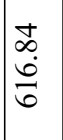 & 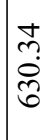 & 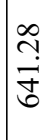 \\
\hline 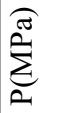 & $\begin{array}{l}\hat{0} \\
0\end{array}$ & 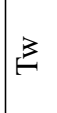 & $\mid \begin{array}{r}\hat{b} \\
\infty \\
\infty \\
\cdots\end{array}$ & 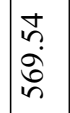 & 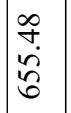 & $\mid \begin{array}{l}\stackrel{\partial}{ } \\
\dot{0} \\
\dot{\rho}\end{array}$ & $\begin{array}{l}\text { రె } \\
\stackrel{2}{2} \\
\approx\end{array}$ & 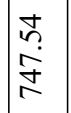 & $\frac{\partial}{\vec{a}}$ & \begin{tabular}{l}
$\vec{m}$ \\
$\tilde{\sigma}$ \\
\multirow{2}{*}{}
\end{tabular} & $\begin{array}{l}7 \\
\circ \\
2\end{array}$ & $\begin{array}{l}\infty \\
\infty \\
\tilde{\lambda} \\
\infty\end{array}$ & $\begin{array}{l}\stackrel{0}{1} \\
\dot{\vec{n}} \\
\infty\end{array}$ & $\left|\begin{array}{c}\hat{b} \\
\dot{\mathbb{N}} \\
\hat{\infty}\end{array}\right|$ & 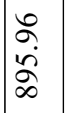 & \begin{tabular}{l}
0 \\
\multirow{2}{*}{} \\
$\stackrel{\text { a }}{ }$
\end{tabular} & $\mid \begin{array}{l}\vec{b} \\
\stackrel{j}{\alpha} \\
\alpha\end{array}$ & $\begin{array}{l}\hat{\alpha} \\
\tilde{n} \\
\alpha\end{array}$ & $\mid \begin{array}{l}0 \\
0 \\
\infty \\
0 \\
0\end{array}$ & $\begin{array}{l}\vec{m} \\
\underset{i}{\infty} \\
\infty \\
\sigma\end{array}$ & $\approx$ & 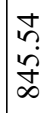 \\
\hline $\begin{array}{l}0 \\
0 \\
0 \\
0 \\
0\end{array}$ & in & $\stackrel{\rho}{a}$ & $N$ & $\vec{\infty}$ & $\overrightarrow{ \pm}$ & กิ & तु. & 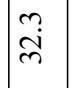 & $\mid \begin{array}{c}m \\
\infty \\
m \\
m\end{array}$ & $\mid \begin{array}{l}\dot{J} \\
\dot{f}\end{array}$ & $\begin{array}{l}t \\
\dot{B}\end{array}$ & \begin{tabular}{l}
$n$ \\
\hdashline \\
\hdashline \\
$n$
\end{tabular} & $\begin{array}{l}n \\
i \\
\delta\end{array}$ & $\left|\begin{array}{l}n \\
\infty \\
0 \\
0\end{array}\right|$ & $\begin{array}{l}0 \\
\stackrel{+}{i}\end{array}$ & $\begin{array}{l}0 \\
\dot{\infty} \\
\infty\end{array}$ & $\hat{\infty}$ & $\bar{i}$ & $\begin{array}{l}\infty \\
\infty \\
\infty\end{array}$ & $\begin{array}{l}\infty \\
\dot{0} \\
\dot{0}\end{array}$ & 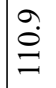 & $\hat{\sigma}$ \\
\hline
\end{tabular}




\begin{tabular}{|c|c|c|c|c|c|c|c|c|c|c|c|c|c|c|c|c|c|c|c|c|c|c|}
\hline & & $\vec{z}$ & 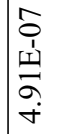 & $\begin{array}{c}\hat{0} \\
1 \\
1 \\
\underline{1} \\
+ \\
+\end{array}$ & $\begin{array}{l}\hat{1} \\
0 \\
1 \\
1 \\
b \\
b \\
\dot{+}\end{array}$ & 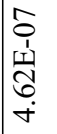 & 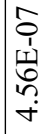 & 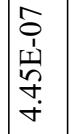 & 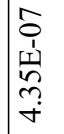 & 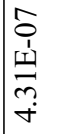 & \begin{tabular}{l}
$\hat{o}$ \\
$\hat{1}$ \\
$\underline{1}$ \\
\\
\hdashline \\
$\dot{\gamma}$
\end{tabular} & 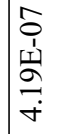 & $\begin{array}{l}\hat{O} \\
\stackrel{1}{1} \\
\stackrel{1}{N} \\
\stackrel{+}{+}\end{array}$ & 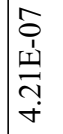 & 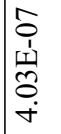 & $\begin{array}{l}\hat{\sigma} \\
\hat{1} \\
\underline{1} \\
\hat{n} \\
\dot{m}\end{array}$ & 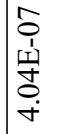 & $\begin{array}{c}\hat{o} \\
\dot{1} \\
\hat{1} \\
\hat{0} \\
\dot{r}\end{array}$ & $\mid \begin{array}{l}\hat{O} \\
\hat{1} \\
\stackrel{1}{\Delta} \\
\dot{\sigma} \\
\dot{+}\end{array}$ & \begin{tabular}{|l}
$\hat{o}$ \\
$\hat{1}$ \\
$\underline{1}$ \\
$\hat{\sigma}$ \\
$\dot{n}$
\end{tabular} & 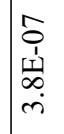 & 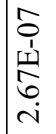 \\
\hline & & $\stackrel{\oplus}{\infty}$ & 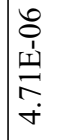 & $\begin{array}{l}8 \\
0 \\
1 \\
\\
\\
n\end{array}$ & $\begin{array}{l}0 \\
0 \\
1 \\
1 \\
\vdots \\
\vdots \\
i \\
i\end{array}$ & 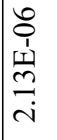 & 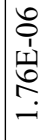 & 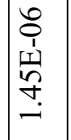 & 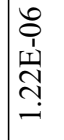 & 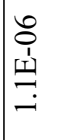 & 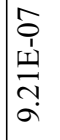 & $\mid \begin{array}{l}\hat{a} \\
\hat{1} \\
\underline{1} \\
\hat{a} \\
\infty\end{array}$ & 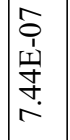 & 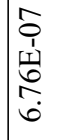 & 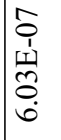 & $\begin{array}{l}\hat{s} \\
\hat{1} \\
\underline{1} \\
\tilde{m} \\
i n\end{array}$ & 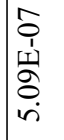 & $\begin{array}{l}\hat{o} \\
\hat{1} \\
\underline{1} \\
\dot{\sigma} \\
\dot{+}\end{array}$ & 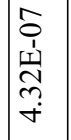 & 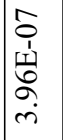 & 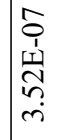 & 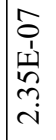 \\
\hline & & $\vec{D}$ & 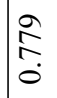 & $\begin{array}{l}\overrightarrow{8} \\
\stackrel{0}{0} \\
0\end{array}$ & $\stackrel{\sqrt{n}}{\stackrel{n}{a}}$ & 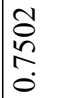 & $\stackrel{i}{\stackrel{2}{f}}$ & 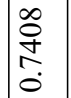 & 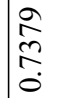 & 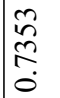 & 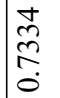 & 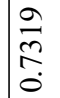 & $\mid \begin{array}{c}\hat{0} \\
\hat{n} \\
0 \\
0\end{array}$ & 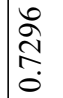 & $\begin{array}{l}\infty \\
\stackrel{\infty}{\mathbb{N}} \\
\stackrel{N}{0}\end{array}$ & $\begin{array}{l}\vec{\infty} \\
\stackrel{N}{0} \\
\dot{0}\end{array}$ & $\begin{array}{l}\stackrel{n}{\hat{N}} \\
\stackrel{N}{0} \\
\stackrel{0}{*}\end{array}$ & 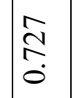 & 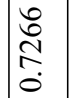 & $\begin{array}{l}\stackrel{\mathfrak{d}}{\mathrm{N}} \\
\stackrel{N}{0}\end{array}$ & 离 & 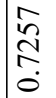 \\
\hline & & $\mathscr{J}$ & 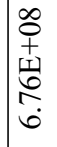 & $\begin{array}{l}\infty \\
0 \\
+ \\
\text { t1 } \\
\text { ñ } \\
\text { r }\end{array}$ & 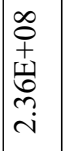 & $\mid \begin{array}{l}\infty \\
0 \\
+ \\
1 \\
\vdots \\
2 \\
\\
-1\end{array}$ & 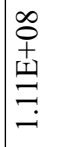 & $\mid \begin{array}{l}2 \\
\infty \\
n \\
\tilde{n} \\
\infty \\
\infty \\
⿱ 乛\end{array}$ & $\begin{array}{l}8 \\
8 \\
0 \\
8 \\
\text { in } \\
\text { in }\end{array}$ & 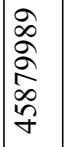 & 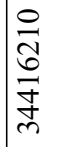 & 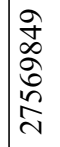 & 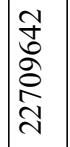 & $\begin{array}{l}\tilde{0} \\
\tilde{\delta} \\
\tilde{N} \\
\infty \\
\infty \\
-\end{array}$ & $\mid \begin{array}{l}\mathbb{D} \\
\stackrel{D}{\infty} \\
\infty \\
\infty \\
\infty \\
\tilde{n} \\
-\end{array}$ & 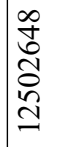 & $\begin{array}{l}0 \\
\tilde{2} \\
\tilde{2} \\
\stackrel{\partial}{0} \\
ٍ\end{array}$ & $\begin{array}{l}\text { ò } \\
\text { } \\
\delta \\
\sigma \\
\sigma\end{array}$ & $\begin{array}{l}\hat{n} \\
\hat{n} \\
\tilde{\infty} \\
\infty\end{array}$ & $\frac{0}{\frac{1}{5}}$ & $\begin{array}{l}\stackrel{P}{+} \\
\infty \\
\infty \\
\infty \\
\infty \\
\infty\end{array}$ & 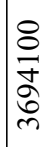 \\
\hline & & t & 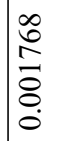 & 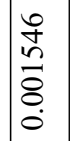 & 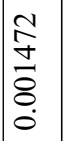 & 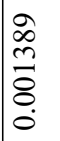 & 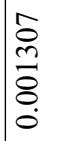 & $\mid \begin{array}{c}\tilde{Z} \\
\underset{\Xi}{\Xi} \\
\stackrel{8}{0} \\
\dot{0}\end{array}$ & 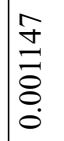 & $\mid \begin{array}{l}\infty \\
0 \\
0 \\
0 \\
0 \\
0 \\
0\end{array}$ & $\begin{array}{l}\tilde{\partial} \\
\tilde{\delta} \\
\stackrel{0}{0} \\
0 \\
0\end{array}$ & $\frac{\tilde{o}}{0}$ & 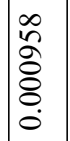 & 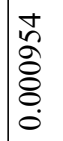 & 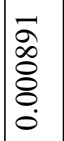 & $\begin{array}{l}\tilde{n} \\
\infty \\
o \\
0 \\
0 \\
0\end{array}$ & $\begin{array}{l}\tilde{\alpha} \\
\hat{\infty} \\
0 \\
0 \\
0 \\
0\end{array}$ & $\begin{array}{l}\tilde{O} \\
\tilde{O} \\
\delta \\
\delta \\
0 \\
0\end{array}$ & 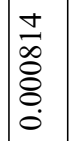 & 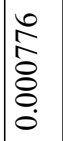 & 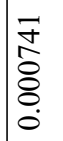 & $\begin{array}{l}\frac{m}{n} \\
\frac{n}{8} \\
0 \\
0\end{array}$ \\
\hline & & $\approx$ & $\stackrel{n}{g}$ & $\begin{array}{l}n \\
n \\
m \\
m\end{array}$ & 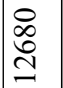 & 芯 & 过 & 帘 & \begin{tabular}{l}
8 \\
$\varnothing$ \\
\hdashline \\
\hdashline
\end{tabular} & $\frac{\hat{\partial}}{\partial}$ & $\begin{array}{l}\hat{b} \\
\approx \\
\approx\end{array}$ & $\hat{\kappa}$ & $\vec{m}$ & $\begin{array}{l}0 \\
8 \\
8\end{array}$ & $\begin{array}{l}q \\
\substack{f \\
\infty \\
\infty}\end{array}$ & $\begin{array}{l}\hat{0} \\
\infty \\
\infty\end{array}$ & $\underset{⿱}{\stackrel{f}{\infty}}$ & 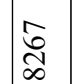 & ठे & $\frac{1}{2}$ & $\underset{\substack{2 \\
\curvearrowright}}{\stackrel{\infty}{2}}$ & $\hat{\infty}$ \\
\hline లే & రิ & 苫 & 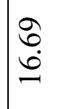 & 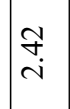 & 苂 & 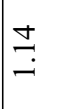 & $\begin{array}{l}\infty \\
\infty \\
0 \\
0\end{array}$ & $\begin{array}{l}n \\
0 \\
0\end{array}$ & $\stackrel{m}{0}$ & ?n & $\stackrel{n}{\stackrel{2}{0}}$ & $\stackrel{+}{n}$ & $\stackrel{+}{\circ}$ & $\stackrel{0}{\stackrel{0}{0}}$ & ले & $\stackrel{7}{\circ}$ & 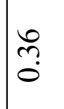 & \begin{tabular}{l}
$n$ \\
\multirow{2}{0}{}
\end{tabular} & $\left|\begin{array}{l}n \\
\tilde{n} \\
0\end{array}\right|$ & $\stackrel{\infty}{\infty}$ & חొ & 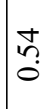 \\
\hline $\begin{array}{l} \pm \\
\sigma \\
\frac{\overrightarrow{0}}{\Xi}\end{array}$ & $\begin{array}{l}\frac{1}{\hat{n}} \\
\stackrel{2}{8} \\
0 \\
0\end{array}$ & $\vec{z}$ & 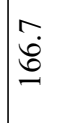 & $\begin{array}{l}\mathcal{f} \\
\text { g. }\end{array}$ & $\begin{array}{l}\mathbb{U} \\
\dot{b} \\
\dot{b} \\
\text {. }\end{array}$ & $\begin{array}{l}\hat{a} \\
\infty \\
i \\
i\end{array}$ & $\overrightarrow{\widehat{\vec{\lambda}}}$ & $\begin{array}{l}2 \\
\infty \\
\infty\end{array}$ & 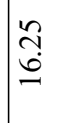 & $\begin{array}{l}\mathfrak{6} \\
\dot{ \pm} \\
\dot{0}\end{array}$ & $\begin{array}{l}\vec{b} \\
\text { i }\end{array}$ & $\begin{array}{l}\infty \\
\stackrel{\infty}{0} \\
\stackrel{\text { in }}{ }\end{array}$ & $\stackrel{m}{=}$ & $\stackrel{\Xi}{=}$ & $\mid \begin{array}{l}\infty \\
n \\
0 \\
0\end{array}$ & $\stackrel{0}{0}$ & $\stackrel{ \pm}{\stackrel{\oplus}{\Theta}}$ & $\hat{\alpha}$ & $\hat{a}$ & त̂̀ & $\hat{\sigma}$ & 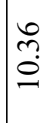 \\
\hline $\begin{array}{l}\stackrel{\mathscr{z}}{\underline{\Delta}} \\
\frac{\vec{\omega}}{\Xi}\end{array}$ & 突 & 营 & 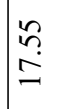 & $\stackrel{\infty}{\vec{i}}$ & $\stackrel{\infty}{\stackrel{\infty}{\rightarrow}}$ & $\stackrel{\cong}{\rightleftharpoons}$ & 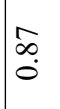 & $\stackrel{\Re}{\stackrel{0}{0}}$ & 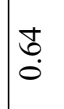 & in & $\tilde{n}$ & $\tilde{n}$ & $\bar{n}$ & $\hat{n}$ & $\tilde{n}$ & $\tilde{n}$ & $\stackrel{+}{n}$ & $\hat{n}$ & ñn & $\tilde{n}$ & $\tilde{n}$ & $\stackrel{\widetilde{I}}{-}$ \\
\hline$\underset{\Xi}{\stackrel{\Xi}{\Xi}}$ & D্ & 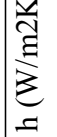 & 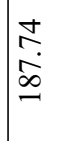 & $\frac{\tilde{6}}{\dot{6}}$ & $\begin{array}{l}\infty \\
\dot{g} \\
\dot{q}\end{array}$ & $\mid$\begin{tabular}{l}
$\infty$ \\
$\tilde{n}$ \\
$\mathfrak{f}$ \\
\cline { 1 - 1 }
\end{tabular} & $\frac{\infty}{0}$ & $\frac{\hat{\alpha}}{\bar{m}}$ & $\begin{array}{l}\infty \\
\infty \\
\infty \\
\infty\end{array}$ & $\stackrel{+}{\stackrel{\sim}{\sim}}$ & $\begin{array}{l}\hat{\infty} \\
\stackrel{\sim}{\sim}\end{array}$ & 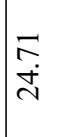 & 猋 & $\begin{array}{l}\hat{0} \\
\ddot{i}\end{array}$ & $\begin{array}{l}\tilde{N} \\
\stackrel{\sim}{+}\end{array}$ & $\begin{array}{l}\infty \\
\infty \\
\end{array}$ & $\begin{array}{l}\stackrel{n}{\stackrel{n}{d}} \\
\stackrel{d}{d}\end{array}$ & $\begin{array}{l}\hat{\alpha} \\
\dot{d}\end{array}$ & $\vec{Z}$ & $\begin{array}{l}\tilde{n} \\
\stackrel{\sim}{\sim}\end{array}$ & $\begin{array}{l}\stackrel{\Xi}{0} \\
\stackrel{+}{\sim}\end{array}$ & $\begin{array}{l}n \\
\infty \\
\infty \\
\infty \\
i\end{array}$ \\
\hline$\sum_{\text {至 }}$ & $\stackrel{0}{0}$ & $\begin{array}{l}\text { đิ } \\
\text { है } \\
= \\
=\end{array}$ & 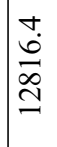 & $\begin{array}{l}\underset{0}{0} \\
\widetilde{\delta} \\
ٍ \\
=\end{array}$ & $\stackrel{\check{\hat{\sigma}}}{\stackrel{\vec{n}}{=}}$ & 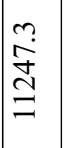 & סै & 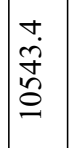 & $\begin{array}{l}n \\
\infty \\
\infty \\
0 \\
0\end{array}$ & ठิ & î̀ & 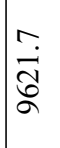 & $\begin{array}{l}n \\
m \\
\vec{m} \\
\sigma\end{array}$ & $\begin{array}{l}\infty \\
\infty \\
2\end{array}$ & $\begin{array}{l}\hat{a} \\
\hat{b} \\
\vec{\sigma}\end{array}$ & $\begin{array}{l}\stackrel{+}{b} \\
\stackrel{2}{\infty} \\
\infty\end{array}$ & $\stackrel{+}{\stackrel{\dot{\gamma}}{+}}$ & $\begin{array}{l}\infty \\
\dot{a} \\
\alpha \\
\sigma\end{array}$ & $\begin{array}{l}0 \\
\stackrel{8}{0} \\
\stackrel{0}{0} \\
0\end{array}$ & $\begin{array}{l}\hat{b} \\
\sim \\
\infty \\
\infty\end{array}$ & $\begin{array}{l}\overrightarrow{1} \\
\infty \\
\infty \\
\infty\end{array}$ & 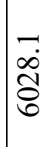 \\
\hline $\begin{array}{l}\sum_{\pi} \\
0 \\
0 \\
0 \\
0\end{array}$ & $\bar{\Xi}$ & $\vec{F}$ & $\begin{array}{l}0 \\
ٌ \\
\infty \\
0 \\
0 \\
ల\end{array}$ & $\begin{array}{l}\stackrel{a}{\vec{i}} \\
\stackrel{m}{m}\end{array}$ & $\begin{array}{l}\infty \\
\infty \\
\infty \\
\tilde{n} \\
n\end{array}$ & 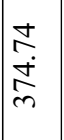 & $\begin{array}{l}\infty \\
\dot{d} \\
\dot{m}\end{array}$ & 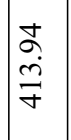 & $\begin{array}{l}\stackrel{0}{-} \\
\underset{\sim}{\tilde{\gamma}} \\
\text { f }\end{array}$ & $\mid \begin{array}{l}8 \\
\dot{q} \\
\dot{q}\end{array}$ & $\begin{array}{l}\text { gे } \\
\dot{0} \\
\text { o }\end{array}$ & $\mid \begin{array}{l}\infty \\
\stackrel{\infty}{i} \\
\infty \\
\infty \\
\dot{\infty}\end{array}$ & $\begin{array}{l}r \\
\stackrel{2}{\infty} \\
\infty \\
2 \\
\sigma\end{array}$ & $\begin{array}{l}\stackrel{\circ}{+} \\
\stackrel{+}{n}\end{array}$ & in & $\begin{array}{l}\infty \\
\stackrel{+}{4} \\
n\end{array}$ & 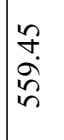 & $\begin{array}{l}7 \\
\underset{D}{+} \\
i n\end{array}$ & $\mid \begin{array}{l}\tilde{b} \\
\infty \\
\infty \\
\infty \\
n\end{array}$ & $\begin{array}{l}2 \\
i \\
i \\
\delta\end{array}$ & 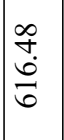 & \\
\hline$\sum_{0}^{\pi}$ & $\begin{array}{l}\infty \\
n \\
0\end{array}$ & 3 & $\underset{\substack{n\\
}}{\stackrel{n}{n}}$ & 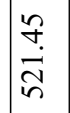 & $\begin{array}{l}n \\
\infty \\
\dot{\infty} \\
\infty \\
\infty\end{array}$ & $\begin{array}{l}m \\
\frac{9}{0} \\
\dot{0}\end{array}$ & $\begin{array}{l}\hat{a} \\
\text { ठे }\end{array}$ & $\mid \begin{array}{l}\infty \\
\stackrel{\mathbb{Z}}{Z}\end{array}$ & 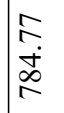 & $\mid \begin{array}{l}\infty \\
m \\
\infty \\
\infty\end{array}$ & $\frac{n}{n}$ & 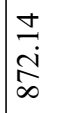 & $\begin{array}{l}\tilde{N} \\
\stackrel{\infty}{\infty} \\
\infty\end{array}$ & 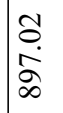 & 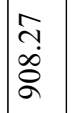 & $\begin{array}{l}\widehat{3} \\
\stackrel{0}{0} \\
\alpha\end{array}$ & $\mid \begin{array}{l}\infty \\
\infty \\
\infty \\
\alpha \\
\alpha \\
\alpha\end{array}$ & $\begin{array}{l}\tilde{\alpha} \\
\infty \\
\infty \\
\tilde{\alpha}\end{array}$ & $\begin{array}{l}0 \\
1 \\
0 \\
n \\
n\end{array}$ & तె & $\begin{array}{l}\overline{0} \\
10 \\
0 \\
\sigma\end{array}$ & $\begin{array}{l}\tilde{\delta} \\
\infty \\
\dot{0} \\
\infty \\
\infty\end{array}$ \\
\hline $\begin{array}{l}0 \\
\mathscr{Z} \\
\tilde{z} \\
\tilde{z}\end{array}$ & in & $\stackrel{\rho}{a}$ & $N$ & $\vec{\infty}$ & $\overrightarrow{ \pm}$ & 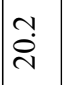 & ֶֶ. & $\begin{array}{l}n \\
\tilde{n}\end{array}$ & $\begin{array}{c}m \\
\infty \\
\infty \\
m\end{array}$ & $\mid \begin{array}{l}+ \\
\dot{J}\end{array}$ & $\begin{array}{l}t \\
\dot{8}\end{array}$ & $\begin{array}{l}n \\
0 \\
i \\
n\end{array}$ & $\mid \begin{array}{l}n \\
i \\
0\end{array}$ & $\begin{array}{l}n \\
\infty \\
\infty \\
0\end{array}$ & $\begin{array}{l}0 \\
\stackrel{+}{T}\end{array}$ & $\begin{array}{l}0 \\
\dot{0} \\
\infty\end{array}$ & $\hat{\infty}$ & $\hat{a}$ & $\left|\begin{array}{l}\infty \\
\infty \\
\infty\end{array}\right|$ & $\begin{array}{l}\infty \\
\dot{0} \\
\stackrel{0}{0}\end{array}$ & $\stackrel{\varrho}{\grave{O}}$ & \\
\hline
\end{tabular}


